TWRS Equipment Engineering

5. Proj./Prog./Dept./Div.:

94C-EWW-369 / Hydrogen

Monitoring / TWRS / N2022

8. Originator Remarks:

The SHMS-E Shop Acceptance Test Report is being routed for approval and release.

11. Receiver Remarks: 11A. Design Baseline Document? [] Yes [X] No

\begin{tabular}{l|r}
\hline 3. From: (Originating Organization) & 4. Related EDT No.: \\
Safety Projects & 600193 \\
\hline
\end{tabular}

6. Design Authority/ Design Agent/Cog. Engr::

TC Schneider / MF Erhart
7. Purchase Order No.:

\section{$\mathrm{MCH}-\mathrm{SBX}-478656$}

9. Equip./Component No.: N/A

10. System/Bldg./Facility: 241 General

12. Major Assm. Dwg. No.: H-14-100837

13. Permit/Permit Application No.: $N / A$

14. Required Response Date: 9-28-97



8D-7400-172-2 (05/96) GEF097 


\title{
STANDARD-E HYDROGEN MONITORING SYSTEM SHOP ACCEPTANCE TEST REPORT
}

\author{
TC Schneider
}

Numatec Hanford Corporation, Richland, WA 99352

U.S. Department of Energy Contract DE-AC06-96RL13200

$\begin{array}{lll}\text { EDT/ECN: } & \text { EDT-600197 } & \text { UC: } 2030 \\ \text { Org Code: } & 8 C 460 & \text { Charge Code: }{ }^{N 2022} \\ \text { B\&R Code: } & \text { EW3120072 } & \text { Tota1 Pages: } 562 w_{10-2-97}\end{array}$

Key Words: Gas Monitoring, Hydrogen Monitoring, Standard Hydrogen Monitoring System, SHMS-E

Abstract: The purpose of this report is to document that the Standard-E Hydrogen Monitoring Systems (SHMS-E), fabricated by Mid-Columbia Engineering (MCE) for installation on the Waste Tank Farms in the Hanford 200 Areas, are constructed as intended by the design. The ATP performance will verify proper system fabrication.

TRADEMARK DISCLAIMER. Reference herein to any specific commercial product, process, or service by trade name, trademark, manufacturer, or otherwise, does not necessarily constitute or imply its endorsement, recommendation, or favoring by the United states Government or any agency thereof or its contractors or subcontractors.

Printed in the United States of America. To obtain copies of this document, contact: WHC/BCS Document Control Services, P.0. BoX 1970, Mailstop H6-08, Richland UA 99352, Phone (509) 372-2420; Fax (509) 376-4989.

STANDARD-E HYOROGEN MONITORING SYSTEM

SHOP ACCEPTANCE TEST REPORT

Rev. 0

APPROVAL DESIGNATOR Q

T.C. Schneider

Mechanical Systems Engineering

Numatec Hanford Corporation, Richland Wa. 99352

U.S. Department of Energy Contract DE-AC06-96RL13200

September, 1997 
TABLE OF CONTENTS



2.0 DESCRIPTION OF TEST ......................... Id

3.0 TEST METHOD AND TEST EqUTPMENT ............... le



5.0 DISPOSITION OF TEST ITEMS ..................... If

6.0 ATTACHMENTS AND APPENDIXES .............. If

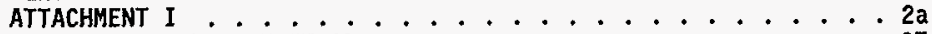

APPENDIX A (UNIT 1) ................... 37

APPENDIX B (UNIT 2) ............... . . . . . 67

APPENDIX C (UNIT 3 ) .................. 97

APPENDIX D (UNIT 4 ) .................. 127

APPENDIX E (UNIT 5 ) . . . . . . . . . . . 157

APPENDIX $F$ (UNIT 6$) \ldots \ldots \ldots . \ldots . \ldots 187$

APPENDIX G (UNIT 7 ) ........................ 217

APPENDIX H (UNIT 8) .............. 247

APPENDIX I (UNIT 9) ................. 277

APPENDIX J (UNIT 10) ............... 307

APPENDIX K (UNIT 11) . . . . . . . . . 337

APPENDIX $L$ (UNIT 12 ) $\ldots \ldots \ldots \ldots$

APPENDIX M (UNIT 13) ............... . . 397

APPENDIX N (UNIT 14) . . . . . . . . . . . 427



APPENDIX P (UNIT 16) . . . . . . . . . . 487

APPENDIX Q (UNIT 17) ................. 517 


\subsection{INTRODUCTION}

\subsection{Background}

Tank Waste Remediation Systems (TWRS) Safety Programs has funded project W-369, Flammable Gas Watch List (FGWL) Tank Hydrogen Monitors, to install Standard Hydrogen Monitoring Systems (SHMS) on all the identified FGWL tanks. Also, additional tanks may require hydrogen monitoring. HNF-SP-1193, Flammab7e Gas Project Topical Report (Johnson et al. 1997) discusses the methodology and criteria requiring additiona] selected waste tank vapor space characterization.

Procurement specification WHC-S-471, Standard-E Hydrogen Monitoring System Cabinet a Gas Characterization and Monitoring System (GCM) (Schneider. Tate 1996) in conjunction with the functional design criteria identified in HNF-SD-WM-FDC-054, Gas Characterization and Monitoring System Functional Design Criteria (Schneider 1997) were used to procure equipment engineering design and equipment fabrication services to support the additional gas monitoring requirements.

\subsection{Scope}

The scope of this test report documents the final hardware acceptance testing performed at the vendor facility to assure equipment construction to approved design.

\subsection{DESCRIPTION OF TEST}

The performance of WHC-SD-WM-ATP-191, Standard-E Hydrogen Monitoring System Shop Acceptance Test Procedure was used to qualify the vendor fabricated SHMS assemblies to the approved system design. The ATP was used to assure that the system equipment components, such as, tubing, valves, filters, instruments, alarm systems and computer systems were properly fabricated into the required systems. The ATP was used to further assure that the system components would perform the required monitoring functions when installed on the selected FGWL waste tanks. This ATP assured the functional capabilities of the equipment.

Following this vendor and subsequent field installation ATPs, the operational calibrations of the process instrumentation will be performed under the guidance of approved maintenance procedures. The operational calibrations of the analytical equipment will be performed under a combination of approved maintenance and vendor procedures using certified standard gases. The SHMS will be operated under the direction of approved operations procedures. 


\subsection{TEST METHOD AND TEST EQUIPMENT}

This test utilized a step by step procedure to assure that the system components were assembled per the approved system design drawings. The test checked the electrical and pneumatic connections to verify functional correctness and operability. Additional equipment testing is documented in the system procurement and certified vendor information files.

The test equipment used to measure the required process parameters was calibrated using standards traceable to the National Institute of Standards and Testing (NIST) standards.

\subsection{TEST RESULTS AND CONCLUSIONS}

All testing was satisfactorily completed. Several test exceptions were prepared to document and track closure of discrepancies discovered during testing. There were three types of test exceptions prepared. Those test exceptions are included in the attached appendixes.

The first type of test exception documented several testing procedural errors. The initial performance of the ATP disclosed several minor errors which were documented by test exceptions and corrected by engineering change notices (ECNs).

The second type of test exception documented fabrication hardware errors. The systems were incorrectly supplied with an undersized air conditioning unit. The test exception was resolved by the vendor supplying the correct size air conditioning units.

The third type of test exception documented equipment labelling issues. The equipment labelling scheme was revised by TWRS following the system design and initial fabrication. Test exceptions were prepared for the individual cabinets to track the correction of equipment labeliing prior to field installation and operation. A QA nonconformance report (NCR) \#54563, QA Log \# TWQA-97-027 was subsequently prepared to track the installation of the revised equipment labelling. The NCR label tracking permitted the test exceptions to be resolved for this test. 


\subsection{DISPOSITION OF TEST ITEMS}

The equipment inspected and tested by WHC-SD-WM-ATP-191 performed as expected and was received by Numatec Hanford Corporation for future installation on selected FGWL waste tanks.

\subsection{ATTACHMENTS AND APPENDIXES}

The completed ATP is included as an attachment to this test report. The individual cabinet test procedures are included in the ATP as appendixes A through $Q$ for complete documentation of the testing activities. 
HNF - SD - WM-ATR - 191

Rev. 0

Page 2a

\section{ATTACHMENT I}

This page intentionally left blank 
STANDARD-E HYDROGEN MONITORING SYSTEM

SHOP ACCEPTANCE TEST PROCEDURE

REV. 0

APPROVAL DESIGNATOR SQ

Issued by

TWRS Safety Programs Engineering Support

October 28, 1996 
1.0 INSTRUCTION SECTION .................. 3

1.1 PURPOSE AND SCOPE . . . . . . . . . ..... 3

1.2 REFERENCES ..................... 3

1.3 RESPONSIBILITIES . . . . . . . . . . . . . . . . . 4

1.4 SYSTEM DESCRIPTION .................. 7

1.5 TEST CONDITIONS AND EQUIPMENT REQUIRED $\ldots \ldots 7 . \ldots 7$

1.6 ACCEPTANCE TEST INSTRUCTIONS $\ldots \ldots . . . . . . . . .8$

1.7 ACCEPTANCE TEST PROCEDURE . . . . . . . . . . . . 11



2.1 TEST DATA SHEETS ......................... 35

2.2 TEST EXCEPTION SHEET . . . . . . . . . . . . 36

2.3 TEST LOG SHEET . . . . . . . . . . . . 36



TEST EXECUTION SHEET . . . . . . . . . . . . 38

PREREQUISITES AND INITIAL TEST CONDITIONS ........... 39

TEST DATA SHEET . . . . . . . . . . . . . . . . . . . . 41

TEST EXCEPTION SHEET ................... 65

TEST LOG. ............................. 66

APPENDIX B . . . . . . . . . . . . . . . . . 67

TEST EXECUTION SHEET .................. 68



TEST DATA SHEET . . . . . . . . . . . . . . . . 71

TEST EXCEPTION SHEET .......................... 95

TEST LOG .......................... 96

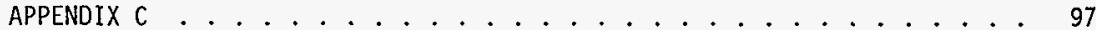

TEST EXECUTION SHEET . . . . . . . . . . . . . . . . . 98

PREREQUISITES AND INITIAL TEST CONDITIONS ........... 99

TEST DATA SHEET ....................... 101

TEST EXCEPTION SHEET . . . . . . . . . . . . . . 125

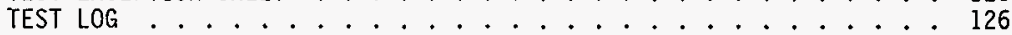



TEST EXECUTION SHEET . . . . . . . . . . . 128

PREREQUISITES AND INITIAL TEST CONDITIONS . . . . . . . . . . 129

TEST DATA SHEET ................... 131

TEST EXCEPTION SHEET . . . . . . . . . . . . . 155



APPENDIX E . . . . . . . . . . . . . . . . . . . 157



PREREQUISITES AND INITIAL TEST CONDITIONS . . . ....... 159

TEST DATA SHEET ................... 161

TEST EXCEPTION SHEET . . . . . . . . . . . . . . 185

TEST LOG ....................... . . . . 186 
APPENDIX F . . . . . . . . . . . . . . . . 187

TEST EXECUTION SHEET . . . . . . . . . . . . . 188

PREREQUISITES AND INITIAL TEST CONDITIONS ................ 189

TEST DATA SHEET . . . . . . . . . . . . . . . . . 191

TEST EXCEPTION SHEET . . . . . . . . . . . . 215



APPENDIX G . . . . . . . . . . . . . 217

TEST EXECUTION SHEET ........................... 218

PREREQUISITES AND INITIAL TEST CONDITIONS .......... 219

TEST DATA SHEET . . . . . . . . . . . . . . 221

TEST EXCEPTION SHEET . . . . . . . . . . . . . . . . . . 245

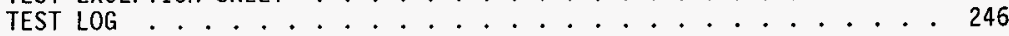

APPENDIX H . . . . . . . . . . . . . . . . . . . 247

TEST EXECUTION SHEET . . . . . . . . . . 248

PREREQUISITES AND INITIAL TEST CONDITIONS ......... 249

TEST DATA SHEET . . . . . . . . . . . . . . . 251

TEST EXCEPTION SHEET . . . . . . . . . . . . . 275

TEST LOG . . . . . . . . . . . . . . . . 276

APPENDIX I . . . . . . . . . . . . . . . 277

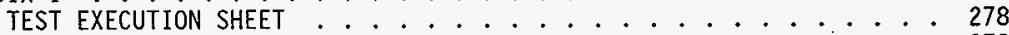

PREREQUISITES AND INITIAL TEST CONDITIONS ......... 279

TEST DATA SHEET ......................... 281

TEST EXCEPTION SHEET . . . . . . . . . . . . . . 305



APPENDIX J . . . . . . . . . . . . . . . . . 307

TEST EXECUTION SHEET $\ldots . \ldots . \ldots 308$

PREREQUISITES AND INITIAL TEST CONDITIONS . . . . . . . 309

TEST DATA SHEET ........................ 311

TEST EXCEPTION SHEET . . . . . . . . . . . . . . . . 335



APPENDIX K . . . . . . . . . . . . . . . . . 337

TEST EXECUTION SHEET . . . . . . . . . . . 338

PREREQUISITES AND INITIAL TEST CONDITIONS ............. 339

TEST DATA SHEET . . . . . . . . . . . . . . 341

TEST EXCEPTION SHEET . . . . . . . . . . . . . . 365



APPENDIX $L \ldots \ldots \ldots$. . . . . . . . . . . . . . . 367

TEST EXECUTION SHEET . . . . . . . . . . . . . 368

PREREQUISITES AND INITIAL TEST CONDITIONS . . . . . . . . . 369

TEST DATA SHEET .......................... 371






APPENDIX M . . . . . . . . . . . . . . . . . . 397

TEST EXECUTION SHEET . . . . . . . . . . . . . . . 398

PREREQUISITES AND INITIAL TEST CONDITIONS . . . . . . . . . . . . . 399

TEST DATA SHEET . . . . . . . . . . . . . . . . 4 401

TEST EXCEPTION SHEET ...................... . . 425

TEST LOG ....................... . . . . . . . . . . . . . . . . .

APPENDIX N . . . . . . . . . . . . . . . . . . . 4 427

TEST EXECUTION SHEET . . . . . . . . . . . . . . . . . . . . 428

PREREQUISITES AND INITIAL TEST CONDITIONS . . . . . . . . . . . 429

TEST DATA SHEET . . . . . . . . . . . . . . . . . . . . . . 431

TEST EXCEPTION SHEET . . . . . . . . . . . . . . . 455

TEST LOG . . . . . . . . . . . . . . . . . . . . . . . 456

APPENDIX 0 . . . . . . . . . . . . . . . . . . . . . 4 457

TEST EXECUTION SHEET

PREREQUISITES AND INITIAL TEST CONDITIONS . . . . . . . . . . . 459

TEST DATA SHEET . . . . . . . . . . . . . . . . . . 461

TEST EXCEPTION SHEET ...................... . 485

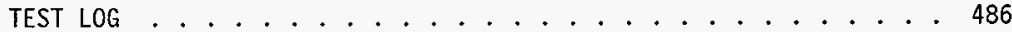

APPENDIX P . . . . . . . . . . . . . . . . . . . . . 4 487

TEST EXECUTION SHEET . . . . . . . . . . . . . . . 488

PREREQUISITES AND INITIAL TEST CONDITIONS . . . . . . . . . 489

TEST DATA SHEET ........................... 491

TEST EXCEPTION SHEET . . . . . . . . . . . . . . . . . . 515

TEST LOG . . . . . . . . . . . . . . . . . . . . . . . . . 516

APPENDIX Q . . . . . . . . . . . . . . . . . . . . 517

TEST EXECUTION SHEET

PREREQUISITES AND INITIAL TEST CONDITIONS . . . . . . . . . . . . 519

TEST DATA SHEET .......................... 521

TEST EXCEPTION SHEET ...................... . . 545






\section{STANDARD-E HYDROGEN MONITORING SYSTEM SHOP ACCEPTANCE TEST PROCEDURE}

\subsection{INSTRUCTION SECTION}

\subsection{PURPOSE AND SCOPE}

The purpose of this document is to demonstrate that the Standard-E Hydrogen Monitoring Systems (SHMS-E) fabricated and provided by Mid Columbia Engineering are constructed as intended by design.

\subsection{REFERENCES}

1.2.1 The following drawings are provided as reference for the test performance.

- H-2-818214 Standard-B Hydrogen Monitoring System Gas Bottle Rack

- H-14-100835, Standard-E Hydrogen Monitoring System Piping \& Instrumentation Diagram

- H-14-100836, Standard-E Hydrogen Monitoring System One-Line, Elementary Diagrams

- H-14-100837, Standard-E Hydrogen Monitoring System Cabinet Assembly

- H-14-100838, Standard-E Hydrogen Monitoring System Wiring Diagram

- H-14-100839, Standard-E Hydrogen Monitoring System Loop Diagrams

- H-14-100840, Standard-E Hydrogen Monitoring System Interior Panels \& Brackets

- H-14-100841, Standard-E Hydrogen Monitoring System Instrument Pane] Assembly

- H-14-100842, Standard-E Hydrogen Monitoring System Cabinet Modifications

- H-14-100843, Standard-E Hydrogen Monitoring System PLC Ladder Diagram

- H-14-100845, Standard-E Hydrogen Monitoring System Sample Pump Stand Assembly 
1.2.2 The listed procedures are provided as reference for the test performance.

- None listed.

\subsection{RESPONSIBILITIES}

Each company or organization participating in the conduct of this ATP will designate personnel to assume the responsibilities and duties as defined herein for their respective roles. The names of these designees shall be provided to the recorder for listing on the recorder's copy of the Test Execution Sheet prior to the performance of any part of this ATP. Each of the SHMS-E units tested at the vendor site will include a separated TEST EXECUTION SHEET and TEST DATA SHEETS to document the testing.

\subsubsection{NHC PROJECT ENGINEER}

* Designate a test director.

* Act as liaison between the participants in acceptance testing.

* Distribute the approved testing schedule as soon as possible, but at least two days prior to testing.

* Ensure preliminary system alignment and inspection has been completed.

* Schedule and conduct a pre-ATP meeting with test participants prior to start of testing.

* Notify the persons performing and witnessing the test prior to the start of testing.

* Notify all concerned parties when a change is made in the testing schedule.

* Sign Test Execution Sheet when ATP is approved and accepted.

* Take necessary action to clear exceptions to the ATP.

* Sign Exception Sheet when exception has been resolved.

* Prepare and issue an Acceptance Test Report for the approved and accepted ATP. 


\subsubsection{TEST DIRECTOR}

* Coordinate all acceptance testing.

* Confirm that the preliminary testing, alignment and inspection of the system or portion of the system to be tested has been completed.

* Stop any test which may cause damage to the system until the test procedure has been revised.

* Approve field changes to the ATP.

* Obtain revisions to the ATP, as necessary, to comply with authorized field changes or to accommodate existing field conditions.

* Evaluate recorded data, discrepancies, and exceptions.

* Obtain from the NHC project engineer, any information or changes necessary to clear or resolve objections.

* Sign Test Execution Sheet when ATP has been performed on each of the systems.

* Sign Test Exception Sheet when retest has been executed and accepted.

* Obtain required signatures on the ATP master prior to reproduction and distribution.

\subsubsection{WITNESSES (Provided by Participating Organizations)}

* Witness the tests.

* Evaluate results of testing.

* Assist the Test Director when requested.

* Sign Test Exception Sheet as a witness.

* Sign Test Exception Sheet as a witness when retest has been executed and accepted. 


\subsubsection{RECORDER}

* Perform all recording duties in black ink.

* Record names of all designated personnel on recorder's copy of ATP prior to start of testing.

* Observe tests, record test data and maintain test log.

* Sign the Test Exception Sheet as the recorder.

* Initial and date every test step on the recorder's copy of the test data sheet. On tables where there is not room for both the initial and date, date may be entered once in space provided at bottom of column if a11 the testing was performed on the same day.

* Record authorized field changes to the ATP.

* Record exceptions and test steps that are not performed on the Test Exception Sheet. Have the information transferred in ink or typed to the Master Exception Sheet $(s)$. Additional Exception Sheets are to be added as needed.

* Orally notify the test director at time the objection is made.

* Assign page number to Data Sheets and Exception Sheets after ATP is complete. Record Page numbers for these items and make corrections, as necessary, to page numbers shown for these pages in the index.

* Transfer the final test results with recorder's signature and dates for each step to the master in ink or type. Submit the completed master to the Test Director for approval signatures and distribution. Retain the recorder's copy and a copy of the master in the field project files.

\subsubsection{FABRICATION CONTRACTOR}

* Organize and perform this acceptance test under coordination of the Test Director.

* Confirm that all equipment required for performing this test (as listed in Section 1.5) will be available at start of testing.

* Provide equipment required for performing this acceptance test, which has not been designated as being provided by others. 
* Request in writing from the project engineer those services, materials, or equipment that have been designated as being supplied by the Department of Energy (DOE) or others.

\subsubsection{OCCUPATIONAL SAFETY AND HEALTH}

Individuals shall carry out their assigned work in a safe manner to protect themselves, others, and the equipment from undue hazards and to prevent damage to property and environment. The fabrication facility manager shall assure the safety of all activities to prevent injury or property damage. Performance of test activities sha?l always include safety and health concerns for the participants. Any hazard identified during the performance of the ATP shall be reported to the Test Director and the facility manager where the test is performed. A daily pretest safety briefing will be held with all test participants and documented in the Test Log.

\subsection{SYSTEM DESCRIPTION}

The primary function of the standard-E hydrogen monitoring system is to monitor specifically for hydrogen in the waste tank atmosphere which may also contain (but not be limited to) unknown quantities of air, nitrous oxide, ammonia, water vapor, carbon dioxide, carbon monoxide, and other gaseous constituents.

The SHMS consists of hydrogen specific monitors, a grab sampler to collect samples for laboratory analysis, a dual column gas chromatograph, a multi-gas analyzer, and the gas sample deljvery and process support system of vaives, filters and tubing necessary to support the operation of the instrumentation. This system will be located in a cabinet placed at the tank of interest. The vapor space will be sampled from a riser probe or from the tank primary ventilation system ducting.

\subsection{TEST CONDITIONS AND EQUIPMENT REQUIRED}

The Contractor shall supply all test equipment unless otherwise noted. The following 1 ist is provided as an aid and is not intended to be all an exhaustive 1 ist. Record pertinent information such as model number, serial number and calibration information as required in the Test Log.

- Multi-meter (AC volts, DC volts, DC current and ohms)

- Assorted hand tools

- Hand held stop watch

- Heat gun 
- Electrical test lead jumper wire

- 12 volt DC 0.5 ampere power supply

- Thermometer

- Trouble light (simulated heat trace load)

- 1 each 1/16" SS Swagelok union

- 7 each 1/16" SS Swagelok cap

- 6 each 1/4" SS Swagelok plugs

- Bottle of high purity nitrogen with regulator adjustable to 80 psig.

- Bottle of high purity helium with regulator adjustable to 80 psig.

- 20 feet of flexible $1 / 4$ inch poly tubing and $1 / 4$ inch Swagelok tube fittings to provide a nitrogen gas source for system tubing verification.

- Bottle and regulator of hydrogen calibration gas. Provided by the buyer.

- Bottle and regulator of methane calibration gas. Provided by the buyer.

- Bottle and regulator of ammonia calibration gas. Provided by the buyer.

\subsection{ACCEPTANCE TEST INSTRUCTIONS}

Acceptance testing is to be conducted in accordance with the steps and requirements specified in this procedure. Any required changes must be authorized in accordance with approved change control procedures.

\subsubsection{MINOR EDITORIAL PROCEDURAL CHANGES}

A minor editorial change is defined as a change which is typographical in nature and does not aiter the technical intent of the procedure. Minor field changes can be made in pen and ink and require the approval of the Test Director and Fabrication Contractor. 


\subsubsection{MAJOR PROCEDURAL CHANGES}

A major procedural change is defined as a change which affects the technical intent of the procedure or affects the system quality or safety. Major procedural changes require written approval of the Project Engineer, Test Director, Fabrication Contractor, and Flour Daniel Northwest (FDN) Quality. Major field changes shall also be recorded as a test exception.

\subsubsection{TEST PERFORMANCE}

The acceptance test procedures detailed in Section 1.7 shall be performed sequentially. The major subsections may be performed out of sequence per the direction of the test director if the intent of the test is not compromised. As required by Section 1.3.4, the recorder will initial and date every test step in the space provided on the recorder's copy of the ATP as each step is completed. Any step that requires verification of data must also be recorded on the Test Data Sheet.

\subsubsection{TEST EXCEPTIONS}

Exceptions to the ATP are sequentially numbered and recorded on individual Exception Sheets. This enables case-by-case resolution, recording, approval, and distribution of each exception.

1.6.4.1 Process a Test Exception Sheet when an exception is identified during testing. An example of a test exception would be when the system, fabricated per the design media, does not perform as expected.

* Number each exception sequentially as it occurs and record it on a Test Exception Sheet.

* Enter name and/or organization of objecting party for each exception.

* Enter a description and a planned action to resolve each exception when such determination is made.

* Provide a log entry of the sequential exception number to identify additional detail as required. 
1.6.4.2 Resolve the Test Exception per the following outlined actions. * Record the action taken to resolve each exception. Action taken may not be the same as $p l a n n e d$ action.

* When the action taken results in an acceptable retest, sign and date the Acceptable Retest Performed section of the Exception Sheet.

* When action taken does not involve an acceptable retest, provide a detailed explanation of why the retest action was not acceptable, and what additional plans are required. The explanation may include why the system should be Accepted-As-Is. The Project Engineer then signs and dates the Exception Resolved section and obtains any other approvals required.

1.6.4.3 Distribute requisite copies of the completed Test Exception Sheets to the client.

\subsubsection{TEST APPROVAL}

Obtain approval of the test performance upon completion of the test procedure. Since the ATP may be performed on several systems, each Test Execution Sheet will stand alone as approval for the system under test. The ATP will be finally approved when all the outstanding tests have been performed and the Acceptance Test Report is prepared. The test will be approved by checking the proper response on the Test Execution Sheet under NHC TEST APPROVAL AND ACCEPTANCE with or without exceptions.

1.6.5.1 The test may be approved without test exceptions .

* Check applicable space on Test Execution Sheet to show that the ATP has been performed and no exceptions have been recorded.

* Sign and date Test Execution Sheet in the spaces provided.

* Distribute requisite copies as directed by the client and send master of ATP to the client.

1.6.5.2 The test may be approved with exceptions resolved.

* Check applicable space on Test Execution Sheet to show that the ATP has been performed with exceptions recorded and resolved.

* Sign and date Test Execution Sheet in the spaces provided.

* Distribute requisite copies as directed by the client and send master of ATP to the client. 
1 6.5.3 The test may be approved with test exceptions outstanding.

* Check applicable space on Test Execution Sheet to show that the ATP has been performed with exceptions recorded, part or all of which are presently outstanding, unresolved.

* Sign and date Test Execution Sheet in the spaces provided.

* Distribute requisite copies as directed by the client and send master of ATP to the client.

\subsection{ACCEPTANCE TEST PROCEDURE}

\subsubsection{ELECTRICAL SYSTEMS}

This procedure section will demonstrate that the electrical devices have been wired and function properly. The process automatic controls and alarms will be simulated and functionally tested following the pneumatic system testing, since they require that the system be operational for a complete test.

\subsubsection{Mains Power}

The following steps verify that the correct main AC power wiring has been properly installed to the appropriate terminals.

1.7.1.1.1 Verify resistance between system ground and cabinet enclosure is less than one (1) ohm.

1.7.1.1.2 ENERGIZE the temporary $208 \mathrm{Vac}, 1$ phase power source to the Standard-E Hydrogen Monitoring System. Refer to the wiring diagram, H-14-100838, for termination information.

1.7.1.1.3 Verify line voltages, $L 1, L 2$, and $N$, at the terminal block.

\section{7 .1 .2 HVAC}

The following steps verify that the heating and air conditioning features of the enclosure have been properly installed and wired.

1.7.1.2.1 ADJUST air conditioner (VTP-AC-*60) temperature control to a value at least $5^{\circ} \mathrm{F}$ above the ambient temperature.

1.7.1.2.2 CLOSE circuit breaker CB-1/2. 
1.7.1.2.3 Verify the air conditioner circulation fan is operating and the heater is oN.

1.7.1.2.4 ADJUST air conditioner temperature control to a value at least $5^{\circ} \mathrm{F}$ below the ambient temperature.

\section{Note: If ambient temperature is below Air Conditioner's minimum setting, a heat gun may be used to heat the $A / C^{\prime} s$ temperature probe.}

1.7.1.2.5 Verify air conditioner heater turns OFF and the compressor turns ON.

1.7.1.2.6. ADJUST Air Conditioner temperature control to $80^{\circ} \mathrm{F}$.

1.7.1.2.7 OPEN circuit breaker CB-1/2.

\subsubsection{Sample Pump} wired.

The following steps verify that the sample pump has been properly

1.7.1.3.1 CLOSE circuit breaker CB-3/4 .

1.7.1.3.2 Verify pump turns $\mathrm{ON}$.

1.7.1.3.3 OPEN circuit breaker CB-3/4.

\subsubsection{Lighting and GFCI}

The following steps verify the proper wiring of the light and GFCI receptacles.

1.7.1.4.I CLOSE circuit breaker CB-6.

1.7.1.4.2 TURN ON the enclosure light via the lamp housing switch and the front panel ENCLOSURE LIGHT switch.

1.7.1.4.3 Verify the enclosure light is $\mathbf{O N}$.

1.7.1.4.4 Verify power is being supplied to the cabinet dual GFCI receptacles and record voltage readings.

1.7.1.4.5 TEST GFCI capability by depressing "TEST" button on each receptacle. Verify zero voltage across receptacle hot and neutral. Reset the GFCI receptacles. 
1.7.1.4.6 TURN OFF the enclosure light via the front pane] ENCLOSURE LIGHT switch.

1.7.1.4.7 OPEN circuit breaker CB-6.

\subsubsection{Instrument Power}

The following steps verify the enclosure instrument power wiring.

1.7.1.5.1 CLOSE circuit breaker CB-7.

1.7.1.5.2 Verify power is being supplied to the isolation transformer ISO XFMER. MEASURE and RECORD the primary and secondary voltages and the secondary neutral to ground. Measure the primary voltage at the feeder breaker and the secondary voltage at the fuse bus.

1.7.1.5.3 CLOSE fused switch FU-1.

1.7.1.5.4 MEASURE and RECORD the VTP-PS-*50 DC output voltage.

\subsubsection{Cabinet Alarm System}

1.7.1.6.1 CLOSE the fused switch FU-9 and FU-10.

1.7.1.6.2 PUSH the ALARM RESET pushbutton VTP-PB-*51 followed by the HORN ACKNOWLEDGE pushbutton VTP-PB- $* 50$. (Due to 1 ack of operating inputs the PLC will indicate existing alarm conditions.)

1.7.1.6.3 PUSH and HOLD the ALARM TEST pushbutton VTP-PB-*52. Verify the following conditions exist:

* Horn VTP-YAH-*50 is ON.

* High Hydrogen Beacon VTP-NAH-*55 is ON.

* Trouble Beacon VTP-XA-*63 is ON.

* A11 front door and internal panel indicating lights EXCEPT the HYDROGEN MONITOR CAL VTP-PBL $-* 54$ and GRAB SAMPLE VTP-PBL $-* 59$ are ON.

1.7.1.6.4 RELEASE the ALARM TEST pushbutton VTP-PB-*52.

1.7.1.6.5 PUSH the ALARM RESET pushbutton VTP-PB-*51 followed by the HORN ACKNOWLEDGE push button VTP-PB-*51. Verify a11 inactive alarms are reset. 
1.7.1.6.6 OPEN fused switches FU-9 and FU-10.

NOTE: THE INDIVIDUAL ALARM FUNCTIONS WILL BE VERIFIED IN THE FOLLOWING SECTIONS WHERE THEY OCCUR WITH THE PROCESS.

\subsubsection{Flow Alarm System}

The following steps verify the flow alarm system wiring and the function of the flow alarm.

1.7.1.7.1 CLOSE fused switches FU-3, FU-9, FU-10 and FU-13.

1.7.1.7.2 PUSH the ALARM RESET pushbution VTP-PB-*51 followed by the HORN ACKNOWLEDGE push button VTP-PB-*51. Verify that the sample low flow alarm light VTP-FAL-*57 on the enclosure door is illuminated. (The reset function of this alarm will be checked with system flow.)

1.7.1.7.3 OPEN VTP-SV-*20 and VTP-SV-*22 to balance the pressure across VTPFIT-*57.

1.7.1.7.4 Visually verify that VTP-FIT-*57 and VTP-FSL -557 are operating.

1.7.1.7.5 MEASURE and RECORD the DC voltage between VTP-MUX-*70 terminals 5+ and 6 -.

NOTE: This step is not applicable for the SHMS-E in NORMAL configuration.

1.7.1.7.6 CLOSE VTP-SV-*20 and VTP-SV $-\star 22$.

1.7.1.7.7 OPEN fused switches FU-3, FU-9, FU-10 and FU-13.

1.7.1.8 Hydrogen Sensors

The following steps verify the hydrogen sensor system wiring.

1.7.1.8.1 CLOSE fused switches FU-2, FU-4, FU-5, FU-9 and FU-10.

1.7.1.8.2 PUSH the ALARM RESET pushbutton VTP-PB-*51 followed by the HORN ACKNOWLEDGE push button VTP-PB-*51. Verify that the high hydrogen beacon VTP-NAK - 55 and the enclosure door high hydrogen 1 amp VTP$\mathrm{NAH}-* 54$ are extinguished. 
1.7.1.8.3 Visually verify instrument VTP-MUX-*70, VTP-NIT-*54, VTP-NIT-*55, VTP-NR-*54 and VTP-YYC-*01 are powered.

NOTE: VTP-NR-*54 is not applicable for the SHMS-E in ANALYTICAL configuration and VTP-MUX-*70 is not applicable for the SHMS-E NORMAL configuration.

1.7.1.8.4 OPEN fused switches FU-2, FU-4, FU-5, FU-9 and FU-10.

\subsubsection{Heat Trace Control}

The following steps verify the operation of the sample gas and calibration and carrier gas heat trace control systems and the associated alarms. Push the HORN ACKNOWLEDGE pushbutton VTP-PB- $* 50$ as necessary to silence the alarms during power up and power down of the instruments.

The sample gas and calibration gas heat trace will not be installed during this test. A simulated heat trace load, a trouble light, wi11 be connected to the heat trace power wires provided in the cast terminal box on the side of the enclosure. The light will provide the heat source to verify that the controller temperature sensing element responds to a change in temperature.

\section{Sample Gas Heat Trace}

1.7.1.9.1 INSTALL a simulated heat trace load for temperature controller VTP-TIC $-* 50$.

1.7.1.9.2 CLOSE circuit breaker CB-5 and fused switches FU-6, FU-7, FU-9 and FU-10 and PUSH the ALARM RESET pushbutton VTP-PB-*51 followed by the HORN ACKNOWLEDGE push button VTP-PB-*5I to clear any alarms.

1.7.1.9.3 Visually verify controllers VTP-TIC-*50 and VTP-TIC-*56 displays are indicating the expected nominal temperature.

1.7.1.9.4 ADJUST the VTP-TIC-*50 and VTP-TIC-*56 setpoint 2 , as necessary, to a value below the indicated temperature and push VTP-PB-*51 to clear the SAMP/CAL GAS TEMP LO front panel a] arm lamp VTP-TAL $-* 50$.

1.7.1.9.5 ADJUST VTP-TIC-*50 temperature set point number 1 to a value at least 20 degrees above ambient temperature as indicated by the controlter.

1.7.1.9.6 ADJUST VTP-TIC-*50 temperature set point number 2 to a value 5 degrees less than set point number 1 . 
1.7.1.9.7 VERIFY that the Horn is active and the cabinet door light VTP-TAL*50 SAMP/CAL GAS TEMP 10 is ON. PUSH the horn acknowledge pushbutton VTP-PB-*51.

1.7.1.9.8 Visually verify operation of heat trace VTP-HT-*50 circuit. The trouble light should be $O N$ and the indicated temperature should increase as the sensing element $(T / C)$ is brought close to the light until the temperature matches the setpoint, when the light is extinguished. Move the $T / C$ away from the 1 ight.

1.7.1.9.9 ADJUST VTP-TIC-*50 temperature set point 2 to 20 degrees below indicated temperature.

1.7.1.9.10 PUSH the ALARM RESET pushbutton VTP-PB-*51 and verify that the cabinet door light VTP-TAL $* 50$ SAMP/CAL GAS TEMP LO is OFF.

1.7.1.9.11 ADJUST VTP-TIC- $* 50$ temperature set point 1 to $120^{\circ} \mathrm{F}$ and set point 2 to $65^{\circ} \mathrm{F}$ (or temporarily below the indicated temperature to clear the alarm lamp VTP-TAL-*50).

1.7.1.9.12 OPEN CB-5 and REMOVE the simulated heat trace load from VTP-TIC*50.

\section{Calibration Gas Heat Trace}

1.7.1.9.13 INSTALL a simulated heat trace load for temperature controller VTP-TIC $-* 56$.

\section{7 .1 .9 .14 CLOSE CB-5.}

1.7.1.9.15 ADJUST VTP-TIC-*56 temperature set point number 1 to a value at least 20 degrees above ambient temperature as indicated by the controller.

1.7.1.9.16 ADJUST VTP-TIC-*56 temperature set point number 2 to a value 5 degrees less than set point number 1 .

1.7.1.9.17 VERIFY that the Horn is active and the cabinet door 1ight VTP-TAL*50 SAMP/CAL GAS TEMP LO is ON. PUSH the HORN ACKNOWLEDGE pushbutton VTP-PB-*51.

1.7.1.9.18 Visually verify operation of heat trace VTP-HT-*56 circuit. The trouble light should be $O N$ and the indicated temperature should increase as the sensing element $(T / C)$ is brought close to the light until the temperature matches the setpoint, when the light is extinguished. Move the $\mathrm{T} / \mathrm{C}$ away from the light. 
1.7.1.9.19 ADJUST VTP-TIC-*56 temperature set point 2 to 20 degrees below indicated temperature.

1.7.1.9.20 PUSH the ALARM RESET pushbutton VTP-PB $* 51$ and verify that the cabinet door light VTP-TAL $* 50$ SAMP/CAL GAS TEMP LO is OFF.

1.7.1.9.21 ADJUST VTP-TIC- $* 56$ temperature set point 1 to $120^{\circ} \mathrm{F}$ and set point 2 to $65^{\circ} \mathrm{F}$ (and RESET VTP-TIC-*50 setpoint 2 to $65^{\circ} \mathrm{F}$ as required).

1.7.1.9.22 OPEN $\mathrm{CB}-5$ and fused switches FU-6 and FU-7.

1.7.1.9.23 REMOVE the simulated heat trace load from VTP-TIC-*56.

\section{Cabinet Temperature}

1.7.1.9.24 CLOSE fused switch FU-8 and visually verify controller VTP-TIS-*62 display is indicating the expected nominal temperature.

1.7.1.9.25 ADJUST VTP-TIS-*62 temperature set point number 1 to a value at least 10 degrees above ambient temperature as indicated by the controller.

1.7.1.9.26 ADJUST VTP-TIS-*62 temperature set point number 2 to a value 10 degrees less than the indicated ambient temperature.

1.7.1.9.27 PUSH the ALARM RESET pushbutton VTP-PB-*51 followed by the HORN ACKNOWLEDGE push button VTP-PB-*51 to clear any alarms. Verify that the enclosure door CABINET TEMP HI/L0 1 amp VTP-TAHL-*62 is extinguished.

1.7.1.9.28 ADJUST VTP-TIS-*62 temperature set point 2 to 10 degrees above indicated temperature.

1.7.1.9.29 VERIFY that the horn is active and the cabinet door light VTPTAHL $* 62$ CABINET TEMP HI/LO is ON. PUSH the HORN ACKNOWLEDGE pushbutton VTP-PB- $* 51$.

1.7.1.9.30 ADJUST VTP-TIS-*62 temperature set point number 2 to a value 10 degrees less than the indicated ambient temperature.

1.7.1.9.31 PUSH the ALARM RESET pushbutton VTP-PB-*51 followed by the HORN ACKNOWLEDGE push button VTP-PB-*51 to clear any alarms. Verify that the enclosure door CABINET TEMP HI/LO 1 amp VTP-TAHL-*62 is extinguished.

1.7.1.9.32 ADJUST VTP-TIS-*62 temperature set point 1 to 10 degrees below indicated temperature. 
1.7.1.9.33 VERIFY that the horn is active and the cabinet door light VTPTAHL-*62 CABINET TEMP HI/LO is ON. PUSH the HORN ACKNOWLEDGE pushbutton VTP-PB-*51.

1.7.1.9.34 ADJUST VTP-TIS-*62 temperature set point 1 to $85^{\circ} \mathrm{F}$ and set point 2 to $60^{\circ} \mathrm{F}$.

1.7.1.9.35 OPEN fused switches FU-8, FU-9 and FU-10.

1.7.1.10 Differential Pressure Transmitter

The following steps verify that VTP-PDIT-*60 instrumentation signals have been properly wired.

1.7.1.10.1 REMOVE the $1 / 8$ inch calibration port plugs from the high and low side ports of VIP-PDIT-*60 to balance the pressure across the instrument.

1.7.1.10.2 CLOSE fused switch FU-14 and visually verify that the pressure transmitter VTP-PDIT-*60 display is operating. RECORD the indicated value on the data sheet.

1.7.1.10.3 MEASURE and RECORD the DC voltage between VTP-MUX-*70 terminals $7+$ and 8 -.

NOTE: This step is not applicable for the SHMS-E in NORMAL configuration.

1.7.1.10.4 REPLACE the $1 / 8$ inch calibration port plugs on the high and low side ports of VTP-PDIT-*60.

1.7.1.10.5 OPEN the fused switch FU-14.

1.7.1.11 Gas Chromatograph System power.

The following steps verify that VTP-MON-*60 has been provided

1.7.1.11.1 CLOSE the fused switch FU-11.

1.7.1.11.2 PUSH the power pushbutton on the front of VTP-MON-*50, and verify that the power indicator is illuminated.

1.7.1.11.3 OPEN the fused switch FU-11 and verify that the power indicator is extinguished. 


\subsubsection{Multi-Gas Analyzer System} power.

The following steps verify that VTP-NIT- $* 52$ has been provided

1.7.1.12.1 CLOSE the fused switch FU-12.

1.7.1.12.2 Turn ON the power switch on VTP-NIT-*52, and verify that the front panel digital display is energized.

1.7.1.12.3 OPEN the fused switch FU-12 and verify that VTP-NIT-*52 is deenergized.

\subsubsection{Personal Computer Systems}

The following steps verify that VTP-PC $-* 60$ and VTP-PC-*70 have been provided power.

1.7.1.13.1 CLOSE CB-6 to energize the dual GFCI receptacles in the bottom of the enclosure.

1.7.1.13.2 Provide power for the two PCs, VTP-PC- *60 and VTP-PC-*70 and VERIFY that both are powered.

1.7.1.13.3 TURN OFF the instrument power switches and OPEN CB-6 to deenergize the systems.

\subsubsection{INTRINSIC SAFETY CIRCUITS}

This procedure section will demonstrate that the intrinsic safety circuits have been wired and labeled properly. It is not in the scope of this procedure to test the intrinsic safety barriers themselves, but to assure that they are installed per code requirements.

1.7.2.1 Verify INTRINSIC SAFETY APPARATUS nameplates are located near intrinsic safety devices.

Intrinsic Safety Devices:

VTP-NE-*54 VTP-EB-*54 VTP-NIT-*54

VTP-NE- *55 VTP-EB-*55 VTP-NIT-*55 
1.7.2.2 Verify intrinsic safety wiring is labeled per NFPA-70 Section 504-80 and ISA RP 12.6.

Intrinsic Safety Wiring: (Wiring between the following devices 7abeled "INTRINSIC SAFETY WIRING")

VTP-EB-*54 / VTP-NE $-* 54$

VTP-EB- $* 55 /$ VTP-NE $-* 55$

\subsubsection{REVIEW}

Check that all steps in the electrical sections 1.7 .1 and 1.7 .2 have been completed, and that the data sheets have been properly signed, dated and commented.

\subsubsection{PNEUMATIC SYSTEMS}

This procedure will demonstrate that all process 1 ines and devices function to the design intent. This section must be performed after the electrical verification since it will use some of the instruments that have been tested.

\subsubsection{Initial Conditions}

The initial conditions will establish the power and pneumatic alignment to perform the pneumatic system verifications.

NOTE: At any time during the breaker and fused switch alignment that an alarm is received, acknowledge the alarm by pushing the HORN ACKNOWLEDGE pushbutton, VTP-PB-*50, on the enclosure front door.

1.7.4.1.1 Verify the following breaker, fused switch line up.

Enclosure Breakers OPEN $\mathrm{CB}-1 / 2, \mathrm{CB}-3 / 4$ and $\mathrm{CB}-5$

Enclosure Breakers CLOSED CB-6 and CB-7

Enclosure Fused SW. OPEN FU-11, FU-12, and FU-15

Enclosure Fused SW. CLOSED FU-1 THRU FU-10, FU-13 and FU-14 
1.7.4.1.2 Verify the following valve line up. All valves listed have a VTPprefix which will be omitted for convenience.

The following listed valves shall be CLOSED:

$\begin{array}{llllll}\text { SV }-* 07 & \text { SV-*08 } & \text { SV-*09 } & \text { SV-*10 } & \text { SV-*12 } & \text { SV-*15 } \\ \text { SV-*18 } & \text { SV-*19 } & \text { SV-*21 } & \text { SV-*23 } & \text { SV-*54 } & \text { SV-*55 } \\ \text { SV }-* 60 & \text { SV-*63 } & \text { SV-*64 } & \text { SV-*65 } & \text { SV-*66 } & \text { SV-*68 } \\ \text { SV-*70 } & \text { SV-*80 } & \text { SV-*81 } & & & \end{array}$

The following listed valves or valve assemblies shall be OPEN:

$\begin{array}{llllll}\text { SV-*03 } & \text { SV }-* 05 & \text { SV }-* 06 & \text { SV-*11 } & \text { SV-*16 } & \text { SV-*17 } \\ \text { SV-*20 } & \text { SV-*22 } & \text { SV -*24 } & \text { SV-*25 } & \text { SV-*61 } & \text { SV-*62 } \\ \text { SV-*67 } & \text { SV-*71 } & \text { FIV-*52 } & \text { FIV-*56 } & \text { FIV-*70 } & \end{array}$

1.7.4.1.3 Disconnect the $1 / 16^{\prime \prime}$ gas lines from the back of VTP-MON-*60 and cap all the lines except the ones labeled SAMPLE IN.

1.7.4.1.4 Disconnect the flexible lines labeled SAMPLE IN and SAMPLE RETURN from the back of VTP-NIT-*52.

1.7.4.1.5 Plug the vent side of filter VTP-FLT-*63 outside the enclosure.

\subsubsection{Main Sample Flow Loop}

This section will establish a nominal main sample flow and verify the correct operation of the system flow and pressure readout components.

1.7.4.2.1 CLOSE CB-3/4 to energize the sample pump VTP-P- $* 50$ and adjust the flow control valve VTP-SV-*24 for a nominal reading on VTP-FIT-*57 of +5 inch $\mathrm{H}_{2} \mathrm{O}$ to establish a nominal 1 CFM main sample flow.

1.7.4.2.2 READ and RECORD the filter differential pressure indicated by VTPPDI-*51. Verify that it is within the expected range.

1.7.4.2.3 READ and RECORD the system pressure indicated by VTP-PI-*53.

Verify that it is within the expected range. 
1.7.4.2.4 PUSH the ALARM RESET pushbutton VTP-PB-*51 and verify that al1 system alarms are reset. There should be no beacons or panel 1 amps 1 ighted and the horn should be silent.

NOTE: If the ambient temperature is outside the process alarm temperature ranges, adjust the temperature alarm setpoints to clear the alarms. Use the TEST LOG as the control mechanism to restore any alarm setpoints.

1.7.4.2.5 CLOSE VTP-SV-*16 and verify that VTP-FIT- $* 57$ reads near $\mathrm{O}$ " $\mathrm{H}_{2} \mathrm{O}$, the sample low flow alarm light VTP-FAL $* 57$ is illuminated and the al arm horn is activated.

1.7.4.2.6 OPEN VTP-SV-* 16 and verify that the horn is silenced and the sample low flow alarm light VTP-FAL-*57 is still illuminated.

1.7.4.2.7 PUSH the ALARM RESET pushbutton VTP-PB $* 51$ and verify that al1 system alarms are reset.

1.7.4.2.8 CLOSE VTP-SV-*25 and verify that VTP-FIT-*57 reads near 0 " $\mathrm{H}_{2} \mathrm{O}$, the sample low flow alarm light VTP-FAL-*57 is illuminated, the alarm horn is activated, trouble beacon VTP-XA-*63 is ON and there is 0 Vac across VTP-YY-*71 terminals 3 and 4.

1.7.4.2.9 OPEN VTP-SV-*25 and verify that the horn is silenced, the sample low flow alarm light VTP-FAL $* 57$ is still illuminated, trouble beacon VTP-XA-*63 is OFF and there is 120 Vac across VTP-YY $-* 71$ terminals 3 and 4 .

1.7.4.2.10 PUSH the ALARM RESET pushbutton VTP-PB-*51 and verify that all system alarms are reset.

1.7.4.2.11 OPEN VTP-SV-*54, VTP-SV-*66 and read the VTP-NE-*54 cell pressure on VTP-PDIT $-* 60$. Verify that it is within the expected range.

1.7.4.2.12 CLOSE VTP-SV-*54 and OPEN VTP-SV-*63 to vent the VTP-PDIT-*60 pressure. Verify that it reads nominaliy $\mathrm{O} \quad \mathrm{H}_{2} \mathrm{O}$.

1.7.4.2.13 CLOSE VTP-SV-*63 and OPEN VTP-SV-*55, and read the VTP-NE-*55 cell pressure on VTP-PDIT-*60. Verify that it is within the expected range.

1.7.4.2.14 CLOSE VTP-SV-*55 and OPEN VTP-SV $-* 64$ to vent the VTP-PDIT-*60 pressure. Verify that it reads nominally $\mathrm{O} " \mathrm{H}_{2} \mathrm{O}$.

1.7.4.2.15 CLOSE VTP-SV-*64 and VTP-SV-*66. 


\subsubsection{Grab Sample Flow Loop}

This section will verify the proper operation of the grab sample flow loop with manual and automatic operation. The automatic grab sample will also provide a functional verification of the hydrogen cells, high hydrogen alarm and remote high hydrogen alarm contacts.

1.7.4.3.1 READ and RECORD the indication on VTP-PDI-*51.

NOTE: It is important to perform the following steps rapidly since the grab sample period is set for nominally 5 minutes.

1.7.4.3.2 PUSH the GRAB SAMPLE pushbutton VTP-PB-*59 on the internal panel door to initiate a manual grab sample and start a stop watch.

1.7.4.3.3 Verify that the GRAB SAMPLE VTP-PBL-*59 and RESET SAMPLER VTP-PBL*58 and VTP-YAL-*58 1 amps are 117 uminated and that there is no flow indicated by VTP-FIV-*52.

1.7.4.3.4 OPEN VTP-SV-* 15 and adjust VTP-FIV-*52 for nominal flow of $10 \mathrm{CFH}$.

1.7.4.3.5 READ and RECORD the indication on VTP-PDI-*51. Verify that it is slightly higher than recorded in step 1.7.4.3.1.

1.7.4.3.6 CLOSE VTP-SV-* 15 and verify that the flow indicated by VTP-FIV-*52 goes to zero.

1.7.4.3.7 OPEN VTP-SV-* 15 and verify that the flow returns.

1.7.4.3.8 Verify, with the stop watch, that the grab sample flow stops after nominally 5 minutes, and the GRAB SAMPLE lamp VTP-PBL-*59 is extinguished.

1.7.4.3.9 PUSH the RESET SAMPLER pushbutton VTP-PB-*58 to RESET SAMPLER 1 amps VTP-PBL $-* 58$ and VTP-YAL- $* 58$.

\subsubsection{4 $\underline{\mathrm{H}}_{2}$ Cell Calibration Loop}

1.7.4.4.1 Connect a regulated bottle of nominally $1 \% \mathrm{H}_{2}$ in balance air or nitrogen to the $\mathrm{H}_{2}$ calibration gas port on the side of the enclosure.

1.7.4.4.2 OPEN the bottle isolation valve and adjust the bottle to provide a low pressure gas source of 10 psig or less.

1.7.4.4.3 OPEN VTP-SV-* 18 and adjust VTP-FIV-*56 for a indicated flow of nominally $2 \mathrm{CFH}$. 
HNF-SD-WM-ATR- 191

Rev. 0

Page 24

1.7.4.4.4 Verify that the following conditions exist:

* The $\mathrm{H}_{2}$ indicating transmitter VTP-NIT-*54 displays an increase in $\mathrm{H}_{2}$ concentration above $0.625 \%$

* The red High $\mathrm{H}_{2}$ beacon VTP-NAH-*55 is 111 uminated

* The enclosure door High $\mathrm{H}_{2} 7 \mathrm{amp}$ VTP-NAH-*54 is 111 uminated

* The GRAB SAMPLE and RESET ${ }^{2}$ SAMPLER 1 amps, VTP-PBL $* 59$, VTP-PBL*58 and VTP-YAL $-* 58$ respectively, are illuminated

* VTP $-F I V-* 52$ indicates flow

* The horn is sounding

* The remote $\mathrm{High} \mathrm{H}_{2}$ contacts between TB2-21 and TB2-22 are OPEN

1.7.4.4.5 PUSH the HORN ACKNOWLEDGE pushbutton VTP-PB-*50 to silence the horn.

1.7.4.4.6 CLOSE VTP-SV-* 18 and verify that VTP-FIV-*56 indicates no flow and the following conditions exist:

* The $\mathrm{H}_{2}$ indicating transmitter VTP-NIT-*54 displays a decrease in $\mathrm{H}_{2}$ concentration below $0.625 \%$

* The red $\mathrm{High} \mathrm{H}_{2}$ beacon VTP-NAH-*55 is extinguished

* The enclosure door High $\mathrm{H}_{3} 1 \mathrm{amp}$ VTP-NAH-*54 is illuminated

* The RESET SAMPLER lamps, VTP-PBL-*58 and VTP-YAL-*58

respectively, are illuminated

* The remote High $\mathrm{H}_{2}$ contacts between $\mathrm{TB} 2-21$ and TB2-22 are CLOSED

1.7.4.4.7 PUSH the RESET SAMPLER pushbutton VTP-PB-*58 and verify that the RESET SAMPLER 1 amps VTP-PBL-*58 and VTP-YAL-*58 are extinguished.

1.7.4.4.8 PUSH the ALARM RESET pushbutton VTP-PB-*51 and verify that the enclosure door High $\mathrm{H}_{2}$ lamp VTP-NAH- $* 54$ is extinguished.

1.7.4.4.9 OPEN VTP-SV-* 19 and adjust VTP-FIV-*56 for a indicated flow of nominally $2 \mathrm{CFH}$.

1.7.4.4.10 Verify that the following conditions exist:

* The $\mathrm{H}_{2}$ indicating transmitter VTP-NIT-*55 displays an increase

in $\mathrm{H}_{2}$ concentration above $0.625 \%$

* The red High $\mathrm{H}_{3}$ beacon VTP-NAH-*55 is illuminated

* The enclosure door High $\mathrm{H}_{2}$ lamp VTP-NAH-*54 is 171 uminated

* The GRAB SAMPLE and RESET ${ }^{2}$ SAMPLER 1 amps, VTP-PBL-*59, VTP-PBL*58 and VTP-YAL-*58 respectively, are illuminated

* VTP-FIV -*52 indicates flow

* The horn is sounding

* The remote High $\mathrm{H}_{2}$ contacts between TB2-21 and TB2-22 are OPEN 
1.7.4.4.11 PUSH the HORN ACKNOWLEDGE pushbutton VTP-PB-*50 to silence the horn.

1.7.4.4.12 CLOSE VTP-SV-* 19 and verify that VTP-FIV-*56 indicates no flow and the following conditions exist:

* The $\mathrm{H}_{2}$ indicating transmitter VTP-NIT-*55 displays a decrease

in $\mathrm{H}_{2}$ concentration below $0.625 \%$

* The red High $\mathrm{H}_{2}$ beacon VTP-NIT-*55 is extinguished

* The enclosure door High $\mathrm{H}_{2}$ lamp VTP-NAH-*54 is illuminated

* The RESET SAMPLER 1amps, VTP-PBL-*58 and VTP-YAL-*58 respectively, are illuminated

* The remote High $\mathrm{H}_{2}$ contacts between TB2-21 and TB2-22 are CLOSED

1.7.4.4.13 PUSH the RESET SAMPLER pushbutton VTP-PB-*58 and verify that the RESET SAMPLER 1 amps VTP-PBL $* 58$ and VTP-YAL $* * 58$ are extinguished.

1.7.4.4.14 PUSH the ALARM RESET pushbutton VTP-PB-*51 and verify that the enclosure door High $\mathrm{H}_{2}$ lamp VTP-NAH-*54 is extinguished.

1.7.4.4.15 CLOSE the cal. gas bottle isolation valve and disconnect it from the cal. gas port on the side of the enclosure.

1.7.4.4.16 PUSH the HYDROGEN MONITOR CAL pushbutton VTP-PB-*54 and verify that the amber trouble light VTP-XA-*63 and enclosure door light VTP-PBL $-* 54$ are 117 uminated and the remote trouble alarm contacts at TB2 23 and 24 are OPEN.

1.7.4.4.17 PUSH again the HYDROGEN MONITOR CAL pushbutton VTP-PB-*54 and verify that the amber trouble light VTP-XA-*63 and enclosure door light VTP-PBL-*54 are extinguished and the remote trouble alarm contacts at TB2 23 and 24 are CLOSED.

1.7.4.5 Gas Chromatograph Instrument Tubing and Cal. Gas Loop

1.7.4.5.1 REMOVE the plug from filter VTP-FLT-*63 on the outside of the enclosure, connect a regulated bottle of pure $\mathrm{N}_{2}$ to that filter and adjust the $\mathrm{N}_{2}$ bottle regulator for a pressure between 3 and 5 psig.

1.7.4.5.2 Verify that there is no gas flow from either the $\mathrm{H}_{2}$ cal. gas port on the side of the enclosure or the 1/16" SAMPLE "A" IN Tine disconnected from VTP-MON-*60.

1.7.4.5.3 REMOVE the cap from the 1/16" COLUMN "A" VENT line at the VTP-MON*60 and verify that gas flows from that line.

1.7.4.5.4 REPLACE the $1 / 16$ " COLUMN "A" VENT line cap. 
1.7.4.5.5 REMOVE the cap from the 1/16" COLUMN "B" VENT 7 ine at the VTP-MON${ }^{*} 60$ and verify that gas flows from that $T$ ine.

1.7.4.5.6 REPLACE the $1 / 16$ " COLUMN "B" VENT line cap.

1.7.4.5.7 REMOVE the cap from the $1 / 16^{\prime \prime}$ SAMPLE VENT 1 ine at the VTP-MON-*60 and verify that gas flows from that line.

1.7.4.5.8 REPLACE the $1 / 16^{*}$ SAMPLE VENT 1 ine cap.

1.7.4.5.9 INSTALL a temporary jumper between VTP-YY-*70 terminals 1 and 2 in order to energize VTP-SOV-*61 and VTP-SOV-*63.

1.7.4.5.10 CLOSE FU-15 to energize VTP-SOV-*61 and VTP-SOV-*63 and verify that gas flows from the $\mathrm{H}_{2}$ cal, gas port on the side of the cabinet and the SAMPLE "A" IN 1ine. (The alternate port may need to be plugged or capped to determine flows due to small line orifices.)

1.7.4.5.11 PLUG the $\mathrm{H}_{2}$ cal. gas port on the side of the cabinet and cap the SAMPLE " $A$ " IN Tine.

1.7.4.5.12 OPEN (slow1y) VTP-SV-*63 and read and record the pressure indicated on VTP-PDIT- $\star 60$. Verify that the pressure in $" \mathrm{H}_{2} \mathrm{O}$ is nominaily equal to the regulated test gas.

1.7.4.5.13 OPEN VTP-SV-*64 and verify that gas flows from the $1 / 16$ " SAMPLE "B" IN line at VTP-MON-*60.

1.7.4.5.14 CLOSE the test gas isolation valve and REMOVE the connection to the VTP-FLT-*63.

1.7.4.5.15 CLOSE VTP-SV-*63 and VTP-SV-*64.

1.7.4.5.16 OPEN FU-15 to deenergize VTP-SOV-*61 and VTP-SOV-*63 and REMOVE the temporary jumper between VTP-YY-*70 terminals 1 and 2 .

1.7.4.5.17 INSTALL a $1 / 16$ " union between the SAMPLE "A" IN and the SAMPLE VENT $T$ ines at VTP-MON-*60.

1.7.4.5.18 OPEN VTP-SV-*60 and verify that there is no flow indicated by VTP$\mathrm{FI}-* 60$.

1.7.4.5.19 CONNECT a temporary $12 \mathrm{VdC}$ power supply to the leads from VTP-SOV*60. Verify that with the solenoid energized that there is still no flow indicated by VTP-FI-*60.

1.7.4.5.20 OPEN VTP-SV-*68 and adjust VTP-SV-*67 for nominal1y $50 \mathrm{ccm}$ as indicated on VTP-FI-*60. 
1.7.4.5.21 TURN OFF the temporary $12 \mathrm{Vdc}$ power supply and verify that the flow indicated on VTP-FI-*60 decreases toward zero.

1.7.4.5.22 CLOSE VTP-SV-*60 and VTP-SV-*68 to isolate the GC main flow loop.

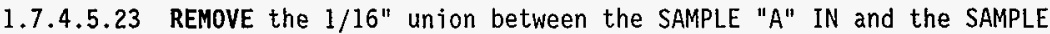
VENT lines at VTP-MON-*60.

1.7.4.5.24 CONNECT a regulated bottle of pure $\mathrm{N}_{2}$ to the $\mathrm{N}_{2} \mathrm{O}$ cal. gas inlet port on the side of the enclosure and adjust the $\mathrm{N}_{2}$ bottle regulator for a pressure between 3 and 5 psig.

1.7.4.5.25 Verify that no gas flows from the SAMPLE "B" IN 7 ine at VTP-MON${ }^{*} 60$ or filter VTP-FLT-*63 on the enclosure side panel.

1.7.4.5.26 INSTALL a temporary jumper between VTP-YY-*70 terminals 3 and 4 in order to energize VTP-SOV-*62 and VTP-SOV-*64.

1.7.4.5.27 CLOSE FU-15 to energize VTP-SOV-*62 and VTP-SOV-*64 and verify that gas flows from the SAMPLE "B" IN 1 ine at VTP-MON-*60 and filter VTP-FLT-*63. (The alternate port may need to be temporarily plugged or capped to determine flows due to small Tine orifices.)

1.7.4.5.28 CLOSE the test gas isolation valve and REMOVE the connection from the $\mathrm{N}_{2} \mathrm{O}$ cal. gas port.

1.7.4.5.29 OPEN FU-15 to deenergize VTP-SOV-*62 and VTP-SOV-*64.

1.7.4.5.30 REMOVE the temporary jumper between VTP-YY-*70 terminals 3 and 4 .

1.7.4.5.31 INSTALL a $1 / 16$ " union between the SAMPLE "B" IN and the SAMPLE VENT 7 ines at VTP-MON-*60.

1.7.4.5.32 OPEN VTP-SV-*60 and verify that there is no flow indicated by VTP$\mathrm{FI}-{ }^{*} 60$.

1.7.4.5.33 TURN ON the temporary $12 \mathrm{Vdc}$ power supply to the leads from VTPSOV $* * 60$. Verify that with the solenoid energized that there is still no flow indicated by VTP-FI-*60.

1.7.4.5.34 OPEN VTP-SV-*68 and adjust VTP-SV-*67 for nominally $50 \mathrm{ccm}$ as indicated on VTP-FI-*60.

1.7.4.5.35 DISCONNECT the temporary $12 \mathrm{Vdc}$ power supply and verify that the flow indicated on VTP-FI-*60 decreases toward zero.

1.7.4.5.36 CLOSE VTP-SV-*60 and VTP-SV-*68 to isolate the GC main flow loop. 
1.7.4.5.37 REMOVE the 1/16" union between the SAMPLE "B" IN and the SAMPLE VENT lines at VTP-MON-*60.

1.7.4.5.38 OPEN CB-3/4 to deenergize the sample pump VTP-P-*50 and ACKNOWLEDGE any process alarms received.

\subsubsection{Multi-Gas Analyzer Instrument Tubing and Cal. Gas Loop}

1.7.4.6.1 VISUALLY VERIFY that the sample delivery tubing for VTP-NIT-*52 from VTP-FLT-*50 to VTP-SOV-*71 and from the tee between VTP-FLT*52 and VTP-FLT-*53 to the VTP-NIT-*52 SAMPLE OUTLET is installed correctly per the P\&ID.

1.7.4.6.2 CONNECT a regulated bottle of pure $\mathrm{N}_{2}$ to the $\mathrm{NH}_{3}$ cal gas inlet port on the side of the enclosure and adjust the $\mathrm{N}_{2}$ bottle regulator for a pressure between 3 and $5 \mathrm{psig.}$

1.7.4.6.3 Verify that no gas flows from the SAMPLE IN 1 ine at VTP-NIT-*52 or filter VTP-FLT-*63.

1.7.4.6.4 INSTALL a temporary jumper between VTP-YY-*70 terminals 5 and 6 in order to energize VTP-SOV-*71 and VTP-SOV-*73.

1.7.4.6.5 CLOSE FU-15 to energize VTP-SOV-*71 and VTP-SOV-*73 and verify that gas flows from the SAMPLE IN 1 ine at VTP-NIT-*52 and from filter VTP-FLT-*63, and the filter flow is indicated by VTP-FIV*70. (The alternate port may need to be temporarily plugged or capped to determine flows due to small line orifices.)

1.7.4.6.6 PLUG the outlet of filter VTP-FLT-*63 on the side of the enclosure and the SAMPLE VENT on VTP-MON-*60.

1.7.4.6.7 OPEN VTP-SV-*66 and VTP-SV-*70, temporarily cap the SAMPLE IN Tine at VTP-NIT-*52 and read and record the pressure displayed on VTPPDIT-*60. Verify that the pressure in " $\mathrm{H}_{2} \mathrm{O}$ is nominally equal to the regulated test gas.

1.7.4.6.8 REMOVE the temporary cap from the SAMPLE IN 1 ine and CLOSE VTP-SV*66 and VTP-SV-*70.

1.7.4.6.9 REMOVE the plug from the outlet of filter VTP-FLT-*63.

1.7.4.6.10 CLOSE the test gas bottle isolation valve and DISCONNECT the 1 ine from the $\mathrm{NH}_{3} \mathrm{Cal}$. gas inlet port on the side of the enclosure.

1.7.4.6.11 CLOSE VTP-SV-*05 and VTP-SV-*16. 
1.7.4.6.12 REMOVE the plug from VTP-SV-*07, CONNECT the reguTated bottle of pure $\mathrm{N}_{2}$ to VTP-SV-*07 and adjust the $\mathrm{N}_{2}$ bottle regulator for.a pressure between 2 and 4 psig.

1.7.4.6.13 OPEN (slowly) VTP-SV-*07 to equalize the system pressure. Verify that no gas flows from the SAMPLE IN or SAMPLE OUT lines at VTPNIT $-* 52$.

1.7.4.6.14 OPEN VTP-SV-*09 and verify that still no gas flows from the SAMPLE IN or SAMPLE OUT lines.

1.7.4.6.15 OPEN FU-15 to deenergize VTP-SOV-*71 and VTP-SOV-*73 and verify that gas flows from the SAMPLE IN but not the SAMPLE OUT 1 ine,

1.7.4.6.16 CLOSE VTP-SV-*09 and verify that no gas exits from the SAMPLE IN line.

1.7.4.6.17 OPEN VTP-SV-*10 and verify that gas flows from the SAMPLE OUT but not the SAMPLE IN line.

1.7.4.6.18 CLOSE the test gas bottle isolation valve, remove the connection from VTP-SV-*07 and replace the plug on VTP-SV-*07.

1.7.4.6.19 CLOSE VTP-SV-*07 and VTP-SV-*10.

1.7.4.6.20 OPEN VTP-SV $-* 05$ and VTP-SV-* 16 .

1.7.4.6.21 REMOVE the temporary jumper between VTP-YY-*70 terminals 5 and 6 .

\subsubsection{Carrier Gas Supply Lines}

1.7.4.7.1 CONNECT a regulated bottle of pure $\mathrm{N}_{2}$ to the $\mathrm{N}_{2}$ carrier gas inlet port on the side of the enclosure and adjust the $\mathrm{N}_{2}$ bottle regulator for a pressure between 3 and 5 psig.

1.7.4.7.2 REMOVE the plug from the $1 / 16^{\prime \prime}$ tubing labeTed $N_{2}$ CARRIER at VTPMON-*60 and VERIFY that gas flows from the tube.

1.7.4.7.3 CLOSE the test bottle isolation valve and DISCONNECT it from the $\mathrm{N}_{2}$ carrier gas inlet port on the side of the enclosure.

1.7.4.7.4 CONNECT a regulated bottle of pure $\mathrm{N}_{2}$ to the He carrier gas inlet port on the side of the enclosure and adjust the $\mathrm{N}_{2}$ bottle regulator for a pressure between 3 and $5 \mathrm{psig.}$

1.7.4.7.5 REMOVE the plug from the $1 / 16^{\prime \prime}$ tubing labeled He CARRIER at VTPMON-*60 and VERIFY that gas flows from the tube. 


\subsection{CLOSE the test bottle isolation valve and DISCONNECT it from the} He carrier gas inlet port on the side of the enclosure.

\subsubsection{REVIEW}

Check that all steps in the pneumatic sections 1.7.4.1 through 1.7.4.6 have been completed, and that the data sheets have been properly signed, dated and commented. Reset any temperature alarm setpoints that were temporarily reset in Section 1.7.4.2 to clear system alarms.

\subsubsection{FUNCTIONAL TESTS}

This section of the procedure will only be applicable for the SHMS-E in the ANALYTICAL configuration. This section will install the "Gas Chromatograph and its computer, and the Multi-Gas Analyzer and its computer. The procedure will demonstrate the instrument's ability to perform normal system sampling activities and detect calibration gases provided through the calibration ports. This test will only verify the equipment operation with commercial analytical software. This section must be performed after the electrical and pneumatic verifications since it will use those instruments and systems.

\subsubsection{Initial Conditions}

The initial conditions will establish the power and pneumatic alignment to perform the analytical system functional verifications.

NOTE: At any time during the breaker and fused switch alignment that an al arm is received, acknowledge the alarm by pushing the HORN ACKNOWLEDGE pushbutton, VTP-PB- $* 50$, on the enclosure front door.

1.7.6.1.1 Verify the following breaker, fused switch line up.

Enclosure Breakers OPEN

Enclosure Breakers CLOSED

Enclosure Fused SW. OPEN

Enclosure Fused SW. CLOSED
$\mathrm{CB}-1 / 2, \mathrm{CB}-3 / 4$ and $\mathrm{CB}-5$

CB- 6 and $C B-7$

FU-11, FU-12, and FU-15

FU-1 THRU FU-10, FU-13 and FU-14 
1.7.6.1.2 Verify the following valve line up. All valves 7 isted have a VTPprefix which will be omitted for convenience.

The following listed valves shall be CLOSED:

$\begin{array}{llllll}S V-* 07 & S V-* 08 & S V-* 09 & S V-* 10 & S V-* 12 & S V-* 15 \\ S V-* 18 & S V-* 19 & S V-* 21 & S V-* 23 & S V-* 54 & S V-* 55 \\ S V-* 60 & S V-* 63 & S V-* 64 & S V-* 65 & S V-* 66 & S V-* 68 \\ S V-* 70 & S V-* 80 & S V-* 81 & & & \end{array}$

The following listed valves or valve assemblies shall be OPEN:

$\begin{array}{llllll}\text { SV }-* 03 & \text { SV-*05 } & \text { SV-*06 } & \text { SV-*11 } & \text { SV-*16 } & \text { SV-*17 } \\ \text { SV }-* 20 & \text { SV }-* 22 & \text { SV } * 24 & \text { SV-*25 } & \text { SV-*61 } & \text { SV-*62 } \\ \text { SV-*67 } & \text { SV-*71 } & \text { FIV-*52 } & \text { FIV-*56 } & \text { FIV-*70 } & .\end{array}$

1.7.6.1.3 Verify the installation of the gas chromatograph, VTP-MON-*60 per the following:

* $1 / 16^{\prime \prime}$ gas lines connected to the back of VTP-MON-*60

* A bottie of ultra-pure $N_{2}$ with a regulator set to 80 psig connected to the $\mathrm{N}_{2}$ carrier gas port on the side of the enciosure. * A bottle of ultra-pure He with a regulator set to 80 psig connected to the He carrier gas port on the side of the enclosure. * Power supply cord connected to VTP-MON-*60

* Solenoid VTP-SOV-*60 wiring connected to the back of VTP-MON$* 60$

* Computer RS-232 interface cable connected between VTP-PC-*60 and VTP-MON-*60

* Computer VTP-PC-*60 installed and plugged into the GFCI receptacle

1.7.6.1.4 Verify the installation of the Multi-Gas Analyzer, VTP-NIT-*52 per the following:

* $4 \mathrm{~mm}$ flexible lines labeled SAMPLE IN and SAMPLE RETURN connected to the back of VTP-NIT- $* 52$

* Power cord connected to VTP-NIT-*52

* Computer VTP-PC-*70 installed and plugged into the GFCI receptacle

1.7.6.1.5 Connect a low pressure $(5 \mathrm{psig}) \mathrm{H}_{2} \mathrm{cal}$. gas source to the enclosure cal. gas port.

1.7.6.1.6 Connect a low pressure (5 psig) $\mathrm{CH}_{4} \mathrm{Cal}$. gas source to the enclosure $\mathrm{N}_{2} \mathrm{O}$ cal. gas port. 
1.7.6.1.7 Connect a low pressure (5 psig) $\mathrm{NH}_{3} \mathrm{cal}$. gas source to the enclosure cal. gas port.

\subsubsection{Establish Main and Auxiliary Loop Flows}

1.7.6.2.1 CLOSE FU-11 and PUSH the power button on VTP-MON-*60. Verify the instrument is powered.

1.7.6.2.2 POWER VTP-PC-*60 and establish communications and a sampling routine per the Test Director's guidance.

1.7.6.2.3 CLOSE FU-12 and PUSH the power button on VTP-NIT-*52. Verify the instrument is powered.

1.7.6.2.4 POWER VTP-PC-*70 and verify that the system can be booted up.

1.7.6.2.5 CLOSE CB-3/4 to energize the sample pump VTP-P-*50 and adjust the flow control valve VTP-SV-*24 for a nominal reading on VTP-FIT-*57 of +1 inch $\mathrm{H}_{2} \mathrm{O}$ to establish a low main sample flow.

1.7.6.2.6 OPEN VTP-SV-*09 and VTP-SV-*10 to establish a Multi-Gas Analyzer flow path.

1.7.6.2.7 OPEN VTP-SV-*60, VTP-SV-*63, VTP-SV-*65 and VTP-SV-*68 to establish a GC loop flow path.

1.7.6.2.8 Establish a sampling routine for VTP-NIT-*52 per the Test Director's guidance.

1.7.6.2.9 OPEN (slowly) VTP-SV-*24 to increase the main sample flow to nominally 0.5 CFM with a reading of $+3 " \mathrm{H}_{2} \mathrm{O}$ on VTP-FIT-*57.

1.7.6.2.10 ADJUST VTP-SV-*67 for a flow reading on VTP-FI-*60 of 10 to 30 $\mathrm{ccm}$.

1.7.6.2.11 READ and RECORD the GC differentia] pressure indicated on VTPPDIT-*60. Verify that the pressure is less than $175{ }^{\prime \prime} \mathrm{H}_{2} \mathrm{O}$.

1.7.6.2.12 OPEN VTP-SV-*64 and CLOSE VTP-SV-*63.

1.7.6.2.13 READ and RECORD the GC differential pressure indicated on VTPPDIT-*60. Verify that the pressure is less than $175 \mathrm{HH}_{2} \mathrm{O}$.

1.7.6.2.14 CLOSE VTP-SV-*65 and OPEN VTP-SV-*66.

1.7.6.2.15 READ and RECORD the GC inlet "B" pressure indicated on VTP-PDIT*60. This pressure will be used to establish $\mathrm{CH}_{4}$ calibration gas flow. 
1.7.6.2.16 OPEN VTP-SV-*63 and CLOSE VTP-SV-*64.

1.7.6.2.17 READ and RECORD the GC inlet "A" pressure indicated on VTP-PDIT*60. This pressure will be used to establish $\mathrm{H}_{2}$ calibration gas flow.

1.7.6.2.18 INSTALL a jumper between VTP-YY-*70 terminats 1 and 2 to provide $\mathrm{H}_{2}$ calibration gas.

1.7.6.2.19 CLOSE FU-15 to energize VTP-SOV-*61 and VTP-SOV-*63.

1.7.6.2.20 ADJUST VTP-SV-*61 to provide the GC inlet "A" pressure indication to within $5 \mathrm{MH}_{2} \mathrm{O}$ of the reading recorded in step 1.7.6.2.15.

1.7.6.2.21 CALIBRATE the channel "A" column per the Test Directors guidance and obtain and record three successive measured concentration values. Also record the cal. gas concentration.

1.7.6.2.22 OPEN FU-15 to deenergize VTP-SOV-*61 and VTP-SOV-*63.

1.7.6.2.23 REMOVE the jumper from between VTP-YY-*70 terminals 1 and 2 .

1.7.6.2.24 OPEN VTP-SV-*64 and CLOSE VTP-SV-*63.

1.7.6.2.25 INSTALL a jumper between VTP-YY $-* 70$ terminals 3 and 4 to provide $\mathrm{CH}_{4}$ calibration gas.

1.7.6.2.26 CLOSE FU-15 to energize VTP-SOV-*62 and VTP-SOV-*64.

1.7.6.2.27 ADJUST VTP-SV-*62 to provide the GC inlet "B" pressure indication to within $5 \mathrm{H}_{2} \mathrm{O}$ of the reading recorded in step 1.7.6.2.15.

1.7.6.2.28 CALIBRATE the channe] "B" column per the Test Directors guidance and OBTAIN and RECORD three successive measured concentration values. Also record the cal. gas concentration.

1.7.6.2.29 OPEN FU-15 to deenergize VTP-SOV-*62 and VTP-SOV-*64.

1.7.6.2.30 REMOVE the jumper from between VTP-YY-*70 terminals 3 and 4 .

1.7.6.2.31 CLOSE VTP-SV-*64 and OPEN VTP-SV-*70.

1.7.6.2.32 MEASURE and RECORD the VTP-NIT-*52 in 1 et pressure on VTP-PDIT-*60 during a sample.

1.7.6.2.33 INSTALL a jumper between VTP-YY-*70 terminals 5 and 6 to provide $\mathrm{NH}_{3}$ calibration gas.

1.7.6.2.34 CLOSE FU-15 to energize VTP-SOV-*71 and VTP-SOV-*73. 
1.7.6.2.35 ESTABLISH a cal. gas bypass flow of nominally $1.5 \mathrm{cfh}$ as indicated on VTP-FIV-*70.

1.7.6.2.36 ADJUST VTP-SV-*71 to provide the same VTP-NIT-*52 sample inlet pressure \pm 5 " $\mathrm{H}_{2} \mathrm{O}$ as measured during a sample in step 1.7.6.2.32.

1.7.6.2.37 OBTAIN and RECORD three successive measured concentration values from VTP-NIT-*52.

1.7.6.2.38 OPEN FU-15 to deenergize VTP-SOV-*71 and VTP-SOV-*73.

1.7.6.2.39 REMOVE the jumper between VTP-YY-*70 terminals 5 and 6 to provide $\mathrm{NH}_{3}$ calibration gas.

\subsubsection{SYSTEM SHUTDOWN}

The following section will secure the SHMS following the ATP performance. It is assumed that the system configuration is the condition following Section 1.7.6.

1.7.7.1 REMOVE the $\mathrm{H}_{2}$ cal. gas source from the enclosure cal. gas port.

1.7.7.2 REMOVE the $\mathrm{CH}_{4}$ cal. gas source from the enclosure $\mathrm{N}_{2} \mathrm{O}$ cal. gas port.

1.7.7.3 REMOVE the $\mathrm{NH}_{3}$ cal. gas source from the enclosure cal. gas port.

1.7.7.4 SECURE and TURN OFF all the analytical instruments and computer systems.

1.7.7.5 CLOSE the $\mathrm{N}_{2}$ carrier gas bottle isolation valve and disconnect and cap the $\mathrm{N}_{2}$ carrier gas 1 ine at the enclosure.

1.7.7.6 CLOSE the He carrier gas bottle isolation valve and disconnect and cap the He carrier gas line at the enclosure.

1.7.7.7 OPEN all the system circuit breakers and fused switches.

1.7.7.8 CLOSE all the system valves with the exception of the following flow control valves:

VTP-FIV-*52 VTP-FIV-*56 VTP-FIV-*70 VTP-SV-*24

VTP-SV-*61 VTP-SV-*62 VTP-SV-*67 VTP-SV-*71

1.7.7.9 DISCONNECT the sample pump from the SHMS enclosure.

1.7.7.10 DEENERGIZE and DISCONNECT the power source to the SHMS under test. 


\subsection{TEST EXECUTION}

The test execution section will establish Appendixes to contain the TEST EXECUTION SHEET, the TEST INITIAL CONDITIONS check sheet, all TEST DATA SHEETS, all TEST EXCEPTION SHEETS and a 11 the TEST LOG SHEETS for the individual tests performed. The TEST EXCEPTION SHEETS and TEST LOG SHEETS may be numbered with alpha suffixes for sequential pages. Since there are two configurations of the SHMS-E, NORMAL and ANALYTICAL, some of the test steps or sections will not be applicable to the system being tested. The test steps within the sections that are not applicable will be annotated with N/A and signed and dated.

\subsection{TEST DATA SHEETS}

The Test Data Sheets are used to document any procedure step requiring verification. A description of the data sheet format follows.

1. Date--Record the date the test is performed.

2. Title of Test--There are two tests being performed by this procedure, the Electrical Systems test and the Pneumatic Systems test.

3. SHMS Unit Number--Record the unit numbers for the Standard-E Hydrogen Monitoring System, e.g. VTP-PNL-*05N and the fabrication control serial number.

4. Test Equipment and $S / N$ or $\mathrm{Cal}$. No.--Record the serial numbers and calibration control number of any device used during the tests.

5. Test Performed By--Print the name of the craftsman performing the test.

6. Procedure Number--This column contains the test steps requiring verification.

7. Item--This column contains the item or function being verified, e.g., Pump, Air Conditioner, Heater, etc.

8. Value--This column contains the quantitative or qualitative measure of the item being verified, i.e. a line voltage may have a value of $120 \mathrm{~V}$, whereas a pump may have a value of ON or OFF.

9. Required Condition--This column indicates the anticipated value of the item being measured.

10. Accept/Reject--Indicate whether the value obtained is acceptable in comparison with the Range.

11. Comment--If the value is rejected, give a justification for denial. 


\subsection{TEST EXCEPTION SHEET}

Exception Data Sheets are used to document exceptions to the test procedure. Actions taken regarding disposition are noted on the exception sheet. Typical dispositions are:

1. Test approved with exception (i.e. rerun of the acceptance test unnecessary).

2. Entire acceptance test to be repeated after the discrepancy has been corrected.

3. Acceptance Test Procedure step(s) affected to be repeated after the discrepancy has been corrected.

\subsection{TEST LOG SHEET}

Test Log Sheets are used to document test start and stop times and to document any other notes concerning the execution of the Acceptance Test Procedure. 
HNF-SD - WM-ATR- 191

Rev, 0

Page 37

\section{APPENDIX A}


APPENDIX A

PAGE $A-3$

Rev, 0

Page 39

SHMS Unit Number:

\section{PREREQUISITES AND INITIAL TEST CONDITIONS}

The following conditions shall exist at the start of the acceptance testing. Initial and date to verify that each of the following items have been accomplished.

m.p.s Systems being tested have been inspected for workmanship and for iz.n-96. $y \cdot 5 \cdot 96$ compliance with design.

m.s m. Continuity and megger tests have been performed on portions of the $12-12-96 \frac{21 /-56}{116}$ electrical and instrument systems being tested, as required.

mas Leak tests on the pneumatic systems have been performed.

$4 \pi, 21.3$

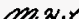
The following circuit breakers and fuses are installed per the

$11 \cdot 5 \cdot 96$ specified size and are open

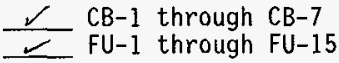

$92 \times .3$

$12+17-9 !$

M. 2NB

All test instruments have a currently valid calibration stamp attached that indicates a calibration traceable to the National Institute of Standards and Technology.

mans The following process and control instrument systems have been

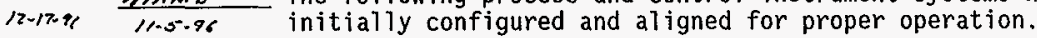

FIT-*57, FSL-*57, NIT-*54, NIT $-* 55,(N R-* 54)$, PDIT-*60, TIC $-* 50$, TIC $-* 56$, TIS $-* 62$, YYC $-* 01$ NOTE: NR-*54 is not used in the ANALYTICAL configuration.

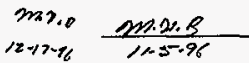

Personnel responsible for directing and witnessing the performance of the tests described in this ATP have read and understand appropriate certified vendor information (CVI) pertaining to the operation of the equipment to be tested.

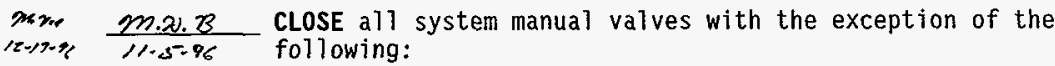

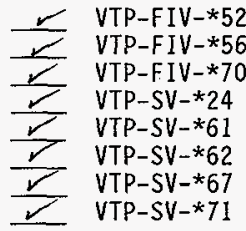


APPENDIX A

m.Ms $20.21 ; 208$ Vac 1 phase 15-20 ampere temporary power source has been $11-5.96$ connected, but not energized, to the appropriate TB1 terminals per H-14-100838.

Verify by signature and date that all prerequisites have been met.

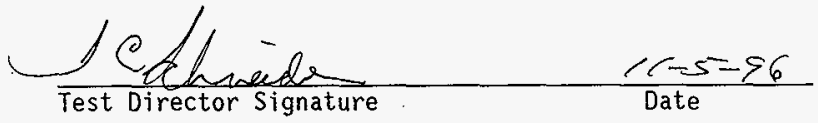


TEST DATA SHEET

\begin{tabular}{|c|c|c|c|c|c|}
\hline \multicolumn{3}{|c|}{ Date: $\quad 1 /-5.96$} & \multicolumn{3}{|c|}{ SHMS Unit Number: VTP-PNC-10SA } \\
\hline \multicolumn{3}{|c|}{$\begin{array}{l}\text { Title of Test: } \\
\text { Electrical Systems }\end{array}$} & \multirow{2}{*}{\multicolumn{3}{|c|}{$\begin{array}{l}\text { Test Equipment and } S / N \text { or Cal. No.: } \\
\text { Fluke volt / ohm meter } \\
\text { s/w } 44620364 \\
\text { model } 77 \\
\text { Cal dede date } 10.97\end{array}$}} \\
\hline \multicolumn{3}{|c|}{$\begin{array}{c}\text { Test Perforned By: See Test Persannal } \\
\text { Paye } 38\end{array}$} & & & \\
\hline $\begin{array}{l}\text { Procedure No. } \\
\text { Initial/Date }\end{array}$ & Item & value & $\begin{array}{c}\text { Requi red } \\
\text { Condition }\end{array}$ & $(A / R)$ & comment \\
\hline \multicolumn{6}{|c|}{ Section 1.7 .1 .1 verifies the systern Mains Power. } \\
\hline $\begin{array}{l}1.7 .1 .1 .1 \\
M . \beta\end{array}$ & Resistance & 0.1 & $<1$ ohm & $A$ & \\
\hline $\begin{array}{l}1.7 .1 .1 .2 \\
m . x_{3}\end{array}$ & $\begin{array}{l}\text { Temp. power } \\
208 \text { Vac } \\
\end{array}$ & N/A & Energized & A & \\
\hline $\begin{array}{l}1.7 .1 .1 .3 \\
m .2 m B \\
\end{array}$ & $\begin{array}{l}\text { Line Vac: } \\
\text { L1-L2 } \\
\text { LI-Gnd } \\
\text { L2-Gnd } \\
N-G \text { and }\end{array}$ & $\frac{\frac{212.3 \mathrm{Vac}}{120.4 \mathrm{~V}_{a c}}}{\frac{121.5 \mathrm{~V}_{\mathrm{rec}}}{0}}$ & $\begin{array}{l}\text { L1-L2: } 208 \\
\operatorname{Vac}+10 /-1 \% \\
\mathrm{~L} 1, \mathrm{~L} 2: 120 \\
\operatorname{Vac} \pm 5 \% \\
\mathrm{~N}: 0 \mathrm{VaC} \\
\end{array}$ & $A$ & . \\
\hline \multicolumn{6}{|c|}{ Section 1.7.1.2 verifies the enciosure HVAC system. } \\
\hline $\begin{array}{l}1.7 .1 .2 .1 \\
m .3 B \\
\end{array}$ & AC Adjusted & $N / A$ & N/A & A & \\
\hline $\begin{array}{l}1.7 .1 .2 .2 \\
\max B\end{array}$ & $\begin{array}{l}\text { CLOSE } \\
\text { CB-1/2 } \\
\end{array}$ & N/A & $N / A$ & A & \\
\hline $\begin{array}{l}1.7 .1 .2 .3 \\
2 x .0 \\
\end{array}$ & Heater ON & $O N$ & Heater ON & A & \\
\hline $\begin{array}{l}1.7 .1 .2 .4 \\
\operatorname{mix}_{x}\end{array}$ & AC Adjusted & N/A & $N / A$ & A & \\
\hline $\begin{array}{l}1.7 .1 .2 .5 \\
\text { gmaxis } \\
\end{array}$ & $\begin{array}{l}\text { Heater 0FF } \\
\text { Cooling ON }\end{array}$ & $\frac{O K}{O N}$ & $\begin{array}{l}\text { Heater OFF } \\
\text { Cooling ON } \\
\end{array}$ & A mat & $\begin{array}{l}\text { Wronge unit okming } \\
\text { tee Tert excostapion? }\end{array}$ \\
\hline 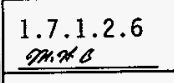 & AC Adjusted & N/A & $N / A$ & A & \\
\hline $\begin{array}{l}1.7 .1 .2 .7 \\
\text { min.p.8 }\end{array}$ & $\begin{array}{l}\text { OPEN } \\
\mathrm{CB}-1 / 2\end{array}$ & $N / A$ & $N / A$ & A & \\
\hline Section 1.7 & 3 verifies & sample pur & np is properly & connec & ed. \\
\hline $\begin{array}{l}1.7 .1 .3 .1 \\
m . n \pi \\
\end{array}$ & $\begin{array}{l}\text { CLOSE } \\
\mathrm{CB}-3 / 4 \\
\end{array}$ & $N / A$ & $N / A$ & A & \\
\hline 1.7.1.3.3.2 & $\begin{array}{l}\text { VTP-P-*50 } \\
\text { ON. }\end{array}$ & - & $\begin{array}{l}\text { VTP-P }-* 50 \\
\text { ON }\end{array}$ & A & \\
\hline
\end{tabular}


TEST DATA SHEET

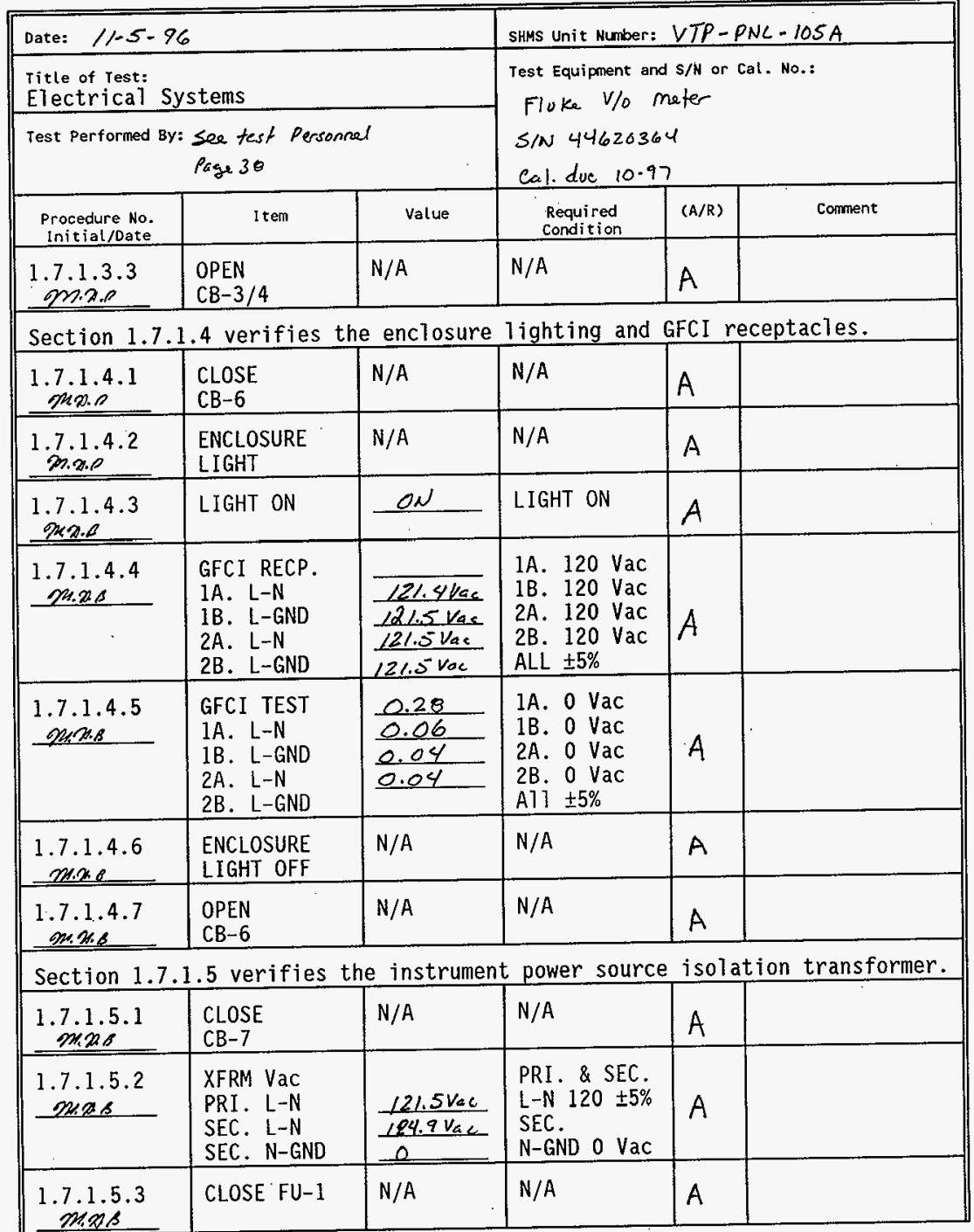


TEST DATA SHEET

\begin{tabular}{|c|c|c|c|c|c|}
\hline \multicolumn{3}{|c|}{ Date: $11-5 \cdot 96$} & \multicolumn{3}{|c|}{ SHMS Unit Number: VTP - PNL $-105 \mathrm{~A}$} \\
\hline \multicolumn{3}{|c|}{$\begin{array}{l}\text { Titte of Test: } \\
\text { Electrical Systems }\end{array}$} & \multirow{2}{*}{\multicolumn{3}{|c|}{$\begin{array}{l}\text { Test Equipment and } S / \mathrm{N} \text { or Cal. No.: } \\
\text { Fluke V/o meter } \\
\text { S/N } 44620364 \\
\text { Cal. due dote } 10.97\end{array}$}} \\
\hline \multicolumn{3}{|c|}{$\begin{aligned} & \text { Test Performed By: } \text { See test Personnel } \\
& \text { Page } 30\end{aligned}$} & & & \\
\hline $\begin{array}{l}\text { Procedure No. } \\
\text { Initial/Date }\end{array}$ & Item & Value & $\begin{array}{l}\text { Required } \\
\text { Condition }\end{array}$ & $(A / R)$ & Comment \\
\hline $\begin{array}{l}1.7 .1 .5 .4 \\
\text { gn.2.B. }\end{array}$ & $\begin{array}{l}\text { VTP-PS } * 50 \\
\text { OUTPUT Vdc }\end{array}$ & 24.00 & $24 \pm 0.1 \mathrm{Vdc}$ & $A$ & \\
\hline \multicolumn{6}{|c|}{ Section 1.7.1.6 verifies the enclosure general al arm system. } \\
\hline $\begin{array}{l}1.7 .1 .6 .1 \\
m .2 .8 .1 \\
\end{array}$ & $\begin{array}{l}\text { CLOSE FU-9 \& } \\
\text { FU-10 }\end{array}$ & $N / A$ & $N / A$ & A & \\
\hline $\begin{array}{l}1.7 .1 .6 .2 \\
\text { m.n.e. }\end{array}$ & $\begin{array}{l}\text { PUSH PB-*51 } \\
\& \text { PB-*50 }\end{array}$ & $N / A$ & $N / A$ & $A$ & \\
\hline 1.7 .1 .6 .3 & $\begin{array}{l}\text { ALARM TEST } \\
\text { VERIFY ALARM } \\
\text { CONDITIONS }\end{array}$ & $\begin{array}{l}o k \\
o k \\
O k \\
o k \\
o k \\
o k \\
O K \\
O K \\
O K \\
O K \\
O K \\
\end{array}$ & $\begin{array}{ll}\text { YAH-*50 } & \text { ON } \\
\text { NAH-*55 } & \text { ON } \\
\text { XA-*63 } & \text { ON } \\
\text { NAH-*54 } & \text { ON } \\
\text { FAL-*57 } & \text { ON } \\
\text { TAHL-*62 ON } \\
\text { TAL-*50 ON } \\
\text { YAL-*58 } & \text { ON } \\
\text { PBL-*58 } & \text { ON } \\
\text { PBL-*54 OFF } \\
\text { PBL-*59 } & \text { OFF } \\
\end{array}$ & A & \\
\hline $\begin{array}{l}1.7 .1 .6 .4 \\
\text { m.x.s. }\end{array}$ & $\begin{array}{l}\text { END ALARM } \\
\text { TEST }\end{array}$ & $N / A$ & $N / A$ & A & \\
\hline 1.7 .1 .6 .5 & RESET ALARMS & $N / A$ & N/A & A & \\
\hline $\begin{array}{l}1.7 .1 .6 .6 \\
m_{0.0}\end{array}$ & $\begin{array}{l}\text { OPEN FU-9 \& } \\
\text { FU-10 }\end{array}$ & $N / A$ & N/A & A & \\
\hline \multicolumn{6}{|c|}{ Section 1.7.1.7 verifies the flow alarm system. } \\
\hline$\frac{1.7 .1 .7 .1}{m \operatorname{mos}_{8}}$ & $\begin{array}{l}\text { CLOSE FU-3, } \\
\text { FU-9, FU-10 } \\
\& \text { FU-13 }\end{array}$ & N/A & $N / A$ & A & \\
\hline $\begin{array}{l}1.7 .1 .7 .2 \\
0.0 .0 \\
\end{array}$ & RESET ALARMS & ok & FAL- $* 57$ ON & A & \\
\hline $\begin{array}{l}1.7 .1 .7 .3 \\
\text { min.2.0 }\end{array}$ & $\begin{array}{l}\text { OPEN SV }-\star 20 \\
\& S V-* 22\end{array}$ & N/A & N/A & A & \\
\hline
\end{tabular}


renos

APPENDIX A

TEST DATA SHEET

\begin{tabular}{|c|c|c|c|c|c|}
\hline \multicolumn{3}{|c|}{ Date: $1 /-5-96$} & \multicolumn{3}{|c|}{ SHMS Unit Number: VTP,$P_{N L}-105 A$} \\
\hline \multicolumn{3}{|c|}{$\begin{array}{l}\text { Tirle of rest: } \\
\text { Electrical Systems }\end{array}$} & \multirow{2}{*}{\multicolumn{3}{|c|}{$\begin{array}{l}\text { Test Equipment and } S / N \text { or Cal. No.: } \\
\text { Fluke V/O moter } \\
\text { SIN } 44620364 \\
\text { due } 10.97\end{array}$}} \\
\hline \multicolumn{3}{|c|}{$\begin{aligned} & \text { Test Performed By: } \text { See test Personnel } \\
& \text { Page } 38\end{aligned}$} & & & \\
\hline $\begin{array}{l}\text { Procedure No. } \\
\text { Initial/Date }\end{array}$ & Item & value & $\begin{array}{l}\text { Required } \\
\text { Condition } \\
\end{array}$ & $(A / R)$ & Corment \\
\hline $\begin{array}{l}1.7 .1 .7 .4 \\
m .2 .2\end{array}$ & $\begin{array}{l}\text { FIT-*57 \& } \\
\text { FSL }-* 57 \\
\text { POWERED }\end{array}$ & ok & $\begin{array}{l}\text { FIT-*57 ON } \\
\text { FSL-*57 ON }\end{array}$ & A & \\
\hline $\begin{array}{l}1.7 .1 .7 .5 \\
m_{1.2}\end{array}$ & $\begin{array}{l}\text { MUX-*70 } \\
\text { TB5+/TB6- }\end{array}$ & $0.994 \mathrm{Var}$ & $1.0 \pm 0.2 \mathrm{Vdc}$ & $A$ & \\
\hline $\begin{array}{l}1.7 .1 .7 .6 \\
M . x . B\end{array}$ & $\begin{array}{l}\text { CLOSE SV }-* 20 \\
\& \text { SV-*22 }\end{array}$ & N/A & N/A & A & \\
\hline $\begin{array}{l}1.7 .1 .7 .7 \\
M . x . B\end{array}$ & $\begin{array}{l}\text { OPEN FU-3, } \\
\text { FU-9, FU-10 } \\
\& \text { FU-13 }\end{array}$ & N/A & N/A & A & \\
\hline \multicolumn{6}{|c|}{ Section 1.7 .1 .8 verifies the $\mathrm{H}_{2}$ monitor wiring. } \\
\hline $\begin{array}{l}1.7 .1 .8 .1 \\
\text { oni.x.B }\end{array}$ & $\begin{array}{l}\text { CLOSE FU-2, } \\
\text { FU-4, FU-5, } \\
\text { FU-9 \& FU-10 }\end{array}$ & N/A & $N / A$ & A & \\
\hline $\begin{array}{l}1.7 .1 .8 .2 \\
m x B \\
\end{array}$ & RESET ALARMS & OK & $\begin{array}{l}\mathrm{NAH}-* 54 \text { OFF } \\
\mathrm{NAH}-* 55 \text { OFF }\end{array}$ & $A$ & \\
\hline $\begin{array}{l}1.7 .1 .8 .3 \\
m .28 B\end{array}$ & $\begin{array}{l}\text { INSTRUMENTS } \\
\text { POWERED }\end{array}$ &  & $\begin{array}{l}\text { MUX-*70 ON } \\
\text { NIT }-* 54 \text { ON } \\
\text { NIT-*55 ON } \\
\text { NR }-* 54 \text { ON } \\
\text { YYC-*01 ON } \\
\end{array}$ & $A$ & \\
\hline $\begin{array}{l}1.7 .1 .8 .4 \\
0.2 .8\end{array}$ & $\begin{array}{l}\text { OPEN FU-2, } \\
\text { FU-4, FU-5, } \\
\text { FU-9 \& FU-10 }\end{array}$ & N/A & $N / A$ & A & \\
\hline \multicolumn{6}{|c|}{ Section 1.7.1.9 verifies the SHMS-E heat trace control system. } \\
\hline $\begin{array}{l}1.7 .1 .9 .1 \\
7 m, x \cdot 1 \\
\end{array}$ & $\begin{array}{l}\text { INSTALL LOAD } \\
\text { VTP-TIC-*50 }\end{array}$ & N/A & $\mathrm{N} / \mathrm{A}$ & A & \\
\hline $\begin{array}{l}1.7 .1 .9 .2 \\
m . x .13\end{array}$ & $\begin{array}{l}\text { CLOSE CB-5, } \\
\text { FU-6, FU-7, } \\
\text { FU-9 \& FU-10 } \\
\text { and RESET } \\
\text { ALARMS }\end{array}$ & N/A & $N / A$ & A & \\
\hline
\end{tabular}


TEST DATA SHEET

\begin{tabular}{|c|c|c|c|c|c|}
\hline \multicolumn{3}{|l|}{ Date: $1 / \cdot 5 \cdot 96$} & \multicolumn{3}{|c|}{ SHMS Unit Nunber: VTP - PNL - $105 \mathrm{~A}$} \\
\hline \multicolumn{3}{|c|}{$\begin{array}{l}\text { Title of Test: } \\
\text { Electrical Systems }\end{array}$} & \multirow{2}{*}{\multicolumn{3}{|c|}{$\begin{array}{l}\text { Test Equipment and } \mathrm{S} / \mathrm{N} \text { or Cal. No.: } \\
N / A\end{array}$}} \\
\hline \multicolumn{3}{|c|}{$\begin{array}{c}\text { Test Performed By: See test Personnal } \\
\text { Page } 30\end{array}$} & & & \\
\hline $\begin{array}{l}\text { Procedure No. } \\
\text { Initial/Date }\end{array}$ & Item & value & $\begin{array}{l}\text { Required } \\
\text { condition }\end{array}$ & $\langle A / R\rangle$ & corment \\
\hline $\begin{array}{l}1.7 .1 .9 .3 \\
m m_{2 . \beta}\end{array}$ & $\begin{array}{l}\text { TIC }-* 50 \& \\
\text { TIC }-* 56 \\
\text { NOMINAL TEMP }\end{array}$ & $\frac{63^{\circ} \mathrm{F}}{62^{\circ} \mathrm{F}}$ & $\begin{array}{l}\text { TIC }-* 50 \\
\text { TIC }-* 56 \\
\text { TEMPERATURE }\end{array}$ & A & \\
\hline $\begin{array}{l}1.7 .1 .9 .4 \\
\text { mix.s. }\end{array}$ & $\begin{array}{l}\text { ADJ TIC } * * 50 \\
\& \text { TIC }-* 56 \\
\text { SP2 \& RESET } \\
\text { ALARMS }\end{array}$ & $N / A$ & $N / A$ & A & \\
\hline $\begin{array}{l}1.7 .1 .9 .5 \\
m_{B}\end{array}$ & $\begin{array}{l}\text { ADJ TIC }-* 50 \\
S P P_{1}\end{array}$ & $N / A$ & $N / A$ & $A$ & \\
\hline 1.7 .1 .9 .6 & $\begin{array}{l}\text { ADJ TIC } * 50 \\
\text { SP } 2\end{array}$ & $N / A$ & $\mathrm{~N} / \mathrm{A}$ & $A$ & \\
\hline$\frac{1.7 .1 .9 .7}{\mathscr{2} x \beta}$ & $\begin{array}{l}\text { TAL }-* 50 \& \\
\text { HORN ACTIVE }\end{array}$ & $\frac{O K}{O O K}$ & $\begin{array}{l}\text { TAL-*50 ON } \\
\text { HORN ON } \\
\text { ACKNOWLEDGE }\end{array}$ & $A$ & \\
\hline $\begin{array}{l}1.7 .1 .9 .8 \\
m \% 8\end{array}$ & $\begin{array}{l}\text { TIC }-* 50 \\
\text { OPERATES }\end{array}$ & ok & $\begin{array}{l}\text { TIC }-* 50 \\
\text { OPERATES }\end{array}$ & $A$ & \\
\hline $\begin{array}{l}1.7 .1 .9 .9 \\
\text { minB }\end{array}$ & $\begin{array}{l}\text { ADJ TIC-*50 } \\
\text { SP } 2\end{array}$ & $N / A$ & $N / A$ & $A$ & \\
\hline $\begin{array}{l}1.7 .1 .9 .10 \\
\mathscr{H} \mathscr{K} B\end{array}$ & RESET ALARM & $\Delta K$ & $\mathrm{TAL}-\star 50$ OFF & $A$ & \\
\hline $\begin{array}{l}1.7 .1 .9 .11 \\
74.218\end{array}$ & $\begin{array}{l}\text { ADJ TIC-*50 } \\
S P 1 \& S P 2\end{array}$ & $N / A$ & $N / A$ & $A$ & \\
\hline $\begin{array}{r}1.7 .1 .9 .12 \\
\text { M. } 2.8 \\
\end{array}$ & $\begin{array}{l}\text { OPEN CB-5 \& } \\
\text { REMOVE LOAD }\end{array}$ & $N / A$ & $N / A$ & A & \\
\hline $\begin{array}{l}1.7 .1 .9 .13 \\
m_{1} \mathscr{x}_{3}\end{array}$ & $\begin{array}{l}\text { INSTALL LOAD } \\
\text { VTP-TIC-*56 }\end{array}$ & $N / A$ & $N / A$ & A & \\
\hline $\begin{array}{l}1.7 .1 .9 .14 \\
2 m . X^{3} \\
\end{array}$ & CLOSE CB-5 & $N / A$ & $N / A$ & A & \\
\hline $\begin{array}{l}1.7 .1 .9 .15 \\
m, z y\end{array}$ & $\begin{array}{l}\text { ADJ TIC-*56 } \\
\text { SP } 1\end{array}$ & $N / A$ & $N / A$ & A & \\
\hline $\begin{array}{l}1.7 .1 .9 .16 \\
m \approx B\end{array}$ & $\begin{array}{l}\text { ADJ TIC-*56 } \\
\text { SP } 2\end{array}$ & N/A & $N / A$ & A & \\
\hline
\end{tabular}


TEST DATA SHEET

\begin{tabular}{|c|c|c|c|c|c|}
\hline \multicolumn{3}{|c|}{ Date: $11-5-96$} & \multicolumn{3}{|c|}{ SHMS Unit Number: VTP-PNL $-105 A$} \\
\hline \multicolumn{3}{|c|}{$\begin{array}{l}\text { Title of rest: } \\
\text { Electrical Systems }\end{array}$} & \multirow{2}{*}{\multicolumn{3}{|c|}{$\begin{array}{l}\text { Test Equipment and } \mathrm{s} / \mathrm{N} \text { or Cal. No.: } \\
N / \mathrm{A}\end{array}$}} \\
\hline \multicolumn{3}{|c|}{$\begin{array}{c}\text { Test Performed By: See test Personnel } \\
\text { Page } 30\end{array}$} & & & \\
\hline $\begin{array}{l}\text { Procedure No. } \\
\text { Initial /Date } \\
\end{array}$ & Item & Value & $\begin{array}{l}\text { Required } \\
\text { Condition }\end{array}$ & $(A / R)$ & Comment \\
\hline $\begin{array}{l}1.7 .1 .9 .17 \\
m .1 .3\end{array}$ & $\begin{array}{l}\text { TAL }-* 50 \& \\
\text { HORN ACTIVE }\end{array}$ & $\frac{\text { ok }}{\text { ok }}$ & $\begin{array}{l}\text { TAL- *50 ON } \\
\text { HORN ON } \\
\text { ACKNOWLEDGE }\end{array}$ & A & . \\
\hline $\begin{array}{r}1.7 .1 .9 .18 \\
9.21 .8 \\
\end{array}$ & $\begin{array}{l}\text { TIC }-* 56 \\
\text { OPERATES } \\
\end{array}$ & ok & $\begin{array}{l}\text { TIC }-* 56 \\
\text { OPERATES }\end{array}$ & A & \\
\hline $\begin{array}{l}1.7 .1 .9 .19 \\
D_{B} \cdot B^{\circ}\end{array}$ & $\begin{array}{l}\text { ADJ TIC }-* 56 \\
\text { SP } 2\end{array}$ & $N / A$ & $N / A$ & A & \\
\hline m.x.B. & RESET ALARM & ok & $\mathrm{TAL}-* 50$ OFF & A & \\
\hline 1.7 .1 .9 .21 & $\begin{array}{l}A D J T I C-* 56 \\
S P 1 \& \text { SP } 2\end{array}$ & $N / A$ & $N / A$ & A & \\
\hline $\begin{array}{l}1.7 .1 .9 .22 \\
74.21 \\
\end{array}$ & $\begin{array}{l}\text { OPEN CB-5, } \\
\text { FU-6 \& FU-7 }\end{array}$ & $N / A$ & $N / A$ & $A$ & \\
\hline $\begin{array}{l}1.7 .1 .9 .23 \\
m_{213} \\
\end{array}$ & $\begin{array}{l}\text { REMOVE LOAD } \\
\text { TIC-*56 }\end{array}$ & $N / A$ & $N / A$ & A & \\
\hline $\begin{array}{l}1.7 .1 .9 .24 \\
\end{array}$ & $\begin{array}{l}\text { CLOSE FU-8 } \\
\text { TIS }-* 62 \\
\text { NOMINAL TEMP }\end{array}$ & $68 \%$ & $\begin{array}{l}\text { TIS }-* 62 \\
\text { TEMP }\end{array}$ & $A$ & \\
\hline $\begin{array}{l}1.7 .1 .9 .25 \\
M .8 B \\
\end{array}$ & $\begin{array}{l}\text { ADJ TIS }-* 62 \\
\text { SP } 1\end{array}$ & $N / A$ & $N / A$ & A & \\
\hline $\begin{array}{l}1.7 .1 .9 .26 \\
m .2 .8 \\
\end{array}$ & $\begin{array}{l}\text { ADJ TIS }-* 62 \\
\text { SP } 2\end{array}$ & $N / A$ & $N / A$ & $A$ & \\
\hline 1.7 .1 .9 .27 & RESET ALARMS & ok & $\begin{array}{l}\text { TAHL-*62 } \\
\text { OFF }\end{array}$ & A & \\
\hline 1.7 .1 .9 .28 & $\begin{array}{l}\text { ADJ TIS *62 } \\
\text { SP } 2\end{array}$ & $N / A$ & $N / A$ & $A$ & \\
\hline 1.7 .1 .9 .29 & $\begin{array}{l}\text { TAHL }-* 62 \text { \& } \\
\text { HORN ACTIVE }\end{array}$ & $\frac{O K}{O K}$ & $\begin{array}{l}\text { TAHL }-* 62 \text { ON } \\
\text { HORN ON } \\
\text { ACKNOWLEDGE }\end{array}$ & $A$ & \\
\hline $\begin{array}{l}1.7 .1 .9 .30 \\
\operatorname{mix} 6 \\
\end{array}$ & $\begin{array}{l}\text { ADJ TIS }-* 62 \\
\text { SP } 2\end{array}$ & $N / A$ & $N / A$ & A & \\
\hline
\end{tabular}


TEST DATA SHEET

\begin{tabular}{|c|c|c|c|c|c|}
\hline \multicolumn{3}{|c|}{ Date: $11-5 \cdot 96$} & \multicolumn{3}{|c|}{ SHMS Unit Number: VTP $-P_{N L}-105 \mathrm{~A}$} \\
\hline \multicolumn{3}{|c|}{$\begin{array}{l}\text { Title of rest: } \\
\text { Electrical Systems }\end{array}$} & \multirow{2}{*}{\multicolumn{3}{|c|}{$\begin{array}{l}\text { rest Equipment and } S / N \text { or Cal. No.: } \\
\text { Fluke V/o mets. } \\
S / N 44620364 \\
C_{a} / \text { due } 10.47\end{array}$}} \\
\hline \multicolumn{3}{|c|}{$\begin{array}{c}\text { Test Performed By: See Test Personnel } \\
\text { Page } 3 \theta\end{array}$} & & & \\
\hline $\begin{array}{l}\text { Procedure No. } \\
\text { Initial/Date }\end{array}$ & Item & Value & $\begin{array}{l}\text { Required } \\
\text { Condition }\end{array}$ & $(A / R)$ & comment \\
\hline $\begin{array}{l}1.7 .1 .9 .31 \\
2 m .3 .3 \\
\end{array}$ & RESET ALARMS & ok & $\begin{array}{l}\text { TAHL-*62 } \\
\text { OFF }\end{array}$ & A & \\
\hline 1.7 .1 .9 .32 & $\begin{array}{l}\text { ADJ TIS-*62 } \\
\text { SP } 1\end{array}$ & N/A & N/A & A & \\
\hline $\begin{array}{l}1.7 .1 .9 .33 \\
3 x .8\end{array}$ & $\begin{array}{l}\text { TAHL-*62 \& } \\
\text { HORN ACTIVE }\end{array}$ & $\frac{O K}{O K}$ & $\begin{array}{l}\text { TAHL-*62 ON } \\
\text { HORN ON } \\
\text { ACKNOWLEDGE }\end{array}$ & A & \\
\hline 1.7 .1 .9 .34 & $\begin{array}{l}A D J \text { TIS } * 62 \\
S P 1 \& S P 2\end{array}$ & N/A & $N / A$ & A & \\
\hline $\begin{array}{l}1.7 .1 .9 .35 \\
x_{1 . R} \\
\end{array}$ & $\begin{array}{l}\text { OPEN FU-8, } \\
\text { FU-9 \& FU-10 }\end{array}$ & N/A & N/A & A & \\
\hline \multicolumn{6}{|c|}{ Section 1.7 .1 .10 verifies the differential pressure transmitter system } \\
\hline $\begin{array}{l}1.7 .1 .10 .1 \\
\text { m.x. }\end{array}$ & $\begin{array}{l}\text { REMOVE PLUGS } \\
\text { BAL PDIT-*60 }\end{array}$ & $N / A$ & $N / A$ & A & \\
\hline$\frac{1.7 .1 .10 .2}{9.2 .8}$ & $\begin{array}{l}\text { CLOSE FU-14 } \\
\text { REC PDIT-*60 } \\
\text { PRESSURE } \\
\end{array}$ & 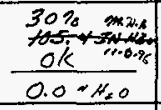 & $\begin{array}{l}\text { PDIT-*60 } \\
\text { PRESSURE } \\
0 \pm I^{\prime \prime} \mathrm{H}_{2} \mathrm{O} \\
\end{array}$ & A & . \\
\hline $\begin{array}{l}1.7 .1 .10 .3 \\
x . x^{2}\end{array}$ & $\begin{array}{l}\text { MUX-*70 Vdc } \\
\text { TB7+ \& TB8- }\end{array}$ & $2.145 \mathrm{Vd}$ & $\begin{array}{l}\text { MUX }-* 70 \text { Vdc } \\
2.2 \pm 0.1\end{array}$ & A & \\
\hline$\frac{1.7 .1 .10 .4}{2 \times 8}$ & $\begin{array}{l}\text { REPLACE } \\
\text { PLUGS ON } \\
\text { PDIT }-* 60 \\
\end{array}$ & N/A & $N / A$ & A & \\
\hline $\begin{array}{l}1.7 .1 .10 .5 \\
m .2 . B \\
\end{array}$ & OPEN FU-14 & $N / A$ & $N / A$ & A & \\
\hline \multicolumn{6}{|c|}{ Section 1.7 .1 .11 verifies the gas chromatograph power } \\
\hline $\begin{array}{l}1.7 .1 .11 .1 \\
x_{0} B \\
\end{array}$ & CLOSE FU-11 & $N / A$ & $N / A$ & A & \\
\hline $\begin{array}{l}1.7 .1 .11 .2 \\
m_{2} .8\end{array}$ & $\begin{array}{l}\text { POWER } \\
\text { MON-*60 }\end{array}$ & QK & $\begin{array}{l}\text { MON }-* 60 \\
\text { POWERED } \\
\end{array}$ & A & \\
\hline $\begin{array}{l}1.7 .1 .11 .3 \\
2 M_{B} B\end{array}$ & OPEN FU-11 & N/A & $N / A$ & A & \\
\hline
\end{tabular}




\section{TEST DATA SHEET}

\begin{tabular}{|c|c|c|c|c|c|}
\hline \multicolumn{3}{|c|}{ Date: $11-5-96$} & \multicolumn{3}{|c|}{ SHMS Unit Number: VTP-PNL-105A } \\
\hline \multicolumn{3}{|c|}{$\begin{array}{l}\text { Title of Iest: } \\
\text { Electrical Systems }\end{array}$} & \multirow{2}{*}{\multicolumn{3}{|c|}{$\begin{array}{l}\text { Test Equipment and } S / N \text { or Cal. No.: } \\
\text { Fluke V/O meter } \\
S / W 44620364 \\
\text { Cal. due } 10.99\end{array}$}} \\
\hline \multicolumn{3}{|c|}{$\begin{aligned} & \text { Test Performed By: } \text { See Test Personnel } \\
& \text { Page } 38\end{aligned}$} & & & \\
\hline $\begin{array}{l}\text { Procedure No. } \\
\text { Initial/Date }\end{array}$ & Item & value & $\begin{array}{l}\text { Required } \\
\text { Condition }\end{array}$ & $(A / R)$ & Comment \\
\hline \multicolumn{6}{|c|}{ Section 1.7.1.12 verifies the multi gas analyzer power. } \\
\hline $\begin{array}{l}1.7 .1 .12 .1 \\
m .2 .3 \\
\end{array}$ & CLOSE FU-12 & $\mathrm{N} / \mathrm{A}$ & N/A & A & \\
\hline $\begin{array}{l}1.7 .1 .12 .2 \\
m x_{x}\end{array}$ & $\begin{array}{l}\text { POWER } \\
\text { NIT }-* 52\end{array}$ & ak & $\begin{array}{l}\text { NIT }-* 52 \\
\text { POWERED } \\
\end{array}$ & $A$ & \\
\hline $\begin{array}{l}1.7 .1 .12 .3 \\
x \times B\end{array}$ & OPEN FU-12 & $N / A$ & $N / A$ & $A$ & \\
\hline \multicolumn{6}{|c|}{ Section 1.7.1.13 verifies the personnel computer power. } \\
\hline $\begin{array}{l}1.7 .1 .13 .1 \\
2.8\end{array}$ & CLOSE CB-6 & $N / A$ & $N / A$ & A & \\
\hline $\begin{array}{l}1.7 .1 .13 .2 \\
\text { m.9.8 } \\
\end{array}$ & $\begin{array}{l}\text { POWER PC-*60 } \\
\& P C-\star 70\end{array}$ & $\frac{o k}{0 K}$ & $\begin{array}{ll}P C-* 60 & O N \\
P C-* 70 & O N \\
\end{array}$ & A & \\
\hline $\begin{array}{l}1.7 .1 .13 .3 \\
2 m .8\end{array}$ & $\begin{array}{l}\text { TURN INST. } \\
\text { OFF \& } \\
\text { OPEN CB-6 }\end{array}$ & - ok & $\begin{array}{ll}P C-* 60 & \text { OFF } \\
P C-* 70 & \text { OFF }\end{array}$ & A & \\
\hline
\end{tabular}

a11 11.5 .96 
APPENDIX A

PAGE $A-13$

TEST DATA SHEET

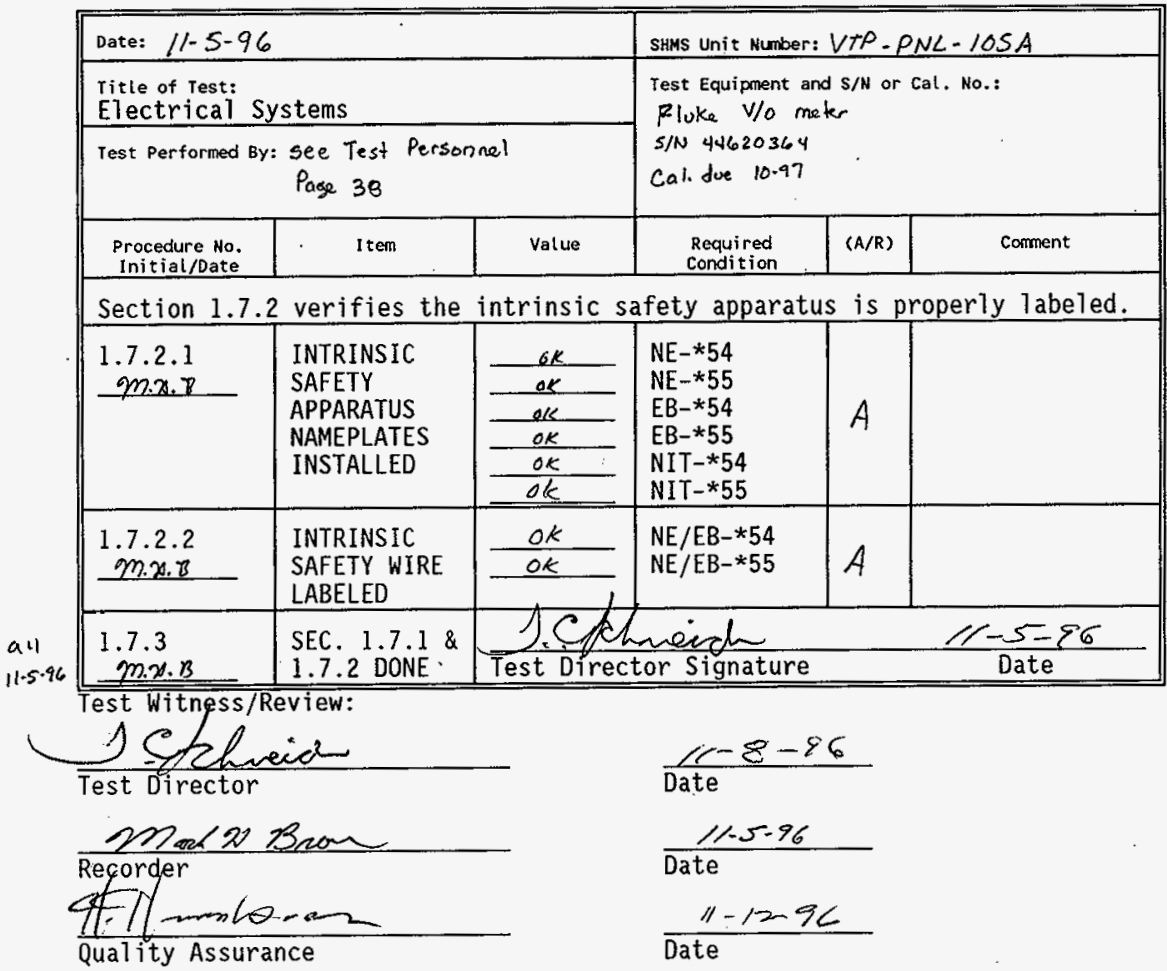


TEST DATA SHEET

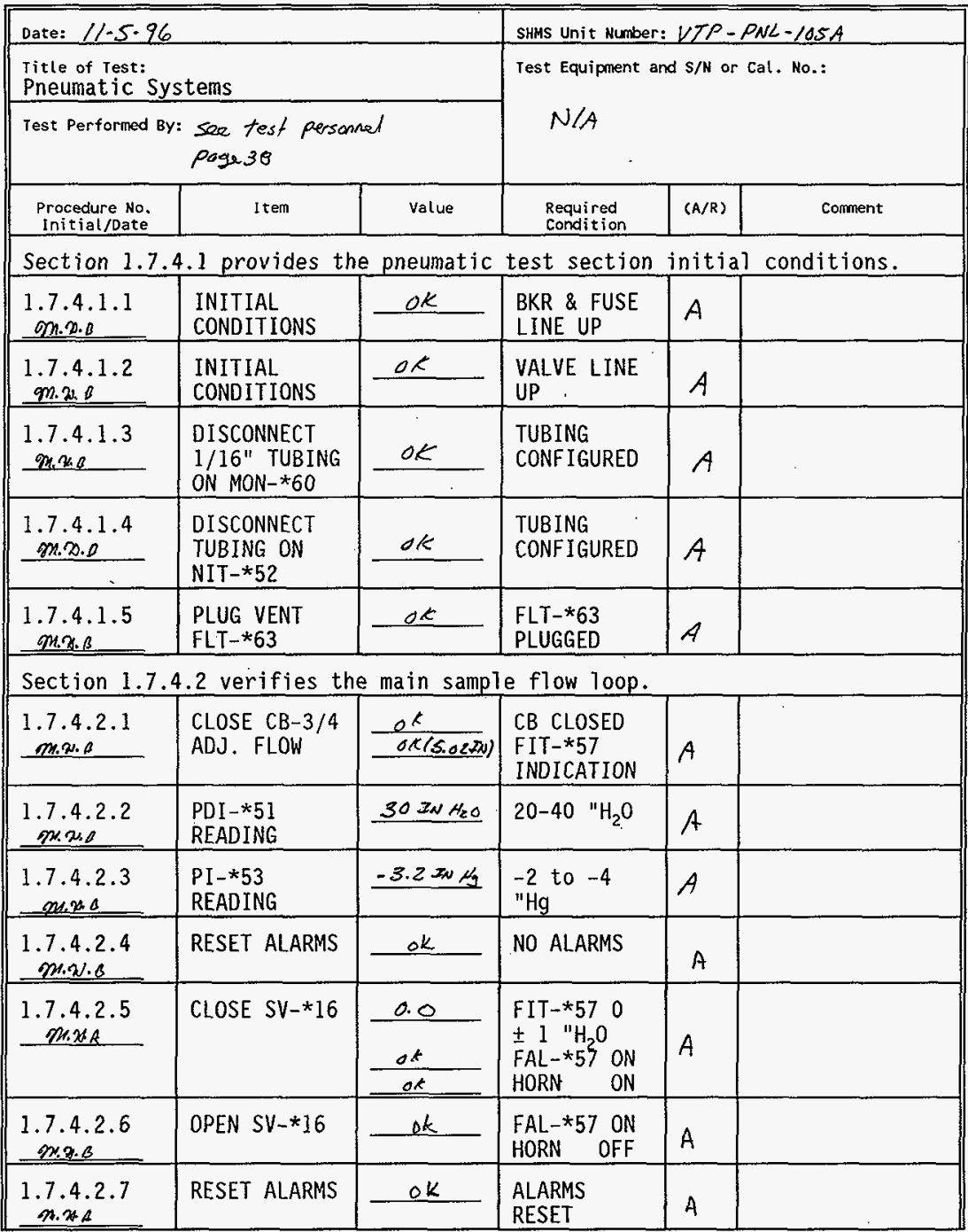


TEST DATA SHEET

\begin{tabular}{|c|c|c|c|c|c|}
\hline \multicolumn{3}{|l|}{ Date: $11-5.96$} & \multicolumn{3}{|c|}{ SHMS Unit Number: $V T P-P N L-105 A$} \\
\hline \multicolumn{3}{|c|}{$\begin{array}{l}\text { Title of Test: } \\
\text { Pneumatic Systems }\end{array}$} & \multirow{2}{*}{\multicolumn{3}{|c|}{$\begin{array}{l}\text { Test Equipment and } S / N \text { or Cal. No.: } \\
\qquad N / A\end{array}$}} \\
\hline \multicolumn{3}{|c|}{$\begin{aligned} \text { Test Performed By: } & \text { See Test Peersonnel } \\
& \rho_{\text {age }} 30\end{aligned}$} & & & \\
\hline $\begin{array}{l}\text { Procedure No. } \\
\text { Initial/Date }\end{array}$ & I tem & value & $\begin{array}{l}\text { Required } \\
\text { Condition }\end{array}$ & $(A / R)$ & Comment \\
\hline 1.7 .4 .2 .8 & CLOSE SV $-* 25$ & -0.3 & $\begin{array}{lll}\mathrm{FIT}-* 57 & 0 \\
+1 & \end{array}$ & & See \\
\hline & & $\frac{O k}{O K}$ & $\begin{array}{ll}\text { FAL-*57 } & \text { ON } \\
\text { HORN } & \text { ON } \\
\end{array}$ & A & $\begin{array}{l}\text { Rev OB } \\
\text { mase } 12-17.92\end{array}$ \\
\hline 1.7 .4 .2 .9 & OPEN SV-*25 & ok & FAL-*57 ON & A. & see Rev oB \\
\hline M.x.B & & & HORN OFF & & $9.240 \quad 12 \cdot 17-96$ \\
\hline$\frac{1.7 .4 .2 .10}{20.2 .0}$ & RESEI ALARMS & $-\ldots . t$ & $\begin{array}{l}\text { ALARMS } \\
\text { RESET } \\
\end{array}$ & $A$ & $\begin{array}{l}\text { see Red, } O \beta \\
\text { mans } 1 z-17-96\end{array}$ \\
\hline $\begin{array}{l}1.7 .4 .2 .11 \\
2 \mathbb{2 1 . 3}\end{array}$ & $\begin{array}{l}\text { OPEN SV-*54 } \\
\& \text { SV-*66 } \\
\text { READ PRESS. } \\
\text { PDIT-*60 }\end{array}$ & $\frac{0 K}{-43.620120}$ & $\begin{array}{l}\text { VALVES OPEN } \\
-27 \text { to }-82 \\
" \mathrm{H}_{2} \mathrm{O}\end{array}$ & A & \\
\hline $\begin{array}{l}1.7 .4 .2 .12 \\
m_{x . \beta}\end{array}$ & $\begin{array}{l}\text { CLOSE SV }-* 54 \\
\text { OPEN SV } * 63 \\
\text { READ PRESS. } \\
\text { PDIT-* } 60 \\
\end{array}$ & $\frac{0 k}{0.09 \mu_{20}}$ & $\begin{array}{l}\text { VALVES } \\
\text { OPERATED } \\
0 \pm 1 \quad \mathrm{H}_{2} \mathrm{O}\end{array}$ & $A$ & 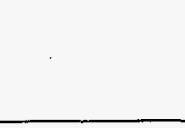 \\
\hline$\frac{1.7 .4 .2 .13}{2 m i x}$ & $\begin{array}{l}\text { CLOSE SV }-* 63 \\
\text { OPEN SV } * 55 \\
\text { READ PRESS. } \\
\text { PDIT-* } 60 \\
\end{array}$ & $\frac{0 R}{-45.08 * 200}$ & $\begin{array}{l}\text { VALVES } \\
\text { OPERATED } \\
-27 \text { to }-82 \\
" \mathrm{H}_{2} \mathrm{O}\end{array}$ & A & \\
\hline $\begin{array}{l}1.7 .4 .2 .14 \\
m_{1 . R}\end{array}$ & $\begin{array}{l}\text { CLOSE SV }-* 55 \\
\text { OPEN SV } * 64 \\
\text { READ PRESS. } \\
\text { PDIT-*60 }\end{array}$ & $\frac{0 k}{0.0974460}$ & $\begin{array}{l}\text { VALVES } \\
\text { OPERATED } \\
0 \pm 1 \quad \mathrm{H}_{2} \mathrm{O}\end{array}$ & A & \\
\hline $\begin{array}{l}1.7 .4 .2 .15 \\
{ }_{2}, x_{B} \\
\end{array}$ & $\begin{array}{l}\text { CLOSE SV-*64 } \\
\& \text { SV-*66 }\end{array}$ & $N / A$ & $N / A$. & A & \\
\hline Section 1.7 & .3 verifies th & grab samp & flow loop. & & \\
\hline $\begin{array}{l}1.7 .4 .3 .1 \\
\text { M.\%.B. }\end{array}$ & READ PDI $* 51$ & $29 " \mathrm{H}_{2} \mathrm{O}$ & $20-40 \quad \mathrm{H}_{2} \mathrm{O}$ & A & \\
\hline $\begin{array}{l}1.7 .4 .3 .2 \\
m .2 .3\end{array}$ & $\begin{array}{l}\text { PUSH PB }-* 59 \\
\text { GRAB SAMPLE }\end{array}$ & oll & \begin{tabular}{|l} 
START \\
STOPWATCH \\
\end{tabular} & A & \\
\hline
\end{tabular}


TEST DATA SHEET

\begin{tabular}{|c|c|c|c|c|c|}
\hline \multicolumn{3}{|c|}{ Date: $12-17-96$} & \multicolumn{3}{|c|}{ SHMS Unit Number: VTP - PNL-105A } \\
\hline \multicolumn{3}{|c|}{$\begin{array}{l}\text { Title of Test: } \\
\text { Pneumatic Systems }\end{array}$} & \multirow{2}{*}{\multicolumn{3}{|c|}{$\begin{array}{l}\text { Test Equipment and S/N or Cal. No.: } \\
\text { Fluke Vlo meke } \\
S / N \text { \&4620364 } \\
\text { c4l due } 10.97\end{array}$}} \\
\hline \multicolumn{3}{|c|}{$\begin{aligned} \text { Test Performed By: } & \text { See Test Personnas } \\
& \text { Page } 30\end{aligned}$} & & & \\
\hline $\begin{array}{l}\text { Procedure No. } \\
\text { Initial/Date }\end{array}$ & Item & Value & $\begin{array}{l}\text { Required } \\
\text { Condition }\end{array}$ & $(A / R)$ & Comment \\
\hline $\begin{array}{l}1.7 .4 .2 .8 \\
\text { mes. } \\
\end{array}$ & \multirow[t]{2}{*}{ CLOSE SV-*25 } & -0 & \multirow{2}{*}{$\begin{array}{l}\text { FIT-*57 } 0 \\
\pm 1 \quad " H_{2} 0 \\
\text { FAL-*57 ON } \\
\text { HORN ON } \\
\text { XA-*63 ON } \\
\text { YY-*71 } \\
\text { TB } 3-4 \text { OV } \\
\end{array}$} & \multirow[b]{2}{*}{$A$} & \\
\hline $1 z-17-\infty 6$ & & $\frac{\frac{\partial k}{\partial K}}{\frac{\partial K}{O V}}$ & & & \\
\hline $\begin{array}{l}1.7 .4 .2 .9 \\
m .20,12 \times 7.26 \\
\end{array}$ & OPEN SV-*25 & $\frac{\frac{. k}{4 k}}{\frac{0 .}{118.5 \mathrm{~V}}}$ & $\begin{array}{l}\text { FAL }-* 57 \text { ON } \\
\text { HORN OFF } \\
\text { XA-*63 OFF } \\
\text { YY }-* 71 \\
\text { TB } 3 \sim 4 \\
\end{array}$ & $A$ & \\
\hline $\operatorname{mim}_{1.8} .4 .2 .10$ & RESET ALARMS & $d K$ & $\begin{array}{l}\text { ALARMS } \\
\text { RESET }\end{array}$ & A & \\
\hline 1.7 .4 .2 .11 & $\begin{array}{l}\text { OPEN SV }-* 54 \\
\& \text { SV -*66 } \\
\text { READ PRESS. } \\
\text { PDIT-*60 }\end{array}$ & & $\begin{array}{l}\text { VALVES OPEN } \\
-27 \text { to }-82 \\
" \mathrm{H}_{2} \mathrm{O}\end{array}$ & & \\
\hline 1.7 .4 .2 .12 & $\begin{array}{l}\text { CLOSE SV }-* 54 \\
\text { OPEN SV } * 63 \\
\text { READ PRESS. } \\
\text { PDIT-*60 }\end{array}$ & & $\begin{array}{l}\text { VALVES } \\
\text { OPERATED } \\
0 \pm 1 \quad " \mathrm{H}_{2} \mathrm{O}\end{array}$ & & \\
\hline 1.7 .4 .2 .13 & $\begin{array}{l}\text { CLOSE SV-*63 } \\
\text { OPEN SV }-* 55 \\
\text { READ PRESS. } \\
\text { PDIT-*60 }\end{array}$ & & $\begin{array}{l}\text { VALVES } \\
\text { OPERATED } \\
-27 \text { to }-82 \\
" \mathrm{H}_{2} \mathrm{O}\end{array}$ & & \\
\hline 1.7 .4 .2 .14 & $\begin{array}{l}\text { CLOSE SV-*55 } \\
\text { OPEN SV-*64 } \\
\text { READ PRESS. } \\
\text { PDIT-*60 }\end{array}$ & & $\begin{array}{l}\text { VALVES } \\
\text { OPERATED } \\
\mathrm{O} \pm 1 \quad \mathrm{H}_{2} \mathrm{O}\end{array}$ & & \\
\hline 1.7 .4 .2 .15 & $\begin{array}{l}\text { CLOSE SV-*64 } \\
\& \text { SV }-* 66\end{array}$ & N/A & $N / A$ & & \\
\hline \multicolumn{6}{|c|}{ Section 1.7 .4 .3 verifies the grab sample flow loop. } \\
\hline $1.7 \cdot 4 \cdot 3 \cdot 1$ & READ PDI $* 51$ & & $20-40 \quad " \mathrm{H}_{2} \mathrm{O}$ & & \\
\hline
\end{tabular}


TEST DATA SHEET

\begin{tabular}{|c|c|c|c|c|c|}
\hline \multicolumn{3}{|c|}{ Date: $11-5.96$} & \multicolumn{3}{|c|}{ SHMS Unit Number: VTP-PNL-105A } \\
\hline \multicolumn{3}{|c|}{$\begin{array}{l}\text { Title of Test: } \\
\text { Pneumatic Systems }\end{array}$} & \multirow{2}{*}{\multicolumn{3}{|c|}{$\begin{array}{l}\text { Test Equipment and } S / \mathrm{N} \text { or Cal. No.: } \\
\qquad \mathrm{N} / \mathrm{A}\end{array}$}} \\
\hline \multicolumn{3}{|c|}{$\begin{array}{l}\text { Test Performed By: see test personnel } \\
\text { page } 38\end{array}$} & & & \\
\hline $\begin{array}{l}\text { Procedure No. } \\
\text { Initial/Date }\end{array}$ & Item & Value & $\begin{array}{l}\text { Required } \\
\text { Condition }\end{array}$ & $(A / R)$ & Comment \\
\hline $\begin{array}{l}1.7 .4 .3 .3 \\
\text { M.x.3 }\end{array}$ & $\begin{array}{l}\text { GRAB SAMPLE } \\
\text { LAMPS ON } \\
\text { READ FIV-*52 }\end{array}$ & 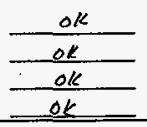 & $\begin{array}{l}\text { PBL }-* 58 \text { ON } \\
\text { PBL }-* 59 \text { ON } \\
\text { YAL }-* 58 \text { ON } \\
\text { FIV }-* 52 \quad 0 \\
\end{array}$ & A & \\
\hline $\begin{array}{l}1.7 .4 .3 .4 \\
0 \% .7 .0 \\
\end{array}$ & $\begin{array}{l}\text { OPEN SV }-\star 15 \\
\& \text { ADJ. FIV- } \\
* 52\end{array}$ & ok & $\begin{array}{l}\text { SV }-* 15 \text { OPEN } \\
\text { FIV }-* 52 \\
\text { FLOW } 10 \text { CFH }\end{array}$ & $A$ & \\
\hline $\begin{array}{l}1.7 .4 .3 .5 \\
M 9.00 .0\end{array}$ & READ PDI $-* 51$ & $32.7^{\prime \prime} \mathrm{HzO}$ & $\begin{array}{l}\text { PDI } * 51 \\
\text { HIGHER THAN } \\
1.7 .4 .3 .1 \\
\end{array}$ & A & \\
\hline $\begin{array}{l}1.7 .4 .3 .6 \\
n .2 . n\end{array}$ & CLOSE SV-*15 & $\Delta K$ & FIV $-* 52 \quad 0$ & $A$ & \\
\hline $\begin{array}{l}1.7 .4 .3 .7 \\
\text { m.x.e }\end{array}$ & OPEN SV $-\star 15$ & OK & $\begin{array}{l}\text { FIV -*52 } \\
\text { FLOW }\end{array}$ & A & \\
\hline $\begin{array}{l}1.7 .4 .3 .8 \\
m \cdot 2 . B\end{array}$ & $\begin{array}{l}\text { PBL }-* 59 \\
\text { GRAB SAMPLE } \\
\text { TIME }\end{array}$ & 5.02 & $\begin{array}{l}\text { PBL }-* 59 \text { OFF } \\
\text { STOP WATCH } \\
5 \pm .5 \mathrm{MIN} \\
\end{array}$ & $A$ & \\
\hline $\begin{array}{l}1.7 .4 .3 .9 \\
\text { m.2.0. }\end{array}$ & $\begin{array}{l}\text { PB-*58 RESET } \\
\text { SAMPLER }\end{array}$ & $\begin{array}{l}\text { ok } \\
\text { OK }\end{array}$ & $\begin{array}{l}\mathrm{PBL}-* 58 \text { OFF } \\
\text { YAL-*58 OFF }\end{array}$ & A & \\
\hline \multicolumn{6}{|c|}{ Section 1.7.4.4 verifies the $\mathrm{H}_{2}$ cell calibration loop. } \\
\hline $\begin{array}{l}1.7 .4 .4 .1 \\
m .21 .8 \\
\end{array}$ & $\begin{array}{l}\text { CONNECT } \mathrm{H}_{2} \\
\text { CAL GAS }\end{array}$ & N/A & N/A & A & \\
\hline $\begin{array}{l}1.7 .4 .4 .2 \\
m x .3 \\
\end{array}$ & $\begin{array}{l}\text { OPEN ISO } \\
\text { VALVE }\end{array}$ & ok & $<10$ PSIG & A & \\
\hline $\begin{array}{l}1.7 .4 .4 .3 \\
m_{1} x .3 . \\
\end{array}$ & $\begin{array}{l}\text { OPEN SV-*18 } \\
\text { ADJ. FIV-*56 }\end{array}$ & 2 & $2 \pm .1 \mathrm{CFH}$ & $A$ & \\
\hline
\end{tabular}


APPENDIX A

TEST DATA SHEET

\begin{tabular}{|c|c|c|c|c|c|}
\hline \multicolumn{3}{|l|}{ Date: $11-5.96$} & \multicolumn{3}{|c|}{ SHMS Unit Number: $V T P-P N L-105 \mathrm{~A}$} \\
\hline \multicolumn{3}{|c|}{$\begin{array}{l}\text { Title of Test: } \\
\text { Pneumatic Systems }\end{array}$} & \multirow{2}{*}{\multicolumn{3}{|c|}{$\begin{array}{l}\text { rest Equipment and } S / N \text { or Cal. No.: } \\
\text { Floke V/o meter } \\
S / N 44620364 \\
\text { Cal. due } 10.97\end{array}$}} \\
\hline \multicolumn{3}{|c|}{$\begin{aligned} \text { Test Performed By: } & \text { sea test Persionnad } \\
& \text { page } 38\end{aligned}$} & & & \\
\hline $\begin{array}{l}\text { Procedure No. } \\
\text { Initial/Date }\end{array}$ & Item & Value & $\begin{array}{l}\text { Required } \\
\text { Condition } \\
\end{array}$ & $(A / R)$ & Comment \\
\hline $\begin{array}{l}1.7 .4 .4 .4 \\
\text { nexpes }\end{array}$ & $\begin{array}{l}\text { VERIFY } \\
\text { CONDITIONS }\end{array}$ & 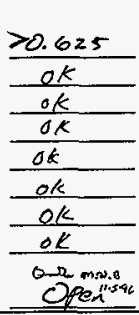 & $\begin{array}{l}\text { NIT-*54 } \\
>.625 \% \mathrm{H}_{2} \\
\text { NAH-*55 ON } \\
\text { NAH-*54 ON } \\
\text { PBL-*59 ON } \\
\text { PBL-*58 ON } \\
\text { YAL-*58 ON } \\
\text { FIV-*52 FLO } \\
\text { HORN ON } \\
\text { TB2-21/22 } \\
\text { OPEN }\end{array}$ & A & \\
\hline $\begin{array}{l}1.7 .4 .4 .5 \\
2 \times, 9.8 \\
\end{array}$ & $\begin{array}{l}\text { ACKNOWLEDGE } \\
\text { HORN }\end{array}$ & $N / A$ & $N / A$ & A & \\
\hline $\begin{array}{l}1.7 .4 .4 .6 \\
0.2 .8 \\
\end{array}$ & $\begin{array}{l}\text { CLOSE SV-*18 } \\
\text { VERIFY } \\
\text { CONDITIONS }\end{array}$ & $\begin{array}{l}0 K \\
0.58 \% \\
0 K \\
O K \\
O K \\
O K \\
O K \\
O \Omega \\
\end{array}$ & $\begin{array}{l}\text { FIV-*56 0 } \\
\text { NIT-*54 } \\
<.625 \% \mathrm{H}_{2} \\
\text { NAH-*55 OFF } \\
\text { NAH-*54 ON } \\
\text { PBL-*58 ON } \\
\text { YAL-*58 ON } \\
\text { TB2-21/22 } \\
\text { CLOSED } \\
\end{array}$ & $A$ & \\
\hline $\begin{array}{l}1.7 .4 .4 .7 \\
\mathscr{x}_{n}, 0.8 \\
\end{array}$ & $\begin{array}{l}\text { PUSH RESET } \\
\text { SAMPLER PB- } \\
* 58\end{array}$ & OE & $\begin{array}{l}\text { PBL-*58 } 0 \mathrm{FF} \\
\text { YAL-*58 } \\
\text { OFF }\end{array}$ & $A$ & \\
\hline $\begin{array}{l}1.7 .4 .4 .8 \\
\text { ginge } \\
\end{array}$ & RESET ALARM & $O K$ & $\mathrm{NAH}-* 54$ OFF & $A$ & \\
\hline $\begin{array}{l}1.7 .4 .4 .9 \\
2 \operatorname{2x.20} 8 \\
\end{array}$ & $\begin{array}{l}\text { OPEN SV }-* 19 \\
\text { ADJ. FIV }-* 56 \\
\end{array}$ & $\begin{array}{l}2 \text { CFम } \\
\text { ol }\end{array}$ & $2 \pm .1 \mathrm{CFH}$ & A & \\
\hline
\end{tabular}

all $11 \cdot 5 \cdot 94$ 
APPENDIX A

TEST DATA SHEET

\begin{tabular}{|c|c|c|c|c|c|}
\hline \multicolumn{3}{|c|}{ Date: $11-5.96$} & \multicolumn{3}{|c|}{ SHMS Unit Number: VTP-PNL - $105 A$} \\
\hline \multicolumn{3}{|c|}{$\begin{array}{l}\text { Title of Test: } \\
\text { Pneumatic Systems }\end{array}$} & \multirow{2}{*}{\multicolumn{3}{|c|}{ 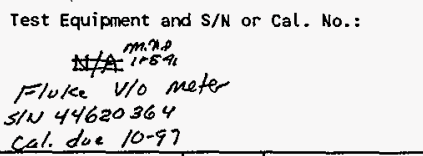 }} \\
\hline \multicolumn{3}{|c|}{ 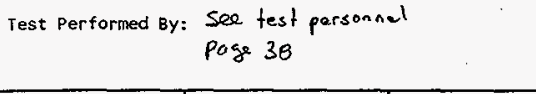 } & & & \\
\hline $\begin{array}{l}\text { Procedure No. } \\
\text { Initial/Date }\end{array}$ & I tem & value & $\begin{array}{l}\text { Required } \\
\text { Condition }\end{array}$ & $(A / R)$ & Comment \\
\hline $\begin{array}{l}1.7 .4 .4 .10 \\
2 \pi .3 \\
\end{array}$ & $\begin{array}{l}\text { VERIFY } \\
\text { CONDITIONS }\end{array}$ & 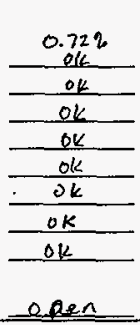 & $\begin{array}{l}\text { NIT-*545S } 12.2 \\
>.625 \% \mathrm{H}_{2} \\
\text { NAH-*55 ON } \\
\text { NAH-*54 ON } \\
\text { PBL-*59 ON } \\
\text { PBL-*58 ON } \\
\text { YAL-*58 ON } \\
\text { FIV-*52 FLO } \\
\text { HORN ON } \\
\text { TB2-21/22 } \\
\text { OPEN }\end{array}$ & A & \\
\hline $\begin{array}{l}1.7 .4 .4 .11 \\
2 \times 1.8 \\
\end{array}$ & $\begin{array}{l}\text { ACKNOWLEDGE } \\
\text { HORN }\end{array}$ & $N / A$ & $N / A$ & $A$ & \\
\hline $\begin{array}{l}1.7 .4 .4 .12 \\
21.8 .8 \\
\end{array}$ & $\begin{array}{l}\text { CLOSE SV-* } 19 \\
\text { VERIFY } \\
\text { CONDITIONS }\end{array}$ & 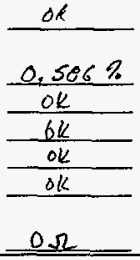 & $\begin{array}{l}\text { FIV-*56 } \\
\text { NIT-*5455 } \\
<.625 \% \mathrm{H}_{32} \\
\text { NAH-*55 OFF } \\
\text { NAH-*54 ON } \\
\text { PBL-*58 ON } \\
\text { YAL-*58 ON } \\
\text { TB2-21/22 } \\
\text { CLOSED }\end{array}$ & * & \\
\hline $\begin{array}{l}1.7 .4 .4 .13 \\
2 \times 1.3 .3 \\
\end{array}$ & $\begin{array}{l}\text { PUSH RESET } \\
\text { SAMPLER PB- } \\
* 58\end{array}$ & $-\frac{o k}{o k}$ & $\begin{array}{l}\text { PBL-*58 OFF } \\
\text { YAL }-* 58 \text { OFF }\end{array}$ & A & \\
\hline $\begin{array}{l}1.7 .4 .4 .14 \\
2 m .3 .1 \\
\end{array}$ & RESET ALARM & - ok & $\mathrm{NAH}-* 54$ OFF & A & \\
\hline $\begin{array}{l}1.7 .4 .4 .15 \\
91.2 .8 \\
\end{array}$ & $\begin{array}{l}\text { DISCONNECT } \\
\text { CAL. GAS }\end{array}$ & $N / A$ & $N / A$ & A & \\
\hline $\begin{array}{l}1.7 .4 .4 .16 \\
\text { m.x }\end{array}$ & $\begin{array}{l}\text { PUSH PB }-* 54 \\
\text { VERIFY } \\
\text { CONDITIONS }\end{array}$ & $\begin{array}{r}-O K \\
O K \\
\text { OPON } \\
\end{array}$ & $\begin{array}{l}\text { XA-*63 ON } \\
\text { PBL }-* 54 \text { ON } \\
\text { TB2-23/24 } \\
\text { OPEN }\end{array}$ & $A$ & \\
\hline $\begin{array}{l}1.7 .4 .4 .17 \\
\text { mas } \\
\end{array}$ & $\begin{array}{l}\text { PUSH PB-*54 } \\
\text { VERIFY } \\
\text { CONDITIONS }\end{array}$ & $\begin{array}{l}\frac{o k}{o k} \\
0 \Omega\end{array}$ & $\begin{array}{l}\text { XA-*63 OFF } \\
\text { PBL-*54 OFF } \\
\text { TB2-23/24 } \\
\text { CLOSED }\end{array}$ & A & \\
\hline
\end{tabular}




\section{TEST DATA SHEET}

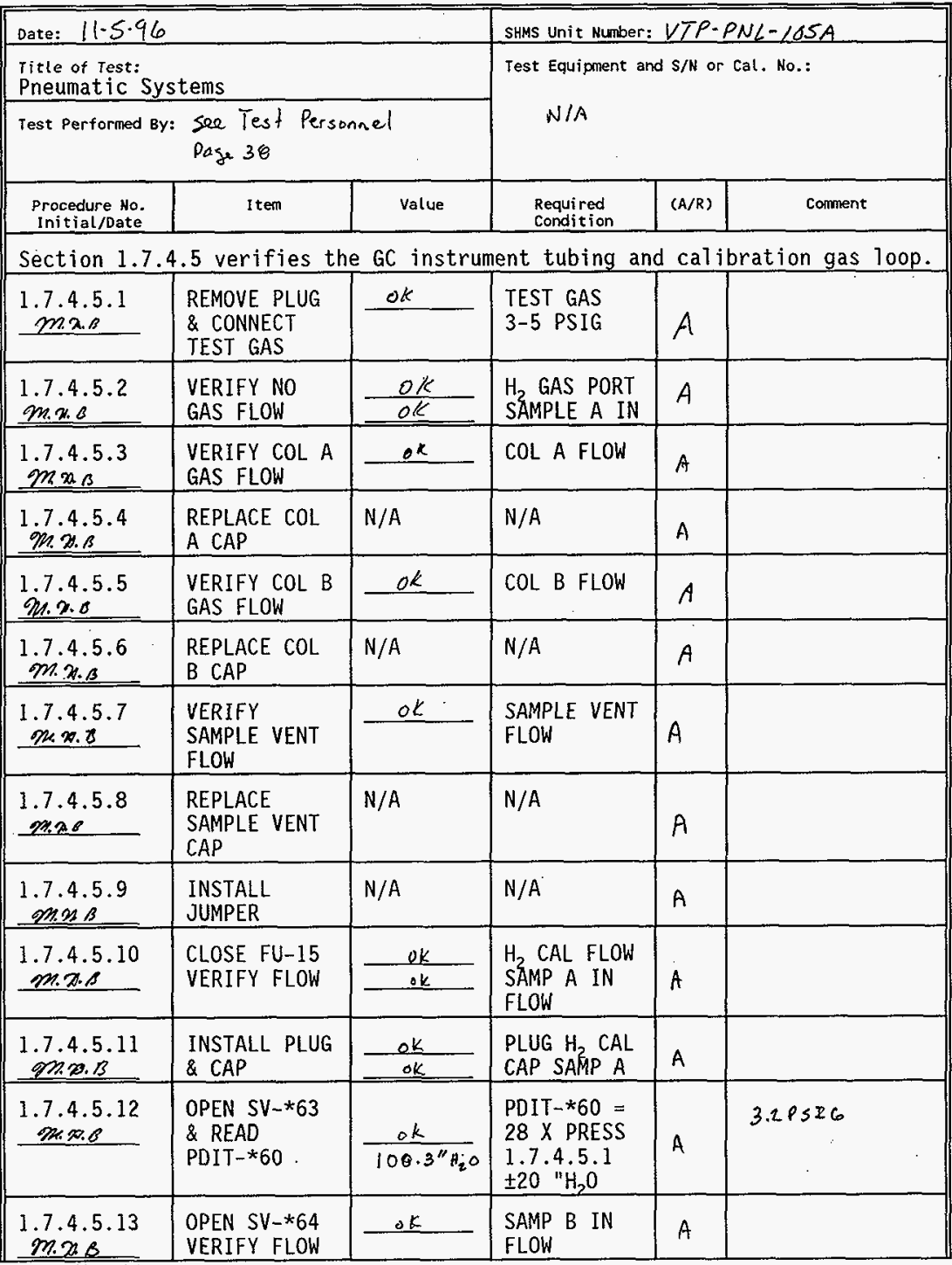


TEST DATA SHEET

\begin{tabular}{|c|c|c|c|c|c|}
\hline \multicolumn{3}{|l|}{ Date: $11.5 \cdot 96$} & \multicolumn{3}{|c|}{ SHMS Unit Number: VTP-PNL-10.5A } \\
\hline \multicolumn{3}{|c|}{$\begin{array}{l}\text { Title of Test: } \\
\text { Pneumatic Systems }\end{array}$} & \multirow{2}{*}{\multicolumn{3}{|c|}{$\begin{array}{l}\text { Test Equipment and } S / N \text { or Cal. No.: } \\
\text { N/A }\end{array}$}} \\
\hline \multicolumn{3}{|c|}{ 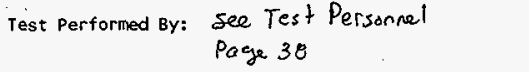 } & & & \\
\hline $\begin{array}{l}\text { Procedure No. } \\
\text { Initial/Date }\end{array}$ & I tem & Value & $\begin{array}{l}\text { Required } \\
\text { Condition }\end{array}$ & $(A / R)$ & Comment \\
\hline $\begin{array}{l}1.7 .4 .5 .14 \\
2 \text { m.x.B. } \\
\end{array}$ & $\begin{array}{l}\text { REMOVE TEST } \\
\text { GAS }\end{array}$ & $N / A$ & $N / A$ & A & \\
\hline $\begin{array}{l}1.7 .4 .5 .15 \\
M .0 .0 \\
\end{array}$ & $\begin{array}{l}\text { CLOSE SV }-* 63 \\
\& \text { SV }-* 64\end{array}$ & $N / A$ & $N / A$ & $A$ & \\
\hline $\begin{array}{l}1.7 .4 .5 .16 \\
2020 \\
\end{array}$ & $\begin{array}{l}\text { OPEN FU-15 } \\
\text { REMOVE } \\
\text { JUMPER }\end{array}$ & $N / A$ & $N / A$ & $A$ & \\
\hline $\begin{array}{l}1.7 .4 .5 .17 \\
\text { sp.X.3. } \\
\end{array}$ & $\begin{array}{l}\text { INSTALL } \\
\text { SAMP A UNION }\end{array}$ & $N / A$ & $N / A$ & A & \\
\hline $\begin{array}{l}1.7 .4 .5 .18 \\
\text { m.3.1. } \\
\end{array}$ & $\begin{array}{l}\text { OPEN SV }-* 60 \\
\text { VERIFY FLOW }\end{array}$ & ok & $\begin{array}{l}\text { FI }-\star 60 \\
\text { NO FLOW }\end{array}$ & A & \\
\hline $\begin{array}{l}1.7 .4 .5 .19 \\
-23 B \\
\end{array}$ & $\begin{array}{l}\text { POWER SOV- } \\
* 60 \text {, VERIFY } \\
\text { NO FLOW }\end{array}$ & $O K$ & $\begin{array}{l}\text { FI }-\star 60 \\
\text { NO FLOW }\end{array}$ & A & \\
\hline $\begin{array}{l}1.7 .4 .5 .20 \\
\text { Ox. } 3.8\end{array}$ & $\begin{array}{l}\text { OPEN SV-*68 } \\
\text { ADJ SV-*67 }\end{array}$ & $50 \mathrm{ccm}$ & $\begin{array}{l}\mathrm{FI}-* 60 \\
50 \mathrm{CCM}\end{array}$ & A & \\
\hline $\begin{array}{l}1.7 .4 .5 .21 \\
m_{1} x_{3} \\
\end{array}$ & $\begin{array}{l}\text { DEENERGIZE } \\
\text { SOV }-* 60\end{array}$ & ok & $\begin{array}{l}\text { FI-*60 FLOW } \\
\text { DECREASES }\end{array}$ & A & \\
\hline $\begin{array}{l}1.7 .4 .5 .22 \\
9.20 \\
\end{array}$ & $\begin{array}{l}\text { CLOSE SV-*60 } \\
\& \text { SV-*68 }\end{array}$ & $N / A$ & $N / A$ & A & \\
\hline $\begin{array}{l}1.7 .4 .5 .23 \\
x B \\
\end{array}$ & $\begin{array}{l}\text { REMOVE } \\
\text { SAMP A UNION }\end{array}$ & $N / A$ & $N / A$ & A & \\
\hline $\begin{array}{l}1.7 .4 .5 .24 \\
92.20 .3 \\
\end{array}$ & $\begin{array}{l}\text { CONNECT TEST } \\
\text { GAS } N_{2} 0 \text { PORT } \\
\end{array}$ & $O K$ & $\begin{array}{l}\text { TEST GAS } \\
3-5 \text { PSIG } \\
\end{array}$ & $A$ & \\
\hline $\begin{array}{l}1.7 .4 .5 .25 \\
M .2 .3 \\
\end{array}$ & $\begin{array}{l}\text { VERIFY NO } \\
\text { FLOW }\end{array}$ & $\frac{\text { ok }}{\text { ok }}$ & $\begin{array}{l}\text { SAMP B IN \& } \\
\text { FLT-*63 } \\
\text { NO FLOW }\end{array}$ & A & \\
\hline $\begin{array}{l}1.7 .4 .5 .26 \\
\mathrm{PMBOB}_{3} \\
\end{array}$ & $\begin{array}{l}\text { INSTALL } \\
\text { JUMPER }\end{array}$ & $N / A$ & $N / A$ & $A$ & \\
\hline $\begin{array}{l}1.7 .4 .5 .27 \\
\not 2 \pi B \text { B } \\
\end{array}$ & $\begin{array}{l}\text { CLOSE FU-15 } \\
\text { VERIFY FLOW }\end{array}$ & $\frac{O K}{\Delta K}$ & $\begin{array}{l}\text { SAMP B IN } \\
\text { FLT } * 63 \\
\text { FLOW }\end{array}$ & A & \\
\hline
\end{tabular}




\section{TEST DATA SHEET}

\begin{tabular}{|c|c|c|c|c|c|}
\hline \multicolumn{3}{|l|}{ Date: $11-5.96$} & \multicolumn{3}{|c|}{ SHMS Unit Number: VTP-PNL-105A } \\
\hline \multicolumn{3}{|c|}{$\begin{array}{l}\text { Title of rest: } \\
\text { Pneumatic Systems }\end{array}$} & \multirow{2}{*}{\multicolumn{3}{|c|}{$\begin{array}{l}\text { Test Equipment and } \mathrm{s} / \mathrm{N} \text { or Cal. No.: } \\
\text { N/A }\end{array}$}} \\
\hline \multicolumn{3}{|c|}{$\begin{array}{c}\text { Test Performed By: see Test Personnel } \\
\text { pag } 38\end{array}$} & & & \\
\hline $\begin{array}{l}\text { Procedure No. } \\
\text { Initial/Date }\end{array}$ & Item & Value & $\begin{array}{l}\text { Required } \\
\text { Condition } \\
\end{array}$ & $(A / R)$ & Comment \\
\hline $\begin{array}{l}1.7 .4 .5 .28 \\
M .2 .8 \\
\end{array}$ & $\begin{array}{l}\text { DISCONNECT } \\
\text { TEST GAS } \\
\end{array}$ & $N / A$ & $N / A$ & A & \\
\hline $\begin{array}{l}1.7 .4 .5 .29 \\
\operatorname{man} \times B .8 \\
\end{array}$ & OPEN FU-15 & $N / A$ & $N / A$ & A & \\
\hline $\begin{array}{l}1.7 .4 .5 .30 \\
m \times \cdot B \\
\end{array}$ & $\begin{array}{l}\text { REMOVE } \\
\text { JUMPER }\end{array}$ & $N / A$ & $N / A$ & $A$ & \\
\hline $\begin{array}{l}1.7 .4 .5 .31 \\
9 x \cdot B \cdot B \\
\end{array}$ & $\begin{array}{l}\text { INSTALL } \\
\text { SAMP B UNION }\end{array}$ & $N / A$ & N/A & $A$ & \\
\hline $\begin{array}{l}1.7 .4 .5 .32 \\
0 \times 3 \times 13 \\
\end{array}$ & $\begin{array}{l}\text { OPEN SV }-* 60 \\
\text { VERIFY FLOW }\end{array}$ & $o k$ & $\begin{array}{l}\text { FI-*60 } \\
\text { NO FLOW }\end{array}$ & A & \\
\hline $\begin{array}{l}1.7 .4 .5 .33 \\
2.3 . \beta \\
\end{array}$ & $\begin{array}{l}\text { POWER SON- } \\
* 60, \text { VERIFY } \\
\text { NO FLOW }\end{array}$ & DK. & $\begin{array}{l}\text { FI }-* 60 \\
\text { NO FLOW }\end{array}$ & A & \\
\hline $\begin{array}{l}1.7 .4 .5 .34 \\
2.2 .8 \\
\end{array}$ & $\begin{array}{l}\text { OPEN SV } * 68 \\
\& \text { ADJ SV-*67 }\end{array}$ & $50 \mathrm{cem}$ & $\begin{array}{l}\mathrm{FI}-* 60 \\
50 \mathrm{CCM} \\
\end{array}$ & $A$ & \\
\hline 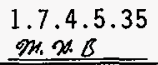 & $\begin{array}{l}\text { DEENERGIZE } \\
\text { SOV }-\star 60\end{array}$ & ok & $\begin{array}{l}\text { FI-*60 FLOW } \\
\text { DECREASES }\end{array}$ & A & \\
\hline $\begin{array}{l}1.7 .4 .5 .36 \\
X B \\
\end{array}$ & $\begin{array}{l}\text { CLOSE SV }-* 60 \\
\& \text { SV }-* 68\end{array}$ & $N / A$ & $N / A$ & A & \\
\hline $\begin{array}{l}1.7 .4 .5 .37 \\
3 x . x 6 \\
\end{array}$ & $\begin{array}{l}\text { REMOVE } \\
\text { SAMP B UNION }\end{array}$ & $N / A$ & $N / A$ & $A$ & \\
\hline $\begin{array}{l}1.7 .4 .5 .38 \\
\text { Mx. } 3.8 \\
\end{array}$ & $\begin{array}{l}\text { OPEN CB-3/4 } \\
\text { ACK. ALARMS }\end{array}$ & $N / A$ & $N / A$ & $A$ & \\
\hline \multicolumn{6}{|c|}{$\begin{array}{l}\text { Section } 1.7 .4 .6 \text { verifies the multi gas analyzer instrument tubing and } \\
\text { calibration gas loop. }\end{array}$} \\
\hline $\begin{array}{l}1.7 .4 .6 .1 \\
\\
\end{array}$ & $\begin{array}{l}\text { VERIFY } \\
\text { NIT * } 52 \\
\text { SAMPLE LINES }\end{array}$ & $N / A$ & $N / A$ & A & \\
\hline $\begin{array}{l}1.7 .4 .6 .2 \\
M .2 .8 \\
\end{array}$ & $\begin{array}{l}\text { CONNECT TEST } \\
\text { GAS NH } \text { NORT }_{3} \text { POR }\end{array}$ & $o k$ & $\begin{array}{l}\text { TEST GAS } \\
3-5 \text { PSIG }\end{array}$ & A & \\
\hline $\begin{array}{l}1.7 .4 .6 .3 \\
\text { \#H. }_{0} .8\end{array}$ & $\begin{array}{l}\text { VERIFY NO } \\
\text { FLOW }\end{array}$ & ok & $\begin{array}{l}\text { SAMPLE IN } \\
\text { FLT-*63 }\end{array}$ & A & \\
\hline
\end{tabular}


APPENDIX A

TEST DATA SHEET

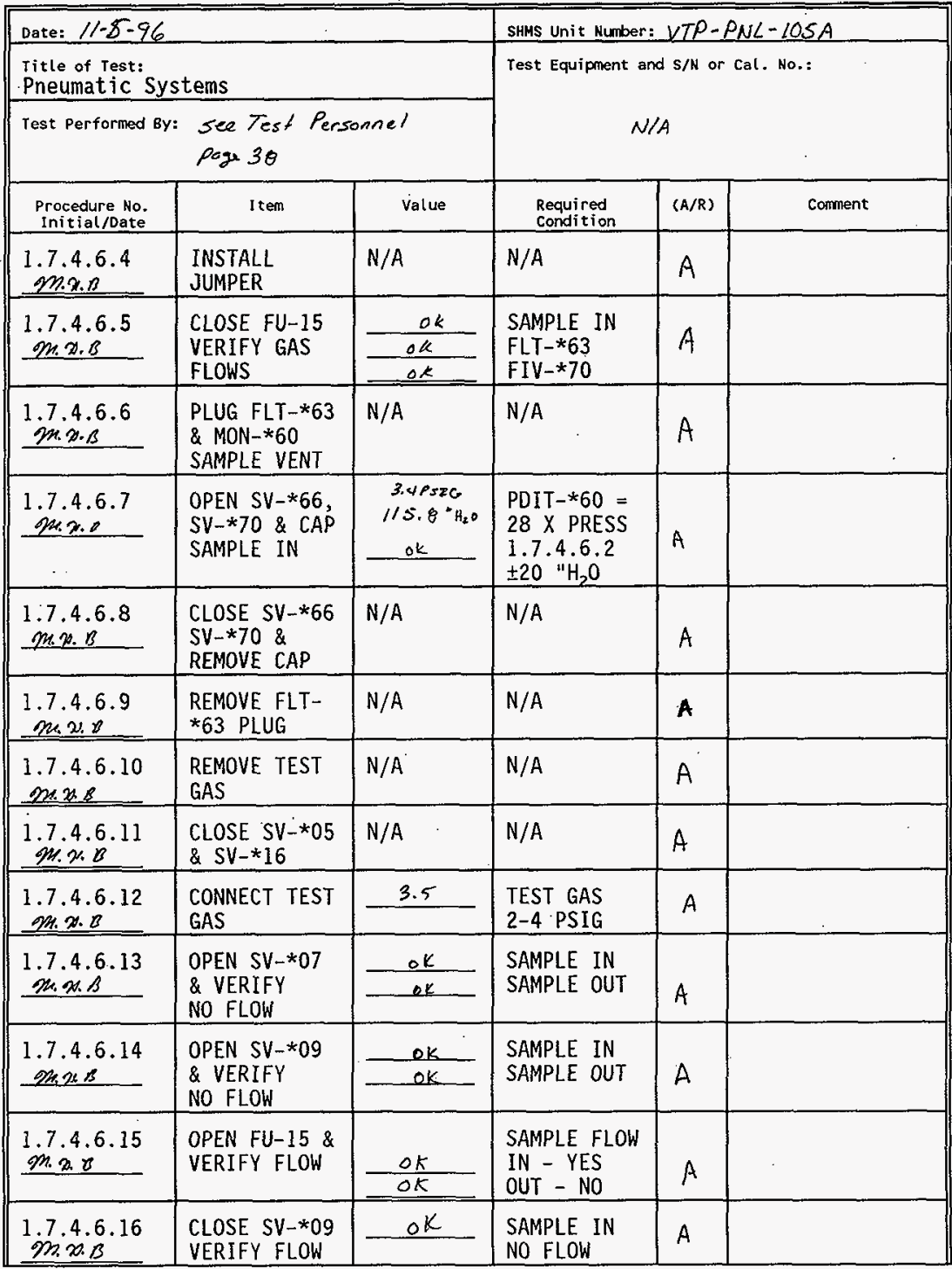


TEST DATA SHEET

\begin{tabular}{|c|c|c|c|c|c|}
\hline \multicolumn{3}{|l|}{ Date: $11.5 \cdot 96$} & \multicolumn{3}{|c|}{ SHMS Unit Number: $V T P-P N L-10 S A$} \\
\hline \multicolumn{3}{|c|}{$\begin{array}{l}\text { Title of Test: } \\
\text { Pneumatic Systems }\end{array}$} & \multirow{2}{*}{\multicolumn{3}{|c|}{$\begin{array}{l}\text { Test Equipment and } S / N \text { or Cal. No.: } \\
\text { N/A }\end{array}$}} \\
\hline \multicolumn{3}{|c|}{$\begin{aligned} & \text { Test Performed By: } \text { see Test Personnel } \\
& \text { Page } 30\end{aligned}$} & & & \\
\hline $\begin{array}{l}\text { Procedure No. } \\
\text { Initial/Date }\end{array}$ & Item & Value & $\begin{array}{l}\text { Required } \\
\text { Condition }\end{array}$ & $(A / R)$ & Comment \\
\hline $\begin{array}{l}1.7 .4 .6 .17 \\
\text { m.3.B. }\end{array}$ & $\begin{array}{l}\text { OPEN SV-*10 } \\
\text { VERIFY FLOW }\end{array}$ & $\frac{O K}{O K}$ & $\begin{array}{l}\text { SAMPLE FLOW } \\
\text { IN - NO } \\
\text { OUT - YES }\end{array}$ & A & \\
\hline $\begin{array}{l}1.7 .4 .6 .18 \\
\text { m.x.n } \\
\end{array}$ & $\begin{array}{l}\text { REMOVE TEST } \\
\text { GAS \& PLUG } \\
\text { SV }-* 07\end{array}$ & $N / A$ & $N / A$ & A & \\
\hline $\begin{array}{l}1.7 .4 .6 .19 \\
\text { m.x.8 }\end{array}$ & $\begin{array}{l}\text { CLOSE SV-*07 } \\
\& \text { SV }-* 10\end{array}$ & $N / A$ & N/A & $A$ & \\
\hline $\begin{array}{l}1.7 .4 .6 .20 \\
m .31 .13\end{array}$ & $\begin{array}{l}\text { OPEN SV-*08 } \\
\& \text { SV-* } 16\end{array}$ & $N / A$ & $N / A$ & A & v \\
\hline $\begin{array}{l}1.7 .4 .6 .21 \\
m .2 .8 \\
\end{array}$ & $\begin{array}{l}\text { REMOVE } \\
\text { JUMPER }\end{array}$ & N/A & $N / A$ & $A$ & \\
\hline \multicolumn{6}{|c|}{ Section 1.7.4.7 verifies the carrier gas supply lines. } \\
\hline $\begin{array}{l}1.7 .4 .7 .1 \\
\end{array}$ & $\begin{array}{l}\text { CONNECT TEST } \\
\text { GAS }\end{array}$ & $3.5 P I_{6}$ & 3-5 PSIG & A & \\
\hline $\begin{array}{l}1.7 .4 .7 .2 \\
m .8\end{array}$ & $\begin{array}{l}\text { REMOVE PLUG } \\
\& \text { VERIFY } \\
\text { FLOW } \\
\end{array}$ & $O K$ & $\begin{array}{l}N_{2} \text { CARRIER } \\
\text { GASS FLOWS }\end{array}$ & A & \\
\hline $\begin{array}{l}1.7 .4 .7 .3 \\
m .2 .8 \\
\end{array}$ & $\begin{array}{l}\text { DISCONNECT } \\
\text { TEST GAS } \\
\end{array}$ & $\mathrm{N} / \mathrm{A}$ & $N / A$ & A & \\
\hline $\begin{array}{l}1.7 .4 .7 .4 \\
m .7 .8 \\
\end{array}$ & $\begin{array}{l}\text { CONNECT TEST } \\
\text { GAS }\end{array}$ & 3.SPSIG & 3-5 PSIG & A & \\
\hline $\begin{array}{l}1.7 .4 .7 .5 \\
m .20 .8 \\
\end{array}$ & $\begin{array}{l}\text { REMOVE PLUG } \\
\text { \& VERIFY } \\
\text { FLOW }\end{array}$ & ok & $\begin{array}{l}\text { He CARRIER } \\
\text { GAS FLOWS }\end{array}$ & A & \\
\hline $\begin{array}{l}1.7 .4 .7 .6 \\
m .2 .0 \\
\end{array}$ & $\begin{array}{l}\text { DISCONNECT } \\
\text { TEST GAS }\end{array}$ & $N / A$ & $N / A$ & A & \\
\hline $\begin{array}{l}1.7 .5 \\
m .2 .8\end{array}$ & $\begin{array}{l}\text { TEST SEC. } \\
1.7 .4 \text { DONE }\end{array}$ & $\frac{10}{\text { Test Direc }}$ & $\begin{array}{l}\text { Ohaved } \\
\text { or Signature }\end{array}$ & & $\frac{-8-86}{\text { Date }}$ \\
\hline
\end{tabular}

all 11-5-96 
APPENDIX A

TEST DATA SHEET

\begin{tabular}{|c|c|c|c|c|c|}
\hline Date: $\| 1-8$. & & & SHMS Unit Hunt & VTP & $O S A$ \\
\hline $\begin{array}{l}\text { Title of Test: } \\
\text { Pneumatic } S\end{array}$ & tems & & Test Equipment & $\mathrm{S} / \mathrm{N}$ & \\
\hline Test Perforned & $\begin{array}{l}\text { see Test Per } \\
\text { Page } 38\end{array}$ & mel & $N / A$ & & \\
\hline $\begin{array}{l}\text { Procedure No. } \\
\text { Initial/Date }\end{array}$ & Item & value & $\begin{array}{l}\text { Required } \\
\text { Condition }\end{array}$ & $(A / R)$ & Comment \\
\hline $\begin{array}{l}1.7 .6 \\
9 m .21 . B .\end{array}$ & $\begin{array}{l}\text { THIS SECTION } \\
\text { IF, NOT }\end{array}$ & $\begin{array}{l}S \text { ONLY FOR } \\
\text { BLE, N/A S } \\
\text { Se } \\
\text { Signature }\end{array}$ & $\begin{array}{l}\text { THE SHMS-E } \\
\text { EPS AND TES }\end{array}$ & $\begin{array}{l}\text { THE } \\
\text { IREC } \\
\text { Con. } \\
\text { Date } \\
\end{array}$ & $\begin{array}{l}\text { CAL MODE. } \\
\text { L SIGN. }\end{array}$ \\
\hline 1.7 .6 .1 .1 & $\begin{array}{l}\text { VERIFY BKR } \\
\text { LINE UP }\end{array}$ & $N / A$ & $N / A$ & A & \\
\hline $\begin{array}{l}1.7 .6 .1 .2 \\
m .2 .0^{2}\end{array}$ & $\begin{array}{l}\text { VERIFY VALVE } \\
\text { LINE UP }:\end{array}$ & $N / A$ & $N / A$ & A & \\
\hline$\underset{\substack{1.7 .6 .0\\
}}{1.6 .1 .3}$ & $\begin{array}{l}\text { VERIFY MON- } \\
\text { *60 INSTALL }\end{array}$ & $N / A$ & $N / A$ & $A$ & \\
\hline $\begin{array}{l}1.7 .6 .1 .4 \\
\text { mone }\end{array}$ & $\begin{array}{l}\text { VERIFY NIT- } \\
\text { *52 INSTALL }\end{array}$ & $N / A$ & $N / A$ & $A$ & \\
\hline $\begin{array}{c}1.7 .6 .1 .5 \\
\text { m.x. }\end{array}$ & $\begin{array}{l}\text { CONNECT } \mathrm{H}_{2} \\
\text { CAL GAS }\end{array}$ & $N / A$ & $N / A$ & $A$ & \\
\hline $\begin{array}{c}1.7 .6 .1 .6 \\
\text { m.2.3. }\end{array}$ & $\begin{array}{l}\text { CONNECT } \mathrm{CH}_{4} \\
\text { CAL GAS }\end{array}$ & $N / A$ & $N / A$ & A & \\
\hline $\begin{array}{l}1.7 .6 .1 .7 \\
\operatorname{mon} 3\end{array}$ & $\begin{array}{l}\text { CONNECT } \mathrm{NH}_{3} \\
\text { CAL GAS }\end{array}$ & $N / A$ & $N / A$ & $A$ & \\
\hline Section 1.7 & 2 establishes & the main a & d auxiliary & op $f$ & \\
\hline $\begin{array}{l}1.7 .6 .2 .1 \\
\text { m.m.0 }\end{array}$ & CLOSE FU-11 & ok & $\begin{array}{l}\text { MON-*60 } \\
\text { POWERED }\end{array}$ & $A$ & \\
\hline $\begin{array}{l}1.7 .6 .2 .2 \\
m . x .0^{2}\end{array}$ & POWER PC $-* 60$ & ok & $\begin{array}{l}\text { ESTABLISH } \\
\text { SAMPLING } \\
\text { ROUTINE }\end{array}$ & $A$ & \\
\hline $\begin{array}{l}1.7 .6 .2 .3 \\
\text { mm..8 } \\
\end{array}$ & CLOSE FU-12 & _. & $\begin{array}{l}\text { NIT-*52 } \\
\text { POWERED }\end{array}$ & $A$ & \\
\hline $\begin{array}{c}1.7 .6 .2 .4 \\
\operatorname{mos} \\
\end{array}$ & POWER PC -*70 & - ex & $\begin{array}{l}P C-* 70 \\
\text { BOOTED UP }\end{array}$ & $A$ & \\
\hline$\underset{m_{2.0}}{1.7 .6 .2 .5}$ & $\begin{array}{l}\text { CLOSE CB-3/4 } \\
\text { ADJUST FLOW }\end{array}$ & $\frac{0 k}{1.0{ }^{4} \mathrm{H}_{2} \mathrm{O}}$ & $\begin{array}{l}\mathrm{FIT}-* 57 \\
1 \pm .1 \mathrm{H} \mathrm{H}_{2} \mathrm{l}\end{array}$ & $A$ & \\
\hline
\end{tabular}


TEST DATA SHEET

\begin{tabular}{|c|c|c|c|c|c|}
\hline \multicolumn{3}{|l|}{ Date: $11-8-96$} & \multicolumn{3}{|c|}{ SHMS Unit Number: VTP - PNL - 105A } \\
\hline \multicolumn{3}{|c|}{$\begin{array}{l}\text { Title of Test: } \\
\text { Pneumatic Systems }\end{array}$} & \multirow{2}{*}{\multicolumn{3}{|c|}{$\begin{array}{l}\text { Test Equipment and } S / \mathrm{N} \text { or Cal. No.: } \\
\text { N/A }\end{array}$}} \\
\hline \multicolumn{3}{|c|}{$\begin{array}{c}\text { Test Performed By: see Test Personnel } \\
\text { Page } 36\end{array}$} & & & \\
\hline $\begin{array}{l}\text { Procedure No. } \\
\text { Initial/Date }\end{array}$ & Item & value & $\begin{array}{l}\text { Required } \\
\text { Condition }\end{array}$ & $(A / R)$ & Comment \\
\hline $\begin{array}{c}1.7 .6 .2 .6 \\
\text { m.x. }\end{array}$ & $\begin{array}{l}\text { OPEN SV-*09 } \\
\& \text { SV-*10 }\end{array}$ & $N / A$ & $N / A$ & $A$ & \\
\hline $\begin{array}{l}1.7 .6 .2 .7 \\
m .8 .6 \\
\end{array}$ & $\begin{array}{l}\text { OPEN SV-*60, } \\
63,65 \& 68\end{array}$ & $N / A$ & $N / A$ & $A$ & \\
\hline $\begin{array}{l}1.7 .6 .2 .8 \\
n .7 .3 \\
\end{array}$ & NIT-*52 & ok & $\begin{array}{l}\text { ESTABLISH } \\
\text { SAMPLING } \\
\text { ROUTINE } \\
\end{array}$ & $A$ & \\
\hline $\begin{array}{l}1.7 .6 .2 .9 \\
\text { m.x.e. } \\
\end{array}$ & $\begin{array}{l}\text { OPEN SV }-* 24 \\
\text { ADJ FLOW }\end{array}$ & $2.9^{*} \mathrm{H}, 0$ & $\begin{array}{l}\mathrm{FIT}-* 57 \\
3 \pm .1 " \mathrm{H}_{2} \mathrm{O} \\
\end{array}$ & A & \\
\hline $\begin{array}{l}1.7 .6 .2 .10 \\
m . y .0 \\
\end{array}$ & $\begin{array}{l}\text { ADJ FLOW } \\
\text { SV-*67 }\end{array}$ & $30 \mathrm{ccm}$ & $\begin{array}{l}\mathrm{FI}-* 60 \\
5-10 \mathrm{ccm}\end{array}$ & & $\begin{array}{l}4 \sec \text { exceetstsen } 22 \\
E C N 634645\end{array}$ \\
\hline $\begin{array}{l}1.7 .6 .2 .11 \\
\text { m.x.B. } \\
\end{array}$ & $\begin{array}{l}\text { RECORD } \\
\text { PDIT-*60 }\end{array}$ & $135.1 " \mathrm{H}_{20}$ & $\begin{array}{l}100-250 \\
" \mathrm{H}_{2} \mathrm{O}\end{array}$ & $A$ & ECN634645 \\
\hline $\begin{array}{l}1.7 .6 .2 .12 \\
m x .0 \\
\end{array}$ & $\begin{array}{l}\text { OPEN SV }-* 64 \\
\text { CLOSE SV }-* 63\end{array}$ & $N / A$ & $N / A$ & $A$ & \\
\hline $\begin{array}{l}1.7 .6 .2 .13 \\
m \times .8 \\
\end{array}$ & $\begin{array}{l}\text { RECORD } \\
\text { PDIT-*60 }\end{array}$ & $136.1 \% 140$ & $\begin{array}{l}100-250 \\
1 " \mathrm{H}_{2} \mathrm{O}\end{array}$ & $A$ & EeN 634645 \\
\hline $\begin{array}{l}1.7 .6 .2 .14 \\
m .2 .1 \\
\end{array}$ & $\begin{array}{l}\text { CLOSE SV-*65 } \\
\text { OPEN SV-*66 }\end{array}$ & $N / A$ & $N / A$ & $A$ & \\
\hline $\begin{array}{l}1.7 .6 .2 .15 \\
\text { gm.x. } \\
\end{array}$ & $\begin{array}{l}\text { RECORD B IN } \\
\text { PDIT }-* 60\end{array}$ & $=28,00^{\circ} \mathrm{H}=0$ & 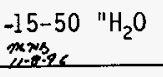 & $A$ & ECN 634645 \\
\hline $\begin{array}{l}1.7 .6 .2 .16 \\
\text { m.2.x. } \\
\end{array}$ & $\begin{array}{l}\text { OPEN SV }-* 63 \\
\text { CLOSE SV-*64 }\end{array}$ & $N / A$ & $N / A$ & A & \\
\hline $\begin{array}{l}1.7 .6 .2 .17 \\
m \times . \beta \\
\end{array}$ & $\begin{array}{l}\text { RECORD A IN } \\
\text { PDIT-*60 }\end{array}$ & $.28 .50 \%$ & 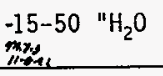 & $A$ & ECN 634645 \\
\hline $\begin{array}{l}1.7 .6 .2 .18 \\
\text { m.n.p } \\
\end{array}$ & $\begin{array}{l}\text { INSTALL } \\
\text { JUMPER } \\
\end{array}$ & $N / A$ & $N / A$ & A & \\
\hline $\begin{array}{l}1.7 .6 .2 .19 \\
\text { m.2. } \\
\end{array}$ & CLOSE FU-15 & $N / A$ & $N / A$ & $A$ & \\
\hline $\begin{array}{l}1.7 .6 .2 .20 \\
\operatorname{mon}^{\circ} B \\
\end{array}$ & $\begin{array}{l}\text { ADJ SV-*61 \& } \\
\text { RECORD } \\
\text { PDIT-*60 }\end{array}$ & $-27.80^{\prime \prime} \mathrm{H}^{\circ}$ & $\begin{array}{l} \pm 5^{\prime \prime} \mathrm{H}_{2} \mathrm{O} \text { of } \\
1.7 .6 .2 .17\end{array}$ & A & \\
\hline
\end{tabular}




\section{TEST DATA SHEET}

\begin{tabular}{|c|c|c|c|c|c|}
\hline \multicolumn{3}{|c|}{ Date: $11-8 \cdot 96$} & \multicolumn{3}{|c|}{ SHMS Unit Number: VTP - PNL - $105 \mathrm{~A}$} \\
\hline \multicolumn{3}{|c|}{$\begin{array}{l}\text { Title of Test: } \\
\text { Pneumatic Systems }\end{array}$} & \multirow{2}{*}{\multicolumn{3}{|c|}{ 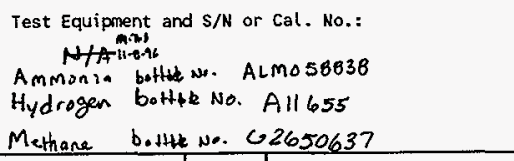 }} \\
\hline \multicolumn{3}{|c|}{$\begin{aligned} \text { Test Performed By: } & \text { See Test Personnel } \\
& \text { Page } 38\end{aligned}$} & & & \\
\hline $\begin{array}{l}\text { Procedure Ho. } \\
\text { initial/Date }\end{array}$ & Item & value & $\begin{array}{l}\text { Required } \\
\text { Condition }\end{array}$ & $(A / R)$ & Coment \\
\hline$\frac{1.7 .6 .2 .21}{m \cdot 2 \cdot s^{2}}$ & $\begin{array}{l}\text { CAL MON-*60 } \\
\text { CHANNEL A }\end{array}$ & $\begin{array}{l}10440.42 \mathrm{pPM} \\
10423.36 \mathrm{PPm} \\
10498.65 \mathrm{PPM}\end{array}$ & $\begin{array}{l}\text { READINGS } \\
\text { WITHIN } \pm 5 \%\end{array}$ & A & $\begin{array}{l}10,500 \mathrm{PPm} \\
\text { Cal. Gas } \\
\text { Hydrogem }\end{array}$ \\
\hline $\begin{array}{l}1.7 .6 .2 .22 \\
\text { m.p.o. } \\
\end{array}$ & OPEN FU-15 & $N / A$ & N/A & $A$ & \\
\hline $\begin{array}{l}1.7 .6 .2 .23 \\
\text { m.x.s } \\
\end{array}$ & $\begin{array}{l}\text { REMOVE } \\
\text { JUMPER }\end{array}$ & $N / A$ & N/A & $A$ & \\
\hline $\begin{array}{l}1.7 .6 .2 .24 \\
\text { m.x.0. }\end{array}$ & $\begin{array}{l}\text { OPEN SV }-* 64 \\
\text { CLOSE SV-*63 }\end{array}$ & N/A & N/A & A & \\
\hline $\begin{array}{l}1.7 .6 .2 .25 \\
m .2 .8 \\
\end{array}$ & $\begin{array}{l}\text { INSTALL } \\
\text { JUMPER }\end{array}$ & $N / A$ & N/A & A & \\
\hline $\begin{array}{l}1.7 .6 .2 .26 \\
\text { mis.2.s } \\
\end{array}$ & CLOSE FU-15 & N/A & N/A & A & \\
\hline $\begin{array}{l}1.7 .6 .2 .27 \\
2 M .2 . B \\
\end{array}$ & $\begin{array}{l}\text { ADJ SV }-* 62 \& \\
\text { RECORD } \\
\text { PDIT-*60 }\end{array}$ & $-26.6^{\prime \prime} \mathrm{H}_{6} \mathrm{O}$ & 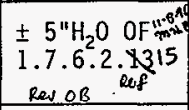 & A & $\begin{array}{l}10,000 \mathrm{PPM} \\
\text { Chi. Ges } \\
\text { methene }\end{array}$ \\
\hline$\frac{1.7 .6 .2 .28}{m . p . s}$ & $\begin{array}{l}\text { CAL MON }-* 60 \\
\text { CHANNEL B }\end{array}$ & $\frac{9977.21 P P M}{10009.009 P P M}$ & $\begin{array}{l}\text { READINGS } \\
\text { WITHIN } \pm 5 \%\end{array}$ & A & \\
\hline $\begin{array}{l}1.7 .6 .2 .29 \\
\text { m.2.s } \\
\end{array}$ & OPEN FU-15 & $N / A$ & N/A & $A$. & \\
\hline $\begin{array}{l}1.7 .6 .2 .30 \\
\text { mip.s }\end{array}$ & $\begin{array}{l}\text { REMOVE } \\
\text { JUMPER }\end{array}$ & $\mathrm{N} / \mathrm{A}$ & N/A & $A$ & \\
\hline $\begin{array}{l}1.7 .6 .2 .31 \\
m \cdot x^{2}\end{array}$ & $\begin{array}{l}\text { CLOSE SV }-* 64 \\
\text { OPEN SV-*70 }\end{array}$ & N/A & N/A & A & . \\
\hline $\begin{array}{l}1.7 .6 .2 .32 \\
\text { mip.p }\end{array}$ & $\begin{array}{l}\text { RECORD } \\
\text { PDIT }-* 60 \\
\text { NIT } * 52 \text { IN }\end{array}$ & $-5.98 e^{4} \mathrm{H}^{\circ}$ & $\begin{array}{l}O \text { to }_{0}-30 \mathrm{H}_{2} \mathrm{~S} \\
+5=50-\mathrm{H}_{2} \mathrm{O}\end{array}$ & & $\begin{array}{l}\text { *Eception } \\
\text { Ref Ren os } \\
\text { ECN } 634645,63\end{array}$ \\
\hline $\begin{array}{l}1.7 .6 .2 .33 \\
m_{1 . \beta} \cdot 3\end{array}$ & $\begin{array}{l}\text { INSTALL } \\
\text { JUMPER }\end{array}$ & $N / A$ & $N / A$ & $A$ & \\
\hline $\begin{array}{l}1.7 .6 .2 .34 \\
\text { mir.s. }\end{array}$ & CLOSE FU-15 & $\mathrm{N} / \mathrm{A}$ & $N / A$ & A & \\
\hline
\end{tabular}


TEST DATA SHEET

\begin{tabular}{|c|c|c|c|c|c|}
\hline \multicolumn{3}{|c|}{ Date: $11-8-96$} & \multicolumn{3}{|c|}{ SHMS Unit Number: VTP $P N L-105 A$} \\
\hline \multicolumn{3}{|c|}{$\begin{array}{l}\text { Title of rest: } \\
\text { Pneumatic Systems }\end{array}$} & \multirow{2}{*}{\multicolumn{3}{|c|}{$\begin{array}{l}\text { Test Equipnent and } S / \mathrm{N} \text { or Cal. No.: } \\
\text { Ammonia bottld No. ALmos8836 }\end{array}$}} \\
\hline \multicolumn{3}{|c|}{$\begin{array}{c}\text { Test Performed By: See Test Personnel } \\
\text { Pay } 30\end{array}$} & & & \\
\hline $\begin{array}{l}\text { Procedure No. } \\
\text { Initial/Date }\end{array}$ & I ten & Value & $\begin{array}{l}\text { Required } \\
\text { Condition } \\
\end{array}$ & $(A / R)$ & Comment \\
\hline $\begin{array}{l}1.7 .6 .2 .35 \\
\text { m.21.8 }\end{array}$ & $\begin{array}{l}\text { ESTABLISH } \\
\text { BYPASS FLOW }\end{array}$ & $1.5 \mathrm{cfh}$ & $\begin{array}{l}\text { FIV }-* 70 \\
1.5 \pm .2 \mathrm{cfh}\end{array}$ & A & \\
\hline $\begin{array}{l}1.7 .6 .2 .36 \\
\text { m.7.B }\end{array}$ & $\begin{array}{l}\text { ADJ SV }-* 71 \\
\text { NIT-*52 } \\
\text { SAMPLE IN }\end{array}$ & $-4.99^{\circ} \mathrm{H}_{2} \mathrm{O}$ & $\begin{array}{l} \pm 5 \quad " \mathrm{H}_{2} \mathrm{O} \quad \mathrm{OF} \\
1.7 .6 .2 .32\end{array}$ & A & \\
\hline$\frac{1.7 .6 .2 .37}{m .20 .8}$ & $\begin{array}{ll}\text { RECORD } & \\
\text { NIT } * 52 & \text { mank } \\
\text { SAMPLES } & \\
\end{array}$ & $\begin{array}{l}\frac{35.9}{25.7 \mathrm{PPM}} \\
\frac{35.9 \mathrm{PPM}}{36.3 \mathrm{PPM}} \\
\end{array}$ & $\begin{array}{l}\text { READINGS } \\
\text { WITHIN } \pm 5 \%\end{array}$ & A & 97. PPM \\
\hline $\begin{array}{l}1.7 .6 .2 .38 \\
32.3^{2} \\
\end{array}$ & OPEN FU-15 & $N / A$ & $N / A$ & A & \\
\hline $\begin{array}{l}1.7 .6 .2 .39 \\
\text { m.p.B. }\end{array}$ & $\begin{array}{l}\text { REMOVE } \\
\text { JUMPER }\end{array}$ & N/A & $N / A$ & A & \\
\hline \multicolumn{6}{|c|}{ Section 1.7 .7 provides for test system shutdown. } \\
\hline $\begin{array}{l}1.7 .7 .1 \\
\text { miv. } 1 \\
\end{array}$ & $\begin{array}{l}\text { DISCONNECT } \\
\mathrm{H}_{2} \text { CAL GAS } \\
\end{array}$ & $N / A$ & N/A & A & \\
\hline $\begin{array}{l}1.7 .7 .2 \\
\text { m.p. }{ }^{3} \\
\end{array}$ & $\begin{array}{l}\text { DISCONNECT } \\
\mathrm{CH}_{6} \text { CAL GAS } \\
\end{array}$ & $N / A$ & $N / A$ & A & \\
\hline $\begin{array}{l}1.7 .7 .3 \\
m \% \cdot 8 \\
\end{array}$ & $\begin{array}{l}\text { DISCONNECT } \\
\mathrm{NH}_{3} \text { CAL GAS } \\
\end{array}$ & $N / A$ & N/A & $A$ & \\
\hline $\begin{array}{l}1.7 .7 .4 \\
\text { m.x. }\end{array}$ & $\begin{array}{l}\text { SECURE \& } \\
\text { TURN OFF } \\
\text { ANA. INST.S } \\
\end{array}$ & $N / A$ & $N / A$ & $A$ & \\
\hline $\begin{array}{l}1.7 .7 .5 \\
\text { m.x.s. }\end{array}$ & $\begin{array}{l}\text { DISCONNECT } \\
\mathrm{N}_{2} \text { CARRIER } \\
\end{array}$ & $N / A$ & $N / A$ & A & \\
\hline $\begin{array}{l}1.7 .7 .6 \\
\text { m.x.0 } \\
\end{array}$ & $\begin{array}{l}\text { DISCONNECT } \\
\text { He CARRIER } \\
\end{array}$ & $N / A$ & $N / A$ & $A$ & \\
\hline $\begin{array}{r}1.7 .7 .7 \\
\text { m.2. }\end{array}$ & $\begin{array}{l}\text { OPEN ALL } \\
\text { BREAKERS \& } \\
\text { FUSES } \\
\end{array}$ & $N / A$ & $N / A$ & A & \\
\hline $\begin{array}{l}1.7 .7 .8 \\
\text { m.jes }\end{array}$ & $\begin{array}{l}\text { CLOSE LISTED } \\
\text { SYS. VALVES }\end{array}$ & N/A & N/A & A & \\
\hline
\end{tabular}


APPENDIX A

PAGE A-29
Rent o

COPY
HNF-SD-WH-ATR-191

Rev. 0

Page 64

TEST DATA SHEET

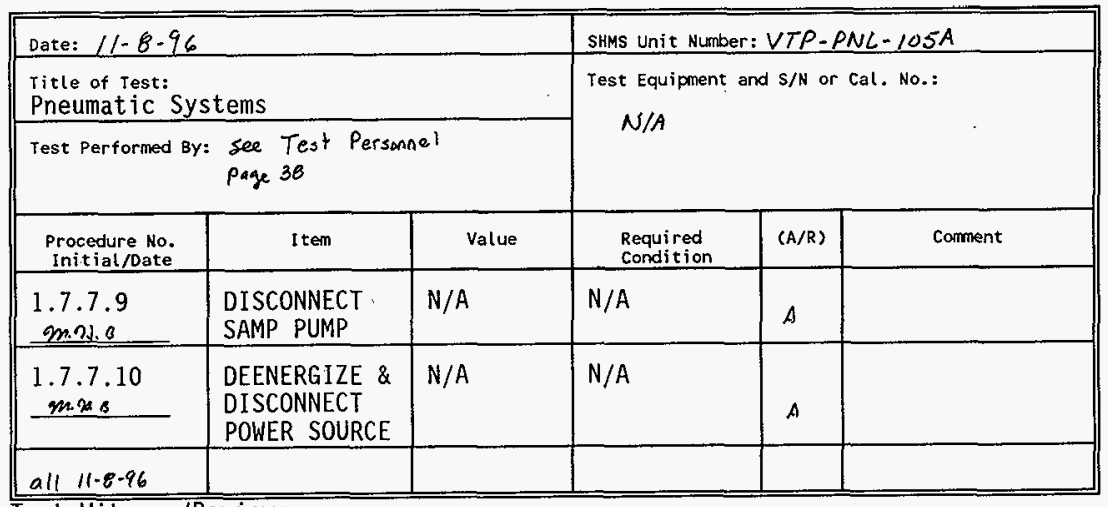

Test Witness/Review:
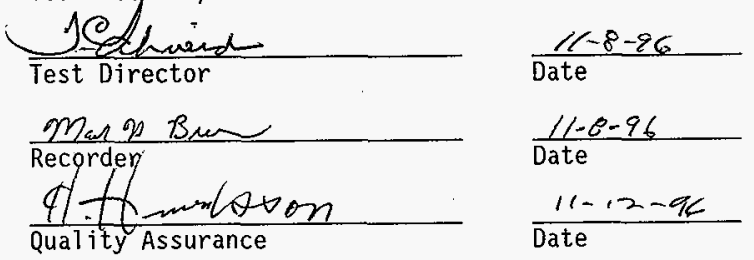
APPENDIX A

PAGE $A-30$ meson

$$
\begin{array}{r}
\text { HNF-SD-HM-ATR- } 191 \\
\text { ReV. } 0 \\
\text { Page } 65 \mathrm{~A}
\end{array}
$$

COPY

TEST EXCEPTION SHEET

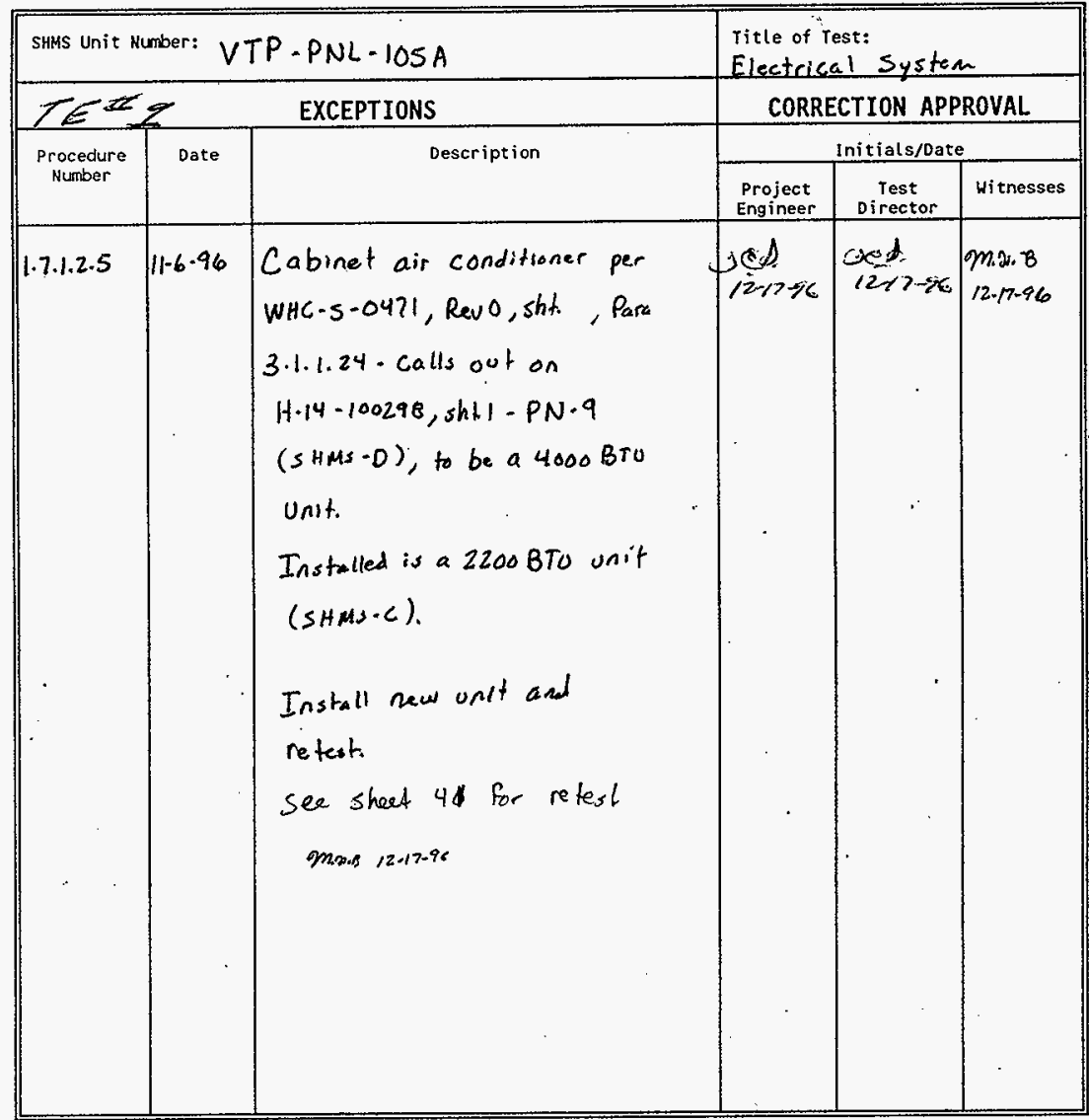

TEST APPROVED WITH EXCEPTIONS

SChwinn

WHC Project Engineer

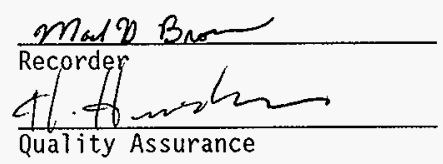

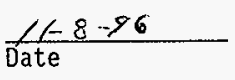

$\frac{11-8-96}{\text { Date }}$

$\frac{i 1-12-\pi k}{\text { Date }}$ 


\section{TEST EXCEPTION SHEET}

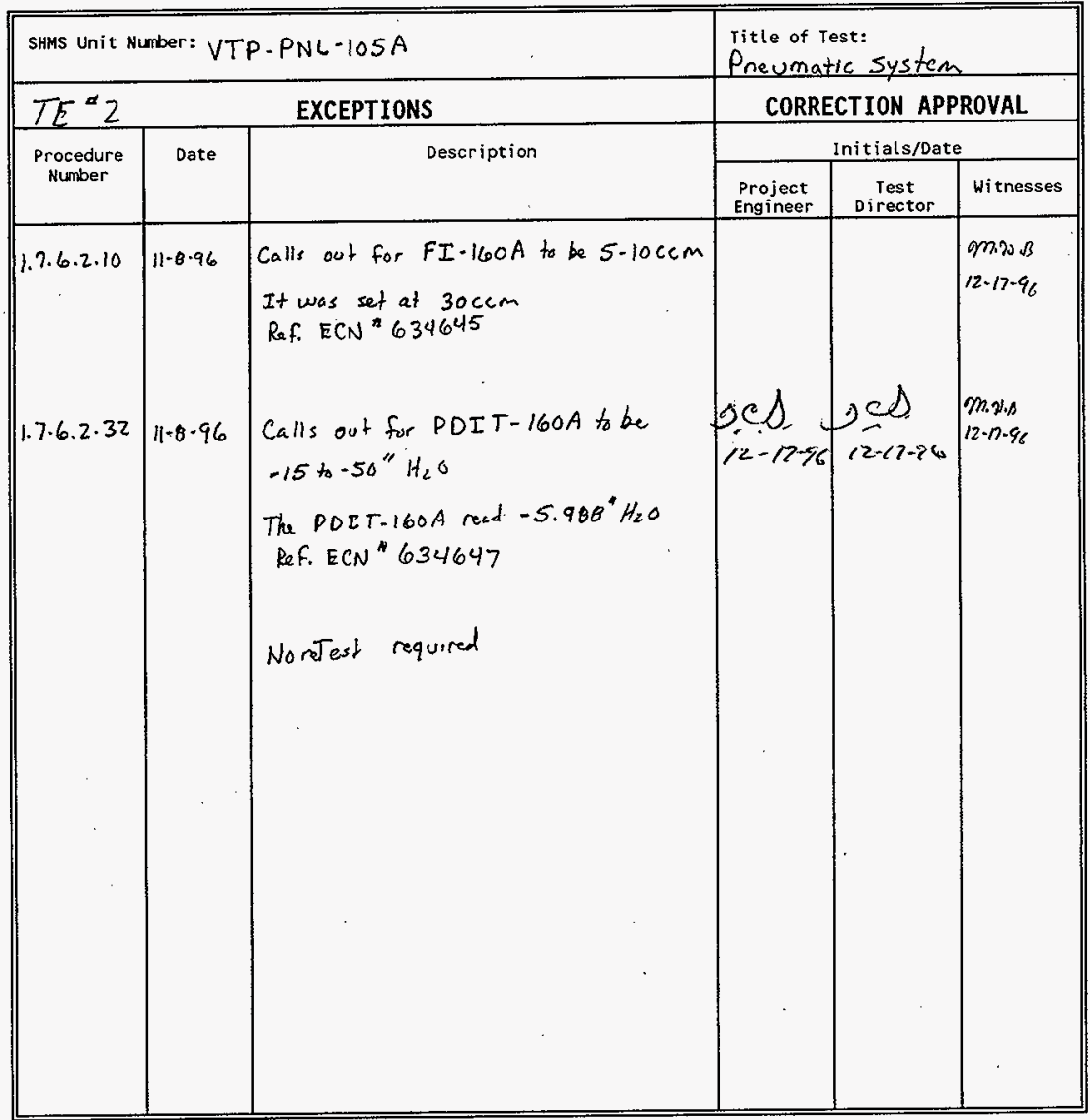

\section{TEST APPROVED WITH EXCEPTIONS}

As sho Project Engineer

$\frac{16-8-86}{\text { Date }}$

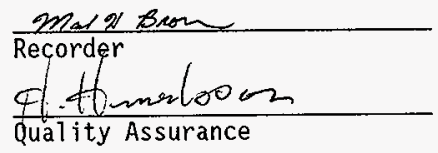

$\frac{11-8-76}{\text { Date }}$

$\frac{i 1-12-96}{\text { Date }}$ 
APPENDIX A

PAGE A-32

$$
\begin{array}{r}
\text { HNF-SD-WM-ATR- } 191 \\
\text { ReV. } 0 \\
\text { Page } 66
\end{array}
$$

TEST LOG .

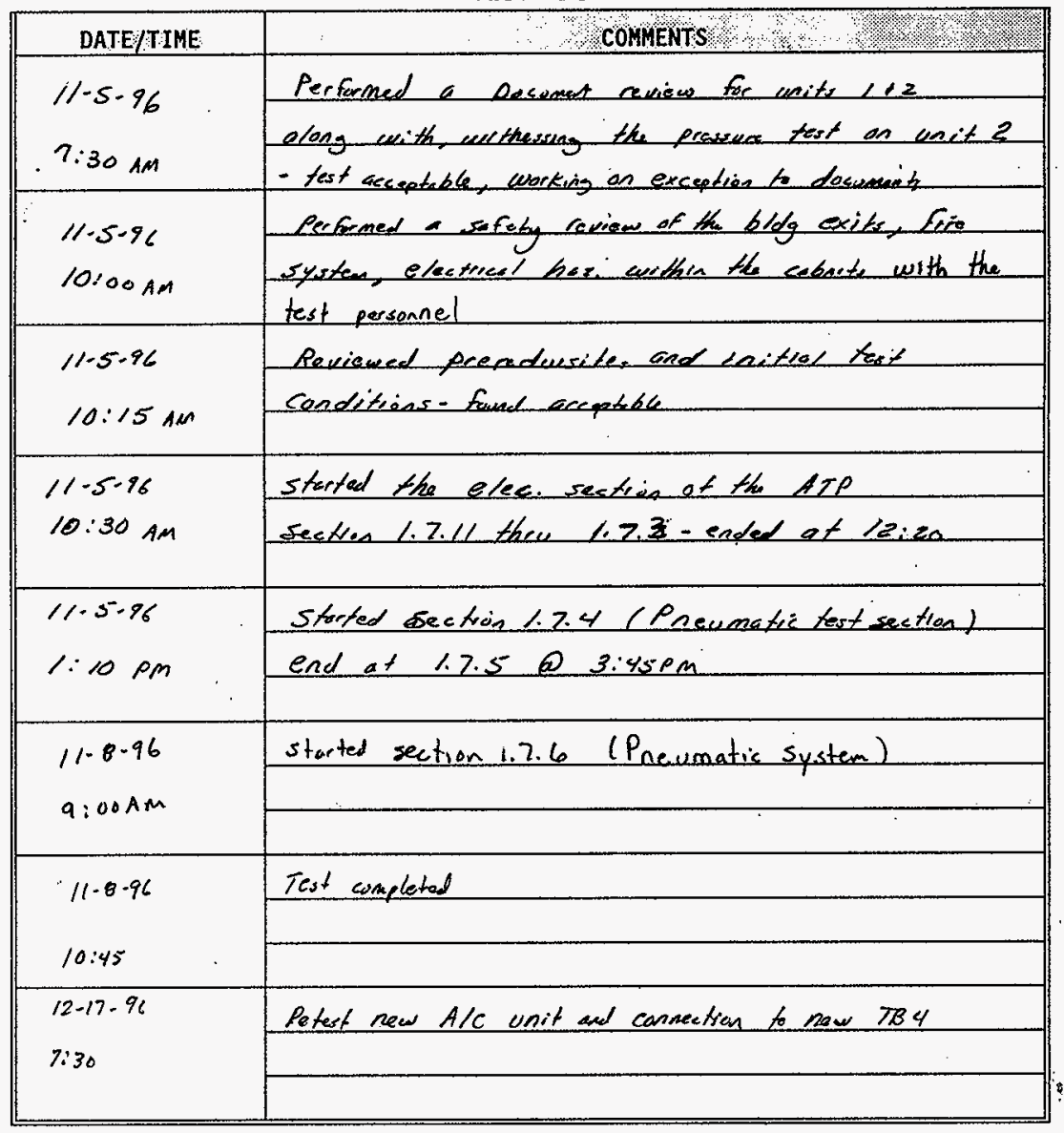


APPENDIX B 
TEST EXECUTION SHEET

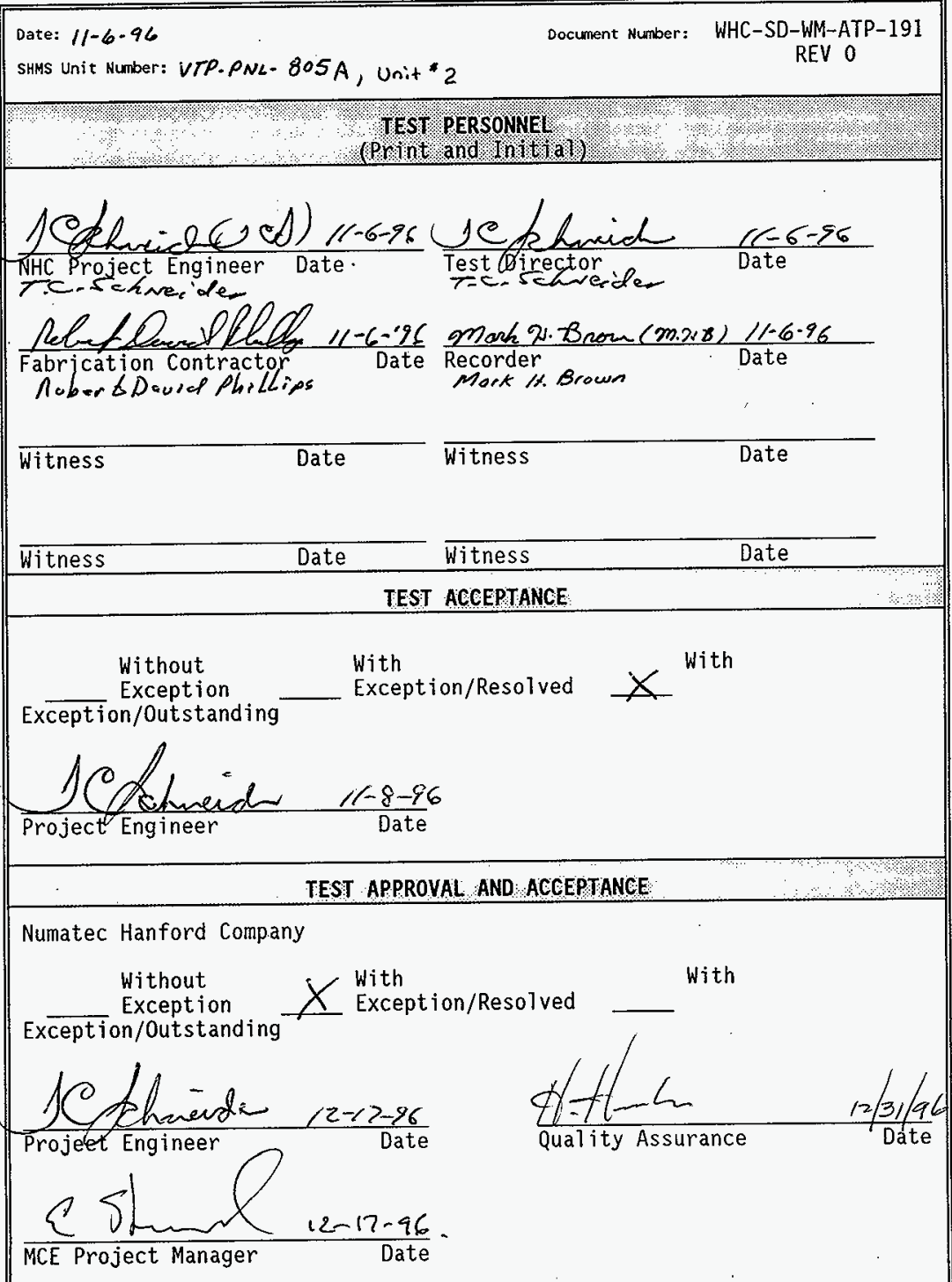


APPENDIX B

SHMS Unit Number:

\section{PREREQUISITES AND INITIAL TEST CONDITIONS}

The following conditions shall exist at the start of the acceptance testing. Initial and date to verify that each of the following items have been accomp i i shed. $\underset{m=n, B}{m} \frac{M, 2, B}{\text { Systems being tested have been inspected for workmanship and for }}$ compliance with design.

m.s.B. Continuity and megger tests have been performed on portions of the electrical and instrument systems being tested, as required.

m.s. Leak tests on the pneumatic systems have been performed.

$1 \%-17 \cdot \%$ $m x . B$ The following circuit breakers and fuses are installed per the specified size and are open

\begin{tabular}{ll} 
OK & CB-1 through $\mathrm{CB}-7$ \\
\hline OK & FU-1 through FU-15
\end{tabular}

ma. m.s. All test instruments have a currently valid calibration stamp attached that indicates a calibration traceable to the National Institute of Standards and Technology.

ma.n m. The following process and control instrument systems have been initially configured and aligned for proper operation.

FIT-*57, FSL $-\star 57$, NIT $-\star 54$, NIT $* * 55,(N R-* 54)$, PDIT $* * 60$, TIC $-\star 50$, TIC-*56, TIS-*62, YYC-*01 NOTE: NR-*54 is not used in the ANALYTICAL configuration.

$m x \pi$

$17 \times>-9$

$m .3$ Personne] responsible for directing and witnessing the performance of the tests described in this ATP have read and understand appropriate certified vendor information (CVI) pertaining to the operation of the equipment to be tested.

M.s.s CLOSE all system manual vaives with the exception of the following:

$$
\begin{aligned}
& \text { oK VTP-FIV }-* 52 \\
& \text { ok VTP-FIV-*56 } \\
& \text { OK VTP-FIV }-* 70 \\
& \text { ok VTP-SV-*24 } \\
& \text { OK VTP-SV-*61 } \\
& \text { eK VTP-SV-*62 } \\
& \text { ok VTP-SV-*67 } \\
& \text { ak VTP-SV-*71 }
\end{aligned}
$$


APPENDIX B

Onis

\begin{abstract}
$\operatorname{man}$
m. N.

208 Vac 1 phase (15-20 ampere temporary power source has been

$17+27$

11.5 .96 connected, but not energized, to the appropriate TBI terminals per H-14-100838.
\end{abstract}

Verify by signature and date that all prerequisites have been met.

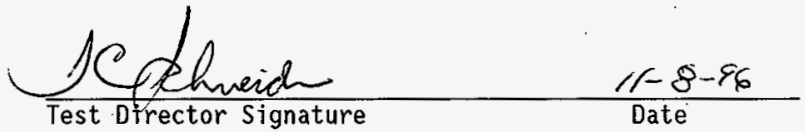




\section{TEST DATA SHEET}

\begin{tabular}{|c|c|c|c|c|c|}
\hline \multicolumn{3}{|c|}{ Date: $11-6-96$} & \multicolumn{3}{|c|}{ SHMS Unit Number: VTP-PNL $-80 S A$} \\
\hline \multicolumn{3}{|c|}{$\begin{array}{l}\text { Title of Test: } \\
\text { Electrical systems }\end{array}$} & \multirow{2}{*}{\multicolumn{3}{|c|}{$\begin{array}{l}\text { Test Equipment and } \mathrm{S} / \mathrm{N} \text { or Cal. No.: } \\
\text { Fluke V/O meter } \\
S / N 44620564 \\
\text { Cal. due. } 10.97\end{array}$}} \\
\hline \multicolumn{3}{|c|}{$\begin{array}{l}\text { Test Performed By: see test Personmel } \\
\text { Page } 68\end{array}$} & & & \\
\hline $\begin{array}{l}\text { Procedure No. } \\
\text { Initial/Date }\end{array}$ & Item & value & $\begin{array}{l}\text { Required } \\
\text { Condition } \\
\end{array}$ & $(A / R)$ & Comment \\
\hline \multicolumn{6}{|c|}{ Section 1.7.1.1 verifies the system Mains Power. } \\
\hline $\begin{array}{c}1.7 .1 .1 .1 \\
\text { m.m.n }\end{array}$ & Resistance & $0.2 \Omega$ & $<1$ ohm & $A$ & \\
\hline 1.7 .1 .1 .2 & $\begin{array}{l}\text { Temp. power } \\
208 \mathrm{Vac}\end{array}$ & $N / A$ & Energized & A & \\
\hline $\begin{array}{l}1.7 .1 .1 .3 \\
\text { mane }^{2}\end{array}$ & $\begin{array}{l}\text { Line Vac: } \\
\text { L1-L2 } \\
\text { L1-Gnd } \\
\text { L2-Gnd } \\
N \text {-Gnd }\end{array}$ & $\begin{array}{c}\frac{211.2 \mathrm{Vac}}{121.3 \mathrm{Vac}} \\
\frac{121.3 \mathrm{Vac}}{0.0} \\
.0\end{array}$ & $\begin{array}{l}L 1-L 2: 208 \\
\text { Vac }+10 /-1 \% \\
L 1, L 2: 120 \\
\text { Vac } \pm 5 \% \\
N: 0 \text { Vac }\end{array}$ & $A$ & \\
\hline \multicolumn{6}{|c|}{ Section 1.7 .1 .2 verifies the enclosure HVAC system. } \\
\hline $\begin{array}{l}1.7 .1 .2 .1 \\
m x \beta\end{array}$ & AC Adjusted & $N / A$ & N/A & A & \\
\hline $\begin{array}{l}1.7 .1 .2 .2 \\
7.2 .6\end{array}$ & $\begin{array}{l}\text { CLOSE } \\
\text { CB-1/2 }\end{array}$ & $N / A$ & $N / A$ & A & \\
\hline $\begin{array}{l}1.7 .1 .2 .3 \\
m x_{B}\end{array}$ & Heater ON & ek & Heater ON & $A$ & \\
\hline $\begin{array}{l}1.7 .1 .2 .4 \\
m x \beta\end{array}$ & AC Adjusted & $N / A$ & $N / A$ & A & \\
\hline $\begin{array}{l}1.7 .1 .2 .5 \\
m x .8\end{array}$ & $\begin{array}{l}\text { Heater OFF } \\
\text { Cooling ON }\end{array}$ & ok & $\begin{array}{l}\text { Heater OFF } \\
\text { Cooling ON }\end{array}$ & A & 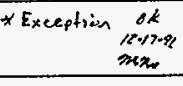 \\
\hline $\begin{array}{l}1.7 .1 .2 .6 \\
\mathrm{mis}\end{array}$ & AC Adjusted & $N / A$ & N/A & A & . \\
\hline $\begin{array}{l}1.7 .1 .2 .7 \\
2.8\end{array}$ & $\begin{array}{l}\text { OPEN } \\
\mathrm{CB}-1 / 2 \\
\end{array}$ & $N / A$ & $N / A$ & A & \\
\hline \multicolumn{6}{|c|}{ Section 1.7.1.3 verifies the sample pump is properly connected. } \\
\hline $\begin{array}{c}1.7 .1 .3 .1 \\
m .0 .0 \\
\end{array}$ & $\begin{array}{l}\text { CLOSE } \\
\text { CB-3/4 }\end{array}$ & $\mathrm{N} / \mathrm{A}$ & N/A & A & \\
\hline $\begin{array}{c}1.7 .1 .3 .2 \\
\text { min.. }\end{array}$ & $\begin{array}{l}\text { VTP-P }-* 50 \\
\text { ON }\end{array}$ & ok & $\begin{array}{l}V T P-P-\star 50 \\
O N\end{array}$ & $A$ & \\
\hline
\end{tabular}


APPENDIX B

$\therefore$ and

HNF - SD - WM-ATR - 191

PAGE B- 6

\section{TEST DATA SHEET}

\begin{tabular}{|c|c|c|c|c|c|}
\hline \multicolumn{3}{|c|}{ Date: 11.6 .96} & \multicolumn{3}{|c|}{ SHMS Unit Number: VTP - PNL - $805 A$} \\
\hline \multicolumn{3}{|c|}{$\begin{array}{l}\text { Title of Test: } \\
\text { Electrical Systems }\end{array}$} & \multirow{2}{*}{\multicolumn{3}{|c|}{$\begin{array}{l}\text { Test Equipment and } \mathrm{S} / \mathrm{N} \text { or Cal. No.: } \\
\text { Fluke } \mathrm{V} / 0 \text { meter } \\
\text { SIN } 44620364 \\
\text { Cal doe } 10-97\end{array}$}} \\
\hline \multicolumn{3}{|c|}{ 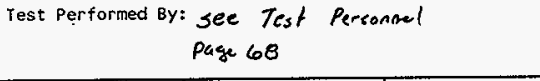 } & & & \\
\hline $\begin{array}{l}\text { Procedure No. } \\
\text { Initial/Date }\end{array}$ & Item & Value & $\begin{array}{c}\text { Required } \\
\text { Condition }\end{array}$ & $(A / R)$ & Comment \\
\hline $\begin{array}{l}1.7 .1 .3 .3 \\
\text { nomas } \\
\end{array}$ & $\begin{array}{l}\text { OPEN } \\
\text { CB-3/4 }\end{array}$ & $N / A$ & $\mathrm{~N} / \mathrm{A}$ & A & \\
\hline \multicolumn{6}{|c|}{ Section 1.7 .1 .4 verifies the enclosure lighting and GFCI receptacles. } \\
\hline $\begin{array}{l}1.7 .1 .4 .1 \\
2028 \\
\end{array}$ & $\begin{array}{l}\text { CLOSE } \\
\text { CB-6 }\end{array}$ & $N / A$ & N/A & A & \\
\hline $\begin{array}{c}1.7 .1 .4 .2 \\
m x .3 \\
\end{array}$ & $\begin{array}{l}\text { ENCLOSURE } \\
\text { LIGHT }\end{array}$ & $N / A$ & $N / A$ & A & \\
\hline $\begin{array}{c}1.7 .1 .4 .3 \\
\text { m.1.s. }\end{array}$ & LIGHT ON & & LIGHT ON & & \\
\hline $\begin{array}{l}1.7 .1 .4 .4 \\
m x:\end{array}$ & $\begin{array}{l}\text { GFCI RECP. } \\
1 A . L-N \\
1 B . L-G N D \\
2 A . L-N \\
\text { 2B. L-GND } \\
\end{array}$ & $\frac{\frac{120.7 \mathrm{Vac}}{120.6 \mathrm{Vac}}}{\frac{120.6 \mathrm{Vacc}}{120.6 \mathrm{Vac}}}$ & $\begin{array}{ll}1 A . & 120 \\
1 B . & \mathrm{VaC} \\
2 \mathrm{Ba} & 120 \mathrm{VaC} \\
2 \mathrm{~A} . & 120 \mathrm{VaC} \\
\text { ALL } \pm 5 \% & \end{array}$ & A & $\begin{array}{l}A A-G=0.0 V_{C C} \\
{ }_{N-G} A=0.0 V_{G C}\end{array}$ \\
\hline $\begin{array}{l}1.7 .1 .4 .5 \\
\text { masp }\end{array}$ & $\begin{array}{l}\text { GFCI TEST } \\
1 A . L-N \\
1 B . L-G N D \\
2 A . L-N \\
2 B . L-G N D \\
\end{array}$ & $\frac{\frac{0.5 v_{4 c}}{0.5 v_{4 c}}}{\frac{0.0 v_{a c}}{0.3 v_{a c}}}$ & 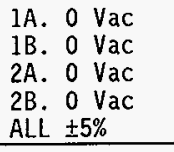 & A & \\
\hline $\begin{array}{l}1.7 .1 .4 .6 \\
\text { mxio. } \\
\end{array}$ & $\begin{array}{l}\text { ENCLOSURE } \\
\text { LIGHT OFF } \\
\end{array}$ & $N / A$ & $N / A$ & $A$ & \\
\hline $\begin{array}{l}1.7 .1 .4 .7 \\
\text { m.x.s. } \\
\end{array}$ & $\begin{array}{l}\text { OPEN } \\
\text { CB-6 }\end{array}$ & $\mathrm{N} / \mathrm{A}$ & $\mathrm{N} / \mathrm{A}$ & A & \\
\hline \multicolumn{6}{|c|}{ Section 1.7.1.5 verifies the instrument power source isolation transformer } \\
\hline $\begin{array}{l}1.7 .1 .5 .1 \\
2 \pi .8 \\
\end{array}$ & $\begin{array}{l}\text { CLOSE } \\
\text { CB-7 }\end{array}$ & $N / A$ & $N / A$ & A & \\
\hline $\begin{array}{l}1.7 .1 .5 .2 \\
m \times .8 \\
\end{array}$ & $\begin{array}{l}\text { XFRM VaC } \\
\text { PRI. L-N } \\
\text { SEC. L-N } \\
\text { SEC. N-GND } \\
\end{array}$ & $\frac{\frac{121.2 V_{a c}}{124.5 V_{A c}}}{0.7 V_{a c}}$ & $\begin{array}{l}\text { PRI. \& SEC. } \\
\text { L-N } 120 \pm 5 \% \\
\text { SEC. } \\
\text { N-GND O VaC }\end{array}$ & $A$ & \\
\hline $\begin{array}{l}1.7 .1 .5 .3 \\
20.8 \\
\end{array}$ & CLOSE FU-1 & $N / A$ & $N / A$ & $A$ & \\
\hline
\end{tabular}


TEST DATA SHEET

\begin{tabular}{|c|c|c|c|c|c|}
\hline \multicolumn{3}{|c|}{ Date: $1 /-6-96$} & \multicolumn{3}{|c|}{ SHMS Unit Number: VTP-PNL- $8 \Omega 5 A$} \\
\hline \multicolumn{3}{|c|}{$\begin{array}{l}\text { Title of rest: } \\
\text { Electrical Systems }\end{array}$} & \multirow{2}{*}{\multicolumn{3}{|c|}{$\begin{array}{l}\text { Test Equipment and } S / N \text { or Cal. No.: } \\
\text { F/uke V/o mater } \\
S / N 4 / 620364 \\
\text { Cal. doe. } 10.97\end{array}$}} \\
\hline \multicolumn{3}{|c|}{ 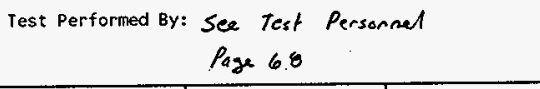 } & & & \\
\hline $\begin{array}{c}\text { Procedure No. } \\
\text { Initial/Date }\end{array}$ & Item & Value & $\begin{array}{l}\text { Required } \\
\text { Condition }\end{array}$ & $(A / R)$ & Comment \\
\hline $\begin{array}{l}1.7 .1 .5 .4 \\
\text { m.p.s }\end{array}$ & $\begin{array}{l}\text { VTP-PS-*50 } \\
\text { OUTPUT Vdc } \\
\end{array}$ & $23.99 \mathrm{Vde}$ & $24 \pm 0.1 \mathrm{Vdc}$ & $A$ & \\
\hline \multicolumn{6}{|c|}{ Section 1.7.1.6 verifies the enclosure general alarm system. } \\
\hline 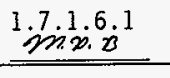 & $\begin{array}{l}\text { CLOSE FU-9 \& } \\
\text { FU-10 }\end{array}$ & $N / A$ & $N / A$ & $A$ & \\
\hline $\begin{array}{l}1.7 .1 .6 .2 \\
M x . B .\end{array}$ & $\begin{array}{l}\text { PUSH PB }-* 51 \\
\& \mathrm{~PB}-* 50\end{array}$ & $N / A$ & $N / A$ & A & \\
\hline $\begin{array}{l}1.7 .1 .6 .3 \\
\text { mix.s. }\end{array}$ & $\begin{array}{l}\text { ALARM TEST } \\
\text { VERIFY ALARM } \\
\text { CONDITIONS }\end{array}$ & 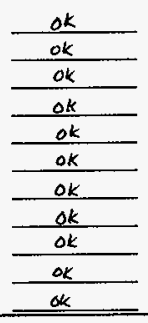 & $\begin{array}{ll}\text { YAH-*50 } & \text { ON } \\
\text { NAH-*55 } & \text { ON } \\
\text { XA-*63 } & \text { ON } \\
\text { NAH-*54 } & \text { ON } \\
\text { FAL-*57 } & \text { ON } \\
\text { TAHL-*62 ON } \\
\text { TAL-*50 ON } \\
\text { YAL-*58 } & \text { ON } \\
\text { PBL-*58 } & \text { ON } \\
\text { PBL-*54 OFF } \\
\text { PBL-*59 } & \text { OFF } \\
\end{array}$ & $A$ & \\
\hline $\begin{array}{l}1.7 .1 .6 .4 \\
\text { maxs }\end{array}$ & $\begin{array}{l}\text { END ALARM } \\
\text { TEST }\end{array}$ & N/A & $N / A$ & $A$ & \\
\hline $\begin{array}{l}1.7 .1 .6 .5 \\
\text { m.x.s }\end{array}$ & RESET ALARMS & N/A & $N / A$ & $A$ & \\
\hline $\begin{array}{l}1.7 .1 .6 .6 \\
\text { muxe }\end{array}$ & $\begin{array}{l}\text { OPEN FU-9 \& } \\
\text { FU-10 }\end{array}$ & N/A & $N / A$ & A & \\
\hline \multicolumn{6}{|c|}{ Section 1.7.1.7 verifies the flow al arm system. } \\
\hline $\begin{array}{l}1.7 .1 .7 .1 \\
\text { minss }\end{array}$ & $\begin{array}{l}\text { CLOSE FU-3, } \\
\text { FU-9, FU-10 } \\
\& \text { FU-13 } \\
\end{array}$ & N/A & N/A & A & \\
\hline $\begin{array}{l}1.7 .1 .7 .2 \\
\text { m.v.B }\end{array}$ & RESET ALARMS & $\longrightarrow k$ & FAL- $* 57$ ON & A & \\
\hline $\operatorname{log.8.8}_{\text {m.7. }}^{1.7 .1 .7 .3}$ & $\begin{array}{l}\text { OPEN SV }-* 20 \\
\& \text { SV }-* 22\end{array}$ & N/A & N/A & A & \\
\hline
\end{tabular}


TEST DATA SHEET

\begin{tabular}{|c|c|c|c|c|c|}
\hline \multicolumn{3}{|c|}{ Date: $\quad 11-6-96$} & \multicolumn{3}{|c|}{ SHMS Unit Number: $V T P-P N L-805 A$} \\
\hline \multicolumn{3}{|c|}{$\begin{array}{l}\text { Title of Test: } \\
\text { Electrical Systems }\end{array}$} & \multirow{2}{*}{\multicolumn{3}{|c|}{$\begin{array}{l}\text { Test Equipment and } S / N \text { or Cal. No.: } \\
\text { m/A mos. } \\
\text { F/uke V/O meter } \\
\text { S/N44620364, cal duc. } 10.97\end{array}$}} \\
\hline \multicolumn{3}{|c|}{$\begin{aligned} & \text { Test Performed By: } \text { see test Personmel } \\
& \text { Page } 68\end{aligned}$} & & & \\
\hline $\begin{array}{l}\text { Procedure No. } \\
\text { Initial/Date }\end{array}$ & Item & Value & $\begin{array}{l}\text { Required } \\
\text { condition } \\
\end{array}$ & $(A / R)$ & Comment \\
\hline $\begin{array}{l}1.7 .1 .7 .4 \\
\text { mingen }\end{array}$ & $\begin{array}{l}\text { FIT-*57 \& } \\
\text { FSL }-* 57 \\
\text { POWERED }\end{array}$ & ok & $\begin{array}{l}\text { FIT-*57 ON } \\
\text { FSL-*57 ON }\end{array}$ & $A$ & \\
\hline $\begin{array}{l}1.7 .1 .7 .5 \\
\text { m.x. }\end{array}$ & $\begin{array}{l}\text { MuX-*70 } \\
\text { TB5+/TB6- }\end{array}$ & $0.98 \mathrm{Vdc}$ & $1.0 \pm 0.2 \mathrm{Vdc}$ & A & \\
\hline $\begin{array}{l}1.7 .1 .7 .6 \\
\text { min: } 8\end{array}$ & $\begin{array}{l}\text { CLOSE SV-*20 } \\
\& \text { SV } * * 22\end{array}$ & N/A & $N / A$ & $A$ & \\
\hline 1.7 .1 .7 .7 & $\begin{array}{l}\text { OPEN FU-3, } \\
\text { FU-9, FU-10 } \\
\& \text { FU-13 }\end{array}$ & N/A & $N / A$ & $A$ & \\
\hline \multicolumn{6}{|c|}{ Section 1.7 .1 .8 verifies the $\mathrm{H}_{2}$ monitor wiring. } \\
\hline 1.7 .1 .8 .1 & $\begin{array}{l}\text { CLOSE FU-2, } \\
\text { FU-4, FU-5, } \\
\text { FU-9 \& FU-10 }\end{array}$ & $\mathrm{N} / \mathrm{A}$ & N/A & A & \\
\hline $\begin{array}{l}1.7 .1 .8 .2 \\
\text { mixs }\end{array}$ & RESET ALARMS & ok & $\begin{array}{l}\text { NAH }-* 54 \text { OFF } \\
\text { NAH }-* 55 \text { OFF }\end{array}$ & A & \\
\hline $\begin{array}{l}1.7 .1 .8 .3 \\
\text { min.s }\end{array}$ & $\begin{array}{l}\text { INSTRUMENTS } \\
\text { POWERED }\end{array}$ & $\begin{array}{c}\frac{o k}{0 k} \\
\text { ok } \\
\text { N/A } \\
\text { ok } \\
\end{array}$ & $\begin{array}{l}\text { MUX }-* 70 \text { ON } \\
\text { NIT }-* 54 \text { ON } \\
\text { NIT }-* 55 \text { ON } \\
\text { NR }-* 54 \text { ON } \\
\text { YYC }-* 01 \text { ON } \\
\end{array}$ & & $\begin{array}{l}\text { NR. ES4A not } \\
\text { in this unit. }\end{array}$ \\
\hline$\frac{1.7 .1 .8 .4}{m \times 8}$ & $\begin{array}{l}\text { OPEN FU-2, } \\
\text { FU-4, FU-5, } \\
\text { FU-9 \& FU-10 }\end{array}$ & $N / A$ & N/A & $A^{\prime}$ & \\
\hline \multicolumn{6}{|c|}{ Section 1.7.1.9 verifies the SHMS-E heat trace control system. } \\
\hline $\begin{array}{l}1.7 .1 .9 .1 \\
m . x .0 \\
\end{array}$ & $\begin{array}{l}\text { INSTALL LOAD } \\
\text { VTP-TIC-*50 }\end{array}$ & $N / A$ & $\mathrm{~N} / \mathrm{A}$ & A & \\
\hline${ }_{10.0 .3}^{7.1 .9 .2}$ & $\begin{array}{l}\text { CLOSE CB-5, } \\
\text { FU-6, FU-7, } \\
\text { FU-9 \& FU-10 } \\
\text { and RESET } \\
\text { ALARMS }\end{array}$ & $N / A$ & $N / A$ & A & \\
\hline
\end{tabular}


TEST DATA SHEET

\begin{tabular}{|c|c|c|c|c|c|}
\hline \multicolumn{3}{|l|}{ Date: $11-6.96$} & \multicolumn{3}{|c|}{ SHMS Unit Number: $V T P-P_{N L}-805 \mathrm{~A}$} \\
\hline \multicolumn{3}{|c|}{$\begin{array}{l}\text { Title of Test: } \\
\text { Electrical Systems }\end{array}$} & \multirow{2}{*}{\multicolumn{3}{|c|}{$\begin{array}{l}\text { Test Equipment and } S / N \text { or cal. No.: } \\
\text { Fluke V/o meter } \\
\text { S/N } 44620364 \\
\text { Cal doe. } 10.97\end{array}$}} \\
\hline \multicolumn{3}{|c|}{$\begin{aligned} & \text { Test Performed By: } \text { See Test Personnel } \\
& \text { Page } 68\end{aligned}$} & & & \\
\hline $\begin{array}{c}\text { Procedure No. } \\
\text { Initial/Date }\end{array}$ & Item & vatue & $\begin{array}{l}\text { Required } \\
\text { Condition } \\
\end{array}$ & $(A / R)$ & Comment \\
\hline $\begin{array}{l}1.7 .1 .9 .3 \\
\end{array}$ & $\begin{array}{l}\text { TIC }-* 50 \& \\
\text { TIC-*56 } \\
\text { NOMINAL TEMP }\end{array}$ & $\frac{64 \%}{64 \text { of }}$ & $\begin{array}{l}\text { TIC }-* 50 \\
\text { TIC }-* 56 \\
\text { TEMPERATURE } \\
\end{array}$ & $A$ & \\
\hline $\begin{array}{l}1.7 .1 .9 .4 \\
m \times 8\end{array}$ & $\begin{array}{l}\text { ADJ TIC }-\star 50 \\
\& \text { TIC }-\star 56 \\
\text { SP2 \& RESET } \\
\text { ALARMS } \\
\end{array}$ & $N / A$ & N/A & $A$ & \\
\hline $\begin{array}{l}1.7 .1 .9 .5 \\
\text { m.p. }\end{array}$ & $\begin{array}{l}\text { ADJ TIC-*50 } \\
\text { SP } 1\end{array}$ & N/A & $N / A$ & A & \\
\hline $\begin{array}{l}1.7 .1 .9 .6 \\
\end{array}$ & $\begin{array}{l}\text { ADJ TIC }-* 50 \\
\text { SP 2 }\end{array}$ & $N / A$ & N/A & A & \\
\hline $\begin{array}{l}1.7 .1 .9 .7 \\
m . x .0\end{array}$ & $\begin{array}{l}\text { TAL-*50\& } \\
\text { HORN ACTIVE }\end{array}$ & $\frac{o k}{-o k}$ & $\begin{array}{l}\text { TAL-*50 ON } \\
\text { HORN ON } \\
\text { ACKNOWLEDGE } \\
\end{array}$ & A & \\
\hline $\begin{array}{l}1.7 .1 .9 .8 \\
\text { mang. }\end{array}$ & $\begin{array}{l}\text { TIC }-* 50 \\
\text { OPERATES } \\
\end{array}$ & ok & $\begin{array}{l}\text { TIC-*50 } \\
\text { OPERATES }\end{array}$ & A & \\
\hline $\begin{array}{l}1.7 .1 .9 .9 \\
m . x^{m}\end{array}$ & $\begin{array}{l}\text { ADJ TIC-*50 } \\
\text { SP } 2\end{array}$ & $N / A$ & $\mathrm{~N} / \mathrm{A}$ & A & \\
\hline $\begin{array}{l}1.7 .1 .9 .10 \\
m \times \cdot s\end{array}$ & RESET ALARM & _. $\partial k$ & TAL-*50 OFF & $A$ & \\
\hline $\begin{array}{l}1.7 .1 .9 .11 \\
\text { mm.8 } \\
\end{array}$ & $\begin{array}{l}\text { ADJ TIC-*50 } \\
S P 1 \& S P 2 \\
\end{array}$ & N/A & N/A & $A$ & \\
\hline $\begin{array}{l}1.7 .1 .9 .12 \\
\end{array}$ & $\begin{array}{l}\text { OPEN CB-5 \& } \\
\text { REMOVE LOAD } \\
\end{array}$ & N/A & $N / A$ & A & \\
\hline $\begin{array}{r}1.7 .1 .9 .13 \\
-20.213 \\
\end{array}$ & $\begin{array}{l}\text { INSTALL LOAD } \\
\text { VTP-TIC-*56 } \\
\end{array}$ & $N / A$ & $N / A$ & A & \\
\hline $\begin{array}{l}1.7 .1 .9 .14 \\
-m .2 .0 \\
\end{array}$ & CLOSE CB-5 & N/A & N/A & A & \\
\hline $\begin{array}{l}1.7 .1 .9 .15 \\
\text { m.x.p. }\end{array}$ & $\begin{array}{l}\text { ADJ TIC }-* 56 \\
\text { SP } 1\end{array}$ & N/A & $N / A$ & A & \\
\hline $\begin{array}{l}1.7 .1 .9 .16 \\
9 x .8\end{array}$ & $\begin{array}{l}\text { ADJ TIC-*56 } \\
\text { SP } 2\end{array}$ & N/A & $N / A$ & A & \\
\hline
\end{tabular}


TEST DATA SHEET

\begin{tabular}{|c|c|c|c|c|c|}
\hline \multicolumn{3}{|c|}{ Date: $1 / .6-96$} & \multicolumn{3}{|c|}{ SHMS Unit Number: VTP $-P_{N L}-805 \mathrm{~A}$} \\
\hline \multicolumn{3}{|c|}{$\begin{array}{l}\text { Title of Test: } \\
\text { Electrical Systems }\end{array}$} & \multirow{2}{*}{\multicolumn{3}{|c|}{$\begin{array}{l}\text { Test Equipment and } s / N \text { or Cal. No.: } \\
\text { N/A }\end{array}$}} \\
\hline \multicolumn{3}{|c|}{$\begin{aligned} & \text { Test Performed By: } \text { See Test Personned } \\
& \text { Page } 68\end{aligned}$} & & & \\
\hline $\begin{array}{c}\text { Procedure No. } \\
\text { Initial/0ate } \\
\end{array}$ & Item & Value & $\begin{array}{l}\text { Required } \\
\text { Condition } \\
\end{array}$ & $(A / R)$ & Comment \\
\hline $\begin{array}{l}1.7 .1 .9 .17 \\
\text { man. }\end{array}$ & $\begin{array}{l}\text { TAL-*50 \& } \\
\text { HORN ACTIVE }\end{array}$ & $\begin{array}{l}\frac{O K}{O K} \\
O K \\
O K\end{array}$ & $\begin{array}{l}\text { TAL }-* 50 \text { ON } \\
\text { HORN ON } \\
\text { ACKNOWLEDGE }\end{array}$ & $A$ & \\
\hline $\begin{array}{l}1.7 .1 .9 .18 \\
m x_{1}\end{array}$ & $\begin{array}{l}\text { TIC }-* 56 \\
\text { OPERATES }\end{array}$ & ek & $\begin{array}{l}\text { TIC }-* 56 \\
\text { OPERATES }\end{array}$ & $A$ & \\
\hline $\begin{array}{l}1.7 .1 .9 .19 \\
\text { on } 2.3 \\
\end{array}$ & $\begin{array}{l}\text { ADJ TIC-*56 } \\
\text { SP } 2\end{array}$ & $N / A$ & $N / A$ & $A$ & \\
\hline $\begin{array}{l}1.7 .1 .9 .20 \\
2 \times 3.8 \\
\end{array}$ & RESET ALARM & ok & $\mathrm{TAL}-\star 50 \quad 0 \mathrm{FF}$ & $A$ & \\
\hline $\begin{array}{l}1.7 .1 .9 .21 \\
\operatorname{ming}^{2}\end{array}$ & $\begin{array}{l}\text { ADJ TIC }-* 56 \\
\text { SP } 1 \& \text { SP 2 }\end{array}$ & $N / A$ & N/A & $A$ & \\
\hline $\begin{array}{l}1.7 .1 .9 .22 \\
\text { mas. }\end{array}$ & $\begin{array}{l}\text { OPEN CB-5, } \\
\text { FU-6 \& FU-7 }\end{array}$ & $N / A$ & $N / A$ & $A$ & \\
\hline $\begin{array}{l}1.7 .1 .9 .23 \\
\text { min.e }\end{array}$ & $\begin{array}{l}\text { REMOVE LOAD } \\
\text { TIC }-* 56\end{array}$ & $\mathrm{~N} / \mathrm{A}$ & N/A & $A$ & \\
\hline$\frac{1.7 .1 .9 .24}{m .0 .0}$ & $\begin{array}{l}\text { CLOSE FU-8 } \\
\text { TIS-*62 } \\
\text { NOMINAL TEMP } \\
\end{array}$ & ok & $\begin{array}{l}\text { TIS }-* 62 \\
\text { TEMP }\end{array}$ & $A$ & \\
\hline $\begin{array}{l}1.7 .1 .9 .25 \\
m_{2.3} \\
\end{array}$ & $\begin{array}{l}\text { ADJ TIS-*62 } \\
\text { SP } 1\end{array}$ & $N / A$ & $N / A$ & A & \\
\hline $\begin{array}{l}1.7 .1 .9 .26 \\
\text { nyas.3... } \\
\end{array}$ & $\begin{array}{l}\text { ADJ TIS-*62 } \\
\text { SP } 2\end{array}$ & $N / A$ & N/A & $A$ & \\
\hline $\begin{array}{l}1.7 .1 .9 .27 \\
\text { mxn }\end{array}$ & RESET ALARMS & ok & $\begin{array}{l}\text { TAHL }-* 62 \\
\text { OFF }\end{array}$ & $A$ & \\
\hline $\begin{array}{l}1.7 .1 .9 .28 \\
\text { nine.e }\end{array}$ & $\begin{array}{l}\text { ADJ TIS-*62 } \\
\text { SP } 2\end{array}$ & $N / A$ & N/A & A & \\
\hline$\frac{1.7 .1 .9 .29}{\operatorname{mix.0}}$ & $\begin{array}{l}\text { TAHL }-* 62 \& \\
\text { HORN ACTIVE }\end{array}$ & $\frac{o k}{\Delta a k}$ & $\begin{array}{l}\text { TAHL }-* 62 \text { ON } \\
\text { HORN ON } \\
\text { ACKNOWLEDGE } \\
\end{array}$ & $A$ & \\
\hline $\begin{array}{l}1.7 .1 .9 .30 \\
\text { maxps. } \\
\end{array}$ & $\begin{array}{l}\text { ADJ TIS-*62 } \\
\text { SP } 2\end{array}$ & N/A & $N / A$ & $A$ & \\
\hline
\end{tabular}


TEST DATA SHEET

\begin{tabular}{|c|c|c|c|c|c|}
\hline \multicolumn{3}{|c|}{ Date: $1 / .6 \cdot 96$} & \multicolumn{3}{|c|}{ SHMS Unit Number: VTP-PNL-805A } \\
\hline \multicolumn{3}{|c|}{$\begin{array}{l}\text { Title of Test: } \\
\text { Electrical Systems }\end{array}$} & \multirow{2}{*}{\multicolumn{3}{|c|}{$\begin{array}{l}\text { Test Equiprient and s/N or Cal. No.: } \\
F / u K e \text { V/O meter } \\
\text { S/N } 4 / 620364 \\
\mathrm{Cal} \text { due. } 10.97\end{array}$}} \\
\hline \multicolumn{3}{|c|}{$\begin{array}{l}\text { Test Performed By: See Test Personnel } \\
\text { Page } 68\end{array}$} & & & \\
\hline $\begin{array}{l}\text { Procedure No. } \\
\text { initial/Date }\end{array}$ & Item & Value & $\begin{array}{l}\text { Required } \\
\text { Condition }\end{array}$ & $(A / R)$ & Comment \\
\hline $\begin{array}{l}1.7 .1 .9 .31 \\
\text { m.p. } \\
\end{array}$ & RESET ALARMS & $0 k$ & $\begin{array}{l}\text { TAHL-*62 } \\
\text { OFF }\end{array}$ & $A$ & \\
\hline $\begin{array}{l}1.7 .1 .9 .32 \\
24.0 .8 \\
\end{array}$ & $\begin{array}{l}\text { ADJ TIS }-* 62 \\
S P_{1}\end{array}$ & $N / A$ & $N / A$ & $A$ & \\
\hline $\begin{array}{l}1.7 .1 .9 .33 \\
m_{10.0} \\
\end{array}$ & $\begin{array}{l}\text { TAHL }-* 62 \& \text {. } \\
\text { HORN ACTIVE }\end{array}$ & $\frac{0 k}{0 k}$ & $\begin{array}{l}\text { TAHL-*62 ON } \\
\text { HORN ON } \\
\text { ACKNOWLEOGE }\end{array}$ & $A$ & \\
\hline $\begin{array}{l}1.7 .1 .9 .34 \\
73 . x_{0} \\
\end{array}$ & $\begin{array}{l}\text { ADJ TIS-*62 } \\
\text { SP } 1 \& \text { SP } 2\end{array}$ & $N / A$ & $N / A$ & $A$ & \\
\hline $\begin{array}{l}1.7 .1 .9 .35 \\
m .00 \\
\end{array}$ & $\begin{array}{l}\text { OPEN FU-8, } \\
\text { FU-9 \& FU-10 }\end{array}$ & $N / A$ & $N / A$ & $A$ & \\
\hline \multicolumn{6}{|c|}{ Section 1.7.1.10 verifies the differential pressure transmitter system. } \\
\hline $\begin{array}{l}1.7 .1 .10 .1 \\
\operatorname{mos}_{2}\end{array}$ & $\begin{array}{l}\text { REMOVE PLUGS } \\
\text { BAL PDIT-*60 }\end{array}$ & N/A & $N / A$ & $A$ & \\
\hline $\begin{array}{l}1.7 .1 .10 .2 \\
m \infty .1\end{array}$ & $\begin{array}{l}\text { CLOSE FU-14 } \\
\text { REC PDIT-* } 60 \\
\text { PRESSURE }\end{array}$ & $=0.0195$ & $\begin{array}{l}\text { POIT-*60 } \\
\text { PRESSURE } \\
0 \pm 1 " \mathrm{H}_{2} \mathrm{O}\end{array}$ & $A$ & \\
\hline $\begin{array}{l}1.7 .1 .10 .3 \\
\text { mo. }\end{array}$ & $\begin{array}{l}\text { MUX-*70 Vdc } \\
\text { TB7+ \& TB8- }\end{array}$ & $2.154 \mathrm{~V}_{10}$ & $\begin{array}{l}\text { MUX }-* 70 \mathrm{Vdc} \\
2.2 \pm 0.1 \\
\end{array}$ & $A$ & \\
\hline$\frac{1.7 .1 .10 .4}{2 m \cdot 2 \cdot 8}$ & $\begin{array}{l}\text { REPLACE } \\
\text { PLUGS ON } \\
\text { PDIT-*60 }\end{array}$ & N/A & $N / A$ & $A$ & \\
\hline $\begin{array}{l}1.7 .1 .10 .5 \\
\text { Mxp.s } \\
\end{array}$ & OPEN FU-14 & N/A & $N / A$ & $A$ & \\
\hline \multicolumn{6}{|c|}{ Section 1.7 .1 .11 verifies the gas chromatograph power. } \\
\hline $\begin{array}{l}1.7 .1 .11 .1 \\
\text { max.83 } \\
\end{array}$ & CLOSE FU-11 & $N / A$ & $N / A$ & $A$ & \\
\hline $\begin{array}{l}1.7 .1 .11 .2 \\
222.6 \\
\end{array}$ & $\begin{array}{l}\text { POWER } \\
\text { MON-*60 }\end{array}$ & $124.6 V_{a c}$ & $\begin{array}{l}\text { MON-*60 } \\
\text { POWERED }\end{array}$ & $A$ & \\
\hline $\begin{array}{l}1.7 .1 .11 .3 \\
7 \pi \approx .0\end{array}$ & OPEN FU-11 & $N / A$ & $N / A$ & A & \\
\hline
\end{tabular}


TEST DATA SHEET

\begin{tabular}{|c|c|c|c|c|c|}
\hline \multicolumn{3}{|c|}{ Date: $\quad / 1-6-96$} & \multicolumn{3}{|c|}{ SHMS Unit Number: VTP-PNL $-805 \mathrm{~A}$} \\
\hline \multicolumn{3}{|c|}{$\begin{array}{l}\text { Title of rest: } \\
\text { Electrical Systems }\end{array}$} & \multirow{2}{*}{\multicolumn{3}{|c|}{$\begin{array}{l}\text { Test Equipment and S/N or Cal. No.: } \\
\text { F/uke V/O meter } \\
S / N 44620364 \\
C a / \text { doe. } 10.97\end{array}$}} \\
\hline \multicolumn{3}{|c|}{$\begin{aligned} \text { Test Performed By: See Test Personnel } & \text { Poge } 60\end{aligned}$} & & & \\
\hline $\begin{array}{l}\text { Procedure No. } \\
\text { Initial/Date }\end{array}$ & Item & value & $\begin{array}{l}\text { Required } \\
\text { Condition }\end{array}$ & $(A / R)$ & Comment \\
\hline \multicolumn{6}{|c|}{ Section 1.7.1.12 verifies the multi gas analyzer power. } \\
\hline $\begin{array}{l}1.7 .1 .12 .1 \\
\text { mx.8 } \\
\end{array}$ & CLOSE FU-12 & $N / A$ & $N / A$ & $A$ & \\
\hline $\begin{array}{l}1.7 .1 .12 .2 \\
m \times . n \\
\end{array}$ & $\begin{array}{l}\text { POWER } \\
\text { NIT }-* 52\end{array}$ & 124.5 Vou & $\begin{array}{l}\text { NIT }-* 52 \\
\text { POWERED } \\
\end{array}$ & A & \\
\hline $\begin{array}{l}1.7 .1 .12 .3 \\
\min x \\
\end{array}$ & OPEN FU-12 & $N / A$ & $N / A$ & A & \\
\hline \multicolumn{6}{|c|}{ Section 1.7 .1 .13 verifies the personnel computer power. } \\
\hline $\begin{array}{l}1.7 .1 .13 .1 \\
\text { m.x. } \\
\end{array}$ & CLOSE CB-6 & $N / A$ & $N / A$ & $A$ & \\
\hline $\begin{array}{l}1.7 .1 .13 .2 \\
m_{n_{3}, 0} \\
\end{array}$ & $\begin{array}{l}\text { POWER PC-*60 } \\
\& P C-* 70\end{array}$ & ok & $\begin{array}{ll}P C-* 60 & O N \\
P C-* 70 & O N \\
\end{array}$ & $A$ & \\
\hline $\begin{array}{l}1.7 .1 .13 .3 \\
\ln x . x \\
\end{array}$ & $\begin{array}{l}\text { TURN INST. } \\
\text { OFF \& } \\
\text { OPEN CB-6 }\end{array}$ & ok & $\begin{array}{ll}P C-* 60 & \text { OFF } \\
P C-* 70 & \text { OFF }\end{array}$ & $A$ & \\
\hline
\end{tabular}

al1 $11-6+96$ 
TEST DATA SHEET

\begin{tabular}{|c|c|c|c|c|c|}
\hline \multicolumn{3}{|c|}{ Date: 11.6 .96} & \multicolumn{3}{|c|}{ SHMS Unit Number: $V T P-P N L-B 05 A$} \\
\hline \multicolumn{3}{|c|}{$\begin{array}{l}\text { Title of Test: } \\
\text { Electrical Systems }\end{array}$} & \multirow{2}{*}{\multicolumn{3}{|c|}{$\begin{array}{l}\text { Test Equipment and S/N or Cal. No.: } \\
N / A\end{array}$}} \\
\hline \multicolumn{3}{|c|}{$\begin{array}{c}\text { Test Performed By: see Test Personnel } \\
\text { Page } 68\end{array}$} & & & \\
\hline $\begin{array}{c}\text { Procedure No. } \\
\text { Initial/Date } \\
\end{array}$ & I tem & Value & $\begin{array}{l}\text { Required } \\
\text { Condition }\end{array}$ & $(A / R)$ & Comment \\
\hline \multicolumn{6}{|c|}{ Section 1.7 .2 verifies the intrinsic safety apparatus is properly labeied } \\
\hline $\begin{array}{l}11.6 .96 \\
1.7 .2 .1 \\
m 2 x .8 \\
\end{array}$ & $\begin{array}{l}\text { INTRINSIC } \\
\text { SAFETY } \\
\text { APPARATUS } \\
\text { NAMEPLATES } \\
\text { INSTALLED }\end{array}$ & $\begin{array}{c}\frac{0 k}{0 k} \\
\frac{0 k}{0 k} \\
0 k \\
0 k\end{array}$ & $\begin{array}{l}N E-* 54 \\
N E-* 55 \\
E B-* 54 \\
E B-* 55 \\
N I T-* 54 \\
N I T-* 55\end{array}$ & A & \\
\hline $\begin{array}{l}117^{-6-96} .2 .2 \\
m .20 .8 \\
\end{array}$ & $\begin{array}{l}\text { INTRINSIC } \\
\text { SAFETY WIRE } \\
\text { LABELED } \\
\end{array}$ & OK & $\begin{array}{l}\text { NE/EB-*54 } \\
\text { NE/EB-*55 }\end{array}$ & $A$ & \\
\hline $\begin{array}{l}11.7^{-6} .96 \\
n \times 2 \times 6 \\
\end{array}$ & $\begin{array}{l}\text { SEC. } 1.7 .1 \text { \& } \\
1.7 .2 \text { DONE }\end{array}$ & $\frac{1}{\text { Test Di }}$ & Lar Signatu & & $=8-86$ \\
\hline
\end{tabular}

Test Witness/Review:
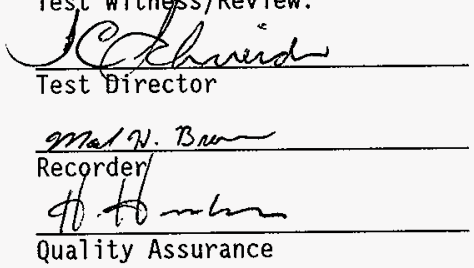

$\frac{11-8-96}{\text { Date }}$

$\frac{11 \cdot 6 \cdot 96}{\text { Date }}$

$\frac{11-12-96}{\text { Date }}$ 
TEST DATA SHEET

\begin{tabular}{|c|c|c|c|c|c|}
\hline \multicolumn{3}{|c|}{ Date: $11-6-96$} & \multicolumn{3}{|c|}{ SHMS Unit Number: $V T P-P N L-805 A$} \\
\hline \multicolumn{3}{|c|}{$\begin{array}{l}\text { Title of Test: } \\
\text { Pneumatic Systems }\end{array}$} & \multirow{2}{*}{\multicolumn{3}{|c|}{$\begin{array}{l}\text { Test Equipment and } S / N \text { or Cal. No.: } \\
\text { N/A }\end{array}$}} \\
\hline \multicolumn{3}{|c|}{$\begin{array}{r}\text { Test Performed By: See } \\
\text { Paye }\end{array}$} & & & \\
\hline $\begin{array}{l}\text { Procedure No. } \\
\text { Initial/Date }\end{array}$ & Item & value & $\begin{array}{l}\text { Required } \\
\text { Condition }\end{array}$ & $(A / R)$ & Comment \\
\hline \multicolumn{6}{|c|}{ Section 1.7 .4 .1 provides the pneumatic test section initial conditions } \\
\hline $\begin{array}{l}1.7 .4 .1 .1 \\
m .2 .3 \\
\end{array}$ & $\begin{array}{l}\text { INITIAL } \\
\text { CONDITIONS } \\
\end{array}$ & ot & $\begin{array}{l}\text { BKR \& FUSE } \\
\text { LINE UP } \\
\end{array}$ & $A$ & \\
\hline $\begin{array}{l}1.7 .4 .1 .2 \\
m . p .8 \\
\end{array}$ & $\begin{array}{l}\text { INITIAL } \\
\text { CONDITIONS }\end{array}$ & $-\Delta k$ & $\begin{array}{l}\text { VALVE LINE } \\
\text { UP }\end{array}$ & A & \\
\hline $\begin{array}{l}1.7 .4 .1 .3 \\
m .2 .8\end{array}$ & $\begin{array}{l}\text { DISCONNECT } \\
1 / 16^{" ~ T U B I N G} \\
\text { ON MON-*60 }\end{array}$ & ok & $\begin{array}{l}\text { TUBING } \\
\text { CONFIGURED }\end{array}$ & $A$ & \\
\hline $\begin{array}{l}1.7 .4 .1 .4 \\
24.0 .3\end{array}$ & $\begin{array}{l}\text { DISCONNECT } \\
\text { TUBING ON } \\
\text { NIT } * 52 \\
\end{array}$ & - ak & $\begin{array}{l}\text { TUBING } \\
\text { CONFIGURED }\end{array}$ & $A$ & \\
\hline $\begin{array}{l}1.7 .4 .1 .5 \\
m 2.6 \\
\end{array}$ & $\begin{array}{l}\text { PLUG VENT } \\
\text { FLT }-* 63\end{array}$ & $d K$ & $\begin{array}{l}\text { FLT-*63 } \\
\text { PLUGGED }\end{array}$ & $A$ & \\
\hline \multicolumn{6}{|c|}{ Section 1.7 .4 .2 verifies the main sample flow loop. } \\
\hline $\begin{array}{l}1.7 .4 .2 .1 \\
7.0 .8 \\
\end{array}$ & $\begin{array}{l}\text { CLOSE CB-3/4 } \\
\text { ADJ. FLOW }\end{array}$ & $\frac{0 / 1}{5.01}$ & $\begin{array}{l}\text { CB CLOSED } \\
\text { FIT } * 57 \\
\text { INDICATION }\end{array}$ & A & \\
\hline $\begin{array}{l}1.7 .4 .2 .2 \\
229.8 \\
\end{array}$ & $\begin{array}{l}\text { PDI-*51 } \\
\text { READING } \\
\end{array}$ & $27 \% " 1 / 20$ & $20-40 " \mathrm{H}_{2} \mathrm{O}$ & A & \\
\hline $\begin{array}{l}1.7 .4 .2 .3 \\
m .2 .8 \\
\end{array}$ & $\begin{array}{l}\text { PI }-* 53 \\
\text { READING }\end{array}$ & $\frac{5.001 .686}{-2.0 \% 1 \mathrm{Hg}}$ & $\begin{array}{l}-2 \text { to }-4 \\
" \mathrm{Hg}\end{array}$ & A & \\
\hline $\begin{array}{l}1.7 .4 .2 .4 \\
\text { mas }\end{array}$ & RESET ALARMS & $O K$ & NO ALARMS & A & \\
\hline $\begin{array}{l}1.7 .4 .2 .5 \\
21.04 \\
\end{array}$ & CLOSE SV-*16 & $\begin{array}{l}\frac{0.025^{*} 400}{0 k} \\
\frac{0 k}{0 k}\end{array}$ & $\begin{array}{ll}\mathrm{FIT}-* 57 & 0 \\
\pm 1 \quad " \mathrm{H}_{2} \mathrm{O} & \\
\text { FAL } * 57 & \text { ON } \\
\text { HORN } & \text { ON } \\
\end{array}$ & A & \\
\hline $\begin{array}{l}1.7 .4 .2 .6 \\
\text { xux.5 } \\
\end{array}$ & OPEN SV-*16 & ok & $\begin{array}{l}\text { FAL-*57 ON } \\
\text { HORN OFF } \\
\end{array}$ & A & \\
\hline $\begin{array}{l}1.7 .4 .2 .7 \\
\text { ming }\end{array}$ & RESET ALARMS & ok & $\begin{array}{l}\text { ALARMS } \\
\text { RESET }\end{array}$ & A & \\
\hline
\end{tabular}


TEST DATA SHEET

\begin{tabular}{|c|c|c|c|c|c|}
\hline Date: $11-6.94$ & & & SHMS Unit Number & VTP- & $P_{N L}-805 A$ \\
\hline $\begin{array}{l}\text { Title of Test: } \\
\text { Pneumatic Sys }\end{array}$ & tems & & Test Equipment ar & $\mathrm{S} / \mathrm{N}$ or & Cal. No.: \\
\hline Test Performed By: & $\begin{array}{l}\text { See Test Per } \\
\text { Poge } 60\end{array}$ & inned & $N / A$ & & \\
\hline $\begin{array}{c}\text { Procedure No. } \\
\text { Initial/Date }\end{array}$ & Iten & value & $\begin{array}{l}\text { Required } \\
\text { condition } \\
\end{array}$ & $(A / R)$ & Comment \\
\hline 1.7 .4 .2 .8 & CLOSE SV-*25 & 0.03 & $\mathrm{FIT}-* 570$ & & see Rev OB \\
\hline mot & & OKK & $\begin{array}{ll} \pm 1 & +12 \\
\text { FAL }-* 57 & \text { ON } \\
\text { HORN } & \text { ON } \\
\end{array}$ & $A$ & $m 2.8 \quad 12 \cdot 17-91$ \\
\hline$\frac{1.7 .4 .2 .9}{102806}$ & OPEN SV-*25 & ok & $\begin{array}{l}F A L-* 57 \text { ON } \\
\text { HORN OFF }\end{array}$ & $A$ & $\begin{array}{l}\text { see } \operatorname{Ras} \Delta B \\
m \text { mis } 12-17.96\end{array}$ \\
\hline$\frac{1.7 .4 .2 .10}{3.2 .0 k}$ & RESET_ALARMS & $\Delta k$ & $\begin{array}{l}\text { ALARMS } \\
\text { RESET }\end{array}$ & $A$ & $\frac{\text { see Rov } O B}{\text { mans } 12-17-96}$ \\
\hline $\begin{array}{l}1.7 .4 .2 .11 \\
\text { mps }\end{array}$ & $\begin{array}{l}\text { OPEN SV-*54 } \\
\& \text { SV-*66 } \\
\text { READ PRESS. } \\
\text { PDIT-*60 }\end{array}$ & $\frac{0 k}{-42.74 \% 20}$ & $\begin{array}{l}\text { VALVES OPEN } \\
-27 \text { to }-82 \\
" \mathrm{H}_{2} \mathrm{O}\end{array}$ & $A$ & \\
\hline $\begin{array}{l}1.7 .4 .2 .12 \\
\text { m.x. }\end{array}$ & $\begin{array}{l}\text { CLOSE SV } * 54 \\
\text { OPEN SV } * 63 \\
\text { READ PRESS. } \\
\text { PDIT-*60 } \\
\end{array}$ & $\frac{k}{-0.01}$ & $\begin{array}{l}\text { VALVES } \\
\text { OPERATED } \\
0 \pm 1 \quad \mathrm{H}_{2} \mathrm{O}\end{array}$ & $A$ & \\
\hline $\begin{array}{l}1.7 .4 .2 .13 \\
2 m . x .8 \\
\end{array}$ & $\begin{array}{l}\text { CLOSE SV } * 633 \\
\text { OPEN SV } * 55 \\
\text { READ PRESS. } \\
\text { PDIT-*60 } \\
\end{array}$ & $\frac{0 k}{-44 \cdot 3^{41120}}$ & $\begin{array}{l}\text { VALVES } \\
\text { OPERATED } \\
-27 \text { to }-82 \\
" \mathrm{H}_{2} \mathrm{O} \\
\end{array}$ & A & \\
\hline $\begin{array}{l}1.7 .4 .2 .14 \\
\min x e^{2}\end{array}$ & $\begin{array}{l}\text { CLOSE SV-*55 } \\
\text { OPEN SV-*64 } \\
\text { READ PRESS. } \\
\text { PDIT-*60 }\end{array}$ & $\frac{O X}{-0.02^{\circ} \mathrm{H}_{2} \mathrm{O}}$ & $\begin{array}{l}\text { VALVES } \\
\text { OPERATED } \\
0 \pm 1 \quad " \mathrm{H}_{2} \mathrm{O}\end{array}$ & $A$ & \\
\hline $\begin{array}{l}1.7 .4 .2 .15 \\
\text { mase }\end{array}$ & $\begin{array}{l}\text { CLOSE SV-*64 } \\
\& \text { SV-*66 }\end{array}$ & $N / A$ & $N / A$ & $A$ & \\
\hline Section 1.7 & 3 verifies t & grab samp & flow loop. & & \\
\hline $\begin{array}{c}1.7 .4 .3 .1 \\
\text { nex } 2\end{array}$ & READ PDI $* 51$ & $27.5^{n} \mathrm{H}_{2} \mathrm{O}$ & $20-40 " \mathrm{H}_{2} \mathrm{O}$ & $A$ & \\
\hline $\begin{array}{l}1.7 .4 .3 .2 \\
\text { m.p.r. }\end{array}$ & $\begin{array}{l}\text { PUSH PB }-* 59 \\
\text { GRAB SAMPLE }\end{array}$ & $d E$ & $\begin{array}{l}\text { START } \\
\text { STOPWATCH }\end{array}$ & $A$ & \\
\hline
\end{tabular}

all $11-6-96$ 
TEST DATA SHEET

\begin{tabular}{|c|c|c|c|c|c|}
\hline Date: $11-6$ & & & SHMS Unit Number: & $V T P$ & $-805 A$ \\
\hline $\begin{array}{l}\text { Title of Test: } \\
\text { Pneumatic } \mathrm{S}\end{array}$ & tems & & $\begin{array}{l}\text { Test Equipment ar } \\
\text { Fluke V/o me }\end{array}$ & $\mathrm{S} / \mathrm{N}$ or & \\
\hline Test Performed & $\begin{array}{l}\text { see Test Pe } \\
\text { Pere } 60\end{array}$ & soand & $\begin{array}{l}S / N \quad 446203 \\
\text { Cal. doe } 10-9\end{array}$ & & \\
\hline $\begin{array}{c}\text { Procedure No. } \\
\text { initial/Date }\end{array}$ & item & value & $\begin{array}{l}\text { Required } \\
\text { Condition } \\
\end{array}$ & $(A / R)$ & Comment \\
\hline $\begin{array}{l}1.7 .4 .2 .8 \\
m .3 B\end{array}$ & CLOSE SV-*25 & 0 & $\begin{array}{l}\text { FIT-*57 } \\
\pm 1 " H, 0\end{array}$ & & \\
\hline $12-77-96$ & & $\frac{\frac{O K}{O K}}{\frac{O K}{O V}}$ & $\begin{array}{ll}\text { FAL-*57 ON } \\
\text { HORN } & \text { ON } \\
\text { XA-*63 } & \text { ON } \\
\text { YY-*71 } & \\
\text { TB } 3-4 & \text { OV } \\
\end{array}$ & A & \\
\hline $\begin{array}{l}1.7 .4 .2 .9 \\
m .21 .8\end{array}$ & OPEN SV $* 25$ & & $\begin{array}{l}\text { FAL- *57 ON } \\
\text { HORN OFF }\end{array}$ & & \\
\hline 12.17 .91 & & $\begin{array}{c}0 k \\
120.4 \\
\end{array}$ & \begin{tabular}{|l} 
XA-*63 \\
YYF $-* 71$ \\
TB $3-4 \quad 120 \mathrm{~V}$ \\
\end{tabular} & A & \\
\hline $\begin{array}{l}1.7 .4 .2 .10 \\
\text { mpase } 12-17-9 \mathrm{c} \\
\end{array}$ & RESET ALARMS & $\partial k$ & $\begin{array}{l}\text { ALARMS } \\
\text { RESET }\end{array}$ & A & \\
\hline 1.7 .4 .2 .11 & $\begin{array}{l}\text { OPEN SV } * 54 \\
\& \text { SV } * 66 \\
\text { READ PRESS. } \\
\text { PDIT-*60 }\end{array}$ & & $\begin{array}{l}\text { VALVES OPEN } \\
-27 \text { to }-82 \\
" \mathrm{H}_{2} \mathrm{O}\end{array}$ & & \\
\hline 1.7 .4 .2 .12 & $\begin{array}{l}\text { CLOSE SV }-* 54 \\
\text { OPEN SV } * 63 \\
\text { READ PRESS. } \\
\text { PDIT }-* 60 \\
\end{array}$ & & $\begin{array}{l}\text { VALVES } \\
\text { OPERATED } \\
0 \pm 1 \quad \mathrm{H}_{2} \mathrm{O}\end{array}$ & & \\
\hline 1.7 .4 .2 .13 & $\begin{array}{l}\text { CLOSE SV-*63 } \\
\text { OPEN SV-*55 } \\
\text { READ PRESS. } \\
\text { PDIT-* } 60 \\
\end{array}$ & & $\begin{array}{l}\text { VALVES } \\
\text { OPERATED } \\
-27 \text { to }-82 \\
" \mathrm{H}_{2} \mathrm{O} \\
\end{array}$ & & \\
\hline 1.7 .4 .2 .14 & $\begin{array}{l}\text { CLOSE SV-*55 } \\
\text { OPEN SV }-* 64 \\
\text { READ PRESS. } \\
\text { PDIT-*60 } \\
\end{array}$ & & $\begin{array}{l}\text { VALVES } \\
\text { OPERATED } \\
0 \pm 1 \quad \text { " } \mathrm{H}_{2} \mathrm{O}\end{array}$ & & \\
\hline 1.7 .4 .2 .15 & $\begin{array}{l}\text { CLOSE SV-*64 } \\
\& \text { SV-*66 }\end{array}$ & $N / A$ & N/A & & \\
\hline 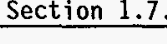 & 3 & Yrov & gop. & & \\
\hline 1.7 .4 .3 .1 & READ PDI $-* 51$ & & $20-40 \quad " \mathrm{H}_{2} \mathrm{O}$ & & \\
\hline
\end{tabular}


TEST DATA SHEET

\begin{tabular}{|c|c|c|c|c|c|}
\hline Date: $11-6-9$ & & & SHMS Unit Number: & VTP. & $305 A$ \\
\hline $\begin{array}{l}\text { Title of rest: } \\
\text { Pneumatic } S \\
\end{array}$ & tems & & Test Equipment an & $S / N$ or & \\
\hline Test Performed B & $\begin{array}{l}\text { See Test Pers } \\
\text { Page } 60\end{array}$ & innel & $N / A$ & & \\
\hline $\begin{array}{c}\text { Procedure No. } \\
\text { Initial/oate } \\
\end{array}$ & Itern & Value & $\begin{array}{l}\text { Required } \\
\text { Condition } \\
\end{array}$ & $(A / R)$ & comment \\
\hline $\begin{array}{l}1.7 .4 .3 .3 \\
\max ^{2} \\
\end{array}$ & $\begin{array}{l}\text { GRAB SAMPLE } \\
\text { LAMPS ON } \\
\text { READ FIV-*52 }\end{array}$ & $\begin{array}{l}\frac{o k}{0 k} \\
\frac{o k}{0 k}\end{array}$ & $\begin{array}{lr}\mathrm{PBL}-* 58 & \text { ON } \\
\mathrm{PBL}-* 59 & \text { ON } \\
\mathrm{YAL}-* 58 & \text { ON } \\
\text { FIV }-* 52 & 0 \\
\end{array}$ & A & \\
\hline $\begin{array}{l}1.7 .4 .3 .4 \\
m . x .8\end{array}$ & $\begin{array}{l}\text { OPEN SV }-* 15 \\
\& \text { ADJ. FIV- } \\
* 52\end{array}$ & $\frac{a k}{10 \text { crut }}$ & $\begin{array}{l}\text { SV }-* 15 \text { OPEN } \\
\text { FIV-*52 } \\
\text { FLOW } 10 \mathrm{CFH} \\
\end{array}$ & $A$ & \\
\hline $\begin{array}{l}1.7 .4 .3 .5 \\
\text { an.n.s. }\end{array}$ & READ PDI $-* 51$ & $31^{\prime \prime} \mathrm{H}_{2} \mathrm{O}$ & $\begin{array}{l}\text { PDI }-* 51 \\
\text { HIGHER THAN } \\
1.7 .4 .3 .1 \\
\end{array}$ & $A$ & \\
\hline $\begin{array}{l}1.7 .4 .3 .6 \\
\text { nex } x_{8} \\
\end{array}$ & CLOSE SV-* 15 & ok & FIV $-* 520$ & A & \\
\hline $\begin{array}{c}1.7 .4 .3 .7 \\
\text { n.2.7. } \\
\end{array}$ & OPEN SV-*15 &.$k$ & $\begin{array}{l}\text { FIV-*52 } \\
\text { FLOW }\end{array}$ & A & \\
\hline $\begin{array}{c}1.7 .4 .3 .8 \\
\operatorname{mos} .8\end{array}$ & $\begin{array}{l}\text { PBL }-* 59 \\
\text { GRAB SAMPLE } \\
\text { TIME } \\
\end{array}$ & $\frac{d k}{4.59 \mathrm{~min}}$ & $\begin{array}{l}\text { PBL-*59 OFF } \\
\text { STOP WATCH } \\
5 \pm .5 \text { MIN } \\
\end{array}$ & $A$ & \\
\hline $\begin{array}{c}1.7 .4 .3 .9 \\
3 \pi 2.83 \\
\end{array}$ & $\begin{array}{l}\text { PB-*58 RESET } \\
\text { SAMPLER }\end{array}$ & $\frac{a k}{O K}$ & $\begin{array}{l}\mathrm{PBL}-* 58 \text { OFF } \\
\text { YAL-*58 } \\
\end{array}$ & $A$ & \\
\hline Section 1.7 & 4 verifies & $\mathrm{H}_{2} \mathrm{cell} \mathrm{ca}$ & libration 70 & & \\
\hline $\begin{array}{l}1.7 .4 .4 .1 \\
m .8 .8 \\
\end{array}$ & $\begin{array}{l}\text { CONNECT } \mathrm{H}_{2} \\
\text { CAL GAS }\end{array}$ & $N / A$ & N/A & A & \\
\hline $\begin{array}{c}1.7 .4 .4 .2 \\
12.918\end{array}$ & $\begin{array}{l}\text { OPEN ISO } \\
\text { VALVE }\end{array}$ & $5 P=I G$ & $<10$ PSIG & A & \\
\hline $\begin{array}{l}1.7 .4 .4 .3 \\
\text { mis }\end{array}$ & $\begin{array}{l}\text { OPEN SV-* } 18 \\
\text { ADJ. FIV-*56 }\end{array}$ & $2 \mathrm{CFH}$ & $2 \pm .1 \mathrm{CFH}$ & A & \\
\hline
\end{tabular}

d11 $11-6.96$ 


\section{TEST DATA SHEET}

\begin{tabular}{|c|c|c|c|c|c|}
\hline \multicolumn{3}{|c|}{ Date: $11-6-96$} & \multicolumn{3}{|c|}{ SHMS Unit Number: VTP.PNL-BOSA } \\
\hline \multicolumn{3}{|c|}{$\begin{array}{l}\text { Title of Test: } \\
\text { Pneumatic Systems }\end{array}$} & \multirow{2}{*}{\multicolumn{3}{|c|}{$\begin{array}{l}\text { rest Equipment and } S / N \text { or Cal. No.: } \\
\text { Fluke V/O meter } \\
\text { SN } 44620364 \\
\text { Cal. due } 10-97\end{array}$}} \\
\hline \multirow{2}{*}{$\begin{array}{l}\text { Test Performed By } \\
\begin{array}{c}\text { Procedure No. } \\
\text { Initial/Date }\end{array} \\
\end{array}$} & \multicolumn{2}{|c|}{$\begin{array}{l}\text { See Test Personnel } \\
\text { Pagn } 68\end{array}$} & & & \\
\hline & I tem & Value & $\begin{array}{l}\text { Required } \\
\text { Condition }\end{array}$ & $(A / R)$ & Comment \\
\hline $\begin{array}{l}1.7 .4 .4 .4 \\
m x . n \\
\end{array}$ & $\begin{array}{l}\text { VERIFY } \\
\text { CONDITIONS }\end{array}$ & 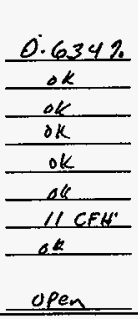 & $\begin{array}{l}\mathrm{NIT}-* 54 \\
>.625 \% \mathrm{H}_{2} \\
\text { NAH-*55 ON } \\
\text { NAH-*54 ON } \\
\text { PBL-*59 ON } \\
\text { PBL-*58 ON } \\
\text { YAL-*58 ON } \\
\text { FIV-*52 FLO } \\
\text { HORN ON } \\
\text { TB2-21/22 } \\
\text { OPEN } \\
\end{array}$ & A & \\
\hline $\begin{array}{l}1.7 .4 .4 .5 \\
\sin x \\
\end{array}$ & $\begin{array}{l}\text { ACKNOWLEDGE } \\
\text { HORN }\end{array}$ & $N / A$ & $N / A$ & A & \\
\hline $\begin{array}{l}1.7 .4 .4 .6 \\
\min \end{array}$ & $\begin{array}{l}\text { CLOSE SV-*18 } \\
\text { VERIFY } \\
\text { CONDITIONS }\end{array}$ & $\begin{array}{l}0.610 \\
0 k \\
0 k \\
0 k \\
04 \\
0.02 \Omega\end{array}$ & $\begin{array}{l}\text { FIV }-* 56 \quad 0 \\
\text { NIT-*54 } \\
<.625 \% \mathrm{H}_{2} \\
\mathrm{NAH}-* 55 \text { OFF } \\
\mathrm{NAH}-* 54 \text { ON } \\
\mathrm{PBL}-* 58 \text { ON } \\
\text { YAL-*58 ON } \\
\text { TB2-21/22 } \\
\text { CLOSED }\end{array}$ & A & \\
\hline $\begin{array}{l}1.7 .4 .4 .7 \\
2400\end{array}$ & $\begin{array}{l}\text { PUSH RESET } \\
\text { SAMPLER PB- } \\
\star 58 \\
\end{array}$ & $\frac{\Delta K}{O K}$ & $\begin{array}{ll}\text { PBL-*58 } & \text { OFF } \\
\text { YAL-*58 } & \text { OFF }\end{array}$ & $A$ & \\
\hline $\begin{array}{c}1.7 .4 .4 .8 \\
1.2 .8 \\
\end{array}$ & RESET ALARM & . ok & $\mathrm{NAH}-* 54$ OFF & A & \\
\hline $\begin{array}{l}1.7 .4 .4 .9 \\
7 \times 70 \\
\end{array}$ & $\begin{array}{l}\text { OPEN SV-*19 } \\
\text { ADJ. FIV-*56 }\end{array}$ & $2 C F H$ & $2 \pm .1 \mathrm{CFH}$ & A & \\
\hline
\end{tabular}

all $11-6-96$ 
TEST DATA SHEET

\begin{tabular}{|c|c|c|c|c|c|}
\hline \multicolumn{3}{|l|}{ Date: $11.6-96$} & \multicolumn{3}{|c|}{ SHMS Unit Number: VTP.PNL-BOSA } \\
\hline \multicolumn{3}{|c|}{$\begin{array}{l}\text { Title of Test: } \\
\text { Pneumatic Systems }\end{array}$} & \multirow{2}{*}{\multicolumn{3}{|c|}{ 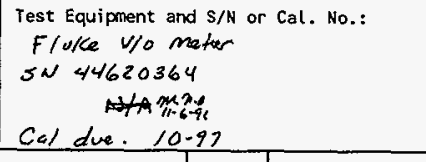 }} \\
\hline \multicolumn{3}{|c|}{$\begin{aligned} \text { Test Performed By: } & \text { see Test Porsonnel } \\
& \text { Page } 68\end{aligned}$} & & & \\
\hline $\begin{array}{l}\text { Procedure No. } \\
\text { Initial/oate }\end{array}$ & Item & value & $\begin{array}{l}\text { Required } \\
\text { Condition }\end{array}$ & $(A / R)$ & Comment \\
\hline $\begin{array}{l}1.7 .4 .4 .10 \\
m_{12.2} \\
\end{array}$ & $\begin{array}{l}\text { VERIFY } \\
\text { CONDITIONS }\end{array}$ & $\begin{array}{l}\frac{0.630}{0 K} \\
0 K \\
0 K \\
0 K \\
0 K \\
0 K \\
0 K \\
\text { OPa } \\
\end{array}$ & $\begin{array}{l}\text { NIT-*54 55 } 522^{204} \\
>.625 \% \mathrm{H}_{2} \\
\text { NAH-*55 ON } \\
\text { NAH-*54 ON } \\
\text { PBL-*59 ON } \\
\text { PBL-*58 ON } \\
\text { YAL-*58 ON } \\
\text { FIV-*52 FLO } \\
\text { HORN ON } \\
\text { TB2-21/22 } \\
\text { OPEN }\end{array}$ & A & \\
\hline $\begin{array}{l}1.7 .4 .4 .11 \\
-\sin 2.8 \\
\end{array}$ & $\begin{array}{l}\text { ACKNOWLEDGE } \\
\text { HORN }\end{array}$ & $N / A$ & $N / A$ & $\alpha$ & \\
\hline $\begin{array}{l}1.7 .4 .4 .12 \\
\text { mine }\end{array}$ & $\begin{array}{l}\text { CLOSE SV-*19 } \\
\text { VERIFY } \\
\text { CONDITIONS }\end{array}$ & $\begin{array}{l}0.609 \\
0.0 k \\
0 k \\
0 K \\
0 k \\
0.03 \Omega\end{array}$ & $\begin{array}{l}\text { FIV-*56 } 0 \\
\text { NIT-*54 55 } \\
<.625 \% \mathrm{H}_{2} \\
\mathrm{NAH}-* 55 \mathrm{OFF} \\
\mathrm{NAH}-* 54 \text { ON } \\
\text { PBL-*58 ON } \\
\text { YAL-*58 ON } \\
\text { TB2-21/22 } \\
\text { CLOSED } \\
\end{array}$ & $A$ & \\
\hline $\begin{array}{l}1.7 .4 .4 .13 \\
1 \times .78 \\
\end{array}$ & $\begin{array}{l}\text { PUSH RESET } \\
\text { SAMPLER PB- } \\
* 58 \\
\end{array}$ & $\frac{\sigma k}{o k}$ & $\begin{array}{l}\mathrm{PBL}-* 58 \text { OFF } \\
\mathrm{YAL}-* 58 \text { OFF }\end{array}$ & $A$ & \\
\hline $\begin{array}{l}1.7 .4 .4 .14 \\
\text { mins. } \\
\end{array}$ & RESET ALARM & $\Delta k$ & $\mathrm{NAH}-* 54$ OFF & A & \\
\hline $\begin{array}{l}1.7 .4 .4 .15 \\
\text { m.3.s. }\end{array}$ & $\begin{array}{l}\text { DISCONNECT } \\
\text { CAL. GAS }\end{array}$ & $N / A$ & $N / A$ & A & \\
\hline $\begin{array}{l}1.7 .4 .4 .16 \\
\text { minn }\end{array}$ & $\begin{array}{l}\text { PUSH PB }-* 54 \\
\text { VERIFY } \\
\text { CONDITIONS }\end{array}$ & $\begin{array}{l}\text { ok } \\
\text { ole } \\
\text { oper }\end{array}$ & $\begin{array}{l}X A-* 63 \text { ON } \\
\text { PBL-*54 ON } \\
\text { TB2-23/24 } \\
\text { OPEN } \\
\end{array}$ & $A$ & \\
\hline $\begin{array}{l}1.7 .4 .4 .17 \\
\text { m.2.0 }\end{array}$ & $\begin{array}{l}\text { PUSH PB }-* 54 \\
\text { VERIFY } \\
\text { CONDITIONS }\end{array}$ & $\begin{array}{l}\text { ok } \\
o k \\
0.02 \Omega\end{array}$ & $\begin{array}{l}X A-* 63 \text { OFF } \\
\text { PBL } * 54 \text { OFF } \\
\text { TB2-23/24 } \\
\text { CLOSED }\end{array}$ & A & \\
\hline
\end{tabular}


TEST DATA SHEET

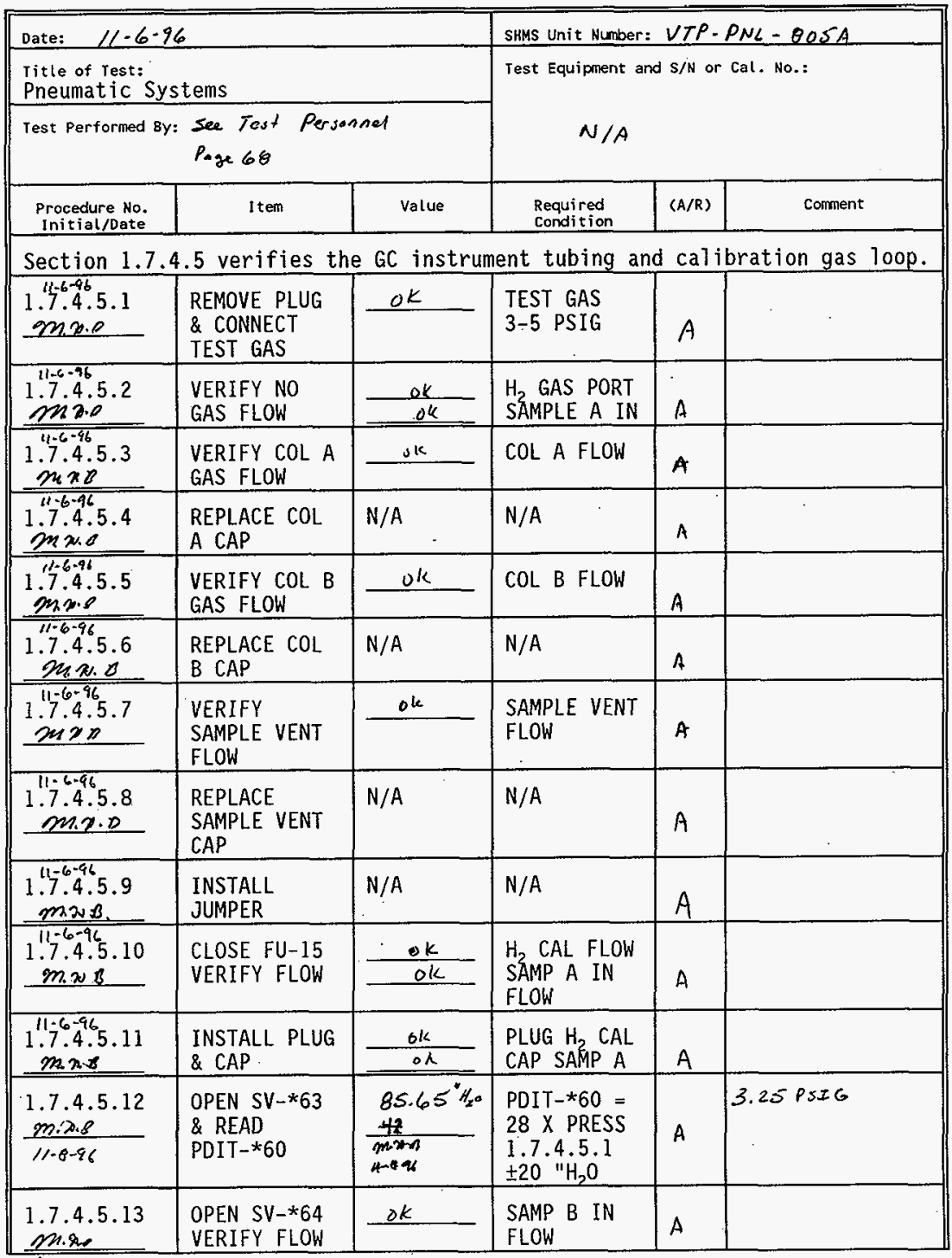


APPENDIX B

TEST DATA SHEET

\begin{tabular}{|c|c|c|c|c|c|}
\hline \multicolumn{3}{|l|}{ Date: $11-8.96$} & \multicolumn{3}{|c|}{ SHMS Unit Number: VTP.PNL-805A } \\
\hline \multicolumn{3}{|c|}{$\begin{array}{l}\text { Title of Test: } \\
\text { Pneumatic Systems }\end{array}$} & \multirow{2}{*}{\multicolumn{3}{|c|}{$\begin{array}{l}\text { Test Equipnent and } S / \mathbb{N} \text { or Cal, No.: } \\
\text { N/A }\end{array}$}} \\
\hline \multicolumn{3}{|c|}{$\begin{aligned} \text { Test Performed By: } & \text { see Test Personnal } \\
& \text { Pag, } 68\end{aligned}$} & & & \\
\hline $\begin{array}{c}\text { Procedure No. } \\
\text { Initial/Date }\end{array}$ & Item & Value & $\begin{array}{l}\text { Required } \\
\text { Condition }\end{array}$ & $(A / R)$ & Comment \\
\hline 1.7 .4 .5 .14 & $\begin{array}{l}\text { REMOVE TEST } \\
\text { GAS }\end{array}$ & $N / A$ & $N / A$ & A & \\
\hline $\begin{array}{l}1.7 .4 .5 .15 \\
\text { ming.e }\end{array}$ & $\begin{array}{l}\text { CLOSE SV-*63 } \\
\& S V-* 64\end{array}$ & $N / A$ & N/A & $A$ & \\
\hline $\begin{array}{l}1.7 .4 .5 .16 \\
\max _{0.0} \\
\end{array}$ & $\begin{array}{l}\text { OPEN FU-15 } \\
\text { REMOVE } \\
\text { JUMPER } \\
\end{array}$ & N/A & $N / A$ & $A$ & \\
\hline $\begin{array}{l}1.7 .4 .5 .17 \\
\text { gnxes. }\end{array}$ & $\begin{array}{l}\text { INSTALL } \\
\text { SAMP A UNION }\end{array}$ & N/A & N/A & A & \\
\hline $\begin{array}{l}1.7 .4 .5 .18 \\
\end{array}$ & $\begin{array}{l}\text { OPEN SV-*60 } \\
\text { VERIFY FLOW }\end{array}$ & ok & $\begin{array}{l}\text { FI-*60 } \\
\text { NO FLOW }\end{array}$ & A & $\cdot$ \\
\hline 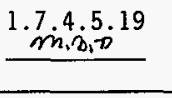 & $\begin{array}{l}\text { POWER SOV- } \\
* 60, \text { VERIFY } \\
\text { NO FLOW }\end{array}$ & ok & $\begin{array}{l}\text { FI-*60 } \\
\text { NO FLOW }\end{array}$ & $A$ & \\
\hline $\begin{array}{l}1.7 .4 .5 .20 \\
\text { m.r.p } \\
\end{array}$ & $\begin{array}{l}\text { OPEN SV-*68 } \\
\text { ADJ SV-*67 }\end{array}$ & $\partial U$ & $\begin{array}{l}F I-* 60 \\
50 \mathrm{CCM} \\
\end{array}$ & $A$ & \\
\hline $\begin{array}{l}1.7 .4 .5 .21 \\
m .21 .0 \\
\end{array}$ & $\begin{array}{l}\text { DEENERGIZE } \\
\text { SOV-*60 }\end{array}$ & ok & $\begin{array}{l}\text { FI-*60 FLOW } \\
\text { DECREASES }\end{array}$ & A & \\
\hline $\begin{array}{r}1.7 .4 .5 .22 \\
m .2 B^{B} \\
\end{array}$ & $\begin{array}{l}\text { CLOSE SV-*60 } \\
\& \text { SV-*68 }\end{array}$ & $N / A$ & $N / A$ & A & \\
\hline $\begin{array}{l}1.7 .4 .5 .23 \\
\operatorname{mins}^{2} \\
\end{array}$ & $\begin{array}{l}\text { REMOVE } \\
\text { SAMP A UNION }\end{array}$ & $N / A$ & $\mathrm{~N} / \mathrm{A}$ & A & \\
\hline $\begin{array}{l}1.7 .4 .5 .24 \\
x .0 \\
\end{array}$ & $\begin{array}{l}\text { CONNECT TEST } \\
\text { GAS } \mathrm{N}_{2} \mathrm{O} \text { PORT } \\
\end{array}$ & $3 P_{3 I_{6}}$ & $\begin{array}{l}\text { TEST GAS } \\
3-5 \text { PSIG } \\
\end{array}$ & $A$ & \\
\hline $\begin{array}{l}1.7 .4 .5 .25 \\
\text { m.s.a. } \\
\end{array}$ & $\begin{array}{l}\text { VERIFY NO } \\
\text { FLOW }\end{array}$ & ok & $\begin{array}{l}\text { SAMP B IN \& } \\
\text { FLT-*63 } \\
\text { NO FLOW }\end{array}$ & 9 & \\
\hline $\begin{array}{l}1.7 .4 .5 .26 \\
\text { mixpo }\end{array}$ & $\begin{array}{l}\text { INSTALL } \\
\text { JUMPER } \\
\end{array}$ & $N / A$ & N/A & A & \\
\hline$\underset{\text { mins }}{1.7 .4 .5 .27}$ & $\begin{array}{l}\text { CLOSE FU-15 } \\
\text { VERIFY FLOW }\end{array}$ & $\begin{array}{l}\text { OK } \\
\text { OK } \\
\end{array}$ & $\begin{array}{l}\text { SAMP B IN } \\
\text { FLT-*63 } \\
\text { FLOW }\end{array}$ & A & \\
\hline
\end{tabular}


TEST DATA SHEET

\begin{tabular}{|c|c|c|c|c|c|}
\hline \multicolumn{3}{|l|}{ Date: $/ 1-8.96$} & \multicolumn{3}{|c|}{ SHMS Unit Number: VTP - PNL $-805 A$} \\
\hline \multicolumn{3}{|c|}{$\begin{array}{l}\text { Title of Test: } \\
\text { Pneumatic Systems }\end{array}$} & \multirow{2}{*}{\multicolumn{3}{|c|}{$\begin{array}{l}\text { rest Equipment and } S / N \text { or Cal. No.: } \\
N / A\end{array}$}} \\
\hline \multicolumn{3}{|c|}{$\begin{aligned} \text { rest Performed 8y: } & \text { See Test Personnal } \\
& \text { Page } 68\end{aligned}$} & & & \\
\hline $\begin{array}{l}\text { Procedure No. } \\
\text { Initial/Date }\end{array}$ & 1 tem & value & $\begin{array}{l}\text { Required } \\
\text { Condition }\end{array}$ & $(A / R)$ & Comment \\
\hline 1.7 .4 .5 .28 & $\begin{array}{l}\text { DISCONNECT } \\
\text { TEST GAS }\end{array}$ & $N / A$ & $N / A$ & A & \\
\hline $\begin{array}{l}1.7 .4 .5 .29 \\
m . x \beta\end{array}$ & OPEN FU-15 & $N / A$ & $N / A$ & A & \\
\hline $\begin{array}{l}7.4 .5 .30 \\
m 0^{0}\end{array}$ & $\begin{array}{l}\text { REMOVE } \\
\text { JUMPER }\end{array}$ & $N / A$ & $N / A$ & A & \\
\hline $\begin{array}{l}1.7 .4 .5 .31 \\
\min x \cdot x^{3}\end{array}$ & $\begin{array}{l}\text { INSTALL } \\
\text { SAMP B UNION }\end{array}$ & $N / A$ & $N / A$ & A & \\
\hline $\begin{array}{r}1.7 .4 .5 .32 \\
m .013 \\
\end{array}$ & $\begin{array}{l}\text { OPEN SV }-* 60 \\
\text { VERIFY FLOW }\end{array}$ & $\frac{\text { m. } 210}{.001000}$ & $\begin{array}{l}\text { FI-*60 } \\
\text { NO FLOW }\end{array}$ & A & \\
\hline $\begin{array}{l}1.7 .4 .5 .33 \\
\text { min.p.p }\end{array}$ & $\begin{array}{l}\text { POWER SOV- } \\
* 60 \text {, VERIFY } \\
\text { NO FLOW }\end{array}$ & $\frac{M x D}{\text { No flow }}$ & $\begin{array}{l}\text { FI-*60 } \\
\text { NO FLOW }\end{array}$ & A & \\
\hline $\begin{array}{l}1.7 .4 .5 .34 \\
m p \cdot B\end{array}$ & $\begin{array}{l}\text { OPEN SV }-* 68 \\
\& \text { ADJ SV }-* 67 \\
\end{array}$ & $\frac{m \cdot p}{o n}$ & $\begin{array}{l}\mathrm{FI}-* 60 \\
50 \mathrm{CCM} \\
\end{array}$ & A & \\
\hline $\begin{array}{l}1.7 .4 .5 .35 \\
2 m 3.8 \\
\end{array}$ & $\begin{array}{l}\text { DEENERGIZE } \\
\text { SOV }-* 60\end{array}$ & $\frac{g n \cdot 2 \cdot 0}{8 K}$ & $\begin{array}{l}\text { FI-*60 FLOW } \\
\text { DECREASES } \\
\end{array}$ & $A$ & \\
\hline $\operatorname{mins}^{7.4 .5 .36}$ & $\begin{array}{l}\text { CLOSE SV-*60 } \\
\& \text { SV-*68 }\end{array}$ & $N / A$ & $N / A$ & A & \\
\hline $\begin{array}{l}1.7 .4 .5 .37 \\
2 \times 2.33\end{array}$ & $\begin{array}{l}\text { REMOVE } \\
\text { SAMP B UNION }\end{array}$ & $N / A$ & $N / A$ & $A$ & \\
\hline $\begin{array}{l}1.7 .4 .5 .38 \\
\text { m.x.8. }\end{array}$ & $\begin{array}{l}\text { OPEN CB-3/4 } \\
\text { ACK. ALARMS }\end{array}$ & $\mathrm{N} / \mathrm{A}$ & $\mathrm{N} / \mathrm{A}$ & $A$ & \\
\hline \multicolumn{6}{|c|}{$\begin{array}{l}\text { Section } 1.7 .4 .6 \text { verifies the multi gas analyzer instrument tubing and } \\
\text { calibration gas loop. }\end{array}$} \\
\hline $\begin{array}{l}1.7 .4 .6 .1 \\
m . x .73 \\
\end{array}$ & $\begin{array}{l}\text { VERIFY } \\
\text { NIT-*52 } \\
\text { SAMPLE LINES }\end{array}$ & $\mathrm{N} / \mathrm{A}$ & $N / A$ & A & \\
\hline $\begin{array}{l}1.7 .4 .6 .2 \\
m . p . n \\
\end{array}$ & $\begin{array}{l}\text { CONNECT TEST } \\
\text { GAS } \mathrm{NH}_{3} \text { PORT }\end{array}$ & $3.5 P \leq I 6$ & $\begin{array}{l}\text { TEST GAS } \\
3-5 \text { PSIG } \\
\end{array}$ & A & \\
\hline $\begin{array}{l}1.7 .4 .6 .3 \\
\operatorname{mine}_{0}\end{array}$ & $\begin{array}{l}\text { VERIFY NO } \\
\text { FLOW }\end{array}$ & $\frac{\operatorname{sak}}{\Delta k}$ & $\begin{array}{l}\text { SAMPLE IN } \\
\text { FLT-*63 }\end{array}$ & A & \\
\hline
\end{tabular}




\section{TEST DATA SHEET}

\begin{tabular}{|c|c|c|c|c|c|}
\hline \multicolumn{3}{|l|}{ Date: $11-8.96$} & \multicolumn{3}{|c|}{ SHMS Unit Number: VTP-PNL-805A } \\
\hline \multicolumn{3}{|c|}{$\begin{array}{l}\text { Title of Test: } \\
\text { Pneumatic Systems }\end{array}$} & \multirow{2}{*}{\multicolumn{3}{|c|}{$\begin{array}{l}\text { Test Equipment and } S / \mathrm{N} \text { or Cal. No.: } \\
\text { N/A }\end{array}$}} \\
\hline \multicolumn{3}{|c|}{$\begin{aligned} \text { Test Performed By: } & \text { See Test Personnel } \\
& \text { Page } 68\end{aligned}$} & & & \\
\hline $\begin{array}{l}\text { Procedure No. } \\
\text { Initial/Date }\end{array}$ & Item & Value & $\begin{array}{l}\text { Required } \\
\text { Condition } \\
\end{array}$ & $(A / R)$ & Comment \\
\hline $\begin{array}{l}1.7 .4 .6 .4 \\
M .00\end{array}$ & $\begin{array}{l}\text { INSTALL } \\
\text { JUMPER }\end{array}$ & $N / A$ & N/A & $A$ & \\
\hline $\begin{array}{l}1.7 .4 .6 .5 \\
\min x .0\end{array}$ & $\begin{array}{l}\text { CLOSE FU-15 } \\
\text { VERIFY GAS } \\
\text { FLOWS }\end{array}$ & $\begin{array}{l}\text { ok } \\
\text { ok } \\
\text { oll } \\
\end{array}$ & $\begin{array}{l}\text { SAMPLE IN } \\
\text { FLT }-* 63 \\
\text { FIV }-* 70\end{array}$ & $A$ & \\
\hline$\frac{1.7 .4 .6 .6}{m .8 .6}$ & $\begin{array}{l}\text { PLUG FLT-*63 } \\
\text { \& MON-*60 } \\
\text { SAMPLE VENT }\end{array}$ & $N / A$ & $N / A$ & A & \\
\hline $\begin{array}{l}1.7 .4 .6 .7 \\
m .0 .0\end{array}$ & $\begin{array}{l}\text { OPEN SV-*66, } \\
\text { SV-*70 \& CAP } \\
\text { SAMPLE IN }\end{array}$ & 134. $1^{n} \mathrm{H}_{2} \mathrm{O}$ & $\begin{array}{l}\text { PDIT-*60 = } \\
28 \times \text { PRESS } \\
1.7 .4 .6 .2 \\
\pm 20 \mathrm{H}_{2} \mathrm{O}\end{array}$ & A & $4 P \leq 36$ \\
\hline $\begin{array}{l}1.7 .4 .6 .8 \\
m .8 .8\end{array}$ & $\begin{array}{l}\text { CLOSE SV-*66 } \\
\text { SV-*70 \& } \\
\text { REMOVE CAP }\end{array}$ & $N / A$ & $N / A$ & $A$ & \\
\hline $\begin{array}{l}1.7 .4 .6 .9 \\
\min >0\end{array}$ & $\begin{array}{l}\text { REMOVE FLT- } \\
* 63 \text { PLUG }\end{array}$ & $N / A$ & $N / A$ & $A$ & \\
\hline $\begin{array}{l}1.7 .4 .6 .10 \\
\text { nxinen }\end{array}$ & $\begin{array}{l}\text { REMOVE TEST } \\
\text { GAS }\end{array}$ & $N / A$ & $N / A$ & A & \\
\hline $\begin{array}{l}1.7 .4 .6 .11 \\
\end{array}$ & $\begin{array}{l}\text { CLOSE SV-*05 } \\
\& \text { SV }-* 16\end{array}$ & $N / A$ & $N / A$ & A & \\
\hline $\begin{array}{l}1.7 .4 .6 .12 \\
\sin \times 10 \\
\end{array}$ & $\begin{array}{l}\text { CONNECT TEST } \\
\text { GAS }\end{array}$ & $2.25 P$ SIG & $\begin{array}{l}\text { TEST GAS } \\
2-4 \text { PSIG } \\
\end{array}$ & A & \\
\hline $\begin{array}{l}1.7 .4 .6 .13 \\
m .21 .33 \\
\end{array}$ & $\begin{array}{l}\text { OPEN SV-*07 } \\
\& \text { VERIFY } \\
\text { NO FLOW } \\
\end{array}$ & $\frac{\text { ok }}{\text { oll }}$ & $\begin{array}{l}\text { SAMPLE IN } \\
\text { SAMPLE OUT }\end{array}$ & A & . \\
\hline $\begin{array}{l}1.7 .4 .6 .14 \\
m . n . n\end{array}$ & $\begin{array}{l}\text { OPEN SV }-* 09 \\
\text { \& VERIFY } \\
\text { NO FLOW }\end{array}$ & $\frac{o k}{o k}$ & $\begin{array}{l}\text { SAMPLE IN } \\
\text { SAMPLE OUT }\end{array}$ & $A$ & \\
\hline $\begin{array}{l}1.7 .4 .6 .15 \\
\text { mingon }^{2}\end{array}$ & $\begin{array}{l}\text { OPEN FU-15 \& } \\
\text { VERIFY FLOW }\end{array}$ & $\begin{array}{l}\text { ok } \\
Q K \\
\end{array}$ & $\begin{array}{l}\text { SAMPLE FLOW } \\
\text { IN - YES } \\
\text { OUT - NO } \\
\end{array}$ & A & \\
\hline $\begin{array}{l}1.7 .4 .6 .16 \\
m 26\end{array}$ & $\begin{array}{l}\text { CLOSE SV-*09 } \\
\text { VERIFY FLOW }\end{array}$ & -ok & $\begin{array}{l}\text { SAMPLE IN } \\
\text { NO FLOW }\end{array}$ & $\theta$ & \\
\hline
\end{tabular}


TEST DATA SHEET

\begin{tabular}{|c|c|c|c|c|c|}
\hline & \multicolumn{3}{|c|}{ SHMS Unit Number: VTP-PNL $-805 A$} \\
\hline \multicolumn{3}{|c|}{$\begin{array}{l}\text { Title of Test: } \\
\text { Pneumatic Systems }\end{array}$} & \multirow{2}{*}{\multicolumn{3}{|c|}{$\begin{array}{l}\text { Test Equipment and } \mathrm{s} / \mathrm{N} \text { or Cal. No.: } \\
N / A\end{array}$}} \\
\hline \multicolumn{3}{|c|}{$\begin{aligned} \text { Test Perforned By: } & \text { Test Personnal } \\
& \text { Page } 68\end{aligned}$} & & & \\
\hline $\begin{array}{l}\text { Procedure No. } \\
\text { Initial/Date } \\
\end{array}$ & Item & value & $\begin{array}{l}\text { Required } \\
\text { Condition }\end{array}$ & $(A / R)$ & Comment \\
\hline $\begin{array}{l}1.7 .4 .6 .17 \\
\text { nux.e. }\end{array}$ & $\begin{array}{l}\text { OPEN SV-*10 } \\
\text { VERIFY FLOW }\end{array}$ & $O K$ & $\begin{array}{l}\text { SAMPLE FLOW } \\
\text { IN - NO } \\
\text { OUT - YES } \\
\end{array}$ & $A$ & \\
\hline $\begin{array}{l}1.7 .4 .6 .18 \\
m .0 .0\end{array}$ & $\begin{array}{l}\text { REMOVE TEST } \\
\text { GAS \& PLUG } \\
\text { SV-*07 }\end{array}$ & $N / A$ & N/A & A & \\
\hline $\begin{array}{l}1.7 .4 .6 .19 \\
m .218 \\
\end{array}$ & $\begin{array}{l}\text { CLOSE. SV }-* 07 \\
\& \text { SV }-* 10\end{array}$ & $N / A$ & N/A & A & \\
\hline $\begin{array}{l}1.7 .4 .6 .20 \\
\text { m. p.o. } \\
\end{array}$ & $\begin{array}{l}\text { OPEN SV-*05 } \\
\& \text { SV-*16 }\end{array}$ & $N / A$ & $\mathrm{~N} / \mathrm{A}$ & A & \\
\hline $\begin{array}{l}1.7 .4 .6 .21 \\
m .2 B \\
\end{array}$ & $\begin{array}{l}\text { REMOVE } \\
\text { JUMPER }\end{array}$ & $N / A$ & N/A & $A$ & \\
\hline \multicolumn{6}{|c|}{ Section 1.7.4.7 verifies the carrier gas supply lines. } \\
\hline $\begin{array}{l}1.7 .4 .7 .1 \\
m .2 p \\
\end{array}$ & $\begin{array}{l}\text { CONNECT TEST } \\
\text { GAS }\end{array}$ & ok & 3-5 PSIG & A & $4 P 326$ \\
\hline $\begin{array}{r}1.7 .4 .7 .2 \\
m \times n \\
\end{array}$ & $\begin{array}{l}\text { REMOVE PLUG } \\
\& \text { VERIFY } \\
\text { FLOW } \\
\end{array}$ & ok & $\begin{array}{l}N_{2} \text { CARRIER } \\
\text { GAS FLOWS }\end{array}$ & A & \\
\hline $\begin{array}{c}1.7 .4 .7 .3 \\
m v p \\
\end{array}$ & $\begin{array}{l}\text { DISCONNECT } \\
\text { TEST GAS }\end{array}$ & $\mathrm{N} / \mathrm{A}$ & N/A & 1 & \\
\hline $\begin{array}{l}1.7 .4 .7 .4 \\
\text { m.2. }\end{array}$ & $\begin{array}{l}\text { CONNECT TEST } \\
\text { GAS } \\
\end{array}$ & ok & 3-5 PSIG & $A$ & yestc \\
\hline $\begin{array}{l}1.7 .4 .7 .5 \\
-2 \times 2.8\end{array}$ & $\begin{array}{l}\text { REMOVE PLUG } \\
\text { \& VERIFY } \\
\text { FLOW } \\
\end{array}$ & ok & $\begin{array}{l}\text { He CARRIER } \\
\text { GAS FLOWS }\end{array}$ & $A$ & \\
\hline $\begin{array}{l}1.7 .4 .7 .6 \\
2 \text { m.P. } \\
\end{array}$ & $\begin{array}{l}\text { DISCONNECT } \\
\text { TEST GAS } \\
\end{array}$ & $N / A$ & N/A & $A$ & \\
\hline $\begin{array}{l}1.7 .5 \\
m m 8\end{array}$ & $\begin{array}{l}\text { TEST SEC. } \\
1.7 .4 \text { DONE }\end{array}$ & Test $0_{i}$ & Luecre & & $\frac{1 / 8-96}{\text { Date }}$ \\
\hline
\end{tabular}

a.11 $11-8-96$ 
TEST DATA SHEET

\begin{tabular}{|c|c|c|c|c|c|}
\hline \multicolumn{3}{|c|}{ Date: $11-8.96$} & \multicolumn{3}{|c|}{ SHMS Unit Number: VTP - PNL - BOSA } \\
\hline \multicolumn{3}{|c|}{$\begin{array}{l}\text { Title of Test: } \\
\text { Pneumatic Systems }\end{array}$} & \multirow{2}{*}{\multicolumn{3}{|c|}{ 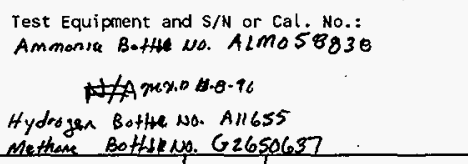 }} \\
\hline \multicolumn{3}{|c|}{$\begin{aligned} \text { Test Performed By: } & \text { see Test Personnel } \\
& \text { Paxe } 68\end{aligned}$} & & & \\
\hline $\begin{array}{c}\text { Procedure No. } \\
\text { Initial/Date }\end{array}$ & Item & value & $\begin{array}{l}\text { Required } \\
\text { Condition }\end{array}$ & $(A / R)$ & Comment \\
\hline $\begin{array}{l}1.7 .6 \\
m .2 .8\end{array}$ & \multicolumn{5}{|c|}{ 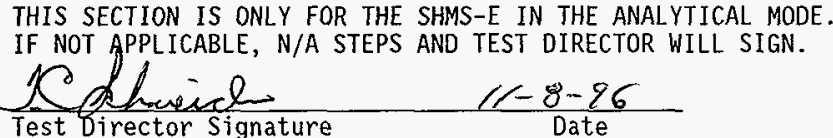 } \\
\hline $\begin{array}{l}1.7 \cdot 6 \cdot 1 \cdot 1 \\
m \cdot 1 \cdot B\end{array}$ & $\begin{array}{l}\text { VERIFY BKR } \\
\text { LINE UP }\end{array}$ & N/A & N/A & ${ }_{11-8-96}^{A}$ & \\
\hline $\begin{array}{l}1.7 .6 .1 .2 \\
m .2 . \beta\end{array}$ & $\begin{array}{l}\text { VERIFY VALVE } \\
\text { LINE UP }\end{array}$ & N/A & N/A & $\begin{array}{c}A \\
1 /-8.96\end{array}$ & \\
\hline $\begin{array}{c}1.7 .6 .1 .3 \\
m x_{0} \cdot x_{3} \\
\end{array}$ & $\begin{array}{l}\text { VERIFY MON- } \\
* 60 \text { INSTALL } \\
\end{array}$ & $N / A$ & $N / A$ & ${ }_{1}^{A}-89$ & \\
\hline $\begin{array}{l}1.7 .6 .1 .4 \\
m . x e\end{array}$ & $\begin{array}{l}\text { VERIFY NIT- } \\
* 52 \text { INSTALL } \\
\end{array}$ & N/A & $N / A$ & A & \\
\hline $\begin{array}{l}1.7 .6 .1 .5 \\
\text { m.x.0 }\end{array}$ & $\begin{array}{l}\text { CONNECT } \mathrm{H}_{2} \\
\text { CAL GAS }\end{array}$ & N/A & $N / A$ & A & \\
\hline $\begin{array}{l}1.7 .6 .1 .6 \\
m .2 .0\end{array}$ & $\begin{array}{l}\text { CONNECT } \mathrm{CH}_{4} \\
\text { CAL GAS }\end{array}$ & N/A & N/A & A & \\
\hline $\begin{array}{c}1.7 .6 .1 .7 \\
\text { om.7.0 }\end{array}$ & $\begin{array}{l}\text { CONNECT } \mathrm{NH}_{3} \\
\text { CAL GAS }\end{array}$ & $N / A$ & N/A & A & \\
\hline \multicolumn{6}{|c|}{ Section 1.7.6.2 establishes the main and auxiliary loop flows. } \\
\hline $\begin{array}{c}1.7 .6 .2 .1 \\
\text { mines }\end{array}$ & CLOSE FU-11 & ok & $\begin{array}{l}\text { MON-*60 } \\
\text { POWERED }\end{array}$ & A & \\
\hline $\begin{array}{l}1.7 .6 .2 .2 \\
\text { mox.s }\end{array}$ & POWER PC $-* 60$ & $-a \alpha_{L}$ & $\begin{array}{l}\text { ESTABLISH } \\
\text { SAMPLING } \\
\text { ROUTINE } \\
\end{array}$ & A & \\
\hline $\begin{array}{c}1.7 .6 .2 .3 \\
\text { gupe }\end{array}$ & CLOSE FU-12 & $\pi k$ & $\begin{array}{l}\text { NIT }-* 52 \\
\text { POWERED }\end{array}$ & A & \\
\hline 1.7 .6 .2 .4 & POWER PC $-* 70$ & $\therefore O K$ & $\begin{array}{l}P C-* 70 \\
\text { BOOTED UP }\end{array}$ & A & \\
\hline $\begin{array}{c}1.7 .6 .2 .5 \\
\text { mines }\end{array}$ & $\begin{array}{l}\text { CLOSE CB- } 3 / 4 \\
\text { ADJUST FLOW }\end{array}$ & $\begin{array}{c}1.09 \times 1 / 20 \\
0.4\end{array}$ & $\begin{array}{l}\mathrm{FIT}-* 57 \\
1 \pm .1 \mathrm{MH} \mathrm{H}_{2} \mathrm{O}\end{array}$ & $A$ & \\
\hline
\end{tabular}




\section{TEST DATA SHEET}

\begin{tabular}{|c|c|c|c|c|c|}
\hline \multicolumn{3}{|c|}{ Date: $11-6.96$} & \multicolumn{3}{|c|}{ SHMS Unit Number: VTP-PNL $-80 S A$} \\
\hline \multicolumn{3}{|c|}{$\begin{array}{l}\text { Title of Test: } \\
\text { Pneumatic Systems }\end{array}$} & \multirow{2}{*}{\multicolumn{3}{|c|}{$\begin{array}{l}\text { Test Equipment and } S / \mathrm{N} \text { or Cal. No.: } \\
\text { Ammonia Bothd NO. ALmo58838 }\end{array}$}} \\
\hline \multicolumn{3}{|c|}{$\begin{aligned} \text { Test Performed By: } & \text { See Test Personnel } \\
& \text { Page } 68\end{aligned}$} & & & \\
\hline $\begin{array}{l}\text { Procedure No. } \\
\text { Initial/Date }\end{array}$ & Item & value & $\begin{array}{l}\text { Required } \\
\text { Condition } \\
\end{array}$ & $(A / R)$ & Comment \\
\hline $\begin{array}{c}1.7 .6 .2 .6 \\
\text { min.e }\end{array}$ & $\begin{array}{l}\text { OPEN SV-*09 } \\
\& \text { SV }-* 10\end{array}$ & N/A & $N / A$ & $A$ & \\
\hline $\begin{array}{l}1.7 .6 .2 .7 \\
\text { mine. } \\
\end{array}$ & $\begin{array}{l}\text { OPEN SV-*60, } \\
63,65 \& 68\end{array}$ & N/A & $N / A$ & $A$ & \\
\hline $1.7 \dot{m i n}^{6} 0^{2} .8$ & NIT $-* 52$ & ok & $\begin{array}{l}\text { ESTABLISH } \\
\text { SAMPLING } \\
\text { ROUTINE }\end{array}$ & A & \\
\hline $\begin{array}{l}1.7 .6 .2 .9 \\
\text { mex. }\end{array}$ & $\begin{array}{l}\text { OPEN SV-*24 } \\
\text { ADJ FLOW }\end{array}$ & $2.9^{*} \mathrm{H}_{2} \mathrm{C}$ & $\begin{array}{l}\mathrm{FIT}-* 57 \\
3 \pm .1 " \mathrm{H}_{2} \mathrm{O}\end{array}$ & A & \\
\hline $\begin{array}{l}1.7 .6 .2 .10 \\
\min _{0.0} \\
\end{array}$ & $\begin{array}{l}\text { ADJ FLOW } \\
\text { SV-*67 }\end{array}$ & 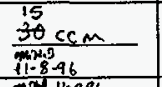 & $\begin{array}{l}\mathrm{FI}-* 60 \\
5-10 \mathrm{ccm}\end{array}$ & A & 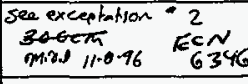 \\
\hline $\begin{array}{l}1.7 .6 .2 .11 \\
m_{m .0}\end{array}$ & $\begin{array}{l}\text { RECORD } \\
\text { POIT }-* 60\end{array}$ & 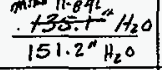 & $\begin{array}{l}100-250 \\
{ }^{\prime \prime} \mathrm{H}_{2} \mathrm{O}\end{array}$ & A & Eon 634645 \\
\hline 1.7 .6 .2 .12 & $\begin{array}{l}\text { OPEN SV }-* 64 \\
\text { CLOSE SV-*63 }\end{array}$ & $N / A$ & $N / A$ & A & \\
\hline $\begin{array}{r}1.7 .6 .2 .13 \\
\text { m2.0. } \\
\end{array}$ & $\begin{array}{l}\text { RECORD } \\
\text { PDIT }-* 60\end{array}$ & 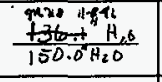 & $\begin{array}{l}100-250 \\
" \mathrm{H}_{2} \mathrm{O}\end{array}$ & A & $\operatorname{ExN} 634645$ \\
\hline $\begin{array}{l}1.7 .6 .2 .14 \\
\text { mx.6. } \\
\end{array}$ & $\begin{array}{l}\text { CLOSE SV }-* 65 \\
\text { OPEN SV-*66. }\end{array}$ & $N / A$ & $N / A$ & A & \\
\hline $\begin{array}{l}1.7 .6 .2 .15 \\
m \times x_{3}\end{array}$ & $\begin{array}{l}\text { RECORD B IN } \\
\text { POIT-* } 60 \\
\end{array}$ & 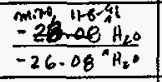 & $-15-50 \quad " \mathrm{H}_{2} \mathrm{O}$ & A & $\operatorname{Ex} 634645$ \\
\hline $\begin{array}{l}1.7 \cdot 6.2 .16 \\
m_{20.8}\end{array}$ & $\begin{array}{l}\text { OPEN SV }-* 63 \\
\text { CLOSE SV-*64 }\end{array}$ & $N / A$ & $N / A$ & A & \\
\hline $\begin{array}{l}1.7 .6 .2 .17 \\
x_{0.3}\end{array}$ & $\begin{array}{l}\text { RECORD A IN } \\
\text { PDIT } * 60\end{array}$ & $-26.17^{n} \mathrm{H}$ & $-15-50 \quad " \mathrm{H}_{2} \mathrm{O}$ & $\Lambda$ & $E \mathrm{CN} 634645$ \\
\hline $\begin{array}{l}1.7 .6 .2 .18 \\
\operatorname{mog.8}\end{array}$ & $\begin{array}{l}\text { INSTALL } \\
\text { JUMPER }\end{array}$ & $N / A$ & $N / A$ & $A$ & \\
\hline $\begin{array}{l}1.7 .6 .2 .19 \\
m \times 3 .\end{array}$ & CLOSE FU-15 & N/A & $N / A$ & A & \\
\hline $\begin{array}{l}1.7 .6 .2 .20 \\
m_{1 x .0}\end{array}$ & $\begin{array}{l}\text { ADJ SV }-* 61 \& \\
\text { RECORD } \\
\text { PDIT }-* 60\end{array}$ & $-26.00^{4} \mathrm{H}_{2}$ & $\begin{array}{l} \pm 5 " \mathrm{H}_{2} \mathrm{O} \text { OF } \\
1.7 .6 .2 .17\end{array}$ & $A$ & \\
\hline
\end{tabular}


TEST DATA SHEET

\begin{tabular}{|c|c|c|c|c|c|}
\hline \multicolumn{3}{|l|}{ Date: $11-6-96$} & \multicolumn{3}{|c|}{ SHMS Unit Number: VTP-PNL $-805 A$} \\
\hline \multicolumn{3}{|c|}{$\begin{array}{l}\text { Title of Test: } \\
\text { Pneumatic Systems }\end{array}$} & \multirow{2}{*}{\multicolumn{3}{|c|}{$\begin{array}{l}\text { Test Equipment and } \mathrm{s} / \mathrm{N} \text { or Cal. No.: } \\
\text { Ammonia Botfle No. ALmo58830 }\end{array}$}} \\
\hline Test Performed By: & $\begin{array}{l}\text { See Test Pers } \\
\text { Page } 68\end{array}$ & nel & & & \\
\hline $\begin{array}{l}\text { Procedure No. } \\
\text { Initial/oate }\end{array}$ & I tem & Vatue & $\begin{array}{l}\text { Required } \\
\text { condition }\end{array}$ & $(A / R)$ & Comment \\
\hline $\begin{array}{l}1.7 .6 .2 .21 \\
m . x . s \\
\end{array}$ & $\begin{array}{l}\text { CAL MON }-* 60 \\
\text { CHANNEL A }\end{array}$ & $\begin{array}{l}10,544.51 \mathrm{PPM} \\
10,545.58 \mathrm{PPP} \\
10,537.18 \mathrm{PPm}\end{array}$ & $\begin{array}{l}\text { READINGS } \\
\text { WITHIN } \pm 5 \%\end{array}$ & $A$ & . \\
\hline $\begin{array}{l}1.7 .6 .2 .22 \\
\text { m.x.6. }\end{array}$ & OPEN FU-15 & $\mathrm{N} / \mathrm{A}$ & $N / A$ & A & \\
\hline $\begin{array}{l}1.7 .6 .2 .23 \\
m_{x} x \cdot p^{-3}\end{array}$ & $\begin{array}{l}\text { REMOVE } \\
\text { JUMPER } \\
\end{array}$ & $N / A$ & $N / A$ & $A$ & \\
\hline $\begin{array}{l}1.7 .6 .2 .24 \\
m_{B} x_{0} \\
\end{array}$ & $\begin{array}{l}\text { OPEN SV }-* 64 \\
\text { CLOSE SV-*63 }\end{array}$ & $N / A$ & $N / A$ & $A$ & \\
\hline $\begin{array}{l}1.7 .6 .2 .25 \\
\text { ixi.x. }\end{array}$ & $\begin{array}{l}\text { INSTALL } \\
\text { JUMPER }\end{array}$ & $N / A$ & $N / A$ & A & \\
\hline $\begin{array}{l}1.7 .6 .2 .26 \\
\text { min. }\end{array}$ & CLOSE FU-15 & $N / A$ & $N / A$ & A & \\
\hline $\begin{array}{l}1.7 .6 .2 .27 \\
\operatorname{mg} 9.8 \\
\end{array}$ & $\begin{array}{l}\text { ADJ SV-*62 \& } \\
\text { RECORD } \\
\text { PDIT-*60 }\end{array}$ & $-25.28^{\circ} \mathrm{H}_{2} \mathrm{O}$ & $\begin{array}{l} \pm 5^{11} \mathrm{H}_{2} \mathrm{O} \text { OF } \\
1.7 .6 .2 .135\end{array}$ & A & $\begin{array}{l}\text { ECN } 634647 \\
\text { ECN } 634645\end{array}$ \\
\hline $\begin{array}{l}1.7 .6 .2 .28 \\
\text { M.7. }\end{array}$ & $\begin{array}{l}\text { CAL MON-*60 } \\
\text { CHANNEL B }\end{array}$ & $\begin{array}{r}10,056.47 \mathrm{PPm} \\
0,091.29 \mathrm{pPP} \\
10,132.26 \mathrm{Pm} \\
\end{array}$ & $\begin{array}{l}\text { READINGS } \\
\text { WITHIN } \pm 5 \%\end{array}$ & $A$ & \\
\hline $\begin{array}{l}1.7 .6 .2 .29 \\
\text { mon.s } \\
\end{array}$ & OPEN FU-15 & $N / A$ & $N / A$ & $A$ & \\
\hline $\begin{array}{l}1.7 .6 .2 .30 \\
m_{2,0}\end{array}$ & $\begin{array}{l}\text { REMOVE } \\
\text { JUMPER } \\
\end{array}$ & N/A & $N / A$ & $A$ & \\
\hline$\underline{m p \hat{\beta}^{2.31}}$ & $\begin{array}{l}\text { CLOSE SV-*64 } \\
\text { OPEN SV-*70 }\end{array}$ & $\mathrm{N} / \mathrm{A}$ & N/A & A & \\
\hline $\begin{array}{l}1.7 .6 .2 .32 \\
m .2 . n \\
\end{array}$ & $\begin{array}{l}\text { RECORD } \\
\text { PDIT-*60 } \\
\text { NIT-*52 IN } \\
\end{array}$ & $-24.00 \mathrm{~A} \mathrm{H}_{2} \mathrm{O}$ & $-15-50 \quad " \mathrm{H}_{2} \mathrm{O}$ & A & $\begin{array}{l}\text { Ecan } 634647 \\
\text { red } 634645\end{array}$ \\
\hline $\begin{array}{l}1.7 .6 .2 .33 \\
m .2 .8 \\
\end{array}$ & $\begin{array}{l}\text { INSTALL } \\
\text { JUMPER }\end{array}$ & N/A & $N / A$ & $A$ & \\
\hline $\begin{array}{l}1.7 .6 .2 .34 \\
m x_{B}\end{array}$ & CLOSE FU-15 & $\mathrm{N} / \mathrm{A}$ & N/A & $A$ & \\
\hline
\end{tabular}


TEST DATA SHEET

\begin{tabular}{|c|c|c|c|c|c|}
\hline \multicolumn{3}{|c|}{ Date: $1 /-6-96$} & \multicolumn{3}{|c|}{ SHMS Unit Number: VTP-PNL-80SA } \\
\hline \multicolumn{3}{|c|}{$\begin{array}{l}\text { Title of Test: } \\
\text { Pneumatic Systems }\end{array}$} & \multirow{2}{*}{\multicolumn{3}{|c|}{$\begin{array}{l}\text { Test Equipment and } \mathrm{s} / \mathrm{N} \text { or Cal. No.: } \\
\text { N/A }\end{array}$}} \\
\hline \multicolumn{3}{|c|}{$\begin{aligned} & \text { Test Performed By: } \text { See Test Personnel } \\
& \text { Page } 68\end{aligned}$} & & & \\
\hline $\begin{array}{l}\text { Procedure No. } \\
\text { Initial/Date }\end{array}$ & Item & Value & $\begin{array}{l}\text { Required } \\
\text { Condition }\end{array}$ & $(A / R)$ & Comment \\
\hline $\begin{array}{l}1.7 .6 .2 .35 \\
m_{2 x} .0 \\
\end{array}$ & $\begin{array}{l}\text { ESTABLISH } \\
\text { BYPASS FLOW }\end{array}$ & $15 \mathrm{cFh}$ & $\begin{array}{l}\text { FIV }-* 70 \\
1.5 \pm .2 \mathrm{cfh}\end{array}$ & A & \\
\hline$\frac{1.7 .6 .2 .36}{2 x_{0.0}}$ & $\begin{array}{l}\text { ADJ SV-*71 } \\
\text { NIT }-* 52 \\
\text { SAMPLE IN }\end{array}$ & $-20.67^{\circ} \mathrm{H}_{8} \mathrm{O}$ & $\begin{array}{l} \pm 5 \quad \mathrm{H}_{2} \mathrm{O} \quad \mathrm{OF} \\
1.7 .6 .2 .32\end{array}$ & A & \\
\hline $\begin{array}{l}1.7 .6 .2 .37 \\
02 x .8 \\
\end{array}$ & $\begin{array}{l}\text { RECORD } \\
\text { NIT }-\star 52 \\
\text { SAMPLES }\end{array}$ & $\begin{array}{l}32.5 \mathrm{PPM} \\
\frac{32.2 \mathrm{PPM}}{32.1 \mathrm{PPPM}} \\
\end{array}$ & $\begin{array}{l}\text { READINGS } \\
\text { WITHIN } \pm 5 \%\end{array}$ & $A$ & \\
\hline $\begin{array}{l}1.7 .6 .2 .38 \\
\operatorname{mins}_{2.8}\end{array}$ & OPEN FU-15 & $N / A$ & N/A & A & \\
\hline $\begin{array}{l}1.7 .6 .2 .39 \\
\text { m.x.s. }\end{array}$ & $\begin{array}{l}\text { REMOVE } \\
\text { JUMPER }\end{array}$ & $N / A$ & $N / A$ & $A$ & \\
\hline \multicolumn{6}{|c|}{ Section 1.7.7 provides for test system shutdown. } \\
\hline $\begin{array}{r}1.7 .7 .1 \\
m x .00 \\
\end{array}$ & $\begin{array}{l}\text { DISCONNECT } \\
\mathrm{H}_{2} \text { CAL GAS }\end{array}$ & $N / A$ & $N / A$ & A & \\
\hline $\begin{array}{l}1.7 .7 .2 \\
m .2 .8\end{array}$ & $\begin{array}{l}\text { DISCONNECT } \\
\mathrm{CH}_{,} \text {CAL GAS }\end{array}$ & $N / A$ & $N / A$ & $A$ & \\
\hline $\begin{array}{c}1.7 .7 .3 \\
\text { m.x.B. }\end{array}$ & $\begin{array}{l}\text { DISCONNECT } \\
\mathrm{NH}_{3} \text { CAL GAS }\end{array}$ & $N / A$ & $N / A$ & A & \\
\hline $\begin{array}{l}1.7 .7 .4 \\
m .7 .73 \\
\end{array}$ & $\begin{array}{l}\text { SECURE \& } \\
\text { TURN OFF } \\
\text { ANA. INST.S }\end{array}$ & $N / A$ & $N / A$ & A & \\
\hline $\begin{array}{l}1.7 .7 .5 \\
m \text {. }\end{array}$ & $\begin{array}{l}\text { DISCONNECT } \\
\mathrm{N}_{2} \text { CARRIER }\end{array}$ & $N / A$ & $N / A$ & A & \\
\hline $\begin{array}{l}1.7 .7 .6 \\
m \times B\end{array}$ & $\begin{array}{l}\text { DISCONNECT } \\
\text { He CARRIER }\end{array}$ & $N / A$ & $N / A$ & A & \\
\hline $\begin{array}{l}1.7 .7 .7 \\
\text { m. B B }\end{array}$ & $\begin{array}{l}\text { OPEN ALL } \\
\text { BREAKERS \& } \\
\text { FUSES }\end{array}$ & $N / A$ & N/A & A & \\
\hline $\begin{array}{c}1.7 .7 .8 \\
m \times n .3 \\
\end{array}$ & $\begin{array}{l}\text { CLOSE LISTED } \\
\text { SYS. VALVES }\end{array}$ & $N / A$ & $N / A$ & A & \\
\hline
\end{tabular}


TEST DATA SHEET

\begin{tabular}{|c|c|c|c|c|c|}
\hline \multicolumn{3}{|c|}{ Date: $\quad 1 /-6-96$} & \multicolumn{3}{|c|}{ SHMS Unit Number: VTP.PNL $=805 A$} \\
\hline \multicolumn{3}{|c|}{$\begin{array}{l}\text { Title of Test: } \\
\text { Pneumatic Systems }\end{array}$} & \multirow{2}{*}{\multicolumn{3}{|c|}{ Test Equipment and $\mathrm{S} / \mathrm{N}$ or Cal. No.: }} \\
\hline \multicolumn{3}{|c|}{$\begin{aligned} \text { Test Performed By: } & \text { see Test Personnal } \\
& \text { Page } 60\end{aligned}$} & & & \\
\hline $\begin{array}{l}\text { Procedure No. } \\
\text { Initial/Date }\end{array}$ & Item & Value & $\begin{array}{l}\text { Required } \\
\text { Condition }\end{array}$ & $(A / R)$ & Comment \\
\hline $\begin{array}{l}1.7 .7 .9 \\
\operatorname{man} x \cdot 0\end{array}$ & $\begin{array}{l}\text { DISCONNECT } \\
\text { SAMP PUMP } \\
\end{array}$ & $N / A$ & $N / A$ & A & \\
\hline $\begin{array}{l}1.7 .7 .10 \\
m .2 .3\end{array}$ & $\begin{array}{l}\text { DEENERGIZE \& } \\
\text { DISCONNECT } \\
\text { POWER SOURCE } \\
\end{array}$ & $N / A$ & $N / A$ & A & \\
\hline all $11-8-96$ & & & & & \\
\hline
\end{tabular}

Test Witness/Review:

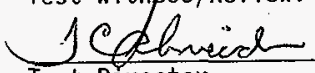

Test Director

Mad n Bror

Recorder

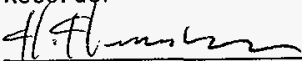

Quality Assurance $\frac{11-8-26}{\text { Date }}$

$\frac{11-8-96}{\text { Date }}$

$1-2-96$

Date 
APPENDIX B

PAGE B-30

$$
\begin{array}{r}
\text { HNF-SD-WM-ATR-191 } \\
\text { ReV. } 0 \\
\text { Page } 95 \mathrm{~A}
\end{array}
$$

TEST EXCEPTION SHEET

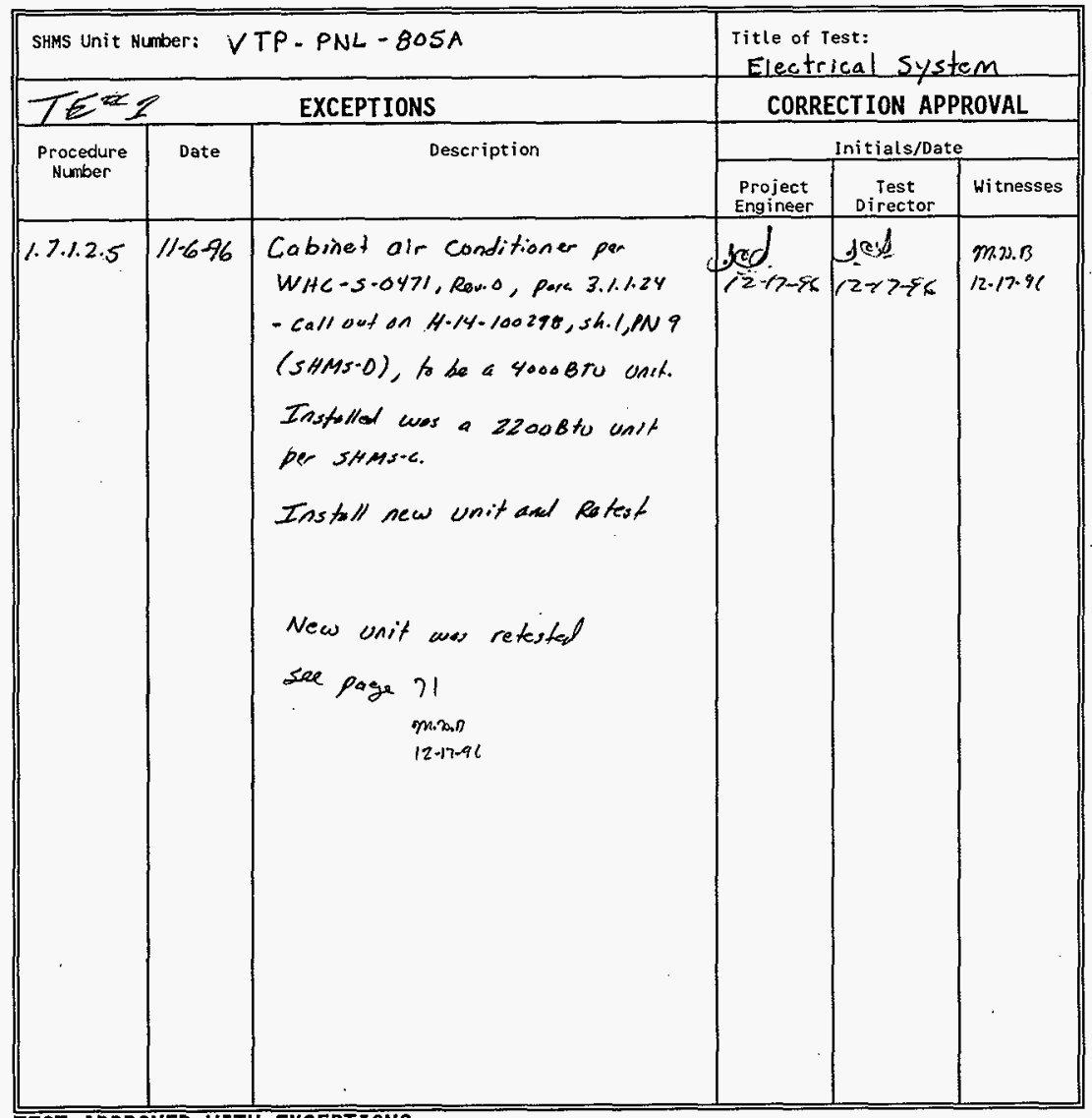

TEST APPROVED WITH EXCEPTIONS

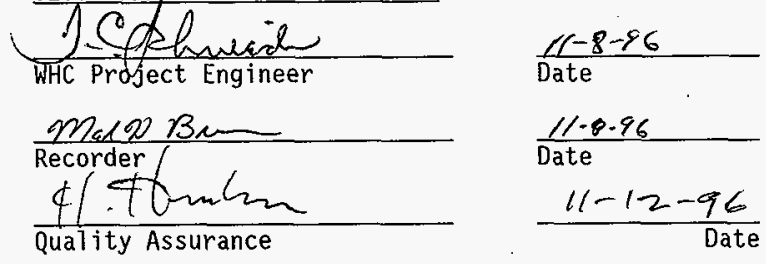


APPENDIX $A \cdot B$ mas

PAGE $X-1, \quad 11-8-86$
RECORD

COPY

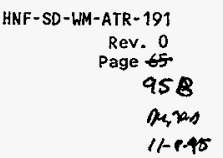

TEST EXCEPTION SHEET

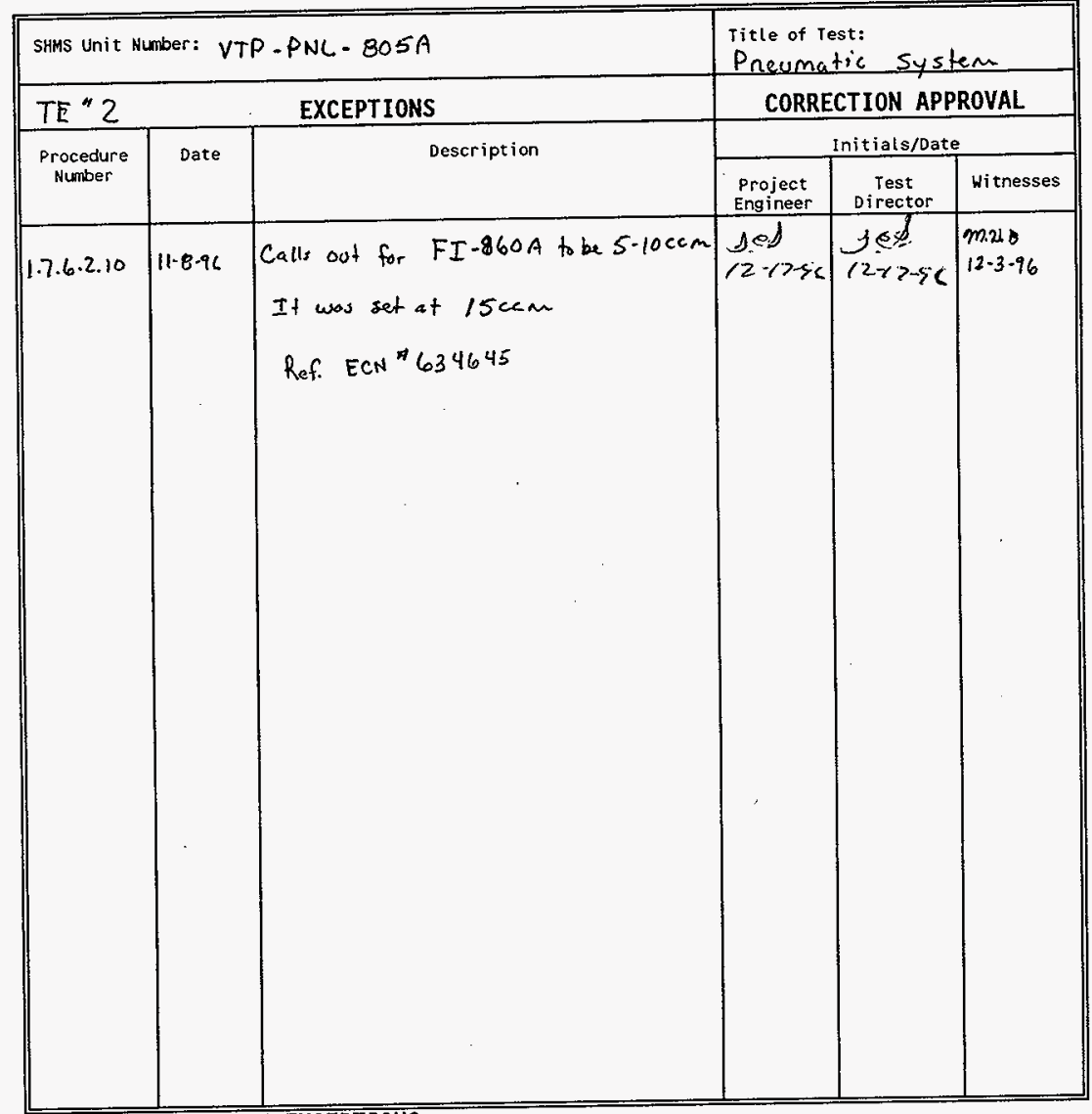

TEST APPROVED WITH EXCEPTIONS
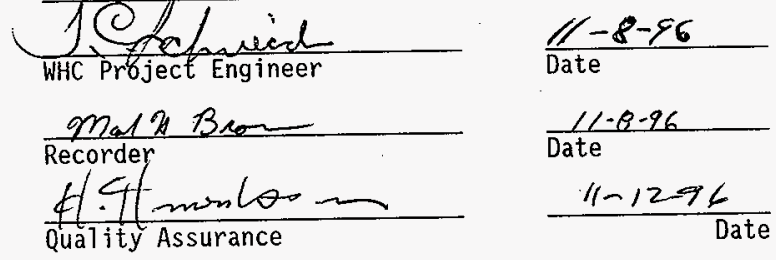

$\frac{11-\theta-96}{\text { Date }}$

$\frac{11-12-96}{\text { Date }}$ 
APPENDIX B

PAGE B-32
HNF-SD-WH-ATR-191

Rev. 0
Page $96 \mathrm{~A}$

TEST LOG

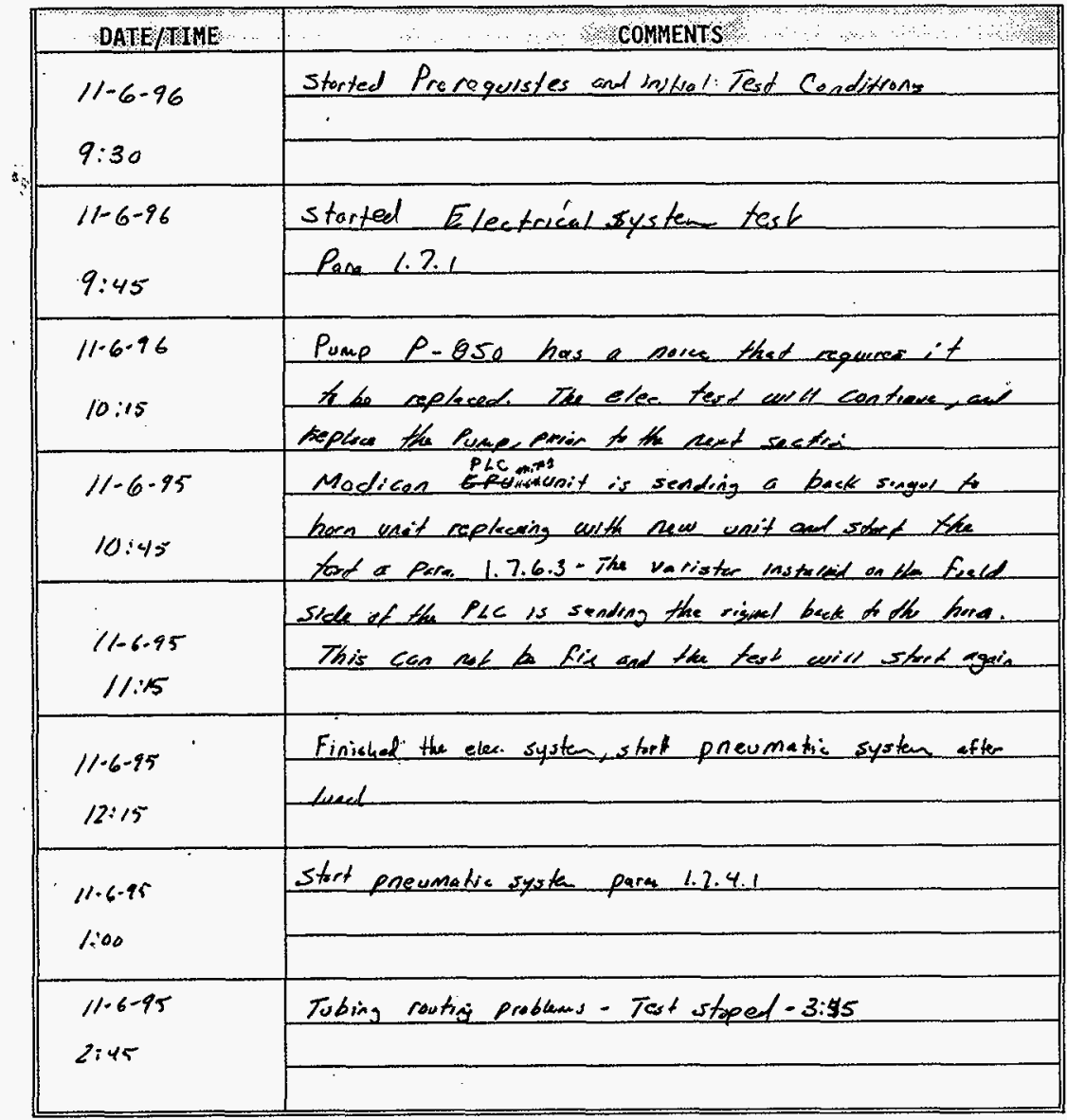


APPENDIX B

PAGE B-33.
HNF-SD-WM-ATR- 191

Rev. 0
Page $96 \mathbb{R}$

TEST LOG

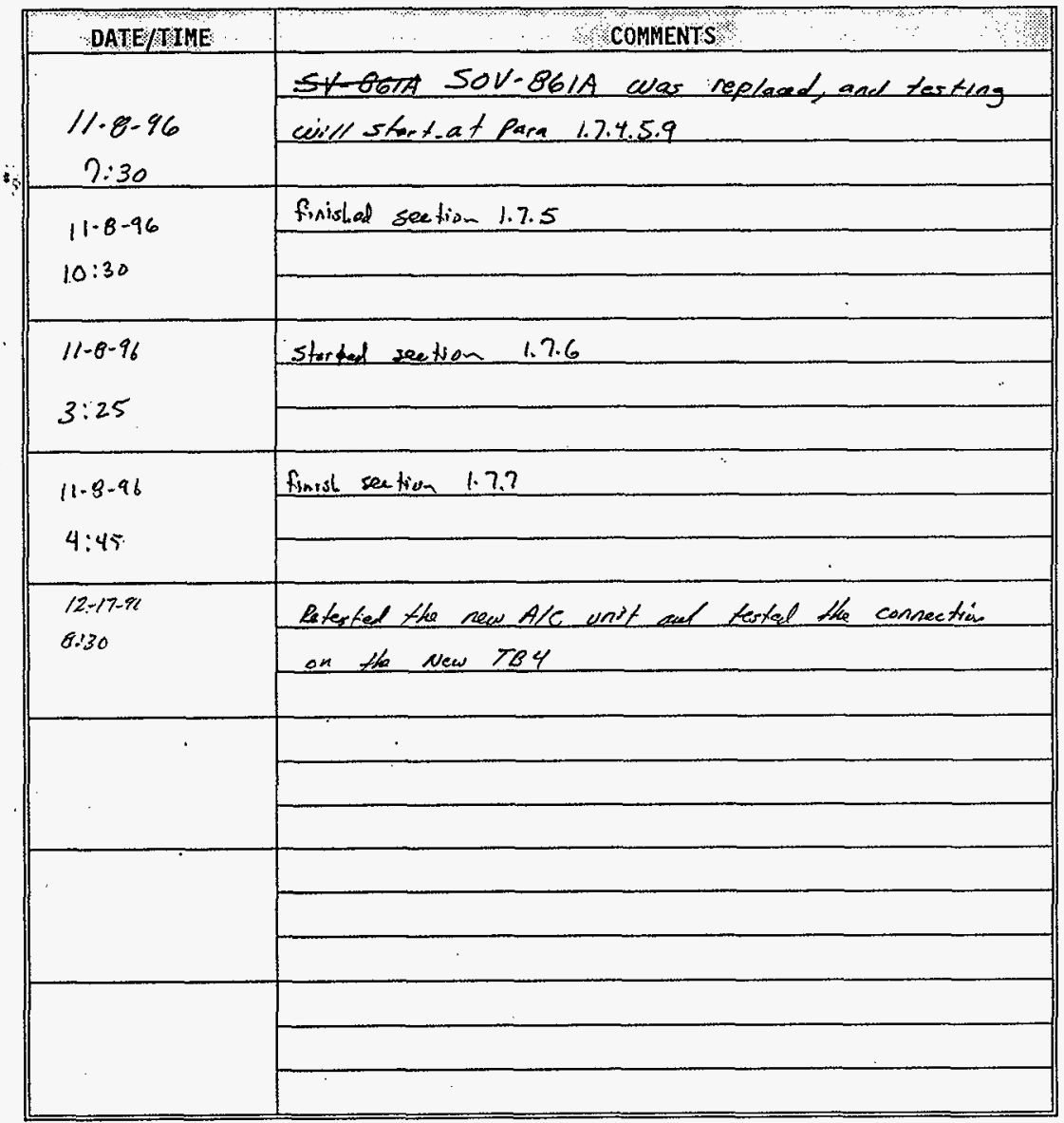


APPENDIX C

PAGE $\mathrm{C}-/$
Cat

on
HNF-SD-WM-ATR-191

Rev. 0

Page 97

APPENDIX C 
TEST EXECUTION SHEET

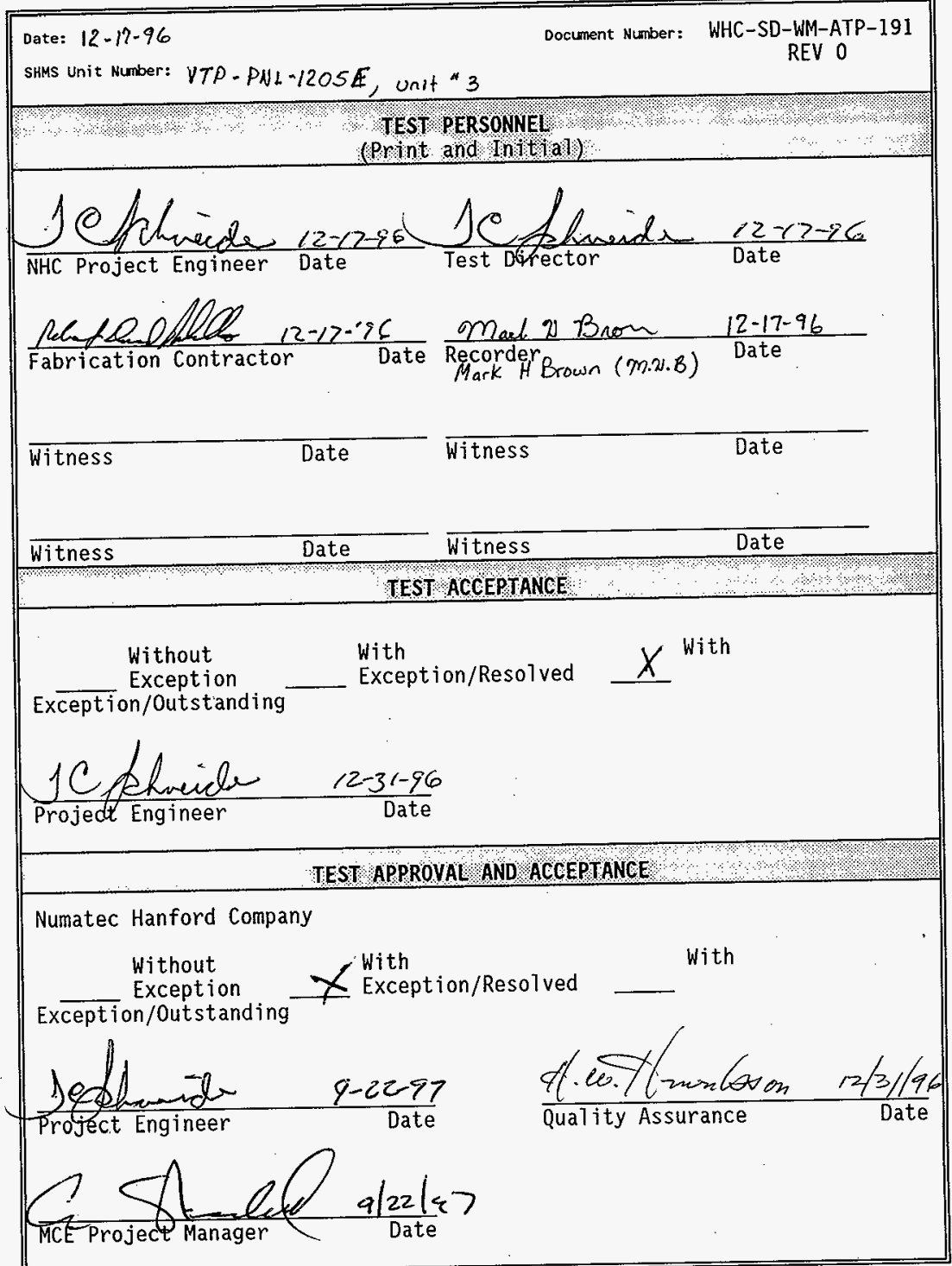


SHMS Unit Number: VTP - PNL-12OSE

\section{PREREQUISITES AND INITIAL TEST CONDITIONS}

The following conditions shall exist at the start of the acceptance testing. Initial and date to verify that each of the following items have been accomplished.

m.2.B Systems being tested have been inspected for workmanship and for 12-17-96 compliance with design. / With exception of Id tages. MHB 12-17-96 sen $T E * 1$

mas Continuity and megger tests have been performed on portions of the $12-17-16$ electrical and instrument systems being tested, as required.

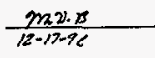

mola $12+17-96$ Leak tests on the pneumatic systems have been performed.

The following circuit breakers and fuses are installed per the specified size and are open

$\mathrm{CB}-1$ through $\mathrm{CB}-7$

FU-1 through FU-15

$m \cdot 2 \cdot n$

Al] test instruments have a currently valid calibration stamp attached that indicates a calibration traceable to the National Institute of Standards and Technology.

$m \times \cdot n$

$12+17.96$

The following process and control instrument systems have been initially configured and aligned for proper operation.

FIT-*57, FSL $-\star 57$, NIT-*54, NIT-*55, (NR-*54), PDIT-*60, TIC $-\star 50$, TIC $-* 56$, TIS $-* 62, Y Y C-* 01$ NOTE: NR-*54 is not used in the ANALYTICAL configuration.

m.r.p Personnel responsible for directing and witnessing the performance of the tests described in this ATP have read and understand appropriate certified vendor information (CVI) pertaining to the operation of the equipment to be tested.

m.9.0 CLOSE all system manual valves with the exception of the

$12 \cdot 17-96$ following:

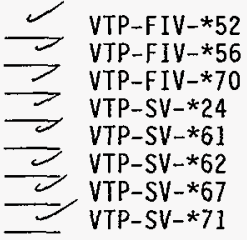
port, but vented to atmosphere for testing. 
208 Vac 1 phase 15-20 ampere temporary power source has been

$12 \cdot 17-96$ connected, but not energized, to the appropriate TBl terminals per H-14-100838.

Verify by signature and date that all prerequisites have been met.

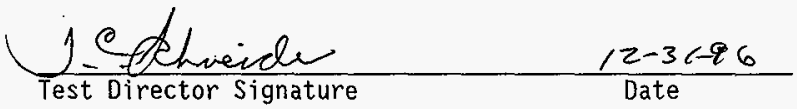


TEST DATA SHEET

\begin{tabular}{|c|c|c|c|c|c|}
\hline \multicolumn{3}{|c|}{ Date: $12-17.96$} & \multicolumn{3}{|c|}{ SHMS Unit Number: VTP - PNL -1205E } \\
\hline \multicolumn{3}{|c|}{$\begin{array}{l}\text { ritle of Test: } \\
\text { Electrical Systems }\end{array}$} & \multirow{2}{*}{\multicolumn{3}{|c|}{$\begin{array}{l}\text { Test Equipment and } S / N \text { or Cal. No.: } \\
\text { Fluke V/o meter } \\
S / N 44620364 \\
\text { Cal. doe } 10.97\end{array}$}} \\
\hline \multicolumn{3}{|c|}{$\begin{array}{c}\text { Test Performed By: see Test Personnal } \\
\text { Page } 98\end{array}$} & & & \\
\hline $\begin{array}{l}\text { Procedure No. } \\
\text { Initial/Date }\end{array}$ & Item & Value & $\begin{array}{r}\text { Required } \\
\text { Condition } \\
\end{array}$ & $(A / R)$ & Comment \\
\hline \multicolumn{6}{|c|}{ Section 1.7.1.1 verifies the system Mains Power. } \\
\hline $\begin{array}{l}1.7 .1 .1 .1 \\
m x_{1}\end{array}$ & Resistance & 0.02 & $<\mathrm{lohm}$ & A & \\
\hline 1.7 .1 .1 .2 & $\begin{array}{l}\text { Temp power } \\
208 \text { Vac }\end{array}$ & N/A & Energized & $A$ & \\
\hline${ }^{1.7 .1 .1 .3}$ & $\begin{array}{l}\text { Line Vac: } \\
\text { LI-L2 } \\
\text { L1-Gind } \\
\text { L2-Gnd } \\
N-\text { Gnd }\end{array}$ & $\begin{array}{l}\frac{207.8 V_{a c}}{179.1 V_{c c}} \\
\frac{119.3 V_{a c}}{0.0}\end{array}$ & $\begin{array}{l}\text { L1-L2: } 208 \\
\text { Vac }+10 /-1 \% \\
L 1, L 2: 120 \\
\text { Vac } \pm 5 \% \\
N: 0 \text { Vac }\end{array}$ & $A$ & \\
\hline \multicolumn{6}{|c|}{ Section 1.7 .1 .2 verifies the enclosure HVAC system. } \\
\hline 1.7 .1 .2 .1 & AC Adjusted & $N / A$ & N/A & A & \\
\hline $\begin{array}{c}1.7 .1 .2 .2 \\
m . x \\
\end{array}$ & $\begin{array}{l}\text { CLOSE } \\
\text { CB-1/2 }\end{array}$ & $N / A$ & $N / A$ & A & \\
\hline $\begin{array}{l}1.7 .1 .2 .3 \\
m .2 B\end{array}$ & Heater ON & $\mathrm{ON}$ & Heater ON & $A$ & \\
\hline $\begin{array}{l}1.7 .1 .2 .4 \\
m \times .8\end{array}$ & AC Adjusted & $N / A$ & N/A & A & \\
\hline $\begin{array}{l}1.7 .1 .2 .5 \\
\text { m.x.8 }\end{array}$ & $\begin{array}{l}\text { Heater OFF } \\
\text { Cooling ON }\end{array}$ & $\begin{array}{l}\text { OfF } \\
\text { ON }\end{array}$ & $\begin{array}{l}\text { Heater OFF } \\
\text { Cooling ON }\end{array}$ & A & \\
\hline $\begin{array}{l}1.7 .1 .2 .6 \\
\text { m.x.B }\end{array}$ & AC Adjusted & $N / A$ & $N / A$ & A & \\
\hline $\begin{array}{l}1.7 .1 .2 .7 \\
m i . B\end{array}$ & $\begin{array}{l}\text { OPEN } \\
\text { CB- } 1 / 2 \\
\end{array}$ & $N / A$ & $N / A$ & A & \\
\hline \multicolumn{6}{|c|}{ Section 1.7 .1 .3 verifies the sample pump is properly connected. } \\
\hline $\begin{array}{r}1.7 .1 .3 .1 \\
m . x . B \\
\end{array}$ & $\begin{array}{l}\text { CLOSE } \\
\text { CB-3/4 }\end{array}$ & $N / A$ & $N / A$ & $A$ & \\
\hline $\begin{array}{l}1.7 .1 .3 .2 \\
m x \cdot x \\
\end{array}$ & $\begin{array}{l}\text { VTP-P }-\star 50 \\
\text { ON }\end{array}$ & $O K$ & $\begin{array}{l}\text { VTP-P-*50 } \\
\text { ON }\end{array}$ & $A$ & \\
\hline
\end{tabular}


TEST DATA SHEET

\begin{tabular}{|c|c|c|c|c|c|}
\hline \multicolumn{3}{|c|}{ Date: $\quad 12-17.96$} & \multicolumn{3}{|c|}{ SHMS Unit Number: VTP-PNL-1205E } \\
\hline \multicolumn{3}{|c|}{$\begin{array}{l}\text { Title of rest: } \\
\text { Electrical Systems }\end{array}$} & \multirow{2}{*}{\multicolumn{3}{|c|}{$\begin{array}{l}\text { Test Equipment and } \mathrm{S} / \mathrm{N} \text { or Cal. No.: } \\
\text { Fluke V/o meter } \\
S / N 4462036^{4} \\
\text { Cal. due } 10-97\end{array}$}} \\
\hline \multicolumn{3}{|c|}{$\begin{aligned} \text { Test Performed By: } & \text { See Test Persunnal } \\
& \text { Page } 98\end{aligned}$} & & & \\
\hline $\begin{array}{l}\text { Procedure No. } \\
\text { Initial/Date }\end{array}$ & Iten & Value & $\begin{array}{l}\text { Required } \\
\text { Condition } \\
\end{array}$ & $(\mathrm{A} / \mathrm{R})$ & Comment \\
\hline $\begin{array}{c}1.7 .1 .3 .3 \\
m \times B\end{array}$ & $\begin{array}{l}\text { OPEN } \\
\text { CB-3/4 }\end{array}$ & $N / A$ & N/A & A & \\
\hline \multicolumn{6}{|c|}{ Section 1.7 .1 .4 verifies the enclosure lighting and GFCI receptacles. } \\
\hline $\begin{array}{l}1.7 .1 .4 .1 \\
1\end{array}$ & $\begin{array}{l}\text { CLOSE } \\
\text { CB-6 }\end{array}$ & $N / A$ & $N / A$ & $A$ & \\
\hline 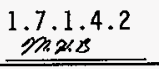 & $\begin{array}{l}\text { ENCLOSURE } \\
\text { LIGHT }\end{array}$ & N/A & N/A & A & \\
\hline 1.7 .1 .4 .3 & LIGHT ON & ON & LIGHT ON & $A$ & \\
\hline $\begin{array}{l}1.7 .1 .4 .4 \\
m . x .3\end{array}$ & $\begin{array}{l}\text { GFCI RECP. } \\
\text { IA. L-N } \\
\text { IB. L-GND } \\
2 A . L-N \\
\text { 2B. L-GND } \\
\end{array}$ & $\frac{\frac{119.2 V_{\text {ec }}}{U 19.3 V_{\text {ac }}}}{\frac{119.3 V_{a c}}{119.3 V_{a c}}}$ & $\begin{array}{l}\text { 1A. } 120 \mathrm{Vac} \\
1 \mathrm{~B} .120 \mathrm{Vac} \\
2 \mathrm{~A} .120 \mathrm{VaC} \\
2 \mathrm{~B} .120 \mathrm{Vac} \\
\mathrm{ALL} \pm 5 \%\end{array}$ & A & \\
\hline $\begin{array}{l}1.7 .1 .4 .5 \\
m . x \cdot B\end{array}$ & $\begin{array}{l}\text { GFCI TEST } \\
1 A . L-N \\
1 B . L-G N D \\
2 A . L-N \\
2 B . \quad L-G N D \\
\end{array}$ & $\frac{\frac{0 . V_{c c}}{1.6 V_{c c}}}{\frac{0.4 V_{c c}}{0.2 V_{a c}}}$ & $\begin{array}{l}1 \mathrm{~A} .0 \mathrm{Vac} \\
1 \mathrm{~B} .0 \mathrm{VaC} \\
2 \mathrm{~A} .0 \mathrm{VaC} \\
2 \mathrm{~B} .0 \mathrm{VaC} \\
\mathrm{ALL} \pm 5 \%\end{array}$ & A & \\
\hline$m .7 .1 .4 .6$ & $\begin{array}{l}\text { ENCLOSURE } \\
\text { LIGHT OFF }\end{array}$ & N/A & N/A & A & \\
\hline $\begin{array}{l}1.7 .1 .4 .7 \\
93.2 . B^{4}\end{array}$ & $\begin{array}{l}\text { OPEN } \\
\text { CB-6 }\end{array}$ & $\mathrm{N} / \mathrm{A}$ & N/A & A & \\
\hline \multicolumn{6}{|c|}{ Section 1.7.1.5 verifies the instrument power source isolation transformer. } \\
\hline $\begin{array}{l}1.7 .1 .5 .1 \\
m \cdot 2 \cdot B \\
\end{array}$ & $\begin{array}{l}\text { CLOSE } \\
\text { CB-7 }\end{array}$ & N/A & N/A & $A$ & \\
\hline $\begin{array}{l}1.7 .1 .5 .2 \\
m_{n .8} .8 \\
\end{array}$ & $\begin{array}{l}\text { XFRM } V a c \\
\text { PRI. L-N } \\
\text { SEC. L-N } \\
\text { SEC. N-GND } \\
\end{array}$ & $\frac{\frac{119.8 \mathrm{Vac}}{123.1 \mathrm{Vac}}}{0.2 \mathrm{Vac}}$ & $\begin{array}{l}\text { PRI. \& SEC. } \\
\text { L-N } 120 \pm 5 \% \\
\text { SEC. } \\
\text { N-GND O VaC }\end{array}$ & A & \\
\hline $\begin{array}{l}1.7: 1.5 .3 \\
\text { m.x. }\end{array}$ & CLOSE FU-1 & $N / A$ & $N / A$ & $A$ & \\
\hline
\end{tabular}


TEST DATA SHEET

\begin{tabular}{|c|c|c|c|c|c|}
\hline \multicolumn{3}{|c|}{ Date: $12-17-96$} & \multicolumn{3}{|c|}{ SHMS Unit Number: VTP - PNL-1205E } \\
\hline \multicolumn{3}{|c|}{$\begin{array}{l}\text { ritle of Test: } \\
\text { Electrical Systems }\end{array}$} & \multirow{2}{*}{\multicolumn{3}{|c|}{$\begin{array}{l}\text { rest Equipment and } S / N \text { or Cal. No.: } \\
\text { Fluke V/O meter } \\
S / N 44620364 \\
\text { Cal. due. } 10.97\end{array}$}} \\
\hline \multicolumn{3}{|c|}{$\begin{aligned} \text { rest Performed By: } & \text { See Test Personinal } \\
& \text { Page } 98\end{aligned}$} & & & \\
\hline $\begin{array}{c}\text { Procedure No. } \\
\text { Initial/Date } \\
\end{array}$ & I tem & Value & $\begin{array}{l}\text { Required } \\
\text { Condition } \\
\end{array}$ & $(A / R)$ & Comment \\
\hline $\begin{array}{l}1.7 .1 .5 .4 \\
\text { m.x.s } \\
\end{array}$ & $\begin{array}{l}\text { VTP-PS-*50 } \\
\text { OUTPUT Vdc }\end{array}$ & $24.03 \mathrm{Vdr}$ & $24 \pm 0.1 \mathrm{Vdc}$ & A & \\
\hline \multicolumn{6}{|c|}{ Section 1.7.1.6 verifies the enclosure general al arm system. } \\
\hline $\begin{array}{l}1.7 .1 .6 .1 \\
m_{2 \times B} \\
\end{array}$ & $\begin{array}{l}\text { CLOSE FU-9 \& } \\
\text { FU-10 }\end{array}$ & N/A & N/A & A & \\
\hline $\begin{array}{l}1.7 .1 .6 .2 \\
m . \beta^{2}\end{array}$ & $\begin{array}{l}\text { PUSH PB-*51 } \\
\& \text { PB-*50 }\end{array}$ & N/A & N/A & A & \\
\hline $1.7 \cdot 1.6 .3$ & $\begin{array}{l}\text { ALARM TEST } \\
\text { VERIFY ALARM } \\
\text { CONDITIONS }\end{array}$ & $\begin{array}{l}-O K \\
O K \\
O K \\
O K \\
O K \\
O K \\
O K \\
O K \\
O K \\
O K \\
O K \\
O\end{array}$ & $\begin{array}{ll}\text { YAH-*50 } & \text { ON } \\
\text { NAH-*55 } & \text { ON } \\
\text { XA-*63 } & \text { ON } \\
\text { NAH-*54 } & \text { ON } \\
\text { FAL-*57 } & \text { ON } \\
\text { TAHL-*62 ON } \\
\text { TAL-*50 } & \text { ON } \\
\text { YAL-*58 } & \text { ON } \\
\text { PBL-*58 } & \text { ON } \\
\text { PBL-*54 OFF } & \text { OFF } \\
\text { PBL-*59 } & \text { OFF } \\
\end{array}$ & $A$ & 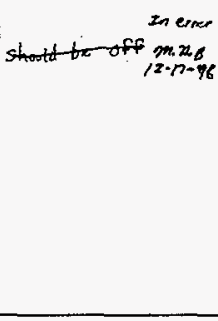 \\
\hline $\begin{array}{c}1.7 .1 .6 .4 \\
202.9\end{array}$ & $\begin{array}{l}\text { END ALARM } \\
\text { TEST } \\
\end{array}$ & N/A & N/A & A & \\
\hline $\begin{array}{l}1.7 .1 .6 .5 \\
\text { moses }\end{array}$ & RESET ALARMS & $N / A$ & $N / A$ & $A$ & \\
\hline $\begin{array}{c}1.7 .1 .6 .6 \\
32 x^{2} \\
\end{array}$ & $\begin{array}{l}\text { OPEN FU-9 \& } \\
\text { FU-10 }\end{array}$ & N/A & N/A & A & \\
\hline \multicolumn{6}{|c|}{ Section 1.7.1.7 verifies the flow alarm system. } \\
\hline $\begin{array}{l}1.7 .1 .7 .1 \\
\text { mases }\end{array}$ & $\begin{array}{l}\text { CLOSE FU-3, } \\
\text { FU-9, FU-10 } \\
\& \text { FU-13 } \\
\end{array}$ & N/A & $N / A$ & A & \\
\hline $\begin{array}{r}1.7 .1 .7 .2 \\
m=2.0 \\
\end{array}$ & RESET ALARMS & ON & FAL $-* 57$ ON & A & \\
\hline $\begin{array}{c}1.7 .1 .7 .3 \\
m x .8 \\
\end{array}$ & $\begin{array}{l}\text { OPEN SV }-* 20 \\
\& \text { SV-*22 }\end{array}$ & $N / A$ & $N / A$ & A & \\
\hline
\end{tabular}


APPENDIX $C$

TEST DATA SHEET

\begin{tabular}{|c|c|c|c|c|c|}
\hline \multicolumn{3}{|c|}{ Date: $\quad 12-17-96$} & \multicolumn{3}{|c|}{ SHMS Unit Number: VTP - PNL $-1205 E$} \\
\hline \multicolumn{3}{|c|}{$\begin{array}{l}\text { Title' of Test: } \\
\text { Electrical Systems }\end{array}$} & \multirow{2}{*}{\multicolumn{3}{|c|}{$\begin{array}{l}\text { Test Equipment and } S / N \text { or Cal. No.: } \\
\text { Fluke V/O meter } \\
S / N 44620364 \\
\text { Cal. due } 10.47\end{array}$}} \\
\hline \multicolumn{3}{|c|}{$\begin{array}{c}\text { Test Performed By: See Test Personnol } \\
\text { Page } 98\end{array}$} & & & \\
\hline $\begin{array}{l}\text { Procedure No. } \\
\text { Initial/Date }\end{array}$ & Item & value & $\begin{array}{l}\text { Required } \\
\text { Condition }\end{array}$ & $(A / R)$ & Comment \\
\hline $\min ^{1.7 .1 .7 .4}$ & $\begin{array}{l}\text { FIT-*57 \& } \\
\text { FSL-*57 } \\
\text { POWERED }\end{array}$ & $-\frac{O N}{O N}$ & $\begin{array}{l}\text { FIT-*57 ON } \\
\text { FSL-*57 ON }\end{array}$ & A & \\
\hline $\begin{array}{l}1.7 .1 .7 .5 \\
m . n . b \\
\end{array}$ & $\begin{array}{l}\text { MUX-*70 } \\
\text { TB5+/TB6- }\end{array}$ & $1,0 \mathrm{Vde}$ & $1.0 \pm 0.2 \mathrm{Vdc}$ & $A$ & \\
\hline $\begin{array}{l}1.7 .1 .7 .6 \\
m_{313}\end{array}$ & $\begin{array}{l}\text { CLOSE SV-*20 } \\
\& \text { SV }-\star 22\end{array}$ & $N / A$ & $N / A$ & $A$ & \\
\hline $\begin{array}{l}1.7 .1 .7 .7 \\
m .8 .8 \\
\end{array}$ & $\begin{array}{l}\text { OPEN FU-3, } \\
\text { FU-9, FU-10 } \\
\& \text { FU-13 }\end{array}$ & $N / A$ & $N / A$ & $A$ & \\
\hline \multicolumn{6}{|c|}{ Section 1.7.1.8 verifies the $\mathrm{H}_{2}$ monitor wiring. } \\
\hline $\begin{array}{l}1.7 .1 .8 .1 \\
\text { mx.s }\end{array}$ & $\begin{array}{l}\text { CLOSE FU-2, } \\
\text { FU-4, FU-5, } \\
\text { FU-9 \& FU-10 }\end{array}$ & N/A & $N / A$ & $A$ & \\
\hline $\begin{array}{r}1.7 .1 .8 .2 \\
.22 .0 \\
\end{array}$ & RESET ALARMS & $\frac{O K}{O K}$ & $\begin{array}{l}\mathrm{NAH}-* 54 \text { OFF } \\
\text { NAH- } * 55 \text { OFF }\end{array}$ & A & \\
\hline $\begin{array}{l}1.7 .8 .3 \\
\text { m.N.B }\end{array}$ & $\begin{array}{l}\text { INSTRUMENTS } \\
\text { POWERED }\end{array}$ & $\frac{\frac{O N}{O N}}{\frac{O N}{N / A}} \frac{O N}{O N}$ & $\begin{array}{l}\text { MUX-*70 ON } \\
\text { NIT-*54 ON } \\
\text { NIT-*55 ON } \\
\text { NR-*54 ON } \\
\text { YYC-*01 ON }\end{array}$ & $A$ & \\
\hline $\begin{array}{l}1.7 .1 .8 .4 \\
m .2 . B \\
\end{array}$ & $\begin{array}{l}\text { OPEN FU-2, } \\
\text { FU-4, FU-5, } \\
\text { FU-9 \& FU-10 }\end{array}$ & $N / A$ & $N / A$ & A & \\
\hline \multicolumn{6}{|c|}{ Section 1.7.1.9 verifies the SHMS-E heat trace control system. } \\
\hline $\begin{array}{l}1.7 .1 .9 .1 \\
\operatorname{mix.\beta } \\
\end{array}$ & $\begin{array}{l}\text { INSTALL LOAD } \\
\text { VTP-TIC-*50 }\end{array}$ & $N / A$ & $N / A$ & A & \\
\hline $\begin{array}{l}1.7 .1 .9 .2 \\
m x^{\circ}\end{array}$ & $\begin{array}{l}\text { CLOSE CB-5, } \\
\text { FU-6, FU-7, } \\
\text { FU-9 \& FU-10 } \\
\text { and RESET } \\
\text { ALARMS }\end{array}$ & $N / A$ & $N / A$ & $A$ & \\
\hline
\end{tabular}


APPENDIX $C$

TEST DATA SHEET

\begin{tabular}{|c|c|c|c|c|c|}
\hline Date: $12-17-$ & & & SHMS Unit Number & $V T P$ - & $205 E$ \\
\hline $\begin{array}{l}\text { Title of rest: } \\
\text { Electrical }\end{array}$ & stems & & Test Equipment ar & $\begin{array}{l}\mathrm{S} / \mathrm{N} \text { or } \\
\text { meter }\end{array}$ & \\
\hline Test Performed B & $\begin{array}{l}\text { see Test Pe } \\
\text { Page } 98\end{array}$ & Sonnal & $\begin{array}{l}\operatorname{Sin} 446203 \\
\text { Cal. due } 10\end{array}$ & 47 & \\
\hline $\begin{array}{l}\text { Procedure No. } \\
\text { Initial/Date }\end{array}$ & Item & value & $\begin{array}{l}\text { Required } \\
\text { condition }\end{array}$ & (A/R) & Comment \\
\hline$\frac{1.7 .1 .9 .3}{m x .8}$ & $\begin{array}{l}\text { TIC-*50 \& } \\
\text { TIC-*56 } \\
\text { NOMINAL TEMP }\end{array}$ & $\frac{620 F}{600 F}$ & $\begin{array}{l}\text { TIC }-\star 50 \\
\text { TIC }-* 56 \\
\text { TEMPERATURE }\end{array}$ & $A$ & \\
\hline 1.7 .1 .9 .4 & $\begin{array}{l}\text { ADJ TIC } * 50 \\
\& \text { TIC-*56 } \\
\text { SP2 \& RESET } \\
\text { ALARMS }\end{array}$ & $N / A$ & $N / A$ & $A$ & \\
\hline $\begin{array}{r}1.7 .1 .9 .5 \\
m 2 x . B \\
\end{array}$ & $\begin{array}{l}\text { ADJ TIC }-* 50 \\
\text { SP } 1\end{array}$ & $N / A$ & $N / A$ & $A$ & \\
\hline $\begin{array}{l}1.7 .1 .9 .6 \\
\text { on.2.8 }\end{array}$ & $\begin{array}{l}\text { ADJ TIC }-* 50 \\
\text { SP } 2\end{array}$ & $N / A$ & $N / A$ & $A$ & \\
\hline $\begin{array}{l}1.7 .1 .9 .7 \\
m .28\end{array}$ & $\begin{array}{l}\text { TAL-*50 \& } \\
\text { HORN ACTIVE }\end{array}$ & $\frac{d O}{O K}$ & $\begin{array}{l}\text { TAL-*50 ON } \\
\text { HORN ON } \\
\text { ACKNOWLEDGE }\end{array}$ & $A$ & \\
\hline $\begin{array}{c}1.7 .1 .9 .8 \\
m .3 .3\end{array}$ & $\begin{array}{l}\text { TIC }-* 50 \\
\text { OPERATES }\end{array}$ & $-O K$ & $\begin{array}{l}\text { TIC }-* 50 \\
\text { OPERATES }\end{array}$ & $A$ & \\
\hline $\begin{array}{l}1.7 .1 .9 .9 \\
138 B\end{array}$ & $\begin{array}{l}A D J_{2} T I C-* 50 \\
S P 2\end{array}$ & $N / A$ & $N / A$ & A & \\
\hline $\begin{array}{c}1.7 .1 .9 .10 \\
\end{array}$ & RESET ALARM & $O K$ & $\mathrm{TAL}-* 50$ OFF & $A$ & \\
\hline $\begin{array}{l}1.7 .1 .9 .11 \\
\end{array}$ & $\begin{array}{l}\text { ADJ TIC } * 550 \\
S P 1 \& S P 2\end{array}$ & $N / A$ & $N / A$ & A & \\
\hline $\begin{array}{l}1.7 .1 .9 .12 \\
\text { mins. }\end{array}$ & $\begin{array}{l}\text { OPEN CB-5 \& } \\
\text { REMOVE LOAD }\end{array}$ & $\mathrm{N} / \mathrm{A}$ & N/A & $A$ & \\
\hline $\begin{array}{c}1.7 .1 .9 .13 \\
\text { m.2.1. }\end{array}$ & $\begin{array}{l}\text { INSTALL LOAD } \\
\text { VTP-TIC-*56 }\end{array}$ & $N / A$ & $N / A$ & A & \\
\hline $\begin{array}{l}1.7 .1 .9 .14 \\
\text { m.D. }\end{array}$ & CLOSE CB-5 & $N / A$ & N/A & A & \\
\hline $\begin{array}{l}1.7 .1 .9 .15 \\
\text { m.2.B. }\end{array}$ & $\begin{array}{l}\text { ADJ TIC-*56 } \\
\text { SP } 1\end{array}$ & $N / A$ & $N / A$ & A & \\
\hline $\begin{array}{l}1.7 .1 .9 .16 \\
m \lambda . \beta\end{array}$ & $\begin{array}{l}\text { ADJ TIC }-* 56 \\
\text { SP } 2\end{array}$ & $N / A$ & $N / A$ & A & \\
\hline
\end{tabular}


TEST DATA SHEET

\begin{tabular}{|c|c|c|c|c|c|}
\hline \multicolumn{3}{|c|}{ Date: $12-17-96$} & \multicolumn{3}{|c|}{ SHMS Unit Number: VTP-PNL-1205E } \\
\hline \multicolumn{3}{|c|}{$\begin{array}{l}\text { Title of Test: } \\
\text { Electrical Systems }\end{array}$} & \multirow{2}{*}{\multicolumn{3}{|c|}{$\begin{array}{l}\text { Test Equipnent and } s / \mathrm{N} \text { or Cal. No.: } \\
\qquad N / A\end{array}$}} \\
\hline \multicolumn{3}{|c|}{$\begin{aligned} \text { Test Performed By: } & \text { See Test Personn.l } \\
& \text { Page } 98\end{aligned}$} & & & \\
\hline $\begin{array}{c}\text { Procedure No. } \\
\text { Initial/Date } \\
\end{array}$ & I tem & value & $\begin{array}{c}\text { Required } \\
\text { Condition }\end{array}$ & $(A / R)$ & comnent \\
\hline $\begin{array}{l}1.7 .1 .9 .17 \\
\text { m.x.3 }\end{array}$ & $\begin{array}{l}\text { TAL }-* 50 \& \\
\text { HORN ACTIVE }\end{array}$ & $\frac{O N}{O N}$ & $\begin{array}{l}\text { TAL }-* 50 \text { ON } \\
\text { HORN ON } \\
\text { ACKNOWLEDGE } \\
\end{array}$ & $A$ & \\
\hline $\begin{array}{c}1.7 .1 .9 .18 \\
m 2.8 \\
\end{array}$ & $\begin{array}{l}\text { TIC }-* 56 \\
\text { OPERATES } \\
\end{array}$ & ON & $\begin{array}{l}\text { TIC }-* 56 \\
\text { OPERATES } \\
\end{array}$ & A & \\
\hline $\begin{array}{l}1.7 .1 .9 .19 \\
\text { m.2. B } \\
\end{array}$ & $\begin{array}{l}\text { ADJ TIC-*56 } \\
\text { SP 2 }\end{array}$ & $N / A$ & $N / A$ & A & \\
\hline $\begin{array}{l}1.7 .1 .9 .20 \\
m .2 . A^{2}\end{array}$ & RESET ALARM & - ok & $T A L-\star 50$ OFF & A & \\
\hline $\begin{array}{l}1.7 .1 .9 .21 \\
m .2 .8 \\
\end{array}$ & $\begin{array}{l}\text { ADJ TIC }-* 56 \\
S P 1 \& S P 2\end{array}$ & N/A & $N / A$ & $A$ & \\
\hline $\begin{array}{l}1.7 .1 .9 .22 \\
m . \lambda_{. B} \\
\end{array}$ & $\begin{array}{l}\text { OPEN CB-5, } \\
\text { FU-6 \& FU-7 }\end{array}$ & $N / A$ & $N / A$ & A & \\
\hline $\begin{array}{l}1.7 .1 .9 .23 \\
\end{array}$ & $\begin{array}{l}\text { REMOVE LOAD } \\
\text { TIC }-* 56\end{array}$ & $N / A$ & $N / A$ & A & \\
\hline $\begin{array}{l}1.7 .1 .9 .24 \\
m \pi .3\end{array}$ & $\begin{array}{l}\text { CLOSE FU-8 } \\
\text { TIS }-{ }^{*} 62 \\
\text { NOMINAL TEMP }\end{array}$ & $74^{\circ} \mathrm{F}$ & $\begin{array}{l}\text { TIS }-* 62 \\
\text { TEMP }\end{array}$ & $A$ & \\
\hline $\begin{array}{l}1.7 .1 .9 .25 \\
m_{A . B}\end{array}$ & $\begin{array}{l}\text { ADJ TIS-*62 } \\
\text { SP } 1\end{array}$ & N/A & N/A & A & \\
\hline $\begin{array}{l}1.7 .1 .9 .26 \\
m .3 . B \\
\end{array}$ & $\begin{array}{l}\text { ADJ TIS-*62 } \\
\text { SP 2 }\end{array}$ & $N / A$ & N/A & A & \\
\hline $\begin{array}{c}1.7 .1 .9 .27 \\
m_{D . B} B \\
\end{array}$ & RESET ALARMS & - OK & $\begin{array}{l}\text { TAHL }-* 62 \\
\text { OFF }\end{array}$ & A & \\
\hline $\begin{array}{l}1.7 .1 .9 .28 \\
\end{array}$ & $\begin{array}{l}\text { ADJ TIS }-* 62 \\
\text { SP } 2\end{array}$ & $N / A$ & N/A & $A$ & \\
\hline$\frac{1.7 .1 .9 .29}{m .30 .8}$ & $\begin{array}{l}\text { TAHL-*62 \& } \\
\text { HORN ACTIVE }\end{array}$ & $\begin{array}{r}\frac{O N}{O N} \\
O N \\
O K \\
\end{array}$ & $\begin{array}{l}\text { TAHL }-* 62 \text { ON } \\
\text { HORN ON } \\
\text { ACKNOWLEDGE } \\
\end{array}$ & A & \\
\hline $\begin{array}{l}7.1 .9 .30 \\
m_{2} \cdot \beta \\
\end{array}$ & $\begin{array}{l}\text { ADJ TIS }-* 62 \\
\text { SP 2 }\end{array}$ & N/A & N/A & A & \\
\hline
\end{tabular}


TEST DATA SHEET

\begin{tabular}{|c|c|c|c|c|c|}
\hline \multicolumn{3}{|c|}{ Date: $\quad 12-17-96$} & \multicolumn{3}{|c|}{ SHMS Unit Number: VTP-PNL - $1205 E$} \\
\hline \multicolumn{3}{|c|}{$\begin{array}{l}\text { Iitle of Jest: } \\
\text { Electrical Systems }\end{array}$} & \multirow{2}{*}{\multicolumn{3}{|c|}{ 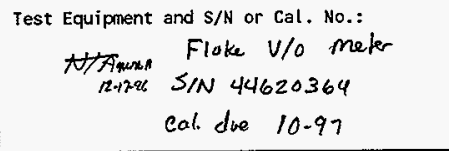 }} \\
\hline \multicolumn{3}{|c|}{$\begin{aligned} \text { Test Performed By: See Test Personnal } & \text { Poge } 98\end{aligned}$} & & & \\
\hline $\begin{array}{l}\text { Procedure No. } \\
\text { Injtial/Date }\end{array}$ & Item & value & $\begin{array}{l}\text { Required } \\
\text { condition } \\
\end{array}$ & (A/R) & Corment \\
\hline $\begin{array}{l}1.7 .1 .9 .31 \\
\text { m.x.B }\end{array}$ & RESET ALARMS & ok & $\begin{array}{l}\text { TAHL-*62 } \\
\text { OFF }\end{array}$ & A & \\
\hline $\begin{array}{c}1.7 .1 .9 .32 \\
m p \cdot s \\
\end{array}$ & $\begin{array}{l}\text { ADJ TIS-*62 } \\
\text { SP }{ }_{1}\end{array}$ & N/A & $N / A$ & $A$ & \\
\hline $\begin{array}{l}1.7 .1 .9 .33 \\
\min 2.3\end{array}$ & $\begin{array}{l}\text { TAHL-*62 \& } \\
\text { HORN ACTIVE }\end{array}$ & $\frac{\frac{O N}{\partial N}}{\Delta K}$ & $\begin{array}{l}\text { TAHL }-* 62 \text { ON } \\
\text { HORN ON } \\
\text { ACKNOWLEDGE }\end{array}$ & A & \\
\hline $\begin{array}{l}1.7 .1 .9 .34 \\
m \times B\end{array}$ & $\begin{array}{l}\text { ADJ TIS-*62 } \\
S P 1 \& S P 2\end{array}$ & N/A & N/A & A & \\
\hline $\begin{array}{l}1.7 .9 .35 \\
m_{x} \times{ }^{2}\end{array}$ & $\begin{array}{l}\text { OPEN FU-8, } \\
\text { FU-9 \& FU-10 }\end{array}$ & N/A & N/A & A & \\
\hline \multicolumn{6}{|c|}{ Section 1.7.1.10 verifies the differential pressure transmitter system. } \\
\hline $\begin{array}{l}1.7 .1 .10 .1 \\
\text { m.x.s }\end{array}$ & $\begin{array}{l}\text { REMOVE PLUGS } \\
\text { BAL PDIT-*60 }\end{array}$ & N/A & N/A & A & \\
\hline $\begin{array}{l}1.7 .1 .10 .2 \\
m . n . \beta \\
\end{array}$ & $\begin{array}{l}\text { CLOSE FU-14 } \\
\text { REC PDIT-*60 } \\
\text { PRESSURE }\end{array}$ & $0.0078^{\prime \prime} \mathrm{H}_{20}$ & $\begin{array}{l}\text { PDIT-*60 } \\
\text { PRESSURE } \\
0 \pm 1 " \mathrm{H}_{2} 0 \\
\end{array}$ & A & \\
\hline $\begin{array}{l}1.7 .1 .10 .3 \\
\text { M.x.B. }\end{array}$ & $\begin{array}{l}\text { MUX-*70 Vdc } \\
\text { TB7+ \& TB8- } \\
\end{array}$ & $2.200 \mathrm{Vdc}$ & $\begin{array}{l}\text { MUX-*70 Vdc } \\
2.2 \pm 0.1\end{array}$ & A & \\
\hline $\begin{array}{l}1.7 .1 .10 .4 \\
m . x .8 \\
\end{array}$ & $\begin{array}{l}\text { REPLACE } \\
\text { PLUGS ON } \\
\text { PDIT }-* 60 \\
\end{array}$ & N/A & N/A & A & \\
\hline 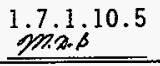 & OPEN FU-14 & $N / A$ & $\mathrm{~N} / \mathrm{A}$ & A & \\
\hline \multicolumn{6}{|c|}{ Section 1.7.1.11 verifies the gas chromatograph power } \\
\hline $\begin{array}{l}1.7 .1 .11 .1 \\
M \text { M... }\end{array}$ & CLOSE FU-11 & $N / A$ & $N / A$ & A & \\
\hline $\begin{array}{l}1.7 .1 .11 .2 \\
m .2 . \beta \\
\end{array}$ & $\begin{array}{l}\text { POWER } \\
\text { MON-*60 }\end{array}$ & OK & $\begin{array}{l}\text { MON-*60 } \\
\text { POWERED } \\
\end{array}$ & A & \\
\hline $\begin{array}{l}1.7 .1 .11 .3 \\
m 2.8 \\
\end{array}$ & OPEN FU-11 & N/A & $N / A$ & A & \\
\hline
\end{tabular}




\section{TEST DATA SHEET}

\begin{tabular}{|c|c|c|c|c|c|}
\hline \multicolumn{3}{|c|}{ Date: $12-17-96$} & \multicolumn{3}{|c|}{ SHMS Unit Number: VTP-PNL-120.5E } \\
\hline \multicolumn{3}{|c|}{$\begin{array}{l}\text { Title of Test: } \\
\text { Electrical Systems }\end{array}$} & \multirow{2}{*}{\multicolumn{3}{|c|}{$\begin{array}{r}\text { Test Equipment and S/N or Cal. No.: } \\
\text { Floke V/o meter } \\
\text { N/A S/N } 44620364 \\
\min _{\text {Iethes }} \text { Cal. due } 10-97\end{array}$}} \\
\hline \multicolumn{3}{|c|}{$\begin{aligned} \text { Test Performed By: } & \text { see Test Persoanal } \\
& \text { Page } 98\end{aligned}$} & & & \\
\hline $\begin{array}{c}\text { Procedure No. } \\
\text { Initial/Date } \\
\end{array}$ & Item & Value & $\begin{array}{l}\text { Required } \\
\text { Condition }\end{array}$ & $(A / R)$ & Comment \\
\hline \multicolumn{6}{|c|}{ Section 1.7.1.12 verifies the multi gas analyzer power. } \\
\hline $\begin{array}{l}1.7 .1 .12 .1 \\
\text { m.x.s } \\
\end{array}$ & CLOSE FU-12 & N/A & N/A & A & \\
\hline $\begin{array}{l}1.7 .1 .12 .2 \\
\text { m.x.s } \\
\end{array}$ & $\begin{array}{l}\text { POWER } \\
\text { NIT }-* 52\end{array}$ & $122.5 \mathrm{Vac}$ & $\begin{array}{l}\text { NIT-*52 } \\
\text { POWERED }\end{array}$ & A & \\
\hline $\begin{array}{l}1.7 .1 .12 .3 \\
\min x .8\end{array}$ & OPEN FU-12 & N/A & N/A & A & \\
\hline \multicolumn{6}{|c|}{ Section 1.7.1.13 verifies the personnel computer power. } \\
\hline 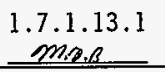 & CLOSE CB-6 & $N / A$ & $N / A$ & A & \\
\hline $\begin{array}{l}1.7 .1 .13 .2 \\
\text { m.x.B. }\end{array}$ & $\begin{array}{l}\text { POWER PC-*60 } \\
\& P C-* 70\end{array}$ & $\begin{array}{l}\text { OK } \\
\text { OK }\end{array}$ & $\begin{array}{ll}P C-* 60 & 0 N \\
P C-* 70 & O N \\
\end{array}$ & A & \\
\hline $\begin{array}{l}1.7 .1 .13 .3 \\
m \infty . \beta\end{array}$ & $\begin{array}{l}\text { TURN INST. } \\
\text { OFF \& } \\
\text { OPEN CB-6 }\end{array}$ & $\frac{o k}{o k}$ & $\begin{array}{ll}P C-* 60 & 0 F \\
P C-* 70 & O F\end{array}$ & A & \\
\hline
\end{tabular}

all 12+17-96 
TEST DATA SHEET

\begin{tabular}{|c|c|c|c|c|c|}
\hline \multicolumn{3}{|c|}{ Date: $12-17-96$} & \multicolumn{3}{|c|}{ SHMS Unit Number: $V T P-P N L-12059$} \\
\hline \multicolumn{3}{|c|}{$\begin{array}{l}\text { Title of Test: } \\
\text { Electrical Systems }\end{array}$} & \multirow{2}{*}{\multicolumn{3}{|c|}{$\begin{array}{l}\text { Test Equipment and } S / N \text { or Cal. No.: } \\
N / A\end{array}$}} \\
\hline \multicolumn{3}{|c|}{$\begin{aligned} \text { Test Performed By: } & \text { See Test Personnal } \\
& \text { Page } 90\end{aligned}$} & & & \\
\hline $\begin{array}{l}\text { Procedure No. } \\
\text { Initial/Date }\end{array}$ & Iten & Value & $\begin{array}{r}\text { Required } \\
\text { Condition } \\
\end{array}$ & $(A / R)$ & Comment \\
\hline \multicolumn{6}{|c|}{ Section 1.7 .2 verifies the intrinsic safety apparatus is properly 1 abeled. } \\
\hline$\frac{1 \cdot 7 \dot{2}^{2} y^{1}}{q-z-87}$ & $\begin{array}{l}\text { INTRINSIC } \\
\text { SAFETY } \\
\text { APPARATUS } \\
\text { NAMEPLATES } \\
\text { INSTALLED }\end{array}$ & $\frac{\sim}{\sim}$ & $\begin{array}{l}\text { NE- } * 54 \\
\text { NE- } * 55 \\
E B-* 54 \\
\text { EB-*55 } \\
\text { NIT-*54 } \\
\text { NIT }-* 55 \\
\end{array}$ & & $\operatorname{Sen} T E^{*} 1$ \\
\hline $\begin{array}{l}1.7 .2 .2 \\
\frac{9 m \cdot 2 \cdot \beta}{12-17-96}\end{array}$ & $\begin{array}{l}\text { INTRINSIC } \\
\text { SAFETY WIRE } \\
\text { LABELED }\end{array}$ & ok & $\begin{array}{l}N E / E B-* 54 \\
N E / E B-* 55\end{array}$ & $A$ & \\
\hline 1.7 .3 & $\begin{array}{l}\text { SEC. } 1.7 .1 \text { \& } \\
1.7 .2 \text { DONE }\end{array}$ & $\frac{1000}{\text { Test Dired }}$ & or Signature & & $\frac{12-17-96}{\text { Date }}$ \\
\hline
\end{tabular}

Test Witness/Review:
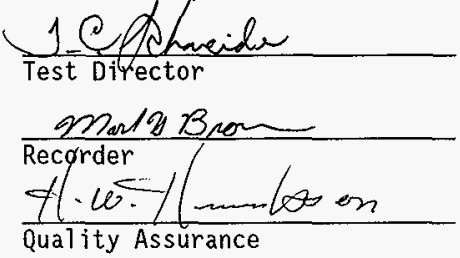

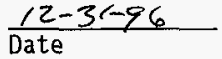

$\frac{12-17-96}{\text { Date }}$

$\frac{12-3 /-96}{\text { Date }}$ 
TEST DATA SHEET

\begin{tabular}{|c|c|c|c|c|c|}
\hline \multicolumn{3}{|l|}{ Date: $12-17-96$} & \multicolumn{3}{|c|}{ SHMS Unit Number: VTP - PNL-1205E } \\
\hline \multicolumn{3}{|c|}{$\begin{array}{l}\text { Iitle of Test: } \\
\text { Pneumatic Systems }\end{array}$} & \multirow{2}{*}{\multicolumn{3}{|c|}{$\begin{array}{l}\text { Test Equipment and } S / \mathrm{N} \text { or cal. No.: } \\
N / A\end{array}$}} \\
\hline \multicolumn{3}{|c|}{$\begin{aligned} \text { Test Performed By: } & \text { See Test Personnal } \\
& \text { Page } 98\end{aligned}$} & & & \\
\hline $\begin{array}{c}\text { Procedure No. } \\
\text { initial/Date }\end{array}$ & Item & value & $\begin{array}{l}\text { Required } \\
\text { Condition }\end{array}$ & $(A / R)$ & Comment \\
\hline \multicolumn{6}{|c|}{ Section 1.7.4.1 provides the pneumatic test section initial conditions. } \\
\hline $\begin{array}{c}1.7 .4 .1 .1 \\
9.2 .13 \\
\end{array}$ & $\begin{array}{l}\text { INITIAL } \\
\text { CONDITIONS }\end{array}$ & ok & $\begin{array}{l}\text { BKR \& FUSE } \\
\text { LINE UP }\end{array}$ & $A$ & \\
\hline $\begin{array}{l}1.7 .4 .1 .2 \\
\text { mo.s } \\
\end{array}$ & $\begin{array}{l}\text { INITIAL } \\
\text { CONDITIONS }\end{array}$ & OK & $\begin{array}{l}\text { VALLVE LINE } \\
\text { UP }\end{array}$ & A & \\
\hline$\frac{1.7 .4 .1 .3}{\sin }$ & $\begin{array}{l}\text { DISCONNECT } \\
1 / 16 " \text { TUBING } \\
\text { ON MON-*60 }\end{array}$ & $\Delta K$ & $\begin{array}{l}\text { TUBING } \\
\text { CONFIGURED }\end{array}$ & A & \\
\hline $\begin{array}{l}1.7 .4 .1 .4 \\
m .8\end{array}$ & $\begin{array}{l}\text { DISCONNECT } \\
\text { TUBING ON } \\
\text { NIT-*52 }\end{array}$ & OK & $\begin{array}{l}\text { TUBING } \\
\text { CONFIGURED }\end{array}$ & A & \\
\hline $\begin{array}{c}1.7 .4 .1 .5 \\
m 22 B \\
\end{array}$ & $\begin{array}{l}\text { PLUG VENT } \\
\text { FLT }-* 63\end{array}$ & ok & $\begin{array}{l}\text { FLT-*63 } \\
\text { PLUGGED }\end{array}$ & $A$ & \\
\hline \multicolumn{6}{|c|}{ Section 1.7 .4 .2 verifies the main sample flow loop. } \\
\hline $\begin{array}{l}1.7 .4 .2 .1 \\
\text { m.m.7. }\end{array}$ & $\begin{array}{l}\text { CLOSE CB-3/4 } \\
\text { ADJ. FLOW }\end{array}$ & $\frac{\mathrm{OK}}{5.0 " \mathrm{H}_{2} \mathrm{O}}$ & $\begin{array}{l}\text { CB CLOSED } \\
\text { FIT-* } 57 \\
\text { INDICATION }\end{array}$ & A & \\
\hline $\begin{array}{l}1.7 .4 .2 .2 \\
m .2 s\end{array}$ & $\begin{array}{l}\text { PDI } * 51 \\
\text { READING }\end{array}$ & $25^{\prime \prime} \mathrm{H}_{2} \mathrm{O}$ & $20-40 \quad " \mathrm{H}_{2} \mathrm{O}$ & A & \\
\hline $\begin{array}{l}1.7 .4 .2 .3 \\
\end{array}$ & $\begin{array}{l}\text { PI }-* 53 \\
\text { READING }\end{array}$ & $-2.5^{\circ} \mathrm{Hg}$ & $\begin{array}{l}-2 \text { to }-4 \\
" \mathrm{Hg}\end{array}$ & 4 & \\
\hline $\begin{array}{l}1.7 .4 .2 .4 \\
2 x \cdot x \cdot x^{2}\end{array}$ & RESET ALARMS & $\partial K$ & NO ALARMS & A & \\
\hline $\begin{array}{l}1.7 .4 .2 .5 \\
m .2 B\end{array}$ & CLOSE SV-*16 & $\frac{0.05^{-1} \mathrm{H}_{2} \mathrm{O}}{\frac{\mathrm{ON}}{\mathrm{ON}}}$ & $\begin{array}{l}\text { FIT-*57 } 0 \\
\pm 1 \quad " \mathrm{H}_{2} \mathrm{O} \\
\text { FAL-*57 } \\
\text { HONN } \\
\text { HON } \\
\end{array}$ & A & \\
\hline $\begin{array}{l}1.7 .4 .2 .6 \\
\text { m.x.s } \\
\end{array}$ & OPEN SV-*16 & ok & $\begin{array}{l}\text { FAL_*57 ON } \\
\text { HORN } \quad \text { OFF } \\
\end{array}$ & A & \\
\hline $\begin{array}{c}1.7 .4 .2 .7 \\
m \cdot 2 . B\end{array}$ & RESET ALARMS & OK & $\begin{array}{l}\text { ALARMS } \\
\text { RESET } \\
\end{array}$ & $A$ & \\
\hline
\end{tabular}


TEST DATA SHEET

\begin{tabular}{|c|c|c|c|c|c|}
\hline \multicolumn{3}{|c|}{ Date: $\quad 12-17-96$} & \multicolumn{3}{|c|}{ SHMS unit Number: VTP.PNL - $1205 E$} \\
\hline \multicolumn{3}{|c|}{$\begin{array}{l}\text { Title of rest: } \\
\text { Pneumatic Systems }\end{array}$} & \multirow{2}{*}{\multicolumn{3}{|c|}{$\begin{array}{l}\text { Test Equipment and } \mathrm{S} / \mathrm{N} \text { or Cal. No.: } \\
\text { N/A }\end{array}$}} \\
\hline \multicolumn{3}{|c|}{$\begin{aligned} \text { Test Performed By: See Test Personnal } & \text { See } \\
& \text { Page } 96\end{aligned}$} & & & \\
\hline $\begin{array}{l}\text { Procedure No. } \\
\text { Initial/Date }\end{array}$ & Item & value & $\begin{array}{l}\text { Required } \\
\text { Condition }\end{array}$ & $(A / R)$ & Corment \\
\hline $\begin{array}{l}1.7 .4 .2 .8 \\
m .8 . \\
\end{array}$ & CLOSE SV-*25 & $\begin{array}{l}0.02 \\
\text { on } \\
\text { on } \\
\text { on } \\
0 . \text { Vac }\end{array}$ & $\begin{array}{ll}\mathrm{FIT}-* 57 & 0 \\
\pm 1 \quad " \mathrm{H}_{2} 0 & \\
\text { FAL-*57 } & \text { ON } \\
\text { HORN } & \text { ON } \\
X A-* 63 & \text { ON } \\
\text { YY-*71 } & \\
\text { TB } 3-4 & \text { OV } \\
\end{array}$ & $A$ & \\
\hline $\begin{array}{l}1.7 .4 .2 .9 \\
2 x .2 \\
\end{array}$ & OPEN SV-*25 & $\frac{\frac{o k}{o k}}{\frac{o k}{110.3 \mathrm{Vac}}}$ & $\begin{array}{ll}\mathrm{FAL}-* 57 & \text { ON } \\
\text { HORN } & \text { OFF } \\
\text { XA-*63 } & \text { OFF } \\
\text { YY-*71 } & \\
\text { TB } 3-4 & 120 \mathrm{~V}\end{array}$ & $A$ & \\
\hline $\begin{array}{l}1.7 .4 .2 .10 \\
\text { mxs }\end{array}$ & RESET ALARMS & ok & $\begin{array}{l}\text { ALARMS } \\
\text { RESET }\end{array}$ & $A$ & \\
\hline $\begin{array}{l}1.7 .4 .2 .11 \\
-\max . B \\
\end{array}$ & $\begin{array}{l}\text { OPEN SV-*54 } \\
\& \text { SV-*66 } \\
\text { READ PRESS. } \\
\text { PDIT-*60 } \\
\end{array}$ & $\begin{array}{r}\mathrm{OK} \\
-37.5^{\circ} \mathrm{H}_{2} \mathrm{O} \\
\end{array}$ & $\begin{array}{l}\text { VALVES OPEN } \\
-27 \text { to }-82 \\
" \mathrm{H}_{2} \mathrm{O}\end{array}$ & $A$ & \\
\hline $\begin{array}{l}1.7 .4 .2 .12 \\
-m 28 \\
\end{array}$ & $\begin{array}{l}\text { CLOSE SV }-* 54 \\
\text { OPEN SV } * 63 \\
\text { READ PRESS } \\
\text { PDIT-* } 60 \\
\end{array}$ & 0.06 & $\begin{array}{l}\text { VALVES } \\
\text { OPERATED } \\
0 \pm 1 \quad " \mathrm{H}_{2} \mathrm{O}\end{array}$ & $A$ & \\
\hline $\begin{array}{l}1.7 .4 .2 .13 \\
-2.2 . x \\
\end{array}$ & $\begin{array}{l}\text { CLOSE SV }-* 63 \\
\text { OPEN SV } * 55 \\
\text { READ PRESS } \\
\text { PDIT }-* 60 \\
\end{array}$ & $\frac{O K}{-36 \cdot 8 \% H_{2}}$ & $\begin{array}{l}\text { VALVES } \\
\text { OPERATED } \\
-27 \text { to }-82 \\
" \mathrm{H}_{2} \mathrm{O}\end{array}$ & A & \\
\hline $\begin{array}{l}1.7 .4 .2 .14 \\
m .2 .8 \\
\end{array}$ & $\begin{array}{l}\text { CLOSE SV-*55 } \\
\text { OPEN SV } * 64 \\
\text { READ PRESS } \\
\text { PDIT-* } 60 \\
\end{array}$ & $0.0 K$ & $\begin{array}{l}\text { VALVES } \\
\text { OPERATED } \\
0 \pm 1 " \mathrm{H}_{2} \mathrm{O}\end{array}$ & A & \\
\hline $\begin{array}{l}1.7 .4 .2 .15 \\
m .2 . B \\
\end{array}$ & $\begin{array}{l}\text { CLOSE SV-*64 } \\
\& \text { SV }-* 66\end{array}$ & $N / A$ & $N / A$ & A & \\
\hline \multicolumn{6}{|c|}{ Section 1.7 .4 .3 verifies the grab sample flow loop. } \\
\hline $\begin{array}{l}1.7 .4 .3 .1 \\
2.2 .3 \\
\end{array}$ & READ PDI $* 51$ & $25^{\prime \prime} \mathrm{H}_{2} \mathrm{O}$ & $20-40 " \mathrm{H}_{2} \mathrm{O}$ & $A$ & \\
\hline
\end{tabular}


TEST DATA SHEET

\begin{tabular}{|c|c|c|c|c|c|}
\hline \multicolumn{3}{|c|}{ Date: $\quad 12-17-96$} & \multicolumn{3}{|c|}{ SHMS Unit Number: VTP-PNL-1205E } \\
\hline \multicolumn{3}{|c|}{$\begin{array}{l}\text { Iitle of rest: } \\
\text { Pneumatic Systems }\end{array}$} & \multirow{2}{*}{\multicolumn{3}{|c|}{$\begin{array}{l}\text { Test Equipment and } S / N \text { or cal. No.: } \\
N / A\end{array}$}} \\
\hline \multicolumn{3}{|c|}{$\begin{aligned} \text { Test Performed By: } & \text { See Test Personnal } \\
& \text { Page } 98\end{aligned}$} & & & \\
\hline $\begin{array}{l}\text { Procedure No. } \\
\text { Initial/oate }\end{array}$ & Item & Value & $\begin{array}{l}\text { Required } \\
\text { Condition } \\
\end{array}$ & $(A / R)$ & Comment \\
\hline $\begin{array}{c}1.7 .4 .3 .2 \\
m .2 .0 \\
\end{array}$ & $\begin{array}{l}\text { PUSH PB-*59 } \\
\text { GRAB SAMPLE } \\
\end{array}$ & or & $\begin{array}{l}\text { START } \\
\text { STOPWATCH }\end{array}$ & A & \\
\hline $\begin{array}{l}1.7 .4 .3 .3 \\
\text { gxp.13. }\end{array}$ & $\begin{array}{l}\text { GRAB SAMPLE } \\
\text { LAMPS ON } \\
\text { READ FIV }-* 52\end{array}$ & $\begin{array}{l}\frac{O N}{O N} \\
\frac{O N}{O K} \\
O K\end{array}$ & 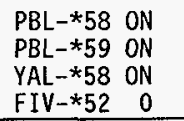 & A & \\
\hline $\begin{array}{c}1.7 .4 .3 .4 \\
2 m .28\end{array}$ & $\begin{array}{l}\text { OPEN SV } * \text { * } 15 \\
\& \text { ADJ. FIV - } \\
* 52\end{array}$ & OK & $\begin{array}{l}\text { SV }-* 15 \text { OPEN } \\
\text { FIV }-* 52 \\
\text { FLOW } 10 \text { CFH }\end{array}$ & $A$ & \\
\hline $\begin{array}{c}1.7 .4 .3 .5 \\
x y\end{array}$ & READ PDI $-* 51$ & $27.5^{4} H_{20}$ & $\begin{array}{l}\text { PDI }-* 51 \\
\text { HIGHER THAN } \\
1.7 .4 .3 .1 \\
\end{array}$ & $A$ & \\
\hline $\begin{array}{l}1.7 .4 .3 .6 \\
2.3 .3 \\
\end{array}$ & CLOSE SV-*15 & ok & FIV $-* 52 \quad 0$ & A & \\
\hline $\begin{array}{l}1.7 .4 .3 .7 \\
02 x .8\end{array}$ & OPEN SV-* 15 & _ok & $\begin{array}{l}\text { FIV }-* 52 \\
\text { FLOW }\end{array}$ & A & \\
\hline $\begin{array}{c}1.7 .4 .3 .8 \\
20.2 .8 \\
\end{array}$ & $\begin{array}{l}\text { PBL }-* 59 \\
\text { GRAB SAMPLE } \\
\text { TIME }\end{array}$ & 5.00 & $\begin{array}{l}\text { PBL }-* 59 \text { OFF } \\
\text { STOP WATCH } \\
5 \pm .5 \text { MIN }\end{array}$ & A & \\
\hline $\begin{array}{c}1.7 .4 .3 .9 \\
20.0 \\
\end{array}$ & $\begin{array}{l}\text { PB-*58 RESET } \\
\text { SAMPLER }\end{array}$ & $\frac{o k}{o k}$ & $\begin{array}{l}\text { PBL-*58 OFF } \\
\text { YAL-*58 OFF }\end{array}$ & A & \\
\hline \multicolumn{6}{|c|}{ Section 1.7.4.4 verifies the $\mathrm{H}_{2}$ cell calibration loop } \\
\hline $\begin{array}{r}1.7 .4 .4 .1 \\
m . x . B \\
\end{array}$ & $\begin{array}{l}\text { CONNECT } \mathrm{H}_{2} \\
\text { CAL GAS }\end{array}$ & N/A & $N / A$ & A & \\
\hline $\begin{array}{l}1.7 .4 .4 .2 \\
m 2.2 . B^{2}\end{array}$ & $\begin{array}{l}\text { OPEN ISO } \\
\text { VALVE }\end{array}$ & SPSIG & $<10$ PSIG & A & \\
\hline $\begin{array}{l}1.7 .4 .4 .3 \\
m 2.0\end{array}$ & $\begin{array}{l}\text { OPEN SV-*18 } \\
\text { ADJ. FIV-*56 }\end{array}$ & $2 \mathrm{CFH}$ & $2 \pm .1 \mathrm{CFH}$ & A & \\
\hline
\end{tabular}


APPENDIX $C$

PAGE $\mathrm{C}-17$

TEST DATA SHEET

\begin{tabular}{|c|c|c|c|c|c|}
\hline \multicolumn{3}{|c|}{ Date: $12-17-96$} & \multicolumn{3}{|c|}{ SHMS Unit Number: VTP-PNL-1205E } \\
\hline \multicolumn{3}{|c|}{$\begin{array}{l}\text { Title of Test: } \\
\text { Pneumatic Systems }\end{array}$} & \multirow{2}{*}{\multicolumn{3}{|c|}{$\begin{array}{l}\text { Test Equipment and } S / N \text { or Cal. No.: } \\
\text { Fluke V/O meter } \\
S / N \text { 44620364 } \\
\text { Cal due } 10.97\end{array}$}} \\
\hline \multicolumn{3}{|c|}{$\begin{aligned} \text { Test Performed sy: See Test Personnal } & \text { Tage } 90 \\
& \text { Pa }\end{aligned}$} & & & \\
\hline $\begin{array}{l}\text { Procedure No. } \\
\text { Initial/Date }\end{array}$ & Item & Value & $\begin{array}{l}\text { Required } \\
\text { Condition } \\
\end{array}$ & $(A / R)$ & Corment \\
\hline $\begin{array}{l}1.7 .4 .4 .4 \\
m .8 .6 \\
\end{array}$ & $\begin{array}{l}\text { VERIFY } \\
\text { CONDITIONS }\end{array}$ & 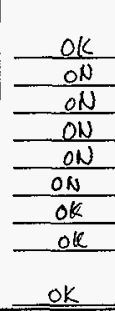 & $\begin{array}{l}\mathrm{NIT}-* 54 \\
>-625 \% \mathrm{H}_{2} \\
\mathrm{NAH}-* 55 \mathrm{ON} \\
\text { NAH-*54 ON } \\
\mathrm{PBL}-* 59 \text { ON } \\
\mathrm{PBL}-* 58 \text { ON } \\
\text { YAL-*58 ON } \\
\text { FIV-*52 FLO } \\
\text { HORN ON } \\
\text { TB2-2I/22 } \\
\text { OPEN }\end{array}$ & $A$ & \\
\hline $\begin{array}{l}1.7 .4 .4 .5 \\
\text { on.2.8 } \\
\end{array}$ & $\begin{array}{l}\text { ACKNOWLEDGE } \\
\text { HORN }\end{array}$ & $N / A$ & N/A & $\Delta$ & \\
\hline $\begin{array}{l}1.7 .4 .4 .6 \\
m . x . B\end{array}$ & $\begin{array}{l}\text { CLOSE SV-*18 } \\
\text { VERIFY } \\
\text { CONDITIONS }\end{array}$ & $\begin{array}{l}O K \\
-O K \\
O N \\
O N \\
O N \\
O N \\
O K \\
\end{array}$ & $\begin{array}{l}\text { FIV }-* 56 \quad 0 \\
\text { NIT-*554 } \\
<.625 \% \mathrm{H}_{2} \\
\text { NAH- } * 55 \text { OFF } \\
\text { NAH-*54 ON } \\
\text { PBL-*58 ON } \\
\text { YAL-*58 ON } \\
\text { TB2-21/22 } \\
\text { CLOSED } \\
\end{array}$ & A & \\
\hline $\begin{array}{r}1.7 .4 .4 .7 \\
m .3 .3\end{array}$ & $\begin{array}{l}\text { PUSH RESET } \\
\text { SAMPLER PB- } \\
* 58\end{array}$ & OR & $\begin{array}{l}\text { PBL-*58 OFF } \\
\text { YAL-*58 OFF }\end{array}$ & $A$ & \\
\hline $\begin{array}{l}1.7 .4 .4 .8 \\
2 \text { gno.B. } \\
\end{array}$ & RESET ALARM & ok & $\mathrm{NAH}-* 54$ OFF & A & \\
\hline $\begin{array}{l}1.7 .4 .4 .9 \\
m x \cdot B \\
\end{array}$ & $\begin{array}{l}\text { OPEN SV }-* 19 \\
\text { ADJ. FIV-*56 }\end{array}$ & $\underline{O E}$ & $2 \pm .1 \mathrm{CFH}$ & A & \\
\hline
\end{tabular}

$0 / 1 / 2-17-96$ 
TEST DATA SHEET

\begin{tabular}{|c|c|c|c|c|c|}
\hline \multicolumn{3}{|c|}{ Date: $\quad 12-17-96$} & \multicolumn{3}{|c|}{ SHMS Unit Number: VIP-PNL-12OSE } \\
\hline \multicolumn{3}{|c|}{$\begin{array}{l}\text { Iitle of rest: } \\
\text { Preumatic Systems }\end{array}$} & \multirow{2}{*}{\multicolumn{3}{|c|}{$\begin{array}{l}\text { Test Equipment and S/N or cal. No.: } \\
\text { Floke V/o meter } \\
S / N 44620364 \\
\text { Cal. due } 10.97\end{array}$}} \\
\hline \multicolumn{3}{|c|}{$\begin{array}{c}\text { Test Performed By: See Test Personnal } \\
\text { Page } 98\end{array}$} & & & \\
\hline $\begin{array}{l}\text { Procedure No. } \\
\text { Initial/Date }\end{array}$ & I tem & Value & $\begin{array}{l}\text { Required } \\
\text { Condition }\end{array}$ & $(A / R)$ & comment \\
\hline$\frac{1.7 .4 .4 .10}{\dot{x}_{x} \cdot 3}$ & $\begin{array}{l}\text { VERIFY } \\
\text { CONDITIONS }\end{array}$ & $\begin{array}{l}O K \\
O K \\
O K \\
O K \\
O K \\
O K \\
O K \\
O K \\
O K \\
\end{array}$ & 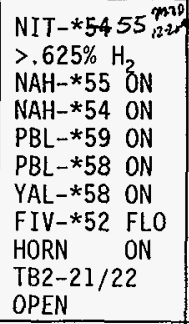 & $A$ & \\
\hline $\begin{array}{l}1.7 .4 .4 .11 \\
\text { mins }\end{array}$ & $\begin{array}{l}\text { ACKNOWLEDGE } \\
\text { HORN }\end{array}$ & $N / A$ & $N / A$ & A & \\
\hline$\frac{1.7 .4 .4 .12}{m_{2.8} .8}$ & $\begin{array}{l}\text { CLOSE SV-*19 } \\
\text { VERIFY } \\
\text { CONDITIONS }\end{array}$ & $\begin{array}{l}\text { OK } \\
\text { OK } \\
\text { OK } \\
\text { OK } \\
\text { OK } \\
\text { OK } \\
\text { OK } \\
\end{array}$ & 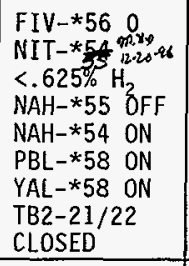 & $A$ & \\
\hline$\frac{1.7 .4 .4 .13}{m .2 .8}$ & $\begin{array}{l}\text { PUSH RESET } \\
\text { SAMPLER PB- } \\
* 58\end{array}$ & $\frac{o k}{\Delta K}$ & $\begin{array}{l}\text { PBL-*58 OFF } \\
\text { YAL-*58 OFF }\end{array}$ & A & \\
\hline $\begin{array}{l}1.7 .4 .4 .14 \\
\end{array}$ & RESET ALARM & $O K$ & $\mathrm{NAH}-* 54$ OFF & A & \\
\hline $\begin{array}{l}1.7 .4 .4 .15 \\
\text { m.x.s }\end{array}$ & $\begin{array}{l}\text { DISCONNECT } \\
\text { CAL. GAS }\end{array}$ & $N / A$ & $N / A$ & A & \\
\hline$\frac{1.7 .4 .4 .16}{m_{1.3 .5}}$ & $\begin{array}{l}\text { PUSH PB- } * 54 \\
\text { VERIFY } \\
\text { CONDITIONS }\end{array}$ & $\frac{O K}{O K}$ & $\begin{array}{l}X A-* 63 \text { ON } \\
\text { PBL-*54 ON } \\
\text { TB2-23/24 } \\
\text { OPEN } \\
\end{array}$ & A & \\
\hline$\frac{1.7 .4 .4 .17}{m n \beta^{2}}$ & $\begin{array}{l}\text { PUSH PB- } * 54 \\
\text { VERIFY } \\
\text { CONDITIONS }\end{array}$ & $\begin{array}{l}O K \\
O K \\
O K \\
\end{array}$ & $\begin{array}{l}\text { XA-*63 OFF } \\
\text { PBL-*54 OFF } \\
\text { TB2-23/24 } \\
\text { CLOSED }\end{array}$ & A & \\
\hline
\end{tabular}


APPENDIX $\mathrm{C}$

TEST DATA SHEET

\begin{tabular}{|c|c|c|c|c|c|}
\hline \multicolumn{3}{|c|}{ Date: $12-17-96$} & \multicolumn{3}{|c|}{ SHMS unit Number: VTP-PNL-1205E } \\
\hline \multicolumn{3}{|c|}{$\begin{array}{l}\text { Title of rest: } \\
\text { Pneumatic Systems }\end{array}$} & \multirow{2}{*}{\multicolumn{3}{|c|}{$\begin{array}{l}\text { Test Equipnent and } S / N \text { or Cal. No.: } \\
\qquad N / A\end{array}$}} \\
\hline \multicolumn{3}{|c|}{$\begin{aligned} \text { Test Performed By: See Test Personnol } & \text { Tage } 98 \\
& \text { Page }\end{aligned}$} & & & \\
\hline $\begin{array}{l}\text { Procedure No. } \\
\text { Initial/Date }\end{array}$ & Item & Value & $\begin{array}{l}\text { Required } \\
\text { Condition }\end{array}$ & $(A / R)$ & Comment \\
\hline \multicolumn{6}{|c|}{ Section 1.7.4.5 verifies the GC instrument tubing and calibration gas $100 p$. } \\
\hline$\frac{1.7 .4 .5 .1}{m .28}$ & $\begin{array}{l}\text { REMOVE PLUG } \\
\& \text { CONNECT } \\
\text { TEST GAS } \\
\end{array}$ & _k & $\begin{array}{l}\text { TEST GAS } \\
3-5 \text { PSIG }\end{array}$ & A & \\
\hline $\begin{array}{l}1.7 .4 .5 .2 \\
\min x \\
\end{array}$ & $\begin{array}{l}\text { VERIFY NO } \\
\text { GAS FLOW }\end{array}$ & $\frac{O K}{O K}$ & $\begin{array}{l}\mathrm{H}_{2} \text { GAS PORT } \\
\text { SAMPLE A IN }\end{array}$ & A & \\
\hline $\begin{array}{c}1.7 .4 .5 .3 \\
m x .8\end{array}$ & $\begin{array}{l}\text { VERIFY COL A } \\
\text { GAS FLOW }\end{array}$ & ok & COL A FLOW & A & \\
\hline $\begin{array}{l}1.7 .4 .5 .4 \\
\text { mans.6 }\end{array}$ & $\begin{array}{l}\text { REPLACE COL } \\
\text { A CAP }\end{array}$ & $N / A$ & $N / A$ & A & \\
\hline 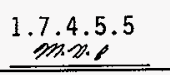 & $\begin{array}{l}\text { VERIFY COL B } \\
\text { GAS FLOW }\end{array}$ & $o K$ & COL B FLOW & A & \\
\hline $\begin{array}{l}1.7 .4 .5 .6 \\
\text { mas }\end{array}$ & $\begin{array}{l}\text { REPLACE COL } \\
\text { B CAP }\end{array}$ & N/A & N/A & A & \\
\hline $\begin{array}{r}1.7 .4 .5 .7 \\
\text { m.2.0.6 } \\
\end{array}$ & $\begin{array}{l}\text { VERIFY } \\
\text { SAMPLE VENT } \\
\text { FLOW }\end{array}$ & 0 & $\begin{array}{l}\text { SAMPLE VENT } \\
\text { FLOW }\end{array}$ & A & \\
\hline $\begin{array}{l}1.7 .4 .5 .8 \\
m .9 .8\end{array}$ & $\begin{array}{l}\text { REPLACE } \\
\text { SAMPLE VENT } \\
\text { CAP } \\
\end{array}$ & $N / A$ & N/A & A & \\
\hline $\begin{array}{c}1.7 .4 .5 .9 \\
\text { m.p.8. }\end{array}$ & $\begin{array}{l}\text { INSTALL } \\
\text { JUMPER } \\
\end{array}$ & $N / A$ & N/A & A & \\
\hline$\frac{1.7 .4 .5 .10}{m_{0.3 . \beta}}$ & $\begin{array}{l}\text { CLOSE FU-15 } \\
\text { VERIFY FLOW }\end{array}$ & $\frac{O K}{O K}$ & $\begin{array}{l}\mathrm{H}_{2} \text { CAL FLOW } \\
\text { SAMP A IN } \\
\text { FLOW }\end{array}$ & $A$ & \\
\hline $\begin{array}{l}1.7 .4 .5 .11 \\
m .20 .13 \\
\end{array}$ & $\begin{array}{l}\text { INSTALL PLUG } \\
\& \text { CAP }\end{array}$ & $\frac{O K}{O K}$ & $\begin{array}{l}\text { PLUG } \mathrm{H}_{2} \text { CAL } \\
\text { CAP SAMP A }\end{array}$ & $A$ & \\
\hline $\begin{array}{l}1.7 .4 .5 .12 \\
m .0 .8 \\
\end{array}$ & $\begin{array}{l}\text { OPEN SV }-* 63 \\
\& \text { READ } \\
\text { PDIT }-* 60\end{array}$ & $\frac{181^{4} \mathrm{HzO}}{\approx 605 t}$ & $\begin{array}{l}\text { PDIT-*60 = } \\
28 \times \text { PRESS } \\
1.7 .4 .5 .1 \\
\pm 20 \quad " \mathrm{H}_{2} \mathrm{O}\end{array}$ & A & \\
\hline $\begin{array}{c}1.7 .4 .5 .13 \\
m \times B .3 \\
\end{array}$ & $\begin{array}{l}\text { OPEN SV-*64 } \\
\text { VERIFY FLOW }\end{array}$ & $o k$ & $\begin{array}{l}\text { SAMP B IN } \\
\text { FLOW }\end{array}$ & A & \\
\hline
\end{tabular}


TEST DATA SHEET

\begin{tabular}{|c|c|c|c|c|c|}
\hline Date: $\quad 12-17-9$ & & & SHMS Unit Number & VTP & $1205 E$ \\
\hline $\begin{array}{l}\text { Iitle of rest: } \\
\text { Pneumatic } S\end{array}$ & tems & & Test Equipment a & $S / N$ or & \\
\hline Test Performed B & $\begin{array}{l}\text { See Test Pers } \\
\text { Page } 98\end{array}$ & & & & \\
\hline $\begin{array}{l}\text { Procedure No. } \\
\text { Initial/Date }\end{array}$ & Item & value & $\begin{array}{l}\text { Required } \\
\text { condition }\end{array}$ & $(A / R)$ & Corment \\
\hline $\begin{array}{c}1.7 .4 .5 .14 \\
m \text { MN. } \\
\end{array}$ & $\begin{array}{l}\text { REMOVE TEST } \\
\text { GAS }\end{array}$ & $N / A$ & N/A & $A$ & \\
\hline $\begin{array}{l}1.7 .4 .5 .15 \\
1 \text { m.s.e. }\end{array}$ & $\begin{array}{l}\text { CLOSE SV-*63 } \\
\& \text { SV }-* 64\end{array}$ & $N / A$ & $N / A$ & $A$ & \\
\hline $\begin{array}{l}1.7 .4 .5 .16 \\
m .2 .8\end{array}$ & $\begin{array}{l}\text { OPEN FU-15 } \\
\text { REMOVE } \\
\text { JUMPER } \\
\end{array}$ & $N / A$ & $N / A$ & $A$ & \\
\hline $\begin{array}{l}1.7 .4 .5 .17 \\
\text { m.s.s }\end{array}$ & $\begin{array}{l}\text { INSTALL } \\
\text { SAMP A UNION }\end{array}$ & $N / A$ & $\mathrm{~N} / \mathrm{A}$ & $A$ & \\
\hline $\begin{array}{l}1.7 .4 .5 .18 \\
m .2 \\
\end{array}$ & $\begin{array}{l}\text { OPEN SV-*60 } \\
\text { VERIFY FLOW }\end{array}$ & OK & $\begin{array}{l}\text { FI-*60 } \\
\text { NO FLOW }\end{array}$ & $A$ & \\
\hline$\frac{1.7 .4 .5 .19}{-m .8}$ & $\begin{array}{l}\text { POWER SOV- } \\
\text { *60, VERIFY } \\
\text { NO FLOW } \\
\end{array}$ & OK & $\begin{array}{l}\text { FI-*60 } \\
\text { NO FLOW }\end{array}$ & A & \\
\hline $\begin{array}{l}1.7 .4 .5 .20 \\
\text { m.p.8 } \\
\end{array}$ & $\begin{array}{l}\text { OPEN SV }-* 68 \\
\text { ADJ SV-*67 }\end{array}$ & OK & $\begin{array}{l}F I-* 60 \\
50 \mathrm{CCM}\end{array}$ & $A$ & \\
\hline $\begin{array}{c}1.7 .4 .5 .21 \\
m_{x .8}\end{array}$ & $\begin{array}{l}\text { DEENERGIZE } \\
\text { SOV } * 60\end{array}$ & $O K$ & $\begin{array}{l}\text { FI-*60 FLOW } \\
\text { DECREASES } \\
\end{array}$ & A & \\
\hline $\begin{array}{c}1.7 .4 .5 .22 \\
\min _{0.3} \\
\end{array}$ & $\begin{array}{l}\text { CLOSE SV-*60 } \\
\& \text { SV }-* 68\end{array}$ & $N / A$ & N/A & A & \\
\hline $\begin{array}{l}1.7 .4 .5 .23 \\
\end{array}$ & $\begin{array}{l}\text { REMOVE } \\
\text { SAMP A UNION }\end{array}$ & $N / A$ & N/A & A & \\
\hline $\begin{array}{l}1.7 .4 .5 .24 \\
\min _{1} \text { n }^{2} \\
\end{array}$ & $\begin{array}{l}\text { CONNECT TEST } \\
\text { GAS } \mathrm{N}_{2} \mathrm{O} \text { PORT } \\
\end{array}$ & OK & $\begin{array}{l}\text { TEST GAS } \\
3-5 \text { PSIG } \\
\end{array}$ & $A$ & \\
\hline$\frac{1.7 .4 .5 .25}{\text { m.2.8 }}$ & $\begin{array}{l}\text { VERIFY NO } \\
\text { FLOW }\end{array}$ & $\frac{O K}{O E}$ & $\begin{array}{l}\text { SAMP B IN \& } \\
\text { FLT-*63 } \\
\text { NO FLOW }\end{array}$ & A & \\
\hline $\begin{array}{c}1.7 .4 .5 .26 \\
\end{array}$ & $\begin{array}{l}\text { INSTALL } \\
\text { JUMPER }\end{array}$ & $\mathrm{N} / \mathrm{A}$ & N/A & $A$ & \\
\hline $\begin{array}{l}1.7 .4 .5 .27 \\
m \text { m..3 }\end{array}$ & $\begin{array}{l}\text { CLOSE FU-15 } \\
\text { VERIFY FLOW }\end{array}$ & - ok & $\begin{array}{l}\text { SAMP B IN } \\
\text { FLT-* } 63 \\
\text { FLOW }\end{array}$ & $A$ & \\
\hline
\end{tabular}


TEST DATA SHEET

\begin{tabular}{|c|c|c|c|c|c|}
\hline \multicolumn{3}{|c|}{ Date: $12-17-96$} & \multicolumn{3}{|c|}{ SHMs Unit Number: VTP - PNL-12OSE } \\
\hline \multicolumn{3}{|c|}{$\begin{array}{l}\text { ritle of rest: } \\
\text { Pneumatic Systems }\end{array}$} & \multirow{2}{*}{\multicolumn{3}{|c|}{$\begin{array}{l}\text { Test Equipment and } S / \mathrm{N} \text { or Cal. No.: } \\
N / A\end{array}$}} \\
\hline \multicolumn{3}{|c|}{$\begin{aligned} \text { Test Performed sy: } & \text { see Test Personnal } \\
& \text { Page } 9 e\end{aligned}$} & & & \\
\hline $\begin{array}{l}\text { Procedure No. } \\
\text { Initial/Date }\end{array}$ & I tem & Value & $\begin{array}{l}\text { Required } \\
\text { Condition }\end{array}$ & $(A / R)$ & Conment \\
\hline $\begin{array}{l}1.7 .4 .5 .28 \\
\max _{8}\end{array}$ & $\begin{array}{l}\text { DISCONNECT } \\
\text { TEST GAS }\end{array}$ & N/A & $N / A$ & A & \\
\hline $\begin{array}{l}1.7 .4 .5 .29 \\
\end{array}$ & OPEN FU-15 & $N / A$ & $N / A$ & A & \\
\hline $\begin{array}{l}1.7 .4 .5 .30 \\
=m 2.8 \\
\end{array}$ & $\begin{array}{l}\text { REMOVE } \\
\text { JUMPER }\end{array}$ & $N / A$ & $N / A$ & $A$ & \\
\hline $\begin{array}{l}1.7 .4 .5 .31 \\
\min x\end{array}$ & $\begin{array}{l}\text { INSTALL } \\
\text { SAMP B UNION }\end{array}$ & N/A & $N / A$ & A & \\
\hline $\begin{array}{l}1.7 .4 .5 .32 \\
1862.8 \\
\end{array}$ & $\begin{array}{l}\text { OPEN SV-*60 } \\
\text { VERIFY FLOW }\end{array}$ & ok & $\begin{array}{l}\text { FI-*60 } \\
\text { NO FLOW }\end{array}$ & $A$ & \\
\hline 1.7 .4 .5 .33 & $\begin{array}{l}\text { POWER SOV- } \\
* 60, \text { VERIFY } \\
\text { NO FLOW }\end{array}$ & ok & $\begin{array}{l}\text { FI-*60 } \\
\text { NO FLOW }\end{array}$ & $A$ & \\
\hline $\begin{array}{l}1.7 .4 .5 .34 \\
12.2 .8 \\
\end{array}$ & $\begin{array}{l}\text { OPEN SV-*68 } \\
\text { \& ADJ SV-*67 }\end{array}$ & $\Delta K$ & $\begin{array}{r}F I-* 60 \\
50 \mathrm{CCM} \\
\end{array}$ & $A$ & \\
\hline $\begin{array}{c}1.7 .4 .5 .35 \\
3.2 .3\end{array}$ & $\begin{array}{l}\text { DEENERGIZE } \\
\text { SOV-*60 }\end{array}$ & ot & $\begin{array}{l}\text { FI }-* 60 \text { FLOW } \\
\text { DECREASES }\end{array}$ & $A$ & \\
\hline $\begin{array}{l}1.7 .4 .5 .36 \\
\min _{2}{ }^{\circ} \\
\end{array}$ & $\begin{array}{l}\text { CLOSE SV-*60 } \\
\& \text { SV-*68 }\end{array}$ & $N / A$ & $N / A$ & A & \\
\hline $\begin{array}{l}1.7 .4 .5 .37 \\
\operatorname{mos} 2 . \\
\end{array}$ & $\begin{array}{l}\text { REMOVE } \\
\text { SAMP B UNION }\end{array}$ & $N / A$ & $\mathrm{~N} / \mathrm{A}$ & A & \\
\hline $\begin{array}{l}1.7 .4 .5 .38 \\
\text { m.n. } \\
\end{array}$ & $\begin{array}{l}\text { OPEN CB-3/4 } \\
\text { ACK. ALARMS } \\
\end{array}$ & $N / A$ & $N / A$ & $A$ & \\
\hline \multicolumn{6}{|c|}{$\begin{array}{l}\text { Section } 1.7 .4 .6 \text { verifies the multi gas analyzer instrument tubing and } \\
\text { calibration gas loop. }\end{array}$} \\
\hline $\begin{array}{c}1.7 .4 .6 .1 \\
\text { mns-s }\end{array}$ & $\begin{array}{l}\text { VERIFY } \\
\text { NIT } * 52 \\
\text { SAMPLE LINES }\end{array}$ & N/A & N/A & $A$ & \\
\hline $\begin{array}{l}1.7 .4 .6 .2 \\
\text { monses }\end{array}$ & $\begin{array}{l}\text { CONNECT TEST } \\
\text { GAS } \mathrm{NH}_{3} \text { PORT }\end{array}$ & ok & $\begin{array}{l}\text { TEST GAS } \\
3-5 \text { PSIG } \\
\end{array}$ & $A$ & \\
\hline $\begin{array}{l}1.7 .4 .6 .3 \\
\text { m.m.s. } \\
\end{array}$ & $\begin{array}{l}\text { VERIFY NO } \\
\text { FLOW }\end{array}$ & $O K$ & $\begin{array}{l}\text { SAMPLE IN } \\
\text { FLT-*63 }\end{array}$ & $A$ & \\
\hline
\end{tabular}


TEST DATA SHEET

\begin{tabular}{|c|c|c|c|c|c|}
\hline \multicolumn{3}{|c|}{ Date: $\quad 12-17-96$} & \multicolumn{3}{|c|}{ SHMS Unit Number: VTP-PNL-1205E } \\
\hline \multicolumn{3}{|c|}{$\begin{array}{l}\text { Title of rest: } \\
\text { Pneumatic Systems }\end{array}$} & \multirow{2}{*}{\multicolumn{3}{|c|}{$\begin{array}{l}\text { Test Equipment and } s / N \text { or Cal. No.: } \\
N / A\end{array}$}} \\
\hline \multicolumn{3}{|c|}{$\begin{aligned} \text { Test Performed By: See Test Personnal } \\
\text { Page ge }\end{aligned}$} & & & \\
\hline $\begin{array}{c}\text { Procedure No. } \\
\text { Initial/Date }\end{array}$ & Item & Value & $\begin{array}{l}\text { Required } \\
\text { Condition } \\
\end{array}$ & $(A / R)$ & comment \\
\hline $\begin{array}{l}1.7 .4 .6 .4 \\
m .0 .8\end{array}$ & $\begin{array}{l}\text { INSTALL } \\
\text { JUMPER }\end{array}$ & $N / A$ & $N / A$ & A & \\
\hline $\begin{array}{l}1.7 .4 .6 .5 \\
m .3 .8 \\
\end{array}$ & $\begin{array}{l}\text { CLOSE FU-15 } \\
\text { VERIFY GAS } \\
\text { FLOWS }\end{array}$ & $\begin{array}{c}\text { OK } \\
\text { OK } \\
\text { OK } \\
\end{array}$ & $\begin{array}{l}\text { SAMPLE IN } \\
\text { FLT }-* 63 \\
\text { FIV-*70 }\end{array}$ & $A$ & \\
\hline $\begin{array}{r}1.7 .4 .6 .6 \\
2 x x .3 \\
\end{array}$ & $\begin{array}{l}\text { PLUG FLT }-* 63 \\
\& \text { MON-*60 } \\
\text { SAMPLE VENT }\end{array}$ & $N / A$ & $N / A$ & $A$ & \\
\hline $\begin{array}{l}1.7 .4 .6 .7 \\
m .2 .6\end{array}$ & $\begin{array}{l}\text { OPEN SV-*66, } \\
\text { SV-*70 \& CAP } \\
\text { SAMPLE IN }\end{array}$ & $\begin{aligned} & 143.0^{4} \mathrm{H}_{2} \mathrm{O} \\
= & 4.6 \mathrm{PSI}\end{aligned}$ & $\begin{array}{l}\text { PDIT }-* 60= \\
28 \times \text { PRESS } \\
1.7 .4 .6 .2 \\
\pm 20{ }^{\prime \prime} \mathrm{H}_{2} \mathrm{O}\end{array}$ & A & \\
\hline $\begin{array}{l}1.7 .4 .6 .8 \\
m 2.8 .\end{array}$ & $\begin{array}{l}\text { CLOSE SV-*66 } \\
\text { SV-*70 \& } \\
\text { REMOVE CAP }\end{array}$ & $N / A$ & $N / A$ & A & \\
\hline $\begin{array}{c}1.7 .4 .6 .9 \\
\text { m.2.s. } \\
\end{array}$ & $\begin{array}{l}\text { REMOVE FLT- } \\
* 63 \text { PLUG }\end{array}$ & $N / A$ & $N / A$ & $A$ & \\
\hline $\begin{array}{r}1.7 .4 .6 .10 \\
m .2 .3 \\
\end{array}$ & $\begin{array}{l}\text { REMOVE TEST } \\
\text { GAS }\end{array}$ & $N / A$ & $N / A$ & $A$ & \\
\hline $\begin{array}{l}1.7 .4 .6 .11 \\
m .3 . \beta \\
\end{array}$ & $\begin{array}{l}\text { CLOSE SV-*05 } \\
\& \text { SV }-* 16\end{array}$ & $N / A$ & $N / A$ & A & \\
\hline $\begin{array}{c}1.7 .4 \cdot 6 \cdot 12 \\
m \cdot 2 \cdot B^{2} \\
\end{array}$ & $\begin{array}{l}\text { CONNECT TEST } \\
\text { GAS }\end{array}$ & ok & $\begin{array}{l}\text { TEST GAS } \\
2-4 \text { PSIG } \\
\end{array}$ & A & \\
\hline$\frac{1.7 .4 .6 .13}{m .2 .3}$ & $\begin{array}{l}\text { OPEN SV-*07 } \\
\& \text { VERIFY } \\
\text { NO FLOW }\end{array}$ & - ok & $\begin{array}{l}\text { SAMPLE IN } \\
\text { SAMPLE OUT }\end{array}$ & A & \\
\hline $\begin{array}{l}1.7 .4 .6 .14 \\
m .24 .8 \\
\end{array}$ & $\begin{array}{l}\text { OPEN SV }-* 09 \\
\& \text { VERIFY } \\
\text { NO FLOW }\end{array}$ & - ok & $\begin{array}{l}\text { SAMPLE IN } \\
\text { SAMPLE OUT }\end{array}$ & $A$ & \\
\hline $\begin{array}{c}1.7 .4 .6 .15 \\
m . x .3 \\
\end{array}$ & $\begin{array}{l}\text { OPEN FU-15 \& } \\
\text { VERIFY FLOW }\end{array}$ & $\frac{O K}{O K}$ & $\begin{array}{l}\text { SAMPLE FLOW } \\
\text { IN - YES } \\
\text { OUT - NO } \\
\end{array}$ & A & \\
\hline $\begin{array}{l}1.7 .4 .6 .16 \\
m, p . B \\
\end{array}$ & $\begin{array}{l}\text { CLOSE SV-*09 } \\
\text { VERIFY FLOW }\end{array}$ & ok & $\begin{array}{l}\text { SAMPLE IN } \\
\text { NO FLOW }\end{array}$ & A & \\
\hline
\end{tabular}


TEST DATA SHEET

\begin{tabular}{|c|c|c|c|c|c|}
\hline \multicolumn{3}{|c|}{ Date: $\quad 12-17-96$} & \multicolumn{3}{|c|}{ SHMS Unit Number: VTP. PNL-1205E } \\
\hline \multicolumn{3}{|c|}{$\begin{array}{l}\text { Iirle of Test: } \\
\text { Pneumatic Systems }\end{array}$} & \multirow{2}{*}{\multicolumn{3}{|c|}{$\begin{array}{l}\text { Test Equipment and } S / N \text { or cal. No.: } \\
\qquad N / A\end{array}$}} \\
\hline \multicolumn{3}{|c|}{$\begin{array}{c}\text { rest Performed By: see Test Personnal } \\
\text { Poge } 98\end{array}$} & & & \\
\hline $\begin{array}{l}\text { Procedure No. } \\
\text { Initjal/Date }\end{array}$ & Item & value & $\begin{array}{l}\begin{array}{l}\text { Required } \\
\text { Condition }\end{array} \\
\end{array}$ & $(A / R)$ & Comment \\
\hline $\begin{array}{l}1.7 .4 .6 .17 \\
m 28.17\end{array}$ & $\begin{array}{l}\text { OPEN SV-*10 } \\
\text { VERIFY FLOW }\end{array}$ & - ok & $\begin{array}{l}\text { SAMPLE FLOW } \\
\text { IN - NO } \\
\text { OUT - YES } \\
\end{array}$ & A & \\
\hline $\begin{array}{l}1.7 .4 .6 .18 \\
m .8\end{array}$ & $\begin{array}{l}\text { REMOVE TEST } \\
\text { GAS \& PLUG } \\
\text { SV-*07 }\end{array}$ & N/A & N/A & A & \\
\hline $\begin{array}{l}1.7 .4 .6 .19 \\
\min ^{2}\end{array}$ & $\begin{array}{l}\text { CLOSE SV-*07 } \\
\& \text { SV }-* 10\end{array}$ & N/A & $N / A$ & A & \\
\hline $\begin{array}{l}1.7 .4 .6 .20 \\
m, 3.8 \\
\end{array}$ & $\begin{array}{l}\text { OPEN SV-*05 } \\
\& \text { SV-*16 }\end{array}$ & $N / A$ & $N / A$ & A & \\
\hline $\begin{array}{c}1.7 .4 .6 .21 \\
\text { on } x_{1 . B}\end{array}$ & $\begin{array}{l}\text { REMOVE } \\
\text { JUMPER }\end{array}$ & $N / A$ & $N / A$ & A & \\
\hline \multicolumn{6}{|c|}{ Section 1.7 .4 .7 verifies the carrier gas supply lines. } \\
\hline $\begin{array}{l}1.7 .4 .7 .1 \\
\text { m.2.0 }\end{array}$ & $\begin{array}{l}\text { CONNECT TEST } \\
\text { GAS }\end{array}$ & ok & 3-5 PSIG & A & \\
\hline $\begin{array}{l}1.7 .4 .7 .2 \\
-m .2 . B^{2} \\
\end{array}$ & $\begin{array}{l}\text { REMOVE PLUG } \\
\& \text { VERIFY } \\
\text { FLOW } \\
\end{array}$ & ok & $\begin{array}{l}\mathrm{N}_{3} \text { CARRIER } \\
\text { GAS FLOWS }\end{array}$ & A & \\
\hline $\begin{array}{c}1.7 .4 .7 .3 \\
m x_{B}\end{array}$ & $\begin{array}{l}\text { DISCONNECT } \\
\text { TEST GAS }\end{array}$ & $N / A$ & N/A & A & \\
\hline $\begin{array}{c}1.7 .4 .7 .4 \\
m x .6 \\
\end{array}$ & $\begin{array}{l}\text { CONNECT TEST } \\
\text { GAS }\end{array}$ & ok & $3-5$ PSIG & A & \\
\hline $\begin{array}{c}1.7 .4 .7 .5 \\
\end{array}$ & $\begin{array}{l}\text { REMOVE PLUG } \\
\& \text { VERIFY } \\
\text { FLOW }\end{array}$ & OK & $\begin{array}{l}\text { He CARRIER } \\
\text { GAS FLOWS }\end{array}$ & $A$ & \\
\hline $\begin{array}{c}1.7 .4 .7 .6 \\
\text { ins.i. }\end{array}$ & $\begin{array}{l}\text { DISCONNECT } \\
\text { TEST GAS }\end{array}$ & N/A & N/A & A & \\
\hline $\begin{array}{l}1.7 .5 \\
\text { mas } \\
\end{array}$ & $\begin{array}{l}\text { TEST SEC. } \\
1.7 .4 \text { DONE }\end{array}$ & $\frac{1}{\text { Test Dir }}$ & wid & & $\frac{12-17-96}{\text { Date }}$ \\
\hline
\end{tabular}


APPENDIX C

TEST DATA SHEET

\begin{tabular}{|c|c|c|c|c|c|}
\hline \multicolumn{3}{|c|}{ Date: $12-18-96$} & \multicolumn{3}{|c|}{ SHMS Unit Nunber: VTP.PNL-1205E } \\
\hline \multicolumn{3}{|c|}{$\begin{array}{l}\text { Title of rest: } \\
\text { Pneumatic Systems }\end{array}$} & \multirow{2}{*}{\multicolumn{3}{|c|}{ 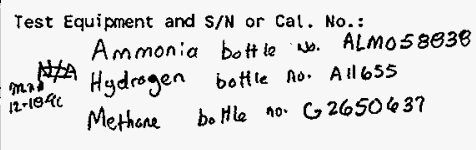 }} \\
\hline \multicolumn{3}{|c|}{$\begin{aligned} & \text { Test Performed By: } \text { see Test Personnd } \\
& \text { Page } 98\end{aligned}$} & & & \\
\hline $\begin{array}{l}\text { Procedure No. } \\
\text { Initial/Date }\end{array}$ & Item & value & $\begin{array}{l}\text { Required } \\
\text { Condition }\end{array}$ & $(A / R)$ & coment \\
\hline $\begin{array}{l}1.7 .6 \\
m .2 . \beta\end{array}$ & \multicolumn{5}{|c|}{$\begin{array}{l}\text { THIS SECTION IS ONLY FOR THE SHMS-E IN THE ANALYTICAL MODE. } \\
\text { IF NOT APPLICABLE, N/A STEPS AND TEST DIRECTOR WILL SIGN. }\end{array}$} \\
\hline & \multicolumn{3}{|c|}{ Test Director Signature } & \multicolumn{2}{|l|}{ Date } \\
\hline $\begin{array}{c}1.7 .6 .1 .1 \\
\operatorname{man} 3\end{array}$ & $\begin{array}{l}\text { VERIFY BKR } \\
\text { LINE UP }\end{array}$ & N/A & $N / A$ & A & \\
\hline $\begin{array}{l}1.7 .6 .1 .2 \\
m .0 .0\end{array}$ & $\begin{array}{l}\text { VERIFY VALVE } \\
\text { LINE UP }\end{array}$ & N/A & N/A & A & \\
\hline $\begin{array}{l}1.7 .6 .1 .3 \\
m_{0.13}\end{array}$ & $\begin{array}{l}\text { VERIFY MON- } \\
* 60 \text { INSTALL }\end{array}$ & N/A & N/A & A & \\
\hline $\begin{array}{l}1.7 .6 .1 .4 \\
m_{2.8 .5} \\
\end{array}$ & $\begin{array}{l}\text { VERIFY NIT- } \\
* 52 \text { INSTALL } \\
\end{array}$ & $N / A$ & $N / A$ & A & \\
\hline $\begin{array}{l}1.7 .6 .1 .5 \\
m \times 0^{2} \\
\end{array}$ & $\begin{array}{l}\text { CONNECT } \mathrm{H}_{2} \\
\text { CAL GAS }\end{array}$ & $N / A$ & N/A & A & \\
\hline $\begin{array}{l}1.7 .6 .1 .6 \\
\text { moss } \\
\end{array}$ & $\begin{array}{l}\text { CONNECT } \mathrm{CH}_{4} \\
\text { CAL GAS }\end{array}$ & $\mathrm{N} / \mathrm{A}$ & $N / A$ & A & \\
\hline $\begin{array}{l}1.7 .6 .1 .7 \\
0 \text { m.8. }\end{array}$ & $\begin{array}{l}\text { CONNECT } \mathrm{NH}_{3} \\
\text { CAL GAS }\end{array}$ & $N / A$ & N/A & A & \\
\hline \multicolumn{6}{|c|}{ Section 1.7.6.2 establishes the main and auxiliary loop flows. } \\
\hline $\begin{array}{c}1.7 .6 .2 .1 \\
m p B \\
\end{array}$ & CLOSE FU-11 & $O K$ & $\begin{array}{l}\text { MON }-* 60 \\
\text { POWERED } \\
\end{array}$ & $A$ & \\
\hline $\begin{array}{l}1.7 .6 .2 .2 \\
\text { m.x.s }\end{array}$ & POWER PC $-* 60$ & $O K$ & $\begin{array}{l}\text { ESTABLISH } \\
\text { SAMPLING } \\
\text { ROUTINE } \\
\end{array}$ & A & \\
\hline $\begin{array}{l}1.7 .6 .2 .3 \\
\text { m.x.x.B }\end{array}$ & CLOSE FU-12 & OK & $\begin{array}{l}\text { NIT-*52 } \\
\text { POWERED } \\
\end{array}$ & A & \\
\hline $\begin{array}{l}1.7 .6 .2 .4 \\
m \times 1\end{array}$ & POWER PC $-* 70$ & ok & $\begin{array}{l}\text { PC }-* 70 \\
\text { BOOTED UP } \\
\end{array}$ & $A$ & \\
\hline $\begin{array}{c}1.7 .6 .2 .5 \\
\text { mas }\end{array}$ & $\begin{array}{l}\text { CLOSE CB-3/4 } \\
\text { ADJUST FLOW }\end{array}$ & ok & $\begin{array}{l}\text { FIT-*57 } \\
1 \pm .1 \quad \mathrm{H}_{2} \mathrm{O}\end{array}$ & $A$ & \\
\hline
\end{tabular}


APPENDIX C

TEST DATA SHEET

\begin{tabular}{|c|c|c|c|c|c|}
\hline \multicolumn{3}{|c|}{ Date: $\quad 12 \cdot 18 \cdot 96$} & \multicolumn{3}{|c|}{ SHMS Unit Number: VTP-PNL-1205E } \\
\hline \multicolumn{3}{|c|}{$\begin{array}{l}\text { Title of Test: } \\
\text { Pneumatic Systems }\end{array}$} & \multirow{2}{*}{\multicolumn{3}{|c|}{ 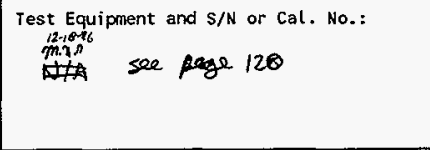 }} \\
\hline \multicolumn{3}{|c|}{$\begin{aligned} \text { rest Performed By: } & \text { see Test Personnal } \\
& \text { Page } 98\end{aligned}$} & & & \\
\hline $\begin{array}{c}\text { Procedure No. } \\
\text { Initial/oate }\end{array}$ & Item & Value & $\begin{array}{l}\text { Required } \\
\text { Condition }\end{array}$ & $(A / R)$ & Corment \\
\hline $\begin{array}{l}1.7 .6 .2 .6 \\
m_{x}, 3\end{array}$ & $\begin{array}{l}\text { OPEN SV-*09 } \\
\& \text { SV }-* 10\end{array}$ & $N / A$ & N/A & A & \\
\hline $\begin{array}{c}1.7 .6 .2 .7 \\
m . p B\end{array}$ & $\begin{array}{l}\text { OPEN SV-*60, } \\
63,65 \& 68\end{array}$ & $N / A$ & $N / A$ & A & \\
\hline $\begin{array}{l}1.7 .6 .2 .8 \\
m .2 . B\end{array}$ & NIT-*52 & ok & $\begin{array}{l}\text { ESTABLISH } \\
\text { SAMPLING } \\
\text { ROUTINE } \\
\end{array}$ & A & \\
\hline $\begin{array}{l}1.7 .6 .2 .9 \\
m{ }_{2 . B} \\
\end{array}$ & $\begin{array}{l}\text { OPEN SV }-* 24 \\
\text { ADJ FLOW }\end{array}$ & $\frac{\mathrm{OK}}{2.96^{\prime \prime} \mathrm{H}_{2} \mathrm{O}}$ & $\begin{array}{l}\mathrm{FIT}-* 57 \\
3 \pm .1 \quad \mathrm{HH}_{2} \mathrm{O}\end{array}$ & A & \\
\hline $\begin{array}{l}1.7 .6 .2 .10 \\
\text { MM.v.B. }\end{array}$ & $\begin{array}{l}\text { ADJ FLOW } \\
\text { SV-*67 }\end{array}$ & $20 \mathrm{~cm}$ & $\begin{array}{l}F I-* 60 \\
10-30 \mathrm{ccm}\end{array}$ & A & \\
\hline $\begin{array}{l}1.7 .6 .2 .11 \\
\text { om. } 2.3 . \\
\end{array}$ & $\begin{array}{l}\text { RECORD } \\
\text { PDIT-*60 } \\
\end{array}$ & $165^{\circ \prime} \mathrm{H}_{2} \mathrm{O}$ & $<175 \quad \mathrm{H}_{2} \mathrm{O}$ & A & \\
\hline $\begin{array}{l}1.7 .6 .2 .12 \\
m, 2,0\end{array}$ & $\begin{array}{l}\text { OPEN SV }-* 64 \\
\text { CLOSE SV-*63 }\end{array}$ & $N / A$ & N/A & $A$ & \\
\hline $\begin{array}{l}1.7 .6 .2 .13 \\
m .7 .8 \\
\end{array}$ & $\begin{array}{l}\text { RECORD } \\
\text { PDIT }-* 60 \\
\end{array}$ & $158.8^{\prime \prime} \mathrm{H}_{20}$ & $<175 \quad " \mathrm{H}_{2} \mathrm{O}$ & $A$ & \\
\hline$\frac{1.7 .6 .2 .14}{m_{m .2}}$ & $\begin{array}{l}\text { CLOSE SV-*65 } \\
\text { OPEN SV }-* 66\end{array}$ & $N / A$ & $N / A$ & A & \\
\hline $\begin{array}{l}1.7 .6 .2 .15 \\
\text { M.N.B } \\
\end{array}$ & $\begin{array}{l}\text { RECORD B IN } \\
\text { PDIT- } * 60 \\
\end{array}$ & $-23.6 " 1 / 20$ & $\begin{array}{l}-15 \text { to }-50 \\
" \mathrm{H}_{2} \mathrm{O}\end{array}$ & $A$ & \\
\hline $\begin{array}{c}1.7 .6 .2 .16 \\
\text { M.X.B } \\
\end{array}$ & $\begin{array}{l}\text { OPEN SV }-* 63 \\
\text { CLOSE SV }-* 64\end{array}$ & $N / A$ & $N / A$ & A & \\
\hline $\begin{array}{c}1.7 .6 .2 .17 \\
m . x \cdot B \\
\end{array}$ & $\begin{array}{l}\text { RECORD A IN } \\
\text { PDIT }-* 60\end{array}$ & -23.58 & $\begin{array}{l}-15 \text { to }-50 \\
" \mathrm{H}_{2} \mathrm{O}\end{array}$ & A & \\
\hline $\begin{array}{c}1.7 .6 .2 .18 \\
\text { gnx.x } \\
\end{array}$ & $\begin{array}{l}\text { INSTALL } \\
\text { JUMPER } \\
\end{array}$ & $N / A$ & $N / A$ & A & \\
\hline $\begin{array}{l}1.7 .6 .2 .19 \\
m . \not 1 B \\
\end{array}$ & CLOSE FU- 15 & $N / A$ & $N / A$ & A & \\
\hline$\frac{1.7 .6 .2 .20}{m \cdot x \cdot 8}$ & $\begin{array}{l}\text { ADJ SV }-* 61 \& \\
\text { RECORD } \\
\text { PDIT } * * 60\end{array}$ & $=19.72{ }^{4} \mathrm{H}_{6} \mathrm{O}$ & $\begin{array}{l} \pm 5^{\prime \prime} \mathrm{H}_{2} 0 \mathrm{OF} \\
1.7 .6 .2 .17\end{array}$ & $A$ & \\
\hline
\end{tabular}


TEST DATA SHEET

\begin{tabular}{|c|c|c|c|c|c|}
\hline \multicolumn{3}{|c|}{ Date: $12-10-96$} & \multicolumn{3}{|c|}{ SHMS Unit Number: VTP-PNL-1205E } \\
\hline \multicolumn{3}{|c|}{$\begin{array}{l}\text { Title of Test: } \\
\text { Pneumatic Systems }\end{array}$} & \multirow{2}{*}{\multicolumn{3}{|c|}{$\begin{array}{l}\text { Test Equipment and } s / N \text { or cal. No.: } \\
\text { See page } 120\end{array}$}} \\
\hline \multicolumn{3}{|c|}{$\begin{aligned} & \text { Test Performed By: } \text { see Test Personnal } \\
& \text { Page } 99 .\end{aligned}$} & & & \\
\hline $\begin{array}{l}\text { Procedure No. } \\
\text { Initial/Date }\end{array}$ & Item & Value & $\begin{array}{l}\text { Required } \\
\text { Condition }\end{array}$ & $(A / R)$ & Corment \\
\hline$\frac{1.7 .6 .2 .21}{m .2 .0}$ & $\begin{array}{l}\text { CAL MON-*60 } \\
\text { CHANNEL A }\end{array}$ & $\begin{array}{l}10455.9 \mathrm{PPM} \\
10486.9 \mathrm{PPM} \\
10467.5 \mathrm{PPM}\end{array}$ & $\begin{array}{l}\text { READINGS } \\
\text { WITHIN } \pm 5 \%\end{array}$ & $A$ & Hydragen \\
\hline $\begin{array}{c}1.7 .6 .2 .22 \\
m . \mu .1 \\
\end{array}$ & OPEN FU-15 & $\mathrm{N} / \mathrm{A}$ & $N / A$ & A & \\
\hline $\begin{array}{l}1.7 .6 .2 .23 \\
\operatorname{mix.5} \\
\end{array}$ & $\begin{array}{l}\text { REMOVE } \\
\text { JUMPER }\end{array}$ & $N / A$ & N/A & A & \\
\hline $\begin{array}{l}1.7 .6 .2 .24 \\
92.8 \\
\end{array}$ & $\begin{array}{l}\text { OPEN SV }-* 64 \\
\text { CLOSE SV }-* 63\end{array}$ & N/A & N/A & $A$ & \\
\hline $\begin{array}{l}1.7 .6 .2 .25 \\
\mathrm{m.2.8}\end{array}$ & $\begin{array}{l}\text { INSTALL } \\
\text { JUMPER } \\
\end{array}$ & $N / A$ & $N / A$ & $A$ & \\
\hline $\begin{array}{l}1.7 .6 .2 .26 \\
\text { m.b.s. }\end{array}$ & CLOSE FU-15 & $N / A$ & $N / A$ & A & \\
\hline $\begin{array}{c}1.7 .6 .2 .27 \\
m . n .8 \\
\end{array}$ & $\begin{array}{l}\text { ADJ SV-*62 \& } \\
\text { RECORD } \\
\text { PDIT-*60 }\end{array}$ & $-19.9^{\circ} \mathrm{H} 2 \mathrm{O}$ & $\begin{array}{l} \pm 5 " \mathrm{H}_{2} \mathrm{OOF} \\
1.7 .6 .2 .15\end{array}$ & A & \\
\hline $\begin{array}{l}1.7 .6 .2 .28 \\
m . x . B \\
\end{array}$ & $\begin{array}{l}\text { CAL MON-*60 } \\
\text { CHANNEL B }\end{array}$ & $\begin{array}{l}9979.7 \mathrm{PPM} \\
9987.5 \mathrm{PPM} \\
9990.6 \mathrm{PPM}\end{array}$ & $\begin{array}{l}\text { READINGS } \\
\text { WITHIN } \pm 5 \%\end{array}$ & $A$ & Me thame \\
\hline $\begin{array}{l}1.7 .6 .2 .29 \\
M .21 .3 \\
\end{array}$ & OPEN FU-15 & $N / A$ & $N / A$ & $A$ & \\
\hline $\begin{array}{l}1.7 .6 .2 .30 \\
m .21 B^{3} \\
\end{array}$ & $\begin{array}{l}\text { REMOVE } \\
\text { JUMPER }\end{array}$ & $N / A$ & N/A & $A$ & \\
\hline $\begin{array}{l}1.7 .6 .2 .31 \\
m_{2.0}\end{array}$ & $\begin{array}{l}\text { CLOSE SV-*64 } \\
\text { OPEN SV-*70 }\end{array}$ & $N / A$ & N/A & A & \\
\hline $\begin{array}{l}1.7 .6 .2 .32 \\
m . m_{0.3} \\
\end{array}$ & $\begin{array}{l}\text { RECORD } \\
\text { PDIT-*60 } \\
\text { NIT-*52 IN } \\
\end{array}$ & $-10.0^{4} \mathrm{H}_{2} \mathrm{O}$ & $\begin{array}{l}0 \text { to }-30 \\
" \mathrm{H}_{3} \mathrm{O}\end{array}$ & $A$ & \\
\hline $\begin{array}{l}1.7 .6 .2 .33 \\
\text { inxo.s. }\end{array}$ & $\begin{array}{l}\text { INSTALL } \\
\text { JUMPER }\end{array}$ & $N / A$ & $N / A$ & $A$ & \\
\hline 1.7 .6 .2 .34 & CLOSE FU-15 & $N / A$ & N/A & $A$ & \\
\hline
\end{tabular}




\section{TEST DATA SHEET}

\begin{tabular}{|c|c|c|c|c|c|}
\hline \multicolumn{3}{|c|}{ Date: $12-18-96$} & \multicolumn{3}{|c|}{ SHMs Unit Number: VTP. PNL $-1205 \mathrm{~A}$} \\
\hline \multicolumn{3}{|c|}{$\begin{array}{l}\text { Title of Test: } \\
\text { Pneumatic Systems }\end{array}$} & \multirow{2}{*}{\multicolumn{3}{|c|}{$\begin{array}{l}\text { Test Equipment and } S / N \text { or Cal. No.: } \\
n x y \\
2.10 .96\end{array}$}} \\
\hline \multicolumn{3}{|c|}{$\begin{aligned} & \text { Test Performed By: } \text { see Test Personnal } \\
& \text { Page } 98\end{aligned}$} & & & \\
\hline $\begin{array}{l}\text { Procedure No. } \\
\text { Initial/Date }\end{array}$ & I tem & value & $\begin{array}{l}\text { Required } \\
\text { Condition }\end{array}$ & $(A / R)$ & Corment \\
\hline $\begin{array}{l}1.7 .6 .2 .35 \\
\end{array}$ & $\begin{array}{l}\text { ESTABLISH } \\
\text { BYPASS FLOW }\end{array}$ & $1.7 \mathrm{chh}$ & $\begin{array}{l}F I V-* 70 \\
1.5 \pm .2 \mathrm{cfh}\end{array}$ & A & \\
\hline $\begin{array}{l}1.7 .6 .2 .36 \\
2 n x-13\end{array}$ & $\begin{array}{l}\text { ADJ SV-*71 } \\
\text { NIT }-* 52 \\
\text { SAMPLE IN }\end{array}$ & $-12.3^{\circ} \mathrm{H}_{2} \mathrm{O}$ & $\begin{array}{l} \pm 5 \quad \mathrm{H}_{2} \mathrm{O} \text { OF } \\
1.7 .6 .2 .32\end{array}$ & A & \\
\hline $\begin{array}{l}1.7 .6 .2 .37 \\
27.2 .1 \\
\end{array}$ & $\begin{array}{l}\text { RECORD } \\
\text { NIT }-* 52 \\
\text { SAMPLES }\end{array}$ & $\frac{34.5 \mathrm{PPM}}{35.1 \mathrm{PPM}}$ & $\begin{array}{l}\text { READINGS } \\
\text { WITHIN } \pm 5 \%\end{array}$ & A & Ammonia \\
\hline $\begin{array}{l}1.7 .6 .2 .38 \\
\text { m.x. } x^{38} \\
\end{array}$ & OPEN FU-15 & $N / A$ & $N / A$ & $A$ & \\
\hline $\begin{array}{l}1.7 .6 .2 .39 \\
=920.8 \\
\end{array}$ & $\begin{array}{l}\text { REMOVE } \\
\text { JUMPER }\end{array}$ & $N / A$ & $N / A$ & A & \\
\hline \multicolumn{6}{|c|}{ Section 1.7 .7 provides for test system shutdown. } \\
\hline $\begin{array}{l}1.7 .1 \\
9 \dot{x}_{3.6} \\
\end{array}$ & $\begin{array}{l}\text { DISCONNECT } \\
\mathrm{H}_{2} \text { CAL GAS }\end{array}$ & $N / A$ & $N / A$ & A & \\
\hline $\begin{array}{l}1.7 .7 .2 \\
9 x, 86 \\
\end{array}$ & $\begin{array}{l}\text { DISCONNECT } \\
\mathrm{CH}_{6} \text { CAL GAS }\end{array}$ & $N / A$ & $N / A$ & A & \\
\hline $\begin{array}{r}1.7 .7 .3 \\
92.83 \\
\end{array}$ & $\begin{array}{l}\text { DISCONNECT } \\
\mathrm{NH}_{3} \text { CAL GAS }\end{array}$ & $N / A$ & $N / A$ & A & \\
\hline $\begin{array}{l}1.7 .7 .4 \\
M\end{array}$ & $\begin{array}{l}\text { SECURE \& } \\
\text { TURN OFF } \\
\text { ANA. INST.S } \\
\end{array}$ & $N / A$ & $N / A$ & $A$ & \\
\hline $\begin{array}{l}1.7 .7 .5 \\
M 2 x . B \\
\end{array}$ & $\begin{array}{l}\text { DISCONNECT } \\
\mathrm{N}_{2} \text { CARRIER }\end{array}$ & $N / A$ & $N / A$ & A & \\
\hline $\begin{array}{r}1.7 .7 .6 \\
2 \text { M.P.B. } \\
\end{array}$ & $\begin{array}{l}\text { DISCONNECT } \\
\text { He CARRIER } \\
\end{array}$ & $N / A$ & $N / A$ & A & \\
\hline $\begin{array}{r}1.7 .7 .7 \\
2028 \\
\end{array}$ & $\begin{array}{l}\text { OPEN ALL } \\
\text { BREAKERS \& } \\
\text { FUSES }\end{array}$ & N/A & $N / A$ & A & \\
\hline 1.7 .7 .8 & $\begin{array}{l}\text { CLOSE LISTED } \\
\text { SYS. VALVES } \\
\end{array}$ & $N / A$ & N/A & A & \\
\hline
\end{tabular}


APPENDIX C

PAGE C- 28
RECON

COPY
HNF-SD-WM-ATR-191

Rev. 0
Page 124

TEST DATA SHEET

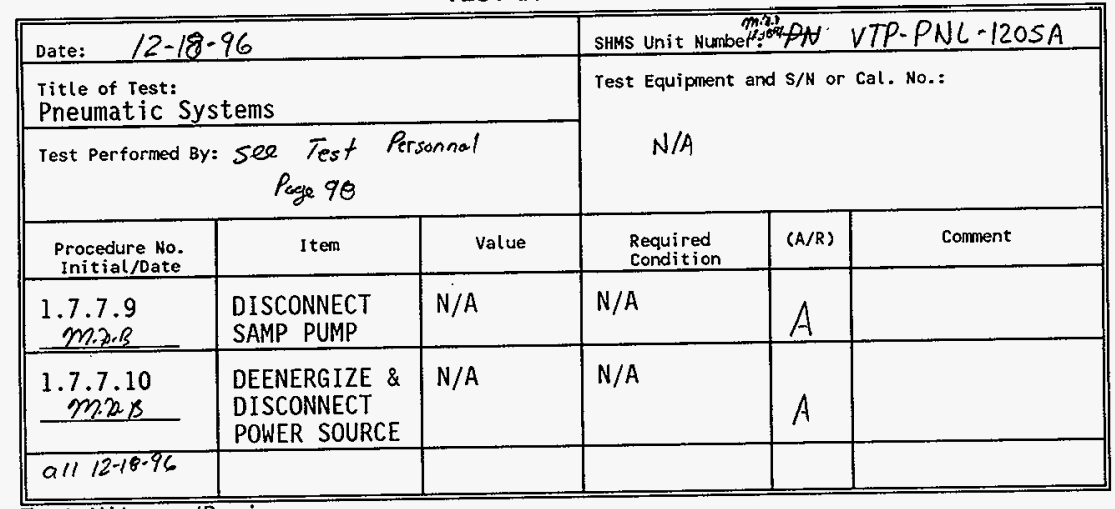

Test Witness/Review:
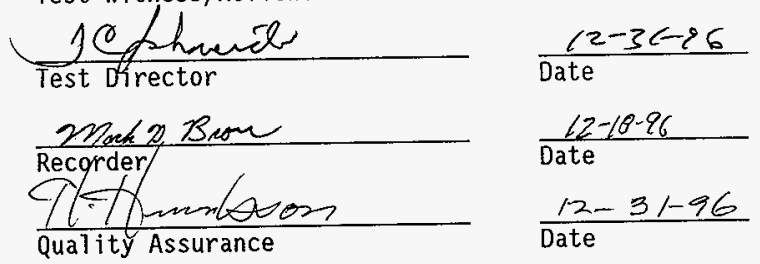
APPENDIX $\mathrm{C}$

PAGE C- 29

$$
\begin{array}{r}
\text { HNF-SD-WH-ATR- } 191 \\
\text { Rev. } 0 \\
\text { Page } 125
\end{array}
$$

TEST EXCEPTION SHEET

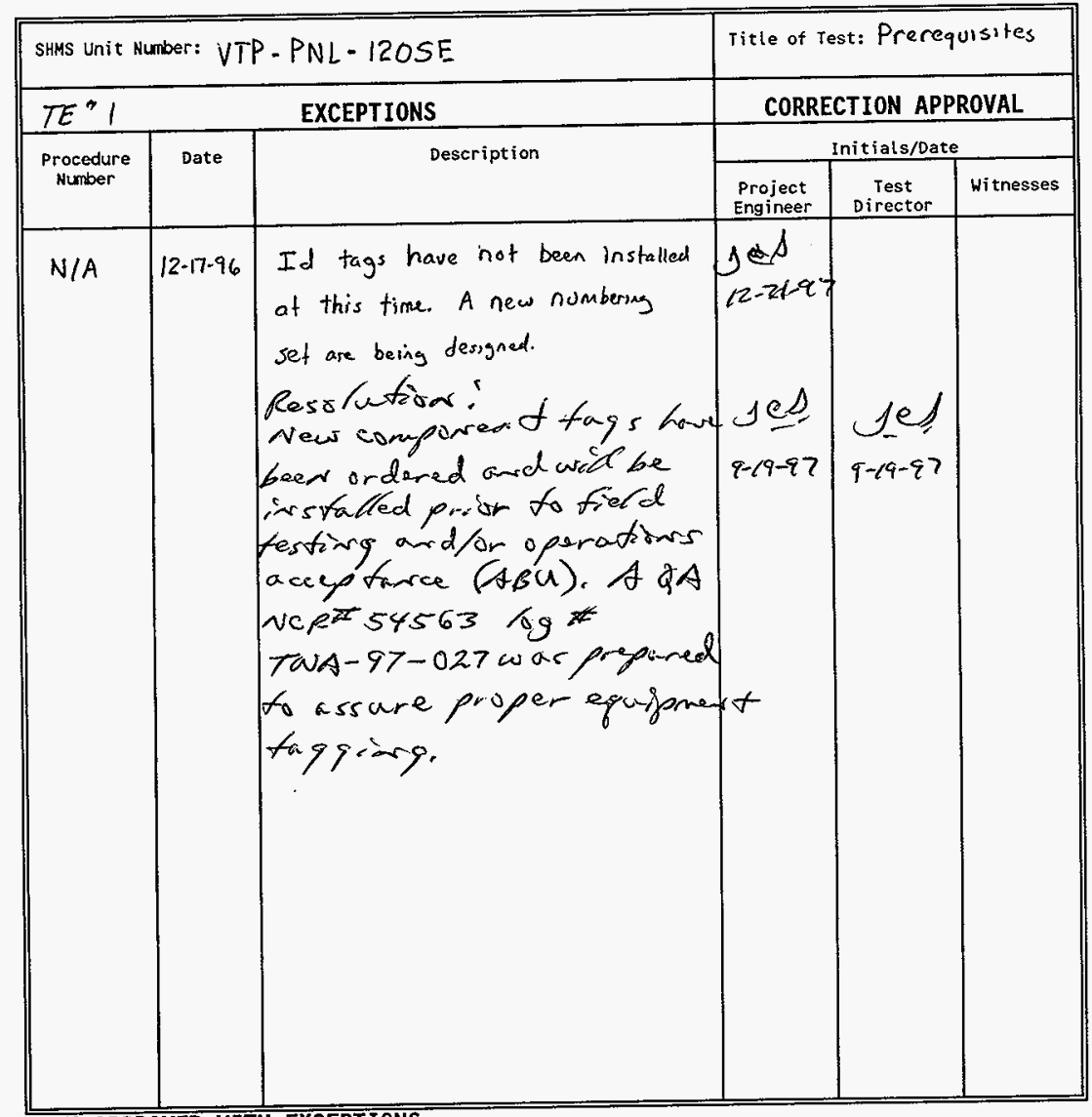

TEST APPROVED WITH EXCEPTIONS

1Cheswerde

WHC Project Engineer

opal X Brow

Recorder

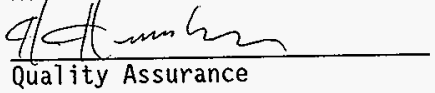

$\frac{12-31-86}{\text { Date }}$

$\frac{12-1796}{\text { Date }}$

$\frac{12-3 /-96}{\text { Date }}$ 
TEST LOG

\begin{tabular}{|c|c|}
\hline DATE/TIME & COMMENTS \\
\hline $12-17-96$ & Started ATP, along with the safen tolk on the shims \\
\hline $1: 30$ & unit weith test personnel \\
\hline $12-17-96$ & stoped at Pare 1.7 .6 \\
\hline $5: 00$ & \\
\hline $12 \cdot 18-96$ & Storted Parn 1.7 .6 \\
\hline $7: 30$ & \\
\hline $12-18-16$ & Finished ATP \\
\hline $2: 00$ & \\
\hline & \\
\hline & \\
\hline & \\
\hline & \\
\hline & \\
\hline & \\
\hline & \\
\hline & \\
\hline & \\
\hline & \\
\hline & \\
\hline & \\
\hline
\end{tabular}


Page 127

APPENDIX D 
TEST EXECUTION SHEET

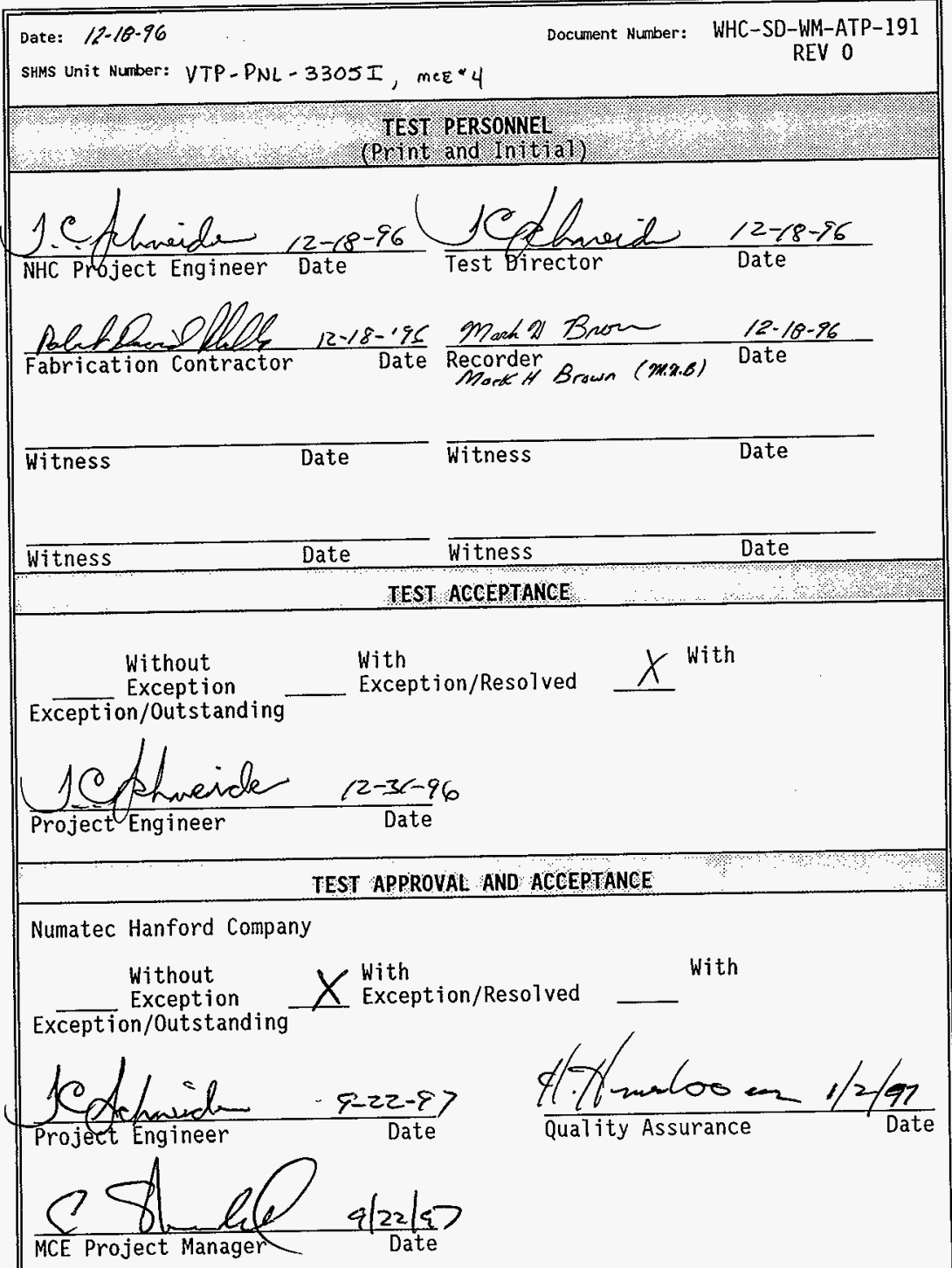


SHMS Unit Number:

\section{PREREQUISITES AND INITIAL TEST CONDITIONS}

The following conditions shall exist at the start of the acceptance testing. Initial and date to verify that each of the following items have been accomplished.

M.s Systems being tested have been inspected for workmanship and for $12-18-96$ compliance with design./ exception of $2 d$ tags $-\pi^{2+1}$ ma.s 12-18-96

mx.o_Continuity and megger tests have been performed on portions of the $12 \times 10-96$ electrical and instrument systems being tested, as required.

mo.s_Leak tests on the pneumatic systems have been performed.

The following circuit breakers and fuses are installed per the $12-10-96$ specified size and are open

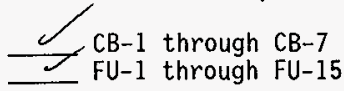

$\frac{m x x}{12-1096}$

A11 test instruments have a currentiy valjd calibration stamp attached that indicates a calibration traceable to the National Institute of Standards and Technology.

max The following process and control instrument systems have been $12-10-76$ initially configured and aligned for proper operation.

FIT-*57, FSL-*57, NIT-*54, NIT-*55, (NR-*54), PDIT-*60, TIC-*50, TIC $-* 56$, TIS $-* 62, Y Y C-* 01$ NOTE: NR $-* 54$ is not used in the ANALYTICAL configuration.

mas Personnel responsible for directing and witnessing the performance $12-10-86$ of the tests described in this ATP have read and understand appropriate certified vendor information (CVI) pertaining to the operation of the equipment to be tested.

CLOSE a1? system manual valves with the exception of the following:

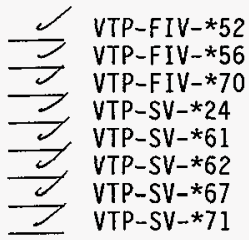


208 Vac 1 phase 15-20 ampere temporary power source has been $12-18-96$ connected, but not energized, to the appropriate TBI terminals per H-14-100838.

Verify by signature and date that all prerequisites have been met.

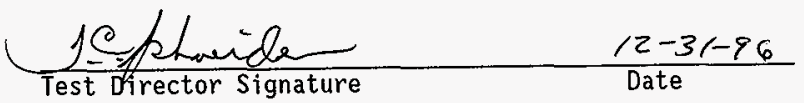


TEST DATA SHEET

\begin{tabular}{|c|c|c|c|c|c|}
\hline \multicolumn{3}{|c|}{ Date: $12-18-46$} & \multicolumn{3}{|c|}{ SHMS Unit Number: VTP-PNL-3305I } \\
\hline \multicolumn{3}{|c|}{$\begin{array}{l}\text { Title of Test: } \\
\text { Electrical Systems }\end{array}$} & \multirow{2}{*}{\multicolumn{3}{|c|}{$\begin{array}{l}\text { Test Equipment and S/N or Cal. No.: } \\
\text { Fluke V/o meter } \\
\text { S/N } 44620364 \\
\text { Cal. due. } 10.97\end{array}$}} \\
\hline \multicolumn{3}{|c|}{$\begin{array}{c}\text { Test Performed By: See Test Personnel } \\
\text { Page } 128\end{array}$} & & & \\
\hline $\begin{array}{l}\text { Procedure No. } \\
\text { Initial/Date } \\
\end{array}$ & Item & Value & $\begin{array}{c}\text { Required } \\
\text { Condition }\end{array}$ & $(A / R)$ & corment \\
\hline \multicolumn{6}{|c|}{ Section 1.7.1.1 verifies the system Mains Power. } \\
\hline $\begin{array}{l}1.7 .1 .1 .1 \\
\end{array}$ & Resistance & 0.1 & $<1$ ohm & A & \\
\hline $\begin{array}{l}1.7 .1 .1 .2 \\
=0.8\end{array}$ & $\begin{array}{l}\text { Temp power } \\
208 \mathrm{Vac}\end{array}$ & $N / A$ & Energized & A & \\
\hline${ }_{1}^{1.7 .1 .1 .3}$ & $\begin{array}{l}\text { Line Vac: } \\
\text { L1-L2 } \\
\text { L1-Gind } \\
\text { L2-Gnd } \\
\mathrm{N}-\text { Gnd }\end{array}$ & $\frac{\frac{209.1 V_{a c}}{118.7 V_{a c}}}{\frac{120.3 V_{a c}}{0}}$ & $\begin{array}{l}\text { L1-L2: } 208 \\
\text { Vac }+10 /-1 \% \\
\text { L1, L2: } 120 \\
\text { Vac } \pm 5 \% \\
N: 0 \text { Vac } \\
\end{array}$ & A & \\
\hline \multicolumn{6}{|c|}{ Section 1.7 .1 .2 verifies the enclosure HVAC system. } \\
\hline $\begin{array}{l}1.7 .1 \dot{m}^{2} .1 \\
\end{array}$ & AC Adjusted & $N / A$ & N/A & $A$ & \\
\hline $\begin{array}{r}1.7 .1 .2 .2 \\
\end{array}$ & $\begin{array}{l}\text { CLOSE } \\
\text { CB-1/2 }\end{array}$ & N/A & $N / A$ & A & \\
\hline $\begin{array}{l}1.7 .1 .2 .3 \\
-\quad 2038 \\
\end{array}$ & Heater oN & ak & Heater ON & A & \\
\hline $\begin{array}{l}1.7 .1 .2 .4 \\
m_{3} B^{2} \\
\end{array}$ & AC Adjusted & N/A & N/A & $A$ & \\
\hline $\begin{array}{l}1.7 .1 .2 .5 \\
m .3 . B \\
\end{array}$ & $\begin{array}{l}\text { Heater OFF } \\
\text { Cooling ON }\end{array}$ & $\begin{array}{r}O K \\
O K \\
\end{array}$ & $\begin{array}{l}\text { Heater OFF } \\
\text { Cooling ON }\end{array}$ & A & \\
\hline $\begin{array}{r}1.7 .1 .2 .6 \\
\end{array}$ & AC Adjusted & N/A & N/A & A & \\
\hline $\begin{array}{c}1.7 .1 .2 .7 \\
M . x . M \\
\end{array}$ & $\begin{array}{l}\text { OPEN } \\
\text { CB-1/2 }\end{array}$ & N/A & N/A & A & \\
\hline \multicolumn{6}{|c|}{ Section 1.7 .1 .3 verifies the sample pump is properly connected. } \\
\hline $\begin{array}{l}1.7 .1 .3 .1 \\
\text { m.x.B. }\end{array}$ & $\begin{array}{l}\text { CLOSE } \\
\text { CB-3/4 } \\
\end{array}$ & N/A & N/A & A & \\
\hline $\begin{array}{l}1.7 .1 .3 .2 \\
m .0 .8\end{array}$ & $\begin{array}{l}\text { VTP-P-*50 } \\
\text { ON }\end{array}$ & OK & $\begin{array}{l}\text { VTP-P }-* 50 \\
0 N\end{array}$ & A & \\
\hline
\end{tabular}


APPENDIX D

TEST DATA SHEET

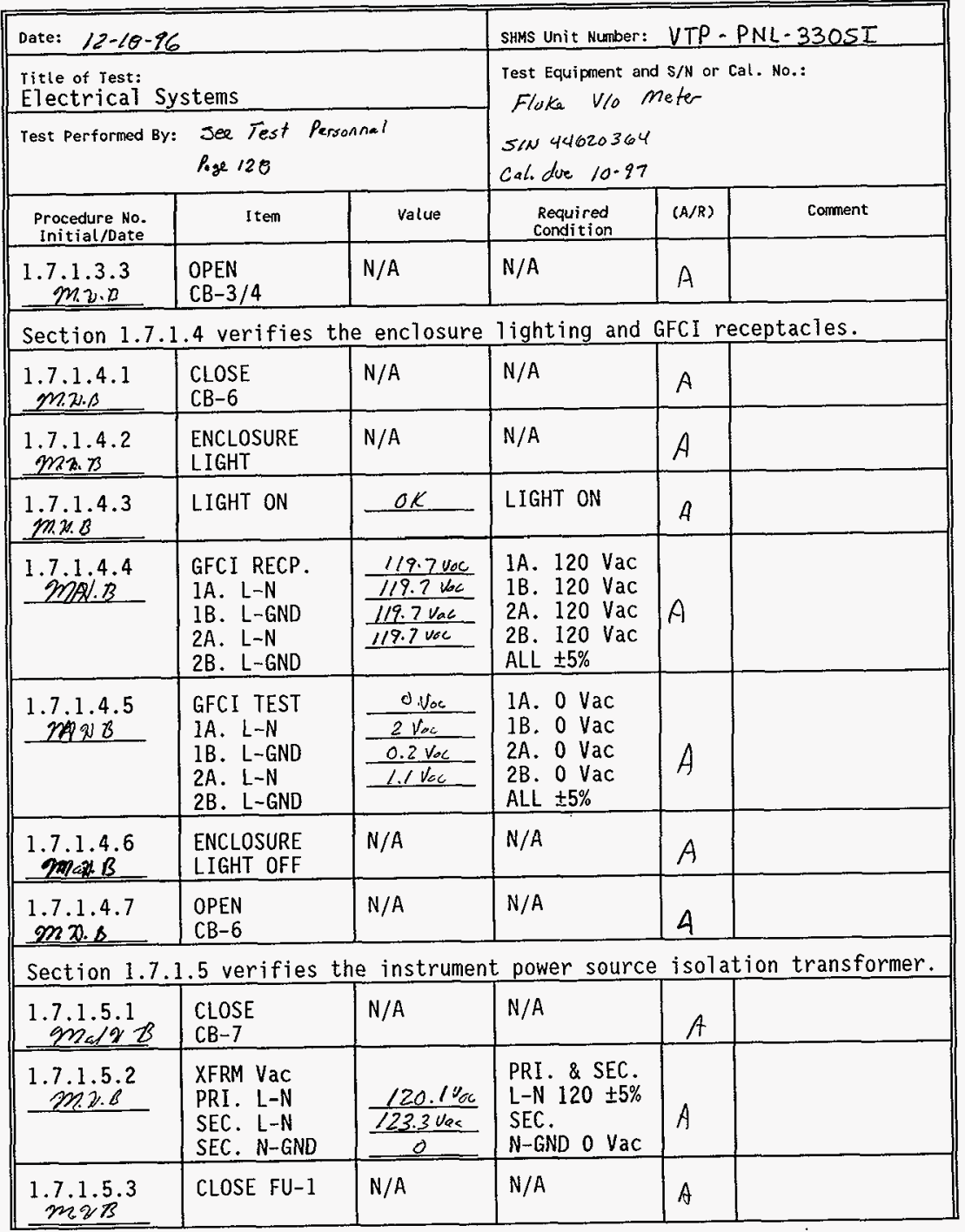


TEST DATA SHEET

\begin{tabular}{|c|c|c|c|c|c|}
\hline \multicolumn{3}{|c|}{ Date: $\quad 12-18-96$} & \multicolumn{3}{|c|}{ SHMS Unit Number: VTP-PNL - 330SI } \\
\hline \multicolumn{3}{|c|}{$\begin{array}{l}\text { Title of Test: } \\
\text { Electrical Systems }\end{array}$} & \multirow{2}{*}{\multicolumn{3}{|c|}{$\begin{array}{l}\text { Test Equipment and } S / N \text { or Cal. No.: } \\
\text { Fluke V/o meter } \\
S / N \$ 14620364 \\
\text { Cal, due. } 10-97\end{array}$}} \\
\hline \multicolumn{3}{|c|}{$\begin{array}{c}\text { Test Performed By: See Test Pessonnol } \\
\text { Page } 128\end{array}$} & & & \\
\hline $\begin{array}{l}\text { Procedure No. } \\
\text { Initial/Oate }\end{array}$ & Item & value & $\begin{array}{l}\text { Required } \\
\text { Condition } \\
\end{array}$ & $(A / R)$ & Comment \\
\hline $\begin{array}{l}1.7 .1 .5 .4 \\
\text { m.2.8 }\end{array}$ & $\begin{array}{l}\text { VTP-PS } * 50 \\
\text { OUTPUT Vdc } \\
\end{array}$ & $24.09 \mathrm{Vtc}$ & $24 \pm 0.1 \mathrm{Vdc}$ & $A$ & \\
\hline $\begin{array}{l}1.7 .1 .6 .1 \\
\text { mig.s } \\
\end{array}$ & $\begin{array}{l}\text { CLOSE FU-9 \& } \\
\text { FU-10 }\end{array}$ & $N / A$ & $N / A$ & A & \\
\hline $\begin{array}{l}1.7 .1 .6 .2 \\
2 \operatorname{mos} . \beta\end{array}$ & $\begin{array}{l}\text { PUSH PB-*51 } \\
\& \text { PB }-* 50\end{array}$ & N/A & $N / A$ & $A$ & \\
\hline $\begin{array}{l}1.7 .1 .6 .3 \\
\text { on. } 21.5\end{array}$ & $\begin{array}{l}\text { ALARM TEST } \\
\text { VERIFY ALARM } \\
\text { CONDITIONS }\end{array}$ & $\begin{array}{l}O N \\
O N \\
O N \\
O N \\
O N \\
O N \\
O N \\
O N \\
O N \\
\frac{O N}{O K} \\
O K \\
\end{array}$ & $\begin{array}{ll}\text { YAH-*50 } & \text { ON } \\
\text { NAH-*55 } & \text { ON } \\
\text { XA-*63 } & \text { ON } \\
\text { NAH-*54 } & \text { ON } \\
\text { FAL-*57 } & \text { ON } \\
\text { TAHL-*62 } & \text { ON } \\
\text { TAL-*50 } & \text { ON } \\
\text { YAL-*58 } & \text { ON } \\
\text { PBL-*58 } & \text { ON } \\
\text { PBL-*54 } & \text { OFF } \\
\text { PBL-*59 } & \text { OFF } \\
\end{array}$ & $A$ & \\
\hline $\begin{array}{l}1.7 .1 .6 .4 \\
\end{array}$ & $\begin{array}{l}\text { END ALARM } \\
\text { TEST }\end{array}$ & N/A & $N / A$ & $A$ & \\
\hline m.1.6.5 & RESET ALARMS & $N / A$ & N/A & $A$ & \\
\hline 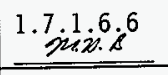 & $\begin{array}{l}\text { OPEN FU-9 \& } \\
\text { FU-10 }\end{array}$ & $N / A$ & $N / A$ & $A$ & \\
\hline \multicolumn{6}{|c|}{ Section 1.7.1.7 verifies the flow alarm system. } \\
\hline $1.7 \cdot 1.7 \cdot 1$ & $\begin{array}{l}\text { CLOSE FU-3, } \\
\text { FU-9,. FU-10 } \\
\& \text { FU-13 } \\
\end{array}$ & N/A & $N / A$ & $A$ & \\
\hline 2.7 .1 .7 .2 & RESET ALARMS & $O K$ & $\mathrm{FAL}-\star 57$ ON & A & \\
\hline $\begin{array}{l}1.7 .1 .7 .3 \\
m \cdot x \cdot B \\
\end{array}$ & $\begin{array}{l}\text { OPEN SV-*20 } \\
\& \text { SV-*22 }\end{array}$ & $N / A$ & N/A & $A$ & \\
\hline
\end{tabular}


TEST DATA SHEET

\begin{tabular}{|c|c|c|c|c|c|}
\hline \multicolumn{3}{|c|}{ Date: $12-18-96$} & \multicolumn{3}{|c|}{ SHMS Unit Number: VTP-PNL-3305I } \\
\hline \multicolumn{3}{|c|}{$\begin{array}{l}\text { Title of Test: } \\
\text { Electrical Systems }\end{array}$} & \multirow{2}{*}{\multicolumn{3}{|c|}{$\begin{array}{l}\text { Test Equipment and } S / \mathrm{N} \text { or Cal. No.: } \\
\text { Fluke v/o mefor } \\
\text { S/N } 44620364 \\
\text { Cal. due } 10.97\end{array}$}} \\
\hline \multicolumn{3}{|c|}{$\begin{array}{c}\text { Test Performed By: See Test Pessennal } \\
\text { Page } 128\end{array}$} & & & \\
\hline $\begin{array}{l}\text { Procedure No. } \\
\text { Initial/Date. }\end{array}$ & Item & Value & $\begin{array}{r}\text { Required } \\
\text { Condition } \\
\end{array}$ & $(A / R)$ & Corment \\
\hline $\begin{array}{l}1.7 .1 .7 .4 \\
m \geq 0\end{array}$ & $\begin{array}{l}\text { FIT }-* 57 \quad \& \\
\text { FSL }-* 57 \\
\text { POWERED }\end{array}$ & OK & $\begin{array}{l}\text { FIT-*57 ON } \\
\text { FSL-*57 ON }\end{array}$ & A & \\
\hline 1.7 .1 .7 .5 & $\begin{array}{l}\text { MUX-*70 } \\
\text { TB5+/TB6- }\end{array}$ & $0.999 \mathrm{VLd}$ & $1.0 \pm 0.2 \mathrm{Vdc}$ & $A$ & \\
\hline $\begin{array}{l}1.7 .1 .7 .6 \\
\text { m.2.0 }\end{array}$ & $\begin{array}{l}\text { CLOSE SV-*20 } \\
\& \text { SV } * 222\end{array}$ & N/A & $N / A$ & A & \\
\hline $\begin{array}{l}1.7 .1 .7 .7 \\
\end{array}$ & $\begin{array}{l}\text { OPEN FU-3, } \\
\text { FU-9, FU-10 } \\
\& \text { FU-13 }\end{array}$ & N/A & $N / A$ & A & \\
\hline \multicolumn{6}{|c|}{ Section 1.7 .1 .8 verifies the $\mathrm{H}_{2}$ monitor wiring. } \\
\hline $\begin{array}{l}1.7 .1 .8 .1 \\
m_{2}\end{array}$ & $\begin{array}{l}\text { CLOSE FU-2, } \\
\text { FU-4, FU-5, } \\
\text { FU-9 \& FU-10 }\end{array}$ & $N / A$ & N/A & $A$ & \\
\hline $\begin{array}{l}1.7 .1 .8 .2 \\
M .2 B\end{array}$ & RESET ALARMS & $\frac{O K}{O K}$ & $\begin{array}{l}\mathrm{NAH}-* 54 \text { OFF } \\
\mathrm{NAH}-* 55 \text { OFF } \\
\end{array}$ & $A$ & \\
\hline $\begin{array}{l}1.7 .1 .8 .3 \\
\text { m.x }\end{array}$ & $\begin{array}{l}\text { INSTRUMENTS } \\
\text { POWERED }\end{array}$ & $\begin{array}{l}\frac{O K}{O K} \\
\frac{O K}{O K} \\
N / A \\
d K \\
\end{array}$ & $\begin{array}{l}\text { MUX-*70 ON } \\
\text { NIT }-* 54 \text { ON } \\
\text { NIT }-* 55 \text { ON } \\
\text { NR }-* 54 \text { ON } \\
\text { YYC-*01 ON } \\
\end{array}$ & $A$ & \\
\hline $\begin{array}{l}1.7 .1 .8 .4 \\
m 23.8 \\
\end{array}$ & $\begin{array}{l}\text { OPEN FU-2, } \\
\text { FU-4, FU-5, } \\
\text { FU-9 \& FU-10 }\end{array}$ & N/A & N/A & A & \\
\hline \multicolumn{6}{|c|}{ Section 1.7.1.9 verifies the SHMS-E heat trace control system. } \\
\hline $\begin{array}{l}1.7 .1 .9 .1 \\
m .2 \times 8 .\end{array}$ & $\begin{array}{l}\text { INSTALL LOAD } \\
\text { VTP-TIC-*50 }\end{array}$ & $N / A$ & N/A & $A$ & \\
\hline${ }_{m \rightarrow x}^{1.7 .1 .9 .2}$ & $\begin{array}{l}\text { CLOSE CB-5, } \\
\text { FU-6, FU-7, } \\
\text { FU-9 \& FU-10 } \\
\text { and RESET } \\
\text { ALARMS }\end{array}$ & $N / A$ & N/A & A & \\
\hline
\end{tabular}


TEST DATA SHEET

\begin{tabular}{|c|c|c|c|c|c|}
\hline Date: $12-18-9$ & & & SHMS Unit Number: & $V T P$. & $3305 I$ \\
\hline $\begin{array}{l}\text { Titte of Test: } \\
\text { Electrical }\end{array}$ & stems & & Test Equipment ar & $S / N$ or & \\
\hline Test Performed & $\begin{array}{l}\text { See Test Perso } \\
\text { Page } 128\end{array}$ & & $N / A$ & & \\
\hline $\begin{array}{l}\text { Procedure No. } \\
\text { Initial/Date }\end{array}$ & Item & value & $\begin{array}{c}\text { Required } \\
\text { condition } \\
\end{array}$ & $(A / R)$ & Comment \\
\hline $\begin{array}{l}1.7 .1 .9 .3 \\
\end{array}$ & $\begin{array}{l}\text { TIC-*50 \& } \\
\text { TIC-*56 } \\
\text { NOMINAL TEMP }\end{array}$ & $\begin{array}{r}67 . \mathrm{F} \\
660 \mathrm{P} \\
\end{array}$ & $\begin{array}{l}\text { TIC }-* 50 \\
\text { TIC }-* 56 \\
\text { TEMPERATURE } \\
\end{array}$ & $A$ & \\
\hline $\begin{array}{l}1.7 .1 .9 .4 \\
m_{1} x_{0} B\end{array}$ & $\begin{array}{l}\text { ADJ TIC }-* 50 \\
\& \text { TIC }-* 56 \\
\text { SP2 \& RESET } \\
\text { ALARMS }\end{array}$ & $N / A$ & $N / A$ & $A$ & \\
\hline $\begin{array}{l}1.7 .1 .9 .5 \\
2 \operatorname{mos}\end{array}$ & $\begin{array}{l}\text { ADJ TIC-*50 } \\
\text { SP } 1 \\
\end{array}$ & $N / A$ & $N / A$ & $A$ & \\
\hline $\begin{array}{c}1.7 .1 .9 .6 \\
m .8 .8 \\
\end{array}$ & $\begin{array}{l}\text { ADJ TIC-*50 } \\
S P 2\end{array}$ & $N / A$ & $\mathrm{~N} / \mathrm{A}$ & $A$ & \\
\hline $\begin{array}{c}1.7 .1 .9 .7 \\
m . \lambda . n\end{array}$ & $\begin{array}{l}\text { TAL-*50 \& } \\
\text { HORN ACTIVE }\end{array}$ & $\frac{\partial k}{o^{k}}$ & $\begin{array}{l}\text { TAL-*50 ON } \\
\text { HORN ON } \\
\text { ACKNOWLEDGE }\end{array}$ & $A$ & \\
\hline $\begin{array}{c}1.7 .1 .9 .8 \\
\text { m.x.s. }\end{array}$ & \begin{tabular}{|l} 
TIC- $* 50$ \\
OPERATES \\
\end{tabular} & ok & $\begin{array}{l}\text { TIC }-* 50 \\
\text { OPERATES } \\
\end{array}$ & $A$ & \\
\hline $\begin{array}{c}1.7 .1 .9 .9 \\
m 2.8 \\
\end{array}$ & $\begin{array}{l}A D J \text { TIC-*50 } \\
\text { SP 2 }\end{array}$ & $N / A$ & N/A & $A$ & 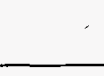 \\
\hline 1.7 .1 .9 .10 & RESET ALARM & ox & TAL- $* 50$ OFF & $A$ & \\
\hline $\begin{array}{l}1.7 .1 .9 .11 \\
\text { m.x.B } \\
\end{array}$ & $\begin{array}{l}\text { ADJ TIC }-* 50 \\
\text { SP } 1 \& S P \quad 2 \\
\end{array}$ & $N / A$ & $N / A$ & $A$ & \\
\hline $\begin{array}{l}7.1 .9 .12 \\
\text { m.7.8. }\end{array}$ & $\begin{array}{l}\text { OPEN CB-5 \& } \\
\text { REMOVE LOAD } \\
\end{array}$ & $N / A$ & N/A & A & \\
\hline $\begin{array}{l}1.7 .1 .9 .13 \\
\text { mo.s } \\
\end{array}$ & $\begin{array}{l}\text { INSTALL LOAD } \\
\text { VTP-TIC }-* 56 \\
\end{array}$ & $N / A$ & $N / A$ & $A$ & \\
\hline $\begin{array}{l}1.7 .1 .9 .14 \\
m \rightarrow \operatorname{mos} \\
\end{array}$ & CLOSE CB-5 & N/A & $N / A$ & A & \\
\hline $\begin{array}{l}1.7 .1 .9 .15 \\
\text { m.x.8 }\end{array}$ & $\begin{array}{l}\text { ADJ TIC-*56 } \\
\text { SP } 1\end{array}$ & N/A & N/A & A & \\
\hline $\begin{array}{l}1.7 .1 .9 .16 \\
\text { min.e }\end{array}$ & $\begin{array}{l}\text { ADJ TIC }-* 56 \\
\text { SP } 2\end{array}$ & $N / A$ & $N / A$ & A & \\
\hline
\end{tabular}


TEST DATA SHEET

\begin{tabular}{|c|c|c|c|c|c|}
\hline \multicolumn{3}{|c|}{ Date: $\quad 12-18-96$} & SHMS Unit Mumber: & \multicolumn{2}{|c|}{ VTP-PNL-330SI } \\
\hline \multicolumn{3}{|c|}{$\begin{array}{l}\text { Title of rest: } \\
\text { Electrical Systems }\end{array}$} & \multirow{2}{*}{\multicolumn{3}{|c|}{$\begin{array}{l}\text { Test Equipment and } s / \mathbb{N} \text { or Cal. No.: } \\
N / A\end{array}$}} \\
\hline \multicolumn{3}{|c|}{ 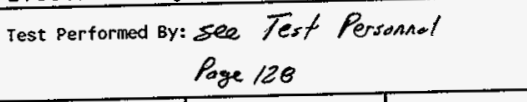 } & & & \\
\hline $\begin{array}{l}\text { Procedure Ho. } \\
\text { Initial/Date }\end{array}$ & Item & Value & $\begin{array}{l}\text { Required } \\
\text { Condition } \\
\end{array}$ & $(A / R)$ & comment \\
\hline$\frac{1.7 .1 .9 .17}{m \cdot x \cdot B}$ & $\begin{array}{l}\text { TAL-*50 \& } \\
\text { HORN ACTIVE }\end{array}$ & $\begin{array}{l}\frac{O K}{O K} \\
O K \\
O K\end{array}$ & $\begin{array}{l}\text { TAL-*50 ON } \\
\text { HORN ON } \\
\text { ACKNOWLEDGE }\end{array}$ & $A$ & \\
\hline $\begin{array}{l}1.7 .1 .9 .18 \\
\end{array}$ & $\begin{array}{l}\text { TIC- } * 56 \\
\text { OPERATES }\end{array}$ & de & $\begin{array}{l}\text { TIC }-* 56 \\
\text { OPERATES }\end{array}$ & $A$ & \\
\hline $\begin{array}{l}1.7 .1 .9 .19 \\
\text { mins } \\
\end{array}$ & $\begin{array}{l}\text { ADJ TIC-*56 } \\
\text { SP } 2\end{array}$ & $N / A$ & $N / A$ & A & \\
\hline $\begin{array}{l}1.7 .1 .9 .20 \\
\end{array}$ & RESET ALARM & OK & $T A L-* 50$ OFF & $A$ & \\
\hline$\underline{\min }_{1.0}$ & $\begin{array}{l}A D J T I C-* 56 \\
S P 1 \& S P 2\end{array}$ & $\mathrm{~N} / \mathrm{A}$ & N/A & $A$ & \\
\hline $\begin{array}{l}1.7 .1 .9 .22 \\
\text { m.2.3. } \\
\end{array}$ & $\begin{array}{l}\text { OPEN CB-5, } \\
\text { FU-6 \& FU-7 } \\
\end{array}$ & $N / A$ & N/A & $A$ & \\
\hline $\begin{array}{l}1.7 .1 .9 .23 \\
m .2 .8 \\
\end{array}$ & $\begin{array}{l}\text { REMOVE LOAD } \\
\text { TIC } * 56\end{array}$ & $N / A$ & $N / A$ & $A$ & \\
\hline$\frac{1.1 .9 .24}{m 2 . B^{2}}$ & $\begin{array}{l}\text { CLOSE FU-8 } \\
\text { TIS-*62 } \\
\text { NOMINAL TEMP }\end{array}$ & $690 \mathrm{~F}$ & $\begin{array}{l}\text { TIS-*62 } \\
\text { TEMP }\end{array}$ & $A$ & \\
\hline $\begin{array}{l}1.7 .1 .9 .25 \\
\max .8 \\
\end{array}$ & $\begin{array}{l}\text { ADJ TIS-*62 } \\
\text { SP } 1\end{array}$ & $N / A$ & $N / A$ & A & \\
\hline 1.7 .1 .9 .26 & $\begin{array}{l}\text { ADJ TIS }-* 62 \\
\text { SP 2 } \\
\end{array}$ & $N / A$ & $N / A$ & $A$ & \\
\hline $\begin{array}{l}1.7 .9 .927 \\
2.3\end{array}$ & RESET ALARMS & $a^{k}$ & $\begin{array}{l}\text { TAHL }-* 62 \\
\text { OFF }\end{array}$ & A & \\
\hline $\begin{array}{l}1.7 .1 .9 .28 \\
m .0 .0 \\
\end{array}$ & $\begin{array}{l}\text { ADJ TIS-*62 } \\
\text { SP 2 } \\
\end{array}$ & $N / A$ & $N / A$ & A & \\
\hline$\frac{1.7 .1 .9 .29}{2 \pi 2.8}$ & $\begin{array}{l}\text { TAHL-*62 \& } \\
\text { HORN ACTIVE }\end{array}$ & $\frac{O K}{0 K}$ & $\begin{array}{l}\text { TAHL-*62 ON } \\
\text { HORN ON } \\
\text { ACKNOWLEDGE }\end{array}$ & $A$ & \\
\hline 1.7 .1 .9 .30 & $\begin{array}{l}\text { ADJ TIS }-* 62 \\
\text { SP } 2\end{array}$ & $N / A$ & $N / A$ & $A$ & \\
\hline
\end{tabular}


TEST DATA SHEET

\begin{tabular}{|c|c|c|c|c|c|}
\hline \multicolumn{3}{|c|}{ Date: $\quad 12-18-96$} & \multicolumn{3}{|c|}{ SHMS Unit Number: VTP - PNL $-3305 \mathrm{I}$} \\
\hline \multicolumn{3}{|c|}{$\begin{array}{l}\text { Title of rest: } \\
\text { Electrical Systems }\end{array}$} & \multirow{2}{*}{\multicolumn{3}{|c|}{$\begin{array}{l}\text { Test Equipnent and } S / N \text { or cal. No.: } \\
\text { Floke vio meter } \\
S / N 44620364 \\
\text { Cal due } 10.97\end{array}$}} \\
\hline \multicolumn{3}{|c|}{$\begin{aligned} & \text { Test Performed By: } \text { See Test Personnol } \\
& \text { Poge } 128\end{aligned}$} & & & \\
\hline $\begin{array}{l}\text { Procedure No. } \\
\text { Initial/Date }\end{array}$ & I tem & Value & $\begin{array}{l}\text { Required } \\
\text { Condition } \\
\end{array}$ & $(A / R)$ & Comment \\
\hline $\begin{array}{c}1.7 .1 .9 .31 \\
m .2 .3 \\
\end{array}$ & RESET ALARMS & $\sigma k$ & $\begin{array}{l}\text { TAHL }-* 62 \\
0 \mathrm{FF}\end{array}$ & A & \\
\hline $\begin{array}{l}1.7 .1 .9 .32 \\
m x . B \\
\end{array}$ & $\begin{array}{l}\text { AOJ TIS }-* 62 \\
\text { SP } 1\end{array}$ & $N / A$ & $N / A$ & A & \\
\hline$\frac{1.7 .1 .9 .33}{\text { minos }}$ & $\begin{array}{l}\text { TAHL -*62 \& } \\
\text { HORN ACTIVE }\end{array}$ & $\begin{array}{l}\text { OK } \\
\text { OK } \\
\text { OK }\end{array}$ & $\begin{array}{l}\text { TAHL-*62 ON } \\
\text { HORN ON } \\
\text { ACKNOWLEDGE }\end{array}$ & $A$ & \\
\hline $\begin{array}{l}1.7 .1 .9 .34 \\
\operatorname{gxp} x \cdot 3\end{array}$ & $\begin{array}{l}\text { ADJ TIS-*62 } \\
S P \text { I \& SP 2 }\end{array}$ & $N / A$ & $\mathrm{~N} / \mathrm{A}$ & $A$ & \\
\hline 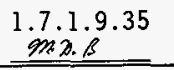 & $\begin{array}{l}\text { OPEN FU-8, } \\
\text { FU-9 \& FU-10 }\end{array}$ & $N / A$ & $N / A$ & A & \\
\hline \multicolumn{6}{|c|}{ Section 1.7.1.10 verifies the differential pressure transmitter system. } \\
\hline 1.7 .1 .10 .1 & $\begin{array}{l}\text { REMOVE PLUGS } \\
\text { BAL PDIT-*60 } \\
\end{array}$ & $N / A$ & $N / A$ & A & \\
\hline $\operatorname{mis}^{1.7 .1 .10 .2}$ & $\begin{array}{l}\text { CLOSE FU- } 14 \\
\text { REC PDIT-*60 } \\
\text { PRESSURE } \\
\end{array}$ & $0.001 \mathrm{H}_{2}^{\circ}$ & $\begin{array}{l}\text { PDIT-*60 } \\
\text { PRESSURE } \\
0 \pm 1 " \mathrm{H}_{2} \mathrm{O}\end{array}$ & A & \\
\hline 1.7 .1 .10 .3 & $\begin{array}{l}\text { MUX-*70 Vdc } \\
\text { TB7+ \& TB8- }\end{array}$ & $2.15 \mathrm{Vdc}$ & $\begin{array}{l}\text { MUX-*70 Vdc } \\
2.2 \pm 0.1\end{array}$ & A & \\
\hline $\begin{array}{l}1.7 .1 .10 .4 \\
m .20 .13 \\
\end{array}$ & $\begin{array}{l}\text { REPLACE } \\
\text { PLUGS ON } \\
\text { PDIT } * 60 \\
\end{array}$ & $N / A$ & $N / A$ & $A$ & \\
\hline $\begin{array}{l}1.7 .1 .10 .5 \\
\text { M.M.B. } \\
\end{array}$ & OPEN FU-14 & $N / A$ & N/A & A & \\
\hline \multicolumn{6}{|c|}{ Section 1.7.1.11 verifies the gas chromatograph power. } \\
\hline $\begin{array}{l}1.7 .1 .11 .1 \\
\text { mins }\end{array}$ & CLOSE FU-11 & $N / A$ & $N / A$ & A & \\
\hline $\begin{array}{c}1.7 .1 .11 .2 \\
m_{x .3} \\
\end{array}$ & $\begin{array}{l}\text { POWER } \\
\text { MON-*60 } \\
\end{array}$ & ok & $\begin{array}{l}\text { MON-*60 } \\
\text { POWERED } \\
\end{array}$ & $A$ & \\
\hline${ }_{0.7 . x \cdot b}^{1.7 .11 .3}$ & OPEN FU-11 & $N / A$ & N/A & A & \\
\hline
\end{tabular}


TEST DATA SHEET

\begin{tabular}{|c|c|c|c|c|c|}
\hline \multicolumn{3}{|c|}{ Date: $\quad 12-18-96$} & \multicolumn{3}{|c|}{ SHMS Unit Number: VTP-PNL-3305I } \\
\hline \multicolumn{3}{|c|}{$\begin{array}{l}\text { ritle of rest: } \\
\text { Electrical Systems }\end{array}$} & \multirow{2}{*}{\multicolumn{3}{|c|}{$\begin{array}{l}\text { Test Equiprnent and } S / \mathrm{N} \text { or Cal. No.: } \\
\text { N/A }\end{array}$}} \\
\hline \multicolumn{3}{|c|}{$\begin{aligned} \text { Test Performed By: } & \text { see Test Personnel } \\
& \text { Page } 128\end{aligned}$} & & & \\
\hline $\begin{array}{l}\text { Procedure No. } \\
\text { Initial/Oate }\end{array}$ & I tem & Value & $\begin{array}{l}\text { Required } \\
\text { Condition }\end{array}$ & $(A / R)$ & comment \\
\hline \multicolumn{6}{|c|}{ Section 1.7 .1 .12 verifies the multi gas analyzer power. } \\
\hline $\begin{array}{c}1.7 .1 .12 .1 \\
m 2.8 \\
\end{array}$ & CLOSE FU-12 & $N / A$ & $N / A$ & A & \\
\hline $\begin{array}{c}1.7 .1 .12 .2 \\
\text { sinsep } \\
\end{array}$ & $\begin{array}{l}\text { POWER } \\
\text { NIT }-* 52\end{array}$ & $O K$ & $\begin{array}{l}\text { NIT-*52 } \\
\text { POWERED } \\
\end{array}$ & $A$ & \\
\hline 1.7 int.in. 12.3 & OPEN FU-12 & $N / A$ & $N / A$ & A & \\
\hline \multicolumn{6}{|c|}{ Section 1.7.1.13 verifies the personnel computer power. } \\
\hline $\begin{array}{l}1.7 .1 .13 .1 \\
2 \times 2 \times 1 \\
\end{array}$ & CLOSE CB-6 & $N / A$ & $N / A$ & $A$ & \\
\hline $\begin{array}{l}1.7 .1 .13 .2 \\
32.28 \\
\end{array}$ & $\begin{array}{l}\text { POWER PC-*60 } \\
\& P C-* 70\end{array}$ & $\frac{k}{0 k}$ & $\begin{array}{ll}P C-* 60 & O N \\
P C-* 70 & O N \\
\end{array}$ & $A$ & \\
\hline $\begin{array}{l}1.7 .1 .13 .3 \\
\text { m.2. }\end{array}$ & $\begin{array}{l}\text { TURN INST. } \\
\text { OFF \& } \\
\text { OPEN CB-6 }\end{array}$ & $\frac{O K}{O K}$ & $\begin{array}{ll}\mathrm{PC}-\star 60 & \text { OFF } \\
\mathrm{PC}-* 70 & \text { OFF }\end{array}$ & $A$ & \\
\hline
\end{tabular}

all $12 \cdot 18-96$ 
TEST DATA SHEET

\begin{tabular}{|c|c|c|c|c|c|}
\hline \multicolumn{3}{|l|}{ Date: $12-18-96$} & SHMS Unit Number: & \multicolumn{2}{|c|}{ VTP-PNL-3305I } \\
\hline \multicolumn{3}{|c|}{$\begin{array}{l}\text { Title of Test: } \\
\text { ETectrical Systems }\end{array}$} & \multirow{2}{*}{\multicolumn{3}{|c|}{$\begin{array}{l}\text { Test Equipment and } S / \mathrm{N} \text { or Cal. No.: } \\
\text { N/A }\end{array}$}} \\
\hline \multicolumn{3}{|c|}{$\begin{aligned} \text { Test Performed By: Sea Test Personn-1 } & \text { Page } 128\end{aligned}$} & & & \\
\hline $\begin{array}{l}\text { Procedure No. } \\
\text { Initial/Date }\end{array}$ & Item & Value & $\begin{array}{c}\text { Required } \\
\text { Condition }\end{array}$ & $(A / R)$ & Comment \\
\hline \multicolumn{6}{|c|}{ Section 1.7 .2 verifies the intrinsic safety apparatus is properiy labeled } \\
\hline 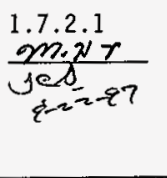 & $\begin{array}{l}\text { INTRINSIC } \\
\text { SAFETY } \\
\text { APPARATUS } \\
\text { NAMEPLATES } \\
\text { INSTALLED }\end{array}$ & $\frac{\pi}{4}$ & $\begin{array}{l}\text { NE-*54 } \\
\text { NE-*55 } \\
E B-* 54 \\
E B-* 55 \\
\text { NIT-*54 } \\
\text { NIT-*55 }\end{array}$ & & $\operatorname{sen} T E^{*} 1$ \\
\hline $\begin{array}{l}1.7 .2 .2 \\
\frac{2.2 .13}{12-18-96}\end{array}$ & $\begin{array}{l}\text { INTRINSIC } \\
\text { SAFETY WIRE } \\
\text { LABELED }\end{array}$ & -ok & $\begin{array}{l}\mathrm{NE} / \mathrm{EB}-* 54 \\
\mathrm{NE} / \mathrm{EB}-* 55\end{array}$ & A & \\
\hline 1.7 .3 & $\begin{array}{l}\text { SEC. 1.7.1 \& } \\
1.7 .2 \text { DONE }\end{array}$ & Test Di & meider & & $\frac{12-18-86}{\text { Date }}$ \\
\hline
\end{tabular}

\section{Test Witness/Review:}

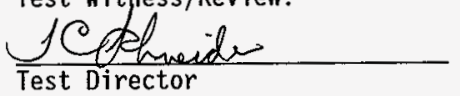

malio Brow

Recorder

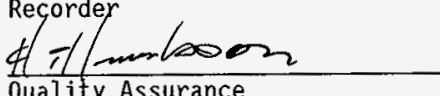

$\frac{1 z-3<-86}{\text { Date }}$

$\frac{12.31 .96}{\text { Date }}$

$\frac{1-2-77}{\text { Date }}$ 
TEST DATA SHEET

\begin{tabular}{|c|c|c|c|c|c|}
\hline \multicolumn{3}{|c|}{ Date: $\quad 12-19-96$} & \multicolumn{3}{|c|}{ SHMS Unit Number: VTP.PNL-330SI } \\
\hline \multicolumn{3}{|c|}{$\begin{array}{l}\text { Title of rest: } \\
\text { Pneumatic Systems }\end{array}$} & \multirow{2}{*}{\multicolumn{3}{|c|}{$\begin{array}{l}\text { Test Equipment and } s / \mathrm{N} \text { or Cal. No.: } \\
N / A\end{array}$}} \\
\hline \multicolumn{3}{|c|}{$\begin{array}{c}\text { Test Performed By: see Test Personnal } \\
\text { lage } 128\end{array}$} & & & \\
\hline $\begin{array}{l}\text { Procedure No. } \\
\text { initial/Date }\end{array}$ & Item & Value & $\begin{array}{l}\text { Required } \\
\text { Condition } \\
\end{array}$ & $(\mathrm{A} / \mathrm{R})$ & Comment \\
\hline \multicolumn{6}{|c|}{ Section 1.7.4.1 provides the pneumatic test section initial conditions. } \\
\hline $\begin{array}{l}1.7 .4 .1 .1 \\
m .3 .3\end{array}$ & $\begin{array}{l}\text { INITIAL } \\
\text { CONDITIONS }\end{array}$ & ok & $\begin{array}{l}\text { BKR \& FUSE } \\
\text { LINE UP }\end{array}$ & A & \\
\hline $\begin{array}{l}1.7 .4 .1 .2 \\
\min 2.6 \\
\end{array}$ & $\begin{array}{l}\text { INITIAL } \\
\text { CONDITIONS }\end{array}$ & ok & $\begin{array}{l}\text { VALVE LINE } \\
\text { UP }\end{array}$ & A & \\
\hline $\begin{array}{l}1.7 .4 .1 .3 \\
\operatorname{ma.8}\end{array}$ & $\begin{array}{l}\text { DISCONNECT } \\
1 / 16^{\prime \prime} \text { TUB ING } \\
\text { ON MON-*60 } \\
\end{array}$ & OK & $\begin{array}{l}\text { TUBING } \\
\text { CONFIGURED }\end{array}$ & A & \\
\hline $\begin{array}{l}1.7 .4 .1 .4 \\
2 x-1\end{array}$ & $\begin{array}{l}\text { DISCONNECT } \\
\text { TUBING ON } \\
\text { NIT }-\star 52\end{array}$ & ok & $\begin{array}{l}\text { TUBING } \\
\text { CONFIGURED }\end{array}$ & $A$ & \\
\hline $\begin{array}{l}1.7 .4 .1 .5 \\
m \times 8\end{array}$ & $\begin{array}{l}\text { PLUG VENT } \\
\text { FLT }-* 63\end{array}$ & ok & $\begin{array}{l}\text { FLT-*63 } \\
\text { PLUGGED }\end{array}$ & A & \\
\hline \multicolumn{6}{|c|}{ Section 1.7.4.2 verifies the main sample flow loop. } \\
\hline $\begin{array}{l}1.7 .4 .2 .1 \\
m .20 .8\end{array}$ & $\begin{array}{l}\text { CLOSE CB-3/4 } \\
\text { ADJ. FLOW }\end{array}$ & ok & $\begin{array}{l}\text { CB CLOSEO } \\
\text { FIT-*57 } \\
\text { INDICATION }\end{array}$ & A & \\
\hline $\begin{array}{l}1.7 .4 .2 .2 \\
m .2 .1\end{array}$ & $\begin{array}{l}\text { PDI }-* 51 \\
\text { READING } \\
\end{array}$ & $27 " 1 / 20$ & $20-40 " \mathrm{H}_{2} \mathrm{O}$ & A & \\
\hline $\begin{array}{l}1.7 .4 .2 .3 \\
20.2 .8 \\
\end{array}$ & $\begin{array}{l}\text { PI }-* 53 \\
\text { READING }\end{array}$ & $-2.6 \mathrm{Hg}$ & $\begin{array}{l}-2 \text { to }-4 \\
" \mathrm{Hg}\end{array}$ & A & \\
\hline $\begin{array}{l}1.7 .4 .2 .4 \\
\text { gm:g.B }\end{array}$ & RESET ALARMS & ok & NO ALARMS & A & \\
\hline $\begin{array}{l}1.7 .4 .2 .5 \\
9.7 .8\end{array}$ & CLOSE SV-*16 & $\begin{array}{l}0.03 \\
\text { OKK } \\
\text { OKK } \\
\end{array}$ & $\begin{array}{l}\text { FIT }-* 570 \\
\pm 1 \quad " \mathrm{H}_{2} 0 \\
\text { FAL }-* 57 \text { ON } \\
\text { HORN ON } \\
\end{array}$ & A & \\
\hline $\begin{array}{l}1.7 .4 .2 .6 \\
\end{array}$ & OPEN SV-*16 & ok & $\begin{array}{l}\text { FAL }-* 57 \text { ON } \\
\text { HORN OFF }\end{array}$ & $A$ & \\
\hline $1.7 \cdot x_{p \cdot B^{4}}^{2.7}$ & RESET ALARMS & ok & $\begin{array}{l}\text { ALARMS } \\
\text { RESET }\end{array}$ & $A$ & \\
\hline
\end{tabular}




\section{TEST DATA SHEET}

\begin{tabular}{|c|c|c|c|c|c|}
\hline \multicolumn{3}{|c|}{ Date: $12-19-96$} & \multicolumn{3}{|c|}{ SHMS Unit Number: VTP-PNL-330SI } \\
\hline \multicolumn{3}{|c|}{$\begin{array}{l}\text { Title of rest: } \\
\text { Pneumatic Systems }\end{array}$} & \multirow{2}{*}{\multicolumn{3}{|c|}{$\begin{array}{l}\text { Test Equipment and } \mathrm{S} / \mathrm{N} \text { or Cal. No.: } \\
\text { Fluke v/0 meter } \\
S / N 44620364 \\
\text { Cal. doe } 10-97\end{array}$}} \\
\hline \multicolumn{3}{|c|}{$\begin{array}{l}\text { Test Performed By: see Test Personnal } \\
\qquad \text { Page } 120\end{array}$} & & & \\
\hline $\begin{array}{l}\text { Procedure No. } \\
\text { Initial/Date }\end{array}$ & Item & value & $\begin{array}{l}\text { Required } \\
\text { Condition } \\
\end{array}$ & $(A / R)$ & Corment \\
\hline $\begin{array}{l}1.7 .4 .2 .8 \\
\text { mas. }\end{array}$ & CLOSE SV-*25 & $\begin{array}{l}0.00 \\
\frac{0 K}{0 K} \\
0 \mathrm{~K} \\
2.6 \mathrm{Vac}\end{array}$ & $\begin{array}{l}\text { FIT-*57 } 0 \\
\pm I \quad " H_{2} 0 \\
\text { FAL-*57 ON } \\
\text { HORN } \\
\text { XA-*63 ON } \\
\text { YY-*71 } \\
\text { TB 3-4 ON } \\
\end{array}$ & $A$ & \\
\hline $\begin{array}{l}1.7 .4 .2 .9 \\
\text { miax }\end{array}$ & OPEN SV-*25 & $\frac{\frac{o k}{o k}}{\frac{o k}{120.1 V_{a c}}}$ & $\begin{array}{l}\text { FAL }-* 57 \text { ON } \\
\text { HORN OFF } \\
\text { XA-*63 OFF } \\
Y Y-* 71 \\
\text { TB } 3-4 \quad 120 \mathrm{~V} \\
\end{array}$ & $A$ & \\
\hline $\operatorname{mixh}^{1.7 .4 .2 .10}$ & RESET ALARMS & ok & $\begin{array}{l}\text { ALARMS } \\
\text { RESET }\end{array}$ & A & \\
\hline$\frac{1.7 .4 .2 .11}{\min _{0.3}}$ & $\begin{array}{l}\text { OPEN SV-*54 } \\
\& \text { SV-*66 } \\
\text { READ PRESS. } \\
\text { PDIT-*60 }\end{array}$ & $\frac{\partial K}{-41.42^{\alpha 11400}}$ & $\begin{array}{l}\text { VALVES OPEN } \\
-27 \text { to }-82 \\
" \mathrm{H}_{2} \mathrm{O}\end{array}$ & $A$ & \\
\hline$\frac{1.7 .4 .2 .12}{m \cdot D \cdot R^{2}}$ & $\begin{array}{l}\text { CLOSE SV }-* 54 \\
\text { OPEN SV }-* 63 \\
\text { READ PRESS. } \\
\text { PDIT-*60 } \\
\end{array}$ & $\begin{array}{l}0 K \\
0.00 \\
\end{array}$ & $\begin{array}{l}\text { VALVES } \\
\text { OPERATED } \\
0 \pm 1 " \mathrm{H}_{2} \mathrm{O}\end{array}$ & A & \\
\hline $\begin{array}{l}1.7 .4 .2 .13 \\
m .2 . s\end{array}$ & $\begin{array}{l}\text { CLOSE SV-*63 } \\
\text { OPEN SV-*55 } \\
\text { READ PRESS. } \\
\text { PDIT-*60 } \\
\end{array}$ & $\begin{array}{l}\text { OK } \\
-43.0 \\
\end{array}$ & $\begin{array}{l}\text { VALVES } \\
\text { OPERATED } \\
-27 \text { to }-82 \\
\mathrm{H}_{2} \mathrm{O} \\
\end{array}$ & $A$ & \\
\hline $\begin{array}{l}1.7 .4 .2 .14 \\
\text { mins }\end{array}$ & $\begin{array}{l}\text { CLOSE SV }-* 55 \\
\text { OPEN SV } * 64 \\
\text { READ PRESS. } \\
\text { PDIT } * 60\end{array}$ & 0 & $\begin{array}{l}\text { VALVES } \\
\text { OPERATED } \\
0 \pm 1 " \mathrm{H}_{2} 0\end{array}$ & A & \\
\hline $\begin{array}{l}1.7 .4 .2 .15 \\
m x \cdot A \\
\end{array}$ & $\begin{array}{l}\text { CLOSE SV }-* 64 \\
\& \text { SV-*66 }\end{array}$ & $N / A$ & $N / A$ & $A$ & \\
\hline $\begin{array}{l}\text { Section } 1.7 \\
1.7 .4 \cdot 3 \cdot 1 \\
\text { min. } \dot{B}^{1} \\
\end{array}$ & $\frac{3 \text { verifies th }}{\text { READ PDI }-* 51}$ & $\frac{\text { grab samp }}{27^{\text {"HaO }}}$ & $\frac{\text { e flow } 100 p}{20-40 " \mathrm{H}_{2} \mathrm{O}}$ & $A$ & \\
\hline
\end{tabular}


TEST DATA SHEET

\begin{tabular}{|c|c|c|c|c|c|}
\hline \multicolumn{3}{|c|}{ Date: 12.19 .96} & \multicolumn{3}{|c|}{ SHMs Unit Number: VTP-PNL-330SI } \\
\hline \multicolumn{3}{|c|}{$\begin{array}{l}\text { Title of Iest: } \\
\text { Pneumatic Systems }\end{array}$} & \multirow{2}{*}{\multicolumn{3}{|c|}{$\begin{array}{l}\text { Test Equipment and } \mathrm{s} / \mathrm{N} \text { or Cal. No.: } \\
N / A\end{array}$}} \\
\hline \multicolumn{3}{|c|}{$\begin{aligned} \text { Test Performed By: } & \text { see Test Personnal } \\
& \text { Page } 12 \theta\end{aligned}$} & & & \\
\hline $\begin{array}{l}\text { Procedure No. } \\
\text { Initial/Date }\end{array}$ & I tem & Value & $\begin{array}{l}\text { Required } \\
\text { condition }\end{array}$ & $(A / R)$ & Comment \\
\hline $\begin{array}{l}1.7 .4 .3 .2 \\
m .7 .8 s \\
\end{array}$ & $\begin{array}{l}\text { PUSH PB-*59 } \\
\text { GRAB SAMPLE }\end{array}$ & $O K$ & $\begin{array}{l}\text { START } \\
\text { STOPWATCH }\end{array}$ & $A$ & \\
\hline $\begin{array}{l}1.7 .4 .3 .3 \\
m .2 x .8\end{array}$ & $\begin{array}{l}\text { GRAB SAMPLE } \\
\text { LAMPS ON } \\
\text { READ FIV }-* 52\end{array}$ & $\begin{array}{c}\frac{O K}{O K} \\
O K \\
O K \\
\end{array}$ & $\begin{array}{l}\text { PBL-*58 ON } \\
\text { PBL }-* 59 \text { ON } \\
\text { YAL }-* 58 \text { ON } \\
\text { FIV }-* 52 \quad 0 \\
\end{array}$ & A & \\
\hline $\begin{array}{l}1.7 .4 .3 .4 \\
M .2 . B\end{array}$ & $\begin{array}{l}\text { OPEN SV }-* 15 \\
\& \text { ADJ. FIV } \\
* 52\end{array}$ & $10 \mathrm{CF}$ & $\begin{array}{l}\text { SV-* } 15 \text { OPEN } \\
\text { FIV-*52 } \\
\text { FLOW } 10 \mathrm{CFH}\end{array}$ & $A$ & \\
\hline $\begin{array}{l}1.7 .4 .3 .5 \\
2 m .2 .0\end{array}$ & READ PDI $-* 51$ & $27.5^{\circ} \mathrm{H}_{2} \mathrm{O}$ & $\begin{array}{l}\text { PDI }-* 51 \\
\text { HIGHER THAN } \\
1.7 .4 .3 .1 \\
\end{array}$ & A & \\
\hline $\begin{array}{c}1.7 .4 .3 .6 \\
x . B . B \\
\end{array}$ & CLOSE SV-*15 & 0 & FIV $-\star 52 \quad 0$ & $A$ & \\
\hline $\begin{array}{r}1.7 .4 .3 .7 \\
\text { m. }\end{array}$ & OPEN SV $-* 15$ & ok & $\begin{array}{l}\text { FIV }-* 52 \\
\text { FLOW }\end{array}$ & A & \\
\hline $\begin{array}{l}1.7 .4 .3 .8 \\
\min ^{2} x\end{array}$ & $\begin{array}{l}\text { PBL }-* 59 \\
\text { GRAB SAMPLE } \\
\text { TIME }\end{array}$ & $\begin{array}{l}.0 K \\
5.00 \\
\end{array}$ & $\begin{array}{l}\text { PBL } * 59 \text { OFF } \\
\text { STOP WATCH } \\
5 \pm .5 \text { MIN }\end{array}$ & $A$ & \\
\hline $\begin{array}{l}1.7 .4 .3 .9 \\
\text { m.A. }{ }^{3} \\
\end{array}$ & $\begin{array}{l}\text { PB }-* 58 \text { RESET } \\
\text { SAMPLER }\end{array}$ & ok & $\begin{array}{l}\mathrm{PBL}-\star 58 \\
\text { OFF } \\
\text { YAL-*58 OFF } \\
\end{array}$ & $A$ & \\
\hline \multicolumn{6}{|c|}{ Section 1.7.4.4 verifies the $\mathrm{H}_{2}$ cell calibration loop. } \\
\hline $\begin{array}{l}1.7 .4 .4 .1 \\
20.8 .8\end{array}$ & $\begin{array}{l}\text { CONNECT } H_{2} \\
\text { CAL GAS }\end{array}$ & $N / A$ & $N / A$ & $A$ & \\
\hline $\begin{array}{l}1.7 .4 .4 .2 \\
\text { m.p.ps }\end{array}$ & $\begin{array}{l}\text { OPEN ISO } \\
\text { VALVE }\end{array}$ & OK & $<10$ PSIG & A & \\
\hline $\begin{array}{c}1.7 .4 .4 .3 \\
\text { im.p. } \\
\end{array}$ & $\begin{array}{l}\text { OPEN SV-*18 } \\
\text { ADJ. FIV-*56 }\end{array}$ & $2 \mathrm{CFU}$ & $2 \pm .1 \mathrm{CFH}$ & A & \\
\hline
\end{tabular}

abl 12-19-96 
TEST DATA SHEET

\begin{tabular}{|c|c|c|c|c|c|}
\hline \multicolumn{3}{|c|}{ Date: $\quad 12-19-96$} & \multicolumn{3}{|c|}{ SHMS Unit Number: VTP-PNL-3305I } \\
\hline \multicolumn{3}{|c|}{$\begin{array}{l}\text { Title of Test: } \\
\text { Pneumatic Systems }\end{array}$} & \multirow{2}{*}{\multicolumn{3}{|c|}{$\begin{array}{l}\text { Test Equipment and } S / \mathrm{N} \text { or Cal. No.: } \\
\text { F/u/a V/o meter } \\
5 / N 44620364 \\
\text { eol. due } 10-97\end{array}$}} \\
\hline \multicolumn{3}{|c|}{$\begin{array}{c}\text { Test Performed By: See Test Personnel } \\
\text { Page } 128\end{array}$} & & & \\
\hline $\begin{array}{l}\text { Procedure No. } \\
\text { Initial/Date }\end{array}$ & Item & Value & $\begin{array}{l}\text { Required } \\
\text { Condition }\end{array}$ & $(A / R)$ & Comment \\
\hline $\begin{array}{l}1.7 .4 .4 .4 \\
9 m .2 .8 \\
\end{array}$ & $\begin{array}{l}\text { VERIFY } \\
\text { CONDITIONS }\end{array}$ & $\frac{\frac{0.629 \% H_{2}}{\frac{O K}{O K}}}{\frac{\frac{O K}{O K}}{\frac{O K}{O K}}}$ & $\begin{array}{l}\text { NIT-*54 } \\
>.625 \% \mathrm{H}_{2} \\
\text { NAH-*55 ON } \\
\text { NAH-*54 ON } \\
\text { PBL-*59 ON } \\
\text { PBL-*58 ON } \\
\text { YAL-*58 ON } \\
\text { FIV-*52 FLO } \\
\text { HORN ON } \\
\text { TB2-21/22 } \\
\text { OPEN } \\
\end{array}$ & $A$ & \\
\hline $\begin{array}{l}1.7 .4 .4 .5 \\
\text { mix } 10 \\
\end{array}$ & $\begin{array}{l}\text { ACKNOWLEDGE } \\
\text { HORN }\end{array}$ & $N / A$ & $N / A$ & A & \\
\hline $\begin{array}{l}1.7 .4 .4 .6 \\
M 20\end{array}$ & $\begin{array}{l}\text { CLOSE SV-*18 } \\
\text { VERIFY } \\
\text { CONDITIONS }\end{array}$ & $\begin{array}{l}-\frac{O K}{0.602} \\
\frac{0 K}{0 K} \\
\frac{O K}{O K} \\
\frac{O K}{0} \\
\end{array}$ & $\begin{array}{l}\text { FIV }-* 560 \\
\text { NIT-*54 } \\
<.625 \% \mathrm{H}_{2} \\
\text { NAH- } * 55 \text { OFF } \\
\text { NAH-*54 ON } \\
\text { PBL-*58 ON } \\
\text { YAL }-* 58 \text { ON } \\
\text { TB2-21/22 } \\
\text { CLOSED } \\
\end{array}$ & A & \\
\hline $\begin{array}{c}1.7 .4 .4 .7 \\
\text { m2.0. } \\
\end{array}$ & $\begin{array}{l}\text { PUSH RESET } \\
\text { SAMPLER PB- } \\
* 58\end{array}$ & $-\frac{O R}{O K}$ & $\begin{array}{l}\mathrm{PBL}-\star 58 \text { OFF } \\
\text { YAL }-\star 58 \text { OFF }\end{array}$ & A & \\
\hline $\begin{array}{c}1.7 .4 .4 .8 \\
2 \times 20 \\
\end{array}$ & RESET ALARM & LE & $\mathrm{NAH}-\star 54$ OFF & $A$ & \\
\hline $\begin{array}{l}1.7 .4 .4 .9 \\
\not \sin R \\
\end{array}$ & $\begin{array}{l}\text { OPEN SV-*19 } \\
\text { ADJ. FIV-*56 }\end{array}$ & $2 \mathrm{CFH}$ & $2 \pm .1 \mathrm{CFH}$ & A & \\
\hline
\end{tabular}

al1 $12-19-26$ 
TEST DATA SHEET

\begin{tabular}{|c|c|c|c|c|c|}
\hline \multicolumn{3}{|c|}{ Date: $\quad 12 \cdot 19-96$} & \multicolumn{3}{|c|}{ SHMS Unit Number: VTP - PNL -3305 I } \\
\hline \multicolumn{3}{|c|}{$\begin{array}{l}\text { ritle of rest: } \\
\text { Pneumatic Systems }\end{array}$} & \multirow{2}{*}{\multicolumn{3}{|c|}{$\begin{array}{l}\text { Test Equipment and } S / \mathrm{N} \text { or Cal. No.: } \\
\text { Fluke V/o Meter } \\
S / N 44620364 \\
\text { Cai. due } 10-97\end{array}$}} \\
\hline Test Performed B & $\begin{array}{l}\text { see Test Perse } \\
\text { Page } 128\end{array}$ & & & & \\
\hline $\begin{array}{l}\text { Procedure No. } \\
\text { Initial/Date }\end{array}$ & Item & value & $\begin{array}{l}\text { Requi red } \\
\text { Condition }\end{array}$ & $(A / R)$ & Corment \\
\hline$\frac{1.7 .4 .4 .10}{x+x .8}$ & $\begin{array}{l}\text { VERIFY } \\
\text { CONDITIONS }\end{array}$ & 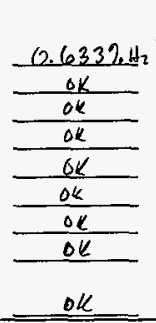 & 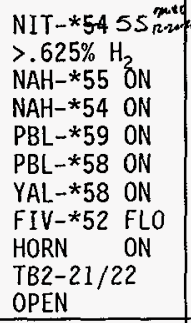 & A & \\
\hline $\begin{array}{l}1.7 .4 .4 .11 \\
\text { nin.s }\end{array}$ & $\begin{array}{l}\text { ACKNOWLEDGE } \\
\text { HORN }\end{array}$ & N/A & N/A & A & \\
\hline$\frac{1.7 .4 .4 .12}{20,2.8}$ & $\begin{array}{l}\text { CLOSE SV-*19 } \\
\text { VERIFY } \\
\text { CONDITIONS }\end{array}$ & $\frac{\frac{O K}{0.60 t}}{\frac{0 K}{O k}} \frac{0 k}{\frac{O K}{O K}}$ & 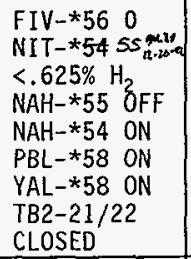 & A & \\
\hline $\begin{array}{l}1.7 .4 .4 .13 \\
m .7 . e\end{array}$ & $\begin{array}{l}\text { PUSH RESET } \\
\text { SAMPLER PB- } \\
* 58\end{array}$ & -ok & $\begin{array}{l}\text { PBL-*58 OFF } \\
\text { YAL-*58 OFF }\end{array}$ & $A$ & \\
\hline $\begin{array}{r}7.4 .4 .14 \\
m_{3 . \beta} \\
\end{array}$ & RESET ALARM & or & NAH $-* 54$ OFF & A & \\
\hline$\frac{1.7 .4 .4 .15}{2 \operatorname{sen}}$ & $\begin{array}{l}\text { DISCONNECT } \\
\text { CAL. GAS }\end{array}$ & N/A & N/A & A & \\
\hline$\frac{1.7 .4 .4 .16}{\operatorname{mip.p}}$ & $\begin{array}{l}\text { PUSH PB-*54 } \\
\text { VERIFY } \\
\text { CONDITIONS }\end{array}$ & $\frac{\text { ok }}{\text { or }}$ & $\begin{array}{l}X A-* 63 \text { ON } \\
\text { PBL-*54 ON } \\
\text { TB2-23/24 } \\
\text { OPEN }\end{array}$ & $A$ & \\
\hline $\begin{array}{l}1.7 .4 .4 .17 \\
\min 2.8\end{array}$ & $\begin{array}{l}\text { PUSH PB }-* 54 \\
\text { VERIFY } \\
\text { CONDITIONS }\end{array}$ & ox & $\begin{array}{l}X A-* 63 \text { OFF } \\
\text { PBL-*54 OFF } \\
\text { TB2-23/24 } \\
\text { CLOSED }\end{array}$ & $A$ & \\
\hline
\end{tabular}


TEST DATA SHEET

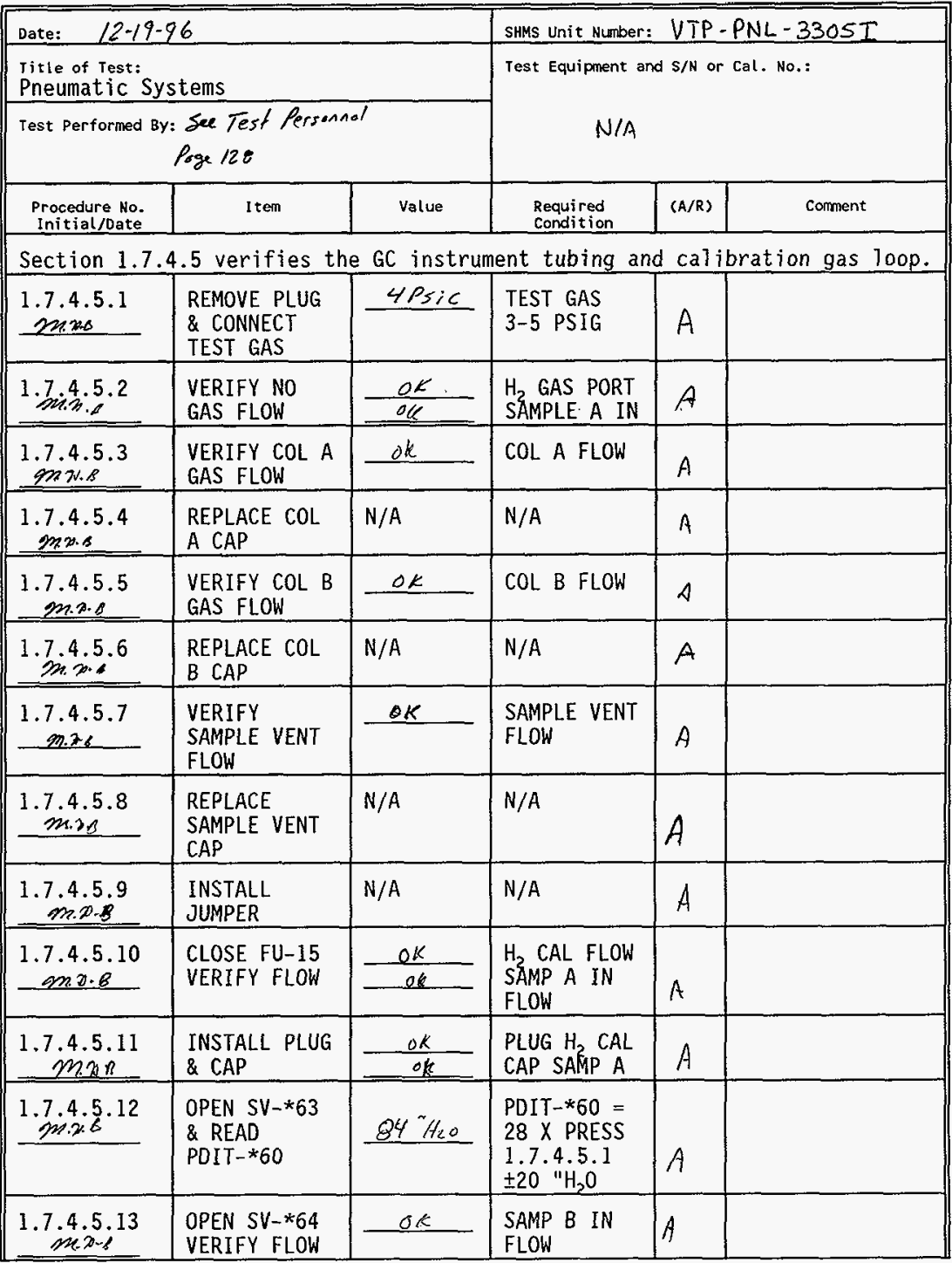


TEST DATA SHEET

\begin{tabular}{|c|c|c|c|c|c|}
\hline \multirow{2}{*}{\multicolumn{3}{|c|}{$\begin{array}{l}\text { Date: } \quad 12-19-96 \\
\text { Titte of Test: } \\
\text { Pneumatic Systems }\end{array}$}} & \multicolumn{3}{|c|}{ SHMS Unit Number: VTP - PNL $-3305 I$} \\
\hline & & & \multirow{2}{*}{\multicolumn{3}{|c|}{$\begin{array}{l}\text { Test Equipment and } S / N \text { or Cal. No.: } \\
N / A\end{array}$}} \\
\hline \multicolumn{3}{|c|}{$\begin{aligned} & \text { Test Performed sy: } \text { see Test Personnal } \\
& \text { Page } 128\end{aligned}$} & & & \\
\hline $\begin{array}{l}\text { Procedure No. } \\
\text { Initial/Date }\end{array}$ & I tem & Value & $\begin{array}{l}\text { Required } \\
\text { Condition }\end{array}$ & $(A / R)$ & comment \\
\hline$\frac{1.7 .4 .5 .14}{m \times s}$ & $\begin{array}{l}\text { REMOVE TEST } \\
\text { GAS }\end{array}$ & $\mathrm{N} / \mathrm{A}$ & N/A & A & \\
\hline $\begin{array}{l}1.7 .4 .5 .15 \\
x_{1,2 . \beta}\end{array}$ & $\begin{array}{l}\text { CLOSE SV-*63 } \\
\& \text { SV-*64 }\end{array}$ & $N / A$ & N/A & A & \\
\hline$\frac{1.7 .4 .5 .16}{2 x .28}$ & $\begin{array}{l}\text { OPEN FU-15 } \\
\text { REMOVE } \\
\text { JUMPER } \\
\end{array}$ & $N / A$ & $N / A$ & A & \\
\hline $\begin{array}{l}1.7 .4 .5 .17 \\
m_{x-\beta} \\
\end{array}$ & $\begin{array}{l}\text { INSTALL } \\
\text { SAMP A UNION }\end{array}$ & N/A & $N / A$ & A & \\
\hline $\begin{array}{l}1.7 .4 .5 .18 \\
\end{array}$ & $\begin{array}{l}\text { OPEN SV-*60 } \\
\text { VERIFY FLOW }\end{array}$ & $O K$ & $\begin{array}{l}\text { FI-*60 } \\
\text { NO FLOW }\end{array}$ & A & \\
\hline$\frac{1.7 .4 .5 .19}{m \text { m.3. }}$ & $\begin{array}{l}\text { POWER SOV- } \\
\star 60 \text {, VERIFY } \\
\text { NO FLOW }\end{array}$ & ok & $\begin{array}{l}\text { FI-*60 } \\
\text { NO FLOW }\end{array}$ & A & \\
\hline $\begin{array}{l}1.7 .4 .5 .20 \\
m, x, B\end{array}$ & $\begin{array}{l}\text { OPEN SV-*68 } \\
A 0 J \text { SV-*67 }\end{array}$ & OK & $\begin{array}{l}\mathrm{FI}-* 60 \\
50 \mathrm{CCM} \\
\end{array}$ & A & \\
\hline $\begin{array}{l}1.7 .4 .5 .21 \\
m=0 \pi \\
\end{array}$ & $\begin{array}{l}\text { DEENERGIZE } \\
\text { SOV }-* 60 \\
\end{array}$ & ok & $\begin{array}{l}\text { FI }-* 60 \text { FLOW } \\
\text { DECREASES }\end{array}$ & $A$ & \\
\hline $\begin{array}{l}1.7 .4 .5 .22 \\
M .2 .0 \\
\end{array}$ & $\begin{array}{l}\text { CLOSE SV-*60 } \\
\& \text { SV }-* 68\end{array}$ & $N / A$ & N/A & $A$ & \\
\hline $\begin{array}{r}1.7 .4 .5 .23 \\
m \cdot x \cdot p \\
\end{array}$ & $\begin{array}{l}\text { REMOVE } \\
\text { SAMP A UNION }\end{array}$ & N/A & $\mathrm{N} / \mathrm{A}$ & $A$ & \\
\hline$\frac{1.7 .4 .5 .24}{m \cdot 20: B^{2}}$ & $\begin{array}{l}\text { CONNECT TEST } \\
\text { GAS } \mathrm{N}_{2} \mathrm{O} \text { PORT }\end{array}$ & $d K$ & $\begin{array}{l}\text { TEST GAS } \\
3-5 \text { PSIG }\end{array}$ & A & \\
\hline$\frac{1.7 .4 .5 .25}{m .2 .8}$ & $\begin{array}{l}\text { VERIFY NO } \\
\text { FLOW }\end{array}$ & $\frac{O K}{\partial K}$ & $\begin{array}{l}\text { SAMP B IN \& } \\
\text { FLT } * 63 \\
\text { NO FLOW }\end{array}$ & A & \\
\hline $\begin{array}{l}1.7 .4 .5 .26 \\
M .3 . B \\
\end{array}$ & $\begin{array}{l}\text { INSTALL } \\
\text { JUMPER }\end{array}$ & $N / A$ & $\mathrm{~N} / \mathrm{A}$ & $A$ & \\
\hline $\begin{array}{l}1.7 .4 .5 .27 \\
\sin 2.0\end{array}$ & $\begin{array}{l}\text { CLOSE FU-15 } \\
\text { VERIFY FLOW }\end{array}$ & $\frac{O K}{\partial k}$ & $\begin{array}{l}\text { SAMP B IN } \\
\text { FLT-* } 63 \\
\text { FLOW }\end{array}$ & A & \\
\hline
\end{tabular}


TEST DATA SHEET

\begin{tabular}{|c|c|c|c|c|c|}
\hline \multirow{2}{*}{\multicolumn{3}{|c|}{$\begin{array}{l}\text { Date: } \quad 12-19-96 \\
\text { Titte of Test: } \\
\text { Pneumatic Systems }\end{array}$}} & \multicolumn{3}{|c|}{ SHMS Unit Number: VTP.PNL-3305T } \\
\hline & & & \multirow{2}{*}{\multicolumn{3}{|c|}{$\begin{array}{l}\text { Test Equipment and } S / \mathrm{N} \text { or Cal. No.: } \\
\text { N/A }\end{array}$}} \\
\hline \multicolumn{3}{|c|}{$\begin{aligned} \text { Test Performed 8y: } & \text { soe Test Personned } \\
& \text { Page } 128\end{aligned}$} & & & \\
\hline $\begin{array}{l}\text { Procedure No. } \\
\text { Initial/Date }\end{array}$ & Item & Value & $\begin{array}{l}\text { Required } \\
\text { Condition }\end{array}$ & $(A / R)$ & Comment \\
\hline$\frac{1.7 .4 .5 .28}{m_{1.0 .0}}$ & $\begin{array}{l}\text { DISCONNECT } \\
\text { TEST GAS }\end{array}$ & $N / A$ & N/A & $A$ & \\
\hline 1.7 .4 .5 .29 & OPEN FU-15 & $N / A$ & N/A & $A$ & \\
\hline $\begin{array}{l}1.7 .4 .5 .30 \\
\text { onise. }\end{array}$ & $\begin{array}{l}\text { REMOVE } \\
\text { JUMPER }\end{array}$ & $N / A$ & $N / A$ & A & \\
\hline $\begin{array}{c}1.7 .4 .5 .31 \\
\text { min.x.4 } \\
\end{array}$ & $\begin{array}{l}\text { INSTALL } \\
\text { SAMP B UNION }\end{array}$ & $N / A$ & $\mathrm{~N} / \mathrm{A}$ & $A$ & \\
\hline $\begin{array}{c}1.7 .4 .5 .32 \\
m=2.8 \\
\end{array}$ & $\begin{array}{l}\text { OPEN SV-*60 } \\
\text { VERIFY FLOW }\end{array}$ & OK & $\begin{array}{l}\text { FI-*60 } \\
\text { NO FLOW }\end{array}$ & A & \\
\hline$\frac{1.7 .4 .5 .33}{\operatorname{mos} 28}$ & $\begin{array}{l}\text { POWER SOV- } \\
* 60, \text { VERIFY } \\
\text { NO FLOW } \\
\end{array}$ & OK & $\begin{array}{l}\text { FI-*60 } \\
\text { NO FLOW }\end{array}$ & $A$ & \\
\hline $\begin{array}{c}1.7 .4 .5 .34 \\
\text { mive }\end{array}$ & $\begin{array}{l}\text { OPEN SV }-* 68 \\
\& \text { ADJ SV }-* 67\end{array}$ & ak & $\begin{array}{l}\mathrm{FI}-* 60 \\
50 \mathrm{CCM}\end{array}$ & A & \\
\hline $\begin{array}{l}1.7 .4 .5 .35 \\
\min _{. B}\end{array}$ & $\begin{array}{l}\text { DEENERGIZE } \\
\text { SOV } * 60\end{array}$ & $O C$ & $\begin{array}{l}\text { FI-*60 FLOW } \\
\text { DECREASES }\end{array}$ & $A$ & \\
\hline $\begin{array}{c}1.7 .4 .5 .36 \\
m .9 .9 \\
\end{array}$ & $\begin{array}{l}\text { CLOSE SV-*60 } \\
\& \text { SV }-* 68\end{array}$ & $N / A$ & $\mathrm{~N} / \mathrm{A}$ & $A$ & \\
\hline $\min _{7.83} \cdot 4.5 .37$ & $\begin{array}{l}\text { REMOVE } \\
\text { SAMP B UNION }\end{array}$ & $N / A$ & $N / A$ & A & \\
\hline $\begin{array}{l}1.7 .4 .5 .38 \\
m .10 \\
\end{array}$ & $\begin{array}{l}\text { OPEN CB- } 3 / 4 \\
\text { ACK. ALARMS } \\
\end{array}$ & $N / A$ & $N / A$ & A & \\
\hline \multicolumn{6}{|c|}{$\begin{array}{l}\text { Section } 1.7 .4 .6 \text { verifies the multi gas analyzer instrument tubing and } \\
\text { calibration gas loop. }\end{array}$} \\
\hline $\begin{array}{l}1.7 .4 .6 .1 \\
m .2 .3\end{array}$ & $\begin{array}{l}\text { VERIFY } \\
\text { NIT-*52 } \\
\text { SAMPLE LINES } \\
\end{array}$ & $N / A$ & $N / A$ & $A$ & \\
\hline $\begin{array}{l}1.7 .4 .6 .2 \\
m \times s\end{array}$ & $\begin{array}{l}\text { CONNECT TEST } \\
\text { GAS NH } \\
\end{array}$ & ok & $\begin{array}{l}\text { TEST GAS } \\
3-5 \text { PSIG } \\
\end{array}$ & $A$ & \\
\hline $\begin{array}{c}1.7 .4 .6 .3 \\
\text { miny.s }\end{array}$ & $\begin{array}{l}\text { VERIFY NO } \\
\text { FLOW }\end{array}$ & $\frac{x a k}{04}$ & $\begin{array}{l}\text { SAMPLE IN } \\
\text { FLT }-* 63\end{array}$ & A & \\
\hline
\end{tabular}


TEST DATA SHEET

\begin{tabular}{|c|c|c|c|c|c|}
\hline \multicolumn{3}{|c|}{ Date: $\quad 12-19-96$} & \multicolumn{3}{|c|}{ SHMS Unit Number: VTP-PNL-3305I } \\
\hline \multicolumn{3}{|c|}{$\begin{array}{l}\text { Title of Test: } \\
\text { Pneumatic Systems }\end{array}$} & \multirow{2}{*}{\multicolumn{3}{|c|}{$\begin{array}{l}\text { Test Equipment and } S / N \text { or Cal. No.: } \\
N / A\end{array}$}} \\
\hline \multicolumn{3}{|c|}{$\begin{aligned} & \text { Test Perforned By: } \text { see Test Personnd } \\
& \text { Poge } 128\end{aligned}$} & & & \\
\hline $\begin{array}{l}\text { Procedure No. } \\
\text { Initial/Date }\end{array}$ & Item & value & $\begin{array}{l}\text { Required } \\
\text { condition }\end{array}$ & $(A / R)$ & Comment \\
\hline $\begin{array}{c}1.7 .4 .6 .4 \\
\text { ma.n }\end{array}$ & $\begin{array}{l}\text { INSTALL } \\
\text { JUMPER }\end{array}$ & N/A & N/A & A & \\
\hline $\begin{array}{c}1.7 .4 .6 .5 \\
m 8 . R\end{array}$ & $\begin{array}{l}\text { CLOSE FU-15 } \\
\text { VERIFY GAS } \\
\text { FLOWS }\end{array}$ & $\frac{O K}{O K}$ & $\begin{array}{l}\text { SAMPLE IN } \\
\text { FLT-*63 } \\
\text { FIV-*70 } \\
\end{array}$ & A & \\
\hline $\begin{array}{c}1.7 .4 .6 .6 \\
m 0.8 \\
\end{array}$ & $\begin{array}{l}\text { PLUG FLT-*63 } \\
\& \text { MON-*60 } \\
\text { SAMPLE VENT }\end{array}$ & N/A & N/A & A & \\
\hline $\begin{array}{l}1.7 .4 .6 .7 \\
\min _{3}\end{array}$ & $\begin{array}{l}\text { OPEN SV }-* 66 \\
\text { SV-*70 \& CAP } \\
\text { SAMPLE IN }\end{array}$ & $136^{4} \mathrm{H}_{2} \mathrm{O}$ & $\begin{array}{l}\text { PDIT-*60 = } \\
28 \times \text { PRESS } \\
1.7 .4 .6 .2 \\
\pm 20{ }^{11 \mathrm{H}_{2} \mathrm{O}}\end{array}$ & A & \\
\hline $\begin{array}{l}1.7 .4 .6 .8 \\
\operatorname{mix}\end{array}$ & $\begin{array}{l}\text { CLOSE SV-*66 } \\
\text { SV-*70 \& } \\
\text { REMOVE CAP } \\
\end{array}$ & N/A & N/A & $A$ & \\
\hline $\begin{array}{c}1.7 .4 .6 .9 \\
m . \lambda B \\
\end{array}$ & $\begin{array}{l}\text { REMOVE FLT- } \\
* 63 \text { PLUG } \\
\end{array}$ & N/A & N/A & A & \\
\hline $\begin{array}{c}1.7 .4 .6 .10 \\
m .2 .8 \\
\end{array}$ & $\begin{array}{l}\text { REMOVE TEST } \\
\text { GAS }\end{array}$ & N/A & $N / A$ & A & \\
\hline $\begin{array}{c}1.7 .4 .6 .11 \\
m .3 . \\
\end{array}$ & $\begin{array}{l}\text { CLOSE SV-*05 } \\
\& \text { SV-*16 }\end{array}$ & N/A & N/A & $A$ & \\
\hline $\begin{array}{l}1.7 .4 .6 .12 \\
M .2 .13 \\
\end{array}$ & $\begin{array}{l}\text { CONNECT TEST } \\
\text { GAS } \\
\end{array}$ & $4 P_{516}$ & $\begin{array}{l}\text { TEST GAS } \\
2-4 \text { PSIG } \\
\end{array}$ & $A$ & \\
\hline $\begin{array}{l}1.7 .4 .6 .13 \\
m . x . B \\
\end{array}$ & $\begin{array}{l}\text { OPEN SV }-* 07 \\
\& \text { VERIFY } \\
\text { NO FLOW } \\
\end{array}$ & $\frac{O K}{O K}$ & $\begin{array}{l}\text { SAMPLE IN } \\
\text { SAMPLE OUT }\end{array}$ & A & \\
\hline$\frac{1.7 .4 .6 .14}{3 \times 3.3}$ & $\begin{array}{l}\text { OPEN SV }-* 09 \\
\& \text { VERIFY } \\
\text { NO FLOW } \\
\end{array}$ & $\frac{O K}{O K}$ & $\begin{array}{l}\text { SAMPLE IN } \\
\text { SAMPLE OUT }\end{array}$ & A & \\
\hline $\begin{array}{l}1.7 .4 .6 .15 \\
m .3, n \\
\end{array}$ & $\begin{array}{l}\text { OPEN FU-15 \& } \\
\text { VERIFY FLOW }\end{array}$ & $O K$ & $\begin{array}{l}\text { SAMPLE FLOW } \\
\text { IN - YES } \\
\text { OUT - NO } \\
\end{array}$ & A & \\
\hline $\begin{array}{c}1.7 .4 .6 .16 \\
\text { mN. }\end{array}$ & $\begin{array}{l}\text { CLOSE SV-*09 } \\
\text { VERIFY FLOW }\end{array}$ & ok & $\begin{array}{l}\text { SAMPLE IN } \\
\text { NO FLOW }\end{array}$ & A & \\
\hline
\end{tabular}


TEST DATA SHEET

\begin{tabular}{|c|c|c|c|c|c|}
\hline \multicolumn{3}{|c|}{ Date: $\quad 12-19-96$} & \multicolumn{3}{|c|}{ SHMS Unit Number: VTP.PNL-3305I } \\
\hline \multicolumn{3}{|c|}{$\begin{array}{l}\text { Title of Test: } \\
\text { Pneumatic Systems }\end{array}$} & \multirow{2}{*}{\multicolumn{3}{|c|}{$\begin{array}{l}\text { Test Equipment and } s / M \text { or cal. No.: } \\
\qquad N / A\end{array}$}} \\
\hline \multicolumn{3}{|c|}{$\begin{aligned} \text { Test Performed by: } & \text { See Test Personnal } \\
& \text { Page } 12 e\end{aligned}$} & & & \\
\hline $\begin{array}{l}\text { Procedure No. } \\
\text { Initial/Date }\end{array}$ & Item & Value & $\begin{array}{l}\text { Required } \\
\text { Condition }\end{array}$ & $(A / R)$ & Comment \\
\hline$\frac{1.7 .4 .6 .17}{m_{n .3}}$ & $\begin{array}{l}\text { OPEN SV-* } 10 \\
\text { VERIFY FLOW }\end{array}$ & $\frac{\partial K}{O K}$ & $\begin{array}{l}\text { SAMPLE FLOW } \\
\text { IN - NO } \\
\text { OUT - YES }\end{array}$ & $A$ & \\
\hline $\begin{array}{r}1.7 .4 .6 .18 \\
m \cdot 2.3 \\
\end{array}$ & $\begin{array}{l}\text { REMOVE TEST } \\
\text { GAS \& PLUG } \\
\text { SV }-* 07\end{array}$ & N/A & $N / A$ & A & \\
\hline$\frac{1.7 .4 .6 .19}{\mathrm{m.ms}}$ & $\begin{array}{l}\text { CLOSE SV-*07 } \\
\& \text { SV-* } 10\end{array}$ & $N / A$ & $N / A$ & $A$ & \\
\hline $\begin{array}{c}1.7 .4 .6 .20 \\
m_{2} . B . B\end{array}$ & $\begin{array}{l}\text { OPEN SV }-* 05 \\
\& \text { SV-*16 }\end{array}$ & $N / A$ & $\mathrm{~N} / \mathrm{A}$ & A & \\
\hline $\begin{array}{l}1.7 .4 .6 .21 \\
2 m .8 \\
\end{array}$ & $\begin{array}{l}\text { REMOVE } \\
\text { JUMPER } \\
\end{array}$ & $N / A$ & $N / A$ & A & \\
\hline \multicolumn{6}{|c|}{ Section 1.7.4.7 verifies the carrier gas supply lines. } \\
\hline $\begin{array}{c}1.7 .4 .7 .1 \\
\operatorname{man.B}\end{array}$ & $\begin{array}{l}\text { CONNECT TEST } \\
\text { GAS }\end{array}$ & ok & 3-5 PSIG & A & \\
\hline$\frac{1.7 .4 .7 .2}{m .2 .8}$ & $\begin{array}{l}\text { REMOVE PLUG } \\
\& \text { VERIFY } \\
\text { FLOW } \\
\end{array}$ & die & $\begin{array}{l}\mathrm{N}_{3} \text { CARRIER } \\
\text { GAS FLOWS }\end{array}$ & $A$ & \\
\hline $\begin{array}{c}1.7 .4 .7 .3 \\
m \cdot x \cdot x^{2}\end{array}$ & $\begin{array}{l}\text { DISCONNECT } \\
\text { TEST GAS }\end{array}$ & $N / A$ & $N / A$ & $A$ & \\
\hline $\begin{array}{l}1.7 .4 .7 .4 \\
m_{2.20}\end{array}$ & $\begin{array}{l}\text { CONNECT TEST } \\
\text { GAS }\end{array}$ & ok & 3-5 PSIG & $A$ & \\
\hline $\begin{array}{l}1.7 .4 .7 .5 \\
m \times 1 .\end{array}$ & $\begin{array}{l}\text { REMOVE PLUG } \\
\& \text { VERIFY } \\
\text { FLOW } \\
\end{array}$ & $8 k$ & $\begin{array}{l}\text { He CARRIER } \\
\text { GAS FLOWS }\end{array}$ & $A$ & \\
\hline $\begin{array}{l}1.7 .4 .7 .6 \\
m .7 .03 \\
\end{array}$ & $\begin{array}{l}\text { DISCONNECT } \\
\text { TEST GAS }\end{array}$ & $N / A$ & $\mathrm{~N} / \mathrm{A}$ & $A$ & \\
\hline $\begin{array}{l}1.7 .5 \\
779.2 . \mathrm{B} \\
\end{array}$ & $\begin{array}{l}\text { TEST SEC. } \\
1.7 .4 \text { DONE }\end{array}$ & $\frac{10}{\text { Test Dir }}$ & oreide & & $\frac{12-19-96}{\text { Date }}$ \\
\hline
\end{tabular}


TEST DATA SHEET

\begin{tabular}{|c|c|c|c|c|c|}
\hline \multicolumn{3}{|c|}{ Date: $\quad 12-19-96$} & \multicolumn{3}{|c|}{ SHMS Unit Number: VTP - PNL-3305I } \\
\hline \multicolumn{3}{|c|}{$\begin{array}{l}\text { Title of Test: } \\
\text { Pneumatic Systems }\end{array}$} & \multirow{2}{*}{\multicolumn{3}{|c|}{$\begin{array}{l}\text { Test Equipment and S/N or Cal. No.: } \\
\text { Ammonia botthe No. ALmo58830 } \\
\text { Hydrogen bottle No. All655 } \\
\text { Methene bottle No. } 62650637\end{array}$}} \\
\hline \multicolumn{3}{|c|}{$\begin{aligned} \text { Test Performed By: } & \text { see Test Personnol } \\
& \text { Poge } 128\end{aligned}$} & & & \\
\hline $\begin{array}{l}\text { Procedure No. } \\
\text { Initial/Date }\end{array}$ & I tem & value & $\begin{array}{l}\text { Required } \\
\text { Condition }\end{array}$ & $(A / R)$ & Corment \\
\hline $\begin{array}{l}1.7 .6 \\
\text { m.n.n }\end{array}$ & \multicolumn{5}{|c|}{$\begin{array}{l}\text { THIS SECTION IS ONLY FOR THE SHMS-E IN THE ANALYTICAL MODE. } \\
\text { IF NOT APPLICABLE, N/A STEPS AND TEST DIRECTOR WILL SIGN. }\end{array}$} \\
\hline & Test Director & Signature & & Date & \\
\hline $\begin{array}{c}1.7 .6 .1 .1 \\
\text { mx.e }\end{array}$ & $\begin{array}{l}\text { VERIFY BKR } \\
\text { LINE UP }\end{array}$ & N/A & N/A & A & \\
\hline $\min ^{1.7 .6 .1 .2}$ & $\begin{array}{l}\text { VERIFY VALVE } \\
\text { LINE UP }\end{array}$ & $N / A$ & $N / A$ & A & \\
\hline$\underbrace{1.7 .6 .1 .3}_{\text {mos }}$ & $\begin{array}{l}\text { VERIFY MON- } \\
* 60 \text { INSTALL }\end{array}$ & $N / A$ & $N / A$ & A & \\
\hline $\begin{array}{c}1.7 .6 .1 .4 \\
\max \end{array}$ & $\begin{array}{l}\text { VERIFY NIT- } \\
\text { *52 INSTALL }\end{array}$ & $N / A$ & N/A & $A$ & \\
\hline $\begin{array}{c}1.7 .6 .1 .5 \\
2 \mathrm{mas}^{2}\end{array}$ & $\begin{array}{l}\text { CONNECT } \mathrm{H}_{2} \\
\text { CAL GAS }\end{array}$ & $N / A$ & $N / A$ & A & \\
\hline $\begin{array}{l}1.7 .6 .1 .6 \\
\operatorname{m.25}\end{array}$ & $\begin{array}{l}\text { CONNECT } \mathrm{CH}_{4} \\
\text { CAL GAS }\end{array}$ & N/A & N/A & A & \\
\hline $\begin{array}{l}1.7 .6 .1 .7 \\
\text { M.N. }\end{array}$ & $\begin{array}{l}\text { CONNECT } \mathrm{NH}_{3} \\
\text { CAL GAS }\end{array}$ & $N / A$ & N/A & A & \\
\hline Section 1. & 2 establishes & he main & auxiliary & p & \\
\hline $\begin{array}{l}1.7 .6 .2 .1 \\
\text { m.p.s }\end{array}$ & CLOSE FU-11 & ok & $\begin{array}{l}\text { MON-*60 } \\
\text { POWERED }\end{array}$ & A & \\
\hline $\begin{array}{l}1.7 .6 .2 .2 \\
m .2 . x\end{array}$ & POWER PC $-* 60$ & $O Q$ & $\begin{array}{l}\text { ESTABLISH } \\
\text { SAMPLING } \\
\text { ROUTINE } \\
\end{array}$ & $A$ & \\
\hline $\begin{array}{l}1.7 .6 .2 .3 \\
\text { mine }\end{array}$ & CLOSE FU-12 & ok & $\begin{array}{l}\text { NIT-*552 } \\
\text { POWERED } \\
\end{array}$ & A & \\
\hline $\begin{array}{c}1.7 .6 .2 .4 \\
m . x \\
\end{array}$ & POWER PC $-* 70$ & ok & $\begin{array}{l}\text { PC-*70 } \\
\text { BO0TED UP }\end{array}$ & $A$ & \\
\hline $\begin{array}{l}1.7 .6 .2 .5 \\
\text { am.2.n }\end{array}$ & $\begin{array}{l}\text { CLOSE CB-3/4 } \\
\text { ADJUST FLOW }\end{array}$ & $1.1 " \mathrm{H} / 2 \mathrm{O}$ & $\begin{array}{l}\mathrm{FIT}-\star 57 \\
1 \pm .1 \quad \mathrm{H}_{2} \mathrm{O}\end{array}$ & A & \\
\hline
\end{tabular}


APPENDIX D

TEST DATA SHEET

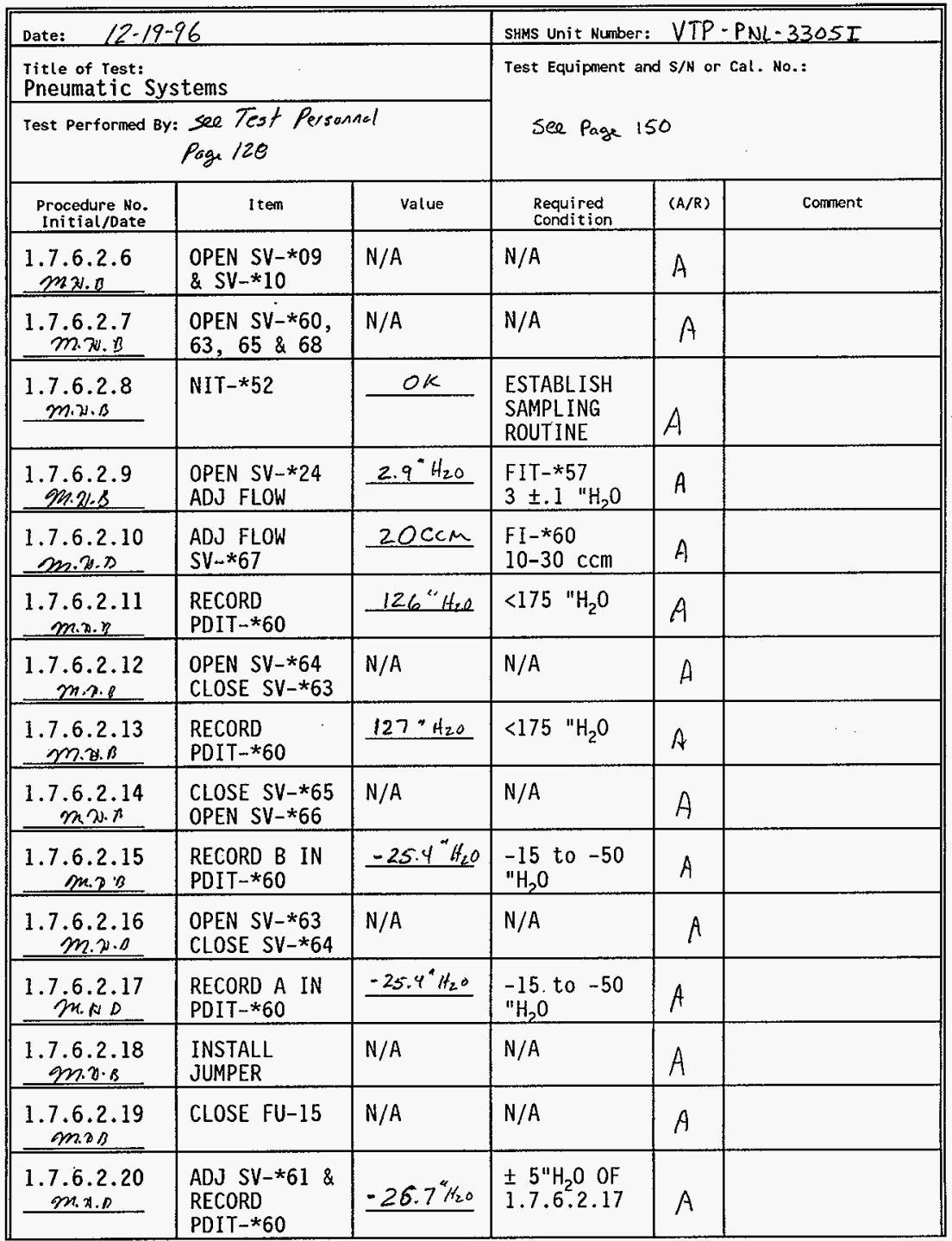


TEST DATA SHEET

\begin{tabular}{|c|c|c|c|c|c|}
\hline \multicolumn{3}{|c|}{ Date: $\quad 12-19-96$} & \multicolumn{3}{|c|}{ SHMS Unit Number: VTP-PNL-330SI } \\
\hline \multicolumn{3}{|c|}{$\begin{array}{l}\text { Title of Test: } \\
\text { Pneumatic Systems }\end{array}$} & \multirow{2}{*}{\multicolumn{3}{|c|}{$\begin{array}{l}\text { Test Equipment and S/N or Cal. No.: } \\
\text { See page } 150\end{array}$}} \\
\hline \multicolumn{3}{|c|}{$\begin{aligned} & \text { Test Performed By: } \text { See Test Personnd } \\
& \text { loge } 12 e\end{aligned}$} & & & \\
\hline $\begin{array}{l}\text { Procedure No. } \\
\text { Initial/Date }\end{array}$ & Item & value & $\begin{array}{l}\text { Required } \\
\text { Condition }\end{array}$ & $(A / R)$ & Coment \\
\hline $\begin{array}{c}1.7 .6 .2 .21 \\
m .2 .8 \\
\end{array}$ & $\begin{array}{l}\text { CAL MON-*60 } \\
\text { CHANNEL A }\end{array}$ & $\begin{array}{l}10480.5 \mathrm{PPM} \\
10533.6 \mathrm{PPM} \\
105.42 .3 \mathrm{PPM}\end{array}$ & $\begin{array}{l}\text { READINGS } \\
\text { WITHIN } \pm 5 \%\end{array}$ & $A$ & Hydregen \\
\hline $\begin{array}{c}1.7 .6 .2 .22 \\
m .2 .0 \\
\end{array}$ & OPEN FU-15 & $N / A$ & N/A & A & \\
\hline $\begin{array}{l}1.7 .6 .2 .23 \\
\text { m.7. . B } \\
\end{array}$ & $\begin{array}{l}\text { REMOVE } \\
\text { JUMPER }\end{array}$ & $N / A$ & N/A & A & \\
\hline $\begin{array}{l}1.7 .6 .2 .24 \\
m x \cdot B \\
\end{array}$ & $\begin{array}{l}\text { OPEN SV }-* 64 \\
\text { CLOSE SV }-* 63\end{array}$ & $N / A$ & N/A & A & \\
\hline $\begin{array}{l}1.7 .6 .2 .25 \\
\text { gn.n.B. }\end{array}$ & $\begin{array}{l}\text { INSTALL } \\
\text { JUMPER }\end{array}$ & $N / A$ & $N / A$ & $A$ & \\
\hline $\begin{array}{c}1.7 .6 .2 .26 \\
\sin x \cdot 0 \\
\end{array}$ & CLOSE FU-15 & $N / A$ & $N / A$ & A & \\
\hline $\begin{array}{l}1.7 .6 .2 .27 \\
m \cdot x-s\end{array}$ & $\begin{array}{l}\text { ADJ SV }-* 62 \& \\
\text { RECORD } \\
\text { PDIT }-* 60\end{array}$ & $-21.0^{\prime \prime} \mathrm{H}_{2} \mathrm{O}$ & $\begin{array}{l} \pm 5^{\prime \prime} \mathrm{H}_{2} \mathrm{O} \text { OF } \\
1.7 .6 .2 .15\end{array}$ & $A$ & \\
\hline $\begin{array}{l}1.7 .6 .2 .28 \\
m .3\end{array}$ & $\begin{array}{l}\text { CAL MON -*60 } \\
\text { CHANNEL B }\end{array}$ & $\begin{array}{l}10026.3 \mathrm{PPp} \\
\frac{10180.8 \mathrm{PPM}}{10441.1 \mathrm{PPM}} \\
\end{array}$ & $\begin{array}{l}\text { READINGS } \\
\text { WITHIN } \pm 5 \%\end{array}$ & $A$ & Methane \\
\hline $\begin{array}{l}1.7 .6 .2 .29 \\
m . x .2 \\
\end{array}$ & OPEN FU-15 & $N / A$ & N/A & $A$ & \\
\hline $\begin{array}{l}1.7 .6 .2 .30 \\
m>\cdot B\end{array}$ & $\begin{array}{l}\text { REMOVE } \\
\text { JUMPER }\end{array}$ & $\mathrm{N} / \mathrm{A}$ & $N / A$ & $A$ & \\
\hline $\begin{array}{l}1.7 .6 .2 .31 \\
m \cdot x \cdot p\end{array}$ & $\begin{array}{l}\text { CLOSE SV }-* 64 \\
\text { OPEN SV }-* 70\end{array}$ & $N / A$ & $N / A$ & A & \\
\hline $\begin{array}{l}1.7 .6 .2 .32 \\
\text { on.2..8 }\end{array}$ & $\begin{array}{l}\text { RECORD } \\
\text { PDIT-*60 } \\
\text { NIT-*52 IN }\end{array}$ & $-10.6^{4} 400$ & $\begin{array}{l}0 \text { to }-30 \\
" \mathrm{H}_{2} \mathrm{O}\end{array}$ & A & \\
\hline $\begin{array}{l}1.7 .6 .2 .33 \\
92.2 .0 \\
\end{array}$ & $\begin{array}{l}\text { INSTALL } \\
\text { JUMPER } \\
\end{array}$ & $N / A$ & $N / A$ & $A$ & \\
\hline 1.7 .6 .2 .34 & CLOSE FU-15 & $N / A$ & $N / A$ & $A$ & \\
\hline
\end{tabular}


TEST DATA SHEET

\begin{tabular}{|c|c|c|c|c|c|}
\hline \multicolumn{3}{|c|}{ Date: $\quad 12-19-96$} & \multicolumn{3}{|c|}{ SHMs Unit Nunber: VTP-PNL-330SI } \\
\hline \multicolumn{3}{|c|}{$\begin{array}{l}\text { Title of Test: } \\
\text { Pneumatic Systems }\end{array}$} & \multirow{2}{*}{\multicolumn{3}{|c|}{$\begin{array}{l}\text { Test Equipment and } \mathrm{S} / \mathrm{N} \text { or cal. No.: } \\
\text { See page } 150\end{array}$}} \\
\hline \multicolumn{3}{|c|}{$\begin{array}{c}\text { Test Performed By: See Test Personcal } \\
\text { Page } 128\end{array}$} & & & \\
\hline $\begin{array}{l}\text { Procedure No. } \\
\text { Initial/Date }\end{array}$ & Item & value & $\begin{array}{l}\text { Required } \\
\text { Condition } \\
\end{array}$ & $(A / R)$ & corment \\
\hline$\underbrace{1.7 .6 .3}_{\text {m. }}$ & $\begin{array}{l}\text { ESTABLISH } \\
\text { BYPASS FLOW }\end{array}$ & $1.5 \mathrm{cfh}$ & $\begin{array}{l}\text { FIV }-* 70 \\
1.5 \pm .2 \mathrm{cfh}\end{array}$ & $A$ & \\
\hline $\begin{array}{l}1.7 .6 .2 .36 \\
m .0 .0 \\
\end{array}$ & $\begin{array}{l}\text { ADJ SV-*71 } \\
\text { NIT-*52 } \\
\text { SAMPLE IN }\end{array}$ & $-12.4{ }^{\prime \prime} \mathrm{H}_{2} \mathrm{O}$ & $\begin{array}{l} \pm 5 \quad " \mathrm{H}_{2} \mathrm{O} \quad \mathrm{OF} \\
1.7 .6 .2 .32\end{array}$ & A & \\
\hline$\frac{1.7 .6 .2 .37}{m 32.6}$ & $\begin{array}{l}\text { RECORD } \\
\text { NIT-*52 } \\
\text { SAMPLES } \\
\end{array}$ & $\frac{\frac{44.1 \mathrm{em}}{44.4 \mathrm{pm}}}{44.6 \mathrm{ppm}}$ & $\begin{array}{l}\text { READINGS } \\
\text { WITHIN } \pm 5 \%\end{array}$ & $A$ & Ammonia \\
\hline $\begin{array}{l}1.7 .6 .2 .38 \\
\text { m3.8 } \\
\end{array}$ & OPEN FU-15 & $N / A$ & N/A & A & \\
\hline$\underbrace{1.7 .6 .39}_{1.7 x}$ & $\begin{array}{l}\text { REMOVE } \\
\text { JUMPER }\end{array}$ & N/A & N/A & A & \\
\hline \multicolumn{6}{|c|}{ Section 1.7 .7 provides for test system shutdown. } \\
\hline $\begin{array}{l}1.7 .7 .1 \\
\quad m_{13.3} \cdot 3 \\
\end{array}$ & $\begin{array}{l}\text { DISCONNECT } \\
\mathrm{H}_{2} \text { CAL GAS }\end{array}$ & N/A & N/A & A & \\
\hline $\begin{array}{r}1.7 .7 .2 \\
m .9 \cdot 8 \\
\end{array}$ & $\begin{array}{l}\text { DISCONNECT } \\
\mathrm{CH}_{6} \text { CAL GAS }\end{array}$ & $N / A$ & $N / A$ & A & \\
\hline $\begin{array}{r}1.7 .7 .3 \\
m \% \cdot B \\
\end{array}$ & $\begin{array}{l}\text { DISCONNECT } \\
\mathrm{NH}_{3} \text { CAL GAS }\end{array}$ & $N / A$ & N/A & A & \\
\hline $\begin{array}{l}1.7 .7 .4 \\
92.2 .0 \\
\end{array}$ & $\begin{array}{l}\text { SECURE \& } \\
\text { TURN OFF } \\
\text { ANA. INST.S }\end{array}$ & $N / A$ & $N / A^{\circ}$ & $A$ & \\
\hline $\begin{array}{r}1.7 .7 .5 \\
m .248 \\
\end{array}$ & $\begin{array}{l}\text { DISCONNECT } \\
\mathrm{N}_{2} \text { CARRIER }\end{array}$ & N/A & N/A & $A$ & \\
\hline $\begin{array}{l}1.7 .7 .6 \\
\text { 9n.2. }\end{array}$ & $\begin{array}{l}\text { DISCONNECT } \\
\text { He CARRIER } \\
\end{array}$ & $N / A$ & N/A & A & \\
\hline $\begin{array}{l}1.7 .7 .7 \\
m i x . x\end{array}$ & $\begin{array}{l}\text { OPEN ALL } \\
\text { BREAKERS \& } \\
\text { FUSES } \\
\end{array}$ & N/A & N/A & $A$ & \\
\hline $\begin{array}{r}1.7 .7 .8 \\
90.0 .8 \\
\end{array}$ & $\begin{array}{l}\text { CLOSE LISTED } \\
\text { SYS. VALVES }\end{array}$ & $N / A$ & $N / A$ & A & \\
\hline
\end{tabular}

all $12-19-96$ 
APPENDIX D

PAGE D-28 and
ny y
HNF-SD-WM-ATR- 191

Rev. 0

TEST DATA SHEET

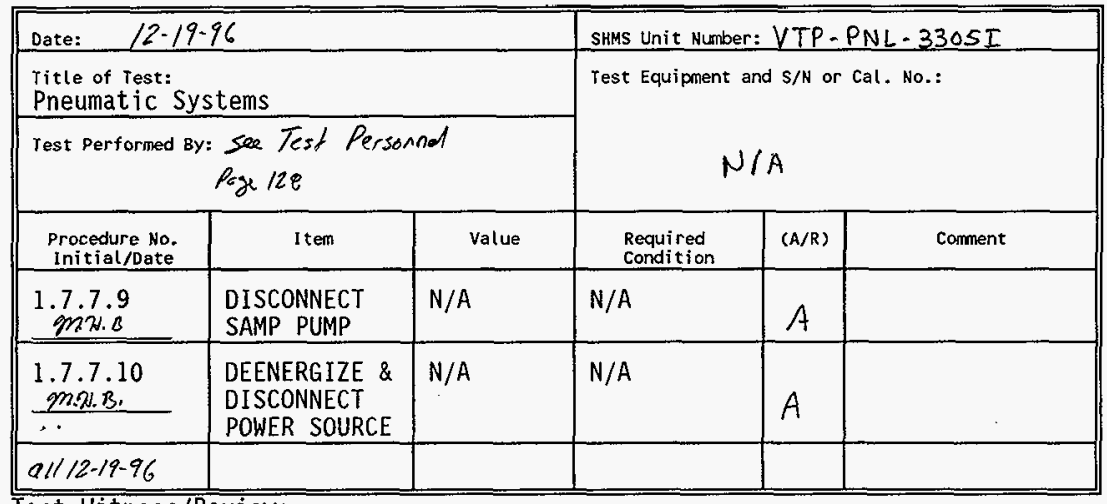

Test Witness/Review:

$\underbrace{10 \text { Acheide }}_{\text {Test Director }} \frac{12-3 /-96}{\text { Date }}$

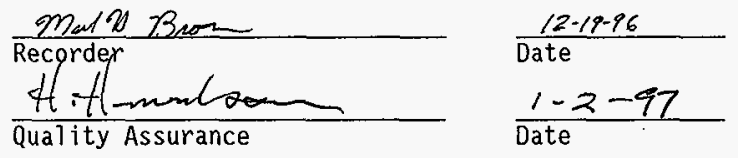


APPENDIX D

PAGE D-29
HNF -SD-WM-ATR- 191
ReV. 0

Rev. 0

TEST EXCEPTION SHEET

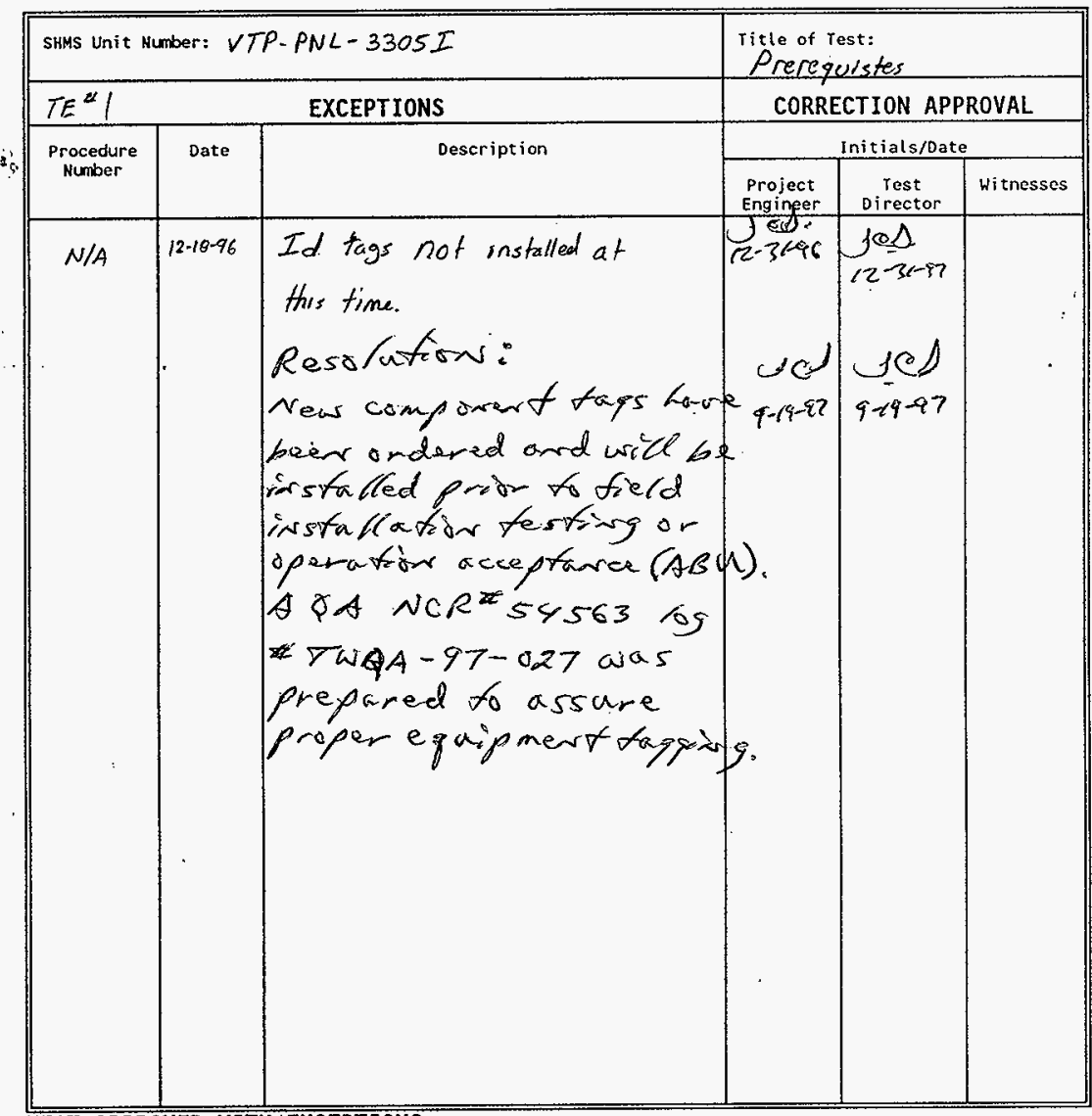

TEST APPROVED WITH EXCEPTIONS
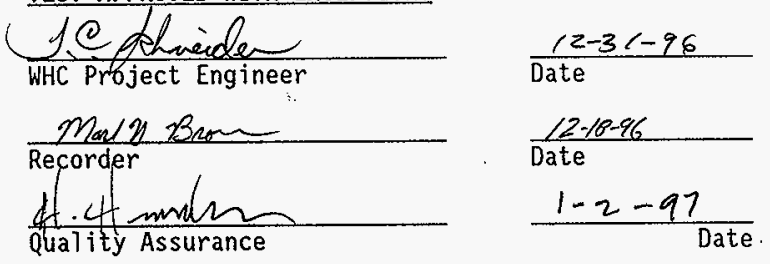
TEST LOG

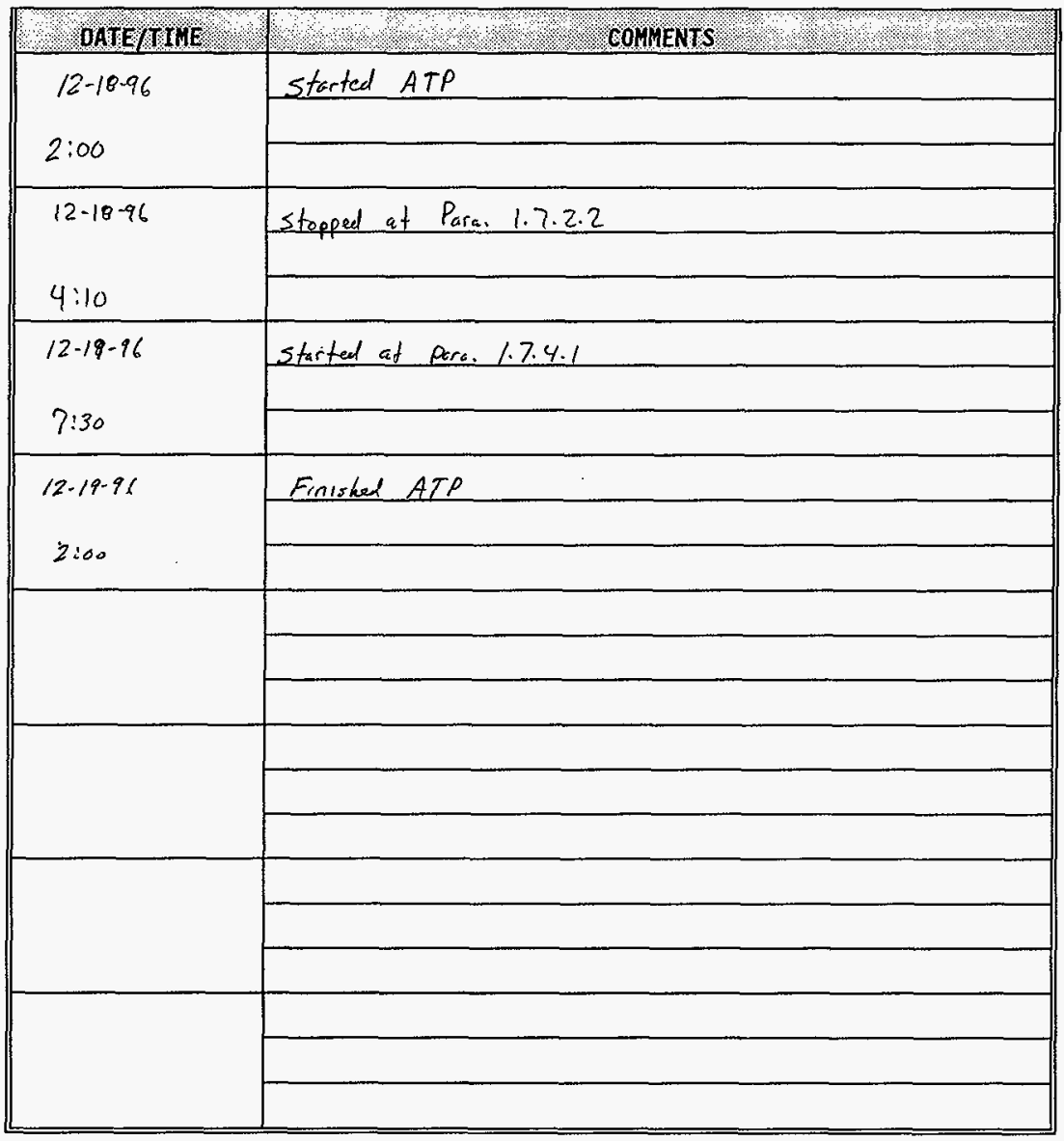




\section{TEST EXECUTION SHEET}

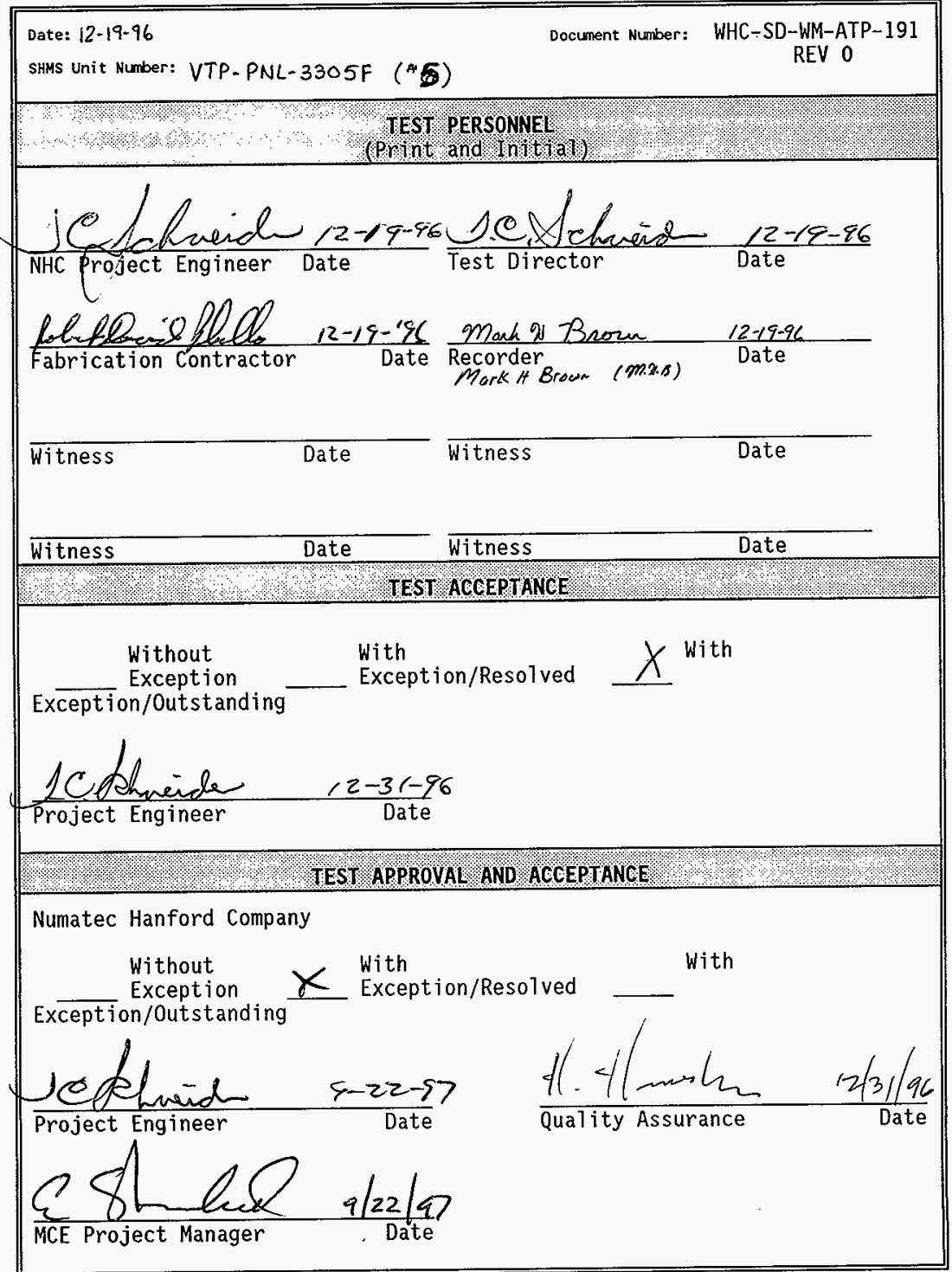


SHMS Unit Number:

\section{PREREQUISITES AND INITIAL TEST CONDITIONS}

The following conditions shall exist at the start of the acceptance testing. Initial and date to verify that each of the following items have been accomplished.

m.M.B Systems being tested have been inspected for workmanship and for 12-19-96 comp]iance with design. / with the exception of Id tags. TE"1 m: $12+9.96$

m.2.B Continuity and megger tests have been performed on portions of the $12 \cdot 19.96$ electrical and instrument systems being tested, as required.

m.x.8 Leak tests on the pneumatic systems have been performed.

$12 \cdot 19-16$

The following circuit breakers and fuses are installed per the specified size and are open

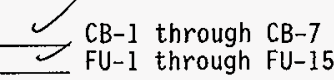

$M, D \cdot B$

All test instruments have a currently valjd calibration stamp

$12 \cdot 19 \cdot 86$ attached that indicates a calibration traceable to the National Institute of Standards and Technology.

mo.s The following process and control instrument systems have been 12-20-96 initially configured and aligned for proper operation.

FIT-*57, FSL $-* 57$, NIT $-* 54$, NIT-*55, (NR-*54), PDIT-*60, TIC $-* 50$, TIC $-* 56$, TIS $-* 62, Y Y C-* 01$ NOTE: NR-*54 is not used in the ANALYTICAL configuration.

ons.s Personnel responsible for directing and witnessing the performance of the tests described in this ATP have read and understand appropriate certified vendor information (CVI) pertaining to the operation of the equipment to be tested.

$\frac{m .27 .6}{12-20-26}$ CLOSE all system manual valves with the exception of the following:

$$
\begin{aligned}
& \text { VTP-FIV-*52 } \\
& \text { VTP-FIV-*56 } \\
& \text { VTP-FIV-*70 } \\
& \text { VTP-SV-*24 } \\
& \text { VTP-SV-*61 } \\
& \text { VTP-SV-*62 } \\
& \text { VTP-SV-*67 } \\
& \text { VTP-SV-*71 }
\end{aligned}
$$
port, but vented to atmosphere for testing. 
m.s.

12.20096
208 Vac 1 phase 15-20 ampere temporary power source has been connected, but not energized, to the appropriate TBI terminals per H-14-100838.

Verify by signature and date that all prerequisites have been met.

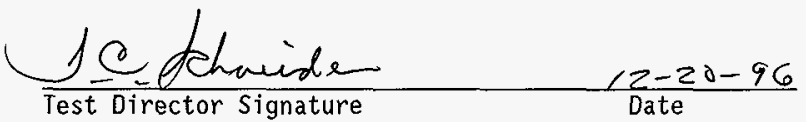




\section{TEST DATA SHEET}

\begin{tabular}{|c|c|c|c|c|c|}
\hline \multicolumn{3}{|c|}{ Date: $12.20-96$} & \multicolumn{3}{|c|}{ SHMS Unit Number: VTP-PNL-3305F } \\
\hline \multicolumn{3}{|c|}{$\begin{array}{l}\text { Title of rest: } \\
\text { Electrical Systems }\end{array}$} & \multirow{2}{*}{\multicolumn{3}{|c|}{$\begin{array}{l}\text { Test Equipment and S/N or Cal. Ho.: } \\
\text { Fluke V/O meter } \\
\text { SIN } 44620364 \\
\text { Cal. due } 10.97\end{array}$}} \\
\hline \multicolumn{3}{|c|}{$\begin{array}{c}\text { Test Perforned By: See Test Personnal } \\
\text { Page 158 }\end{array}$} & & & \\
\hline $\begin{array}{l}\text { Procedure No. } \\
\text { initial/Date } \\
\end{array}$ & item & value & $\begin{array}{l}\text { Required } \\
\text { Condition }\end{array}$ & $(A / R)$ & comment \\
\hline \multicolumn{6}{|c|}{ Section 1.7.1.1 verifies the system Mains Power. } \\
\hline $\begin{array}{l}1.7 .1 .1 .1 \\
\text { mas. }\end{array}$ & Resistance & 0.1 & $<1$ ohm & A & \\
\hline $\begin{array}{l}1.7 .1 .1 .2 \\
m \lambda . p \\
\end{array}$ & $\begin{array}{l}\text { Temp. power } \\
208 \mathrm{Vac}\end{array}$ & $N / A$ & Energized & A & \\
\hline$\frac{1.7 .1 .1 .3}{m x .8}$ & $\begin{array}{l}\text { Line Vac: } \\
\text { L1-L2 } \\
\text { L1-Gnd } \\
\text { L2-Gnd } \\
\mathrm{N}-\mathrm{Gnd}\end{array}$ & $\frac{\frac{200.6 \mathrm{Vac}}{119.6 \mathrm{~V}_{\mathrm{ac}}}}{\frac{119.9 \mathrm{~V}_{\mathrm{ac}}}{0}}$ & $\begin{array}{l}\text { L1-L2: } 208 \\
\text { Vac }+10 /-1 \% \\
\text { L1, L2: } 120 \\
\text { Vac } \pm 5 \% \\
N: 0 \text { Vac }\end{array}$ & A & \\
\hline \multicolumn{6}{|c|}{ Section 1.7 .1 .2 verifies the enclosure HVAC system. } \\
\hline $\begin{array}{l}1.7 .1 .2 .1 \\
m 28 \\
\end{array}$ & AC Adjusted & N/A & $N / A$ & A & \\
\hline $\begin{array}{l}1.7 .1 .2 .2 \\
\text { mo.B } \\
\end{array}$ & $\begin{array}{l}\text { CLOSE } \\
\text { CB-1/2 } \\
\end{array}$ & N/A & N/A & A & \\
\hline $\begin{array}{l}1.7 .1 .2 .3 \\
m .8 .8 \\
\end{array}$ & Heater ON & OK & Heater ON & A & \\
\hline $\begin{array}{l}1.7 .1 .2 .4 \\
M x \cdot \beta\end{array}$ & AC Adjusted & $N / A$ & N/A & A & \\
\hline $\begin{array}{l}1.7 .1 .2 .5 \\
\text { m.x. } 13\end{array}$ & $\begin{array}{l}\text { Heater OFF } \\
\text { Cooling ON } \\
\end{array}$ & $\frac{O K}{O K}$ & $\begin{array}{l}\text { Heater OFF } \\
\text { Cooling ON } \\
\end{array}$ & A & \\
\hline 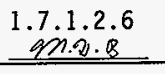 & AC Adjusted & $N / A$ & N/A & $A$ & \\
\hline$m_{m} x^{2}-i^{7}$ & $\begin{array}{l}\text { OPEN } \\
\mathrm{CB}-1 / 2\end{array}$ & N/A & $N / A$ & A & \\
\hline \multicolumn{6}{|c|}{ Section 1.7.1.3 verifies the sample pump is properly connected. } \\
\hline $\begin{array}{l}1.7 .1 .3 .1 \\
m a \cdot B\end{array}$ & $\begin{array}{l}\text { CLOSE } \\
\text { CB-3/4 }\end{array}$ & N/A & $N / A$ & $A$ & \\
\hline $\begin{array}{r}1.7 .1 .3 .2 \\
\text { min.s } \\
\end{array}$ & $\begin{array}{l}\text { VTP-P- *50 } \\
0 N\end{array}$ & $A$ & $\begin{array}{l}\text { VTP-P-*50 } \\
\text { ON }\end{array}$ & A & \\
\hline
\end{tabular}


TEST DATA SHEET

\begin{tabular}{|c|c|c|c|c|c|}
\hline \multicolumn{3}{|c|}{ Date: $12-20-96$} & \multicolumn{3}{|c|}{ SHMS Unit Number: VTP-PNL-330SF } \\
\hline \multicolumn{3}{|c|}{$\begin{array}{l}\text { Title of Test: } \\
\text { Electrical Systems }\end{array}$} & \multirow{2}{*}{\multicolumn{3}{|c|}{$\begin{array}{l}\text { Test Equipment and } S / \mathrm{N} \text { or Cal. No.: } \\
\text { see page } 161\end{array}$}} \\
\hline \multicolumn{3}{|c|}{$\begin{aligned} & \text { Test Performed By: } \text { see Test Personncl } \\
& \text { Page } 156\end{aligned}$} & & & \\
\hline $\begin{array}{l}\text { Procedure No. } \\
\text { Initial/Date }\end{array}$ & Item & value & $\begin{array}{l}\text { Required } \\
\text { Condition }\end{array}$ & $(A / R)$ & coment \\
\hline $\begin{array}{r}1.7 .1 .3 .3 \\
m 2.0 \\
\end{array}$ & $\begin{array}{l}\text { OPEN } \\
\text { CB-3/4 }\end{array}$ & N/A & $N / A$ & $A$ & \\
\hline \multicolumn{6}{|c|}{ Section 1.7.1.4 verifies the enclosure lighting and GFCI receptacles. } \\
\hline $\begin{array}{l}1.7 .1 .4 .1 \\
m_{2 \times .3} \\
\end{array}$ & $\begin{array}{l}\text { CLOSE } \\
\text { CB-6 }\end{array}$ & N/A & N/A & $A$ & \\
\hline $\begin{array}{l}7.1 .4 .2 \\
\end{array}$ & $\begin{array}{l}\text { ENCLOSURE } \\
\text { LIGHT }\end{array}$ & $N / A$ & $N / A$ & $A$ & \\
\hline 1.7 .1 .4 .3 & LIGHT ON & $A$ & LIGHT ON & A & \\
\hline$\frac{1.7 .1 .4 .4}{\min x \cdot 1}$ & $\begin{array}{l}\text { GFCI RECP. } \\
1 A . L-N \\
1 B . \text { L-GND } \\
2 A . \text { L-N } \\
2 B . \text { L-GND }\end{array}$ & $\frac{\frac{119.5 V_{a c}}{119.3 V_{a c}}}{\frac{119.4 V_{a c}}{119.5 V_{a c}}}$ & $\begin{array}{l}\text { 1A. } 120 \mathrm{Vac} \\
1 \mathrm{~B} .120 \mathrm{VaC} \\
2 \mathrm{~A} .120 \mathrm{VaC} \\
2 B .120 \mathrm{VaC} \\
\text { ALL } \pm 5 \%\end{array}$ & A & \\
\hline $\min ^{1.7 .1 .4 .5}$ & $\begin{array}{l}\text { GFCI TEST } \\
\text { 1A. L-N } \\
\text { 1B. L-GND } \\
2 A . L-N \\
2 B . L-G N D \\
\end{array}$ & $\frac{\frac{0.0 v_{0 c}}{48.0 v_{c c}}}{\frac{0.27 v_{a c}}{0.4 v_{c c}}}$ & $\begin{array}{l}1 A .0 \mathrm{Vac} \\
1 \mathrm{~B} .0 \mathrm{Vac} \\
2 \mathrm{~A} .0 \mathrm{Vac} \\
2 \mathrm{~B} .0 \mathrm{Vac} \\
\text { ALL } \pm 5 \%\end{array}$ & A & \\
\hline $\begin{array}{c}1.7 .1 .4 .6 \\
m \times x .8 \\
\end{array}$ & $\begin{array}{l}\text { ENCLOSURE } \\
\text { LIGHT OFF }\end{array}$ & N/A & $\mathrm{N} / \mathrm{A}$ & $A$ & \\
\hline $\begin{array}{l}1.7 .1 .4 .7 \\
\text { min.1.8. } \\
\end{array}$ & $\begin{array}{l}\text { OPEN } \\
\text { CB-6 }\end{array}$ & N/A & $N / A$ & A & \\
\hline \multicolumn{6}{|c|}{ Section 1.7.1.5 verifies the instrument power source isolation transformer. } \\
\hline $\begin{array}{l}1.7 .1 .5 .1 \\
\text { in. }\end{array}$ & $\begin{array}{l}\text { CLOSE } \\
\text { CB-7 }\end{array}$ & N/A & $N / A$ & $A$ & \\
\hline $\begin{array}{l}1.7 .1 .5 .2 \\
n \in m-1\end{array}$ & $\begin{array}{l}\text { XFRM Vac } \\
\text { PRI. L-N } \\
\text { SEC. L-N } \\
\text { SEC. N-GND } \\
\end{array}$ & $\frac{\frac{119.6 \mathrm{Vac}}{120.0 \mathrm{Vac}}}{0.0 \mathrm{~V}_{\mathrm{ac}}}$ & $\begin{array}{l}\text { PRI. \& SEC. } \\
\text { L-N } 120 \pm 5 \% \\
\text { SEC. } \\
\text { N-GND O Vac. }\end{array}$ & $A$ & \\
\hline $\begin{array}{l}1.7 .1 .5 .3 \\
\text { opu.p B } \\
\end{array}$ & CLOSE FU-1 & $N / A$ & $N / A$ & $A$ & \\
\hline
\end{tabular}


TEST DATA SHEET

\begin{tabular}{|c|c|c|c|c|c|}
\hline \multicolumn{3}{|c|}{ Date: $\quad 12-20-96$} & \multicolumn{3}{|c|}{ SHMS Unit Number: VTP-PNL-3305F } \\
\hline \multicolumn{3}{|c|}{$\begin{array}{l}\text { Title of Test: } \\
\text { Electrical Systems }\end{array}$} & \multirow{2}{*}{\multicolumn{3}{|c|}{$\begin{array}{l}\text { Test Equipment and } \mathrm{S} / \mathrm{N} \text { or Cal. No.: } \\
\text { See Poge } 161\end{array}$}} \\
\hline \multicolumn{3}{|c|}{$\begin{aligned} \text { Test Performed By: See Test Personnal } \\
\text { Page } 150\end{aligned}$} & & & \\
\hline $\begin{array}{c}\text { Procedure No. } \\
\text { Initial/Date } \\
\end{array}$ & Item & value & $\begin{array}{c}\text { Required } \\
\text { condition }\end{array}$ & $(A / R)$ & Comment \\
\hline $\begin{array}{c}1.7 .1 .5 .4 \\
m .2 . \Omega \\
\end{array}$ & $\begin{array}{l}\text { VTP-PS-*50 } \\
\text { OUTPUT Vdc } \\
\end{array}$ & $23.90 \mathrm{Vdt}$ & $24 \pm 0.1 \mathrm{Vdc}$ & $\theta$ & \\
\hline \multicolumn{6}{|c|}{ Section 1.7.1.6 verifies the enclosure general al arm system. } \\
\hline $\begin{array}{l}1.7 .1 .6 .1 \\
\end{array}$ & $\begin{array}{l}\text { CLOSE FU-9 \& } \\
\text { FU-10 }\end{array}$ & $N / A$ & N/A & $A$ & \\
\hline $\begin{array}{l}1.7 .1 .6 .2 \\
m_{x p}\end{array}$ & $\begin{array}{l}\text { PUSH PB-*51 } \\
\& \text { PB }-* 50 \\
\end{array}$ & $N / A$ & $N / A$ & A & \\
\hline $\begin{array}{l}1.7 .1 .6 .3 \\
\end{array}$ & $\begin{array}{l}\text { ALARM TEST } \\
\text { VERIFY ALARM } \\
\text { CONDITIONS }\end{array}$ & 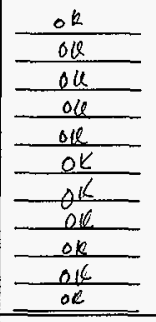 & $\begin{array}{ll}\text { YAH-*50 } & \text { ON } \\
\text { NAH-*55 } & \text { ON } \\
\text { XA-*63 } & \text { ON } \\
\text { NAH-*54 } & \text { ON } \\
\text { FAL-*57 } & \text { ON } \\
\text { TAHL-*62 ON } \\
\text { TAL-*50 } & \text { ON } \\
\text { YAL-*58 } & \text { ON } \\
\text { PBL-*58 } & \text { ON } \\
\text { PBL-*54 } & \text { OFF } \\
\text { PBL-*59 } & \text { OFF } \\
\end{array}$ & A & \\
\hline $\begin{array}{l}1.7 .1 .6 .4 \\
\text { Snine }\end{array}$ & $\begin{array}{l}\text { END ALARM } \\
\text { TEST }\end{array}$ & N/A & N/A & $A$ & \\
\hline $\begin{array}{l}1.7 .1 .6 .5 \\
m n^{\circ} \Omega \\
\end{array}$ & RESET ALARMS & N/A & $N / A$ & A & \\
\hline $\begin{array}{c}1.7 .1 .6 .6 \\
m \geqslant .0 \\
\end{array}$ & $\begin{array}{l}\text { OPEN FU-9 \& } \\
\text { FU-10 }\end{array}$ & $N / A$ & $N / A$ & A & \\
\hline \multicolumn{6}{|c|}{ Section 1.7.1.7 verifies the flow alarm system. } \\
\hline 1.7 .1 .7 .1 & $\begin{array}{l}\text { CLOSE FU-3, } \\
\text { FU-9, FU-10 } \\
\& \quad F U-13 \\
\end{array}$ & N/A & N/A & A & \\
\hline $\begin{array}{l}1.7 .1 .7 .2 \\
m x \cdot B \\
\end{array}$ & RESET ALARMS & ok & $\mathrm{FAL}-* 57$ ON & $A$ & \\
\hline $\begin{array}{l}1.7 .1 .7 .3 \\
M 1.2 \\
\end{array}$ & $\begin{array}{l}\text { OPEN SV }-* 20 \\
\& \text { SV }-* 22\end{array}$ & $N / A$ & $N / A$ & A & \\
\hline
\end{tabular}


TEST DATA SHEET

\begin{tabular}{|c|c|c|c|c|c|}
\hline \multicolumn{3}{|c|}{ Date: $\quad 12-20 \cdot 96$} & \multicolumn{3}{|c|}{ SHMS Unit Number: VTP-PNL - 3305F } \\
\hline \multicolumn{3}{|c|}{$\begin{array}{l}\text { Title of Test: } \\
\text { Electrical Systems }\end{array}$} & \multirow{2}{*}{\multicolumn{3}{|c|}{$\begin{array}{l}\text { Test Equipment and } S / N \text { or Cal. No.: } \\
\text { see Page } 161\end{array}$}} \\
\hline \multicolumn{3}{|c|}{$\begin{aligned} \text { Test Performed By: } & \text { see Test Personnal } \\
& \text { Page } 15 \%\end{aligned}$} & & & \\
\hline $\begin{array}{l}\text { Procedure No. } \\
\text { Initial/Date }\end{array}$ & Item & value & $\begin{array}{l}\text { Required } \\
\text { Condition }\end{array}$ & $(A / R)$ & comment \\
\hline$\frac{1.7 .1 .7 .4}{\min \beta}$ & $\begin{array}{l}\text { FIT }-* 57 \quad \& \\
\text { FSL } * 57 \\
\text { POWERED }\end{array}$ & $\frac{o k}{0 k}$ & $\begin{array}{l}\text { FIT-*57. ON } \\
\text { FSL-*57 ON }\end{array}$ & A & \\
\hline $\begin{array}{c}1.7 .1 .7 .5 \\
\end{array}$ & $\begin{array}{l}\text { MUX-*70 } \\
\text { TB5+/TB6- }\end{array}$ & $1.01 \mathrm{VLC}$ & $1.0 \pm 0.2 \mathrm{VdC}$ & $A$ & \\
\hline $\begin{array}{c}1.7 .1 .7 .6 \\
m_{1}, \beta \\
\end{array}$ & $\begin{array}{l}\text { CLOSE SV }-* 20 \\
\& \text { SV }-* 22\end{array}$ & N/A & $\mathrm{N} / \mathrm{A}$ & A & \\
\hline $\begin{array}{c}1.7 .1 .7 .7 \\
m .20\end{array}$ & $\begin{array}{l}\text { OPEN FU-3, } \\
\text { FU-9, FU-10 } \\
\& \text { FU-13 }\end{array}$ & $\mathrm{N} / \mathrm{A}$ & N/A & A & \\
\hline \multicolumn{6}{|c|}{ Section 1.7 .1 .8 verifies the $\mathrm{H}_{2}$ monitor wiring. } \\
\hline $\begin{array}{l}1.7 .1 .8 .1 \\
m i n n\end{array}$ & $\begin{array}{l}\text { CLOSE FU-2, } \\
\text { FU-4, FU-5, } \\
\text { FU-9 \& FU-10 }\end{array}$ & N/A & N/A & A & \\
\hline $\begin{array}{l}1.7 .1 .8 .2 \\
\text { minn }\end{array}$ & RESET ALARMS & ok & $\begin{array}{l}\mathrm{NAH}-* 54 \text { OFF } \\
\mathrm{NAH}-* 55 \text { OFF } \\
\end{array}$ & A & \\
\hline $\begin{array}{l}1.7 .1 .8 .3 \\
m . x+\beta\end{array}$ & $\begin{array}{l}\text { INSTRUMENTS } \\
\text { POWERED }\end{array}$ & $\begin{array}{c}\frac{O K}{O K K} \\
\frac{O K}{O N} \\
N / A \\
O K\end{array}$ & $\begin{array}{l}\text { MUX-*70 ON } \\
\text { NIT-*54 ON } \\
\text { NIT-*55 ON } \\
\text { NR }-* 54 \text { ON } \\
\text { YYC-*01 ON }\end{array}$ & A & \\
\hline $\begin{array}{l}1.7 .1 .8 .4 \\
M . \not B P \\
\end{array}$ & $\begin{array}{l}\text { OPEN FU-2, } \\
\text { FU-4, FU-5, } \\
\text { FU-9 \& FU-10 }\end{array}$ & N/A & N/A & A & \\
\hline \multicolumn{6}{|c|}{ Section 1.7 .1 .9 verifies the SHMS-E heat trace control system. } \\
\hline $\begin{array}{c}1.7 .1 .9 .1 \\
\end{array}$ & $\begin{array}{l}\text { INSTALL LOAD } \\
\text { VTP-TIC-*50 }\end{array}$ & N/A & N/A & A & \\
\hline $\begin{array}{l}1.7 .1 .9 .2 \\
m B B\end{array}$ & $\begin{array}{l}\text { CLOSE CB-5, } \\
\text { FU-6, FU-7, } \\
\text { FU-9 \& FU-10 } \\
\text { and RESET } \\
\text { ALARMS }\end{array}$ & N/A & N/A & $A$ & . \\
\hline
\end{tabular}


TEST DATA SHEET

\begin{tabular}{|c|c|c|c|c|c|}
\hline \multicolumn{3}{|c|}{ Date: $\quad 12-20-96$} & \multicolumn{3}{|c|}{ SHMS Unit Number: VTP-PNL-3305F } \\
\hline \multicolumn{3}{|c|}{$\begin{array}{l}\text { Title of rest: } \\
\text { Electrical Systems }\end{array}$} & \multirow{2}{*}{\multicolumn{3}{|c|}{$\begin{array}{l}\text { Test Equipment and } S / N \text { or Cal. No.: } \\
\text { N/A }\end{array}$}} \\
\hline \multicolumn{3}{|c|}{$\begin{aligned} \text { Test Performed By: } & \text { See Test Personnal } \\
& \text { Page 158 }\end{aligned}$} & & & \\
\hline $\begin{array}{c}\text { Procedure No. } \\
\text { Initial/Date } \\
\end{array}$ & Item & Value & $\begin{array}{c}\text { Required } \\
\text { Condition }\end{array}$ & $(A / R)$ & Comment \\
\hline $\begin{array}{l}1.7 .1 .9 .3 \\
\text { mmins }_{3}\end{array}$ & $\begin{array}{l}\text { TIC }-* 50 \& \\
\text { TIC } * 566 \\
\text { NOMINAL TEMP }\end{array}$ & $\begin{array}{r}65^{\circ} \mathrm{p} \\
62 \circ \mathrm{p} \\
\end{array}$ & $\begin{array}{l}\text { TIC }-* 50 \\
\text { TIC-*56 } \\
\text { TEMPERATURE }\end{array}$ & $A$ & \\
\hline $\begin{array}{c}1.7 .1 .9 .4 \\
m .2,3\end{array}$ & $\begin{array}{l}\text { ADJ TIC }-* 50 \\
\& \text { TIC }-* 56 \\
\text { SP2 \& RESET } \\
\text { ALARMS } \\
\end{array}$ & $N / A$ & $N / A$ & A & \\
\hline $\begin{array}{c}1.7 .1 .9 .5 \\
\mathrm{~m}>\mathrm{B} \\
\end{array}$ & $\begin{array}{l}\text { ADJ TIC }-* 50 \\
\text { SP } 1\end{array}$ & $N / A$ & $N / A$ & A & \\
\hline${ }_{m i n}^{1.7} \dot{x}^{9.6}$ & $\begin{array}{l}\text { ADJ TIC }-* 50 \\
\text { SP } 2\end{array}$ & $N / A$ & $N / A$ & A & \\
\hline $\begin{array}{l}1.7 .1 .9 .7 \\
m \cdot x_{1} \cdot B^{2}\end{array}$ & $\begin{array}{l}\text { TAL-*50\& } \\
\text { HORN ACTIVE }\end{array}$ & $\begin{array}{c}\text { ok } \\
\text { ok } \\
\text { ole } \\
\end{array}$ & $\begin{array}{l}\text { TAL-*50 ON } \\
\text { HORN ON } \\
\text { ACKNOWLEDGE }\end{array}$ & $A$ & \\
\hline 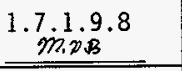 & $\begin{array}{l}\text { TIC-*50 } \\
\text { OPERATES }\end{array}$ & ok & $\begin{array}{l}\text { TIC }-\star 50 \\
\text { OPERATES } \\
\end{array}$ & $A$ & \\
\hline $\begin{array}{l}1.7 .1 .9 .9 \\
m, \infty .0 \\
\end{array}$ & $\begin{array}{l}\text { ADJ TIC-*50 } \\
\text { SP 2 }\end{array}$ & N/A & N/A & A & \\
\hline $\begin{array}{l}1.7 .1 .9 .10 \\
\operatorname{mix}_{1} \\
\end{array}$ & RESET ALARM & ok & $\mathrm{TAL}-* 50$ OFF & A & \\
\hline $\begin{array}{l}1.7 .1 .9 .11 \\
\text { mins } \\
\end{array}$ & $\begin{array}{l}A D J \text { TIC }-* 50 \\
S P 1 \& S P 2\end{array}$ & N/A & N/A & A & \\
\hline $\begin{array}{l}1.7 .1 .9 .12 \\
\text { m.t. } \\
\end{array}$ & $\begin{array}{l}\text { OPEN CB-5 \& } \\
\text { REMOVE LOAD }\end{array}$ & N/A & $N / A$ & A & \\
\hline$\frac{1.7 .1 .9 .13}{m . p .3}$ & $\begin{array}{l}\text { INSTALL LOAD } \\
\text { VTP-TIC-*56 }\end{array}$ & N/A & N/A & $A$ & \\
\hline $\begin{array}{l}1.7 .1 .9 .14 \\
\text { M.x.e } \\
\end{array}$ & CLOSE CB-5 & N/A & $N / A$ & $A$ & \\
\hline $\begin{array}{l}1.7 .1 .9 .15 \\
2 m . x . B \\
\end{array}$ & $\begin{array}{l}\text { ADJ TIC-*56 } \\
\text { SP } 1\end{array}$ & $N / A$ & $N / A$ & $A$ & \\
\hline $\begin{array}{l}1.7 .1 .9 .16 \\
\sin x \\
\end{array}$ & $\begin{array}{l}\text { ADJ TIC }-* 56 \\
\text { SP } 2\end{array}$ & $N / A$ & $N / A$ & $A$ & \\
\hline
\end{tabular}




\section{TEST DATA SHEET}

\begin{tabular}{|c|c|c|c|c|c|}
\hline \multicolumn{3}{|c|}{ Date: $\quad 12-20-96$} & \multicolumn{3}{|c|}{ SHMS Unit Number: VTP-PNL-3305F } \\
\hline \multicolumn{3}{|c|}{$\begin{array}{l}\text { Title of Test: } \\
\text { Electrical Systems }\end{array}$} & \multirow{2}{*}{\multicolumn{3}{|c|}{$\begin{array}{l}\text { Test Equipment and } S / N \text { or Cal. No.: } \\
N / A\end{array}$}} \\
\hline \multicolumn{3}{|c|}{$\begin{aligned} & \text { Iest Performed By: } \text { see Test Persomnal } \\
& \text { Page } 158\end{aligned}$} & & & \\
\hline $\begin{array}{l}\text { Procedure No. } \\
\text { Initial/Date }\end{array}$ & item & Value & $\begin{array}{l}\text { Required } \\
\text { Condition } \\
\end{array}$ & $(A / R)$ & Comment \\
\hline$\frac{1.7 .1 .9 .17}{-m .3}$ & $\begin{array}{l}\text { TAL-*50\& } \\
\text { HORN ACTIVE }\end{array}$ & $\begin{array}{l}\text { OK } \\
\text { OK } \\
\text { OK } \\
=\end{array}$ & $\begin{array}{l}\text { TAL }-* 50 \text { ON } \\
\text { HORN ON } \\
\text { ACKNOWLEDGE }\end{array}$ & $A$ & \\
\hline $\begin{array}{l}1.7 .1 .9 .18 \\
\text { mise }\end{array}$ & $\begin{array}{l}\text { TIC }-* 56 \\
\text { OPERATES }\end{array}$ & ok & $\begin{array}{l}\text { TIC- } * 56 \\
\text { OPERATES } \\
\end{array}$ & $A$ & \\
\hline $\begin{array}{c}1.7 .1 .9 .19 \\
m_{2, B}, 3 \\
\end{array}$ & $\begin{array}{l}\text { ADJ TIC-*56 } \\
\text { SP } 2\end{array}$ & $N / A$ & N/A & $A$ & \\
\hline $\begin{array}{l}1.7 .1 .9 .20 \\
=2.2 .0 \\
\end{array}$ & RESET ALARM & $m+n$ & TAL $-* 50$ OFF & A & \\
\hline $\begin{array}{l}1.7 .1 .9 .21 \\
\text { OM.N.B. } \\
\end{array}$ & $\begin{array}{l}A D J \text { TIC-*56 } \\
S P 1 \& S P 22 \\
\end{array}$ & N/A & N/A & $A$ & \\
\hline $\begin{array}{l}1.7 .1 .9 .22 \\
-m \cdot 1 \cdot B \\
\end{array}$ & $\begin{array}{l}\text { OPEN CB-5, } \\
\text { FU-6 \& FU-7 }\end{array}$ & $N / A$ & N/A & $A$ & \\
\hline $\begin{array}{r}1.7 .1 .9 .23 \\
\text { m.n.B. } \\
\end{array}$ & $\begin{array}{l}\text { REMOVE LOAD } \\
\text { TIC-*56 }\end{array}$ & $N / A$ & N/A & $A$ & \\
\hline $\begin{array}{c}1.7 .1 .9 .24 \\
9 m .8 . B \\
\end{array}$ & $\begin{array}{l}\text { CLOSE FU-8 } \\
\text { TIS-*62 } \\
\text { NOMINAL TEMP } \\
\end{array}$ & $65^{\circ} \mathrm{F}$ & $\begin{array}{l}\text { TIS }-* 62 \\
\text { TEMP }\end{array}$ & A & \\
\hline $\begin{array}{c}1.7 .1 .9 .25 \\
m^{n . n} \\
\end{array}$ & $\begin{array}{l}\text { ADJ TIS }-* 62 \\
\text { SP } 1\end{array}$ & N/A & $N / A$ & A & \\
\hline $\begin{array}{l}1.7 .1 .9 .26 \\
\text { m.o.0. } \\
\end{array}$ & $\begin{array}{l}\text { ADJ TIS-*62 } \\
\text { SP } 2\end{array}$ & N/A & N/A & A & \\
\hline $\begin{array}{l}1.7 .1 .9 .27 \\
\text { m.n.s }\end{array}$ & RESET ALARMS & OK & $\begin{array}{l}\text { TAHL-*62 } \\
\text { OFF }\end{array}$ & $A$ & \\
\hline $\begin{array}{l}1.7 .1 .9 .28 \\
\text { m.x.8 } \\
\end{array}$ & $\begin{array}{l}\text { ADJ TIS }-* 62 \\
\text { SP } 2\end{array}$ & $N / A$ & $N / A$ & A & \\
\hline $\begin{array}{l}1.7 .1 .9 .29 \\
\text { mxip }\end{array}$ & $\begin{array}{l}\text { TAHL-*62 \& } \\
\text { HORN ACTIVE }\end{array}$ & $\frac{O K}{O K}$ & $\begin{array}{l}\text { TAHLL* } 62 \text { ON } \\
\text { HORN ON } \\
\text { ACKNOWLEDGE } \\
\end{array}$ & A & \\
\hline $\begin{array}{c}1.7 .1 .9 .30 \\
\text { m.x.B }\end{array}$ & $\begin{array}{l}\text { ADJ TIS-*62 } \\
\text { SP } 2\end{array}$ & $N / A$ & N/A & A & \\
\hline
\end{tabular}


TEST DATA SHEET

\begin{tabular}{|c|c|c|c|c|c|}
\hline \multicolumn{3}{|c|}{$12-20-96$} & \multicolumn{3}{|c|}{ SHMS Unit Number: VIP-PNL-3305F } \\
\hline \multicolumn{3}{|c|}{$\begin{array}{l}\text { Title of rest: } \\
\text { Electrical Systems }\end{array}$} & \multirow{2}{*}{\multicolumn{3}{|c|}{$\begin{array}{l}\text { Test Equipment and S/N or Cal. No.: } \\
\text { see page } 161\end{array}$}} \\
\hline \multicolumn{3}{|c|}{$\begin{aligned} \text { Test Performed By: See Test Personnal } \\
\text { Page } 158\end{aligned}$} & & & \\
\hline $\begin{array}{l}\text { Procedure No. } \\
\text { Initial/Date } \\
\end{array}$ & Item & Value & $\begin{array}{l}\text { Required } \\
\text { Condition } \\
\end{array}$ & $(A / R)$ & Comment \\
\hline $\begin{array}{l}1.1 .9 .31 \\
\text { min. }\end{array}$ & RESET ALARMS & ok & $\begin{array}{l}\text { TAHL-*62 } \\
\text { OFF } \\
\end{array}$ & A & \\
\hline $\begin{array}{l}\text { mNB } \\
\end{array}$ & $\begin{array}{l}\text { ADJ TIS-*62 } \\
\text { SP } 1\end{array}$ & $N / A$ & N/A & $A$ & \\
\hline$\frac{1.7 .1 .9 .33}{m \cdot B}$ & $\begin{array}{l}\text { TAHL-*62 \& } \\
\text { HORN ACTIVE }\end{array}$ & $\begin{array}{l}o K \\
O K \\
O K \\
\end{array}$ & $\begin{array}{l}\text { TAHL }-* 62 \text { ON } \\
\text { HORN ON } \\
\text { ACKNOWLEDGE } \\
\end{array}$ & $A$ & \\
\hline $\begin{array}{c}1.7 .1 .9 .34 \\
\mathrm{mg.B3} \\
\end{array}$ & $\begin{array}{l}\text { ADJ TIS-*62 } \\
S P 1 \& S P 2\end{array}$ & $\mathrm{~N} / \mathrm{A}$ & $N / A$ & A & \\
\hline $\operatorname{mas}_{1.1 .9 .35}$ & $\begin{array}{l}\text { OPEN FU-8, } \\
\text { FU-9 \& FU-10 }\end{array}$ & $N / A$ & N/A & $A$ & \\
\hline \multicolumn{6}{|c|}{ Section 1.7.1.10 verifies the differential pressure transmitter system. } \\
\hline $\begin{array}{l}1.7 .1 .10 .1 \\
m .23 .8 \\
\end{array}$ & $\begin{array}{l}\text { REMOVE PLUGS } \\
\text { BAL PDIT }-\star 60 \\
\end{array}$ & $N / A$ & N/A & $A$ & \\
\hline$\frac{1.7 .1 .10 .2}{\min x}$ & $\begin{array}{l}\text { CLOSE FU-14 } \\
\text { REC PDIT-*60 } \\
\text { PRESSURE }\end{array}$ & $0.000^{\circ} \mathrm{H}_{2} \mathrm{O}$ & $\begin{array}{l}\text { PDIT-*60 } \\
\text { PRESSURE } \\
0 \pm 1 " \mathrm{H}_{2} \mathrm{O} \\
\end{array}$ & $A$ & \\
\hline $\begin{array}{l}1.7 .1 .10 .3 \\
m: D \cdot B \\
\end{array}$ & $\begin{array}{l}\text { MUX-*70 VdC } \\
\text { TB7+ \& TB8- }\end{array}$ & 2.20 & $\begin{array}{l}\text { MUX-*70 Vdc } \\
2.2 \pm 0.1 \\
\end{array}$ & A & \\
\hline $\begin{array}{l}1.7 .1 .10 .4 \\
m_{1} \mathrm{v} .13 \\
\end{array}$ & $\begin{array}{l}\text { REPLACE } \\
\text { PLUGS ON } \\
\text { PDIT } * 60 \\
\end{array}$ & $N / A$ & $N / A$ & A & \\
\hline $\operatorname{min.s}^{1.7 .1 .10 .5}$ & OPEN FU-14 & $N / A$ & $N / A$ & $A$ & \\
\hline \multicolumn{6}{|c|}{ Section 1.7.1.11 verifies the gas chromatograph power. } \\
\hline $\begin{array}{l}1.7 .1 .11 .1 \\
M M . N . B \\
\end{array}$ & CLOSE FU-11 & N/A & $N / A$ & $A$ & \\
\hline $\begin{array}{l}1.7 .1 .11 .2 \\
\text { mo.s }\end{array}$ & $\begin{array}{l}\text { POWER } \\
\text { MON-*60 }\end{array}$ & ok & $\begin{array}{l}\text { MON-*60 } \\
\text { POWERED }\end{array}$ & A & \\
\hline $\begin{array}{l}1.7 .1 .11 .3 \\
\text { mnn.s.3 }\end{array}$ & OPEN FU-11 & N/A & $N / A$ & A & \\
\hline
\end{tabular}


TEST DATA SHEET

\begin{tabular}{|c|c|c|c|c|c|}
\hline \multicolumn{3}{|c|}{ Date: $\quad 12 \cdot 20 \cdot 96$} & \multicolumn{3}{|c|}{ SHMS Unit Number: VTP-PNL-3305F } \\
\hline \multicolumn{3}{|c|}{$\begin{array}{l}\text { Title of Test: } \\
\text { Electrical Systems }\end{array}$} & \multicolumn{3}{|c|}{ Test Equipment and $\mathrm{S} / \mathrm{N}$ or Cal. No.: } \\
\hline \multicolumn{3}{|c|}{$\begin{array}{c}\text { Test Performed By: See Test Personnal } \\
\text { Page } 158\end{array}$} & \multicolumn{3}{|c|}{$N / A$} \\
\hline $\begin{array}{c}\text { Procedure No. } \\
\text { Initial/Date }\end{array}$ & Item & Value & $\begin{array}{l}\text { Required } \\
\text { Condition }\end{array}$ & $(A / R)$ & Comment \\
\hline \multicolumn{6}{|c|}{ Section 1.7 .1 .12 verifies the multi gas analyzer power. } \\
\hline $\begin{array}{c}1.7 .1 .12 .1 \\
m .8\end{array}$ & CLOSE FU-12 & $N / A$ & $N / A$ & A & \\
\hline $\begin{array}{l}1.7 .1 .12 .2 \\
m x .3 \\
\end{array}$ & $\begin{array}{l}\text { POWER } \\
\text { NIT } * 52\end{array}$ & ok & $\begin{array}{l}\text { NIT }-* 52 \\
\text { POWERED } \\
\end{array}$ & $A$ & \\
\hline $\begin{array}{c}1.7 .1 .12 .3 \\
m_{m \infty n} \\
\end{array}$ & OPEN FU-12 & $N / A$ & $N / A$ & $A$ & \\
\hline \multicolumn{6}{|c|}{ Section 1.7 .1 .13 verifies the personnel computer power. } \\
\hline $\begin{array}{l}1.7 .1 .13 .1 \\
\end{array}$ & CLOSE CB-6 & $N / A$ & $N / A$ & A & \\
\hline $\begin{array}{c}1.7 .1 .13 .2 \\
m_{1 \times B} . B \\
\end{array}$ & $\begin{array}{l}\text { P0WER PC-*60 } \\
\& \text { PC }-* 70\end{array}$ & ok & $\begin{array}{ll}P C-* 60 & O N \\
P C-* 70 & O N \\
\end{array}$ & A & \\
\hline $\begin{array}{l}1.7 .1 .13 .3 \\
\text { maxs } \\
\end{array}$ & $\begin{array}{l}\text { TURN INST. } \\
\text { OFF \& } \\
\text { OPEN CB-6 }\end{array}$ & $\frac{O K}{O K}$ & $\begin{array}{ll}P C-* 60 & \text { OFF } \\
P C-* 70 & \text { OFF }\end{array}$ & $A$ & \\
\hline
\end{tabular}

a) $12-20-46$ 
TEST DATA SHEET

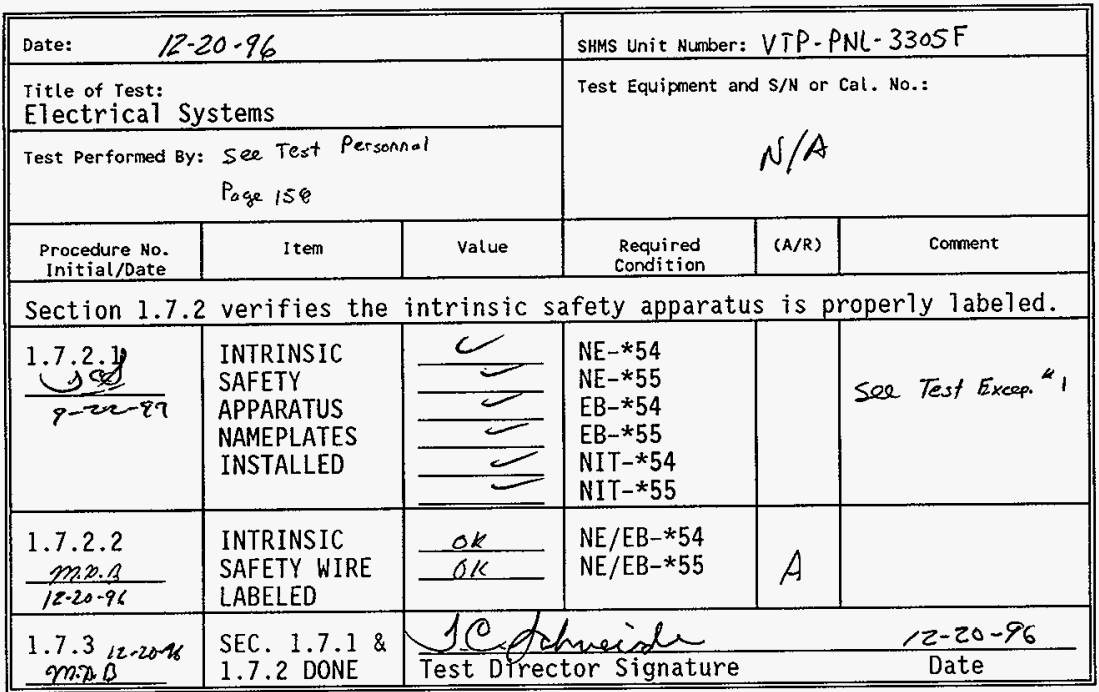

Test Witneps/Review:
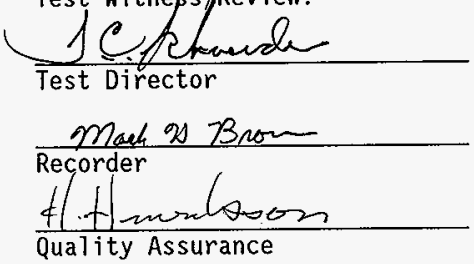

\section{$\frac{12-3<-96}{\text { Date }}$}

$12-20-96$

Date

$\frac{12-31-96}{\text { Date }}$ 
TEST DATA SHEET

\begin{tabular}{|c|c|c|c|c|c|}
\hline \multicolumn{3}{|c|}{ Date: $12-20-96$} & \multicolumn{3}{|c|}{ SHMS Unit Number: VTP-PNL $-3305 F$} \\
\hline \multicolumn{3}{|c|}{$\begin{array}{l}\text { Iitle of rest: } \\
\text { Pneumatic Systems }\end{array}$} & \multirow{2}{*}{\multicolumn{3}{|c|}{$\begin{array}{l}\text { Test Equipment and } S / \mathrm{N} \text { or Cal. No.: } \\
\text { N/A }\end{array}$}} \\
\hline \multicolumn{3}{|c|}{$\begin{aligned} & \text { Test Performed By: } \text { see Test Personnal } \\
& \text { Page } 158\end{aligned}$} & & & \\
\hline $\begin{array}{l}\text { Procedure No. } \\
\text { Initial/Date }\end{array}$ & Item & Value & $\begin{array}{l}\text { Required } \\
\text { Condition }\end{array}$ & $(A / R)$ & Corment \\
\hline \multicolumn{6}{|c|}{ Section 1.7.4.1 provides the pneumatic test section initial conditions. } \\
\hline $\begin{array}{l}1.7 .4 .1 .1 \\
m_{10.8} \\
\end{array}$ & $\begin{array}{l}\text { INITIAL } \\
\text { CONDITIONS }\end{array}$ & ok & $\begin{array}{l}\text { BKR \& FUSE } \\
\text { LINE UP }\end{array}$ & $A$ & \\
\hline $1.7,4.1 .2$ & $\begin{array}{l}\text { INITIAL } \\
\text { CONDITIONS }\end{array}$ & OK & $\begin{array}{l}\text { VALVE LINE } \\
\text { UP }\end{array}$ & A & \\
\hline$\frac{1.7 .4 .1 .3}{2 m_{3} / 3}$ & $\begin{array}{l}\text { DISCONNECT } \\
1 / 16^{\prime \prime} \text { TUBING } \\
\text { ON MON-*60 }\end{array}$ & OK & $\begin{array}{l}\text { TUBING } \\
\text { CONFIGURED }\end{array}$ & $A$ & \\
\hline $\begin{array}{l}1.7 .4 .1 .4 \\
m, 2, x^{2}\end{array}$ & $\begin{array}{l}\text { DISCONNECT } \\
\text { TUBING ON } \\
\text { NIT }-* 52\end{array}$ & ok & $\begin{array}{l}\text { TUBING } \\
\text { CONFIGURED }\end{array}$ & A & \\
\hline $\begin{array}{l}1.7 .4 .1 .5 \\
\text { mi.x. } \\
\end{array}$ & $\begin{array}{l}\text { PLUG VENT } \\
\text { FLT }-* 63\end{array}$ & ok & $\begin{array}{l}\text { FLT-*63 } \\
\text { PLUGGED }\end{array}$ & A & \\
\hline \multicolumn{6}{|c|}{ Section 1.7.4.2 verifies the main sample flow loop. } \\
\hline $\begin{array}{l}1.7 .4 .2 .1 \\
\text { mo.s } \\
\end{array}$ & $\begin{array}{l}\text { CLOSE CB-3/4 } \\
\text { ADJ. FLOW }\end{array}$ & $\frac{\partial k}{\partial u}$ & $\begin{array}{l}\text { CB CLOSED } \\
\text { FIT-*57 } \\
\text { INDICATION }\end{array}$ & A & \\
\hline $\begin{array}{l}1.7 .4 .2 .2 \\
m .2 .3 \\
\end{array}$ & $\begin{array}{l}\text { PDI }-* 51 \\
\text { READING } \\
\end{array}$ & $27^{\circ} 1 t_{20}$ & $20-40 " \mathrm{H}_{2} \mathrm{O}$ & A & \\
\hline $\begin{array}{c}1.7 .4 .2 .3 \\
m .3 .8 \\
\end{array}$ & $\begin{array}{l}\text { PI }-* 53 \\
\text { READING }\end{array}$ & $-2.6^{\circ} \mathrm{H}_{2}$ & $\begin{array}{l}-2 \text { to }-4 \\
" \mathrm{Hg}\end{array}$ & A & \\
\hline $\begin{array}{c}1.7 .4 .2 .4 \\
m 3 . B \\
\end{array}$ & RESET ALARMS & OK & NO ALARMS & $A$ & \\
\hline $\begin{array}{r}1.7 .4 .2 .5 \\
m n_{1} x_{D} \\
\end{array}$ & CLOSE SV-*16 & $\begin{array}{l}0.13^{*} H_{0} \\
\frac{O 4}{O K} \\
\end{array}$ & 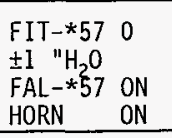 & A & \\
\hline $\begin{array}{c}1.7 .4 .2 .6 \\
m, 2 \infty\end{array}$ & OPEN SV-*16 & ok & $\begin{array}{l}\text { FAL-*57 ON } \\
\text { HORN OFF } \\
\end{array}$ & A & \\
\hline $\begin{array}{l}1.7 .4 .2 .7 \\
\text { ming.0 }\end{array}$ & RESET ALARMS & ok & $\begin{array}{l}\text { ALARMS } \\
\text { RESET }\end{array}$ & A & \\
\hline
\end{tabular}


TEST DATA SHEET

\begin{tabular}{|c|c|c|c|c|c|}
\hline \multicolumn{3}{|c|}{ Date: $\quad 12-20-96$} & \multicolumn{3}{|c|}{ SHMS Unit Number: VTP- $\rho_{N L}-3305 F$} \\
\hline \multicolumn{3}{|c|}{$\begin{array}{l}\text { Title of Test: } \\
\text { Pneumatic Systems }\end{array}$} & \multirow{2}{*}{\multicolumn{3}{|c|}{$\begin{array}{l}\text { Test Equipment and S/N or Cal. No.: } \\
\text { See page } 16 i\end{array}$}} \\
\hline \multicolumn{3}{|c|}{$\begin{aligned} & \text { Test Performed By: } \text { See Test Personnel } \\
& \text { Page } 158\end{aligned}$} & & & \\
\hline $\begin{array}{l}\text { Procedure No. } \\
\text { Initial/Date }\end{array}$ & Item & value & $\begin{array}{l}\text { Required } \\
\text { Condition } \\
\end{array}$ & $(A / R)$ & Comment \\
\hline $\begin{array}{c}1.7 .4 .2 .8 \\
32.2 .8 \\
\end{array}$ & CLOSE SV-*25 & 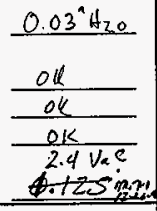 & 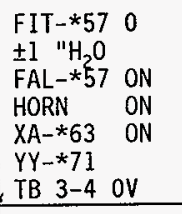 & $A$ & \\
\hline $\begin{array}{l}1.7 .4 .2 .9 \\
m . D . p\end{array}$ & OPEN SV-*25 & $\frac{\frac{O K}{O K}}{\frac{O K}{126.2 V_{a C}}}$ & 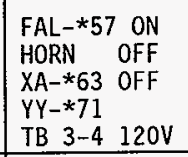 & $A$ & \\
\hline $\begin{array}{c}1.7 .4 .2 .10 \\
m 2 \cdot \beta \\
\end{array}$ & RESET ALARMS & OK & $\begin{array}{l}\text { ALARMS } \\
\text { RESET }\end{array}$ & $A$ & \\
\hline$\frac{1.7 .4 .2 .11}{m .0 . \beta}$ & $\begin{array}{l}\text { OPEN SV-*54 } \\
\& \text { SV-*66 } \\
\text { READ PRESS. } \\
\text { PDIT-*60 } \\
\end{array}$ & $\frac{\partial k}{-42.00}$ & $\begin{array}{l}\text { VALVES OPEN } \\
-27 \text { to }-82 \\
" \mathrm{H}_{3} \mathrm{O}\end{array}$ & A & \\
\hline$\frac{1.7 .4 .2 .12}{\operatorname{man}}$ & $\begin{array}{l}\text { CLOSE SV-*54 } \\
\text { OPEN SV-*63 } \\
\text { READ PRESS. } \\
\text { PDIT-*60 } \\
\end{array}$ & $\frac{0 \mathrm{~K}}{-0.004^{4} \mathrm{H}_{30}}$ & $\begin{array}{l}\text { VALVES } \\
\text { OPERATED } \\
0 \pm 1 " \mathrm{H}_{2} \mathrm{O}\end{array}$ & $A$ & \\
\hline $\begin{array}{l}1.7 .4 .2 .13 \\
m: 2.3 \\
\end{array}$ & $\begin{array}{l}\text { CLOSE SV-*63 } \\
\text { OPEN SV-*55 } \\
\text { READ PRESS. } \\
\text { PDIT-*60 } \\
\end{array}$ & $\frac{O K}{=43.4^{\circ} \mathrm{H}_{2} \mathrm{O}}$ & $\begin{array}{l}\text { VALVES } \\
\text { OPERATED } \\
-27 \text { to }-82 \\
=\mathrm{H}_{2} \mathrm{O} \\
\end{array}$ & A & \\
\hline$\frac{1.7 .4 .2 .14}{\min 8}$ & $\begin{array}{l}\text { CLOSE SV }-* 55 \\
\text { OPEN SV }-* 64 \\
\text { READ PRESS. } \\
\text { PDIT-*60 } \\
\end{array}$ & $\frac{0 k}{0.004^{4} H_{20}}$ & $\begin{array}{l}\text { VALVES } \\
\text { OPERATED } \\
0 \pm 1 " \mathrm{H}_{2} \mathrm{O}\end{array}$ & $A$ & \\
\hline $\begin{array}{l}1.7 .4 .2 .15 \\
m 2.0 \\
\end{array}$ & $\begin{array}{l}\text { CLOSE SV-*64 } \\
\& \text { SV-*66 }\end{array}$ & $N / A$ & N/A & A & \\
\hline \multicolumn{6}{|c|}{ Section 1.7.4.3 verifies the grab sample flow loop. } \\
\hline $\begin{array}{c}1.7 .4 .3 .1 \\
m . x . \beta \\
\end{array}$ & READ PDI-*51 & $\frac{O R}{27^{4} / A_{20}}$ & $20-40 \quad " \mathrm{H}_{2} \mathrm{O}$ & A & \\
\hline
\end{tabular}


TEST DATA SHEET

\begin{tabular}{|c|c|c|c|c|c|}
\hline \multicolumn{3}{|c|}{ Date: $\quad / 2 \cdot 20-96$} & \multicolumn{3}{|c|}{ SHMS Unit Number: VTP-PNL-330SF } \\
\hline \multicolumn{3}{|c|}{$\begin{array}{l}\text { Title of Test: } \\
\text { Pneumatic Systems }\end{array}$} & \multirow{2}{*}{\multicolumn{3}{|c|}{$\begin{array}{l}\text { Test Equipnent and } S / \mathrm{N} \text { or Cal. No.: } \\
\text { N/A }\end{array}$}} \\
\hline \multicolumn{3}{|c|}{$\begin{aligned} \text { Test Performed By: } & \text { See Test Personnd } \\
& \text { Page 15e }\end{aligned}$} & & & \\
\hline $\begin{array}{l}\text { Procedure No. } \\
\text { Injitial/Date }\end{array}$ & Item & Value & $\begin{array}{l}\text { Required } \\
\text { Condition } \\
\end{array}$ & $(A / R)$ & corment \\
\hline $\begin{array}{l}1.7 .4 .3 .2 \\
m_{n} \nu_{1.3} \\
\end{array}$ & $\begin{array}{l}\text { PUSH PB-*59 } \\
\text { GRAB SAMPLE }\end{array}$ & Ox & $\begin{array}{l}\text { START } \\
\text { STOPWATCH }\end{array}$ & A & \\
\hline $\begin{array}{r}1.7 .4 .3 .3 \\
\text { op.4.8. } \\
\end{array}$ & $\begin{array}{l}\text { GRAB SAMPLE } \\
\text { LAMPS ON } \\
\text { READ FIV }-* 52\end{array}$ & $\begin{array}{l}\text { ok } \\
\text { ok } \\
\text { all } \\
\text { o }\end{array}$ & $\begin{array}{l}P B L-* 58 \text { ON } \\
P B L-* 59 \text { ON } \\
Y A L-* 58 \text { ON } \\
\text { FIV }-* 52 \quad 0 \\
\end{array}$ & A & \\
\hline$\frac{1.7 .4 .3 .4}{m 20.3}$ & $\begin{array}{l}\text { OPEN SV-*15 } \\
\& \text { ADJ. FIV }- \\
\star 52\end{array}$ & $\frac{0}{10 \mathrm{CFH}}$ & $\begin{array}{l}\text { SV-* } 15 \text { OPEN } \\
\text { FIV } * 52 \\
\text { FLOW } 10 \text { CFH }\end{array}$ & A & \\
\hline $\begin{array}{l}1.7 .4 .3 .5 \\
m i 2 . \\
\end{array}$ & READ PDI $-* 51$ & $30^{\prime \prime} \mathrm{H}_{2} \mathrm{O}$ & $\begin{array}{l}\text { PDI } * * 51 \\
\text { HIGHER THAN } \\
1.7 .4 .3 .1 \\
\end{array}$ & A & \\
\hline $\begin{array}{l}1.7 .4 .3 .6 \\
\text { mase } \\
\end{array}$ & CLOSE SV-* 15 & 0 & FIV $-* 520$ & A & \\
\hline $\begin{array}{l}1.7 .4 .3 .7 \\
m x .3 \\
\end{array}$ & OPEN SV-* 15 & $10^{\circ} \mathrm{Flt}$ & $\begin{array}{l}\text { FIV }-* 52 \\
\text { FLOW }\end{array}$ & $A$ & \\
\hline $\begin{array}{l}1.7 .4 .3 .8 \\
m i n\end{array}$ & $\begin{array}{l}\text { PBL }-* 59 \\
\text { GRAB SAMPLE } \\
\text { TIME }\end{array}$ & $5.41 \mathrm{sec}$ & $\begin{array}{l}\text { PBL }-* 59 \text { OFF } \\
\text { STOP WATCH } \\
5 \pm .5 \mathrm{MIN} \\
\end{array}$ & A & \\
\hline $\begin{array}{l}1.7 .4 .3 .9 \\
9 \mathrm{ma3}\end{array}$ & $\begin{array}{l}\text { PB } * 58 \text { RESET } \\
\text { SAMPLER } \\
\end{array}$ & - & $\begin{array}{l}\mathrm{PBL}-* 58 \text { OFF } \\
\mathrm{YAL}-* 58 \text { OFF } \\
\end{array}$ & A & \\
\hline \multicolumn{6}{|c|}{ Section 1.7.4.4 verifies the $\mathrm{H}_{2}$ cell calibration loop } \\
\hline $\begin{array}{l}1.7 .4 .4 .1 \\
\text { manes. }\end{array}$ & $\begin{array}{l}\text { CONNECT } \mathrm{H}_{2} \\
\text { CAL GAS }\end{array}$ & $N / A$ & N/A & $A$ & \\
\hline $\begin{array}{l}1.7 .4 .4 .2 \\
\sin 8 \\
\end{array}$ & $\begin{array}{l}\text { OPEN ISO } \\
\text { VALVE }\end{array}$ & $2.75 P_{316}$ & $<10$ PSIG & A & \\
\hline $\begin{array}{l}1.7 .4 .4 .3 \\
m 0.8\end{array}$ & $\begin{array}{l}\text { OPEN SV-*18 } \\
\text { ADJ. FIV-*56. }\end{array}$ & $2 \mathrm{CFH}$ & $2 \pm .1 \mathrm{CFH}$ & A & \\
\hline
\end{tabular}

all $12-20-96$ 
TEST DATA SHEET

\begin{tabular}{|c|c|c|c|c|c|}
\hline \multicolumn{3}{|c|}{ Date: $\quad 12-20-96$} & \multicolumn{3}{|c|}{ SHMS Unit Number: VTP-PNL-3305F } \\
\hline \multicolumn{3}{|c|}{$\begin{array}{l}\text { Title of Test: } \\
\text { Pneumatic Systems }\end{array}$} & \multirow{2}{*}{\multicolumn{3}{|c|}{$\begin{array}{l}\text { Test Equipment and } \mathrm{S} / \mathrm{N} \text { or Cal. No.: } \\
\text { see Page } 161\end{array}$}} \\
\hline \multicolumn{3}{|c|}{$\begin{aligned} \text { Test Performed By: } & \text { See Test Personnal } \\
& \text { Page } 158\end{aligned}$} & & & \\
\hline $\begin{array}{l}\text { Procedure No. } \\
\text { Initial/Date }\end{array}$ & Item & Value & $\begin{array}{l}\text { Required } \\
\text { Condition }\end{array}$ & $(A / R)$ & Comment \\
\hline $\begin{array}{l}1.7 .4 .4 .4 \\
m p .8\end{array}$ & $\begin{array}{l}\text { VERIFY } \\
\text { CONDITIONS }\end{array}$ & 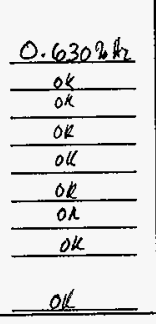 & $\begin{array}{l}\text { NIT-*54 } \\
>-525 \% \mathrm{H}_{2} \\
\text { NAH-*55 ON } \\
\text { NAH-*54 ON } \\
\text { PBL-*59 ON } \\
\text { PBL-*58 ON } \\
\text { YAL-*58 ON } \\
\text { FIV-*52 FLO } \\
\text { HORN ON } \\
\text { TB2-21/22 } \\
\text { OPEN } \\
\end{array}$ & A & \\
\hline $\begin{array}{l}1.7 .4 .4 .5 \\
m . x \cdot B\end{array}$ & $\begin{array}{l}\text { ACKNOWLEDGE } \\
\text { HORN }\end{array}$ & $N / A$ & $\mathrm{~N} / \mathrm{A}$ & A & \\
\hline $\begin{array}{l}1.7 .4 .4 .6 \\
\text { onxy }\end{array}$ & $\begin{array}{l}\text { CLOSE SV-*18 } \\
\text { VERIFY } \\
\text { CONDITIONS }\end{array}$ & $\begin{array}{l}\frac{0.599 \% .42}{\frac{o k}{o k}} \\
\frac{\frac{o k}{o k}}{\frac{O K}{o k}} \\
\frac{O k}{2}\end{array}$ & $\begin{array}{l}\text { FIV-*56 } 0 \\
\text { NIT-*54 } \\
<.625 \% \mathrm{H}_{2} \\
\text { NAH-*55 OFF } \\
\text { NAH- } * 54 \text { ON } \\
\text { PBL-*58 ON } \\
\text { YAL-*58 ON } \\
\text { TB2-21/22 } \\
\text { CLOSED } \\
\end{array}$ & & \\
\hline$\frac{1.7 .4 .4 .7}{-9000}$ & $\begin{array}{l}\text { PUSH RESET } \\
\text { SAMPLER PB- } \\
\star 58 \\
\end{array}$ & ok & $\begin{array}{l}\text { PBL-*58 OFF } \\
\text { YAL-*58 OFF }\end{array}$ & A & \\
\hline $\begin{array}{c}1.7 .4 .4 .8 \\
\operatorname{mos} \\
\end{array}$ & RESET ALARM & _ok & $\mathrm{NAH}-\star 54$ OFF & $A$ & \\
\hline 1.7 .4 .4 .9 & $\begin{array}{l}\text { OPEN SV-*19 } \\
\text { ADJ. FIV-*56 }\end{array}$ & 2 & $2 \pm .1 \mathrm{CFH}$ & $A$ & \\
\hline
\end{tabular}

al1 $12 \cdot 20-96$ 
TEST DATA SHEET

\begin{tabular}{|c|c|c|c|c|c|}
\hline \multicolumn{3}{|c|}{ Date: $\quad 12-20-96$} & \multicolumn{3}{|c|}{ SHMS Unit Number: VTP - PNL - 3305F } \\
\hline \multicolumn{3}{|c|}{$\begin{array}{l}\text { Title of Test: } \\
\text { Pneumatic Systems }\end{array}$} & \multirow{2}{*}{\multicolumn{3}{|c|}{$\begin{array}{l}\text { Test Equipment and } S / \mathrm{N} \text { or Cal. No.: } \\
\text { See Page } 161\end{array}$}} \\
\hline \multicolumn{3}{|c|}{$\begin{array}{c}\text { Test Performed By: see Test Personned } \\
\text { Page } 15 e\end{array}$} & & & \\
\hline $\begin{array}{l}\text { Procedure No. } \\
\text { Initial/Date }\end{array}$ & Item & Value & $\begin{array}{l}\begin{array}{l}\text { Required } \\
\text { Condition }\end{array} \\
\end{array}$ & $(A / R)$ & Comment \\
\hline $\begin{array}{l}1.7 .4 .4 .10 \\
\text { M.x.B. } \\
\end{array}$ & $\begin{array}{l}\text { VERIFY } \\
\text { CONDITIONS }\end{array}$ & 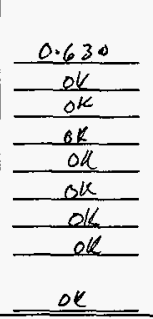 & $\begin{array}{l}\text { NIT-*54 SS } \\
>.625 \% \mathrm{H}^{-3} \\
\text { NAH-*55 ON } \\
\text { NAH-*54 ON } \\
\text { PBL-*59 ON } \\
\text { PBL-*58 ON } \\
\text { YAL-*58 ON } \\
\text { FIV-*52 FL0 } \\
\text { HORN ON } \\
\text { TB2-21/22 } \\
\text { OPEN }\end{array}$ & $A$ & · \\
\hline $\begin{array}{r}1.7 .4 .4 .11 \\
m: 0.0 \\
\end{array}$ & $\begin{array}{l}\text { ACKNOWLEDGE } \\
\text { HORN }\end{array}$ & $N / A$ & $N / A$ & $A$ & \\
\hline $\begin{array}{l}1.7 .4 .4 .12 \\
m x .8\end{array}$ & $\begin{array}{l}\text { CLOSE SV-*19 } \\
\text { VERIFY } \\
\text { CONDITIONS }\end{array}$ & 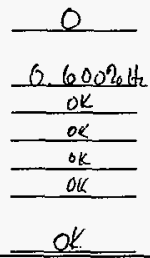 & $\begin{array}{l}\text { FIV } * 56 \text { o } \\
\text { NIT-*54 } 55 \text { m. } \\
<.625 \% \mathrm{H}_{2} \\
\text { NAH-*55 OFF } \\
\text { NAH-*54 ON } \\
\text { PBL-*58 ON } \\
\text { YAL-*58 ON } \\
\text { TB2-21/22 } \\
\text { CLOSED }\end{array}$ & $A$ & \\
\hline $\begin{array}{c}1.7 .4 .4 .13 \\
m .0 \\
\end{array}$ & $\begin{array}{l}\text { PUSH RESET } \\
\text { SAMPLER PB- } \\
* 58\end{array}$ & $\sigma k$ & $\begin{array}{l}\text { PBL }-* 58 \text { OFF } \\
\text { YAL }-* 58 \text { OFF }\end{array}$ & $A$ & \\
\hline $\begin{array}{l}1.7 .4 .4 .14 \\
m 2.0 \\
\end{array}$ & RESET ALARM & ok & $\mathrm{NAH}-* 54$ OFF & $A$ & \\
\hline $\begin{array}{l}1.7 .4 .4 .15 \\
\text { m.n. } \\
\end{array}$ & $\begin{array}{l}\text { DISCONNECT } \\
\text { CAL. GAS }\end{array}$ & $N / A$ & $N / A$ & $A$ & \\
\hline $\begin{array}{c}1.7 .4 .4 .16 \\
m . x . p \\
\end{array}$ & $\begin{array}{l}\text { PUSH PB } * 54 \\
\text { VERIFY } \\
\text { CONDITIONS }\end{array}$ & 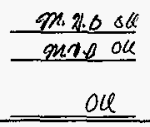 & $\begin{array}{l}X A-* 63 \text { ON } \\
\text { PBL-*54 ON } \\
\text { TB2-23/24 } \\
\text { OPEN }\end{array}$ & $A$ & \\
\hline $\begin{array}{l}1.7 .4 .4 .17 \\
3 x .8 \\
\end{array}$ & $\begin{array}{l}\text { PUSH PB-*54 } \\
\text { VERIFY } \\
\text { CONDITIONS }\end{array}$ & $\begin{array}{r}-\frac{O K}{O K} \\
-O K \\
\end{array}$ & $\begin{array}{l}X A-* 63 \text { OFF } \\
\text { PBL } * 54 \text { OFF } \\
\text { TB2-23/24 } \\
\text { CLOSED }\end{array}$ & $A$ & \\
\hline
\end{tabular}


TEST DATA SHEET

\begin{tabular}{|c|c|c|c|c|c|}
\hline \multicolumn{3}{|c|}{$12-20-96$} & \multicolumn{3}{|c|}{ SHMS Unit Number: VTP-PNL $-3305 \mathrm{~F}$} \\
\hline \multicolumn{3}{|c|}{$\begin{array}{l}\text { Title of Test: } \\
\text { Pneumatic Systems }\end{array}$} & \multirow{2}{*}{\multicolumn{3}{|c|}{$\begin{array}{l}\text { Test Equiprient and S/N or Cal. No.: } \\
\text { See Page } 161\end{array}$}} \\
\hline \multicolumn{3}{|c|}{$\begin{aligned} \text { Test Performed By: } & \text { So Test Personnal } \\
& \text { Page } 150\end{aligned}$} & & & \\
\hline $\begin{array}{l}\text { Procedure No. } \\
\text { Initial/Date } \\
\end{array}$ & Item & value & $\begin{array}{l}\text { Required } \\
\text { Condition }\end{array}$ & $(A / R)$ & Comment \\
\hline \multicolumn{6}{|c|}{ Section 1.7 .4 .5 verifies the GC instrument tubing and calibration gas } \\
\hline $\begin{array}{l}1.7 .4 .5 .1 \\
m \times .3\end{array}$ & $\begin{array}{l}\text { REMOVE PLUG } \\
\text { \& CONNECT } \\
\text { TEST GAS } \\
\end{array}$ & $\frac{\text { ore }}{3 P 506}$ & $\begin{array}{l}\text { TEST GAS } \\
3-5 \text { PSIG }\end{array}$ & $A$ & \\
\hline $\begin{array}{l}1.7 .4 .5 .2 \\
\text { ginpo }\end{array}$ & $\begin{array}{l}\text { VERIFY NO } \\
\text { GAS FLOW }\end{array}$ & $\begin{array}{l}m a n s \\
m_{1} x_{1}\end{array}$ & $\begin{array}{l}\mathrm{H}_{2} \text { GAS PORT } \\
\text { SAMPLE A IN }\end{array}$ & A & \\
\hline $\begin{array}{l}1.7 .4 .5 .3 \\
m \text { m.n.s. } \\
\end{array}$ & $\begin{array}{l}\text { VERIFY COL A } \\
\text { GAS FLOW }\end{array}$ & - od & COL A FLOW & $A$ & \\
\hline $\begin{array}{l}1.7 .4 .5 .4 \\
m x . D\end{array}$ & $\begin{array}{l}\text { REPLACE COL } \\
\text { A CAP }\end{array}$ & $N / A$ & $N / A$ & $A$ & \\
\hline $\begin{array}{l}1.7 .4 .5 .5 \\
\text { mone }\end{array}$ & $\begin{array}{l}\text { VERIFY COL B } \\
\text { GAS FLOW }\end{array}$ & or & COL B FLOW & $A$ & \\
\hline $\begin{array}{l}1.7 .4 .5 .6 \\
\text { ong.D } \\
\end{array}$ & $\begin{array}{l}\text { REPLACE COL } \\
\text { B CAP }\end{array}$ & $N / A$ & $N / A$ & A & \\
\hline $\begin{array}{l}1.7 .4 .5 .7 \\
-\min x+\beta\end{array}$ & $\begin{array}{l}\text { VERIFY } \\
\text { SAMPLE VENT } \\
\text { FLOW } \\
\end{array}$ & ok & $\begin{array}{l}\text { SAMPLE VENT } \\
\text { FLOW }\end{array}$ & $A$ & \\
\hline $\begin{array}{l}1.7 .4 .5 .8 \\
\text { mane }\end{array}$ & $\begin{array}{l}\text { REPLACE } \\
\text { SAMPLE VENT } \\
\text { CAP }\end{array}$ & $N / A$ & $N / A$ & $A$ & \\
\hline $\begin{array}{l}1.7 .4 .5 .9 \\
\min _{10}{ }^{2} \\
\end{array}$ & $\begin{array}{l}\text { INSTALL } \\
\text { JUMPER } \\
\end{array}$ & $N / A$ & $N / A$ & A & \\
\hline $\begin{array}{l}1.7 .4 .5 .10 \\
-m .0 \\
\end{array}$ & $\begin{array}{l}\text { CLOSE FU-15 } \\
\text { VERIFY FLOW }\end{array}$ & $\frac{m k}{O K}$ & $\begin{array}{l}\mathrm{H}_{2} \text { CAL FLOW } \\
\text { SAMP A IN } \\
\text { FLOW }\end{array}$ & A & \\
\hline $\begin{array}{l}1.7 .4 .5 .11 \\
=0 m e \\
\end{array}$ & $\begin{array}{l}\text { INSTALL PLUG } \\
\& \text { CAP } \\
\end{array}$ & - ok & $\begin{array}{l}\text { PLUG } \mathrm{H}_{2} \text { CAL } \\
\text { CAP SAMP A } \\
\end{array}$ & A & \\
\hline $\begin{array}{c}1.7 .4 .5 .12 \\
-m 2 . n \\
\end{array}$ & $\begin{array}{l}\text { OPEN SV-*63 } \\
\& \text { READ } \\
\text { PDIT }-* 60\end{array}$ & 80.' $\mathrm{H}_{2} \mathrm{O}$ & $\begin{array}{l}\text { PDIT }-* 60= \\
28 \times \text { PRESS } \\
1.7 .4 .5 .1 \\
\pm 20 \mathrm{H}_{2} \mathrm{O} \\
\end{array}$ & A & $\frac{28}{\frac{20}{35}}$ \\
\hline $\begin{array}{l}1.7 .4 .5 .13 \\
m_{M} x \cdot \beta \\
\end{array}$ & $\begin{array}{l}\text { OPEN SV }-* 64 \\
\text { VERIFY FLOW }\end{array}$ & ok & $\begin{array}{l}\text { SAMP B IN } \\
\text { FLOW }\end{array}$ & $A$ & \\
\hline
\end{tabular}


TEST DATA SHEET

\begin{tabular}{|c|c|c|c|c|c|}
\hline \multicolumn{3}{|c|}{ Date: $12-20-96$} & \multicolumn{3}{|c|}{ SHMS Unit Number: VTP - PNL - 3305F } \\
\hline \multicolumn{3}{|c|}{$\begin{array}{l}\text { Title of Test: } \\
\text { Pneumatic Systems }\end{array}$} & \multirow{2}{*}{\multicolumn{3}{|c|}{$\begin{array}{l}\text { Test Equipnent and } \mathrm{S} / \mathrm{N} \text { or Cal. No.: } \\
\text { See Page } 161\end{array}$}} \\
\hline \multicolumn{3}{|c|}{$\begin{aligned} \text { Test Performed By: } & \text { see Test Personnol } \\
& \text { Page } 150\end{aligned}$} & & & \\
\hline $\begin{array}{l}\text { Procedure No. } \\
\text { Initial/Date }\end{array}$ & Item & Value & $\begin{array}{l}\text { Requi red } \\
\text { Condition }\end{array}$ & $(A / R)$ & Comment \\
\hline $\begin{array}{l}1.7 .4 .5 .14 \\
m 2.8\end{array}$ & $\begin{array}{l}\text { REMOVE TEST } \\
\text { GAS }\end{array}$ & N/A & N/A & A & \\
\hline $\begin{array}{r}1.7 .4 .5 .15 \\
m_{n \rightarrow D} \\
\end{array}$ & $\begin{array}{l}\text { CLOSE SV-*63 } \\
\& \text { SV-*64 }\end{array}$ & $N / A$ & N/A & A & \\
\hline $\begin{array}{l}1.7 .4 .5 .16 \\
m . p \cdot D^{-1}\end{array}$ & $\begin{array}{l}\text { OPEN FU-15 } \\
\text { REMOVE } \\
\text { JUMPER }\end{array}$ & $N / A$ & N/A & A & \\
\hline $\begin{array}{l}1.7 .4 .5 .17 \\
m .2 . p \\
\end{array}$ & $\begin{array}{l}\text { INSTALL } \\
\text { SAMP A UNION }\end{array}$ & $N / A$ & $N / A$ & A & \\
\hline $\begin{array}{l}1.7 .4 .5 .18 \\
=9.7 .0 \\
\end{array}$ & $\begin{array}{l}\text { OPEN SV }-\star 60 \\
\text { VERIFY FLOW }\end{array}$ & Dk & $\begin{array}{l}\text { FI-*60 } \\
\text { NO FLOW }\end{array}$ & A & \\
\hline $\begin{array}{l}1.7 .4 .5 .19 \\
m 0.0\end{array}$ & $\begin{array}{l}\text { POWER SOV- } \\
\star 60 \text {, VERIFY } \\
\text { NO FLOW }\end{array}$ & $O K$ & $\begin{array}{l}\text { FI-*60 } \\
\text { NO FLOW }\end{array}$ & $A$ & \\
\hline $\begin{array}{l}1.7 .4 .5 .20 \\
\operatorname{min.p}\end{array}$ & $\begin{array}{l}\text { OPEN SV }-* 68 \\
\text { ADJ SV }-* 67\end{array}$ & $-500 \mathrm{~cm}$ & $\begin{array}{l}\mathrm{FI}-* 60 \\
50 \mathrm{CCM}\end{array}$ & $A$ & \\
\hline $\begin{array}{l}1.7 .4 .5 .21 \\
9 m .2 .5 \\
\end{array}$ & $\begin{array}{l}\text { DEENERGIZE } \\
\text { SOV-* } 60\end{array}$ & $O K$ & $\begin{array}{l}\text { FI-*60 FLOW } \\
\text { DECREASES } \\
\end{array}$ & A & \\
\hline 1.7 .4 .5 .22 & $\begin{array}{l}\text { CLOSE SV-*60 } \\
\& \text { SV }-* 68 \\
\end{array}$ & $N / A$ & N/A & A & \\
\hline $\begin{array}{l}7.4 .5 .23 \\
\end{array}$ & $\begin{array}{l}\text { REMOVE } \\
\text { SAMP A UNION }\end{array}$ & $N / A$ & $N / A$ & $A$ & \\
\hline $\begin{array}{l}1.7 .4 .5 .24 \\
m .2 .3 \\
\end{array}$ & $\begin{array}{l}\text { CONNECT TEST } \\
\text { GAS } \mathrm{N}_{2} \mathrm{O} \text { PORT } \\
\end{array}$ & 3PSiE & $\begin{array}{l}\text { TEST GAS } \\
3-5 \text { PSIG }\end{array}$ & $A$ & \\
\hline $\begin{array}{l}1.7 .4 .5 .25 \\
\text { m.0.0 }\end{array}$ & $\begin{array}{l}\text { VERIFY NO } \\
\text { FLOW }\end{array}$ & $-\frac{O K}{O K}$ & $\begin{array}{l}\text { SAMP B IN \& } \\
\text { FLT-*63 } \\
\text { NO FLOW }\end{array}$ & $A$ & \\
\hline 1.7 .4 .5 .26 & $\begin{array}{l}\text { INSTALL } \\
\text { JUMPER } \\
\end{array}$ & $N / A$ & $N / A$ & A & \\
\hline $\begin{array}{l}1.7 .4 .5 .27 \\
2 x 2.2\end{array}$ & $\begin{array}{l}\text { CLOSE FU-15 } \\
\text { VERIFY FLOW }\end{array}$ & $\frac{o^{K}}{\Delta K}$ & $\begin{array}{l}\text { SAMP B IN } \\
\text { FLT-*63 } \\
\text { FLOW }\end{array}$ & A & \\
\hline
\end{tabular}


APPENDIX E

TEST DATA SHEET

\begin{tabular}{|c|c|c|c|c|c|}
\hline \multicolumn{3}{|c|}{ Date: $\quad 12-20-96$} & \multicolumn{3}{|c|}{ SHMS Unit Number: VTP-PNL-330SF } \\
\hline \multicolumn{3}{|c|}{$\begin{array}{l}\text { Title of Test: } \\
\text { Pneumatic Systems }\end{array}$} & \multirow{2}{*}{\multicolumn{3}{|c|}{$\begin{array}{l}\text { Test Equipment and S/N or Cal. No.: } \\
\text { See paye } 161\end{array}$}} \\
\hline \multicolumn{3}{|c|}{$\begin{aligned} & \text { Test Performed By: } \text { see Test Personnel } \\
& \text { Page } 15 e \\
&\end{aligned}$} & & & \\
\hline $\begin{array}{c}\text { Procedure No. } \\
\text { Initial/Date }\end{array}$ & Item & Value & $\begin{array}{l}\text { Required } \\
\text { Condition }\end{array}$ & $(A / R)$ & Comment \\
\hline $\begin{array}{r}1.7 .4 .5 .28 \\
m_{m .2 .3} \\
\end{array}$ & $\begin{array}{l}\text { DISCONNECT } \\
\text { TEST GAS } \\
\end{array}$ & $N / A$ & $N / A$ & A) & \\
\hline $\begin{array}{l}1.7 .4 .5 .29 \\
\text { m.x.s } \\
\end{array}$ & OPEN FU-15 & $\mathrm{N} / \mathrm{A}$ & $N / A$ & $A$ & \\
\hline $\begin{array}{l}1.7 .4 .5 .30 \\
\min \times \\
\end{array}$ & $\begin{array}{l}\text { REMOVE } \\
\text { JUMPER } \\
\end{array}$ & $N / A$ & N/A & $\theta$ & \\
\hline $\begin{array}{l}1.7 .4 .5 .31 \\
m . x .3 \\
\end{array}$ & $\begin{array}{l}\text { INSTALL } \\
\text { SAMP B UNION }\end{array}$ & N/A & N/A & $A$ & \\
\hline $\begin{array}{c}1.7 .4 .5 .32 \\
\text { m.x.p } \\
\end{array}$ & $\begin{array}{l}\text { OPEN SV }-* 60 \\
\text { VERIFY FLOW }\end{array}$ & OK & $\begin{array}{l}F I-* 60 \\
\text { NO FLOW }\end{array}$ & $A$ & \\
\hline $\begin{array}{l}1.7 .4 .5 .33 \\
\text { m.x.8 } \\
\end{array}$ & $\begin{array}{l}\text { POWER SOV- } \\
* 60 \text {, VERIFY } \\
\text { NO FLOW }\end{array}$ & OL & $\begin{array}{l}\text { FI-*60 } \\
\text { NO FLOW }\end{array}$ & A & \\
\hline $\begin{array}{c}1.7 .4 .5 .34 \\
m . x .8 \\
\end{array}$ & $\begin{array}{l}\text { OPEN SV-*68 } \\
\& \text { ADJ SV-*67 }\end{array}$ & ove & $\begin{array}{l}\mathrm{FI}-* 60 \\
50 \mathrm{CCM} \\
\end{array}$ & $A$ & \\
\hline $\begin{array}{l}1.7 .4 .5 .35 \\
m .20 \\
\end{array}$ & $\begin{array}{l}\text { DEENERGIZE } \\
\text { SOV-*60 }\end{array}$ & ok & $\begin{array}{l}\text { FI-*60 FLOW } \\
\text { DECREASES }\end{array}$ & A & \\
\hline $\begin{array}{l}1.7 .4 .5 .36 \\
\min _{x \rightarrow 3} \\
\end{array}$ & $\begin{array}{l}\text { CLOSE SV-*60 } \\
\& \text { SV }-* 68 \\
\end{array}$ & N/A & $\mathrm{N} / \mathrm{A}$ & A & \\
\hline $\begin{array}{l}1.7 .4 .5 .37 \\
\text { min.8 } \\
\end{array}$ & $\begin{array}{l}\text { REMOVE } \\
\text { SAMP B UNION }\end{array}$ & $N / A$ & N/A & $A$ & \\
\hline $\begin{array}{l}1.7 .4 .5 .38 \\
\text { m.x.B. } \\
\end{array}$ & $\begin{array}{l}\text { OPEN CB-3/4 } \\
\text { ACK. ALARMS } \\
\end{array}$ & $N / A$ & N/A & A & \\
\hline \multicolumn{6}{|c|}{$\begin{array}{l}\text { Section } 1.7 .4 .6 \text { verifies the multi gas analyzer instrument tubing and } \\
\text { calibration gas loop. }\end{array}$} \\
\hline $\begin{array}{l}1.7 .4 .6 .1 \\
M . x \cdot B\end{array}$ & $\begin{array}{l}\text { VERIFY } \\
\text { NIT-*52 } \\
\text { SAMPLE LINES } \\
\end{array}$ & $N / A$ & $\mathrm{~N} / \mathrm{A}$ & $A$ & \\
\hline $\begin{array}{l}1.7 .4 .6 .2 \\
m .2 . n \\
\end{array}$ & $\begin{array}{l}\text { CONNECT TEST } \\
\text { GAS NH } 3 \text { PORT }\end{array}$ & 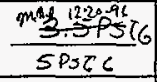 & $\begin{array}{l}\text { TEST GAS } \\
\text { 3-5 PSIG } \\
\end{array}$ & A & \\
\hline $\begin{array}{r}1.7 .4 .6 .3 \\
\text { m.3.0.0 } \\
\end{array}$ & $\begin{array}{l}\text { VERIFY NO } \\
\text { FLOW }\end{array}$ & $\frac{O Q}{O K}$ & $\begin{array}{l}\text { SAMPLE IN } \\
\text { FLT }-* 63\end{array}$ & A & \\
\hline
\end{tabular}


TEST DATA SHEET

\begin{tabular}{|c|c|c|c|c|c|}
\hline \multirow{2}{*}{\multicolumn{3}{|c|}{$\begin{array}{l}\text { Date: } 12-20-96 \\
\text { ritle of Test: } \\
\text { Pneumatic Systems } \\
\end{array}$}} & \multicolumn{3}{|c|}{ SHMS Unit Number: VTP-PNL $-3305 F$} \\
\hline & & & \multirow{2}{*}{\multicolumn{3}{|c|}{$\begin{array}{l}\text { Test Equipment and } S / \mathrm{N} \text { or Cal. No.: } \\
N / A\end{array}$}} \\
\hline \multicolumn{3}{|c|}{$\begin{aligned} \text { Test Performed By: } & \text { See Test Personnal } \\
& \text { Page } 158\end{aligned}$} & & & \\
\hline $\begin{array}{c}\text { Procedure No. } \\
\text { Initial/Date } \\
\end{array}$ & Item & Value & $\begin{array}{l}\text { Required } \\
\text { condition } \\
\end{array}$ & $(A / R)$ & Comment \\
\hline $\begin{array}{l}1.7 .4 .6 .4 \\
m m_{0.0} \\
\end{array}$ & $\begin{array}{l}\text { INSTALL } \\
\text { JUMPER }\end{array}$ & $N / A$ & $N / A$ & A & \\
\hline $\begin{array}{l}1.7 .4 .6 .5 \\
m i .0\end{array}$ & $\begin{array}{l}\text { CLOSE FU-15 } \\
\text { VERIFY GAS } \\
\text { FLOWS }\end{array}$ & $\frac{O K}{O O K}$ & $\begin{array}{l}\text { SAMPLE IN } \\
\text { FLT }-* 63 \\
\text { FIV }-* 70\end{array}$ & A & \\
\hline $\begin{array}{l}1.7 .4 .6 .6 \\
m i n .0 \\
\end{array}$ & $\begin{array}{l}\text { PLUG FLT }-* 63 \\
\& \text { MON-*60 } \\
\text { SAMPLE VENT }\end{array}$ & N/A & N/A & A & \\
\hline $\begin{array}{l}1.7 .4 .6 .7 \\
m .2 .8 \\
\end{array}$ & $\begin{array}{l}\text { OPEN SV-*66, } \\
\text { SV-*70 \& CAP } \\
\text { SAMPLE IN }\end{array}$ & $162 \% 120$ & $\begin{array}{l}\text { PDIT-*60= } \\
28 \times \text { PRESS } \\
1.7 .4 .6 .2 \\
\pm 20 " \mathrm{H}_{2} \mathrm{O} \\
\end{array}$ & A & $\frac{\frac{5}{28}}{\frac{5}{140}}$ \\
\hline $\begin{array}{r}1.7 .4 .6 .8 \\
m_{i} .0 .1 \\
\end{array}$ & $\begin{array}{l}\text { CLOSE SV-*66 } \\
\text { SV-*70 \& } \\
\text { REMOVE CAP }\end{array}$ & N/A & $N / A$ & A & \\
\hline $\begin{array}{r}1.7 .4 .6 .9 \\
m_{1} v .82 \\
\end{array}$ & $\begin{array}{l}\text { REMOVE FLT- } \\
* 63 \text { PLUG }\end{array}$ & $N / A$ & $N / A$ & $A$ & \\
\hline $\begin{array}{l}1.7 .4 .6 .10 \\
m_{1} . p \\
\end{array}$ & $\begin{array}{l}\text { REMOVE TEST } \\
\text { GAS }\end{array}$ & $N / A$ & $N / A$ & A & \\
\hline $\begin{array}{l}1.7 .4 .6 .11 \\
m 9.7 .8 \\
\end{array}$ & $\begin{array}{l}\text { CLOSE SV-*05 } \\
\& \text { SV }-* 16\end{array}$ & $N / A$ & $N / A$ & A & \\
\hline $\begin{array}{l}1.7 .4 .6 .12 \\
\dot{m i n . \beta} \\
\end{array}$ & $\begin{array}{l}\text { CONNECT TEST } \\
\text { GAS }\end{array}$ & 40 sile & $\begin{array}{l}\text { TEST GAS } \\
2-4 \text { PSIG } \\
\end{array}$ & A & \\
\hline $\begin{array}{l}1.7 .4 .6 .13 \\
\text { m.x.s } \\
\end{array}$ & $\begin{array}{l}\text { OPEN SV-*07 } \\
\& \text { VERIFY } \\
\text { NO FLOW } \\
\end{array}$ & - or & $\begin{array}{l}\text { SAMPLE IN } \\
\text { SAMPLE OUT }\end{array}$ & $A$ & \\
\hline $\begin{array}{c}1.7 .4 .6 .14 \\
m 20\end{array}$ & $\begin{array}{l}\text { OPEN SV-*09 } \\
\& \text { VERIFY } \\
\text { NO FLOW } \\
\end{array}$ & $\frac{O K}{\text { OKC }}$ & $\begin{array}{l}\text { SAMPLE IN } \\
\text { SAMPLE OUT }\end{array}$ & A & \\
\hline $\begin{array}{l}1.7 .4 .6 .15 \\
2 \operatorname{min.0} \\
\end{array}$ & $\begin{array}{l}\text { OPEN FU-15 \& } \\
\text { VERIFY FLOW }\end{array}$ & OU & $\begin{array}{l}\text { SAMPLE FLOW } \\
\text { IN - YES } \\
\text { OUT - NO } \\
\end{array}$ & $A$ & \\
\hline $\begin{array}{l}1.7 .4 .6 .16 \\
\end{array}$ & $\begin{array}{l}\text { CLOSE SV-*09 } \\
\text { VERIFY FLOW }\end{array}$ & $\partial$ & $\begin{array}{l}\text { SAMPLE IN } \\
\text { NO FLOW }\end{array}$ & 4 & \\
\hline
\end{tabular}


TEST DATA SHEET

\begin{tabular}{|c|c|c|c|c|c|}
\hline \multicolumn{3}{|c|}{ Date: $\quad 12-20-96$} & \multicolumn{3}{|c|}{ SHMS Unit Number: VTP-PNL-3305F } \\
\hline \multicolumn{3}{|c|}{$\begin{array}{l}\text { Title of Test: } \\
\text { Pneumatic Systems }\end{array}$} & \multirow{2}{*}{\multicolumn{3}{|c|}{$\begin{array}{l}\text { Test Equipment and } S / \mathrm{N} \text { or Cal. No.: } \\
\text { See page } 161\end{array}$}} \\
\hline \multicolumn{3}{|c|}{ 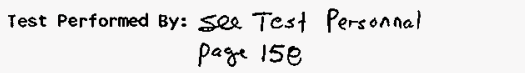 } & & & \\
\hline $\begin{array}{l}\text { Procedure No. } \\
\text { Initial/oate }\end{array}$ & I tem & Value & $\begin{array}{l}\text { Required } \\
\text { condition }\end{array}$ & $(A / R)$ & Comment \\
\hline$\frac{1.7 .4 .6 .17}{m_{2.0}}$ & $\begin{array}{l}\text { OPEN SV-*10 } \\
\text { VERIFY FLOW }\end{array}$ & $\frac{O K}{B K}$ & $\begin{array}{l}\text { SAMPLE FLOW } \\
\text { IN - NO } \\
\text { OUT - YES } \\
\end{array}$ & A & \\
\hline$\frac{1.7 .4 .6 .18}{m_{2}}$ & $\begin{array}{l}\text { REMOVE TEST } \\
\text { GAS \& PLUG } \\
\text { SV } * 07\end{array}$ & $N / A$ & N/A & A & \\
\hline $\begin{array}{l}1.7 .4 .6 .19 \\
m .2 .0 \\
\end{array}$ & $\begin{array}{l}\text { CLOSE SV-*07 } \\
\& \text { SV }-* 10\end{array}$ & $N / A$ & N/A & $A$ & \\
\hline $\begin{array}{l}1.7 .4 .6 .20 \\
\text { on.i.e }\end{array}$ & $\begin{array}{l}\text { OPEN SV }-* 05 \\
\& \text { SV }-* 16\end{array}$ & $N / A$ & $N / A$ & A & \\
\hline $\begin{array}{l}1.7 .4 .6 .21 \\
\operatorname{min.p.0} \\
\end{array}$ & $\begin{array}{l}\text { REMOVE } \\
\text { JUMPER }\end{array}$ & $\mathrm{N} / \mathrm{A}$ & N/A & $A$ & \\
\hline \multicolumn{6}{|c|}{ Section 1.7.4.7 verifies the carrier gas supply lines. } \\
\hline $\begin{array}{l}1.7 .4 .7 .1 \\
m .2 .3 \\
\end{array}$ & $\begin{array}{l}\text { CONNECT TEST } \\
\text { GAS }\end{array}$ & $4 P S T C$ & 3-5 PSIG & A & \\
\hline $\begin{array}{l}1.7 .4 .7 .2 \\
\mathbb{M} .2 . B \\
\end{array}$ & $\begin{array}{l}\text { REMOVE PLUG } \\
\text { \& VERIFY } \\
\text { FLOW }\end{array}$ & ok & $\begin{array}{l}\mathrm{N}_{2} \text { CARRIER } \\
\text { GAS FLOWS }\end{array}$ & $A$ & \\
\hline $\begin{array}{l}1.7 .4 .7 .3 \\
m_{2} \gamma \beta \\
\end{array}$ & $\begin{array}{l}\text { DISCONNECT } \\
\text { TEST GAS }\end{array}$ & $N / A$ & $\mathrm{~N} / \mathrm{A}$ & A & \\
\hline $\begin{array}{l}1.7 .4 .7 .4 \\
\text { mxis }\end{array}$ & $\begin{array}{l}\text { CONNECT TEST } \\
\text { GAS }\end{array}$ & $4 \rho$ sis & 3-5 PSIG & $A$ & \\
\hline $\begin{array}{l}1.7 .4 .7 .5 \\
m .2 . p\end{array}$ & $\begin{array}{l}\text { REMOVE PLUG } \\
\& \text { VERIFY } \\
\text { FLOW }\end{array}$ & ok & $\begin{array}{l}\text { He CARRIER } \\
\text { GAS FLOWS }\end{array}$ & $A$ & \\
\hline $\begin{array}{l}1.7 .4 .7 .6 \\
m .3 . s \\
\end{array}$ & $\begin{array}{l}\text { DISCONNECT } \\
\text { TEST GAS }\end{array}$ & N/A & N/A & A & \\
\hline $\begin{array}{l}1.7 .5 \\
g m i n s\end{array}$ & $\begin{array}{l}\text { TEST SEC. } \\
1.7 .4 \text { DONE }\end{array}$ & $\frac{\text { jege }}{\text { Test Dire }}$ & weido & & $\frac{2-30-96}{\text { Date }}$ \\
\hline
\end{tabular}


TEST DATA SHEET

\begin{tabular}{|c|c|c|c|c|c|}
\hline \multicolumn{3}{|c|}{ Date: $\quad 12-20-96$} & \multicolumn{3}{|c|}{ SHMS Unit Number: VTP - PNL - 3305F } \\
\hline \multicolumn{3}{|c|}{$\begin{array}{l}\text { Title of rest: } \\
\text { Pneumatic Systems }\end{array}$} & \multirow{2}{*}{\multicolumn{3}{|c|}{$\begin{array}{l}\text { Test Equipment and } S / N \text { or Cal. No.: } \\
\text { N/A }\end{array}$}} \\
\hline \multicolumn{3}{|c|}{$\begin{aligned} \text { Test Performed By: } & \text { See Test Personnal } \\
& \text { Page } 158\end{aligned}$} & & & \\
\hline $\begin{array}{l}\text { Procedure No. } \\
\text { Initial/Date }\end{array}$ & Item & Value & $\begin{array}{l}\text { Required } \\
\text { Condition } \\
\end{array}$ & $(A / R)$ & Comment \\
\hline $\begin{array}{l}1.7 .6 \\
M \cdot 2, B\end{array}$ & \multicolumn{5}{|c|}{$\begin{array}{l}\text { THIS SECTION IS ONLY FOR THE SHMS-E IN THE ANALYTICAL MODE. } \\
\text { IF NOT APPLICABLE, N/A STEPS AND TEST DIRECTOR WILL SIGN. } \\
\text { 1C } \\
\text { Test Director Signature } \\
\end{array}$} \\
\hline $\begin{array}{l}1.7 .6 .1 .1 \\
m .2 .8 \\
\end{array}$ & $\begin{array}{l}\text { VERIFY BKR } \\
\text { LINE UP }\end{array}$ & N/A & $N / A$ & A & \\
\hline $\begin{array}{l}1.7 .6 .1 .2 \\
\operatorname{mix}_{1} \cdot B\end{array}$ & $\begin{array}{l}\text { VERIFY VALVE } \\
\text { LINE UP }\end{array}$ & $\mathrm{N} / \mathrm{A}$ & N/A & A & \\
\hline $\begin{array}{c}1.7 .6 .1 .3 \\
m .20 .8 \\
\end{array}$ & $\begin{array}{l}\text { VERIFY MON- } \\
* 60 \text { INSTALL } \\
\end{array}$ & $N / A$ & $\mathrm{~N} / \mathrm{A}$ & A & \\
\hline $\begin{array}{l}1.7 .6 .1 .4 \\
\text { maß }\end{array}$ & $\begin{array}{l}\text { VERIFY NIT- } \\
\star 52 \text { INSTALL } \\
\end{array}$ & $\mathrm{N} / \mathrm{A}$ & N/A & A & \\
\hline $\begin{array}{l}1.7 .6 .1 .5 \\
\text { mant }\end{array}$ & $\begin{array}{l}\text { CONNECT } \mathrm{H}_{2} \\
\text { CAL GAS }\end{array}$ & $N / A$ & $N / A$ & A & \\
\hline moxs $^{1.7 .6 .1 .6}$ & $\begin{array}{l}\text { CONNECT } \mathrm{CH}_{4} \\
\text { CAL GAS }\end{array}$ & $N / A$ & $N / A$ & A & \\
\hline $\begin{array}{l}1.7 .6 .1 .7 \\
{ }^{2} \rightarrow R\end{array}$ & $\begin{array}{l}\text { CONNECT } \mathrm{NH}_{3} \\
\text { CAL GAS }\end{array}$ & $N / A$ & N/A & $A$ & \\
\hline \multicolumn{6}{|c|}{ Section 1.7.6.2 establishes the main and auxiliary loop flows. } \\
\hline $\begin{array}{l}1.7 .6 .2 .1 \\
m x .0\end{array}$ & CLOSE FU-11 & oK & $\begin{array}{l}\text { MON-*60 } \\
\text { POWERED }\end{array}$ & A & \\
\hline $\begin{array}{l}1.7 .6 .2 .2 \\
m \infty .8\end{array}$ & POWER PC-*60 & ok & $\begin{array}{l}\text { ESTABLISH } \\
\text { SAMPLING } \\
\text { ROUTINE } \\
\end{array}$ & A & \\
\hline $\begin{array}{c}1.7 .6 .2 .3 \\
\text { m.x. }\end{array}$ & CLOSE FU-12 & $-o c$ & $\begin{array}{l}\text { NIT }-* 52 \\
\text { POWERED } \\
\end{array}$ & $A$ & \\
\hline $\begin{array}{c}1.7 .6 .2 .4 \\
m .2 .1 \\
\end{array}$ & POWER PC-*70 & ote & $\begin{array}{l}\text { PC-*70 } \\
\text { BOOTED UP } \\
\end{array}$ & A & \\
\hline $\begin{array}{l}1.7 .6 .2 .5 \\
m m_{2} R\end{array}$ & $\begin{array}{l}\text { CLOSE CB-3/4 } \\
\text { ADJUST FLOW }\end{array}$ & $\frac{O K}{r^{M}+H_{0}}$ & $\begin{array}{l}\mathrm{FIT}-* 57 \\
\mathrm{I} \pm .1 \quad \mathrm{H} \mathrm{H}_{3} \mathrm{O}\end{array}$ & $A$ & \\
\hline
\end{tabular}


TEST DATA SHEET

\begin{tabular}{|c|c|c|c|c|c|}
\hline \multicolumn{3}{|c|}{ Date: $12 \cdot 20-96$} & \multicolumn{3}{|c|}{ SHMS Unit Number: VTP - PNL-3305F } \\
\hline \multicolumn{3}{|c|}{$\begin{array}{l}\text { Title of Test: } \\
\text { Pneumatic Systems }\end{array}$} & \multirow{2}{*}{\multicolumn{3}{|c|}{$\begin{array}{l}\text { Test Equipnent and } S / \mathrm{N} \text { or Cal. No.: } \\
\text { N/A }\end{array}$}} \\
\hline \multicolumn{3}{|c|}{$\begin{aligned} \text { Test Performed By: } & \text { See Test Personnal } \\
& \text { Page } 158\end{aligned}$} & & & \\
\hline $\begin{array}{l}\text { Procedure No. } \\
\text { Initial/Date }\end{array}$ & 1 tem & Value & $\begin{array}{l}\text { Required } \\
\text { Condition } \\
\end{array}$ & $(A / R)$ & Comment \\
\hline 1.7 .6 .2 .6 & $\begin{array}{l}\text { OPEN SV-*09 } \\
\& S V-* 10\end{array}$ & $N / A$ & N/A & A & \\
\hline $\begin{array}{c}1.7 .6 .2 .7 \\
\text { m.7. } \\
\end{array}$ & $\begin{array}{l}\text { OPEN SV-*60, } \\
63,65 \& 68\end{array}$ & $N / A$ & $\mathrm{~N} / \mathrm{A}$ & A & \\
\hline $\min _{\text {min }}^{1.7 .6 .2 .8}$ & NIT $-\star 52$ & ok & $\begin{array}{l}\text { ESTABLISH } \\
\text { SAMPLING } \\
\text { ROUTINE }\end{array}$ & $A$ & \\
\hline $\begin{array}{l}1.7 .6 .2 .9 \\
\text { on.p.o }\end{array}$ & $\begin{array}{l}\text { OPEN SV-*24 } \\
\text { ADJ FLOW }\end{array}$ & $2.99^{\circ} \mathrm{Hz}_{\mathrm{z}} \mathrm{O}$ & $\begin{array}{l}\text { FIT-*57 } \\
3 \pm .1 \quad \mathrm{H}_{2} \mathrm{O}\end{array}$ & A & \\
\hline$\underset{m .7 .0}{1.7 .6 .2 .10}$ & $\begin{array}{l}\text { ADJ FLOW } \\
\text { SV } * * 67\end{array}$ & $20 \mathrm{cen}$ & $\begin{array}{l}\mathrm{FI}-* 60 \\
10-30 \mathrm{ccm}\end{array}$ & A & \\
\hline $\begin{array}{l}1.7 .6 .2 .11 \\
\min _{B} B \\
\end{array}$ & $\begin{array}{l}\text { RECORD } \\
\text { PDIT }-\star 60\end{array}$ & $136.2^{\circ}$ tho & $<175 \quad \mathrm{H}_{2} \mathrm{O}$ & A & \\
\hline $\begin{array}{l}1.7 .6 .2 .12 \\
9 m, x x_{3} \\
\end{array}$ & $\begin{array}{l}\text { OPEN SV }-* 64 \\
\text { CLOSE SV-*63 }\end{array}$ & N/A & $N / A$ & $A$ & \\
\hline $\begin{array}{l}1.7 .6 .2 .13 \\
\text { munes } \\
\end{array}$ & $\begin{array}{l}\text { RECORD } \\
\text { PDIT }-* 60\end{array}$ & $137^{4} \mathrm{H}(2)$ & $<175 \quad " \mathrm{H}_{2} \mathrm{O}$ & $A$ & \\
\hline $\operatorname{lm}_{m .0} .7 .6 .2 .14$ & $\begin{array}{l}\text { CLOSE SV }-* 65 \\
\text { OPEN SV }-* 66\end{array}$ & $N / A$ & N/A & A & \\
\hline $\begin{array}{l}1.7 .6 .2 .15 \\
m x .0 \\
\end{array}$ & $\begin{array}{l}\text { RECORD B IN } \\
\text { PDIT-*60 }\end{array}$ & $-25.8^{\prime \prime} H_{2} \mathrm{O}$ & $\begin{array}{l}-15 \text { to }-50 \\
" \mathrm{H}_{2} \mathrm{O}\end{array}$ & $A$ & \\
\hline $\begin{array}{l}1.7 .6 .2 .16 \\
m .2 . B \\
\end{array}$ & $\begin{array}{l}\text { OPEN SV }-* 63 \\
\text { CLOSE SV }-* 64\end{array}$ & $N / A$ & N/A & $A$ & \\
\hline $\begin{array}{l}1.7 .6 .2 .17 \\
\text { gnd.s. } \\
\end{array}$ & $\begin{array}{l}\text { RECORD A IN } \\
\text { PDIT-*60 }\end{array}$ & $-25.0^{-1} H_{2} O$ & $\begin{array}{l}-15 \text { to }-50 \\
" \mathrm{H}_{2} \mathrm{O}\end{array}$ & A & \\
\hline${ }_{m}^{1.7 .6 .2 .18}$ & $\begin{array}{l}\text { INSTALL } \\
\text { JUMPER }\end{array}$ & $N / A$ & $\mathrm{~N} / \mathrm{A}$ & $A$ & \\
\hline $\begin{array}{c}1.7 .6 .2 .19 \\
\text { min.s } \\
\end{array}$ & CLOSE FU-15 & $N / A$ & N/A & $A$ & \\
\hline$\frac{1.7 .6 .2 .20}{m \cdot 2 \cdot 3}$ & $\begin{array}{l}\text { ADJ SV-*61 \& } \\
\text { RECORD } \\
\text { PDIT-*60 }\end{array}$ & $-20.8^{\prime} \mathrm{H}_{2} \mathrm{O}$ & $\begin{array}{l} \pm 5^{\prime \prime} \mathrm{H}_{2} \mathrm{O} \text { OF } \\
1.7 .6 .2 .17\end{array}$ & A & \\
\hline
\end{tabular}


TEST DATA SHEET

\begin{tabular}{|c|c|c|c|c|c|}
\hline \multicolumn{3}{|c|}{ Date: $12-20-96$} & \multicolumn{3}{|c|}{ SHMS unit Number: VTP.PNL-3305F } \\
\hline \multicolumn{3}{|c|}{$\begin{array}{l}\text { Title of Test: } \\
\text { Pneumatic Systems }\end{array}$} & \multirow{2}{*}{\multicolumn{3}{|c|}{$\begin{array}{l}\text { Test Equipment and } S / \mathrm{N} \text { or Cal. No.: } \\
\text { Ammonia bottol No. Almo58830 } \\
\text { Hydroger bottal No. Al1655 } \\
\text { Methere bottel No. G } 2650637\end{array}$}} \\
\hline \multicolumn{3}{|c|}{$\begin{array}{c}\text { Test Performed By: } \\
\text { Soe Test Personnal } \\
\text { Page } 158\end{array}$} & & & \\
\hline $\begin{array}{l}\text { Procedure No. } \\
\text { Initial/Date }\end{array}$ & Item & Value & $\begin{array}{l}\text { Required } \\
\text { Condition }\end{array}$ & $(A / R)$ & Corment \\
\hline $\begin{array}{l}1.7 .6 .2 .21 \\
29.213 \\
\end{array}$ & $\begin{array}{l}\text { CAL MON-*60 } \\
\text { CHANNEL A }\end{array}$ & $\begin{array}{l}10428.4 \mathrm{pm} \\
10500.4 \mathrm{pm} \\
10515.02 \mathrm{~m}\end{array}$ & $\begin{array}{l}\text { READINGS } \\
\text { WITHIN } \pm 5 \%\end{array}$ & A & Hydregan \\
\hline $\begin{array}{l}1.7 .6 .2 .22 \\
m x \cdot 0 \\
\end{array}$ & OPEN FU-15 & $N / A$ & $N / A$ & A & \\
\hline $\begin{array}{l}1.7 .6 .2 .23 \\
\text { min. B } \\
\end{array}$ & $\begin{array}{l}\text { REMOVE } \\
\text { JUMPER }\end{array}$ & $N / A$ & $N / A$ & A & \\
\hline $\begin{array}{c}1.7 .6 .2 .24 \\
m_{0.0} \\
\end{array}$ & $\begin{array}{l}\text { OPEN SV-*64 } \\
\text { CLOSE SV-*63 }\end{array}$ & $N / A$ & $N / A$ & A & \\
\hline $\begin{array}{c}1.7 .6 .2 .25 \\
m_{m i n}-R \\
\end{array}$ & $\begin{array}{l}\text { INSTALL } \\
\text { JUMPER }\end{array}$ & N/A & N/A & A & \\
\hline $\begin{array}{l}1.7 .6 .2 .26 \\
\text { gmin.p }\end{array}$ & CLOSE FU-15 & $N / A$ & $N / A$ & A & \\
\hline $\begin{array}{l}1.7 .6 .2 .27 \\
\text { miv. }\end{array}$ & $\begin{array}{l}\text { ADJ SV }-* 62 \& \\
\text { RECORD } \\
\text { PDIT }-* 60\end{array}$ & $-21.0^{\circ} H_{x \Delta}$ & $\begin{array}{l} \pm 5 \mathrm{H}_{2} \mathrm{O} \text { OF } \\
1.7 .6 .2 .15\end{array}$ & $A$ & \\
\hline $\begin{array}{c}1.7 .6 .2 .28 \\
m .2 .8 \\
\end{array}$ & $\begin{array}{l}\text { CAL MON-*60 } \\
\text { CHANNEL B }\end{array}$ & $\frac{9978.9788 \mathrm{~m}}{9974.6979 \mathrm{~m}}$ & $\begin{array}{l}\text { READINGS } \\
\text { WITHIN } \pm 5 \%\end{array}$ & $A$ & Methore \\
\hline $\begin{array}{l}1.7 .6 .2 .29 \\
2 \pi .3 \\
\end{array}$ & OPEN FU-15 & N/A & N/A & A & \\
\hline $\begin{array}{l}1.7 .6 .2 .30 \\
m 2.0 \\
\end{array}$ & $\begin{array}{l}\text { REMOVE } \\
\text { JUMPER } \\
\end{array}$ & $N / A$ & N/A & $A$ & \\
\hline $\begin{array}{c}1.7 .6 .2 .31 \\
m m_{0} \beta \\
\end{array}$ & $\begin{array}{l}\text { CLOSE SV }-* 64 \\
\text { OPEN SV }-* 70\end{array}$ & $N / A$ & $N / A$ & A & \\
\hline $\begin{array}{l}1.7 .6 .2 .32 \\
m=2 . \beta\end{array}$ & $\begin{array}{l}\text { RECORD } \\
\text { PDIT }-* 60 \\
\text { NIT }-* 52 \text { IN }\end{array}$ & $-9.0^{\circ 1} \mathrm{H}_{2} \mathrm{O}$ & $\begin{array}{l}0 \text { to }-30 \\
" \mathrm{H}_{2} \mathrm{O}\end{array}$ & A & \\
\hline $\begin{array}{l}1.7 .6 .2 .33 \\
m-2.3 \\
\end{array}$ & $\begin{array}{l}\text { INSTALL } \\
\text { JUMPER } \\
\end{array}$ & N/A & $N / A$ & A & \\
\hline $\begin{array}{l}1.7 .6 .2 .34 \\
m \mathcal{B} \beta\end{array}$ & CLOSE FU-15 & N/A & N/A & A & \\
\hline
\end{tabular}


TEST DATA SHEET

\begin{tabular}{|c|c|c|c|c|c|}
\hline \multicolumn{3}{|c|}{ Date: $12-20-96$} & \multicolumn{3}{|c|}{ SHMS Unit Number: VTP-PNL-330BF } \\
\hline \multicolumn{3}{|c|}{$\begin{array}{l}\text { Title of Test: } \\
\text { Pneumatic Systems }\end{array}$} & \multirow{2}{*}{\multicolumn{3}{|c|}{$\begin{array}{l}\text { Test Equipment and } S / \mathrm{N} \text { or Cal. No.: } \\
\text { See Page } 182\end{array}$}} \\
\hline \multicolumn{3}{|c|}{$\begin{aligned} \text { Test Performed By: } & \text { Ses Tesł Persomal } \\
& \text { Page } 158\end{aligned}$} & & & \\
\hline $\begin{array}{l}\text { Procedure No. } \\
\text { Initial/Date }\end{array}$ & 1 tem & Value & $\begin{array}{l}\text { Required } \\
\text { Condition }\end{array}$ & $(A / R)$ & Comment \\
\hline $\begin{array}{l}1.7 .6 .2 .35 \\
m .2 . B \\
\end{array}$ & $\begin{array}{l}\text { ESTABLISH } \\
\text { BYPASS FLOW }\end{array}$ & $1.7 \mathrm{efh}$ & $\begin{array}{l}\text { FIV }-* 70 \\
1.5 \pm .2 \mathrm{cfh}\end{array}$ & A & \\
\hline $\begin{array}{l}1.7 .6 .2 .36 \\
m .7 .3\end{array}$ & $\begin{array}{l}\text { ADJ SV }-* 71 \\
\text { NIT }-* 52 \\
\text { SAMPLE IN }\end{array}$ & $-12.0 \%$ & $\begin{array}{l} \pm 5 \quad " \mathrm{H}_{2} \mathrm{O} \quad \mathrm{OF} \\
1.7 .6 .2 .32\end{array}$ & A & \\
\hline$\frac{1.7 \cdot 6 \cdot 2.37}{m \cdot D^{3}}$ & $\begin{array}{l}\text { RECORD } \\
\text { NIT }-* 52 \\
\text { SAMPLES }\end{array}$ & $\begin{array}{r}\frac{32.3 \mathrm{PPM}}{37.6 \mathrm{PPM}} \\
\frac{37.9 \mathrm{PPM}}{27 .}\end{array}$ & $\begin{array}{l}\text { READINGS } \\
\text { WITHIN } \pm 5 \%\end{array}$ & A & Ammoniá \\
\hline $\begin{array}{l}1.7 .6 .2 .38 \\
2 x_{1} B \\
\end{array}$ & OPEN FU-15 & $N / A$ & $N / A$ & A & \\
\hline $\begin{array}{l}1.7 .6 .2 .39 \\
m .2 . B \\
\end{array}$ & $\begin{array}{l}\text { REMOVE } \\
\text { JUMPER }\end{array}$ & N/A & N/A & A & \\
\hline \multicolumn{6}{|c|}{ Section 1.7.7 provides for test system shutdown. } \\
\hline $\begin{array}{l}1.7 .7 .1 \\
\text { m.7. }\end{array}$ & $\begin{array}{l}\text { DISCONNECT } \\
\mathrm{H}_{2} \text { CAL GAS }\end{array}$ & N/A & $N / A$ & A & \\
\hline $\begin{array}{l}1.7 .7 .2 \\
2 M .2 . B \\
\end{array}$ & $\begin{array}{l}\text { DISCONNECT } \\
\mathrm{CH}_{6} \text { CAL GAS } \\
\end{array}$ & N/A & $N / A$ & A & \\
\hline $\begin{array}{l}1.7 .7 .3 \\
9 m .7 .8 \\
\end{array}$ & $\begin{array}{l}\text { DISCONNECT } \\
\mathrm{NH}_{3} \text { CAL GAS }\end{array}$ & $\mathrm{N} / \mathrm{A}$ & N/A & A & \\
\hline $\begin{array}{l}1.7 .7 .4 \\
M .218\end{array}$ & $\begin{array}{l}\text { SECURE \& } \\
\text { TURN OFF } \\
\text { ANA. INST.S }\end{array}$ & N/A & N/A & A & \\
\hline $\begin{array}{r}1.7 .7 .5 \\
M P B B \\
\end{array}$ & $\begin{array}{l}\text { DISCONNECT } \\
\mathrm{N}_{2} \text { CARRIER } \\
\end{array}$ & $\mathrm{N} / \mathrm{A}$ & N/A & A & \\
\hline $\begin{array}{r}1.7 .7 .6 \\
20.21 B \\
\end{array}$ & $\begin{array}{l}\text { DISCONNECT } \\
\text { He CARRIER } \\
\end{array}$ & $N / A$ & $N / A$ & A & \\
\hline $\begin{array}{l}1.7 .7 .7 \\
2 m .2 \times 3\end{array}$ & $\begin{array}{l}\text { OPEN ALL } \\
\text { BREAKERS \& } \\
\text { FUSES }\end{array}$ & $N / A$ & N/A & $A$ & \\
\hline 1.7 .7 .8 & $\begin{array}{l}\text { CLOSE LISTED } \\
\text { SYS. VALVES }\end{array}$ & N/A & $N / A$ & A & \\
\hline
\end{tabular}


APPENDIX E

PAGE E-28
NF - SD - WM - STR - 191

Rage 184

TEST DATA SHEET

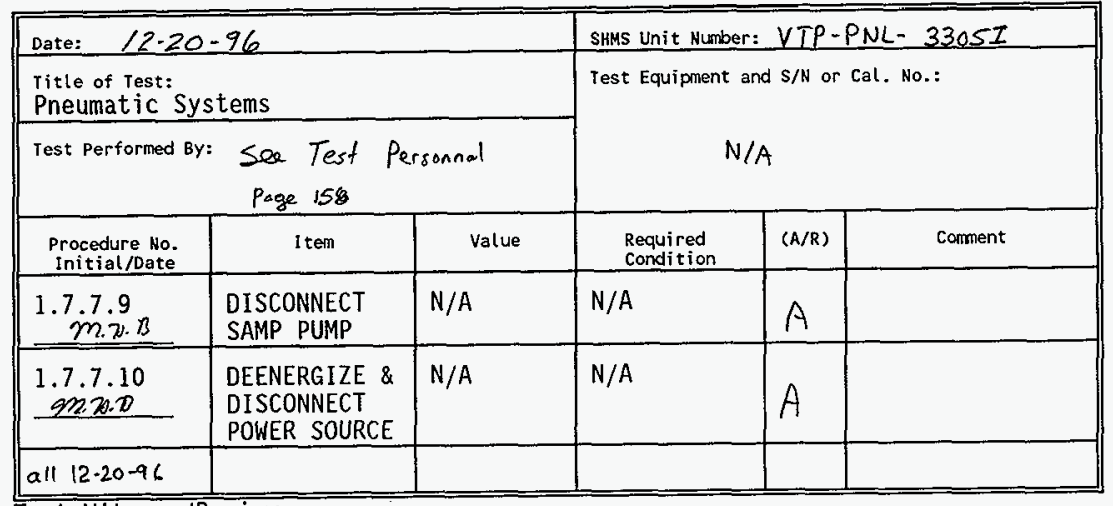

Test Witness/Review:
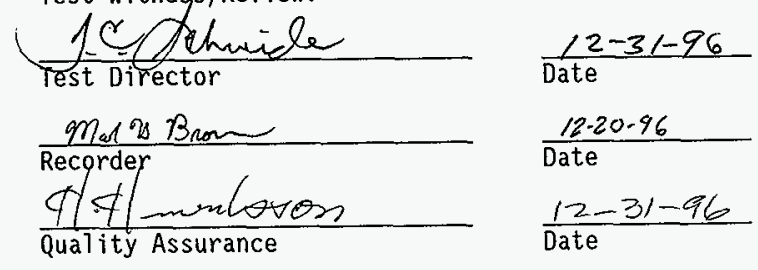
APPENDIX E

HNF-SD-WM-ATR - 191

Rage 185

PAGE E-29

TEST EXCEPTION SHEET

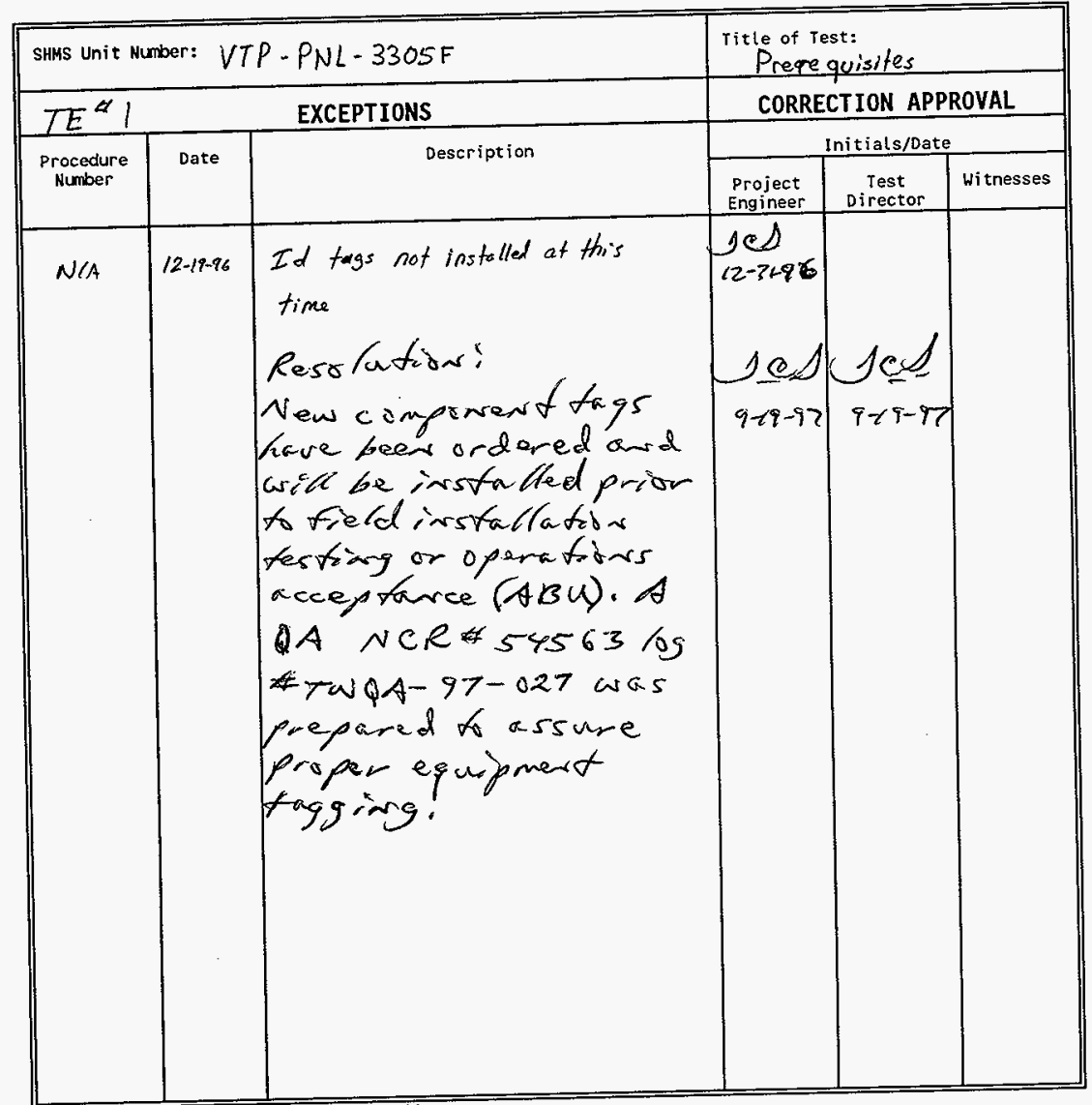

TEST APPROVED WITH EXCEPTIONS

$$
\begin{aligned}
& \frac{10 \text { S/menle }}{\text { WHC Project Engineer }} \frac{12-3 /-96}{\text { Date }} \\
& \text { make } 2 \text { prom } \\
& \text { Recorder } \\
& \frac{12 \cdot 20-96}{\text { Date }} \\
& \frac{\text { Et }}{\text { Quality Assurance }} \\
& \frac{12-3 /-96}{\text { Date }}
\end{aligned}
$$


APPENDIX E

PAGE E-30
HNF-SD-WM-ATR-191 Rev. 0

Page 186

TEST LOG

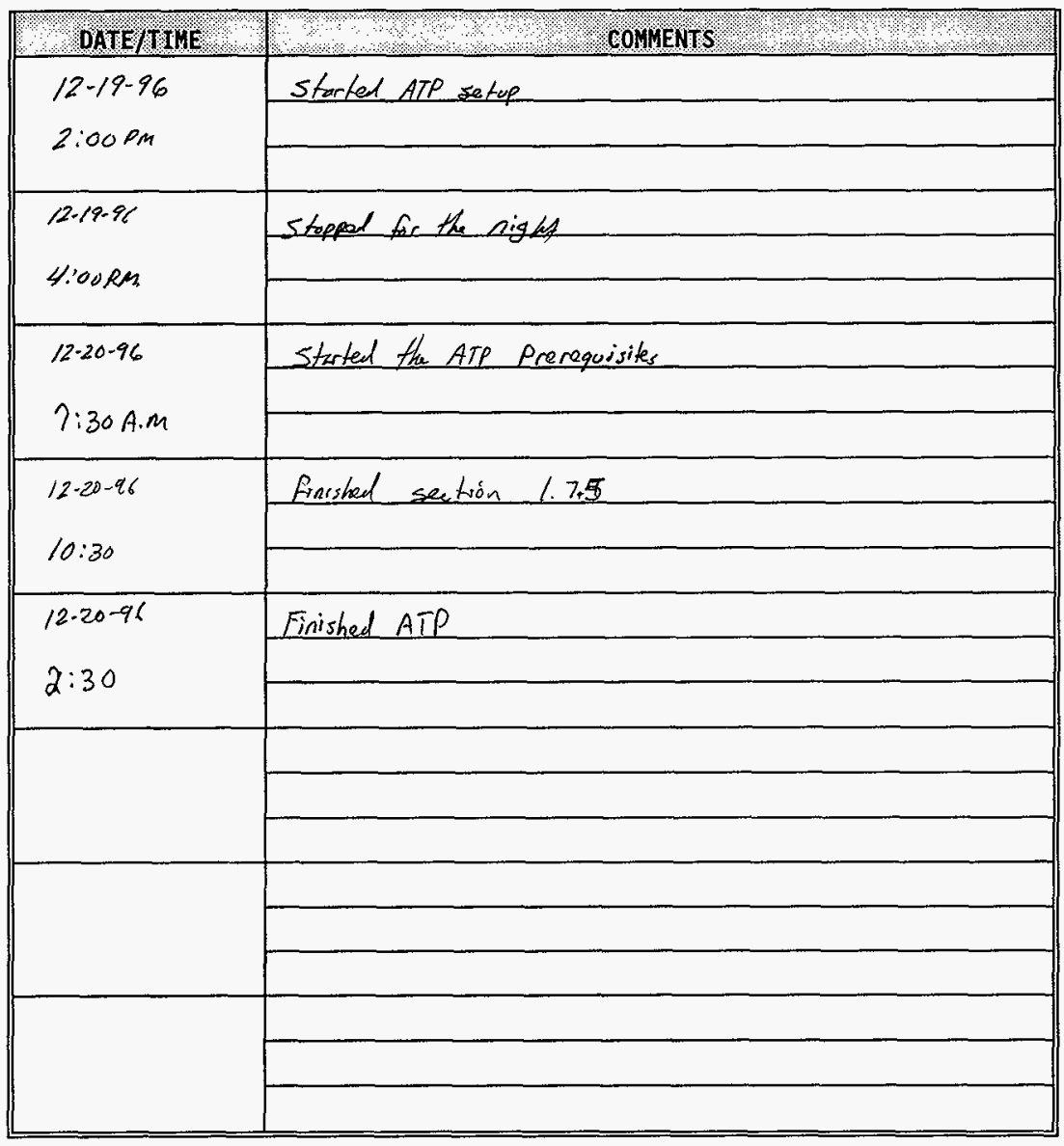


APPENDIX $F$

PAGE F-/
HNF-SD-WM-ATR-191

Rev. 0

Page 187

\section{APPENDIX F}




\begin{tabular}{|c|c|c|}
\hline $\begin{array}{l}\text { Date: } \angle-/ S=97 \\
\text { SHMs Unit Number: } \pm 6\end{array}$ & $\begin{array}{l}5 \% 102 \\
\text { Gin I. Dolat }\end{array}$ & $\begin{array}{c}\text { WHC-SD-WM-ATP-191 } \\
\text { REV } 0\end{array}$ \\
\hline
\end{tabular}

Tom Schwerde- $1 e) / 1-4-9$ NHC Project Engineer Date

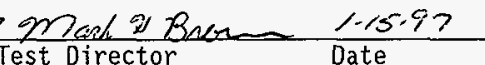

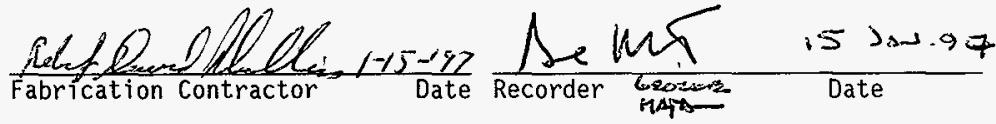
Witness Date Witness Date

Witness Date Witness Date

\section{TEST ACCEDTRNCE}

Exception/Outstanding $\quad$ With $\quad$ Exception/Resolved $\quad X$ With

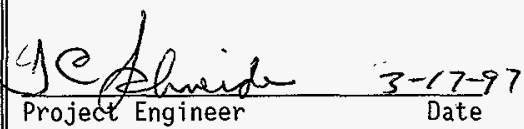

\section{TEST APPROUA A ANU ACCEPTANCE}

Numatec Hanford Company

Exception/Outstanding

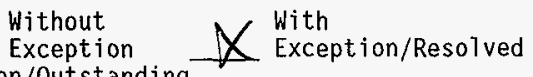

With

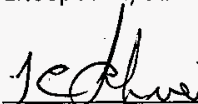
Project Engineer $\frac{9-22-97}{\text { Date }}$

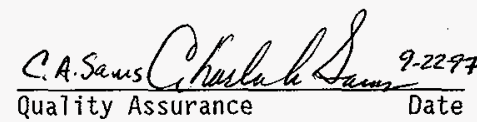
$\frac{1}{\text { MCE Project }}+8 / 22 / 97$ 
SHMS Unit Number:

\section{PREREQUISITES AND INITIAL TEST CONDITIONS}

The following conditions shall exist at the start of the acceptance testing. Initial and date to verify that each of the following items have been accomplished.

ian $1.51 \mathrm{n}$ Systems being tested have been inspected for workmanship and for compliance with design.

en ash continuity and megger tests have been performed on portions of the electrical and instrument systems being tested, as required.

on $1 / 3 / 17$ Leak tests on the pneumatic systems have been performed.

on is lat The following circuit breakers and fuses are installed per the specified size and are open

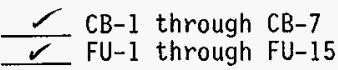

Ln $1.51 s]$ All test instruments have a currently valid calibration stamp attached that indicates a calibration traceable to the National Institute of Standards and Technology.

on $1.5 / 97$ The following process and control instrument systems have been initially configured and aligned for proper operation.

FIT-*57, FSL-*57, NIT-*54, NIT-*55,' (NR-*54), PDIT-*60,' TIC-*50, TIC-*56,,$T I S-* 62$, YYC $-* 01$ NOTE: NR-*54 is not used in the ANALYTICAL configuration.

Gn $1.51,7$ Personnel responsible for directing and witnessing the performance of the tests described in this ATP have read and understand appropriate certified vendor information (CVI) pertaining to the operation of the equipment to be tested.

on 1.5/97 CLOSE all system manual valves with the exception of the following:

\begin{tabular}{|c|c|}
\hline 1 & VTP-FIV $-* 52$ \\
\hline$\sqrt{\prime}$ & VTP-FIV-*56 \\
\hline 7 & VTP-FIV $-* 70$ \\
\hline 7 & VTP-SV-*24 \\
\hline 7 & VTP-SV-*61 \\
\hline 7 & VTP-SV-*62 \\
\hline $\bar{V}$ & VTP-SV-*67 \\
\hline 6 & VTP-SV $-* 7$ \\
\hline
\end{tabular}

GM $1 / 5 / 97$ The sample pump VTP-P-*50, connected to the cabinet sample out port, but vented to atmosphere for testing. 
Ga $1 / 5 / 97208$ Vac 1 phase 15-20 ampere temporary power source has been connected, but not energized, to the appropriate TBl terminals per H-14-100838.

Verify by signature and date that all prerequisites have been met.

$\frac{9 \text { and o }-B \text { non }}{\text { Test Director Signature }} \frac{1-15-97}{\text { Date }}$


TEST DATA SHEET

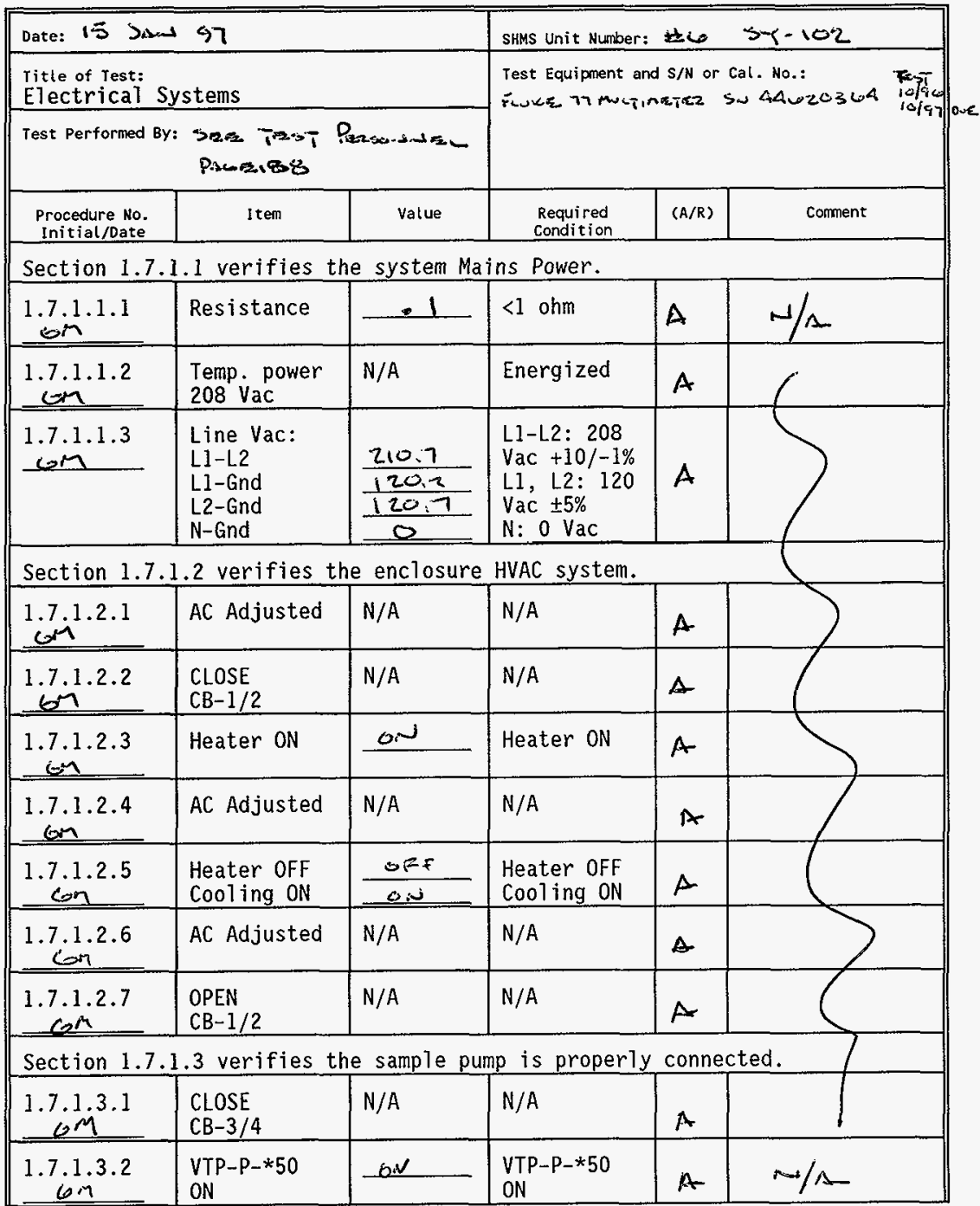


TEST DATA SHEET

\begin{tabular}{|c|c|c|c|c|c|}
\hline \multicolumn{3}{|c|}{ Date: is >Au 1497} & \multicolumn{3}{|c|}{ SHMS Unit Number: 0 s-i-102 } \\
\hline \multicolumn{3}{|c|}{$\begin{array}{l}\text { Titte of Test: } \\
\text { Electrical Systems }\end{array}$} & \multirow{2}{*}{\multicolumn{3}{|c|}{ 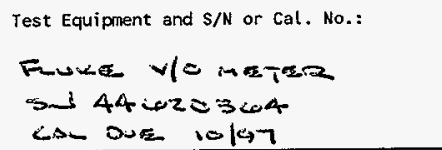 }} \\
\hline \multicolumn{3}{|c|}{ 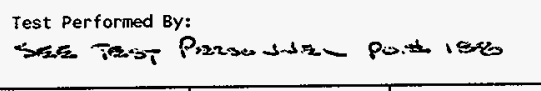 } & & & \\
\hline $\begin{array}{l}\text { Procedure No. } \\
\text { Initial/Date }\end{array}$ & Iten & value & $\begin{array}{r}\text { Required } \\
\text { Condition } \\
\end{array}$ & $(A / R)$ & Comment \\
\hline $\begin{array}{c}1.7 .1 .3 .3 \\
6 \mathrm{~m}\end{array}$ & $\begin{array}{l}\text { OPEN } \\
\text { CB-3/4 }\end{array}$ & $N / A$ & N/A & 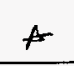 & \\
\hline \multicolumn{6}{|c|}{ Section 1.7.1.4 verifies the enclosure lighting and GFCI receptacles. } \\
\hline$\frac{1.7 .1 .4 .1}{67}$ & $\begin{array}{l}\text { CLOSE } \\
\text { CB-6 }\end{array}$ & N/A & N/A & A & \\
\hline $\begin{array}{c}1.7 .1 .4 .2 \\
6 m \\
\end{array}$ & $\begin{array}{l}\text { ENCLOSURE } \\
\text { LIGHT }\end{array}$ & N/A & N/A & $A$ & \\
\hline 1.7 .1 .4 .3 & LIGHT ON & o.t & LIGHT ON & & \\
\hline$\frac{1.7 .1 .4 .4}{\operatorname{en}^{2}}$ & $\begin{array}{l}\text { GFCI RECP. } \\
1 A . L-N \\
1 B . L-G N D \\
2 A . L-N \\
\text { 2B. L-GND } \\
\end{array}$ & $\frac{\frac{180,5}{120.37}}{\frac{120.41}{120.27}}$ & $\begin{array}{l}\text { 1A. } 120 \mathrm{Vac} \\
1 \mathrm{~B} .120 \mathrm{Vac} \\
2 \mathrm{~A} .120 \mathrm{Vac} \\
2 B .120 \mathrm{Vac} \\
\text { ALL } \pm 5 \%\end{array}$ & A & \\
\hline$\frac{1.7 .1 .4 .5}{\operatorname{coin}}$ & $\begin{array}{l}\text { GFCI TEST } \\
1 A . L-N \\
1 B . L-G N D \\
2 A . L-N \\
\text { 2B. L-GND } \\
\end{array}$ & 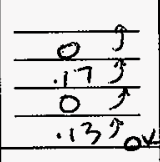 & $\begin{array}{l}\text { 1A. } 0 \text { Vac } \\
1 B .0 \text { Vac } \\
2 A .00 \mathrm{Vac} \\
2 B .00 \mathrm{Vac} \\
A L \mathrm{~L} \pm 5 \% \\
\end{array}$ & $A$ & \\
\hline $\begin{array}{c}1.7 .1 .4 .6 \\
6.1\end{array}$ & $\begin{array}{l}\text { ENCLOSURE } \\
\text { LIGHT OFF } \\
\end{array}$ & N/A & N/A & $A$ & \\
\hline $\begin{array}{c}1.7 .1 .4 .7 \\
62 \\
\end{array}$ & $\begin{array}{l}\text { OPEN } \\
\text { CB-6 } \\
\end{array}$ & N/A & N/A & $\Delta$ & / \\
\hline \multicolumn{6}{|c|}{ Section 1.7.1.5 verifies the instrument power source } \\
\hline$\frac{1.7 .1 .5 .1}{6.2}$ & $\begin{array}{l}\text { CLOSE } \\
\text { CB-7 }\end{array}$ & N/A & N/A & A & \\
\hline$\frac{1.7 .1 .5 .2}{6 \mathrm{~m}}$ & $\begin{array}{l}\text { XFRM Vac } \\
\text { PRI. L-N } \\
\text { SEC. L-N } \\
\text { SEC. N-GND } \\
\end{array}$ & $\frac{120.5}{124.1}$ & $\begin{array}{l}\text { PRI. \& SEC. } \\
\text { L-N } 120 \pm 5 \% \\
\text { SEC. } \\
\text { N-GND } 0 \text { Vac }\end{array}$ & A & $\downarrow$ \\
\hline$\frac{1.7 .1 .5 .3}{6 n^{3}}$ & CLOSE FU-1 & N/A & N/A & $A$ & $\omega / \Delta$ \\
\hline
\end{tabular}


TEST DATA SHEET

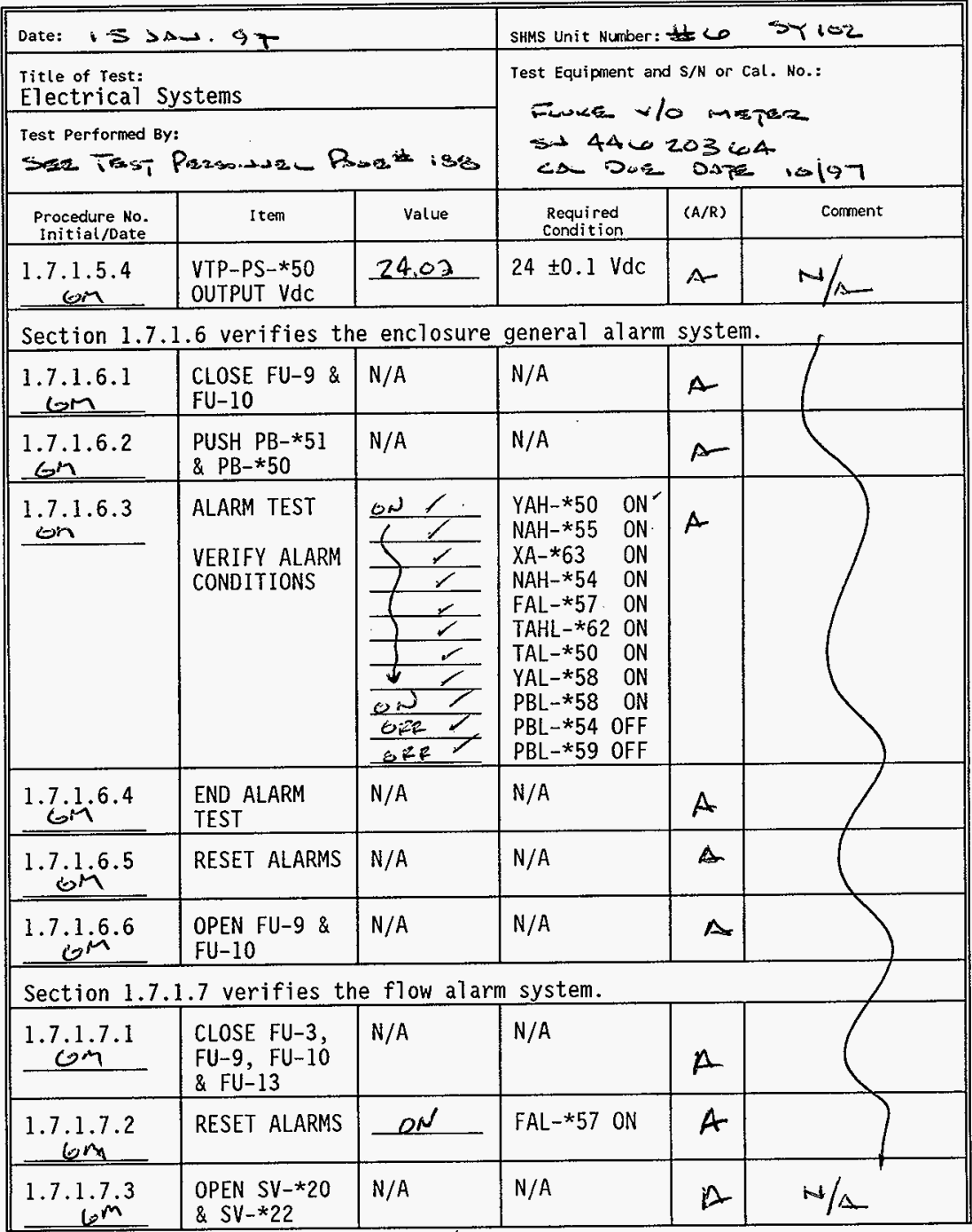


TEST DATA SHEET

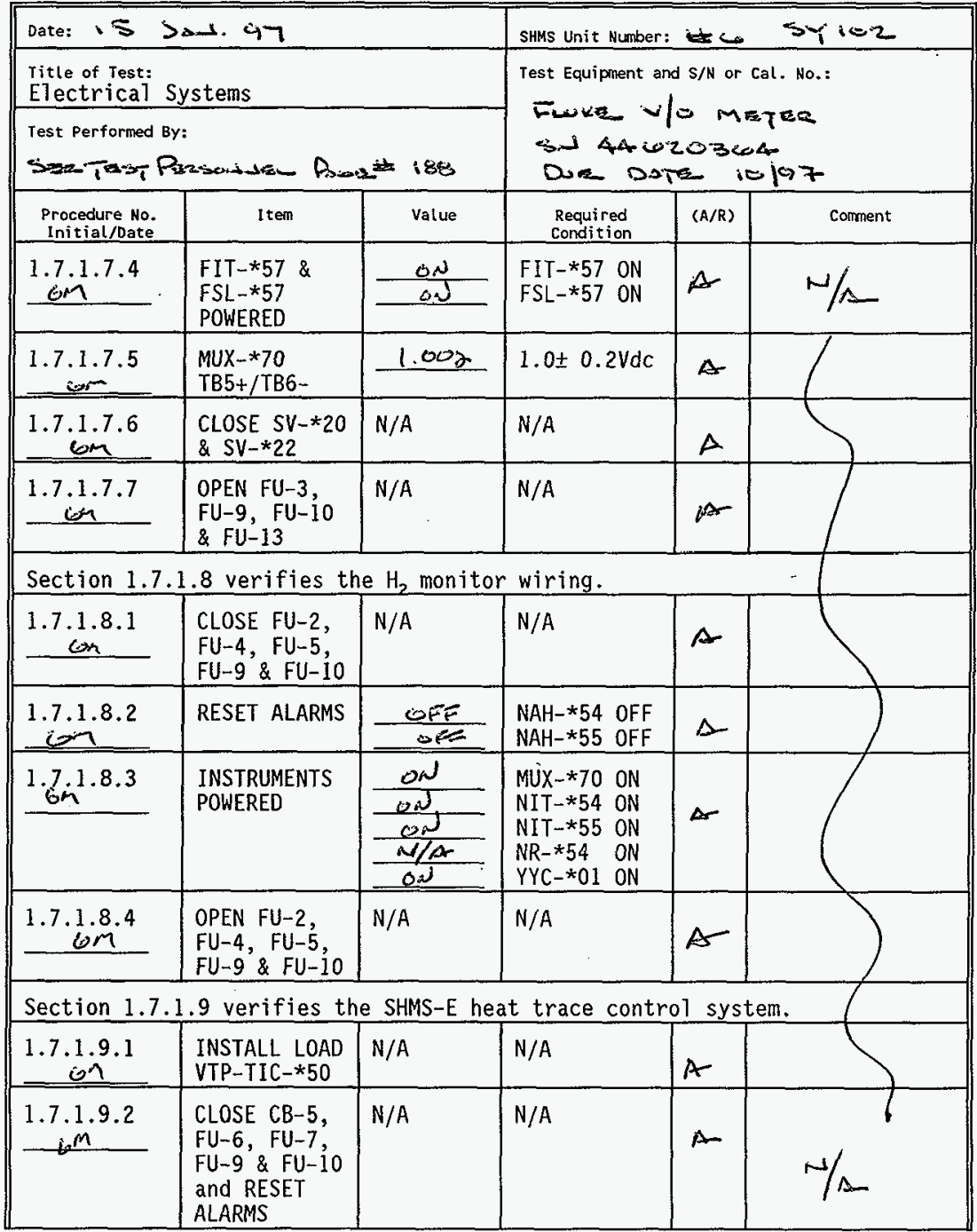


TEST DATA SHEET

\begin{tabular}{|c|c|c|c|c|c|}
\hline \multicolumn{3}{|c|}{ Date: is $>397$} & \multicolumn{3}{|c|}{ SHMS Unit Number: twe SYlO2 } \\
\hline \multicolumn{3}{|c|}{$\begin{array}{l}\text { Title of Test: } \\
\text { Electrical Systems }\end{array}$} & \multirow{2}{*}{\multicolumn{3}{|c|}{$\begin{array}{l}\text { Test Equipment and } S / \mathrm{N} \text { or Cal. No.: } \\
-\mathrm{S} / \mathrm{S}\end{array}$}} \\
\hline \multicolumn{3}{|c|}{ 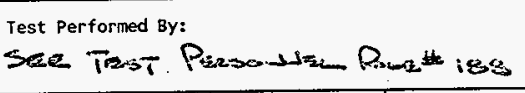 } & & & \\
\hline $\begin{array}{l}\text { Procedure No. } \\
\text { Initial/Date }\end{array}$ & Item & value & $\begin{array}{l}\text { Required } \\
\text { Condition }\end{array}$ & $(A / R)$ & Corment \\
\hline 1.7 .1 .9 .3 & $\begin{array}{l}\text { TIC-*50 \& } \\
\text { TIC-*56 } \\
\text { NOMINAL TEMP } \\
\end{array}$ & ok & $\begin{array}{l}\text { TIC }-* 50 \\
\text { TIC }-* 56 \\
\text { TEMPERATURE }\end{array}$ & $\Delta$ & \\
\hline $\begin{array}{l}1.7 .1 .9 .4 \\
\cos \end{array}$ & $\begin{array}{l}\text { ADJ TIC }-* 50 \\
\& \text { TIC }-* 56 \\
\text { SP2 \& RESET } \\
\text { ALARMS }\end{array}$ & N/A & N/A & A & \\
\hline$\frac{1.7 .1 .9 .5}{6 m}$ & $\begin{array}{l}\text { ADJ TIC-*50 } \\
\text { SP } 1\end{array}$ & $N / A$ & N/A & $A$ & \\
\hline $\begin{array}{l}1.7 .1 .9 .6 \\
\end{array}$ & $\begin{array}{l}A D J \\
S P 1 C-* 50 \\
S P\end{array}$ & N/A & N/A & $A$ & \\
\hline $\begin{array}{l}1.7 .1 .9 .7 \\
6\end{array}$ & $\begin{array}{l}\text { TAL-*50\& } \\
\text { HORN ACTIVE }\end{array}$ & $\frac{\cos }{\infty}$ & $\begin{array}{l}\text { TAL-*50 ON } \\
\text { HORN ON } \\
\text { ACKNOWLEDGE } \\
\end{array}$ & $A$ & \\
\hline $\begin{array}{l}1.7 .1 .9 .8 \\
\mathrm{Gm}\end{array}$ & $\begin{array}{l}\text { TIC }-* 50 \\
\text { OPERATES }\end{array}$ & ok & $\begin{array}{l}\text { TIC-*50 } \\
\text { OPERATES }\end{array}$ & $A$ & \\
\hline $\begin{array}{l}1.7 .1 .9 .9 \\
\end{array}$ & $\begin{array}{l}\text { ADJ TIC-*50 } \\
S P 2\end{array}$ & $N / A$ & N/A & $\Delta$ & \\
\hline $\begin{array}{c}1.7 .1 .9 .10 \\
\end{array}$ & RESET ALARM & ORP & TAL-*50 OFF & $\Delta$ & \\
\hline$\frac{1.7 .1 .9 .11}{6 \mathrm{~m}}$ & $\begin{array}{l}\text { ADJ TIC }-* 50 \\
S P 1 \& S P 2\end{array}$ & $\mathrm{~N} / \mathrm{A}$ & $N / A$ & $A$ & \\
\hline$\frac{1.7 .1 .9 .12}{\operatorname{lon}}$ & $\begin{array}{l}\text { OPEN CB-5 \& } \\
\text { REMOVE LOAD }\end{array}$ & N/A & N/A & $A$ & \\
\hline $\begin{array}{c}1.7 .1 .9 .13 \\
6 n \\
\end{array}$ & $\begin{array}{l}\text { INSTALL LOAD } \\
\text { VTP-TIC-*56 }\end{array}$ & N/A & $N / A$ & $\Delta$ & \\
\hline 1.7 .1 .9 .14 & CLOSE CB-5 & N/A & N/A & $\Delta$ & \\
\hline$\frac{1.7 .1 .9 .15}{6 m}$ & $\begin{array}{l}A D J \\
\text { SP I I }-* 56\end{array}$ & $\mathrm{~N} / \mathrm{A}$ & N/A & A & \\
\hline $\begin{array}{r}1.7 .1 .9 .16 \\
\end{array}$ & $\begin{array}{l}\text { ADJ TIC }-* 56 \\
\text { SP } 2\end{array}$ & $N / A$ & $N / A$ & A & $\mathrm{N} / \mathrm{A}$ \\
\hline
\end{tabular}


TEST DATA SHEET

\begin{tabular}{|c|c|c|c|c|c|}
\hline Date: is 3 & S. 97 & & SHMS Unit Number & $\$ 6$ & $5 \%: 02$ \\
\hline $\begin{array}{l}\text { Title of Test: } \\
\text { Electrical }\end{array}$ & stems & & Test Equipment a & $\mathrm{S} / \mathrm{N}$ or & Cal. No \\
\hline $\begin{array}{l}\text { Test Performed } \\
\text { S Toss T }\end{array}$ & 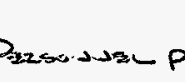 & 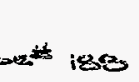 & $\rightarrow / \Delta$ & & \\
\hline $\begin{array}{l}\text { Procedure No- } \\
\text { Initial/Date }\end{array}$ & Item & Value & $\begin{array}{l}\text { Required } \\
\text { Condition }\end{array}$ & $(A / R)$ & Comment \\
\hline $\begin{array}{l}1.7 .1 .9 .17 \\
62\end{array}$ & $\begin{array}{l}\text { TAL-*50 \& } \\
\text { HORN ACTIVE }\end{array}$ & $\frac{\frac{O N}{O N}}{\frac{O N}{0.2}}$ & $\begin{array}{l}\text { TAL-* } 50 \text { ON } \\
\text { HORN ON } \\
\text { ACKNOWLEDGE }\end{array}$ & $A$ & \\
\hline $\begin{array}{c}1.7 .1 .9 .18 \\
6 \mathrm{~min} \\
\end{array}$ & $\begin{array}{l}\text { TIC }-* 56 \\
\text { OPERATES }\end{array}$ & $-\dot{o k}$ & $\begin{array}{l}\text { TIC }-* 56 \\
\text { OPERATES } \\
\end{array}$ & $A$ & \\
\hline $\begin{array}{r}1.7 .1 .9 .19 \\
6 \mathrm{n} \\
\end{array}$ & $\begin{array}{l}\text { ADJ TIC-*56 } \\
\text { SP } 2\end{array}$ & $N / A$ & $N / A$ & 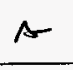 & \\
\hline $\begin{array}{c}1.7 .1 .9 .20 \\
\end{array}$ & RESET ALARM & OFF & TAL $-* 50$ OFF & $A$ & \\
\hline $\begin{array}{c}1.7 .1 .9 .21 \\
\end{array}$ & $\begin{array}{l}\text { ADJ TIC-*56 } \\
\text { SP } 1 \& S P 2\end{array}$ & $N / A$ & $N / A$ & A & \\
\hline $\begin{array}{c}1.7 .1 .9 .22 \\
6 \mathrm{~m} \\
\end{array}$ & $\begin{array}{l}\text { OPEN CB-5, } \\
\text { FU-6 \& FU-7 }\end{array}$ & N/A & $N / A$ & A & \\
\hline $\mathrm{Gm}^{1.7 .1 .9 .23}$ & $\begin{array}{l}\text { REMOVE LOAD } \\
\text { TIC-*56 }\end{array}$ & $N / A$ & $\mathrm{~N} / \mathrm{A}$ & $A$ & \\
\hline$\frac{1.7 .1 .9 .24}{6.1}$ & $\begin{array}{l}\text { CLOSE FU - } \\
\text { TIS }-* 62 \\
\text { NOMINAL TEMP }\end{array}$ & 60 & $\begin{array}{l}\text { TIS }-* 62 \\
\text { TEMP }\end{array}$ & $\Delta$ & \\
\hline $\begin{array}{l}1.7 .1 .9 .25 \\
-1.9 \\
\end{array}$ & $\begin{array}{l}\text { ADJ TIS-*62 } \\
\text { SP } 1\end{array}$ & N/A & $N / A$ & $A$ & \\
\hline $\begin{array}{l}1.7 .1 .9 .26 \\
\end{array}$ & $\begin{array}{l}\text { ADJ TIS }-* 62 \\
\text { SP } 2\end{array}$ & $N / A$ & $N / A$ & $A$ & \\
\hline${ }^{1.7} \dot{6}^{1.9 .27}$ & RESET ALARMS & OFP & $\begin{array}{l}\text { TAHL-*62 } \\
\text { OFF }\end{array}$ & A & \\
\hline $\begin{array}{l}1.7 .1 .9 .28 \\
6 m\end{array}$ & $\begin{array}{l}\text { ADJ TIS-*62 } \\
\text { SP 2 } \\
\end{array}$ & $N / A$ & $N / A$ & $\alpha$ & \\
\hline$\frac{1.7 .1 .9 .29}{6 m}$ & $\begin{array}{l}\text { TAHL-*62 \& } \\
\text { HORN ACTIVE }\end{array}$ & $\frac{0,3}{\frac{0 J}{0 K}}$ & $\begin{array}{l}\text { TAHL-*62 ON } \\
\text { HORN ON } \\
\text { ACKNOWLEDGE }\end{array}$ & A & \\
\hline $\begin{array}{l}1.7 .1 .9 .30 \\
\end{array}$ & $\begin{array}{l}\text { ADJ TIS } * 62 \\
\text { SP } 2\end{array}$ & $N / A$ & $N / A$ & $\Delta$ & \\
\hline
\end{tabular}


TEST DATA SHEET

\begin{tabular}{|c|c|c|c|c|c|}
\hline \multicolumn{3}{|c|}{ Date: is DAD. 97} & \multicolumn{3}{|c|}{ SHMS Unit Number: $6 \mathrm{Sin} 102$} \\
\hline \multicolumn{3}{|c|}{$\begin{array}{l}\text { Title of rest: } \\
\text { Electrical Systems }\end{array}$} & \multirow{2}{*}{\multicolumn{3}{|c|}{ 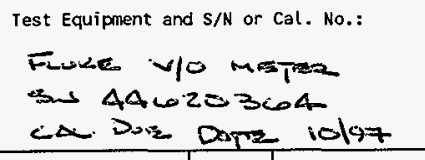 }} \\
\hline \multicolumn{3}{|c|}{ 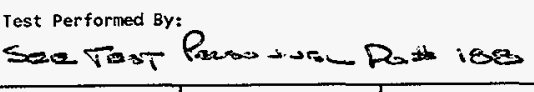 } & & & \\
\hline $\begin{array}{l}\text { Procedure No. } \\
\text { Initial/Date }\end{array}$ & Item & value & $\begin{array}{c}\text { Required } \\
\text { condition }\end{array}$ & $(\mathrm{A} / \mathrm{R})$ & Corment \\
\hline $\begin{array}{l}1.7 .1 .9 .31 \\
0.17\end{array}$ & RESET ALARMS & OFF & $\begin{array}{l}\text { TAHL }-* 62 \\
\text { OFF }\end{array}$ & A & \\
\hline $\operatorname{6in}^{1.7 .1 .32}$ & $\begin{array}{l}\text { ADJ TIS } * 62 \\
\text { SP } 1\end{array}$ & N/A & N/A & A & \\
\hline $\sin ^{1.7 .1 .933}$ & $\begin{array}{l}\text { TAHL-*62 \& } \\
\text { HORN ACTIVE }\end{array}$ & $\frac{003}{0,3}$ & $\begin{array}{l}\text { TAHL }-* 62 \text { ON } \\
\text { HORN ON } \\
\text { ACKNOWLEDGE }\end{array}$ & A & \\
\hline $\operatorname{lon}^{1.7 .1 .9 .34}$ & $\begin{array}{l}\text { ADJ TIS-*62 } \\
\text { SP } 1 \text { \& SP } 2\end{array}$ & N/A & N/A & A & \\
\hline $\mathrm{g}^{1.7 .9 .35}$ & $\begin{array}{l}\text { OPEN FU-8, } \\
\text { FU-9 \& FU-10 }\end{array}$ & N/A & $N / A$ & A & \\
\hline \multicolumn{6}{|c|}{ Section 1.7.1.10 verifies the differential pressure transmitter system } \\
\hline $\mathrm{em}^{1.1 .10 .1}$ & $\begin{array}{l}\text { REMOVE PLUGS } \\
\text { BAL PDIT-*60 }\end{array}$ & N/A & N/A & $\AA$ & \\
\hline$\frac{1.7 .1 .10 .2}{\cos }$ & $\begin{array}{l}\text { CLOSE FU-14 } \\
\text { REC PDIT }-{ }^{*} 60 \\
\text { PRESSURE }\end{array}$ & .015 & $\begin{array}{l}\text { POIT-*60 } \\
\text { PRESSURE } \\
0 \pm 1 " \mathrm{H}_{2} \mathrm{O}\end{array}$ & $\Delta$ & \\
\hline $\mathrm{om}^{1.7}$ & $\begin{array}{l}\text { MUX-*70 Vdc } \\
T B 7+\& \text { TB8- }\end{array}$ & 2.153 & $\begin{array}{l}\text { MUX-*70 Vdc } \\
2.2 \pm 0.1\end{array}$ & A & \\
\hline$\frac{1.7 .1 .10 .4}{6 . m}$ & $\begin{array}{l}\text { REPLACE } \\
\text { PLUGS ON } \\
\text { PDIT-*60 } \\
\end{array}$ & N/A & N/A & A & \\
\hline $\begin{array}{l}1.7 .1 .10 .5 \\
66^{m} \\
\end{array}$ & OPEN FU-14 & N/A & $\mathrm{N} / \mathrm{A}$ & A & \\
\hline \multicolumn{6}{|c|}{ Section 1.7.1.11 verifies the gas chromatograph power } \\
\hline $\begin{array}{r}1.7 .1 .11 \mathrm{~m}^{1} \\
2^{m}\end{array}$ & CLOSE FU-11 & N/A & N/A & A & \\
\hline $\begin{array}{l}1.7 .1 .11 .2 \\
\mathrm{~m}^{\mathrm{m}}\end{array}$ & $\begin{array}{l}\text { POWER } \\
\text { MON-*60 } \\
\end{array}$ & 0,12 & $\begin{array}{l}\text { MON-*60 } \\
\text { POWERED } \\
\end{array}$ & A & \\
\hline$\frac{1.7 .1 .11 .3}{19}$ & OPEN FU-11 & $N / A$ & N/A & $A$ & $\Delta$ \\
\hline
\end{tabular}


PAGE $F-12$
APPENDIX $F$

$$
\begin{array}{r}
\text { HNF-SD-WM-ATR-191 } \\
\text { ReV. } 0 \\
\text { Page } 198
\end{array}
$$

TEST DATA SHEET

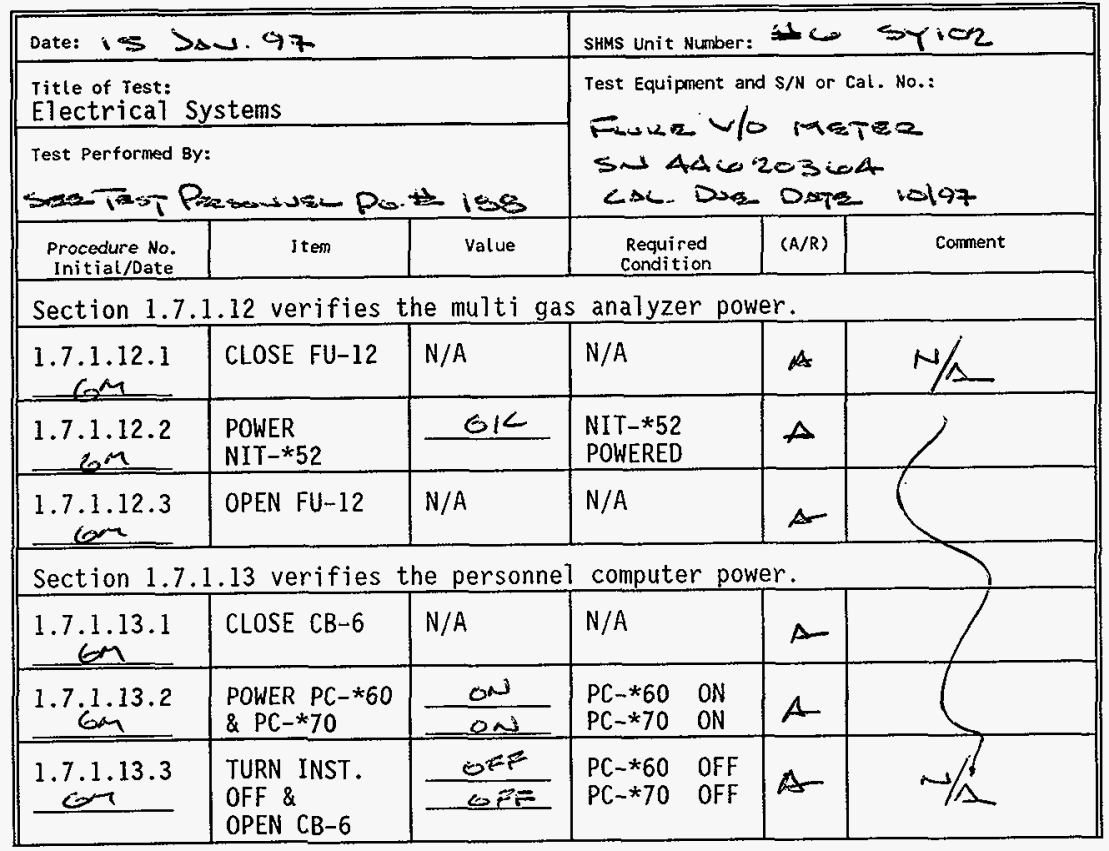

Ant Gin :15/177 
TEST DATA SHEET

\begin{tabular}{|c|c|c|c|c|c|}
\hline \multicolumn{3}{|c|}{ Date: is $s_{s-4} .97$} & \multicolumn{3}{|c|}{ SHMS Unit Number: $\$ 6$ SY 102} \\
\hline \multicolumn{3}{|c|}{$\begin{array}{l}\text { Title of Test: } \\
\text { Electrical Systems }\end{array}$} & \multirow{2}{*}{\multicolumn{3}{|c|}{ 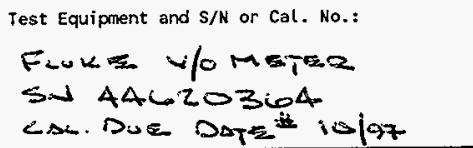 }} \\
\hline \multicolumn{3}{|c|}{ 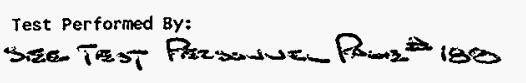 } & & & \\
\hline $\begin{array}{l}\text { Procedure No. } \\
\text { Initial/Date }\end{array}$ & Item & value & $\begin{array}{l}\text { Required } \\
\text { Condition }\end{array}$ & $(A / R)$ & Comment \\
\hline \multicolumn{6}{|c|}{ Section 1.7 .2 verifies the intrinsic safety apparatus is properly labeled. } \\
\hline $1.7 .2 \mathrm{el}$ & \multirow{2}{*}{$\begin{array}{l}\text { INTRINSIC } \\
\text { SAFETY } \\
\text { APPARATUS } \\
\text { NAMEPLATES } \\
\text { INSTALLED }\end{array}$} & & \multirow{2}{*}{$\begin{array}{l}\text { NE-*54 } \\
\text { NE-*55 } \\
\text { EB-*54 } \\
\text { EB-*55 } \\
\text { NIT-*54 } \\
\text { NIT-*55 }\end{array}$} & \multirow[b]{2}{*}{ A } & \multirow[t]{2}{*}{ ExCEDTOSII } \\
\hline $9-2 x-97$ & & 5 & & & \\
\hline $\begin{array}{l}1.7 .2 .2 \\
\frac{\sin }{1 \cos 5-2=27}\end{array}$ & $\begin{array}{l}\text { INTRINSIC } \\
\text { SAFETY WIRE } \\
\text { LABELED }\end{array}$ & $\frac{r}{r}$ & $\begin{array}{l}\mathrm{NE} / \mathrm{EB}-* 54 \\
\mathrm{NE} / \mathrm{EB}-* 55\end{array}$ & A- & $\mathrm{N} / \mathrm{s}$ \\
\hline $\begin{array}{l}1.7 .39-2297 \\
m .0 . B\end{array}$ & $\begin{array}{l}\text { SEC. } 1.7 .1 \text { \& } \\
1.7 .2 \text { DONE }\end{array}$ & $\frac{m_{\text {and }}}{\text { Test Dire }}$ & $\frac{\text { Bra }}{\text { or Signature }}$ & & $\frac{9.28-97}{\text { Date }}$ \\
\hline
\end{tabular}

Test Witness/Review:

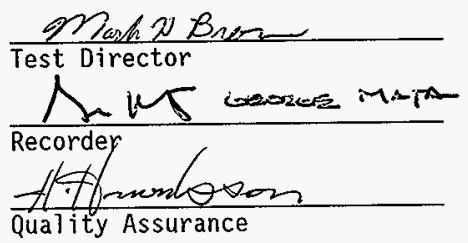

$$
\begin{aligned}
& \frac{1-15-97}{\text { Date }} \\
& \frac{1530-9.97}{\text { Date }} \\
& \frac{1-27-97}{\text { Date }}
\end{aligned}
$$


TEST DATA SHEET

\begin{tabular}{|c|c|c|c|c|c|}
\hline Date: is $>$ & 1. 97 & & SHMS Unit Number & +46 & 3102 \\
\hline $\begin{array}{l}\text { Title of Test: } \\
\text { Pneumatic } S\end{array}$ & tems & & Test Equipment a & $\mathrm{S} / \mathrm{N}$ or & al. No.: \\
\hline $\begin{array}{l}\text { Test Performed } \\
\text { Sose- Tarsi }\end{array}$ & $x \rightarrow d x=P$ & $1 \pm: 88$ & & & \\
\hline $\begin{array}{c}\text { Procedure No. } \\
\text { Initial/Date }\end{array}$ & Item & Value & $\begin{array}{l}\text { Required } \\
\text { Condition }\end{array}$ & $(A / R)$ & Comment \\
\hline Section 1.7 & .1 provides tr & pneumatic & test section & initia & con \\
\hline$\frac{1.7 .4 .1 .1}{6}$ & $\begin{array}{l}\text { INITIAL } \\
\text { CONDITIONS }\end{array}$ & ok & $\begin{array}{l}\text { BKR \& FUSE } \\
\text { LINE UP }\end{array}$ & $\Delta$ & \\
\hline $\begin{array}{c}1.7 .4 .1 .2 \\
\mathrm{gm} \\
\end{array}$ & $\begin{array}{l}\text { INITIAL } \\
\text { CONDITIONS } \\
\end{array}$ & ok & $\begin{array}{l}\text { VALVE LINE } \\
\text { UP }\end{array}$ & $A$ & \\
\hline $\begin{array}{l}1.7 .4 .1 .3 \\
6.4\end{array}$ & $\begin{array}{l}\text { DISCONNECT } \\
1 / 16^{\prime \prime} \text { TUBING } \\
\text { ON MON-*60 }\end{array}$ & OK & $\begin{array}{l}\text { TUBING } \\
\text { CONFIGURED }\end{array}$ & $\Delta$ & \\
\hline $\begin{array}{l}1.7 .4 .1 .4 \\
.04\end{array}$ & $\begin{array}{l}\text { DISCONNECT } \\
\text { TUBING ON } \\
\text { NIT }-\star 52 \\
\end{array}$ & sk & $\begin{array}{l}\text { TUBING } \\
\text { CONF IGURED }\end{array}$ & A & \\
\hline $\begin{array}{c}1.7 .4 .1 .5 \\
\end{array}$ & $\begin{array}{l}\text { PLUG VENT } \\
\text { FLT }{ }^{*} 63\end{array}$ & ok & $\begin{array}{l}\text { FLT-*63 } \\
\text { PLUGGED }\end{array}$ & $\Lambda$ & \\
\hline Section 1.7 & 2 verifies th & main samp & e flow loop. & & \\
\hline $\begin{array}{l}1.7 .4 .2 .1 \\
6\end{array}$ & $\begin{array}{l}\text { CLOSE CB-3/4 } \\
\text { ADJ. FLOW }\end{array}$ & $\frac{\mathrm{CuO}_{2} 7}{0.2}$ & $\begin{array}{l}\text { CB CLOSED } \\
\text { FIT-*57 } \\
\text { INDICATION }\end{array}$ & A & \\
\hline $\begin{array}{c}1.7 .4 .2 .2 \\
64\end{array}$ & $\begin{array}{l}\text { PDI } * 51 \\
\text { READING }\end{array}$ & 27 & $20-40 " \mathrm{H}_{2} \mathrm{O}$ & A & \\
\hline $\begin{array}{c}1.7 .2 .3 \\
\end{array}$ & $\begin{array}{l}\text { PI }-* 53 \\
\text { READING }\end{array}$ & -3 & $\begin{array}{l}-2 \text { to }-4 \\
" \mathrm{Hg}\end{array}$ & $A$ & \\
\hline $\begin{array}{c}1.7 .4 .2 .4 \\
6 \mathrm{~m}\end{array}$ & RESET ALARMS & No & NO ALARMS & $A$ & \\
\hline $1.7 \sin ^{4.5}$ & CLOSE SV $-* 16$ & $\begin{array}{c}0 \\
O N \\
\sigma N\end{array}$ & $\begin{array}{lll}\text { FIT }-* 57 & 0 \\
\pm 1 \quad " H_{2} 0 & \\
\text { FAL }-* 57 & \text { ON } \\
\text { HORN } & \text { ON } \\
\end{array}$ & A & \\
\hline $\mathrm{sin}^{1.7 .6}$ & OPEN SV-*16 & $\frac{\text { on }}{-028}$ & $\begin{array}{l}\text { FAL }-* 57 \text { ON } \\
\text { HORN OFF }\end{array}$ & $\Delta$ & ) \\
\hline $\sin ^{1.7 .2 .7}$ & RESET ALARMS & OK & $\begin{array}{l}\text { ALARMS } \\
\text { RESET } \\
\end{array}$ & $\Delta$ & $1 / 1$ \\
\hline
\end{tabular}


TEST DATA SHEET

\begin{tabular}{|c|c|c|c|c|c|}
\hline \multicolumn{3}{|c|}{ Date: is Sous is } & \multicolumn{3}{|c|}{ SHMS Unit Number: $\mathrm{SY}_{102}$} \\
\hline \multicolumn{3}{|c|}{$\begin{array}{l}\text { Title of rest: } \\
\text { Pneumatic Systems } \\
\end{array}$} & \multirow{2}{*}{\multicolumn{3}{|c|}{ Test Equipment and $S / N$ or Cal. No.: }} \\
\hline \multicolumn{3}{|c|}{ 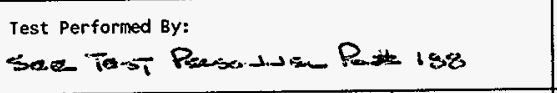 } & & & \\
\hline $\begin{array}{l}\text { Procedure No. } \\
\text { Initial/Date }\end{array}$ & Item & value & $\begin{array}{l}\text { Required } \\
\text { Condition } \\
\end{array}$ & $(A / R)$ & Comment \\
\hline $\begin{array}{l}1.7 .4 .2 .8 \\
\operatorname{con}\end{array}$ & CLOSE SV-*25 & $\begin{array}{c}0 \\
\frac{0 N}{0} \\
0 N \\
0\end{array}$ & $\begin{array}{l}\text { FIT-*57 } 0 \\
\pm 1 \text { " } \mathrm{H}_{2} 0 \\
\text { FAL-*57 ON } \\
\text { HORN } \\
\text { XA-*63 ON } \\
\text { YY-*71 } \\
\text { YY } \\
\text { TB } 3-4 \text { ON } \\
\end{array}$ & A & $\omega / \Delta$ \\
\hline $\begin{array}{l}1.7 .4 .2 .9 \\
6\end{array}$ & OPEN SV $-* 25$ & $\frac{\frac{O N}{O R E}}{\frac{\text { ORE }}{119.1}}$ & $\begin{array}{l}\text { FAL-*57 ON } \\
\text { HORN OFF } \\
\text { XA-*63 OFF } \\
\text { YY-*71 } \\
\text { TB } 3-4 \quad 120 \mathrm{~V} \\
\end{array}$ & $\Delta$ & \\
\hline $\begin{array}{l}1.7 .4 .2 .10 \\
6 \times \\
\end{array}$ & RESET ALARMS & ok & $\begin{array}{l}\text { ALARMS } \\
\text { RESET }\end{array}$ & 1 & \\
\hline $\begin{array}{l}1.7 .4 .2 .11 \\
6.5 \\
\end{array}$ & $\begin{array}{l}\text { OPEN SV-*54 } \\
\& \text { SV-*66 } \\
\text { READ PRESS. } \\
\text { PDIT-*60 } \\
\end{array}$ & $\frac{\nearrow}{-40.75}$ & $\begin{array}{l}\text { VALVES OPEN } \\
-27 \text { to }-82 \\
" \mathrm{H}_{2} \mathrm{O}\end{array}$ & A & \\
\hline$\frac{1.7 .4 .2 .12}{\sigma^{2}}$ & $\begin{array}{l}\text { CLOSE SV }-* 54 \\
\text { OPEN SV }-* 63 \\
\text { READ PRESS. } \\
\text { PDIT-*60 } \\
\end{array}$ & .019 & $\begin{array}{l}\text { VALVES } \\
\text { OPERATED } \\
0 \pm 1 " \mathrm{H}_{2} \mathrm{O}\end{array}$ & $A$ & \\
\hline$\frac{1.7 .4 .2 .13}{6 m}$ & $\begin{array}{l}\text { CLOSE SV }-* 63 \\
\text { OPEN SV-*55 } \\
\text { READ PRESS. } \\
\text { PDIT-*60 } \\
\end{array}$ & $\frac{1}{-42.93}$ & $\begin{array}{l}\text { VALVES } \\
\text { OPERATED } \\
-27 \text { to }-82 \\
" \mathrm{H}_{2} \mathrm{O} \\
\end{array}$ & $A$ & \\
\hline$\frac{1.7 .4 .2 .14}{6 m^{14}}$ & $\begin{array}{l}\text { CLOSE SV }-* 55 \\
\text { OPEN SV }-* 64 \\
\text { READ PRESS. } \\
\text { PDIT-*60 } \\
\end{array}$ & $\frac{V}{.0195}$ & $\begin{array}{l}\text { VALVES } \\
\text { OPERATED } \\
0 \pm 1 " \mathrm{H}_{2} \mathrm{O}\end{array}$ & A & \\
\hline $\begin{array}{c}1.7 .4 .2 .15 \\
\end{array}$ & $\begin{array}{l}\text { CLOSE SV-*64 } \\
\& \text { SV-*66 }\end{array}$ & N/A & $N / A$ & A & \\
\hline \multicolumn{6}{|c|}{ Section 1.7.4.3 verifies the grab sample flow loop. } \\
\hline $\begin{array}{c}1.7 .4 .3 .1 \\
6.7 \\
\end{array}$ & READ PDI $* 51$ & 27 & $20-40 " \mathrm{H}_{2} \mathrm{O}$ & A & $N / S$ \\
\hline
\end{tabular}




\section{TEST DATA SHEET}

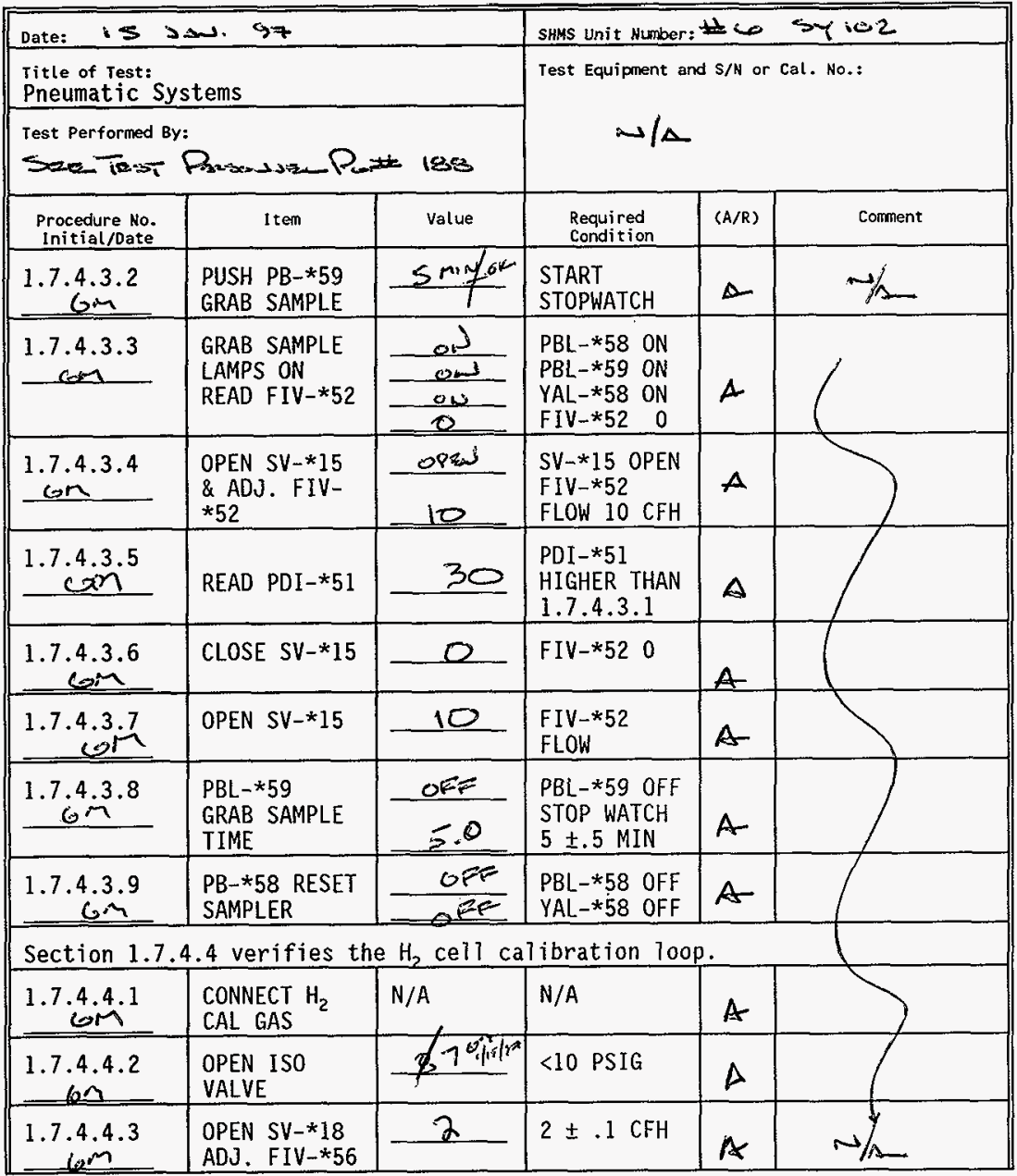

Auc en iliskiz 
TEST DATA SHEET

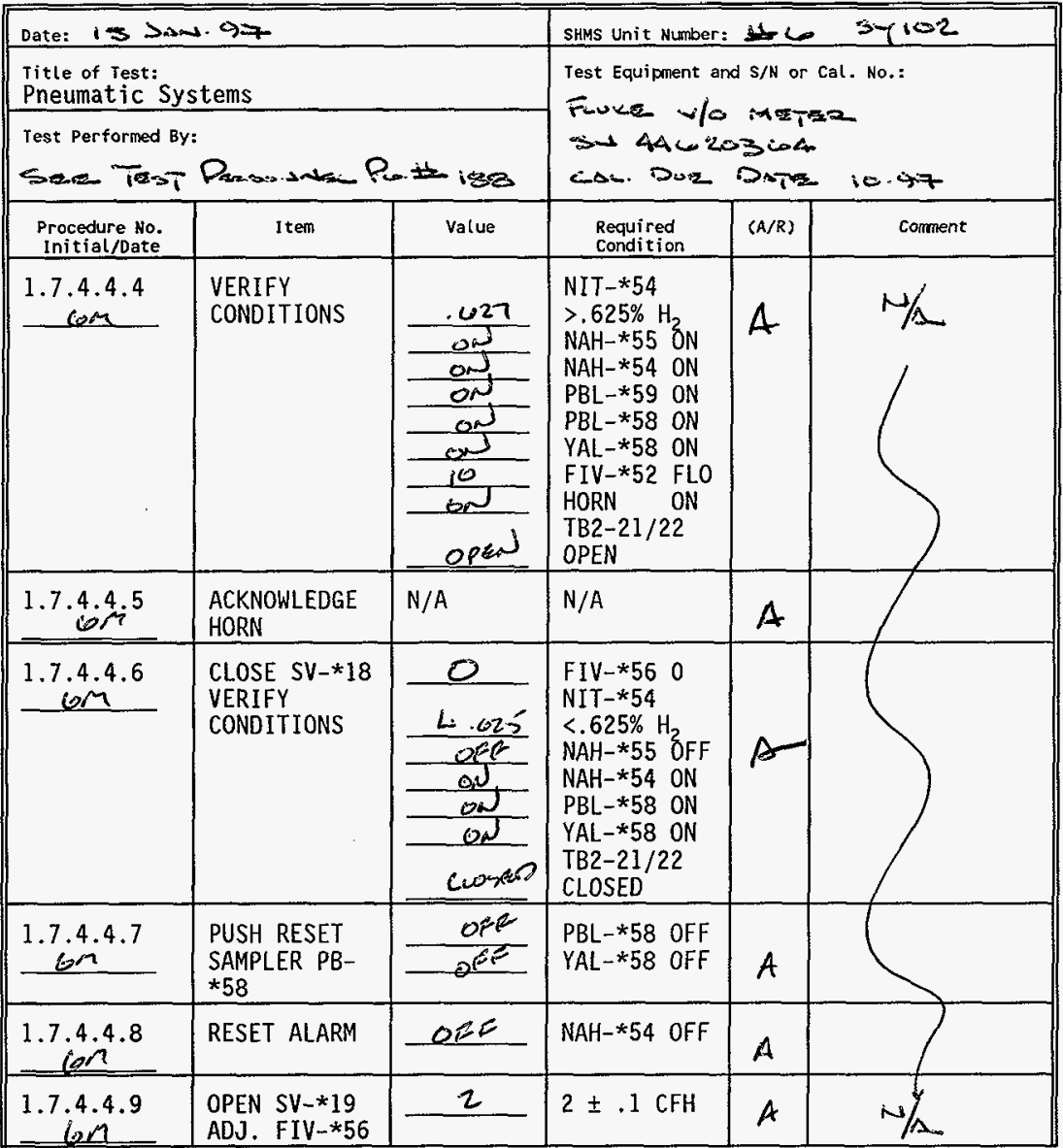

An on ilistiz 
APPENDIX $\mathrm{F}$

TEST DATA SHEET

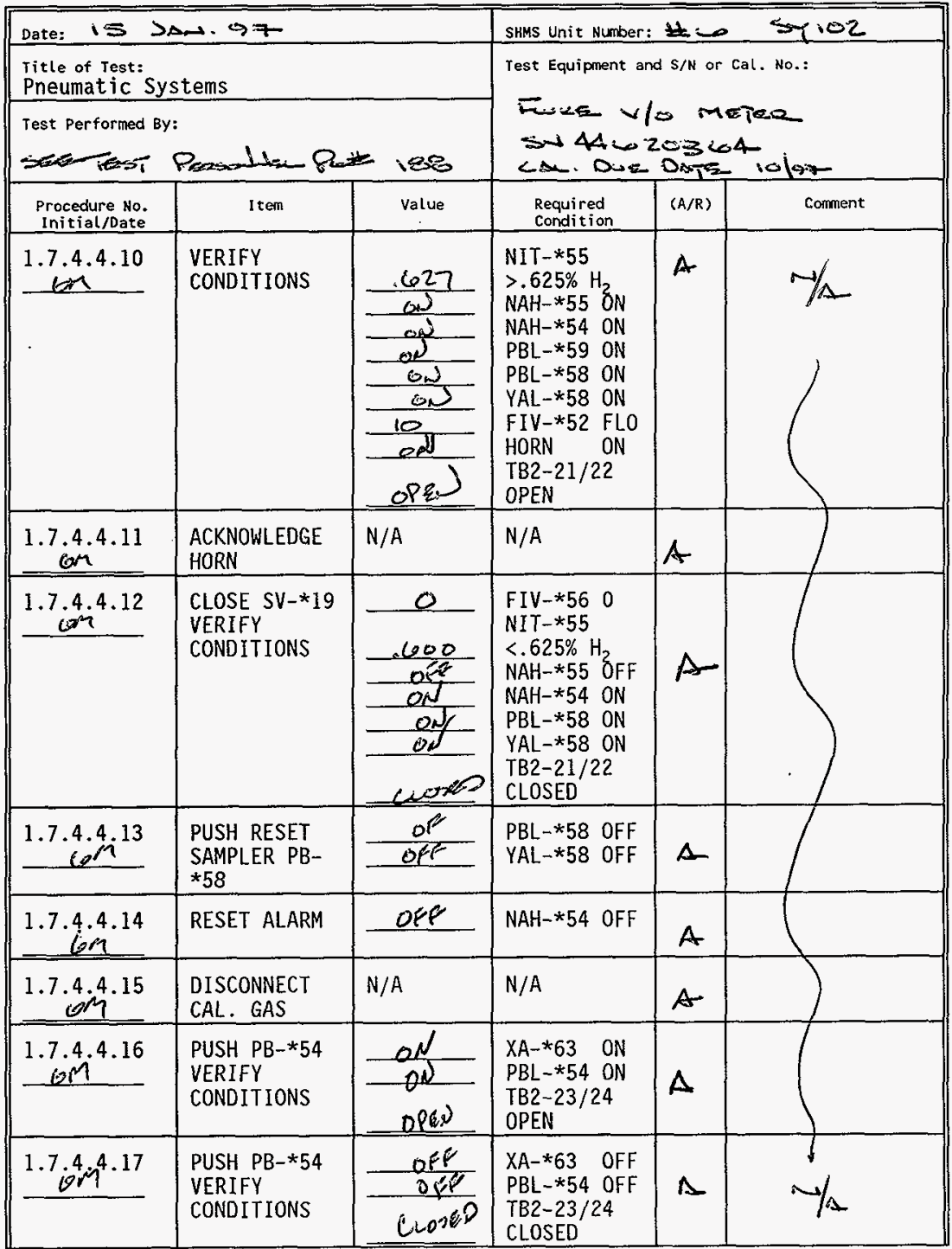


APPENDIX F

HNF-SD-WM-ATR - 191

PAGE F-/ 9

Rage 205

TEST DATA SHEET

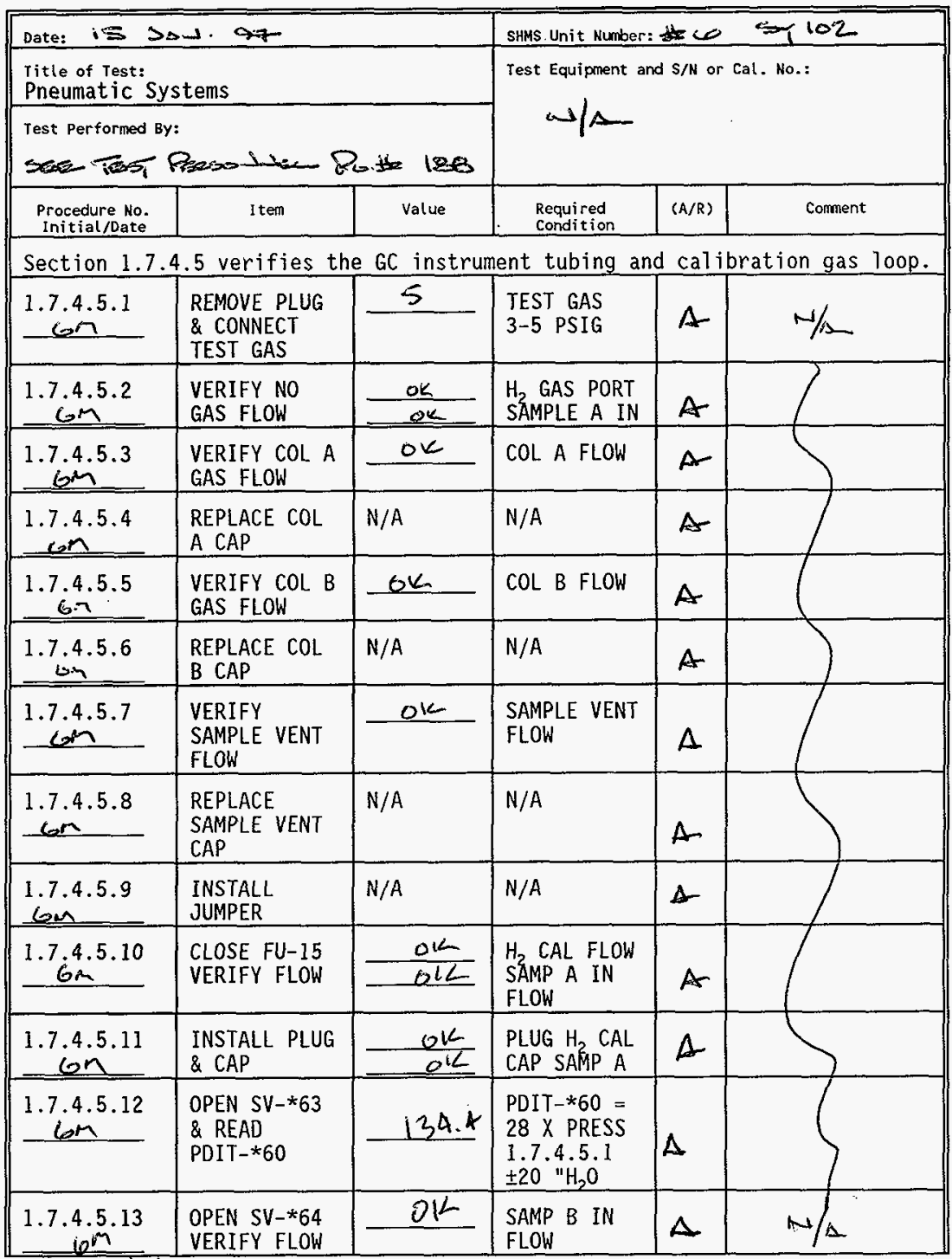




\section{TEST DATA SHEET}

\begin{tabular}{|c|c|c|c|c|c|}
\hline \multicolumn{3}{|c|}{ Date: 1550.97} & \multicolumn{3}{|c|}{ SHMS Unit Number $=20$ Stiviz } \\
\hline \multicolumn{3}{|c|}{$\begin{array}{l}\text { Title of Test: } \\
\text { Pneumatic Systems }\end{array}$} & \multirow{2}{*}{\multicolumn{3}{|c|}{ Test Equipment and $\mathrm{S} / \mathrm{N}$ or Cal. No.: }} \\
\hline \multicolumn{3}{|c|}{ 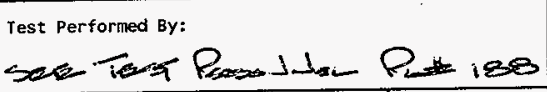 } & & & \\
\hline $\begin{array}{l}\text { Procedure No. } \\
\text { Initial/Date }\end{array}$ & Item & Value & $\begin{array}{l}\text { Required } \\
\text { Condition }\end{array}$ & $(A / R)$ & Comment \\
\hline $\begin{array}{c}1.7 .4 .5 .14 \\
\mathrm{Gm} \\
\end{array}$ & $\begin{array}{l}\text { REMOVE TEST } \\
\text { GAS }\end{array}$ & $N / A$ & $N / A$ & $A$ & \\
\hline $\begin{array}{c}1.7 .4 .5 .15 \\
6.7 . \\
\end{array}$ & $\begin{array}{l}\text { CLOSE SV-*63 } \\
\& \text { SV-*64 }\end{array}$ & $N / A$ & $N / A$ & - & \\
\hline $\begin{array}{l}1.7 .4 .5 .16 \\
\qquad 5^{\circ-1} \\
\end{array}$ & $\begin{array}{l}\text { OPEN FU-15 } \\
\text { REMOVE } \\
\text { JUMPER } \\
\end{array}$ & $N / A$ & $N / A$ & $A$ & \\
\hline $\begin{array}{c}1.7 .4 .5 .17 \\
6 \\
\end{array}$ & $\begin{array}{l}\text { INSTALL } \\
\text { SAMP A UNION }\end{array}$ & $N / A$ & $N / A$ & $A$ & \\
\hline $\begin{array}{c}1.7 .4 .5 .18 \\
\mathrm{~S}^{\mathrm{m}} \\
\end{array}$ & $\begin{array}{l}\text { OPEN SV-*60 } \\
\text { VERIFY FLOW }\end{array}$ & & $\begin{array}{l}\text { FI-*60 } \\
\text { NO FLOW }\end{array}$ & 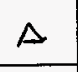 & \\
\hline$\frac{1.7 .4 .5 .19}{\cos }$ & $\begin{array}{l}\text { POWER SOV- } \\
* 60 \text {, VERIFY } \\
\text { NO FLOW } \\
\end{array}$ & 0 & $\begin{array}{l}\text { FI-*60 } \\
\text { NO FLOW }\end{array}$ & & \\
\hline $\begin{array}{l}1.7 .4 .5 .20 \\
\text { C.n } \\
\end{array}$ & $\begin{array}{l}\text { OPEN SV }-* 68 \\
\text { ADJ } S V-* 67\end{array}$ & $\leqslant 0$ & $\begin{array}{l}F I-* 60 \\
50 \mathrm{CCM} \\
\end{array}$ & $A$ & \\
\hline 1.7 .4 .5 .21 & $\begin{array}{l}\text { DEENERGIZE } \\
\text { SOV } * * 60\end{array}$ & () & $\begin{array}{l}\text { FI-*60 FLOW } \\
\text { DECREASES }\end{array}$ & A & \\
\hline $\begin{array}{r}1.7 .4 .5 .22 \\
6 n \\
\end{array}$ & $\begin{array}{l}\text { CLOSE SV }-* 60 \\
\& \text { SV }-* 68\end{array}$ & $N / A$ & $N / A$ & $\Lambda$ & \\
\hline $\begin{array}{r}1.7 .4 .5 \mathrm{~m}^{23} \\
\end{array}$ & $\begin{array}{l}\text { REMOVE } \\
\text { SAMP A UNION }\end{array}$ & $N / A$ & $\mathrm{~N} / \mathrm{A}$ & & \\
\hline$\frac{1.7 .4 .5 .24}{\mathrm{bin}^{2}}$ & $\begin{array}{l}\text { CONNECT TEST } \\
\text { GAS } \mathrm{N}_{2} \mathrm{O} \text { PORT } \\
\end{array}$ & $\alpha$ & $\begin{array}{l}\text { TEST GAS } \\
3-5 \text { PSIG } \\
\end{array}$ & & \\
\hline $\begin{array}{c}1.7 .4 .5 .25 \\
-1.4 \\
\end{array}$ & $\begin{array}{l}\text { VERIFY NO } \\
\text { FLOW }\end{array}$ & $\frac{0.2}{0 K}$ & $\begin{array}{l}\text { SAMP B IN \& } \\
\text { FLT-*63 } \\
\text { NO FLOW }\end{array}$ & & \\
\hline $\begin{array}{r}1.7 .4 .5 .26 \\
\text { iem } \\
\end{array}$ & $\begin{array}{l}\text { INSTALL } \\
\text { JUMPER } \\
\end{array}$ & $N / A$ & $N / A$ & & \\
\hline$\frac{1.7 .4 .5 .27}{\left(a M^{27}\right.}$ & $\begin{array}{l}\text { CLOSE FU-15 } \\
\text { VERIFY FLOW }\end{array}$ & $\frac{016}{6 i^{2}}$ & $\begin{array}{l}\text { SAMP B IN } \\
\text { FLT-*63 } \\
\text { FLOW }\end{array}$ & & 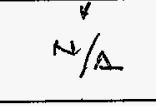 \\
\hline
\end{tabular}


TEST DATA SHEET

\begin{tabular}{|c|c|c|c|c|c|}
\hline \multicolumn{3}{|c|}{ Date: $15>1.97$} & \multicolumn{3}{|c|}{ SHMS Unit Number: $\cong \mathrm{C}^{10 \mathrm{Z}}$} \\
\hline \multicolumn{3}{|c|}{$\begin{array}{l}\text { Title of rest: } \\
\text { Pneumatic Systems }\end{array}$} & \multirow{2}{*}{\multicolumn{3}{|c|}{ Test Equipment and $\mathrm{S} / \mathrm{N}$ or Cal. No.: }} \\
\hline \multicolumn{3}{|c|}{ 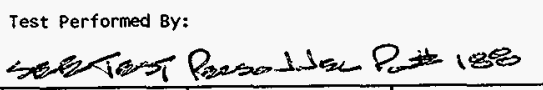 } & & & \\
\hline $\begin{array}{c}\text { Procedure No. } \\
\text { Initial/Date }\end{array}$ & Item & Value & $\begin{array}{l}\text { Required } \\
\text { Condition } \\
\end{array}$ & $(A / R)$ & commen \\
\hline $\begin{array}{l}1.7 .4 .5 .28 \\
\end{array}$ & $\begin{array}{l}\text { DISCONNECT } \\
\text { TEST GAS } \\
\end{array}$ & $N / A$ & $N / A$ & $\Lambda$ & \\
\hline $\begin{array}{c}1.7 .4 .5 .29 \\
.69 \\
\end{array}$ & OPEN FU-15 & $N / A$ & $N / A$ & A & \\
\hline 1.7 .4 .5 .30 & $\begin{array}{l}\text { REMOVE } \\
\text { JUMPER }\end{array}$ & $N / A$ & $N / A$ & $A$ & \\
\hline $\begin{array}{r}1.7 .4 .5 .31 \\
\quad 64 \\
\end{array}$ & $\begin{array}{l}\text { INSTALL } \\
\text { SAMP B UNION }\end{array}$ & $N / A$ & $N / A$ & $A$ & \\
\hline $\begin{array}{c}1.7 .4 .5 .32 \\
1.7 \\
\end{array}$ & $\begin{array}{l}\text { OPEN SV }-* 60 \\
\text { VERIFY FLOW } \\
\end{array}$ & GK & $\begin{array}{l}\text { FI-*60 } \\
\text { NO FLOW }\end{array}$ & $A$ & \\
\hline$\frac{1.7 .4 .5 .33}{60^{m}}$ & $\begin{array}{l}\text { POWER SOV- } \\
* 60, \text { VERIFY } \\
\text { NO FLOW } \\
\end{array}$ & ok & $\begin{array}{l}\text { FI-*60 } \\
\text { NO FLOW }\end{array}$ & $A$ & \\
\hline 1.7 .4 .5 .34 & $\begin{array}{l}\text { OPEN SV }-* 68 \\
\& \text { ADJ SV-*67 }\end{array}$ & 50 & $\begin{array}{l}\mathrm{FI}-* 60 \\
50 \mathrm{cCM} \\
\end{array}$ & A & \\
\hline $\begin{array}{c}1.7 .4 .5 .35 \\
\end{array}$ & $\begin{array}{l}\text { DEENERGIZE } \\
\text { SOV }-* 60\end{array}$ & -ok & $\begin{array}{l}\text { FI-*60 FLOW } \\
\text { DECREASES }\end{array}$ & $A$ & \\
\hline $1.7 \mathrm{bin}^{4.5 .36}$ & $\begin{array}{l}\text { CLOSE SV }-* 60 \\
\& \text { SV }-* 68 \\
\end{array}$ & N/A & N/A & $\Delta$ & \\
\hline $\begin{array}{c}1.7 .4 .5 .37 \\
\end{array}$ & $\begin{array}{l}\text { REMOVE } \\
\text { SAMP B UNION } \\
\end{array}$ & N/A & N/A & $\Delta$ & \\
\hline $\begin{array}{c}1.7 .4 .5 .38 \\
\end{array}$ & $\begin{array}{l}\text { OPEN CB-3/4 } \\
\text { ACK. ALARMS }\end{array}$ & N/A & N/A & $\Delta$ & \\
\hline \multicolumn{6}{|c|}{$\begin{array}{l}\text { Section } 1.7 .4 .6 \text { verifies the multi gas analyzer instrument tubing and } \\
\text { calibration gas loop. }\end{array}$} \\
\hline$\frac{1.7 .4 .6 .1}{6 \times 1}$ & $\begin{array}{l}\text { VERIFY } \\
\text { NIT } * 52 \\
\text { SAMPLE LINES } \\
\end{array}$ & N/A & N/A & $A$ & \\
\hline $\begin{array}{l}1.7 .4 .6 .2 \\
6.77 \\
\end{array}$ & $\begin{array}{l}\text { CONNECT TEST } \\
\text { GAS } \mathrm{NH}_{3} \text { PORT }\end{array}$ & $x$ & $\begin{array}{l}\text { TEST GAS } \\
3-5 \text { PSIG } \\
\end{array}$ & A & \\
\hline 1.7 .4 .6 .3 & $\begin{array}{l}\text { VERIFY NO } \\
\text { FLOW }\end{array}$ & $\frac{0^{2}}{a^{2}}$ & $\begin{array}{l}\text { SAMPLE IN } \\
\text { FLT-*63 }\end{array}$ & $\Delta$ & \\
\hline
\end{tabular}


TEST DATA SHEET

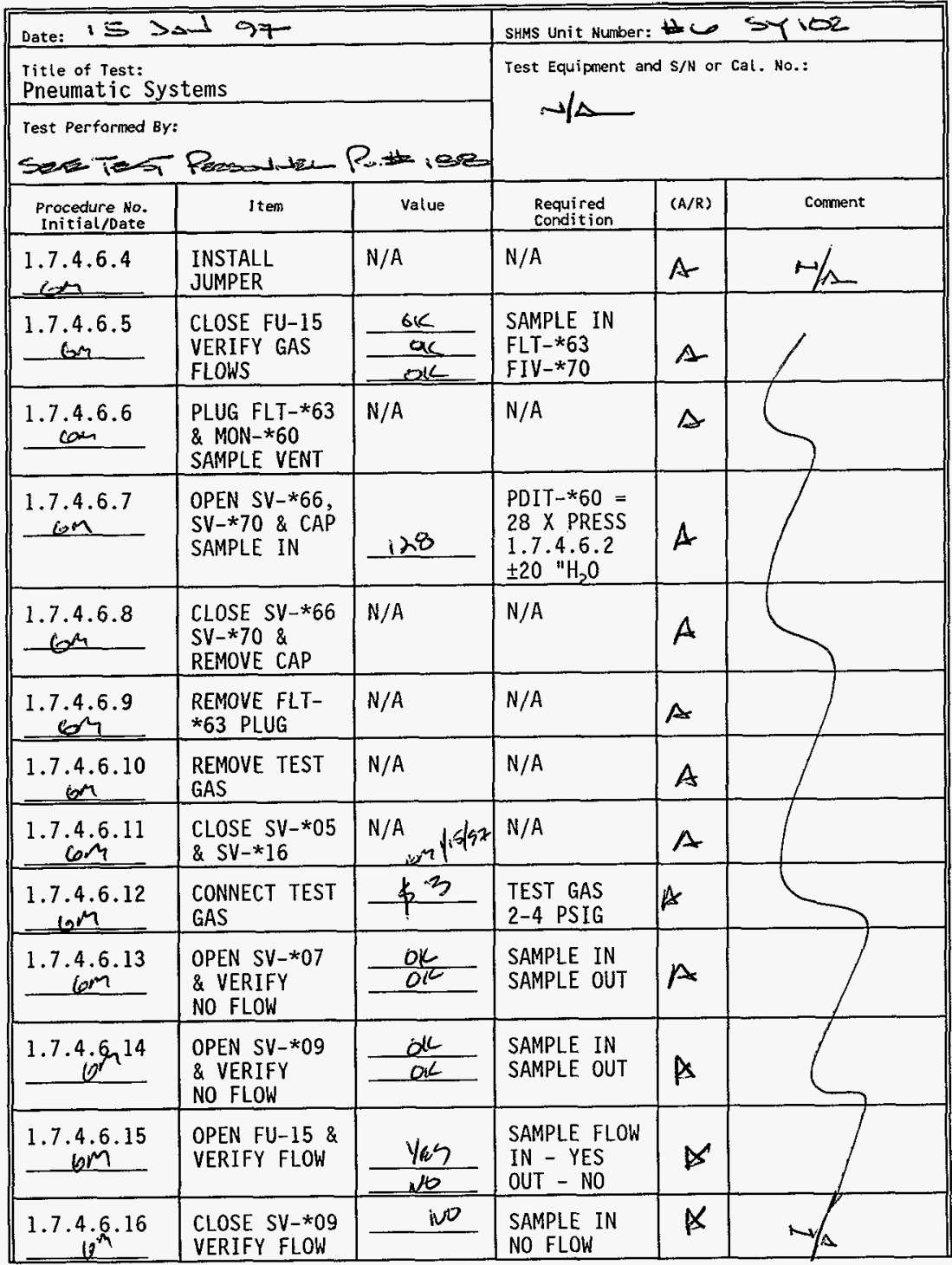




\section{TEST DATA SHEET}

\begin{tabular}{|c|c|c|c|c|c|}
\hline \multicolumn{3}{|c|}{ Date: $15>\rightarrow \rightarrow .97$} & \multicolumn{3}{|c|}{ SHMS Unit Number: to siloz } \\
\hline \multicolumn{3}{|c|}{$\begin{array}{l}\text { Title of Test: } \\
\text { Pneumatic Systems }\end{array}$} & \multirow{2}{*}{\multicolumn{3}{|c|}{ Test Equipment and $\mathrm{S} / \mathrm{N}$ or Cal. No.: }} \\
\hline \multicolumn{3}{|c|}{ Test Performed By: } & & & \\
\hline $\begin{array}{c}\text { Procedure No. } \\
\text { Initial/Date }\end{array}$ & I tem & Value & $\begin{array}{l}\text { Required } \\
\text { Condition }\end{array}$ & $(A / R)$ & comment \\
\hline$\frac{1.7 .4 .6 .17}{69^{-1}}$ & $\begin{array}{l}\text { OPEN SV-*10 } \\
\text { VERIFY FLOW }\end{array}$ & $\frac{n 0}{1.25}$ & $\begin{array}{l}\text { SAMPLE FLOW } \\
\text { IN - NO } \\
\text { OUT - YES } \\
\end{array}$ & $A$ & \\
\hline$\frac{1.7 .4 .6 .18}{6 m}$ & $\begin{array}{l}\text { REMOVE TEST } \\
\text { GAS \& PLUG } \\
\text { SV }-* 07\end{array}$ & $N / A$ & N/A & A & \\
\hline $\begin{array}{c}1.7 .4 .6 .19 \\
\end{array}$ & $\begin{array}{l}\text { CLOSE SV-*07 } \\
\& \text { SV }-\star 10\end{array}$ & N/A & N/A & A & \\
\hline $\begin{array}{r}1.7 .4 .6 .20 \\
\end{array}$ & $\begin{array}{l}\text { OPEN SV }-* 05 \\
\& \text { SV-*16 }\end{array}$ & N/A & N/A & A & \\
\hline $\begin{array}{l}1.7 .4 .6 .21 \\
\end{array}$ & $\begin{array}{l}\text { REMOVE } \\
\text { JUMPER } \\
\end{array}$ & $N / A$ & $N / A$ & $A$ & \\
\hline \multicolumn{6}{|c|}{ Section 1.7.4.7 verifies the carrier gas supply lines. } \\
\hline 1.7 .4 .7 .1 & $\begin{array}{l}\text { CONNECT TEST } \\
\text { GAS }\end{array}$ & 3 & 3-5 PSIG & $\Delta$ & \\
\hline 1.7 .4 .7 .2 & $\begin{array}{l}\text { REMOVE PLUG } \\
\& \text { VERIFY } \\
\text { FLOW } \\
\end{array}$ & $a^{2}$ & $\begin{array}{l}N_{2} \text { CARRIER } \\
\text { GÄS FLOWS }\end{array}$ & A & \\
\hline $\begin{array}{r}1.7 .4 .7 .3 \\
\text { ogm }\end{array}$ & $\begin{array}{l}\text { DISCONNECT } \\
\text { TEST GAS }\end{array}$ & $N / A$ & N/A & $\Delta$ & \\
\hline $\begin{array}{r}1.7 .4 .7 .4 \\
\end{array}$ & $\begin{array}{l}\text { CONNECT TEST } \\
\text { GAS }\end{array}$ & 3 & 3-5 PSIG & $A$ & \\
\hline 1.7 .4 .7 .5 & $\begin{array}{l}\text { REMOVE PLUG } \\
\text { \& VERIFY } \\
\text { FLOW } \\
\end{array}$ & $0^{k}$ & $\begin{array}{l}\text { He CARRIER } \\
\text { GAS FLOWS }\end{array}$ & $A$ & \\
\hline $\begin{array}{c}1.7 .4 .7 .6 \\
6 m^{6} \\
\end{array}$ & $\begin{array}{l}\text { DISCONNECT } \\
\text { TEST GAS } \\
\end{array}$ & $N / A$ & $N / A$ & A & $-1 / \Delta^{2}$ \\
\hline $\begin{array}{r}1.7 .5 \\
6 \mathrm{~m} \\
\end{array}$ & $\begin{array}{l}\text { TEST SEC. } \\
1.7 .4 \text { DONE }\end{array}$ & \multicolumn{4}{|c|}{$\begin{array}{cc}M_{\text {ood }} N \text { Bnes } & 1-15-97 \\
\text { Test Director Signature } & \text { Date }\end{array}$} \\
\hline
\end{tabular}

su GM I.5/8.7 
TEST DATA SHEET

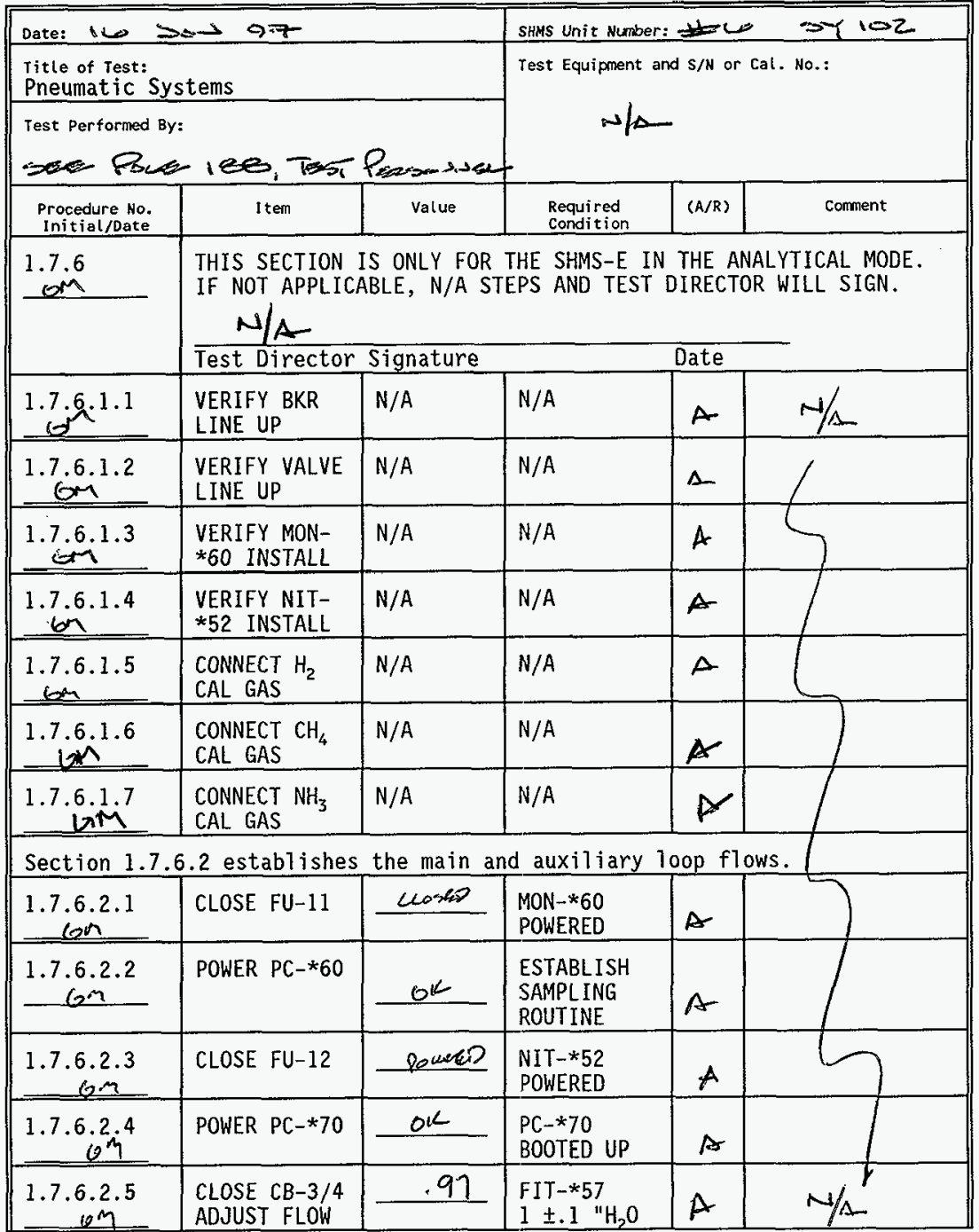


APPENDIX $F$

TEST DATA SHEET

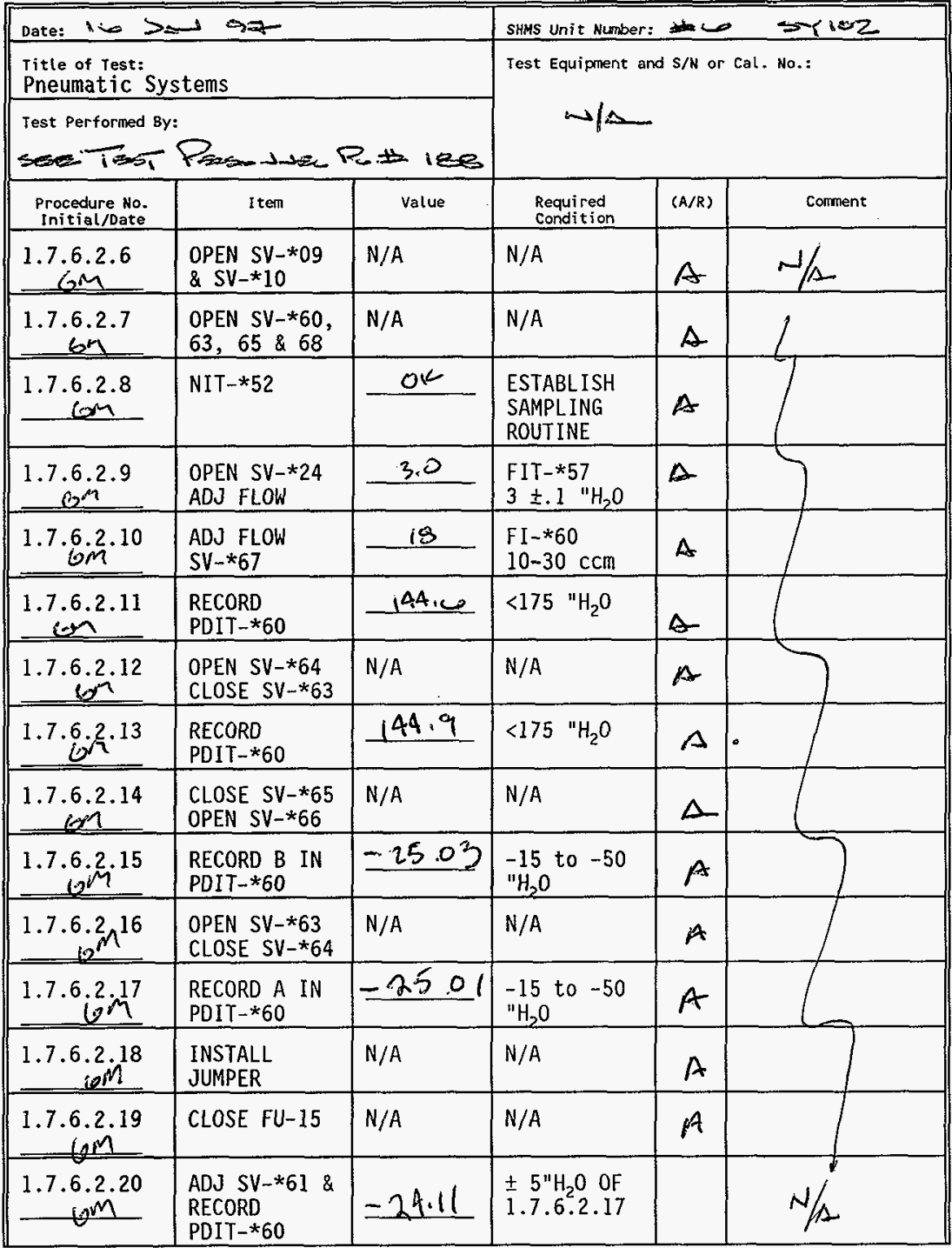


APPENDIX $F$

TEST DATA SHEET

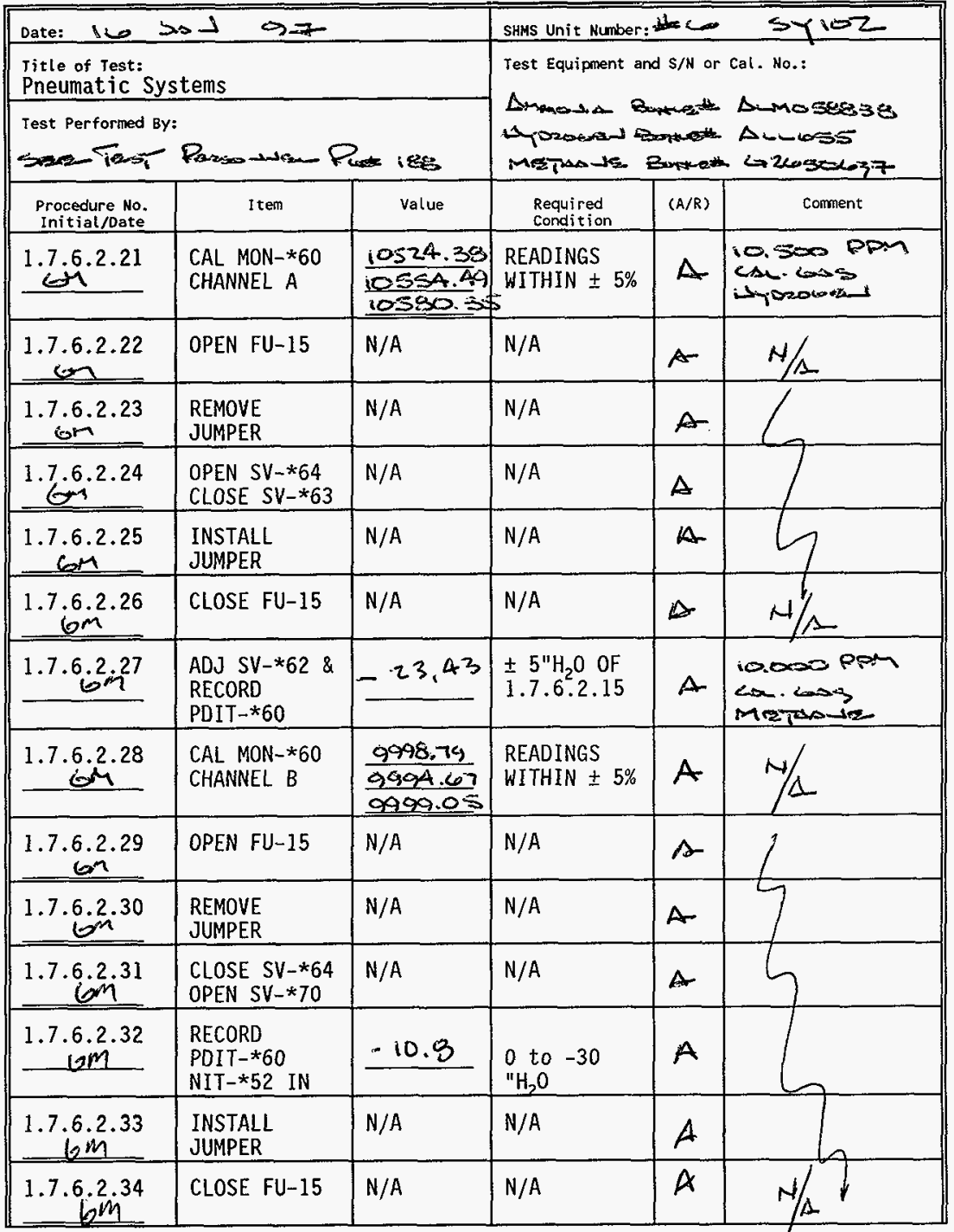

$A=1 / 16197$ 


\section{TEST DATA SHEET}

\begin{tabular}{|c|c|c|c|c|c|}
\hline \multicolumn{3}{|c|}{ Date: io $3 \Delta \rightarrow .97$} & \multicolumn{3}{|c|}{ 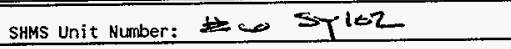 } \\
\hline \multicolumn{3}{|c|}{$\begin{array}{l}\text { Title of Test: } \\
\text { Pneumatic Systems }\end{array}$} & \multirow{2}{*}{\multicolumn{3}{|c|}{$\begin{array}{l}\text { Test Equipment and S/N or Cal. No.: } \\
\text { Dimmonis Bumosesezs }\end{array}$}} \\
\hline \multicolumn{3}{|c|}{ 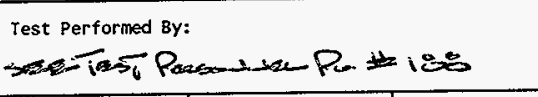 } & & & \\
\hline $\begin{array}{l}\text { Procedure No. } \\
\text { Initial/Date }\end{array}$ & 1 tem & Value & $\begin{array}{l}\text { Required } \\
\text { Condition }\end{array}$ & $(\mathrm{A} / \mathrm{R})$ & Comment \\
\hline 1.7 .6 .2 .35 & $\begin{array}{l}\text { ESTABLISH } \\
\text { BYPASS FLOW } \\
\end{array}$ & 1.4 & $\begin{array}{l}F I V-* 70 \\
1.5 \pm .2 \mathrm{cfh} \\
\end{array}$ & $\Delta$ & \\
\hline$\frac{1.7 .6 .2 .36}{67}$ & $\begin{array}{l}\text { ADJ SV-*71 } \\
\text { NIT_*52 } \\
\text { SAMPLE IN }\end{array}$ & $=14$ & $\begin{array}{l} \pm 5 \mathrm{NH}_{2} \mathrm{O} \mathrm{OF} \\
1.7 .6 .2 .32\end{array}$ & $A$ & \\
\hline $\mathrm{Gi}^{1.7 .6 .2 .37}$ & $\begin{array}{l}\text { RECORD } \\
\text { NIT-*52 } \\
\text { SAMPLES }\end{array}$ & $\begin{array}{l}37.9 \\
38.0 \\
38.3 \\
\end{array}$ & $\begin{array}{l}\text { READINGS } \\
\text { WITHIN } \pm 5 \%\end{array}$ & $A$ & \\
\hline $\begin{array}{l}1.7 .6 .2 .38 \\
\end{array}$ & OPEN FU-15 & N/A & $N / A$ & $A$ & \\
\hline $\begin{array}{c}1.7 .6 .2 .39 \\
\end{array}$ & $\begin{array}{l}\text { REMOVE } \\
\text { JUMPER }\end{array}$ & $N / A$ & $N / A$ & $A$ & \\
\hline \multicolumn{6}{|c|}{ Section 1.7 .7 provides for test system shutdown. } \\
\hline $\begin{array}{r}1.7 .7 .1 \\
\quad 60 M \\
\end{array}$ & $\begin{array}{l}\text { DISCONNECT } \\
\mathrm{H}_{2} \text { CAL GAS }\end{array}$ & $N / A$ & N/A & A & \\
\hline $\begin{array}{r}1.7 .7 .2 \\
\text { com } \\
\end{array}$ & $\begin{array}{l}\text { DISCONNECT } \\
\mathrm{CH}_{6} \text { CAL GAS } \\
\end{array}$ & $N / A$ & N/A & A & \\
\hline $\begin{array}{r}1.7 .7 .3 \\
6 \mathrm{~m} \\
\end{array}$ & $\begin{array}{l}\text { DISCONNECT } \\
\mathrm{NH}_{3} \text { CAL GAS }\end{array}$ & $N / A$ & $N / A$ & $A$ & \\
\hline $\begin{array}{r}1.7 .7 .4 \\
6 \% \\
\end{array}$ & $\begin{array}{l}\text { SECURE \& } \\
\text { TURN OFF } \\
\text { ANA. INST.S } \\
\end{array}$ & $N / A$ & $N / A$ & A & \\
\hline $\begin{array}{r}1.7 .7 .5 \\
6 \mathrm{~m} \\
\end{array}$ & $\begin{array}{l}\text { DISCONNECT } \\
\mathrm{N}_{2} \text { CARRIER } \\
\end{array}$ & $N / A$ & $N / A$ & A & \\
\hline $\begin{array}{r}1.7 .7 .6 \\
6 \mathrm{~m} \\
\end{array}$ & $\begin{array}{l}\text { DISCONNECT } \\
\text { He CARRIER }\end{array}$ & $N / A$ & $N / A$ & $\Delta$ & \\
\hline $\begin{array}{c}1.7 .7 .7 \\
6 \dot{n}^{n}\end{array}$ & $\begin{array}{l}\text { OPEN ALL } \\
\text { BREAKERS \& } \\
\text { FUSES }\end{array}$ & N/A & $N / A$ & $p^{\infty}$ & \\
\hline $\begin{array}{c}1.7 .7 .8 \\
6 m^{8} \\
\end{array}$ & $\begin{array}{l}\text { CLOSE LISTED } \\
\text { SYS. VALVES } \\
\end{array}$ & N/A & $N / A$ & $A$ & $A$ \\
\hline
\end{tabular}


APPENDIX $F$

PAGE F- 28
HNF-SD-WH-ATR-191

Rev. 0
Page 214

TEST DATA SHEET

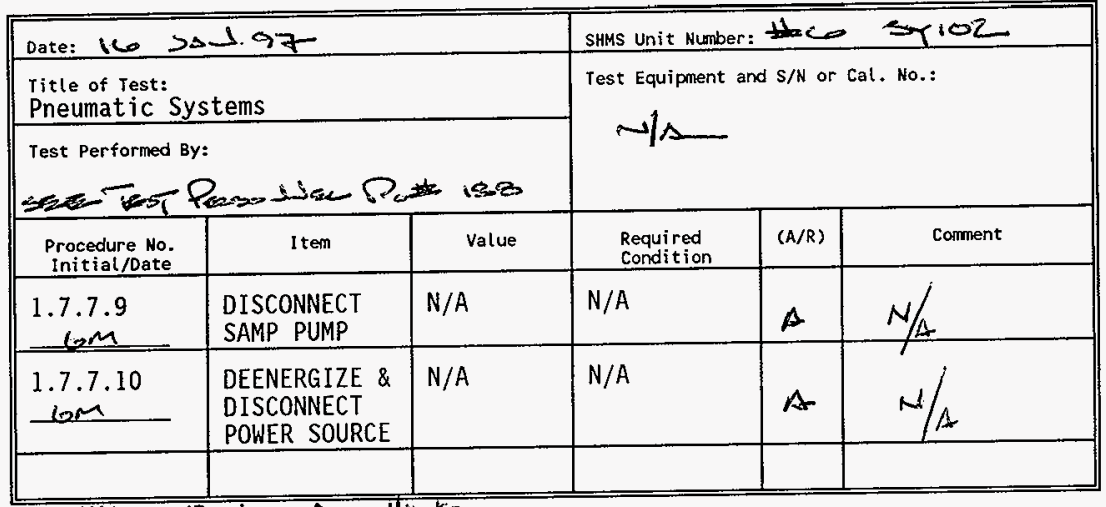

Test Witness/Review: sc The if in

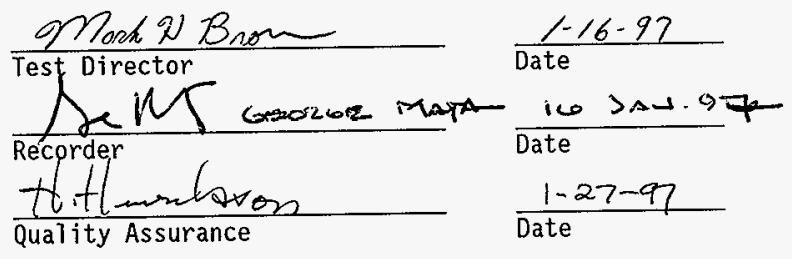


TEST EXCEPTION SHEET

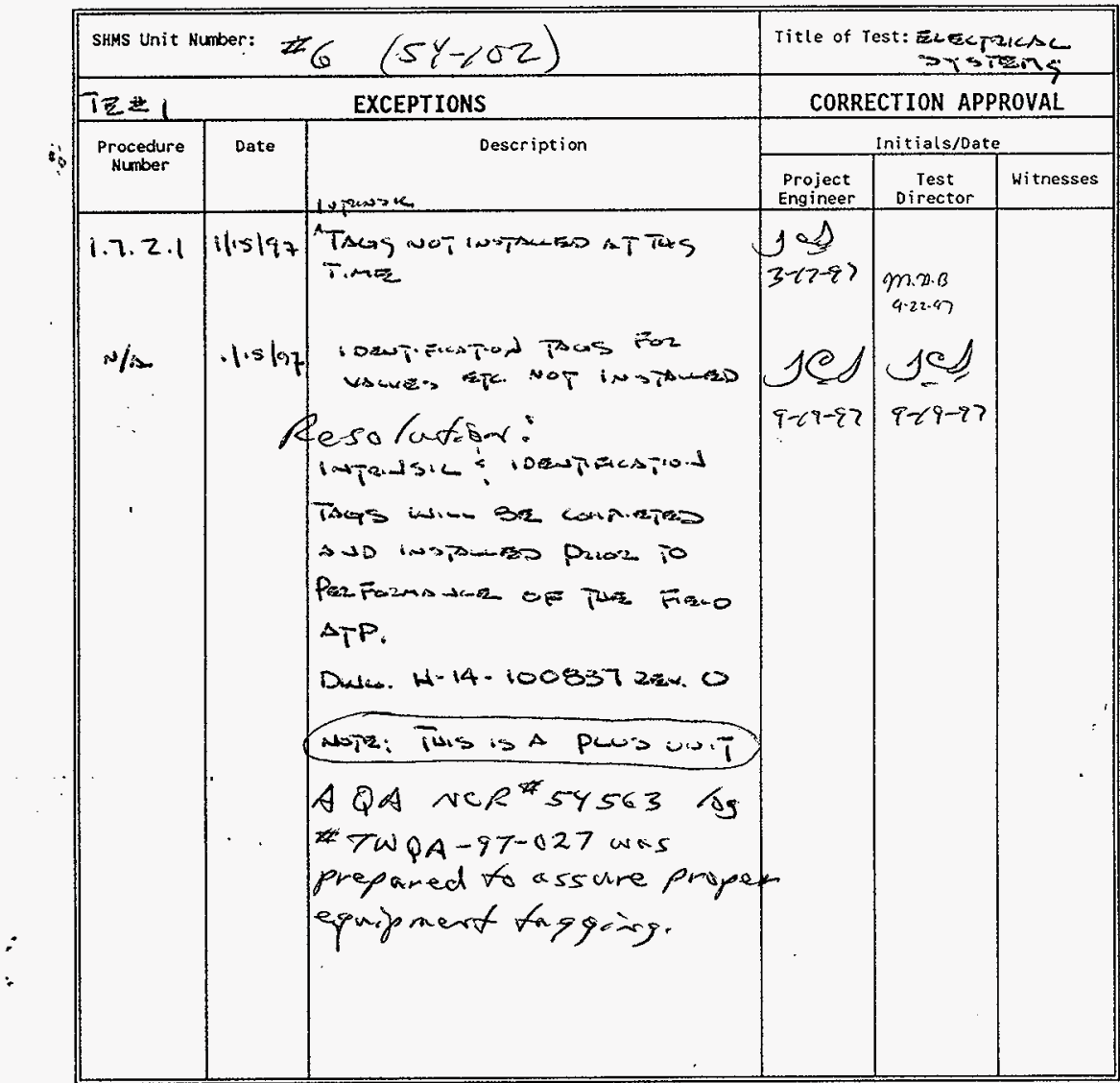

\section{TEST APPBOVVD WITH EXCEPTIONS}

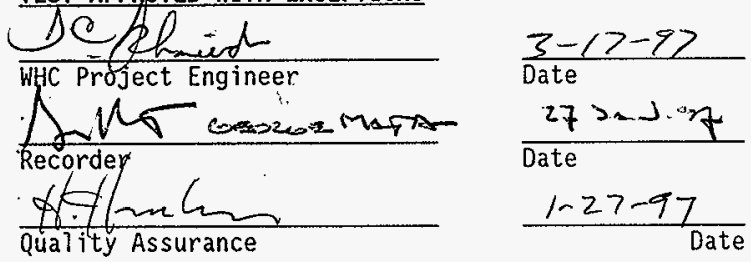


APPENDIX $F$

PAGE F-30
INF - SD - WM-ATR - 191

Rev. 0
Page 216

TEST LOG

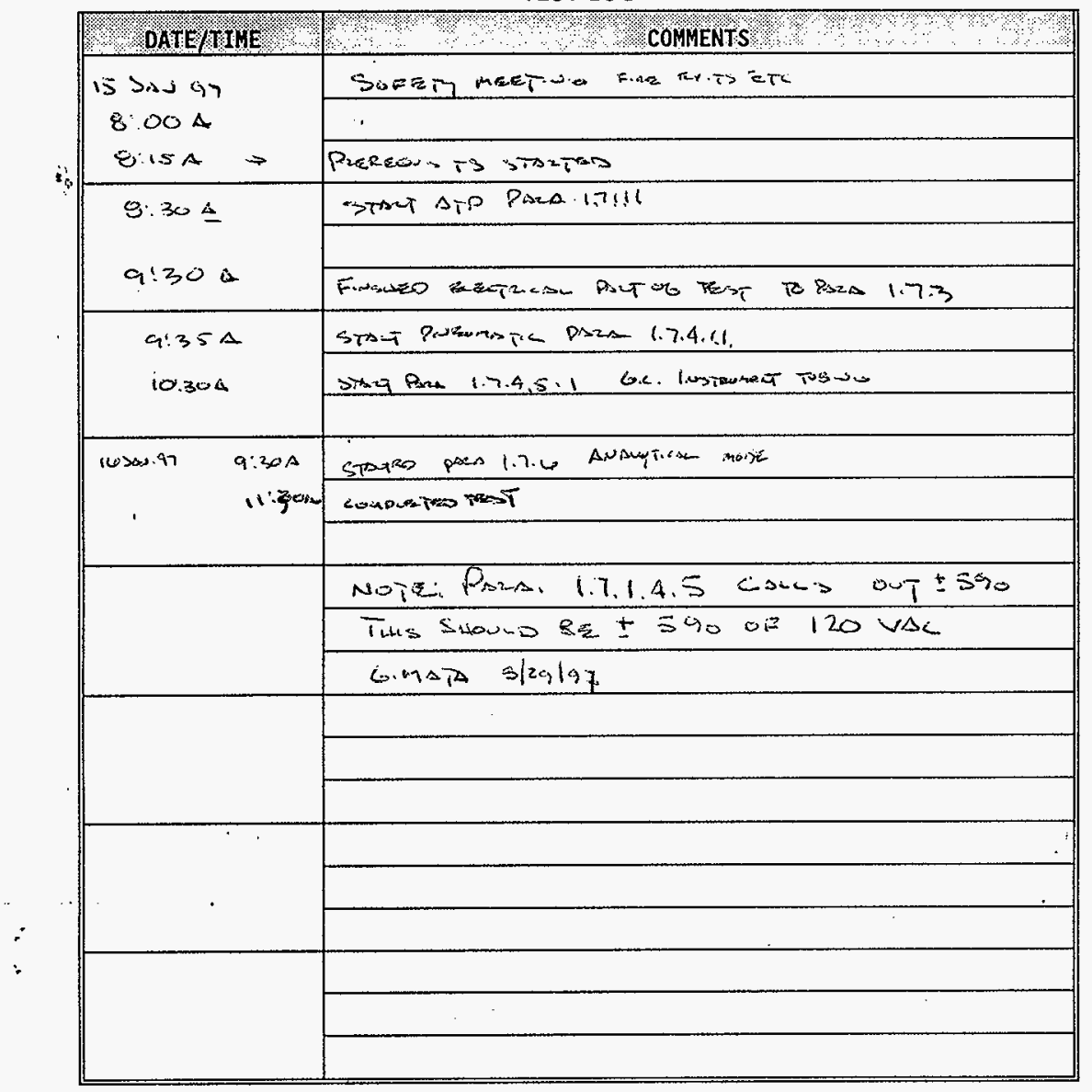


APPENDIX $G$

PAGE G-I
HNF - SD - WM-ATR - 191

Rev. 0

Page 217

APPENDIX G 
TEST EXECUTION SHEET

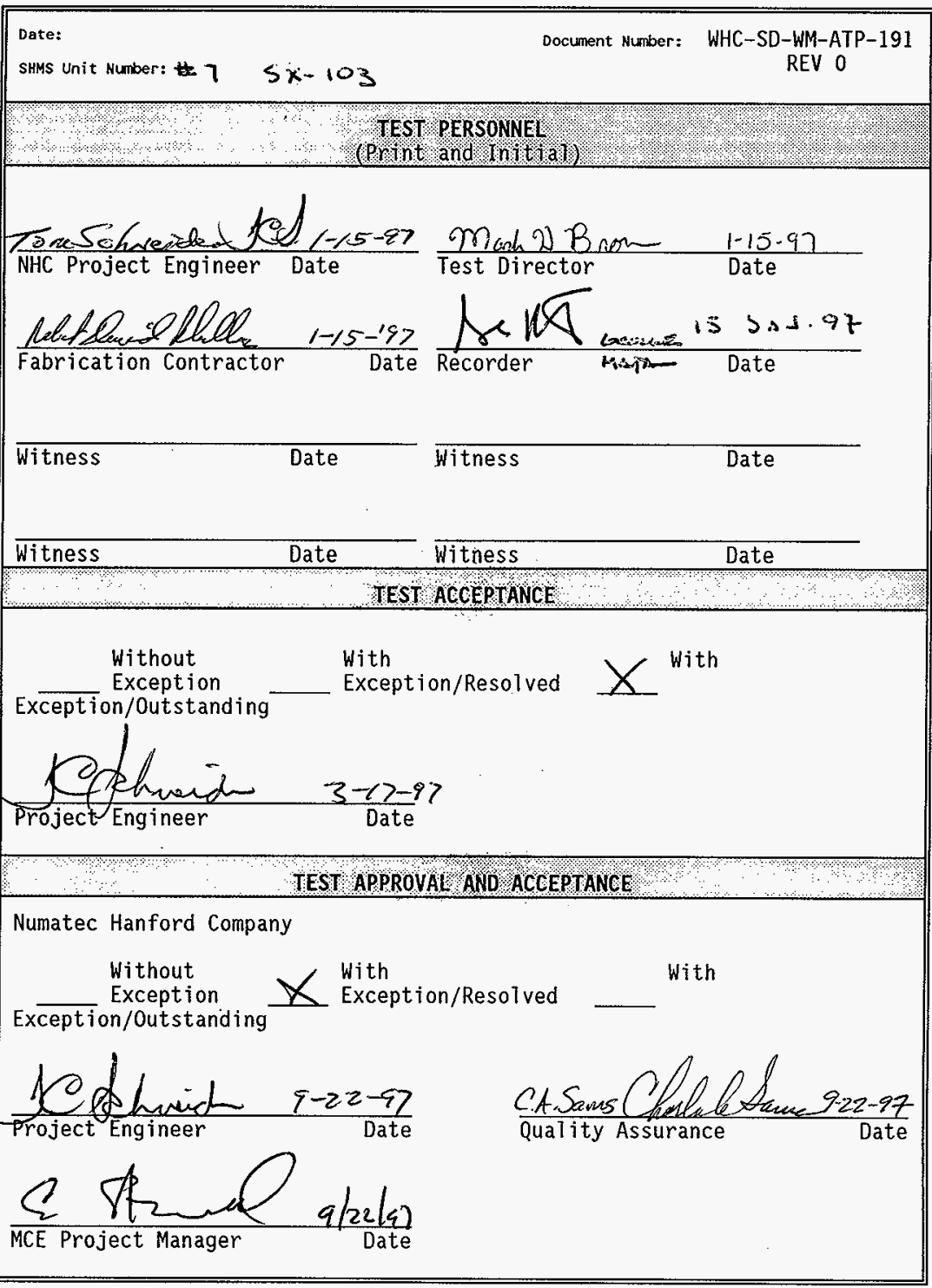


SHMS Unit Number:

\section{PREREQUISITES AND INITIAL TEST CONDITIONS}

The following conditions shall exist at the start of the acceptance testing. Initial and date to verify that each of the following items have been accomplished.

GM Mskit Systems being tested have been inspected for workmanship and for compliance with design.

Gn.t.stiz Continuity and megger tests have been performed on portions of the electrical and instrument systems being tested, as required.

on $12417 t_{\text {Leak }}$ tests on the pneumatic systems have been performed.

CMilis is The following circuit breakers and fuses are installed per the specified size and are open

$$
\begin{array}{lll}
\nearrow & \text { CB-1 through } \mathrm{CB}-7 \\
\nearrow & \text { FU-1 through FU-15 }
\end{array}
$$

I.s liq All test instruments have a currently valid calibration stamp attached that indicates a calibration traceable to the National Institute of Standards and Technology.

$G$ lis/97 The following process and control instrument systems have been initially configured and aligned for proper operation.

FIT-*57, FSL $-* 57$, NIT-*54, NIT-*55, (NR-*54), PDIT-*60, TIC-*50, TIC- $* 56$, TIS $-* 62$, YYC $-* 01$ NOTE: NR $-* 54$ is not used in the ANALYTICAL configuration.

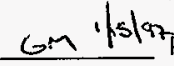

Personnel responsible for directing and witnessing the performance of the tests described in this ATP have read and understand appropriate certified vendor information (CVI) pertaining to the operation of the equipment to be tested.

$6 m \cdot 1 s / i z$ CLOSE all system manual valves with the exception of the following:

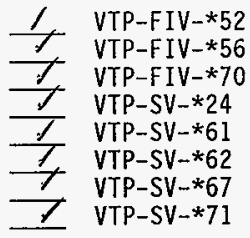

The sample pump VTP-P-*50, connected to the cabinet sample out port, but vented to atmosphere for testing. 
GM $1 / 51^{7} 208 \mathrm{Vac} 1$ phase 15-20 ampere temporary power source has been connected, but not energized, to the appropriate TBI terminals per H-14-100838.

Verify by signature and date that all prerequisites have been met.

\begin{tabular}{cc} 
Man $\%$ Bnon & $1-15-96$ \\
\hline Test Director Signature & Date \\
gmand $x$ Bnown & $1.27-96$
\end{tabular}


TEST DATA SHEET

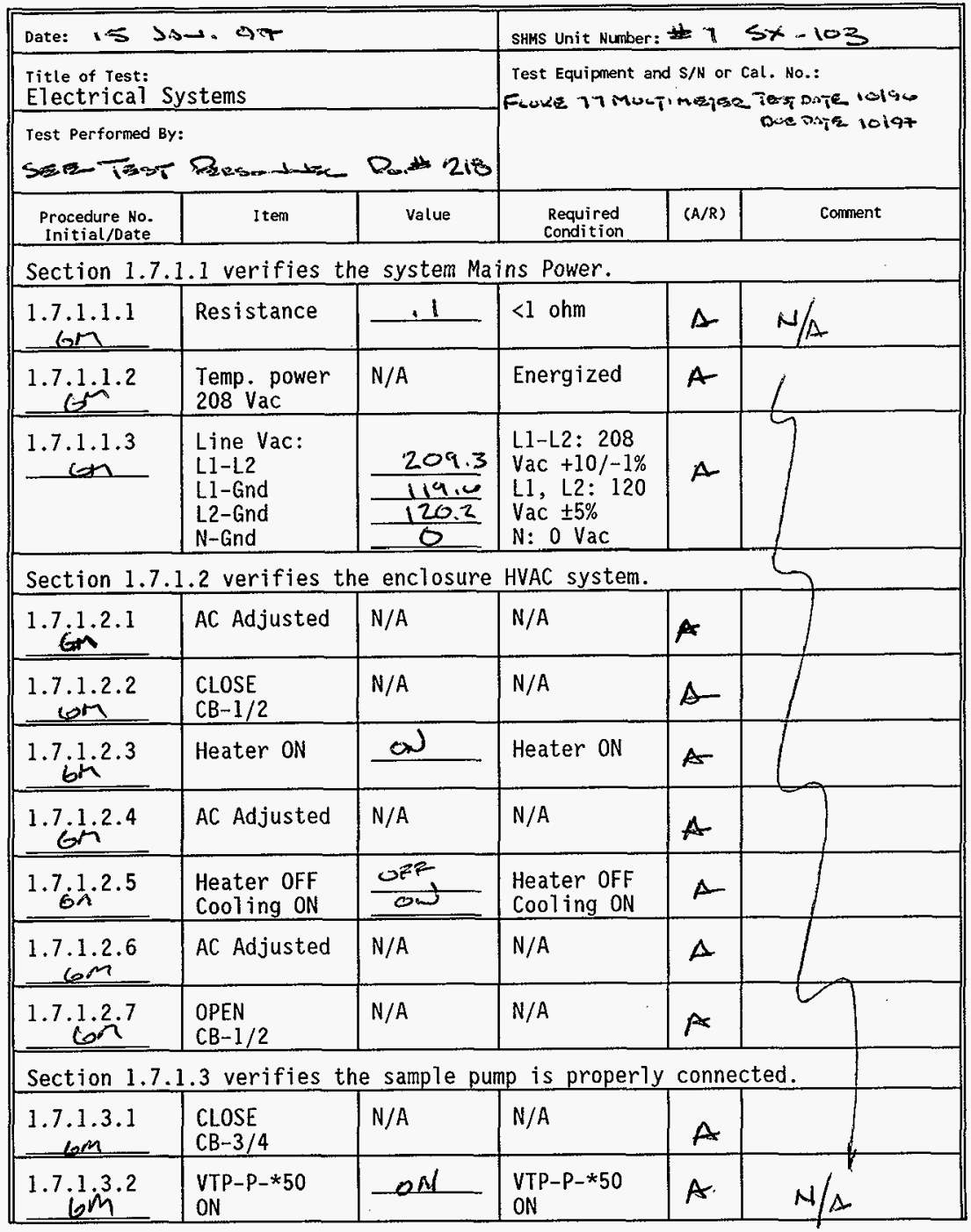


TEST DATA SHEET

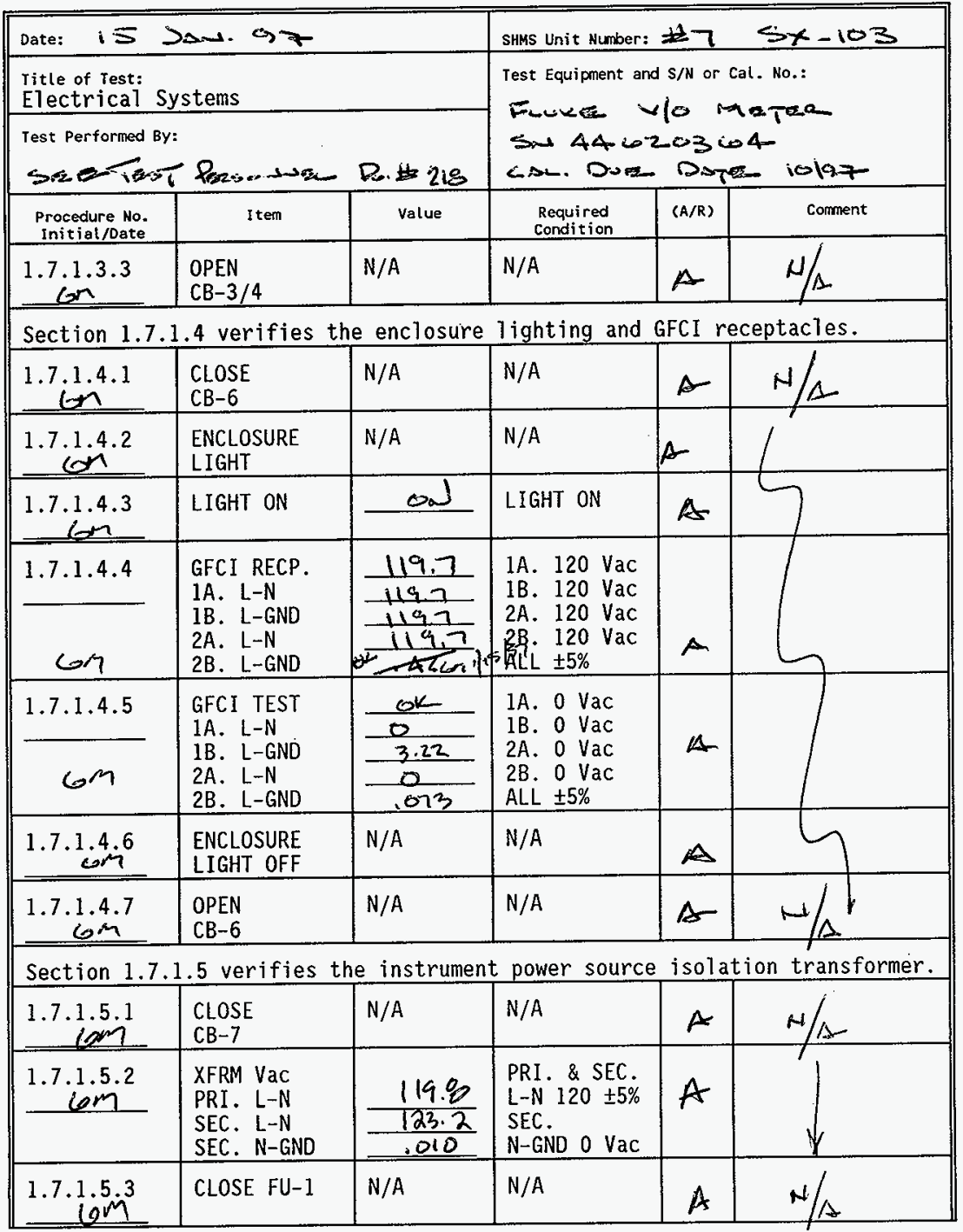


TEST DATA SHEET

\begin{tabular}{|c|c|c|c|c|c|}
\hline \multicolumn{3}{|c|}{ Date: is SA. 97} & \multicolumn{3}{|c|}{ SHMS Unit Number: $7 \quad 3 x-103$} \\
\hline \multicolumn{3}{|c|}{$\begin{array}{l}\text { Title of rest: } \\
\text { Electrical Systems } \\
\end{array}$} & \multirow{2}{*}{\multicolumn{3}{|c|}{ 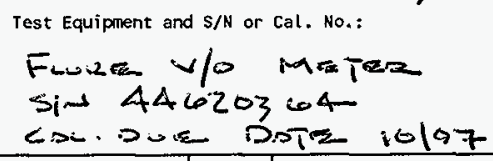 }} \\
\hline \multicolumn{3}{|c|}{ 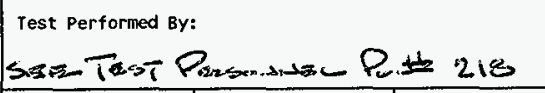 } & & & \\
\hline $\begin{array}{l}\text { Procedure No. } \\
\text { Initial/Date }\end{array}$ & Item & Value & $\begin{array}{l}\text { Required } \\
\text { Condition }\end{array}$ & $(A / R)$ & Comment \\
\hline$\frac{1.7 .1 .5 .4}{\operatorname{con}^{-}}$ & $\begin{array}{l}\text { VTP-PS-*50 } \\
\text { OUTPUT Vdc }\end{array}$ & 23.99 & $24 \pm 0.1 \mathrm{Vdc}$ & $\Delta$ & \\
\hline \multicolumn{6}{|c|}{ Section 1.7.1.6 verifies the enclosure general al arm system. } \\
\hline $\begin{array}{l}1.7 .1 .6 .1 \\
6 \mathrm{~m} \\
\end{array}$ & $\begin{array}{l}\text { CLOSE FU-9 \& } \\
\text { FU-10 }\end{array}$ & $N / A$ & $N / A$ & Ar & \\
\hline $\begin{array}{c}1.7 .1 .6 .2 \\
67\end{array}$ & $\begin{array}{l}\text { PUSH PB }-* 51 \\
\& \text { PB- } * 50\end{array}$ & N/A & N/A & $A$ & \\
\hline $\begin{array}{l}1.7 .1 .6 .3 \\
\text { con }\end{array}$ & $\begin{array}{l}\text { ALARM TEST } \\
\text { VERIFY ALARM } \\
\text { CONDITIONS }\end{array}$ & 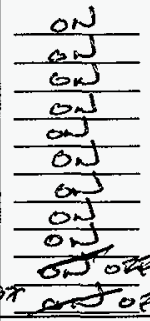 & $\begin{array}{lll}\text { YAH-*550 } & \text { ON } \\
\text { NAH- } * 55 & \text { ON } \\
\text { XA-*63 } & \text { ON } \\
\text { NAH-*544 } & \text { ON } \\
\text { FAL-*57 } & \text { ON } \\
\text { TAHL-*62 } & \text { ON } \\
\text { TAL-*50 } & \text { ON } \\
\text { YAL-*58 } & \text { ON } \\
\text { PBL-*58 } & \text { ON } \\
\text { PBL-*54 } & \text { OFF } \\
P B L-* 59 & \text { OFF } \\
\end{array}$ & $\triangle$ & \\
\hline 1.7 .1 .6 .4 & $\begin{array}{l}\text { END ALARM } \\
\text { TEST }\end{array}$ & N/A & $N / A$ & A & \\
\hline 1.7 .1 .6 .5 & RESET ALARMS & $N / A$ & $N / A$ & $A$ & \\
\hline $\begin{array}{l}1.7 .1 .6 .6 \\
\mathrm{lom} \\
\end{array}$ & $\begin{array}{l}\text { OPEN FU-9 \& } \\
\text { FU-10 }\end{array}$ & N/A & N/A & 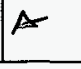 & \\
\hline \multicolumn{6}{|c|}{ Section 1.7.1.7 verifies the flow alarm system. } \\
\hline$\frac{1.7 .1 .7 .1}{6 m^{1}}$ & $\begin{array}{l}\text { CLOSE FU-3, } \\
\text { FU-9, FU-10 } \\
\& \text { FU-13 } \\
\end{array}$ & N/A & N/A & A & \\
\hline $\begin{array}{l}1.7 .7 m^{2} \\
\end{array}$ & RESET ALARMS & $O N$ & FAL-*57 ON & A & \\
\hline 1.7 .1 .7 .3 & $\begin{array}{l}\text { OPEN SV-*20 } \\
\& \text { SV-*22 }\end{array}$ & N/A & N/A & A & $N / A$ \\
\hline
\end{tabular}


APPENDIX G

HNF-SD-WM-ATR-191

PAGE G- 8

TEST DATA SHEET

\begin{tabular}{|c|c|c|c|c|c|}
\hline \multicolumn{3}{|c|}{ Date: is Som. 97} & \multicolumn{3}{|c|}{ SHMS Unit Number: $\pm 7 \leq x-103$} \\
\hline \multicolumn{3}{|c|}{$\begin{array}{l}\text { Title of rest: } \\
\text { Electrical Systems }\end{array}$} & \multirow{2}{*}{\multicolumn{3}{|c|}{ 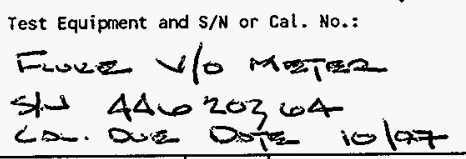 }} \\
\hline \multicolumn{3}{|c|}{ 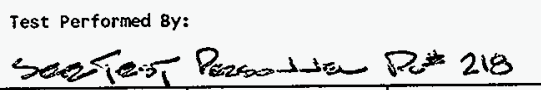 } & & & \\
\hline $\begin{array}{l}\text { Procedure No. } \\
\text { Initial/Date } \\
\end{array}$ & I tem & value & $\begin{array}{c}\text { Required } \\
\text { Condition }\end{array}$ & $\langle A / R\rangle$ & Comment \\
\hline $\begin{array}{c}1.7 .1 .7 .4 \\
\operatorname{cor}\end{array}$ & $\begin{array}{l}\text { FIT-*57 \& } \\
\text { FSL-*57 } \\
\text { POWERED }\end{array}$ & $\frac{Q N}{O N}$ & $\begin{array}{l}\text { FIT-*57 ON } \\
\text { FSL-*57 ON }\end{array}$ & $A$ & \\
\hline $\mathrm{Gm}^{1.7 .5}$ & $\begin{array}{l}\text { MUX-*70 } \\
\text { TB5+/TB6- }\end{array}$ & .998 & $1.0 \pm 0.2 \mathrm{Vdc}$ & $A$ & \\
\hline $\begin{array}{c}1.7 .1 .7 .6 \\
\mathrm{G} \\
\end{array}$ & $\begin{array}{l}\text { CLOSE SV } * 20 \\
\& S V-* 22 \\
\end{array}$ & $N / A$ & $N / A$ & $\leadsto$ & \\
\hline $\begin{array}{l}1.7 .1 .7 .7 \\
-607 \\
\end{array}$ & $\begin{array}{l}\text { OPEN FU-3, } \\
\text { FU-9, FU-10 } \\
\& \text { FU-13 }\end{array}$ & $N / A$ & $N / A$ & $\Delta$ & \\
\hline \multicolumn{6}{|c|}{ Section 1.7 .1 .8 verifies the $\mathrm{H}_{2}$ monitor wiring. } \\
\hline $\begin{array}{c}1.7 .1 .8 .1 \\
6 m\end{array}$ & $\begin{array}{l}\text { CLOSE FU-2, } \\
\text { FU-4, FU-5, } \\
\text { FU-9 \& FU-10 }\end{array}$ & $N / A$ & $N / A$ & 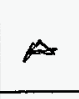 & \\
\hline $1 . \dot{7} \dot{i n j}^{1.2}$ & RESET ALARMS & $\frac{O E F}{D E F}$ & $\begin{array}{ll}\text { NAH- } * 54 & \text { OFF } \\
\text { NAH- } * 55 & \text { OFF } \\
\end{array}$ & $\Delta$ & \\
\hline $\begin{array}{c}1.7 .1 .8 .3 \\
162 \\
\end{array}$ & $\begin{array}{l}\text { INSTRUMENTS } \\
\text { POWERED }\end{array}$ & $\frac{\frac{O N}{O N}}{\frac{\text { ON }}{\text { ON }}}$ & $\begin{array}{ll}M U X-* 70 & \text { ON } \\
\text { NIT-*54 ON } \\
\text { NIT-*55 ON } \\
\text { NR-*54 ON } \\
\text { YYC-*01 ON }\end{array}$ & $A$ & $\cos s e n$ \\
\hline $\begin{array}{c}1.7 .1 .8 .4 \\
-6.7\end{array}$ & $\begin{array}{l}\text { OPEN FU-2, } \\
\text { FU-4, FU-5, } \\
\text { FU-9 \& FU-10 }\end{array}$ & $N / A$ & $N / A$ & $\Delta$ & \\
\hline \multicolumn{6}{|c|}{ Section 1.7.1.9 verifies the SHMS-E heat trace control system. } \\
\hline $\begin{array}{c}1.7 .1 .9 .1 \\
6 \mathrm{~m}^{\mathrm{m}} \\
\end{array}$ & $\begin{array}{l}\text { INSTALL LOAD } \\
\text { VTP-TIC }-* 50\end{array}$ & $N / A$ & $N / A$ & $\Delta$ & \\
\hline $\begin{array}{c}1.7 .1 .9 .2 \\
6.9 \\
\end{array}$ & $\begin{array}{l}\text { CLOSE CB-5, } \\
\text { FU-6, FU-7, } \\
\text { FU-9 \& FU-10 } \\
\text { and RESET } \\
\text { ALARMS }\end{array}$ & $N / A$ & $N / A$ & $A$ & \\
\hline
\end{tabular}


APPENDIX $G$

TEST DATA SHEET

\begin{tabular}{|c|c|c|c|c|c|}
\hline Date: 15 & 97 & & SHMS Unit Number: & +67 & $5 x-103$ \\
\hline $\begin{array}{l}\text { Title of rest: } \\
\text { Electrical }\end{array}$ & stems & & Test Equipnent anc & $S / N$ or & \\
\hline Test Performed B & $P_{\text {tessondsin }}$ & $R_{0}=218$ & $\rightarrow / \Delta$ & & \\
\hline $\begin{array}{l}\text { Procedure No. } \\
\text { Initial/Date }\end{array}$ & Item & Value & $\begin{array}{l}\text { Required } \\
\text { Condition }\end{array}$ & $(A / R)$ & Comment \\
\hline$\frac{1.7 .1 .9 .3}{6.7}$ & $\begin{array}{l}\text { TIC }-* 50 \quad \& \\
\text { TIC }-* 56 \\
\text { NOMINAL TEMP }\end{array}$ & ok & $\begin{array}{l}\text { TIC }-* 50 \\
\text { TIC }-\star 56 \\
\text { TEMPERATURE }\end{array}$ & A & \\
\hline$\frac{1.7 .1 .9 .4}{64}$ & $\begin{array}{l}\text { ADJ TIC }-* 50 \\
\& \text { TIC-*56 } \\
\text { SP2 \& RESET } \\
\text { ALARMS }\end{array}$ & $N / A$ & $N / A$ & $\Delta$ & \\
\hline$\frac{1.7 .1 .9 .5}{6 \mathrm{om}^{2}}$ & $\begin{array}{l}\mathrm{ADJ}_{1} T I C-* 50 \\
S P_{1}\end{array}$ & $N / A$ & $\mathrm{~N} / \mathrm{A}$ & $A$ & \\
\hline $\begin{array}{c}1.7 .1 .9 .6 \\
\mathrm{w}^{4} \\
\end{array}$ & $\begin{array}{l}\text { ADJ TIC }-* 50 \\
\text { SP } 2\end{array}$ & $N / A$ & $N / A$ & $A$ & \\
\hline $\begin{array}{c}1.7 .1 .9 .7 \\
67\end{array}$ & $\begin{array}{l}\text { TAL-*50\& } \\
\text { HORN ACTIVE }\end{array}$ & $\frac{a N}{\frac{a}{a x}}$ & $\begin{array}{l}\text { TAL } * 50 \text { ON } \\
\text { HORN ON } \\
\text { ACKNOWLEDGE }\end{array}$ & $A$ & \\
\hline $\begin{array}{l}1.7 .19^{9.8} \\
\end{array}$ & $\begin{array}{l}\text { TIC-*50 } \\
\text { OPERATES }\end{array}$ & or & $\begin{array}{l}\text { TIC }-* 50 \\
\text { OPERATES }\end{array}$ & $A$ & \\
\hline $\begin{array}{l}1.7 .9 .9 \\
\end{array}$ & $\begin{array}{l}\text { ADJ TIC }-* 50 \\
S P 2\end{array}$ & $N / A$ & N/A & $A$ & \\
\hline $\begin{array}{l}1.7 .1 .9 .10 \\
0^{3}\end{array}$ & RESET ALARM & OFF & TAL $-\star 50$ OFF & A & \\
\hline $\begin{array}{c}1.7 .1 .9 .11 \\
6 \mathrm{~m} \\
\end{array}$ & $\begin{array}{l}\text { ADJ TIC }-* 50 \\
S P 1 \& S P 2 \\
\end{array}$ & $N / A$ & $N / A$ & $\Delta$ & \\
\hline $6^{1.7} m^{9.12}$ & $\begin{array}{l}\text { OPEN CB-5 \& } \\
\text { REMOVE LOAD }\end{array}$ & $N / A$ & $N / A$ & A & \\
\hline $\begin{array}{c}1.7 .9 .13 \\
\end{array}$ & $\begin{array}{l}\text { INSTALL LOAD } \\
\text { VTP-TIC-*56 }\end{array}$ & $N / A$ & $N / A$ & $A$ & \\
\hline $\begin{array}{r}1.7 .1 .9 .14 \\
6 m^{14} \\
\end{array}$ & CLOSE CB-5 & $N / A$ & N/A & A & \\
\hline $\begin{array}{r}1.7 .1 .9 .15 \\
6 n^{5} \\
\end{array}$ & $\begin{array}{l}\text { ADJ TIC-*56 } \\
\text { SP } 1\end{array}$ & $N / A$ & $N / A$ & $A$ & \\
\hline $\begin{array}{r}1.7 .1 .9 .16 \\
6 \mathrm{~m} \\
\end{array}$ & $\begin{array}{l}\text { ADJ TIC-*56 } \\
\text { SP } 2\end{array}$ & $N / A$ & $\mathrm{~N} / \mathrm{A}$ & A & $N / A$ \\
\hline
\end{tabular}


TEST DATA SHEET

\begin{tabular}{|c|c|c|c|c|c|}
\hline \multirow{2}{*}{\multicolumn{3}{|c|}{$\begin{array}{l}\text { Date: } 15 \text { Dom. 9 } \\
\text { Title of Test: } \\
\text { Electrical Systems }\end{array}$}} & \multirow{3}{*}{\multicolumn{3}{|c|}{$\begin{array}{l}\text { SHMS Unit Number: } 7 \quad \leq x \cdot 102 \\
\text { Test Equipment and S/N or cal. No.: }\end{array}$}} \\
\hline & & & & & \\
\hline \multicolumn{3}{|c|}{ 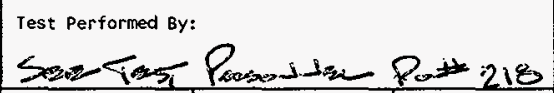 } & & & \\
\hline $\begin{array}{l}\text { Procedure No. } \\
\text { Initial/Date }\end{array}$ & I tem & Value & $\begin{array}{l}\text { Required } \\
\text { Condition }\end{array}$ & $(A / R)$ & Comment \\
\hline$\frac{1.7 .1 .9 .17}{6 g^{m}}$ & $\begin{array}{l}\text { TAL-*50 \& } \\
\text { HORN ACTIVE }\end{array}$ & $\frac{0, j}{0 k}$ & $\begin{array}{l}\text { TAL-*50 ON } \\
\text { HORN ON } \\
\text { ACKNOWLEDGE }\end{array}$ & $A$ & \\
\hline $\begin{array}{l}1.7 .1 .9 .18 \\
6 \mathrm{~cm} \\
\end{array}$ & $\begin{array}{l}\text { TIC }-* 56 \\
\text { OPERATES } \\
\end{array}$ & OK & $\begin{array}{l}\text { TIC }-* 56 \\
\text { OPERATES }\end{array}$ & A & \\
\hline $\begin{array}{l}1.7 .1 .9 .19 \\
6 n \\
\end{array}$ & $\begin{array}{l}\text { ADJ IIC-*56 } \\
\text { SP } 2\end{array}$ & N/A & $N / A$ & $\Delta$ & \\
\hline$\frac{1.7 .1 .9 .20}{6 m}$ & RESET ALARM & OFF & TAL $-\star 50$ OFF & $\triangle$ & \\
\hline $\begin{array}{c}1.7 .1 .9 .21 \\
6.1 \\
\end{array}$ & $\begin{array}{l}\text { ADJ TIC-*56 } \\
\text { SP 1 \& SP 2 }\end{array}$ & N/A & $N / A$ & A & \\
\hline $\begin{array}{r}1.7 .1 .9 .22 \\
6.4 \\
\end{array}$ & $\begin{array}{l}\text { OPEN CB-5, } \\
\text { FU-6 \& FU-7 } \\
\end{array}$ & $N / A$ & N/A & $\Delta$ & \\
\hline $\begin{array}{r}1.7 .1 .9 .23 \\
.01^{2} \\
\end{array}$ & $\begin{array}{l}\text { REMOVE LOAD } \\
\text { TIC }-* 56\end{array}$ & $N / A$ & $N / A$ & $A$ & \\
\hline$\frac{1.7 .1 .9 .24}{6 m^{24}}$ & $\begin{array}{l}\text { CLOSE FU-8 } \\
\text { TIS }-* 62 \\
\text { NOMINAL TEMP }\end{array}$ & ok & $\begin{array}{l}\text { TIS-*62 } \\
\text { TEMP }\end{array}$ & $\curvearrowright$ & \\
\hline $\begin{array}{l}1.7 .1 .9 .25 \\
6 \mathrm{~m}^{2} \\
\end{array}$ & $\begin{array}{l}\text { ADJ TIS-*62 } \\
\text { SP } 1\end{array}$ & $N / A$ & N/A & $A$ & \\
\hline $\begin{array}{c}1.7 .1 .9 .26 \\
6.1 \\
\end{array}$ & $\begin{array}{l}\text { ADJ TIS-*62 } \\
\text { SP } 2\end{array}$ & N/A & N/A & $A$ & \\
\hline $\begin{array}{r}1.7 .1 .9 .27 \\
\mathrm{nim}\end{array}$ & RESET ALARMS & off & $\begin{array}{l}\text { TAHL-*62 } \\
\text { OFF } \\
\end{array}$ & A & \\
\hline $\begin{array}{c}1.7 .1 .9 .28 \\
\mathrm{Gm} \\
\end{array}$ & $\begin{array}{l}\text { ADJ TIS-*62 } \\
\text { SP } 2\end{array}$ & $N / A$ & $N / A$ & A & \\
\hline$\frac{1.7 .1 .9 .29}{\operatorname{com}}$ & $\begin{array}{l}\text { TAHL-*62 \& } \\
\text { HORN ACTIVE }\end{array}$ & $\begin{array}{l}\text { ON } \\
\text { ON } \\
\text { OL } \\
\end{array}$ & $\begin{array}{l}\text { TAHL-*62 ON } \\
\text { HORN ON } \\
\text { ACKNOWLEDGE } \\
\end{array}$ & $A$ & \\
\hline $\begin{array}{r}1.7 .1 .9 .30 \\
\mathrm{am} \\
\end{array}$ & $\begin{array}{l}\text { ADJ TIS-*62 } \\
\text { SP } 2\end{array}$ & N/A & $N / A$ & $A$ & $N / \Delta$ \\
\hline
\end{tabular}


TEST DATA SHEET

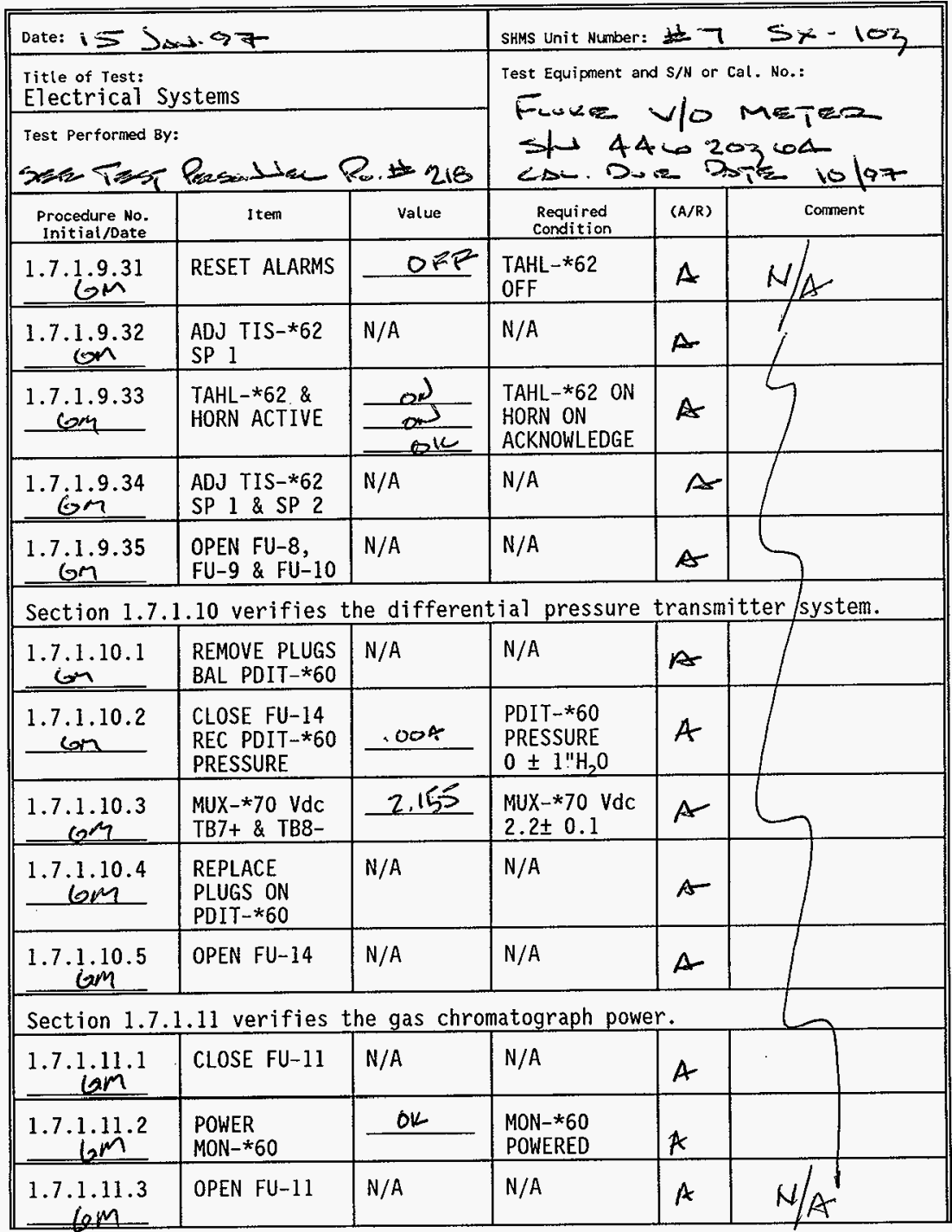


APPENDIX G

PAGE G-/Z

TEST DATA SHEET

\begin{tabular}{|c|c|c|c|c|c|}
\hline \multicolumn{3}{|c|}{ Date: 15 D. $\rightarrow .97$} & \multicolumn{3}{|c|}{ SHMS Unit Number: $75 x-103$} \\
\hline \multicolumn{3}{|c|}{$\begin{array}{l}\text { Title of Test: } \\
\text { Electrical Systems }\end{array}$} & \multirow{2}{*}{\multicolumn{3}{|c|}{ 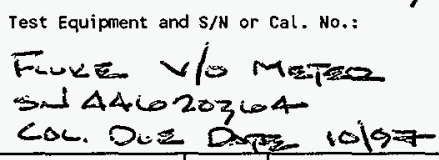 }} \\
\hline \multicolumn{3}{|c|}{ 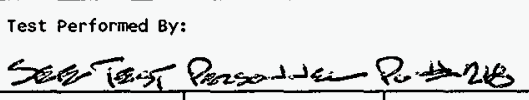 } & & & \\
\hline $\begin{array}{l}\text { Procedure No. } \\
\text { Initial/Date }\end{array}$ & I tem & Value & $\begin{array}{l}\text { Required } \\
\text { Condition }\end{array}$ & $(A / R)$ & Comment \\
\hline \multicolumn{6}{|c|}{ Section 1.7.1.12 verifies the multi gas analyzer power. } \\
\hline $\begin{array}{c}1.7 .1 .12 .1 \\
6 m \\
\end{array}$ & CLOSE FU-12 & $N / A$ & $N / A$ & $\Delta$ & \\
\hline 1.7 .1 .12 .2 & $\begin{array}{l}\text { POWER } \\
\text { NIT }-* 52\end{array}$ & ok & $\begin{array}{l}\text { NIT-*52 } \\
\text { POWERED } \\
\end{array}$ & 1 & \\
\hline $\begin{array}{c}1.7 .1 .12 .3 \\
\mathrm{~cm} \\
\end{array}$ & OPEN FU-12 & N/A & N/A & 䧄 & \\
\hline \multicolumn{6}{|c|}{ Section 1.7.1.13 verifies the personnel computer power. } \\
\hline $\begin{array}{c}1.7 .1 .13 .1 \\
\mathrm{ign}\end{array}$ & CLOSE CB-6 & $N / A$ & $N / A$ & $\Delta$ & \\
\hline $\begin{array}{c}1.7 .1 .13 .2 \\
\end{array}$ & $\begin{array}{l}\text { POWER PC-*60 } \\
\& P C-\star 70\end{array}$ & on & $\begin{array}{ll}P C-* 60 & O N \\
P C-* 70 & O N \\
\end{array}$ & $\Delta$ & \\
\hline $\begin{array}{c}1.7 .1 .13 .3 \\
.6 m \\
\end{array}$ & $\begin{array}{l}\text { TURN INST. } \\
\text { OFF \& } \\
\text { OPEN CB-6 }\end{array}$ & $\frac{O F P}{O P Q}$ & $\begin{array}{ll}P C-* 60 & O F F \\
P C-* 70 & O F F\end{array}$ & $\Delta$ & \\
\hline
\end{tabular}

sic on ifsliz 
TEST DATA SHEET

\begin{tabular}{|c|c|c|c|c|c|}
\hline \multicolumn{3}{|c|}{ Date: $15 \operatorname{Ss} 1.97$} & \multicolumn{3}{|c|}{ SHMS Unit Number: it $>>x-i 03$} \\
\hline \multicolumn{3}{|c|}{$\begin{array}{l}\text { Title of rest: } \\
\text { Electrical Systems }\end{array}$} & \multirow{2}{*}{\multicolumn{3}{|c|}{ 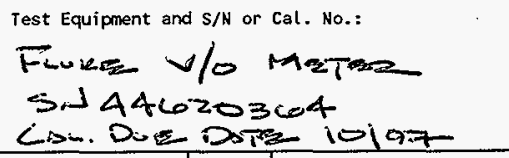 }} \\
\hline \multirow{2}{*}{$\begin{array}{l}\text { Test Performed By: } \\
\text { Procedure No. } \\
\text { Initial/Date }\end{array}$} & \multicolumn{2}{|c|}{ 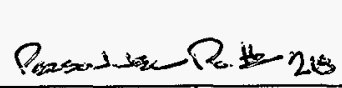 } & & & \\
\hline & Item & Value & $\begin{array}{l}\text { Required } \\
\text { Condition }\end{array}$ & $(A / R)$ & Coment \\
\hline \multicolumn{6}{|c|}{ Section $1.7,2$ verifies the intrinsic safety apparatus is properly labeled. } \\
\hline$\frac{1.7 .2 . j}{9-2 \pi-27}$ & $\begin{array}{l}\text { INTRINSIC } \\
\text { SAFETY } \\
\text { APPARATUS } \\
\text { NAMEPLATES } \\
\text { INSTALLED }\end{array}$ & L & $\begin{array}{l}\text { NE }-* 54 \\
\text { NE }-* 55 \\
\text { EB-*54 } \\
\text { EB-*55 } \\
\text { NIT-*54 } \\
\text { NIT }-* 55\end{array}$ & A & 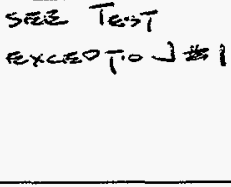 \\
\hline $\begin{array}{l}1.7 .2 .2 \\
\text { Gim ilistis }\end{array}$ & $\begin{array}{l}\text { INTRINSIC } \\
\text { SAFETY WIRE } \\
\text { LABELED }\end{array}$ & 0,0 & $\begin{array}{l}N E / E B-* 54 \\
N E / E B-* 55\end{array}$ & A & \\
\hline $1.7 .3 \mathrm{ming}^{9.27 .1}$ & $\begin{array}{l}\text { SEC. } 1.7 .1 \text { \& } \\
1.7 .2 \text { DONE }\end{array}$ & $\frac{\gamma \text { mand }}{\text { Test Direc }}$ & $\frac{1 \text { Brom }}{\text { or Signatu }}$ & & $\frac{22-97}{\text { Date }}$ \\
\hline
\end{tabular}

Test Witness/Review:

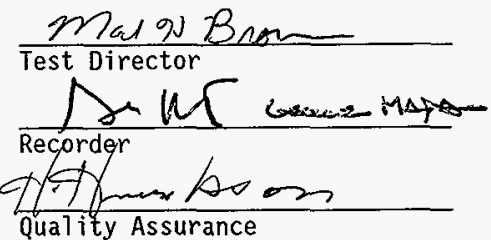

$$
\begin{aligned}
& \frac{1-15-97}{\text { Date }} \\
& \frac{15 \text { sas. } 97}{\text { Date }} \\
& \frac{1-27-97}{\text { Date }}
\end{aligned}
$$


APPENDIX G

PAGE G-1Y
HNF-SD-WM-ATR-191
ReV. 0
Page 230

TEST DATA SHEET

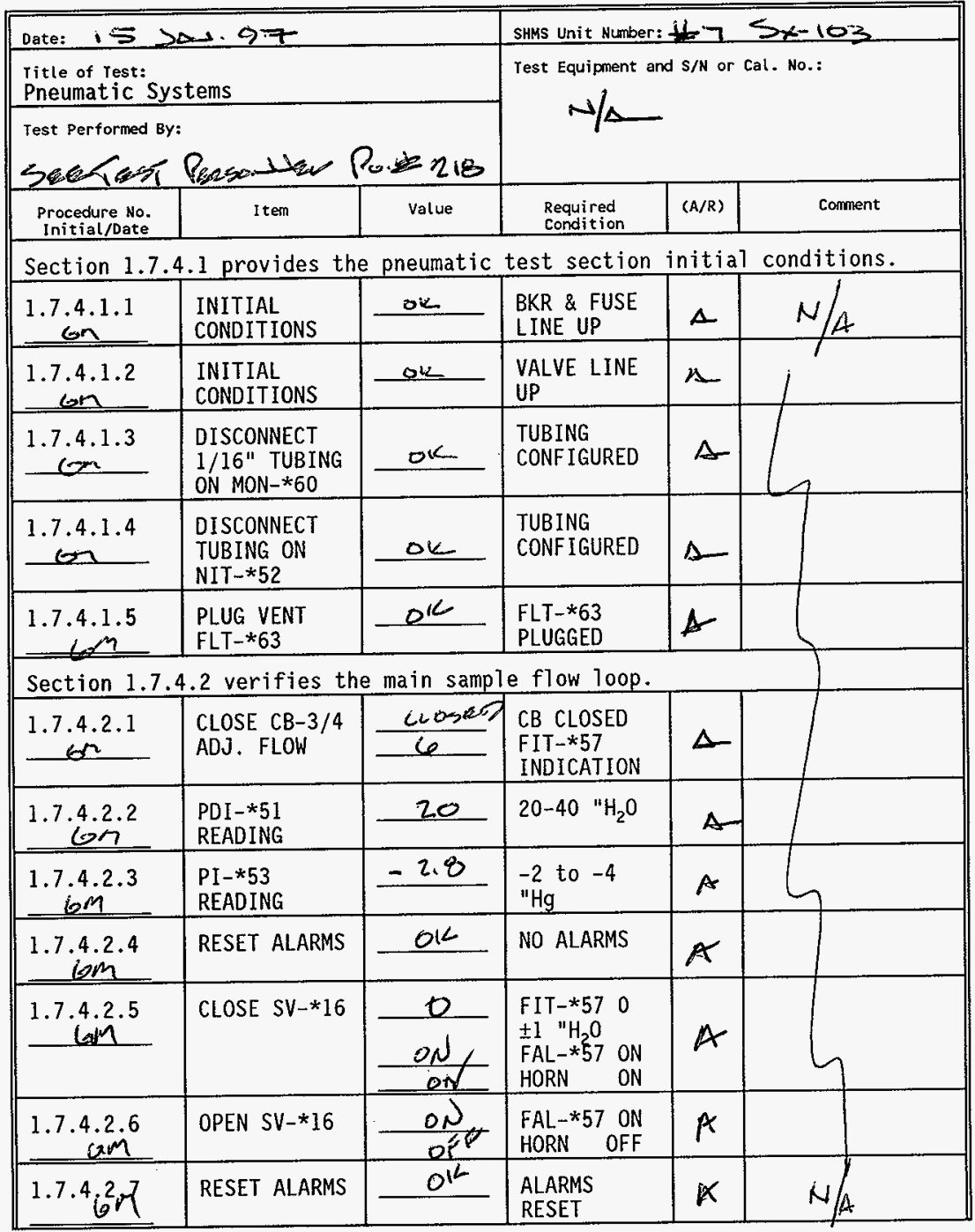

Ne 201.5147 
APPENDIX $G$

TEST DATA SHEET

\begin{tabular}{|c|c|c|c|c|c|}
\hline \multicolumn{3}{|c|}{ Date: .5 JA: 1.97} & \multicolumn{3}{|c|}{ SHMS Unit Number: $\pm 7>x-102$} \\
\hline \multicolumn{3}{|c|}{$\begin{array}{l}\text { Titte of Test: } \\
\text { Pneumatic Systems }\end{array}$} & \multirow{2}{*}{\multicolumn{3}{|c|}{ Test Equipnent and $\mathrm{s} / \mathrm{N}$ or Cal. No.: }} \\
\hline \multicolumn{3}{|c|}{ 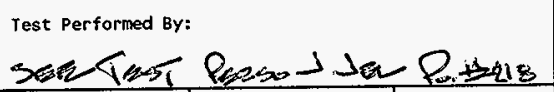 } & & & \\
\hline $\begin{array}{l}\text { Procedure No. } \\
\text { Initial/Date } \\
\end{array}$ & Item & value & $\begin{array}{l}\text { Required } \\
\text { Condition } \\
\end{array}$ & $\langle A / R\rangle$ & Comment \\
\hline $\begin{array}{l}1.7 .4 .2 .8 \\
6.4\end{array}$ & CLOSE SV $-* 25$ & $\begin{array}{c}0 \\
\frac{0.5}{00} \\
\frac{0 N}{24} \\
\end{array}$ & 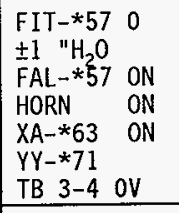 & 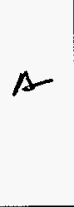 & N/ \\
\hline $\mathrm{eg}^{1.7 .4 .2 .9}$ & OPEN SV-*25 & $\begin{array}{l}\text { of } \\
\text { Ope } \\
\text { Of } \\
120 \\
\end{array}$ & 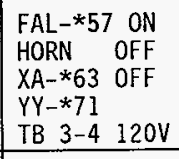 & $A$ & \\
\hline $\begin{array}{r}1.7 .4 .2 .10 \\
67 \\
\end{array}$ & RESET ALARMS & $d x$ & $\begin{array}{l}\text { ALARMS } \\
\text { RESET } \\
\end{array}$ & $A$ & \\
\hline$\frac{1.7 .4 .2 .11}{\operatorname{lom}}$ & $\begin{array}{l}\text { OPEN SV-*54 } \\
\text { \& SV-*66 } \\
\text { READ PRESS. } \\
\text { PDIT-*60 } \\
\end{array}$ & $\frac{-O K}{-40.0}$ & $\begin{array}{l}\text { VALVES OPEN } \\
-27 \text { to }-82 \\
\mathrm{H}_{2} \mathrm{O}\end{array}$ & $A$ & \\
\hline 1.7 .4 .2 .12 & $\begin{array}{l}\text { CLOSE SV-*54 } \\
\text { OPEN SV-*63 } \\
\text { READ PRESS. } \\
\text { PDIT-*60 } \\
\end{array}$ & $\frac{0 k}{0.815 / 4}$ & $\begin{array}{l}\text { VALVES } \\
\text { OPERATED } \\
\text { O } \pm 1 " \mathrm{H}_{2} \mathrm{O}\end{array}$ & A & \\
\hline$\frac{1.7 .4 .2 .13}{\sin ^{2}}$ & $\begin{array}{l}\text { CLOSE SV }-* 63 \\
\text { OPEN SV-*55 } \\
\text { READ PRESS. } \\
\text { PDIT } * * 60 \\
\end{array}$ & $\frac{a}{-42.91}$ & $\begin{array}{l}\text { VALVES } \\
\text { OPERATED } \\
-27 \text { to }-82 \\
" \mathrm{H}_{2} \mathrm{O}\end{array}$ & $A$ & \\
\hline$\frac{1.7 .4 .2 .14}{\mathrm{Gm}}$ & $\begin{array}{l}\text { CLOSE SV-*55 } \\
\text { OPEN SV }-* 64 \\
\text { READ PRESS. } \\
\text { PDIT-*60 } \\
\end{array}$ & $\frac{0 k}{.0000}$ & $\begin{array}{l}\text { VALVES } \\
\text { OPERATED } \\
0 \pm 1 " \mathrm{H}_{2} \mathrm{O}\end{array}$ & $A$ & \\
\hline$\frac{1.7 .4 .2 .15}{6 \mathrm{~m}}$ & $\begin{array}{l}\text { CLOSE SV-*64 } \\
\& \text { SV-*66 }\end{array}$ & $N / A$ & $N / A$ & $A$ & \\
\hline \multicolumn{6}{|c|}{ Section 1.7.4.3 verifies the grab sample flow loop. } \\
\hline $1.7 .4 .3 \mathrm{im}$ & READ PDI-*51 & 25 & $20-40 " \mathrm{H}_{2} \mathrm{O}$ & $A$ & $M / A$ \\
\hline
\end{tabular}




\section{TEST DATA SHEET}

\begin{tabular}{|c|c|c|c|c|c|}
\hline \multicolumn{3}{|c|}{ Date: is >nd.97 } & \multicolumn{3}{|c|}{ SHMS Unit Number: $175 \quad 5 x-103$} \\
\hline \multicolumn{3}{|c|}{$\begin{array}{l}\text { Title of Test: } \\
\text { Pneumatic Systems }\end{array}$} & \multirow{2}{*}{\multicolumn{3}{|c|}{ Test Equipment and $\mathrm{S} / \mathrm{N}$ or Cal. No.: }} \\
\hline \multicolumn{3}{|c|}{ 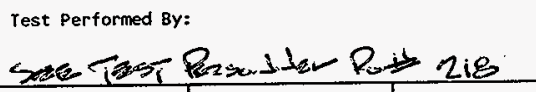 } & & & \\
\hline $\begin{array}{l}\text { Procedure No. } \\
\text { Initial/Date } \\
\end{array}$ & Item & Value & $\begin{array}{l}\text { Required } \\
\text { condition } \\
\end{array}$ & $(A / R)$ & Comment \\
\hline $\begin{array}{c}1.7 .4 .3 .2 \\
6.2 \\
\end{array}$ & $\begin{array}{l}\text { PUSH PB-*59 } \\
\text { GRAB SAMPLE } \\
\end{array}$ & $a k$ & $\begin{array}{l}\text { START } \\
\text { STOPWATCH }\end{array}$ & $A$ & $N / A$ \\
\hline $\begin{array}{c}1.7 .4 .3 .3 \\
\text { on }\end{array}$ & $\begin{array}{l}\text { GRAB SAMPLE } \\
\text { LAMPS ON } \\
\text { READ FIV-*52 }\end{array}$ & $\frac{\frac{o n}{a+b}}{\frac{0 n}{2}}$ & $\begin{array}{l}\text { PBL-*58 ON } \\
\text { PBL-*59 ON } \\
\text { YAL-*58 ON } \\
\text { FIV }-* 52 \quad 0 \\
\end{array}$ & $A$ & 1 \\
\hline $\mathrm{sim}^{1.7 .4 .3 .4}$ & $\begin{array}{l}\text { OPEN SV-*15 } \\
\& \text { ADJ. FIV- } \\
* 52\end{array}$ & $\frac{\text { opkad }}{10}$ & $\begin{array}{l}\text { SV-* } 15 \text { OPEN } \\
\text { FIV-*52 } \\
\text { FLOW } 10 \mathrm{CFH}\end{array}$ & $A$ & \\
\hline 1.7 .4 .3 .5 & READ PDI $-* 51$ & 29 & $\begin{array}{l}\text { PDI }-* 51 \\
\text { HIGHER THAN } \\
1.7 .4 .3 .1 \\
\end{array}$ & $\Delta$ & \\
\hline 1.7 .4 .3 .6 & CLOSE SV-*15 & 0 & FIV $-* 520$ & $A$ & \\
\hline $\begin{array}{c}1.7 .4 .3 .7 \\
\mathrm{om}\end{array}$ & OPEN SV $\ldots 15$ & 10 & $\begin{array}{l}\text { FIV }-* 52 \\
\text { FLOW }\end{array}$ & $\Delta$ & \\
\hline$\frac{1.7 .4 .3 .8}{o m}$ & $\begin{array}{l}\text { PBL }-* 59 \\
\text { GRAB SAMPLE } \\
\text { TIME } \\
\end{array}$ & $\frac{F F}{5}$ & $\begin{array}{l}\text { PBL-*59 OFF } \\
\text { STOP WATCH } \\
5 \pm .5 \text { MIN }\end{array}$ & $\Delta$ & \\
\hline $1.7 \cdot 4 \cdot 3 \cdot 7^{9}$ & $\begin{array}{l}\text { PB-*58 RESET } \\
\text { SAMPLER } \\
\end{array}$ & $\begin{array}{l}\text { off } \\
\text { ofe }\end{array}$ & $\begin{array}{l}\mathrm{PBL}-* 58 \text { OFF } \\
\text { YAL-*58 } 0 \mathrm{FF}\end{array}$ & P & \\
\hline \multicolumn{6}{|c|}{ Section 1.7 .4 .4 verifies the $\mathrm{H}_{2}$ cell calibration loop. } \\
\hline$\frac{1.7 .4 .4 .1}{i^{n}}$ & $\begin{array}{l}\text { CONNECT } \mathrm{H}_{2} \\
\text { CAL GAS }\end{array}$ & $\mathrm{N} / \mathrm{A}$ & $N / A$ & A & \\
\hline $\begin{array}{r}1.7 .4 .4 .2 \\
6 n^{4} \\
\end{array}$ & $\begin{array}{l}\text { OPEN ISO } \\
\text { VALVE }\end{array}$ & 5 & $<10$ PSIG & A & $n$ \\
\hline $\begin{array}{c}1.7 .4 .4 .3 \\
\quad 6 \mathrm{~m} \\
\end{array}$ & $\begin{array}{l}\text { OPEN SV-* } 18 \\
\text { ADJ. FIV-*56 } \\
\end{array}$ & 2 & $2 \pm .1 \mathrm{CFH}$ & $A$ & $N / A^{\prime}$ \\
\hline
\end{tabular}


TEST DATA SHEET

\begin{tabular}{|c|c|c|c|c|c|}
\hline \multicolumn{3}{|c|}{ Date: $15>0.97$} & \multicolumn{3}{|c|}{ SHMS Unit Nunber: $7 \times 103$} \\
\hline \multicolumn{3}{|c|}{$\begin{array}{l}\text { Title of Test: } \\
\text { Pneumatic Systems }\end{array}$} & \multirow{2}{*}{\multicolumn{3}{|c|}{ 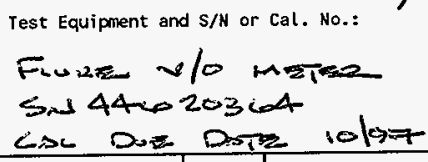 }} \\
\hline \multicolumn{3}{|c|}{$\begin{array}{l}\text { Test Performed By: } \\
213\end{array}$} & & & \\
\hline $\begin{array}{l}\text { Procedure No. } \\
\text { Initial/Date }\end{array}$ & Item & value & $\begin{array}{l}\text { Required } \\
\text { Condition } \\
\end{array}$ & $(A / R)$ & Comment \\
\hline $\begin{array}{l}1.7 .4 .4 .4 \\
6 \mathrm{~m}\end{array}$ & $\begin{array}{l}\text { VERIFY } \\
\text { CONDITIONS }\end{array}$ & 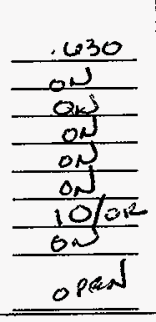 & $\begin{array}{l}\text { NIT-*54 } \\
>.625 \% \mathrm{H}_{2} \\
\text { NAH-*55 ON } \\
\text { NAH-*54 ON } \\
\text { PBL-*59 ON } \\
\text { PBL-*58 ON } \\
\text { YAL-*58 ON } \\
\text { FIV-*52 FLO } \\
\text { HORN ON } \\
\text { TB2-21/22 } \\
\text { OPEN } \\
\end{array}$ & $A$ & r \\
\hline $\begin{array}{l}1.7 .4 .4 .5 \\
\sin \\
\end{array}$ & $\begin{array}{l}\text { ACKNOWLEDGE } \\
\text { HORN }\end{array}$ & $N / A$ & N/A & A & \\
\hline$\frac{1.7 .4 .4 .6}{6 m}$ & $\begin{array}{l}\text { CLOSE SV-*18 } \\
\text { VERIFY } \\
\text { CONDITIONS }\end{array}$ & 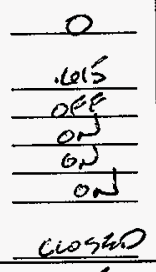 & $\begin{array}{l}\text { FIV-*56 O } \\
\text { NIT-*54 } \\
<.625 \% \mathrm{H}_{2} \\
\text { NAH- } * 55 \text { OFF } \\
\text { NAH- } * 54 \text { ON } \\
\text { PBL-*58 ON } \\
\text { YAL-*58 ON } \\
\text { TB2-21/22 } \\
\text { CLOSED } \\
\end{array}$ & $\Delta$ & \\
\hline $\begin{array}{c}1.7 .4 .4 .7 \\
0\end{array}$ & $\begin{array}{l}\text { PUSH RESET } \\
\text { SAMPLER PB- } \\
* 58 \\
\end{array}$ & $\frac{\text { Of }}{\text { OP }}$ & $\begin{array}{l}\text { PBL-*58 OFF } \\
\text { YAL-*58 OFF }\end{array}$ & $\mathbb{A}$ & \\
\hline $\begin{array}{c}1.7 .4 .4 .8 \\
\end{array}$ & RESET ALARM & ofe & NAH $-\star 54$ OFF & $A$ & \\
\hline $\begin{array}{c}1.7 .4 .4 .9 \\
\text { lom }\end{array}$ & $\begin{array}{l}\text { OPEN SV-* } 19 \\
\text { ADJ. FIV-*56 }\end{array}$ & 2 & $2 \pm .1 \mathrm{CFH}$ & A & $\mathrm{M} / \mathrm{A}$ \\
\hline
\end{tabular}

Aw on lisfit 
TEST DATA SHEET

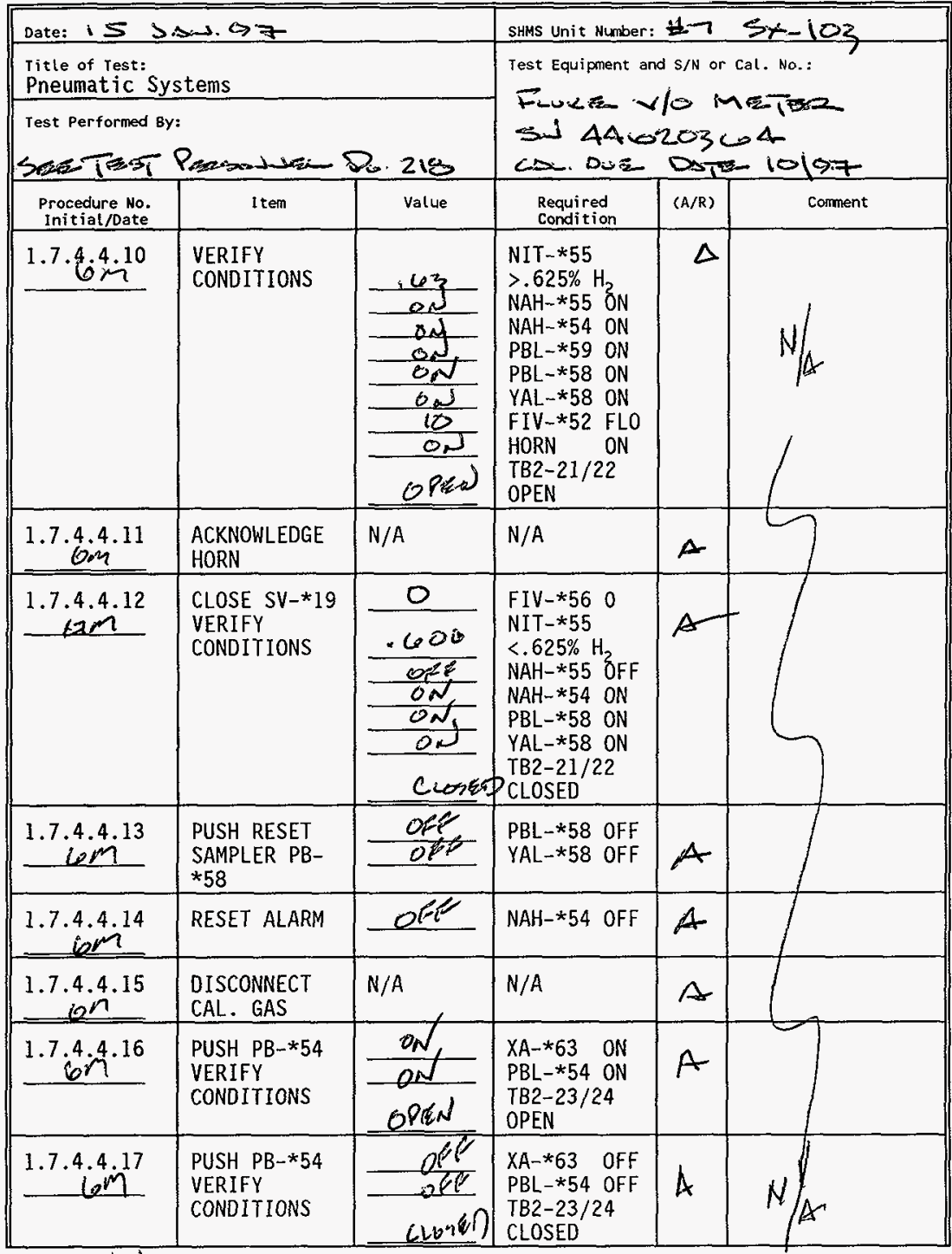


TEST DATA SHEET

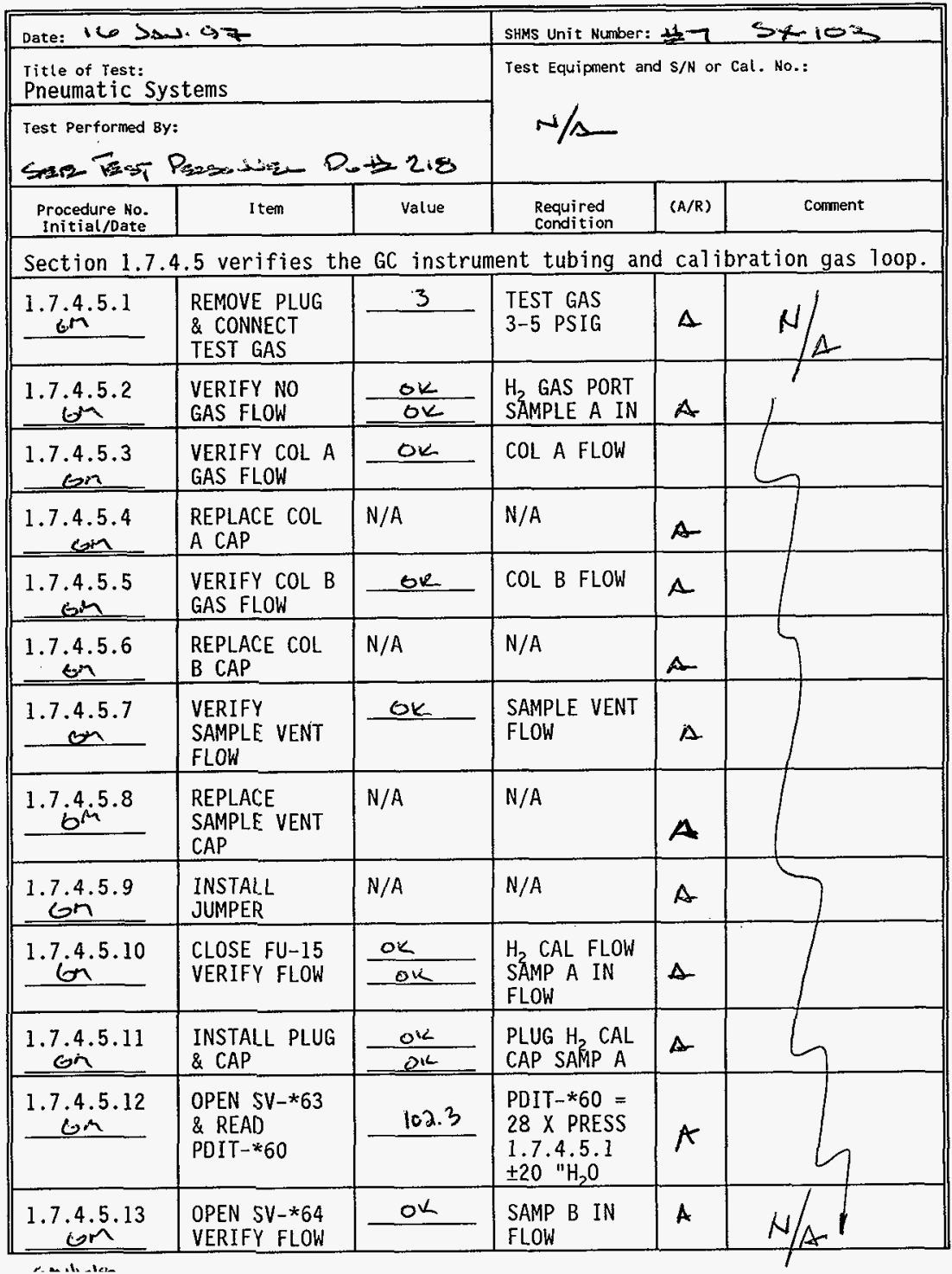


TEST DATA SHEET

\begin{tabular}{|c|c|c|c|c|c|}
\hline Date: ice $>$ & 4.97 & & SHMS Unit Number & \pm 7 & $5 x-103$ \\
\hline $\begin{array}{l}\text { Title of Test: } \\
\text { Pneumatic } S \\
\end{array}$ & tems & & Test Equipment a & $S / N$ or & 1. No.: \\
\hline $\begin{array}{l}\text { Test Performed B } \\
\text { Sezez TES }\end{array}$ & 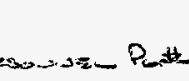 & 215 & $N / A$ & & \\
\hline $\begin{array}{c}\text { Procedure No. } \\
\text { Initial/Date }\end{array}$ & item & Value & $\begin{array}{l}\text { Required } \\
\text { Condition }\end{array}$ & $(A / R)$ & Comment \\
\hline $\begin{array}{l}1.7 .4 .5 .14 \\
\end{array}$ & $\begin{array}{l}\text { REMOVE TEST } \\
\text { GAS }\end{array}$ & $N / A$ & $N / A$ & A & \\
\hline $\begin{array}{l}1.7 .4 .5 .15 \\
\left(x^{n}\right.\end{array}$ & $\begin{array}{l}\text { CLOSE SV-*63 } \\
\& \text { SV }-* 64\end{array}$ & $N / A$ & $N / A$ & $A$ & \\
\hline $\begin{array}{l}1.7 .4 .5 .16 \\
\end{array}$ & $\begin{array}{l}\text { OPEN FU-15 } \\
\text { REMOVE } \\
\text { JUMPER }\end{array}$ & $N / A$ & $N / A$ & $\Delta$ & \\
\hline $\mathrm{Gm}^{1.7 .4 .5 .17}$ & $\begin{array}{l}\text { INSTALL } \\
\text { SAMP A UNION }\end{array}$ & $N / A$ & $N / A$ & $\Delta$ & \\
\hline $\begin{array}{r}1.7 .4 .5 .18 \\
\end{array}$ & $\begin{array}{l}\text { OPEN SV }-* 60 \\
\text { VERIFY FLOW }\end{array}$ & $\alpha$ & $\begin{array}{l}\text { FI-*60 } \\
\text { NO FLOW }\end{array}$ & $A$ & \\
\hline 1.7 .4 .5 .19 & $\begin{array}{l}\text { POWER SOV- } \\
* 60 \text {, VERIFY } \\
\text { NO FLOW }\end{array}$ & OK & $\begin{array}{l}\text { FI-*60 } \\
\text { NO FLOW }\end{array}$ & $A$ & \\
\hline 1.7 .4 .5 .20 & $\begin{array}{l}\text { OPEN SV-*68 } \\
\text { ADJ SV-*67 }\end{array}$ & 50 & $\begin{array}{l}\mathrm{FI}-* 60 \\
50 \mathrm{cCM}\end{array}$ & $\Lambda$ & \\
\hline $\mathrm{Gn}^{1.7 .4 .5 .21}$ & $\begin{array}{l}\text { DEENERGIZE } \\
\text { SOV }-* 60\end{array}$ & ok & $\begin{array}{l}\text { FI }-* 60 \text { FLOW } \\
\text { DECREASES }\end{array}$ & $\Delta$ & \\
\hline$\frac{1.7 .5 .5 .22}{6}$ & $\begin{array}{l}\text { CLOSE SV-*60 } \\
\& \text { SV } * * 68\end{array}$ & $N / A$ & $N / A$ & $A$ & \\
\hline$\underbrace{1.7 .23}_{\text {Gin }}$ & $\begin{array}{l}\text { REMOVE } \\
\text { SAMP A UNION }\end{array}$ & $N / A$ & $N / A$ & $A$ & \\
\hline $\begin{array}{c}1.7 .4 .5 .24 \\
\mathrm{om}^{5}\end{array}$ & $\begin{array}{l}\text { CONNECT TEST } \\
\text { GAS } \mathrm{N}_{2} \mathrm{O} \text { PORT } \\
\end{array}$ & 4 & $\begin{array}{l}\text { TEST GAS } \\
3-5 \text { PSIG }\end{array}$ & $A$ & \\
\hline$\frac{1.7 .4 .5 .25}{6 n}$ & $\begin{array}{l}\text { VERIFY NO } \\
\text { FLOW }\end{array}$ & $\frac{\text { No }}{\text { No }}$ & $\begin{array}{l}\text { SAMP B IN \& } \\
\text { FLT-*63 } \\
\text { NO FLOW }\end{array}$ & $\star$ & \\
\hline $\begin{array}{c}1.7 .4 .5 .26 \\
\mathrm{Cm} \\
\end{array}$ & $\begin{array}{l}\text { INSTALL } \\
\text { JUMPER } \\
\end{array}$ & $N / A$ & $N / A$ & $\triangle$ & \\
\hline$\frac{1.7 .4 .5 .27}{6 m}$ & $\begin{array}{l}\text { CLOSE FU-15 } \\
\text { VERIFY FLOW }\end{array}$ & $\frac{\operatorname{sen}}{\operatorname{son}}$ & $\begin{array}{l}\text { SAMP B IN } \\
\text { FLT }-^{*} 63 \\
\text { FLOW }\end{array}$ & $\hat{i}$ & $N / A$ \\
\hline
\end{tabular}


TEST DATA SHEET

\begin{tabular}{|c|c|c|c|c|c|}
\hline \multicolumn{3}{|c|}{ Date: 16 SS.4.97 } & \multicolumn{3}{|c|}{ SHMS Unit Number: $\quad S x-i \in 3$} \\
\hline \multicolumn{3}{|c|}{$\begin{array}{l}\text { Title of Test: } \\
\text { Pneumatic Systems }\end{array}$} & \multirow{2}{*}{\multicolumn{3}{|c|}{$\begin{array}{l}\text { Test Equipnent and } \mathrm{S} / \mathrm{N} \text { or Cal. No.: } \\
\text { S/A }\end{array}$}} \\
\hline \multicolumn{3}{|c|}{ 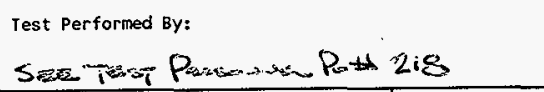 } & & & \\
\hline $\begin{array}{l}\text { Procedure No. } \\
\text { Initial/Date }\end{array}$ & Item & value & $\begin{array}{l}\text { Required } \\
\text { Condition }\end{array}$ & $(A / R)$ & Comment \\
\hline $\mathrm{GM}^{1.7 .4 .5 .28}$ & $\begin{array}{l}\text { DISCONNECT } \\
\text { TEST GAS }\end{array}$ & N/A & $\mathrm{N} / \mathrm{A}$ & $A$ & \\
\hline $\begin{array}{l}1.7 .4 .5 .29 \\
\end{array}$ & OPEN FU-15 & N/A & N/A & $\Delta$ & \\
\hline 1.7 .4 .5 .30 & $\begin{array}{l}\text { REMOVE } \\
\text { JUMPER }\end{array}$ & N/A & $\mathrm{N} / \mathrm{A}$ & $A$ & \\
\hline 1.7.4. G. $^{5.31}$ & $\begin{array}{l}\text { INSTALL } \\
\text { SAMP B UNION }\end{array}$ & N/A & $N / A$ & A & \\
\hline $\begin{array}{c}1.7 .4 .5 .32 \\
0.7 \\
\end{array}$ & $\begin{array}{l}\text { OPEN SV }-* 60 \\
\text { VERIFY FLOW }\end{array}$ & or & $\begin{array}{l}\text { FI }-* 60 \\
\text { NO FLOW }\end{array}$ & 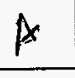 & \\
\hline$\frac{1.7 .4 .5 .33}{6 \text { s. }^{-1}}$ & $\begin{array}{l}\text { POWER SOV- } \\
* 60, \text { VERIFY } \\
\text { NO FLOW }\end{array}$ & ok & $\begin{array}{l}\text { FI }-* 60 \\
\text { NO FLOW }\end{array}$ & $\Lambda$ & \\
\hline $\begin{array}{c}1.7 .4 .5 .34 \\
\end{array}$ & $\begin{array}{l}\text { OPEN SV-*68 } \\
\& \text { ADJ SV-*67 }\end{array}$ & 50 & $\begin{array}{l}F I-* 60 \\
50 \mathrm{CCM} \\
\end{array}$ & $\Delta$ & \\
\hline$\frac{1.7 .4 .5 .35}{6 \omega^{n}}$ & $\begin{array}{l}\text { DEENERGIZE } \\
\text { SOV-*60 }\end{array}$ & ok & $\begin{array}{l}\text { FI-*60 FLOW } \\
\text { DECREASES } \\
\end{array}$ & $\Delta$ & \\
\hline $\begin{array}{c}1.7 .4 .5 .36 \\
64 \\
\end{array}$ & $\begin{array}{l}\text { CLOSE SV-*60 } \\
\& \text { SV-*68 }\end{array}$ & $N / A$ & N/A & A & \\
\hline$\frac{1.7 .4 .5 .37}{62^{m}}$ & $\begin{array}{l}\text { REMOVE } \\
\text { SAMP B UNION }\end{array}$ & N/A & N/A & $A$ & \\
\hline $\begin{array}{c}1.7 .4 .5 .38 \\
69 \\
\end{array}$ & $\begin{array}{l}\text { OPEN CB- } 3 / 4 \\
\text { ACK. ALARMS } \\
\end{array}$ & $\mathrm{N} / \mathrm{A}$ & N/A & $\Delta$ & \\
\hline \multicolumn{6}{|c|}{$\begin{array}{l}\text { Section } 1.7 .4 .6 \text { verifies the multi gas analyzer instrument tubifg and } \\
\text { calibration gas loop. }\end{array}$} \\
\hline$\frac{1.7 .4 .6 .1}{\log }$ & $\begin{array}{l}\text { VERIFY } \\
\text { NIT-*52 } \\
\text { SAMPLE LINES } \\
\end{array}$ & $N / A$ & N/A & $\Delta$ & \\
\hline$\frac{1.7 .4 .6 .2}{\text { in }^{2}}$ & $\begin{array}{l}\text { CONNECT TEST } \\
\text { GAS NH } \\
\end{array}$ & $x$ & $\begin{array}{l}\text { TEST GAS } \\
3-5 \text { PSIG } \\
\end{array}$ & $A$ & \\
\hline $\begin{array}{r}1.7 .4 .6 .3 \\
60^{r} \\
\end{array}$ & $\begin{array}{l}\text { VERIFY NO } \\
\text { FLOW }\end{array}$ & $\frac{o^{k}}{a k}$ & $\begin{array}{l}\text { SAMPLE IN } \\
\text { FLT-*63 }\end{array}$ & $\Delta$ & $M / A$ \\
\hline
\end{tabular}


TEST DATA SHEET

\begin{tabular}{|c|c|c|c|c|c|}
\hline Date: $16=$ & $\therefore .97$ & & SHMS Unit Number: & \pm 7 & $5 x-103$ \\
\hline $\begin{array}{l}\text { Iitle of rest: } \\
\text { Pneumatic } S \mathrm{y}\end{array}$ & tems & & Test Equipment an & $\mathrm{S} / \mathrm{N}$ or & Cal. No.: \\
\hline $\begin{array}{l}\text { Test Performed B } \\
\text { Sea Tosi }\end{array}$ & D. Pot & 218 & $\omega / \Delta$ & & \\
\hline $\begin{array}{c}\text { Procedure No. } \\
\text { Initial/Date } \\
\end{array}$ & Item & value & $\begin{array}{l}\text { Required } \\
\text { condition }\end{array}$ & $(A / R)$ & Comment \\
\hline $\begin{array}{r}1.7 .4 .6 .4 \\
\end{array}$ & $\begin{array}{l}\text { INSTALL } \\
\text { JUMPER }\end{array}$ & $N / A$ & N/A & $A$ & $A$ \\
\hline$\frac{1.7 .4 .6 .5}{6 x}$ & $\begin{array}{l}\text { CLOSE FU-15 } \\
\text { VERIFY GAS } \\
\text { FLOWS }\end{array}$ & $\frac{\frac{o k}{b k}}{0 k}$ & $\begin{array}{l}\text { SAMPLE IN } \\
\text { FLT -*63 } \\
\text { FIV-*70 }\end{array}$ & $\Delta$ & \\
\hline $\begin{array}{l}1.7 .4 .6 .6 \\
\end{array}$ & $\begin{array}{l}\text { PLUG FLT-*63 } \\
\& \text { MON-*60 } \\
\text { SAMPLE VENT }\end{array}$ & $N / A$ & $N / A$ & A & $l$ \\
\hline $\begin{array}{c}1.7 .4 .6 .7 \\
\end{array}$ & $\begin{array}{l}\text { OPEN SV-*66, } \\
\text { SV- } * 70 \& \text { CAP } \\
\text { SAMPLE IN }\end{array}$ & 98.3 & $\begin{array}{l}\text { PDIT-*60 }= \\
28 \times \text { PRESS } \\
1.7 .4 .6 .2 \\
\pm 20 " \mathrm{H}_{2} \mathrm{O}\end{array}$ & so & \\
\hline $\begin{array}{l}1.7 .4 .6 .8 \\
62\end{array}$ & $\begin{array}{l}\text { CLOSE SV }-* 66 \\
\text { SV -*70 \& } \\
\text { REMOVE CAP }\end{array}$ & $N / A$ & $N / A$ & $\Delta$ & \\
\hline $\begin{array}{l}1.7 .4 .6 .9 \\
\end{array}$ & $\begin{array}{l}\text { REMOVE FLT- } \\
* 63 \text { PLUG }\end{array}$ & $N / A$ & N/A & $A$ & \\
\hline $\begin{array}{r}1.7 .4 .6 .10 \\
\end{array}$ & $\begin{array}{l}\text { REMOVE TEST } \\
\text { GAS }\end{array}$ & $N / A$ & N/A & $\Delta$ & \\
\hline $\begin{array}{c}1.7 .4 .6 .11 \\
6 m \\
\end{array}$ & $\begin{array}{l}\text { CLOSE SV-*05 } \\
\& \text { SV-*16 }\end{array}$ & $N / A$ & $N / A$ & $\Delta$ & \\
\hline $\begin{array}{r}1.7 .4 .6 .12 \\
v^{m}\end{array}$ & $\begin{array}{l}\text { CONNECT TEST } \\
\text { GAS }\end{array}$ & 4 & $\begin{array}{l}\text { TEST GAS } \\
2-4 \text { PSIG } \\
\end{array}$ & $\ltimes$ & \\
\hline$\frac{1.7 .4 .6 .13}{6 m}$ & $\begin{array}{l}\text { OPEN SV }-* 07 \\
\& \text { VERIFY } \\
\text { NO FLOW }\end{array}$ & $\frac{10}{100}$ & $\begin{array}{l}\text { SAMPLE IN } \\
\text { SAMPLE OUT }\end{array}$ & A & \\
\hline$\frac{1.7 .4 .6 .14}{i^{2} n^{-1}}$ & $\begin{array}{l}\text { OPEN SV }-* 09 \\
\& \text { VERIFY } \\
\text { NO FLOW }\end{array}$ & $\frac{N O}{N O}$ & $\begin{array}{l}\text { SAMPLE IN } \\
\text { SAMPLE OUT }\end{array}$ & $A$ & \\
\hline$\frac{1.7 .4 .6 .15}{6 m^{-15}}$ & $\begin{array}{l}\text { OPEN FU-15 \& } \\
\text { VERIFY FLOW }\end{array}$ & $\frac{Y E}{N D}$ & $\begin{array}{l}\text { SAMPLE FLOW } \\
\text { IN - YES } \\
\text { OUT - NO } \\
\end{array}$ & $\Delta$ & \\
\hline $\begin{array}{c}1.7 .4 .6 .16 \\
6 \mathrm{n} \\
\end{array}$ & $\begin{array}{l}\text { CLOSE SV-*09 } \\
\text { VERIFY FLOW }\end{array}$ & NO & $\begin{array}{l}\text { SAMPLE IN } \\
\text { NO FLOW }\end{array}$ & $\Delta$ & \\
\hline
\end{tabular}


TEST DATA SHEET

\begin{tabular}{|c|c|c|c|c|c|}
\hline \multicolumn{3}{|c|}{ Date: if S.4.97 } & \multicolumn{3}{|c|}{ SHMS Unit Number: $t 7 \quad 5 x-103$} \\
\hline \multicolumn{3}{|c|}{$\begin{array}{l}\text { Titte of Test: } \\
\text { Pneumatic Systems }\end{array}$} & \multirow{2}{*}{\multicolumn{3}{|c|}{$\begin{array}{l}\text { Test Equipment and S/N or Cal. No.: } \\
\text { / } / \triangle \text {. }\end{array}$}} \\
\hline \multicolumn{3}{|c|}{ 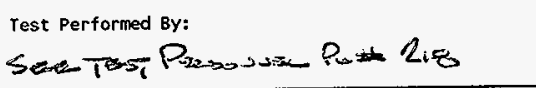 } & & & \\
\hline $\begin{array}{l}\text { Procedure No. } \\
\text { Initial/Date }\end{array}$ & Item & value & $\begin{array}{l}\text { Required } \\
\text { Condition } \\
\end{array}$ & $(A / R)$ & Comment \\
\hline$\frac{1.7 .4 .6 .17}{\operatorname{con}}$ & $\begin{array}{l}\text { OPEN SV }-* 10 \\
\text { VERIFY FLOW }\end{array}$ & No & $\begin{array}{l}\text { SAMPLE FLOW } \\
\text { IN - NO } \\
\text { OUT - YES }\end{array}$ & $\Delta$ & \\
\hline$\frac{1.7 .4 .6 .18}{6^{n+1}}$ & $\begin{array}{l}\text { REMOVE TEST } \\
\text { GAS \& PLUG } \\
\text { SV }-* 07\end{array}$ & N/A & $N / A$ & $\Delta$ & \\
\hline $\begin{array}{c}1.7 .4 .6 .19 \\
\end{array}$ & $\begin{array}{l}\text { CLOSE SV-*07 } \\
\& \text { SV }-* 10\end{array}$ & $N / A$ & $N / A$ & $\Delta$ & \\
\hline 1.7 .4 .6 .20 & $\begin{array}{l}\text { OPEN SV }-* 05 \\
\& \text { SV }-* 16\end{array}$ & $N / A$ & $N / A$ & $\Delta$ & \\
\hline 1.7.4.6.21 & $\begin{array}{l}\text { REMOVE } \\
\text { JUMPER }\end{array}$ & $N / A$ & $N / A$ & $A$ & \\
\hline \multicolumn{6}{|c|}{ Section 1.7.4.7 verifies the carrier gas supply lines. } \\
\hline $\begin{array}{c}1.7 .4 .7 .1 \\
\end{array}$ & $\begin{array}{l}\text { CONNECT TEST } \\
\text { GAS }\end{array}$ & 4 & 3-5 PSIG & $\sim$ & \\
\hline$\frac{1.7 .4 .7 .2}{6.7}$ & $\begin{array}{l}\text { REMOVE PLUG } \\
\& \text { VERIFY } \\
\text { FLOW } \\
\end{array}$ & finos & $\begin{array}{l}\mathrm{N}_{2} \text { CARRIER } \\
\text { GAS FLOWS }\end{array}$ & & \\
\hline $\begin{array}{r}1.7 .4 .7 .3 \\
60 m \\
\end{array}$ & $\begin{array}{l}\text { DISCONNECT } \\
\text { TEST GAS }\end{array}$ & N/A & N/A & $\sim$ & \\
\hline $\begin{array}{c}1.7 .4 .7 .4 \\
\text { on }\end{array}$ & $\begin{array}{l}\text { CONNECT TEST } \\
\text { GAS }\end{array}$ & $\not$ & 3-5 PSIG & 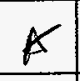 & \\
\hline$\frac{1.7 .4 .7 .5}{\min ^{5}}$ & $\begin{array}{l}\text { REMOVE PLUG } \\
\text { \& VERIFY } \\
\text { FLOW } \\
\end{array}$ & $C=0$ & $\begin{array}{l}\text { He CARRIER } \\
\text { GAS FLOWS }\end{array}$ & $\mathbb{K}$ & 1 \\
\hline $1.7 .4 y^{7.6}$ & $\begin{array}{l}\text { DISCONNECT } \\
\text { TEST GAS }\end{array}$ & $N / A$ & N/A & $\mathbb{B}$ & $N / A^{1}$ \\
\hline $1.7 .5 \mathrm{~m}$ & $\begin{array}{l}\text { TEST SEC. } \\
1.7 .4 \text { DONE }\end{array}$ & \multicolumn{4}{|c|}{$\frac{m_{a 1} \text { I } B \text { now }}{\text { Test Director Signature }} \quad \frac{1-16-96}{\text { Date }}$} \\
\hline
\end{tabular}


TEST DATA SHEET

\begin{tabular}{|c|c|c|c|c|c|}
\hline Date: 17 s. & 1.97 & & SHMS Unit Numbe & \pm 7 & $5 x-103$ \\
\hline $\begin{array}{l}\text { Yitle of Test: } \\
\text { Pneumatic } S\end{array}$ & tems & & Test Equipment & $s / N$ or & Cat, No.: \\
\hline $\begin{array}{l}\text { Test Performed } \mathrm{B} \\
\text { See Tast }\end{array}$ & 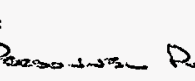 & 2020 & $N / \Delta$ & & \\
\hline $\begin{array}{l}\text { Procedure No. } \\
\text { Initial/Date }\end{array}$ & Item & Value & $\begin{array}{l}\text { Required } \\
\text { condition }\end{array}$ & $(A / R)$ & Comment \\
\hline $\begin{array}{l}1.7 .6 \\
\operatorname{con}\end{array}$ & $\begin{array}{l}\text { THIS SECTION } \\
\text { IF NOT APPLIC } \\
\frac{N / A}{\text { Test Director }}\end{array}$ & $\begin{array}{l}\text { IS ONLY FO } \\
\text { ABLE, N/A } \\
\text { Signature }\end{array}$ & $\begin{array}{l}\text { THE SHMS-E } \\
\text { EPS AND TEST }\end{array}$ & $\begin{array}{l}\text { THE AN } \\
\text { IRECTC } \\
\text { Date }\end{array}$ & $\begin{array}{l}\text { LL MODE. } \\
\text { SIGN. }\end{array}$ \\
\hline 1.7 .6 .1 .1 & $\begin{array}{l}\text { VERIFY BKR } \\
\text { LINE UP }\end{array}$ & N/A & $\mathrm{N} / \mathrm{A}$ & A & \\
\hline$\frac{1.7 .6 .1 .2}{6 \mathrm{~m}^{2}}$ & $\begin{array}{l}\text { VERIFY VALVE } \\
\text { LINE UP } \\
\end{array}$ & $N / A$ & N/A & A & \\
\hline $\begin{array}{l}1.7 .6 .1 .3 \\
\quad . n \\
\end{array}$ & $\begin{array}{l}\text { VERIFY MON- } \\
\text { *60 INSTALL }\end{array}$ & N/A & $N / A$ & $A$ & \\
\hline $\begin{array}{c}1.7 .6 .1 .4 \\
\mathrm{Gm}\end{array}$ & $\begin{array}{l}\text { VERIFY NIT- } \\
* 52 \text { INSTALL } \\
\end{array}$ & N/A & N/A & $A$ & \\
\hline $\begin{array}{c}1.7 .6 .1 .5 \\
6 \mathrm{GM} \\
\end{array}$ & $\begin{array}{l}\text { CONNECT } \mathrm{H}_{2} \\
\text { CAL GAS }\end{array}$ & N/A & $N / A$ & A & \\
\hline $\begin{array}{c}1.7 .6 .1 .6 \\
6 \mathrm{om}\end{array}$ & $\begin{array}{l}\text { CONNECT } \mathrm{CH}_{4} \\
\text { CAL GAS }\end{array}$ & N/A & $N / A$ & A & \\
\hline $\begin{array}{c}1.7 .6 .1 .7 \\
6.7\end{array}$ & $\begin{array}{l}\text { CONNECT NH } \\
\text { CAL GAS } \\
\end{array}$ & $N / A$ & $N / A$ & $A$ & \\
\hline Section 1.7 & .2 establ ishes & the main & d auxiliary & op fl & \\
\hline $\begin{array}{c}1.7 .6 .2 .1 \\
\mathrm{om} \\
\end{array}$ & CLOSE FU-11 & ok & $\begin{array}{l}\text { MON }-* 60 \\
\text { POWERED } \\
\end{array}$ & $A$ & \\
\hline$\frac{1.7 .6 .2 .2}{6 \mathrm{~m}}$ & POWER PC-*60 & $-0^{k}$ & $\begin{array}{l}\text { ESTABLISH } \\
\text { SAMPLING } \\
\text { ROUTINE } \\
\end{array}$ & A & \\
\hline $\begin{array}{r}1.7 .6 .2 .3 \\
0.9\end{array}$ & CLOSE FU-12 & ok & $\begin{array}{l}\text { NIT } * * 52 \\
\text { POWERED } \\
\end{array}$ & $A$ & \\
\hline $\begin{array}{c}1.7 .6 .2 .4 \\
6 \mathrm{~m}^{2} \\
\end{array}$ & POWER PC $-* 70$ & ok & $\begin{array}{l}\text { PC-*70 } \\
\text { BO0TED UP }\end{array}$ & A & \\
\hline 1.7 .6 .2 .5 & $\begin{array}{l}\text { CLOSE CB-3/4 } \\
\text { ADJUST FLOW }\end{array}$ & 1.0 & $\begin{array}{l}\mathrm{FIT}-* 57 \\
1 \pm .1 \mathrm{"H} \mathrm{H}_{2} \mathrm{O}\end{array}$ & A & $N / 4$ \\
\hline
\end{tabular}


APPENDIX G

ReORD

PAGE G-25

Rev. 0.

Page 241

TEST DATA SHEET

\begin{tabular}{|c|c|c|c|c|c|}
\hline \multicolumn{3}{|c|}{ Date: in JS-A. C7 } & \multicolumn{3}{|c|}{ SHMS Unit Number: it $=7 \quad 5 x-103$} \\
\hline \multicolumn{3}{|c|}{$\begin{array}{l}\text { Titie of Test: } \\
\text { Pneumatic Systems }\end{array}$} & \multirow{2}{*}{\multicolumn{3}{|c|}{$\begin{array}{l}\text { Test Equipment and } \mathrm{S} / \mathrm{N} \text { or Cal. No.: } \\
\text { - }\end{array}$}} \\
\hline \multicolumn{3}{|c|}{ 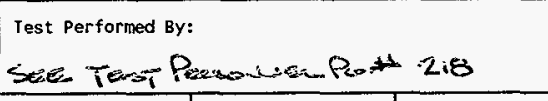 } & & & \\
\hline $\begin{array}{l}\text { Procedure No. } \\
\text { Initial/Date }\end{array}$ & Item & value & $\begin{array}{l}\text { Required } \\
\text { Condition }\end{array}$ & $(A / R)$ & Comment \\
\hline $\begin{array}{r}1.7 .6 .2 .6 \\
\quad 6 \mathrm{~m} \\
\end{array}$ & $\begin{array}{l}\text { OPEN SV-*09 } \\
\& \text { SV }-* 10 \\
\end{array}$ & N/A & $\mathrm{N} / \mathrm{A}$ & A & \\
\hline $\begin{array}{c}1.7 .6 .2 .7 \\
6 \mathrm{Gm} \\
\end{array}$ & $\begin{array}{l}\text { OPEN SV-*60' } \\
63,65^{\prime} \& 68^{\prime}\end{array}$ & $N / A$ & $N / A$ & $A$ & \\
\hline$\frac{1.7 .6 .2 .8}{\operatorname{con}}$ & NIT $-\star 52$ & ok & $\begin{array}{l}\text { ESTABLISH } \\
\text { SAMPLING } \\
\text { ROUTINE }\end{array}$ & $\Delta$ & \\
\hline $\begin{array}{l}1.7 .6 .2 .9 \\
\end{array}$ & $\begin{array}{l}\text { OPEN SV-*24 } \\
\text { ADJ FLOW } \\
\end{array}$ & 3.003 & $\begin{array}{l}\text { FIT-*57 } \\
3 \pm .1 \quad " \mathrm{H}_{2} \mathrm{O} \\
\end{array}$ & $\Delta$ & \\
\hline $\begin{array}{r}1.7 .6 .7^{2.10} \\
\end{array}$ & $\begin{array}{l}\text { ADJ FLOW } \\
\text { SV-*67 }\end{array}$ & 17 & $\begin{array}{l}\mathrm{FI}-* 60 \\
10-30 \mathrm{ccm}\end{array}$ & $\Delta$ & \\
\hline 1.7 .6 .2 .11 & $\begin{array}{l}\text { RECORD } \\
\text { PDIT }-\star 60 \\
\end{array}$ & 141.5 & $<175 \quad " \mathrm{H}_{2} \mathrm{O}$ & $A$ & \\
\hline$\frac{1.7 .6 .2 .12}{6 m}$ & $\begin{array}{l}\text { OPEN SV-*64' } \\
\text { CLOSE SV-*63/ }\end{array}$ & $N / A$ & N/A & A & \\
\hline $\begin{array}{l}1.7 .6 .2 .13 \\
\end{array}$ & $\begin{array}{l}\text { RECORD } \\
\text { PDIT }-* 60\end{array}$ & 183.1 & $<175 \quad \mathrm{H}_{2} \mathrm{O}$ & $A$ & \\
\hline $\begin{array}{c}1.7 .6 .2 .14 \\
\mathrm{bm}^{14} \\
\end{array}$ & $\begin{array}{l}\text { CLOSE SV-*65 } \\
\text { OPEN SV }-* 66 / \\
\end{array}$ & N/A & $N / A$ & $A$ & \\
\hline$\frac{1.7 .6 .2 .15}{\mathrm{~m}^{m}}$ & $\begin{array}{l}\text { RECORD B IN } \\
\text { PDIT }-* 60\end{array}$ & $-24-42$ & $\begin{array}{l}-15 \text { to }-50 \\
\mathrm{H}_{2} \mathrm{O}\end{array}$ & $A$ & \\
\hline 1.7 .6 .2 .16 & $\begin{array}{l}\text { OPEN SV-*63 } \\
\text { CLOSE SV-*64 }\end{array}$ & $N / A$ & N/A & A & \\
\hline $\begin{array}{r}1.7 .6 .2 .17 \\
6 \times 27 \\
\end{array}$ & $\begin{array}{l}\text { RECORD A IN } \\
\text { PDIT }-* 60\end{array}$ & $=24.41$ & $\begin{array}{l}-15 \text { to }-50 \\
" \mathrm{H}_{2} \mathrm{O}\end{array}$ & A & \\
\hline$\frac{1.7 .6 .2 .18}{1 \mathrm{~g}^{\mathrm{m}}}$ & $\begin{array}{l}\text { INSTALL } \\
\text { JUMPER }\end{array}$ & N/A & N/A & $A$ & \\
\hline $\begin{array}{r}1.7 .6 .2 .19 \\
6^{2 m}\end{array}$ & CLOSE FU-15 & N/A & N/A & A & \\
\hline$\frac{1.7 .6 .2 .20}{6 m}$ & $\begin{array}{l}\text { ADJ SV-*61 \& } \\
\text { RECORD } \\
\text { PDIT }-* 60\end{array}$ & -20.73 & $\begin{array}{l} \pm 5^{\prime \prime} \mathrm{H}_{2} \mathrm{O} \text { OF } \\
1.7 .6 .2 .17\end{array}$ & $A$ & $N / A$ \\
\hline
\end{tabular}

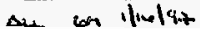


TEST DATA SHEET

\begin{tabular}{|c|c|c|c|c|c|}
\hline \multicolumn{3}{|c|}{ Date: 16 SAn. 47} & \multicolumn{3}{|c|}{ SHMS Unit Number: $=17 \leq x-103$} \\
\hline \multicolumn{3}{|c|}{$\begin{array}{l}\text { Title of Test: } \\
\text { Pneumatic Systems }\end{array}$} & \multirow{2}{*}{\multicolumn{3}{|c|}{ 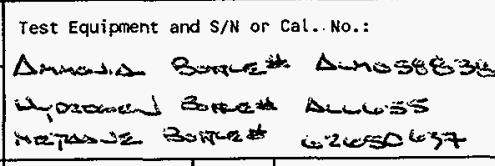 }} \\
\hline \multicolumn{3}{|c|}{ 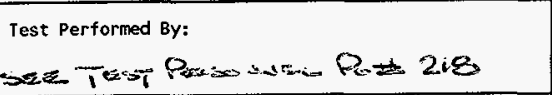 } & & & \\
\hline $\begin{array}{l}\text { Procedure No. } \\
\text { Initial/Date }\end{array}$ & 1 tem & Value & $\begin{array}{l}\text { Required } \\
\text { Condition }\end{array}$ & $(A / R)$ & Corment \\
\hline$\frac{1.7 .6 .2 .21}{\operatorname{son}}$ & $\begin{array}{l}\text { CAL MON }-* 60 \\
\text { CHANNEL } A\end{array}$ & $\frac{\frac{10510}{10511}}{10478}$ & $\begin{array}{l}\text { READINGS } \\
\text { WITHIN } \pm 5 \%\end{array}$ & $A$ & 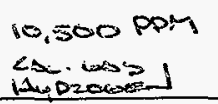 \\
\hline$\frac{1.7 .6 .2 .22}{\mathrm{gin}}$ & OPEN FU-15 & $N / A$ & $\mathrm{~N} / \mathrm{A}$ & A & \\
\hline $\begin{array}{c}1.7 .6 .2 .23 \\
\mathrm{gm} \\
\end{array}$ & $\begin{array}{l}\text { REMOVE } \\
\text { JUMPER }\end{array}$ & N/A & $N / A$ & A & \\
\hline $\mathrm{Gin}^{1.7 .6 .2 .24}$ & $\begin{array}{l}\text { OPEN SV-*64- } \\
\text { CLOSE SV-*63 }\end{array}$ & $N / A$ & $N / A$ & $A$ & \\
\hline $\begin{array}{c}1.7 .6 .2 .25 \\
\mathrm{om} \\
\end{array}$ & $\begin{array}{l}\text { INSTALL } \\
\text { JUMPER } \\
\end{array}$ & $N / A$ & N/A & $\triangle$ & \\
\hline$\frac{1.7 .6 .2 .26}{\cos ^{m}}$ & CLOSE FU-15 & $N / A$ & $N / A$ & $A$ & \\
\hline $\begin{array}{l}1.7 .6 .2 .27 \\
\end{array}$ & $\begin{array}{l}\text { ADJ SV-*62 \& } \\
\text { RECORD } \\
\text { PDIT-*60 }\end{array}$ & 21.55 & $\begin{array}{l} \pm 5^{1 "} \mathrm{H}_{2} \mathrm{O} \text { OF } \\
1.7 .6 .2 .15\end{array}$ & $\wedge$ & 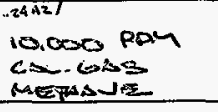 \\
\hline$\frac{1.7 .6 .2 .28}{6.94}$ & $\begin{array}{l}\text { CAL MON-*60 } \\
\text { CHANNEL B }\end{array}$ & $\frac{\frac{9999.4}{0968.1}}{00009.2}$ & $\begin{array}{l}\text { READINGS } \\
\text { WITHIN } \pm 5 \%\end{array}$ & $\Delta$ & \\
\hline $\begin{array}{c}1.7 .6 .2 .29 \\
\sin \\
\end{array}$ & OPEN FU-15 & N/A & N/A & $\Lambda$ & \\
\hline $\begin{array}{l}1.7 .6 .2 .30 \\
\text { orn }\end{array}$ & $\begin{array}{l}\text { REMOVE } \\
\text { JUMPER }\end{array}$ & N/A & N/A & $\Delta$ & \\
\hline$\dot{G}^{1.7 .2 .31}$ & $\begin{array}{l}\text { CLOSE SV-*64 } \\
\text { OPEN SV-*70 }\end{array}$ & $N / A$ & N/A & 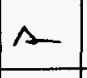 & \\
\hline$\frac{1.7 .6 .2 .32}{6 m}$ & $\begin{array}{l}\text { RECORD } \\
\text { PDIT-*60 } \gamma_{0^{4}} \mu^{\mu *^{2}} \\
\text { NIT-*52 IN }\end{array}$ & -11.56 & $\begin{array}{l}0 \text { to }-30 \\
\mathrm{NH}_{2} \mathrm{O} \\
\end{array}$ & A & \\
\hline $\begin{array}{c}1.7 .6 .2 .33 \\
6 n \\
\end{array}$ & $\begin{array}{l}\text { INSTALL } \\
\text { JUMPER }\end{array}$ & N/A & N/A & A & \\
\hline $\begin{array}{c}1.7 .6 .2 .34 \\
6 \mathrm{~m}\end{array}$ & CLOSE FU-15 & N/A & N/A & A & $H / k \downarrow$ \\
\hline
\end{tabular}


TEST DATA SHEET

\begin{tabular}{|c|c|c|c|c|c|}
\hline \multicolumn{3}{|c|}{ Date: $16 \mathrm{Sam.97}$} & SHMS Unit Number & \multicolumn{2}{|c|}{$5 x-103 \pm 7$} \\
\hline \multicolumn{3}{|c|}{$\begin{array}{l}\text { Title of Test: } \\
\text { Pneumatic Systems }\end{array}$} & \multicolumn{3}{|c|}{ Test Equipment and $\mathrm{S} / \mathrm{N}$ or Cal. No.: } \\
\hline \multicolumn{3}{|c|}{$\begin{array}{l}\text { Test Performed By: } \\
\text { Sexe Test Pezwandin Pot 2is }\end{array}$} & & 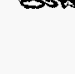 & \\
\hline $\begin{array}{l}\text { Procedure No. } \\
\text { Initial/Date }\end{array}$ & I tem & value & $\begin{array}{l}\text { Required } \\
\text { Condition }\end{array}$ & $(A / R)$ & Comment \\
\hline $\begin{array}{r}1.7 .6 .2 .35 \\
6.9 \\
\end{array}$ & $\begin{array}{l}\text { ESTABLISH } \\
\text { BYPASS FLOW }\end{array}$ & 1.6 & $\begin{array}{l}\text { FIV }-\star 70 \\
1.5 \pm .2 \mathrm{cfh}\end{array}$ & A & \\
\hline$\frac{1.7 .6 .2 .36}{67^{n}}$ & $\begin{array}{l}\text { ADJ SV-*71 } \\
\text { NIT-*52 } \\
\text { SAMPLE IN }\end{array}$ & -16.12 & $\begin{array}{l} \pm 5 \quad \mathrm{H}_{2} \mathrm{O} O \mathrm{OF} \\
1.7 .6 .2 .32\end{array}$ & $A$ & -2.56 \\
\hline $\begin{array}{l}1.7 .6 .2 .37 \\
-6.7 \\
\end{array}$ & $\begin{array}{l}\text { RECORD } \\
\text { NIT-*52 } \\
\text { SAMPLES }\end{array}$ & $\begin{array}{r}38.6 \\
38.7 \\
38.0 \\
\end{array}$ & $\begin{array}{l}\text { READINGS } \\
\text { WITHIN } \pm 5 \%\end{array}$ & $A$ & 97 \\
\hline $\begin{array}{l}1.7 .6 .2 .38 \\
\mathrm{GM}^{2} \\
\end{array}$ & OPEN FU-15 & $N / A$ & N/A & $A$ & \\
\hline $\begin{array}{r}1.7 .6 .2 .39 \\
.09 \\
\end{array}$ & $\begin{array}{l}\text { REMOVE } \\
\text { JUMPER } \\
\end{array}$ & $N / A$ & $N / A$ & $A$ & \\
\hline \multicolumn{6}{|c|}{ Section 1.7 .7 provides for test system shutdown. } \\
\hline 1.7 .7 .1 & $\begin{array}{l}\text { DISCONNECT } \\
\mathrm{H}_{3} \text { CAL GAS }\end{array}$ & $N / A$ & $N / A$ & A & \\
\hline $\begin{array}{l}1.7 .7 .2 \\
\text { on } \\
\end{array}$ & $\begin{array}{l}\text { DISCONNECT } \\
\mathrm{CH}_{6} \text { CAL GAS }\end{array}$ & $N / A$ & $N / A$ & $\Delta$ & \\
\hline $\begin{array}{l}1.7 .7 .3 \\
\operatorname{con}^{n} \\
\end{array}$ & $\begin{array}{l}\text { DISCONNECT } \\
\mathrm{NH}_{3} \text { CAL GAS }\end{array}$ & $N / A$ & $N / A$ & & \\
\hline$\frac{1.7 .7 .4}{6 m}$ & $\begin{array}{l}\text { SECURE \& } \\
\text { TURN OFF } \\
\text { ANA. INST.S }\end{array}$ & $N / A$ & $N / A$ & A & \\
\hline $\begin{array}{r}1.7 .7 .5 \\
\end{array}$ & $\begin{array}{l}\text { DISCONNECT } \\
\mathrm{N}_{2} \text { CARRIER }\end{array}$ & $N / A$ & $N / A$ & $\Delta$ & \\
\hline $1.7 .7 .6 \mathrm{~m}$ & $\begin{array}{l}\text { DISCONNECT } \\
\text { He CARRIER } \\
\end{array}$ & $N / A$ & $N / A$ & $A$ & \\
\hline $\begin{array}{r}1.7 .7 .7 \\
6\end{array}$ & $\begin{array}{l}\text { OPEN ALL } \\
\text { BREAKERS \& } \\
\text { FUSES }\end{array}$ & $N / A$ & $N / A$ & $A$ & \\
\hline $\begin{array}{r}1.7 .7 .8 \\
6 \text { h } \\
\end{array}$ & $\begin{array}{l}\text { CLOSE LISTED } \\
\text { SYS. VALVES }\end{array}$ & $N / A$ & $N / A$ & $\Delta$ & $\infty$ \\
\hline
\end{tabular}

DLC IVIII 
APPENDIX G

PAGE G-28 tan

$\therefore \ldots$.
HWF-SD-WM-ATR- 191

Rev. 0

Page 244

TEST DATA SHEET

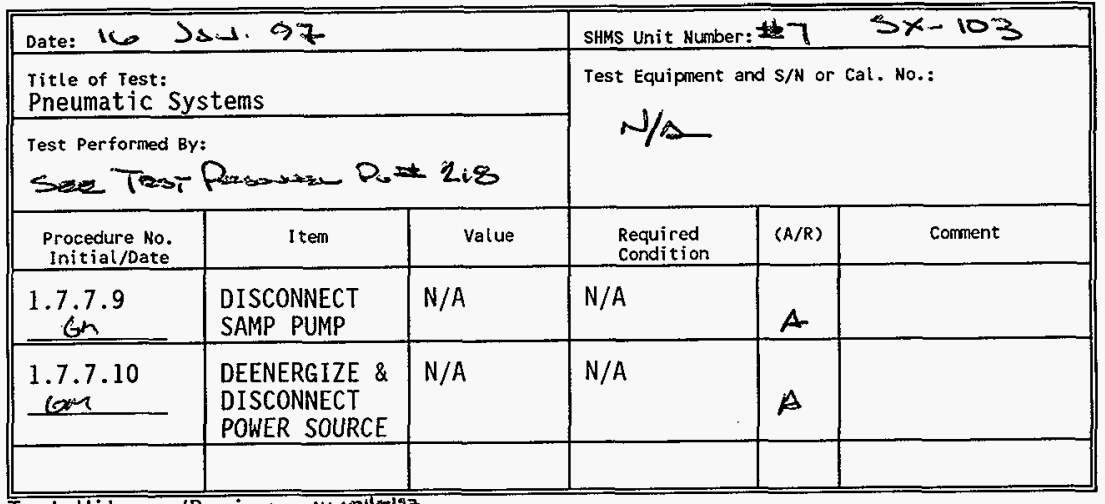

Test Witness/Review:

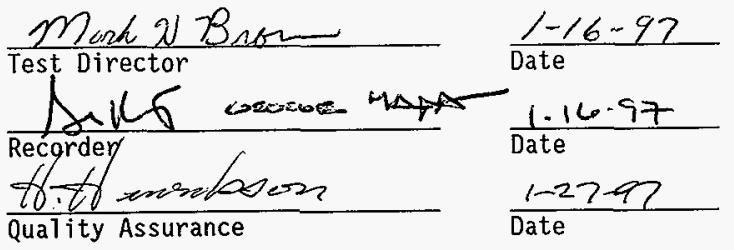


APPENDIX G

PAGE G-29
HNF-SD-WM-ATR- 191
ReV. 0

Rev. 0
Page 245

TEST EXCEPTION SHEET

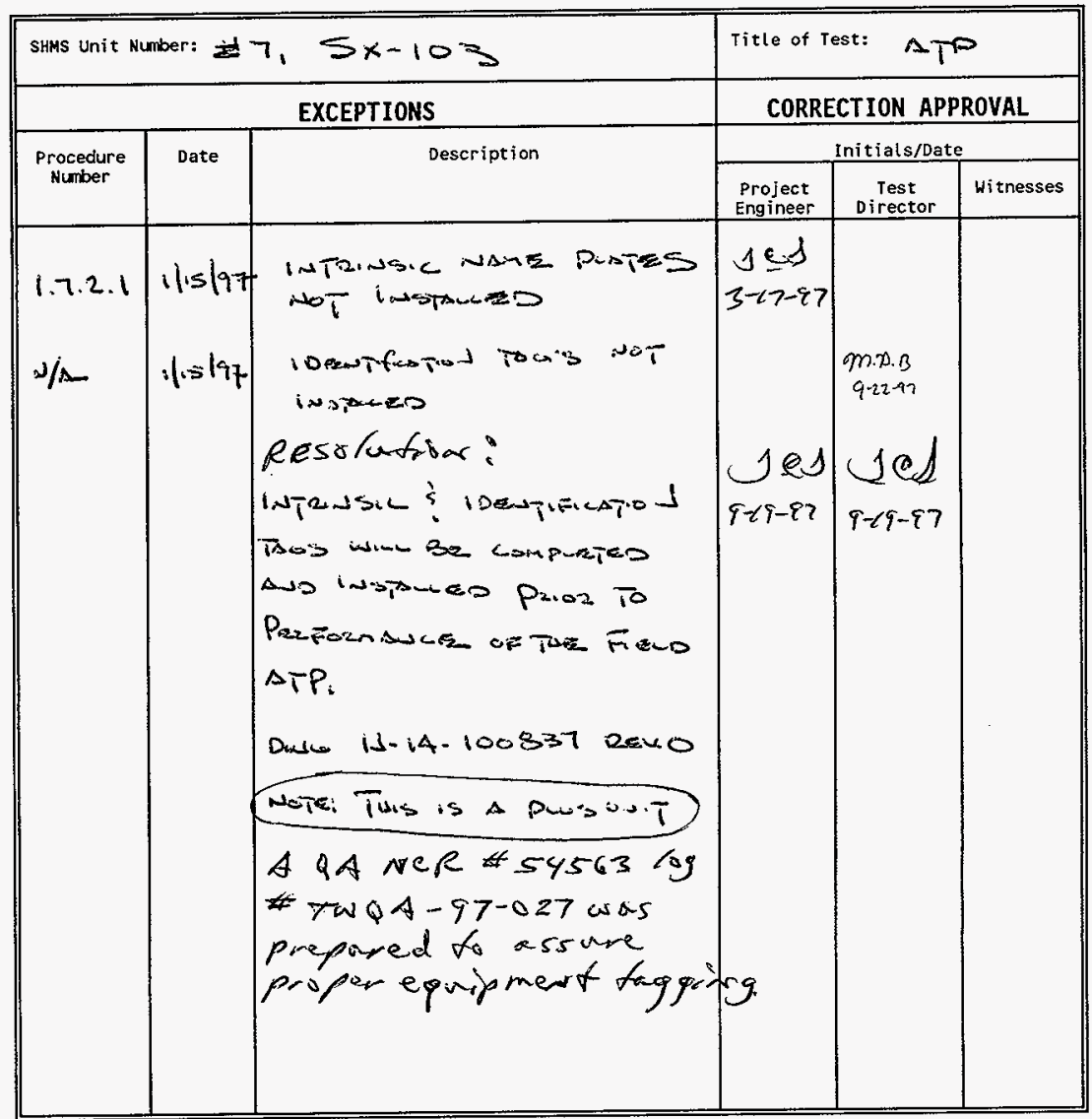

TEST APPROVED WITH EXCEPTIONS

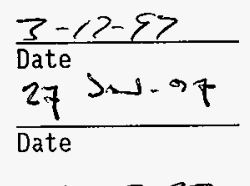

$\frac{1-27-97}{\text { Date }}$ 
APPENDIX $G$

PAGE G-30
HNF-SD-WM-ATR- 191

Rev. 0
Page 246

TEST LOG

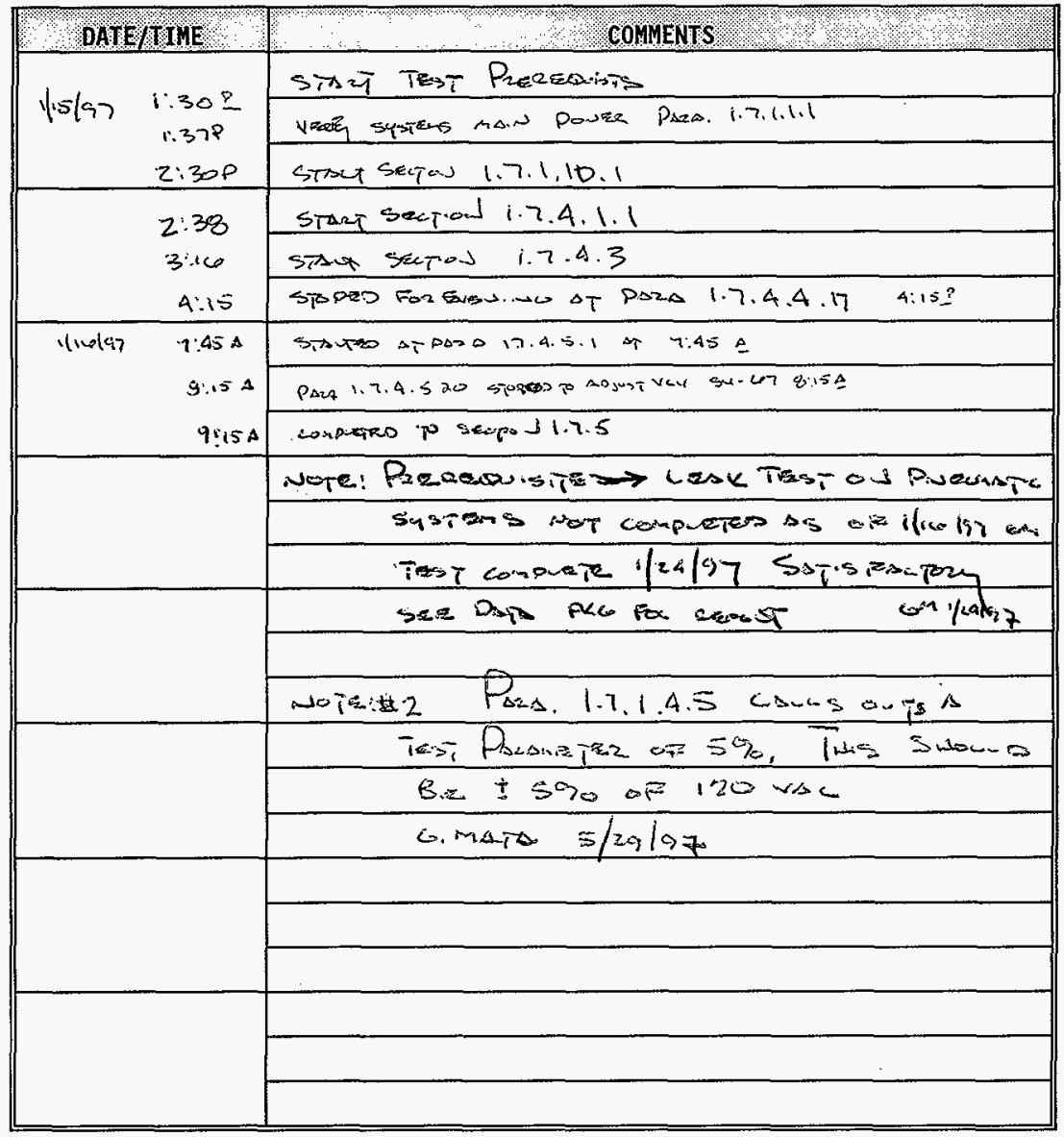

20 
APPENDIX H

PAGE H-I
HWF-SD-WM-ATR - 191

Rev. 0

Page 247

\section{APPENDIX H}

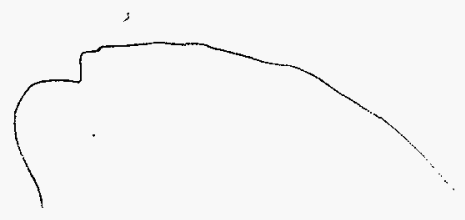


TEST EXECUTION SHEET

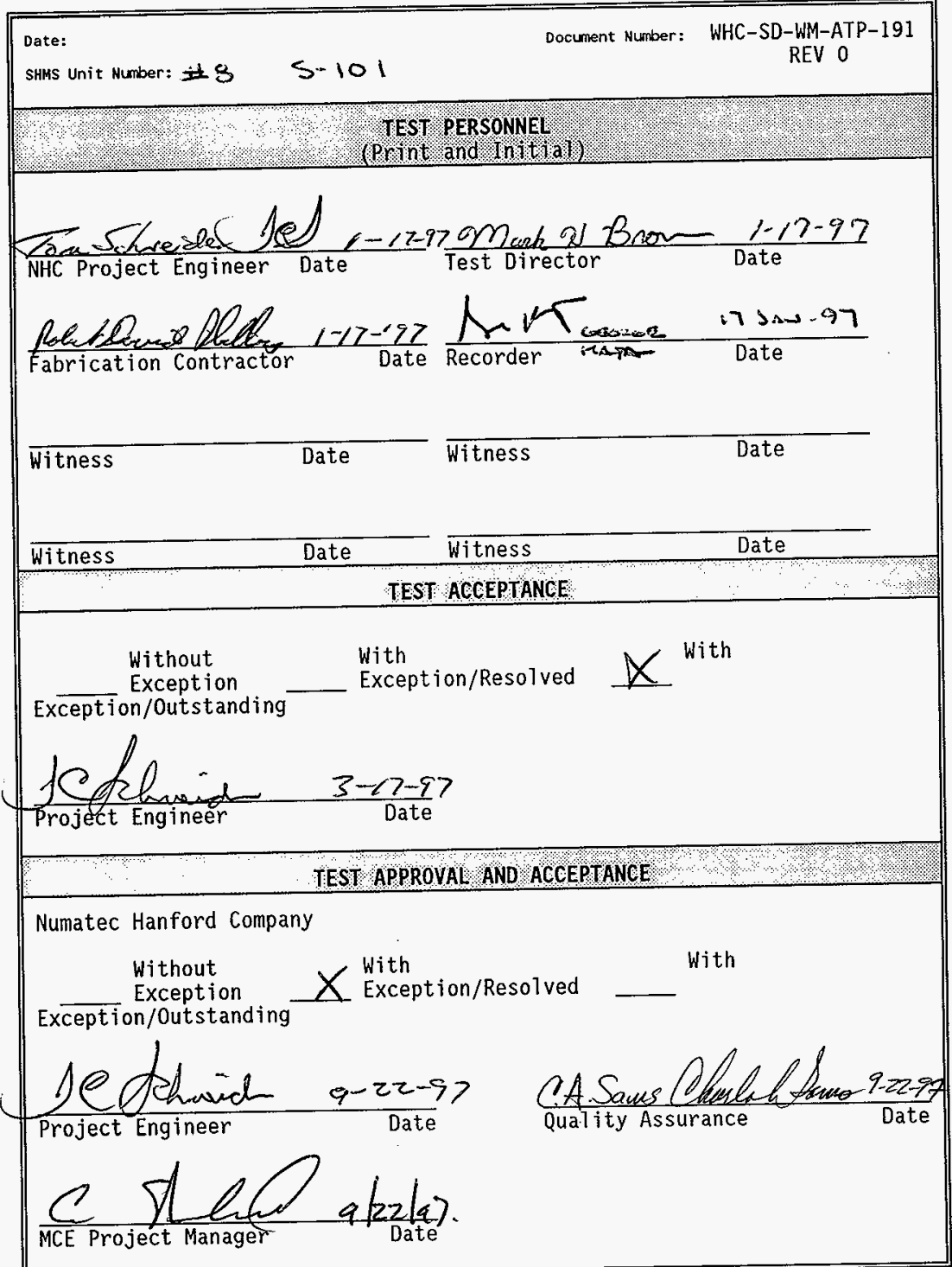


APPENDIX H

PAGE $\mathrm{H}-3$

SHMS Unit Number:

\section{PREREOUISITES AND INITIAL TEST CONDITIONS}

The following conditions shall exist at the start of the acceptance testing. Initial and date to verify that each of the following items have been accomplished.

6n Inlat Systems being tested have been inspected for workmanship and for compliance with design.

un faliz Continuity and megger tests have been performed on portions of the electrical and instrument systems being tested, as required.

en $1 / 24197$ Leak tests on the pneumatic systems have been performed.

gniflat The following circuit breakers and fuses are installed per the specified size and are open

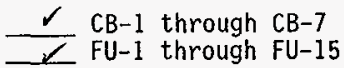

Gn likis 7 All test instruments have a currently valid calibration stamp attached that indicates a calibration traceable to the National Institute of Standards and Technology.

inlyz The following process and control instrument systems have been initiajly configured and aligned for proper operation.

FIT-*57, FSL-*57, NIT-*54, NIT-*55, (NR-*54), PDIT-*60, TIC-*50, TIC $-* 56$, TIS $* * 62$, YYC $-* 01$ NOTE: NR $-* 54$ is not used in the ANALYTICAL configuration.

Gilnkz Personnel responsible for directing and witnessing the performance of the tests described in this ATP have read and understand appropriate certified vendor information (CVI) pertaining to the operation of the equipment to be tested.

on $11.7 \mathrm{kiz}$

CLOSE all system manual valves with the exception of the following:

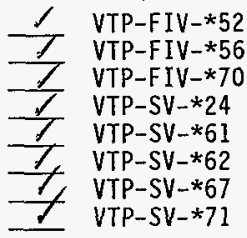

Lam $1 / 9 / 47$ The sample pump VTP-P-*50, connected to the cabinet sample out port, but vented to atmosphere for testing. 
APPENDIX $\mathrm{H}$

PAGE $\mathrm{H}-4$

HNF-SD-WM-ATR-191

Rev. 0

Page 250

con $1 / 7 / 47208$ Vac 1 phase 15-20 ampere temporary power source has been connected, but not energized, to the appropriate TBI terminals per H-14-100838.

Verify by signature and date that all prerequisites have been met.

$\frac{\text { Mand } 213 \text { Ba }_{\text {Dest Director Signature }}}{1-27-87}$




\section{TEST DATA SHEET}

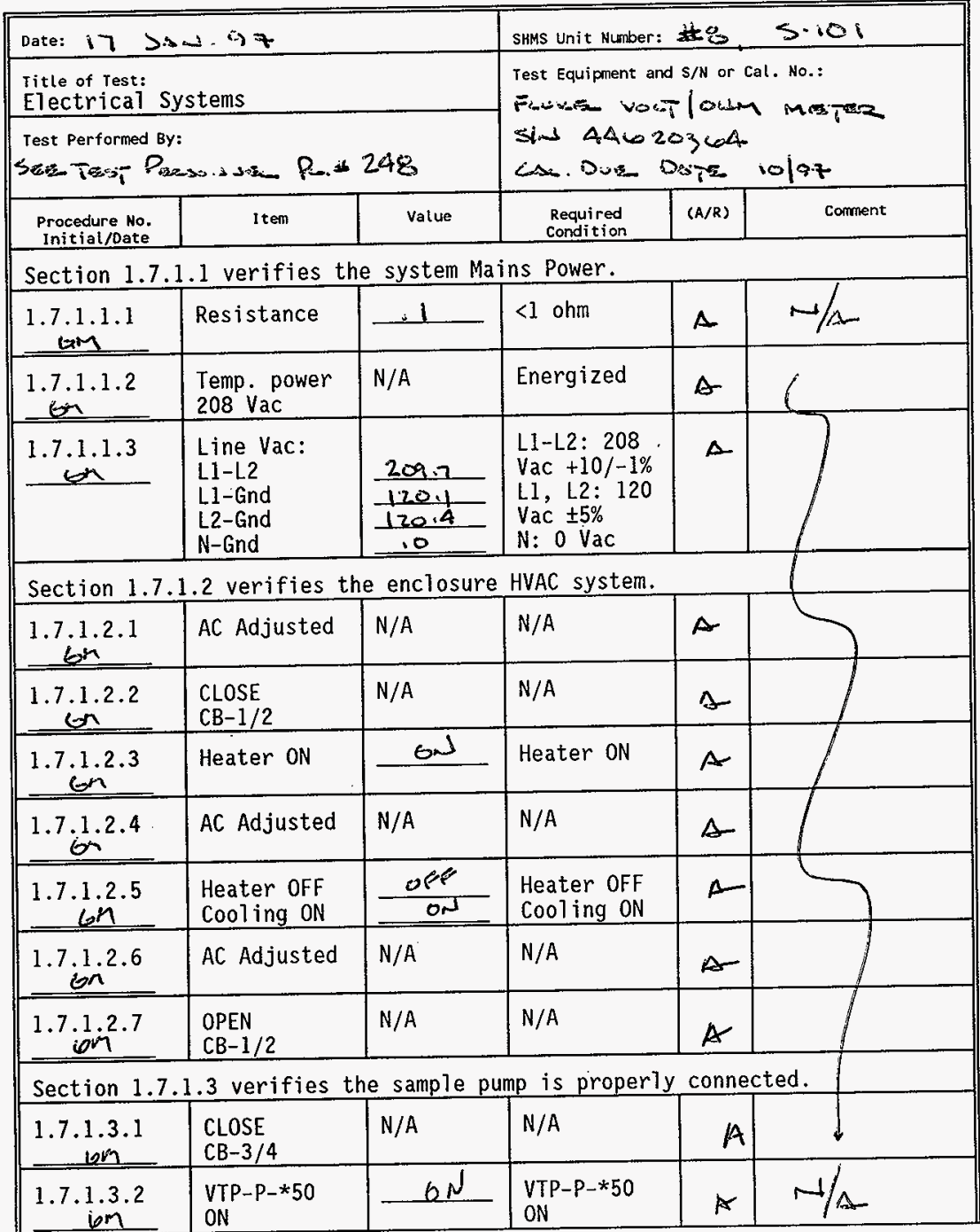


APPENDIX $\mathrm{H}$

TEST DATA SHEET

\begin{tabular}{|c|c|c|c|c|c|}
\hline \multicolumn{3}{|c|}{ Date: $17>0,0.97$} & \multicolumn{3}{|c|}{ SHMS Unit Number: $8, S-101$} \\
\hline \multicolumn{3}{|c|}{$\begin{array}{l}\text { Title of Test: } \\
\text { Electrical Systems }\end{array}$} & \multirow{2}{*}{\multicolumn{3}{|c|}{ 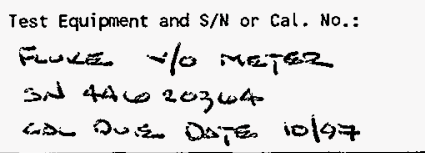 }} \\
\hline \multicolumn{3}{|c|}{ 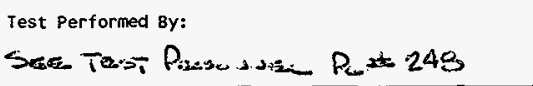 } & & & \\
\hline $\begin{array}{l}\text { Procedure No. } \\
\text { Initial/Date }\end{array}$ & Item & value & $\begin{array}{c}\text { Required } \\
\text { Condition }\end{array}$ & $(A / R)$ & Corment \\
\hline $\begin{array}{l}1.7 .1 .3 .3 \\
2^{m}\end{array}$ & $\begin{array}{l}\text { OPEN } \\
\text { CB-3/4 }\end{array}$ & N/A & N/A & A & \\
\hline \multicolumn{6}{|c|}{ Section 1.7 .1 .4 verifies the enclosure lighting and GFCI receptacles. } \\
\hline $\begin{array}{l}1.7 .1 .4 .1 \\
\mathrm{om}^{m}\end{array}$ & $\begin{array}{l}\text { CLOSE } \\
\text { CB-6 }\end{array}$ & N/A & N/A & A & \\
\hline $\mathrm{Gr}^{1.7 .2}$ & $\begin{array}{l}\text { ENCLOSURE } \\
\text { LIGHT }\end{array}$ & N/A & $N / A$ & $\Delta$ & \\
\hline $\mathrm{gin}^{1.7 .3}$ & LIGHT ON & on & LIGHT ON & $\Delta-$ & \\
\hline$\frac{1.7 .1 .4 .4}{6 M^{4}}$ & $\begin{array}{l}\text { GFCI RECP. } \\
1 A . L-N \\
1 B . L-G N D \\
2 A . L-N \\
2 B . L-G N D\end{array}$ & $\frac{\frac{120.0}{119.9}}{\frac{119.3}{120.1}} \frac{2 k}{2 k}$ & $\begin{array}{l}\text { 1A. } 120 \mathrm{Vac} \\
1 B .120 \mathrm{Vac} \\
2 \mathrm{~A} .120 \mathrm{Vac} \\
2 B .120 \mathrm{Vac} \\
\text { ALL } \pm 5 \%\end{array}$ & 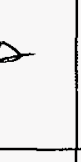 & \\
\hline$\frac{1.7 .1 .4 .5}{6 m}$ & $\begin{array}{l}\text { GFCI TEST } \\
1 A . L-N \\
\text { IB. L-GND } \\
2 A . L-N \\
\text { 2B. L-GND } \\
\end{array}$ & $\frac{\frac{.12}{.29}}{\frac{.12}{.19}}$ & $\begin{array}{l}1 A .0 \text { Vac } \\
1 B .0 \text { Vac } \\
2 A .0 \text { Vac } \\
2 B .0 \text { Vac } \\
A L L \pm 5 \%\end{array}$ & A & \\
\hline $\begin{array}{c}1.7 .1 .4 .6 \\
\end{array}$ & $\begin{array}{l}\text { ENCLOSURE } \\
\text { LIGHT OFF }\end{array}$ & N/A & N/A & $\Delta$ & \\
\hline $\begin{array}{c}1.7 .1 .4 .7 \\
6 \mathrm{~m} \\
\end{array}$ & $\begin{array}{l}\text { OPEN } \\
\text { CB-6 } \\
\end{array}$ & $N / A$ & N/A & A & \\
\hline \multicolumn{6}{|c|}{ Section 1.7.1.5 verifies the instrument power source isolation transformer. } \\
\hline $\begin{array}{r}1.7 .1 .5 .1 \\
6.9 \\
\end{array}$ & $\begin{array}{l}\text { CLOSE } \\
\text { CB-7 }\end{array}$ & N/A & $\mathrm{N} / \mathrm{A}$ & A & ( \\
\hline$\frac{1.7 .1 .5 .2}{6^{n}}$ & $\begin{array}{l}\text { XFRM } V a c \\
\text { PRI. L-N } \\
\text { SEC. L-N } \\
\text { SEC. N-GND } \\
\end{array}$ & $\begin{array}{r}120.2 \\
1237 \\
.012 \\
\end{array}$ & $\begin{array}{l}\text { PRI \& \& SEC. } \\
\text { L-N } 120 \pm 5 \% \\
\text { SEC. } \\
\text { N-GND O Vac }\end{array}$ & A & \\
\hline $1.7 .1 .5 i^{3}$ & CLOSE FU-1 & N/A & $N / A$ & A & \\
\hline
\end{tabular}


TEST DATA SHEET

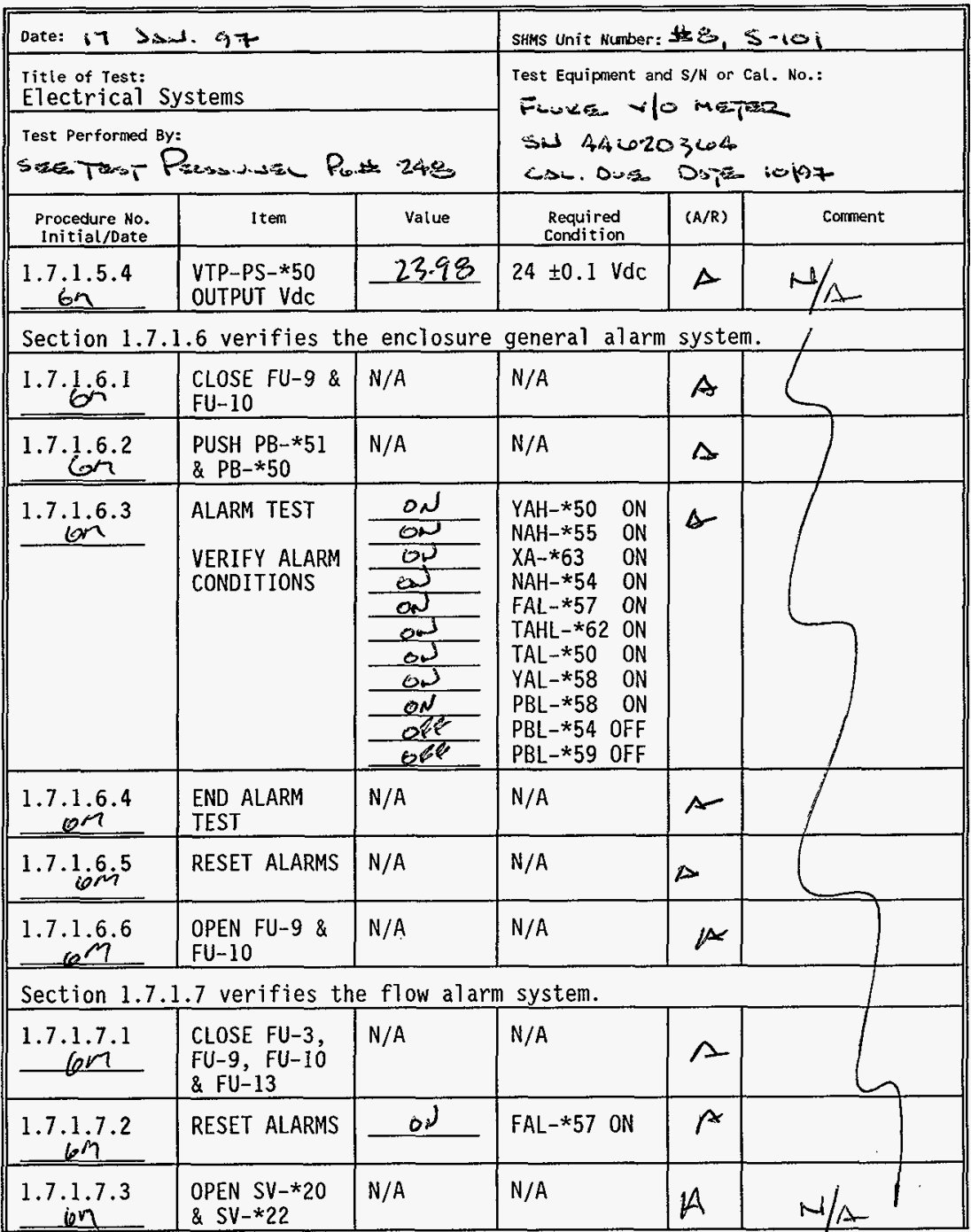


APPENDIX $\mathrm{H}$

TEST DATA SHEET

\begin{tabular}{|c|c|c|c|c|c|}
\hline \multicolumn{3}{|c|}{ Date: i7 >.s. 97} & \multicolumn{3}{|c|}{ SHMs Unit Number: $\$, S-101$} \\
\hline \multicolumn{3}{|c|}{$\begin{array}{l}\text { Title of Test: } \\
\text { Electrical Systems } \\
\end{array}$} & \multirow{2}{*}{\multicolumn{3}{|c|}{ 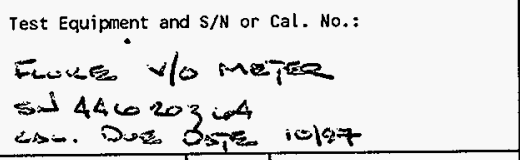 }} \\
\hline \multicolumn{3}{|c|}{ 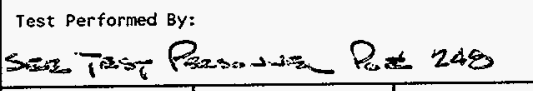 } & & & \\
\hline $\begin{array}{l}\text { Procedure No. } \\
\text { Initial/Date }\end{array}$ & Item & value & $\begin{array}{c}\text { Required } \\
\text { condition }\end{array}$ & $(\mathrm{A} / \mathrm{R})$ & Corment \\
\hline${ }_{0.7}^{1.7}$ & $\begin{array}{l}\text { FIT-*57 \& } \\
\text { FSL } * 57 \\
\text { POWERED }\end{array}$ & $\frac{O D}{O P}$ & $\begin{array}{l}\text { FIT-*57 ON } \\
\text { FSL-*57 ON }\end{array}$ & $\Delta$ & - \\
\hline $\begin{array}{l}1.7 .1 .7 .5 \\
\text { s/es }\end{array}$ & $\begin{array}{l}\text { MUX-*70 } \\
\text { TB5+/TB6- }\end{array}$ & $n / 2$ & $1.0 \pm 0.2 \mathrm{Vdc}$ & & 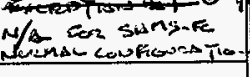 \\
\hline 1.7 .1 .7 .6 & $\begin{array}{l}\text { CLOSE SV }-* 20 \\
\& \text { SV }-* 22\end{array}$ & $N / A$ & $N / A$ & $\Delta$ & \\
\hline $\sin ^{1.7 .1 .7 .7}$ & $\begin{array}{l}\text { OPEN FU-3, } \\
\text { FU-9, FU-10 } \\
\& \text { FU-13 }\end{array}$ & $N / A$ & $N / A$ & $A$ & \\
\hline \multicolumn{6}{|c|}{ Section 1.7 .1 .8 verifies the $\mathrm{H}_{2}$ monitor wiring. } \\
\hline $\sin ^{1.7 .1 .8 .1}$ & $\begin{array}{l}\text { CLOSE FU-2, } \\
\text { FU-4, FU-5, } \\
\text { FU-9 \& FU-10 }\end{array}$ & N/A & N/A & $\Delta$ & \\
\hline $\begin{array}{l}1.7 .1 .8 .2 \\
6\end{array}$ & RESET ALARMS & $\frac{O P F}{O P F}$ & $\begin{array}{l}\text { NAH }-* 54 \text { OFF } \\
\text { NAH-*55 OFF } \\
\end{array}$ & 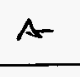 & \\
\hline$\frac{1.7 .1 .8 .3}{6.17}$ & $\begin{array}{l}\text { INSTRUMENTS } \\
\text { POWERED }\end{array}$ & 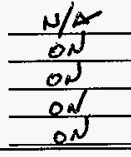 & 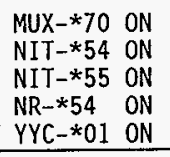 & A & \\
\hline$\frac{1.7 .1 .8 .4}{6 m}$ & $\begin{array}{l}\text { OPEN FU-2, } \\
\text { FU-4, FU-5, } \\
\text { FU-9 \& FU-10 }\end{array}$ & $N / A$ & N/A & $\boldsymbol{A}$ & \\
\hline \multicolumn{6}{|c|}{ Section 1.7.1.9 verifies the SHMS-E heat trace control system } \\
\hline $\begin{array}{c}1.7 .1 .9 .1 \\
6 \mathrm{~m} \\
\end{array}$ & $\begin{array}{l}\text { INSTALL LOAD } \\
\text { VTP-TIC-*50 }\end{array}$ & $N / A$ & $N / A$ & A & \\
\hline $\begin{array}{r}1.7 .1 .9 .2 \\
6 m^{2}\end{array}$ & $\begin{array}{l}\text { CLOSE CB-5, } \\
\text { FU-6, FU-7, } \\
\text { FU-9 \& FU-10 } \\
\text { and RESET } \\
\text { ALARMS }\end{array}$ & $N / A$ & $N / A$ & $A$ & $M / 4$ \\
\hline
\end{tabular}


TEST DATA SHEET

\begin{tabular}{|c|c|c|c|c|c|}
\hline \multicolumn{3}{|c|}{ Date: if Ds.4. 97} & \multicolumn{3}{|c|}{ SHMS Unit Number: $\$ \otimes_{i} \leq-101$} \\
\hline \multicolumn{3}{|c|}{$\begin{array}{l}\text { Title of Test: } \\
\text { Electrical Systems }\end{array}$} & \multirow{2}{*}{\multicolumn{3}{|c|}{ Test Equipment and $\mathrm{S} / \mathrm{N}$ or Cal. No.: }} \\
\hline \multicolumn{3}{|c|}{ 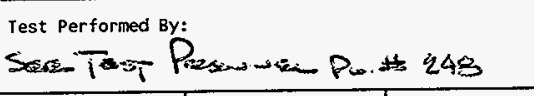 } & & & \\
\hline $\begin{array}{c}\text { Procedure No. } \\
\text { Initial/Date } \\
\end{array}$ & Item & Value & $\begin{array}{l}\text { Required } \\
\text { Condition } \\
\end{array}$ & $(A / R)$ & corment \\
\hline 6.7 .9 .3 & $\begin{array}{l}\text { TIC-*50 \& } \\
\text { TIC-*56 } \\
\text { NOMINAL TEMP }\end{array}$ & $\frac{o k}{o k}$ & $\begin{array}{l}\text { TIC }-* 50 \\
\text { TIC }-\star 56 \\
\text { TEMPERATURE }\end{array}$ & $A$ & $\Delta$ \\
\hline$\frac{1.7 .1 .9 .4}{6 \pi^{2}}$ & $\begin{array}{l}\text { ADJ TIC }-* 50 \\
\& \text { TIC }-* 56 \\
\text { SP2 \& RESET } \\
\text { ALARMS } \\
\end{array}$ & $N / A$ & N/A & $A$ & \\
\hline $\begin{array}{l}1.7 .1 .9 .5 \\
6 m^{2}\end{array}$ & $\begin{array}{l}\text { ADJ TIC }-* 50 \\
\text { SP } 1\end{array}$ & $N / A$ & $N / A$ & A & \\
\hline $\begin{array}{c}1.7 .1 .9 .6 \\
\end{array}$ & $\begin{array}{l}\text { ADJ TIC-*50 } \\
\text { SP } 2\end{array}$ & $N / A$ & N/A & $\wedge$ & \\
\hline $\begin{array}{c}1.7 .1 .9 .7 \\
\end{array}$ & $\begin{array}{l}\text { TAL }-* 50 \& \\
\text { HORN ACTIVE }\end{array}$ & $\frac{o r}{o r}$ & $\begin{array}{l}\text { TAL-*50 ON } \\
\text { HORN ON } \\
\text { ACKNOWLEDGE }\end{array}$ & $A$ & \\
\hline$\frac{1.7 .1 .9 .8}{6 n}$ & $\begin{array}{l}\text { TIC }-\star 50 \\
\text { OPERATES }\end{array}$ & OK & $\begin{array}{l}\text { TIC }-* 50 \\
\text { OPERATES }\end{array}$ & $p$ & \\
\hline $\begin{array}{r}1.7 .1 .9 .9 \\
6 m^{\circ}\end{array}$ & $\begin{array}{l}A D J T I C-* 50 \\
S P 2\end{array}$ & N/A & $\mathrm{N} / \mathrm{A}$ & $\Lambda$ & \\
\hline $\begin{array}{r}1.7 .1 .9 .9^{0} \\
\end{array}$ & RESET ALARM & $0^{41}$ & TAL $-\star 50 \quad$ OFF & $\Delta$ & \\
\hline$\frac{1.7 .1 .9 \mathrm{~m}^{11}}{6}$ & $\begin{array}{l}\text { ADJ TIC- } * 50 \\
S P 1 \& S P 2\end{array}$ & $N / A$ & $N / A$ & $A$ & \\
\hline${ }^{1.7 .1 .9 .12}$ & $\begin{array}{l}\text { OPEN CB-5 \& } \\
\text { REMOVE LOAD } \\
\end{array}$ & $N / A$ & $N / A$ & $A$ & \\
\hline $\begin{array}{c}1.7 .1 .9 .13 \\
6 m^{2} \\
\end{array}$ & $\begin{array}{l}\text { INSTALL LOAD } \\
\text { VTP-TIC-*56 }\end{array}$ & $N / A$ & $N / A$ & P & \\
\hline $\begin{array}{c}1.7 .1 .9 .14 \\
\mathrm{cen}\end{array}$ & CLOSE CB-5 & $N / A$ & $N / A$ & $\mathbb{A s}$ & \\
\hline$\frac{1.7 .1 .9 .15}{6 \text { in }^{-1}}$ & $\begin{array}{l}\text { ADJ TIC-*56 } \\
\text { SP } 1\end{array}$ & $N / A$ & $N / A$ & $\boldsymbol{p}$ & \\
\hline$\frac{1.7 .1 .99 \mathrm{i}^{16}}{6}$ & $\begin{array}{l}\text { ADJ TIC-*56 } \\
\text { SP } 2\end{array}$ & N/A & N/A & 4 & $N / \Delta$ \\
\hline
\end{tabular}


APPENDIX H

TEST DATA SHEET

\begin{tabular}{|c|c|c|c|c|c|}
\hline \multicolumn{3}{|c|}{ Date: $17>9 \rightarrow .97$} & \multicolumn{3}{|c|}{ SHmS Unit Number: 3,5101} \\
\hline \multicolumn{3}{|c|}{$\begin{array}{l}\text { Title of rest: } \\
\text { Electrical Systems }\end{array}$} & \multirow{2}{*}{\multicolumn{3}{|c|}{ Test Equipment and $S / \mathrm{N}$ or Cal. No.: }} \\
\hline \multicolumn{3}{|c|}{ 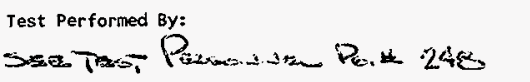 } & & & \\
\hline $\begin{array}{l}\text { Procedure No. } \\
\text { Initial/Date }\end{array}$ & I tem & Value & $\begin{array}{c}\text { Required } \\
\text { Condition }\end{array}$ & $(A / R)$ & Comment \\
\hline$\frac{1.7 .1 .9 .17}{\mathrm{Gm}}$ & $\begin{array}{l}\text { TAL }-* 50 \& \\
\text { HORN ACTIVE }\end{array}$ & $\frac{0 h}{6 \%}$ & $\begin{array}{l}\text { TAL-*50 ON } \\
\text { HORN ON } \\
\text { ACKNOWLEDGE }\end{array}$ & $\Delta$ & \\
\hline $\begin{array}{r}1.7 .1 .9 .18 \\
\operatorname{con} \\
\end{array}$ & $\begin{array}{l}\text { TIC }-* 56 \\
\text { OPERATES } \\
\end{array}$ & $O K$ & $\begin{array}{l}\text { TIC }-* 56 \\
\text { OPERATES }\end{array}$ & 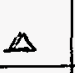 & \\
\hline $\begin{array}{c}1.7 .1 .9 .19 \\
\end{array}$ & $\begin{array}{l}\text { ADJ TIC }-* 56 \\
\text { SP } 2\end{array}$ & $N / A$ & $N / A$ & $A$ & \\
\hline $\begin{array}{l}1.7 .1 .9 .20 \\
\end{array}$ & RESET ALARM & etp & TAL-*50 OFF & $A$ & \\
\hline $\begin{array}{r}1.7 .1 .9 .21 \\
6.9 \\
\end{array}$ & $\begin{array}{l}\text { ADJ TIC }-* 56 \\
\text { SP } 1 \text { \& SP 2 }\end{array}$ & $N / A$ & $N / A$ & $A$ & \\
\hline $\begin{array}{r}1.7 .1 .9 .22 \\
\end{array}$ & $\begin{array}{l}\text { OPEN CB-5, } \\
\text { FU-6 \& FU-7 }\end{array}$ & $N / A$ & $N / A$ & $A$ & \\
\hline $\begin{array}{l}1.7 .1 .9 .23 \\
\operatorname{Com} \\
\end{array}$ & $\begin{array}{l}\text { REMOVE LOAD } \\
\text { TIC }-\star 56\end{array}$ & $\mathrm{~N} / \mathrm{A}$ & $N / A$ & & \\
\hline$\frac{1.7 .1 .9 .24}{67^{-19}}$ & $\begin{array}{l}\text { CLOSE FU-8 } \\
\text { TIS }-* 62 \\
\text { NOMINAL TEMP }\end{array}$ & $0<$ & $\begin{array}{l}\text { TIS }-* 62 \\
\text { TEMP }\end{array}$ & A & \\
\hline $\begin{array}{r}1.7 .1 .9 .25 \\
.0 .2 \\
\end{array}$ & $\begin{array}{l}\text { ADJ TIS }-* 62 \\
\text { SP } 1\end{array}$ & $N / A$ & $N / A$ & $A$ & \\
\hline $\begin{array}{c}1.7 .1 .9 .26 \\
\end{array}$ & $\begin{array}{l}\text { ADJ TIS }-\star 62 \\
\text { SP } 2\end{array}$ & $N / A$ & $N / A$ & 7 & \\
\hline $\begin{array}{r}1.7 .1 .9 \dot{m}^{27} \\
\end{array}$ & RESET ALARMS & OH & $\begin{array}{l}\text { TAHL }-* 62 \\
\text { OFF }\end{array}$ & $A$ & \\
\hline $1.7 .1 .9{ }_{6}^{9} r^{88}$ & $\begin{array}{l}\text { ADJ TIS-*62 } \\
\text { SP } 2\end{array}$ & $N / A$ & $N / A$ & $A$ & \\
\hline$\frac{1.7 .1 .9 .29}{6 n}$ & $\begin{array}{l}\text { TAHL-*62 \& } \\
\text { HORN ACTIVE }\end{array}$ & $\begin{array}{r}02 \\
0 \\
\end{array}$ & $\begin{array}{l}\text { TAHL }-* 62 \text { ON } \\
\text { HORN ON } \\
\text { ACKNOWLEDGE }\end{array}$ & p & \\
\hline $1.7 .1 .9 . n^{30}$ & $\begin{array}{l}\text { ADJ TIS }-* 62 \\
\text { SP } 2\end{array}$ & $N / A$ & $\mathrm{~N} / \mathrm{A}$ & $A$ & $N / A_{-}$ \\
\hline
\end{tabular}


TEST DATA SHEET

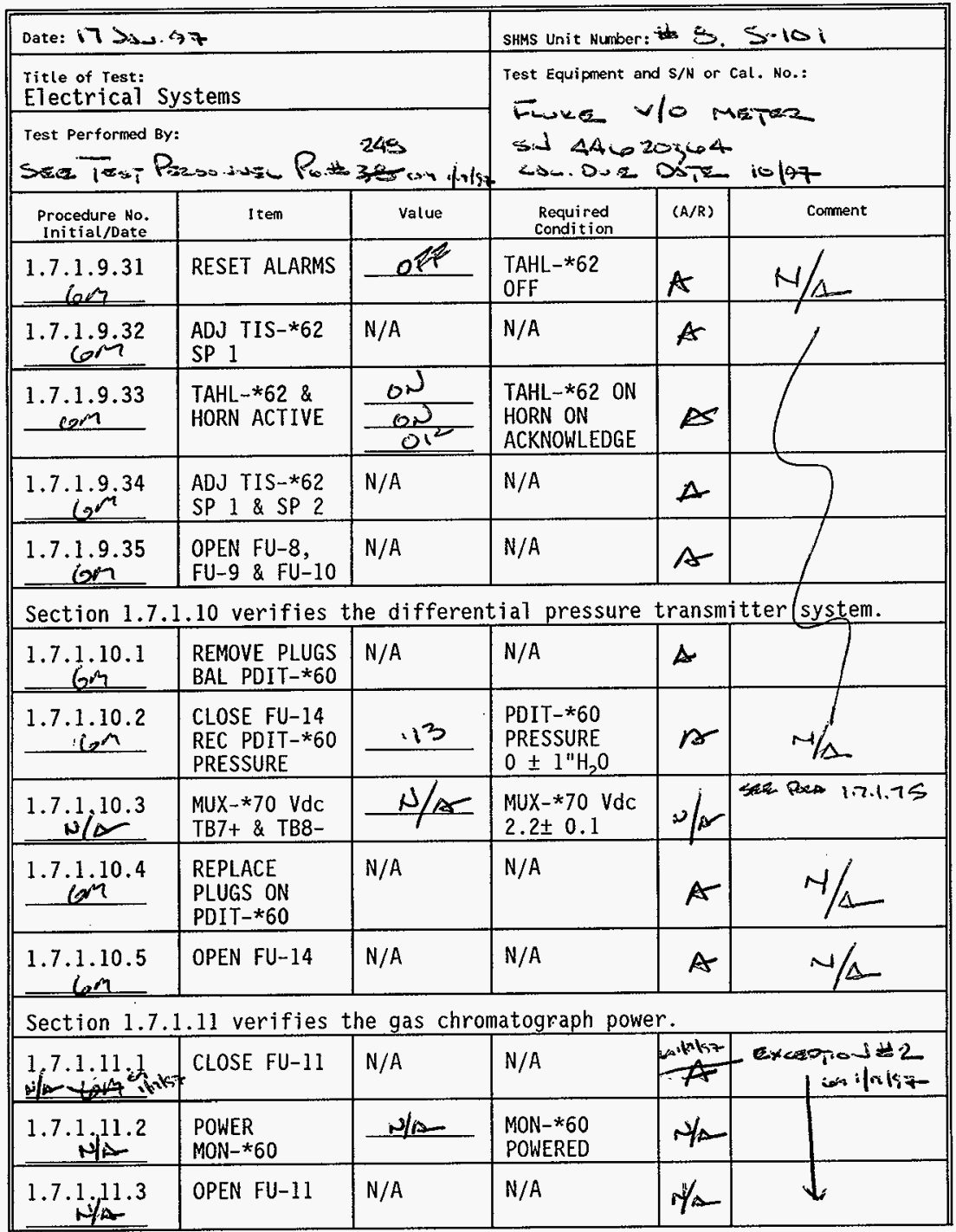




\section{TEST DATA SHEET}

\begin{tabular}{|c|c|c|c|c|c|}
\hline \multicolumn{3}{|c|}{ Date: 17 SA. 97} & \multicolumn{3}{|c|}{ SHMS Unit Number: $z$} \\
\hline \multicolumn{3}{|c|}{$\begin{array}{l}\text { Title of Test: } \\
\text { Electrical Systems }\end{array}$} & \multirow{2}{*}{\multicolumn{3}{|c|}{ Test Equipment and $S / \mathrm{N}$ or Cal. Ho.: }} \\
\hline \multicolumn{3}{|c|}{$\begin{array}{l}\text { Test Performed By: } \\
\end{array}$} & & & \\
\hline $\begin{array}{l}\text { Procedure No. } \\
\text { Initial/Date }\end{array}$ & I tem & Value & $\begin{array}{l}\text { Required } \\
\text { Condition }\end{array}$ & $(A / R)$ & Conr \\
\hline \multicolumn{6}{|c|}{ Section 1.7 .1 .12 verifies the multi gas analyzer power. } \\
\hline 1.7 .1 .12 .1 & CLOSE FU-12 & $N / A$ & N/A & & $\begin{array}{l}5=2 \\
+2\end{array}$ \\
\hline $1.7 .(1.12 .2$ & $\begin{array}{l}\text { POWER } \\
\text { NIT-*52 }\end{array}$ & $\mu / A$ & $\begin{array}{l}\text { NIT-*52 } \\
\text { POWERED }\end{array}$ & & \\
\hline $1.7 / 1.12 .3$ & OPEN FU-12 & $N / A$ & $N / A$ & & \\
\hline \multicolumn{6}{|c|}{ Section 1.7 .1 .13 verifies the personnel computer power. } \\
\hline 1.7 .13 .1 & CLOSE CB-6 & $N / A$ & $N / A$ & & \\
\hline $1.7\{1.13 .2$ & $\begin{array}{l}\text { POWER PC-*60 } \\
\& P C-* 70\end{array}$ & $\frac{N / N}{1 / N-}$ & $\begin{array}{ll}\mathrm{PC}-{ }^{*} 60 & 0 \mathrm{~N} \\
\mathrm{PC}-* 70 & \mathrm{ON} \\
\end{array}$ & & \\
\hline $1.7 \sqrt{13.3}$ & $\begin{array}{l}\text { TURN INST. } \\
\text { OFF \& } \\
\text { OPEN CB-6 }\end{array}$ & $\frac{N / A-}{N / A-}$ & $\begin{array}{ll}P C-* 60 & \text { OFF } \\
P C-* 70 & \text { OFF }\end{array}$ & $3 / 1$ & \\
\hline
\end{tabular}




\section{TEST DATA SHEET}

\begin{tabular}{|c|c|c|c|c|c|}
\hline \multicolumn{3}{|c|}{ Date: if DA. 97} & \multicolumn{3}{|c|}{ SHMS Unit Number: $8,5 . i 01$} \\
\hline \multicolumn{3}{|c|}{$\begin{array}{l}\text { Title of Test: } \\
\text { Electrical Systems }\end{array}$} & \multirow{2}{*}{\multicolumn{3}{|c|}{ 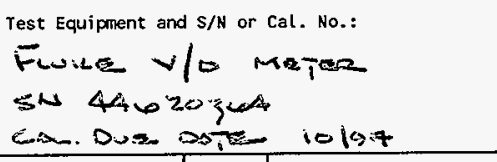 }} \\
\hline \multicolumn{3}{|c|}{$\begin{array}{l}\text { Test Performed By: } \\
248\end{array}$} & & & \\
\hline $\begin{array}{l}\text { Procedure No. } \\
\text { Initial/Date }\end{array}$ & It tem & Value & $\begin{array}{l}\text { Required } \\
\text { Condition } \\
\end{array}$ & $(A / R)$ & Comnent \\
\hline \multicolumn{6}{|c|}{ Section 1.7 .2 verifies the intrinsic safety apparatus is properiy 1 abeled. } \\
\hline$\frac{1.7 j \dot{e}^{2} j^{1}}{\varepsilon-22-\varepsilon 7}$ & $\begin{array}{l}\text { INTRINSIC } \\
\text { SAFETY } \\
\text { APPARATUS } \\
\text { NAMEPLATES } \\
\text { INSTALLED }\end{array}$ & 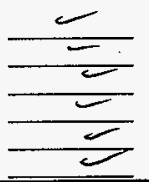 & $\begin{array}{l}N E-* 54 \\
N E-* 55 \\
E B-* 54 \\
E B-* 55 \\
N I T-* 54 \\
N I T-* 55\end{array}$ & A & Ex<apionj 1 \\
\hline 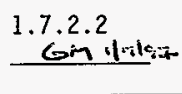 & $\begin{array}{l}\text { INTRINSIC } \\
\text { SAFETY WIRE } \\
\text { LABELED }\end{array}$ & $\frac{a k}{O K}$ & $\begin{array}{l}\mathrm{NE} / \mathrm{EB}-* 54 \\
\mathrm{NE} / \mathrm{EB}-* 55\end{array}$ & 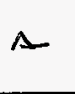 & $-1 / A$ \\
\hline $\begin{array}{l}1.7 .399 .22 \cdot 97 \\
m \cdot 0 \cdot \beta\end{array}$ & $\begin{array}{l}\text { SEC. } 1.7 .1 \text { \& } \\
1.7 .2 \text { DONE }\end{array}$ & $\frac{\text { Mad D }}{\text { Test Direc }}$ & $\frac{\text { Bnow }}{\text { or signature }}$ & & $\frac{9.22 .97}{\text { Date }}$ \\
\hline
\end{tabular}

Test Witness/Review:

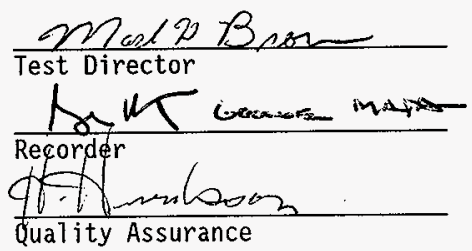

$\frac{1-17-97}{\text { Date }}$
$\frac{17 \text { Das.97 }}{\text { Date }}$
$\frac{1-27-97}{\text { Date }}$


APPENDIX $\mathrm{H}$

HNF-SD-WM-ATR-191
ReV. 0

Rage 260

TEST DATA SHEET

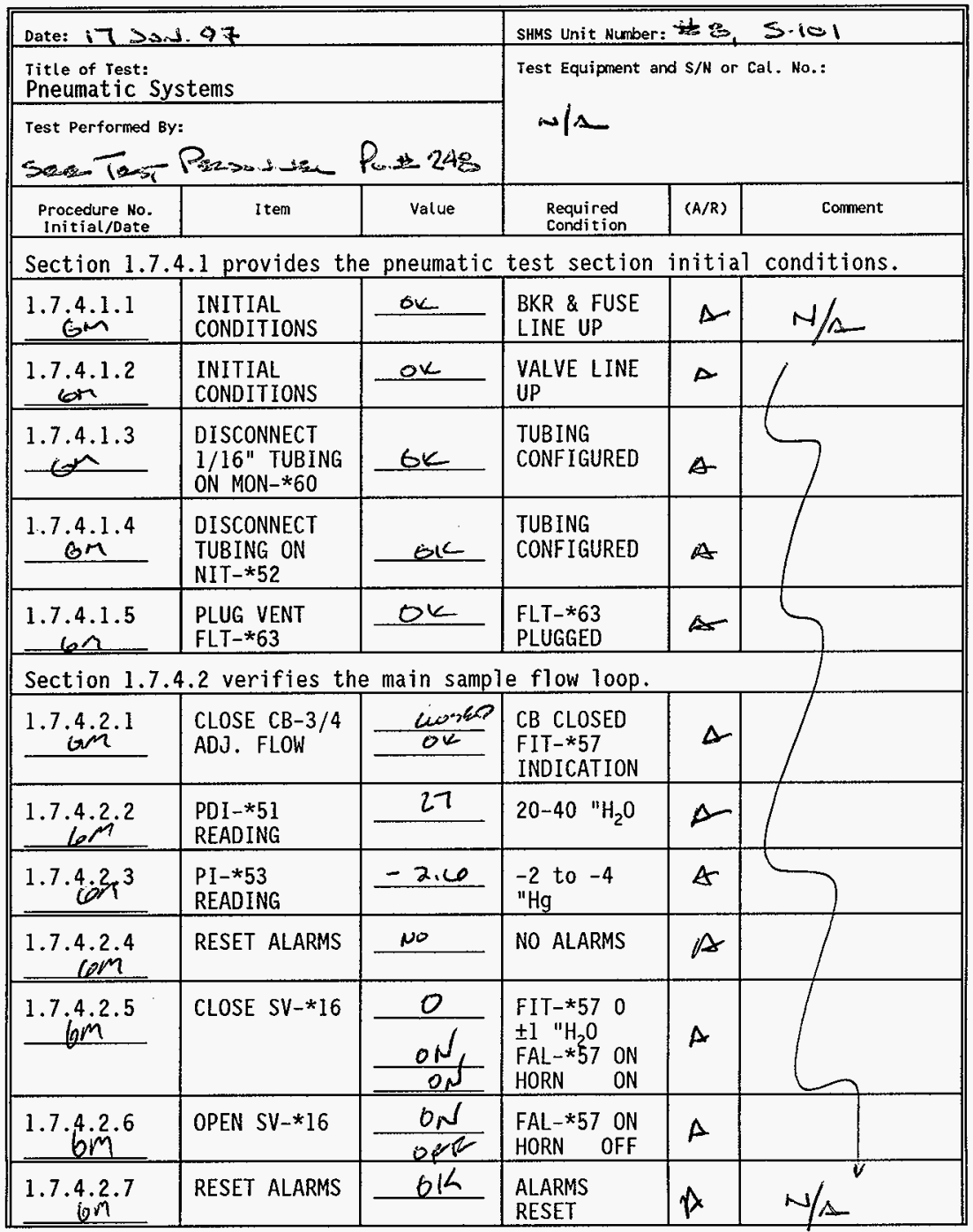

AL 2 inlet G on. 
TEST DATA SHEET

\begin{tabular}{|c|c|c|c|c|c|}
\hline \multicolumn{3}{|c|}{ Date: $17 \supset x^{3}, 97$} & \multicolumn{3}{|c|}{ SHMS Unit Number: $\$$ S.10, } \\
\hline \multicolumn{3}{|c|}{$\begin{array}{l}\text { Title of Test: } \\
\text { Pneumatic Systems }\end{array}$} & \multirow{2}{*}{\multicolumn{3}{|c|}{ Test Equipment and $\mathrm{S} / \mathrm{N}$ or Cal. No.: }} \\
\hline \multicolumn{3}{|c|}{ 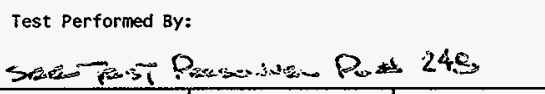 } & & & \\
\hline $\begin{array}{l}\text { Procedure Ho. } \\
\text { Initial/Date }\end{array}$ & Item & value & $\begin{array}{l}\text { Required } \\
\text { condition }\end{array}$ & $(A / R)$ & Comment \\
\hline$\frac{1: 7.4 .2 .8}{o n}$ & CLOSE SV $-* 25$ & 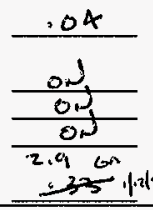 & 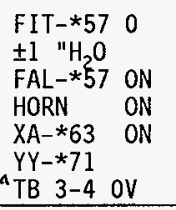 & $\Delta$ & $\begin{aligned} \text { Whats } & Y Y-71-2 \\
& Y Y<-03\end{aligned}$ \\
\hline $\begin{array}{c}1.7 .4 .2 .9 \\
\end{array}$ & OPEN SV-*25 & $\frac{\frac{O N}{O E}}{\frac{O P}{121.4}}$ & $\begin{array}{l}\text { FAL }-* 57 \text { ON } \\
\text { HORN OFF } \\
\text { XA-*63 OFF } \\
\text { YY-*71 } \\
\text { TB } 3-4 \quad 120 \mathrm{~V} \\
\end{array}$ & $\triangle$ & \\
\hline $\begin{array}{c}1.7 .4 .2 .10 \\
.94 \\
\end{array}$ & RESET ALARMS & OK & $\begin{array}{l}\text { ALARMS } \\
\text { RESET }\end{array}$ & $\Delta$ & \\
\hline$\frac{1.7 .4 .2 .11}{\sin ^{2}}$ & $\begin{array}{l}\text { OPEN SV-*5 } A^{\prime} \\
\& \text { SV-*66 } \\
\text { READ PRESS. } \\
\text { PDIT }-* 60 \\
\end{array}$ & $\frac{0+45}{-4.70}$ & $\begin{array}{l}\text { VALVES OPEN } \\
-27 \text { to }-82 \\
" \mathrm{H}_{2} \mathrm{O}\end{array}$ & A & \\
\hline$\frac{1.7 .4 .2 .12}{6 m^{m}}$ & $\begin{array}{l}\text { CLOSE SV }-* 54 \\
\text { OPEN SV-*63/ } \\
\text { READ PRESS. } \\
\text { PDIT-*60 }\end{array}$ & .125 & $\begin{array}{l}\text { VALVES } \\
\text { OPERATED } \\
0 \pm 1 " \mathrm{H}_{2} \mathrm{O}\end{array}$ & A & \\
\hline $\begin{array}{r}1.7 .4 .2 .13 \\
6 m^{13} \\
\end{array}$ & $\begin{array}{l}\text { CLOSE SV-*63 } \\
\text { OPEN SV-*55 } \\
\text { READ PRESS. } \\
\text { PDIT-*60 } \\
\end{array}$ & $\frac{\text { ok }}{-43.27}$ & $\begin{array}{l}\text { VALVES } \\
\text { OPERATED } \\
-27 \text { to }-82 \\
" \mathrm{H}_{2} \mathrm{O} \\
\end{array}$ & A & \\
\hline$\frac{1.7 .4 i^{2.14}}{6}$ & $\begin{array}{l}\text { CLOSE SV }-* 55 y \\
\text { OPEN SV } * 64 \\
\text { READ PRESS. } \\
\text { PDIT-*60 } \\
\end{array}$ & $\frac{.0 K}{.1307}$ & $\begin{array}{l}\text { VALVES } \\
\text { OPERATED } \\
\mathrm{O} \pm 1 " \mathrm{H}_{2} \mathrm{O}\end{array}$ & r & \\
\hline 1.7 .4 .2 .15 & $\begin{array}{l}\text { CLOSE SV-*64 } \\
\& S V-* 66 \quad V\end{array}$ & $N / A$ & $N / A$ & A & \\
\hline \multicolumn{6}{|c|}{ Section 1.7.4.3 verifies the grab sample flow loop. } \\
\hline $\begin{array}{r}1.7 .4 .3 \mathrm{~m}^{1} \\
\end{array}$ & READ PDI $* 51$ & 27 & $20-40 " \mathrm{H}_{2} \mathrm{O}$ & $F$ & from \\
\hline
\end{tabular}


TEST DATA SHEET

\begin{tabular}{|c|c|c|c|c|c|}
\hline Date: i7 $\mathrm{S}$ & 47 & & SHMS Unit Number: & 18 & $3 \cdot 101$ \\
\hline $\begin{array}{l}\text { Title of Test: } \\
\text { Pneumatic S }\end{array}$ & tems & & Test Equipment an & $S / N$ or & 1. No.: \\
\hline $\begin{array}{l}\text { Test Performed } \\
\text { Sez }\end{array}$ & zesecosiz- $D_{0}$ & $=245$ & N/A & & \\
\hline $\begin{array}{l}\text { Procedure No. } \\
\text { Initial/Date }\end{array}$ & Item & Value & $\begin{array}{l}\text { Required } \\
\text { condition }\end{array}$ & $(A / R)$ & Corment \\
\hline $\begin{array}{r}1.7 .4 .3 .2 \\
6 \mathrm{~m} \\
\end{array}$ & $\begin{array}{l}\text { PUSH PB-*59 } \\
\text { GRAB SAMPLE } \\
\end{array}$ & ok & $\begin{array}{l}\text { START } \\
\text { STOPWATCH }\end{array}$ & $A$ & \\
\hline$\frac{1.7 .4 .3 .3}{6 i}$ & $\begin{array}{l}\text { GRAB SAMPLE } \\
\text { LAMPS ON } \\
\text { READ FIV-*52 }\end{array}$ & $\frac{a}{a}$ & $\begin{array}{l}\text { PBL-*58 ON } \\
\text { PBL-*59 ON } \\
\text { YAL-*58 ON } \\
\text { FIV }-* 52 \quad 0 \\
\end{array}$ & $\Delta$ & \\
\hline $\begin{array}{r}1.7 .4 .3 .4 \\
\end{array}$ & $\begin{array}{l}\text { OPEN SV }-* 15 \\
\& \text { ADJ. FIV- } \\
* 52\end{array}$ & $\frac{o p a x}{10}$ & $\begin{array}{l}\text { SV-* } 15 \text { OPEN } \\
\text { FIV-*52 } \\
\text { FLOW } 10 \text { CFH }\end{array}$ & $A$ & \\
\hline$\frac{1.7 .4 .3 .5}{6 r}$ & READ PDI $-* 51$ & 31 & $\begin{array}{l}\text { PDI }-* 51 \\
\text { HIGHER THAN } \\
1.7 .4 .3 .1 \\
\end{array}$ & $\Delta$ & \\
\hline $\begin{array}{c}1.7 .4 .3 .6 \\
\cos ^{6}\end{array}$ & CLOSE SV-*15 & 0 & FIV $-* 520$ & $\beta$ & \\
\hline $\begin{array}{c}1.7 .4 .3 .7 \\
609 \\
\end{array}$ & OPEN SV-*15 & 10 & $\begin{array}{l}\text { FIV-*52 } \\
\text { FLOW } \\
\end{array}$ & $A$ & \\
\hline$\frac{1.7 .4 .3 .8}{6 \mathrm{~m}^{8}}$ & $\begin{array}{l}\text { PBL }-* 59 \\
\text { GRAB SAMPLE } \\
\text { TIME }\end{array}$ & $\frac{\text { ofe }}{4.59}$ & $\begin{array}{l}\text { PBL-*59 OFF } \\
\text { STOP WATCH } \\
5 \pm .5 \text { MIN } \\
\end{array}$ & $\mu$ & \\
\hline $\begin{array}{r}1.7 .4 .3 .9 \\
0 \mathrm{~m}^{2} \\
\end{array}$ & $\begin{array}{l}\text { PB-*58 RESET } \\
\text { SAMPLER }\end{array}$ & $\frac{o H^{2}}{o^{2}}$ & $\begin{array}{l}\mathrm{PBL}-* 58 \text { OFF } \\
\mathrm{YAL}-* 58 \text { OFF }\end{array}$ & $A$ & \\
\hline Section 1.7 & .4 verifies $t$ & $\mathrm{H}_{2} \mathrm{cell}$ & ibration 100 & & \\
\hline $\begin{array}{c}1.7 .4 .4 .1 \\
\mathrm{gin}\end{array}$ & $\begin{array}{l}\text { CONNECT } \mathrm{H}_{2} \\
\text { CAL GAS }\end{array}$ & $N / A$ & $N / A$ & $A$ & \\
\hline \begin{tabular}{|l}
1.7 .4 .4 .2 \\
s.4
\end{tabular} & $\begin{array}{l}\text { OPEN ISO } \\
\text { VALVE }\end{array}$ & 4.5 & $<10$ PSIG & $A$ & \\
\hline $\begin{array}{c}1.7 .4 .4 .3 \\
\text { ion }\end{array}$ & $\begin{array}{l}\text { OPEN SV-*18 } \\
\text { ADJ. FIV-*56 }\end{array}$ & 2 & $2 \pm .1 \mathrm{CFH}$ & $A$ & $M / A$ \\
\hline
\end{tabular}

Au in/57 
TEST DATA SHEET

IItia-

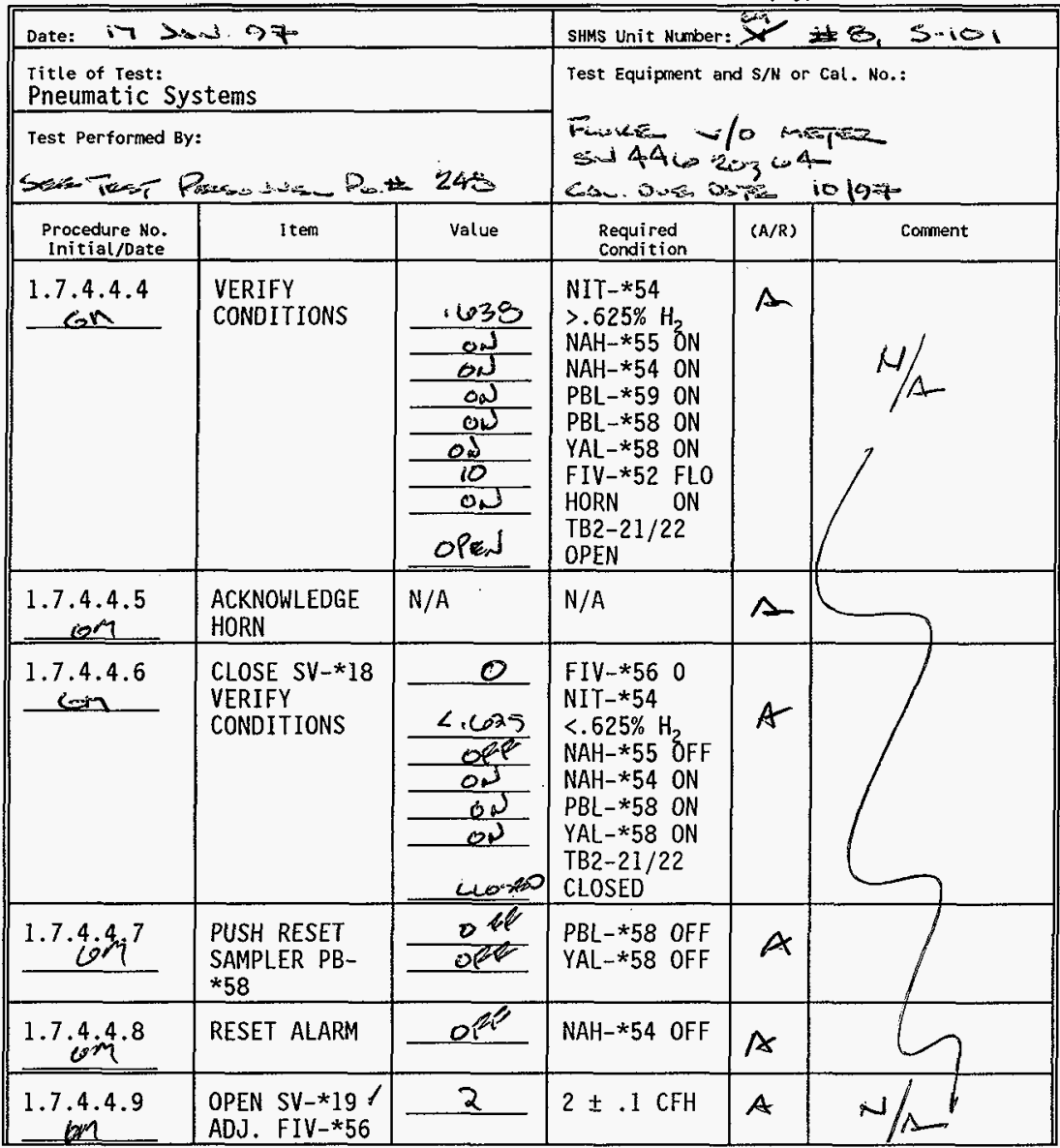

Du lakt en 
TEST DATA SHEET

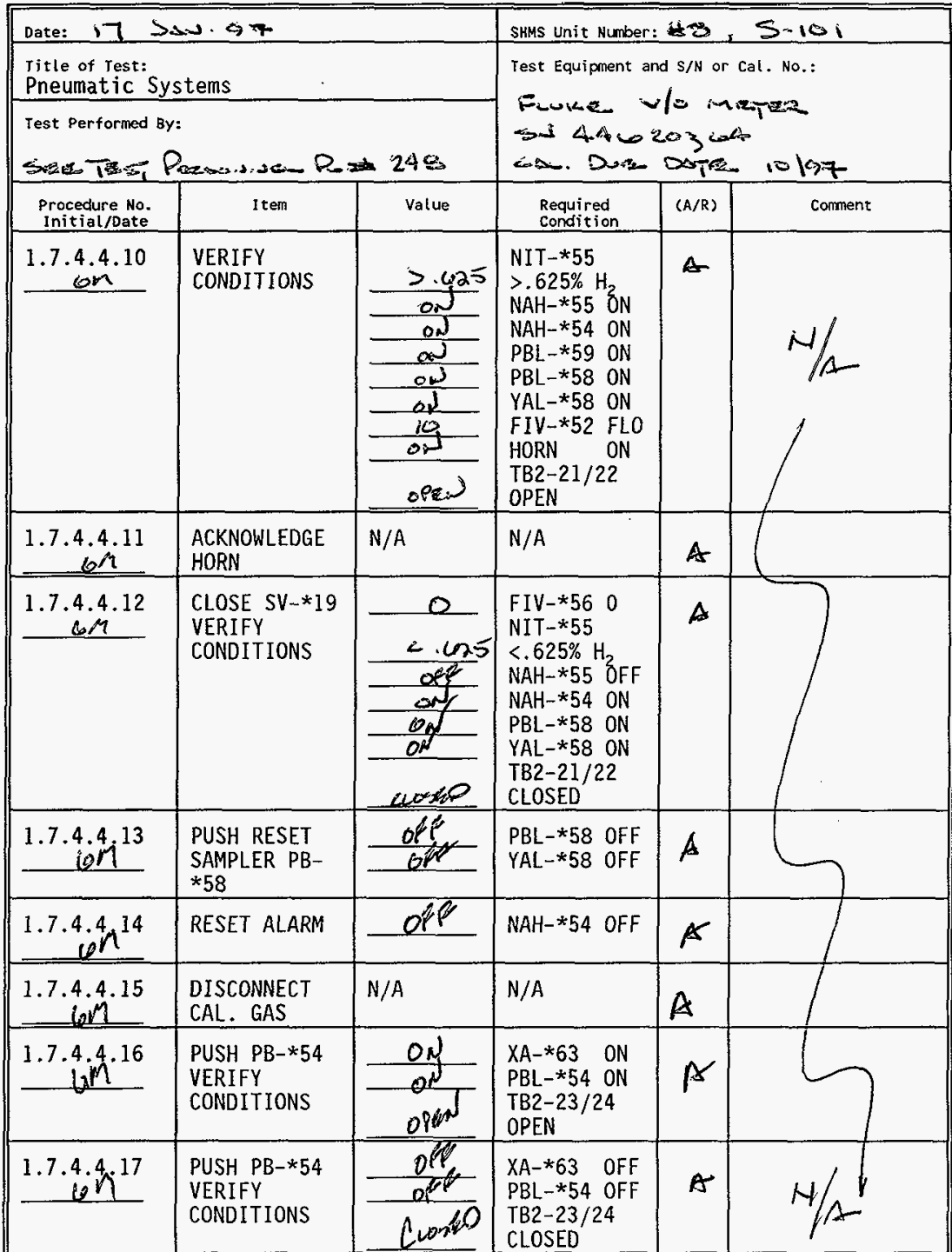

A., ink tom 
APPENDIX $\mathrm{H}$

PAGE $\mathrm{H}-1 \overline{\overline{9}}$

$$
\begin{array}{r}
\text { HNF-SD-WM-ATR- } 191 \\
\text { Rev. } 0 \\
\text { Page } 265
\end{array}
$$

TEST DATA SHEET

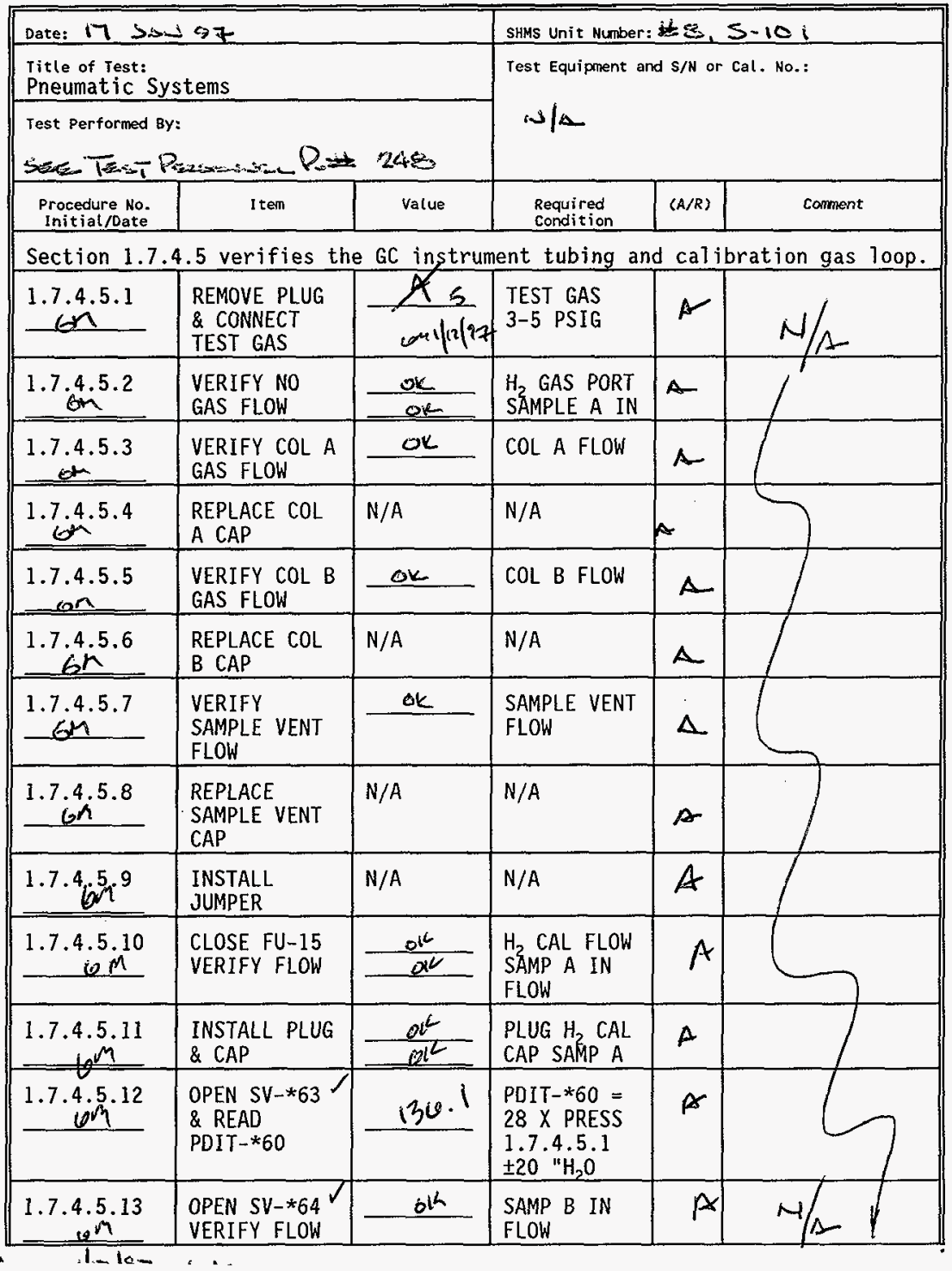


APPENDIX H

TEST DATA SHEET

\begin{tabular}{|c|c|c|c|c|c|}
\hline \multicolumn{3}{|c|}{ Date: 175.4 .47} & \multicolumn{3}{|c|}{ SHMS Unit Number: $\# 3, \mathrm{~S}-101$} \\
\hline \multicolumn{3}{|c|}{$\begin{array}{l}\text { Title of Test: } \\
\text { Pneumatic Systems }\end{array}$} & \multirow{2}{*}{\multicolumn{3}{|c|}{$\begin{array}{l}\text { Test Equipnent and } S / \mathrm{N} \text { or Cal. No.: } \\
\text { N/S }\end{array}$}} \\
\hline \multicolumn{3}{|c|}{ 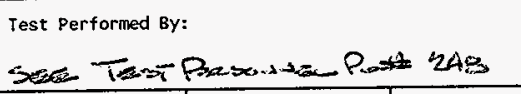 } & & & \\
\hline $\begin{array}{l}\text { Procedure No. } \\
\text { Initial/Date }\end{array}$ & I tern & value & $\begin{array}{l}\text { Required } \\
\text { Condition }\end{array}$ & $(A / R)$ & coment \\
\hline $\begin{array}{l}1.7 .4 .5 .14 \\
\end{array}$ & $\begin{array}{l}\text { REMOVE TEST } \\
\text { GAS } \\
\end{array}$ & N/A & N/A & $\Delta$ & $N / D$ \\
\hline $\begin{array}{r}1.7 .4 .5 .15 \\
\text { gmen }\end{array}$ & $\begin{array}{l}\text { CLOSE SV-*63. } \\
\& \text { SV }-* 64\end{array}$ & $N / A$ & $N / A$ & $A$ & 1 \\
\hline$\frac{1.7 .4 .5 .16}{64}$ & $\begin{array}{l}\text { OPEN FU-15 } \\
\text { REMOVE } \\
\text { JUMPER }\end{array}$ & $N / A$ & $N / A$ & $\Delta$ & \\
\hline $\begin{array}{r}1.7 .4 .5 .17 \\
\end{array}$ & $\begin{array}{l}\text { INSTALL } \\
\text { SAMP A UNION }\end{array}$ & N/A & $N / A$ & $A$ & \\
\hline $\begin{array}{c}1.7 .4 .5 .18 \\
6 n \\
\end{array}$ & $\begin{array}{l}\text { OPEN SV }-* 60 \\
\text { VERIFY FLOW }\end{array}$ & of & $\begin{array}{l}\text { FI-*60 } \\
\text { NO FLOW }\end{array}$ & $A$ & \\
\hline$\frac{1.7 .4 .5 .19}{\operatorname{tn}^{-1}}$ & $\begin{array}{l}\text { POWER SOV- } \\
{ }^{6} 60, \text { VERIFY } \\
\text { NO FLOW } \\
\end{array}$ & or & $\begin{array}{l}\text { FI-*60 } \\
\text { NO FLOW }\end{array}$ & $A$ & \\
\hline 1.7 .4 .5 .20 & $\begin{array}{l}\text { OPEN SV }-* 68 \\
\text { ADJ SV-*67 } \\
\end{array}$ & so & $\begin{array}{l}\mathrm{FI}-* 60 \\
50 \mathrm{CCM} \\
\end{array}$ & $A$ & \\
\hline $\begin{array}{c}1.7 .4 .5 .21 \\
6 n^{2} \\
\end{array}$ & $\begin{array}{l}\text { DEENERGIZE } \\
\text { SOV-*60 }\end{array}$ & OK & $\begin{array}{l}\text { FI-*60 FLOW } \\
\text { DECREASES }\end{array}$ & $\beta$ & \\
\hline$\frac{1.7 .4 .5 n^{22}}{y_{2}}$ & $\begin{array}{l}\text { CLOSE SV-*60 } \\
\& \text { SV-*68 }\end{array}$ & $N / A$ & $N / A$ & A & \\
\hline$\frac{1.7 .4 .5 .23}{y^{m}}$ & $\begin{array}{l}\text { REMOVE } \\
\text { SAMP A UNION } \\
\end{array}$ & N/A & $N / A$ & $A$ & \\
\hline $\begin{array}{c}1.7 .4 .5 .24 \\
6 n n\end{array}$ & $\begin{array}{l}\text { CONNECT TEST } \\
\text { GAS } \mathrm{N}_{2} \mathrm{O} \text { PORT } \\
\end{array}$ & 5 & $\begin{array}{l}\text { TEST GAS } \\
3-5 \text { PSIG } \\
\end{array}$ & 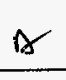 & \\
\hline$\frac{1.7 .4 .5 .25}{0 t^{2}}$ & $\begin{array}{l}\text { VERIFY NO } \\
\text { FLOW }\end{array}$ & $\frac{d^{k}}{O^{K}}$ & $\begin{array}{l}\text { SAMP B IN \& } \\
\text { FLT-*63 } \\
\text { NO FLOW } \\
\end{array}$ & A & \\
\hline$\frac{1.7 .4 .5 .26}{.6 n}$ & $\begin{array}{l}\text { INSTALL } \\
\text { JUMPER } \\
\end{array}$ & $N / A$ & $N / A$ & 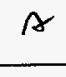 & \\
\hline$\frac{1.7 .4 .5 .27}{6 n}$ & $\begin{array}{l}\text { CLOSE FU-15 } \\
\text { VERIFY FLOW }\end{array}$ & $\frac{0^{2}}{b^{2}}$ & $\begin{array}{l}\text { SAMP B IN } \\
\text { FLT } * 63 \\
\text { FLOW }\end{array}$ & A & \\
\hline
\end{tabular}


APPENDIX $\mathrm{H}$

TEST DATA SHEET

\begin{tabular}{|c|c|c|c|c|c|}
\hline \multicolumn{3}{|c|}{ Date: 17 ग.4. 97} & \multicolumn{3}{|c|}{ SHMS Unit Number: $2, S-10 i$} \\
\hline \multicolumn{3}{|c|}{$\begin{array}{l}\text { ritle of Test: } \\
\text { Pneumatic Systems }\end{array}$} & \multirow{2}{*}{\multicolumn{3}{|c|}{ Test Equipment and $S / \mathrm{N}$ or Cal. No.: }} \\
\hline \multicolumn{3}{|c|}{ 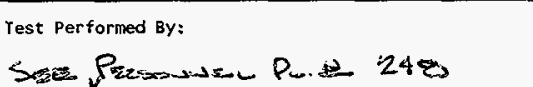 } & & & \\
\hline $\begin{array}{l}\text { Procedure NoT } \\
\text { Initial/Date }\end{array}$ & I tem & value & $\begin{array}{l}\text { Required } \\
\text { Condition } \\
\end{array}$ & $(A / R)$ & Comment \\
\hline $\begin{array}{r}1.7 .4 .5 .28 \\
6 k \\
\end{array}$ & $\begin{array}{l}\text { DISCONNECT } \\
\text { TEST GAS }\end{array}$ & $N / A$ & N/A & $A$ & \\
\hline $\begin{array}{r}1.7 .4 .5 .29 \\
6 n^{4} \\
\end{array}$ & OPEN FU-15 & $N / A$ & $N / A$ & $A$ & \\
\hline $\begin{array}{c}1.7 .4 .5 .30 \\
6 m^{-}\end{array}$ & $\begin{array}{l}\text { REMOVE } \\
\text { JUMPER }\end{array}$ & N/A & $N / A$ & A & \\
\hline$\frac{1.7 .4 .5 .31}{\log ^{4}}$ & $\begin{array}{l}\text { INSTALL } \\
\text { SAMP B UNION }\end{array}$ & N/A & $N / A$ & A & \\
\hline $\begin{array}{r}1.7 .4 .5 .32 \\
19^{3} \\
\end{array}$ & $\begin{array}{l}\text { OPEN SV }-* 60 \\
\text { VERIFY FLOW }\end{array}$ & $x^{2}$ & $\begin{array}{l}\text { FI-*60 } \\
\text { NO FLOW }\end{array}$ & A & \\
\hline$\frac{1.7 .4 .5 .33}{6 x^{4.4}}$ & $\begin{array}{l}\text { POWER SOV- } \\
\star 60 \text {, VERIFY } \\
\text { NO FLOW }\end{array}$ & $a^{2}$ & $\begin{array}{l}\text { FI-*60 } \\
\text { NO FLOW }\end{array}$ & A & \\
\hline $\begin{array}{c}1.7 .4 .5 .34 \\
67^{-14} \\
\end{array}$ & $\begin{array}{l}\text { OPEN SV-*68 } \\
\& \text { ADJ SV-*67 }\end{array}$ & 5s & $\begin{array}{l}\mathrm{FI}-* 60 \\
50 \mathrm{CCM}\end{array}$ & At & \\
\hline $\begin{array}{r}1.7 .4 .5 .35 \\
6.5 \\
\end{array}$ & $\begin{array}{l}\text { DEENERGIZE } \\
\text { SOV }-* 60\end{array}$ & $a^{2}$ & $\begin{array}{l}\text { FI-*60 FLOW } \\
\text { DECREASES }\end{array}$ & As & \\
\hline 1.7 .4 .5 .36 & $\begin{array}{l}\text { CLOSE SV-*60 } \\
\& \text { SV }-* 68\end{array}$ & N/A & $N / A$ & $A$ & \\
\hline $\begin{array}{r}1.7 .4 .5 i^{37} \\
\end{array}$ & $\begin{array}{l}\text { REMOVE } \\
\text { SAMP B UNION }\end{array}$ & N/A & $N / A$ & $\Delta$ & \\
\hline $\begin{array}{r}1.7 .4 .5 .38 \\
0.17 \\
\end{array}$ & $\begin{array}{l}\text { OPEN CB-3/4 } \\
\text { ACK. ALARMS }\end{array}$ & $N / A$ & $N / A$ & N & \\
\hline \multicolumn{6}{|c|}{$\begin{array}{l}\text { Section } 1.7 .4 .6 \text { verifies the multi gas analyzer instrument tubing } \\
\text { calibration gas loop. }\end{array}$} \\
\hline 1.7 .4 .6 .1 & $\begin{array}{l}\text { VERIFY } \\
\text { NIT-*52 } \\
\text { SAMPLE LINES }\end{array}$ & N/A & $N / A$ & 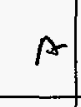 & \\
\hline $\begin{array}{l}1.7 .4 .6 .2 \\
\operatorname{lon}^{4}\end{array}$ & $\begin{array}{l}\text { CONNECT TEST } \\
\text { GAS NH } 3 \text { PORT }\end{array}$ & $-\alpha$ & $\begin{array}{l}\text { TEST GAS } \\
3-5 \text { PSIG }\end{array}$ & A & \\
\hline 1.7 .4 .6 .3 & $\begin{array}{l}\text { VERIFY NO } \\
\text { FLOW }\end{array}$ & $\frac{a^{2}}{a^{2}}$ & $\begin{array}{l}\text { SAMPLE IN } \\
\text { FLT-*63 }\end{array}$ & A & Wh \\
\hline
\end{tabular}

A. llator lan 
TEST DATA SHEET

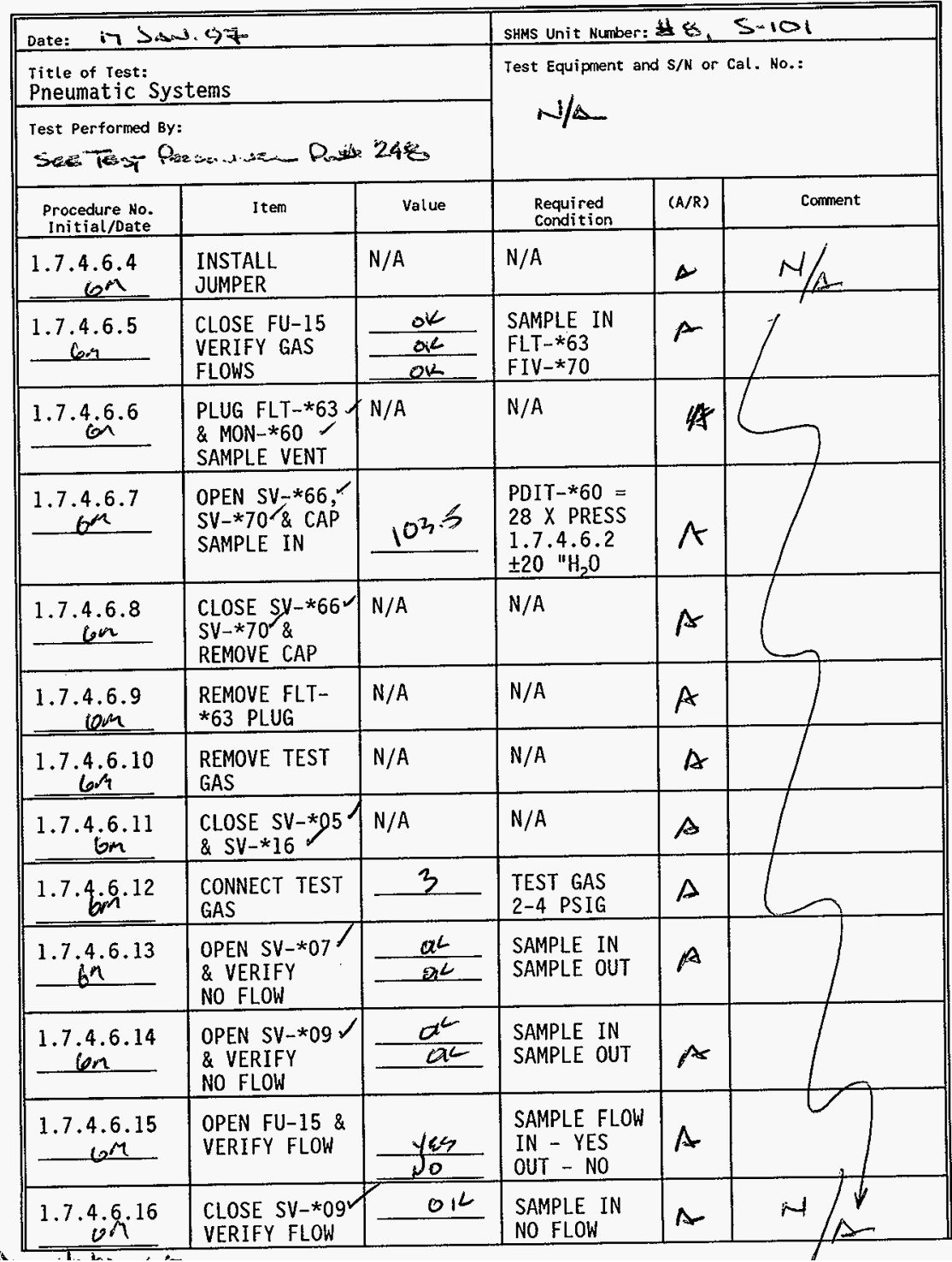




\section{TEST DATA SHEET}

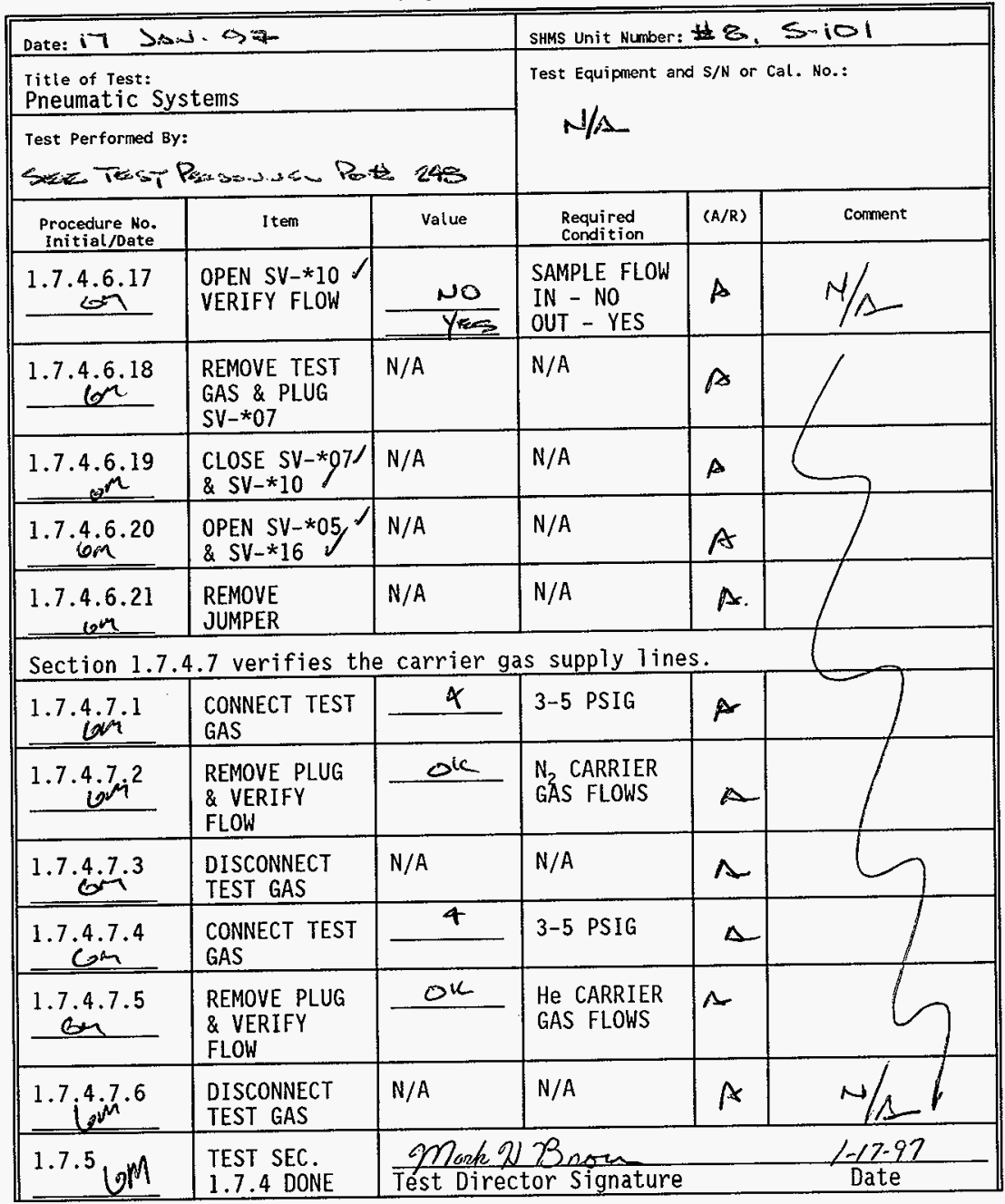

sue inls7 6r 
TEST DATA SHEET

\begin{tabular}{|c|c|c|c|c|c|}
\hline \multicolumn{3}{|c|}{ Date: 173.9 .97} & \multicolumn{3}{|c|}{ SHHS Unit Number: $\$ 8, s-101$} \\
\hline \multicolumn{3}{|c|}{$\begin{array}{l}\text { Title of Test: } \\
\text { Pneumatic Systems }\end{array}$} & \multirow{2}{*}{\multicolumn{3}{|c|}{ Test Equipnent and $\mathrm{S} / \mathrm{N}$ or Cal. No.: }} \\
\hline \multicolumn{3}{|c|}{ Test Performed $B y:$} & & & \\
\hline $\begin{array}{l}\text { Procedure No. } \\
\text { Initial/Date }\end{array}$ & Item & value & $\begin{array}{l}\text { Requi red } \\
\text { Condition }\end{array}$ & $(A / R)$ & Coment \\
\hline 1.7.6 & \multirow{2}{*}{\multicolumn{5}{|c|}{$\begin{array}{l}\text { THIS SECTION IS ONLY FOR THE SHMS-E IN THE ANALYTICAL MODE. } \\
\text { IF NOT APPLICABLE, N/A STEPS AND TEST DIRECTOR WILL SIGN. } \\
\text { Test Director Signature }\end{array}$}} \\
\hline & & & & & \\
\hline 1.7 .6 .1 .1 & $\begin{array}{l}\text { VERIFY BKR } \\
\text { LINE UP }\end{array}$ & $N / A$ & N/A & & \\
\hline 1.76 .1 .2 & $\begin{array}{l}\text { VERIFY VALVE } \\
\text { LINE UP }\end{array}$ & $N / A$ & N/A & & \\
\hline 1.7 .6 .1 .3 & $\begin{array}{l}\text { VERIFY MON- } \\
* 60 \text { INSTALL }\end{array}$ & $N / A$ & N/A & & \\
\hline 1.7 .5 .1 .4 & $\begin{array}{l}\text { VERIFY NIT- } \\
\text { *52 INSTALL } \\
\end{array}$ & N/A & $N / A$ & & . \\
\hline 1.7 .6 .1 .5 & $\begin{array}{l}\text { CONNECT } \mathrm{H}_{2} \\
\text { CAL GAS }\end{array}$ & N/A & $N / A$ & & \\
\hline 1.7 .6 .1 .6 & $\begin{array}{l}\text { CONNECT } \mathrm{CH}_{4} \\
\text { CAL GAS }\end{array}$ & N/A & $N / A$ & & \\
\hline 1.7 .9 .1 .7 & $\begin{array}{l}\text { CONNECT } \mathrm{NH}_{3} \\
\text { CAL GAS }\end{array}$ & N/A & $N / A$ & & \\
\hline Sectifon 1.7 & .2 establishes & the main & d auxiliary & & \\
\hline 1.7 .6 .2 .1 & CLOSE FU-11 & $N / A$ & $\begin{array}{l}\text { MON-*60 } \\
\text { POWERED }\end{array}$ & & \\
\hline 1.76 .2 .2 & POWER PC-*60 & & $\begin{array}{l}\text { ESTABLISH } \\
\text { SAMPLING } \\
\text { ROUTINE } \\
\end{array}$ & & \\
\hline 1.7 .6 .2 .3 & CLOSE FU-12 & & $\begin{array}{l}\text { NIT-*552 } \\
\text { POWERED } \\
\end{array}$ & & \\
\hline 1.7 .6 .2 .4 & POWER PC $-* 70$ & & $\begin{array}{l}P C-* 70 \\
\text { BOOTED UP }\end{array}$ & $k$ & \\
\hline $\begin{array}{l}1.4 .2 .5 \\
\mathrm{~N} / \mathrm{A}^{2}\end{array}$ & $\begin{array}{l}\text { CLOSE CB-3/4 } \\
\text { ADJUST FLOW }\end{array}$ & $-w / a$ & $\begin{array}{l}\mathrm{FIT}-* 57 \\
1 \pm .1 \quad \mathrm{H}_{2} \mathrm{O}\end{array}$ & $\omega / \Delta$ & \\
\hline
\end{tabular}


TEST DATA SHEET

\begin{tabular}{|c|c|c|c|c|c|}
\hline \multirow{2}{*}{\multicolumn{3}{|c|}{\begin{tabular}{|l|} 
Date: $\mathrm{N} / \mathrm{\Lambda}$ \\
Title of Test: \\
Pneumatic Systems \\
\end{tabular}}} & \multicolumn{3}{|c|}{ SHMS Unit Number: } \\
\hline & & & \multirow{2}{*}{\multicolumn{3}{|c|}{ Test Equipment and $\mathrm{S} / \mathrm{N}$ or Cal. No.: }} \\
\hline \multicolumn{3}{|c|}{ Test Performed By: $\mathrm{N} / \mathrm{s}$} & & & \\
\hline $\begin{array}{l}\text { Procedure No. } \\
\text { Initial/Date }\end{array}$ & It tem & Value & $\begin{array}{l}\text { Requi red } \\
\text { Cordition } \\
\end{array}$ & $(A / R)$ & Comment \\
\hline${ }^{1.7} \cdot 6 / s^{2.6}$ & $\begin{array}{l}\text { OPEN SV-*09 } \\
\& \text { SV-*10 }\end{array}$ & $N / A$ & $N / A$ & & \\
\hline 1.7 .6 .2 .7 & $\begin{array}{l}\text { OPEN SV-*60, } \\
63,65 \& 68\end{array}$ & $N / A$ & $N / A$ & & \\
\hline $1.7 .6 \%$ & NIT-*52 & 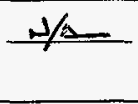 & $\begin{array}{l}\text { ESTABLISH } \\
\text { SAMPLING } \\
\text { ROUTINE }\end{array}$ & & \\
\hline 1.7 .62 .9 & $\begin{array}{l}\text { OPEN SV-*24 } \\
\text { ADJ FLOW }\end{array}$ & $N / N$ & $\begin{array}{l}\text { FIT-*57 } \\
3 \pm .1 \quad \mathrm{H}_{3} \mathrm{O}\end{array}$ & & \\
\hline$1 . 7 . 6 \longdiv { 2 . 1 0 }$ & $\begin{array}{l}\text { ADJ FLOW } \\
\text { SV-*67 }\end{array}$ & $+/$ & $\begin{array}{l}\mathrm{FI}-* 60 \\
10-30 \mathrm{ccm}\end{array}$ & & \\
\hline 1.7 .6$. & $\begin{array}{l}\text { RECORD } \\
\text { PDIT }-\star 60\end{array}$ & $U / A$ & $<175 \quad " \mathrm{H}_{2} \mathrm{O}$ & & \\
\hline 1.7 .6 . & $\begin{array}{l}\text { OPEN SV }-* 64 \\
\text { CLOSE SV }-* 63\end{array}$ & $\mathrm{~N} / \mathrm{A}$ & $N / A$ & & \\
\hline 1.7 .6$. & $\begin{array}{l}\text { RECORD } \\
\text { PDIT } * * 60\end{array}$ & $N / A$ & $<175 \quad " \mathrm{H}_{2} \mathrm{O}$ & & \\
\hline 1.7 .22 & $\begin{array}{l}\text { CLOSE SV-*65 } \\
\text { OPEN SV }-* 66\end{array}$ & $N / A$ & $N / A$ & & \\
\hline 1.7 .6 & $\begin{array}{l}\text { RECORD B IN } \\
\text { PDIT-*60 } \\
\end{array}$ & $N / A$ & $\begin{array}{l}-15 \text { to }-50 \\
" \mathrm{H}_{2} \mathrm{O}\end{array}$ & & \\
\hline$1 . 7 . 6 . 2 \longdiv { 1 6 }$ & $\begin{array}{l}\text { OPEN SV } * * 63 \\
\text { CLOSE SV }-* 64\end{array}$ & $N / A$ & $N / A$ & & \\
\hline 1.7 .6 .2 .17 & $\begin{array}{l}\text { RECORD A IN } \\
\text { PDIT-*60 }\end{array}$ & $N / \Delta$ & $\begin{array}{l}-15 \text { to }-50 \\
" \mathrm{H}_{2} \mathrm{O}\end{array}$ & & \\
\hline 1.7 .6 .2 .18 & $\begin{array}{l}\text { INSTALL } \\
\text { JUMPER }\end{array}$ & $N / A$ & $N / A$ & & \\
\hline 1.7 .6 .2 .19 & CLOSE FU-15 & $N / A$ & $N / A$ & & \\
\hline $\begin{array}{l}1.7 .6 .2 \sqrt{20} \\
N / A\end{array}$ & $\begin{array}{l}\text { ADJ SV }-* 61 \& \\
\text { RECORD } \\
\text { PDIT } * 60\end{array}$ & $N /$ & $\begin{array}{l} \pm 5 " \mathrm{H}_{2} \mathrm{O} \mathrm{OF} \\
1.7 .6 .2 .17\end{array}$ & $J / A$ & \\
\hline
\end{tabular}


TEST DATA SHEET

\begin{tabular}{|c|c|c|c|c|c|}
\hline \multirow{2}{*}{\multicolumn{3}{|c|}{$\begin{array}{l}\text { Date: } \\
\begin{array}{l}\text { Iitle of Test: } \\
\text { Pneumatic Systems }\end{array} \\
\end{array}$}} & \multicolumn{3}{|c|}{ SHMS Unit Number: $\mathrm{N} / \mathrm{A}$} \\
\hline & & & \multirow{2}{*}{\multicolumn{3}{|c|}{ Test Equipment and $S / \mathrm{N}$ or Cal. No.: }} \\
\hline \multicolumn{3}{|c|}{ Test Performed By: } & & & \\
\hline $\begin{array}{l}\text { Procedure No. } \\
\text { Initial/Date }\end{array}$ & I tem & value & $\begin{array}{l}\text { Required } \\
\text { Condition }\end{array}$ & $(A / R)$ & comment \\
\hline$\frac{1.7 .6 .21}{N / N^{2.21}}$ & $\begin{array}{l}\text { CAL MON-*60 } \\
\text { CHANNEL A }\end{array}$ & $N / A$ & $\begin{array}{l}\text { READINGS } \\
\text { WITHIN } \pm 5 \%\end{array}$ & & \\
\hline 1.7 .6 .2 .22 & OPEN FU-15 & $N / A$ & $N / A$ & & \\
\hline 2.23 & $\begin{array}{l}\text { REMOVE } \\
\text { JUMPER } \\
\end{array}$ & $N / A$ & N/A & & \\
\hline$1 . 7 . 6 \longdiv { 2 . 2 4 }$ & $\begin{array}{l}\text { OPEN SV-*64 } \\
\text { CLOSE SV-*63 }\end{array}$ & $N / A$ & $N / A$ & & \\
\hline 2.25 & $\begin{array}{l}\text { INSTALL } \\
\text { JUMPER }\end{array}$ & $N / A$ & $N / A$ & & \\
\hline $1.7 .6 / 2.26$ & CLOSE FU-15 & $N / A$ & $N / A$ & & \\
\hline 1.7 .6 .2 .27 & $\begin{array}{l}\text { ADJ SV-*62 \& } \\
\text { RECORD } \\
\text { PDIT-*60 }\end{array}$ & $N$ & $\begin{array}{l} \pm 5 " \mathrm{H}_{2} \mathrm{O} \text { OF } \\
1.7 .6 .2 .15\end{array}$ & & \\
\hline .2 .28 & $\begin{array}{l}\text { CAL MON-*60 } \\
\text { CHANNEL B }\end{array}$ & & $\begin{array}{l}\text { READINGS } \\
\text { WITHIN } \pm 5 \%\end{array}$ & & \\
\hline$1 . 7 . 6 \longdiv { 2 . 2 9 }$ & OPEN FU-15 & $N / A$ & $N / A$ & & \\
\hline $1.7(6.2 .30$ & $\begin{array}{l}\text { REMOVE } \\
\text { JUMPER } \\
\end{array}$ & $N / A$ & $N / A$ & & \\
\hline 1.7 .6 .2 .31 & $\begin{array}{l}\text { CLOSE SV-*64 } \\
\text { OPEN SV }-* 70\end{array}$ & $N / A$ & $N / A$ & & \\
\hline $1.7(6.2 .32$ & $\begin{array}{l}\text { RECORD } \\
\text { PDIT-*60 } \\
\text { NIT-*52 IN }\end{array}$ & $N / S$ & $\begin{array}{l}0 \text { to }-30 \\
" \mathrm{H}_{2} \mathrm{O}\end{array}$ & & \\
\hline $1.7\{6.2 .33$ & $\begin{array}{l}\text { INSTALL } \\
\text { JUMPER } \\
\end{array}$ & $N / A$ & $N / A$ & $\downarrow$ & \\
\hline $\begin{array}{l}1.7 .5 .34 \\
\mathrm{~N} / \mathrm{N}^{2}\end{array}$ & CLOSE FU-15 & $N / A$ & $N / A$ & $N /$ & \\
\hline
\end{tabular}


TEST DATA SHEET

\begin{tabular}{|c|c|c|c|c|c|}
\hline \multicolumn{3}{|c|}{ Date: $N / 0$} & \multicolumn{3}{|c|}{ Shms unit Number: $\mathrm{N} / \Delta$} \\
\hline \multicolumn{3}{|c|}{$\begin{array}{l}\text { Title of Test: } \\
\text { Pneumatic Systems }\end{array}$} & \multirow{2}{*}{\multicolumn{3}{|c|}{ Test Equipment and $\mathrm{s} / \mathrm{N}$ or Cal. No.: }} \\
\hline \multicolumn{3}{|c|}{ Test Performed 8y: } & & & \\
\hline $\begin{array}{l}\text { Procedure Ho. } \\
\text { Initial/Date } \\
\end{array}$ & Item & value & $\begin{array}{l}\text { Required } \\
\text { Condition } \\
\end{array}$ & $(A / R)$ & Comment \\
\hline $1.7 .6 \cdot 35$ & $\begin{array}{l}\text { ESTABLISH } \\
\text { BYPASS FLOW }\end{array}$ & $N / N$ & $\begin{array}{l}\text { FIV }-* 70 \\
1.5 \pm .2 \mathrm{cfh} \\
\end{array}$ & $N \alpha$ & - \\
\hline$\frac{1.7 .6 .2 .36}{e^{-3}}$ & $\begin{array}{l}\text { ADJ SV-*71 } \\
\text { NIT }-* 52 \\
\text { SAMPLE IN } \\
\end{array}$ & $M$ & $\begin{array}{l} \pm 5 \quad \mathrm{H}_{2} \mathrm{O} \quad \mathrm{OF} \\
1.7 .6 .2 .32\end{array}$ & & \\
\hline 1.7 .6 & $\begin{array}{l}\text { RECORD } \\
\text { NIT-*52 } \\
\text { SAMPLES }\end{array}$ & $\frac{N /}{I A}$ & $\begin{array}{l}\text { READINGS } \\
\text { WITHIN } \pm 5 \%\end{array}$ & & \\
\hline 1.7 .6 .2 .38 & OPEN FU-15 & $N / A$ & $N / A$ & & \\
\hline 1.7 .6 & $\begin{array}{l}\text { REMOVE } \\
\text { JUMPER }\end{array}$ & N/A & $N / A$ & & \\
\hline \multicolumn{6}{|c|}{ Section 1.7 .7 provides for test system shutdown. } \\
\hline 1.7 . & $\begin{array}{l}\text { DISCONNECT } \\
\mathrm{H}_{2} \text { CAL GAS }\end{array}$ & $N / A$ & N/A & & \\
\hline 1.7 .7 .2 & $\begin{array}{l}\text { DISCONNECT } \\
\mathrm{CH}_{a} \text { CAL GAS }\end{array}$ & N/A & $N / A$ & & \\
\hline 1.7 . & $\begin{array}{l}\text { DISCONNECT } \\
\mathrm{NH}_{3} \text { CAL GAS }\end{array}$ & N/A & $N / A$ & & \\
\hline 1.7 .7 & $\begin{array}{l}\text { SECURE \& } \\
\text { TURN OFF } \\
\text { ANA. INST.S } \\
\end{array}$ & $N / A$ & N/A & & \\
\hline $1.7 . \lambda 5$ & $\begin{array}{l}\text { DISCONNECT } \\
\mathrm{N}_{2} \text { CARRIER } \\
\end{array}$ & N/A & N/A & & \\
\hline 1.7 .76 & $\begin{array}{l}\text { DISCONNECT } \\
\text { He CARRIER }\end{array}$ & $N / A$ & N/A & & \\
\hline$\frac{1.7 .7}{\sqrt{3}}$ & $\begin{array}{l}\text { OPEN ALL } \\
\text { BREAKERS \& } \\
\text { FUSES } \\
\end{array}$ & N/A & $N / A$ & & \\
\hline 1.7 .78 & $\begin{array}{l}\text { CLOSE LISTED } \\
\text { SYS. VALVES }\end{array}$ & N/A & N/A & $\mu$ & \\
\hline
\end{tabular}


TEST DATA SHEET

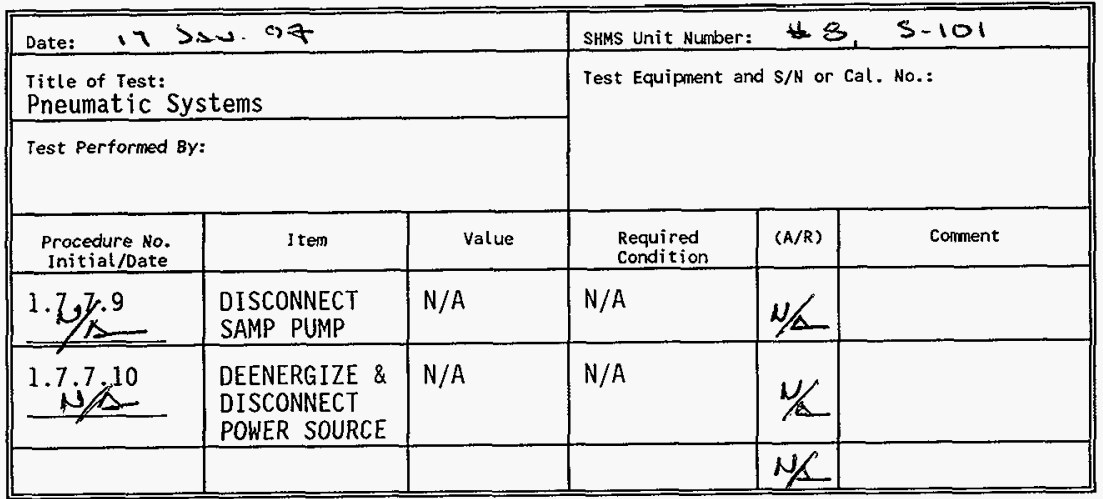

Test Witness/Review:

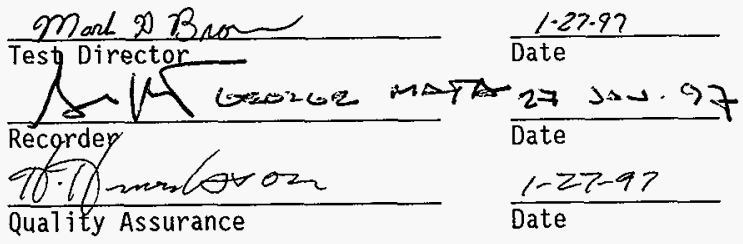


APPENDIX $H$

HNF-SD-WN-ATR-191

PAGE H-29

Rev. 0
Page 275

TEST EXCEPTION SHEET

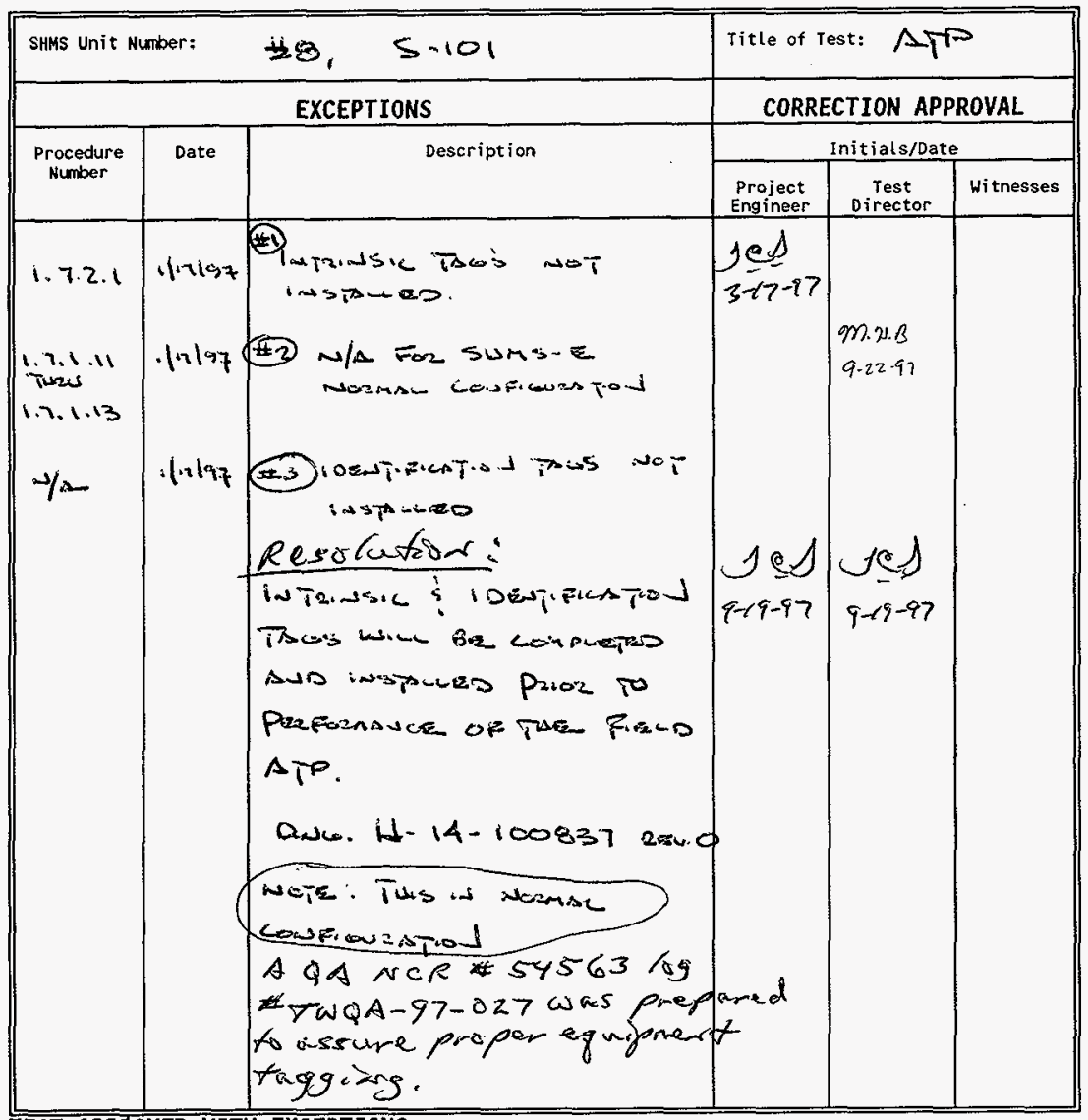

TEST APP ROVED WITH EXCEPTIONS

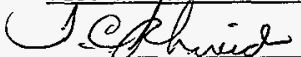

WHC Project Engineer

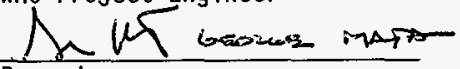

Recorder

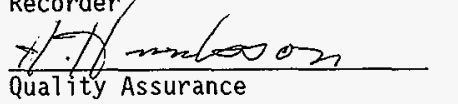

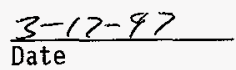

$\frac{27 \sec -97}{\text { Date }}$

$1-27-97$

Date 
APPENDIX $\mathrm{H}$

PAGE H-30
HNF-SD-WM-ATR-191

Rev. 0
Page 276

TEST LOG

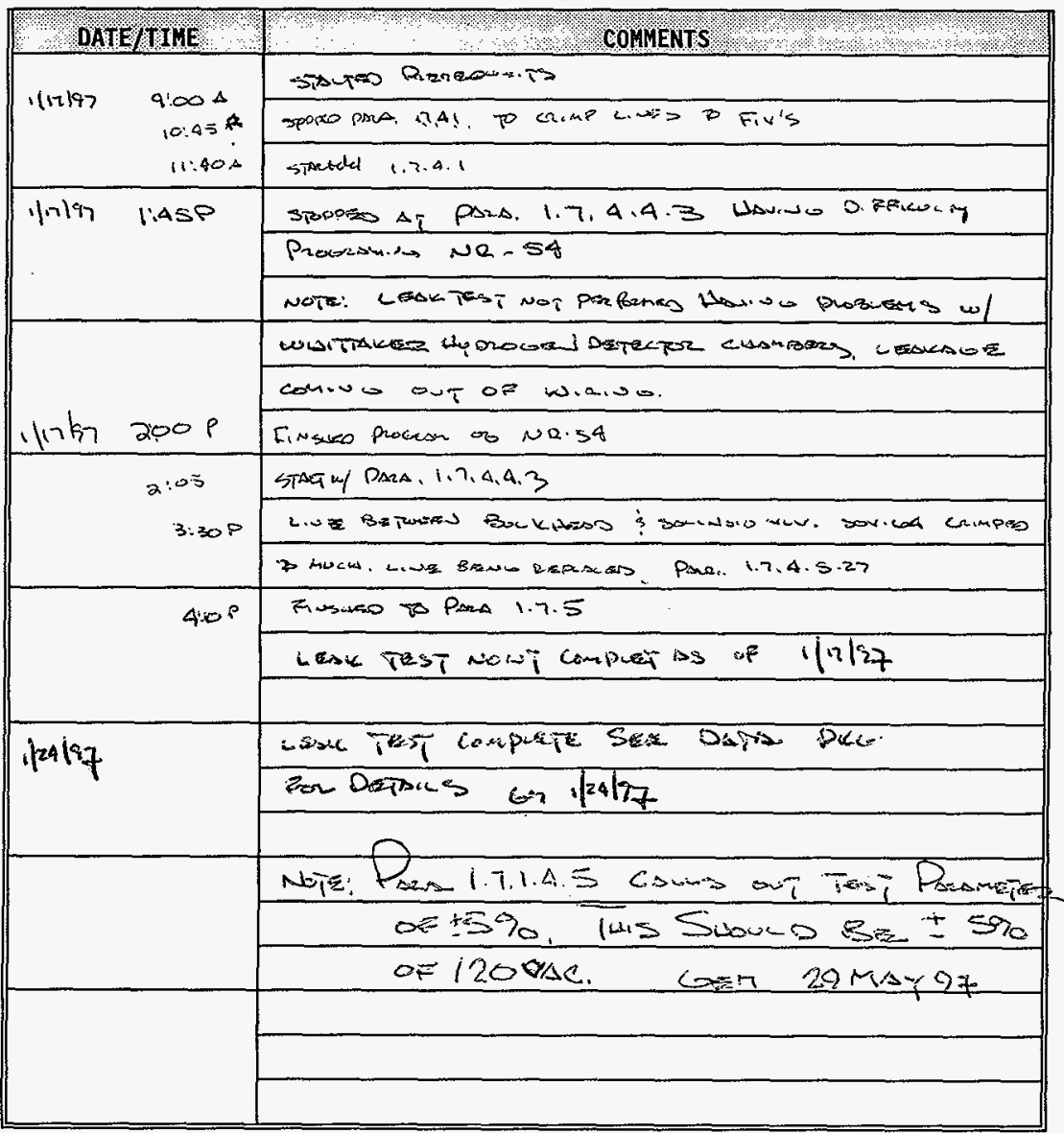


APPENDIX I

PAGE I-/

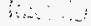

:
HNF - SD-WM-ATR - 191

Rev. 0 Page 277

\section{APPENDIX I}




\section{TEST EXECUTION SHEET}

\begin{tabular}{|c|c|}
\hline $\begin{array}{l}\text { Date: } 24 \text { Fass: } 97 \\
\text { SHMS unit Number: MCRE } \$\end{array}$ & 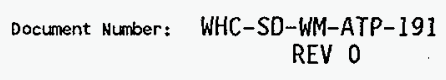 \\
\hline
\end{tabular}

TEST PERSONNEL

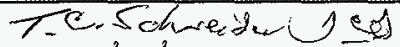

(Print and $1 \mathrm{nt}$ t 1 la)

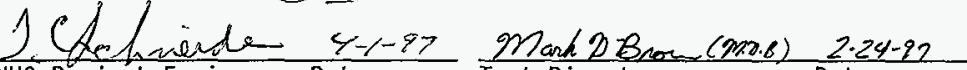

NHC Project Engineer Date Test Director

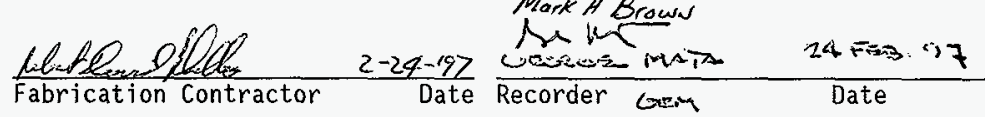

Witness Date Witness Date

Witness Date Witness

\section{TEST ACCEPTANCE}

Without

Exception

Exception/0utstanding

With
Exception/Resolved With

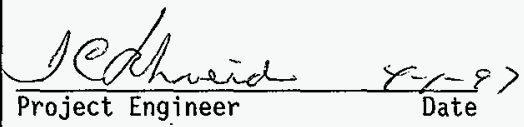

\section{TEST APPROVAL AND ACCEPTANCE}

Numatec Hanford Company

Without
Exception
Exception/Outstanding

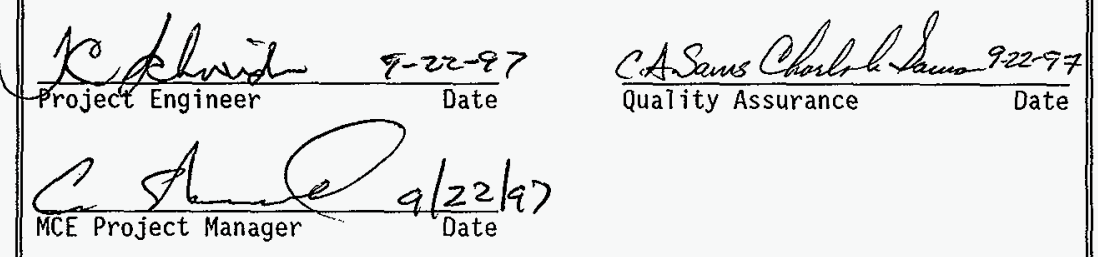


SHMS Unit Number:

\section{PREREQUISITES AND INITIAL TEST CONDITIONS}

The following conditions shall exist at the start of the acceptance testing. Initial and date to verify that each of the following items have been accompl ished.

Gen $2|2 a| 47$ Systems being tested have been inspected for workmanship and for compliance with design. See $T E^{\prime \prime} /$ mas. 2-26-97

Gen 2/24/37 Continuity and megger tests have been performed on portions of the electrical and instrument systems being tested, as required.

$\rightarrow z \mid z a / 3 z$ Leak tests on the pneumatic systems have been performed.

imenzlzalit The following circuit breakers and fuses are installed per the specified size and are open

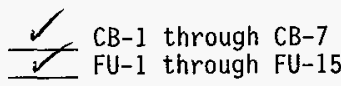

Gen $2 / 24 / 5)$ All test instruments have a currently valid calibration stamp attached that indicates a calibration traceable to the National Institute of Standards and Technology.

Cor $2|24| 77$ The following process and control instrument systems have been initially configured and aligned for proper operation.

FIT-*57, FSL $-* 57$, NIT-*54, NIT-*55, (NR-*54), PDIT-*60, TIC $-* 50$, TIC- $* 56$, TIS $-* 62$, YYC $-* 01$ NOTE: NR $-* 54$ is not used in the ANALYTICAL configuration.

Genzlzotiz Personnel responsible for directing and witnessing the performance of the tests described in this ATP have read and understand appropriate certified vendor information (CVI) pertaining to the operation of the equipment to be tested.

cor zizliz CLOSE all system manual valves with the exception of the following:

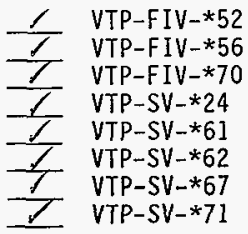

Gen $2 / 24 / 97$ The sample pump VTP-P-*50, connected to the cabinet sample out port, but vented to atmosphere for testing. 
Gean 2/24/19.7 208 Vac 1 phase 15-20 ampere temporary power source has been connected, but not energized, to the appropriate TBI terminals per H-14-100838.

Verify by signature and date that all prerequisites have been met.

$\frac{9 \text { ash } 0 B_{\text {nor }}}{\text { Test Director Signature }} \frac{2-24-97}{\text { Date }}$


TEST DATA SHEET

\begin{tabular}{|c|c|c|c|c|c|}
\hline \multicolumn{3}{|c|}{ Date: 24 Fig3.97 } & \multicolumn{3}{|c|}{ SHMS Unit Number: MCE } \\
\hline \multicolumn{3}{|c|}{$\begin{array}{l}\text { Title of rest: } \\
\text { Electrical Systems }\end{array}$} & \multirow{2}{*}{\multicolumn{3}{|c|}{ 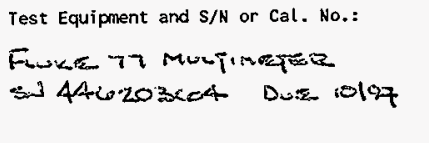 }} \\
\hline \multicolumn{3}{|c|}{ 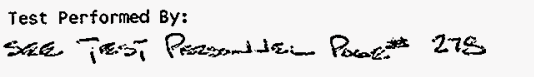 } & & & \\
\hline $\begin{array}{l}\text { Procedure No. } \\
\text { Initial/Date }\end{array}$ & Item & value & $\begin{array}{l}\text { Required } \\
\text { Condition }\end{array}$ & $(A / R)$ & corment \\
\hline \multicolumn{6}{|c|}{ Section 1.7.1.1 verifies the system Mains Power. } \\
\hline $\begin{array}{l}1.7 .1 .1 .1 \\
\text { gean }\end{array}$ & Resistance & .1 & $<1$ ohm & $A$ & $\mathrm{~N}$ \\
\hline $\begin{array}{l}1.7 .1 .1 .2 \\
\text { sein }\end{array}$ & $\begin{array}{l}\text { Temp power } \\
208 \mathrm{Vac}\end{array}$ & N/A & Energized & $A$ & $-\infty /$ \\
\hline $\begin{array}{l}1.7 .1 .1 .3 \\
\operatorname{sem}\end{array}$ & $\begin{array}{l}\text { Line Vac: } \\
\text { LI-L2 } \\
\text { L1-Gnd } \\
\text { L2-Gnd } \\
N-\text { Gnd }\end{array}$ & $\frac{\frac{209.4}{119.7}}{\frac{120.3}{0}}$ & $\begin{array}{l}\lfloor 1-L 2: 208 \\
\text { Vac }+10 /-1 \% \\
L 1,12: 120 \\
\text { Vac } \pm 5 \% \\
N: 0 \text { Vac }\end{array}$ & $A$ & \\
\hline \multicolumn{6}{|c|}{ Section 1.7 .1 .2 verifies the enclosure HVAC system. } \\
\hline sein & AC Adjusted & N/A & $N / A$ & $A$ & $-3 / R$ \\
\hline $\begin{array}{l}1.7 .1 .2 .2 \\
\text { gem }\end{array}$ & $\begin{array}{l}\text { CLOSE } \\
\text { CB-1/2 }\end{array}$ & N/A & N/A & A- & -4 \\
\hline 1.7 .1 .2 .3 & Heater ON & as & Heater ON & $A$ & $N / A$ \\
\hline $\begin{array}{l}1.7 .1 .2 .4 \\
\cos \end{array}$ & AC Adjusted & $N / A$ & N/A & 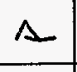 & \\
\hline 1.7 .1 .2 .5 & $\begin{array}{l}\text { Heater OFF } \\
\text { Cooling ON }\end{array}$ & ofe & $\begin{array}{l}\text { Heater OFF } \\
\text { Cooling ON } \\
\end{array}$ & $A$ & \\
\hline $\begin{array}{l}1.7 .1 .2 .6 \\
\end{array}$ & AC Adjusted & $\mathrm{N} / \mathrm{A}$ & $N / A$ & $A$ & \\
\hline 1.7 .1 .2 .7 & $\begin{array}{l}\text { OPEN } \\
\mathrm{CB}-1 / 2\end{array}$ & $N / A$ & $N / A$ & $\Delta$ & \\
\hline \multicolumn{6}{|c|}{ Section 1.7.1.3 verifies the sample pump is properly connected. } \\
\hline $\begin{array}{l}1.7 .1 .3 .1 \\
\end{array}$ & $\begin{array}{l}\text { CLOSE } \\
\text { CB-3/4 }\end{array}$ & $N / A$ & N/A & $\Lambda$ & \\
\hline $\begin{array}{l}1.7 .1 .3 .2 \\
\text { oon }\end{array}$ & $\begin{array}{l}\text { VTP-P-*50 } \\
\text { ON }\end{array}$ & 0.1 & $\begin{array}{l}\text { VTP-P-*50 } \\
\text { ON }\end{array}$ & $\Delta$ & $-1 / 2$ \\
\hline
\end{tabular}




\section{TEST DATA SHEET}

\begin{tabular}{|c|c|c|c|c|c|c|}
\hline \multicolumn{3}{|c|}{ Date: 24 Fes. 27} & \multicolumn{4}{|c|}{ SHMS Unit Number: MCe 9} \\
\hline \multicolumn{3}{|c|}{$\begin{array}{l}\text { Title of Test: } \\
\text { Electrical Systems }\end{array}$} & \multirow{2}{*}{\multicolumn{4}{|c|}{ 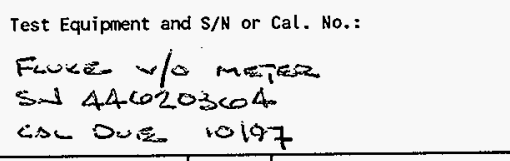 }} \\
\hline \multicolumn{3}{|c|}{ 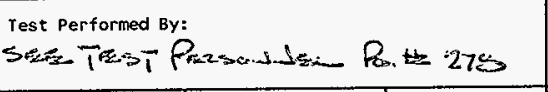 } & & & & \\
\hline $\begin{array}{l}\text { Procedure No. } \\
\text { Initial/Date }\end{array}$ & I tem & Value & $\begin{array}{l}\text { Required } \\
\text { Condition } \\
\end{array}$ & $(A / R)$ & cormer & \\
\hline $\begin{array}{l}1.7 .1 .3 .3 \\
6 \mathrm{~m} \\
\end{array}$ & $\begin{array}{l}\text { OPEN } \\
\mathrm{CB}-3 / 4\end{array}$ & $N / A$ & $N / A$ & 1 & $y_{A}$ & \\
\hline \multicolumn{7}{|c|}{ Section 1.7.1.4 verifies the enclosure lighting and GFCI receptacles. } \\
\hline $\begin{array}{l}1.7 .1 .4 .1 \\
\operatorname{con} \\
\end{array}$ & $\begin{array}{l}\text { CLOSE } \\
\text { CB-6 }\end{array}$ & $N / A$ & $N / A$ & $\Lambda$ & & \\
\hline$\underline{6 g}^{1.7 .1 .2}$ & $\begin{array}{l}\text { ENCLOSURE } \\
\text { LIGHT }\end{array}$ & $N / A$ & N/A & $A$ & & \\
\hline $\begin{array}{l}1.7 .1 .4 .3 \\
6.9 .4\end{array}$ & LIGHT ON & on & LIGHT ON & $A$ & & \\
\hline 1.7 .1 .4 .4 & $\begin{array}{l}\text { GFCI RECP. } \\
\text { IA. L-N } \\
\text { IB. L-GND } \\
2 A . L-N \\
\text { 2B. L-GND } \\
\end{array}$ & $\frac{\frac{189.1}{119.1}}{\frac{119.1}{119.0}}$ & $\begin{array}{l}\text { 1A. } 120 \mathrm{Vac} \\
1 \mathrm{~B} .120 \mathrm{Vac} \\
2 \mathrm{~A} .120 \mathrm{Vac} \\
2 B .120 \mathrm{VaC} \\
\text { ALL } \pm 5 \%\end{array}$ & $\Lambda$ & & \\
\hline $\sin ^{1.7 .1 .4 .5}$ & $\begin{array}{l}\text { GFCI TEST } \\
1 A . L-N \\
1 B . L-G N D \\
2 A . L-N \\
2 B . L-G N D \\
\end{array}$ & $\frac{\frac{40}{6}}{\frac{-351.4}{35}}$ & $\begin{array}{l}1 \mathrm{zaA} 7 \\
1 \mathrm{~A} .0 \mathrm{Vac} \\
1 \mathrm{~B} .0 \mathrm{Vac} \\
2 \mathrm{~A} .0 \mathrm{Vac} \\
2 \mathrm{~B} .00 \mathrm{Vac} \\
\text { ALL } \pm 5 \% \\
\end{array}$ & A & & \\
\hline $\begin{array}{l}1.7 .1 .4 .6 \\
\text { Gom }\end{array}$ & $\begin{array}{l}\text { ENCLOSURE } \\
\text { LIGHT OFF }\end{array}$ & $N / A$ & $N / A$ & $n$ & & \\
\hline $\begin{array}{l}1.7 .1 .4 .7 \\
\end{array}$ & $\begin{array}{l}\text { OPEN } \\
\text { CB-6 } \\
\end{array}$ & $N / A$ & $N / A$ & $\Delta$ & & \\
\hline \multicolumn{7}{|c|}{ Section 1.7.1.5 verifies the instrument power source } \\
\hline 1.7 .1 .5 .1 & $\begin{array}{l}\text { CLOSE } \\
\text { CB-7 } \\
\end{array}$ & $N / A$ & $N / A$ & $A$ & & \\
\hline $\begin{array}{l}1.7 .1 .5 .2 \\
\text { consen }\end{array}$ & $\begin{array}{l}\text { XFRM Vac } \\
\text { PRI. L-N } \\
\text { SEC. L-N } \\
\text { SEC. } N-\text { GND } \\
\end{array}$ & $\frac{120.3}{123.7}$ & $\begin{array}{l}\text { PRI. \& SEC. } \\
\text { L-N } 120 \pm 5 \% \\
\text { SEC. } \\
N \text {-GND O Vac }\end{array}$ & $A$ & & \\
\hline $\begin{array}{l}1.7 .1 .5 .3 \\
6.3\end{array}$ & CLOSE FU-1 & $N / A$ & $N / A$ & $\Delta$ & + & \\
\hline
\end{tabular}


TEST DATA SHEET

\begin{tabular}{|c|c|c|c|c|c|}
\hline Date: $24 F$ & .97 & & SHMS Unit Number: & Me & is 9 \\
\hline $\begin{array}{l}\text { Title of Test: } \\
\text { Electrical }\end{array}$ & stems & & Test Equipment an & $\mathrm{S} / \mathrm{N}$ or & Cal. No.: \\
\hline $\begin{array}{l}\text { Test Performed } \\
\text { SREz Tase- }\end{array}$ & $\operatorname{sen} 1+2 i-R$ & 278 & $\begin{array}{l}\operatorname{Sil} 440020 \\
\angle A L\end{array}$ & $\begin{array}{l}364 \\
0 \times T^{2}\end{array}$ & 10197 \\
\hline $\begin{array}{l}\text { Procedure Ko. } \\
\text { Initial/Date }\end{array}$ & 1 tem & value & $=4$ Sy Required & (A/R) & Comment \\
\hline $\begin{array}{l}1.7 .1 .5 .4 \\
\text { ien }\end{array}$ & $\begin{array}{l}\text { VTP-PS }-* 50 \\
\text { OUTPUT Vdc }\end{array}$ & $\frac{24.5}{24.01}$ & $24 \pm 0.1 \mathrm{Vdc}$ & $\Lambda$ & 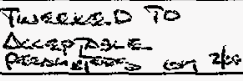 \\
\hline Section 1.7 & .6 verifies $t$ & enciosure & general al arm & syste & \\
\hline isin & $\begin{array}{l}\text { CLOSE FU-9 \& } \\
\text { FU-10 }\end{array}$ & N/A & $N / A$ & $\Delta$ & $M / A$ \\
\hline $\begin{array}{l}1.7 .1 .6 .2 \\
\operatorname{sen}\end{array}$ & $\begin{array}{l}\text { PUSH PB-*51 } \\
\& \text { PB- } * 50\end{array}$ & $N / A$ & $N / A$ & A & \\
\hline $\begin{array}{l}1.7 .1 .6 .3 \\
\text { saxin }\end{array}$ & $\begin{array}{l}\text { ALARM TEST } \\
\text { VERIFY ALARM } \\
\text { CONDITIONS }\end{array}$ & 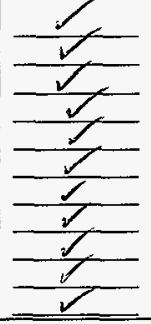 & $\begin{array}{ll}\text { YAH-*50 } & \text { ON } \\
\text { NAH-*55 } & \text { ON } \\
\text { XA-*63 } & \text { ON } \\
\text { NAH-*54 } & \text { ON } \\
\text { FAL }-* 57 & O N \\
\text { TAHL }-* 62 & \text { ON } \\
\text { TAL-*50 } & \text { ON } \\
\text { YAL }-* 58 & \text { ON } \\
\text { PBL }-* 58 & \text { ON } \\
\text { PBL }-* 54 & \text { OFF } \\
\text { PBL }-* 59 & \text { OFF } \\
\end{array}$ & $\Delta$ & \\
\hline $\begin{array}{l}1.7 .1 .6 .4 \\
0.02\end{array}$ & $\begin{array}{l}\text { END ALARM } \\
\text { TEST }\end{array}$ & $N / A$ & $N / A$ & $\Delta$ & \\
\hline sem $^{1.7 .1 .6 .5}$ & RESET ALARMS & $N / A$ & $N / A$ & $A$ & \\
\hline$\underline{G o g}^{1.7 .1 .6}$ & $\begin{array}{l}\text { OPEN FU-9 \& } \\
\text { FU-10 }\end{array}$ & $N / A$ & $N / A$ & & \\
\hline Section 1.7 & verifies $t$ & flow al & system. & & \\
\hline $\begin{array}{l}1.7 .1 .7 .1 \\
602\end{array}$ & $\begin{array}{l}\text { CLOSE FU-3, } \\
\text { FU-9, FU-10 } \\
\& \text { FU-13 }\end{array}$ & N/A & $N / A$ & $\Delta$ & \\
\hline $\begin{array}{l}1.7 .1 .7 .2 \\
- \text { Goin }\end{array}$ & RESET ALARMS & ok & $\mathrm{FAL}-* 57 \mathrm{ON}$ & $\Delta$ & \\
\hline 1.7 .1 .7 .3 & $\begin{array}{l}\text { OPEN SV }-* 20 \\
\& S V-* 22\end{array}$ & $N / A$ & $N / A$ & $A$ & $\omega / \Delta$ \\
\hline
\end{tabular}


TEST DATA SHEET

\begin{tabular}{|c|c|c|c|c|c|}
\hline \multicolumn{3}{|c|}{ Date: 24 Feso 97} & \multicolumn{3}{|c|}{ SHMS Unit Number: Mere } \\
\hline \multicolumn{3}{|c|}{$\begin{array}{l}\text { Title of Test: } \\
\text { Electrical Systems }\end{array}$} & \multirow{2}{*}{\multicolumn{3}{|c|}{ 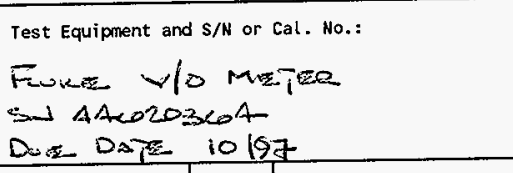 }} \\
\hline \multicolumn{3}{|c|}{ 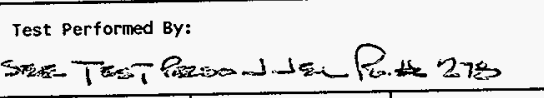 } & & & \\
\hline $\begin{array}{l}\text { Procedure No. } \\
\text { Initial/Date }\end{array}$ & Item & value & $\begin{array}{r}\text { Required } \\
\text { Condition } \\
\end{array}$ & $(A / R)$ & Comment \\
\hline $\begin{array}{l}1.7 .1 .7 .4 \\
\operatorname{sen}\end{array}$ & $\begin{array}{l}\text { FIT-*57 \& } \\
\text { FSL-*57 } \\
\text { POWERED }\end{array}$ & $\frac{O K}{0 K}$ & $\begin{array}{l}\text { FIT-*57 ON } \\
\text { FSL-*57 ON }\end{array}$ & $\Lambda$ & $x^{4 / 2}$ \\
\hline 1.7 .1 .7 .5 & $\begin{array}{l}\text { MUX-*70 } \\
\text { TB5+/TB6- }\end{array}$ & $N / \Delta$ & $1.0 \pm 0.2 \mathrm{Vdc}$ & $N / \Delta$ & 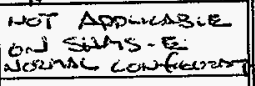 \\
\hline 1.7 .1 .7 .6 & $\begin{array}{l}\text { CLOSE SV-*20 } \\
\& \text { SV }-* 22\end{array}$ & N/A & $\mathrm{N} / \mathrm{A}$ & $\Delta$ & $-1 / s$ \\
\hline $\begin{array}{c}1.7 .1 .7 .7 \\
\end{array}$ & $\begin{array}{l}\text { OPEN FU-3, } \\
\text { FU-9, FU-10 } \\
\& \text { FU-13 }\end{array}$ & $N / A$ & N/A & $\Delta$ & \\
\hline \multicolumn{6}{|c|}{ Section 1.7.1.8 verifies the $\mathrm{H}_{2}$ monitor wiring. } \\
\hline $\begin{array}{c}1.7 .1 .8 .1 \\
\sin \end{array}$ & $\begin{array}{l}\text { CLOSE FU-2, } \\
\text { FU-4, FU-5, } \\
\text { FU-9 \& FU-10 }\end{array}$ & $N / A$ & N/A & $\Delta$ & \\
\hline 1.7 .1 .8 .2 & RESET ALARMS & $\frac{O K}{O K}$ & 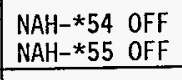 & $A$ & \\
\hline $\begin{array}{l}1.7 .1 .8 .3 \\
\text { ines }\end{array}$ & $\begin{array}{l}\text { INSTRUMENTS } \\
\text { POWERED }\end{array}$ & $\frac{\frac{N / A}{O K}}{\frac{O K}{O K}} \frac{O K}{O K}$ & $\begin{array}{l}\text { MUX-*70 ON } \\
\text { NIT-*54 ON } \\
\text { NIT-*55 ON } \\
\text { NR }-* 54 \text { ON } \\
Y Y C-* 01 \text { ON } \\
\end{array}$ & 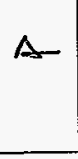 & \\
\hline $\begin{array}{l}1.7 .1 .8 .4 \\
\cos 1\end{array}$ & $\begin{array}{l}\text { OPEN FU-2, } \\
\text { FU-4, FU-5, } \\
\text { FU-9 \& FU-10 }\end{array}$ & N/A & N/A & $\Delta$ & \\
\hline \multicolumn{6}{|c|}{ Section 1.7.1.9 verifies the SHMS-E heat trace control system. } \\
\hline 1.7 .1 .9 .1 & $\begin{array}{l}\text { INSTALL LOAD } \\
\text { VTP-TIC-*50 } \\
\end{array}$ & $N / A$ & $N / A$ & $A$ & \\
\hline $\begin{array}{l}1.7 .1 .9 .2 \\
6 \times 1\end{array}$ & $\begin{array}{l}\text { CLOSE CB-5, } \\
\text { FU-6, FU-7, } \\
\text { FU-9 \& FU-10 } \\
\text { and RESET } \\
\text { ALARMS } \\
\end{array}$ & $N / A$ & $N / A$ & $A$ & $+1 / \Delta$ \\
\hline
\end{tabular}


TEST DATA SHEET

\begin{tabular}{|c|c|c|c|c|c|}
\hline \multicolumn{3}{|c|}{ Date: 24 Fas. 97} & \multicolumn{3}{|c|}{ SHMS Unit Number: MCE $=9$} \\
\hline \multicolumn{3}{|c|}{$\begin{array}{l}\text { Title of Test: } \\
\text { Electrical systems }\end{array}$} & \multirow{2}{*}{\multicolumn{3}{|c|}{ Test Equipment and $\mathrm{s} / \mathrm{N}$ or Cal. No.: }} \\
\hline \multicolumn{3}{|c|}{ Test Performed By: } & & & \\
\hline $\begin{array}{l}\text { Procedure No. } \\
\text { Initial/Date }\end{array}$ & Item & Value & $\begin{array}{l}\text { Required } \\
\text { Condition }\end{array}$ & $(A / R)$ & Comment \\
\hline $\begin{array}{l}1.7 .1 .9 .3 \\
\text { cogn }\end{array}$ & $\begin{array}{l}\text { TIC-*50 \& } \\
\text { TIC-*56 } \\
\text { NOMINAL TEMP }\end{array}$ & $\frac{O K}{O K}$ & $\begin{array}{l}\text { TIC }-* 50 \\
\text { TIC }-* 56 \\
\text { TEMPERATURE }\end{array}$ & $A$ & $1 / \Omega$ \\
\hline $\begin{array}{l}1.7 .1 .9 .4 \\
\text { ofen }\end{array}$ & $\begin{array}{l}\text { ADJ TIC }-* 50 \\
\& \text { TIC }-* 56 \\
\text { SP2 \& RESET } \\
\text { ALARMS } \\
\end{array}$ & $N / A$ & $N / A$ & $A$ & \\
\hline $\begin{array}{l}1.7 .1 .9 .5 \\
\cos \\
\end{array}$ & $\begin{array}{l}\text { ADJ TIC }-* 50 \\
S P P_{1}\end{array}$ & $N / A$ & N/A & A & \\
\hline $\begin{array}{l}1.7 .1 .9 .6 \\
\text { ogin }\end{array}$ & $\begin{array}{l}\text { ADJ TIC } * 50 \\
\text { SP } 2\end{array}$ & N/A & N/A & $A$ & \\
\hline ben & $\begin{array}{l}\text { TAL-*50\& } \\
\text { HORN ACTIVE }\end{array}$ & $\frac{O K}{O K}$ & $\begin{array}{l}\text { TAL-*50 ON } \\
\text { HORN ON } \\
\text { ACKNOWLEDGE } \\
\end{array}$ & $\Delta$ & \\
\hline 1.7 .1 .9 .8 & $\begin{array}{l}\text { TIC }-* 50 \\
\text { OPERATES }\end{array}$ & $\Delta k$ & $\begin{array}{l}\text { TIC }-* 50 \\
\text { OPERATES } \\
\end{array}$ & $\Delta$ & \\
\hline 1.7 .1 .9 .9 & $\begin{array}{l}\text { ADJ TIC-*50 } \\
\mathrm{SP} 2\end{array}$ & N/A & $\mathrm{N} / \mathrm{A}$ & $A$ & \\
\hline $\begin{array}{l}1.7 .1 .9 .10 \\
\text { Gen } \\
\end{array}$ & RESET ALARM & ok & $\mathrm{TAL}-* 50 \quad 0 \mathrm{FF}$ & 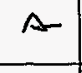 & \\
\hline 1.7 .1 .9 .11 & $\begin{array}{l}\text { ADJ TIC }-* 50 \\
S P 1 \& S P 22\end{array}$ & N/A & N/A & $\wedge$ & \\
\hline 1.7 .1 .9 .12 & $\begin{array}{l}\text { OPEN CB-5 \& } \\
\text { REMOVE LOAD }\end{array}$ & $N / A$ & $N / A$ & $A$ & \\
\hline 1.7 .1 .9 .13 & $\begin{array}{l}\text { INSTALL LOAD } \\
\text { VTP-TIC-*56 }\end{array}$ & $N / A$ & $N / A$ & $A$ & \\
\hline 1.7 .1 .9 .14 & CLOSE CB-5 & $N / A$ & $N / A$ & A & \\
\hline $\begin{array}{l}1.7 .1 .9 .15 \\
6 \cos \\
\end{array}$ & $\begin{array}{l}\text { ADJ TIC-*56 } \\
\text { SP } 1\end{array}$ & $N / A$ & $N / A$ & $\Delta$ & \\
\hline 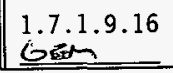 & $\begin{array}{l}\text { ADJ TIC }-* 56 \\
\text { SP } 2\end{array}$ & $N / A$ & $N / A$ & $\Lambda$ & $N H^{4}$ \\
\hline
\end{tabular}


TEST DATA SHEET

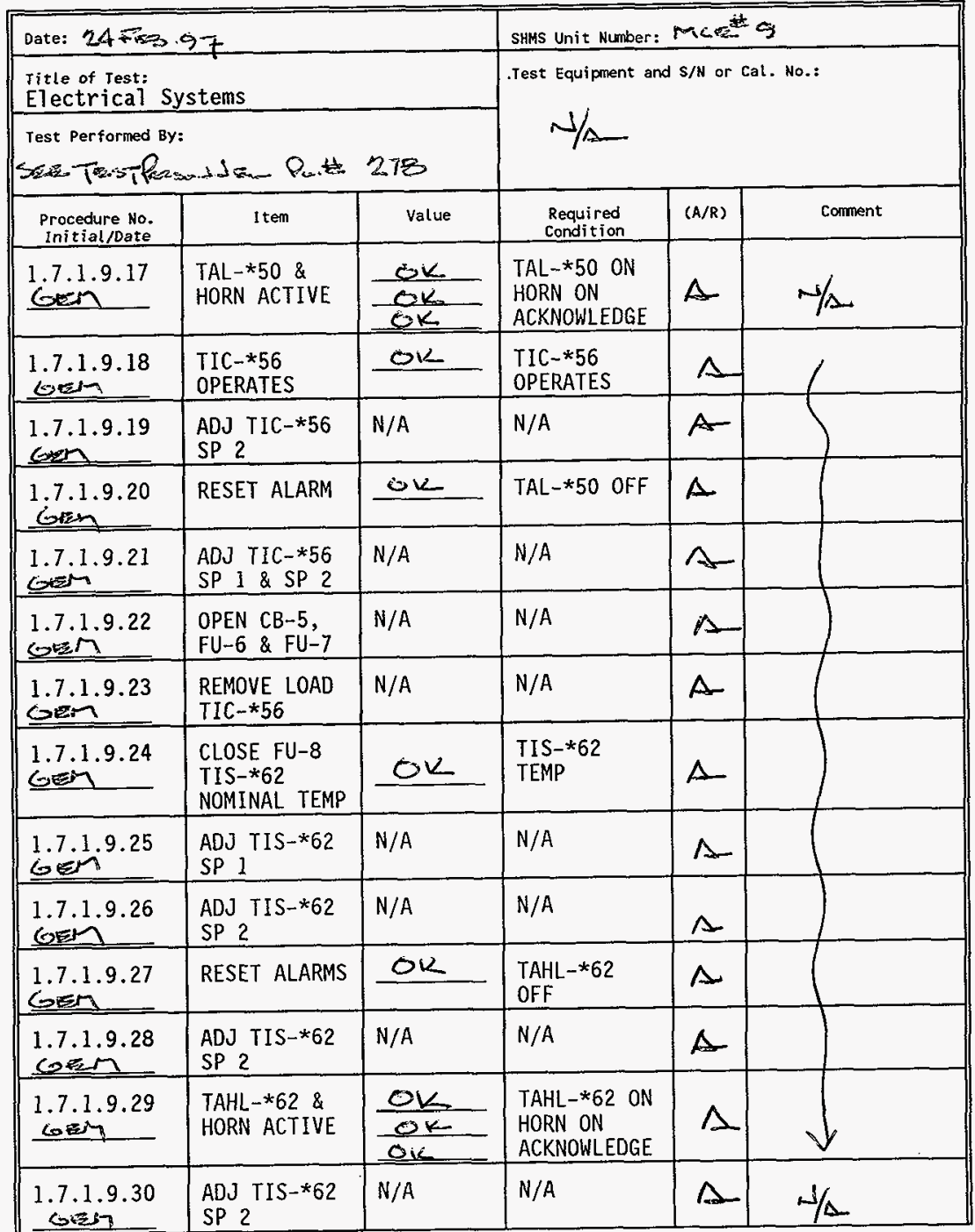


TEST DATA SHEET

\begin{tabular}{|c|c|c|c|c|c|}
\hline \multicolumn{3}{|c|}{ Date: $24=30.97$} & \multicolumn{3}{|c|}{ SHMS Unit Number: Mis it 9} \\
\hline \multicolumn{3}{|c|}{$\begin{array}{l}\text { Title of rest: } \\
\text { Electrical Systems }\end{array}$} & \multirow{2}{*}{\multicolumn{3}{|c|}{ 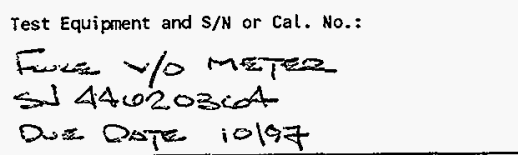 }} \\
\hline \multicolumn{3}{|c|}{ 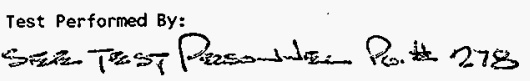 } & & & \\
\hline $\begin{array}{l}\text { Procedure No. } \\
\text { Initial/Date }\end{array}$ & Item & value & $\begin{array}{l}\text { Required } \\
\text { Condition } \\
\end{array}$ & $(A / R)$ & Coment \\
\hline 1.7 .1 .9 .31 & RESET ALARMS & or & $\begin{array}{l}\text { TAHL-*62 } \\
\text { OFF }\end{array}$ & $\Delta$ & $N / \mathrm{s}$ \\
\hline $\begin{array}{l}1.7 .1 .9 .32 \\
\end{array}$ & $\begin{array}{l}\text { ADJ TIS-*62 } \\
\text { SP } 1\end{array}$ & N/A & N/A & $\Delta$ & \\
\hline 1.7 .1 .9 .33 & $\begin{array}{l}\text { TAHL-*62 \& } \\
\text { HORN ACTIVE }\end{array}$ & $\frac{O K}{O K}$ & $\begin{array}{l}\text { TAHL-*62 ON } \\
\text { HORN ON } \\
\text { ACKNOWLEDGE }\end{array}$ & $\mathbb{A}$ & \\
\hline 1.7 .1 .9 .34 & $\begin{array}{l}\text { ADJ TIS-*62 } \\
S P 1 \& S P 2\end{array}$ & N/A & N/A & $A$ & - \\
\hline $\begin{array}{l}1.7 .1 .9 .35 \\
\text { oxim } \\
\end{array}$ & $\begin{array}{l}\text { OPEN FU-8, } \\
\text { FU-9 \& FU-10 }\end{array}$ & N/A & N/A & $\Delta$ & \\
\hline \multicolumn{6}{|c|}{ Section 1.7.1.10 verifies the differential pressure transmitter system. } \\
\hline Gem & $\begin{array}{l}\text { REMOVE PLUGS } \\
\text { BAL PDIT }-* 60 \\
\end{array}$ & $N / A$ & N/A & $\Lambda$ & \\
\hline$\frac{1.7 .1 .10 .2}{\sin ^{10}}$ & $\begin{array}{l}\text { CLOSE FU-14 } \\
\text { REC PDIT }-* 60 \\
\text { PRESSURE }\end{array}$ & .04 & $\begin{array}{l}\text { PDIT-*60 } \\
\text { PRESSURE } \\
0 \pm 1 " \mathrm{H}_{2} \mathrm{O} \\
\end{array}$ & A & $\Delta$ \\
\hline $1.73^{1.10 .3}$ & $\begin{array}{l}\text { MUX }-\star 70 \text { Vdc } \\
\text { TB7+\& TB8- }\end{array}$ & $A / A$ & $\begin{array}{l}\text { MUX }-* 70 \mathrm{Vdc} \\
2.2 \pm 0.1 \\
\end{array}$ & $N / S$ & 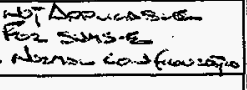 \\
\hline$\frac{1.7 .1 .10 .4}{\text { on }}$ & $\begin{array}{l}\text { REPLACE } \\
\text { PLUGS ON } \\
\text { PDIT }-\star 60 \\
\end{array}$ & $N / A$ & N/A & A & \\
\hline $\begin{array}{l}1.7 .1 .10 .5 \\
\text { Con }\end{array}$ & OPEN . FU-14 & $\mathrm{N} / \mathrm{A}$ & N/A & $\Delta$ & $-1 / \Delta$ \\
\hline \multicolumn{6}{|c|}{ Section 1.7.1.11 verifies the gas chromatograph power. } \\
\hline ר.11.1 & CLOSE FU-11 & N/A & $N / A$ & $\Delta$ & $2 / \Delta$ \\
\hline $\begin{array}{l}1.7 .1 .11 .2 \\
\text { Gesin } \\
\end{array}$ & $\begin{array}{l}\text { POWER } \\
\text { MON-*60 } \\
\end{array}$ & $O R$ & $\begin{array}{l}\text { MON-*60 } \\
\text { POWERED } \\
\end{array}$ & $\Delta$ & 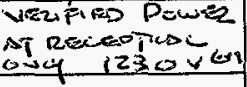 \\
\hline 1.7 .1 .11 .3 & OPEN FU-11 & $N / A$ & N/A & $\Lambda$ & $H$ \\
\hline
\end{tabular}


TEST DATA SHEET

\begin{tabular}{|c|c|c|c|c|c|}
\hline \multicolumn{3}{|c|}{ Date: 24 Fas 97} & \multicolumn{3}{|c|}{ SHMS Unit Number: Mcives 9} \\
\hline \multicolumn{3}{|c|}{$\begin{array}{l}\text { iitle of rest: } \\
\text { Electrical Systems }\end{array}$} & \multirow{2}{*}{\multicolumn{3}{|c|}{ 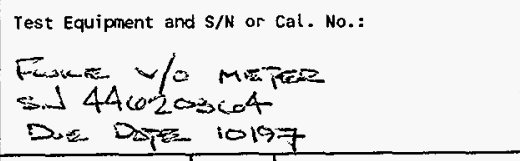 }} \\
\hline \multicolumn{3}{|c|}{ 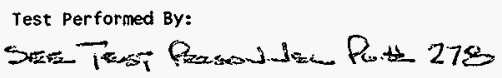 } & & & \\
\hline $\begin{array}{l}\text { Procedure No. } \\
\text { Initial/Date }\end{array}$ & I tem & value & $\begin{array}{l}\text { Required } \\
\text { condition }\end{array}$ & $(A / R)$ & Comment \\
\hline \multicolumn{6}{|c|}{ Section 1.7.1.12 verifies the multi gas analyzer power. } \\
\hline 1.7 .1 .12 .1 & CLOSE FU-12 & N/A & N/A & $\Delta$ & $N / \Lambda$ \\
\hline $\operatorname{sen}^{1.7 .1 .12 .2}$ & $\begin{array}{l}\text { POWER } \\
\text { NIT-*52 }\end{array}$ & OK & $\begin{array}{l}\text { NIT-*52 } \\
\text { POWERED } \\
\end{array}$ & $\Delta$ & 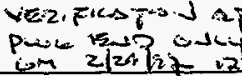 \\
\hline 1.7 .1 .12 .3 & OPEN FU-12 & N/A & $\mathrm{N} / \mathrm{A}$ & $\triangle$ & $\cos ^{2} 2 \operatorname{los} i s$ \\
\hline \multicolumn{6}{|c|}{ Section 1.7.1.13 verifies the personne 1 computer power. } \\
\hline $\begin{array}{l}1.7 .1 .13 .1 \\
6097\end{array}$ & CLOSE CB-6 & N/A & N/A & $\Delta$ & $N / A$ \\
\hline $\begin{array}{l}1.7 .1 .13 .2 \\
69\end{array}$ & $\begin{array}{l}\text { POWER PC-* } 60 \\
\& \text { PC-*70 }\end{array}$ & $\frac{N / A}{N / A}$ & $\begin{array}{ll}P C-* 60 & 0 N \\
P C-* 70 & 0 N\end{array}$ & $\mathrm{~N} / \mathrm{A}$ & $\begin{array}{l}\text { Ref Psen } \\
1.7 .4 .4\end{array}$ \\
\hline 1.7 .1 .13 .3 & $\begin{array}{l}\text { TURN INST. } \\
\text { OFF \& } \\
\text { OPEN CB-6 }\end{array}$ & $\mathrm{N} / \mathrm{A}$ & $\begin{array}{ll}P C-\star 60 & \text { OFF } \\
P C-* 70 & \text { OFF }\end{array}$ & $N / A$ & 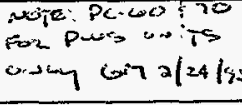 \\
\hline
\end{tabular}

con secis 2/24/197 
TEST DATA SHEET

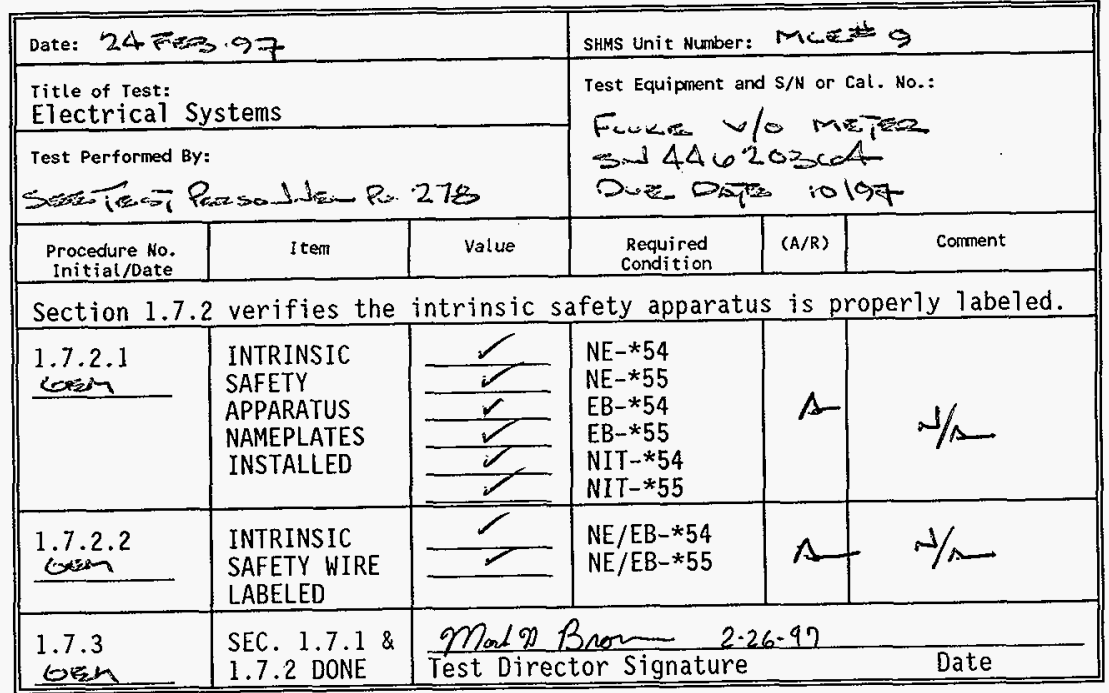

suzalsz Test Witness/Review:
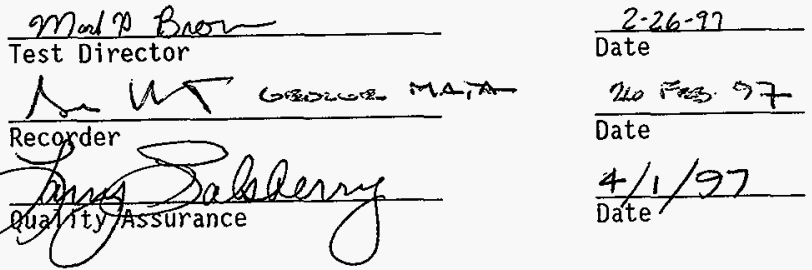
TEST DATA SHEET

\begin{tabular}{|c|c|c|c|c|c|}
\hline \multicolumn{3}{|c|}{ Date: $2478=077$} & \multicolumn{3}{|c|}{ SHMS Unit Number: MCE $\rightarrow$} \\
\hline \multicolumn{3}{|c|}{$\begin{array}{l}\text { Title of Test: } \\
\text { Pneumatic Systems }\end{array}$} & \multirow{2}{*}{\multicolumn{3}{|c|}{ Test Equipnent and $\mathrm{s} / \mathrm{N}$ or $\mathrm{Cal}$. No.: }} \\
\hline \multicolumn{3}{|c|}{ 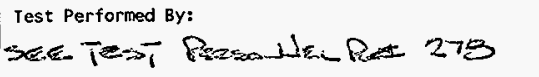 } & & & \\
\hline $\begin{array}{l}\text { Procedure No. } \\
\text { Initial/Date }\end{array}$ & Item & value & $\begin{array}{l}\text { Requi red } \\
\text { Condition }\end{array}$ & $(A / R)$ & Comment \\
\hline \multicolumn{6}{|c|}{ Section 1.7.4.1 provides the pneumatic test section initial conditions } \\
\hline $\begin{array}{l}1.7 .4 .1 .1 \\
\text { oeth }\end{array}$ & $\begin{array}{l}\text { INITIAL } \\
\text { CONDITIONS }\end{array}$ & OKL & $\begin{array}{l}\text { BKR \& FUSE } \\
\text { LINE UP }\end{array}$ & $\Lambda$ & \\
\hline $\begin{array}{l}1.7 .4 .1 .2 \\
60.1 \\
\end{array}$ & $\begin{array}{l}\text { INITIAL } \\
\text { CONDITIONS }\end{array}$ & OL & $\begin{array}{l}\text { VALVE LINE } \\
\text { UP }\end{array}$ & $\Delta$ & \\
\hline $\begin{array}{l}1.7 .4 .1 .3 \\
6.3 \\
\end{array}$ & $\begin{array}{l}\text { DISCONNECT } \\
1 / 16^{11} \text { TUBING } \\
\text { ON MON-*60 }\end{array}$ & $0 k$ & $\begin{array}{l}\text { TUBING } \\
\text { CONFIGURED }\end{array}$ & & \\
\hline $\begin{array}{l}1.7 .4 .1 .4 \\
\text { (x) }\end{array}$ & $\begin{array}{l}\text { DISCONNECT } \\
\text { TUBING ON } \\
\text { NIT } * 52 \\
\end{array}$ & OL & $\begin{array}{l}\text { TUBING } \\
\text { CONFIGURED }\end{array}$ & & \\
\hline $\begin{array}{l}1.7 .4 .1 .5 \\
6 \sin \\
\end{array}$ & $\begin{array}{l}\text { PLUG VENT } \\
\text { FLT-*63 }\end{array}$ & Oir & $\begin{array}{l}\text { FLT-*63 } \\
\text { PLUGGED }\end{array}$ & $A$ & \\
\hline \multicolumn{6}{|c|}{ Section 1.7.4.2 verifies the main sample flow loop. } \\
\hline $\begin{array}{l}1.7 .4 .2 .1 \\
0.2 \operatorname{lin}^{2}\end{array}$ & $\begin{array}{l}\text { CLOSE CB-3/4 } \\
\text { ADJ. FLOW }\end{array}$ & $\frac{9.2}{4.97}$ & $\begin{array}{l}\text { CB CLOSED } \\
\text { FIT } * 57 \\
\text { INDICATION }\end{array}$ & An & \\
\hline $\begin{array}{l}1.7 .4 .2 .2 \\
\end{array}$ & $\begin{array}{l}\text { PDI }-* 51 \\
\text { READING }\end{array}$ & 25 & $20-40 " \mathrm{H}_{2} \mathrm{O}$ & - & \\
\hline $\begin{array}{l}1.7 .4 .2 .3 \\
\text { seg }\end{array}$ & $\begin{array}{l}\text { PI } * \text { *53 } \\
\text { READING }\end{array}$ & -2.4 & $\begin{array}{l}-2 \text { to }-4 \\
" \mathrm{Hg}\end{array}$ & & \\
\hline 1.7 .4 .2 .4 & RESET ALARMS & OK & NO ALARMS & A & \\
\hline $\begin{array}{l}1.7 .4 .2 .5 \\
s=\end{array}$ & CLOSE SV-*16 & 0 & $\begin{array}{l}\text { FIT } * 57 \cdot 0 \\
\pm 1 \quad " H_{2} 0 \\
\text { FAL } * 57 \text { ON } \\
\text { HORN } \quad \text { ON } \\
\end{array}$ & $A$ & \\
\hline $\begin{array}{l}1.7 .4 .2 .6 \\
0.5\end{array}$ & OPEN SV-*16 & $\frac{O K}{O K}$ & $\begin{array}{l}\text { FAL-*57 ON } \\
\text { HORN OFF }\end{array}$ & $\Delta$ & $\underline{2}$ \\
\hline $\begin{array}{l}1.7 .4 .2 .7 \\
605\end{array}$ & RESET ALARMS & $\Delta k$ & $\begin{array}{l}\text { ALARMS } \\
\text { RESET } \\
\end{array}$ & Ar & $-1 / a$ \\
\hline
\end{tabular}

$\cos$ Ane 2/24157. 
TEST DATA SHEET

\begin{tabular}{|c|c|c|c|c|c|}
\hline \multicolumn{3}{|c|}{ Date: $24=03.97$} & \multicolumn{3}{|c|}{ SHMS Unit Number: MCFE } \\
\hline \multicolumn{3}{|c|}{$\begin{array}{l}\text { Title of Test: } \\
\text { Pneumatic Systems }\end{array}$} & \multirow{2}{*}{\multicolumn{3}{|c|}{ Test Equipment and $\mathrm{S} / \mathrm{N}$ or Cal. No.: }} \\
\hline \multicolumn{3}{|c|}{ 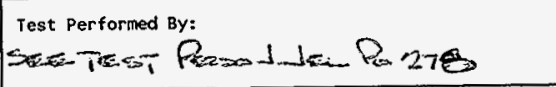 } & & & \\
\hline $\begin{array}{l}\text { Procedure No. } \\
\text { initial/Date }\end{array}$ & Item & Value & $\begin{array}{l}\text { Required } \\
\text { Condition }\end{array}$ & $(A / R)$ & Corment \\
\hline 1.7 .4 .2 .8 & CLOSE SV-*25 & $\frac{O K}{\frac{O K}{O K}} \frac{0 K}{2.1}$ & $\begin{array}{l}\text { FIT-*57 } 0 \\
\pm 1 \text { "H } 0 \\
\text { FAL-*57 ON } \\
\text { HORN ON } \\
\text { XA-*63 ON } \\
\text { YY-*71 } \\
\text { TB } 3-4 \text { OV } \\
\end{array}$ & $\Delta$ & 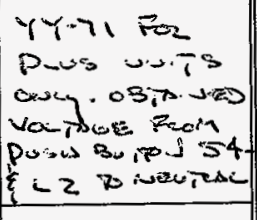 \\
\hline 1.7 .4 .2 .9 & OPEN SV $-* 25-1$ & $\frac{\frac{O K}{O K}}{\frac{0 k}{119.8}}$ & $\begin{array}{l}\text { FAL }-* 57 \text { ON } \\
\text { HORN OFF } \\
\text { XA-*63 OFF } \\
\text { YY-*71 } \\
\text { TB } 3-4 \text { 120V } \\
\end{array}$ & $\Delta$ & \\
\hline $\begin{array}{l}1.7 .4 .2 .10 \\
\text { ser }\end{array}$ & RESET ALARMS & $-O K$ & $\begin{array}{l}\text { ALARMS } \\
\text { RESET }\end{array}$ & $\Delta$ & \\
\hline 1.7 .4 .2 .11 & $\begin{array}{l}\text { OPEN SV }-* 54 \\
\& \text { SV }-* 66 \text { - } \\
\text { READ PRESS. } \\
\text { PDIT } * 60 \\
\end{array}$ & $\frac{o k}{-38.45}$ & $\begin{array}{l}\text { VALVES OPEN } \\
-27 \text { to }-82 \\
" \mathrm{H}_{2} \mathrm{O}\end{array}$ & A- & \\
\hline $\operatorname{lon}^{1.7 .4 .2 .12}$ & $\begin{array}{l}\text { CLOSE SV }-* 54 \\
\text { OPEN SV }-* 63 \\
\text { READ PRESS. } \\
\text { PDIT-*60 } \\
\end{array}$ & $\frac{.0 k}{.0 g}$ & $\begin{array}{l}\text { VALVES } \\
\text { OPERATED } \\
0 \pm I \quad \mathrm{H}_{2} \mathrm{O}\end{array}$ & $A$ & \\
\hline $\begin{array}{l}1.7 .4 .2 .13 \\
\text { oem }\end{array}$ & $\begin{array}{l}\text { CLOSE SV }-* 63 \gamma \\
\text { OPEN SV }-* 55 \\
\text { READ PRESS. } \\
\text { POIT } * 60 \\
\end{array}$ & $\frac{O K}{-39.83}$ & $\begin{array}{l}\text { VALVES } \\
\text { OPERATED } \\
-27 \text { to }-82 \\
" \mathrm{H}_{2} \mathrm{O} \\
\end{array}$ & $A$ & \\
\hline$\frac{1.7 .4 .2 .14}{607}$ & $\begin{array}{l}\text { CLOSE SV }-* 55 \cdot \\
\text { OPEN SV } * 64 \\
\text { READ PRESS. } \\
\text { PDIT-*60 } \\
\end{array}$ & $\begin{array}{l}\text { OK } \\
.02 \\
\end{array}$ & $\begin{array}{l}\text { VALVES } \\
\text { OPERATED } \\
0 \pm 1 " \mathrm{H}_{2} \mathrm{O}\end{array}$ & $A$ & \\
\hline $\begin{array}{l}1.7 .4 .2 .15 \\
\text { oinn } \\
\end{array}$ & $\begin{array}{l}\text { CLOSE SV-*64 } \\
\& \text { SV } * 66 .\end{array}$ & $N / A$ & $N / A$ & $A$ & \\
\hline Section 1.7 & 3 verifies the & grab samp & e flow loop. & & \\
\hline 1.7 .4 .3 .1 & READ PDI $-* 51$ & 25 & $20-40 \quad " \mathrm{H}_{2} \mathrm{O}$ & $A$ & $N / \Delta$ \\
\hline
\end{tabular}


APPENDIX I

TEST DATA SHEET

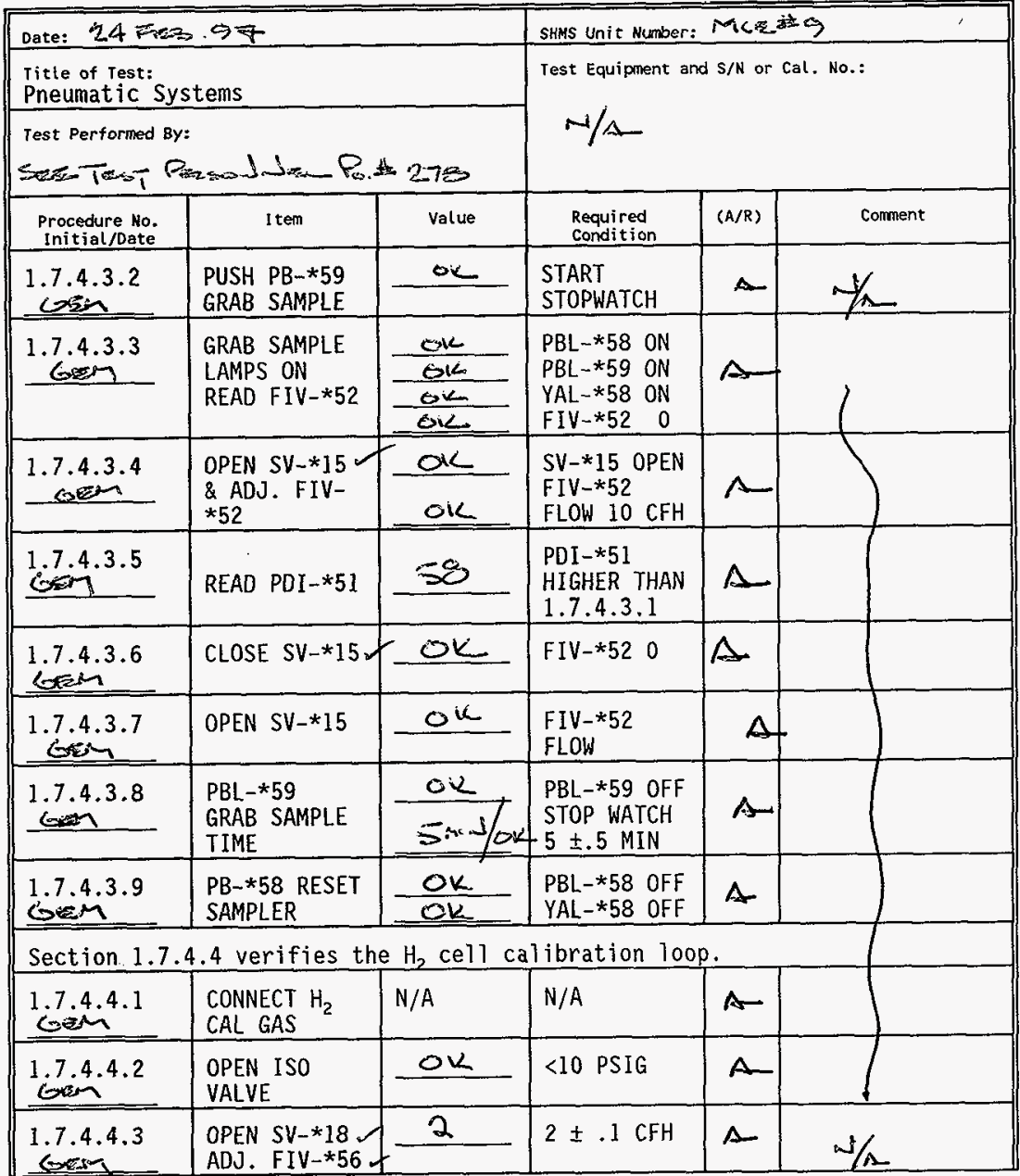

Goen Aun 2124197 
TEST DATA SHEET

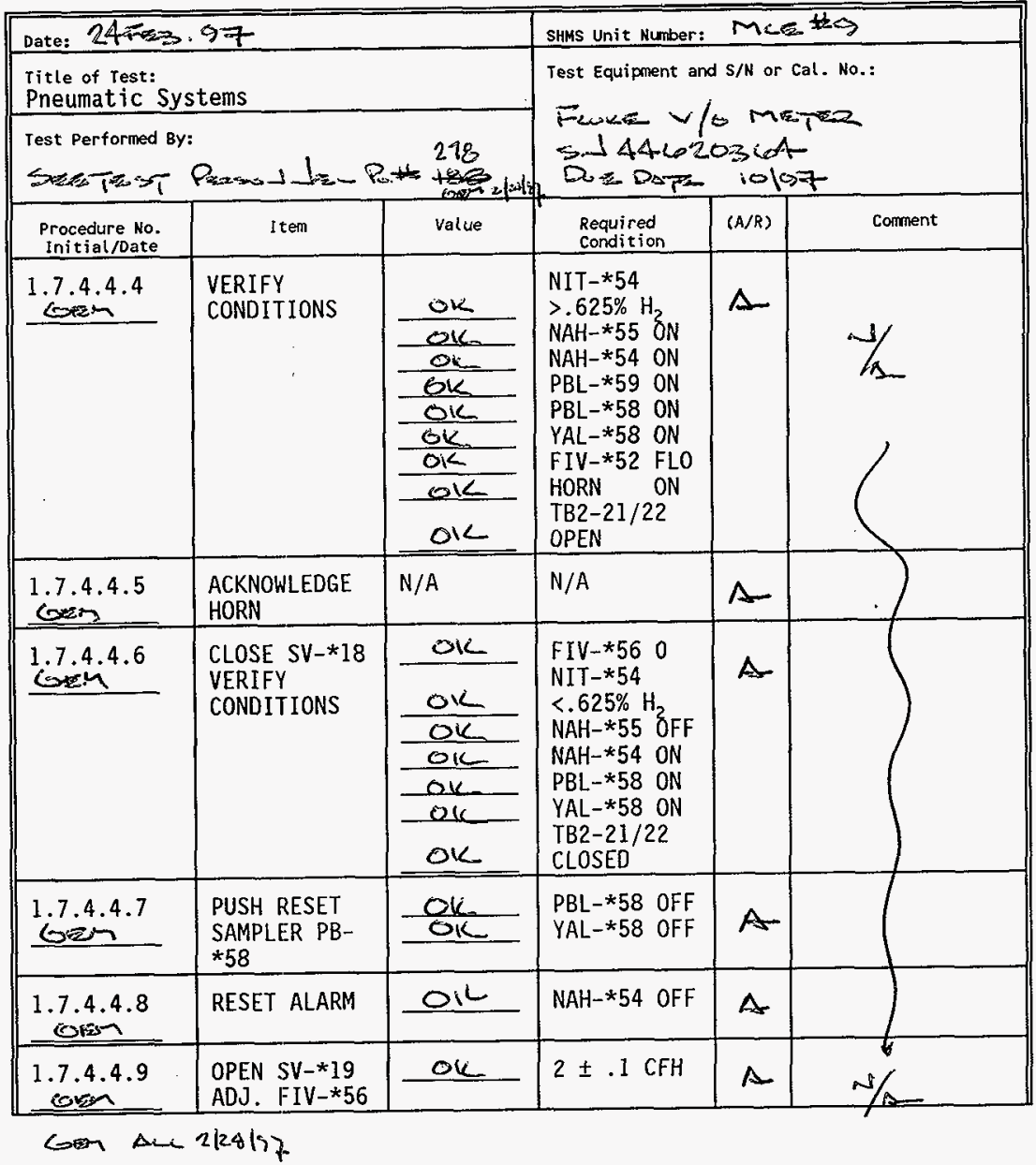


TEST DATA SHEET

\begin{tabular}{|c|c|c|c|c|c|}
\hline \multicolumn{3}{|c|}{ Date: 24 a } & \multicolumn{3}{|c|}{ SHMS Unit Number: Métsos } \\
\hline \multicolumn{3}{|c|}{$\begin{array}{l}\text { Title of Test: } \\
\text { Pneumatic Systems }\end{array}$} & \multirow{2}{*}{\multicolumn{3}{|c|}{ 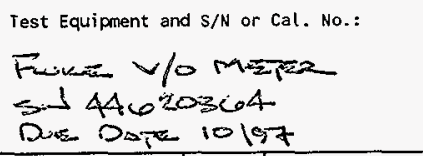 }} \\
\hline \multicolumn{3}{|c|}{ 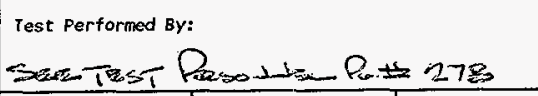 } & & & \\
\hline $\begin{array}{l}\text { Procedure No. } \\
\text { Initial/Date }\end{array}$ & Item & Value & $\begin{array}{l}\text { Required } \\
\text { Condition } \\
\end{array}$ & $(A / R)$ & Comment \\
\hline 68in & $\begin{array}{l}\text { VERIFY } \\
\text { CONDITIONS }\end{array}$ & $\begin{array}{l}\frac{O K}{O k} \\
o k \\
o k \\
\frac{o k}{O k} \\
o k \\
G k \\
o k\end{array}$ & $\begin{array}{l}\text { NIT-*55 } \\
>.625 \% \mathrm{H}_{2} \\
\text { NAH-*55 ON } \\
\text { NAH-*54 ON } \\
\text { PBL-*59 ON } \\
\text { PBL-*58 ON } \\
\text { YAL-*58 ON } \\
\text { FIV-*52 FLO } \\
\text { HORN ON } \\
\text { TB2-21/22 } \\
\text { OPEN } \\
\end{array}$ & $\Delta$ & \\
\hline $\begin{array}{l}1.7 .4 .4 .11 \\
\text {.65? } \\
\end{array}$ & $\begin{array}{l}\text { ACKNOWLEDGE } \\
\text { HORN }\end{array}$ & $N / A$ & $N / A$ & $\Delta$ & \\
\hline$\frac{1.7 .4 .4 .12}{6 x^{2}}$ & $\begin{array}{l}\text { CLOSE SV }-* 19 \\
\text { VERIFY } \\
\text { CONDITIONS }\end{array}$ & $\begin{array}{l}\text { OK } \\
\text { ok } \\
0 k \\
0 k \\
O k \\
O K \\
O K\end{array}$ & $\begin{array}{l}\text { FIV-*56 0 } \\
\text { NIT-*55 } \\
<.625 \% H_{2} \\
\text { NAH-*55 OFF } \\
\text { NAH-*54 ON } \\
\text { PBL-*58 ON } \\
\text { YAL-*58 ON } \\
\text { TB2-21/22 } \\
\text { CLOSED } \\
\end{array}$ & $\Delta$ & \\
\hline 1.7 .4 .4 .13 & $\begin{array}{l}\text { PUSH RESET } \\
\text { SAMPLER PB- } \\
* 58\end{array}$ & $\frac{o k}{a k}$ & $\begin{array}{l}\text { PBL-*58 OFF } \\
\text { YAL-*58 OFF }\end{array}$ & $A$ & \\
\hline${ }^{1} \operatorname{bix}^{7} i^{4.14}$ & RESET ALARM & OK & $\mathrm{NAH}-* 54$ OFF & $\Lambda$ & \\
\hline 1.7 .4 .4 .15 & $\begin{array}{l}\text { DISCONNECT } \\
\text { CAL. GAS }\end{array}$ & N/A & N/A & $\Lambda$ & \\
\hline$\frac{1.7 .4 .4 .16}{607}$ & $\begin{array}{l}\text { PUSH PB-*54 } \\
\text { VERIFY } \\
\text { CONDIYIONS }\end{array}$ & $\frac{O K}{O K}$ & $\begin{array}{l}X A-* 63 \text { ON } \\
\text { PBL-*54 ON } \\
\text { TB2-23/24 } \\
\text { OPEN } \\
\end{array}$ & $\Delta$ & \\
\hline germ $^{1.7 .4 .4 .17}$ & $\begin{array}{l}\text { PUSH PB-*54 } \\
\text { VERIFY } \\
\text { CONDITIONS }\end{array}$ & $\frac{O K}{O K}$ & $\begin{array}{l}\text { XA-*63 OFF } \\
\text { PBL }-* 54 \text { OFF } \\
\text { TB2-23/24 } \\
\text { CLOSED }\end{array}$ & $\Delta$ & $1 /$ \\
\hline
\end{tabular}


TEST DATA SHEET

\begin{tabular}{|c|c|c|c|c|c|}
\hline \multicolumn{3}{|c|}{ Date: $24 \overline{r e s} .97$} & \multicolumn{3}{|c|}{ SHMS Unit Number: Mcazis 9} \\
\hline \multicolumn{3}{|c|}{$\begin{array}{l}\text { ritle of rest: } \\
\text { Pneumatic Systems }\end{array}$} & \multirow{2}{*}{\multicolumn{3}{|c|}{$\begin{array}{l}\text { Test Equipment and } \mathrm{S} / \mathrm{N} \text { or Cal. No.: } \\
\mathrm{S} / \mathrm{\Lambda}\end{array}$}} \\
\hline \multicolumn{3}{|c|}{ 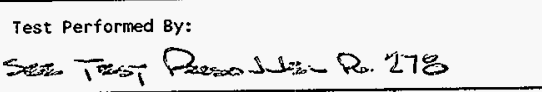 } & & & \\
\hline $\begin{array}{l}\text { Procedure No. } \\
\text { Initial/Date } \\
\end{array}$ & Item & Value & $\begin{array}{l}\text { Required } \\
\text { Condition } \\
\end{array}$ & $(A / R)$ & Corment \\
\hline \multicolumn{6}{|c|}{ Section 1.7.4.5 verifies the GC instrument tubing and calibration gas loop. } \\
\hline 1.7 .4 .5 .1 & $\begin{array}{l}\text { REMOVE PLUG } \\
\& \text { CONNECT } \\
\text { TEST GAS } \\
\end{array}$ & OK & $\begin{array}{l}\text { TEST GAS } \\
3-5 \text { PSIG }\end{array}$ & A & \\
\hline $\begin{array}{l}1.7 .4 .5 .2 \\
6 \times 29\end{array}$ & $\begin{array}{l}\text { VERIFY NO } \\
\text { GAS FLOW }\end{array}$ & $\frac{O K}{O K}$ & $\begin{array}{l}\mathrm{H}_{2} \text { GAS PORT } \\
\text { SAMPLE A IN }\end{array}$ & A & \\
\hline 1.7 .4 .5 .3 & $\begin{array}{l}\text { VERIFY COL A } \\
\text { GAS FLOW }\end{array}$ & ok & COL A FLOW & $\Delta$ & \\
\hline$\frac{1.7 .4 .5 .4}{6 \sin ^{2}}$ & $\begin{array}{l}\text { REPLACE COL } \\
\text { A CAP }\end{array}$ & N/A & N/A & $\Lambda$ & \\
\hline $\begin{array}{l}1.7 .4 .5 .5 \\
\text { Conen }\end{array}$ & $\begin{array}{l}\text { VERIFY COL B } \\
\text { GAS FLOW }\end{array}$ & OR & COL B FLOW & $\Lambda$ & \\
\hline $\begin{array}{l}1.7 .4 .5 .6 \\
\end{array}$ & $\begin{array}{l}\text { REPLACE COL } \\
\text { B CAP }\end{array}$ & N/A & N/A & $\Delta$ & \\
\hline$\frac{1.7 .4 .5 .7}{\sin }$ & $\begin{array}{l}\text { VERIFY } \\
\text { SAMPLE VENT } \\
\text { FLOW }\end{array}$ & ok & $\begin{array}{l}\text { SAMPLE VENT } \\
\text { FLOW }\end{array}$ & $\Delta$ & \\
\hline 1.7 .4 .5 .8 & $\begin{array}{l}\text { REPLACE } \\
\text { SAMPLE VENT } \\
\text { CAP }\end{array}$ & $N / A$ & N/A & A & \\
\hline $\begin{array}{l}1.7 .4 .5 .9 \\
6 \cos ^{2}\end{array}$ & $\begin{array}{l}\text { INSTALL } \\
\text { JUMPER } \\
\end{array}$ & N/A & N/A & $\Delta$ & 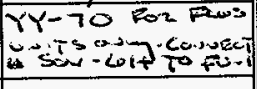 \\
\hline $\begin{array}{l}1.7 .4 .5 .10 \\
\text { Goin }\end{array}$ & $\begin{array}{l}\text { CLOSE FU-15 } \\
\text { VERIFY FLOW }\end{array}$ & $\frac{O K}{O K}$ & $\begin{array}{l}\mathrm{H}_{2} \text { CAL FLOW } \\
\text { SAMP A IN } \\
\text { FLOW }\end{array}$ & $\Delta$ & \\
\hline $\begin{array}{l}1.7 .4 .5 .11 \\
\end{array}$ & $\begin{array}{l}\text { INSTALL PLUG } \\
\& \text { CAP }\end{array}$ & OK & $\begin{array}{l}\text { PLUG } H_{2} \text { CAL } \\
\text { CAP SAMP A } \\
\end{array}$ & $\Delta$ & \\
\hline$\frac{1.7 .4 .5 .12}{6}$ & $\begin{array}{l}\text { OPEN SV }-* 63 \\
\& \text { READ } \\
\text { PDIT } * 60\end{array}$ & 140.5 & $\begin{array}{l}\text { PDIT }-* 60= \\
28 \times \text { PRESS } \\
1.7 .4 .5 .1 \\
\pm 20 " \mathrm{H}_{2} \mathrm{O} \\
\end{array}$ & $A$ & 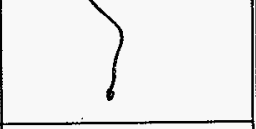 \\
\hline 1.7 .4 .5 .13 & $\begin{array}{l}\text { OPEN SV }-* 64 \\
\text { VERIFY FLOW }\end{array}$ & $O K$ & $\begin{array}{l}\text { SAMP B IN } \\
\text { FLOW }\end{array}$ & $A$ & \\
\hline
\end{tabular}


TEST DATA SHEET

\begin{tabular}{|c|c|c|c|c|c|}
\hline \multicolumn{3}{|c|}{ Date: $24 \overline{F E s} 97$} & \multicolumn{3}{|c|}{ SHMS Unit Number: MLE_to } \\
\hline \multicolumn{3}{|c|}{$\begin{array}{l}\text { Title of Test: } \\
\text { Pneumatic Systems }\end{array}$} & \multirow{2}{*}{\multicolumn{3}{|c|}{ Test Equipment and S/N or Cal. No.: }} \\
\hline \multicolumn{3}{|c|}{ 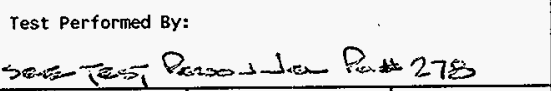 } & & & \\
\hline $\begin{array}{c}\text { Procedure No. } \\
\text { Initial/Date }\end{array}$ & Item & value & $\begin{array}{l}\text { Required } \\
\text { Condition }\end{array}$ & $(A / R)$ & Corment \\
\hline Germ.14 & $\begin{array}{l}\text { REMOVE TEST } \\
\text { GAS }\end{array}$ & N/A & $N / A$ & $\Delta$ & \\
\hline 1.7 .4 .5 .15 & $\begin{array}{l}\text { CLOSE SV }-* 63 \\
\& \text { SV-*64 }\end{array}$ & $N / A$ & $N / A$ & 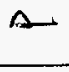 & \\
\hline${ }^{1.7 .4 .5 .16}$ & $\begin{array}{l}\text { OPEN FU-15 } \\
\text { REMOVE } \\
\text { JUMPER }\end{array}$ & N/A & $N / A$ & $\Lambda$ & \\
\hline $\begin{array}{l}1.7 .4 .5 .17 \\
\end{array}$ & $\begin{array}{l}\text { INSTALL } \\
\text { SAMP A UNION }\end{array}$ & N/A & $N / A$ & $\Lambda$ & \\
\hline 1.7 .4 .5 .18 & $\begin{array}{l}\text { OPEN SV } * 60 \\
\text { VERIFY FLOW }\end{array}$ & OK & $\begin{array}{l}\text { FI-*60 } \\
\text { NO FLOW }\end{array}$ & A & \\
\hline 6. & $\begin{array}{l}\text { POWER SOV- } \\
* 60 \text {, VERIFY } \\
\text { NO FLOW }\end{array}$ & OK & $\begin{array}{l}\text { FI-*60 } \\
\text { NO FLOW }\end{array}$ & 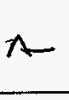 & \\
\hline $\sin ^{1.7 .4 .5 .20}$ & $\begin{array}{l}\text { OPEN SV-*68 } \\
\text { ADJ SV-*67 }\end{array}$ & 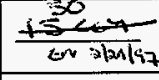 & $\begin{array}{l}\mathrm{FI}-* 60 \\
50 \mathrm{CCM}\end{array}$ & $A$ & 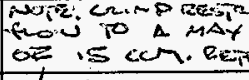 \\
\hline $\begin{array}{l}1.7 .4 .5 .21 \\
6 x=5\end{array}$ & $\begin{array}{l}\text { DEENERGIZE } \\
\text { SOV }-\star 60\end{array}$ & OK & $\begin{array}{l}\text { FI-*60 FLOW } \\
\text { DECREASES } \\
\end{array}$ & $\Lambda$ & crimpers cons \\
\hline 1.7 .4 .5 .22 & $\begin{array}{l}\text { CLOSE SV-*60 } \\
\& \text { SV-*68 }\end{array}$ & N/A & $N / A$ & $\Delta$ & \\
\hline 1.7 .4 .5 .23 & $\begin{array}{l}\text { REMOVE } \\
\text { SAMP A UNION }\end{array}$ & $N / A$ & $N / A$ & A & \\
\hline $\begin{array}{l}1.7 .4 .5 .24 \\
\text { sent }\end{array}$ & $\begin{array}{l}\text { CONNECT TEST } \\
\text { GAS } \mathrm{N}_{2} \mathrm{O} \text { PORT } \\
\end{array}$ & 5 & $\begin{array}{l}\text { TEST GAS } \\
3-5 \text { PSIG } \\
\end{array}$ & $\curvearrowright$ & \\
\hline $\begin{array}{l}1.7 .4 .5 .25 \\
\text { ofen }\end{array}$ & $\begin{array}{l}\text { VERIFY NO } \\
\text { FLOW }\end{array}$ & $\frac{O K}{O L}$ & $\begin{array}{l}\text { SAMP B IN \& } \\
\text { FLT-*63 } \\
\text { NO FLOW } \\
\end{array}$ & $A$ & 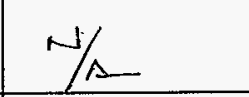 \\
\hline $\begin{array}{l}1.7 .4 .5 .26 \\
6+25 \\
\end{array}$ & $\begin{array}{l}\text { INSTALL } \\
\text { JUMPER }\end{array}$ & $N / A$ & $N / A$ & 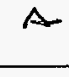 & $602 \cos x$ \\
\hline $\begin{array}{l}1.7 .4 .5 .27 \\
\text { ben }\end{array}$ & $\begin{array}{l}\text { CLOSE FU-15 } \\
\text { VERIFY FLOW }\end{array}$ & ok & $\begin{array}{l}\text { SAMP B IN } \\
\text { FLT }-* 63 \\
\text { FLOW }\end{array}$ & $\Lambda$ & \\
\hline
\end{tabular}


TEST DATA SHEET

\begin{tabular}{|c|c|c|c|c|c|}
\hline \multicolumn{3}{|c|}{ Date: $24 F_{123} .97$} & \multicolumn{3}{|c|}{ SHMS Unit Number: MCEN $Q$} \\
\hline \multicolumn{3}{|c|}{$\begin{array}{l}\text { Title of rest: } \\
\text { Pneumatic Systems }\end{array}$} & \multirow{2}{*}{\multicolumn{3}{|c|}{$\begin{array}{l}\text { Test Equipment and } \mathrm{S} / \mathrm{N} \text { or Cal. No.: } \\
\text { S/S_ }\end{array}$}} \\
\hline \multicolumn{3}{|c|}{ 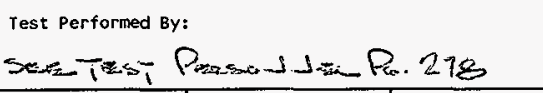 } & & & \\
\hline $\begin{array}{l}\text { Procedure No. } \\
\text { Initial/Date }\end{array}$ & Item & value & $\begin{array}{l}\text { Required } \\
\text { Condition } \\
\end{array}$ & $(A / R)$ & Corment \\
\hline $\begin{array}{l}1.7 .4 .5 .28 \\
\text { g.an }\end{array}$ & $\begin{array}{l}\text { DISCONNECT } \\
\text { TEST GAS }\end{array}$ & $N / A$ & $N / A$ & A & \\
\hline $\begin{array}{l}1.7 .4 .5 .29 \\
\end{array}$ & OPEN FU-15 & $N / A$ & $N / A$ & $\Delta$ & \\
\hline$\underline{\operatorname{sen}}^{1.4 .5 .30}$ & $\begin{array}{l}\text { REMOVE } \\
\text { JUMPER }\end{array}$ & $N / A$ & $N / A$ & A & \\
\hline $\begin{array}{l}1.7 .4 .5 .31 \\
6 \operatorname{sen} \\
\end{array}$ & $\begin{array}{l}\text { INSTALL } \\
\text { SAMP B UNION }\end{array}$ & N/A & $N / A$ & $A$ & \\
\hline $\mathrm{Gesh}^{1.7 .4 .5 .32}$ & $\begin{array}{l}\text { OPEN SV-*60 } \\
\text { VERIFY FLOW }\end{array}$ & ok & $\begin{array}{l}\text { FI }-* 60 \\
\text { NO FLOW }\end{array}$ & $\Delta$ & \\
\hline $67^{1.7 .4 .5 .33}$ & $\begin{array}{l}\text { POWER SOV- }{ }^{-} \\
* 60, \text { VERIFY } \\
\text { NO FLOW } \\
\end{array}$ & - oir & $\begin{array}{l}\text { FI-*60 } \\
\text { NO FLOW }\end{array}$ & $A$ & \\
\hline G.7.4.5.34 & $\begin{array}{l}\text { OPEN SV-*68 } \\
\& \text { ADJ SV-*67y }\end{array}$ & So & $\begin{array}{l}\mathrm{FI}-\star 60 \\
50 \mathrm{CCM} \\
\end{array}$ & $\Delta$ & \\
\hline $6^{1.7 .4 .5 .35}$ & $\begin{array}{l}\text { DEENERGIZE } \\
\text { SOV-*60 }\end{array}$ & 0 & $\begin{array}{l}\text { FI } * * 60 \text { FLOW } \\
\text { DECREASES }\end{array}$ & $A$ & \\
\hline $\begin{array}{l}1.7 .4 .5 .36 \\
\end{array}$ & $\begin{array}{l}\text { CLOSE SV-*60 } \\
\& \text { SV-*68 }\end{array}$ & N/A & $N / A$ & $A$ & \\
\hline $\begin{array}{l}1.7 .4 .5 .37 \\
\end{array}$ & $\begin{array}{l}\text { REMOVE } \\
\text { SAMP B UNION }\end{array}$ & N/A & N/A & $\Delta$ & \\
\hline 1.7 .4 .5 .38 & $\begin{array}{l}\text { OPEN CB-3/4 } \\
\text { ACK. ALARMS }\end{array}$ & N/A & N/A & $\Delta$ & $\mu / \Delta$ \\
\hline \multicolumn{6}{|c|}{$\begin{array}{l}\text { Section } 1.7 .4 .6 \text { verifies the multi gas analyzer instrument tubing and } \\
\text { calibration gas loop. }\end{array}$} \\
\hline $\begin{array}{l}1.7 .4 .6 .1 \\
\text { sonen }\end{array}$ & $\begin{array}{l}\text { VERIFY } \\
\text { NIT }-* 52 \\
\text { SAMPLE LINES }\end{array}$ & N/A & N/A & $\Delta$ & \\
\hline 1.7 .4 .6 .2 & $\begin{array}{l}\text { CONNECT TEST } \\
\text { GAS NH } 3 \text { PORT }\end{array}$ & 5 & $\begin{array}{l}\text { TEST GAS } \\
3-5 \text { PSIG } \\
\end{array}$ & $\Delta$ & 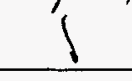 \\
\hline $\operatorname{sen}^{1.7 .4 .6 .3}$ & $\begin{array}{l}\text { VERIFY NO } \\
\text { FLOW }\end{array}$ & $\frac{O k}{0.16}$ & $\begin{array}{l}\text { SAMPLE IN } \\
\text { FLT-*63 }\end{array}$ & $\Delta$ & $\omega$ \\
\hline
\end{tabular}


TEST DATA SHEET

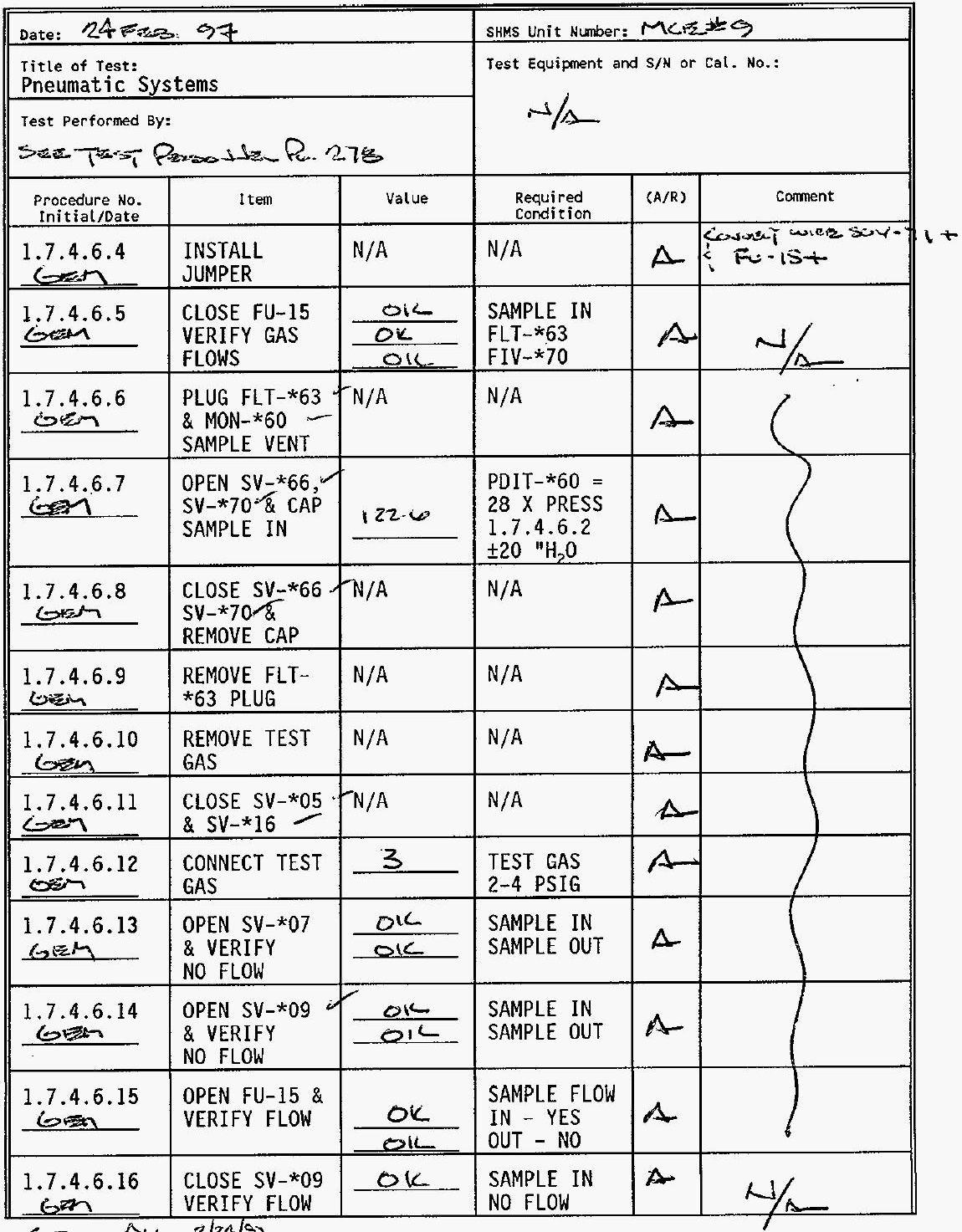


TEST DATA SHEET

\begin{tabular}{|c|c|c|c|c|c|}
\hline \multicolumn{3}{|c|}{ Date: $24 F_{\mathrm{w}} .97$} & \multicolumn{3}{|c|}{ SHMS Unit Number: Mutits } \\
\hline \multicolumn{3}{|c|}{$\begin{array}{l}\text { Title of Test: } \\
\text { Pneumatic Systems }\end{array}$} & \multirow{2}{*}{\multicolumn{3}{|c|}{ Test Equipment and $\mathrm{S} / \mathrm{N}$ or Cal. No.: }} \\
\hline \multicolumn{3}{|c|}{ 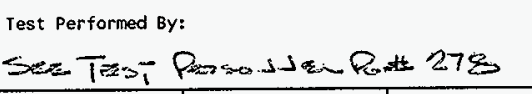 } & & & \\
\hline $\begin{array}{c}\text { Procedure No. } \\
\text { Initial/Date }\end{array}$ & I tem & value & $\begin{array}{l}\text { Required } \\
\text { Condition }\end{array}$ & $(\mathrm{A} / \mathrm{R})$ & comment \\
\hline$\underbrace{1.7 .4 .6 .17}$ & $\begin{array}{l}\text { OPEN SV-*10 } \\
\text { VERIFY FLOW }\end{array}$ & OK & $\begin{array}{l}\text { SAMPLE FLOW } \\
\text { IN - NO } \\
\text { OUT - YES }\end{array}$ & $\Delta$ & $\omega / \Omega$ \\
\hline$\frac{1.7 .4 .6 .18}{\tan }$ & $\begin{array}{l}\text { REMOVE TEST } \\
\text { GAS \& PLUG } \\
\text { SV }-* 07\end{array}$ & $N / A$ & $N / A$ & $\Delta$ & \\
\hline 6.4.6.19 & $\begin{array}{l}\text { CLOSE SV-*07 } \\
\& \text { SV-*10 }\end{array}$ & $N / A$ & $N / A$ & 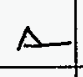 & \\
\hline $\begin{array}{l}1.7 .4 .6 .20 \\
\end{array}$ & $\begin{array}{l}\text { OPEN SV }-* 05 \\
\& S V-* 16\end{array}$ & N/A & $N / A$ & $A$ & \\
\hline $\begin{array}{l}1.7 .4 .6 .21 \\
\text { coten }\end{array}$ & $\begin{array}{l}\text { REMOVE } \\
\text { JUMPER }\end{array}$ & $N / A$ & $N / A$ & $\Lambda$ & \\
\hline \multicolumn{6}{|c|}{ Section 1.7.4.7 verifies the carrier gas supply 1 ines. } \\
\hline i.t.7.7.1 & $\begin{array}{l}\text { CONNECT TEST } \\
\text { GAS }\end{array}$ & $\Sigma$ & 3-5 PSIG & $\Delta$ & \\
\hline $\begin{array}{l}1.7 .4 .7 .2 \\
\text { sizin }\end{array}$ & $\begin{array}{l}\text { REMOVE PLUG } \\
\& \text { VERIFY } \\
\text { FLOW } \\
\end{array}$ & OK & $\begin{array}{l}\mathrm{N}_{2} \text { CARRIER } \\
\text { GAS FLOWS }\end{array}$ & $A$ & \\
\hline $\begin{array}{c}1.7 .4 .7 .3 \\
\end{array}$ & $\begin{array}{l}\text { DISCONNECT } \\
\text { TEST GAS } \\
\end{array}$ & N/A & $N / A$ & $A$ & \\
\hline $\begin{array}{l}1.7 .4 .7 .4 \\
\text { Soxen }\end{array}$ & $\begin{array}{l}\text { CONNECT TEST } \\
\text { GAS }\end{array}$ & 5 & 3-5 PSIG & A & \\
\hline Gem & $\begin{array}{l}\text { REMOVE PLUG } \\
\& \text { VERIFY } \\
\text { FLOW } \\
\end{array}$ & or & $\begin{array}{l}\text { He CARRIER } \\
\text { GAS FLOWS }\end{array}$ & $A$ & \\
\hline$\stackrel{1.4 .7}{\tan }^{1.7 .6}$ & $\begin{array}{l}\text { DISCONNECT } \\
\text { TEST GAS }\end{array}$ & $N / A$ & N/A & $\Delta$ & \\
\hline $\begin{array}{l}1.7 .5 \\
\operatorname{con} \\
\end{array}$ & $\begin{array}{l}\text { TEST SEC. } \\
1.7 .4 \text { DONE }\end{array}$ & \multicolumn{4}{|c|}{$\begin{array}{ll}\text { Marh } 9 \mathrm{~d} \text { Bnow } & 2-26-97 \\
\text { Test Director Signature } & \text { Date }\end{array}$} \\
\hline
\end{tabular}

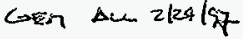


TEST DATA SHEET

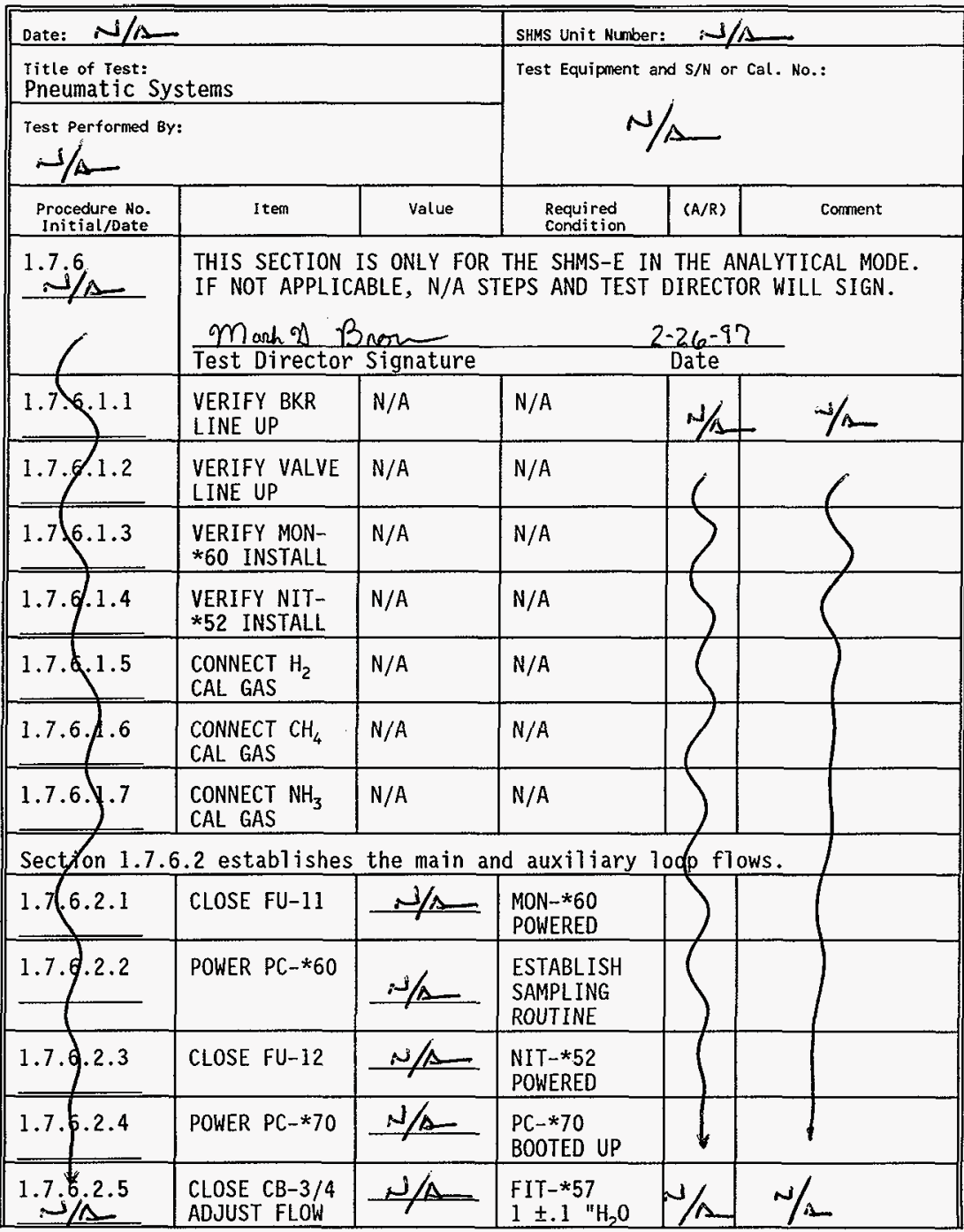


APPENDIX I

TEST DATA SHEET

\begin{tabular}{|c|c|c|c|c|c|}
\hline \multicolumn{3}{|l|}{ Date: $N / \mathbb{A}$} & \multicolumn{3}{|c|}{ SHiMS Unit Number: $\mathrm{N} / \mathrm{A}$} \\
\hline \multicolumn{3}{|c|}{$\begin{array}{l}\text { Title of rest: } \\
\text { Pneumatic Systems }\end{array}$} & \multirow{2}{*}{\multicolumn{3}{|c|}{ Test Equipment and $\mathrm{S} / \mathrm{N}$ or cal. No.: }} \\
\hline \multicolumn{3}{|c|}{ Test Performed By: } & & & \\
\hline $\begin{array}{l}\text { Procedure No. } \\
\text { Initial/Date }\end{array}$ & Item & Value & $\begin{array}{l}\text { Required } \\
\text { Condition }\end{array}$ & $(A / R)$ & cormen \\
\hline $1.73^{6.2 .6}$ & $\begin{array}{l}\text { OPEN SV }-* 09 \\
\& S V-* 10\end{array}$ & $N / A$ & $N / A$ & & $-1 / 2$ \\
\hline $1.7 . \wp^{2.7}$ & $\begin{array}{l}\text { OPEN SV }-* 60 \\
63,65 \& 68\end{array}$ & N/A & N/A & & \\
\hline 1.7 .6 & NIT-*52 & $\frac{1 / A}{1}$ & $\begin{array}{l}\text { ESTABLISH } \\
\text { SAMPL ING } \\
\text { ROUTINE } \\
\end{array}$ & & \\
\hline 1.7 .6$) 2.9$ & $\begin{array}{l}\text { OPEN SV-*24 } \\
\text { ADJ FLOW }\end{array}$ & & $\begin{array}{l}\mathrm{FIT}-* 57 \\
3 \pm .1 " \mathrm{H}_{2} \mathrm{O}\end{array}$ & & \\
\hline 1.7 .5 .2 .10 & $\begin{array}{l}\text { ADJ FLOW } \\
\text { SV-*67 }\end{array}$ & & $\begin{array}{l}\mathrm{FI}-* 60 \\
10-30 \mathrm{ccm} \\
\end{array}$ & & \\
\hline 1.7 .6 & $\begin{array}{l}\text { RECORD } \\
\text { PDIT- } * 60 \\
\end{array}$ & $\pi / 1$ & $<175 \quad \mathrm{H}_{2} \mathrm{O}$ & & \\
\hline 1.7 .9 .2 .12 & $\begin{array}{l}\text { OPEN SV-*64 } \\
\text { CLOSE SV-*63 }\end{array}$ & $N / A$ & N/A & & \\
\hline 1.7 .6 .2 .13 & $\begin{array}{l}\text { RECORD } \\
\text { PDIT-*60 } \\
\end{array}$ & $\omega / A$ & $<175 \quad " \mathrm{H}_{2} \mathrm{O}$ & & \\
\hline $1.7 / 6.2 .14$ & $\begin{array}{l}\text { CLOSE SV }-* 65 \\
\text { OPEN SV }-* 66 \\
\end{array}$ & N/A & $N / A$ & & \\
\hline$1 . 7 . 6 \longdiv { 2 . 1 5 }$ & $\begin{array}{l}\text { RECORD B IN } \\
\text { PDIT }-* 60\end{array}$ & $N / \Lambda$ & $\begin{array}{l}-15 \text { to }-50 \\
" \mathrm{H}_{2} \mathrm{O} \\
\end{array}$ & & \\
\hline 1.7 .22 .16 & $\begin{array}{l}\text { OPEN SV }-* 63 \\
\text { CLOSE SV } * 64 \\
\end{array}$ & $N / A$ & $N / A$ & & \\
\hline $1.7 .6 / 2.17$ & $\begin{array}{l}\text { RECORD A IN } \\
\text { PDIT-*60 }\end{array}$ & $N / \Delta$ & $\begin{array}{l}-15 \text { to }-50 \\
" \mathrm{H}_{3} \mathrm{O}\end{array}$ & & \\
\hline 1.7 .6 .2$) .18$ & $\begin{array}{l}\text { INSTALL } \\
\text { JUMPER } \\
\end{array}$ & N/A & $N / A$ & & \\
\hline 1.7 .6 .2 .19 & CLOSE FU-15 & $N / A$ & $N / A$ & & \\
\hline$\frac{1.7 .6 .2 .20}{\mathrm{H}^{2}}$ & $\begin{array}{l}\text { ADJ SV }-\star 61 \& \\
\text { RECORD } \\
\text { PDIT }-* 60 \\
\end{array}$ & $N / A$ & $\begin{array}{l} \pm 5 " \mathrm{H}_{2} 0 \quad \text { OF } \\
1.7 .6 .2 .17\end{array}$ & $\begin{array}{r}V \\
N\end{array}$ & $N / 2$ \\
\hline
\end{tabular}


TEST DATA SHEET

\begin{tabular}{|c|c|c|c|c|c|}
\hline \multicolumn{3}{|c|}{ Date: N/A } & \multicolumn{3}{|c|}{ SHMS Unit Number: } \\
\hline \multicolumn{3}{|c|}{$\begin{array}{l}\text { Title of Test: } \\
\text { Pneumatic Systems }\end{array}$} & \multirow{2}{*}{\multicolumn{3}{|c|}{ Test Equipment and $\mathrm{S} / \mathrm{N}$ or Cal. No.: }} \\
\hline \multicolumn{3}{|c|}{ Test Performed By: } & & & \\
\hline $\begin{array}{l}\text { Procedure No. } \\
\text { Initial/Date }\end{array}$ & I tem & value & $\begin{array}{l}\text { Required } \\
\text { Condition }\end{array}$ & $(A / R)$ & Comment \\
\hline 1.7.6.2.21 & $\begin{array}{l}\text { CAL MON }-* 60 \\
\text { CHANNEL A }\end{array}$ & & $\begin{array}{l}\text { READINGS } \\
\text { WITHIN } \pm 5 \%\end{array}$ & & \\
\hline 1.76 .2 .22 & OPEN FU-15 & $N / A$ & $N / A$ & & \\
\hline 1.7 .6 & $\begin{array}{l}\text { REMOVE } \\
\text { JUMPER }\end{array}$ & $N / A$ & $N / A$ & & \\
\hline 1.7 .6 .2 .24 & $\begin{array}{l}\text { OPEN SV-*64 } \\
\text { CLOSE SV-*63 }\end{array}$ & $N / A$ & $N / A$ & & \\
\hline 1.7 .6$) 2.25$ & $\begin{array}{l}\text { INSTALL } \\
\text { JUMPER }\end{array}$ & $N / A$ & $N / A$ & & \\
\hline $1.7 . \$ .2 .26$ & CLOSE FU-15 & $\mathrm{N} / \mathrm{A}$ & $\mathrm{N} / \mathrm{A}$ & & \\
\hline$1 . 7 . 6 \longdiv { 2 . 2 7 }$ & $\begin{array}{l}\text { ADJ SV }-* 62 \& \\
\text { RECORD } \\
\text { PDIT-*60 }\end{array}$ & & $\begin{array}{l} \pm 5^{1} \mathrm{H}_{2} \mathrm{O} \text { OF } \\
1.7 .6 .2 .15\end{array}$ & & \\
\hline 1.7 .6 .2 .28 & $\begin{array}{l}\text { CAL MON-*60 } \\
\text { CHANNEL B }\end{array}$ & $\frac{7}{N / A}$ & $\begin{array}{l}\text { READINGS } \\
\text { WITHIN } \pm 5 \%\end{array}$ & & \\
\hline 1.7 .6 .2 .29 & OPEN FU-15 & $\mathrm{N} / \mathrm{A}^{\prime}$ & $N / A$ & & \\
\hline 1.7 .6 .2 .30 & $\begin{array}{l}\text { REMOVE } \\
\text { JUMPER }\end{array}$ & $N / A$ & N/A & & \\
\hline 1.7 .6 .2 .31 & $\begin{array}{l}\text { CLOSE SV }-* 64 \\
\text { OPEN SV }-* 70\end{array}$ & $N / A$ & N/A & & \\
\hline $1.7 .6 / 2.32$ & $\begin{array}{l}\text { RECORD } \\
\text { PDIT }-* 60 \\
\text { NIT }-* 52 \text { IN } \\
\end{array}$ & 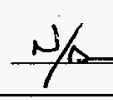 & $\begin{array}{l}0 \text { to }-30 \\
\mathrm{H}_{2} \mathrm{O}\end{array}$ & & \\
\hline 1.7 .6 .2 .33 & $\begin{array}{l}\text { INSTALL } \\
\text { JUMPER }\end{array}$ & $N / A$ & $N / A$ & 6 & \\
\hline $1.7 .6 .^{2.34}$ & CLOSE FU-15 & $N / A$ & $N / A$ & & 1 \\
\hline
\end{tabular}


TEST DATA SHEET

\begin{tabular}{|c|c|c|c|c|c|}
\hline \multirow{2}{*}{\multicolumn{3}{|c|}{\begin{tabular}{|l|} 
Date: $N / \Delta$ \\
Title of Test: \\
Pneumatic Systems \\
\end{tabular}}} & \multicolumn{3}{|c|}{ SHMS Unit Number: $N / \mathrm{A}$} \\
\hline & & & \multirow{2}{*}{\multicolumn{3}{|c|}{ Test Equipment and $S / \mathrm{N}$ or Cal. No.: }} \\
\hline \multicolumn{3}{|c|}{ Test Performed By: } & & & \\
\hline $\begin{array}{l}\text { Procedure No. } \\
\text { Initial/Date }\end{array}$ & Item & value & $\begin{array}{l}\text { Required } \\
\text { condition }\end{array}$ & $(A / R)$ & Coment \\
\hline $1.7 x^{2.35}$ & $\begin{array}{l}\text { ESTABLISH } \\
\text { BYPASS FLOW }\end{array}$ & $N / 1$ & $\begin{array}{l}\text { FIV }-* 70 \\
1.5 \pm .2 \mathrm{cfh}\end{array}$ & & $n / 4$ \\
\hline$\frac{1.7 .6 .2 .36}{4}$ & $\begin{array}{l}\text { ADJ SV-*71 } \\
\text { NIT_*52 } \\
\text { SAMPLE IN }\end{array}$ & & $\begin{array}{l} \pm 5 \quad \mathrm{H}_{2} \mathrm{O} \quad \mathrm{OF} \\
1.7 .6 .2 .32\end{array}$ & & \\
\hline$\frac{1.7 .6 .}{7}$ & $\begin{array}{l}\text { RECORD } \\
\text { NIT-*52 } \\
\text { SAMPLES }\end{array}$ & $\sum_{x / 4}$ & $\begin{array}{l}\text { READINGS } \\
\text { WITHIN } \pm 5 \%\end{array}$ & & \\
\hline 1.7 .6 .2 .38 & OPEN FU-15 & N/A & $N / A$ & & \\
\hline$1 . 7 . 6 \longdiv { 2 . 3 9 }$ & $\begin{array}{l}\text { REMOVE } \\
\text { JUMPER }\end{array}$ & $N / A$ & $N / A$ & & \\
\hline \multicolumn{6}{|c|}{ Sectioh 1.7.7 provides for test system shutdown. } \\
\hline 1.7 . & $\begin{array}{l}\text { DISCONNECT } \\
\mathrm{H}, \text { CAL GAS }\end{array}$ & $N / A$ & $N / A$ & & \\
\hline 1.7 .7 & $\begin{array}{l}\text { DISCONNECT } \\
\mathrm{CH}_{6} \text { CAL GAS }\end{array}$ & $N / A$ & $\mathrm{~N} / \mathrm{A}$ & & \\
\hline 1.7 . & $\begin{array}{l}\text { DISCONNECT } \\
\mathrm{NH}_{3} \text { CAL GAS }\end{array}$ & N/A & $N / A$ & & \\
\hline 1.7 & $\begin{array}{l}\text { SECURE \& } \\
\text { TURN OFF } \\
\text { ANA. INST.S }\end{array}$ & N/A & N/A & & \\
\hline $1.7 .7 / 5$ & $\begin{array}{l}\text { DISCONNECT } \\
\mathrm{N}_{2} \text { CARRIER } \\
\end{array}$ & N/A & $\mathrm{N} / \mathrm{A}$ & & \\
\hline 1.7 .7 .6 & $\begin{array}{l}\text { DISCONNECT } \\
\text { He CARRIER } \\
\end{array}$ & N/A & N/A & & \\
\hline 1.7 .7 .7 & $\begin{array}{l}\text { OPEN ALL } \\
\text { BREAKERS \& } \\
\text { FUSES }\end{array}$ & N/A & N/A & & \\
\hline 1$] .7 .8$ & $\begin{array}{l}\text { CLOSE LISTED } \\
\text { SYS. VALVES }\end{array}$ & $\mathrm{N} / \mathrm{A}$ & N/A & $N / 4$ & $\Delta$ \\
\hline
\end{tabular}


APPENDIX I

PAGE I- 28

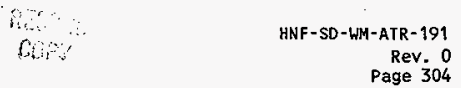

TEST DATA SHEET

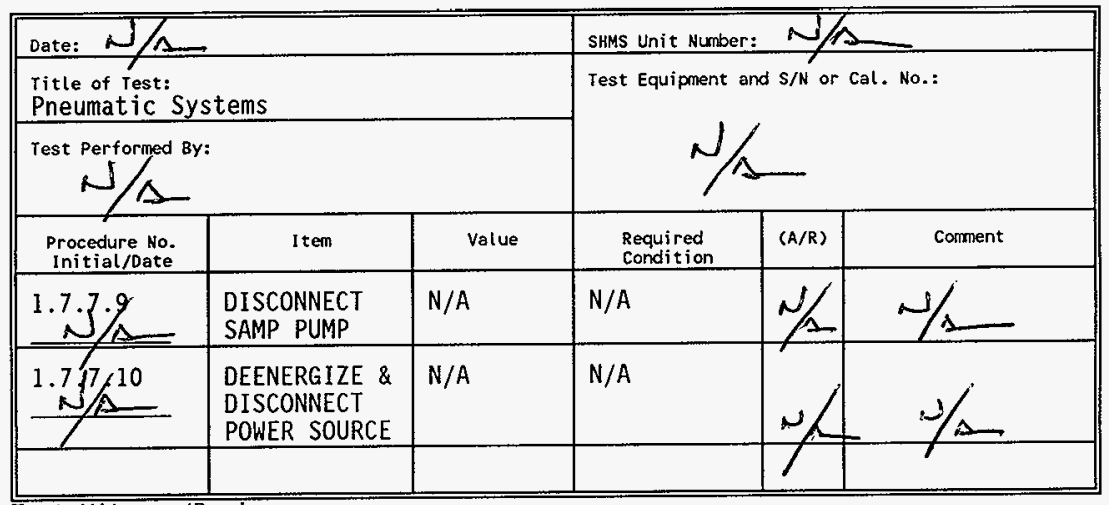

Test Witness/Review:

Mark x Bor

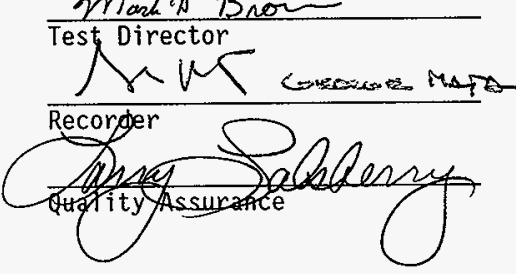

$\frac{2-26-97}{\text { Date }}$

$\frac{260 \text { Fee } .97}{\text { Date }}$

$\frac{4 / 1 / 97}{\text { Date }}$ 


\section{TEST EXCEPTION SHEET}

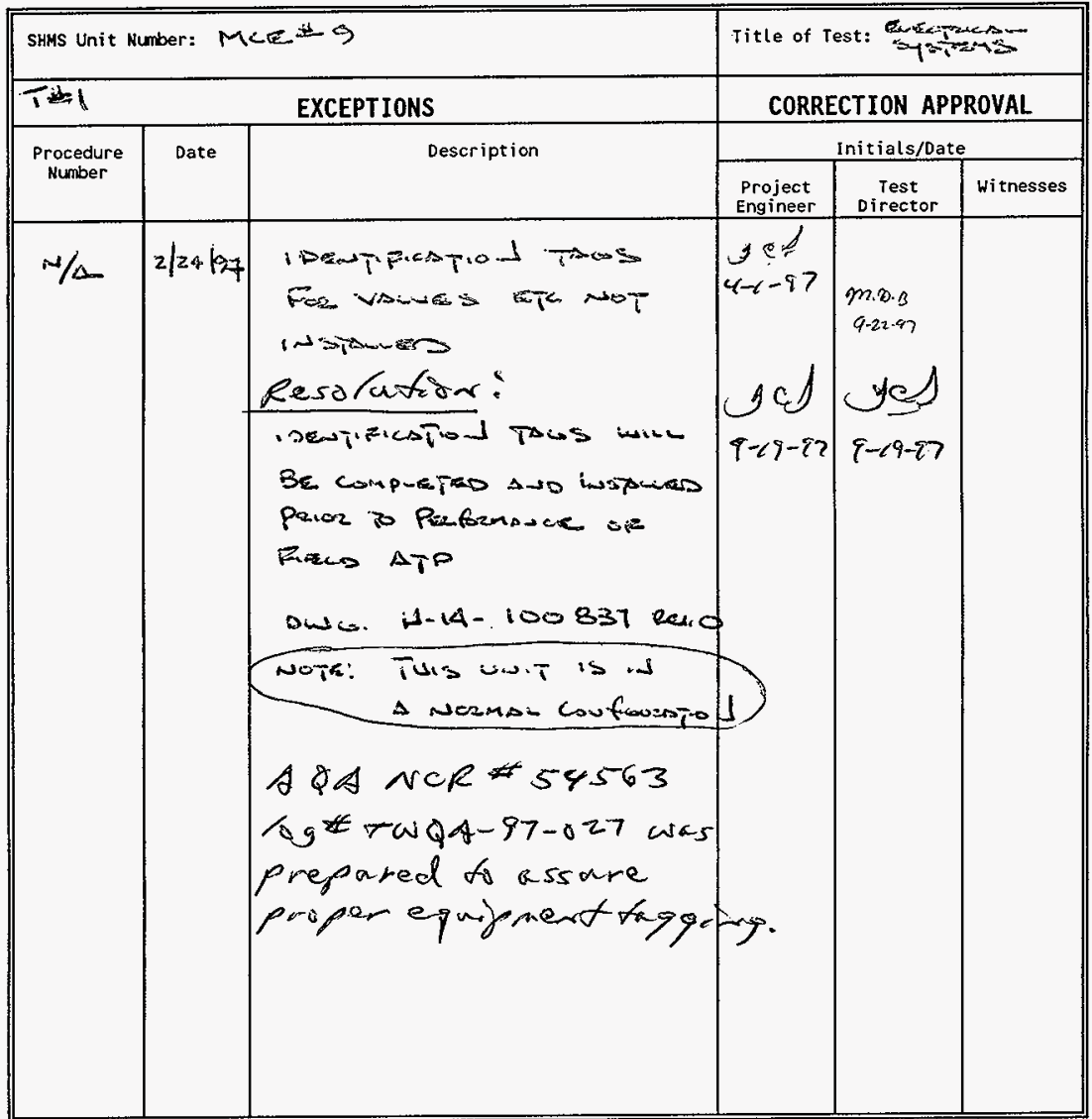

\section{TEST APPROVED WITH EXCEPTIONS}

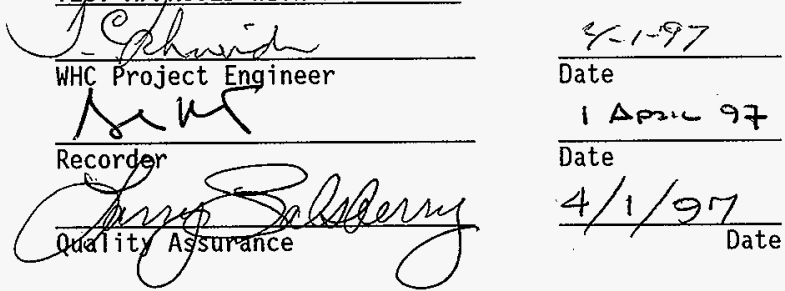


TEST LOG

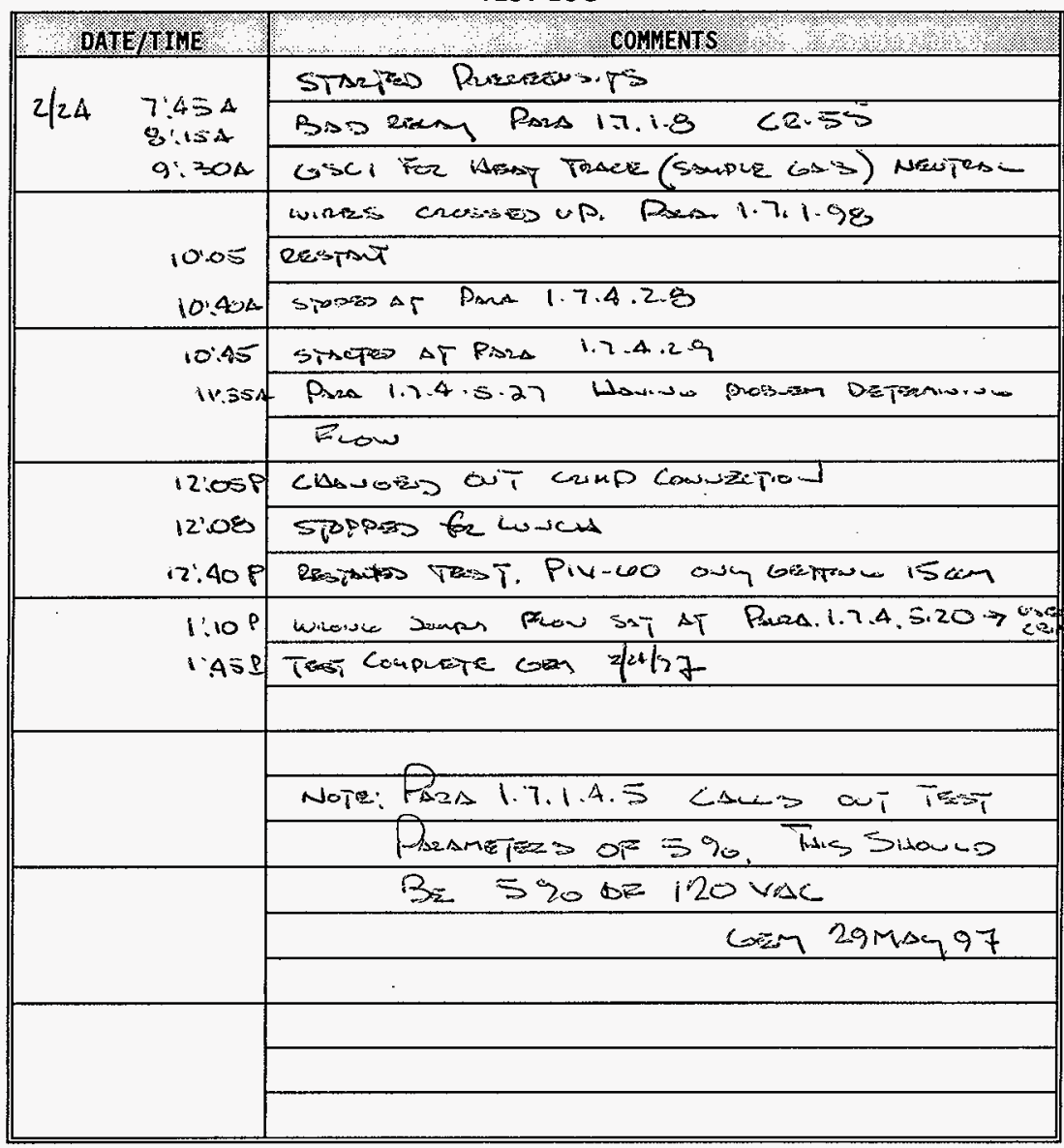


APPENDIX $\mathrm{J}$

PAGE J-/
$H N F-S D-W M-A T R-191$

(a)

a;

$\therefore$

APPENDIX J
Rev. 0

Page 307 
APPENDIX $\mathrm{J}$

PAGE J-2
HNF-SD - WM-ATR-191
ReV. 0

Rev. 0
Page 308

TEST EXECUTION SHEET

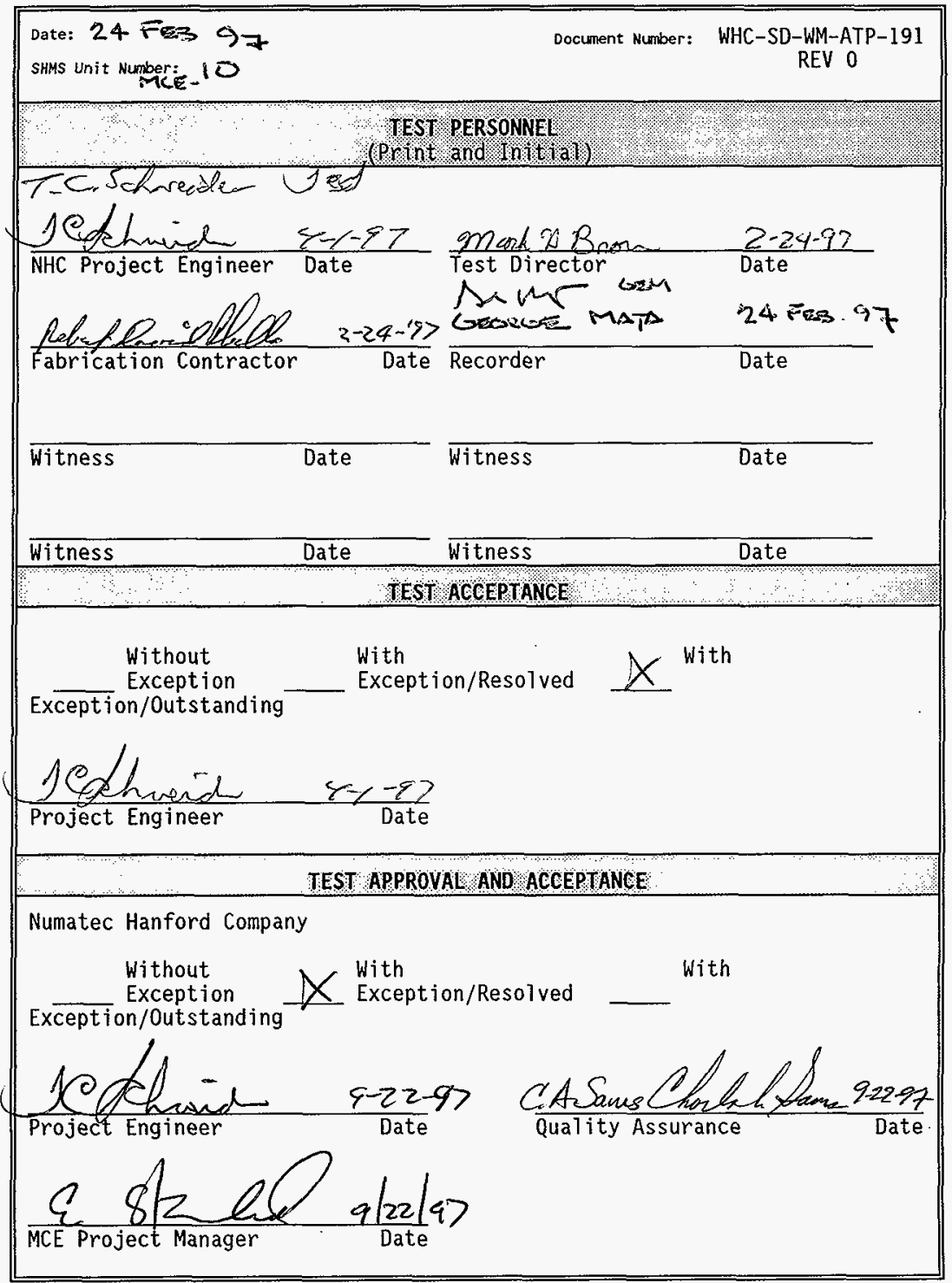


APPENDIX $J$

PAGE J-3

SHMS Unit Number:

\section{PREREQUISITES AND INITIAL TEST CONDITIONS}

The following conditions shall exist at the start of the acceptance testing. Initial and date to verify that each of the following items have been accomplished. Ste TE:

2kenstems being tested have been inspected for workmanship and for compliance with design.

2/24/iffontinuity and megger tests have been performed on portions of the electrical and instrument systems being tested, as required.

$\operatorname{Gen} 2 k+17$ Leak tests on the pneumatic systems have been performed.

SOM $2 / 24 / 97$ The following circuit breakers and fuses are installed per the specified size and are open

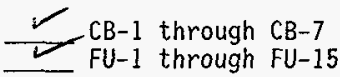

2an $2 / 24 \mid$ S 7 A11 test instruments have a currently valid calibration stamp attached that indicates a calibration traceable to the National Institute of Standards and Technology.

$2 / 24 k 7$ The following process and control instrument systems have been initially configured and aligned for proper operation.

FIT-*57, FSL-*57, NIT-*54, NIT-*55, (NR-*54), PDIT-*60, TIC-*50, TIC-*56, TIS-*62, YYC-*01 NOTE: NR-*54 is not used in the ANALYTICAL configuration.

Gemizalsz Personnel responsible for directing and witnessing the performance of the tests described in this ATP have read and understand appropriate certified vendor information (CVI) pertaining to the operation of the equipment to be tested.

$2 / 24 / 5)$ CLOSE all system manual valves with the exception of the following:

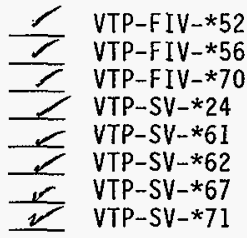

$\tan 2 / 24 \mid 27$ The sample pump VTP-P- $* 50$, connected to the cabinet sample out port, but vented to atmosphere for testing. 
Gen $2 / 24 k 7208$ Vac 1 phase 15-20 ampere temporary power source has been connected, but not energized, to the appropriate TB1 terminals per H-14-100838.

Verify by signature and date that all prerequisites have been met.

$\frac{\text { Mask } 2 \text { Bnon }}{3-28.97}$


APPENDIX J

HNF-SD-WM-ATR-191

Rev.

Page 311

$24 \operatorname{sen} x \sin 7$

TEST DATA SHEET

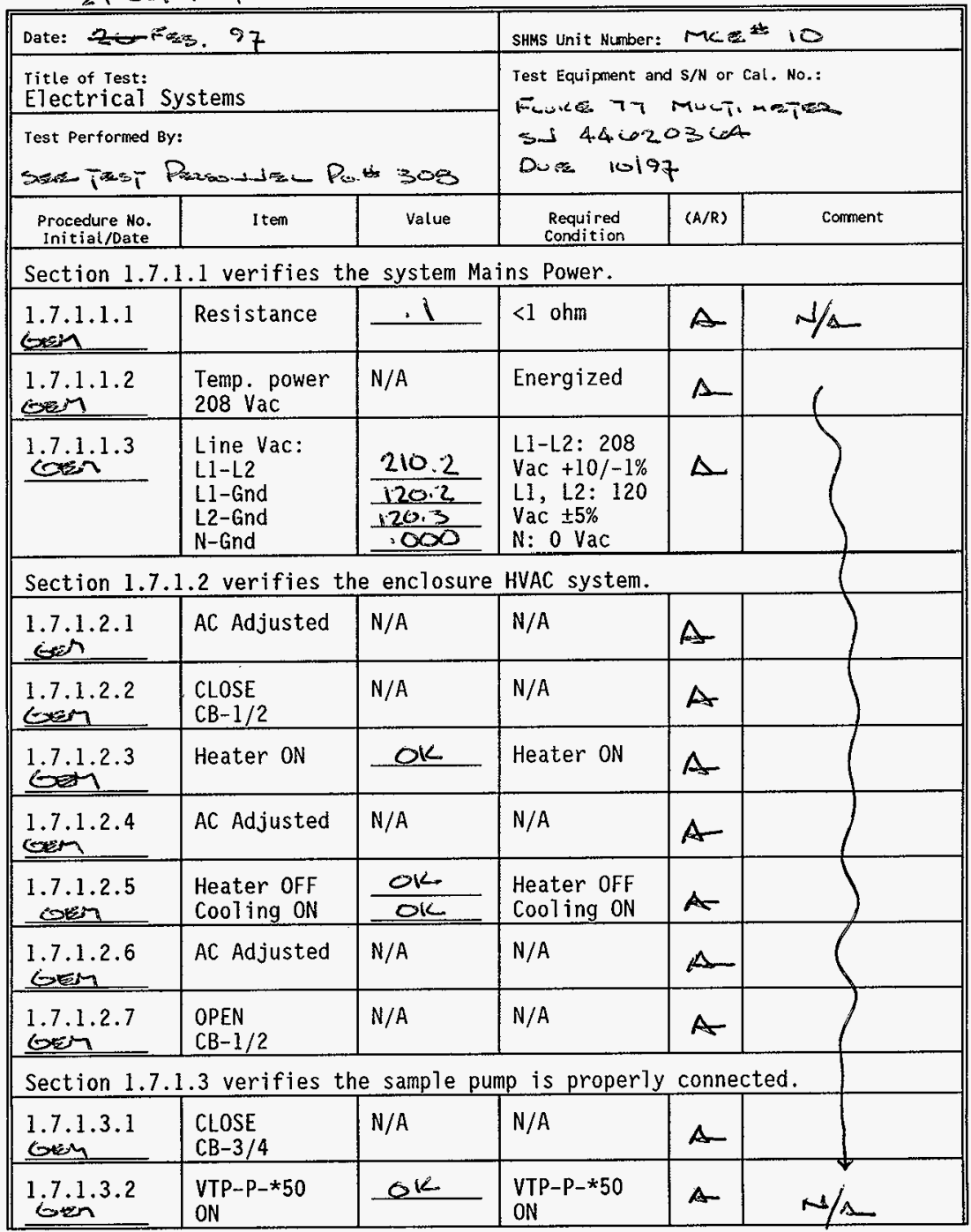

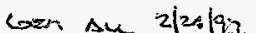




\section{TEST DATA SHEET}

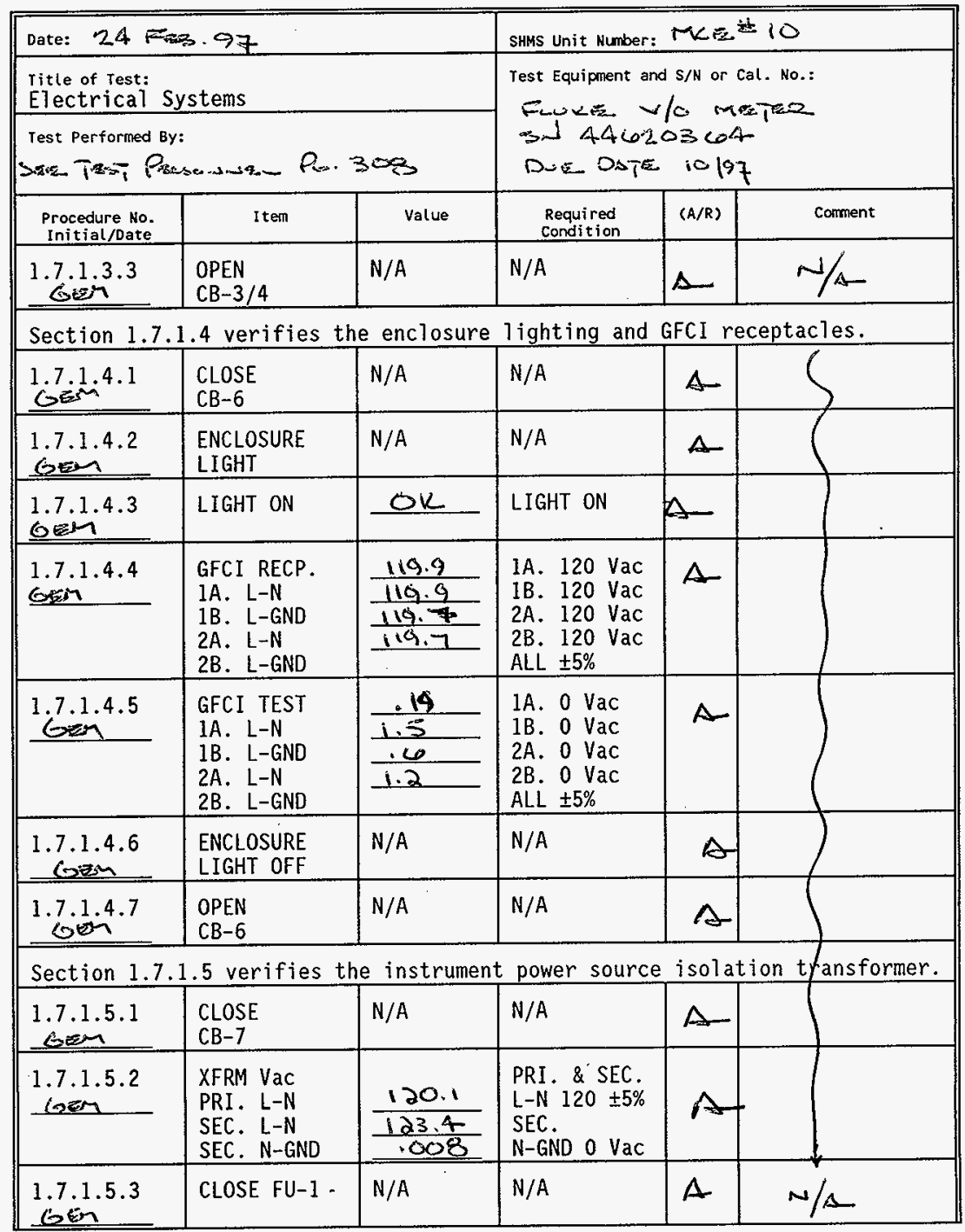


TEST DATA SHEET

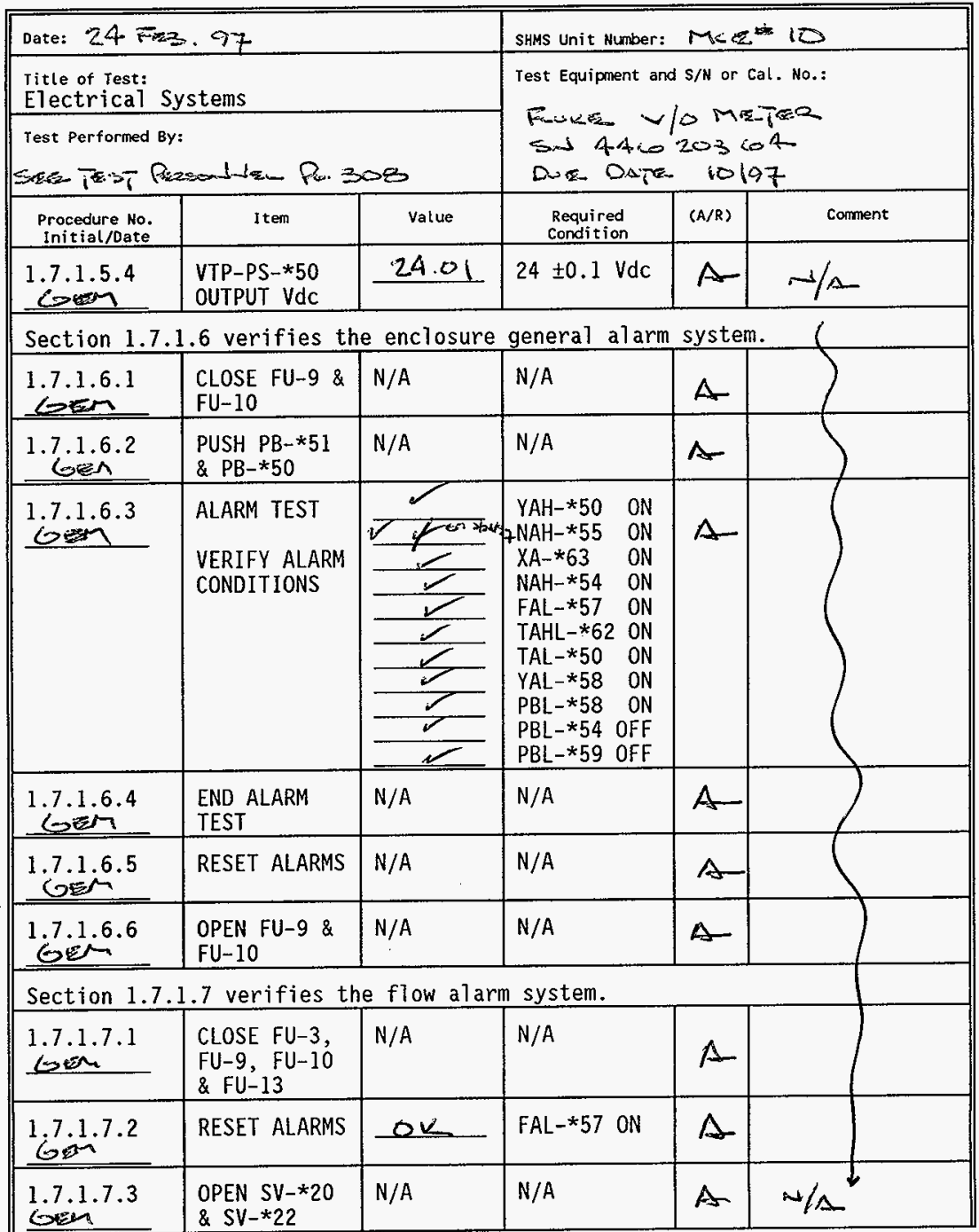


APPENDIX $\mathrm{J}$

TEST DATA SHEET

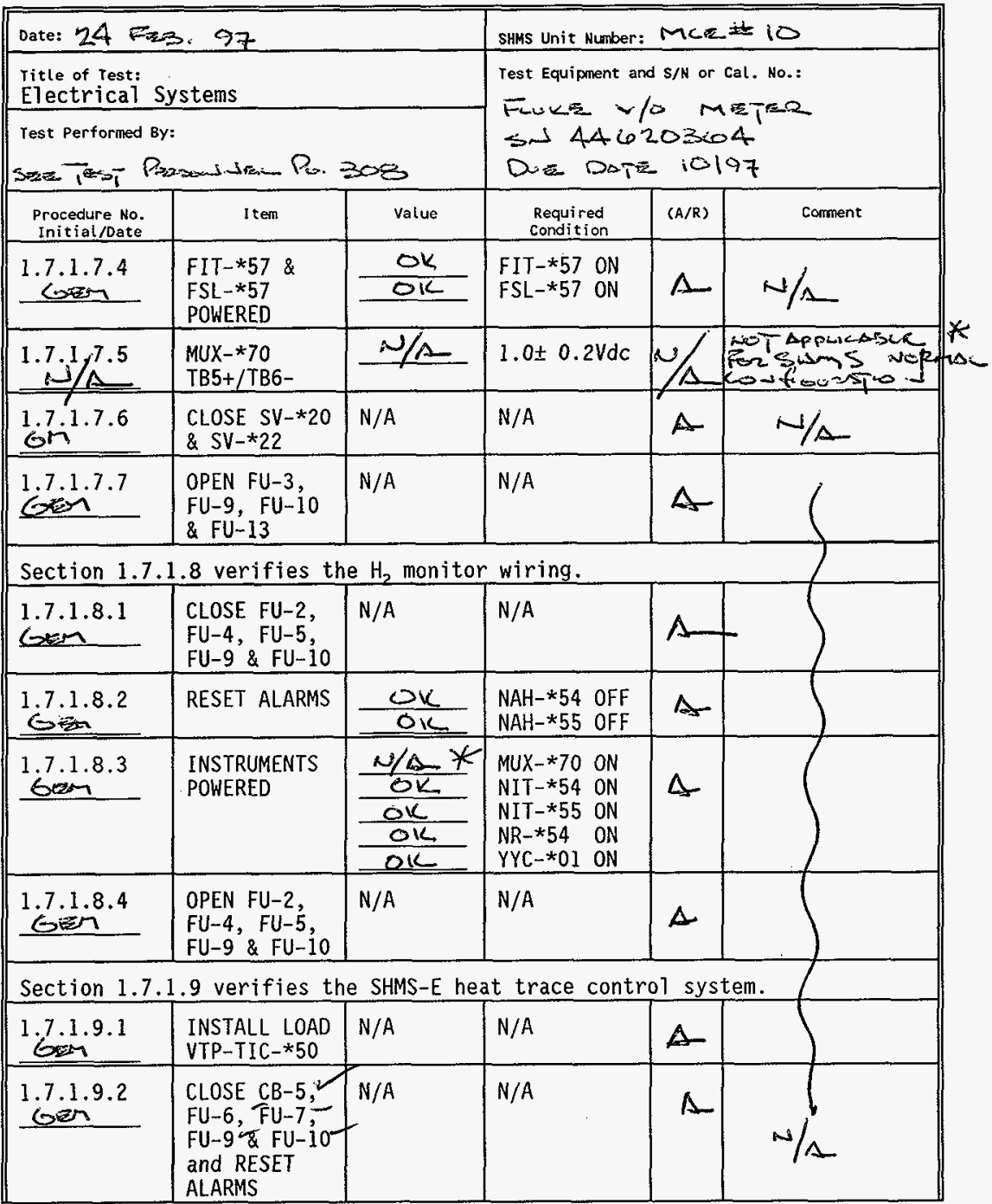


TEST DATA SHEET

\begin{tabular}{|c|c|c|c|c|c|}
\hline \multicolumn{3}{|c|}{ Date: 24 Fos .97} & SHMS Unit Number: & \multicolumn{2}{|c|}{ MGeid 10} \\
\hline \multicolumn{3}{|c|}{$\begin{array}{l}\text { Title of Test: } \\
\text { Electrical Systems }\end{array}$} & \multirow{2}{*}{\multicolumn{3}{|c|}{ rest Equipment and $\mathrm{S} / \mathrm{N}$ or Cal. No.: }} \\
\hline \multicolumn{3}{|c|}{ 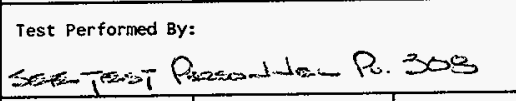 } & & & \\
\hline $\begin{array}{l}\text { Procedure No. } \\
\text { Initial/Date } \\
\end{array}$ & Item & value & $\begin{array}{l}\text { Required } \\
\text { Condition } \\
\end{array}$ & $(A / R)$ & Comment \\
\hline $\begin{array}{l}1.7 .1 .9 .3 \\
\text { 6oing }\end{array}$ & $\begin{array}{l}\text { TIC }-* 50 \text { \& } \\
\text { TIC-*56 } \\
\text { NOMINAL TEMP }\end{array}$ & $\frac{O K}{O K}$ & $\begin{array}{l}\text { TIC }-* 50 \\
\text { TIC }-* 56 \\
\text { TEMPERATURE }\end{array}$ & $\Lambda$ & $N / \Delta$ \\
\hline$\frac{1.7 .1 .9 .4}{6}$ & $\begin{array}{l}\text { ADJ TIC }-\star 50 \\
\& \text { TIC-*56 } \\
\text { SP2 \& RESET } \\
\text { ALARMS } \\
\end{array}$ & $N / A$ & N/A & $A$ & \\
\hline $\begin{array}{l}1.7 .1 .9 .5 \\
\operatorname{con}\end{array}$ & $\begin{array}{l}\mathrm{ADJ}_{\mathrm{TIC}} \mathrm{TI}-* 50 \\
\mathrm{SP} 1\end{array}$ & $N / A$ & N/A & $\Delta$ & \\
\hline $\begin{array}{l}1.7 .1 .9 .6 \\
61\end{array}$ & $\begin{array}{l}\text { ADJ TIC-*50 } \\
\text { SP } 2\end{array}$ & $N / A$ & $N / A$ & $\Delta$ & \\
\hline $6^{7.1 .9 .7}$ & $\begin{array}{l}\text { TAL }-* 50 \& \\
\text { HORN ACTIVE }\end{array}$ & $\frac{O K}{O K}$ & $\begin{array}{l}\text { TAL-*50 ON } \\
\text { HORN ON } \\
\text { ACKNOWLEDGE }\end{array}$ & $A$ & \\
\hline 1.7 .1 .9 .8 & $\begin{array}{l}\text { TIC }-* 50 \\
\text { OPERATES }\end{array}$ & 012 & $\begin{array}{l}\text { TIC }-* 50 \\
\text { OPERATES }\end{array}$ & $A$ & \\
\hline $\begin{array}{l}1.7 .1 .9 .9 \\
\sin \end{array}$ & $\begin{array}{l}\text { ADJ TIC-*50 } \\
\text { SP } 2\end{array}$ & N/A & $N / A$ & $A$ & \\
\hline $\begin{array}{l}1.7 .1 .9 .10 \\
6 \mathrm{n}\end{array}$ & RESET ALARM & OK & $\mathrm{TAL}-* 50$ OFF & $A$ & \\
\hline $\begin{array}{l}1.7 .1 .9 .11 \\
6.57\end{array}$ & $\begin{array}{l}A D J \text { TIC } * 50 \\
S P 1 \& S P 2 \\
\end{array}$ & $N / A$ & $N / A$ & $A$ & \\
\hline $\operatorname{son}^{1.7 .1 .9 .12}$ & $\begin{array}{l}\text { OPEN CB-5 \& } \\
\text { REMOVE LOAD }\end{array}$ & N/A & $N / A$ & $\Delta$ & \\
\hline $\begin{array}{l}1.7 .1 .9 .13 \\
\cos \\
\end{array}$ & $\begin{array}{l}\text { INSTALL LOAD } \\
\text { VTP-TIC-*56 }\end{array}$ & $N / A$ & N/A & $\Lambda$ & \\
\hline Gevin & CLOSE CB-5 & N/A & N/A & $\Lambda$ & \\
\hline gon $^{7.1 .9 .15}$ & $\begin{array}{l}\text { ADJ TIC-*56 } \\
\text { SP } 1\end{array}$ & $N / A$ & N/A & $\Delta$ & \\
\hline $\operatorname{Gg}^{7.1 .9 .16}$ & $\begin{array}{l}\text { ADJ TIC-*56 } \\
\text { SP } 2\end{array}$ & N/A & $N / A$ & $A$ & $\because / \Delta$ \\
\hline
\end{tabular}


TEST DATA SHEET

\begin{tabular}{|c|c|c|c|c|c|}
\hline \multicolumn{3}{|c|}{ Date: 24 Fes. 97} & \multicolumn{3}{|c|}{ SHMS Unit Number: MCE 10} \\
\hline \multicolumn{3}{|c|}{$\begin{array}{l}\text { Title of Test: } \\
\text { Electrical Systems }\end{array}$} & \multirow{2}{*}{\multicolumn{3}{|c|}{ Test Equipment and $\mathrm{S} / \mathrm{N}$ or Cal. No.: }} \\
\hline \multicolumn{3}{|c|}{ 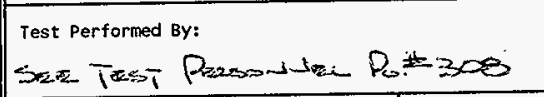 } & & & \\
\hline $\begin{array}{l}\text { Procedure No. } \\
\text { Initial/Date } \\
\end{array}$ & Item & Value & $\begin{array}{l}\text { Required } \\
\text { Condition } \\
\end{array}$ & $(A / R)$ & Carment \\
\hline $\begin{array}{l}1.7 .1 .9 .17 \\
0=0\end{array}$ & $\begin{array}{l}\text { TAL-*50 \& } \\
\text { HORN ACTIVE }\end{array}$ & $\begin{array}{l}o k \\
6 k \\
0 k\end{array}$ & $\begin{array}{l}\text { TAL-*50 ON } \\
\text { HORN ON } \\
\text { ACKNOWLEDGE }\end{array}$ & $\Lambda$ & $\sqrt{2}$ \\
\hline $\begin{array}{l}1.7 .1 .9 .18 \\
\end{array}$ & $\begin{array}{l}\text { TIC }-* 56 \\
\text { OPERATES }\end{array}$ & OK & $\begin{array}{l}\text { TIC-*56 } \\
\text { OPERATES }\end{array}$ & $\triangle$ & \\
\hline $\begin{array}{l}1.7 .1 .9 .19 \\
0\end{array}$ & $\begin{array}{l}\text { ADJ TIC-*56 } \\
\text { SP } 2\end{array}$ & $N / A$ & N/A & $A$ & \\
\hline $\sin ^{1.7 .20}$ & RESET ALARM & OK & TAL $-\star 50 \quad 0 F F$ & $\Delta$ & \\
\hline 1.7 .1 .9 .21 & $\begin{array}{l}\text { ADJ TIC }-* 56 \\
\text { SP } 1 \& S P 2\end{array}$ & $N / A$ & $\mathrm{~N} / \mathrm{A}$ & A & \\
\hline ism & $\begin{array}{l}\text { OPEN CB-5, } \\
\text { FU-6 \& FU-7 }\end{array}$ & $N / A$ & $N / A$ & 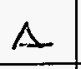 & \\
\hline $\sin ^{1.1 .9 .23}$ & $\begin{array}{l}\text { REMOVE LOAD } \\
\text { TIC-*56 }\end{array}$ & $N / A$ & $N / A$ & $\Delta$ & \\
\hline GEM & $\begin{array}{l}\text { CLOSE FU-8 } \\
\text { TIS-*62 } \\
\text { NOMINAL TEMP }\end{array}$ & OK & $\begin{array}{l}\text { TIS-*62 } \\
\text { TEMP }\end{array}$ & A & \\
\hline $\begin{array}{l}1.7 .9 .25 \\
0+27 \\
\end{array}$ & $\begin{array}{l}\text { ADJ TIS-*62 } \\
\text { SP } 1\end{array}$ & $N / A$ & $N / A$ & $\Delta$ & \\
\hline 1.7 .1 .9 .26 & $\begin{array}{l}\text { ADJ TIS-*62 } \\
\text { SP } 2\end{array}$ & $N / A$ & N/A & $\Delta$ & \\
\hline 1.7 .1 .9 .27 & RESET ALARMS & $O K$ & $\begin{array}{l}\text { TAHL }-* 62 \\
\text { OFF }\end{array}$ & $A$ & \\
\hline 1.7 .1 .9 .28 & $\begin{array}{l}\text { ADJ TIS-*62 } \\
\text { SP } 2\end{array}$ & $N / A$ & N/A & $A$ & \\
\hline $\begin{array}{l}1.7 .1 .9 .29 \\
\sin \end{array}$ & $\begin{array}{l}\text { TAHL }-* 62 \& \\
\text { HORN ACTIVE }\end{array}$ & $\frac{O K}{O K}$ & $\begin{array}{l}\text { TAHL }-* 62 \text { ON } \\
\text { HORN ON } \\
\text { ACKNOWLEDGE }\end{array}$ & $\triangle$ & \\
\hline $\begin{array}{l}1.7 .1 .9 .30 \\
\text { sesm }\end{array}$ & $\begin{array}{l}\text { ADJ TIS }-* 62 \\
\text { SP } 2\end{array}$ & $N / A$ & $N / A$ & $A$ & \\
\hline
\end{tabular}

$\Rightarrow$ Se. rzalis 


\begin{tabular}{|c|c|c|c|c|c|}
\hline \multicolumn{6}{|c|}{ TEST DATA SHEET } \\
\hline \multicolumn{3}{|c|}{ Date: 24 Fres. 97} & \multicolumn{3}{|c|}{ SHMS Unit Number: MCE 10} \\
\hline \multicolumn{3}{|c|}{$\begin{array}{l}\text { Iivle of Test: } \\
\text { Electrical Systems }\end{array}$} & \multirow{2}{*}{\multicolumn{3}{|c|}{ 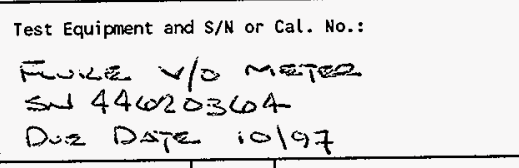 }} \\
\hline \multicolumn{3}{|c|}{ 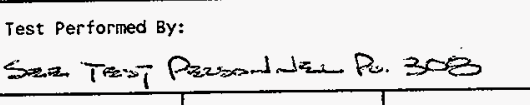 } & & & \\
\hline $\begin{array}{l}\text { Procedure No. } \\
\text { Initial/Date }\end{array}$ & Item & value & $\begin{array}{l}\text { Required } \\
\text { Condition } \\
\end{array}$ & $(A / R)$ & Comment \\
\hline $\begin{array}{l}1.7 .1 .9 .31 \\
\text { getin } \\
\end{array}$ & RESET ALARMS & OK & $\begin{array}{l}\text { TAHL-*62 } \\
\text { OFF } \\
\end{array}$ & $\Lambda$ & $\omega / \alpha$ \\
\hline $\begin{array}{c}1.7 .1 .9 .32 \\
6.96\end{array}$ & $\begin{array}{l}\text { ADJ TIS } * 62 \\
\text { SP } 1\end{array}$ & $N / A$ & $N / A$ & $A$ & \\
\hline $\operatorname{sen}^{1.1 .9 .33}$ & $\begin{array}{l}\text { TAHL-*62 \& } \\
\text { HORN ACTIVE }\end{array}$ & $\frac{O K}{\frac{O K}{O K}}$ & $\begin{array}{l}\text { TAHL-*62 ON } \\
\text { HORN ON } \\
\text { ACKNOWLEDGE } \\
\end{array}$ & $\Delta$ & \\
\hline $\operatorname{sen}^{7.1 .9 .34}$ & $\begin{array}{l}\text { ADJ TIS }-* 62 \\
\text { SP } 1 \& S P 2\end{array}$ & $N / A$ & N/A & $\Delta$ & \\
\hline 1.7.1.9.35 & $\begin{array}{l}\text { OPEN FU-8, } \\
\text { FU-9 \& FU-10 }\end{array}$ & $N / A$ & N/A & $A$ & \\
\hline \multicolumn{6}{|c|}{ Section 1.7.1.10 verifies the differential pressure transmitter system. } \\
\hline $\begin{array}{l}1.7 .1 .10 .1 \\
\mathrm{con}\end{array}$ & $\begin{array}{l}\text { REMOVE PLUGS } \\
\text { BAL PDIT-*60 }\end{array}$ & $N / A$ & N/A & $\Delta$ & \\
\hline$\frac{1.7 .1 .10 .2}{6+1}$ & $\begin{array}{l}\text { CLOSE FU- } 14 \\
\text { REC PDIT-*60 } \\
\text { PRESSURE } \\
\end{array}$ & .19 & $\begin{array}{l}\text { PDIT-*60 } \\
\text { PRESSURE } \\
0 \pm 1 " \mathrm{H}_{2} \mathrm{O} \\
\end{array}$ & $\Delta$ & $\omega / \Delta$ \\
\hline $1.7 .1 .10 .3 / \mathrm{s}$ & $\begin{array}{l}\text { MUX-*70 Vdc } \\
\mathrm{TB} 7+\& \mathrm{~TB} 8- \\
\end{array}$ & $N / \Delta$ & \begin{tabular}{|l|}
$M U X-\star 70 ~ V d c$ \\
$2.2 \pm 0.1$ \\
\end{tabular} & $1 / 2$ & Tot Apencosie \\
\hline$\frac{1.7 .1 .10 .4}{6 \operatorname{sen}}$ & $\begin{array}{l}\text { REPLACE } \\
\text { PLUGS ON } \\
\text { PDIT } * 60 \\
\end{array}$ & N/A & $N / A$ & $\Delta$ & $N / \alpha$ \\
\hline $\begin{array}{c}1.7 .1 .10 .5 \\
\end{array}$ & OPEN FU-14 & $N / A$ & $N / A$ & $\Delta$ & $\omega / \mathrm{s}$ \\
\hline \multicolumn{6}{|c|}{ Section 1.7 .1 .11 verifies the gas chromatograph power } \\
\hline$\frac{1.7 .1 .11 .1}{60}$ & CLOSE FU-11 & $N / A$ & $N / A$ & 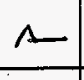 & $N / \Delta$ \\
\hline 1.7 .1 .11 .2 & $\begin{array}{l}\text { POWER } \\
\text { MON-*60 } \\
\end{array}$ & Ore & $\begin{array}{l}\text { MON-*60 } \\
\text { POWERED }\end{array}$ & $A$ & i23 verifin Fü \\
\hline 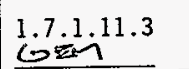 & OPEN FU-11 & $N / A$ & N/A & A & $N / A$ \\
\hline
\end{tabular}


TEST DATA SHEET

\begin{tabular}{|c|c|c|c|c|c|}
\hline \multicolumn{3}{|c|}{ Date: 24 Fos 97} & \multicolumn{3}{|c|}{ SHMS Unit Number: $M C_{-} E_{-}^{\text {Ha }} 10$} \\
\hline \multicolumn{3}{|c|}{$\begin{array}{l}\text { Title of Test: } \\
\text { Electrical Systems }\end{array}$} & \multirow{2}{*}{\multicolumn{3}{|c|}{ 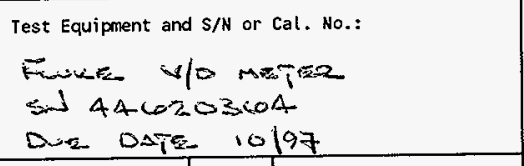 }} \\
\hline \multicolumn{3}{|c|}{ 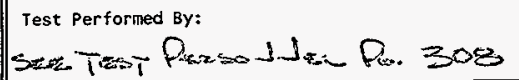 } & & & \\
\hline $\begin{array}{l}\text { Procedure No. } \\
\text { Initial/oate }\end{array}$ & Item & Value & $\begin{array}{l}\text { Required } \\
\text { Condition }\end{array}$ & $(A / R)$ & Comment \\
\hline \multicolumn{6}{|c|}{ Section 1.7 .1 .12 verifies the multi gas analyzer power. } \\
\hline 1.7 .1 .12 .1 & CLOSE FU-12 & $N / A$ & $N / A$ & $\curvearrowright$ & $-3 / \alpha$ \\
\hline${ }_{6 \infty}^{1.7 .1 .12 .2}$ & $\begin{array}{l}\text { POWER } \\
\text { NIT-*52 }\end{array}$ & OK & $\begin{array}{l}\text { NIT }-\star 52 \\
\text { POWERED } \\
\end{array}$ & $\Delta$ & 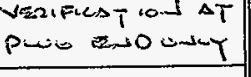 \\
\hline $\begin{array}{l}1.7 .1 .12 .3 \\
\sin ^{2}\end{array}$ & OPEN FU-12. & N/A & $N / A$ & A & $\sim / \Delta$ \\
\hline \multicolumn{6}{|c|}{ Section 1.7.1.13 verifies the personnel computer power. } \\
\hline $\begin{array}{l}1.7 .1 .13 .1 \\
\end{array}$ & CLOSE CB-6 & N/A & N/A & $\triangle$ & $\begin{array}{l}\text { Rer. Pear i.7.1 } \\
\text { Corar } 2 / 24 / 27\end{array}$ \\
\hline 1.7 .1 .13 .2 & $\begin{array}{l}\text { POWER PC }-* 60 \\
\& \text { PC }-* 70\end{array}$ & on & $\begin{array}{ll}\mathrm{PC}-* 60 & \mathrm{ON} \\
\mathrm{PC}-* 70 & \mathrm{ON} \\
\end{array}$ & $\wedge$ & \\
\hline $\begin{array}{l}1.7 .1 .13 .3 \\
\end{array}$ & $\begin{array}{l}\text { TURN INST. } \\
\text { OFF \& } \\
\text { OPEN CB-6 }\end{array}$ & $\frac{O K}{a k}$ & $\begin{array}{ll}\mathrm{PC}-\star 60 & \text { OFF } \\
\mathrm{PC}-* 70 & \text { OFF }\end{array}$ & $\Delta$ & $\eta$ \\
\hline
\end{tabular}


APPENDIX J

PAGE $\mathrm{J}-\mathrm{/} 3$
HNF-SD-WN-ATR- 191

Rev. 0

Page 319

TEST DATA SHEET

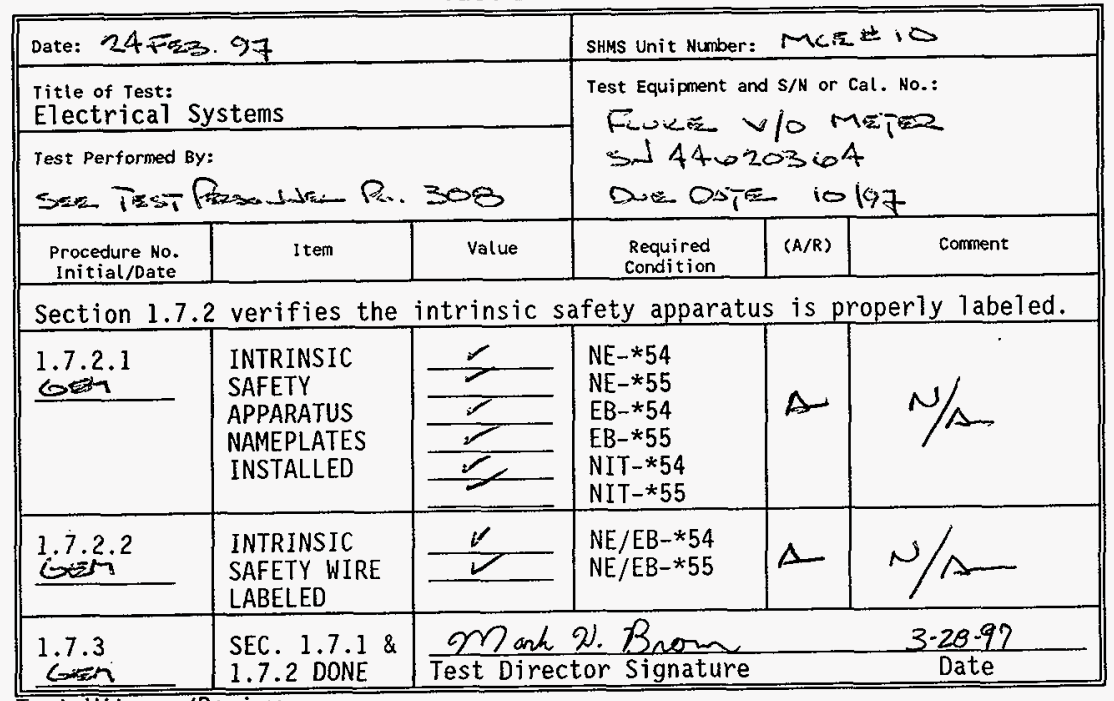

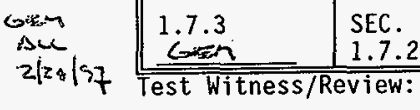

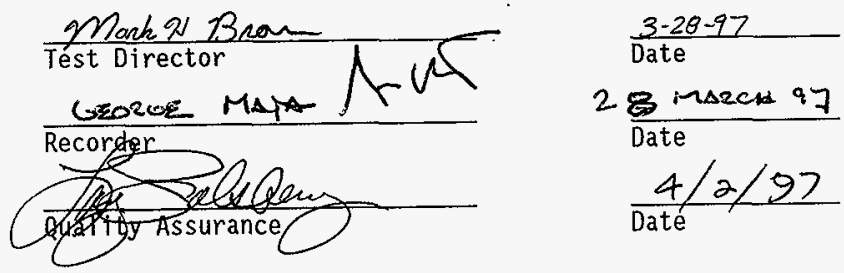


TEST DATA SHEET

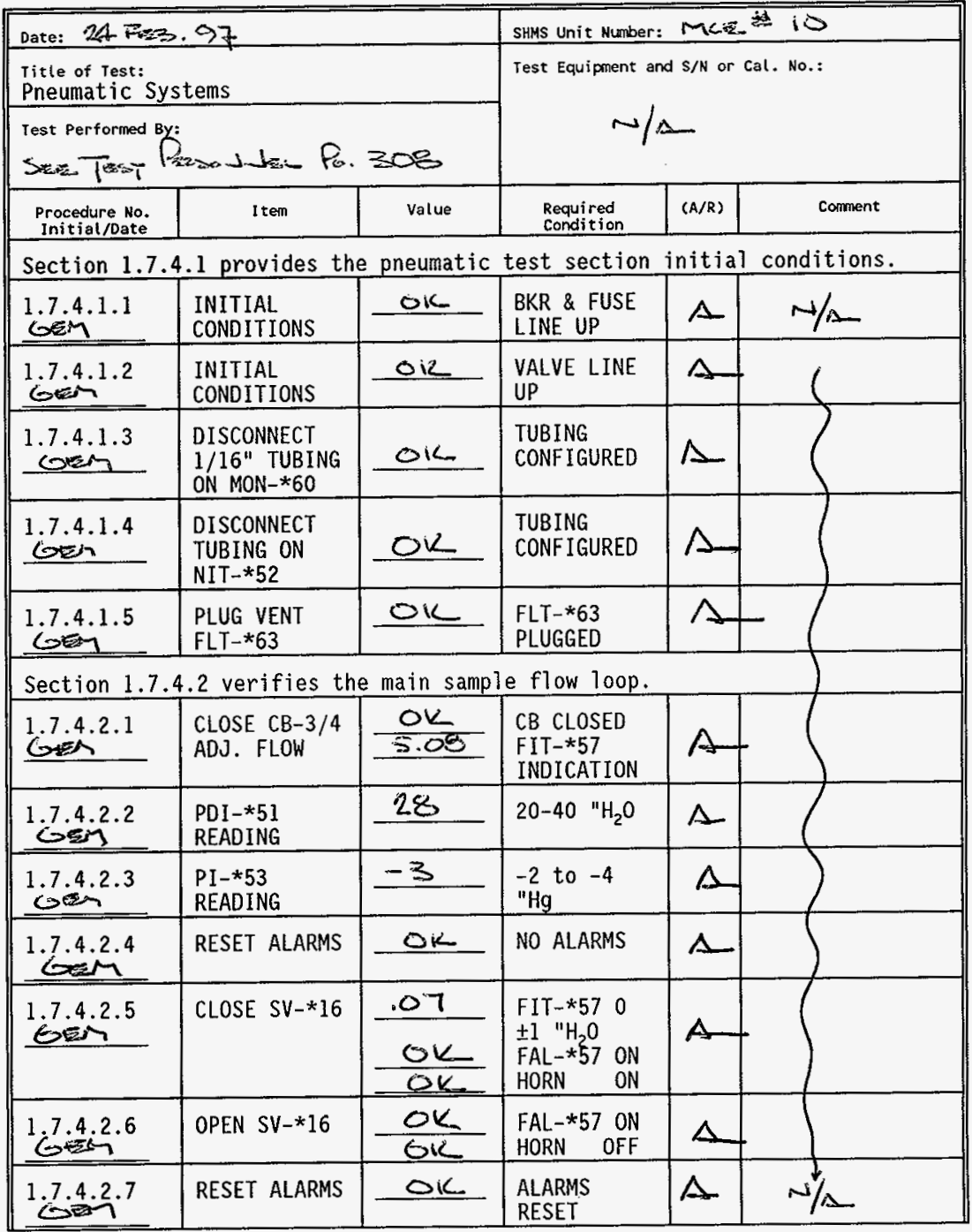


APPENDIX $\mathrm{J}$

TEST DATA SHEET

\begin{tabular}{|c|c|c|c|c|c|}
\hline \multicolumn{3}{|c|}{ Date: $24 \vec{F}=97$} & SHMS Unit Number: & \multicolumn{2}{|c|}{ Mee 10} \\
\hline \multicolumn{3}{|c|}{$\begin{array}{l}\text { Title of Test: } \\
\text { Pneumatic Systems }\end{array}$} & \multirow{2}{*}{\multicolumn{3}{|c|}{ Test Equipment and $\mathrm{S} / \mathrm{N}$ or Cal. No.: }} \\
\hline \multicolumn{3}{|c|}{ 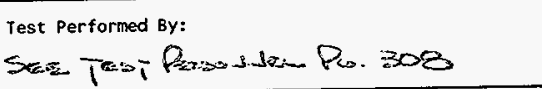 } & & & \\
\hline $\begin{array}{l}\text { Procedure No. } \\
\text { Initial/Date }\end{array}$ & Item & value & $\begin{array}{l}\text { Required } \\
\text { condition }\end{array}$ & $(A / R)$ & Mrazuriżs AT \\
\hline $6 \dot{6}^{2.8}$ & CLOSE SV-*25 & $\frac{.03}{\frac{O K}{O K}} \frac{0}{0}$ & 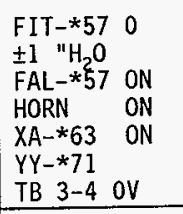 & $A$ & $\begin{array}{l}P B C-54-2 \\
Y Y 71-2 A T \\
P B S 4-\angle 2\end{array}$ \\
\hline 1.7 .4 .2 .9 & OPEN SV-*25 & $\frac{\frac{O K}{O K}}{\frac{O k}{119.7}}$ & 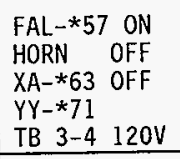 & $\Lambda$ & \\
\hline $\begin{array}{l}1.7 .4 .2 .10 \\
6 i^{2} \\
\end{array}$ & RESET ALARMS & ok & $\begin{array}{l}\text { ALARMS } \\
\text { RESET } \\
\end{array}$ & $\Delta$ & \\
\hline $\begin{array}{l}1.7 .4 .2 .11 \\
605\end{array}$ & $\begin{array}{l}\text { OPEN SV-*54- } \\
\& \text { SV }-* 66- \\
\text { READ PRESS. } \\
\text { PDIT-*60 }\end{array}$ & $\frac{0 k}{-43.44}$ & $\begin{array}{l}\text { VALVES OPEN } \\
-27 \text { to }-82 \\
" \mathrm{H}_{2} \mathrm{O}\end{array}$ & A & \\
\hline $\begin{array}{l}1.7 .4 .2 .12 \\
\end{array}$ & $\begin{array}{l}\text { CLOSE SV-*54 if } \\
\text { OPEN SV }-* 63 \\
\text { READ PRESS. } \\
\text { PDIT-*60 }\end{array}$ & OK & $\begin{array}{l}\text { VALVES } \\
\text { OPERATED } \\
0 \pm 1 " \mathrm{H}_{2} \mathrm{O}\end{array}$ & $\Delta$ & \\
\hline $\operatorname{cosin}^{1.7 .4 .2 .13}$ & $\begin{array}{l}\text { CLOSE SV-*63 } \\
\text { OPEN SV-*554 } \\
\text { READ PRESS. } \\
\text { PDIT-*60 }\end{array}$ & Fok & $\begin{array}{l}\text { VALVES } \\
\text { OPERATED } \\
-27 \text { to }-82 \\
" \mathrm{H}_{2} \mathrm{O} \\
\end{array}$ & $A$ & \\
\hline 1.7 .4 .2 .14 & $\begin{array}{l}\text { CLOSE SV }-* 55^{-1} \\
\text { OPEN SV }-* 64 \\
\text { READ PRESS. } \\
\text { POIT- } * 60 \\
\end{array}$ & $\frac{O K}{.26}$ & $\begin{array}{l}\text { VALVES } \\
\text { OPERATED } \\
0 \pm 1 \quad \mathrm{H}_{2} \mathrm{O}\end{array}$ & $A$ & \\
\hline $\begin{array}{l}1.7 .4 .2 .15 \\
\text { Goth }\end{array}$ & $\begin{array}{l}\text { CLOSE SV-*64 } \\
\& \text { SV-*66 }\end{array}$ & $N / A$ & $N / A$ & $\Delta$ & \\
\hline Section 1. & 3 verifies $t$ & e grab sa & flow loop. & & \\
\hline$\underset{1.7 .4 .3 .1}{\text { ofen }}$ & READ PDI-*51 & 29 & $20-40 \quad " \mathrm{H}_{2} \mathrm{O}$ & $\Lambda$ & $N / A$ \\
\hline
\end{tabular}


TEST DATA SHEET

\begin{tabular}{|c|c|c|c|c|c|}
\hline \multicolumn{3}{|c|}{ Date: $24 \mathrm{Fes}, 97$} & \multicolumn{3}{|c|}{ SHMS Unit Number: Murzis 10} \\
\hline \multicolumn{3}{|c|}{$\begin{array}{l}\text { Title of Test: } \\
\text { Pneumatic Systems }\end{array}$} & \multirow{2}{*}{\multicolumn{3}{|c|}{ Test Equipment and $\mathrm{S} / \mathrm{N}$ or Cal. No.: }} \\
\hline \multicolumn{3}{|c|}{ 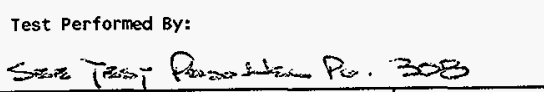 } & & & \\
\hline $\begin{array}{l}\text { Procedure No. } \\
\text { Initial/Date }\end{array}$ & I tem & value & $\begin{array}{l}\text { Required } \\
\text { Condition }\end{array}$ & $(A / R)$ & Corment \\
\hline $\begin{array}{c}1.7 .4 .3 .2 \\
\end{array}$ & $\begin{array}{l}\text { PUSH PB- } * 59 \\
\text { GRAB SAMPLE }\end{array}$ & Lic & $\begin{array}{l}\text { START } \\
\text { STOPWATCH }\end{array}$ & $A$ & $N / \Delta$ \\
\hline $\begin{array}{l}1.7 .4 .3 .3 \\
6 e^{2}=1\end{array}$ & $\begin{array}{l}\text { GRAB SAMPLE } \\
\text { LAMPS ON } \\
\text { READ FIV-*52 }\end{array}$ & $\frac{\frac{O K}{O K}}{\frac{O K}{0}}$ & 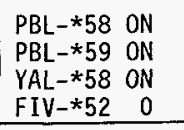 & $\Lambda$ & \\
\hline $1.7 .43^{3.4}$ & $\begin{array}{l}\text { OPEN SV }-* 15 \\
\& \text { ADJ. FIV } \\
* 52\end{array}$ & ok & $\begin{array}{l}\text { SV-* } 15 \text { OPEN } \\
\text { FIV } * 52 \\
\text { FLOW } 10 \mathrm{CFH} \\
\end{array}$ & $\mathbb{A}$ & \\
\hline $6^{1.4 .3 .5}$ & READ PDI $* 51$ & 32.5 & $\begin{array}{l}\text { PDI-*51 } \\
\text { HIGHER THAN } \\
1.7 .4 .3 .1 \\
\end{array}$ & $A$ & \\
\hline 1.4.3.6 & CLOSE SV $-* 15$ if & rok & FIV $-* 520$ & $A$ & \\
\hline $\begin{array}{l}1.7 .4 .3 .7 \\
\text { Soin } \\
\end{array}$ & OPEN SV-*15U & OK & $\begin{array}{l}\text { FIV }-* 52 \\
\text { FLOW } \\
\end{array}$ & A & \\
\hline $\begin{array}{c}1.7 .4 .3 .8 \\
6 \cos \end{array}$ & $\begin{array}{l}\text { PBL-*59 } \\
\text { GRAB SAMPLE } \\
\text { TIME }\end{array}$ & $\frac{O K}{5 / O K}$ & $\begin{array}{l}\text { PBL } * 59 \text { OFF } \\
\text { STOP WATCH } \\
5 \pm .5 \text { MIN } \\
\end{array}$ & $\Delta$ & \\
\hline $\begin{array}{c}1.7 .4 .3 .9 \\
6 \\
\end{array}$ & $\begin{array}{l}\text { PB-*58 RESET } \\
\text { SAMPLER }\end{array}$ & $\frac{O K}{O K}$ & $\begin{array}{l}\text { PBL-*58 OFF } \\
\text { YAL-*58 OFF }\end{array}$ & $A$ & \\
\hline \multicolumn{6}{|c|}{ Section 1.7.4.4 verifies the $\mathrm{H}_{2}$ cell calibration loop. } \\
\hline Gom/orn & $\begin{array}{l}\text { CONNECT } H_{2} \\
\text { CAL GAS }\end{array}$ & $N / A$ & N/A & $A$ & \\
\hline G.7.4.2 & $\begin{array}{l}\text { OPEN ISO } \\
\text { VALVE }\end{array}$ & OK & $<10$ PSIG & $A$ & \\
\hline 1.7.4.4.3 & $\begin{array}{l}\text { OPEN SV-*18 } \\
\text { ADJ. FIV-*56 }\end{array}$ & 2 & $2 \pm .1 \mathrm{CFH}$ & $A$ & $N / A$ \\
\hline \multicolumn{6}{|c|}{ 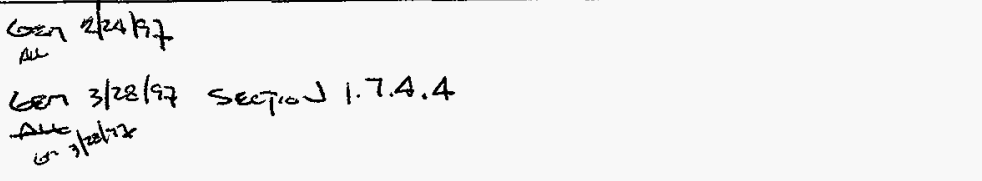 } \\
\hline
\end{tabular}


TEST DATA SHEET

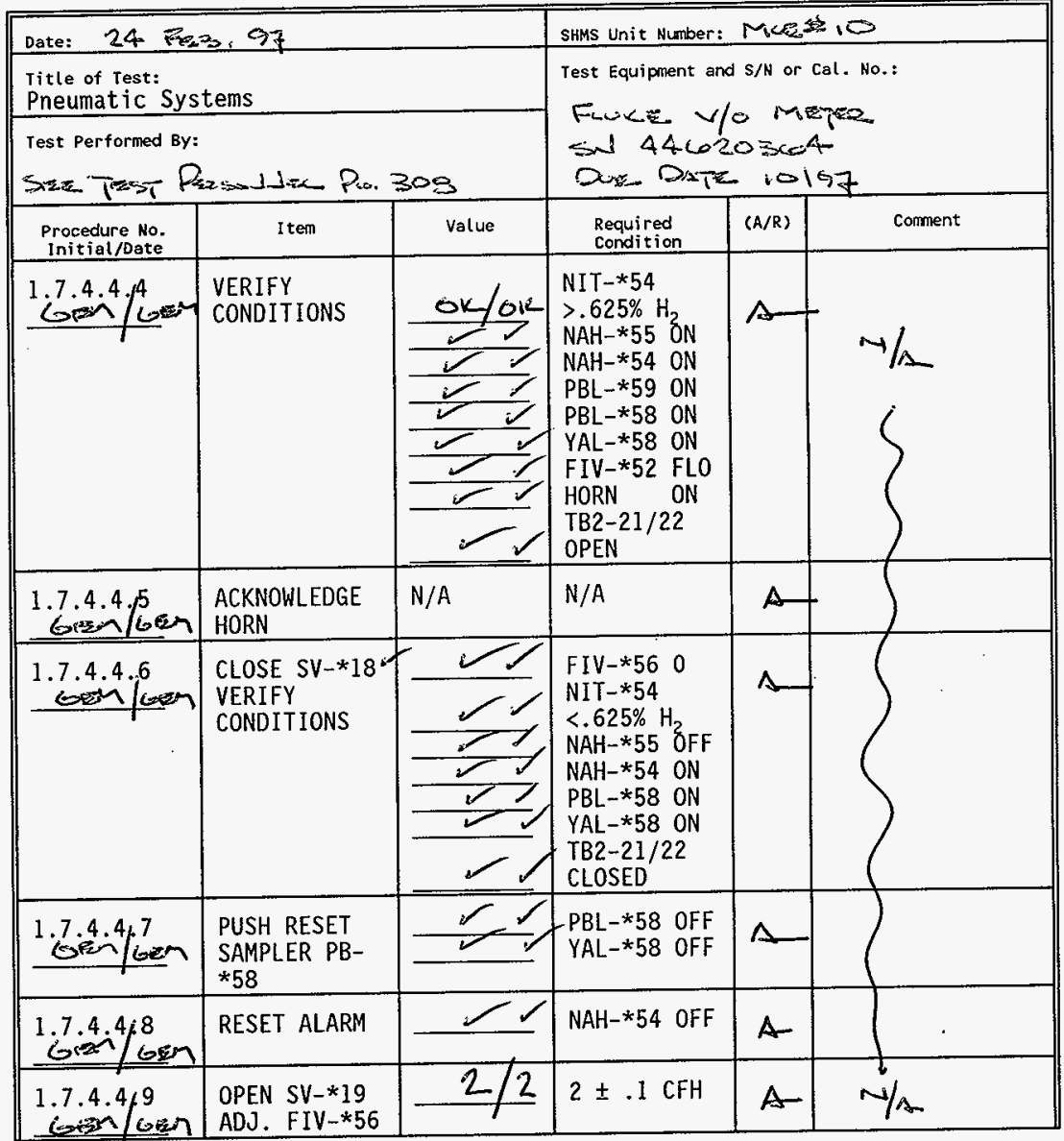

Sen sue 2k4isy

Gein su $3 / 20157$ 
TEST DATA SHEET

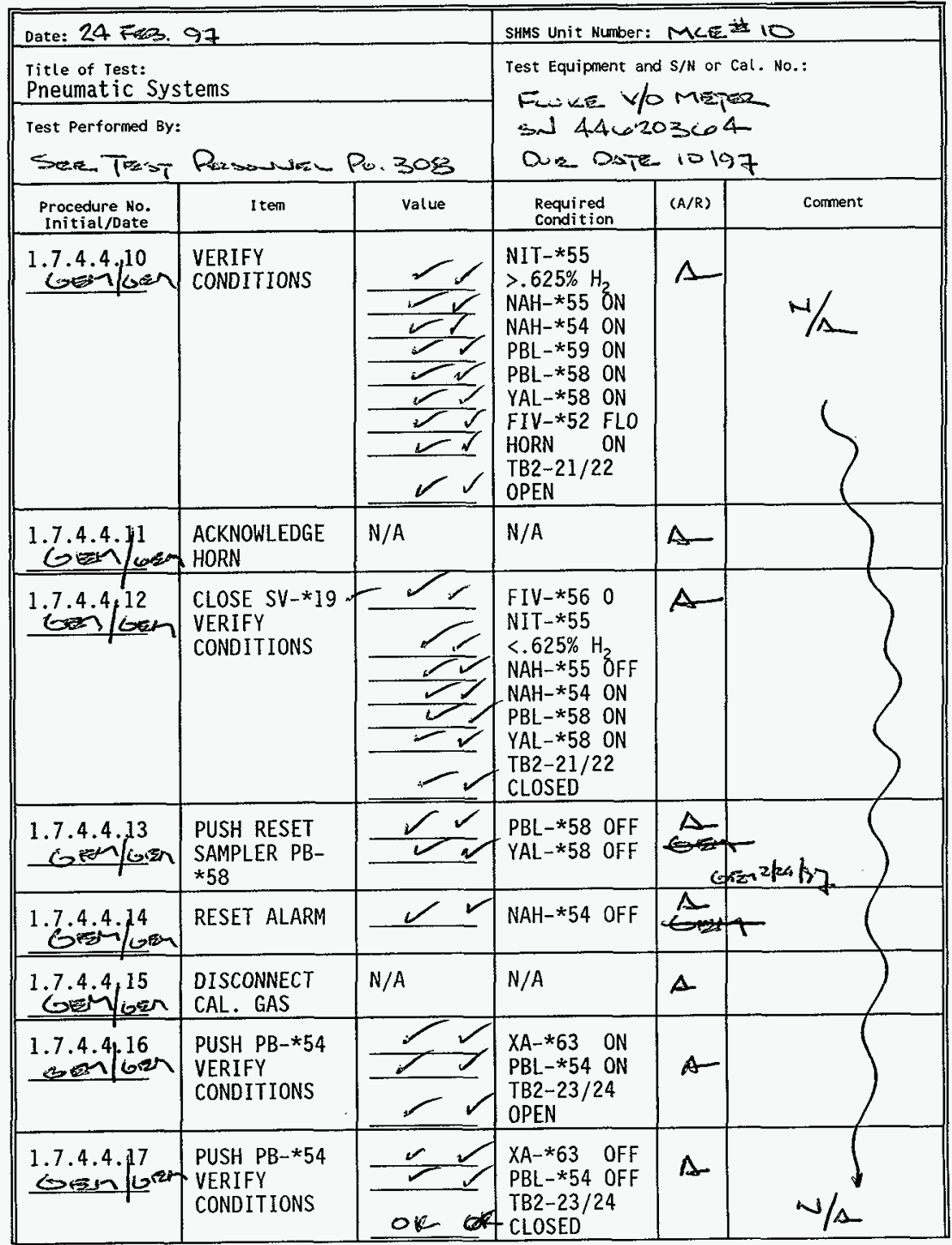


TEST DATA SHEET

\begin{tabular}{|c|c|c|c|c|c|}
\hline \multicolumn{3}{|c|}{ Date: 260 Rxs: 97} & \multicolumn{3}{|c|}{ SHMS Unit Number: MCE 10} \\
\hline \multicolumn{3}{|c|}{$\begin{array}{l}\text { Title of Test: } \\
\text { Pneumatic Systems }\end{array}$} & \multirow{2}{*}{\multicolumn{3}{|c|}{ Test Equipment and $\mathrm{S} / \mathrm{N}$ or Cal. No.: }} \\
\hline \multicolumn{3}{|c|}{$\begin{array}{l}\text { Test performed By: } \\
\text { Sxen Trost Rosoldec D. } 308\end{array}$} & & & \\
\hline $\begin{array}{c}\text { Procedure No. } \\
\text { Initial/Date } \\
\end{array}$ & Item & value & $\begin{array}{l}\text { Required } \\
\text { Condition }\end{array}$ & $(A / R)$ & Comment \\
\hline \multicolumn{6}{|c|}{ Section 1.7.4.5 verifies the GC instrument tubing and calibration gas loop. } \\
\hline $\begin{array}{l}1.7 .4 .5 .1 \\
\operatorname{sen} 2(2 \pi) \\
\end{array}$ & $\begin{array}{l}\text { REMOVE PLUG } \\
\& \text { CONNECT } \\
\text { TEST GAS } \\
\end{array}$ & 5 & $\begin{array}{l}\text { TEST GAS } \\
3-5 \text { PSIG }\end{array}$ & $A$ & $\mathrm{H}$ \\
\hline $\begin{array}{l}1.7 .4 .5 .2 \\
6.07\end{array}$ & $\begin{array}{l}\text { VERIFY NO } \\
\text { GAS FLOW }\end{array}$ & $\frac{O K}{O K}$ & $\begin{array}{l}\mathrm{H}_{2} \text { GAS PORT } \\
\text { SAMPLE A IN }\end{array}$ & $\curvearrowright$ & \\
\hline 1.7.4.5.3 & $\begin{array}{l}\text { VERIFY COL A } \\
\text { GAS FLOW }\end{array}$ & ok & COL A FLOW & A & \\
\hline $\begin{array}{l}1.7 .4 .5 .4 \\
0.01\end{array}$ & $\begin{array}{l}\text { REPLACE COL } \\
\text { A CAP }\end{array}$ & N/A & N/A & $A$ & \\
\hline $\begin{array}{l}1.7 .4 .5 .5 \\
\end{array}$ & $\begin{array}{l}\text { VERIFY COL B } \\
\text { GAS FLOW }\end{array}$ & OK & COL B FLOW & A & \\
\hline $\begin{array}{l}1.7 .4 .5 .6 \\
\text { seing }\end{array}$ & $\begin{array}{l}\text { REPLACE COL } \\
\text { B CAP }\end{array}$ & $N / A$ & N/A & 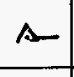 & \\
\hline $\begin{array}{l}1.7 .4 .5 .7 \\
\text { gex-1 }\end{array}$ & $\begin{array}{l}\text { VERIFY } \\
\text { SAMPLE VENT } \\
\text { FLOW }\end{array}$ & ok & $\begin{array}{l}\text { SAMPLE VENT } \\
\text { FLOW }\end{array}$ & $\Delta$ & \\
\hline $\begin{array}{l}1.7 .4 .5 .8 \\
6.7\end{array}$ & $\begin{array}{l}\text { REPLACE } \\
\text { SAMPLE VENT } \\
\text { CAP } \\
\end{array}$ & N/A & $N / A$ & $\Delta$ & \\
\hline 1.7.4.5.9 & $\begin{array}{l}\text { INSTALL } \\
\text { JUMPER } \\
\end{array}$ & $N / A$ & $N / A$ & 1 & $F$ \\
\hline $\begin{array}{l}1.7 .4 .5 .10 \\
\text { cosen }\end{array}$ & $\begin{array}{l}\text { CLOSE FU-15 } \\
\text { VERIFY FLOW }\end{array}$ & $\frac{a k}{o k}$ & $\begin{array}{l}\mathrm{H}_{2} \text { CAL FLOW } \\
\text { SAMP A IN } \\
\text { FLOW }\end{array}$ & $A$ & $1 / \Delta$ \\
\hline 1.7.4.5.11 & $\begin{array}{l}\text { INSTALL PLUG } \\
\& \text { CAP }\end{array}$ & OK & $\begin{array}{l}\text { PLUG } \mathrm{H}_{2} \text { CAL } \\
\text { CAP SAMP A }\end{array}$ & $\Delta-$ & \\
\hline $\begin{array}{l}1.7 .4 .5 .12 \\
\text { exes. }\end{array}$ & $\begin{array}{l}\text { OPEN SV }-* 63 \\
\& \text { READ } \\
\text { PDIT-*60 }\end{array}$ & $\frac{b^{2 / 20192}}{122}$ & $\begin{array}{l}\text { PDIT }-\star 60= \\
28 \times \text { PRESS } \\
1.7 .4 .5 .1 \\
\pm 20 \mathrm{H}_{2} \mathrm{O} \\
\end{array}$ & $\Lambda$ & \\
\hline $\begin{array}{l}1.7 .4 .5 .13 \\
\text { gon }\end{array}$ & $\begin{array}{l}\text { OPEN SV-*64 } \\
\text { VERIFY FLOW }\end{array}$ & ok & $\begin{array}{l}\text { SAMP B IN } \\
\text { FLOW }\end{array}$ & $\Delta$ & $N / \Delta$ \\
\hline
\end{tabular}


TEST DATA SHEET

\begin{tabular}{|c|c|c|c|c|c|}
\hline Date: $26 \mathrm{~F}$ & 0.97 & & SHMS Unit Number: & $m e=$ & -10 \\
\hline $\begin{array}{l}\text { Title of Test: } \\
\text { Pneumatic S }\end{array}$ & tems & & Test Equipment an & $d S / N$ or & Cal. No.: \\
\hline $\begin{array}{l}\text { Test Performed } \\
\text { Seste Tost }\end{array}$ & trososien Pu. & 308 & $\omega / \Delta$ & & \\
\hline $\begin{array}{l}\text { Procedure No. } \\
\text { Initial/Date }\end{array}$ & Item & value & $\begin{array}{l}\text { Required } \\
\text { Condition }\end{array}$ & $(A / R)$ & Comment \\
\hline $\begin{array}{l}1.7 .4 .5 .14 \\
\text { ogen } \\
\end{array}$ & $\begin{array}{l}\text { REMOVE TEST } \\
\text { GAS }\end{array}$ & $N / A$ & $N / A$ & A & $N / s$ \\
\hline $\mathrm{Gg}^{7.4 .5 .15}$ & $\begin{array}{l}\text { CLOSE SV-*632 } \\
\& \text { SV-*64 }\end{array}$ & $\mathrm{N} / \mathrm{A}$ & $N / A$ & $\Delta$ & \\
\hline Gein & $\begin{array}{l}\text { OPEN FU-15 } \\
\text { REMOVE } \\
\text { JUMPER }\end{array}$ & $N / A$ & N/A & A & \\
\hline $\begin{array}{l}1.7 .4 .5 .17 \\
\text { Gen }\end{array}$ & $\begin{array}{l}\text { INSTALL } \\
\text { SAMP A UNION }\end{array}$ & $N / A$ & N/A & $A$ & \\
\hline $\begin{array}{l}1.7 .4 .5 .18 \\
\text { Gom }\end{array}$ & $\begin{array}{l}\text { OPEN SV }-* 60 \\
\text { VERIFY FLOW }\end{array}$ & OK & $\begin{array}{l}\text { FI-*60 } \\
\text { NO FLOW }\end{array}$ & 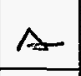 & \\
\hline 1.7.4.5.19 & $\begin{array}{l}\text { POWER SOV- } \\
* 60 \text {, VERIFY } \\
\text { NO FLOW } \\
\end{array}$ & ole & $\begin{array}{l}\text { FI-*60 } \\
\text { NO FLOW }\end{array}$ & $\Delta$ & \\
\hline $\begin{array}{l}1.7 .4 .5 .20 \\
\end{array}$ & $\begin{array}{l}\text { OPEN SV-*68 } \\
\text { ADJ SV-*67 }\end{array}$ & oll & $\begin{array}{l}F I-* 60 \\
50 \mathrm{CCM} \\
\end{array}$ & $\Delta$ & \\
\hline $\begin{array}{l}1.7 .4 .5 .21 \\
\end{array}$ & $\begin{array}{l}\text { DEENERGIZE } \\
\text { SOV-*60 }\end{array}$ & ok & $\begin{array}{l}\text { FI }-{ }^{*} 60 \text { FLOW } \\
\text { DECREASES }\end{array}$ & $\Delta$ & \\
\hline $\begin{array}{l}1.7 .4 .5 .22 \\
\end{array}$ & $\begin{array}{l}\text { CLOSE SV-*60 } \\
\& \text { SV-*68 }\end{array}$ & N/A & $N / A$ & $A$ & \\
\hline 1.7.4.5.23 & $\begin{array}{l}\text { REMOVE } \\
\text { SAMP A UNION }\end{array}$ & $N / A$ & $N / A$ & $A$ & \\
\hline $\begin{array}{l}1.7 .4 .5 .24 \\
\text { igen }\end{array}$ & $\begin{array}{l}\text { CONNECT TEST } \\
\text { GAS } \mathrm{N}_{2} \mathrm{O} \text { PORT } \\
\end{array}$ & 5 & $\begin{array}{l}\text { TEST GAS } \\
3-5 \text { PSIG } \\
\end{array}$ & $\Delta$ & \\
\hline Gen & $\begin{array}{l}\text { VERIFY NO } \\
\text { FLOW }\end{array}$ & $\frac{O K}{O K}$ & $\begin{array}{l}\text { SAMP B IN \& } \\
\text { FLT-*63 } \\
\text { NO FLOW } \\
\end{array}$ & $\Delta$ & $.1 / 2$ \\
\hline $\begin{array}{l}1.7 .4 .5 .26 \\
\text { gon }\end{array}$ & $\begin{array}{l}\text { INSTALL } \\
\text { JUMPER }\end{array}$ & N/A & $\mathrm{N} / \mathrm{A}$ & $\Lambda$ & Sov-cozt ? Futst \\
\hline $\begin{array}{l}1.7 .4 .5 .27 \\
\text { Soen }\end{array}$ & $\begin{array}{l}\text { CLOSE FU-15 } \\
\text { VERIFY FLOW }\end{array}$ & $\frac{\text { ol }}{\text { ox }}$ & $\begin{array}{l}\text { SAMP B IN } \\
\text { FLT-*63 } \\
\text { FLOW }\end{array}$ & $A$ & 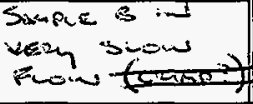 \\
\hline
\end{tabular}


TEST DATA SHEET

\begin{tabular}{|c|c|c|c|c|c|}
\hline \multicolumn{3}{|c|}{ Date: 26PFes. 97} & \multicolumn{3}{|c|}{ SHMS Unit Number: MCE $\$ 10$} \\
\hline \multicolumn{3}{|c|}{$\begin{array}{l}\text { Title of Test: } \\
\text { Pneumatic Systems }\end{array}$} & \multicolumn{3}{|c|}{ Test Equipment and $\mathrm{S} / \mathrm{N}$ or Cal. No.: } \\
\hline \multicolumn{3}{|c|}{ 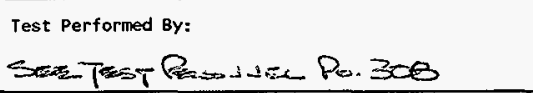 } & \multicolumn{3}{|l|}{$\omega / \Delta$} \\
\hline $\begin{array}{l}\text { Procedure No. } \\
\text { Initial/Date }\end{array}$ & I tem & Value & $\begin{array}{l}\text { Required } \\
\text { Condition }\end{array}$ & $(A / R)$ & Comment \\
\hline $\begin{array}{l}1.7 .4 .5 .28 \\
\text { ovem } \\
\end{array}$ & $\begin{array}{l}\text { DISCONNECT } \\
\text { TEST GAS }\end{array}$ & $N / A$ & $N / A$ & 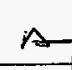 & $n / \Delta$ \\
\hline $\begin{array}{l}1.7 .4 .5 .29 \\
\text { Gen }\end{array}$ & OPEN FU-15 & $N / A$ & $N / A$ & $\wedge$ & \\
\hline $\begin{array}{l}1.7 .4 .5 .30 \\
\text { Giz } \\
\end{array}$ & $\begin{array}{l}\text { REMOVE } \\
\text { JUMPER }\end{array}$ & $N / A$ & $N / A$ & $\Delta$ & \\
\hline $\begin{array}{l}1.7 .4 .5 .31 \\
0.2 \\
\end{array}$ & $\begin{array}{l}\text { INSTALL } \\
\text { SAMP B UNION }\end{array}$ & $N / A$ & $N / A$ & $\Delta$ & \\
\hline $\begin{array}{l}1.7 .4 .5 .32 \\
608 \\
\end{array}$ & $\begin{array}{l}\text { OPEN SV-*60 } \\
\text { VERIFY FLOW }\end{array}$ & oL & $\begin{array}{l}F I-* 60 \\
\text { NO FLOW }\end{array}$ & $\Delta$ & \\
\hline $\begin{array}{l}1.7 .4 .5 .33 \\
\cosh \end{array}$ & $\begin{array}{l}\text { POWER SOV- } \\
\text { *60, VERIFY } \\
\text { NO FLOW }\end{array}$ & ok & $\begin{array}{l}\text { FI }-* 60 \\
\text { NO FLOW }\end{array}$ & $\Delta$ & \\
\hline $\begin{array}{l}1.7 .4 .5 .34 \\
\text { gen } \\
\end{array}$ & $\begin{array}{l}\text { OPEN SV-*68 } \\
\& \text { ADJ SV-*67. }\end{array}$ & ok & $\begin{array}{l}\mathrm{FI}-* 60 \\
50 \mathrm{CCM}\end{array}$ & A & \\
\hline $\begin{array}{l}1.7 .4 .5 .35 \\
6 \underbrace{\circ} \\
\end{array}$ & $\begin{array}{l}\text { DEENERGIZE } \\
\text { SOV-* } 60\end{array}$ & GK & $\begin{array}{l}\text { FI } * * 60 \text { FLOW } \\
\text { DECREASES }\end{array}$ & $\Lambda$ & \\
\hline $\begin{array}{l}1.7 .4 .5 .36 \\
627 \\
\end{array}$ & $\begin{array}{l}\text { CLOSE SV-*60 } \\
\& \text { SV-*68 }\end{array}$ & $\bar{N} / A$ & $N / A$ & $\Delta$ & \\
\hline $\begin{array}{c}1.7 .4 .5 .37 \\
0.7 \\
\end{array}$ & $\begin{array}{l}\text { REMOVE } \\
\text { SAMP B UNION }\end{array}$ & $N / A$ & $N / A$ & $\Delta$ & \\
\hline $\begin{array}{l}1.7 .4 .5 .38 \\
\text { osinn } \\
\end{array}$ & $\begin{array}{l}\text { OPEN CB-3/4 } \\
\text { ACK. ALARMS } \\
\end{array}$ & $N / A$ & $N / A$ & $\Delta$ & \\
\hline \multicolumn{6}{|c|}{$\begin{array}{l}\text { Section } 1.7 .4 .6 \text { verifies the multi gas analyzer instrument tubing and } \\
\text { calibration gas loop. }\end{array}$} \\
\hline $\begin{array}{l}1.7 .4 .6 .1 \\
\text { ingen }\end{array}$ & $\begin{array}{l}\text { VERIFY } \\
\text { NIT-*52 } \\
\text { SAMPLE LINES }\end{array}$ & $N / A$ & $N / A$ & $\Lambda$ & \\
\hline $\begin{array}{l}1.7 .4 .6 .2 \\
\text { Ging }\end{array}$ & $\begin{array}{l}\text { CONNECT TEST } \\
\text { GAS NH } \\
\end{array}$ & 5 & $\begin{array}{l}\text { TEST GAS } \\
3-5 \text { PSIG }\end{array}$ & $\Lambda$ & \\
\hline 1.7 .4 .6 .3 & $\begin{array}{l}\text { VERIFY NO } \\
\text { FLOW }\end{array}$ & $\frac{\text { ok }}{\text { ok }}$ & $\begin{array}{l}\text { SAMPLE IN } \\
\text { FLT-*63 }\end{array}$ & $\Delta$ & $/ \mathrm{s}$ \\
\hline
\end{tabular}


TEST DATA SHEET

\begin{tabular}{|c|c|c|c|c|c|}
\hline \multicolumn{3}{|c|}{ Date: 26 Fes3.97 } & \multicolumn{3}{|c|}{ SHMS Unit Mumber: Mkest 10} \\
\hline \multicolumn{3}{|c|}{$\begin{array}{l}\text { Title of Test: } \\
\text { Pneumatic Systems }\end{array}$} & \multirow{2}{*}{\multicolumn{3}{|c|}{ Test Equipment and $\mathrm{S} / \mathrm{N}$ or Cal. No.: }} \\
\hline \multirow{2}{*}{ 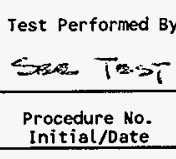 } & \multicolumn{2}{|c|}{ Risolitia $R .308$} & & & \\
\hline & I tem & value & $\begin{array}{l}\text { Required } \\
\text { Condition }\end{array}$ & $(A / R)$ & Comment \\
\hline $\begin{array}{l}1.7 .4 .6 .4 \\
6 \operatorname{lin}^{2} \\
\end{array}$ & $\begin{array}{l}\text { INSTALL } \\
\text { JUMPER } \\
\end{array}$ & $N / A$ & N/A & $\Delta-$ & \\
\hline $\begin{array}{l}1.7 .4 .6 .5 \\
\text { o.9.7 }\end{array}$ & $\begin{array}{l}\text { CLOSE FU- } 15 \\
\text { VERIFY GAS } \\
\text { FLOWS }\end{array}$ & $\begin{array}{l}0 k \\
K \\
K \\
\end{array}$ & $\begin{array}{l}\text { SAMPLE IN } \\
\text { FLT-*63 } \\
\text { FIV-*70 } \\
\end{array}$ & $\Delta$ & $\begin{array}{l}\text { Sinp-iz +N } \\
\text { Nein swow Fw } \\
\text { Fix-70 } \rightarrow+75\end{array}$ \\
\hline 1.7.4.6.6 & $\begin{array}{l}\text { PLUG FLT-* } 63^{-} \\
\& \text { MON-*60 } \\
\text { SAMPLE VENT }\end{array}$ & $\mathrm{N} / \mathrm{A}$ & $N / A$ & A & \\
\hline $\begin{array}{r}1.7 .4 .6 .7 \\
\end{array}$ & $\begin{array}{l}\text { OPEN SV-*66, } \\
\text { SV-*70 \& CAP } \\
\text { SAMPLE IN }\end{array}$ & 133.1 & $\begin{array}{l}\text { PDIT }-* 60= \\
28 \times \text { PRESS } \\
1.7 .4 .6 .2 \\
\pm 20 " \mathrm{H}_{2} \mathrm{O}\end{array}$ & $\Lambda$ & \\
\hline $\begin{array}{l}1.7 .4 .6 .8 \\
\text { Gein }\end{array}$ & $\begin{array}{l}\text { CLOSE SY } \$ * 66 \\
\text { SV-*70 } \% \\
\text { REMOVE CAP }\end{array}$ & $T / A$ & $N / A$ & $\Delta$ & \\
\hline $\begin{array}{l}1.7 .4 .6 .9 \\
\text { Gen } \\
\end{array}$ & $\begin{array}{l}\text { REMOVE FLT- } \\
* 63 \text { PLUG } \\
\end{array}$ & $\mathrm{N} / \mathrm{A}$ & $N / A$ & $\Delta$ & \\
\hline $\begin{array}{l}1.7 .4 .6 .10 \\
\text { Gen }\end{array}$ & $\begin{array}{l}\text { REMOVE TEST } \\
\text { GAS }\end{array}$ & $N / A$ & $N / A$ & $A$ & \\
\hline $\begin{array}{c}1.7 .4 .6 .11 \\
6 \text {.877 } \\
\end{array}$ & $\begin{array}{l}\text { CLOSE SV-*05 } \\
\& \text { SV }-* 16\end{array}$ & N/A & $N / A$ & $\Delta$ & \\
\hline $\begin{array}{l}1.7 .4 .6 .12 \\
\text { Som }\end{array}$ & $\begin{array}{l}\text { CONNECT TEST } \\
\text { GAS }\end{array}$ & 4. & $\begin{array}{l}\text { TEST GAS } \\
2-4 \text { PSIG } \\
\end{array}$ & $\mathcal{A}$ & \\
\hline igin & $\begin{array}{l}\text { OPEN SV-*07 } \\
\text { \& VERIFY } \\
\text { NO FLOW }\end{array}$ & $\begin{array}{l}\text { OK } \\
\text { OK }\end{array}$ & $\begin{array}{l}\text { SAMPLE IN } \\
\text { SAMPLE OUT }\end{array}$ & $A$ & \\
\hline$\frac{1.7 .4 .6 .14}{62 \pi}$ & $\begin{array}{l}\text { OPEN SV }-* 09 \\
\& \text { VERIFY } \\
\text { NO FLOW }\end{array}$ & $\frac{O K}{O K}$ & $\begin{array}{l}\text { SAMPLE IN } \\
\text { SAMPLE OUT }\end{array}$ & $\Lambda$ & \\
\hline $\begin{array}{l}1.7 .4 .6 .16 \\
\text { Oㅅ }\end{array}$ & $\begin{array}{l}\text { CLOSE SV-*09- } \\
\text { VERIFY FLOW }\end{array}$ & Tok & $\begin{array}{l}\text { SAMPLE IN } \\
\text { NO FLOW }\end{array}$ & $A$ & $i$ \\
\hline
\end{tabular}


TEST DATA SHEET

\begin{tabular}{|c|c|c|c|c|c|}
\hline \multicolumn{3}{|c|}{ Date: 260 Fes .97} & \multicolumn{3}{|c|}{ SHMS Unit Number: Murem is } \\
\hline \multicolumn{3}{|c|}{$\begin{array}{l}\text { Title of Test: } \\
\text { Pneumatic Systems }\end{array}$} & \multirow{2}{*}{\multicolumn{3}{|c|}{ Test Equipment and $S / N$ or Cal. No.: }} \\
\hline \multicolumn{3}{|c|}{ 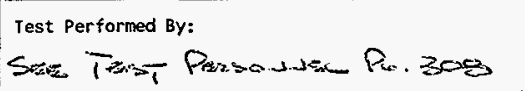 } & & & \\
\hline $\begin{array}{l}\text { Procedure No. } \\
\text { Initial/Date }\end{array}$ & Item & Value & $\begin{array}{l}\text { Required } \\
\text { Condition }\end{array}$ & $(A / R)$ & Comment \\
\hline $\begin{array}{l}1.7 .4 .6 .17 \\
6 \times 3 \\
\end{array}$ & $\begin{array}{l}\text { OPEN SV-*10 } \\
\text { VERIFY FLOW }\end{array}$ & OK & $\begin{array}{l}\text { SAMPLE FLOW } \\
\text { IN - NO } \\
\text { OUT - YES }\end{array}$ & $\Delta$ & A \\
\hline $\begin{array}{l}1.7 .4 .6 .18 \\
6 x=1 \\
\end{array}$ & $\begin{array}{l}\text { REMOVE TEST } \\
\text { GAS \& PLUG } \\
\text { SV-*07 }\end{array}$ & $N / A$ & $N / A$ & $\Delta$ & \\
\hline $\begin{array}{c}1.7 .4 .6 .19 \\
\text { Gron }\end{array}$ & $\begin{array}{l}\text { CLOSE SV-*07 } \\
\& \text { SV-* } 10-\end{array}$ & $N / A$ & $N / A$ & $A$ & \\
\hline $\begin{array}{l}1.7 .4 .6 .20 \\
\text { cons } \\
\end{array}$ & $\begin{array}{l}\text { OPEN SV-*05 } \\
\& S V-* 16\end{array}$ & $N / A$ & $N / A$ & $\Delta$ & \\
\hline $\begin{array}{l}1.7 .4 .6 .21 \\
\mathrm{~S} \\
\end{array}$ & $\begin{array}{l}\text { REMOVE } \\
\text { JUMPER }\end{array}$ & $N / A$ & $N / A$ & $\Delta$ & \\
\hline \multicolumn{6}{|c|}{ Section 1.7.4.7 verifies the carrier gas supply lines. } \\
\hline $\begin{array}{c}1.7 .4 .7 .1 \\
\text { cosem } \\
\end{array}$ & $\begin{array}{l}\text { CONNECT TEST } \\
\text { GAS }\end{array}$ & 4 & $3-5$ PSIG & $\Delta$ & \\
\hline $\begin{array}{l}1.7 .4 .7 .2 \\
\text { (-5inn } \\
\end{array}$ & $\begin{array}{l}\text { REMOVE PLUG } \\
\& \text { VERIFY } \\
\text { FLOW }\end{array}$ & OK. & $\begin{array}{l}\mathrm{N}_{2} \text { CARRIER } \\
\mathrm{GAS} \text { FLOWS }\end{array}$ & $A$ & \\
\hline $\begin{array}{c}1.7 .4 .7 .3 \\
\text { sen } \\
\end{array}$ & $\begin{array}{l}\text { DISCONNECT } \\
\text { TEST GAS } \\
\end{array}$ & $N / A$ & $N / A$ & $A$ & \\
\hline $\begin{array}{c}1.7 .4 .7 .4 \\
0.0 \\
\end{array}$ & $\begin{array}{l}\text { CONNECT TEST } \\
\text { GAS }\end{array}$ & 4 & $3-5$ PSIG & $A$ & \\
\hline $\begin{array}{c}1.7 .4 .7 .5 \\
\text { 6en } \\
\end{array}$ & $\begin{array}{l}\text { REMOVE PLUG } \\
\& \text { VERIFY } \\
\text { FLOW }\end{array}$ & OK & $\begin{array}{l}\text { He CARRIER } \\
\text { GAS FLOWS }\end{array}$ & $\Delta$ & \\
\hline $\begin{array}{l}1.7 .4 .7 .6 \\
612 \\
\end{array}$ & $\begin{array}{l}\text { DISCONNECT } \\
\text { TEST GAS } \\
\end{array}$ & $N / A$ & $N / A$ & $\Lambda$ & $N / \mathrm{A}$ \\
\hline $\begin{array}{l}1.7 .5 \\
\text { Gizh }\end{array}$ & $\begin{array}{l}\text { TEST SEC. } \\
1.7 .4 \text { DONE }\end{array}$ & $\frac{\text { manh }}{\text { Test Dire }}$ & $\frac{13 \text { non }}{\text { or Signature }}$ & & $\frac{28-97}{\text { Date }}$ \\
\hline
\end{tabular}

coen sue zizoliz 
PAGE J-24
APPENDIX $\mathrm{J}$

HNF-SD - WM-ATR $=191$
ReV. 0
Page 330

TEST DATA SHEET

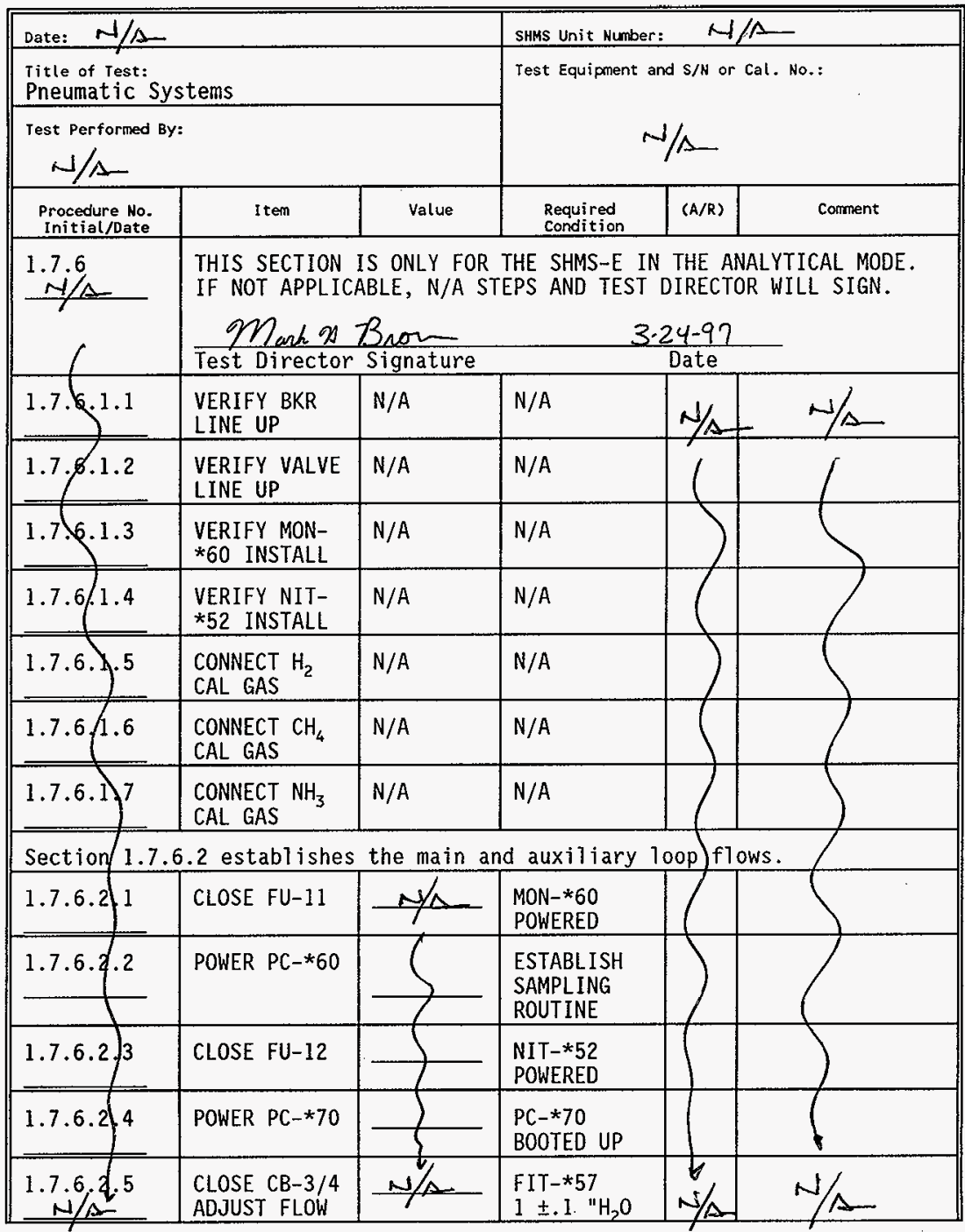


APPENDIX J
PAGE J-25

HNF-SD-WM-ATR-191
ReV. 0
Page 331

TEST DATA SHEET

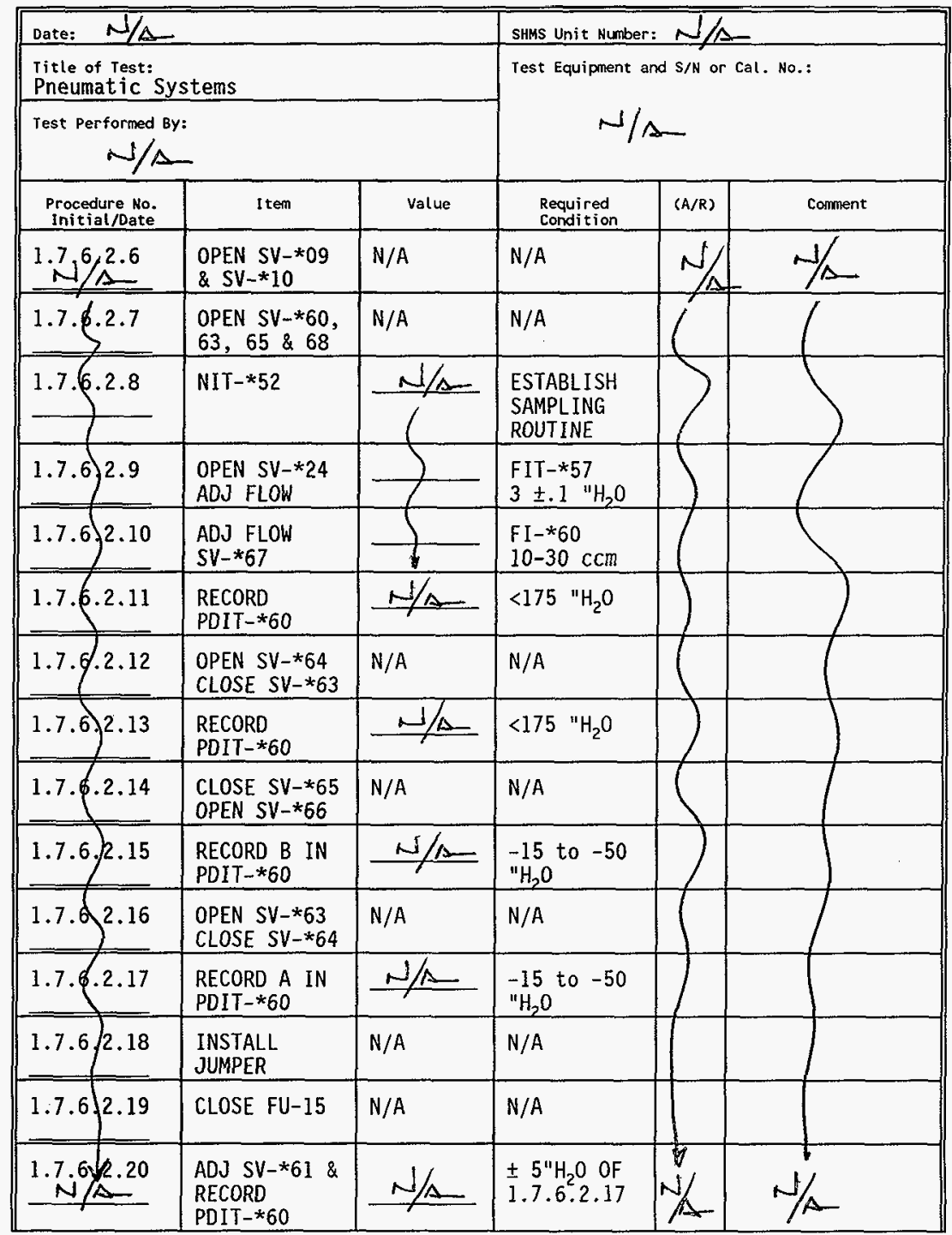


APPENDIX J

TEST DATA SHEET

\begin{tabular}{|c|c|c|c|c|c|c|}
\hline Date: & $\lambda / \alpha$ & & & SHMS Unit Number & $N$ & \\
\hline $\begin{array}{l}\text { Title of } \\
\text { Pneuma }\end{array}$ & $\begin{array}{l}\text { f Test: } \\
\text { atic S }\end{array}$ & tems & & Test Equipment ar & $S C$ or & \\
\hline Test Per & rformed & & & & & \\
\hline $\begin{array}{l}\text { Proced } \\
\text { Initia }\end{array}$ & $\begin{array}{l}\text { lure No. } \\
\text { al/Date }\end{array}$ & I tem & Value & $\begin{array}{l}\text { Required } \\
\text { Condition }\end{array}$ & $(A / R)$ & Comment \\
\hline $1.75^{6}$ & $y^{2.21}$ & $\begin{array}{l}\text { CAL. MON-*60 } \\
\text { CHANNEL A }\end{array}$ & & $\begin{array}{l}\text { READINGS } \\
\text { WITHIN } \pm 5 \%\end{array}$ & & \\
\hline 1.7 .6$. & .2 .22 & OPEN FU-15 & $N / A$ & $N / A$ & & \\
\hline 1.7 .6 & 2.23 & $\begin{array}{l}\text { REMOVE } \\
\text { JUMPER }\end{array}$ & $N / A$ & $N / A$ & & \\
\hline 1.7 .6 & 2.24 & $\begin{array}{l}\text { OPEN SV }-{ }^{*} 64 \\
\text { CLOSE SV }-{ }^{*} 63\end{array}$ & $N / A$ & $N / A$ & & \\
\hline 1.7 .6 & 2.25 & $\begin{array}{l}\text { INSTALL } \\
\text { JUMPER }\end{array}$ & $N / A$ & $N / A$ & & \\
\hline 1.7 .6 & 2.26 & CLOSE FU-15 & $N / A$ & $N / A$ & & \\
\hline 1.7 .6 & 2.27 & $\begin{array}{l}\text { ADJ SV } * 62 \& \\
\text { RECORD } \\
\text { PDIT } * 60\end{array}$ & & $\begin{array}{l} \pm 5^{\prime \prime} \mathrm{H}_{2} \mathrm{O} \text { OF } \\
1.7 .6 .2 .15\end{array}$ & & \\
\hline 1.7 .6 . & .21 .28 & $\begin{array}{l}\text { CAL MON-*60 } \\
\text { CHANNEL B }\end{array}$ & & $\begin{array}{l}\text { READINGS } \\
\text { WITHIN } \pm 5 \%\end{array}$ & & \\
\hline 1.7 .6$. & $\$ .29$ & OPEN FU-15 & N/A & $N / A$ & & \\
\hline 1.7 .6 . & .2 .30 & $\begin{array}{l}\text { REMOVE } \\
\text { JUMPER }\end{array}$ & $N / A$ & $N / A$ & & \\
\hline 1.7 .6$. & 2.31 & $\begin{array}{l}\text { CLOSE SV-*64 } \\
\text { OPEN SV-*70 }\end{array}$ & $N / A$ & $N / A$ & & \\
\hline 1.7 .6 & 2.32 & $\begin{array}{l}\text { RECORD } \\
\text { PDIT-*60 } \\
\text { NIT-*52 IN }\end{array}$ & & $\begin{array}{l}0 \text { to }-30 \\
" \mathrm{H}_{2} \mathrm{O}\end{array}$ & & \\
\hline 1.7 .6 & 2.33 & $\begin{array}{l}\text { INSTALL } \\
\text { JUMPER }\end{array}$ & $N / A$ & $N / A$ & & \\
\hline 1.7 .6 & 2.34 & CLOSE FU-15 & $N / A$ & $N / A$ & & \\
\hline
\end{tabular}


TEST DATA SHEET

\begin{tabular}{|c|c|c|c|c|c|}
\hline \multicolumn{3}{|l|}{ Date: $\mathrm{N} / \mathrm{A}$} & \multicolumn{3}{|c|}{ SHMS Unit Number: $N / \Delta$} \\
\hline \multicolumn{3}{|c|}{$\begin{array}{l}\text { Title of Test: } \\
\text { Pneumatic Systems }\end{array}$} & \multicolumn{3}{|c|}{ Test Equipment and $S / N$ or Cal. No.: } \\
\hline \multicolumn{3}{|c|}{ Test Performed By: } & \multicolumn{3}{|c|}{$N / 1$} \\
\hline $\begin{array}{l}\text { Procedure No. } \\
\text { Initial/0ate } \\
\end{array}$ & I tem & value & $\begin{array}{l}\text { Required } \\
\text { Condition } \\
\end{array}$ & $(A / R)$ & Corment \\
\hline $\mathrm{N}^{1.7 .6}$ & $\begin{array}{l}\text { ESTABLISH } \\
\text { BYPASS FLOW }\end{array}$ & $\frac{N / 2}{1}$ & $\begin{array}{l}\text { FIV-*70 } \\
1.5 \pm .2 \mathrm{cfh} \\
\end{array}$ & & $1 / 1$ \\
\hline $1.7 .6 y^{2.36}$ & $\begin{array}{l}\text { ADJ SV-*71 } \\
\text { NIT-*52 } \\
\text { SAMPLE IN } \\
\end{array}$ & & $\begin{array}{l} \pm 5 \quad \mathrm{H}_{2} \mathrm{O} \quad \mathrm{OF} \\
1.7 .6 .2 .32\end{array}$ & & \\
\hline 1.76 .2 .37 & $\begin{array}{l}\text { RECORD } \\
\text { NIT-*52 } \\
\text { SAMPLES } \\
\end{array}$ & $\pi / x$ & $\begin{array}{l}\text { READINGS } \\
\text { WITHIN } \pm 5 \%\end{array}$ & & \\
\hline $1.7 .6 / 2.38$ & OPEN FU-15 & $N / A$ & N/A & & \\
\hline 1.7 .6 .2 .39 & $\begin{array}{l}\text { REMOVE } \\
\text { JUMPER }\end{array}$ & $N / A$ & $N / A$ & & \\
\hline Sectibn 1.7 & provides for & est syste & shutdown. & & \\
\hline $1.7 / 7.1$ & $\begin{array}{l}\text { DISCONNECT } \\
\mathrm{H}_{2} \text { CAL GAS }\end{array}$ & $N / A$ & $N / A$ & & \\
\hline 1.7. 7.2 & $\begin{array}{l}\text { DISCONNECT } \\
\mathrm{CH}_{6} \text { CAL GAS } \\
\end{array}$ & $N / A$ & N/A & & \\
\hline 1.7.$\} .3$ & $\begin{array}{l}\text { DISCONNECT } \\
\mathrm{NH}_{3} \text { CAL GAS }\end{array}$ & N/A & $N / A$ & & \\
\hline $1.7\{7.4$ & $\begin{array}{l}\text { SECURE \& } \\
\text { TURN OFF } \\
\text { ANA. INST.S }\end{array}$ & $\mathrm{N} / \mathrm{A}$ & N/A & & \\
\hline 1.7 .7 .5 & $\begin{array}{l}\text { DISCONNECT } \\
\mathrm{N}_{2} \text { CARRIER } \\
\end{array}$ & $N / A$ & $N / A$ & & \\
\hline 1.77 .6 & $\begin{array}{l}\text { DISCONNECT } \\
\text { He CARRIER } \\
\end{array}$ & $N / A$ & N/A & & \\
\hline 1.7 .7 .7 & $\begin{array}{l}\text { OPEN ALL } \\
\text { BREAKERS \& } \\
\text { FUSES } \\
\end{array}$ & $N / A$ & $N / A$ & & \\
\hline 1.7.8.8 & $\begin{array}{l}\text { CLOSE LISTED } \\
\text { SYS. VALVES } \\
\end{array}$ & $N / A$ & $N / A$ & $N / \Delta$ & $/ /$ \\
\hline
\end{tabular}


APPENDIX J

PAGE $J-28$
HNF-SD-WH-ATR-191

Rev. 0
Page 334

TEST DATA SHEET

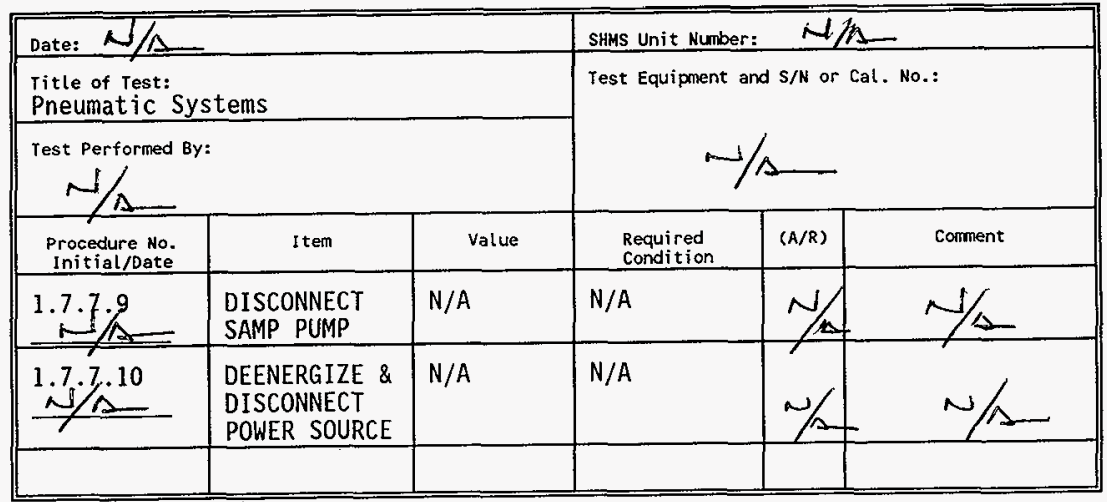

Test Witness/Review:

Mark q 3 nor

Test Director

GEORGE MAYA

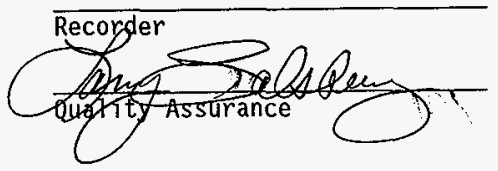

$\frac{3-28-97}{\text { Date }}$

28 M secs 97

Date

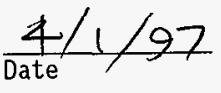


APPENDIX $\mathrm{J}$

PAGE $\mathrm{J}-29$

$$
\begin{array}{r}
\text { HNF-SD-WM-ATR-191 } \\
\text { ReV. } 0 \\
\text { Page } 335 a
\end{array}
$$

TEST EXCEPTION SHEET

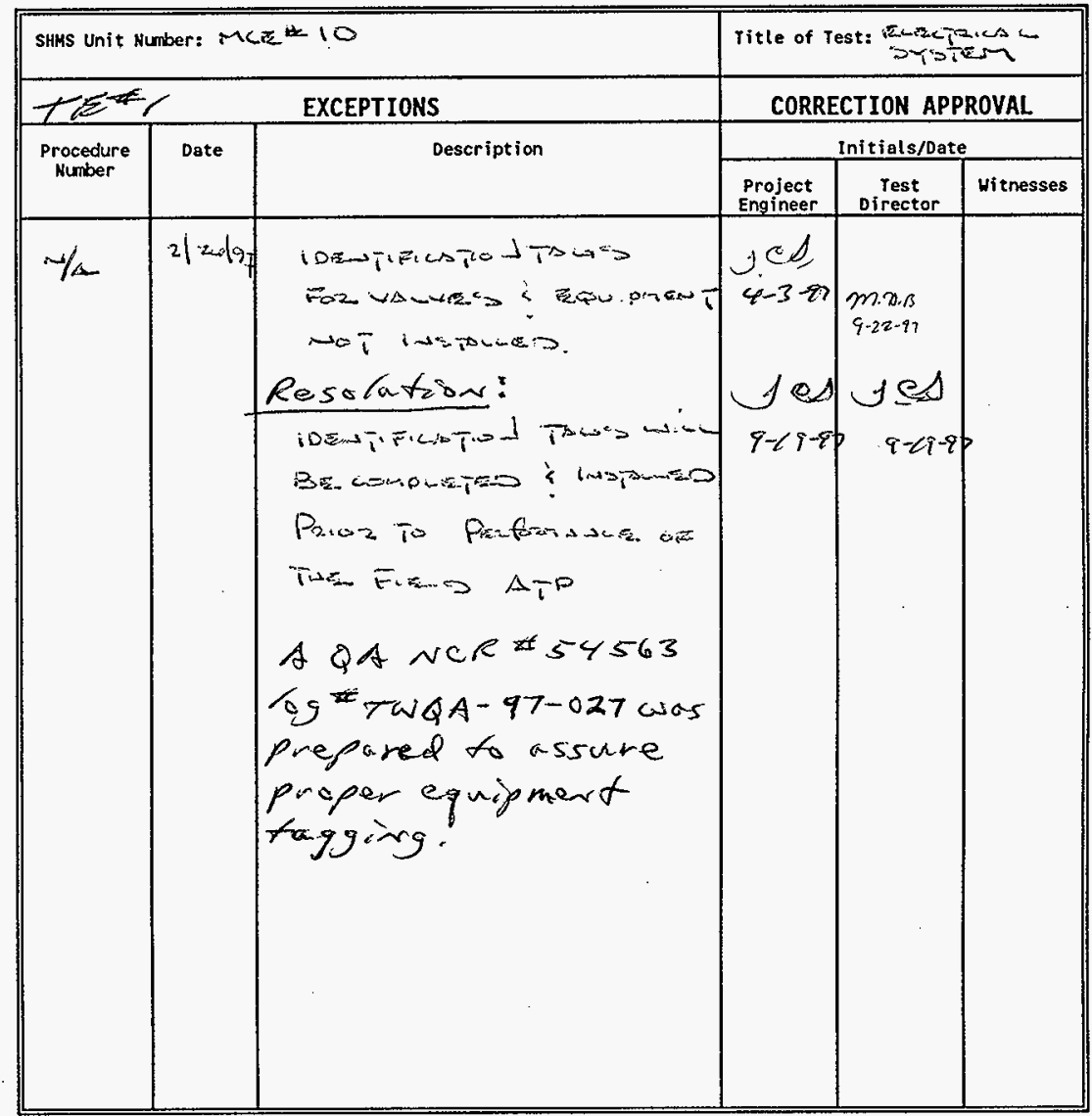

TEST APPROVED WITH EXCEPTIONS

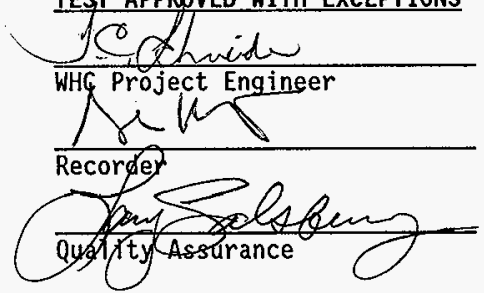

$$
\begin{aligned}
& \frac{4-3-97}{\text { Date }} \\
& \frac{34 p a-97}{\text { Date }} \\
& \frac{4 / 2 / 977}{\text { Date }}
\end{aligned}
$$


APPENDIX $\mathrm{J}$

PAGE $J-3 \circ$

$$
\begin{aligned}
H N F-S D-W M-A I R-191 \\
\text { Rev. } 0 \\
\text { Page } 3356
\end{aligned}
$$

TEST EXCEPTION SHEET

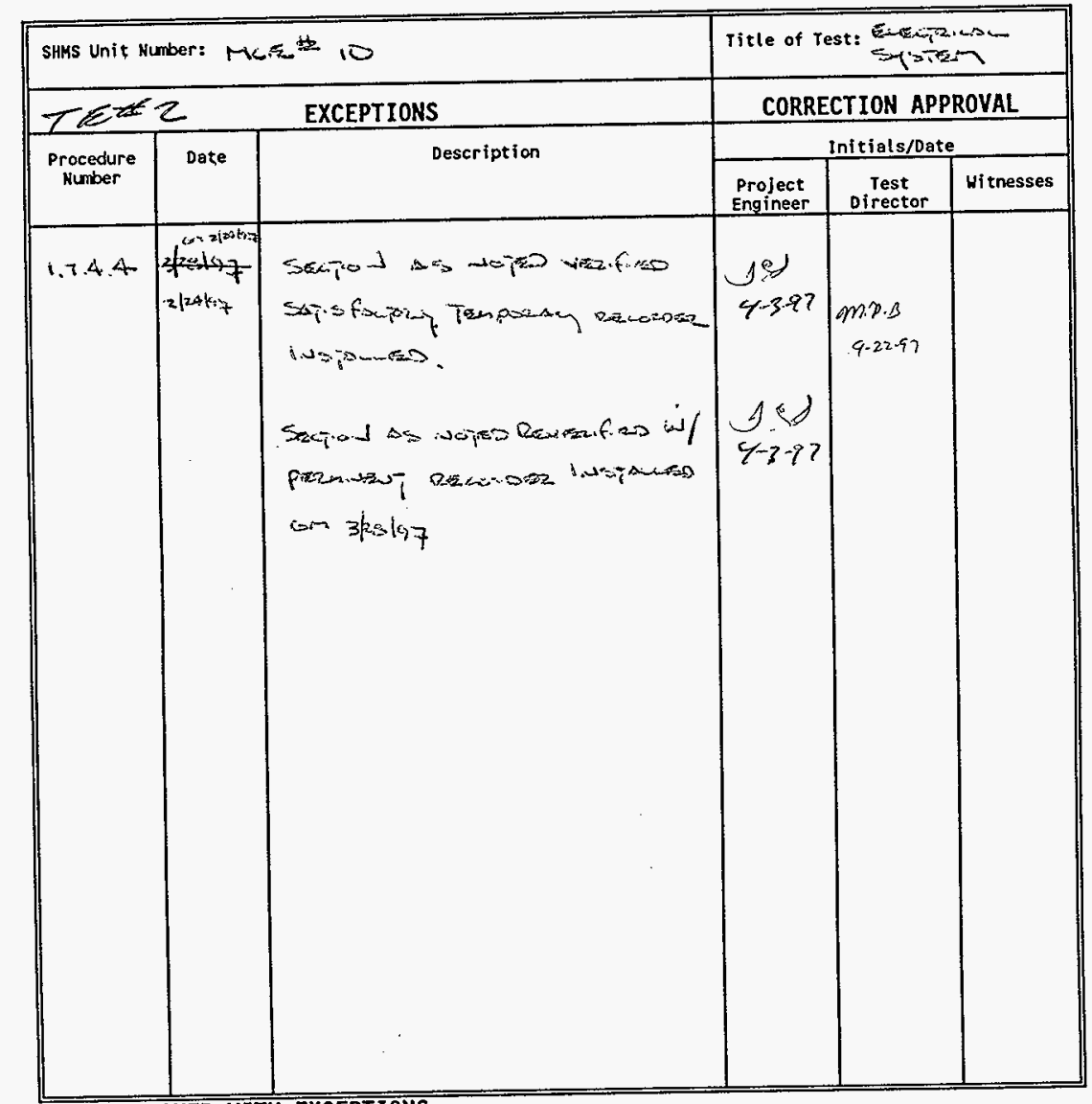

TEST APPROVED WITH EXCEPTIONS

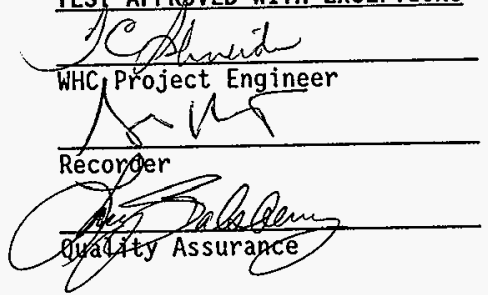

$$
\begin{aligned}
& \frac{4-z-9\rangle}{\text { Date }} \\
& \frac{3 \Delta q-97}{\text { Date }} \\
& \frac{4 / 2 / 97}{\text { Date }}
\end{aligned}
$$




\section{TEST LOG}

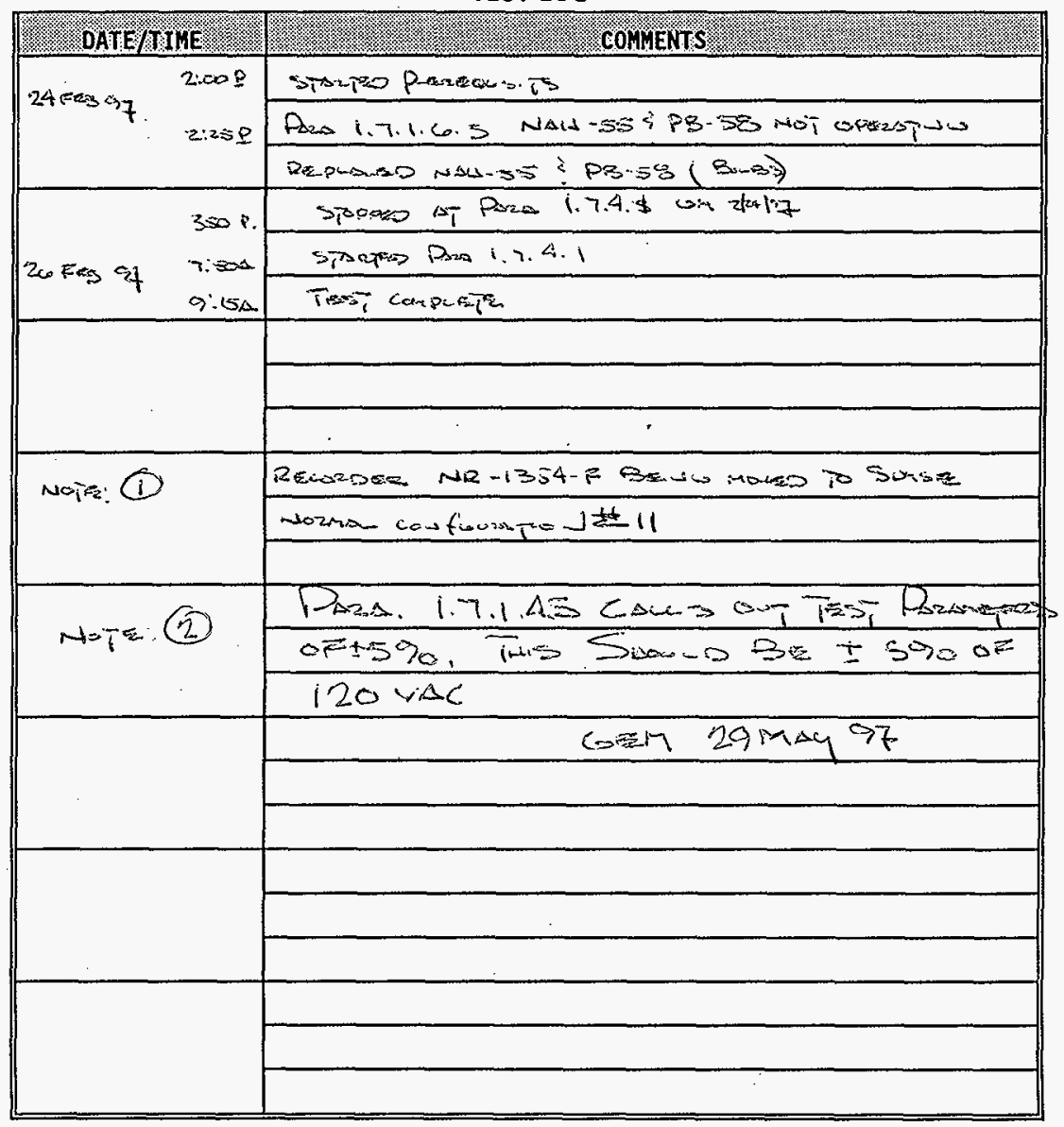


TEST LOG

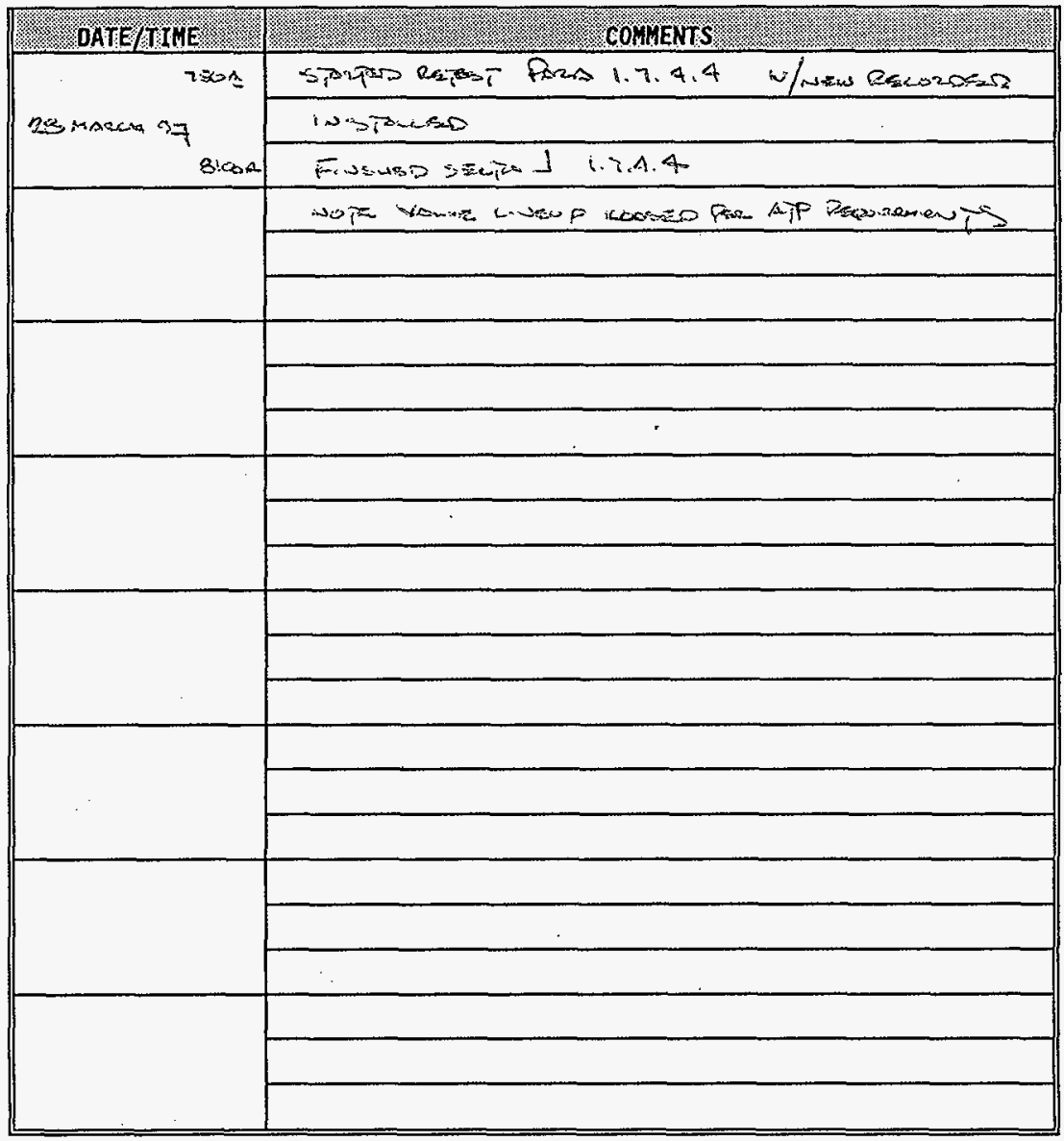


APPENDIX K

PAGE K- $i$
HNF - SD-WM-ATR-191

Rev. 0

Page 337

\section{APPENDIX K}


TEST EXECUTION SHEET

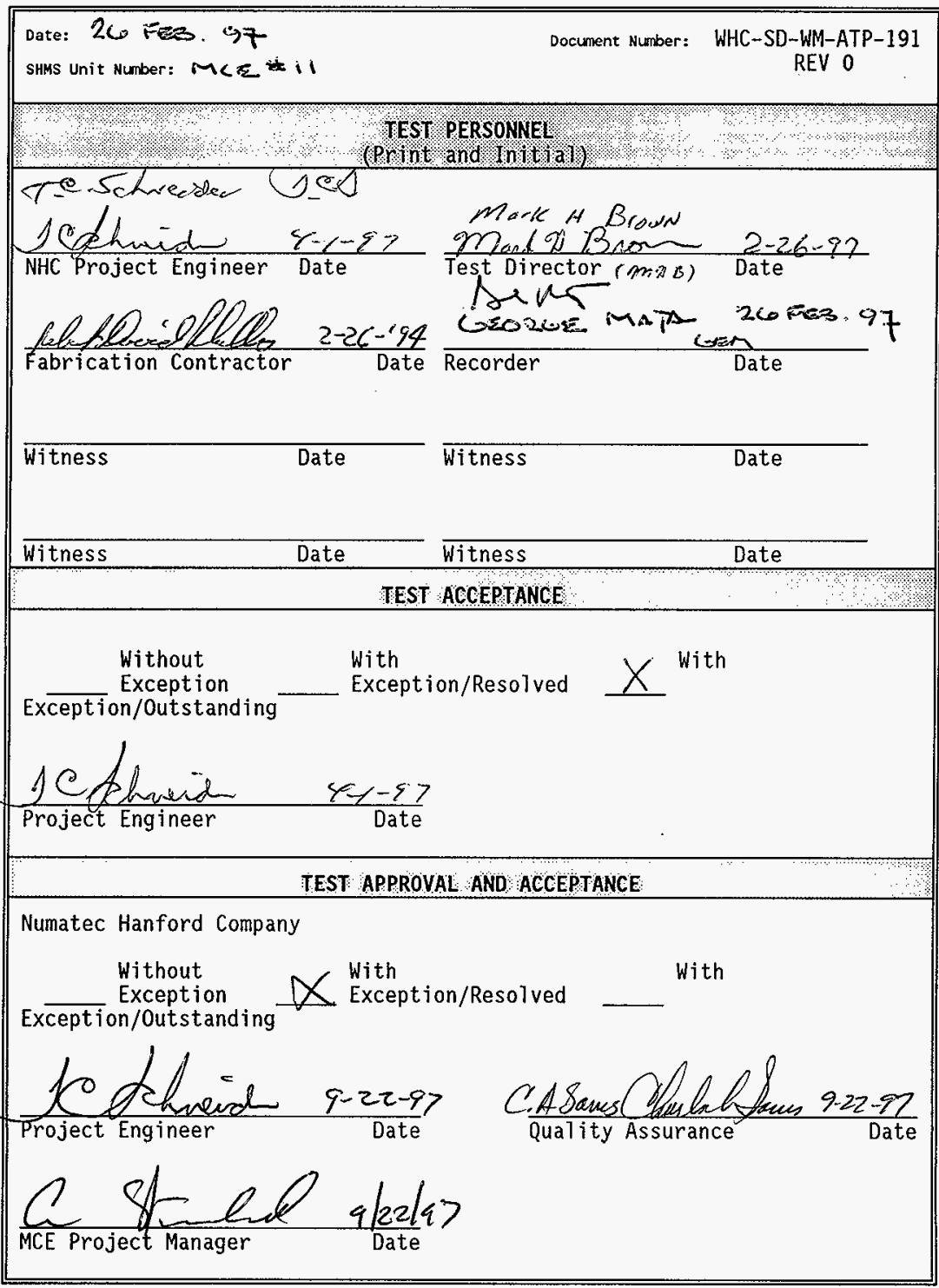


APPENDIX K

PAGE $K-3$

yon
fon

HNF-SD-WH-ATR-191

Rev. 0

page 339

SHMS Unit Number:

\section{PREREQUISITES AND INITIAL TEST CONDITIONS}

The following conditions shall exist at the start of the acceptance testing. Initial and date to verify that each of the following items have been accomplished.

soe Ten: Excosetios:s

wex Systems being tested have been inspected for workmanship and for compliance with design.

Gen $2 / z u / 37$ Continuity and megger tests have been performed on portions of the electrical and instrument systems being tested, as required.

$\sin ^{2}$ kit Leak tests on the pneumatic systems have been performed.

ses $2 / 20 / 7$ The following circuit breakers and fuses are installed per the specified size and are open

Gen zluokifal] test instruments have a currently valid calibration stamp attached that indicates a calibration traceable to the National Institute of Standards and Technology.

6em $2 / 20 / 4$ The following process and control instrument systems have been initially configured and aligned for proper operation.

FIT-*57, FSL-*57, NIT-*54, NIT-*55, (NR-*54), PDIT-*60, TIC $-* 50$, TIC $-* 56$, TIS $-* 62$, YYC $-* 01$ NOTE: NR $-* 54$ is not used in the ANALYTICAL configuration.

Ger z/u/s7 Personne] responsible for directing and witnessing the performance of the tests described in this ATP have read and understand appropriate certified vendor information (CVI) pertaining to the operation of the equipment to be tested.

$\operatorname{con} z / w / 97$ CLOSE all system manual valves with the exception of the following:

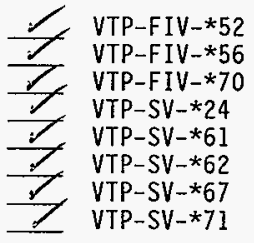

Senz/26/if The sample pump VTP-P-*50, connected to the cabinet sample out port, but vented to atmosphere for testing. 
Gen $4 / 2 u / 2.7208 \mathrm{Vac} 1$ phase 15-20 ampere temporary power source has been connected, but not energized, to the appropriate TB1 terminals per H-14-100838.

Verify by signature and date that all prerequisites have been met.

$\frac{9 \text { Manh 2. Bnor }}{\text { Test Director Signature }} \frac{3-28-97}{\text { Date }}$


TEST DATA SHEET

\begin{tabular}{|c|c|c|c|c|}
\hline \multicolumn{3}{|c|}{ Date: $260 \% .97$} & \multicolumn{2}{|c|}{ SHMS Unit Number: Mcises 11} \\
\hline \multicolumn{3}{|c|}{$\begin{array}{l}\text { Titie of Test: } \\
\text { Electrical Systems }\end{array}$} & \multirow{2}{*}{\multicolumn{2}{|c|}{ 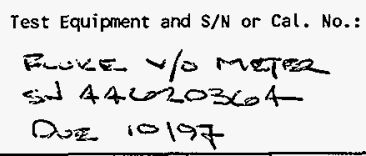 }} \\
\hline \multicolumn{3}{|c|}{ 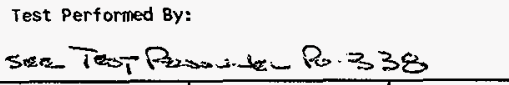 } & & \\
\hline $\begin{array}{l}\text { Procedure No. } \\
\text { Initial/Date }\end{array}$ & Item & Value & $\begin{array}{l}\text { Required } \\
\text { Condition } \\
\end{array}$ & $\langle A / R\rangle$ \\
\hline \multicolumn{5}{|c|}{ Section 1.7 .1 .1 verifies the system Mains Power, } \\
\hline $\begin{array}{l}1.7 .1 .1 .1 \\
\text { conen }\end{array}$ & Resistance & .10 & $<1$ ohm & $A$ \\
\hline $\begin{array}{l}1.7 .1 .1 .2 \\
\text { conen }\end{array}$ & $\begin{array}{l}\text { Temp. power } \\
208 \mathrm{Vac}\end{array}$ & $N / A$ & Energized & $\Delta$ \\
\hline $\begin{array}{l}1.7 .1 .1 .3 \\
\sin \end{array}$ & $\begin{array}{l}\text { Line Vac: } \\
\text { L1-L2 } \\
\text { L1-Gnd } \\
\text { L2-Gnd } \\
\mathrm{N}-\text { Gnd }\end{array}$ & $\frac{211.0}{\frac{121.1}{120.8}} \frac{0}{1}$ & $\begin{array}{l}\mathrm{L} 1-\mathrm{L} 2: 208 \\
\mathrm{Vac}+10 /-1 \% \\
\mathrm{~L} 1, \mathrm{~L} 2: 120 \\
\mathrm{Vac} \pm 5 \% \\
\mathrm{~N}: 0 \mathrm{Vac}\end{array}$ & $\Delta$ \\
\hline \multicolumn{5}{|c|}{ Section 1.7.1.2 verifies the enclosure HVAC system. } \\
\hline 1.7 .1 .2 .1 & AC Adjusted & $N / A$ & $N / A$ & $A$ \\
\hline 1.7 .1 .2 .2 & $\begin{array}{l}\text { CLOSE } \\
\mathrm{CB}-1 / 2\end{array}$ & $N / A$ & $N / A$ & $A-$ \\
\hline $\begin{array}{l}1.7 .1 .2 .3 \\
\end{array}$ & Heater ON & OK & Heater ON & A \\
\hline $\begin{array}{l}1.7 .1 .2 .4 \\
\text { Sien } \\
\end{array}$ & AC Adjusted & $N / A$ & $N / A$ & $\Delta$ \\
\hline $\begin{array}{l}1.7 .1 .2 .5 \\
\sin \end{array}$ & $\begin{array}{l}\text { Heater ofF } \\
\text { Cooling ON }\end{array}$ & ok & $\begin{array}{l}\text { Heater OFF } \\
\text { Cooling ON }\end{array}$ & $\Delta$ \\
\hline $\begin{array}{l}1.7 .1 .2 .6 \\
\text { Lsen } \\
\end{array}$ & AC Adjusted & $N / A$ & $N / A$ & $\Lambda$ \\
\hline $\begin{array}{l}1.7 .1 .2 .7 \\
600 \\
\end{array}$ & $\begin{array}{l}\text { OPEN } \\
\mathrm{CB}-1 / 2 \\
\end{array}$ & $\mathrm{~N} / \mathrm{A}$ & $N / A$ & $\Delta$ \\
\hline \multicolumn{5}{|c|}{ Section 1.7 .1 .3 verifies the sample pump is proper $7 y$ connected. } \\
\hline $\begin{array}{l}1.7 .1 .3 .1 \\
6=2\end{array}$ & $\begin{array}{l}\text { CLOSE } \\
\text { CB-3/4 }\end{array}$ & $\mathrm{N} / \mathrm{A}$ & $N / A$ & $\triangle$ \\
\hline $\begin{array}{l}1.7 .1 .3 .2 \\
6 E^{A}\end{array}$ & $\begin{array}{l}\text { VTP-P }-* 50 \\
\text { ON }\end{array}$ & ok & $\begin{array}{l}\text { VTP-P-*50 } \\
\text { ON }\end{array}$ & $\Delta$ \\
\hline
\end{tabular}


APPENDIX $K$

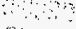

TEST DATA SHEET

\begin{tabular}{|c|c|c|c|c|c|}
\hline \multicolumn{3}{|c|}{ 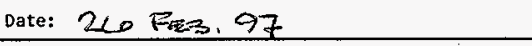 } & \multicolumn{3}{|c|}{ SHMS Unit Number: McE 11} \\
\hline \multicolumn{3}{|c|}{$\begin{array}{l}\text { Title of Test: } \\
\text { Electrical Systems }\end{array}$} & \multirow{2}{*}{\multicolumn{3}{|c|}{ 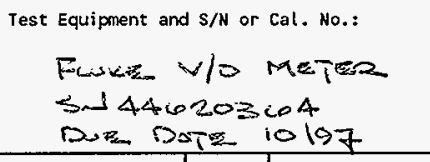 }} \\
\hline \multicolumn{3}{|c|}{ 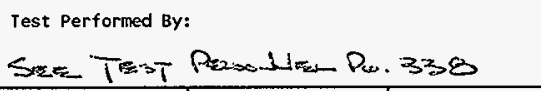 } & & & \\
\hline $\begin{array}{c}\text { Procedure No. } \\
\text { Initial/Date }\end{array}$ & Item & Value & $\begin{array}{l}\text { Required } \\
\text { Condition } \\
\end{array}$ & $(A / R)$ & comment \\
\hline $\begin{array}{l}1.7 .1 .3 .3 \\
\operatorname{ses}\end{array}$ & $\begin{array}{l}\text { OPEN } \\
\text { CB-3/4 }\end{array}$ & N/A & N/A & $\Delta$ & \\
\hline \multicolumn{6}{|c|}{ Section 1.7.1.4 verifies the enclosure lighting and GFCI receptacles. } \\
\hline $\begin{array}{l}1.7 .1 .4 .1 \\
\end{array}$ & $\begin{array}{l}\text { CLOSE } \\
\text { CB-6 }\end{array}$ & N/A & N/A & $\Delta$ & \\
\hline isim & $\begin{array}{l}\text { ENCLOSURE } \\
\text { LIGHT }\end{array}$ & N/A & N/A & $\Delta$ & \\
\hline $\begin{array}{l}1.7 .1 .4 .3 \\
\operatorname{sen}\end{array}$ & LIGHT ON & OK & LIGHT ON & $\Lambda$ & \\
\hline $\begin{array}{l}1.7 .1 .4 .4 \\
\text { gen }\end{array}$ & $\begin{array}{l}\text { GFCI RECP. } \\
\text { IA. L-N } \\
1 B . L-G N D \\
2 A . L-N \\
2 B . L-G N D\end{array}$ & $\frac{\frac{120.6}{120.7}}{\frac{120.9}{120.9}}$ & $\begin{array}{l}\text { 1A. } 120 \mathrm{Vac} \\
1 B .120 \mathrm{Vac} \\
2 \mathrm{~A} .120 \mathrm{VaC} \\
2 B .120 \mathrm{Vac} \\
\text { ALL } \pm 5 \%\end{array}$ & $\Delta$ & \\
\hline ion & $\begin{array}{l}\text { GFCI TEST } \\
1 A . L-N \\
1 B . \text { L-GND } \\
2 A . \text { L-N } \\
\text { 2B. L-GND } \\
\end{array}$ & $\frac{0}{\frac{2}{.3}} \frac{1}{1.1}$ & $\begin{array}{l}1 A .0 \mathrm{Vac} \\
1 \mathrm{~B} .0 \mathrm{Vac} \\
2 \mathrm{~A} .0 \mathrm{Vac} \\
2 B .0 \mathrm{Vac} \\
\text { ALL } \pm 5 \% \\
\end{array}$ & A & \\
\hline${ }_{6}^{7.1 .4 .6}$ & $\begin{array}{l}\text { ENCLOSURE } \\
\text { LIGHT OFF }\end{array}$ & N/A & N/A & 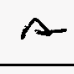 & \\
\hline $\begin{array}{l}1.7 .1 .4 .7 \\
\end{array}$ & $\begin{array}{l}\text { OPEN } \\
\text { CB-6 } \\
\end{array}$ & $\mathrm{N} / \mathrm{A}$ & N/A & A & \\
\hline \multicolumn{6}{|c|}{ Section 1.7.1.5 verifies the instrument power source isolation transformer. } \\
\hline 1.7 .1 .5 .1 & $\begin{array}{l}\text { CLOSE } \\
\text { CB-7 }\end{array}$ & N/A & N/A & $\Delta$ & \\
\hline $\begin{array}{l}1.7 .1 .5 .2 \\
\text { cons. }\end{array}$ & $\begin{array}{l}\text { XFRM Vac } \\
\text { PRI. L-N } \\
\text { SEC. L-N } \\
\text { SEC. N-GND } \\
\end{array}$ & $\frac{\frac{120.5}{124.0}}{.002}$ & $\begin{array}{l}\text { PRI. \& SEC. } \\
\text { L-N } 120 \pm 5 \% \\
\text { SEC. } \\
\text { N-GND O VaC }\end{array}$ & $\Lambda$ & \\
\hline ogen & CLOSE FU-1 & $N / A$ & N/A & A & 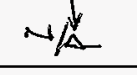 \\
\hline
\end{tabular}


APPENDIX $K$

PAGE KO

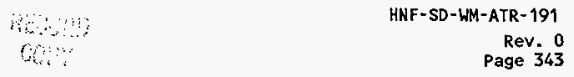

TEST DATA SHEET

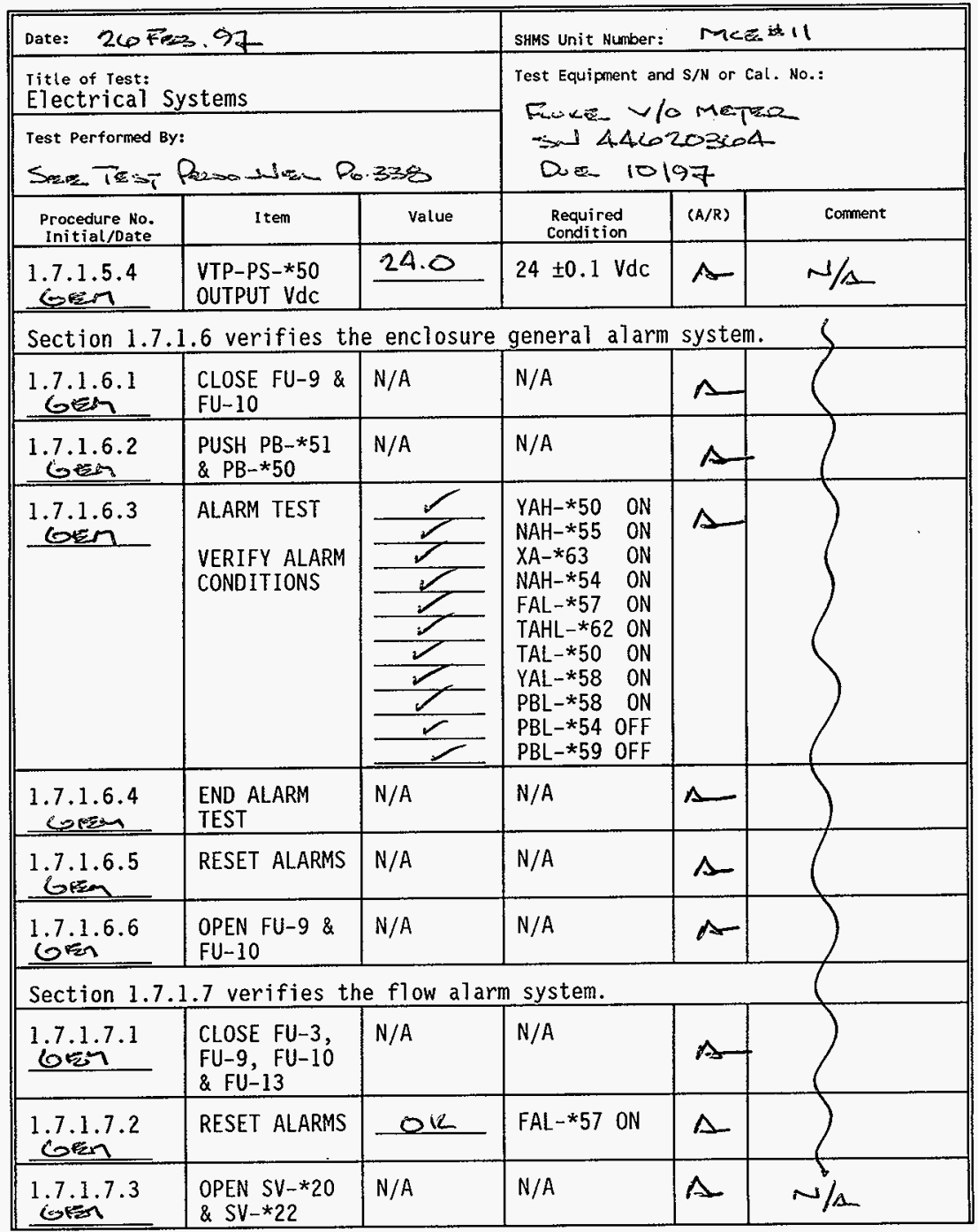

oren sue $2126 / 97$ 
TEST DATA SHEET

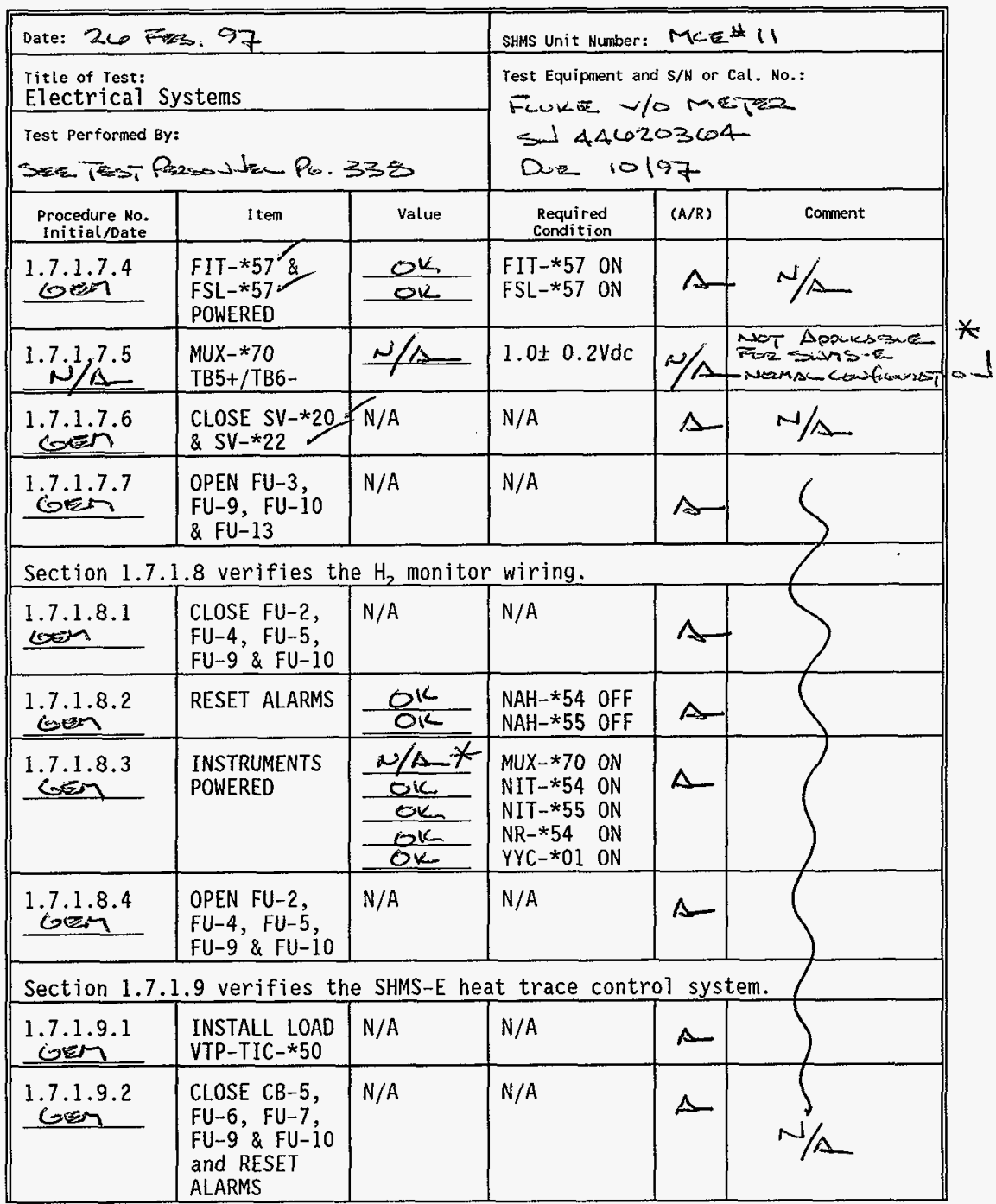

$\operatorname{Erm} 2 / 2 \omega 197$ 
TEST DATA SHEET

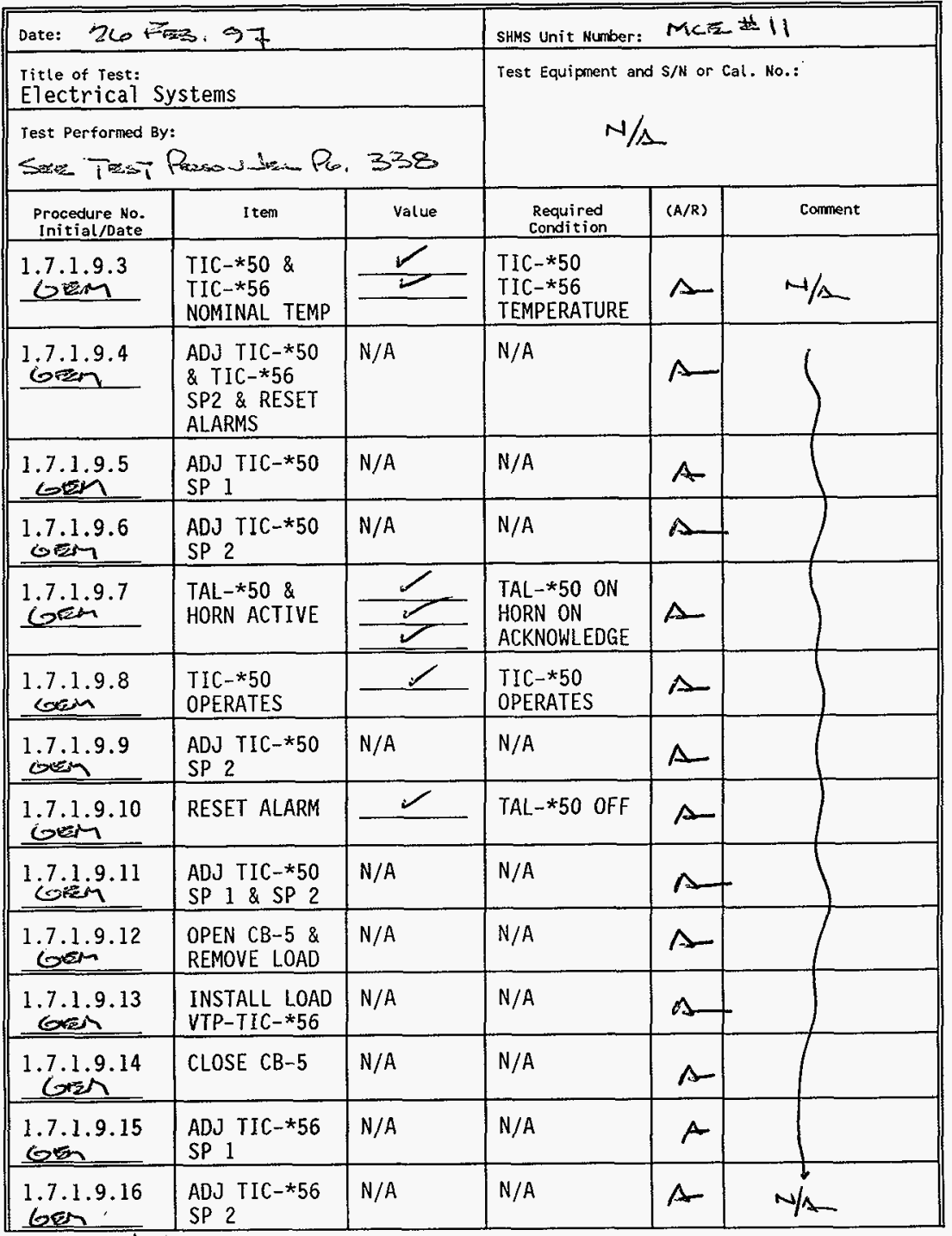


APPENDIX K

HNF-SD-WM-ATR-191

PAGE $K-10$

Rev. 0
Page 346

TEST DATA SHEET

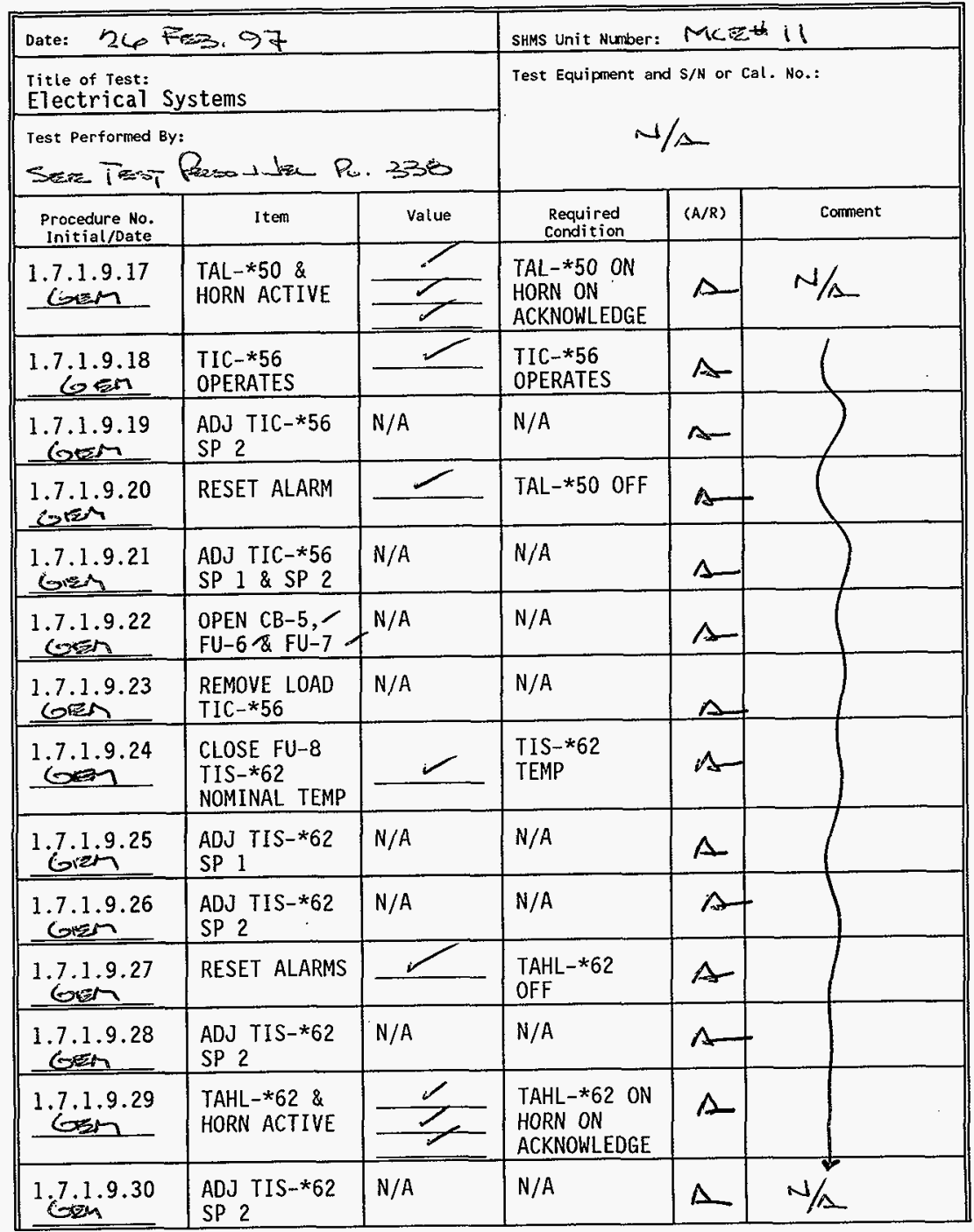

1 A. nl y,olsz 
APPENDIX K

\section{TEST DATA SHEET}

\begin{tabular}{|c|c|c|c|c|c|}
\hline \multicolumn{3}{|c|}{ Date: 260 ans. 97} & \multicolumn{3}{|c|}{ SHMS Unit Number: MK: 11} \\
\hline \multicolumn{3}{|c|}{$\begin{array}{l}\text { Title of Test: } \\
\text { Electrical Systems }\end{array}$} & \multirow{2}{*}{\multicolumn{3}{|c|}{$\begin{array}{l}\text { Test Equipment and s/N or cal. No.: } \\
\text { Fuke v/o Misiee } \\
\text { Sud } 440.20364 \\
\text { Dur } 10197\end{array}$}} \\
\hline \multicolumn{3}{|c|}{ 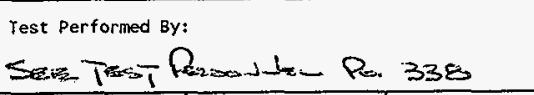 } & & & \\
\hline $\begin{array}{l}\text { Procedure No. } \\
\text { Initial/Date }\end{array}$ & Item & Value & $\begin{array}{l}\text { Required } \\
\text { Condition }\end{array}$ & $(A / R)$ & omment \\
\hline $\begin{array}{l}1.7 .1 .9 .31 \\
\end{array}$ & RESET ALARMS & 1 & $\begin{array}{l}\text { TAHL }-* 62 \\
\text { OFF }\end{array}$ & $\Delta$ & \\
\hline $\begin{array}{c}1.7 .9 .32 \\
0.7\end{array}$ & $\begin{array}{l}\text { ADJ TIS-*62 } \\
\text { SP } 1\end{array}$ & N/A & $N / A$ & $\Delta$ & \\
\hline$\frac{1.1 .9 .33}{6 x=7}$ & $\begin{array}{l}\text { TAHL-*62 \& } \\
\text { HORN ACTIVE }\end{array}$ & 2 & $\begin{array}{l}\text { TAHL-*62 ON } \\
\text { HORN ON } \\
\text { ACKNOWLEDGE }\end{array}$ & $\Delta$ & \\
\hline i.7.1.9.34 & $\begin{array}{l}\text { ADJ TIS }-* 62 \\
\text { SP } 1 \& \text { SP } 2\end{array}$ & N/A & N/A & $A$ & \\
\hline 1.7 .1 .9 .35 & $\begin{array}{l}\text { OPEN FU-8, } \\
\text { FU-9 \& FU-10 }\end{array}$ & N/A & N/A & A & \\
\hline \multicolumn{6}{|c|}{ Section 1.7.1.10 verifies the differential pressure transmitter system. } \\
\hline $\begin{array}{l}1.7 .1 .10 .1 \\
61\end{array}$ & $\begin{array}{l}\text { REMOVE PLUGS } \\
\text { BAL PDIT } * 60 \\
\end{array}$ & N/A & N/A & A- & \\
\hline $\begin{array}{l}1.7 .1 .10 .2 \\
\text { cos }\end{array}$ & $\begin{array}{l}\text { CLOSE FU-14 } \\
\text { REC PDIT-*60 } \\
\text { PRESSURE } \\
\end{array}$ & .1 & $\begin{array}{l}\text { PDIT }-* 60 \\
\text { PRESSURE } \\
0 \pm 1 " \mathrm{H}_{2} \mathrm{O} \\
\end{array}$ & $\Delta$ & \\
\hline $\mathrm{N}^{1.7}$ & $\begin{array}{l}\text { MUX }-* 70 \text { Vdc } \\
\text { TB7+ \& TB8- }\end{array}$ & $N / A$ & $\begin{array}{l}\text { MUX-*70 Vdc } \\
2.2 \pm 0.1\end{array}$ & $\mathrm{~N} / \mathrm{s}$ & 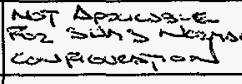 \\
\hline 1.7.1.10.4 & $\begin{array}{l}\text { REPLACE } \\
\text { PLUGS ON } \\
\text { PDIT-*60 } \\
\end{array}$ & N/A & N/A & $\Delta$ & \\
\hline $\begin{array}{l}1.7 .1 .10 .5 \\
\end{array}$ & OPEN FU-14 & N/A & N/A & A & \\
\hline \multicolumn{6}{|c|}{ Section 1.7.1.11 verifies the gas chromatograph power. } \\
\hline 1.7 .1 .11 .1 & CLOSE FU-11 & N/A & N/A & $\mathbb{A}$ & \\
\hline $\begin{array}{l}1.7 .1 .11 .2 \\
\text { Gorm }\end{array}$ & $\begin{array}{l}\text { POWER } \\
\text { MON-* } 60 \\
\end{array}$ & 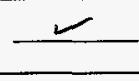 & $\begin{array}{l}\text { MON-**60 } \\
\text { POWERED } \\
\end{array}$ & $\Delta$ & $\begin{array}{l}\text { MenFirg aT } \\
\text { PS-COO }\end{array}$ \\
\hline $\mathrm{Gen}^{1.7 .1 .11 .3}$ & OPEN FU-11 & N/A & N/A & $\Lambda$ & $N / \Delta$ \\
\hline
\end{tabular}


TEST DATA SHEET

\begin{tabular}{|c|c|c|c|c|c|}
\hline \multicolumn{3}{|c|}{ Date: $260 \mathrm{Fez} .97$} & \multicolumn{3}{|c|}{ SHMS Unit Number: MCE $\$ 11$} \\
\hline \multicolumn{3}{|c|}{$\begin{array}{l}\text { Title of rest: } \\
\text { Electrical Systems }\end{array}$} & \multirow{2}{*}{\multicolumn{3}{|c|}{ 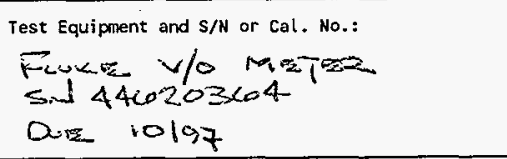 }} \\
\hline \multicolumn{3}{|c|}{ 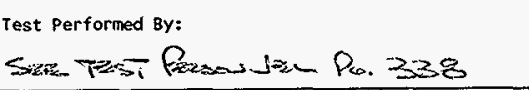 } & & & \\
\hline $\begin{array}{l}\text { Procedure No. } \\
\text { Initial/Date }\end{array}$ & Item & Value & $\begin{array}{l}\text { Required } \\
\text { Condition }\end{array}$ & $(A / R)$ & Comment \\
\hline \multicolumn{6}{|c|}{ Section 1.7.1.12 verifies the multi gas analyzer power. } \\
\hline $\begin{array}{l}1.7 .1 .12 .1 \\
6\end{array}$ & CLOSE FU-12 & N/A & $N / A$ & $\Delta$ & $\mu / \Delta$ \\
\hline $\mathrm{Gitan}^{12.2}$ & $\begin{array}{l}\text { POWER } \\
\text { NIT-*52 }\end{array}$ & $\checkmark$ & $\begin{array}{l}\text { NIT-*52 } \\
\text { POWERED }\end{array}$ & 1 & $\begin{array}{l}\text { VERTFARA AT } \\
P W=123.7 \\
\end{array}$ \\
\hline $\begin{array}{l}1.7 .1 .12 .3 \\
\end{array}$ & OPEN FU-12 & $\mathrm{N} / \mathrm{A}$ & $N / A$ & 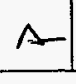 & 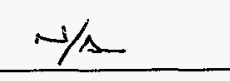 \\
\hline \multicolumn{6}{|c|}{ Section 1.7 .1 .13 verifies the personnel computer power } \\
\hline $\mathrm{G}^{1.1 .13 .1}$ & CLOSE CB- 6 & N/A & $\overline{N / A}$ & 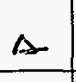 & 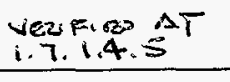 \\
\hline $\begin{array}{l}1.7 .1 .13 .2 \\
\end{array}$ & $\begin{array}{l}\text { POWER PC }-* 60 \\
\& P C-* 70\end{array}$ & 5 & $\begin{array}{ll}\mathrm{PC}-* 60 & \mathrm{ON} \\
\mathrm{PC}-* 70 & \mathrm{ON} \\
\end{array}$ & $\Delta$ & \\
\hline $\begin{array}{l}1.7 .1 .13 .3 \\
\text { getin }\end{array}$ & $\begin{array}{l}\text { TURN INST. } \\
\text { OFF \& } \\
\text { OPEN CB-6 }\end{array}$ & $\frac{1}{2}$ & $\begin{array}{ll}P C-\star 60 & 0 F F \\
P C-* 70 & 0 F F\end{array}$ & 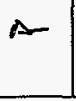 & $\downarrow$ \\
\hline
\end{tabular}

oren suc 2/261i7 
APPENDIX $K$

PAGE $K-/ 3$
HNF-SD-WM-ATR-191

Rev. 0

Page 349

TEST DATA SHEET

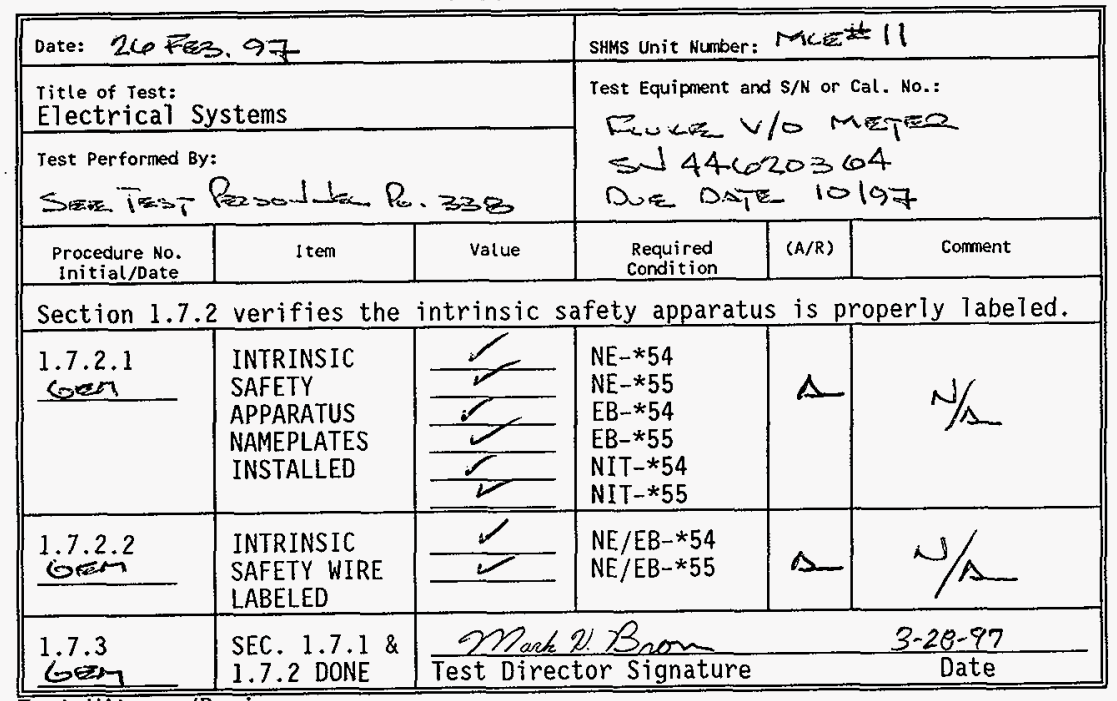

\begin{tabular}{l|l|l} 
ser & 1.7 .3 & SEC. \\
sum & beg & 1.7 .2 \\
zest Witness/Review:
\end{tabular}

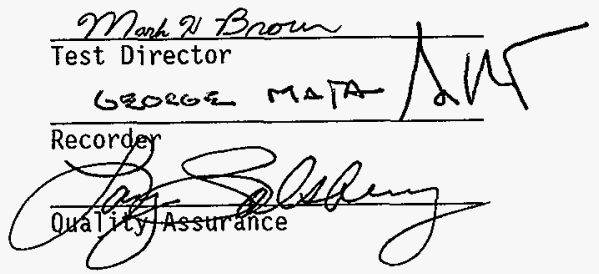

$\frac{3-28-97}{\text { Date }}$

28 maser 97

Date

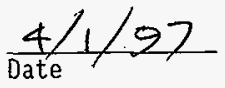


APPENDIX K

PAGE $K-/ 4$

$$
\begin{array}{r}
\text { HNF-SD-WM-ATR- } 191 \\
\text { ReV. } 0 \\
\text { Page } 350
\end{array}
$$

TEST DATA SHEET

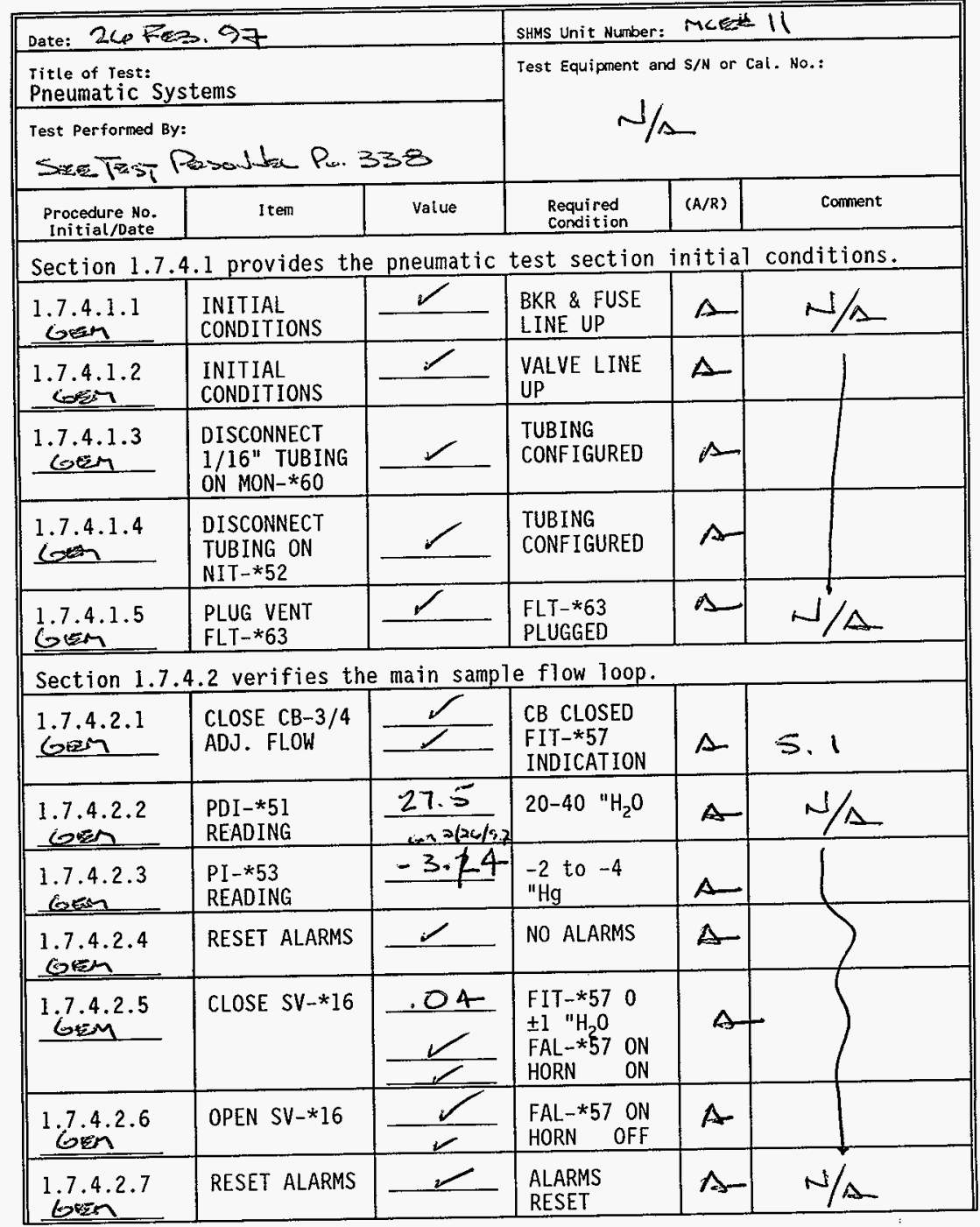

$\operatorname{Gen} \Delta_{2}=2 / 26 / 57$ 


\section{TEST DATA SHEET}

\begin{tabular}{|c|c|c|c|c|c|}
\hline \multicolumn{3}{|c|}{ Date: 26 Fs3.97 } & SHMS Unit Number: & \multicolumn{2}{|c|}{ McE } \\
\hline \multicolumn{3}{|c|}{$\begin{array}{l}\text { Title of Test: } \\
\text { Pneumatic Systems }\end{array}$} & \multirow{2}{*}{\multicolumn{3}{|c|}{ Jest Equipment and $S / \mathrm{N}$ or Cal. No.: }} \\
\hline \multicolumn{3}{|c|}{ 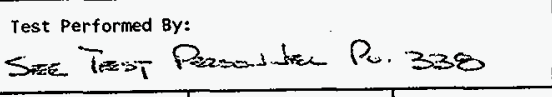 } & & & \\
\hline $\begin{array}{l}\text { Procedure No. } \\
\text { Initial/Date }\end{array}$ & Item & value & $\begin{array}{l}\text { Required } \\
\text { condition } \\
\end{array}$ & $(A / R)$ & comment \\
\hline$\frac{1.7 .4 .2 .8}{6 \sin ^{2}}$ & CLOSE SV-*25 & $\frac{\frac{.04}{2}}{\frac{2}{2}}$ & $\begin{array}{l}\mathrm{FIT}-* 57 \\
\pm 1 \quad \mathrm{H}_{2} \mathrm{O} \\
\text { FAL } * 57 \text { ON } \\
\text { HORN } \\
\text { XA-*63 ON } \\
\text { YY-*71 } \\
\text { TB } 3-4 \text { ON } \\
\end{array}$ & $\Delta$ & 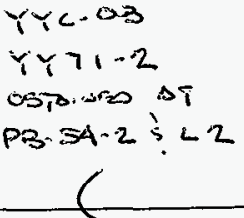 \\
\hline 1.7 .4 .2 .9 & OPEN SV-*25 & $\frac{2}{121.1}$ & $\begin{array}{l}\text { FAL-*57 ON } \\
\text { HORN OFF } \\
\text { XA-*63 OFF } \\
\text { YY-*71 } \\
\text { TB } 3-4 \text { 120V } \\
\end{array}$ & $\Delta$ & $\downarrow$ \\
\hline $\begin{array}{l}1.7 .4 .2 .10 \\
\text { Gen }\end{array}$ & RESET ALARMS & 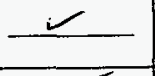 & $\begin{array}{l}\text { ALARMS } \\
\text { RESET }\end{array}$ & $\Delta$ & $N / \Omega$ \\
\hline & $\begin{array}{l}\text { OPEN SV-*54 } \\
\& \text { SV-*66 } \\
\text { READ PRESS. } \\
\text { PDIT-*60 }\end{array}$ & $\frac{7}{-40.19}$ & $\begin{array}{l}\text { VALVES OPEN } \\
-27 \text { to }-82 \\
" \mathrm{H}_{2} \mathrm{O}\end{array}$ & $\triangle$ & \\
\hline$\frac{1.7 .4 .2 .12}{6 \text { ten }}$ & $\begin{array}{l}\text { CLOSE SV }-* 54 \\
\text { OPEN SV }-* 63 \\
\text { READ PRESS. } \\
\text { PDIT-*60 } \\
\end{array}$ & 0.1 & $\begin{array}{l}\text { VALVES } \\
\text { OPERATED } \\
0 \pm 1 \quad \mathrm{H}_{2} \mathrm{O}\end{array}$ & $\Delta$ & \\
\hline 1.7 .4 .2 .13 & $\begin{array}{l}\text { CLOSE SV }-* 63- \\
\text { OPEN SV }-* 55 \\
\text { READ PRESS. } \\
\text { PDIT }-* 60 \\
\end{array}$ & $-42 \cdot 14$ & $\begin{array}{l}\text { VALVES } \\
\text { OPERATED } \\
-27 \text { to }-82 \\
\mathrm{H}_{2} \mathrm{O} \\
\end{array}$ & $A$ & \\
\hline$\underline{1.7 .4 .2 .14}$ & $\begin{array}{l}\text { CLOSE SV }-* 55 \text { क } \\
\text { OPEN SV } * 64 \\
\text { READ PRESS. } \\
\text { PDIT-*60 } \\
\end{array}$ & .12 & $\begin{array}{l}\text { VALVES } \\
\text { OPERATED } \\
0 \pm 1 " \mathrm{H}_{2} \mathrm{O}\end{array}$ & $n$ & \\
\hline $\begin{array}{l}1.7 .4 .2 .15 \\
687^{2} \\
\end{array}$ & $\begin{array}{l}\text { CLOSE SV-*64 } \\
\& \text { SV-*66 }\end{array}$ & N/A & $N / A$ & $\Delta$ & \\
\hline Section 1.7 & 3 verifies $\mathrm{t}$ & grab & flow loop. & & \\
\hline $\begin{array}{l}1.7 .4 .3 .1 \\
\text { cosm }\end{array}$ & READ PDI $* 51$ & 27.5 & $20-40 \quad " \mathrm{H}_{2} \mathrm{O}$ & $\Delta$ & $N / A$ \\
\hline
\end{tabular}




\section{TEST DATA SHEET}

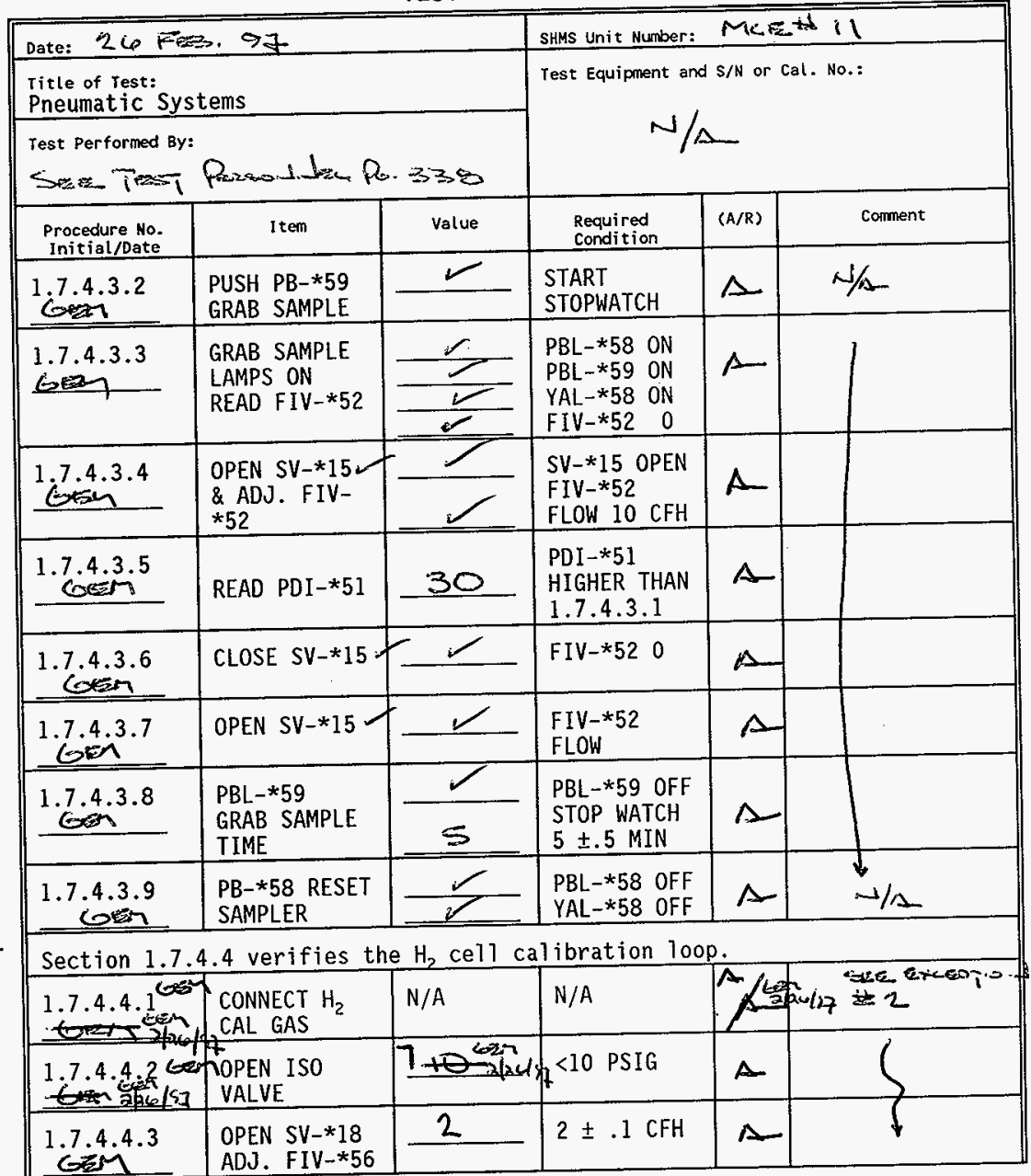

4 con $3 / 28197$ 
TEST DATA SHEET

\begin{tabular}{|c|c|c|c|c|c|}
\hline \multicolumn{3}{|c|}{ Date: 28 MArci 97} & \multicolumn{3}{|c|}{ SHMS Unit Number: MCE 11} \\
\hline \multicolumn{3}{|c|}{$\begin{array}{l}\text { Title of Test: } \\
\text { Pneumatic Systems }\end{array}$} & \multirow{2}{*}{\multicolumn{3}{|c|}{ 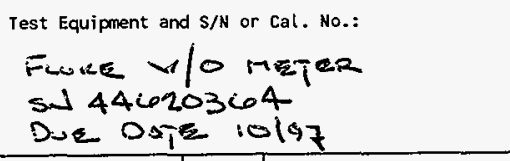 }} \\
\hline \multicolumn{3}{|c|}{$\begin{array}{l}\text { Test Performed By: } \\
\text { See Test Pezsondec Po. } 338\end{array}$} & & & \\
\hline $\begin{array}{l}\text { Procedure No. } \\
\text { Initial/Date }\end{array}$ & I tem & Value & $\begin{array}{l}\text { Required } \\
\text { Condition }\end{array}$ & $(A / R)$ & Comment \\
\hline $\begin{array}{l}1.4 .4 .4 \\
\text { Senta }\end{array}$ & $\begin{array}{l}\text { VERIFY } \\
\text { CONDITIONS }\end{array}$ & & $\begin{array}{l}\mathrm{NIT}-* 54 \\
>.625 \% \mathrm{H}_{2} \\
\text { NAH-*55 ON } \\
\text { NAH-*54 ON } \\
\text { PBL-*59 ON } \\
\text { PBL-*58 ON } \\
\text { YAL-*58 ON } \\
\text { FIV-*52 FLO } \\
\text { HORN ON } \\
\text { TB2-21/22 } \\
\text { OPEN }\end{array}$ & A & $\begin{array}{l}\text { Sae Exceftiol } \\
\pm 2 \\
(\end{array}$ \\
\hline $\begin{array}{l}1.7 .4 .4 .5 \\
-60 n \\
\end{array}$ & $\begin{array}{l}\text { ACKNOWLEDGE } \\
\text { HORN }\end{array}$ & $N / A$ & $N / A$ & $A$ & \\
\hline $\begin{array}{l}1.7 .4 .4 .6 \\
60 \\
\end{array}$ & $\begin{array}{l}\text { CLOSE SV }-* 18 \\
\text { VERIFY } \\
\text { CONDITIONS }\end{array}$ & 2 & $\begin{array}{l}\text { FIV-*56 0 } \\
\text { NIT-*54 } \\
<.625 \% \mathrm{H}_{2} \\
\text { NAH-*55 OFF } \\
\text { NAH-*54 ON } \\
\text { PBL-*58 ON } \\
\text { YAL-*58 ON } \\
\text { TB2-21/22 } \\
\text { CLOSED }\end{array}$ & $A$ & \\
\hline $\begin{array}{l}1.7 .4 .4 .7 \\
\text { - }\end{array}$ & $\begin{array}{l}\text { PUSH RESET } \\
\text { SAMPLER PB- } \\
* 58 \\
\end{array}$ & & $\begin{array}{l}\mathrm{PBL}-* 58 \text { OFF } \\
\text { YAL } * 58 \quad \mathrm{OFF}\end{array}$ & $\Delta$ & \\
\hline $\begin{array}{l}1.7 .4 .4 .8 \\
6.0 \\
\end{array}$ & RESET ALARM & & $\mathrm{NAH}-* 54 \mathrm{OFF}$ & $\Delta$ & \\
\hline $\begin{array}{r}1.7 .4 .4 .9 \\
\end{array}$ & $\begin{array}{l}\text { OPEN SV } * 19 \\
\text { ADJ. FIV-*56 }\end{array}$ & 2 & $2 \pm .1 \mathrm{CFH}$ & $A$ & 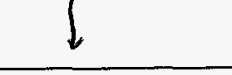 \\
\hline
\end{tabular}


TEST DATA SHEET

\begin{tabular}{|c|c|c|c|c|c|}
\hline \multicolumn{3}{|c|}{ Date: 28 Macus 97} & \multicolumn{3}{|c|}{ SHMS Unit Number: MCE 11} \\
\hline \multicolumn{3}{|c|}{$\begin{array}{l}\text { Title of Test: } \\
\text { Pneumatic Systems }\end{array}$} & \multirow{2}{*}{\multicolumn{3}{|c|}{ 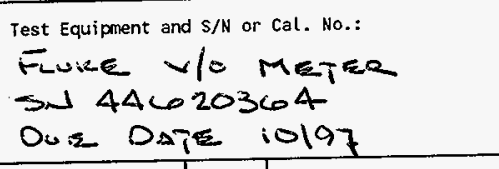 }} \\
\hline \multicolumn{3}{|c|}{ 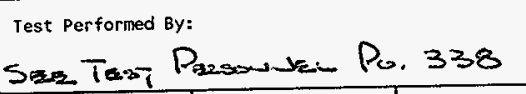 } & & & \\
\hline $\begin{array}{c}\text { Procedure No. } \\
\text { Initial/Date }\end{array}$ & I tem & value & $\begin{array}{l}\text { Required } \\
\text { Condition }\end{array}$ & $(A / R)$ & Comment \\
\hline 1.7 .4 .4 .10 & $\begin{array}{l}\text { VERIFY } \\
\text { CONDITIONS }\end{array}$ & 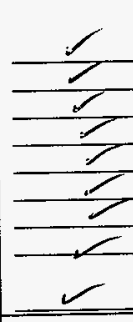 & $\begin{array}{l}\text { NIT-*55 } \\
>.625 \% \mathrm{H}_{2} \\
\text { NAH-*55 ON } \\
\text { NAH-*54 ON } \\
\text { PBL-*59 ON } \\
\text { PBL-*58 ON } \\
\text { YAL-*58 ON } \\
\text { FIV }-* 52 \text { FLO } \\
\text { HORN ON } \\
\text { TB2-21/22 } \\
\text { OPEN } \\
\end{array}$ & $A$ & CEFT' \\
\hline $\begin{array}{c}1.7 .4 .4 .11 \\
\text { Gein }\end{array}$ & $\begin{array}{l}\text { ACKNOWLEDGE } \\
\text { HORN }\end{array}$ & $N / A$ & $N / A$ & $\Lambda$ & \\
\hline $\begin{array}{l}1.7 .4 .4 .12 \\
12\end{array}$ & $\begin{array}{l}\text { CLOSE SV-* } 19 \\
\text { VERIFY } \\
\text { CONDITIONS }\end{array}$ & 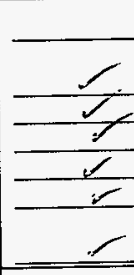 & $\begin{array}{l}\text { FIV-*56 } 0 \\
\text { NIT-*55 } \\
<.625 \% \mathrm{H}_{2} \\
\text { NAH-*55 OFF } \\
\text { NAH-*54 ON } \\
\text { PBL-*58 ON } \\
\text { YAL-*58 ON } \\
\text { TB2-21/22 } \\
\text { CLOSED } \\
\end{array}$ & $\Delta$ & \\
\hline $\begin{array}{c}1.7 .4 .4 .13 \\
6.4\end{array}$ & $\begin{array}{l}\text { PUSH RESET } \\
\text { SAMPLER PB- } \\
* 58 \\
\end{array}$ & $\frac{1}{2}$ & $\begin{array}{l}\text { PBL-*58 OFF } \\
\text { YAL-*58 OFF }\end{array}$ & $\Delta$ & \\
\hline $\begin{array}{l}1.7 .4 .4 .14 \\
\text { gogh }\end{array}$ & RESET ALARM & $r$ & $\mathrm{NAH}-* 54$ OFF & $\Delta$ & \\
\hline $\begin{array}{l}1.7 .4 .4 .15 \\
\text { gogn }\end{array}$ & $\begin{array}{l}\text { DISCONNECT } \\
\text { CAL. GAS }\end{array}$ & $N / A$ & $N / A$ & $\Delta$ & \\
\hline $\begin{array}{l}1.7 .4 .4 .16 \\
\text { gen }\end{array}$ & $\begin{array}{l}\text { PUSH PB-*54 } \\
\text { VERIFY } \\
\text { CONDITIONS }\end{array}$ & $\frac{\pi}{2}$ & $\begin{array}{l}\text { XA-*63 ON } \\
\text { PBL-*54 ON } \\
\text { TB2-23/24 } \\
\text { OPEN } \\
\end{array}$ & A & \\
\hline $\begin{array}{l}1.7 .4 .4 .17 \\
\text { oten }\end{array}$ & $\begin{array}{l}\text { PUSH PB- } * 54 \\
\text { VERIFY } \\
\text { CONDITIONS }\end{array}$ & $\frac{r}{2}$ & $\begin{array}{l}\text { XA-*63 OFF } \\
\text { PBL-*54 OFF } \\
\text { TB2-23/24 } \\
\text { CLOSED } \\
\end{array}$ & 4 & $\downarrow$ \\
\hline
\end{tabular}


APPENDIX K

PAGE K- 19

$$
\begin{array}{r}
H N F-S D-W M-A T R-191 \\
\text { ReV. } 0 \\
\text { Page } 355
\end{array}
$$

TEST DATA SHEET

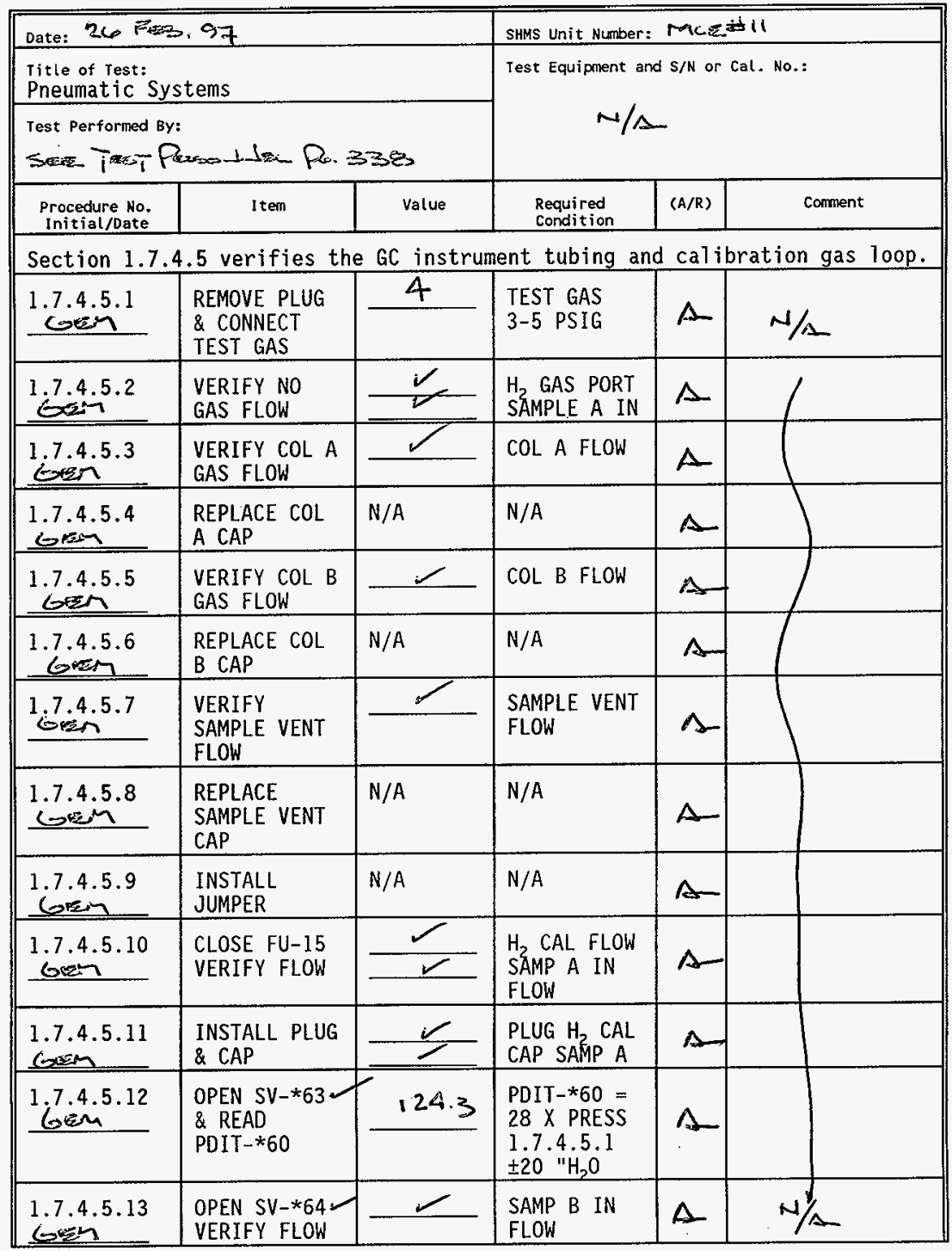


TEST DATA SHEET

\begin{tabular}{|c|c|c|c|c|c|}
\hline \multicolumn{3}{|c|}{ Date: 260 RzB.97 } & \multicolumn{3}{|c|}{ SHMS Unit Number: Mce it il } \\
\hline \multicolumn{3}{|c|}{$\begin{array}{l}\text { Title of Test: } \\
\text { Pneumatic Systems }\end{array}$} & \multicolumn{3}{|c|}{ Test Equipment and $\mathrm{S} / \mathrm{N}$ or Cal. No.: } \\
\hline \multicolumn{3}{|c|}{ 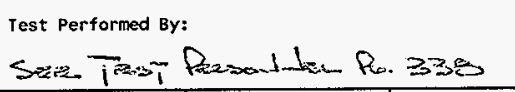 } & \multicolumn{3}{|c|}{$M / \Delta$} \\
\hline $\begin{array}{l}\text { Procedure No- } \\
\text { Initial/Date }\end{array}$ & Item & Value & $\begin{array}{l}\text { Required } \\
\text { Condition }\end{array}$ & $(\mathrm{A} / \mathrm{R})$ & comment \\
\hline $\begin{array}{l}1.7 .4 .5 .14 \\
0 \times 1\end{array}$ & $\begin{array}{l}\text { REMOVE TEST } \\
\text { GAS }\end{array}$ & $N / A$ & $N / A$ & $\Delta$ & $N / \Delta$ \\
\hline $\begin{array}{l}1.7 .4 .5 .15 \\
\text { Gizm } \\
\end{array}$ & $\begin{array}{l}\text { CLOSE SV-*63 } \\
\& \text { SV-*64 }\end{array}$ & $\mathrm{N} / \mathrm{A}$ & $N / A$ & 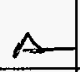 & 1 \\
\hline$\frac{1.7 .4 .5 .16}{(x+16}$ & $\begin{array}{l}\text { OPEN FU-15 } \\
\text { REMOVE } \\
\text { JUMPER }\end{array}$ & $N / A$ & $N / A$ & A & \\
\hline $\begin{array}{l}1.7 .4 .5 .17 \\
\text { Gen } \\
\end{array}$ & $\begin{array}{l}\text { INSTALL } \\
\text { SAMP A UNION }\end{array}$ & $N / A$ & $N / A$ & A & \\
\hline $\begin{array}{l}1.7 .4 .5 .18 \\
62^{2}\end{array}$ & $\begin{array}{l}\text { OPEN SV-*60 } \\
\text { VERIFY FLOW }\end{array}$ & & $\begin{array}{l}\text { FI-*60 } \\
\text { NO FLOW }\end{array}$ & $A$ & \\
\hline $\begin{array}{l}1.7 .4 .5 .19 \\
\text { Sein }\end{array}$ & $\begin{array}{l}\text { POWER SOV- } \\
* 60, \text { VERIFY } \\
\text { NO FLOW }\end{array}$ & & $\begin{array}{l}\text { FI-*60 } \\
\text { NO FLOW }\end{array}$ & $\boldsymbol{A}$ & \\
\hline $\begin{array}{l}1.7 .4 .5 .20 \\
\end{array}$ & $\begin{array}{l}\text { OPEN SV-*68 } \\
\text { ADJ SV }-\star^{*} 67 \\
\end{array}$ & 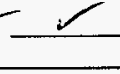 & $\begin{array}{l}\mathrm{FI}-* 60 \\
50 \mathrm{CCM} \\
\end{array}$ & $A$ & \\
\hline $\begin{array}{l}1.7 .4 .5 .21 \\
6+2 \times 1\end{array}$ & $\begin{array}{l}\text { DEENERGIZE } \\
\text { SOV } * * 60\end{array}$ & $r$ & $\begin{array}{l}\text { FI-*60 FLOW } \\
\text { DECREASES }\end{array}$ & A & \\
\hline $\begin{array}{l}1.7 .4 .5 .22 \\
+x=7 \\
\end{array}$ & $\begin{array}{l}\text { CLOSE SV-*60. } \\
\& \text { SV-*68 }\end{array}$ & $-N / A$ & $N / A$ & $\Delta$ & \\
\hline $\begin{array}{c}1.7 .4 .5 .23 \\
6 \mathrm{im} \\
\end{array}$ & $\begin{array}{l}\text { REMOVE } \\
\text { SAMP A UNION }\end{array}$ & $N / A$ & $N / A$ & 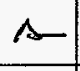 & \\
\hline $\begin{array}{c}1.7 .4 .5 .24 \\
\operatorname{sigm}\end{array}$ & $\begin{array}{l}\text { CONNECT TEST } \\
\text { GAS } N_{2} O \text { PORT }\end{array}$ & 5 & $\begin{array}{l}\text { TEST GAS } \\
3-5 \text { PSIG } \\
\end{array}$ & $\Lambda$ & \\
\hline 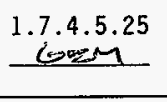 & $\begin{array}{l}\text { VERIFY NO } \\
\text { FLOW }\end{array}$ & $\frac{1}{2}$ & $\begin{array}{l}\text { SAMP B IN \& } \\
\text { FLT }-* 63 \\
\text { NO FLOW }\end{array}$ & $\Delta$ & W \\
\hline $\begin{array}{l}1.7 .4 .5 .26 \\
\text { Gen }\end{array}$ & $\begin{array}{l}\text { INSTALL } \\
\text { JUMPER }\end{array}$ & $N / A$ & $N / A$ & $\Lambda$ & Ser-cot? Fintist \\
\hline $\begin{array}{l}1.7 .4 .5 .27 \\
\text { Gizn }\end{array}$ & $\begin{array}{l}\text { CLOSE FU-15 } \\
\text { VERIFY FLOW }\end{array}$ & $\frac{L}{r}$ & $\begin{array}{l}\text { SAMP B IN } \\
\text { FLT-*63 } \\
\text { FLOW }\end{array}$ & $\Lambda$ & $\begin{array}{l}\text { Sompure B } \\
\text { Simow Fwow }\end{array}$ \\
\hline
\end{tabular}

goren sue z/200187 
TEST DATA SHEET

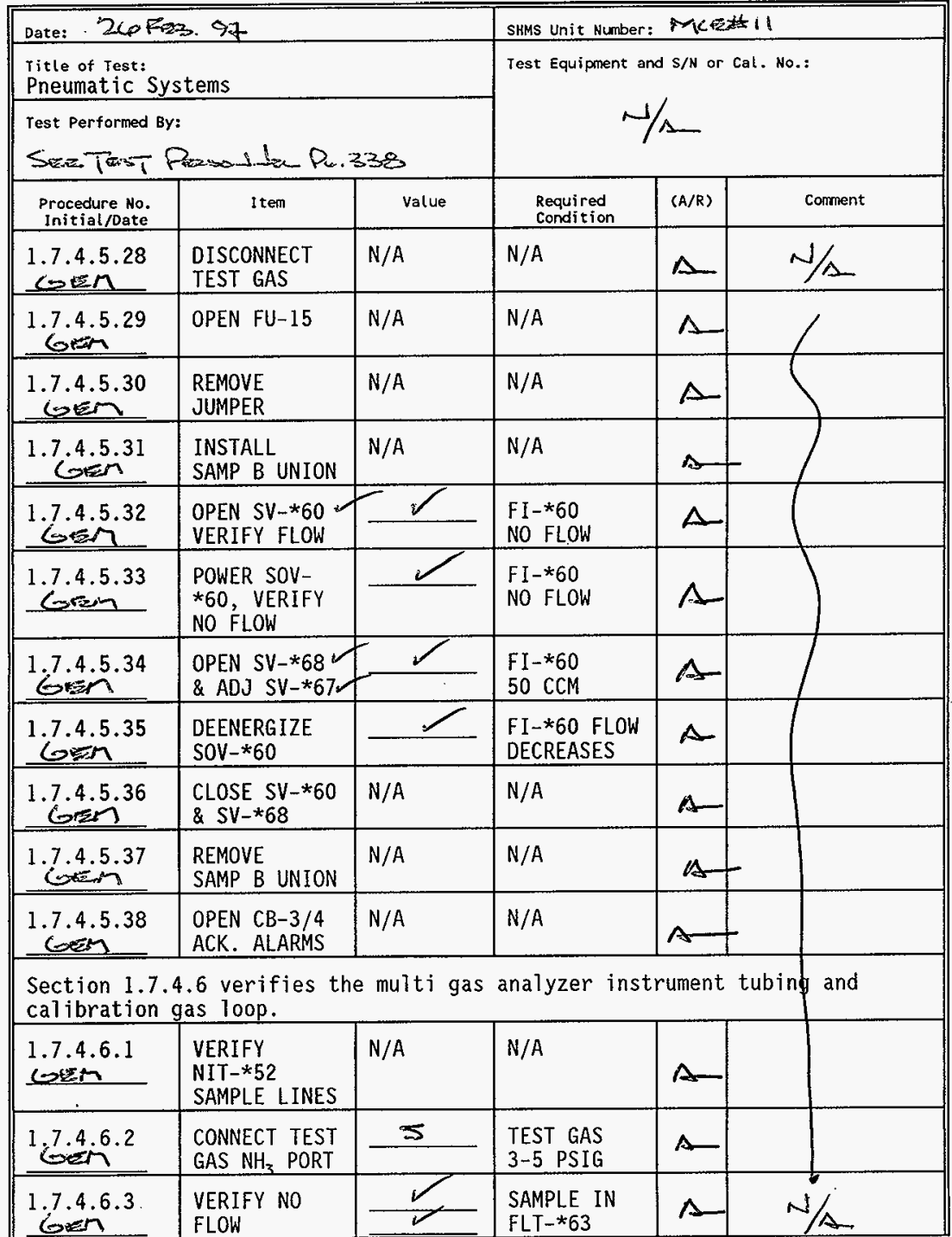


APPENDIX K

PAGE K- 22

$$
\begin{array}{r}
\text { HNF-SD-WM-ATR-191 } \\
\text { Rev. } 0 \\
\text { Page } 358
\end{array}
$$

TEST DATA SHEET

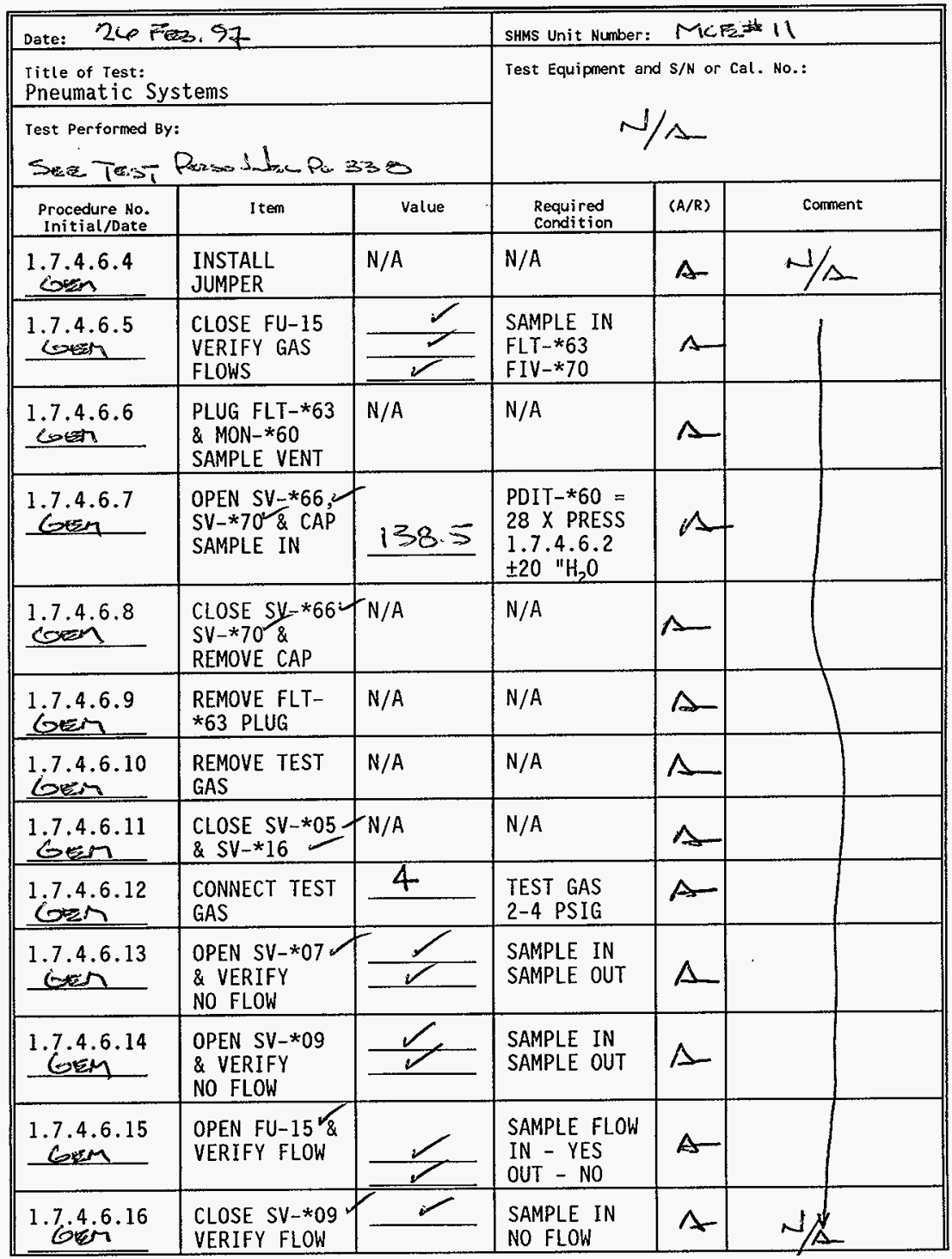


APPENDIX K

HNF-SD-WM-ATR- 191
ReV. 0
Page 359

TEST DATA SHEET

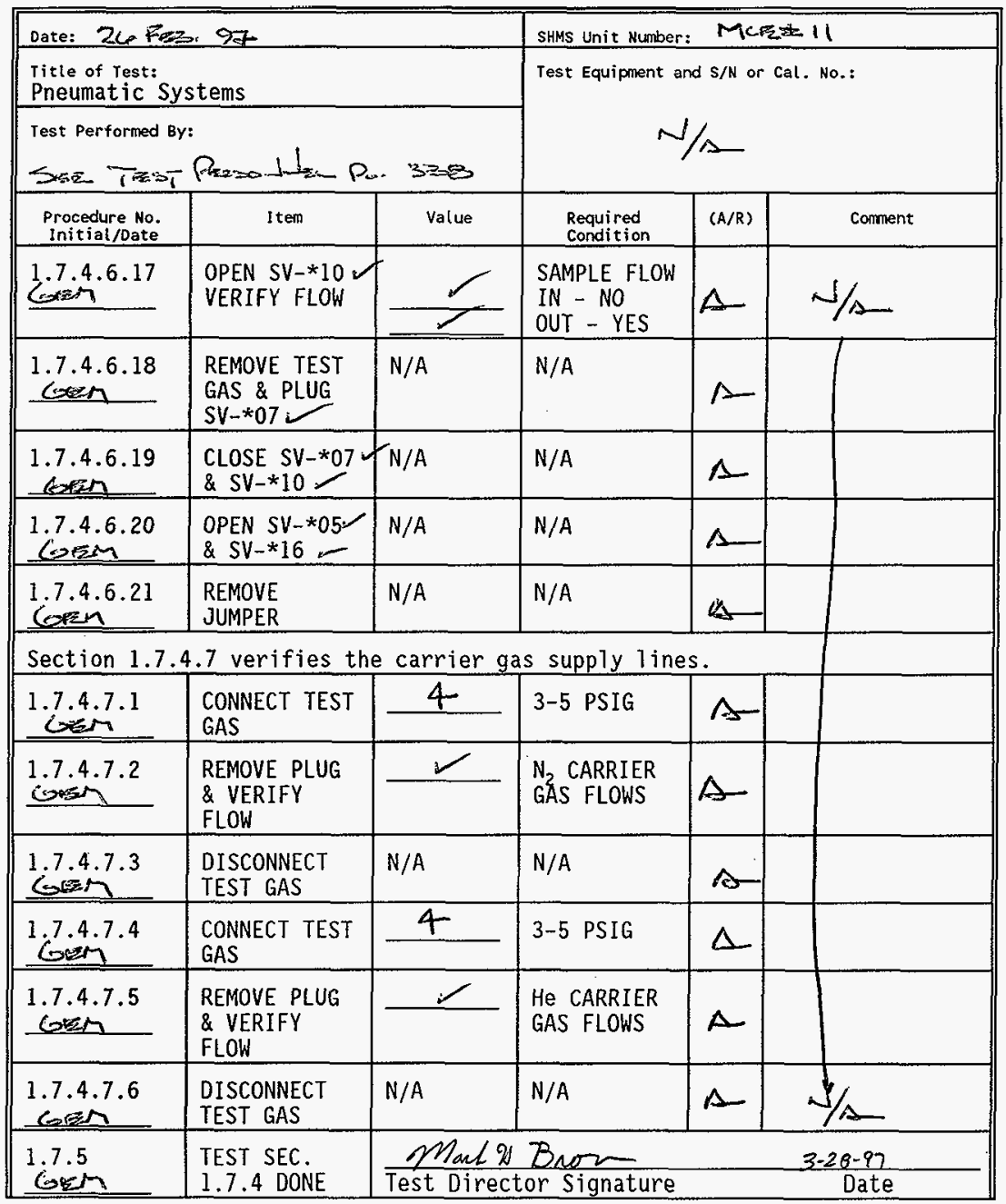

Gen An 2/26/ if 
TEST DATA SHEET

\begin{tabular}{|c|c|c|c|c|c|c|}
\hline \multicolumn{4}{|c|}{ Date: $\mathrm{N} / \mathrm{A}$} & \multicolumn{3}{|c|}{ SHMS Unit Number: $\pi / A$} \\
\hline \multicolumn{4}{|c|}{$\begin{array}{l}\text { Title of Test: } \\
\text { Pneumatic Systems }\end{array}$} & \multirow{2}{*}{\multicolumn{3}{|c|}{ Test Equipnent and $s / \mathrm{N}$ or Cal. No.: }} \\
\hline \multicolumn{4}{|c|}{$\begin{array}{l}\text { Test Performed By: } \\
\text { /S_ }\end{array}$} & & & \\
\hline & $\begin{array}{l}\text { cedure No. } \\
\text { tial/Date } \\
\text { tial }\end{array}$ & I tem & value & $\begin{array}{l}\text { Required } \\
\text { Condition }\end{array}$ & $(A / R)$ & Comment \\
\hline \multicolumn{2}{|c|}{$1.7 .6 / 1$} & \multicolumn{5}{|c|}{ 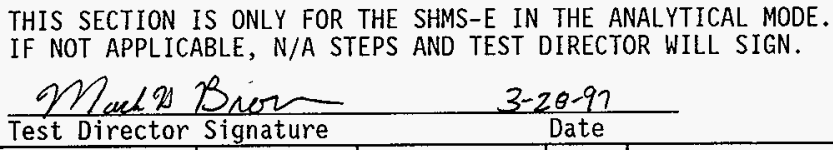 } \\
\hline 1.7 & .9 .1 .1 & $\begin{array}{l}\text { VERIFY BKR } \\
\text { LINE UP }\end{array}$ & N/A & N/A & $N / \Delta$ & $2 / 1$ \\
\hline 1.7. & .6 .1 .2 & $\begin{array}{l}\text { VERIFY VALVE } \\
\text { LINE UP }\end{array}$ & $N / A$ & $N / A$ & 1 & f \\
\hline 1.7. & 6.1 .3 & $\begin{array}{l}\text { VERIFY MON- } \\
* 60 \text { INSTALL }\end{array}$ & $N / A$ & N/A & & \\
\hline 1.7 & 6.1 .4 & $\begin{array}{l}\text { VERIFY NIT- } \\
* 52 \text { INSTALL }\end{array}$ & N/A & N/A & & \\
\hline 1.7 & 6.1 .5 & $\begin{array}{l}\text { CONNECT } \mathrm{H}_{2} \\
\text { CAL GAS }\end{array}$ & N/A & N/A & & \\
\hline 1.7. & .6 .1 .6 & $\begin{array}{l}\text { CONNECT } \mathrm{CH}_{4} \\
\text { CAL GAS }\end{array}$ & $N / A$ & $N / A$ & & \\
\hline 1.7. & .6 .1 .7 & $\begin{array}{l}\text { CONNECT } \mathrm{NH}_{3} \\
\text { CAL GAS }\end{array}$ & N/A & N/A & & \\
\hline \multicolumn{7}{|c|}{ Section 1.7.6.2 establishes the main and auxiliary loop flows. } \\
\hline 1.7 & 6.2 .1 & CLOSE FU-11 & $N / s$ & $\begin{array}{l}\text { MON-*60 } \\
\text { POWERED } \\
\end{array}$ & & \\
\hline 1.7 & 6.2 .2 & POWER PC-*60 & & $\begin{array}{l}\text { ESTABL ISH } \\
\text { SAMPLING } \\
\text { ROUTINE }\end{array}$ & & \\
\hline 1.7 & 6.2 .3 & CLOSE FU-12 & & $\begin{array}{l}\text { NIT-*52 } \\
\text { POWERED } \\
\end{array}$ & & \\
\hline 1.7 & 6.2 .4 & POWER PC $-* 70$ & & $\begin{array}{l}\text { PC-*70 } \\
\text { BOOTED UP }\end{array}$ & & \\
\hline 1.7 & 15.2 .5 & $\begin{array}{l}\text { CLOSE CB-3/4 } \\
\text { ADJUST FLOW }\end{array}$ & $3 / x$ & $\begin{array}{l}\mathrm{FIT}-* 57 \\
1 \pm .1 " \mathrm{H}_{2} \mathrm{O}\end{array}$ & $N / \Delta$ & $+5 / A$ \\
\hline
\end{tabular}


TEST DATA SHEET

\begin{tabular}{|c|c|c|c|c|c|c|}
\hline \multicolumn{4}{|c|}{ Date: $N / \Delta$} & \multicolumn{3}{|c|}{ SHMS Unit Number: $\mathrm{N} / \mathrm{A}$} \\
\hline \multicolumn{4}{|c|}{$\begin{array}{l}\text { Iitle of Test: } \\
\text { Pneumatic Systems }\end{array}$} & \multirow{2}{*}{\multicolumn{3}{|c|}{ Test Equipment and S/N or Cal. No.: }} \\
\hline \multicolumn{4}{|c|}{ Test Performed By: } & & & \\
\hline $\begin{array}{l}\text { Proced } \\
\text { Initia }\end{array}$ & $\begin{array}{l}\text { dure No. } \\
\text { ial/Date }\end{array}$ & I tem & value & $\begin{array}{l}\text { Required } \\
\text { Condition }\end{array}$ & $(A / R)$ & Corment \\
\hline $1.7 \%$ & $A^{2.6}$ & $\begin{array}{l}\text { OPEN SV-*09 } \\
\& \text { SV-*10 }\end{array}$ & $N / A$ & $N / A$ & $N / A$ & $M / \triangle$ \\
\hline 1.7 .6 & 2.7 & $\begin{array}{l}\text { OPEN SV-*60, } \\
63,65 \& 68\end{array}$ & $N / A$ & $N / A$ & & \\
\hline 1.7 .6 & 2.8 & NIT-*52 & $N / A$ & $\begin{array}{l}\text { ESTABLISH } \\
\text { SAMPLING } \\
\text { ROUTINE }\end{array}$ & & \\
\hline 1.7 .6 & 2.9 & $\begin{array}{l}\text { OPEN SV-*24 } \\
\text { ADJ FLOW }\end{array}$ & & $\begin{array}{l}\mathrm{FIT}-\star 57 \\
3 \pm .1 \quad \mathrm{H}_{2} \mathrm{O}\end{array}$ & & \\
\hline 1.7 .6 & 2.10 & $\begin{array}{l}\text { ADJ FLOW } \\
\text { SV-*67 }\end{array}$ & & $\begin{array}{l}\mathrm{FI}-* 60 \\
10-30 \mathrm{ccm}\end{array}$ & & \\
\hline 1.7 .6 & 2.11 & \begin{tabular}{|l} 
RECORD \\
PDIT-*60 \\
\end{tabular} & $N / A$ & $<175 \quad \mathrm{H}_{2} \mathrm{O}$ & & \\
\hline 1.7 .6 & 2.12 & $\begin{array}{l}\text { OPEN SV-*64 } \\
\text { CLOSE SV-*63 }\end{array}$ & $N / A$ & N/A & & \\
\hline 1.7 .6 & 2.13 & \begin{tabular}{|l} 
RECORD \\
PDIT-*60 \\
\end{tabular} & $\pi / \alpha$ & $<175 " \mathrm{H}_{2} \mathrm{O}$ & & \\
\hline 1.7 .6 & .2 .14 & $\begin{array}{l}\text { CLOSE SV } * 65 \\
\text { OPEN SV-*66 } \\
\end{array}$ & $N / A$ & $N / A$ & & \\
\hline 1.7 .6 & .2 .15 & $\begin{array}{l}\text { RECORD B IN } \\
\text { PDIT-*60 }\end{array}$ & $-1 / 1$ & $\begin{array}{l}-15 \text { to }-50 \\
" \mathrm{H}_{2} \mathrm{O}\end{array}$ & & \\
\hline 1.7 .6 & .2 .16 & $\begin{array}{l}\text { OPEN SV-*63 } \\
\text { CLOSE SV-*64 }\end{array}$ & $N / A$ & $N / A$ & & \\
\hline $1.7 . \hbar$ & .2 .17 & $\begin{array}{l}\text { RECORD A IN } \\
\text { PDIT }-* 60\end{array}$ & $\omega / \Delta$ & $\begin{array}{l}-15 \text { to }-50 \\
\mathrm{MH}_{2} \mathrm{O}\end{array}$ & & \\
\hline 1.7 .6 & .2 .18 & $\begin{array}{l}\text { INSTALL } \\
\text { JUMPER } \\
\end{array}$ & $N / A$ & $N / A$ & & \\
\hline $1.7 . \phi$ & .2 .19 & CLOSE FU-15 & N/A & $N / A$ & & \\
\hline$\sqrt{1.7}$ & $\Delta^{2.20}$ & $\begin{array}{l}\text { ADJ SV-*61 \& } \\
\text { RECORD } \\
\text { PDIT -*60 }\end{array}$ & $N / A$ & $\begin{array}{l} \pm 5^{\prime \prime} \mathrm{H}_{2} \mathrm{O} \mathrm{OF} \\
1.7 .6 .2 .17\end{array}$ & & \\
\hline
\end{tabular}


TEST DATA SHEET

\begin{tabular}{|c|c|c|c|c|c|}
\hline \multicolumn{3}{|c|}{ Date: $N / A$} & \multicolumn{3}{|c|}{ SHMS Unit Number: $\Delta / \Delta$} \\
\hline \multicolumn{3}{|c|}{$\begin{array}{l}\text { Title of Test: } \\
\text { Pneumatic Systems }\end{array}$} & \multirow{2}{*}{\multicolumn{3}{|c|}{ Test Equipment and $\mathrm{S} / \mathrm{N}$ or Cal. No.: }} \\
\hline \multicolumn{3}{|c|}{ Test Performed By: } & & & \\
\hline $\begin{array}{l}\text { Procedure No. } \\
\text { Initial/Date }\end{array}$ & Item & value & $\begin{array}{l}\text { Required } \\
\text { condition }\end{array}$ & $(A / R)$ & Comment \\
\hline & $\begin{array}{l}\text { CAL MON-*60 } \\
\text { CHANNEL A }\end{array}$ & $\frac{N / A}{1 / A}$ & $\begin{array}{l}\text { READINGS } \\
\text { WITHIN } \pm 5 \%\end{array}$ & & \\
\hline $1.7 . \$ .2 .22$ & OPEN FU-15 & $N / A$ & $N / A$ & & \\
\hline $1.7 . \$ .2 .23$ & $\begin{array}{l}\text { REMOVE } \\
\text { JUMPER }\end{array}$ & $N / A$ & $N / A$ & & \\
\hline 1.7 .6 .2 .24 & $\begin{array}{l}\text { OPEN SV }-* 64 \\
\text { CLOSE SV-*63 }\end{array}$ & $N / A$ & $N / A$ & & \\
\hline$1 . 7 \longdiv { 6 . 2 . 2 5 }$ & $\begin{array}{l}\text { INSTALL } \\
\text { JUMPER }\end{array}$ & $N / A$ & $N / A$ & & \\
\hline 1.76 .2 .26 & CLOSE FU-15 & N/A & $N / A$ & & \\
\hline 1.7 .6 .2 .27 & $\begin{array}{l}\text { ADJ SV-*62 \& } \\
\text { RECORD } \\
\text { PDIT-*60 }\end{array}$ & $\mathrm{N}$ & $\begin{array}{l} \pm 5^{\prime \prime} \mathrm{H}_{2} \mathrm{OOF} \\
1.7 .6 .2 .15\end{array}$ & & \\
\hline 1.7 .6 .2 .28 & $\begin{array}{l}\text { CAL MON }-* 60 \\
\text { CHANNEL B }\end{array}$ & W & $\begin{array}{l}\text { READINGS } \\
\text { WITHIN } \pm 5 \%\end{array}$ & & \\
\hline 1.7 .6 .2 .29 & OPEN FU-15 & $N / A^{\prime}$ & $N / A$ & & \\
\hline 1.7 .6 .2 .30 & $\begin{array}{l}\text { REMOVE } \\
\text { JUMPER }\end{array}$ & N/A & $N / A$ & & \\
\hline 1.7 .6 .2 .31 & $\begin{array}{l}\text { CLOSE SV }-* 64 \\
\text { OPEN SV-*70 }\end{array}$ & $N / A$ & $\mathrm{~N} / \mathrm{A}$ & & \\
\hline 1.76 .2 .32 & $\begin{array}{l}\text { RECORD } \\
\text { PDIT-*60 } \\
\text { NIT-*52 IN }\end{array}$ & $\Lambda$ & $\begin{array}{l}0 \text { to }-30 \\
1 \mathrm{H}, \mathrm{O}\end{array}$ & & \\
\hline 1.7 .6 .2 .33 & $\begin{array}{l}\text { INSTALL } \\
\text { JUMPER }\end{array}$ & $N / A$ & $N / A$ & & \\
\hline $1.7 \sqrt{6} /^{2.34}$ & CLOSE FU-15 & $N / A$ & $N / A$ & $A$ & $5 / 1$ \\
\hline
\end{tabular}


TEST DATA SHEET

\begin{tabular}{|c|c|c|c|c|c|c|}
\hline \multicolumn{4}{|c|}{ Date: $\mathrm{N} / \mathrm{A}$} & \multicolumn{3}{|c|}{ SHMS Unit Number: $N / A$} \\
\hline \multicolumn{4}{|c|}{$\begin{array}{l}\text { Title of Test: } \\
\text { Pneumatic Systems }\end{array}$} & \multicolumn{3}{|c|}{ Test Equipment and $\mathrm{S} / \mathrm{N}$ or Cal. No.: } \\
\hline \multicolumn{4}{|c|}{ Test Performed By: } & \multicolumn{3}{|c|}{$N / \mathrm{s}$} \\
\hline \multicolumn{2}{|c|}{$\begin{array}{l}\text { Procedure No. } \\
\text { Initial/Date }\end{array}$} & Item & value & $\begin{array}{l}\text { Required } \\
\text { Condition } \\
\end{array}$ & $(A / R)$ & Comment \\
\hline \multicolumn{2}{|c|}{ 1.7.6.2:35 } & $\begin{array}{l}\text { ESTABLISH } \\
\text { BYPASS FLOW }\end{array}$ & $5 / x$ & $\begin{array}{l}\text { FIV }-* 70 \\
1.5 \pm .2 \mathrm{cfh}\end{array}$ & $N / A$ & $N / A$ \\
\hline \multicolumn{2}{|c|}{$1.7 \mathfrak{r}^{6.2 .36}$} & $\begin{array}{l}\text { ADJ SV-*71 } \\
\text { NIT-*52 } \\
\text { SAMPLE IN } \\
\end{array}$ & & $\begin{array}{l} \pm 5 \quad " \mathrm{H}_{2} \mathrm{O} \quad \mathrm{OF} \\
1.7 .6 .2 .32\end{array}$ & & C \\
\hline 1.7 & 6.2 .37 & $\begin{array}{l}\text { RECORD } \\
\text { NIT-*52 } \\
\text { SAMPLES } \\
\end{array}$ & $\frac{1}{w / 2}$ & $\begin{array}{l}\text { READINGS } \\
\text { WITHIN } \pm 5 \%\end{array}$ & & \\
\hline 1.7. & 6.2 .38 & OPEN FU-15 & $\mathrm{N} / \mathrm{A}$ & $N / A$ & & \\
\hline 1.7. & 6.2 .39 & $\begin{array}{l}\text { REMOVE } \\
\text { JUMPER }\end{array}$ & $N / A$ & $N / A$ & & \\
\hline \multicolumn{3}{|r|}{ orovides for } & test system & shutdown. & & \\
\hline 1.7 & 7.1 & $\begin{array}{l}\text { DISCONNECT } \\
\mathrm{H}_{2} \text { CAL GAS }\end{array}$ & N/A & N/A & & \\
\hline 1.7. & 7.2 & $\begin{array}{l}\text { DISCONNECT } \\
\mathrm{CH}_{4} \text { CAL GAS }\end{array}$ & $N / A$ & $N / A$ & & \\
\hline 1.7 . & 7.3 & $\begin{array}{l}\text { DISCONNECT } \\
\mathrm{NH}_{3} \text { CAL GAS }\end{array}$ & N/A & $N / A$ & & \\
\hline 1.7 . & 7.4 & $\begin{array}{l}\text { SECURE \& } \\
\text { TURN OFF } \\
\text { ANA. INST.S }\end{array}$ & $N / A$ & $N / A$ & & \\
\hline 1.7. & 7.5 & $\begin{array}{l}\text { DISCONNECT } \\
\mathrm{N}_{2} \text { CARRIER }\end{array}$ & $N / A$ & $N / A$ & & \\
\hline 1.7 . & 7.6 & $\begin{array}{l}\text { DISCONNECT } \\
\text { He CARRIER }\end{array}$ & $N / A$ & N/A & & \\
\hline 1.7 . & 7.7 & $\begin{array}{l}\text { OPEN ALL } \\
\text { BREAKERS \& } \\
\text { FUSES } \\
\end{array}$ & $N / A$ & N/A & & \\
\hline $\begin{array}{r}1.7 \\
\end{array}$ & 4 & $\begin{array}{l}\text { CLOSE LISTED } \\
\text { SYS. VALVES }\end{array}$ & $N / A$ & $N / A$ & A & $N / A$ \\
\hline
\end{tabular}


APPENDIX $K$

PAGE K-28
INF - SD - WM-ATR - 191

Rev. 0

Page 364

TEST DATA SHEET

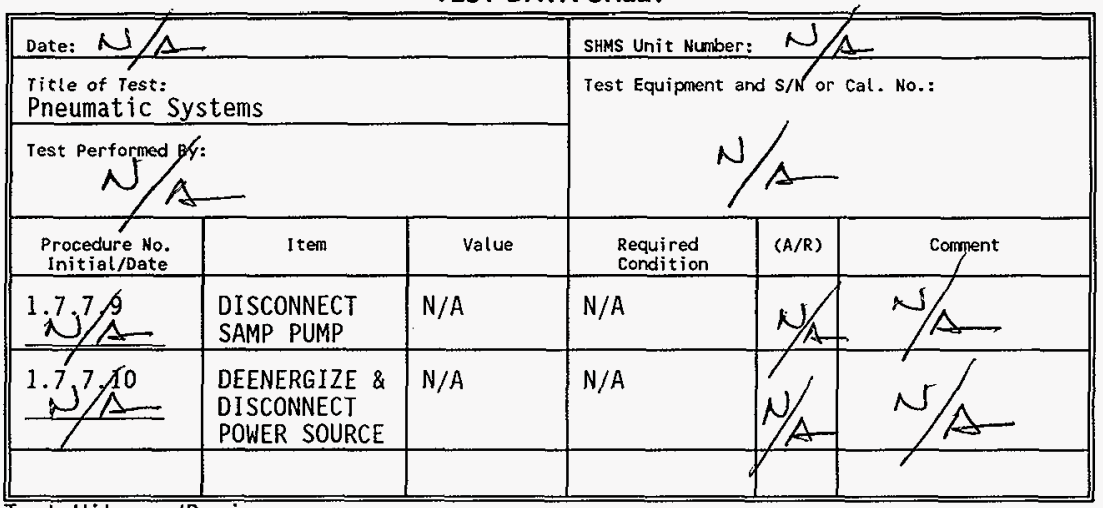

Test Witness/Review:

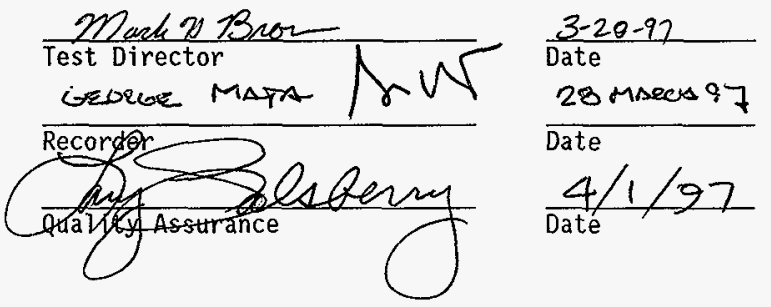


APPENDIX $K$

PAGE K-29

$$
\begin{gathered}
\text { HNF-SD-WM-ATR- } 191 \\
\text { Rev. } 0 \\
\text { Page } 365 \alpha
\end{gathered}
$$

TEST EXCEPTION SHEET

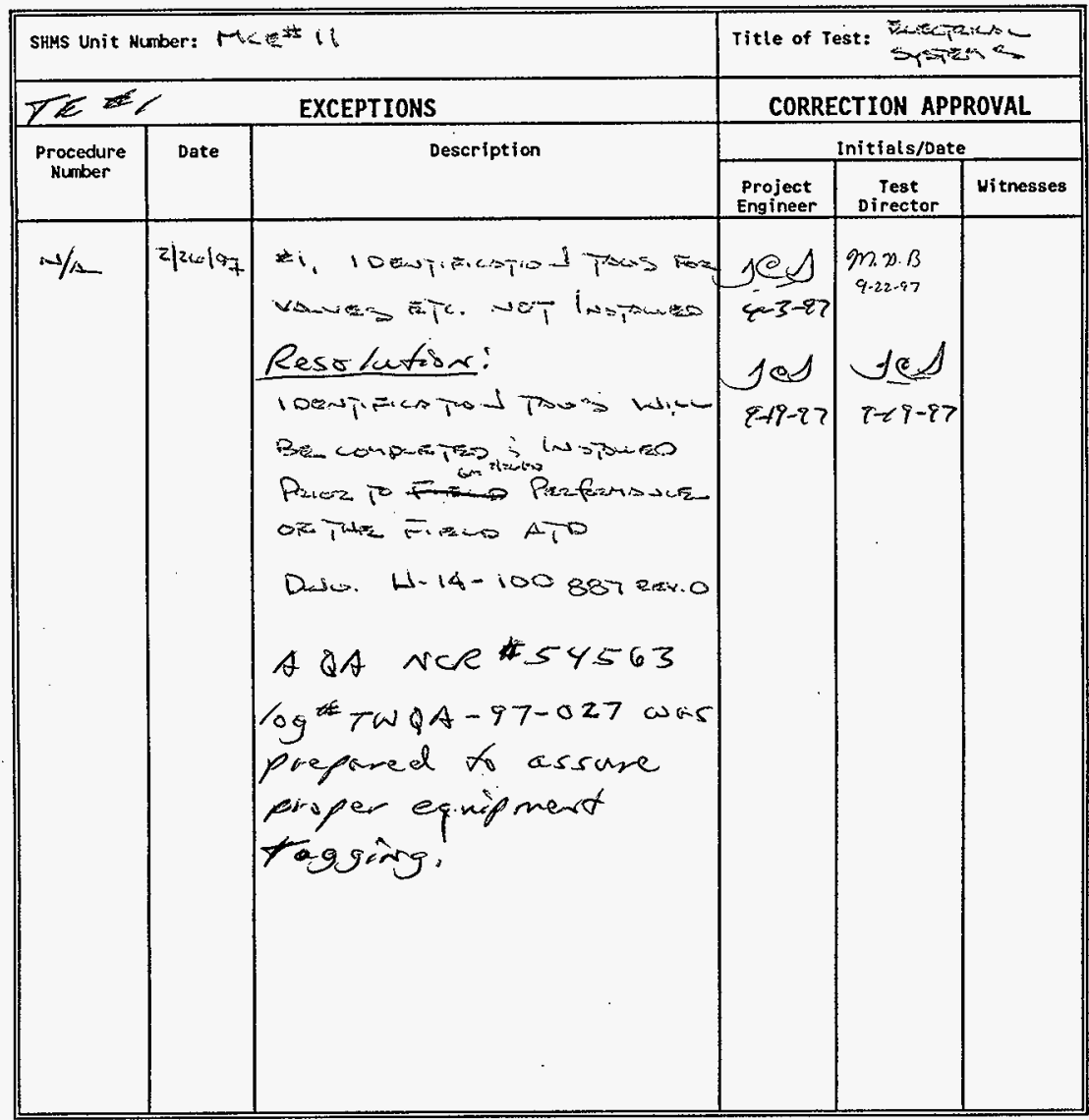

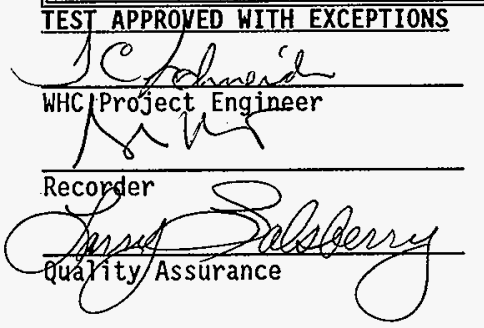

$$
\begin{aligned}
& \frac{4-3-77}{\text { Date }} \\
& \frac{3 \Delta p w 97}{\text { Date }} \\
& \frac{4 / 2 / 97}{\text { Date }}
\end{aligned}
$$


TEST EXCEPTION SHEET

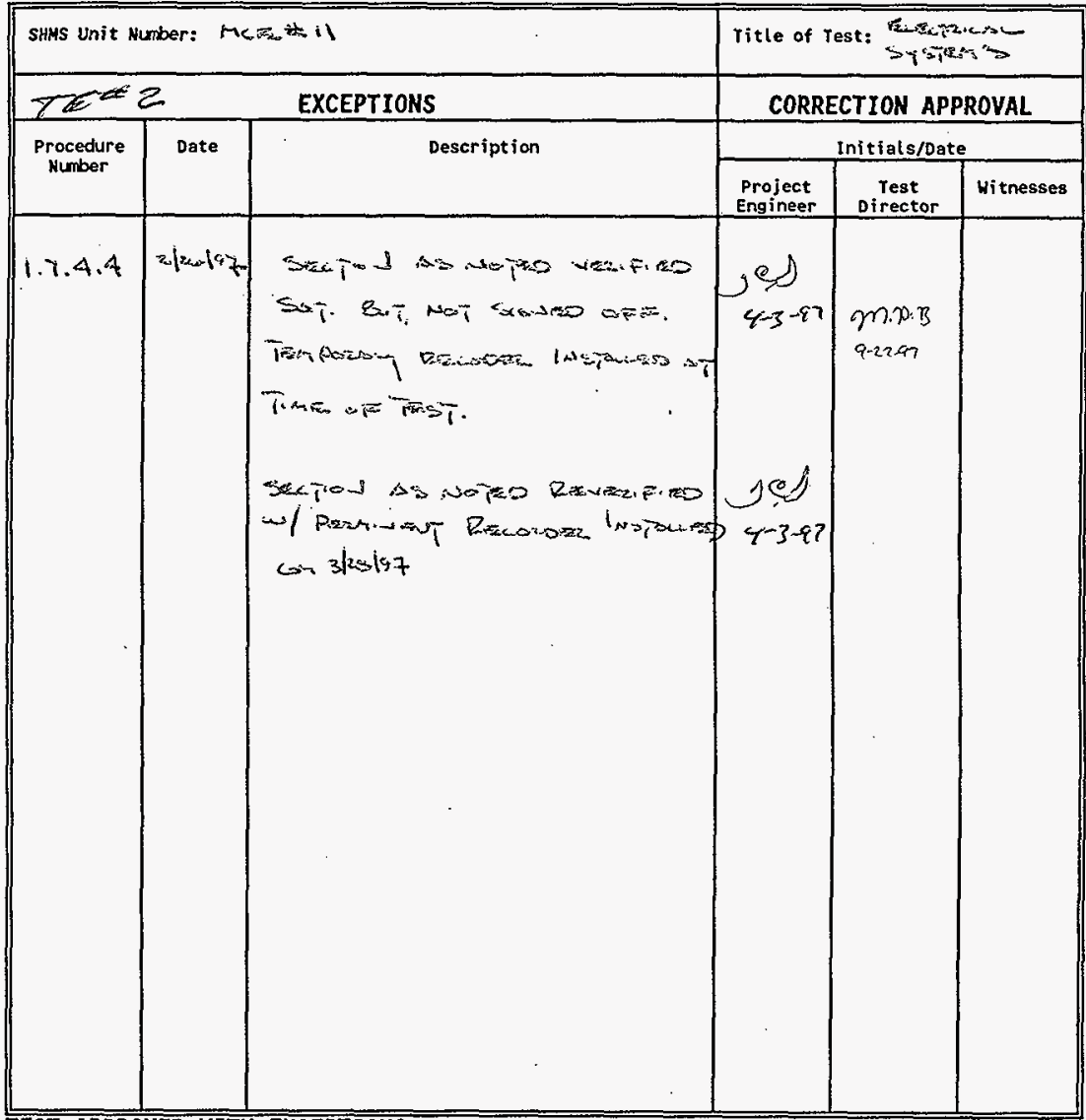

\section{TEST APPRQVED WITH EXCEPTIONS}
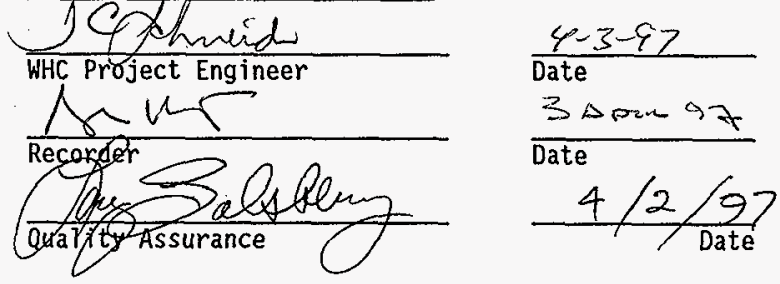
TEST LOG

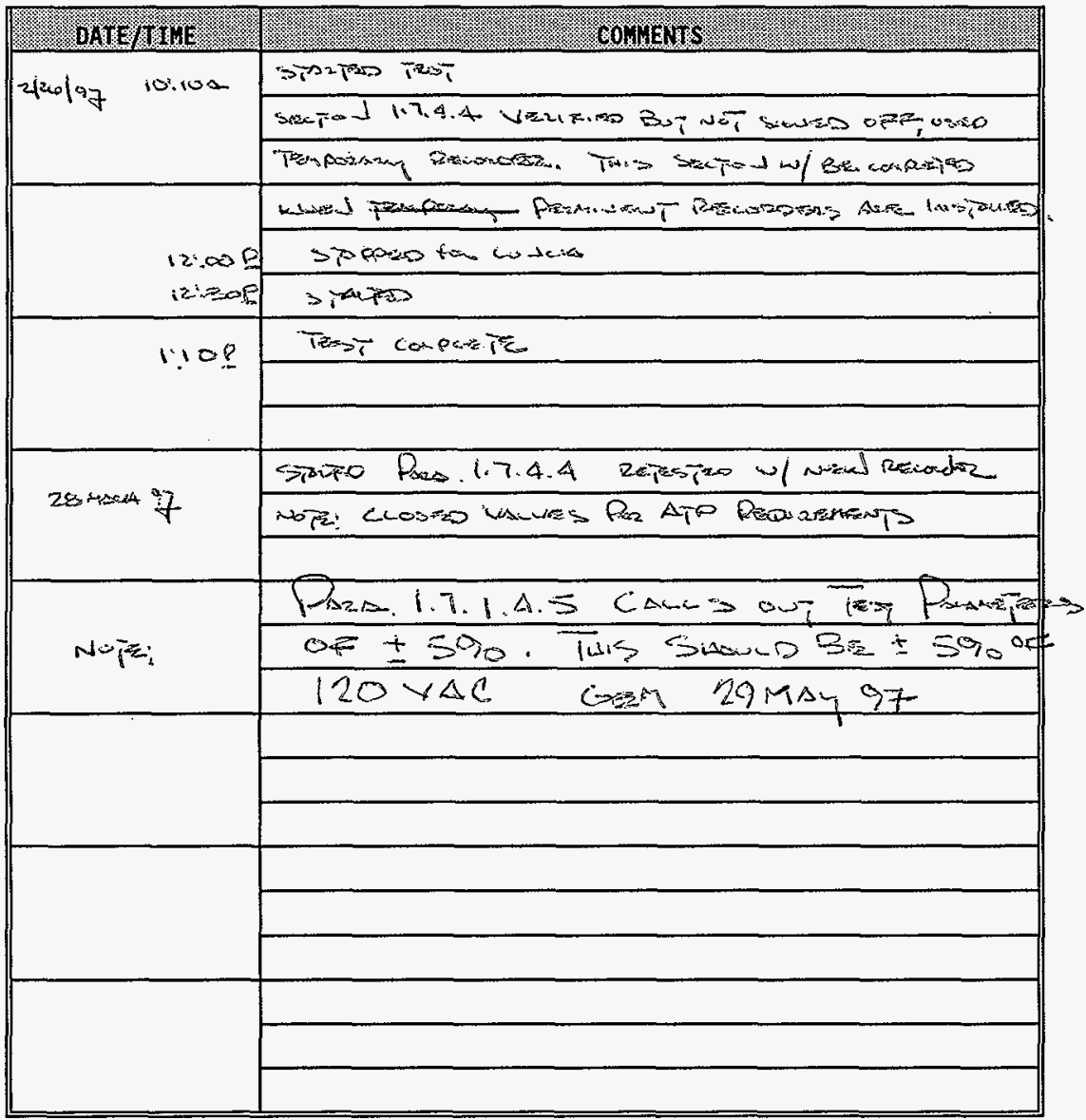


APPENDIX L

PAGE L-/ $\because 4$

a
HNF-SD-WM-ATR - 191

Rev. 0

Page 367

\section{APPENDIX L}


TEST EXECUTION SHEET

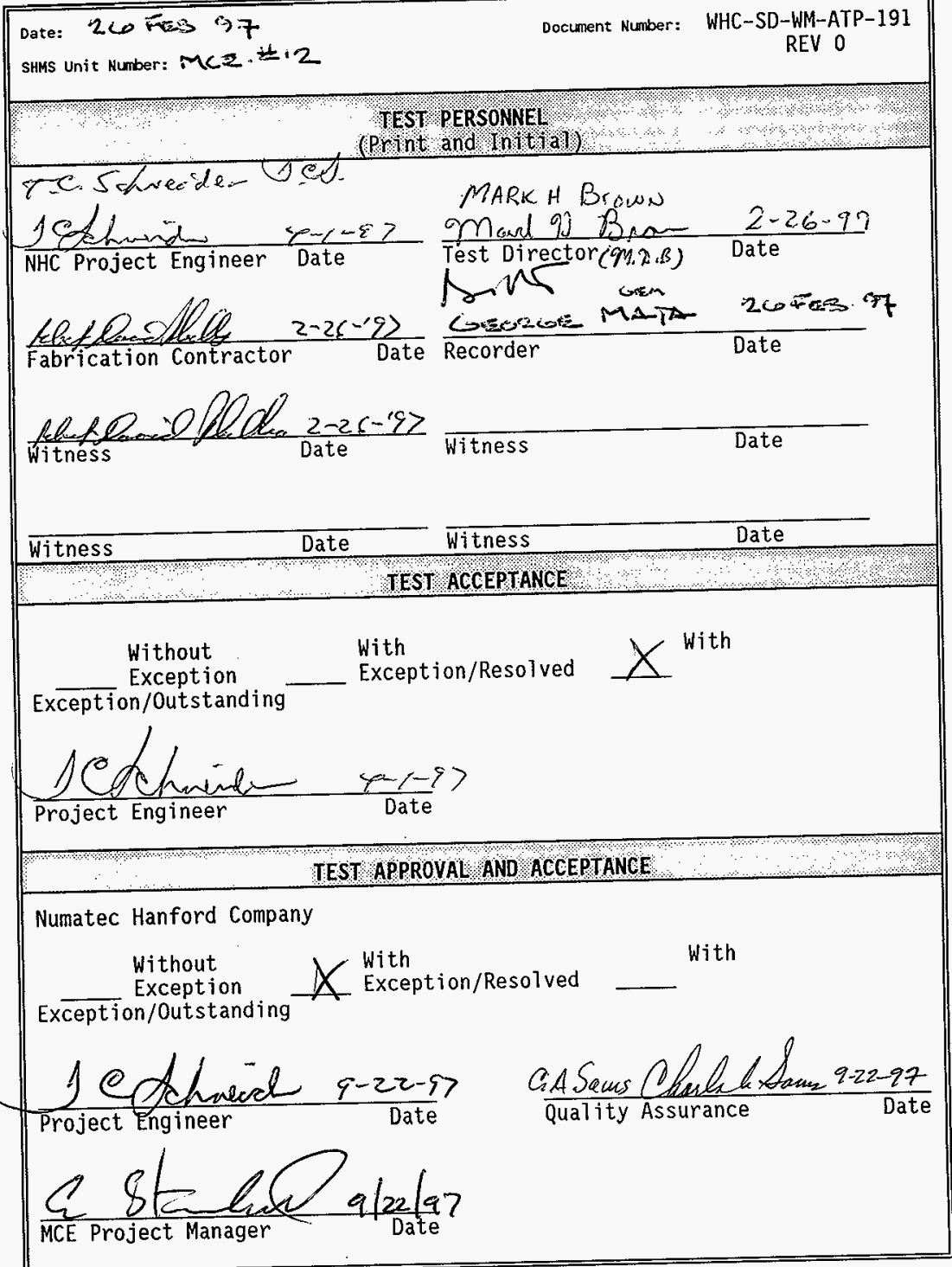


SHMS Unit Number:

\section{PREREQUISITES AND INITIAL TEST CONDITIONS}

The following conditions shall exist at the start of the acceptance testing. Initial and date to verify that each of the following items have been accomplished.

Systems being tested have been inspected for workmanship and for compliance with design.

$6 x_{2} z \mid z w / i z$ Continuity and megger tests have been performed on portions of the electrical and instrument systems being tested, as required.

Gen 2 zukfifleak tests on the pneumatic systems have been performed.

Ganz/2o/rTthe following circuit breakers and fuses are installed per the specified size and are open

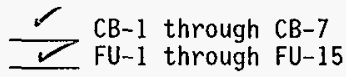

GE्] 2/2ukf All test instruments have a currently valid calibration stamp attached that indicates a calibration traceable to the National Institute of Standards and Technology.

20 $z / 2 / 7$ The following process and control instrument systems have been initially configured and aligned for proper operation.

FIT $-* 57$, FSL $-* 57$, NIT $-\star 54$, NIT $-* 55,(N R-* 54)$, PDIT-*60, TIC $-* 50$, TIC-*56, TIS $-* 62$, YYC $-* 01$ NOTE: NR-*54 is not used in the ANALYTICAL configuration.

canz/zulaz Personnel responsible for directing and witnessing the performance of the tests described in this ATP have read and understand appropriate certified vendor information (CVI) pertaining to the operation of the equipment to be tested.

GenzlablocoSE all system manual valves with the exception of the following:

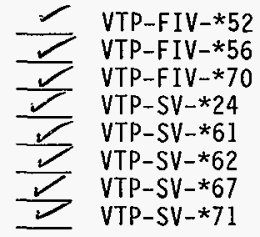

Cenzlutiz The sample pump VTP-P-*50, connected to the cabinet sample out port, but vented to atmosphere for testing. 
208 Vac 1 phase 15-20 ampere temporary power source has been connected, but not energized, to the appropriate $\mathrm{TBl}$ terminals per H-14-100838.

Verify by signature and date that all prerequisites have been met.

$\frac{\text { Manh of Bnowa }}{\text { Test Director Signature }} \frac{3 \cdot 28-97}{\text { Date }}$


TEST DATA SHEET

\begin{tabular}{|c|c|c|c|c|c|c|}
\hline \multicolumn{3}{|c|}{ Date: $260 \overline{F 53} .97$} & \multicolumn{4}{|c|}{ SHMS Unit Number: MCE i 2} \\
\hline \multicolumn{3}{|c|}{$\begin{array}{l}\text { Title of Test: } \\
\text { Electrical Systems }\end{array}$} & \multirow{2}{*}{\multicolumn{4}{|c|}{ 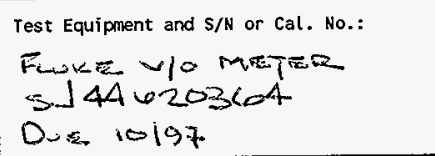 }} \\
\hline \multicolumn{3}{|c|}{ 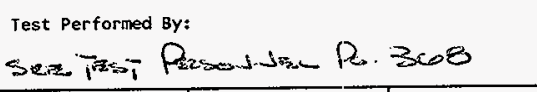 } & & & & \\
\hline $\begin{array}{c}\text { Procedure No. } \\
\text { Initial/Date } \\
\end{array}$ & Item & value & $\begin{array}{l}\text { Required } \\
\text { Condition } \\
\end{array}$ & $(A / R)$ & \multicolumn{2}{|c|}{ Comment } \\
\hline \multicolumn{7}{|c|}{ Section 1.7.1.1 verifies the system Mains Power. } \\
\hline$\underline{1.1 .1 .1}$ & Resistance & .10 & $<1$ ohm & $\Delta$ & \multicolumn{2}{|c|}{$N / \Delta$} \\
\hline $\begin{array}{l}1.7 .1 .1 .2 \\
\text { gen }\end{array}$ & $\begin{array}{l}\text { Temp power } \\
208 \mathrm{Vac}\end{array}$ & N/A & Energized & $A$ & & \\
\hline $\begin{array}{l}1.7 .1 .1 .3 \\
\text { Gizin }\end{array}$ & $\begin{array}{l}\text { Line Vac: } \\
\text { L1-L2 } \\
\text { L1-Gind } \\
\text { L2-Gnd } \\
\mathrm{N}-\text { Gnd }\end{array}$ & $\frac{\frac{208.8}{119.4}}{\frac{119.6}{0}}$ & $\begin{array}{l}\text { L1-L2: } 208 \\
\text { Vac }+10 /-1 \% \\
\text { L1, L2: } 120 \\
\operatorname{Vac} \pm 5 \% \\
\mathrm{~N}: 0 \mathrm{Vac}\end{array}$ & $\Delta$ & & \\
\hline \multicolumn{7}{|c|}{ Section 1.7 .1 .2 verifies the enclosure HVAC system. } \\
\hline $\begin{array}{l}1.7 .1 .2 .1 \\
\end{array}$ & AC Adjusted & N/A & $N / A$ & $\Delta$ & & \\
\hline $\begin{array}{l}1.7 .1 .2 .2 \\
\text { goen }\end{array}$ & $\begin{array}{l}\text { CLOSE } \\
\text { CB-1/2 }\end{array}$ & $N / A$ & N/A & $A$ & & \\
\hline $\begin{array}{l}1.7 .1 .2 .3 \\
\end{array}$ & Heater ON & 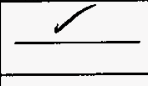 & Heater ON & $\Delta$ & & \\
\hline$\underline{G}^{1.7 .1 .4}$ & AC Adjusted & N/A & $N / A$ & $A$ & & \\
\hline 1.7 .1 .2 .5 & $\begin{array}{l}\text { Heater OFF } \\
\text { Cooling ON }\end{array}$ & $\frac{\nearrow}{\square}$ & $\begin{array}{l}\text { Heater OFF } \\
\text { Cooling ON }\end{array}$ & 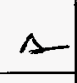 & & \\
\hline $\begin{array}{l}1.7 .1 .2 .6 \\
\end{array}$ & AC Adjusted & N/A & $N / A$ & $A$ & & \\
\hline $\begin{array}{l}1.7 .1 .2 .7 \\
\text { Gizn }\end{array}$ & $\begin{array}{l}\text { OPEN } \\
\text { CB-1/2 }\end{array}$ & N/A & $N / A$ & $A$ & & \\
\hline \multicolumn{7}{|c|}{ Section 1.7.1.3 verifies the sample pump is properly connected. } \\
\hline $\begin{array}{c}1.7 .1 .3 .1 \\
6\end{array}$ & $\begin{array}{l}\text { CLOSE } \\
\text { CB-3/4 }\end{array}$ & $N / A$ & $N / A$ & $A$ & & \\
\hline $\begin{array}{c}1.7 .1 .3 .2 \\
6 \sin \end{array}$ & $\begin{array}{l}\text { VTP-P- } * 50 \\
\text { ON }\end{array}$ & 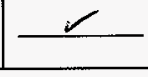 & $\begin{array}{l}\text { VTP-P-*50 } \\
\text { ON }\end{array}$ & $A$ & 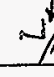 & \\
\hline
\end{tabular}




\section{TEST DATA SHEET}

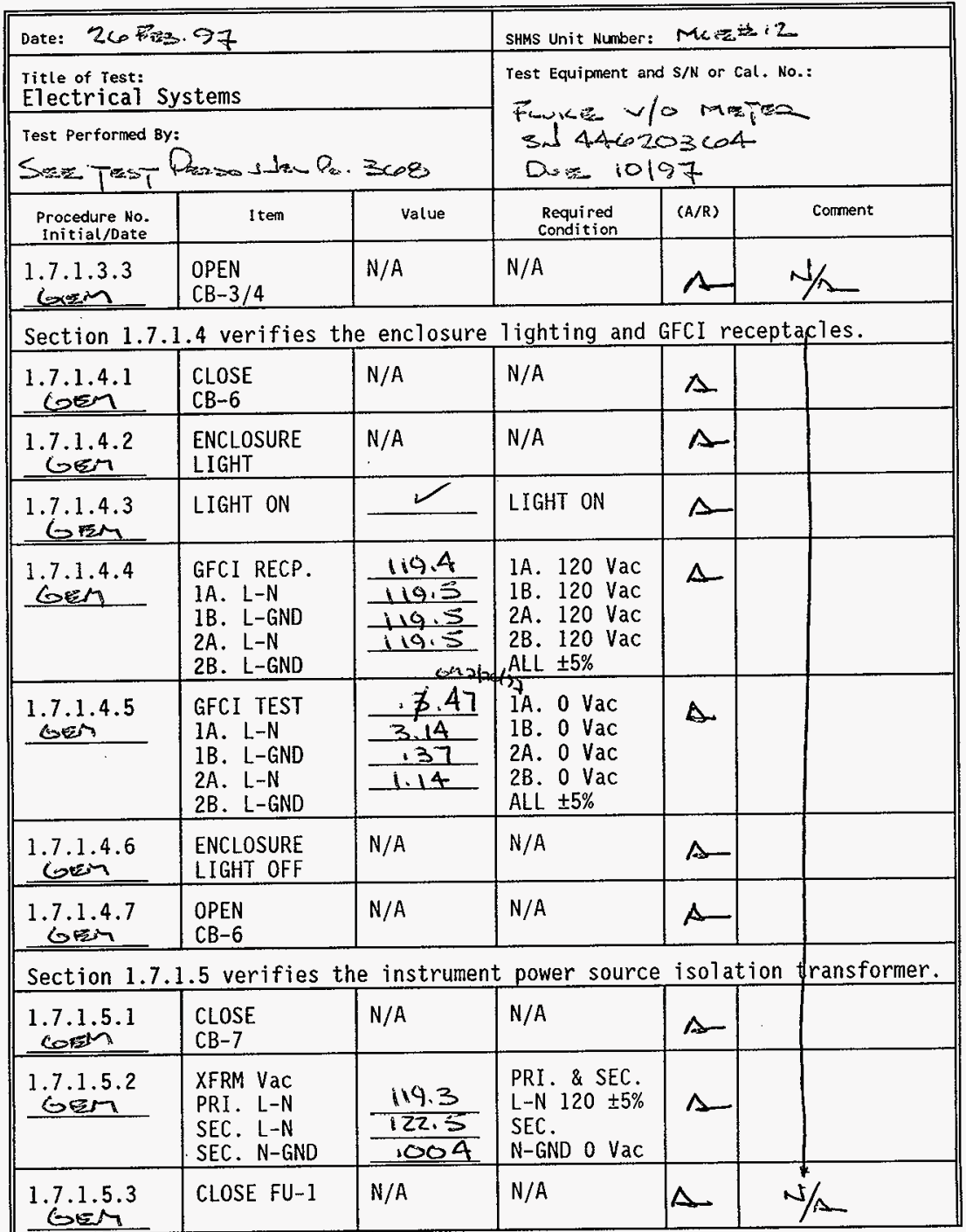


TEST DATA SHEET

\begin{tabular}{|c|c|c|c|c|c|}
\hline \multicolumn{3}{|c|}{ Date: $26=97$} & \multicolumn{3}{|c|}{ SHMS Unit Number: $M K E+12$} \\
\hline \multicolumn{3}{|c|}{$\begin{array}{l}\text { Title of Test: } \\
\text { Electrical Systems }\end{array}$} & \multirow{2}{*}{\multicolumn{3}{|c|}{ 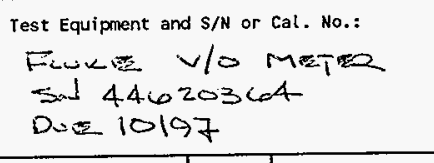 }} \\
\hline \multicolumn{3}{|c|}{ 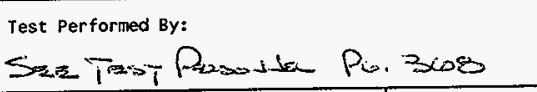 } & & & \\
\hline $\begin{array}{l}\text { Procedure No. } \\
\text { Initial/Date }\end{array}$ & Item & Value & $\begin{array}{l}\text { Required } \\
\text { Condition }\end{array}$ & $(A / R)$ & Comment \\
\hline 1.7 .1 .5 .4 & $\begin{array}{l}\text { VTP-PS } * 50 \\
\text { OUTPUT Vdc }\end{array}$ & 24.02 & $24 \pm 0.1 \mathrm{Vdc}$ & $A$ & $N / \Delta$ \\
\hline \multicolumn{6}{|c|}{ Section 1.7.1.6 verifies the enclosure general alarm system. } \\
\hline $\begin{array}{l}1.7 .1 .6 .1 \\
\text { Gein } \\
\end{array}$ & 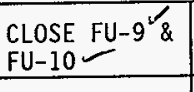 & N/A & N/A & $\Delta$ & \\
\hline $\begin{array}{l}1.7 .1 .6 .2 \\
0.917 \\
\end{array}$ & $\begin{array}{l}\text { PUSH PB-*51 } \\
\& \text { PB-*50 }\end{array}$ & $N / A$ & $\mathrm{~N} / \mathrm{A}$ & $\Delta$ & \\
\hline $\begin{array}{l}1.7 .1 .6 .3 \\
\end{array}$ & $\begin{array}{l}\text { ALARM TEST } \\
\text { VERIFY ALARM } \\
\text { CONDITIONS }\end{array}$ & (2/ & $\begin{array}{ll}\text { YAH-*50 } & \text { ON } \\
\text { NAH } * 55 & \text { ON } \\
X A-* 63 & \text { ON } \\
\text { NAH-*54 } & \text { ON } \\
\text { FAL-*57 } & \text { ON } \\
\text { TAHL-*62 ON } \\
\text { TAL-*50 } & \text { ON } \\
\text { YAL-*58 } & \text { ON } \\
\text { PBL-*58 } & \text { ON } \\
\text { PBL-*54 } & \text { OFF } \\
\text { PBL-*59 } & \text { OFF } \\
\end{array}$ & $\Delta$ & \\
\hline $\begin{array}{c}1.7 .1 .6 .4 \\
\text { Gein }\end{array}$ & $\begin{array}{l}\text { END ALARM } \\
\text { TEST } \\
\end{array}$ & $N / A$ & N/A & $\underline{1}$ & \\
\hline $\begin{array}{l}1.7 .1 .6 .5 \\
\end{array}$ & RESET ALARMS & $N / A$ & $N / A$ & $\Delta$ & \\
\hline 6.1.6.6 & $\begin{array}{l}\text { OPEN FU-9 \& } \\
\text { FU-10 }\end{array}$ & $N / A$ & $N / A$ & $\triangle$ & \\
\hline \multicolumn{6}{|c|}{ Section 1.7.1.7 verifies the flow al arm system. } \\
\hline Gen & $\begin{array}{l}\text { CLOSE, FU-3, } \\
\text { FU-9, FU-10- } \\
\& \text { FU-13 }\end{array}$ & $N / A$ & $N / A$ & $\Delta$ & \\
\hline $\begin{array}{c}1.7 .1 .7 .2 \\
\end{array}$ & RESET ALARMS & $r$ & $F A L-* 57$ ON & $A$ & \\
\hline 1.7 .1 .7 .3 & $\begin{array}{l}\text { OPEN SV-*20 } \\
\& S V-* 22\end{array}$ & N/A & N/A & $\Delta$ & $N / \Delta$ \\
\hline
\end{tabular}


TEST DATA SHEET

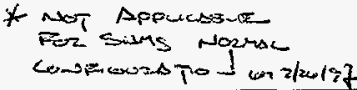

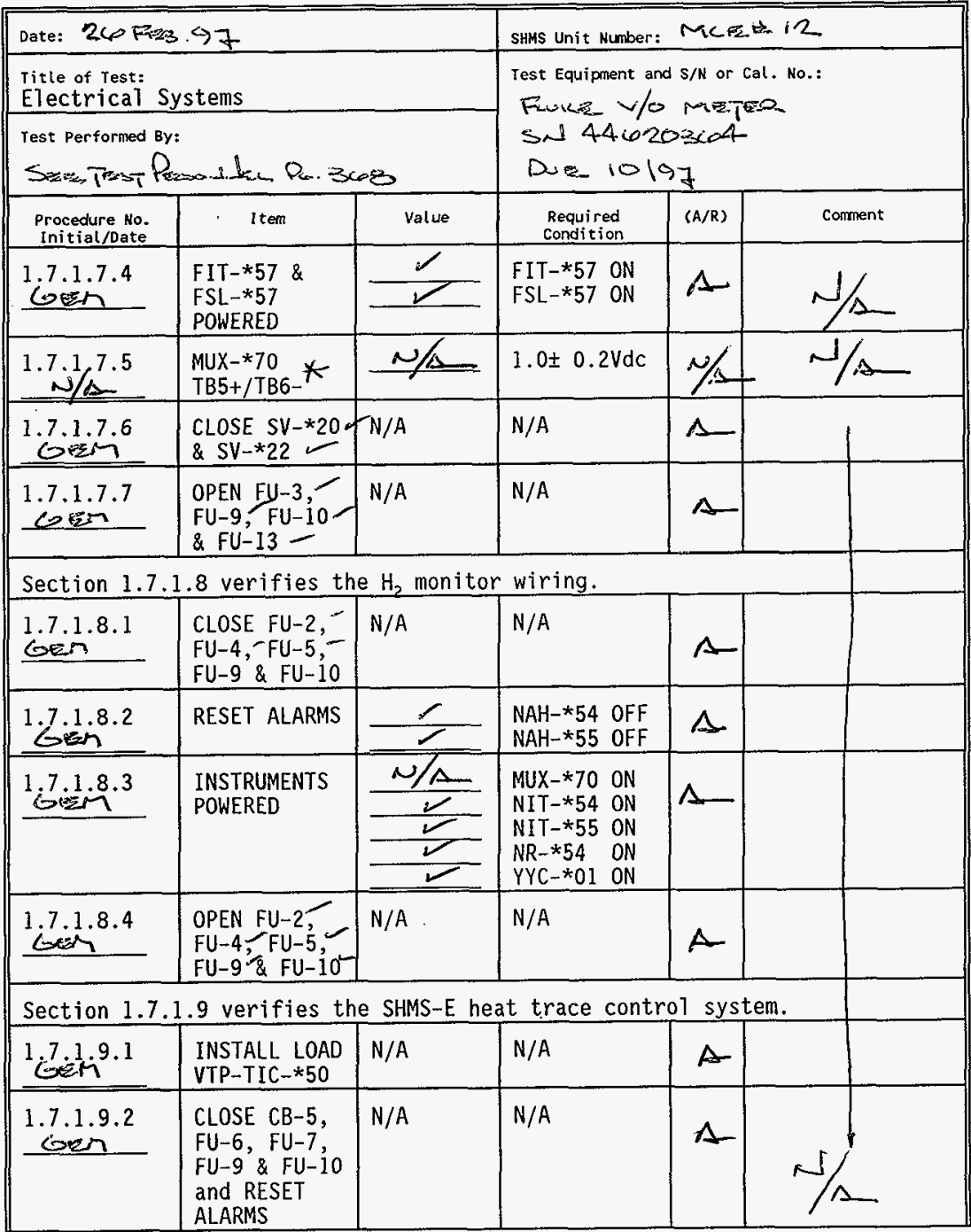

cosen dec zizols) 
APPENDIX L

PAGE L- 9

$$
\begin{array}{r}
\text { HNF-SD- LM-ATR- } 191 \\
\text { ReV. } 0 \\
\text { Page } 375
\end{array}
$$

TEST DATA SHEET

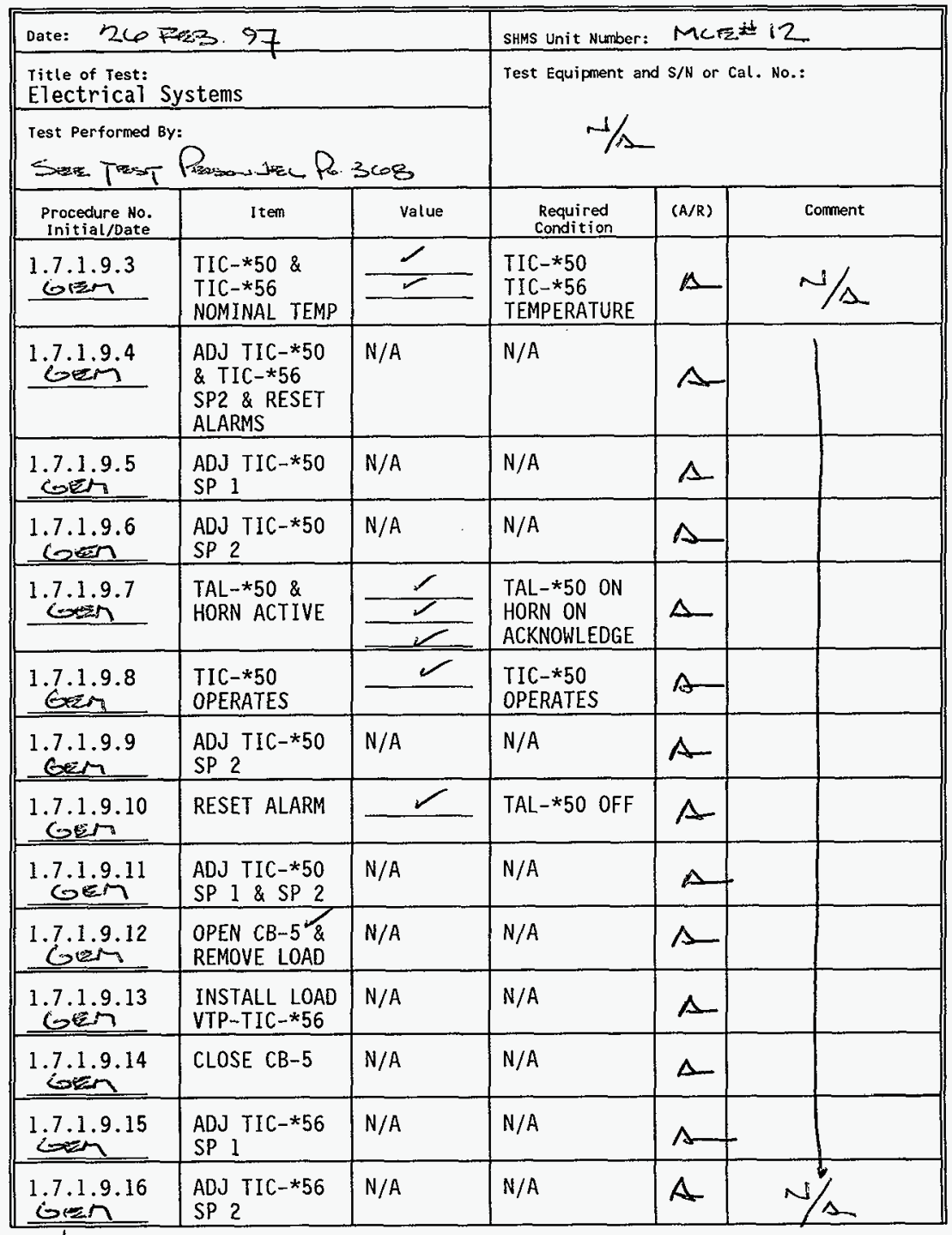


TEST DATA SHEET

\begin{tabular}{|c|c|c|c|c|c|c|}
\hline \multicolumn{3}{|c|}{ Date: 26 Fr3. 97} & \multicolumn{4}{|c|}{ SHMS Unit Number: $M<$ i2 12} \\
\hline \multicolumn{3}{|c|}{$\begin{array}{l}\text { Title of Test: } \\
\text { Electrical Systems }\end{array}$} & \multirow{2}{*}{\multicolumn{4}{|c|}{ Test Equipment and $\mathrm{S} / \mathrm{N}$ or Cal. Ho.: }} \\
\hline \multicolumn{3}{|c|}{ 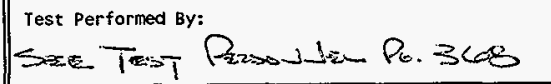 } & & & & \\
\hline $\begin{array}{l}\text { Procedure No. } \\
\text { Initial/oate }\end{array}$ & Item & Value & $\begin{array}{l}\text { Required } \\
\text { Condition } \\
\end{array}$ & $(A / R)$ & & ment \\
\hline 6rim & $\begin{array}{l}\text { TAL }-* 50 \& \\
\text { HORN ACTIVE }\end{array}$ & $\stackrel{2}{2}$ & $\begin{array}{l}\text { TAL-*50 ON } \\
\text { HORN ON } \\
\text { ACKNOWLEDGE }\end{array}$ & $\Delta$ & $N / \Delta$ & \\
\hline $\begin{array}{l}1.7 .1 .9 .18 \\
\text { Geis }\end{array}$ & $\begin{array}{l}\text { TIC }-* 56 \\
\text { OPERATES }\end{array}$ & 2 & $\begin{array}{l}\text { TIC }-* 56 \\
\text { OPERATES }\end{array}$ & $\Delta$ & 1 & \\
\hline Gisio & $\begin{array}{l}\text { ADJ TIC-*56 } \\
\text { SP } 2\end{array}$ & $N / A$ & $N / A$ & $\Delta$ & & \\
\hline $\operatorname{cosin}^{1.7 .1 .9 .20}$ & RESET ALARM & $\mathscr{L}$ & TAL $-* 50$ OFF & $A$ & & \\
\hline $\begin{array}{l}1.7 .1 .9 .21 \\
\end{array}$ & $\begin{array}{l}\text { ADJ TIC-*56 } \\
S P 1 \& S P 2\end{array}$ & $N / A$ & $N / A$ & $A$ & & \\
\hline Gi.in & $\begin{array}{l}\text { OPEN CB-5, } \\
\text { FU-6 \& FU-7 }\end{array}$ & $N / A$ & N/A & $\Delta$ & & \\
\hline $\cos ^{1.7 .1 .9 .23}$ & $\begin{array}{l}\text { REMOVE LOAD } \\
\text { TIC }-* 56\end{array}$ & $N / A$ & N/A & 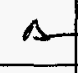 & & \\
\hline$\frac{1.7 .1 .9 .24}{6 m^{2}}$ & $\begin{array}{l}\text { CLOSE FU-8 } \\
\text { TIS } * 62 \\
\text { NOMINAL TEMP }\end{array}$ & & TIS $-* 62$ & $A-$ & & \\
\hline $\begin{array}{l}1.7 .1 .9 .25 \\
\text { com }\end{array}$ & $\begin{array}{l}\text { ADJ TIS-*62 } \\
\text { SP } 1\end{array}$ & $N / A$ & N/A & $A$ & & \\
\hline $\begin{array}{l}1.7 .1 .9 .26 \\
\text { sorin }\end{array}$ & $\begin{array}{l}\text { ADJ TIS-*62 } \\
\text { SP } 2 \\
\end{array}$ & $N / A$ & N/A & $\Delta$ & & \\
\hline $\begin{array}{c}1.7 .1 .9 .27 \\
\end{array}$ & RESET ALARMS & $\sim$ & $\begin{array}{l}\text { TAHL-*62 } \\
\text { OFF }\end{array}$ & $A$ & & \\
\hline $\begin{array}{l}1.7 .1 .9 .28 \\
\end{array}$ & $\begin{array}{l}\text { ADJ TIS-*62 } \\
\text { SP } 2\end{array}$ & $N / A$ & $N / A$ & $A$ & & \\
\hline $\begin{array}{l}1.7 .1 .9 .29 \\
\end{array}$ & $\begin{array}{l}\text { TAHL-*62 \& } \\
\text { HORN ACTIVE }\end{array}$ & $\frac{2}{2}$ & $\begin{array}{l}\text { TAHL-*62 ON } \\
\text { HORN ON } \\
\text { ACKNOWLEDGE }\end{array}$ & $\Delta$ & & \\
\hline 6.1.9.30 & $\begin{array}{l}\text { ADJ TIS }-* 62 \\
\text { SP } 2\end{array}$ & N/A & N/A & $A$ & $5 / A^{\downarrow}$ & \\
\hline
\end{tabular}


TEST DATA SHEET

* NeT Appurascure For sinis Norps

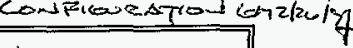

\begin{tabular}{|c|c|c|c|c|c|}
\hline \multicolumn{3}{|c|}{ Date: $26 \rightleftharpoons 5.97$} & \multicolumn{3}{|c|}{ SHMS Unit Number: Mic 12} \\
\hline \multicolumn{3}{|c|}{$\begin{array}{l}\text { Title of rest: } \\
\text { Electrical Systems }\end{array}$} & \multirow{2}{*}{\multicolumn{3}{|c|}{ 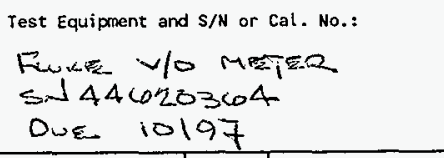 }} \\
\hline \multicolumn{3}{|c|}{ 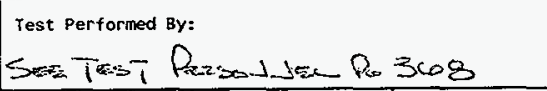 } & & & \\
\hline $\begin{array}{l}\text { Procedure No. } \\
\text { Initial/Date } \\
\end{array}$ & I tem: & Value & $\begin{array}{c}\text { Required } \\
\text { Condition }\end{array}$ & $(A / R)$ & Comment \\
\hline $\begin{array}{l}1.7 .1 .9 .31 \\
\text { Germ } \\
\end{array}$ & RESET ALARMS & 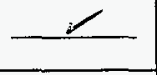 & $\begin{array}{l}\text { TAHL-*62 } \\
\text { OFF }\end{array}$ & & \\
\hline $\begin{array}{l}1.7 .1 .9 .32 \\
\text { Giein } \\
\end{array}$ & $\begin{array}{l}\text { ADJ TIS-*62 } \\
\text { SP } 1\end{array}$ & $N / A$ & $N / A$ & & \\
\hline $\begin{array}{l}1.7 .1 .9 .33 \\
\sin \end{array}$ & $\begin{array}{l}\text { TAHL-*62 \& } \\
\text { HORN ACTIVE }\end{array}$ & 2 & $\begin{array}{l}\text { TAHL }-* 62 \text { ON } \\
\text { HORN ON } \\
\text { ACKNOWLEDGE }\end{array}$ & & \\
\hline $\begin{array}{l}1.7 .1 .9 .34 \\
\end{array}$ & $\begin{array}{l}\text { ADJ TIS }-* 62 \\
S P 1 \& S P 2\end{array}$ & $\mathrm{~N} / \mathrm{A}$ & $N / A$ & & \\
\hline 1.7 .1 .9 .35 & $\begin{array}{l}\text { OPEN FU-8, } \\
\text { FU-9 \& FU-10 }\end{array}$ & $N / A$ & N/A & & \\
\hline \multicolumn{6}{|c|}{ Section 1.7 .1 .10 verifies the differential pressure transmitter system. } \\
\hline$i^{7.1 .10 .1}$ & $\begin{array}{l}\text { REMOVE PLUGS } \\
\text { BAL PDIT-*60 }\end{array}$ & $\mathrm{N} / \mathrm{A}$ & $N / A$ & & \\
\hline $\begin{array}{l}1.7 .1 .10 .2 \\
\text { gen }\end{array}$ & $\begin{array}{l}\text { CLOSE FU-14 } \\
\text { REC PDIT }-* 60 \\
\text { PRESSURE }\end{array}$ & .035 & $\begin{array}{l}\text { PDIT } * 60 \\
\text { PRESSURE } \\
0 \pm 1{ }^{\prime \prime} \mathrm{H}_{2} \mathrm{O}\end{array}$ & & \\
\hline $1.7 \mathrm{jis}^{10.3}$ & $\begin{array}{l}M U X-* 70 \text { VdC } \\
T B 7+\& \text { TB8- }\end{array}$ & $N / \Delta$ & $\begin{array}{l}M U X-\star 70 V \mathrm{Vdc} \\
2.2 \pm 0.1 \pm \\
\end{array}$ & & \\
\hline $\begin{array}{l}1.7 .1 .10 .4 \\
607 \\
\end{array}$ & $\begin{array}{l}\text { REPLACE } \\
\text { PLUGS ON } \\
\text { PDIT-*60 }\end{array}$ & $N / A$ & $N / A$ & & \\
\hline $\begin{array}{l}1.7 .1 .10 .5 \\
601 \\
\end{array}$ & OPEN FU-14 & $N / A$ & $N / A$ & & \\
\hline \multicolumn{6}{|c|}{ Section 1.7.1.11 verifies the gas chromatograph power. } \\
\hline Goin & CLOSE FU- 11 & $N / A$ & $N / A$ & 今 & \\
\hline 1.7 .1 .11 .2 & $\begin{array}{l}\text { POWER } \\
\text { MON }-* 60\end{array}$ & 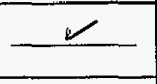 & $\begin{array}{l}\text { MON-*60 } \\
\text { POWERED }\end{array}$ & A & $\begin{array}{l}\text { PS.10O AT } \\
121.9\end{array}$ \\
\hline 1.7 .1 .11 .3 & OPEN FU-11 & $N / A$ & $N / A$ & 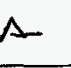 & \\
\hline
\end{tabular}


TEST DATA SHEET

\begin{tabular}{|c|c|c|c|c|c|}
\hline Date: $26 F$ & 3.97 & & SHMS Unit Number: & $\mathrm{MC}$ & \pm 12 \\
\hline $\begin{array}{l}\text { Title of Test: } \\
\text { Electrical }\end{array}$ & stems & & Test Equipment ar & $\mathrm{S} / \mathrm{N}$ or & al. No.: \\
\hline $\begin{array}{l}\text { Test Performed } \\
\text { Sarz-TFest }\end{array}$ & Laxos stem Po. & $3 \times 08$ & $\begin{array}{l}\text { Son } 446 \\
\text { Due } 101\end{array}$ & $\begin{array}{l}0 \times 0 \\
7\end{array}$ & 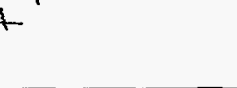 \\
\hline $\begin{array}{l}\text { Procedure No. } \\
\text { initial/Date }\end{array}$ & I tem & value & $\begin{array}{l}\text { Required } \\
\text { Condition }\end{array}$ & $(A / R)$ & Comment \\
\hline Section 1.7 & .12 verifies & he muit & analyzer $p$ & & \\
\hline i.t.12.1 & CLOSE FU-12 & $N / A$ & $N / A$ & $\Delta$ & $N / \Delta$ \\
\hline $\begin{array}{l}1.7 .1 .12 .2 \\
\text { Coten }\end{array}$ & $\begin{array}{l}\text { POWER } \\
\text { NIT }-552\end{array}$ & $\longleftarrow$ & $\begin{array}{l}\text { NIT }-* 52 \\
\text { POWERED }\end{array}$ & $\Lambda$ & 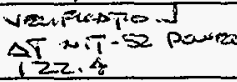 \\
\hline $\operatorname{Eosin}^{1.1 .12 .3}$ & OPEN FU 12 & $N / A$ & $N / A$ & $\Delta$ & $N / \Delta$ \\
\hline Section 1.7 & .13 verifjes & e per & computer por & & \\
\hline $\begin{array}{l}1.7 .1 .13 .1 \\
\text { coten }\end{array}$ & CLOSE CB- 6 & $N / A$ & $N / A$ & $\Lambda$ & $\begin{array}{l}\text { Ver. F. DAD } \\
\text { AT PAss. 1.7.1 }\end{array}$ \\
\hline $\begin{array}{l}1.7 .1 .13 .2 \\
0.24 \\
\end{array}$ & $\begin{array}{l}\text { POWER PC-*60 } \\
\& P C-* 70\end{array}$ & 2 & $\begin{array}{ll}\mathrm{PC}-* 60 & \mathrm{ON} \\
\mathrm{PC}-* 70 & \mathrm{ON} \\
\end{array}$ & A & \\
\hline $\sin ^{1.7 .1 .13 .3}$ & $\begin{array}{l}\text { TURN INST. } \\
\text { OFF \& } \\
\text { OPEN CB-6 }\end{array}$ & 2 & $\begin{array}{ll}P C-* 60 & O F F \\
P C-* 70 & O F F\end{array}$ & $\Lambda$ & $\int$ \\
\hline
\end{tabular}

Gem suc $2 / 26197$ 
TEST DATA SHEET

\begin{tabular}{|c|c|c|c|c|c|}
\hline \multicolumn{3}{|c|}{ Date: 26197} & \multicolumn{3}{|c|}{ SHMS Unit Number: MCED 12} \\
\hline \multicolumn{3}{|c|}{$\begin{array}{l}\text { Title of Test: } \\
\text { Electrical Systems }\end{array}$} & \multirow{2}{*}{\multicolumn{3}{|c|}{$\begin{array}{l}\text { Test Equipment and S/N or Cal. No.: } \\
\text { Fuic } v / 0 \text { meTeR } \\
\text { Sul } 446203604 \\
\text { Diz } 10197\end{array}$}} \\
\hline \multicolumn{3}{|c|}{ 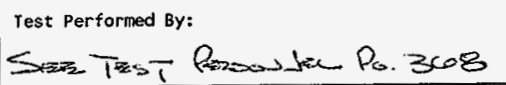 } & & & \\
\hline $\begin{array}{l}\text { Procedure No. } \\
\text { Initial/Date }\end{array}$ & I ten & value & $\begin{array}{c}\text { Required } \\
\text { Condition } \\
\end{array}$ & $(A / R)$ & Comment \\
\hline \multicolumn{6}{|c|}{ Section 1.7 .2 verifies the intrinsic safety apparatus is properly labeled } \\
\hline $\begin{array}{l}1.7 .2 .1 \\
\text { coesin }\end{array}$ & $\begin{array}{l}\text { INTRINSIC } \\
\text { SAFETY } \\
\text { APPARATUS } \\
\text { NAMEPLATES } \\
\text { INSTALLED }\end{array}$ & $\frac{2}{2}$ & $\begin{array}{l}\mathrm{NE}-* 54 \\
\mathrm{NE}-* 55 \\
\mathrm{~EB}-* 54 \\
\mathrm{~EB}-* 55 \\
\mathrm{NIT}-* 54 \\
\text { NIT } * 55\end{array}$ & $A$ & \\
\hline $\begin{array}{l}1.7 .2 .2 \\
621 \\
\end{array}$ & $\begin{array}{l}\text { INTRINSIC } \\
\text { SAFETY WIRE } \\
\text { LABELED }\end{array}$ & $\frac{2}{2}$ & $\begin{array}{l}\text { NE/EB-*54 } \\
\text { NE/EB-*55 }\end{array}$ & $\Delta$ & \\
\hline 6.7.3 & $\begin{array}{l}\text { SEC. } 1.7 .1 \text { \& } \\
1.7 .2 \text { DONE }\end{array}$ & $\frac{\gamma /}{\text { Test Di }}$ & or Signatur & & $\frac{-20-97}{\text { Date }}$ \\
\hline
\end{tabular}

2lowiz Test Witness/Review:
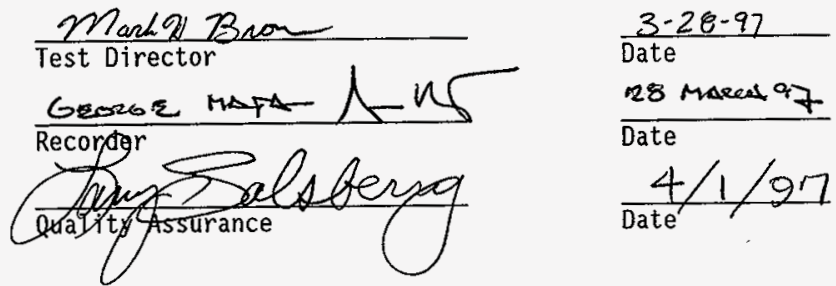
APPENDIX L

PAGE $L-14^{\circ}$
INF - SD-WM-ATR- 191

Rev. 0

TEST DATA SHEET

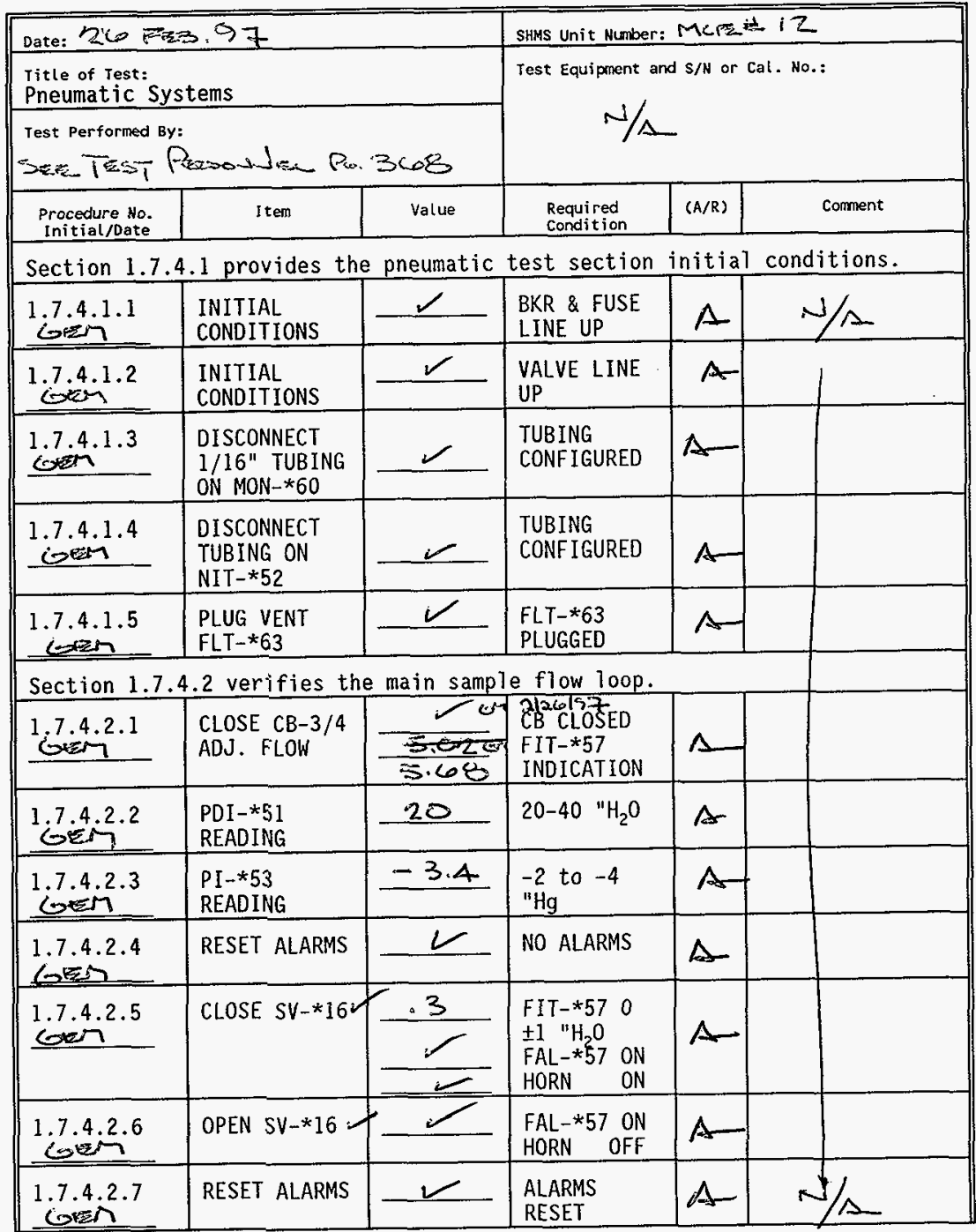

$\cos \Delta \sin _{2}$ zoos 
APPENDIX L

HNF-SD-WM-ATR-191

PAGE L-15

Rev. 0

Page 381

TEST DATA SHEET

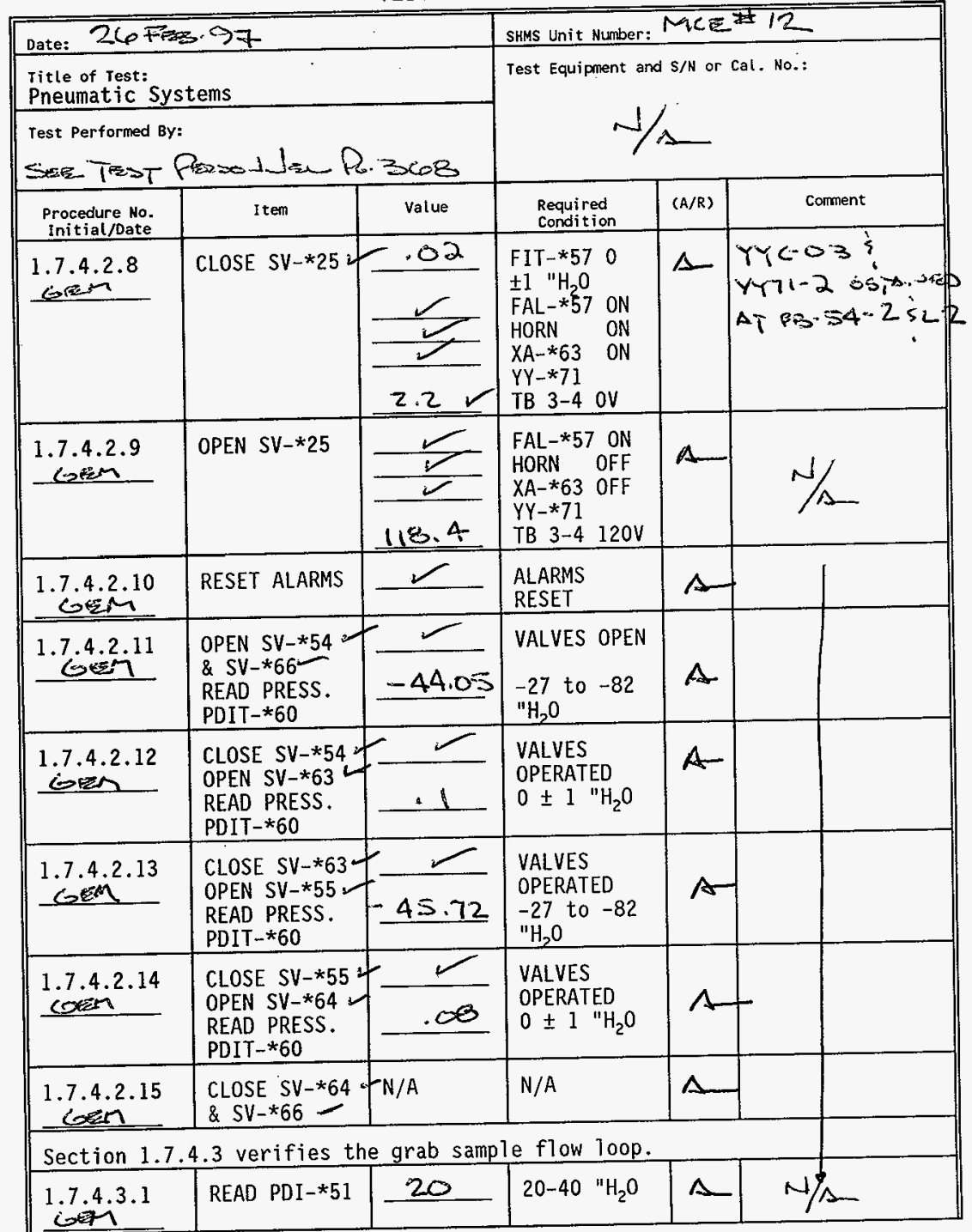

ints las 
TEST DATA SHEET

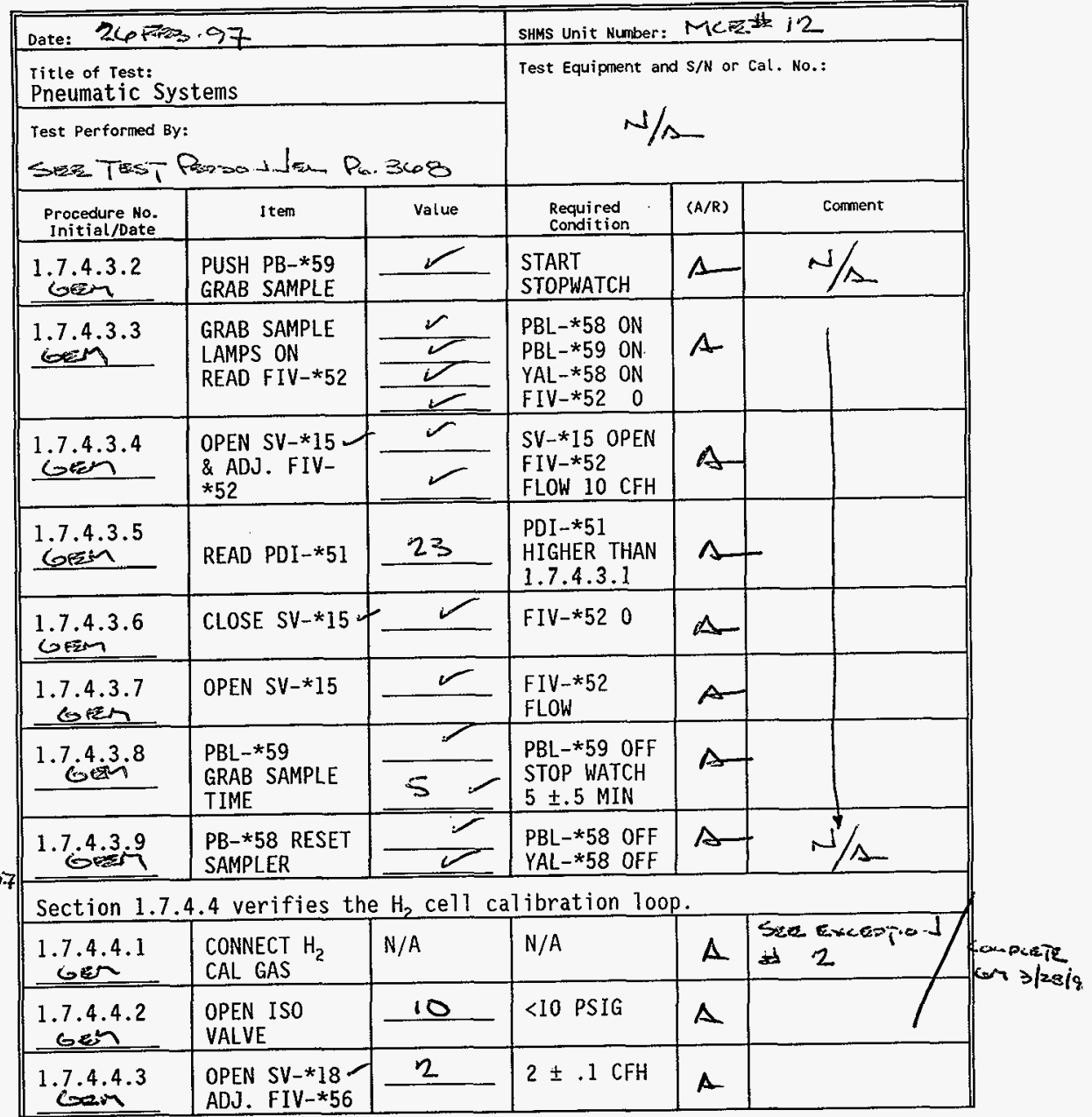

$4 \quad \operatorname{cen}$ sce 3izs/77 
TEST DATA SHEET

\begin{tabular}{|c|c|c|c|c|c|}
\hline \multicolumn{3}{|c|}{ Date: 23 Maecis 97} & \multicolumn{3}{|c|}{ SHMS Unit Mumber: MCE 12} \\
\hline \multicolumn{3}{|c|}{$\begin{array}{l}\text { Title of Test: } \\
\text { Pneumatic Systems }\end{array}$} & \multirow{2}{*}{\multicolumn{3}{|c|}{$\begin{array}{l}\text { Test Equipment and S/N or Cal. No.: } \\
\text { FLUiLE V/O MPTER } \\
\text { SU } 44620364 \\
\text { DUE DOTE } 10197\end{array}$}} \\
\hline \multirow{2}{*}{$\begin{array}{l}\text { Test Performed By } \\
\text { Sae Tasi } \\
\begin{array}{c}\text { Procedure No. } \\
\text { Initial/Date }\end{array} \\
\end{array}$} & \multicolumn{2}{|c|}{ Parsosures Po. 368} & & & \\
\hline & I tem & value & $\begin{array}{l}\text { Required } \\
\text { Condition }\end{array}$ & $(A / R)$ & Comment \\
\hline $\begin{array}{l}1.7 .4 .4 .4 \\
\text { con }\end{array}$ & $\begin{array}{l}\text { VERIFY } \\
\text { CONDITIONS }\end{array}$ & $\frac{\frac{\gamma}{V}}{\frac{V}{V}}$ & $\begin{array}{l}N I T-* 54 \\
>.625 \% H_{2} \\
\text { NAH-*55 ON } \\
\text { NAH }-* 54 \text { ON } \\
\text { PBL-*59 ON } \\
\text { PBL-*58 ON } \\
\text { YAL-*58 ON } \\
\text { FIV } * 52 \text { FLO } \\
\text { HORN ON } \\
\text { TB2-2I/22 } \\
\text { OPEN } \\
\end{array}$ & $A$ & \\
\hline $\begin{array}{l}1.7 .4 .4 .5 \\
\cos \\
\end{array}$ & $\begin{array}{l}\text { ACKNOWLEDGE } \\
\text { HORN }\end{array}$ & $N / A$ & $N / A$ & $A$ & \\
\hline $\cos ^{1.4 .4}$ & $\begin{array}{l}\text { CLOSE SV-*I8 } \\
\text { VERIFY } \\
\text { CONDITIONS }\end{array}$ & 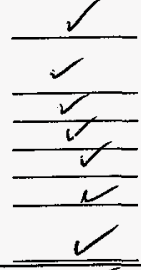 & $\begin{array}{l}\text { FIV-*56 O } \\
\text { NIT }-* 54 \\
<.625 \% \mathrm{H}_{2} \\
\text { NAH-*55 OFF } \\
\text { NAH } * 54 \text { ON } \\
\text { PBL-*58 ON } \\
\text { YAL-*58 ON } \\
\text { TB2-21/22 } \\
\text { CLOSED } \\
\end{array}$ & $A$ & \\
\hline $\begin{array}{l}1.7 .4 .4 .7 \\
\text { Gen }\end{array}$ & $\begin{array}{l}\text { PUSH RESET } \\
\text { SAMPLER PB- } \\
* 58 \\
\end{array}$ & $\frac{\swarrow}{L}$ & $\begin{array}{l}\text { PBL-*58 OFF } \\
\text { YAL-*58 OFF }\end{array}$ & A & \\
\hline $\begin{array}{c}1.7 .4 .4 .8 \\
6 \text { 6in } \\
\end{array}$ & RESET ALARM & $\checkmark$ & $\mathrm{NAH}-\star 54$ OFF & A & \\
\hline $\begin{array}{l}1.7 .4 .4 .9 \\
\cos ^{2}\end{array}$ & $\begin{array}{l}\text { OPEN SV-*19 } \\
\text { ADJ. FIV }-* 56\end{array}$ & 2 & $2 \pm .1 \mathrm{CFH}$ & $A$ & \\
\hline
\end{tabular}

Gey 3128197

su 
TEST DATA SHEET

\begin{tabular}{|c|c|c|c|c|c|}
\hline \multicolumn{3}{|c|}{ Date: 28 mazcis 97} & \multicolumn{3}{|c|}{ SHMS Unit Number: MCE \#12 } \\
\hline \multicolumn{3}{|c|}{$\begin{array}{l}\text { Title of Test: } \\
\text { Pneumatic Systems }\end{array}$} & \multirow{2}{*}{\multicolumn{3}{|c|}{ 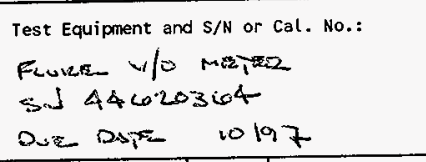 }} \\
\hline \multicolumn{3}{|c|}{$\begin{array}{l}\text { Test Performed By: } \\
\text { Szer Test Parsonden R. } 368\end{array}$} & & & \\
\hline $\begin{array}{l}\text { Procedure No. } \\
\text { Initial/Date }\end{array}$ & Item & value & $\begin{array}{l}\text { Required } \\
\text { Cordition }\end{array}$ & $(A / R)$ & Comment \\
\hline $\begin{array}{l}1.7 .4 .4 .10 \\
\text { Givin }\end{array}$ & $\begin{array}{l}\text { VERIFY } \\
\text { CONDITIONS }\end{array}$ & 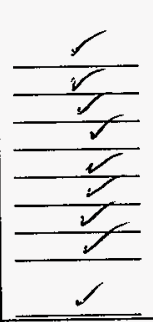 & $\begin{array}{l}\text { NIT-*55 } \\
>625 \% \quad H_{2} \\
\text { NAH-*55 ON } \\
\text { NAH-*54 ON } \\
\text { PBL-*59 ON } \\
\text { PBL-*58 ON } \\
\text { YAL-*58 ON } \\
\text { FIV }-* 52 \quad \text { FLO } \\
\text { HORN ON } \\
\text { TB2-21/22 } \\
\text { OPEN } \\
\end{array}$ & $\Delta$ & \\
\hline$\frac{1.7 .4 .4 .11}{G \mathrm{em}}$ & $\begin{array}{l}\text { ACKNOWLEDGE } \\
\text { HORN }\end{array}$ & N/A & N/A & A & \\
\hline $\begin{array}{c}1.7 .4 .4 .12 \\
\cos \end{array}$ & $\begin{array}{l}\text { CLOSE SV }-* 19 \\
\text { VERIFY } \\
\text { CONDITIONS }\end{array}$ & 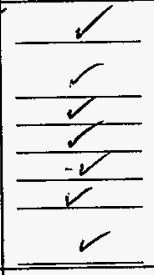 & \begin{tabular}{|l|l|l} 
FIV-*56 & 0 \\
NIT $-* 55$ & \\
$<.625 \% \quad H_{2}$ \\
NAH-*55 OFF \\
NAH-*54 ON \\
PBL-*58 ON \\
YAL-*58 ON \\
TB2-21/22 \\
CL.OSED \\
\end{tabular} & $A$ & \\
\hline $\begin{array}{c}1.7 .4 .4 .13 \\
\end{array}$ & $\begin{array}{l}\text { PUSH RESET } \\
\text { SAMPLER PB- } \\
\star 58 \\
\end{array}$ & $\frac{\swarrow}{r}$ & $\begin{array}{l}\mathrm{PBL}-* 58 \quad 0 \mathrm{FF} \\
\text { YAL-*58 } \mathrm{OFF}\end{array}$ & $A$ & \\
\hline 1.7 .4 .4 .14 & RESET ALARM & $\swarrow$ & $\mathrm{NAH}-\star 54$ OFF & $A$ & \\
\hline 1.7 .4 .4 .15 & $\begin{array}{l}\text { DISCONNECT } \\
\text { CAL. GAS } \\
\end{array}$ & N/A & N/A & $A$ & \\
\hline $\begin{array}{l}1.7 .4 .4 .16 \\
6 i^{2} i^{2} \\
\end{array}$ & $\begin{array}{l}\text { PUSH PB-*54 } \\
\text { VERIFY } \\
\text { CONDITIONS }\end{array}$ & $\begin{array}{r}\swarrow \\
\swarrow \\
\end{array}$ & $\begin{array}{l}X A-* 63 \text { ON } \\
P B L-* 54 \text { ON } \\
T B 2-23 / 24 \\
\text { OPEN } \\
\end{array}$ & $\triangle$ & \\
\hline $\begin{array}{l}1.7 .4 .4 .17 \\
6.2 m\end{array}$ & $\begin{array}{l}\text { PUSH PB-*54 } \\
\text { VERIFY } \\
\text { CONDITIONS }\end{array}$ & $\frac{\swarrow}{K}$ & $\begin{array}{l}\mathrm{XA-} * 63 \text { OFF } \\
\mathrm{PBL}-* 54 \text { OFF } \\
\text { TB2-23/24 } \\
\text { CLOSED }\end{array}$ & $\Delta$ & \\
\hline
\end{tabular}


APPENDIX L

PAGE L-19
HNF-SD-WM-ATR- 191

Rev. 0

TEST DATA SHEET

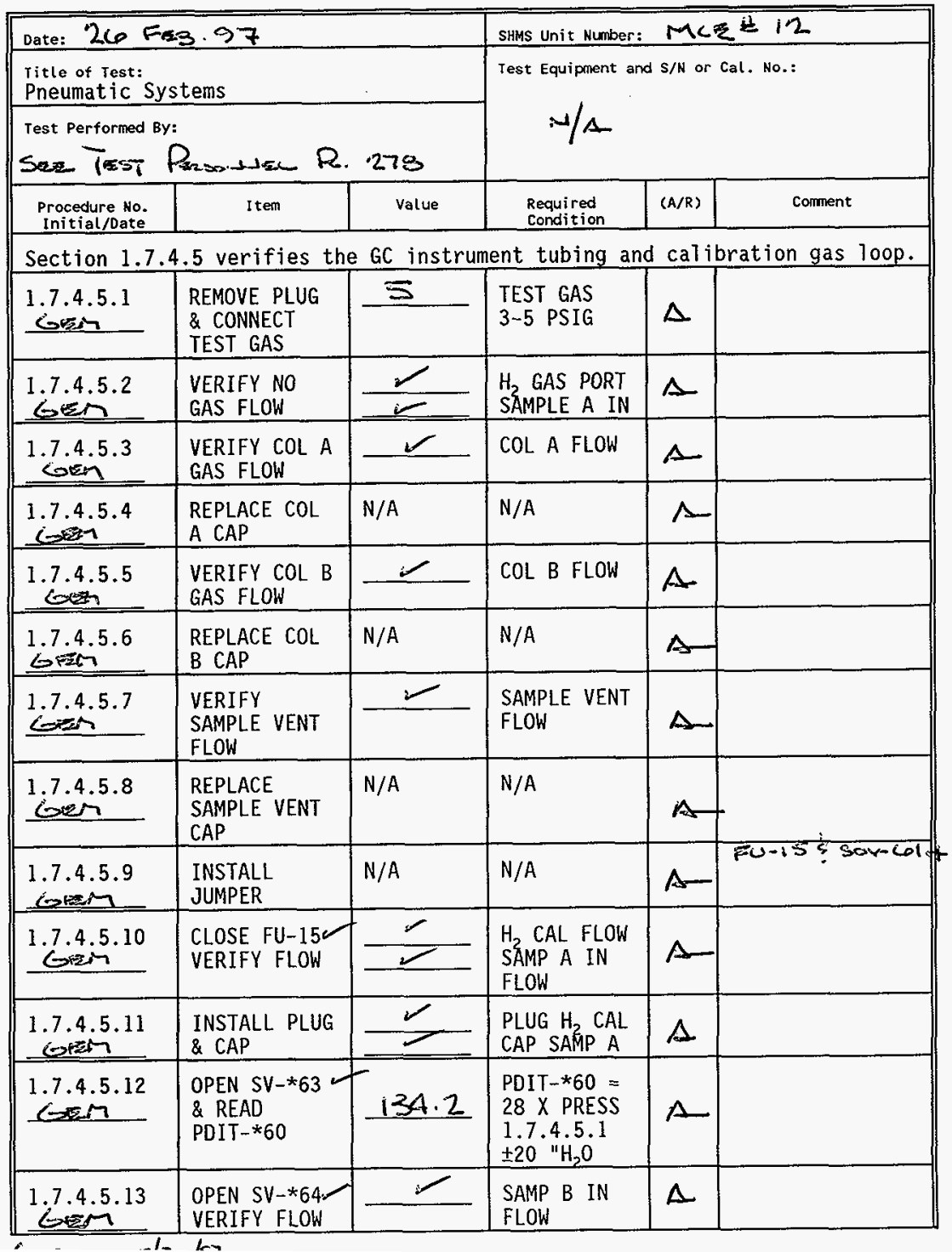


APPENDIX L

TEST DATA SHEET

\begin{tabular}{|c|c|c|c|c|c|}
\hline \multirow{2}{*}{\multicolumn{3}{|c|}{$\begin{array}{l}\text { Date: } 26 \text { Fes } 397 \\
\text { Iitle of rest: } \\
\text { Pneumatic Systems }\end{array}$}} & \multicolumn{3}{|c|}{ SHMS Unit Number: MCEAt 12} \\
\hline & & & \multirow{2}{*}{\multicolumn{3}{|c|}{ Test Equipment and $\mathrm{S} / \mathrm{N}$ or Cal. No.: }} \\
\hline \multicolumn{3}{|c|}{ 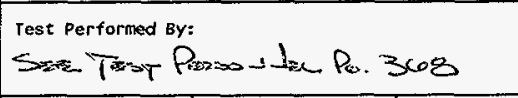 } & & & \\
\hline $\begin{array}{l}\text { Procedure No. } \\
\text { Initial/Date }\end{array}$ & Item & Value & $\begin{array}{l}\text { Required } \\
\text { Condition }\end{array}$ & $(A / R)$ & Corment \\
\hline $\begin{array}{l}1.7 .4 .5 .14 \\
\text { sogen }\end{array}$ & $\begin{array}{l}\text { REMOVE TEST } \\
\text { GAS }\end{array}$ & $N / A$ & N/A & $\Delta$ & $N / \mathrm{s}$ \\
\hline $\begin{array}{l}1.7 .4 .5 .15 \\
\text { Gen }\end{array}$ & $\begin{array}{l}\text { CLOSE SV-*63- } \\
\& \text { SV-*64 }\end{array}$ & N/A & N/A & $\Lambda$ & \\
\hline $\begin{array}{l}1.7 .4 .5 .16 \\
\text { cEn }\end{array}$ & $\begin{array}{l}\text { OPEN FU-15 } \\
\text { REMOVE } \\
\text { JUMPER } \\
\end{array}$ & N/A & $N / A$ & $\mathrm{~A}$ & \\
\hline $\begin{array}{l}1.4 .5 .17 \\
\end{array}$ & $\begin{array}{l}\text { INSTALL } \\
\text { SAMP A UNION } \\
\end{array}$ & $N / A$ & N/A & 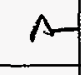 & \\
\hline 1.7 .4 .5 .18 & $\begin{array}{l}\text { OPEN SV-*60 } \\
\text { VERIFY FLOW } \\
\end{array}$ & & $\begin{array}{l}\text { FI-*60 } \\
\text { NO FLOW } \\
\end{array}$ & $A$ & \\
\hline$\frac{1.7 .4 .5 .19}{10}$ & $\begin{array}{l}\text { POWER SOV- } \\
* 60 \text {, VERIFY } \\
\text { NO FLOW } \\
\end{array}$ & & $\begin{array}{l}\text { FI }-* 60 \\
\text { NO FLOW }\end{array}$ & 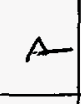 & \\
\hline $\begin{array}{r}1.7 .4 .5 .20 \\
0.27 \\
\end{array}$ & $\begin{array}{l}\text { OPEN SV-*68 } \\
\text { ADJ SV-*67 - } \\
\end{array}$ & 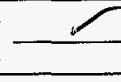 & $\begin{array}{l}F I-* 60 \\
50 \mathrm{CCM}\end{array}$ & $\Delta$ & \\
\hline $\cos ^{1.7 .4 .51}$ & $\begin{array}{l}\text { DEENERGIZE } \\
\text { SOV-*60 } \\
\end{array}$ & & $\begin{array}{l}\text { FI }-* 60 \text { FLOW } \\
\text { DECREASES }\end{array}$ & $\Delta$ & \\
\hline $\begin{array}{l}1.7 .4 .5 .22 \\
\cos \end{array}$ & $\begin{array}{l}\text { CLOSE SV-*60 } \\
\& \text { SV-*68 }\end{array}$ & N/A & N/A & $\Lambda$ & _ \\
\hline $\begin{array}{c}1.7 .4 .5 .23 \\
0.02\end{array}$ & $\begin{array}{l}\text { REMOVE } \\
\text { SAMP A UNION } \\
\end{array}$ & $N / A$ & N/A & $A$ & \\
\hline $\operatorname{cosin}^{1.7 .54}$ & $\begin{array}{l}\text { CONNECT TEST } \\
\text { GAS } \mathrm{N}_{2} \mathrm{O} \text { PORT } \\
\end{array}$ & 5 & $\begin{array}{l}\text { TEST GAS } \\
3-5 \text { PSIG } \\
\end{array}$ & $\Delta$ & \\
\hline$\frac{1.7 .4 .5 .25}{\tan }$ & $\begin{array}{l}\text { VERIFY NO } \\
\text { FLOW }\end{array}$ & -1 & $\begin{array}{l}\text { SAMP B IN \& } \\
\text { FLT-*63 } \\
\text { NO FLOW } \\
\end{array}$ & $\Delta$ & \\
\hline $\begin{array}{l}1.7 .4 .5 .26 \\
\end{array}$ & $\begin{array}{l}\text { INSTALL } \\
\text { JUMPER }\end{array}$ & $\mathrm{N} / \mathrm{A}$ & N/A & $\Lambda$ & 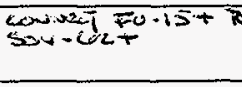 \\
\hline 1.7 .4 .5 .27 & $\begin{array}{l}\text { CLOSE FU-15 } \\
\text { VERIFY FLOW }\end{array}$ & -2 & $\begin{array}{l}\text { SAMP B IN } \\
\text { FLT-*63 } \\
\text { FLOW }\end{array}$ & $\Delta$ & 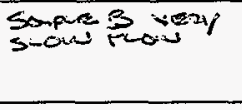 \\
\hline
\end{tabular}

$\cos \operatorname{sic} 2 / 26 / 97$ 
TEST DATA SHEET

\begin{tabular}{|c|c|c|c|c|c|c|}
\hline \multicolumn{3}{|c|}{ Date: 2603.97} & SHMS Unit Number: & \multicolumn{3}{|c|}{ MCFE 12} \\
\hline \multicolumn{3}{|c|}{$\begin{array}{l}\text { Title of Test: } \\
\text { Pneumatic Systems }\end{array}$} & \multirow{2}{*}{\multicolumn{4}{|c|}{ Test Equipment and $S / \mathrm{N}$ or Cal. No.: }} \\
\hline \multicolumn{3}{|c|}{ 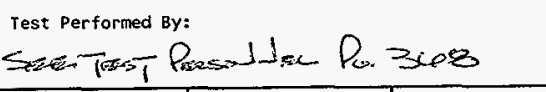 } & & & & \\
\hline $\begin{array}{l}\text { Procedure No. } \\
\text { Initial/Date }\end{array}$ & Item & Value & $\begin{array}{l}\text { Required } \\
\text { condition } \\
\end{array}$ & $(A / R)$ & & Comment \\
\hline $\begin{array}{l}1.7 .4 .5 .28 \\
\text { Gern } \\
\end{array}$ & $\begin{array}{l}\text { DISCONNECT } \\
\text { TEST GAS } \\
\end{array}$ & $N / A$ & $N / A$ & $\Delta$ & & $1 / 1$ \\
\hline $\begin{array}{l}1.7 .4 .5 .29 \\
\end{array}$ & OPEN FU-15 & N/A & $\mathrm{N} / \mathrm{A}$ & $\Lambda$ & & \\
\hline 1.7 .4 .5 .30 & $\begin{array}{l}\text { REMOVE } \\
\text { JUMPER }\end{array}$ & $N / A$ & N/A & $\Delta$ & & \\
\hline $\begin{array}{l}1.7 .4 .5 .31 \\
\mathrm{Eam} \\
\end{array}$ & $\begin{array}{l}\text { INSTALL } \\
\text { SAMP B UNION }\end{array}$ & N/A & $\mathrm{N} / \mathrm{A}$ & A & & \\
\hline $\begin{array}{l}1.7 .4 .5 .32 \\
\text { Gon } \\
\end{array}$ & $\begin{array}{l}\text { OPEN SV-*60 } \\
\text { VERIFY FLOW }\end{array}$ & 2 & $\begin{array}{l}\text { FI-*60 } \\
\text { NO FLOW }\end{array}$ & $\Delta$ & & \\
\hline $\begin{array}{l}1.7 .4 .5 .33 \\
\operatorname{sen} \\
\end{array}$ & $\begin{array}{l}\text { POWER SOV- } \\
* 60, \text { VERIFY } \\
\text { NO FLOW }\end{array}$ & $x^{2}$ & $\begin{array}{l}\text { FI-*60 } \\
\text { NO FLOW }\end{array}$ & $\Delta$ & & \\
\hline$\underline{i}^{1.4 .4 .5 .34}$ & $\begin{array}{l}\text { OPEN SV }-* 68 \\
\& \text { ADJ SV }-* 67\end{array}$ & 2 & $\begin{array}{l}\mathrm{FI}-\star 60 \\
50 \mathrm{cCM} \\
\end{array}$ & $\Lambda$ & & \\
\hline $\begin{array}{l}1.7 .4 .5 .35 \\
\text { Gras } \\
\end{array}$ & $\begin{array}{l}\text { DEENERGIZE } \\
\text { SOV-*60 }\end{array}$ & 2 & $\begin{array}{l}\text { FI-*60 FLOW } \\
\text { DECREASES }\end{array}$ & $\Delta$ & & \\
\hline $\begin{array}{l}1.7 .4 .5 .36 \\
6.97 \\
\end{array}$ & $\begin{array}{l}\text { CLOSE SV-*60- } \\
\& \text { SV-*68- }\end{array}$ & $\mathrm{N} / \mathrm{A}$ & N/A & $\Lambda$ & & \\
\hline $\begin{array}{l}1.7 .4 .5 .37 \\
\cos \\
\end{array}$ & $\begin{array}{l}\text { REMOVE } \\
\text { SAMP B UNION }\end{array}$ & $N / A$ & $\mathrm{~N} / \mathrm{A}$ & $\Delta$ & & \\
\hline $\begin{array}{c}1.7 .4 .5 .38 \\
\text { Gect } \\
\end{array}$ & $\begin{array}{l}\text { OPEN CB-3/4 } \\
\text { ACK. ALARMS }\end{array}$ & $N / A$ & $N / A$ & $\Delta$ & & \\
\hline \multicolumn{6}{|c|}{$\begin{array}{l}\text { Section } 1.7 .4 .6 \text { verifies the multi gas analyzer instrument tubing } \\
\text { calibration gas loop. }\end{array}$} & and \\
\hline 1.7 .4 .6 .1 & $\begin{array}{l}\text { VERIFY } \\
\text { NIT-*52 } \\
\text { SAMPLE LINES } \\
\end{array}$ & N/A & $N / A$ & A & & \\
\hline 1.7 .4 .6 .2 & $\begin{array}{l}\text { CONNECT TEST } \\
\text { GAS NH } \\
\text { PORT }\end{array}$ & $s$ & $\begin{array}{l}\text { TEST GAS } \\
3-5 \text { PSIG } \\
\end{array}$ & $A$ & & \\
\hline $\begin{array}{l}1.4 .6 .3 \\
\text { 乌ign }\end{array}$ & $\begin{array}{l}\text { VERIFY NO } \\
\text { FLOW }\end{array}$ & 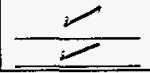 & $\begin{array}{l}\text { SAMPLE IN } \\
\text { FLT-* } 63 \\
\end{array}$ & $\Lambda$ & $N / s$ & \\
\hline
\end{tabular}


APPENDIX L

TEST DATA SHEET

\begin{tabular}{|c|c|c|c|c|c|}
\hline \multicolumn{3}{|c|}{ Date: 2CFFes 97 } & \multicolumn{3}{|c|}{ SHMS Unit Number: Mcke \pm 12} \\
\hline \multicolumn{3}{|c|}{$\begin{array}{l}\text { Iitle of Jest: } \\
\text { Pneumatic Systems }\end{array}$} & \multirow{2}{*}{\multicolumn{3}{|c|}{ Test Equipment and $\mathrm{S} / \mathrm{N}$ or Cal. No.: }} \\
\hline \multicolumn{3}{|c|}{ 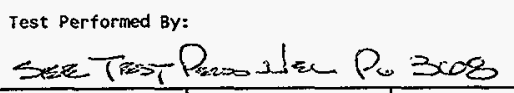 } & & & \\
\hline $\begin{array}{l}\text { Procedure No. } \\
\text { Initial/Date }\end{array}$ & I tem & Value & $\begin{array}{l}\text { Required } \\
\text { Condition }\end{array}$ & $(A / R)$ & Comment \\
\hline $\begin{array}{l}1.7 .4 .6 .4 \\
\sin \end{array}$ & $\begin{array}{l}\text { INSTALL } \\
\text { JUMPER }\end{array}$ & $N / A$ & $N / A$ & $A$ & Funst : soy:- \\
\hline 1.7 .4 .6 .5 & $\begin{array}{l}\text { CLOSE FU-15 } \\
\text { VERIFY GAS } \\
\text { FLOWS }\end{array}$ & 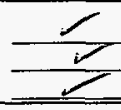 & $\begin{array}{l}\text { SAMPLE IN } \\
\text { FLT-*63 } \\
\text { FIV-*70 }\end{array}$ & $\Delta$ & 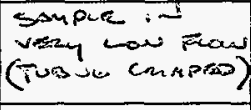 \\
\hline $\begin{array}{l}1.7 .4 .6 .6 \\
\text { Gizin } \\
\end{array}$ & $\begin{array}{l}\text { PLUG FLT } * 63 \\
\& \text { MON } * 60 \\
\text { SAMPLE VENT }\end{array}$ & $\mathbb{N} / A$ & $N / A$ & & \\
\hline 1.7.6.6.7 & $\begin{array}{l}\text { OPEN SV }-* 66, \\
\text { SV }-* 70 * \text { \& CAP } \\
\text { SAMPLE IN }\end{array}$ & 127. & $\begin{array}{l}\text { PDIT }-* 60= \\
28 \times \text { PRESS } \\
1.7 .4 .6 .2 \\
\pm 20 \mathrm{H}_{2} \mathrm{O}\end{array}$ & $A$ & \\
\hline $\begin{array}{l}1.7 .4 .6 .8 \\
\Leftrightarrow \text { 요 }\end{array}$ & $\begin{array}{l}\text { CLOSE SV }=* 66 \\
\text { SV-*70 } \frac{1}{8} \\
\text { REMOVE CAP }\end{array}$ & $-N / A$ & $N / A$ & $A$ & \\
\hline $\begin{array}{c}1.7 .4 .6 .9 \\
\end{array}$ & $\begin{array}{l}\text { REMOVE FLT- } \\
\star 63 \text { PLUG }\end{array}$ & $N / A$ & $N / A$ & A & \\
\hline 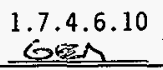 & $\begin{array}{l}\text { REMOVE TEST } \\
\text { GAS }\end{array}$ & $N / A$ & $N / A$ & & \\
\hline $\begin{array}{c}1.7 .4 .6 .11 \\
\text { Erin } \\
\end{array}$ & $\begin{array}{l}\text { CLOSE SV-*05 } \\
\& S V-* 16 \neq\end{array}$ & $N / A$ & $N / A$ & & \\
\hline $\begin{array}{l}1.7 .4 .6 .12 \\
\Leftrightarrow .4 \\
\end{array}$ & $\begin{array}{l}\text { CONNECT TEST } \\
\text { GAS }\end{array}$ & 4 & $\begin{array}{l}\text { TEST GAS } \\
2-4 \text { PSIG } \\
\end{array}$ & 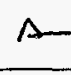 & \\
\hline $\begin{array}{l}1.7 .4 .6 .13 \\
\text { Gen }\end{array}$ & $\begin{array}{l}\text { OPEN SV }-* 07 \\
\& \text { VERIFY } \\
\text { NO FLOW }\end{array}$ & & $\begin{array}{ll}\text { SAMPLE } & \text { IN } \\
\text { SAMPLE } & \text { OUT }\end{array}$ & & \\
\hline $\begin{array}{c}1.7 .4 .6 .14 \\
0^{2 i m} \\
\end{array}$ & $\begin{array}{l}\text { OPEN SV }-* 09: \\
\& \text { VERIFY } \\
\text { NO FLOW }\end{array}$ & - & $\begin{array}{ll}\text { SAMPLE } & \text { IN } \\
\text { SAMPLE OUT }\end{array}$ & & \\
\hline $\begin{array}{l}1.7 .4 .6 .15 \\
\text { Gezn } \\
\end{array}$ & $\begin{array}{l}\text { OPEN FU-15 \& } \\
\text { VERIFY FLOW }\end{array}$ & 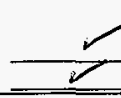 & $\begin{array}{l}\text { SAMPLE FLOW } \\
\text { IN - YES } \\
\text { OUT - NO } \\
\end{array}$ & & \\
\hline $\begin{array}{l}1.7 .4 .6 .16 \\
625 \\
\end{array}$ & $\begin{array}{l}\text { CLOSE SV-*09 } \\
\text { VERIFY FLOW }\end{array}$ & & $\begin{array}{l}\text { SAMPLE IN } \\
\text { NO FLOW }\end{array}$ & $\wedge$ & \\
\hline
\end{tabular}


APPENDIX L

TEST DATA SHEET

\begin{tabular}{|c|c|c|c|c|c|}
\hline \multicolumn{3}{|c|}{ Date: 26 Fs. 97} & \multicolumn{3}{|c|}{ SHMS Unit Number: MCzzat 12} \\
\hline \multicolumn{3}{|c|}{$\begin{array}{l}\text { Title of Test: } \\
\text { Pneumatic Systems }\end{array}$} & \multirow{2}{*}{\multicolumn{3}{|c|}{ Test Equipment and $S / \mathrm{N}$ or Cal. No.: }} \\
\hline \multicolumn{3}{|c|}{ 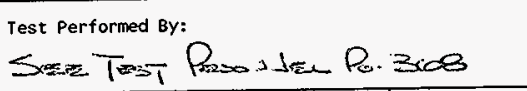 } & & & \\
\hline $\begin{array}{l}\text { Procedure No. } \\
\text { Initial/Date }\end{array}$ & I tem & Value & $\begin{array}{l}\text { Required } \\
\text { Condition }\end{array}$ & $\langle A / R\rangle$ & comment \\
\hline 1.7.4.6.17 & $\begin{array}{l}\text { OPEN SV-*10 } \\
\text { VERIFY FLOW }\end{array}$ & 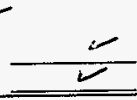 & $\begin{array}{l}\text { SAMPLE FLOW } \\
\text { IN - NO } \\
\text { OUT - YES }\end{array}$ & $\Lambda$ & $N / \mathrm{s}$ \\
\hline $\begin{array}{l}1.7 .4 .6 .18 \\
\text { Goen }\end{array}$ & $\begin{array}{l}\text { REMOVE TEST } \\
\text { GAS \& PLUG } \\
\text { SV-*07 }\end{array}$ & $N / A$ & $N / A$ & $\wedge$ & \\
\hline $\begin{array}{l}1.7 .4 .6 .19 \\
2817 \\
\end{array}$ & $\begin{array}{l}\text { CLOSE SV-*07- } \\
\& \text { SV-*10 }\end{array}$ & $N / A$ & $N / A$ & $\Delta$ & \\
\hline 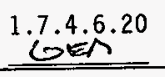 & $\begin{array}{l}\text { OPEN SV-*05 } \\
\& \text { SV-*16- }\end{array}$ & $N / A$ & $\mathrm{~N} / \mathrm{A}$ & 1 & \\
\hline $\begin{array}{c}1.7 .4 .6 .21 \\
\end{array}$ & $\begin{array}{l}\text { REMOVE } \\
\text { JUMPER }\end{array}$ & N/A & N/A & $\Delta$ & \\
\hline \multicolumn{6}{|c|}{ Section 1.7.4.7 verifies the carrier gas supply 1 ines. } \\
\hline $\begin{array}{c}1.7 .4 .7 .1 \\
\text { Gein }\end{array}$ & $\begin{array}{l}\text { CONNECT TEST } \\
\text { GAS }\end{array}$ & 4 & $3-5$ PSIG & $\Delta$ & \\
\hline $\begin{array}{l}1.7 .4 .7 .2 \\
\text { con }\end{array}$ & $\begin{array}{l}\text { REMOVE PLUG } \\
\& \text { VERIFY } \\
\text { FLOW } \\
\end{array}$ & $r$ & $\begin{array}{l}\mathrm{N}_{3} \text { CARRIER } \\
\text { GAS FLOWS }\end{array}$ & $\Lambda$ & \\
\hline 1.7 .4 .7 .3 & $\begin{array}{l}\text { DISCONNECT } \\
\text { TEST GAS }\end{array}$ & $N / A$ & N/A & $A$ & \\
\hline $\begin{array}{l}1.7 .4 .7 .4 \\
\text { Gizi }\end{array}$ & $\begin{array}{l}\text { CONNECT TEST } \\
\text { GAS } \\
\end{array}$ & 4 & 3-5 PSIG & $\Delta$ & \\
\hline 1.7 .4 .7 .5 & $\begin{array}{l}\text { REMOVE PLUG } \\
\text { \& VERIFY } \\
\text { FLOW }\end{array}$ & 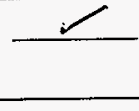 & $\begin{array}{l}\text { He CARRIER } \\
\text { GAS FLOWS }\end{array}$ & $N$ & \\
\hline $\begin{array}{l}1.7 .4 .7 .6 \\
\end{array}$ & $\begin{array}{l}\text { DISCONNECT } \\
\text { TEST GAS }\end{array}$ & $N / A$ & N/A & & 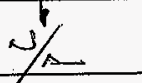 \\
\hline 1.7 .5 Gen & $\begin{array}{l}\text { TEST SEC. } \\
1.7 .4 \text { DONE }\end{array}$ & $\frac{\text { Marh }}{\text { Test Dire }}$ & 2). Boon & & $\frac{3-28-97}{\text { Date }}$ \\
\hline
\end{tabular}

Gren ac 2126157 
APPENDIX L

TEST DATA SHEET

\begin{tabular}{|c|c|c|c|c|c|}
\hline \multicolumn{3}{|c|}{ Date: N/S } & \multicolumn{3}{|c|}{ SHMS Unit Number: N/S } \\
\hline \multicolumn{3}{|c|}{$\begin{array}{l}\text { Title of Test: } \\
\text { Pneumatic Systems }\end{array}$} & \multicolumn{3}{|c|}{ Test Equipment and $\mathrm{S} / \mathrm{N}$ or Cal. No.: } \\
\hline \multicolumn{3}{|c|}{ Test Performed By: } & \multicolumn{3}{|c|}{$N / \Lambda$} \\
\hline $\begin{array}{l}\text { Procedure No. } \\
\text { Initial/oate }\end{array}$ & Item & Value & $\begin{array}{l}\text { Required } \\
\text { condition }\end{array}$ & $(A / R)$ & Comment \\
\hline $1.7 / \mathrm{A}=$ & \multicolumn{5}{|c|}{ 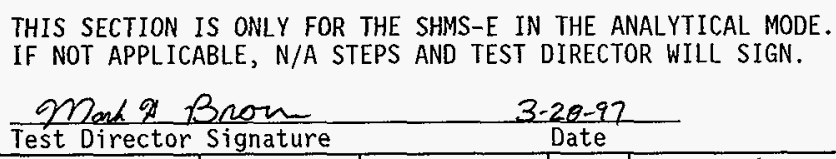 } \\
\hline 1.7 .6 .1 .1 & $\begin{array}{l}\text { VERIFY BKR } \\
\text { LINE UP }\end{array}$ & N/A & $N / A$ & & \\
\hline $1.7 \cdot 6.1 .2$ & $\begin{array}{l}\text { VERIFY VALVE } \\
\text { LINE UP }\end{array}$ & $N / A$ & $N / A$ & & \\
\hline 1.7 .6 .1 .3 & $\begin{array}{l}\text { VERIFY MON- } \\
\text { * } 60 \text { INSTALL }^{2}\end{array}$ & $N / A$ & N/A & & \\
\hline 1.7 .6 .1 .4 & $\begin{array}{l}\text { VERIFY NIT- } \\
* 52 \text { INSTALL }\end{array}$ & N/A & N/A & & \\
\hline 1.7 .6 .1 .5 & $\begin{array}{l}\text { CONNECT } H_{2} \\
\text { CAL GAS }\end{array}$ & N/A & N/A & & \\
\hline 1.7 .6 .1 .6 & $\begin{array}{l}\text { CONNECT } \mathrm{CH}_{4} \\
\text { CAL GAS }\end{array}$ & N/A & $\mathrm{N} / \mathrm{A}$ & & \\
\hline 1.7 .6 .1 .7 & $\begin{array}{l}\text { CONNECT } \mathrm{NH}_{3} \\
\text { CAL GAS }\end{array}$ & N/A & N/A & & \\
\hline \multicolumn{6}{|c|}{ Section 1.7 .6 .2 establishes the main and auxiliary loop flows } \\
\hline 1.7.6.2.1 & CLOSE FU-11 & $N / 1$ & $\begin{array}{l}\text { MON-*60 } \\
\text { POWERED } \\
\end{array}$ & & \\
\hline 1.7 .6 .2 .2 & POWER PC $-* 60$ & & $\begin{array}{l}\text { ESTABLISH } \\
\text { SAMPLING } \\
\text { ROUTINE }\end{array}$ & & \\
\hline 1.7 .6 .2 .3 & CLOSE FU-12 & & $\begin{array}{l}\text { NIT }-* 52 \\
\text { POWERED } \\
\end{array}$ & & \\
\hline 1.7 .6 .2 .4 & POWER PC $-* 70$ & & $\begin{array}{l}\text { PC-*70 } \\
\text { BOOTED UP }\end{array}$ & & \\
\hline 1.6 .2 .5 & $\begin{array}{l}\text { CLOSE CB-3/4 } \\
\text { ADJUST FLOW }\end{array}$ & $N / A$ & $\begin{array}{l}\mathrm{FIT}-* 57 \\
1 \pm .1 \quad \mathrm{H}_{2} \mathrm{O}\end{array}$ & & $N / \Lambda$ \\
\hline
\end{tabular}


APPENDIX L

TEST DATA SHEET

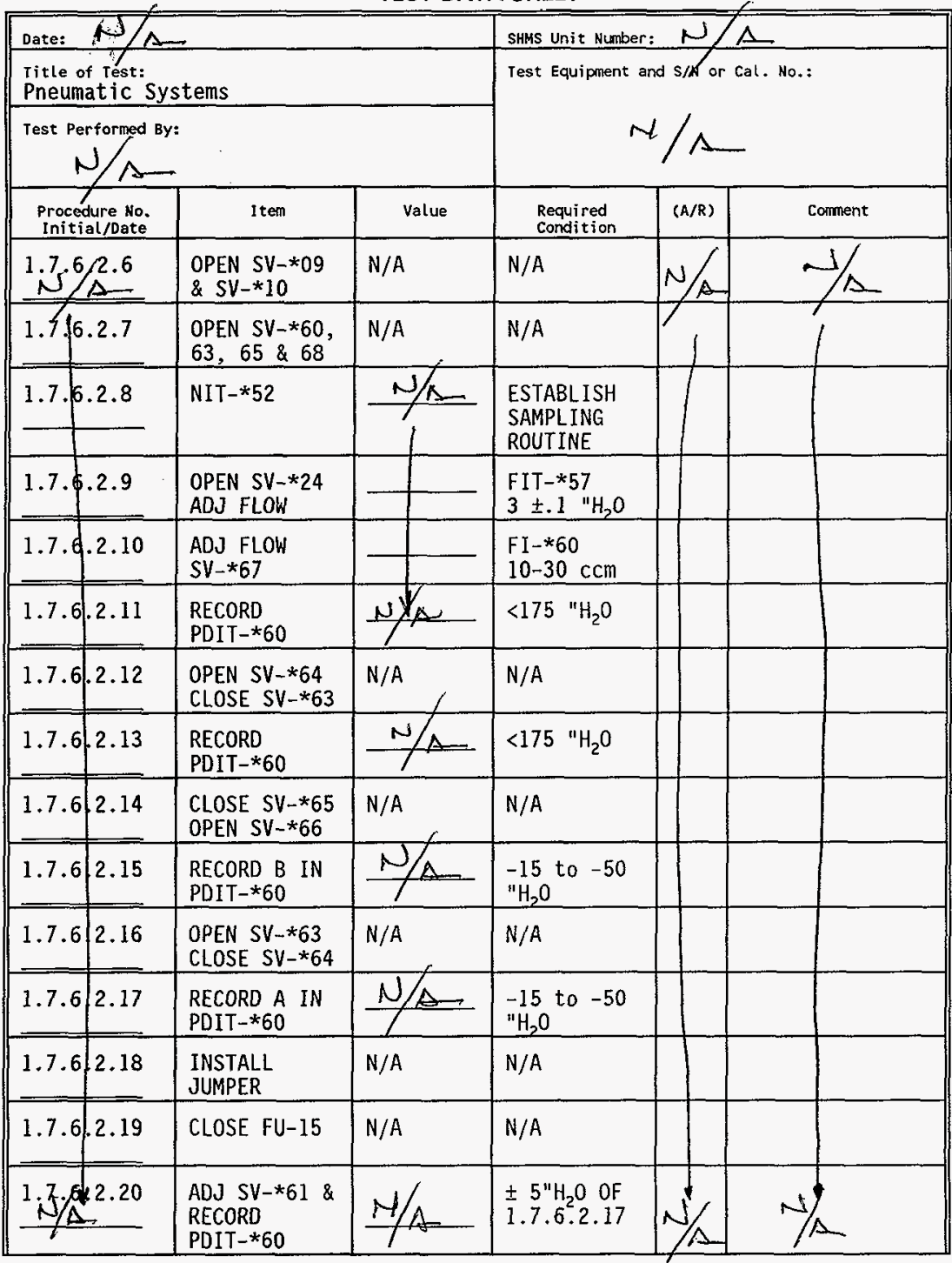


APPENDIX L

TEST DATA SHEET

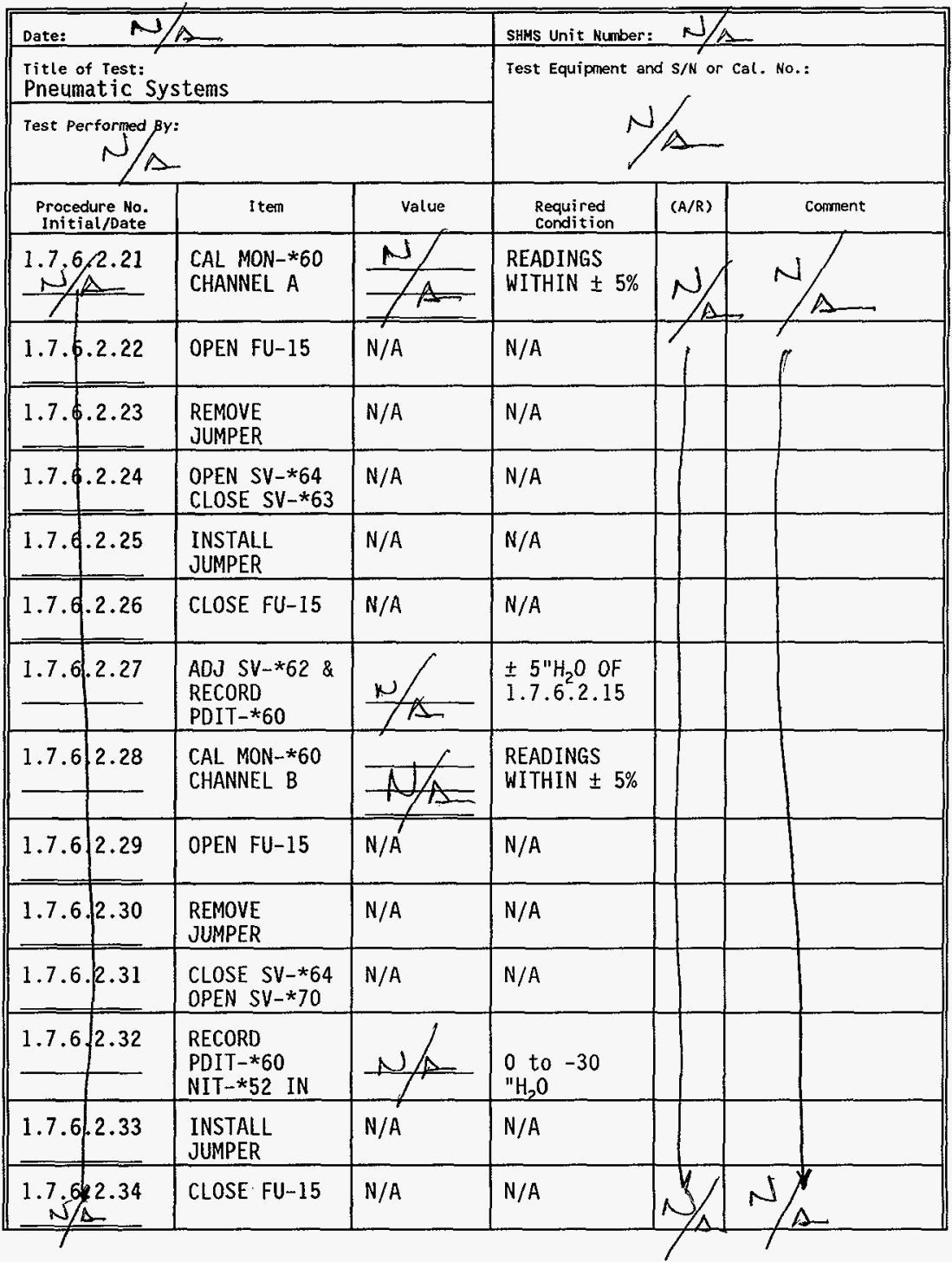


TEST DATA SHEET

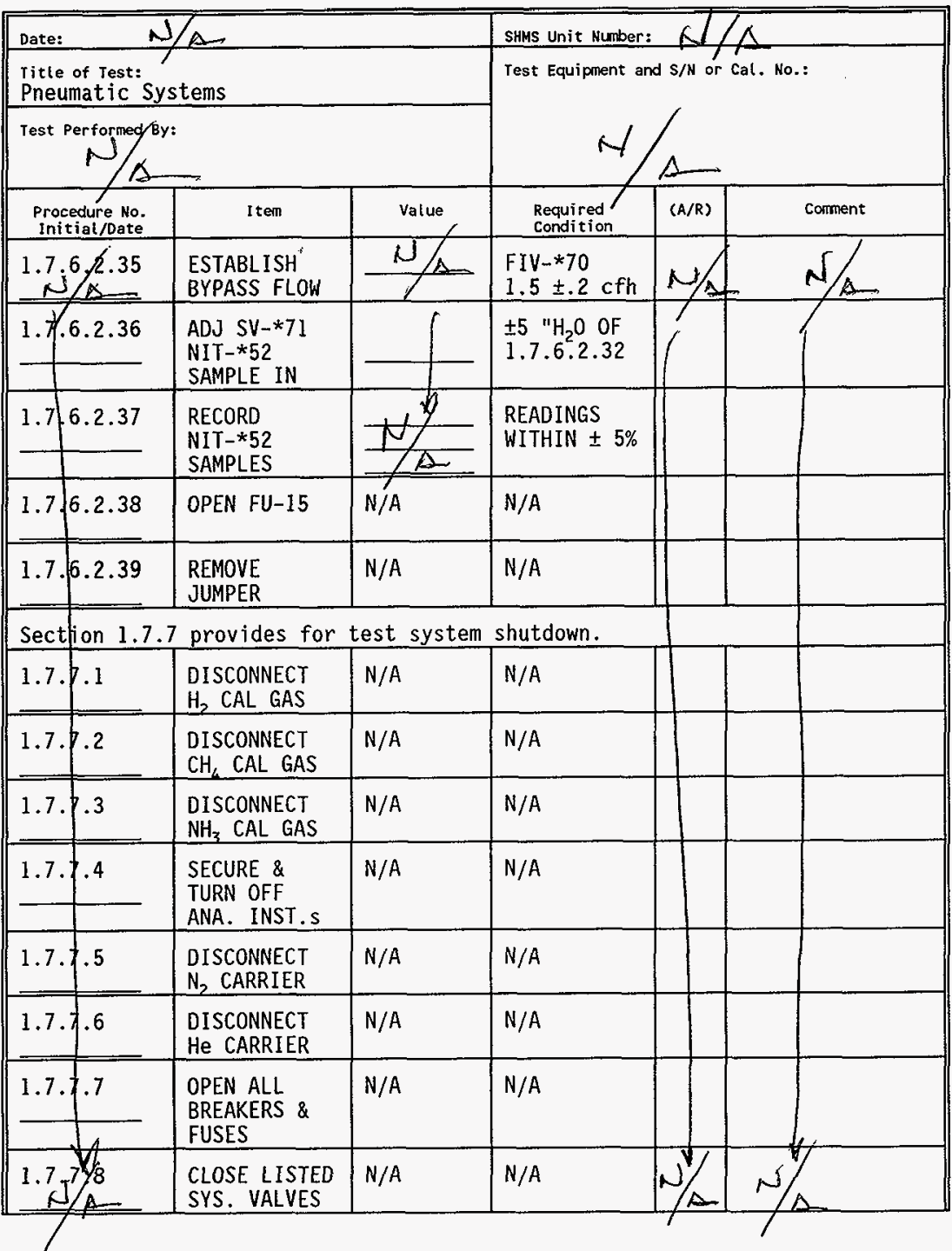


APPENDIX L

PAGE L-2\&
Root:

coly
HNF-SD-WM-ATR- 191

Rev. 0

TEST DATA SHEET

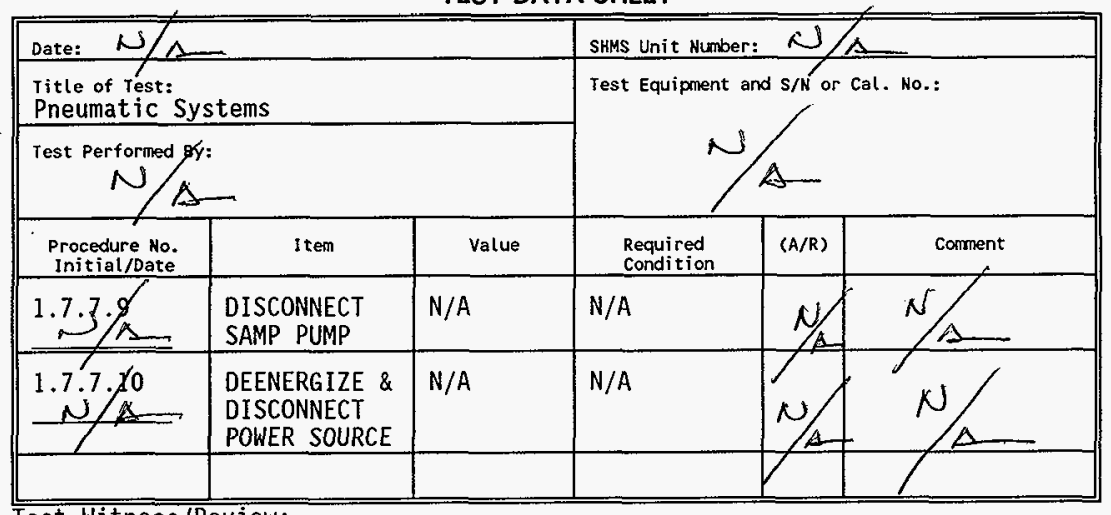

Test Witness/Review:

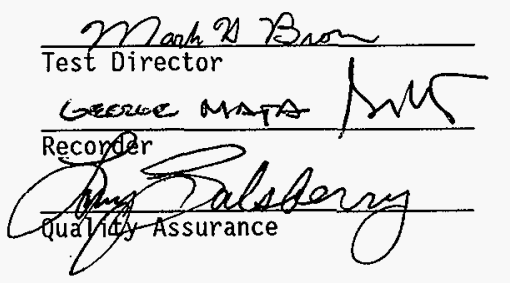

$\frac{3-28-97}{\text { Date }}$

28 mares 97

Date

$\frac{4}{\text { Date }} / 1 / 97$ 
APPENDIX L

PAGE L-29

$$
\begin{array}{r}
\text { HNF-SD-WM-ATR- } 191 \\
\text { Rev. } 0 \\
\text { Page 395a }
\end{array}
$$

TEST EXCEPTION SHEET

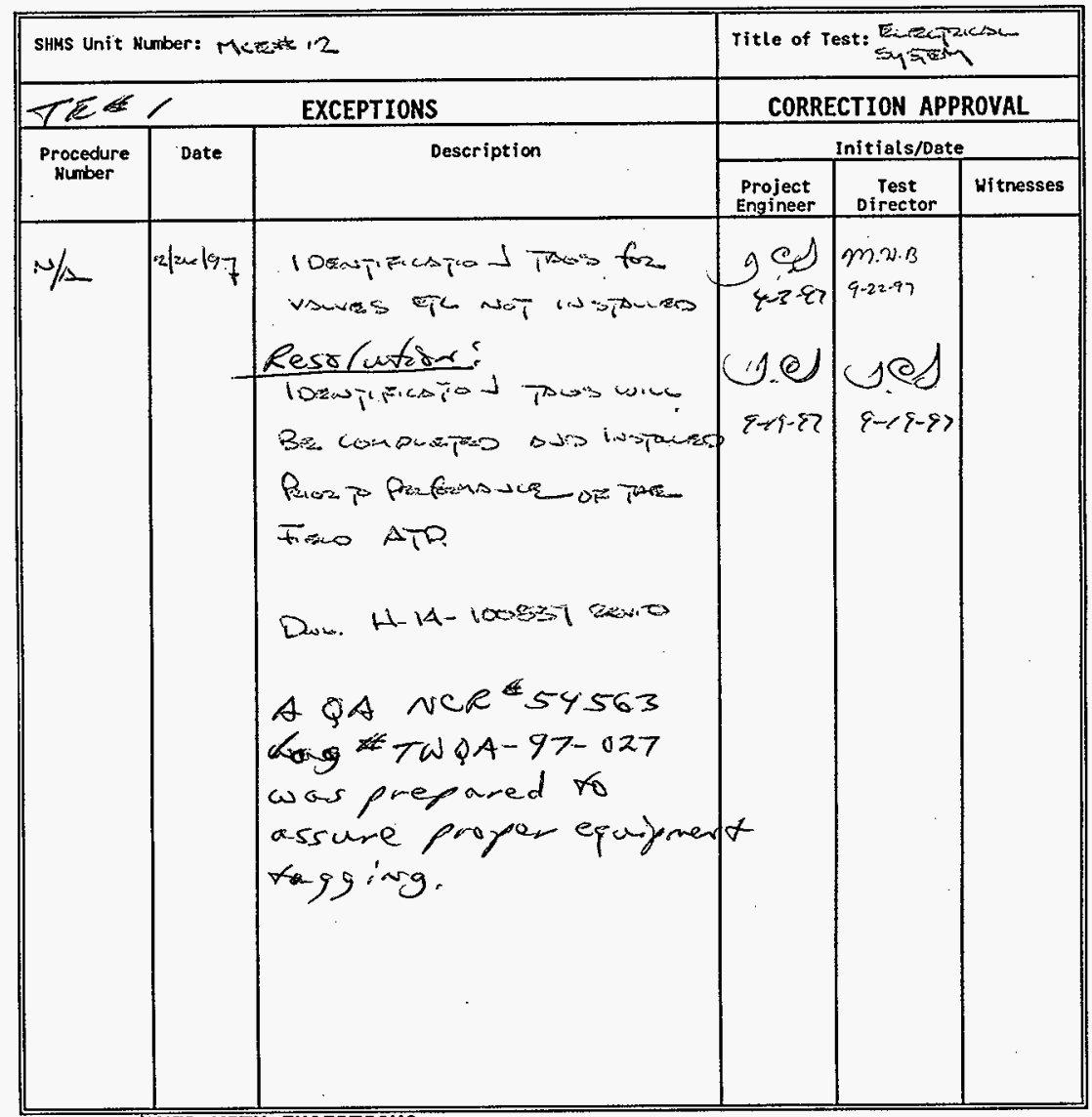

TEST APPROVED WITH EXCEPTIONS

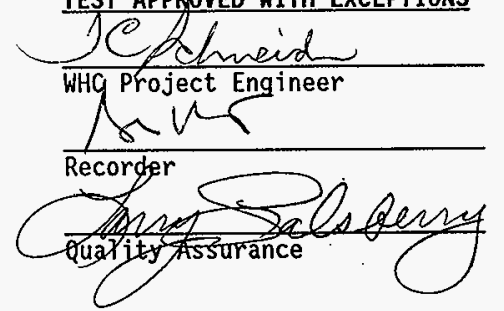

$$
\begin{aligned}
& \frac{y-3-87}{\text { Date }} \\
& 3402-97 \\
& \frac{4 / 2 / 97}{\text { Date }}
\end{aligned}
$$


APPENDIX $L$

UNF-SD - WM-ATR- 191

PAGE L-30

Rev. 0
Page 3956

TEST EXCEPTION SHEET

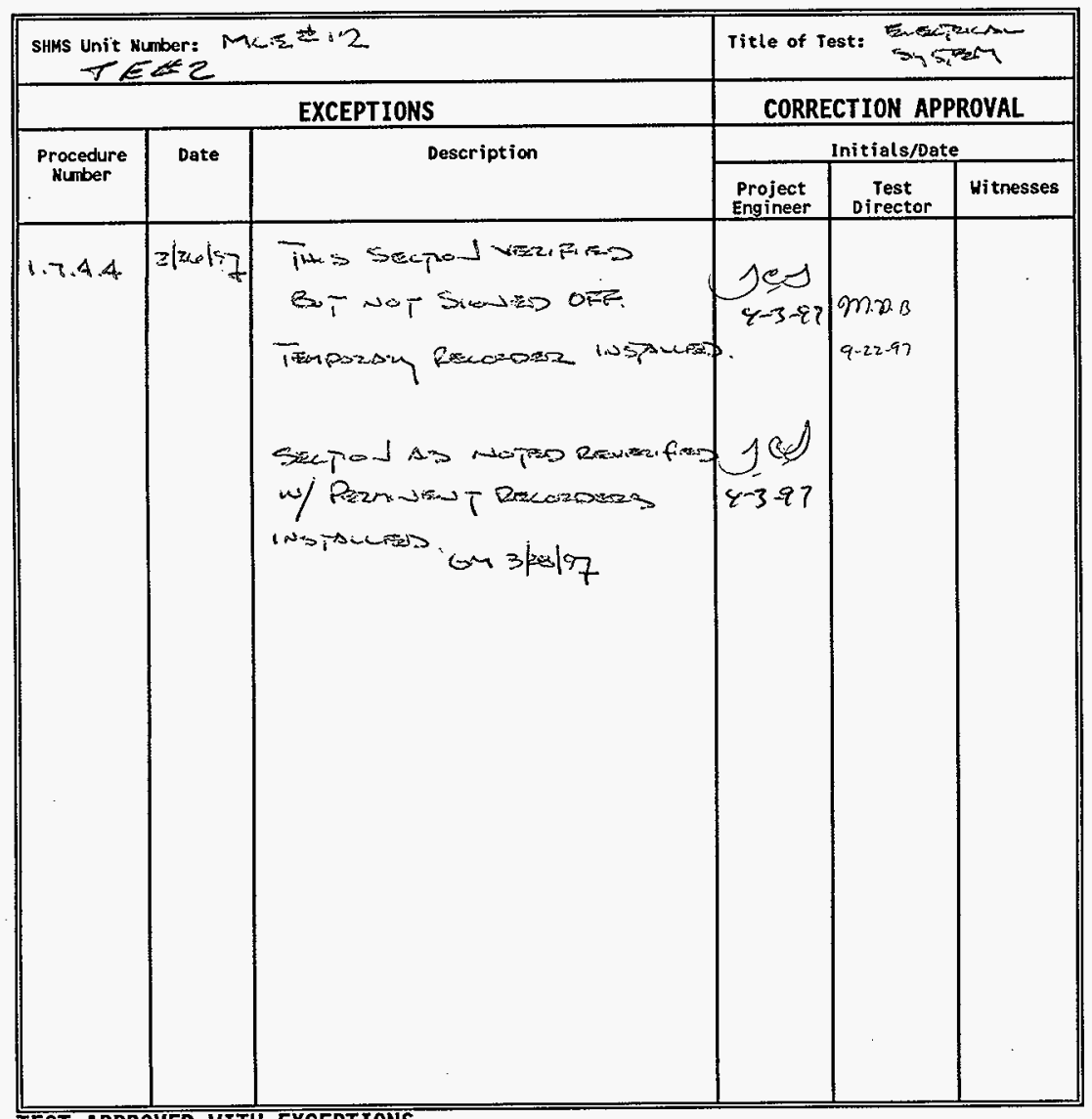

TEST APPROVED WITH EXCEPTIONS
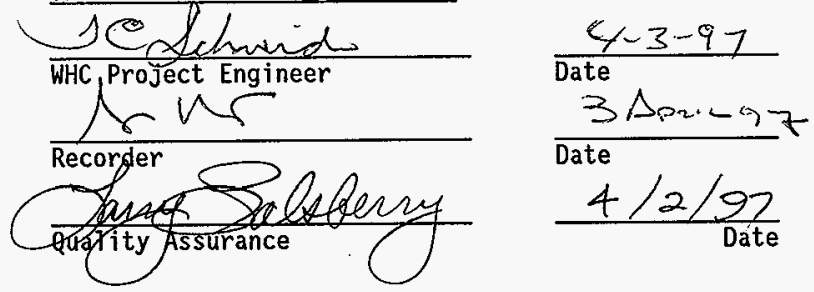
TEST LOG

\begin{tabular}{|c|c|}
\hline DATE/IIME & COMMENTS 4 \\
\hline $2120073 P$ & STOMPO TEST \\
\hline 642126197 & $f+20$ \\
\hline $2: 40 \mathrm{P}$ & 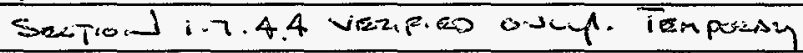 \\
\hline & 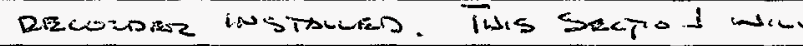 \\
\hline & 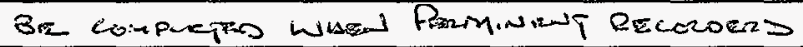 \\
\hline & Ane instiviris. \\
\hline $3: 259$ & Test conpuepess or $2 / 26 / 57$ \\
\hline 28 miven $3: 45 A$ & spent rutsi Pare 1.7.44. \\
\hline & Repeaces Site conse on fiY-56 \\
\hline & NuTABSe TO DOSUST RO 2 CEM \\
\hline & FINSAS TEST AT G:1SA \\
\hline & 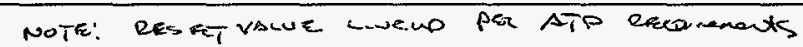 \\
\hline & 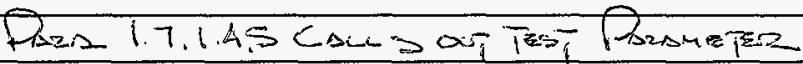 \\
\hline NoTe & 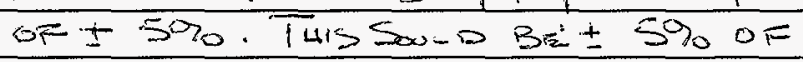 \\
\hline & $120 \mathrm{VAC} \quad$ Gen $29 \mathrm{may} 97$ \\
\hline & \\
\hline & \\
\hline & \\
\hline & \\
\hline & \\
\hline
\end{tabular}


4

at

APPENDIX M 
TEST EXECUTION SHEET

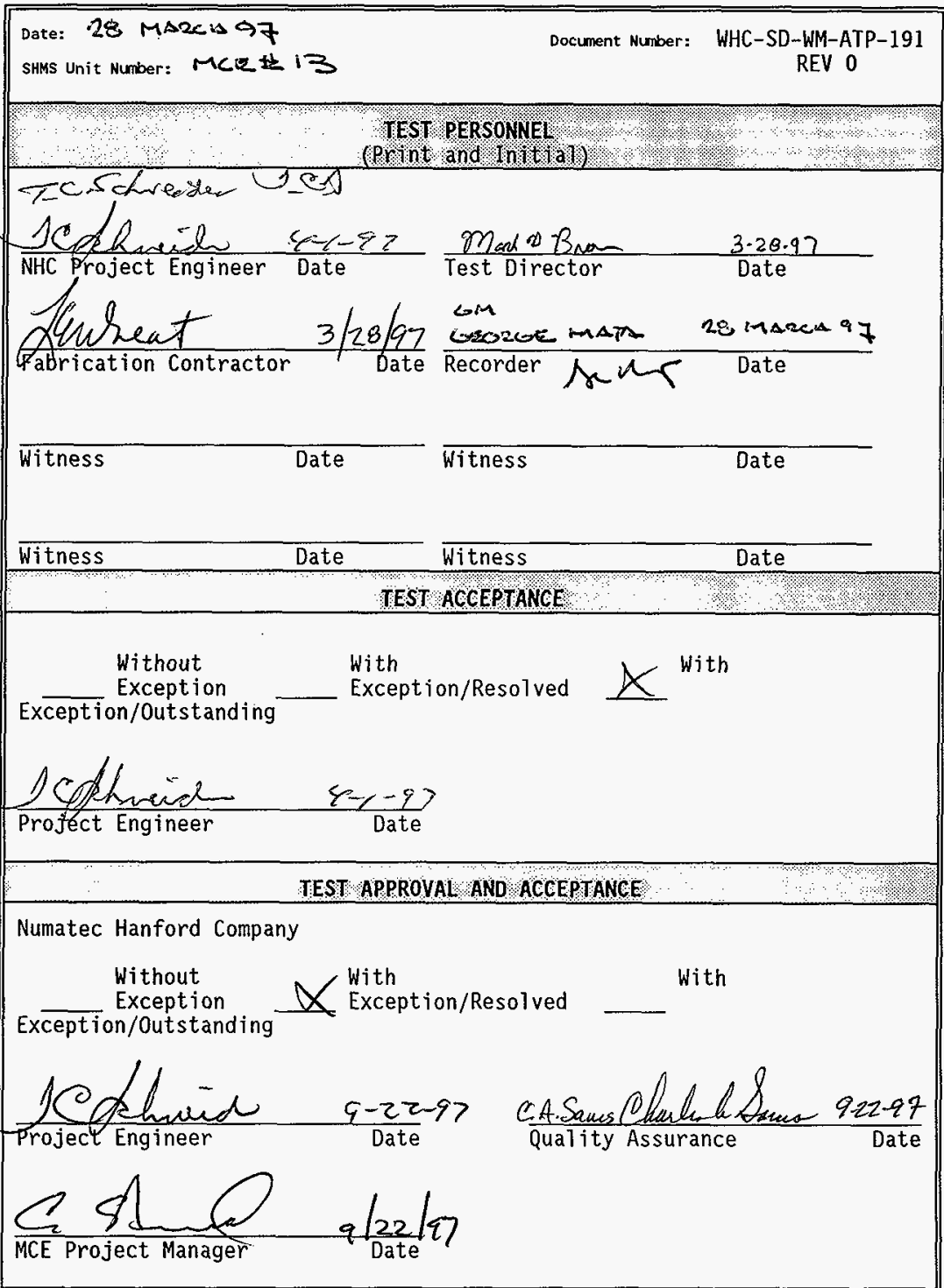


APPENDIX M

PAGE $M-3$

SHMS Unit Number:

\section{PREREQUISITES AND INITIAL TEST CONDITIONS}

The following conditions shall exist at the start of the acceptance testing. Initial and date to verify that each of the following items have been accomplished.

$\cos$

Systems being tested have been inspected for workmanship and for compliance with design.

$\operatorname{con}$ Continuity and megger tests have been performed on portions of the electrical and instrument systems being tested, as required.

Gen Leak tests on the pneumatic systems have been performed.

Gen The following circuit breakers and fuses are installed per the specified size and are open

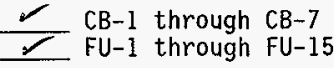

(x)t7)

All test instruments have a currently valid calibration stamp attached that indicates a calibration traceable to the National Institute of Standards and Technology.

Gen The following process and control instrument systems have been initially configured and aligned for proper operation.

FIT-*57, FSL $-* 57$, NIT $* 54$, NIT $-* 55,(N R-* 54)$, PDIT-*60, TIC $-* 50$, TIC $-* 56$, TIS $* 62$, YYC $-* 01$ NOTE: NR $-* 54$ is not used in the ANALYTICAL configuration.

$\operatorname{cen}$

Personnel responsible for directing and witnessing the performance of the tests described in this ATP have read and understand appropriate certified vendor information (CVI) pertaining to the operation of the equipment to be tested.

GeM CLOSE all system manual valves with the exception of the following:

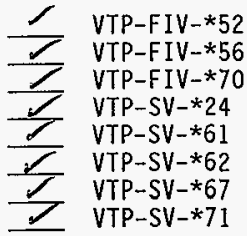

Gom _. The sample pump VTP-P-*50, connected to the cabinet sample out port, but vented to atmosphere for testing. 
Car $208 \mathrm{VaC} 1$ phase 15-20 ampere temporary power source has been 3-28-97 connected, but not energized, to the appropriate TB1 terminals per

Verify by signature and date that all prerequisites have been met.

\begin{tabular}{ll} 
Nad $D$ Bnow & $3 \cdot 28.97$ \\
\hline Test Director Signature & Date
\end{tabular}


TEST DATA SHEET

\begin{tabular}{|c|c|c|c|c|c|}
\hline \multicolumn{3}{|c|}{ Date: 23 mazeis 97} & \multicolumn{3}{|c|}{ SHMS Unit Number: MCE-13 } \\
\hline \multicolumn{3}{|c|}{$\begin{array}{l}\text { Titie of Test: } \\
\text { Electrical Systems }\end{array}$} & \multirow{2}{*}{\multicolumn{3}{|c|}{$\begin{array}{l}\text { Test Equipment and S/N or Cal. No.: } \\
\text { Fulk } / 10 \text { METER } \\
\text { Su } 44620364 \\
\text { Du DATE } 10197\end{array}$}} \\
\hline \multicolumn{3}{|c|}{ 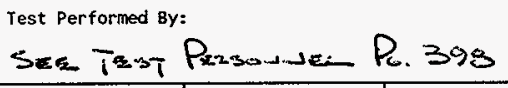 } & & & \\
\hline $\begin{array}{l}\text { Procedure No. } \\
\text { Initial/Date }\end{array}$ & Item & Value & $\begin{array}{l}\text { Required } \\
\text { Condition }\end{array}$ & $(A / R)$ & corment \\
\hline \multicolumn{6}{|c|}{ Section 1.7 .1 .1 verifies the system Mains Power. } \\
\hline $\begin{array}{l}1.7 .1 .1 .1 \\
0 \times 1.1\end{array}$ & Resistance & .1 & $<1$ ohm & $\Delta$ & \\
\hline $\begin{array}{l}1.7 .1 .1 .2 \\
6 x^{2}\end{array}$ & $\begin{array}{l}\text { Temp, power } \\
208 \mathrm{Vac}\end{array}$ & $N / A$ & Energized & $\Delta$ & \\
\hline $\operatorname{cem}^{1.7 .1 .3}$ & $\begin{array}{l}\text { Line Vac: } \\
\text { L1-L2 } \\
\text { L1-Gnd } \\
\text { L2-Gnd } \\
N \text {-Gnd }\end{array}$ & $\frac{\frac{210.1}{120}}{\frac{120.5}{.0}}$ & $\begin{array}{l}\text { L1-L2: } 208 \\
\text { Vac }+10 /-1 \% \\
\text { L1, L2: } 120 \\
\text { Vac } \pm 5 \% \\
N: 0 \text { Vac }\end{array}$ & $A$ & \\
\hline \multicolumn{6}{|c|}{ Section 1.7.1.2 verifies the enclosure HVAC system. } \\
\hline $\begin{array}{c}1.7 .1 .2 .1 \\
6 \sin \end{array}$ & AC Adjusted & N/A & $N / A$ & A & \\
\hline $\begin{array}{l}1.7 .1 .2 .2 \\
\text { lotan }\end{array}$ & $\begin{array}{l}\text { CLOSE } \\
\text { CB-1/2 }\end{array}$ & $N / A$ & N/A & $\Delta$ & \\
\hline $\begin{array}{l}1.7 .1 .2 .3 \\
\text { Gen }\end{array}$ & Heater $\mathrm{ON}$ & $\sim$ & Heater ON & 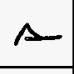 & \\
\hline $\begin{array}{l}1.7 .1 .2 .4 \\
\text { Gen }\end{array}$ & AC Adjusted & N/A & $N / A$ & $\Delta$ & \\
\hline 1.7 .1 .2 .5 & $\begin{array}{l}\text { Heater OFF } \\
\text { Cooling ON }\end{array}$ & $\frac{1}{2}$ & $\begin{array}{l}\text { Heater OFF } \\
\text { Cooling ON }\end{array}$ & $\Delta$ & \\
\hline $\begin{array}{l}\text { Gen } \\
\end{array}$ & AC Adjusted & $N / A$ & $N / A$ & $A$ & \\
\hline $\operatorname{Gen}^{1.7 .1 .2 .7}$ & $\begin{array}{l}\text { OPEN } \\
\mathrm{CB}-1 / 2 \\
\end{array}$ & N/A & $\mathrm{N} / \mathrm{A}$ & An & \\
\hline \multicolumn{6}{|c|}{ Section 1.7.1.3 verifies the sample pump is properly connected. } \\
\hline Gen & $\begin{array}{l}\text { CLOSE } \\
\text { CB-3/4 }\end{array}$ & $N / A$ & N/A & $\Delta$ & $t$ \\
\hline$\underbrace{1.7 .1 .3 .2}_{\text {Géch }}$ & $\begin{array}{l}\text { VTP-P-*50 } \\
\text { ON }\end{array}$ & $r$ & $\begin{array}{l}\text { VTP-P-*50 } \\
\text { ON }\end{array}$ & $\Delta$ & $N / \Delta$ \\
\hline
\end{tabular}


TEST DATA SHEET

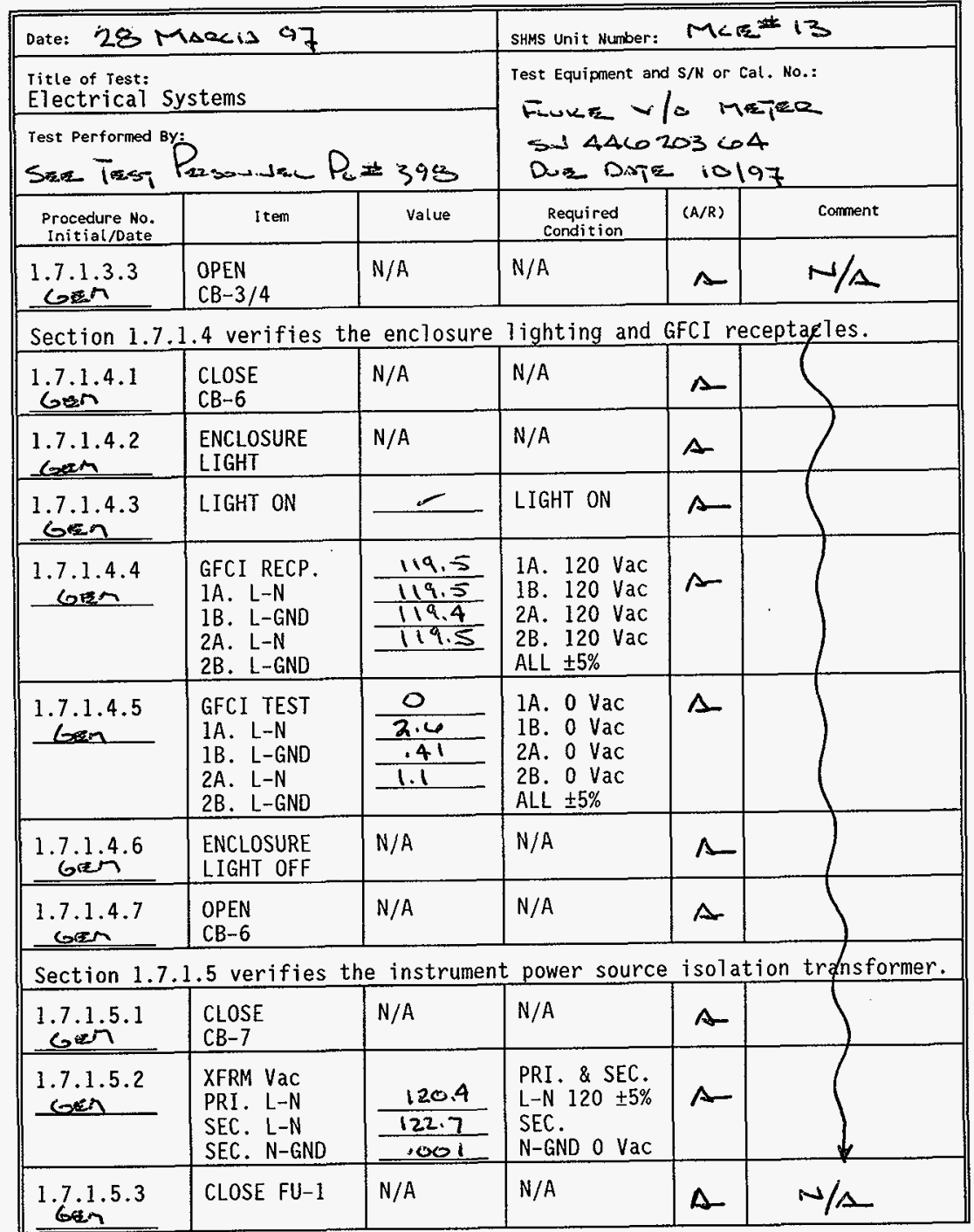


TEST DATA SHEET

\begin{tabular}{|c|c|c|c|c|c|}
\hline \multicolumn{3}{|c|}{ Date: 28 MARCA 97} & \multicolumn{3}{|c|}{ SHMS Unit Number: MCE 13} \\
\hline \multicolumn{3}{|c|}{$\begin{array}{l}\text { Title of Test: } \\
\text { Electrical Systems }\end{array}$} & \multirow{2}{*}{\multicolumn{3}{|c|}{ 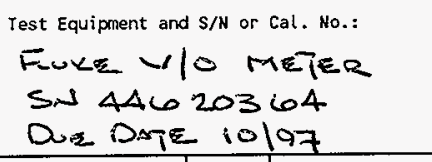 }} \\
\hline \multicolumn{3}{|c|}{ 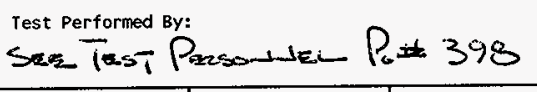 } & & & \\
\hline $\begin{array}{l}\text { Procedure No. } \\
\text { Initial/Date }\end{array}$ & Item & value & $\begin{array}{c}\text { Required } \\
\text { Condition }\end{array}$ & $(A / R)$ & Comment \\
\hline $\begin{array}{l}1.7 .1 .5 .4 \\
6 \pi^{2}\end{array}$ & $\begin{array}{l}\text { VTP-PS-*50 } \\
\text { OUTPUT VdC }\end{array}$ & 23.99 & $24 \pm 0.1 \mathrm{Vdc}$ & $\perp$ & \\
\hline \multicolumn{6}{|c|}{ Section 1.7.1.6 verifies the enclosure general al arm system. ( } \\
\hline $\begin{array}{l}1.7 .1 .6 .1 \\
\text { cosin }\end{array}$ & $\begin{array}{l}\text { CLOSE FU-9 \& } \\
\text { FU-10 }\end{array}$ & $N / A$ & N/A & $A-$ & \\
\hline 1.7.1.6.2 & $\begin{array}{l}\text { PUSH PB-*51 } \\
\& \text { PB- } \star 50\end{array}$ & $N / A$ & N/A & A & \\
\hline $\begin{array}{l}1.7 .1 .6 .3 \\
\text { cozion }\end{array}$ & $\begin{array}{l}\text { ALARM TEST } \\
\text { VERIFY ALARM } \\
\text { CONDITIONS }\end{array}$ & 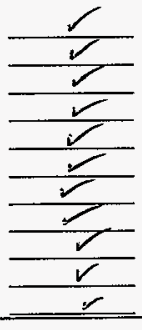 & $\begin{array}{ll}\text { YAH-*50 } & \text { ON } \\
\text { NAH-*55 } & \text { ON } \\
\text { XA-*63 } & \text { ON } \\
N A H-* 54 & \text { ON } \\
\text { FAL-*57 } & \text { ON } \\
\text { TAHL-*62 } & \text { ON } \\
\text { TAL-*50 } & \text { ON } \\
\text { YAL-*58 } & \text { ON } \\
\text { PBL-*58 } & \text { ON } \\
\text { PBL-*54 } & \text { OFF } \\
\text { PBL-*59 } & \text { OFF } \\
\end{array}$ & 1 & \\
\hline $\begin{array}{l}1.7 .1 .6 .4 \\
\operatorname{sen}\end{array}$ & $\begin{array}{l}\text { END ALARM } \\
\text { TEST }\end{array}$ & N/A & $N / A$ & $\Delta$ & \\
\hline $\begin{array}{c}1.7 .1 .6 .5 \\
\text { gen }\end{array}$ & RESET ALARMS & N/A & N/A & $A$ & \\
\hline $\begin{array}{l}1.7 .1 .6 .6 \\
\end{array}$ & $\begin{array}{l}\text { OPEN FU-9 \& } \\
\text { FU-10 }\end{array}$ & N/A & N/A & A & \\
\hline \multicolumn{6}{|c|}{ Section 1.7.1.7 verifies the flow alarm system. } \\
\hline 1.7.1.7.1 & $\begin{array}{l}\text { CLOSE FU-3, } \\
\text { FU-9, FU-10 } \\
\& \text { FU-13 }\end{array}$ & N/A & N/A & $\Lambda$ & \\
\hline $\begin{array}{l}1.7 .1 .7 .2 \\
0 \mathrm{in}\end{array}$ & RESET ALARMS & $\checkmark$ & $\mathrm{FAL}-\star 57$ ON & $A$ & \\
\hline 1.7.1.7.3 & $\begin{array}{l}\text { OPEN SV }-* 20- \\
\& S V-* 22-\end{array}$ & $\mathrm{N} / \mathrm{A}$ & $N / A$ & 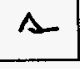 & 1 \\
\hline
\end{tabular}

Gen Au 3/28 k7 
TEST DATA SHEET

\begin{tabular}{|c|c|c|c|c|c|}
\hline \multicolumn{3}{|c|}{ Date: 28 msecs 97} & \multicolumn{3}{|c|}{ SHMS Unit Number: MCE IS } \\
\hline \multicolumn{3}{|c|}{$\begin{array}{l}\text { Title of Test: } \\
\text { Electrical Systems }\end{array}$} & \multirow{2}{*}{\multicolumn{3}{|c|}{$\begin{array}{l}\text { Test Equipment and S/N or Cal. Ho.: } \\
\text { FusizE V lo MEjER } \\
\text { SN } 44620364 \\
\text { Du DATE } 10197\end{array}$}} \\
\hline \multicolumn{3}{|c|}{ 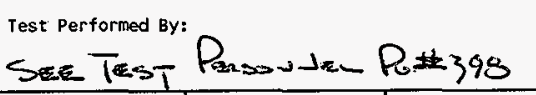 } & & & \\
\hline $\begin{array}{l}\text { Procedure No. } \\
\text { Initial/Date }\end{array}$ & Item & value & $\begin{array}{l}\text { Required } \\
\text { Condition }\end{array}$ & $(A / R)$ & Corment \\
\hline $\mathrm{6en}^{1.7 .1 .7 .4}$ & $\begin{array}{l}\text { FIT-*57 \& } \\
\text { FSL }-* 57 \\
\text { POWERED }\end{array}$ & $\frac{r}{2}$ & $\begin{array}{l}\text { FIT-*57 ON } \\
\text { FSL-*57 ON }\end{array}$ & $\Delta$ & \\
\hline $\begin{array}{l}1.7 .1 .7 .5 \\
\mathrm{H} / \mathrm{A} \\
\end{array}$ & $\begin{array}{l}\text { MUX-*70 } \\
\text { TB5+/TB6- }\end{array}$ & $N / \Delta$ & $1.0 \pm 0.2 \mathrm{Vdc}$ & $N / A$ & 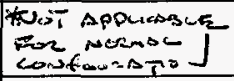 \\
\hline con $^{1.7 .1 .7 .6}$ & $\begin{array}{l}\text { CLOSE SV-*20- } \\
\& \text { SV-*22- }\end{array}$ & $-N / A$ & $N / A$ & 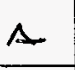 & \\
\hline $\begin{array}{l}1.7 .1 .7 .7 \\
6.27\end{array}$ & $\begin{array}{l}\text { OPEN FU-3, } \\
\text { FU-9, FU-10 } \\
\& \text { FU-13 }\end{array}$ & $N / A$ & $N / A$ & $A$ & \\
\hline \multicolumn{6}{|c|}{ Section 1.7.1.8 verifies the $\mathrm{H}_{2}$ monitor wiring. } \\
\hline $\begin{array}{l}1.7 .1 .8 .1 \\
\end{array}$ & $\begin{array}{l}\text { CLOSE FU-2, } \\
\text { FU-4, FU-5, } \\
\text { FU-9 \& FU-10 }\end{array}$ & $N / A$ & $N / A$ & $\Lambda$ & \\
\hline $\begin{array}{c}1.7 .1 .8 .2 \\
6.7\end{array}$ & RESET ALARMS & $\begin{array}{r}-r \\
r\end{array}$ & $\begin{array}{l}\mathrm{NAH}-* 54 \text { OFF } \\
\mathrm{NAH}-* 55 \text { OFF }\end{array}$ & $\Delta$ & \\
\hline $\begin{array}{l}1.7 .1 .8 .3 \\
625 \\
\end{array}$ & $\begin{array}{l}\text { INSTRUMENTS } \\
\text { POWERED }\end{array}$ & $\frac{\frac{N / \Delta *}{\alpha}}{\frac{L}{L}}$ & $\begin{array}{ll}\text { MUX }-* 70 & \text { ON } \\
\text { NIT }-* 54 & \text { ON } \\
\text { NIT }-* 55 & \text { ON } \\
N R-* 54 & \text { ON } \\
\text { YYC }-* 01 & \text { ON } \\
\end{array}$ & 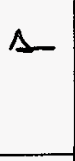 & \\
\hline $\cos ^{1.7 .1 .8}$ & $\begin{array}{l}\text { OPEN FU-2, } \\
\text { FU-4, FU-5, } \\
\text { FU-9 \& FU-10 }\end{array}$ & $N / A$ & $N / A$ & $\Delta$ & \\
\hline \multicolumn{6}{|c|}{ Section 1.7.1.9 verifies the SHMS-E heat trace control system. } \\
\hline $\begin{array}{c}1.7 .1 .9 .1 \\
6 x{ }^{2}\end{array}$ & $\begin{array}{l}\text { INSTALL LOAD } \\
\text { VTP-TIC-*50 }\end{array}$ & $N / A$ & $N / A$ & 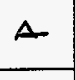 & \\
\hline $\begin{array}{l}1.7 .1 .9 .2 \\
\end{array}$ & $\begin{array}{l}\text { CLOSE CB-5, } \\
\text { FU-6, FU-7, } \\
\text { FU-9 \& FU-10 } \\
\text { and RESET } \\
\text { ALARMS }\end{array}$ & $N / A$ & $N / A$ & A & $/ \Delta$ \\
\hline
\end{tabular}


TEST DATA SHEET

\begin{tabular}{|c|c|c|c|c|c|}
\hline \multicolumn{3}{|c|}{ Date: 28 MARCL 97} & \multicolumn{3}{|c|}{ SHMS Unit Number: MCE } \\
\hline \multicolumn{3}{|c|}{$\begin{array}{l}\text { Iitle of rest: } \\
\text { Electrical Systems }\end{array}$} & \multirow{2}{*}{\multicolumn{3}{|c|}{ Test Equipment and $S / \mathrm{N}$ or Cal. No.: }} \\
\hline \multicolumn{3}{|c|}{$\begin{array}{l}\text { Test Performed By: } \\
\text { Sere Test Papsow.wen Po. } 398\end{array}$} & & & \\
\hline $\begin{array}{c}\text { Procedure No. } \\
\text { Initial/Date }\end{array}$ & Item & value & $\begin{array}{l}\text { Required } \\
\text { Condition } \\
\end{array}$ & $\langle A / R)$ & Comment \\
\hline 1.7 .1 .9 .3 & $\begin{array}{l}\text { TIC }-* 50 \& \\
\text { TIC } * 56 \\
\text { NOMINAL TEMP } \\
\end{array}$ & $-r$ & $\begin{array}{l}\text { TIC }-* 50 \\
\text { TIC }-* 56 \\
\text { TEMPERATURE } \\
\end{array}$ & $A$ & \\
\hline $\begin{array}{l}1.7 .1 .9 .4 \\
6 \operatorname{con}^{2}\end{array}$ & $\begin{array}{l}\text { ADJ TIC }-* 50 \\
\& \text { TIC }-* 56 \\
\text { SP2 \& RESET } \\
\text { ALARMS }\end{array}$ & $N / A$ & $N / A$ & A & \\
\hline Gen & $\begin{array}{l}\text { ADJ TIC-*50 } \\
\text { SP } 1\end{array}$ & N/A & $N / A$ & $\Lambda$ & \\
\hline o. & $\begin{array}{l}\text { ADJ TIC }-* 50 \\
\text { SP } 2\end{array}$ & $N / A$ & $N / A$ & $A$ & \\
\hline 1.7.1.9.7 & $\begin{array}{l}\text { TAL-*50 \& } \\
\text { HORN ACTIVE }\end{array}$ & $\frac{r}{2}$ & $\begin{array}{l}\text { TAL }-* 50 \text { ON } \\
\text { HORN ON } \\
\text { ACKNOWLEDGE }\end{array}$ & $\triangle$ & \\
\hline osen & $\begin{array}{l}\text { TIC }-* 50 \\
\text { OPERATES } \\
\end{array}$ & $\checkmark$ & $\begin{array}{l}\text { TIC }-* 50 \\
\text { OPERATES } \\
\end{array}$ & $\Lambda$ & \\
\hline $\begin{array}{l}1.7 .1 .9 .9 \\
\text { genn }\end{array}$ & $\begin{array}{l}\text { ADJ TIC-*50 } \\
\text { SP } 2\end{array}$ & $N / A$ & $N / A$ & 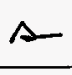 & \\
\hline 1.7 .1 .9 .10 & RESET ALARM & $r$ & TAL $-* 50$ OFF & $\Lambda$ & \\
\hline 1.7.1.9.11 & $\begin{array}{l}\text { ADJ TIC }-* 50 \\
S P \text { 1 \& SP 2 }\end{array}$ & $N / A$ & $N / A$ & $\Delta$ & \\
\hline $\begin{array}{l}1.7 .1 .9 .12 \\
\end{array}$ & $\begin{array}{l}\text { OPEN CB-5 \& } \\
\text { REMOVE LOAD } \\
\end{array}$ & $N / A$ & $N / A$ & $\Delta$ & \\
\hline $\begin{array}{l}1.7 .1 .9 .13 \\
\text { gein } \\
\end{array}$ & $\begin{array}{l}\text { INSTALL LOAD } \\
\text { VTP-TIC } * 56 \\
\end{array}$ & N/A & N/A & $\Lambda$ & \\
\hline $\begin{array}{c}1.7 .1 .9 .14 \\
\mathrm{Gs \textrm {m }} \\
\end{array}$ & CLOSE CB-5 & $N / A$ & $\mathrm{~N} / \mathrm{A}$ & $\Lambda$ & \\
\hline $\begin{array}{l}1.7 .1 .9 .15 \\
6=1\end{array}$ & $\begin{array}{l}\text { ADJ TIC }-* 56 \\
\text { SP } 1\end{array}$ & $N / A$ & $\mathrm{~N} / \mathrm{A}$ & $A$ & \\
\hline $\begin{array}{l}1.7 .1 .9 .16 \\
\operatorname{Gon}^{-1}\end{array}$ & $\begin{array}{l}\mathrm{ADJ}_{\mathrm{T}} \mathrm{TIC}-* 56 \\
\mathrm{SP} 2\end{array}$ & $N / A$ & $N / A$ & 1 & \\
\hline
\end{tabular}


APPENDIX M

PAGE $M-1 O$
HNF-SD-WM-ATR-191
ReV. 0

Rev. 0
page 406

TEST DATA SHEET

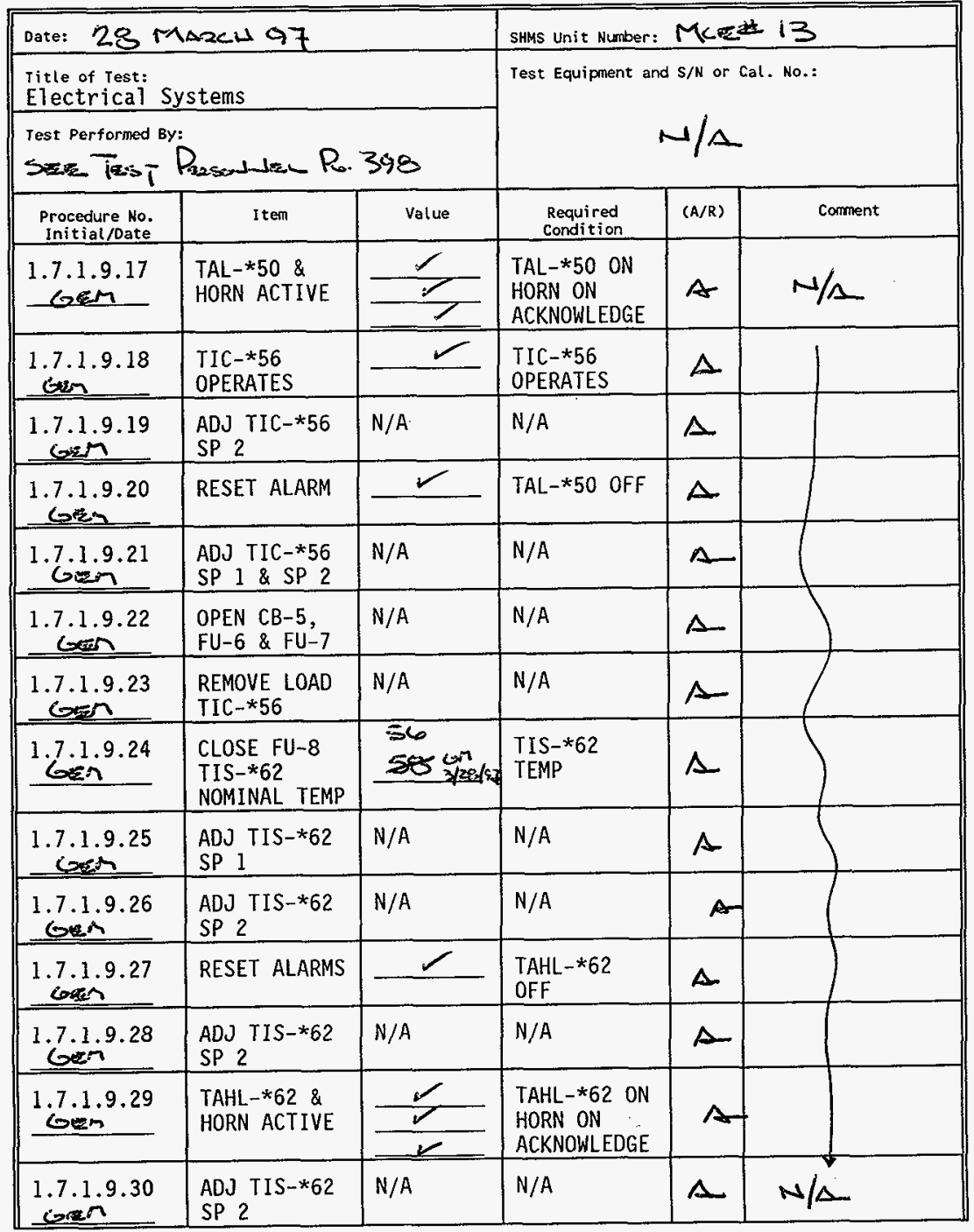

Did n AC L 3/28/97 
APPENDIX $M$

PAGE $M-/ /$
HNF-SD-WM-ATR- 191
REV 0

ReV. 0
Page 407

TEST DATA SHEET

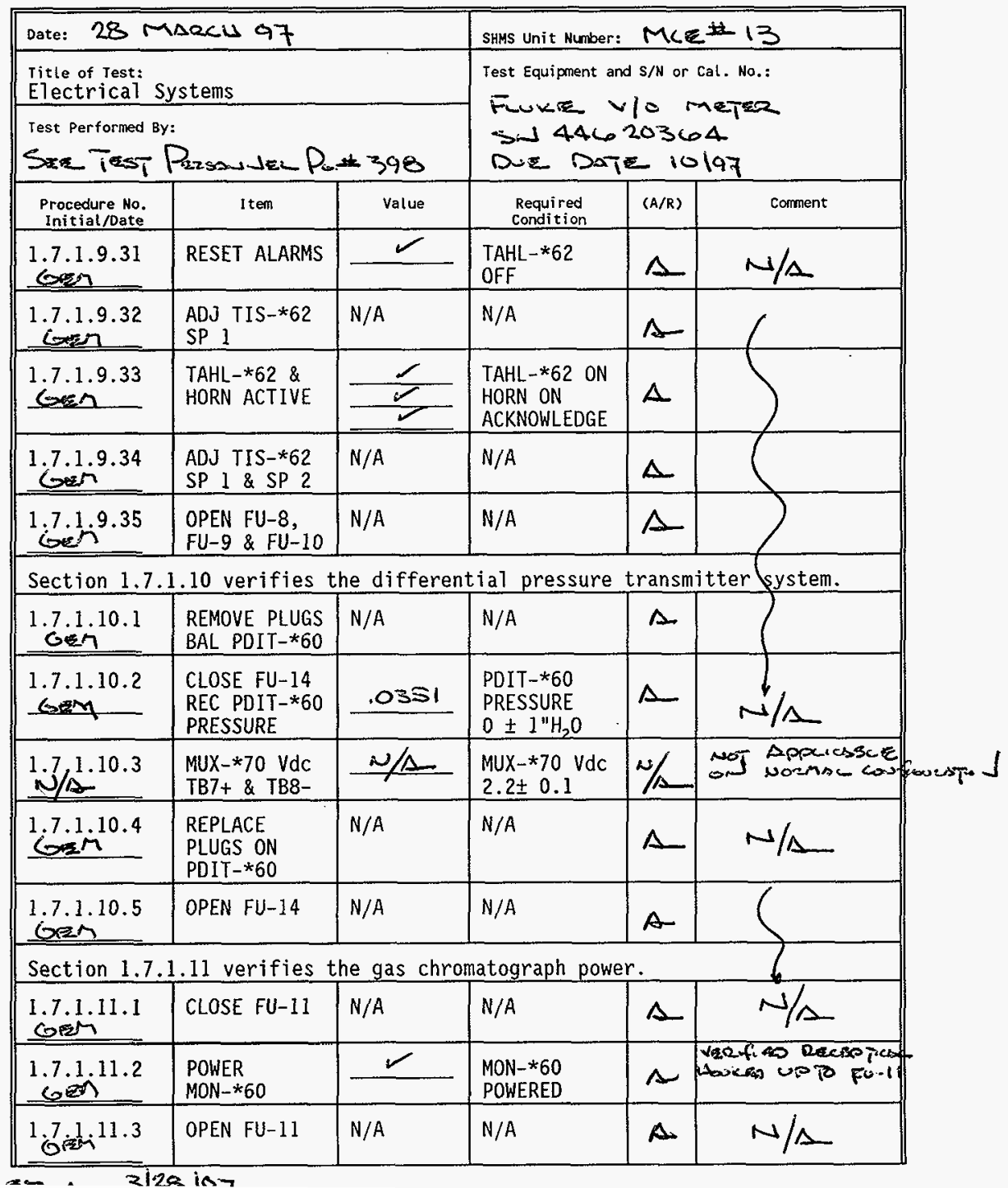


APPENDIX $M$

PAGE $M-12$
INF - SD-WM-ATR-191

Rev. 0

Page 408

TEST DATA SHEET

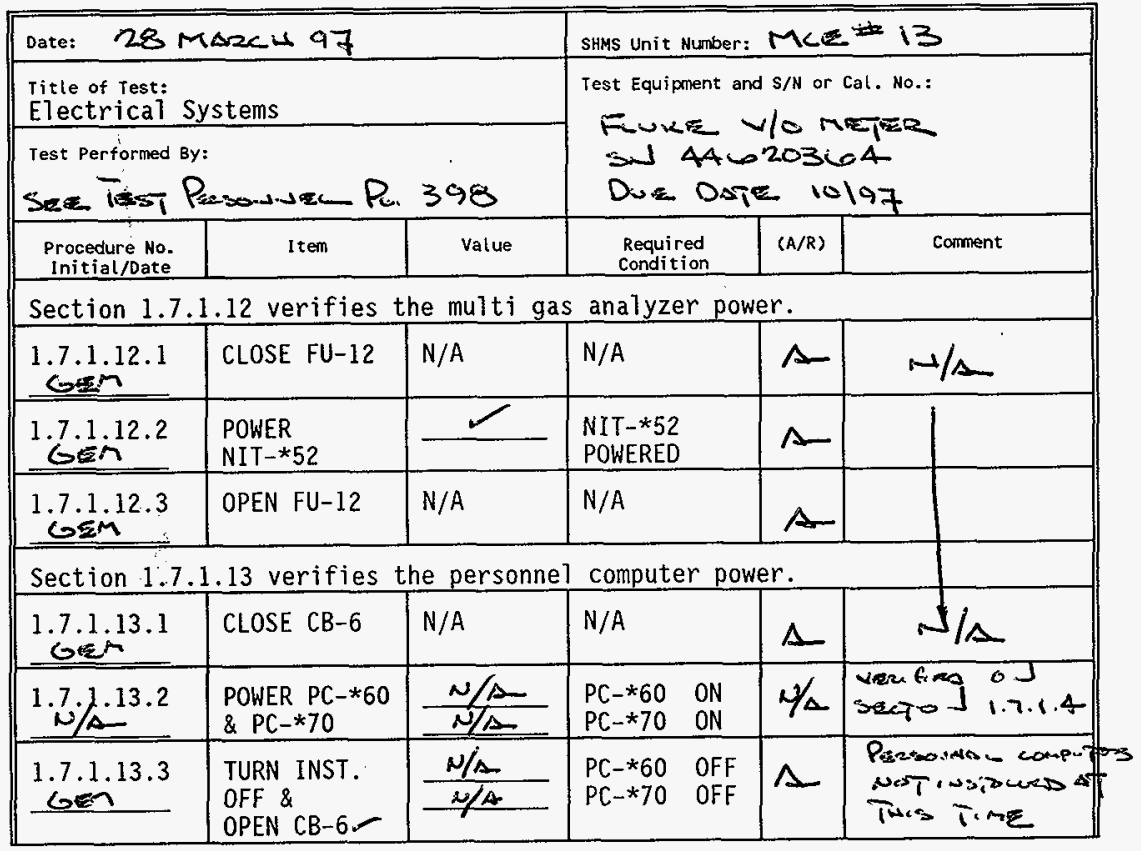

Gem sen 3/28197 
APPENDIX $M$

PAGE $M-13$
HNF-SD-WM-ATR-191

Rev. 0

Page 409

TEST DATA SHEET

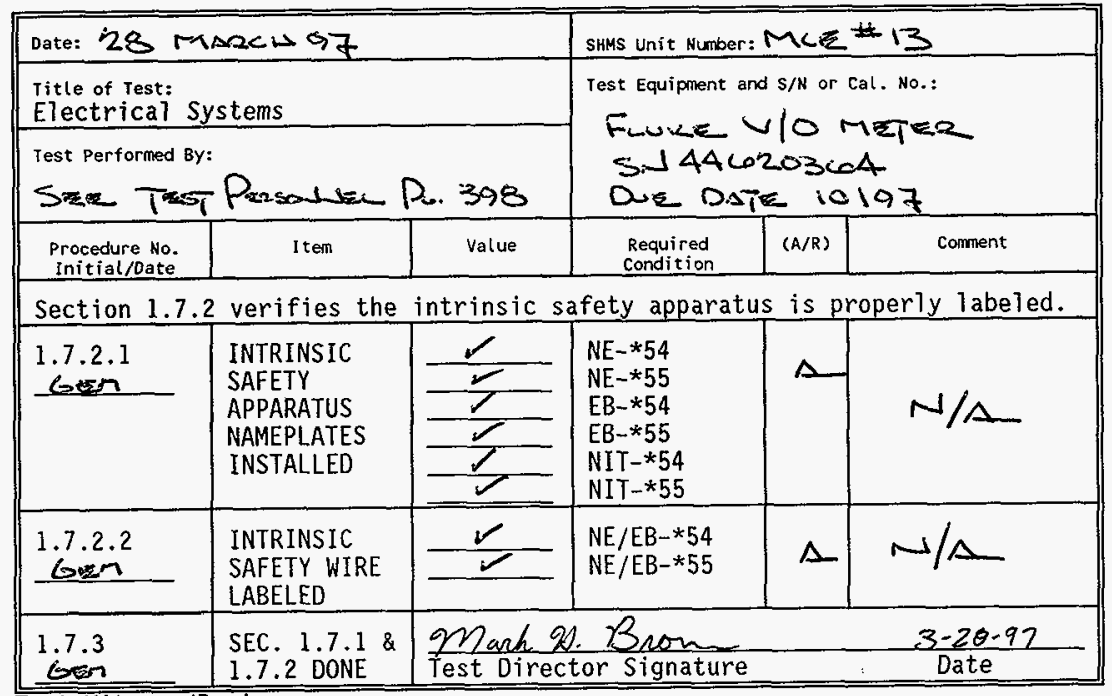

sin -3/28 iq Test Witness/Review:

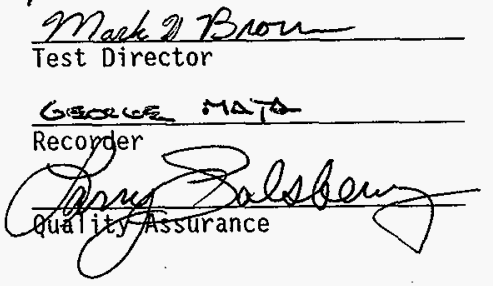

$\frac{3-28-97}{\text { Date }}$

28 marcus 97

Date

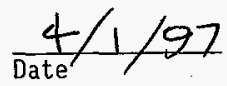


APPENDIX $M$

PAGE M- 14

$$
\begin{array}{r}
\text { HNF-SD - WM-ATR- } 191 \\
\text { Rev. } 0 \\
\text { Page } 410
\end{array}
$$

TEST DATA SHEET

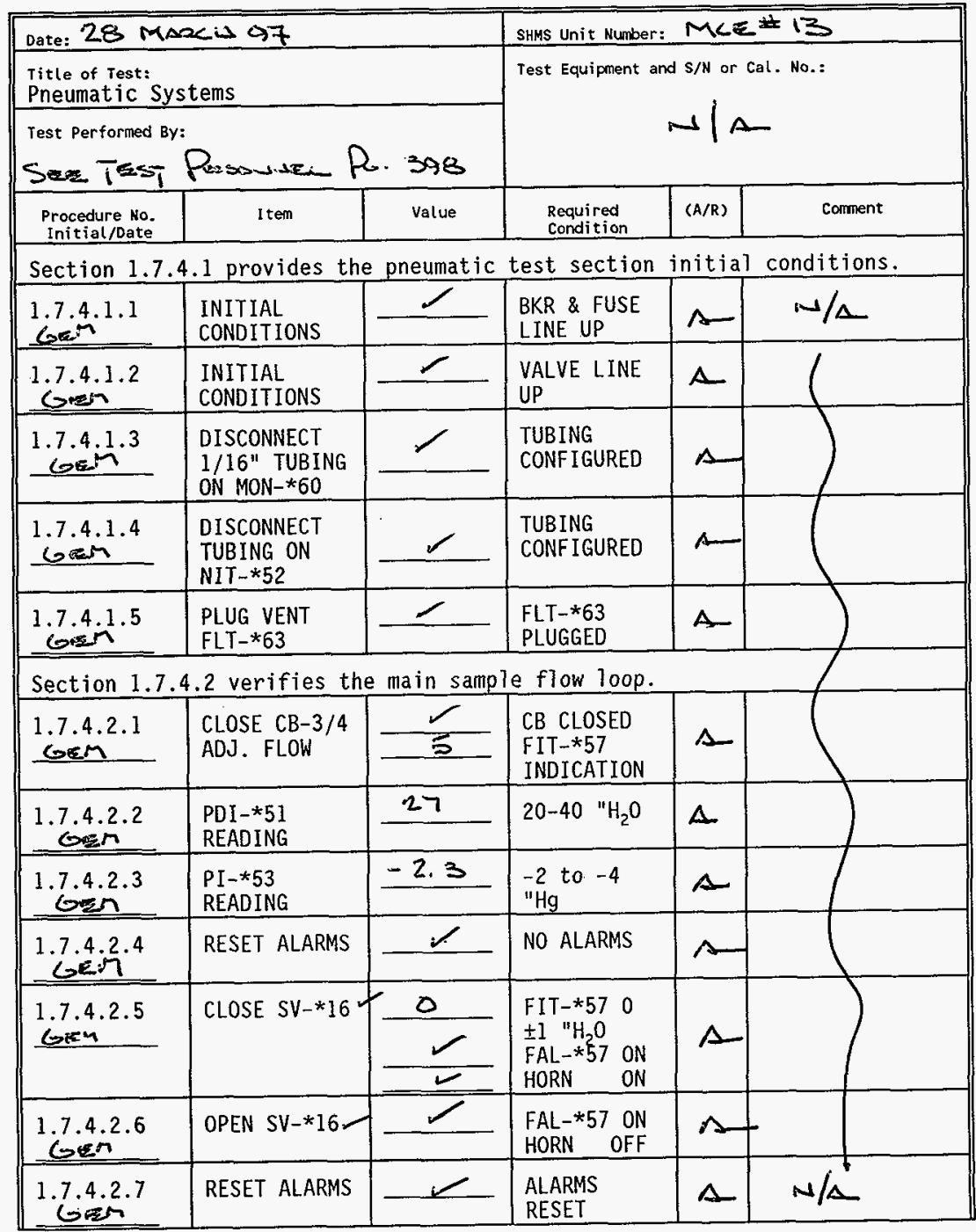

con st sic 3/28/97 
TEST DATA SHEET

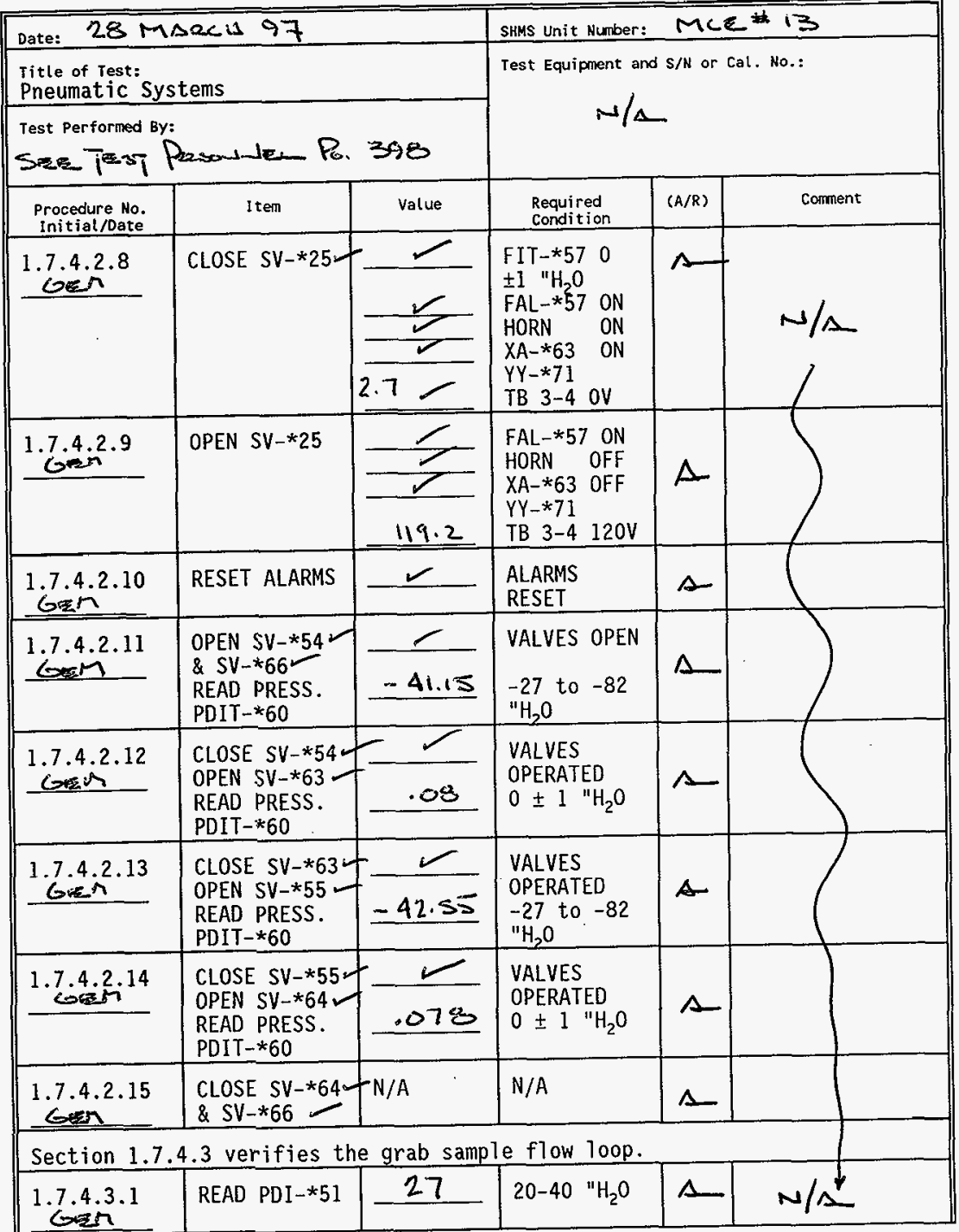


APPENDIX M

TEST DATA SHEET

\begin{tabular}{|c|c|c|c|c|c|}
\hline \multirow{2}{*}{\multicolumn{3}{|c|}{$\begin{array}{l}\text { Date: } 28 \text { Msacis } 97 \\
\text { Title of Test: } \\
\text { Pneumatic Systems }\end{array}$}} & \multicolumn{3}{|c|}{ 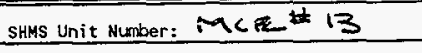 } \\
\hline & & & \multirow{2}{*}{\multicolumn{3}{|c|}{ Test Equipment and $\mathrm{S} / \mathrm{N}$ or Cal. No.: }} \\
\hline \multicolumn{3}{|c|}{ 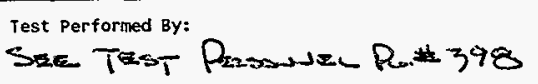 } & & & \\
\hline $\begin{array}{l}\text { Procedure No. } \\
\text { Initial/Date }\end{array}$ & I tem & value & $\begin{array}{l}\text { Required } \\
\text { condition }\end{array}$ & $(A / R)$ & Corment \\
\hline $\operatorname{cosen}^{1.7 .4 .2}$ & $\begin{array}{l}\text { PUSH PB-*59 } \\
\text { GRAB SAMPLE }\end{array}$ & $<$ & $\begin{array}{l}\text { START } \\
\text { STOPWATCH }\end{array}$ & $A$ & \\
\hline 1.7 .4 .3 .3 & $\begin{array}{l}\text { GRAB SAMPLE } \\
\text { LAMPS ON } \\
\text { READ FIV-*52 }\end{array}$ & $\angle$ & $\begin{array}{l}\text { PBL-*58 ON } \\
\text { PBL-*59 ON } \\
\text { YAL-*58 ON } \\
\text { FIV }-* 52 \quad 0 \\
\end{array}$ & 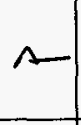 & \\
\hline $\begin{array}{l}1.7 .4 .3 .4 \\
\cos \end{array}$ & $\begin{array}{l}\text { OPEN SV } * 15 \\
\& \text { ADJ. FIV- } \\
* 52\end{array}$ & & $\begin{array}{l}\text { SV-*15 OPEN } \\
\text { FIV-*52 } \\
\text { FLOW } 10 \text { CFH }\end{array}$ & $A-$ & \\
\hline 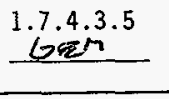 & READ PDI $-* 51$ & 30 & $\begin{array}{l}\text { PDI }-* 51 \\
\text { HIGHER THAN } \\
1.7 .4 .3 .1 \\
\end{array}$ & 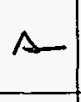 & \\
\hline $\begin{array}{l}1.7 .4 .3 .6 \\
\end{array}$ & CLOSE SV-*15r & 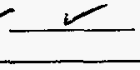 & FIV $-\star 52 \quad 0$ & $\Delta$ & \\
\hline $\begin{array}{r}1.7 .4 .3 .7 \\
6.75 \\
\end{array}$ & OPEN SV-*15 & 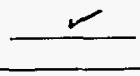 & $\begin{array}{l}\text { FIV-*52 } \\
\text { FLOW }\end{array}$ & $\Delta$ & \\
\hline $\begin{array}{l}1.7 .4 .3 .8 \\
\cos \end{array}$ & $\begin{array}{l}\text { PBL-*59 } \\
\text { GRAB SAMPLE } \\
\text { TIME } \\
\end{array}$ & & $\begin{array}{l}\text { PBL } * 59 \text { OFF } \\
\text { STOP WATCH } \\
5 \pm .5 \mathrm{MIN} \\
\end{array}$ & $A$ & \\
\hline 1.7 .4 .3 .9 & $\begin{array}{l}\text { PB-*58 RESET } \\
\text { SAMPLER }\end{array}$ & $\angle$ & $\begin{array}{l}\text { PBL- } * 58 \text { OFF } \\
\text { YAL }-* 58 \text { OFF }\end{array}$ & $\Lambda$ & \\
\hline \multicolumn{6}{|c|}{ Section 1.7 .4 .4 verifies the $\mathrm{H}_{2}$ cell calibration loop. } \\
\hline $\begin{array}{l}1.7 .4 .4 .1 \\
\end{array}$ & $\begin{array}{l}\text { CONNECT } \mathrm{H}_{2} \\
\text { CAL GAS }\end{array}$ & N/A & $\begin{array}{l}\text { N/A } \\
0 / 97\end{array}$ & $\Delta$ & \\
\hline 1.7 .4 .4 .2 & \begin{tabular}{|l} 
OPEN ISO \\
VALVE \\
\end{tabular} & $\$$ & $<10$ PSIG & $\Delta$ & \\
\hline $\begin{array}{c}1.7 .4 .4 .3 \\
\cos \end{array}$ & $\begin{array}{l}\text { OPEN SV }-* 18 \\
\text { ADJ. FIV }-\star 56\end{array}$ & -2 & $2 \pm .1 \mathrm{CFH}$ & $\Delta$ & \\
\hline
\end{tabular}

Gem Als 3:20/97 
TEST DATA SHEET

\begin{tabular}{|c|c|c|c|c|c|}
\hline \multicolumn{3}{|c|}{ Date: 23 MAac $\Delta 97$} & \multicolumn{3}{|c|}{ SHMS Unit Number: MCE 13} \\
\hline \multicolumn{3}{|c|}{$\begin{array}{l}\text { Title of Test: } \\
\text { Pneumatic Systems }\end{array}$} & \multirow{2}{*}{\multicolumn{3}{|c|}{ 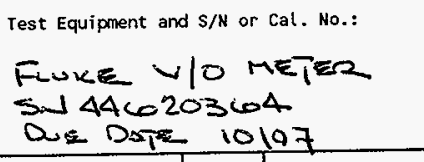 }} \\
\hline \multicolumn{3}{|c|}{$\begin{array}{l}\text { Test Performed By: } \\
\text { Sar TesT Pizsonde- Re } 398\end{array}$} & & & \\
\hline $\begin{array}{l}\text { Procedure No. } \\
\text { Initial/Date }\end{array}$ & Item & Value & $\begin{array}{l}\text { Requi red } \\
\text { Condition } \\
\end{array}$ & $(A / R)$ & comment \\
\hline $\begin{array}{l}1.7 .4 .4 .4 \\
\text { Gein }\end{array}$ & $\begin{array}{l}\text { VERIFY } \\
\text { CONDITIONS }\end{array}$ & $\frac{2}{2}$ & $\begin{array}{l}\mathrm{NIT}-* 54 \\
>-625 \% \mathrm{H}_{2} \\
\mathrm{NAH}-* 55 \mathrm{ON} \\
\mathrm{NAH}-* 54 \text { ON } \\
\mathrm{PBL}-* 59 \mathrm{ON} \\
\mathrm{PBL}-* 58 \text { ON } \\
\text { YAL-*58 ON } \\
\text { FIN-*52 FLO } \\
\text { HORN ON } \\
\text { TB2-21/22 } \\
\text { OPEN } \\
\end{array}$ & $\Lambda$ & \\
\hline isein & $\begin{array}{l}\text { ACKNOWLEDGE } \\
\text { HORN }\end{array}$ & $N / A$ & $N / A$ & $A$ & \\
\hline $\operatorname{los}^{1.7 .4 .4 .6}$ & $\begin{array}{l}\text { CLOSE SV-*18 } \\
\text { VERIFY } \\
\text { CONDITIONS }\end{array}$ & 2 & $\begin{array}{l}\text { FIV }-* 560 \\
\text { NIT-*54 } \\
<.625 \% \mathrm{H}_{2} \\
\text { NAH-*55 OFF } \\
\text { NAH-*54 ON } \\
\text { PBL-*58 ON } \\
\text { YAL-*58 ON } \\
\text { TB2-21/22 } \\
\text { CLOSED } \\
\end{array}$ & $\Delta$ & \\
\hline 1.7 .4 .4 .7 & $\begin{array}{l}\text { PUSH RESET } \\
\text { SAMPLER PB- } \\
* 58\end{array}$ & $\frac{r}{r}$ & $\begin{array}{l}\text { PBL-*58 OFF } \\
\text { YAL-*58 OFF }\end{array}$ & A & \\
\hline 1.7 .4 .4 .8 & RESET ALARM & 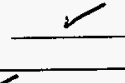 & $\mathrm{NAH}-* 54$ OFF & $A$ & \\
\hline 1.4 .4 .9 & $\begin{array}{l}\text { OPEN SV }-* 19 \\
\text { ADJ. FIV }-* 56\end{array}$ & 2 & $2 \pm .1 \mathrm{CFH}$ & $\Delta$ & $\omega / \Delta$ \\
\hline
\end{tabular}

wen $3 k e f 7$

su 
TEST DATA SHEET

\begin{tabular}{|c|c|c|c|c|c|}
\hline \multicolumn{3}{|c|}{ Date: 28 MD2Cu 97} & \multicolumn{3}{|c|}{ SHMS Unit Number: MCE 13} \\
\hline \multicolumn{3}{|c|}{$\begin{array}{l}\text { Title of Test: } \\
\text { Pneumatic Systems }\end{array}$} & \multirow{2}{*}{\multicolumn{3}{|c|}{ 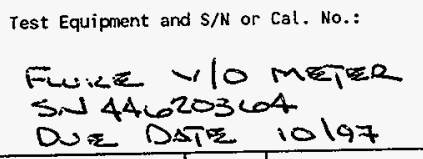 }} \\
\hline \multicolumn{3}{|c|}{ 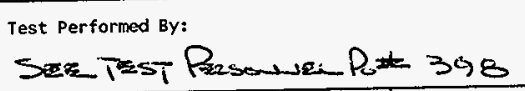 } & & & \\
\hline $\begin{array}{l}\text { Procedure No. } \\
\text { Initial/Date }\end{array}$ & Item & value & $\begin{array}{l}\text { Required } \\
\text { Condition } \\
\end{array}$ & $(A / R)$ & Comment \\
\hline$\underbrace{1.7 .4 .4 .10}$ & $\begin{array}{l}\text { VERIFY } \\
\text { CONDITIONS }\end{array}$ & $\frac{V}{V}$ & $\begin{array}{l}\text { NIT-*55 } \\
>.625 \% \mathrm{H}_{2} \\
\text { NAH-*55 ON } \\
\text { NAH-*54 ON } \\
\text { PBL-*59 ON } \\
\text { PBL-*58 ON } \\
\text { YAL-*58 ON } \\
\text { FIV }-* 52 \quad \text { FLO } \\
\text { HORN ON } \\
\text { TB2-21/22 } \\
\text { OPEN } \\
\end{array}$ & $\Lambda$ & \\
\hline $\begin{array}{l}1.7 .4 .4 .11 \\
\text { Gen } \\
\end{array}$ & $\begin{array}{l}\text { ACKNOWLEDGE } \\
\text { HORN }\end{array}$ & $N / A$ & N/A & $\Delta$ & \\
\hline 1.7 .4 .4 .12 & $\begin{array}{l}\text { CLOSE SV }-* 19 v \\
\text { VERIFY } \\
\text { CONDITIONS }\end{array}$ & 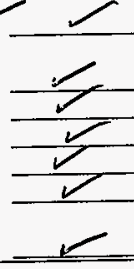 & $\begin{array}{l}\text { FIV-*56 } 0 \\
\text { NIT-*55 } \\
<.625 \% \mathrm{H}_{2} \\
\text { NAH- } * 55 \mathrm{OFF} \\
\text { NAH- } * 54 \text { ON } \\
\text { PBL- } * 58 \text { ON } \\
\text { YAL- } * 58 \text { ON } \\
\text { TB2-21/22 } \\
\text { CLOSED } \\
\end{array}$ & $\Delta$ & \\
\hline $\begin{array}{l}1.7 .4 .4 .13 \\
\text { Gxir }\end{array}$ & $\begin{array}{l}\text { PUSH RESET } \\
\text { SAMPLER PB- } \\
* 58\end{array}$ & & $\begin{array}{l}\text { PBL-*58 OFF } \\
\text { YAL-*58 OFF }\end{array}$ & $\Delta$ & \\
\hline 17.4 .4 .14 & RESET ALARM & & $\mathrm{NAH}-* 54$ OFF & $\Delta$ & \\
\hline 1.7.4.4.15 & $\begin{array}{l}\text { DISCONNECT } \\
\text { CAL. GAS }\end{array}$ & $N / A$ & $N / A$ & $\Delta$ & \\
\hline $\begin{array}{l}1.7 .4 .4 .16 \\
\end{array}$ & $\begin{array}{l}\text { PUSH PB }-* 54 \\
\text { VERIFY } \\
\text { CONDITIONS }\end{array}$ & - & $\begin{array}{l}X A-* 63 \quad \text { ON } \\
P B L-* 54 \text { ON } \\
T B 2-23 / 24 \\
\text { OPEN }\end{array}$ & $\wedge$ & \\
\hline $\begin{array}{l}1.7 .4 .4 .17 \\
\end{array}$ & $\begin{array}{l}\text { PUSH PB-*54 } \\
\text { VERIFY } \\
\text { CONDITIONS }\end{array}$ & 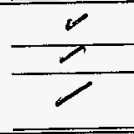 & $\begin{array}{l}X A-* 63 \text { OFF } \\
\text { PBL } * 54 \text { OFF } \\
\text { TB2-23/24 } \\
\text { CLOSED } \\
\end{array}$ & $\Delta$ & $N / \Delta$ \\
\hline
\end{tabular}


APPENDIX $M$

PAGE M- $/ 9$
HNF-SD - WM-ATR - 191

Rev. 0

TEST DATA SHEET

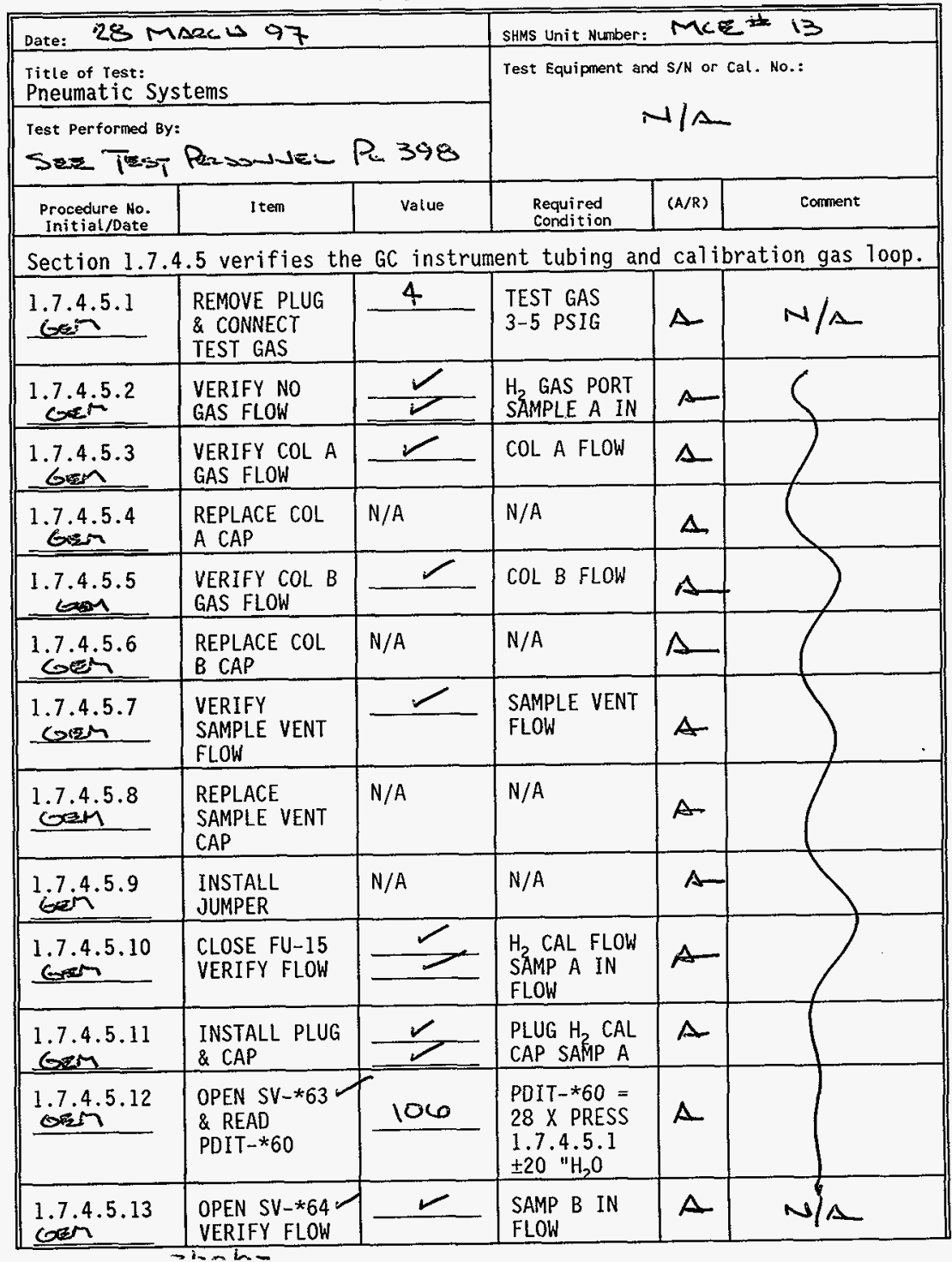


TEST DATA SHEET

\begin{tabular}{|c|c|c|c|c|c|}
\hline \multicolumn{3}{|c|}{ Date: 28 MAesis 97} & \multicolumn{3}{|c|}{ SHMS Unit Number: MCE I3 } \\
\hline \multicolumn{3}{|c|}{$\begin{array}{l}\text { Title of Test: } \\
\text { Pneumatic Systems }\end{array}$} & \multirow{2}{*}{\multicolumn{3}{|c|}{ Test Equipment and $S / \mathrm{N}$ or Cal. No.: }} \\
\hline \multicolumn{3}{|c|}{ 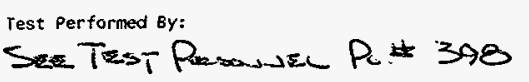 } & & & \\
\hline $\begin{array}{l}\text { Procedure No. } \\
\text { Initial/Date }\end{array}$ & Item & value & $\begin{array}{l}\text { Required } \\
\text { condition } \\
\end{array}$ & $(A / R)$ & Comment \\
\hline $\begin{array}{l}1.7 .4 .5 .14 \\
\text { ogen }\end{array}$ & $\begin{array}{l}\text { REMOVE TEST } \\
\text { GAS }\end{array}$ & N/A & $N / A$ & $\Delta$ & $\Delta$ \\
\hline $\begin{array}{c}1.7 .4 .5 .15 \\
\end{array}$ & $\begin{array}{l}\text { CLOSE SV-*63 } \\
\& \text { SV-*64 }\end{array}$ & $N / A$ & $N / A$ & $A$ & \\
\hline Ger. & $\begin{array}{l}\text { OPEN FU-15 } \\
\text { REMOVE } \\
\text { JUMPER }\end{array}$ & $\mathrm{N} / \mathrm{A}$ & $N / A$ & $A-1$ & \\
\hline $\begin{array}{l}1.7 .4 .5 .17 \\
\text { Genen }\end{array}$ & $\begin{array}{l}\text { INSTALL } \\
\text { SAMP A UNION }\end{array}$ & $N / A$ & $N / A$ & $\Delta$ & \\
\hline 6ein & $\begin{array}{l}\text { OPEN SV } * 60 \\
\text { VERIFY FLOW }\end{array}$ & & $\begin{array}{l}\text { FI-*60 } \\
\text { NO FLOW }\end{array}$ & $\Delta$ & \\
\hline$\frac{1.7 .4 .5 .19}{\mathrm{gen}}$ & $\begin{array}{l}\text { POWER SOV- } \\
\star 60 \text {, VERIFY } \\
\text { NO FLOW } \\
\end{array}$ & & $\begin{array}{l}\text { FI-*60 } \\
\text { NO FLOW }\end{array}$ & $A$ & \\
\hline $\begin{array}{l}1.7 .4 .5 .20 \\
\end{array}$ & $\begin{array}{l}\text { OPEN SV-*68 } \\
\text { ADJ SV-*67 }\end{array}$ & & $\begin{array}{l}\mathrm{FI}-* 60 \\
50 \mathrm{CCM}\end{array}$ & $\Delta$ & \\
\hline $\begin{array}{l}1.7 .4 .5 .21 \\
6.01\end{array}$ & $\begin{array}{l}\text { DEENERGIZE } \\
\text { SOV-*60 }\end{array}$ & 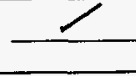 & $\begin{array}{l}\text { FI }-* 60 \text { FLOW } \\
\text { DECREASES }\end{array}$ & $\Lambda$ & \\
\hline 1.7 .4 .5 .22 & $\begin{array}{l}\text { CLOSE SV-*60 } \\
\& S V-* 68=\end{array}$ & $N / A$ & $N / A$ & 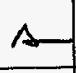 & \\
\hline 1.7 .4 .5 .23 & $\begin{array}{l}\text { REMOVE } \\
\text { SAMP A UNION }\end{array}$ & $N / A$ & $N / A$ & $\Delta$ & \\
\hline $\mathrm{gish}^{1.7 .4 .5 .24}$ & $\begin{array}{l}\text { CONNECT TEST } \\
\text { GAS } \mathrm{N}_{2} \mathrm{O} \text { PORT }\end{array}$ & 4 & $\begin{array}{l}\text { TEST GAS } \\
3-5 \text { PSIG }\end{array}$ & $A$ & \\
\hline 1.7 .4 .5 .25 & $\begin{array}{l}\text { VERIFY NO } \\
\text { FLOW }\end{array}$ & -2 & $\begin{array}{l}\text { SAMP B IN \& } \\
\text { FLT-*63 } \\
\text { NO FLOW }\end{array}$ & $\Delta$ & \\
\hline $\begin{array}{l}1.7 .4 .5 .26 \\
\operatorname{sen} \\
\end{array}$ & $\begin{array}{l}\text { INSTALL } \\
\text { JUMPER }\end{array}$ & $N / A$ & $N / A$ & $\Delta$ & \\
\hline $\begin{array}{l}1.7 .4 .5 .27 \\
\text { gen }\end{array}$ & $\begin{array}{l}\text { CLOSE FU-15 } \\
\text { VERIFY FLOW }\end{array}$ & $\frac{r}{2}$ & $\begin{array}{l}\text { SAMP B IN } \\
\text { FLT-*63 } \\
\text { FLOW }\end{array}$ & $\Lambda$ & \\
\hline
\end{tabular}


APPENDIX $M$

PAGE $M-2 r$
$H N F-S D-H M-A T R-191$
ReV. 0

Rev. 0
Page 417

TEST DATA SHEET

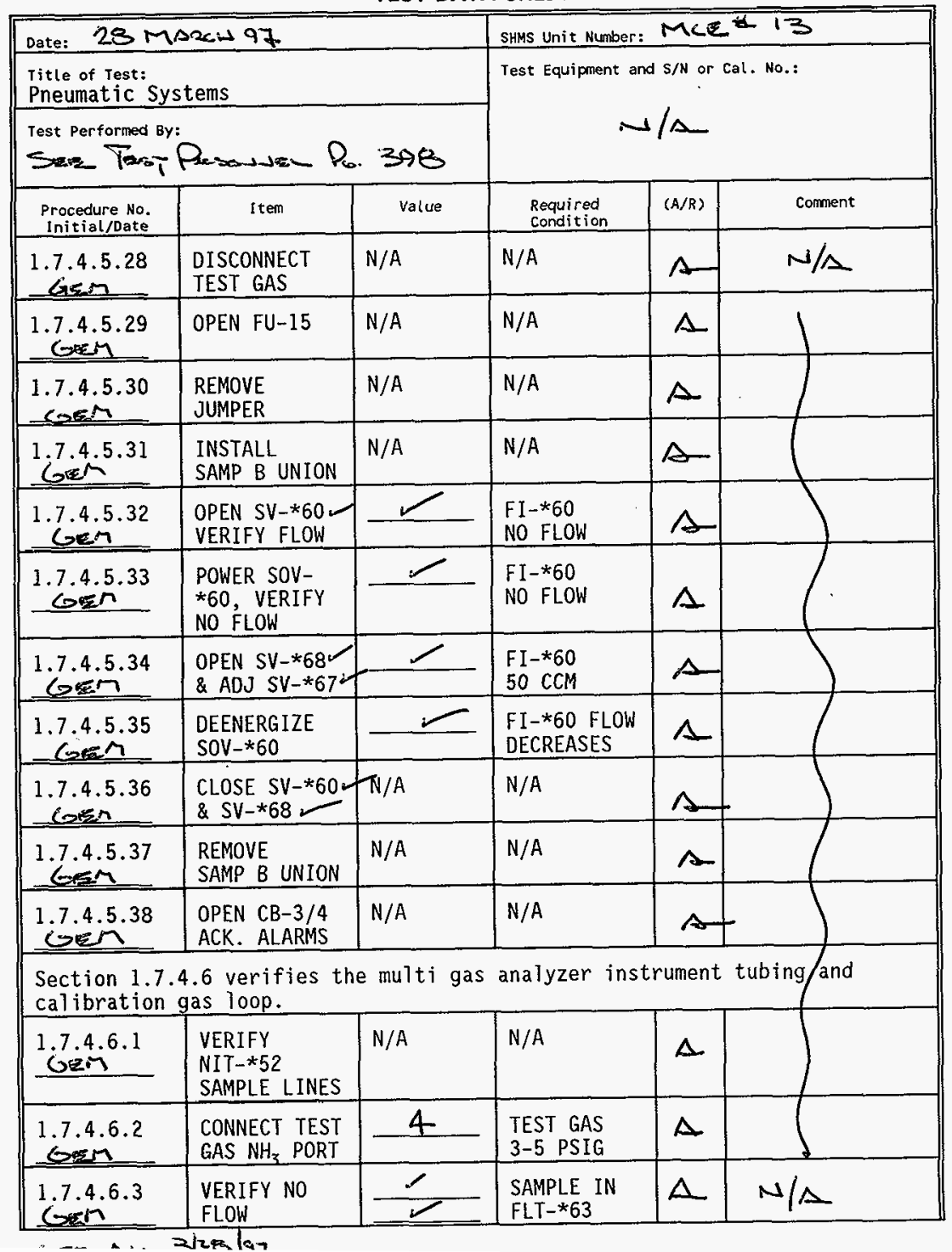


TEST DATA SHEET

\begin{tabular}{|c|c|c|c|c|c|}
\hline \multicolumn{3}{|c|}{ Date: $28 \mathrm{MA2C}+197$} & \multicolumn{3}{|c|}{ SHMS Unit Number: MCE 13} \\
\hline \multicolumn{3}{|c|}{$\begin{array}{l}\text { Title of Test: } \\
\text { Pneumatic Systems }\end{array}$} & \multirow{2}{*}{\multicolumn{3}{|c|}{ Test Equipment and $\mathrm{S} / \mathrm{M}$ or Cal. No.: }} \\
\hline \multicolumn{3}{|c|}{ 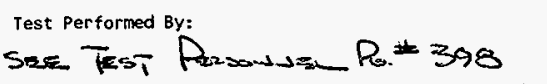 } & & & \\
\hline $\begin{array}{c}\text { Procedure No. } \\
\text { Initial/Date }\end{array}$ & Item & Value & $\begin{array}{l}\text { Required } \\
\text { Condition }\end{array}$ & $(A / R)$ & comment \\
\hline $\begin{array}{c}1.7 .4 .6 .4 \\
(x-9) \\
\end{array}$ & $\begin{array}{l}\text { INSTALL } \\
\text { JUMPER }\end{array}$ & $N / A$ & $N / A$ & 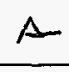 & $\mathrm{N} / \mathrm{s}$ \\
\hline $\begin{array}{l}1.7 .4 .6 .5 \\
6 \in h\end{array}$ & $\begin{array}{l}\text { CLOSE FU-15 } \\
\text { VERIFY GAS } \\
\text { FLOWS }\end{array}$ & $\frac{2}{2}$ & $\begin{array}{l}\text { SAMPLE IN } \\
\text { FLT }-* 63 \\
\text { FIV }-* 70 \\
\end{array}$ & $\Delta$ & \\
\hline $\begin{array}{l}1.7 .4 .6 .6 \\
6 e^{2}\end{array}$ & $\begin{array}{l}\text { PLUG FLT-*63 } \\
\& \text { MON-*60 } \\
\text { SAMPLE VENT }\end{array}$ & N/A & $N / A$ & A & \\
\hline $\begin{array}{l}1.7 .4 .6 .7 \\
\text { 6.2. }\end{array}$ & $\begin{array}{l}\text { OPEN SV-*66, } \\
\text { SV-*70 CAP } \\
\text { SAMPLE IN }\end{array}$ & 105.3 & $\begin{array}{l}\text { POIT-*60 }= \\
28 \times \text { PRESS } \\
1.7 .4 .6 .2 \\
\pm 20 \mathrm{HH}_{2} \mathrm{O}\end{array}$ & $\Delta$ & \\
\hline $\begin{array}{l}1.7 .4 .6 .8 \\
\end{array}$ & $\begin{array}{l}\text { CLOSE SV-*66- } \\
\text { SV-*70 } \\
\text { REMOVE CAP }\end{array}$ & N/A & $N / A$ & $A$ & \\
\hline $\mathrm{Grin}^{1.7 .4 .6 .9}$ & $\begin{array}{l}\text { REMOVE FLT- } \\
\star 63 \text { PLUG } \\
\end{array}$ & N/A & N/A & $\Delta$ & \\
\hline 1.7 .4 .6 .10 & $\begin{array}{l}\text { REMOVE TEST } \\
\text { GAS. }\end{array}$ & N/A & $N / A$ & A & \\
\hline 1.7.4.6.11 & $\begin{array}{l}\text { CLOSE SV-*05 } \\
\& \text { SV-*16 }\end{array}$ & N/A & $N / A$ & $\Delta$ & \\
\hline 1.7.4.6.12 & $\begin{array}{l}\text { CONNECT TEST } \\
\text { GAS }\end{array}$ & 3 & $\begin{array}{l}\text { TEST GAS } \\
2-4 \text { PSIG }\end{array}$ & $\Delta$ & \\
\hline $\begin{array}{l}1.7 .4 .6 .13 \\
6 \times 2 m\end{array}$ & $\begin{array}{l}\text { OPEN SV-*07 } \\
\& \text { VERIFY } \\
\text { NO FLOW }\end{array}$ & $\frac{1}{2}$ & $\begin{array}{l}\text { SAMPLE IN } \\
\text { SAMPLE OUT }\end{array}$ & $\Delta$ & \\
\hline$\frac{1.7 .4 .6 .14}{\operatorname{sen}}$ & $\begin{array}{l}\text { OPEN SV }-* 09 \\
\& \text { VERIFY } \\
\text { NO FLOW }\end{array}$ & $\stackrel{L}{L}$ & $\begin{array}{l}\text { SAMPLE IN } \\
\text { SAMPLE OUT }\end{array}$ & A & \\
\hline$\frac{1.7 .4 .6 .15}{\operatorname{sen}}$ & $\begin{array}{l}\text { OPEN FU-15 \& } \\
\text { VERIFY FLOW }\end{array}$ & & $\begin{array}{l}\text { SAMPLE FLOW } \\
\text { IN - YES } \\
\text { OUT - NO } \\
\end{array}$ & $\Delta$ & \\
\hline 安. & $\begin{array}{l}\text { CLOSE SV-*09 } \\
\text { VERIFY FLOW }\end{array}$ & $-<$ & $\begin{array}{l}\text { SAMPLE IN } \\
\text { NO FLOW }\end{array}$ & $\perp$ & $\omega / \alpha^{b}$ \\
\hline
\end{tabular}


TEST DATA SHEET

\begin{tabular}{|c|c|c|c|c|c|}
\hline \multicolumn{3}{|c|}{ Date: 28 MAncis 97} & \multicolumn{3}{|c|}{ McE 13} \\
\hline \multicolumn{3}{|c|}{$\begin{array}{l}\text { Title of Test: } \\
\text { Pneumatic Systems }\end{array}$} & \multirow{2}{*}{\multicolumn{3}{|c|}{ Test Equipment and $\mathrm{S} / \mathrm{N}$ or Cal. No.: }} \\
\hline \multicolumn{3}{|c|}{ 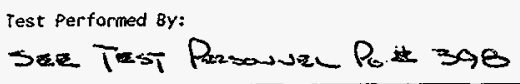 } & & & \\
\hline $\begin{array}{c}\text { Procedure No. } \\
\text { Initial/Date }\end{array}$ & Item & Value & $\begin{array}{l}\text { Reguired } \\
\text { Condition }\end{array}$ & $(A / R)$ & Comment \\
\hline$\frac{1.7 .4 .6 .17}{\mathrm{com}^{+1}}$ & $\begin{array}{l}\text { OPEN SV-*10 } \\
\text { VERIFY FLOW }\end{array}$ & 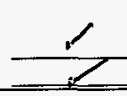 & $\begin{array}{l}\text { SAMPLE FLOW } \\
\text { IN - NO } \\
\text { OUT - YES } \\
\end{array}$ & $\Delta$ & \\
\hline$\frac{1.7 .4 .6 .18}{6.7}$ & $\begin{array}{l}\text { REMOVE TEST } \\
\text { GAS \& PLUG } \\
\text { SV-*07 }\end{array}$ & N/A & N/A & A & \\
\hline $\begin{array}{c}1.7 .4 .6 .19 \\
\end{array}$ & $\begin{array}{l}\text { CLOSE SV-*07 } \\
\& \text { SV-*10 }\end{array}$ & N/A & N/A & $\Lambda$ & \\
\hline $\mathrm{Gem}^{1.7 .4 .6 .20}$ & $\begin{array}{l}\text { OPEN SV-*05 } \\
\& \text { SV }-* 16\end{array}$ & N/A & N/A & $\Lambda$ & \\
\hline $\begin{array}{c}1.7 .4 .6 .21 \\
\end{array}$ & $\begin{array}{l}\text { REMOVE } \\
\text { JUMPER }\end{array}$ & N/A & N/A & $\Delta$ & \\
\hline \multicolumn{6}{|c|}{ Section 1.7.4.7 verifies the carrier gas supply lines. } \\
\hline 2.7 .4 .7 .1 & $\begin{array}{l}\text { CONNECT TEST } \\
\text { GAS }\end{array}$ & 4 & $3-5$ PSIG & $\Delta$ & \\
\hline $\begin{array}{l}1.7 .4 .7 .2 \\
6 x=M\end{array}$ & $\begin{array}{l}\text { REMOVE PLUG } \\
\& \text { VERIFY } \\
\text { FLOW } \\
\end{array}$ & 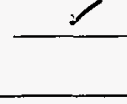 & $\begin{array}{l}\mathrm{N}_{2} \text { CARRIER } \\
\text { GAS FLOWS }\end{array}$ & $\Delta$ & \\
\hline 1.7 .4 .7 .3 & $\begin{array}{l}\text { DISCONNECT } \\
\text { TEST GAS }\end{array}$ & N/A & N/A & $\Delta$ & \\
\hline $\begin{array}{l}1.7 .4 .7 .4 \\
0 x^{2}-n\end{array}$ & $\begin{array}{l}\text { CONNECT TEST } \\
\text { GAS }\end{array}$ & 4 & 3-5 PSIG & $A$ & \\
\hline 1.7 .4 .7 .5 & $\begin{array}{l}\text { REMOVE PLUG } \\
\text { \& VERIFY } \\
\text { FLOW }\end{array}$ & 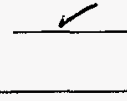 & $\begin{array}{l}\text { He CARRIER } \\
\text { GAS FLOWS }\end{array}$ & $\wedge$ & \\
\hline $\begin{array}{l}1.7 .4 .7 .6 \\
\sin \end{array}$ & $\begin{array}{l}\text { DISCONNECT } \\
\text { TEST GAS }\end{array}$ & N/A & N/A & A & \\
\hline 1.7 .5 & $\begin{array}{l}\text { TEST SEC. } \\
1.7 .4 \text { DONE }\end{array}$ & YMane & $\begin{array}{l}\text { Bi Bnow } \\
\text { tor signature }\end{array}$ & & $\frac{3-28-97}{\text { Date }}$ \\
\hline
\end{tabular}


TEST DATA SHEET

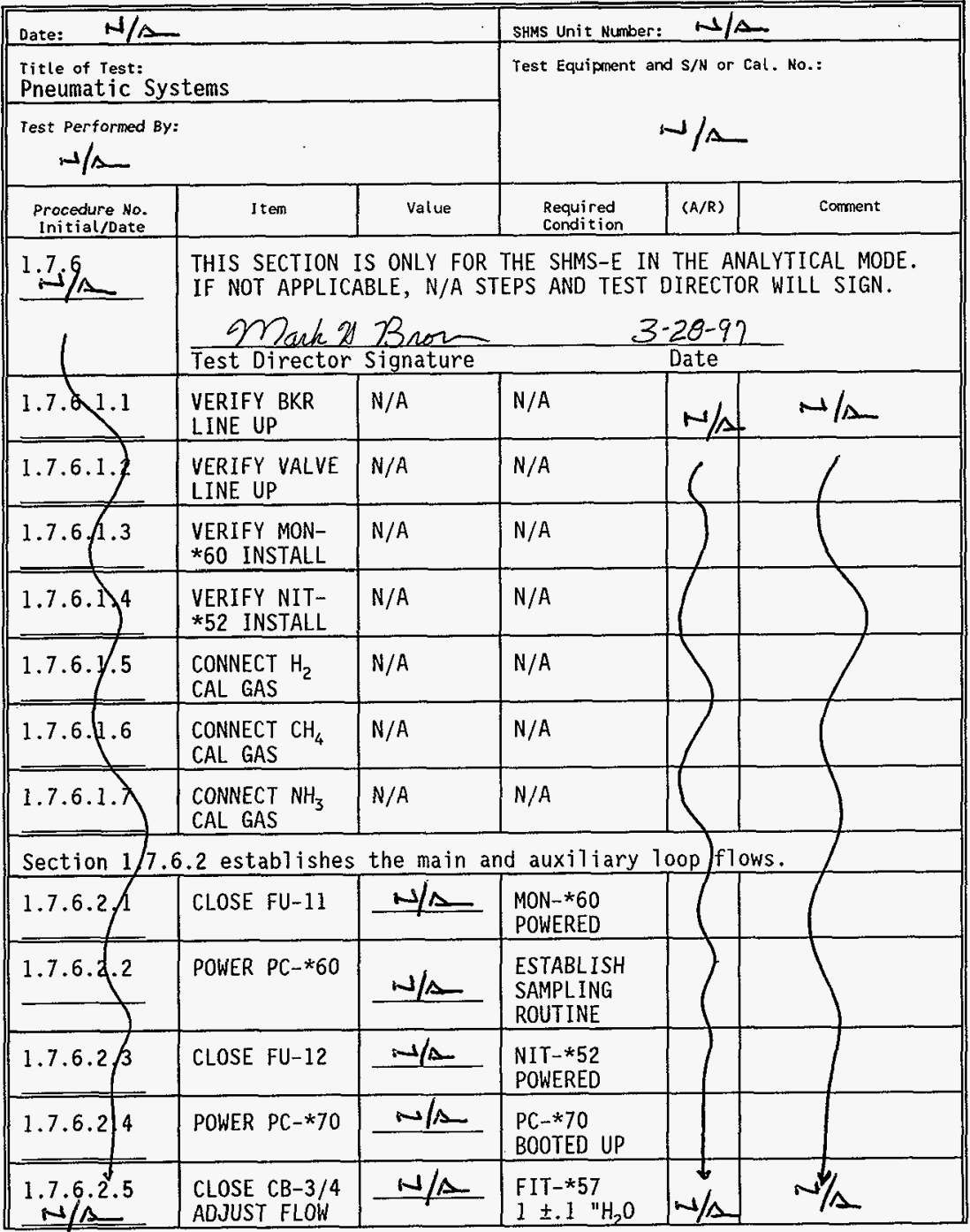


APPENDIX $M$

HNF-SD-WM-ATR-191
ReV. 0

Page 421

TEST DATA SHEET

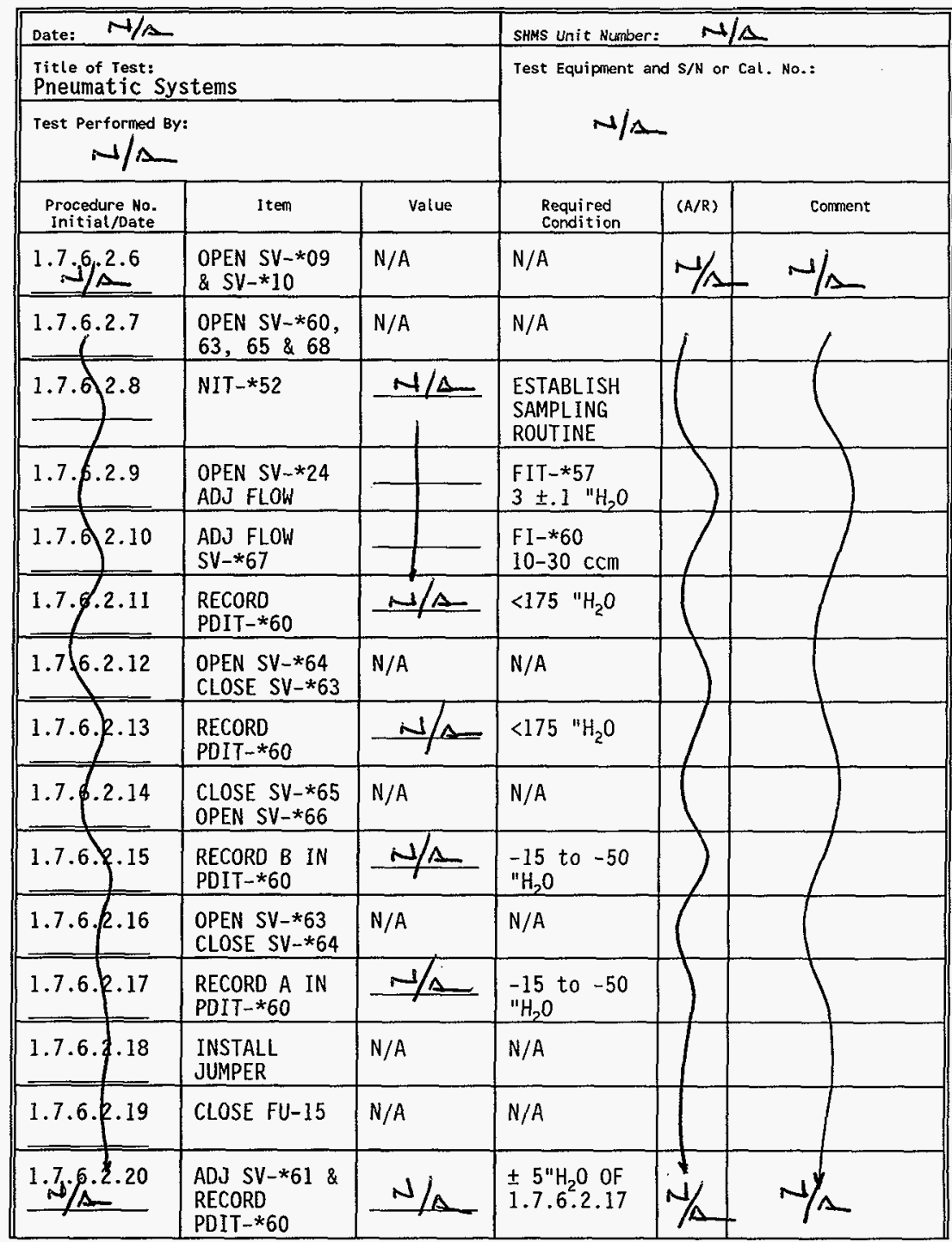


APPENDIX $M$

HNF-SD-WM-ATR-191

PAGE $M-26$

TEST DATA SHEET

\begin{tabular}{|c|c|c|c|c|c|}
\hline Date: $N / \Lambda$ & & & SHAS Unit Number: & $N$ & \\
\hline $\begin{array}{l}\text { ritle of Test: } \\
\text { Pneumatic } S \mathrm{~J}\end{array}$ & tems & & Test Equipment an & $\mathrm{d} / \mathrm{N}$ or & \\
\hline Test Performed B & & & $\mathrm{m} / \mathrm{C}$ & & \\
\hline $\begin{array}{l}\text { Procedure No. } \\
\text { Initial/Date }\end{array}$ & Item & Value & $\begin{array}{l}\text { Required } \\
\text { Condition }\end{array}$ & $(A / R)$ & Comment \\
\hline$\frac{1.7 .6 / s^{2.21}}{1}$ & $\begin{array}{l}\text { CAL MON-*60 } \\
\text { CHANNEL A }\end{array}$ & $\frac{N}{1 / 1}$ & $\begin{array}{l}\text { READINGS } \\
\text { WITHIN } \pm 5 \%\end{array}$ & $\omega$ & \\
\hline 1.76 .2 .22 & OPEN FU-15 & $N / A$ & N/A & & \\
\hline 1.7 .6$. & $\begin{array}{l}\text { REMOVE } \\
\text { JUMPER }\end{array}$ & $N / A$ & N/A & & \\
\hline 1.7 .6 .2 .24 & $\begin{array}{l}\text { OPEN SV }-* 64 \\
\text { CLOSE SV-*63 }\end{array}$ & N/A & N/A & & \\
\hline $\begin{array}{l}1.7 .6 / 2.25 \\
\end{array}$ & $\begin{array}{l}\text { INSTALL } \\
\text { JUMPER } \\
\end{array}$ & N/A & $N / A$ & & \\
\hline 1.7 .5 .2 .26 & CLOSE FU-15 & N/A & $N / A$ & & \\
\hline 1.7 .6 & $\begin{array}{l}\text { ADJ SV-*62 \& } \\
\text { RECORD } \\
\text { PDIT-*60 }\end{array}$ & $-N / S$ & $\begin{array}{l} \pm 5^{\prime \prime} \mathrm{H}_{2} \mathrm{O} \text { OF } \\
1.7 .6 .2 .15\end{array}$ & & \\
\hline 1.7 .6 .2 .18 & $\begin{array}{l}\text { CAL MON-*60 } \\
\text { CHANNEL B }\end{array}$ & $\frac{1}{1 / 1}$ & $\begin{array}{l}\text { READINGS } \\
\text { WITHIN } \pm 5 \%\end{array}$ & & \\
\hline $1.7 .6 / 2.29$ & OPEN FU-15 & $\mathrm{N} / \mathrm{A}^{\prime}$ & $N / A$ & & \\
\hline 1.7 .6 .2 .30 & $\begin{array}{l}\text { REMOVE } \\
\text { JUMPER }\end{array}$ & $N / A$ & $N / A$ & & \\
\hline 1.7 .6 .2 .31 & $\begin{array}{l}\text { CLOSE SV }-* 64 \\
\text { OPEN SV-*70 }\end{array}$ & $N / A$ & $N / A$ & & \\
\hline $1.7 .6 / 2.32$ & $\begin{array}{l}\text { RECORD } \\
\text { PDIT-*60 } \\
\text { NIT-*52 IN }\end{array}$ & $\omega / \Delta$ & $\begin{array}{l}0 \text { to }-30 \\
\mathrm{~N}_{2} \mathrm{O}\end{array}$ & & \\
\hline \begin{tabular}{ll|l}
1.7 .6 & 2.33
\end{tabular} & $\begin{array}{l}\text { INSTALL } \\
\text { JUMPER } \\
\end{array}$ & $N / A$ & $N / A$ & & 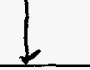 \\
\hline 1.7 .6 .2 .34 & CLOSE FU- 15 & $N / A$ & $N / A$ & $y$ & Is \\
\hline
\end{tabular}


APPENDIX $M$

PAGE M-27

$$
\begin{array}{r}
\text { HNF-SD-LM-ATR-191 } \\
\text { Rev. } 0 \\
\text { Page } 423
\end{array}
$$

TEST DATA SHEET

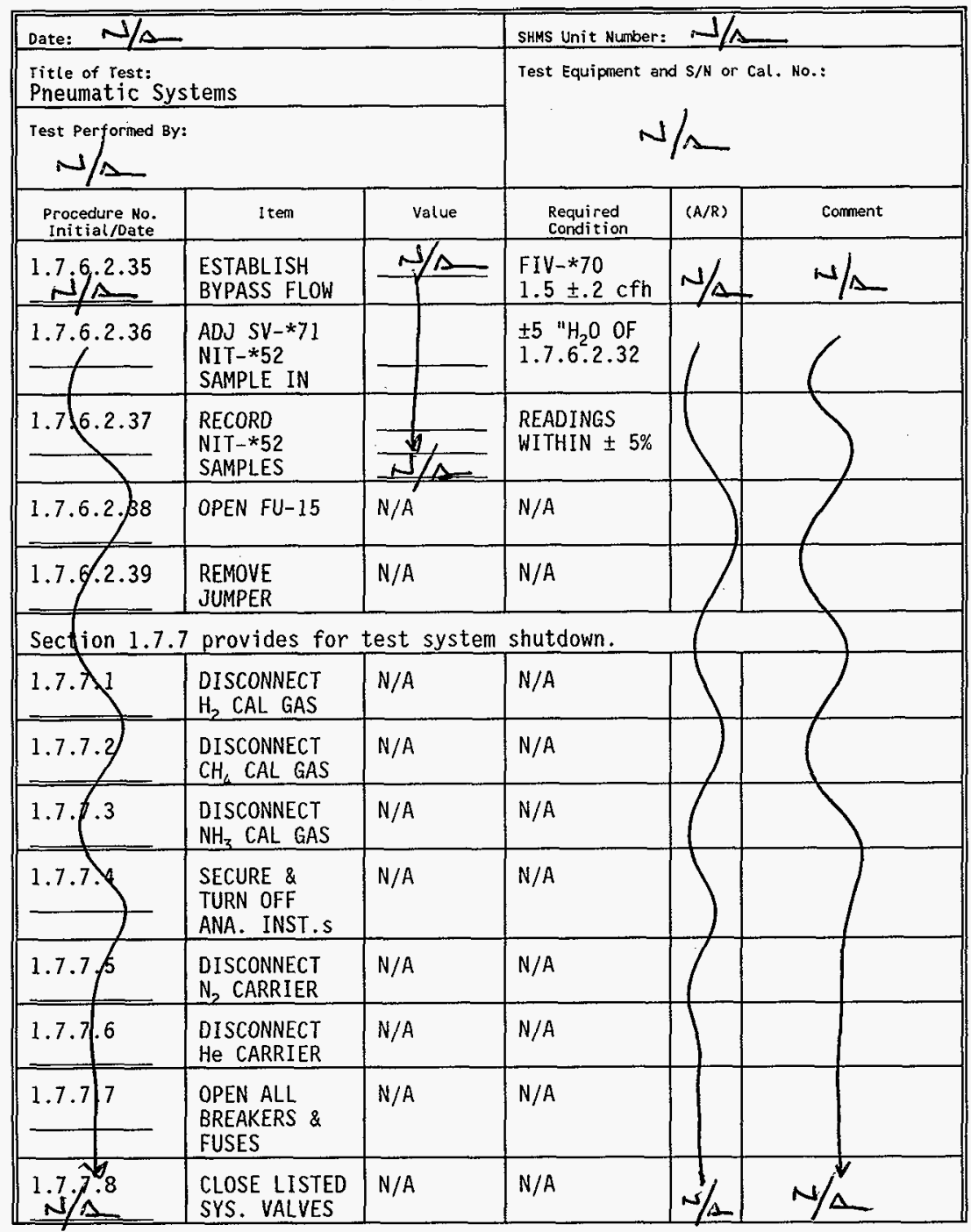


APPENDIX $M$

PAGE M-Z8
Buts:

$\therefore$
HNF-SD-WM-ATR = 191

Rev. 0

Page 424

TEST DATA SHEET

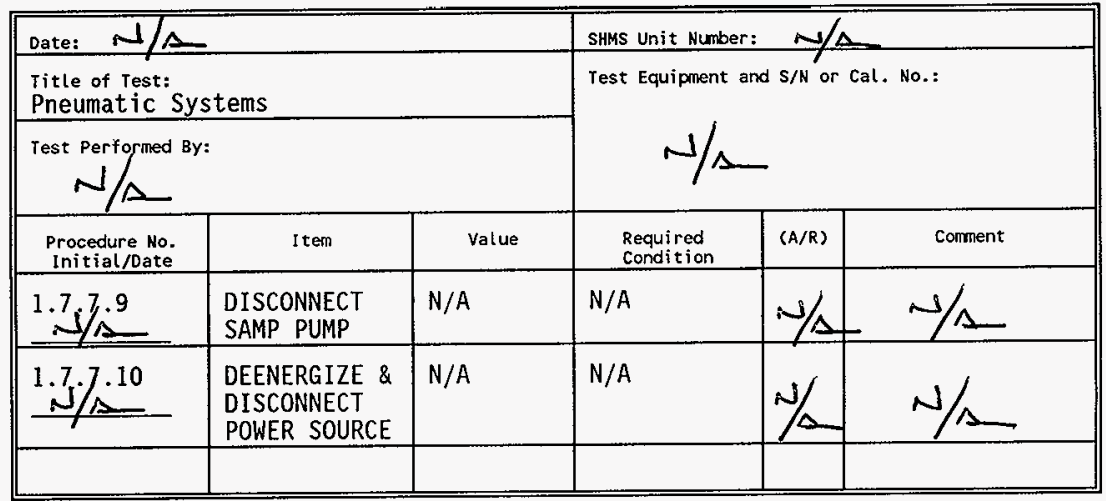

Test Witness/Review:

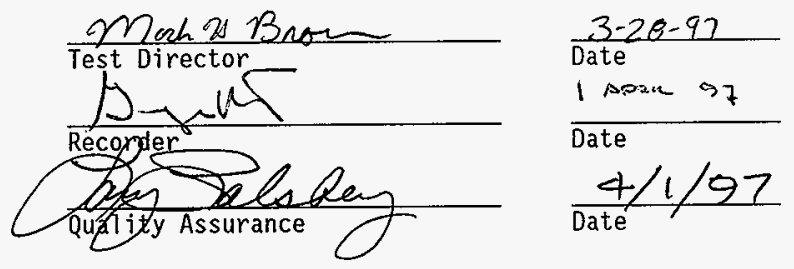


APPENDIX $M$

HWF-SD-WM-ATR- 191

PAGE M-Z9

Rev.
Page 425

TEST EXCEPTION SHEET

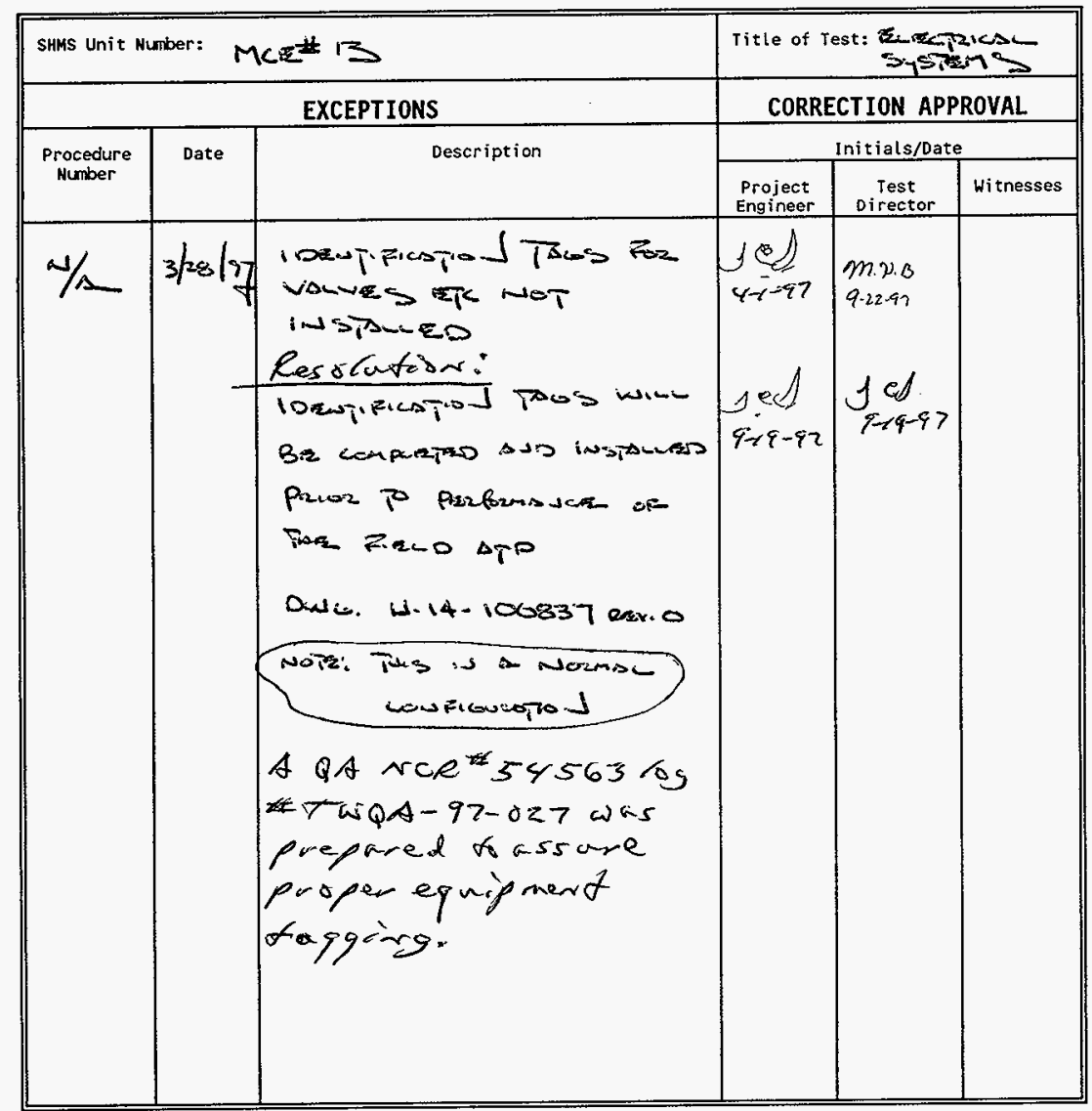

TEST APPROVED WITH EXCEPTIONS

10 Ph and

WHC Project Engineer

$\frac{\zeta-\langle-q\rangle}{\text { Date }}$

IApeic 97

Recorder

Date

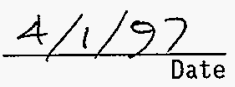


APPENDIX $M$

PAGE $M-30$
INF - SD - WM-ATR- 191

Rev. 0
Page 426

TEST LOG

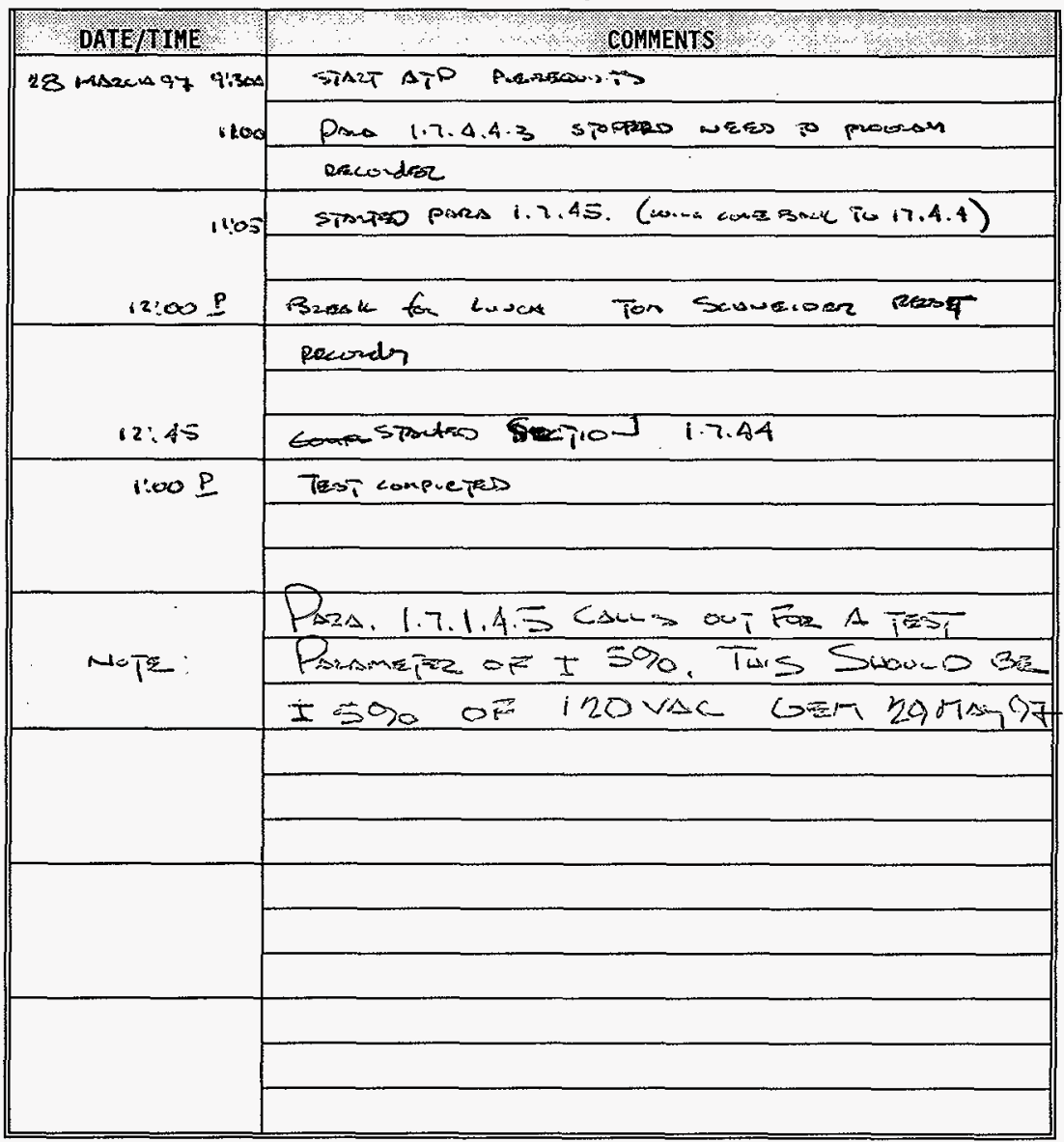


$\therefore \ldots$

$\mathrm{OS}$

APPENDIX N 
TEST EXECUTION SHEET

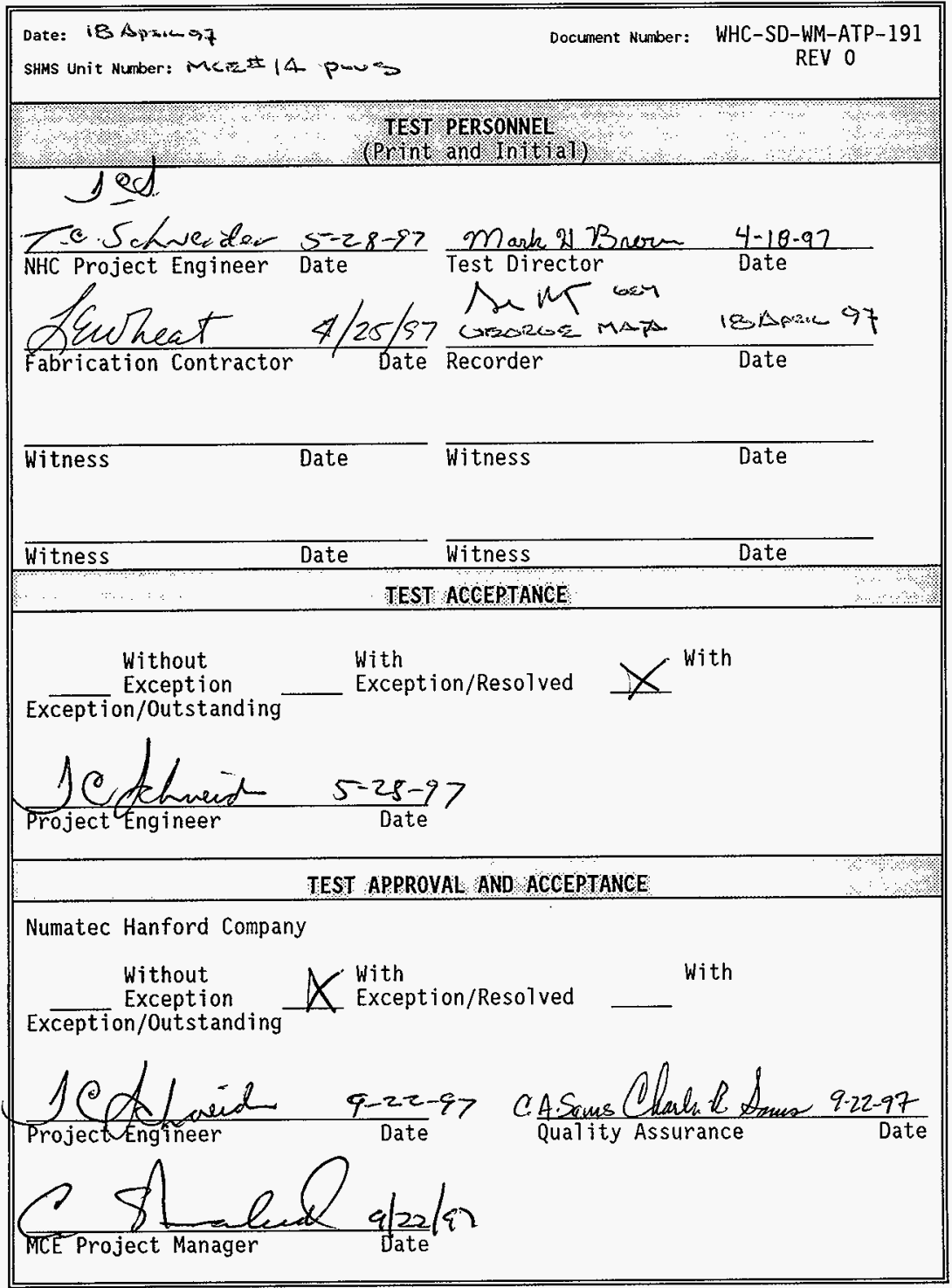


SHMS Unit Number:

\section{PREREQUISITES AND INITIAL TEST CONDITIONS}

The following conditions shall exist at the start of the acceptance testing. Initial and date to verify that each of the following items have been accomplished.

Leir a/fia Systems being tested have been inspected for workmanship and for compliance with design.

cam 4 fos 19 Continuity and megger tests have been performed on portions of the electrical and instrument systems being tested, as required.

Gen 4.18197 Leak tests on the pneumatic systems have been performed.

Gow shisy. The following circuit breakers and fuses are installed per the specified size and are open

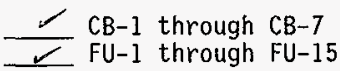

Gon fifeigh All test instruments have a currently valid calibration stamp attached that indicates a calibration traceable to the National Institute of Standards and Technology.

Gealis The following process and control instrument systems have been initially configured and aligned for proper operation.

FIT-*57, FSL-*57, NIT-*54, NIT-*55, (NR-*54), PDIT-*60, TIC-*50, TIC-*56, TIS $-* 62$, YYC $-* 01$ NOTE: NR-*54 is not used in the ANALYTICAL configuration.

Ger 4 ist personnel responsible for directing and witnessing the performance of the tests described in this ATP have read and understand appropriate certified vendor information (CVI) pertaining to the operation of the equipment to be tested.

corer 4 [ie 197 CLOSE all system manual valves with the exception of the following:

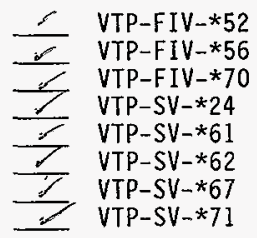

4087 The sample pump VTP-P-*50, connected to the cabinet sample out port, but vented to atmosphere for testing. 
APPENDIX N

PAGE $\mathrm{N}-4$

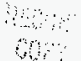

HNF-SD-WM-ATR- 191

Rev. 0

Page 430

ande. 207 Vac 1 phase 15-20 ampere temporary power source has been connected, but not energized, to the appropriate TB1 terminals per H-14-100838.

Verify by signature and date that all prerequisites have been met.

$\frac{\text { Monh } 2 . \beta_{n o r}}{\text { Test Director Signature }} \frac{4-18-97}{\text { Date }}$




\section{TEST DATA SHEET}

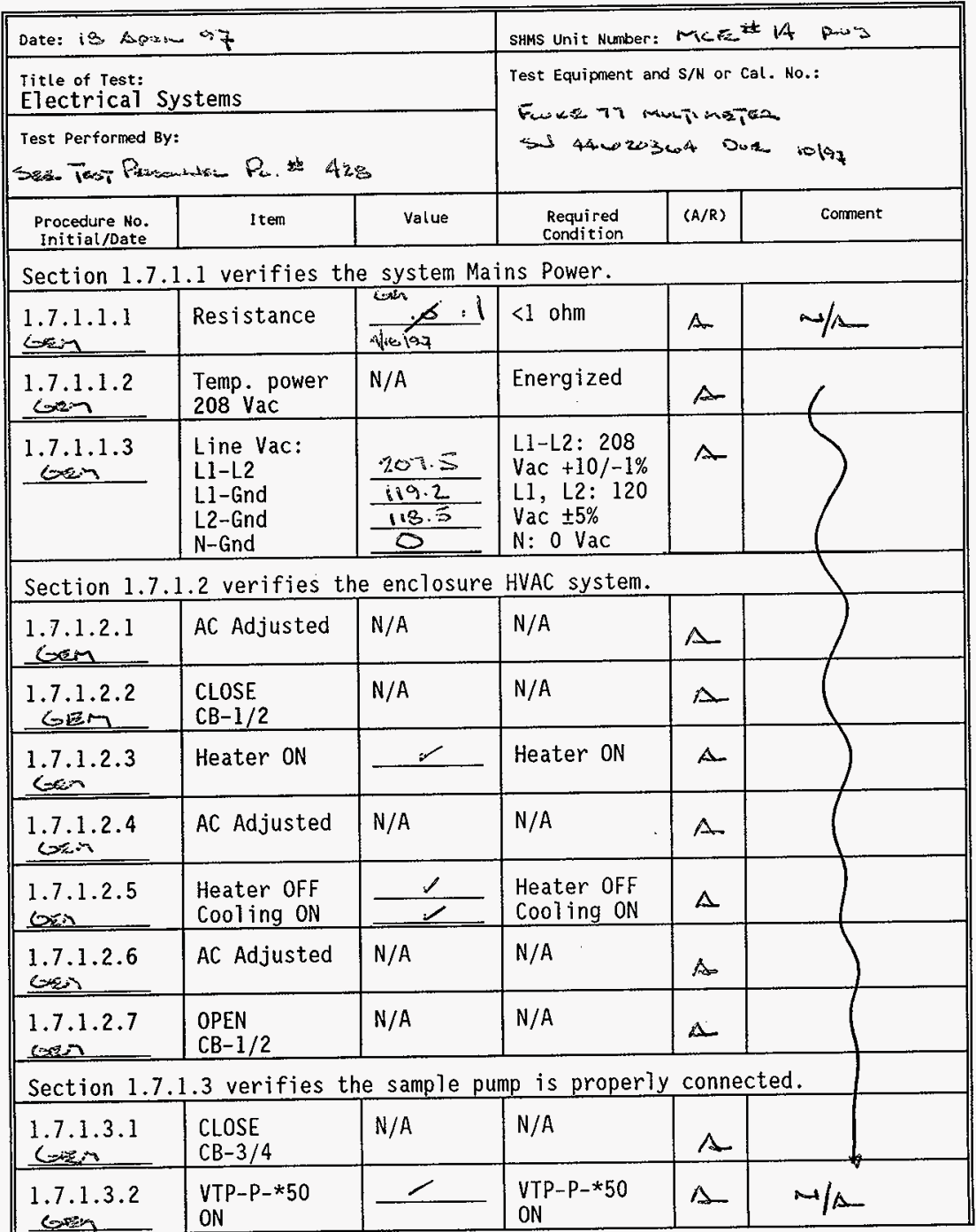


TEST DATA SHEET

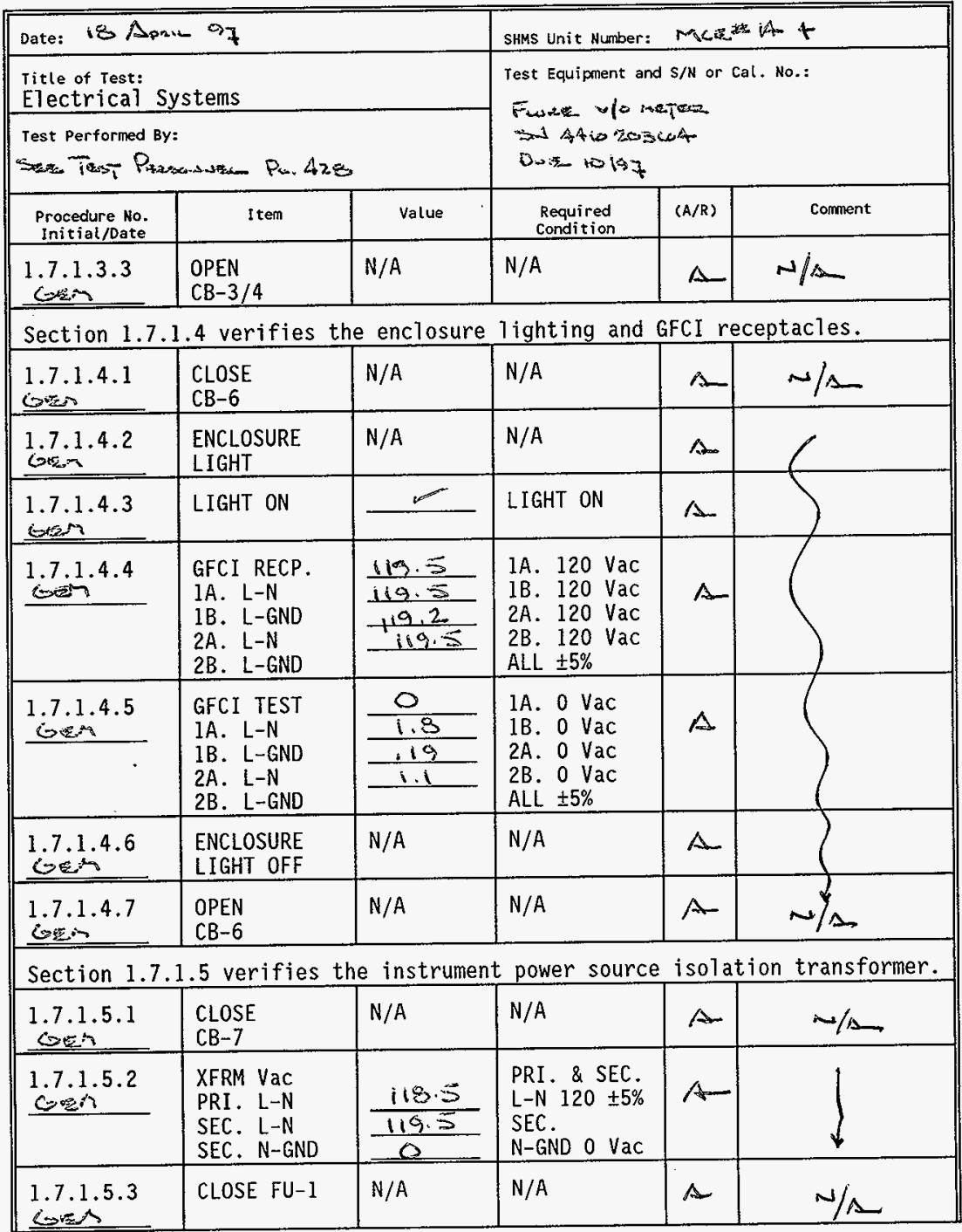


TEST DATA SHEET

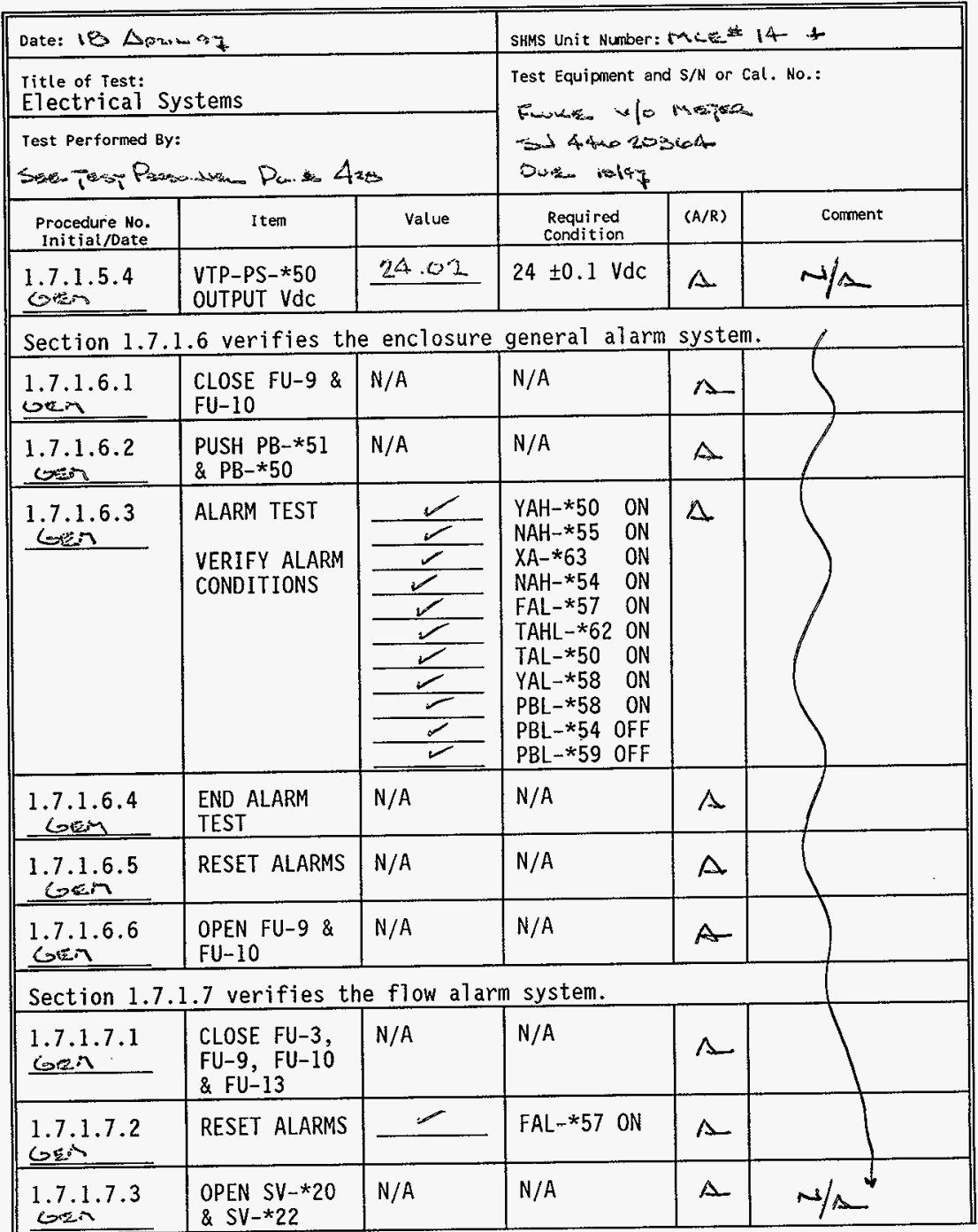




\section{TEST DATA SHEET}

\begin{tabular}{|c|c|c|c|c|c|}
\hline \multicolumn{3}{|c|}{ 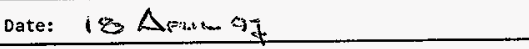 } & \multicolumn{3}{|c|}{ SHMS Unit Number: M<茜 $14+$} \\
\hline \multicolumn{3}{|c|}{$\begin{array}{l}\text { Title of rest: } \\
\text { Electrical Systems }\end{array}$} & \multirow{2}{*}{\multicolumn{3}{|c|}{ 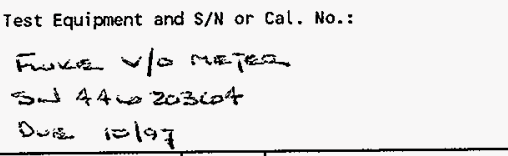 }} \\
\hline \multicolumn{3}{|c|}{$\begin{array}{l}\text { Test Performed By: } \\
\text { sar- Pu. } 4 \text { as }\end{array}$} & & & \\
\hline $\begin{array}{l}\text { Procedure No. } \\
\text { Initial/Date }\end{array}$ & I tem & Value & $\begin{array}{c}\text { Required } \\
\text { Condition }\end{array}$ & $(A / R)$ & Corment \\
\hline $\begin{array}{l}1.7 .1 .7 .4 \\
20.7\end{array}$ & $\begin{array}{l}\text { FIT-*57 \& } \\
\text { FSL-*57 } \\
\text { POWERED }\end{array}$ & $\frac{2}{2}$ & $\begin{array}{l}\text { FIT-*57 ON } \\
\text { FSL-*57 ON }\end{array}$ & $\Lambda$ & \\
\hline $\begin{array}{l}1.7 .1 .7 .5 \\
6.7\end{array}$ & $\begin{array}{l}\text { MUX }-* 70 \\
\text { TB5+/TB6- }\end{array}$ & 1.0 & $1.0 \pm 0.2 \mathrm{Vdc}$ & $\Lambda$ & $\begin{array}{l}\text { TB3.5ice } \\
\text { Meswined At }\end{array}$ \\
\hline $\begin{array}{l}1.7 .1 .7 .6 \\
\text { S.5.7 }\end{array}$ & $\begin{array}{l}\text { CLOSE SV-*20 } \\
\& \text { SV }-* 22\end{array}$ & $N / A$ & $N / A$ & $A$ & \\
\hline $\begin{array}{l}1.7 .1 .7 .7 \\
\cos \end{array}$ & $\begin{array}{l}\text { OPEN FU-3, } \\
\text { FU-9, FU-10 } \\
\& \text { FU-13 }\end{array}$ & N/A & $N / A$ & $\Delta$ & \\
\hline \multicolumn{6}{|c|}{ Section 1.7 .1 .8 verifies the $\mathrm{H}_{2}$ monitor wiring. } \\
\hline $\begin{array}{l}1.7 .1 .8 .1 \\
\text { sein }\end{array}$ & $\begin{array}{l}\text { CLOSE FU-2,- } \\
F U-4, F U-5,- \\
F U-9 \& \text { FU-10- }\end{array}$ & $N / A$ & $N / A$ & A & \\
\hline $\begin{array}{l}1.7 .1 .8 .2 \\
\text { Sxim } \\
\end{array}$ & RESET ALARMS & 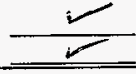 & $\begin{array}{l}\mathrm{NAH}-* 54 \text { OFF } \\
\mathrm{NAH}-* 55 \text { OFF }\end{array}$ & A & \\
\hline 1.7 .1 .8 .3 & $\begin{array}{l}\text { INSTRUMENTS } \\
\text { POWERED }\end{array}$ & $\frac{2}{2 / 2}$ & $\begin{array}{ll}M U X-* 70 & \text { ON } \\
\text { NIT-*54 ON } \\
\text { NIT-*55 ON } \\
\text { NR-*54 ON } \\
Y Y C-* 01 & \text { ON } \\
\end{array}$ & $A$ & 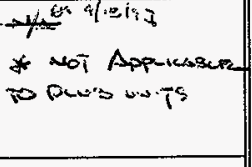 \\
\hline $\begin{array}{l}1.7 .1 .8 .4 \\
\text { gen }\end{array}$ & $\begin{array}{l}\text { OPEN FU-2, } \\
\text { FU-4, FU-5, } \\
\text { FU-9 \& FU-10 }\end{array}$ & $N / A$ & N/A & A & \\
\hline \multicolumn{6}{|c|}{ Section 1.7 .1 .9 verifies the SHMS-E heat trace control system. $\int$} \\
\hline 1.7 .1 .9 .1 & $\begin{array}{l}\text { INSTALL LOAD } \\
\text { VTP-TIC-*50 }\end{array}$ & $N / A$ & $N / A$ & $\Delta$ & \\
\hline $\begin{array}{l}1.7 .1 .9 .2 \\
\text { bien }\end{array}$ & $\begin{array}{l}\text { CLOSE CB-5,- } \\
\text { FU-6, FU-7,- } \\
\text { FU-9 \& FU-10- } \\
\text { and RESET } \\
\text { ALARMS }\end{array}$ & $N / A$ & $N / A$ & $\Delta$ & \\
\hline
\end{tabular}


TEST DATA SHEET

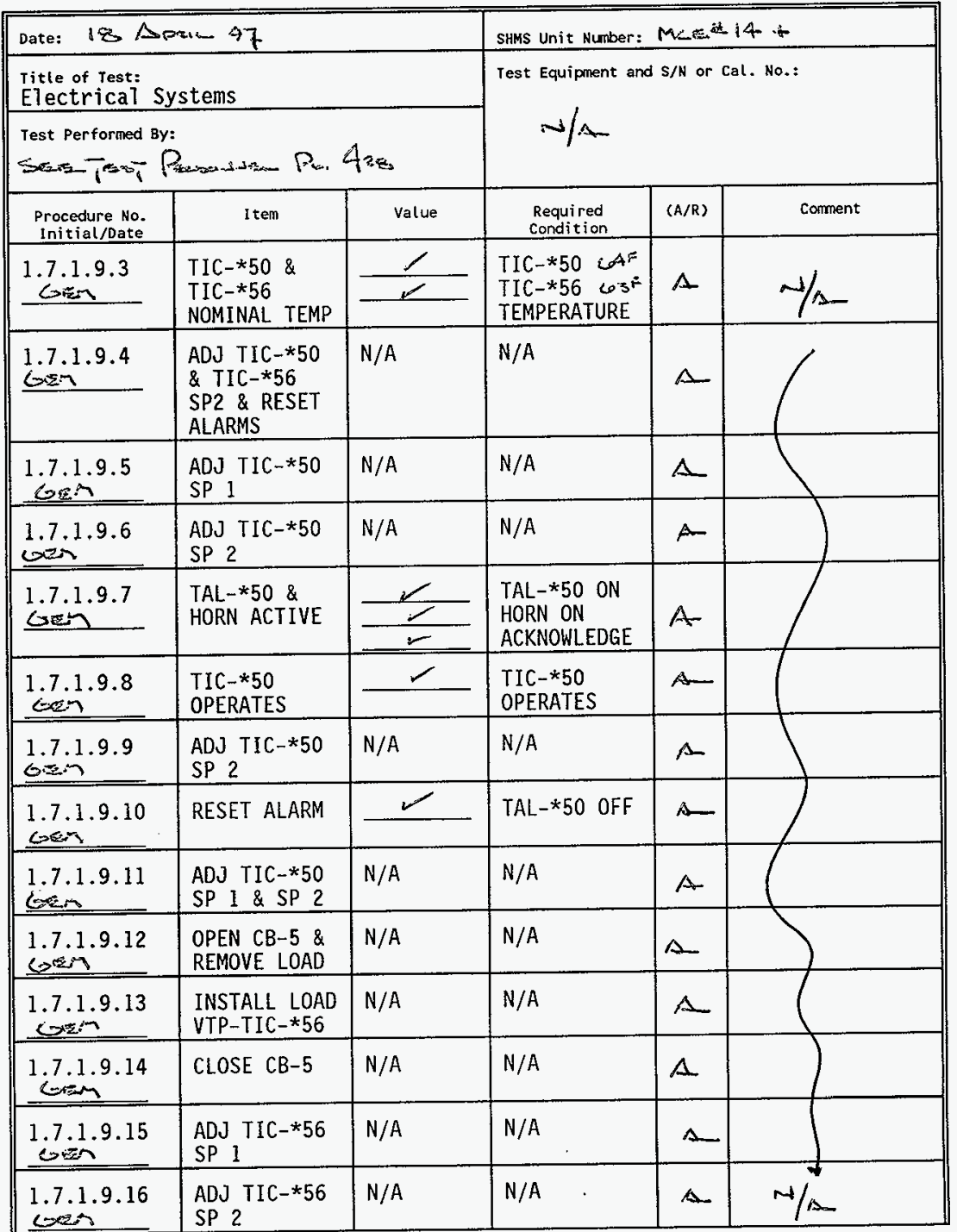


TEST DATA SHEET

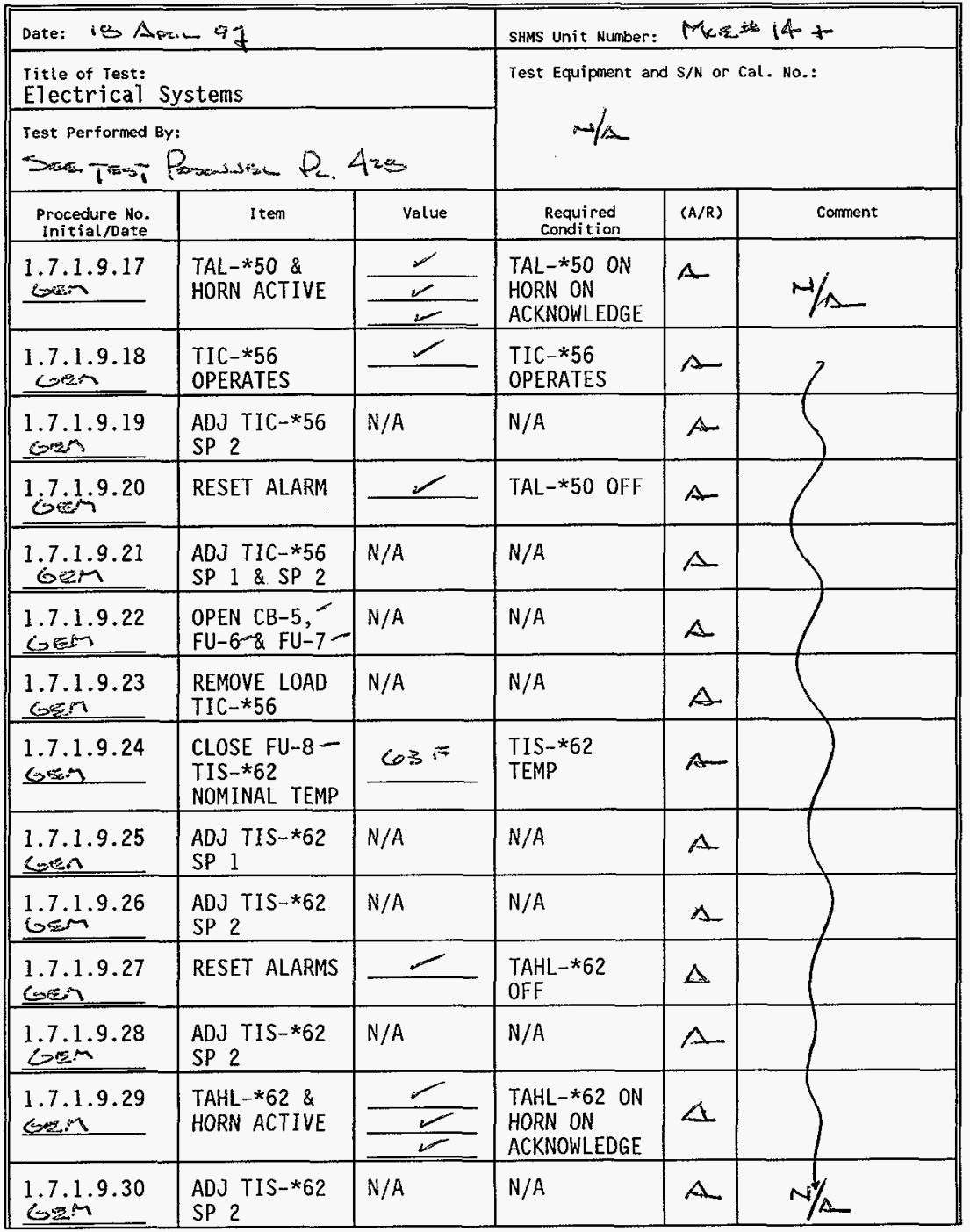


TEST DATA SHEET

\begin{tabular}{|c|c|c|c|c|c|}
\hline \multicolumn{3}{|c|}{ Date: 10 Amin 97} & \multicolumn{3}{|c|}{ SHMS Unit Number: $M_{K} B_{2}$ in $A_{+}+$} \\
\hline \multicolumn{3}{|c|}{$\begin{array}{l}\text { Title of Test: } \\
\text { Electrical Systems }\end{array}$} & \multirow{2}{*}{\multicolumn{3}{|c|}{ 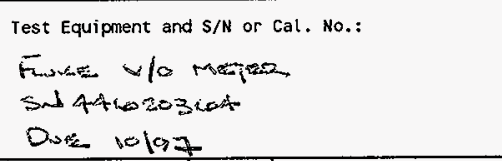 }} \\
\hline \multicolumn{3}{|c|}{ 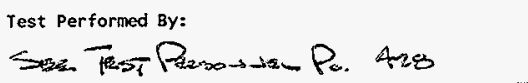 } & & & \\
\hline $\begin{array}{l}\text { Procedure No. } \\
\text { Initial/Date }\end{array}$ & Item & value & $\begin{array}{l}\text { Required } \\
\text { condition }\end{array}$ & $(A / R)$ & Corment \\
\hline $\begin{array}{l}1.7 .1 .9 .31 \\
\end{array}$ & RESET ALARMS & - & $\begin{array}{l}\text { TAHL-*62 } \\
\text { OFF }\end{array}$ & $\Delta$ & \\
\hline $\begin{array}{l}1.7 .1 .9 .32 \\
\text { cxem } \\
\end{array}$ & $\begin{array}{l}\text { ADJ TIS-*62 } \\
\text { SP } 1\end{array}$ & $N / A$ & $N / A$ & A & \\
\hline $\sin ^{1.7 .1 .9 .33}$ & $\begin{array}{l}\text { TAHL-*62 \& } \\
\text { HORN ACTIVE }\end{array}$ & $\frac{1}{2}$ & $\begin{array}{l}\text { TAHL-*62 ON } \\
\text { HORN ON } \\
\text { ACKNOWLEDGE }\end{array}$ & $\triangle$ & \\
\hline $\sin ^{1.7 .1 .9 .34}$ & $\begin{array}{l}\text { ADJ TIS-*62 } \\
\text { SP } 1 \& S P 2\end{array}$ & N/A & $N / A$ & Aि & \\
\hline $\sin ^{1.7 .1 .9 .35}$ & $\begin{array}{l}\text { OPEN FU-8, } \\
\text { FU-9 \& FU-10- }\end{array}$ & $N / A$ & $N / A$ & A & \\
\hline \multicolumn{6}{|c|}{ Section 1.7.1.10 verifies the differential pressure transmitter system. } \\
\hline $\begin{array}{l}1.7 .1 .10 .1 \\
\operatorname{sen} \\
\end{array}$ & $\begin{array}{l}\text { REMOVE PLUGS } \\
\text { BAL PDIT-*60 }\end{array}$ & $N / A$ & N/A & 政 & \\
\hline $\sin ^{1.7 .1 .10 .2}$ & $\begin{array}{l}\text { CLOSE FU-14 } \\
\text { REC PDIT-*60 } \\
\text { PRESSURE }\end{array}$ & -.03 & $\begin{array}{l}\text { PDIT-*60 } \\
\text { PRESSURE } \\
0 \pm 1^{\prime \prime} \mathrm{H}_{2} \mathrm{O} \\
\end{array}$ & $\Delta$ & \\
\hline $\mathrm{Ggen}^{1.7 .1 .10 .3}$ & $\begin{array}{l}\text { MUX-*70 Vdc } \\
\text { TB7+\& TB8- }\end{array}$ & 2.15 & $\begin{array}{l}\text { MUX-*70 Vdc } \\
2.2 \pm 0.1\end{array}$ & $\Delta$ & TB 3.7 .889 \\
\hline $6 z^{1.7 .1 .10 .4}$ & $\begin{array}{l}\text { REPLACE } \\
\text { PLUGS ON } \\
\text { PDIT }-* 60 \\
\end{array}$ & $N / A$ & $N / A$ & A & \\
\hline $\begin{array}{c}1.7 .1 .10 .5 \\
\end{array}$ & OPEN FU-14 & $N / A$ & $\mathrm{~N} / \mathrm{A}$ & A & \\
\hline \multicolumn{6}{|c|}{ Section 1.7.1.11 verifies the gas chromatograph power. } \\
\hline $\begin{array}{l}1.7 .1 .11 .1 \\
102 n\end{array}$ & CLOSE FU-11 & $N / A$ & $N / A$ & $\Delta$ & \\
\hline $\operatorname{Binin}^{11.2}$ & $\begin{array}{l}\text { POWER } \\
\text { MON-*60 } \\
\end{array}$ & $\sim$ & $\begin{array}{l}\text { MON }-* 60 \\
\text { POWERED } \\
\end{array}$ & $\Delta$ & ) \\
\hline $\begin{array}{l}1.7 .1 .11 .3 \\
\cos \end{array}$ & OPEN FU-11 & $N / A$ & $N / A$ & $A$ & \\
\hline
\end{tabular}


APPENDIX $N$

PAGE $N-12$
HWF-SD-WN-ATR-191

Rev. 0

Page 438

TEST DATA SHEET

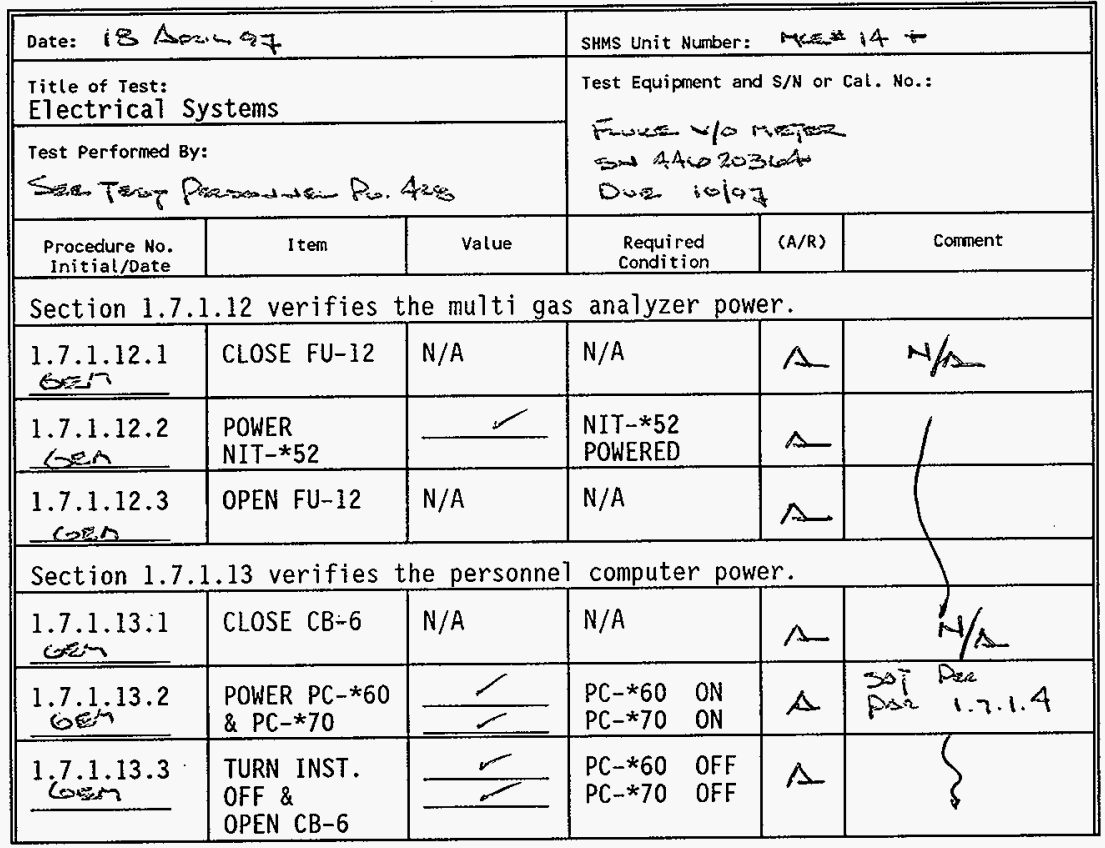

sing A.C $4 / 8 / 87$ 
APPENDIX N

PAGE $\mathrm{N}-13$
HNF-SD-WH-ATR-191

Rev. 0

TEST DATA SHEET

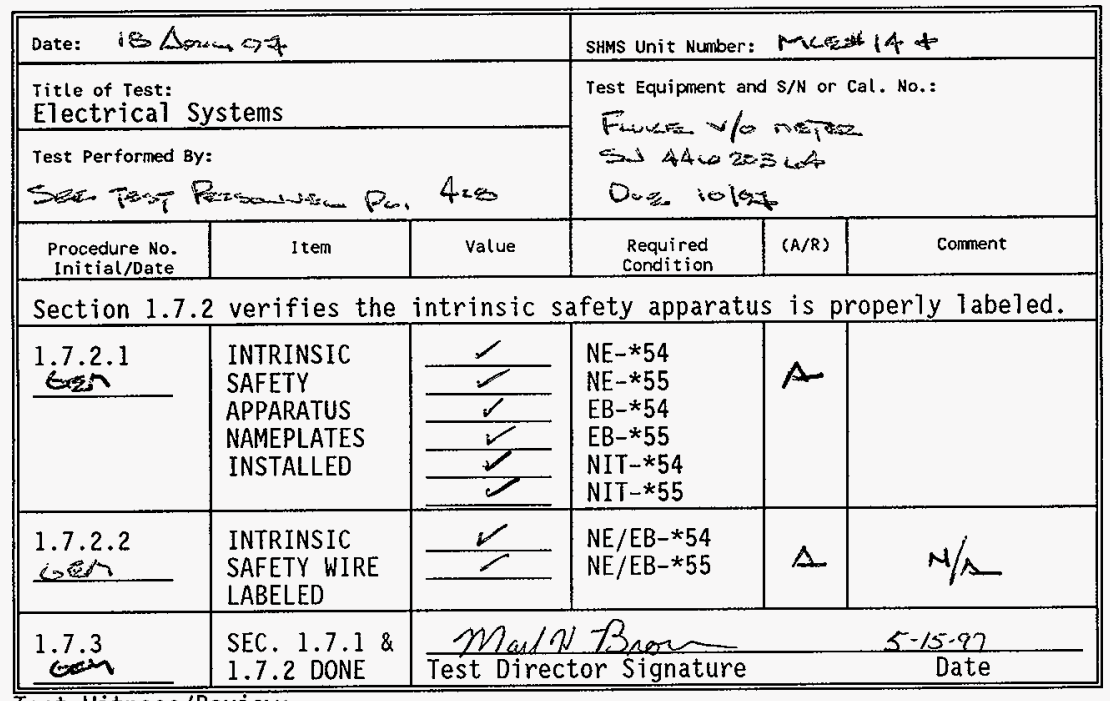

Test Witness/Review:

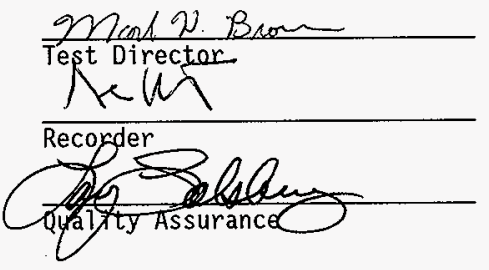

$\frac{5-15-91}{\text { Date }}$

$\frac{\text { co ms y } 97}{\text { Date }}$

$\frac{5 / 28 / 97}{\text { Date }}$ 


\section{TEST DATA SHEET}

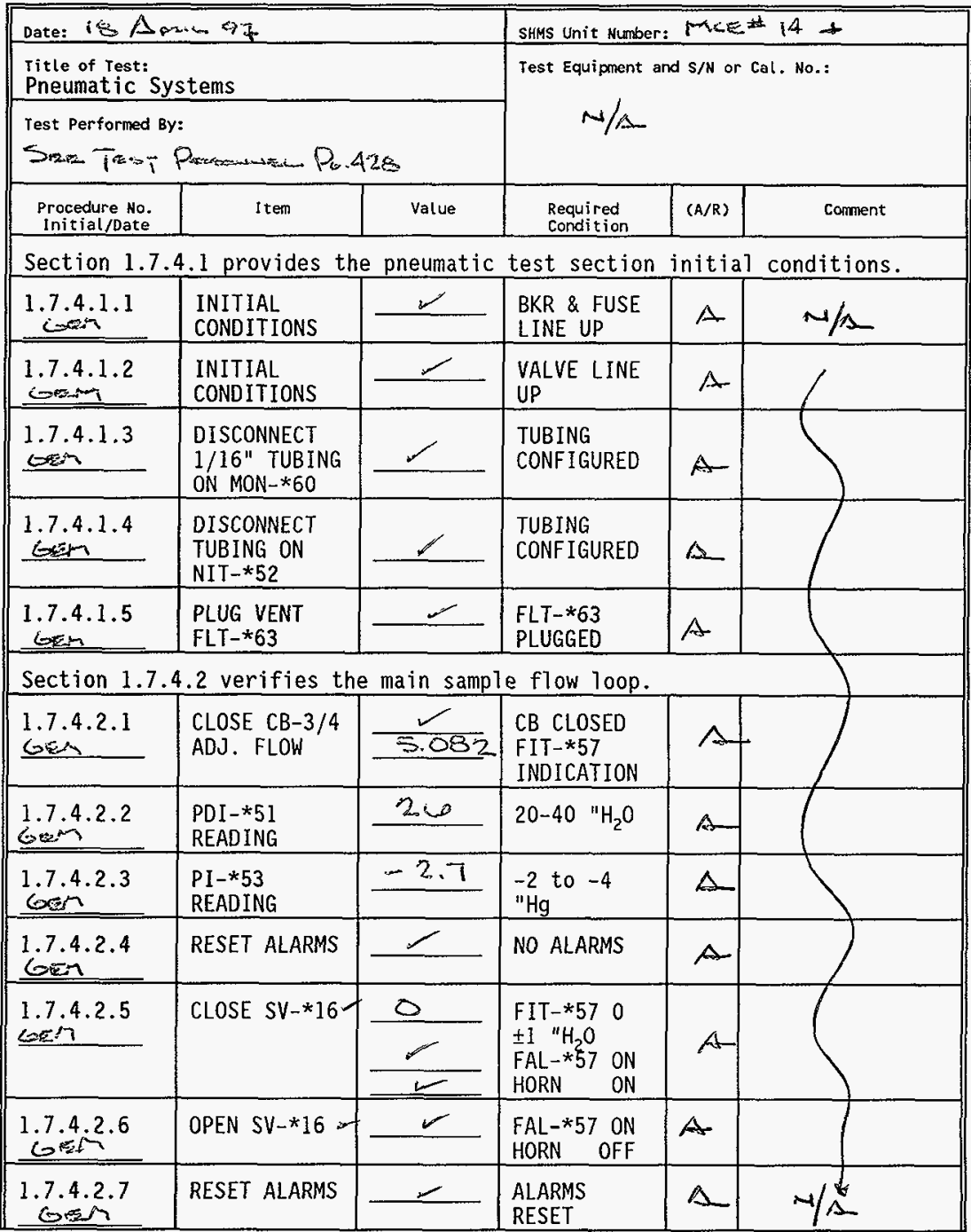


TEST DATA SHEET

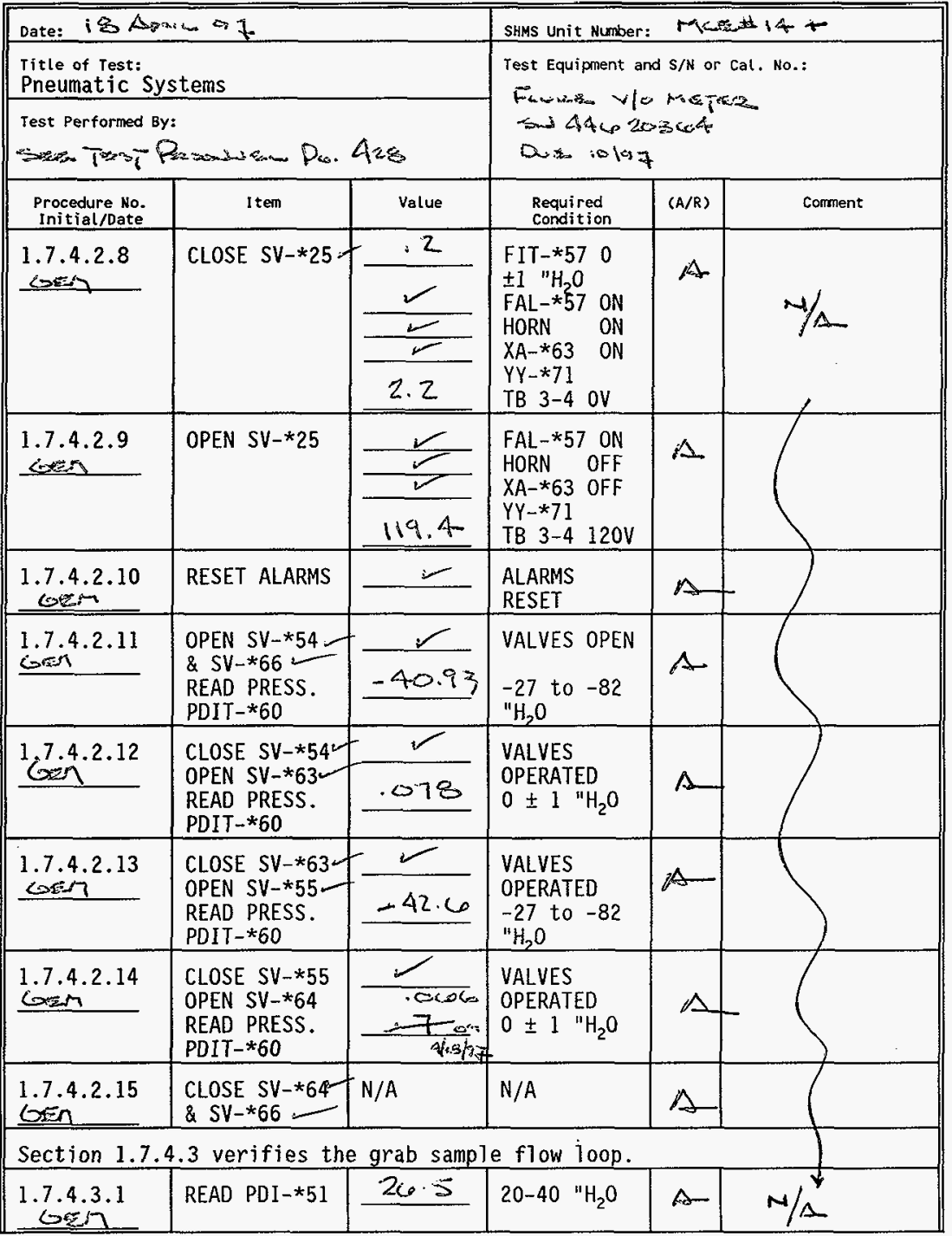

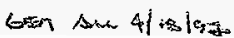


TEST DATA SHEET

\begin{tabular}{|c|c|c|c|c|c|}
\hline \multicolumn{3}{|c|}{ Date: $1 \& \Delta a-m$} & \multicolumn{3}{|c|}{ MuE $1 A_{+}+$} \\
\hline \multicolumn{3}{|c|}{$\begin{array}{l}\text { Title of rest: } \\
\text { Pneumatic Systems }\end{array}$} & \multirow{2}{*}{\multicolumn{3}{|c|}{ Test Equipment and $\mathrm{S} / \mathrm{N}$ or Cal. No.: }} \\
\hline \multicolumn{3}{|c|}{ 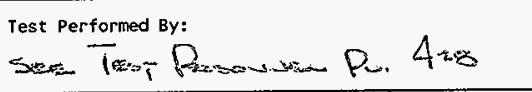 } & & & \\
\hline $\begin{array}{l}\text { Procedure No. } \\
\text { Initial/Date }\end{array}$ & Item & Value & $\begin{array}{l}\text { Required } \\
\text { condition } \\
\end{array}$ & $(A / R)$ & Comment \\
\hline$\underbrace{1.7 .4 .2}_{\text {Lasin }}$ & $\begin{array}{l}\text { PUSH PB-*59 } \\
\text { GRAB SAMPLE }\end{array}$ & 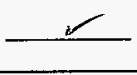 & $\begin{array}{l}\text { START } \\
\text { STOPWATCH }\end{array}$ & $\Lambda$ & $/ 1$ \\
\hline $\begin{array}{l}1.7 .4 .3 .3 \\
60.1\end{array}$ & $\begin{array}{l}\text { GRAB SAMPLE } \\
\text { LAMPS ON } \\
\text { READ FIV-*52 }\end{array}$ & $\frac{2}{2 / 2}$ & $\begin{array}{l}\mathrm{PBL}-* 58 \text { ON } \\
\mathrm{PBL}-* 59 \text { ON } \\
\text { YAL }-* 58 \text { ON } \\
\mathrm{FIV}-* 52 \quad 0 \\
\end{array}$ & $\Delta$ & \\
\hline $\begin{array}{l}1.7 .4 .3 .4 \\
\mathrm{Coc}_{3} \mathrm{~N}^{2}\end{array}$ & $\begin{array}{l}\text { OPEN SV }-* 15 \\
\& \text { ADJ. FIV } \\
\star 52\end{array}$ & $r$ & $\begin{array}{l}\text { SV-* } 15 \text { OPEN } \\
\text { FIV-*52 } \\
\text { FLOW } 10 \mathrm{CFH}\end{array}$ & $A$ & \\
\hline binin $^{7.5}$ & READ PDI $-* 51$ & 30 & $\begin{array}{l}\text { PDI }-* 51 \\
\text { HIGHER THAN } \\
1.7 .4 .3 .1\end{array}$ & $A$ & \\
\hline $\begin{array}{l}1.7 .4 .3 .6 \\
c^{2}+\mathrm{sin}^{-6}\end{array}$ & CLOSE SV-*15 & 0 & FIV $-* 520$ & $A$ & \\
\hline $\begin{array}{l}1.7 .4 .3 .7 \\
6 \sin ^{2}\end{array}$ & OPEN SV-*15 & 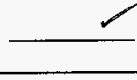 & $\begin{array}{l}\text { FIV }-* 52 \\
\text { FLOW } \\
\end{array}$ & A & \\
\hline 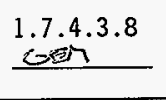 & $\begin{array}{l}\text { PBL }-* 59 \\
\text { GRAB SAMPLE } \\
\text { TIME }\end{array}$ & $\frac{7}{4.50}$ & $\begin{array}{l}\text { PBL }-* 59 \text { OFF } \\
\text { STOP WATCH } \\
5 \pm .5 \text { MIN } \\
\end{array}$ & $\wedge$ & \\
\hline 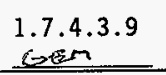 & $\begin{array}{l}\text { PB-*58 RESET } \\
\text { SAMPLER }\end{array}$ & $-\frac{L}{L}$ & $\begin{array}{l}\mathrm{PBL}-* 58 \text { OFF } \\
\text { YAL-*58 OFF }\end{array}$ & $\Lambda$ & \\
\hline \multicolumn{6}{|c|}{ Section 1.7.4.4 verifies the $\mathrm{H}_{2}$ cell calibration loop. } \\
\hline $\begin{array}{l}1.7 .4 .4 .1 \\
\text { GẼ }\end{array}$ & $\begin{array}{l}\text { CONNECT } \mathrm{H}_{2} \\
\text { CAL GAS }\end{array}$ & $N / A$ & $N / A$ & $A$ & \\
\hline $\begin{array}{l}1.7 .4 .4 .2 \\
\text { gen }\end{array}$ & $\begin{array}{l}\text { OPEN ISO } \\
\text { VALVE }\end{array}$ & 5 & $<10$ PSIG & $\curvearrowright$ & 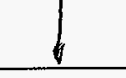 \\
\hline 1.7 .4 .4 .3 & $\begin{array}{l}\text { OPEN SV }-* 18 \\
\text { ADJ. FIV }-* 56\end{array}$ & 2 & $2 \pm .1 \mathrm{CFH}$ & $\Delta$ & $\mathrm{H} / \Delta$ \\
\hline
\end{tabular}




\section{TEST DATA SHEET}

\begin{tabular}{|c|c|c|c|c|c|}
\hline \multicolumn{3}{|c|}{ 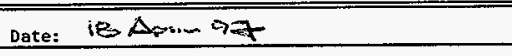 } & \multicolumn{3}{|c|}{ SHMS Unit Number: Mca社 $14-$} \\
\hline \multicolumn{3}{|c|}{$\begin{array}{l}\text { Title of Test: } \\
\text { Pneumatic Systems }\end{array}$} & \multirow{2}{*}{\multicolumn{3}{|c|}{ 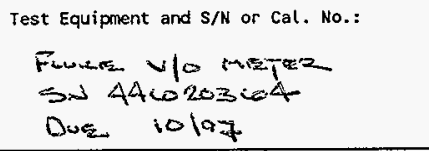 }} \\
\hline \multicolumn{3}{|c|}{ 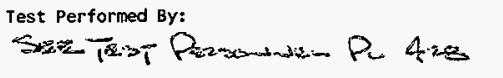 } & & & \\
\hline $\begin{array}{c}\text { Procedure No. } \\
\text { Initial/Date } \\
\end{array}$ & 1 tem & Value & $\begin{array}{l}\text { Required } \\
\text { Condition }\end{array}$ & $(A / R)$ & Comment \\
\hline 1.7 .4 .4 .4 & $\begin{array}{l}\text { VERIFY } \\
\text { CONDITIONS }\end{array}$ & $\frac{2}{2}$ & $\begin{array}{l}\text { NIT-*54 } \\
>.625 \% \quad \mathrm{H}_{2} \\
\text { NAH-*55 ON } \\
\text { NAH-*54 ON } \\
\text { PBL } * 59 \text { ON } \\
\text { PBL } * 58 \text { ON } \\
\text { YAL } * 58 \text { ON } \\
\text { FIV }-* 52 \quad \text { FLO } \\
\text { HORN ON } \\
\text { TB2-21/22 } \\
\text { OPEN } \\
\end{array}$ & $A$ & \\
\hline $\begin{array}{l}1.7 .4 .4 .5 \\
\text { cosir }\end{array}$ & $\begin{array}{l}\text { ACKNOWLEDGE } \\
\text { HORN }\end{array}$ & $\mathrm{N} / \mathrm{A}$ & N/A & $A$ & \\
\hline $\begin{array}{l}1.7 .4 .4 .6 \\
\text { Q }\end{array}$ & $\begin{array}{l}\text { CLOSE SV-*18 } \\
\text { VERIFY } \\
\text { CONDITIONS }\end{array}$ & $\frac{1}{2}$ & $\begin{array}{l}\text { FIV-*56 O } \\
\text { NIT-*54 } \\
<.625 \% \mathrm{H}_{2} \\
\text { NAH-*55 OFF } \\
\text { NAH-*54 ON } \\
\text { PBL }-* 58 \text { ON } \\
\text { YAL }-* 58 \text { ON } \\
\text { TB2-21/22 } \\
\text { CLOSED } \\
\end{array}$ & Ar & \\
\hline $\begin{array}{l}1.7 .4 .4 .7 \\
\cos \end{array}$ & $\begin{array}{l}\text { PUSH RESET } \\
\text { SAMPLER PB- } \\
* 58 \\
\end{array}$ & $\frac{\swarrow}{L}$ & 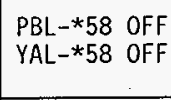 & $A$ & \\
\hline $\begin{array}{l}1.7 .4 .4 .8 \\
\end{array}$ & RESET ALARM & $r$ & $\mathrm{NAH}-* 54$ OFF & $\Delta$ & \\
\hline $\begin{array}{l}1.7 .4 .4 .9 \\
\text { s.t. }\end{array}$ & $\begin{array}{l}\text { OPEN SV-*19. } \\
\text { ADJ. FIV }-* 56 \text {. }\end{array}$ & 2 & $2 \pm .1 \mathrm{CFH}$ & $\Lambda$ & $1 /$ \\
\hline
\end{tabular}

cos tiolst 
TEST DATA SHEET

\begin{tabular}{|c|c|c|c|c|c|}
\hline \multicolumn{3}{|c|}{ Date: 18 Apan 07} & \multicolumn{3}{|c|}{ SHMS Unit Number: Mckz $14+$} \\
\hline \multicolumn{3}{|c|}{$\begin{array}{l}\text { Title of Test: } \\
\text { Pneumatic Systems }\end{array}$} & \multirow{2}{*}{\multicolumn{3}{|c|}{ 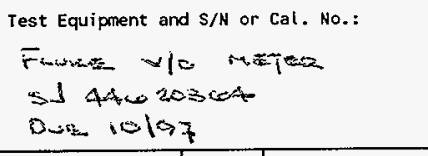 }} \\
\hline \multicolumn{3}{|c|}{ 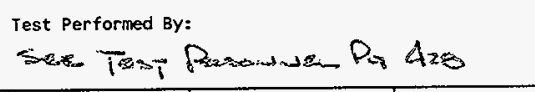 } & & & \\
\hline $\begin{array}{l}\text { Procedure No. } \\
\text { Initial/oate }\end{array}$ & 1 tem & Value & $\begin{array}{l}\text { Required } \\
\text { Condition } \\
\end{array}$ & $(A / R)$ & Corment \\
\hline $\begin{array}{l}1.7 .4 .4 .10 \\
68 m\end{array}$ & $\begin{array}{l}\text { VERIFY } \\
\text { CONDITIONS }\end{array}$ & 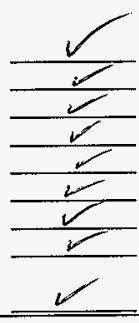 & $\begin{array}{l}\text { NIT-*55 } \\
>>625 \% \mathrm{H}_{2} \\
\text { NAH-*55 ON } \\
\text { NAH-*54 ON } \\
\text { PBL-*59 ON } \\
\text { PBL-*58 ON } \\
\text { YAL-*58 ON } \\
\text { FIV-*52 FLO } \\
\text { HORN ON } \\
\text { TB2-21/22 } \\
\text { OPEN } \\
\end{array}$ & 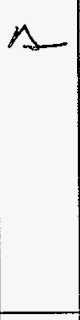 & $\mu$ \\
\hline $\begin{array}{r}1.7 .4 .4 .11 \\
6.23 \\
\end{array}$ & $\begin{array}{l}\text { ACKNOWLEDGE } \\
\text { HORN }\end{array}$ & $N / A$ & N/A & $A-$ & \\
\hline 1.7 .4 .4 .12 & $\begin{array}{l}\text { CLOSE SV-*19: } \\
\text { VERIFY } \\
\text { CONDITIONS }\end{array}$ & $\frac{r}{2}$ & $\begin{array}{l}\text { FIV }-* 56 \quad 0 \\
\text { NIT } * 555 \\
<.625 \% \mathrm{H}_{2} \\
\text { NAH- } * 55 \mathrm{OFF} \\
\text { NAH-*54 ON } \\
\text { PBL-*58 ON } \\
\text { YAL-*58 ON } \\
\text { TB2-21/22 } \\
\text { CLOSED } \\
\end{array}$ & 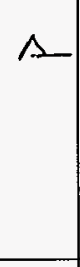 & \\
\hline $\begin{array}{l}1.7 .4 .4 .13 \\
\text { gin }\end{array}$ & $\begin{array}{l}\text { PUSH RESET } \\
\text { SAMPLER PB- } \\
\star 58\end{array}$ & $\frac{r}{r}$ & $\begin{array}{l}\text { PBL- } \star 58 \text { OFF } \\
\text { YAL- } * 58 \text { OFF }\end{array}$ & 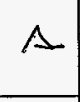 & \\
\hline $\begin{array}{c}1.7 .4 .4 .14 \\
0.2 e_{2} \\
\end{array}$ & RESET ALARM & $\nu$ & NAH $-* 54$ OFF & $\wedge$ & \\
\hline $\begin{array}{l}1.7 .4 .4 .15 \\
0 . \mathrm{cos}^{-17} \\
\end{array}$ & $\begin{array}{l}\text { DISCONNECT } \\
\text { CAL. GAS }\end{array}$ & $N / A$ & $N / A$ & 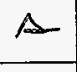 & \\
\hline $\begin{array}{l}1.7 .4 .4 .16 \\
\text { Gein }\end{array}$ & $\begin{array}{l}\text { PUSH PB-*54 } \\
\text { VERIFY } \\
\text { CONDITIONS }\end{array}$ & $\frac{1}{r}$ & $\begin{array}{l}X A-* 63 \text { ON } \\
\text { PBL }-* 54 \text { ON } \\
\text { TB2-23/24 } \\
\text { OPEN } \\
\end{array}$ & $A$ & \\
\hline $\begin{array}{l}1.7 .4 .4 .17 \\
\operatorname{con}\end{array}$ & $\begin{array}{l}\text { PUSH PB-*54 } \\
\text { VERIFY } \\
\text { CONDITIONS }\end{array}$ & $\frac{r}{r}$ & $\begin{array}{l}X A-* 63 \text { OFF } \\
P B L-* 54 \text { OFF } \\
\text { TB2-23/24 } \\
\text { CLOSED }\end{array}$ & $A$ & $N / A$ \\
\hline
\end{tabular}


TEST DATA SHEET

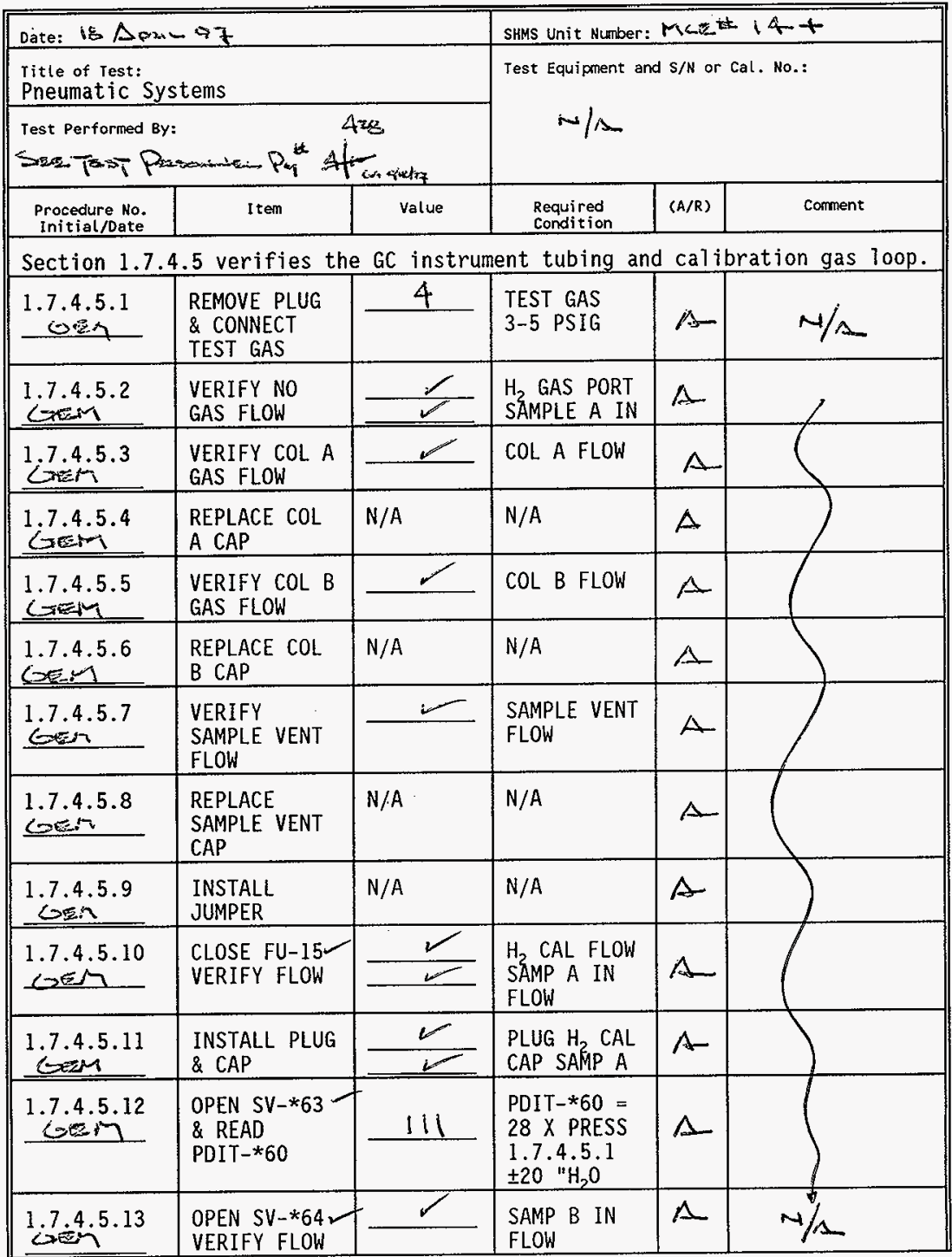


APPENDIX N

TEST DATA SHEET

\begin{tabular}{|c|c|c|c|c|c|}
\hline \multicolumn{3}{|c|}{ Date: is Apsim-97 } & SHMS Unit Number: & \multicolumn{2}{|c|}{ RoE AAt } \\
\hline \multicolumn{3}{|c|}{$\begin{array}{l}\text { Title of rest: } \\
\text { Pneumatic Systems }\end{array}$} & \multirow{2}{*}{\multicolumn{3}{|c|}{$\begin{array}{l}\text { Test Equipment and } S / \mathrm{N} \text { or Cal. No.: } \\
\mathrm{N} / \mathrm{A} \text {. }\end{array}$}} \\
\hline \multicolumn{3}{|c|}{ 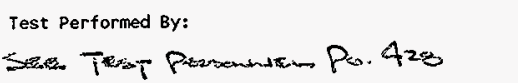 } & & & \\
\hline $\begin{array}{l}\text { Procedure No. } \\
\text { Initial/Date }\end{array}$ & I tem & value & $\begin{array}{l}\text { Required } \\
\text { Condition }\end{array}$ & $(A / R)$ & Corment \\
\hline $\begin{array}{c}1.7 .4 .5 .14 \\
\mathrm{Cs}\end{array}$ & $\begin{array}{l}\text { REMOVE TEST } \\
\text { GAS }\end{array}$ & $N / A$ & $N / A$ & 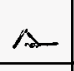 & \\
\hline $\begin{array}{l}1.7 .4 .5 .15 \\
6 \mathrm{em} \\
\end{array}$ & $\begin{array}{l}\text { CLOSE SV }-* 63- \\
\& \text { SV-*64- }\end{array}$ & $\mathrm{F} / \mathrm{A}$ & $N / A$ & $\Delta$ & \\
\hline $\begin{array}{l}1.7 .4 .5 .16 \\
\operatorname{cosen}\end{array}$ & $\begin{array}{l}\text { OPEN FU-15- } \\
\text { REMOVE } \\
\text { JUMPER }\end{array}$ & $N / A$ & $N / A$ & $A$ & \\
\hline$\frac{1.7 .4 .5 .17}{\cos ^{2} \cdot 1}$ & $\begin{array}{l}\text { INSTALL } \\
\text { SAMP A UNION }\end{array}$ & $N / A$ & $N / A$ & $A$ & \\
\hline $\begin{array}{l}1.7 .4 .5 .18 \\
\underline{\cosh }\end{array}$ & $\begin{array}{l}\text { OPEN SV-*60- } \\
\text { VERIFY FLOW }\end{array}$ & 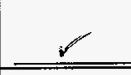 & $\begin{array}{l}\text { FI-*60 } \\
\text { NO FLOW }\end{array}$ & $A$ & \\
\hline$\frac{1.7 .4 .5 .19}{\text { ixis }}$ & $\begin{array}{l}\text { POWER SOV- } \\
* 60, \text { VERIFY } \\
\text { NO FLOW }\end{array}$ & & $\begin{array}{l}\text { FI-*60 } \\
\text { NO FLOW }\end{array}$ & $\Delta$ & \\
\hline$\underline{\text { GE' }}^{1.7 .4 .5 .20}$ & $\begin{array}{l}\text { OPEN SV-*68 } \\
\text { ADJ SV }-* 67\end{array}$ & $\checkmark$ & $\begin{array}{l}\mathrm{FI}-* 60 \\
50 \mathrm{CCM} \\
\end{array}$ & $A$ & \\
\hline $\begin{array}{l}1.7 .4 .5 .21 \\
10.7 \\
\end{array}$ & $\begin{array}{l}\text { DEENERGIZE } \\
\text { SOV-*60 }\end{array}$ & $r$ & $\begin{array}{l}\text { FI-*60 FLOW } \\
\text { DECREASES }\end{array}$ & $\Delta$ & \\
\hline 1.7 .4 .5 .22 & $\begin{array}{l}\text { CLOSE SV-*60- } \\
\& \text { SV-*68 }\end{array}$ & $\mathrm{N} / \mathrm{A}$ & N/A & 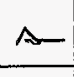 & \\
\hline $\begin{array}{c}1.7 .4 .5 .23 \\
\end{array}$ & $\begin{array}{l}\text { REMOVE } \\
\text { SAMP A UNION }\end{array}$ & N/A & N/A & A & \\
\hline $\operatorname{con}^{1.7 .4 .24}$ & $\begin{array}{l}\text { CONNECT TEST } \\
\text { GAS } N_{2} O \text { PORT }\end{array}$ & 4 & $\begin{array}{l}\text { TEST GAS } \\
3-5 \text { PSIG } \\
\end{array}$ & $A$ & \\
\hline $\sin ^{1.7 .4 .5 .25}$ & $\begin{array}{l}\text { VERIFY NO } \\
\text { FLOW }\end{array}$ & 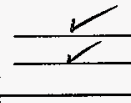 & $\begin{array}{l}\text { SAMP B IN \& } \\
\text { FLT-*63 } \\
\text { NO FLOW }\end{array}$ & $\triangle$ & \\
\hline $\begin{array}{l}1.7 .4 .5 .26 \\
\sin \\
\end{array}$ & $\begin{array}{l}\text { INSTALL } \\
\text { JUMPER } \\
\end{array}$ & $N / A$ & $N / A$ & $\Delta$ & \\
\hline $\begin{array}{l}1.7 .4 .5 .27 \\
\text { 연. }\end{array}$ & $\begin{array}{l}\text { CLOSE FU-15 } \\
\text { VERIFY FLOW }\end{array}$ & $\frac{1}{2}$ & $\begin{array}{l}\text { SAMP B IN } \\
\text { FLT-*63 } \\
\text { FLOW }\end{array}$ & 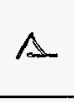 & \\
\hline
\end{tabular}


TEST DATA SHEET

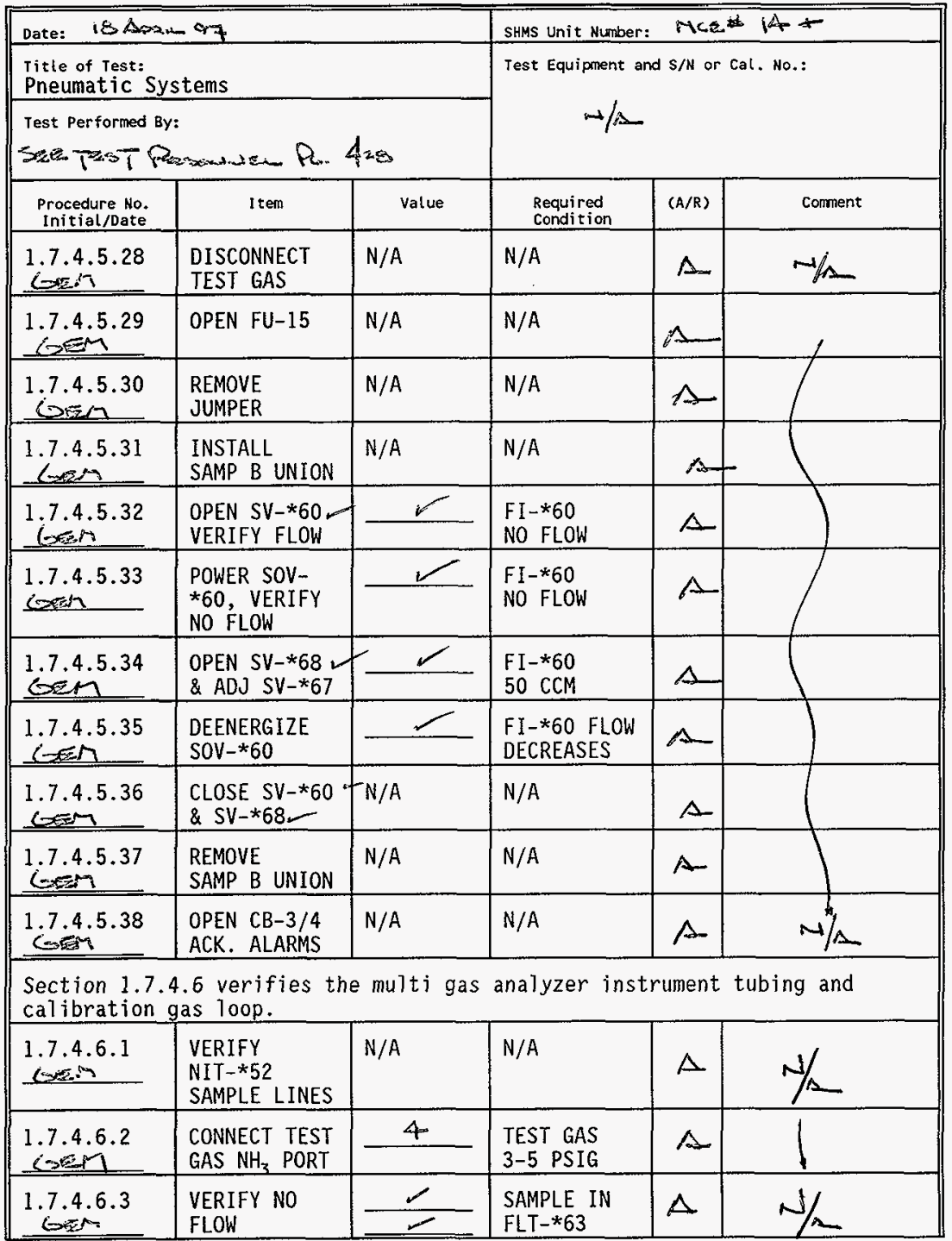


APPENDIX N

TEST DATA SHEET

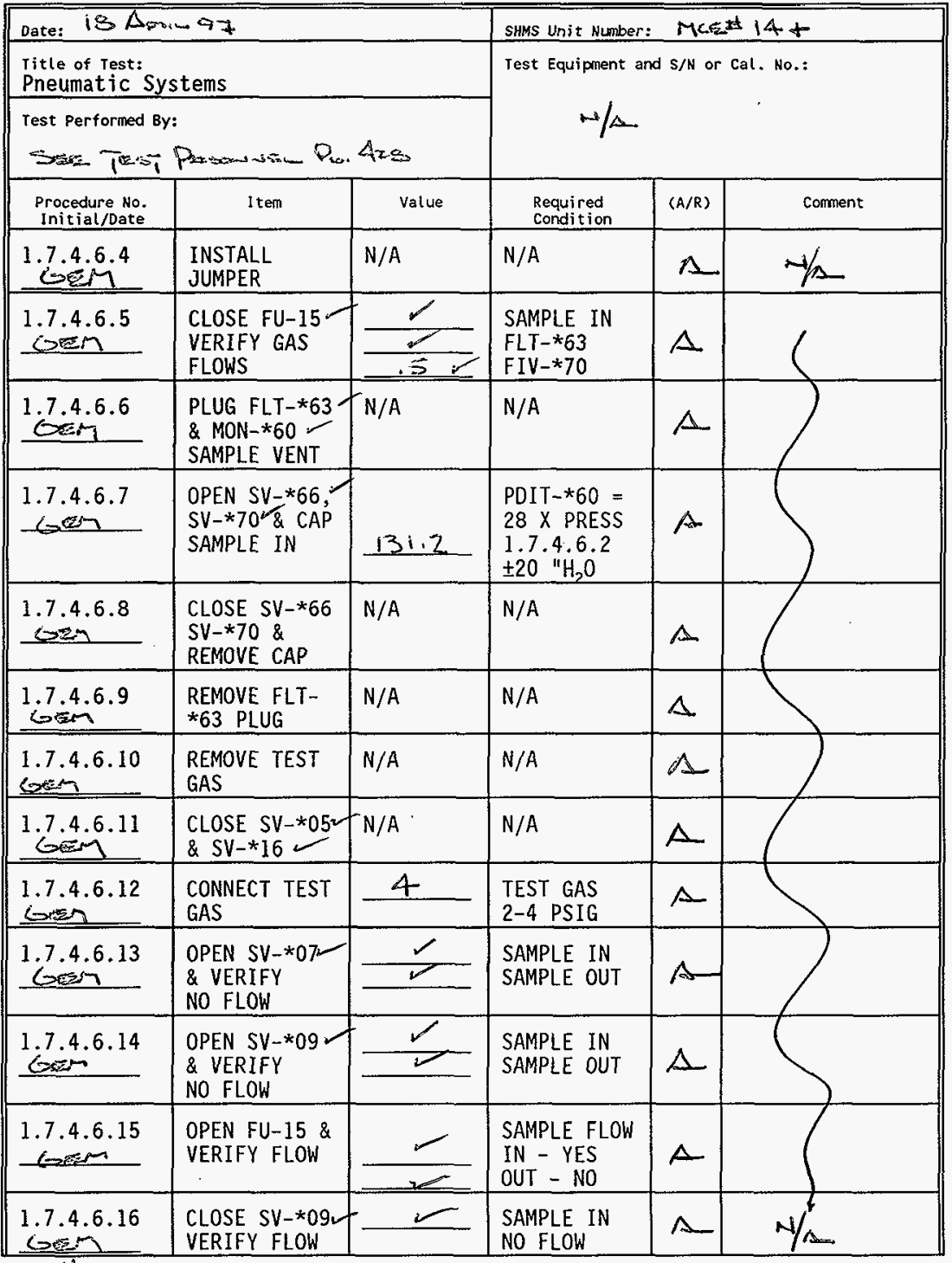


TEST DATA SHEET

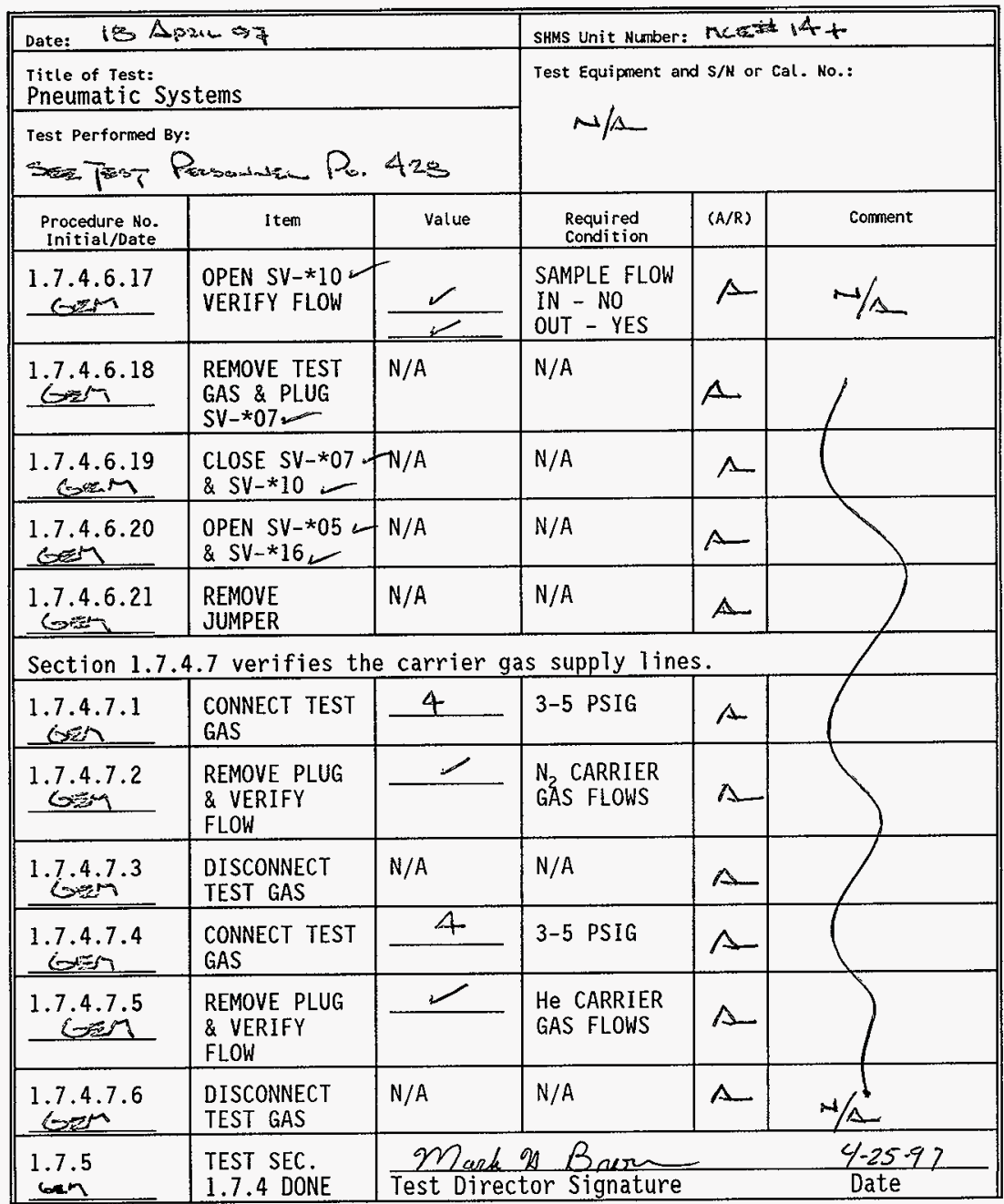

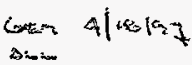


TEST DATA SHEET

\begin{tabular}{|c|c|c|c|c|c|}
\hline \multicolumn{3}{|c|}{ Date: omsy 97} & \multicolumn{3}{|c|}{ SHMS Unit Nunber: M<E $14+$} \\
\hline \multicolumn{3}{|c|}{$\begin{array}{l}\text { Iitle of rest: } \\
\text { Pneumatic Systems }\end{array}$} & \multirow{2}{*}{\multicolumn{3}{|c|}{$\begin{array}{l}\text { Test Equipment and } \mathrm{S} / \mathrm{N} \text { or Cal. No.: } \\
\text { N/A }\end{array}$}} \\
\hline \multicolumn{3}{|c|}{ 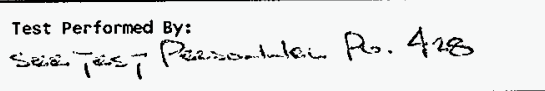 } & & & \\
\hline $\begin{array}{l}\text { Procedure No. } \\
\text { Initial/Date }\end{array}$ & Item & Value & $\begin{array}{l}\text { Required } \\
\text { Condition }\end{array}$ & $(A / R)$ & Comment \\
\hline $\begin{array}{l}1.7 .6 \\
0.1\end{array}$ & \multicolumn{5}{|c|}{$\begin{array}{l}\text { THIS SECTION IS ONLY FOR THE SHMS-E IN THE ANALYTICAL MODE. } \\
\text { IF NOT APPLICABLE, N/A STEPS AND TEST DIRECTOR WILL SIGN. }\end{array}$} \\
\hline & \multicolumn{3}{|c|}{ Test Director Signature } & \multicolumn{2}{|l|}{ Date } \\
\hline $\begin{array}{l}1.7 .6,1.1 \\
\text { iteiti }\end{array}$ & $\begin{array}{l}\text { VERIFY BKR } \\
\text { LINE UP }\end{array}$ & $N / A$ & N/A & $\Delta$ & \\
\hline Geiri. & $\begin{array}{l}\text { VERIFY VALVE } \\
\text { LINE UP } \\
\end{array}$ & $N / A$ & $N / A$ & $A$ & \\
\hline 1.7 .6 .1 .3 & $\begin{array}{l}\text { VERIFY MON- } \\
\star 60 \text { INSTALL }\end{array}$ & $N / A$ & N/A & 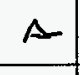 & \\
\hline 1.7 .6 .1 .4 & $\begin{array}{l}\text { VERIFY NIT- } \\
\star 52 \text { INSTALL. }\end{array}$ & N/A & $N / A$ & $\sim$ & \\
\hline$\underline{6}^{1.7 .6 .1 .5}$ & $\begin{array}{l}\text { CONNECT } \mathrm{H}_{2} \\
\text { CAL GAS }\end{array}$ & N/A & $N / A$ & $\Delta$ & \\
\hline $\begin{array}{l}1.7 .6 .1 .6 \\
\end{array}$ & $\begin{array}{l}\text { CONNECT } \mathrm{CH}_{4} \\
\text { CAL GAS }\end{array}$ & N/A & $N / A$ & $\Delta$ & \\
\hline $\begin{array}{l}1.7 .6 .1 .7 \\
\end{array}$ & $\begin{array}{l}\text { CONNECT } \mathrm{NH}_{3} \\
\text { CAL GAS }\end{array}$ & $N / A$ & N/A & $\Delta$ & \\
\hline \multicolumn{6}{|c|}{ Section 1.7.6.2 establishes the main and auxiliary loop flows. } \\
\hline 1.7 .6 .2 .1 & CLOSE FU-11 & 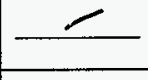 & $\begin{array}{l}\text { MON-*60 } \\
\text { POWERED } \\
\end{array}$ & $\triangle$ & \\
\hline $\begin{array}{l}1.7 .6 .2 .2 \\
\text { equ }\end{array}$ & POWER PC $\rightarrow 60$ & 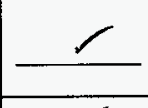 & $\begin{array}{l}\text { ESTABLISH } \\
\text { SAMPLING } \\
\text { ROUT INE } \\
\end{array}$ & 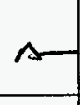 & \\
\hline $\operatorname{sen}^{1.7 .6 .2 .3}$ & CLOSE FU-12 & 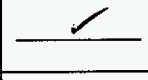 & $\begin{array}{l}\text { NIT-*52 } \\
\text { POWERED } \\
\end{array}$ & $A$ & \\
\hline 1.7 .6 .2 .4 & POWER PC $-* 70$ & 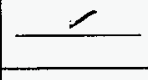 & $\begin{array}{l}\text { PC-*70 } \\
\text { BOOTED UP } \\
\end{array}$ & $\wedge$ & \\
\hline $\begin{array}{l}1.7 .6 .2 .5 \\
\sin \end{array}$ & $\begin{array}{l}\text { CLOSE CB-3/4 } \\
\text { ADJUST FLOW }\end{array}$ & i.o & $\begin{array}{l}\mathrm{FIT}-\star 57 \\
1 \pm .1 " \mathrm{H}_{2} \mathrm{O}\end{array}$ & $\Delta$ & $N / A$ \\
\hline
\end{tabular}


TEST DATA SHEET

\begin{tabular}{|c|c|c|c|c|c|}
\hline \multicolumn{3}{|c|}{ Date: 9 MAT $9 \%$} & \multicolumn{3}{|c|}{ SHMS unit Number: Macis itit } \\
\hline \multicolumn{3}{|c|}{$\begin{array}{l}\text { Iitle of Test: } \\
\text { Pneumatic Systems }\end{array}$} & \multirow{2}{*}{\multicolumn{3}{|c|}{ Test Equipment and $\mathrm{S} / \mathrm{N}$ or Cal. No.: }} \\
\hline \multicolumn{3}{|c|}{ 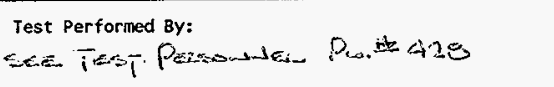 } & & & \\
\hline $\begin{array}{l}\text { Procedure No. } \\
\text { Initial/Date }\end{array}$ & Item & Value & $\begin{array}{l}\text { Required } \\
\text { Condition } \\
\end{array}$ & $(A / R)$ & Comment \\
\hline $\begin{array}{l}1.7 .6 .2 .6 \\
\text { y.s. }\end{array}$ & $\begin{array}{l}\text { OPEN SV-*09. } \\
\& \text { SV-*10 }\end{array}$ & $N / A$ & $N / A$ & $\Delta$ & \\
\hline $\begin{array}{l}1.7 .6 .2 .7 \\
\end{array}$ & $\begin{array}{l}\text { OPEN SV-*60? } \\
63,65 \& 68:\end{array}$ & N/A & $N / A$ & $\Delta$ & \\
\hline $\begin{array}{l}1.7 .6 .2 .8 \\
\end{array}$ & NIT-*52 & 2 & $\begin{array}{l}\text { ESTABLISH } \\
\text { SAMPLING } \\
\text { ROUTINE }\end{array}$ & $d x$ & \\
\hline 1.7 .6 .2 .9 & $\begin{array}{l}\text { OPEN SV-*24 } \\
\text { ADJ FLOW }\end{array}$ & 2.97 & $\begin{array}{l}\mathrm{FIT}-* 57 \\
3 \pm .1 \quad \mathrm{H}_{2} \mathrm{O}\end{array}$ & $A$ & \\
\hline $\begin{array}{l}1.7 .6 .2 .10 \\
\text { ¿ex.7.7 }\end{array}$ & $\begin{array}{l}\text { ADJ FLOW } \\
\text { SV-*67 - }\end{array}$ & 20 & $\begin{array}{l}\mathrm{FI}-* 60 \\
10-30 \mathrm{ccm}\end{array}$ & A & \\
\hline $\begin{array}{l}1.7 .6 .2 .11 \\
0.7\end{array}$ & $\begin{array}{l}\text { RECORD } \\
\text { PDIT } * * 60\end{array}$ & 104.8 & $<175 " \mathrm{H}_{2} \mathrm{O}$ & A & \\
\hline $\begin{array}{l}1.7 .6 .2 .12 \\
\text { sen }\end{array}$ & $\begin{array}{l}\text { OPEN SV-*64.- } \\
\text { CLOSE SV-*63 }\end{array}$ & $N / A$ & $N / A$ & $A$ & \\
\hline $1.7 .6 .1^{2.13}$ & $\begin{array}{l}\text { RECORD } \\
\text { PDIT -*60 }\end{array}$ & 50.5 & $<175 \quad " \mathrm{H}_{2} \mathrm{O}$ & $\leftarrow$ & \\
\hline 1.7 .6 .2 .14 & $\begin{array}{l}\text { CLOSE SV-*65- } \\
\text { OPEN SV-*662 }\end{array}$ & $N / A$ & $N / A$ & 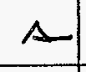 & \\
\hline $\begin{array}{l}1.7 .6 .2 .15 \\
\text { S. }\end{array}$ & $\begin{array}{l}\text { RECORD B IN } \\
\text { PDIT-* } 60\end{array}$ & -25.37 & $\begin{array}{l}-15 \text { to }-50 \\
\mathrm{H}_{2} \mathrm{O}\end{array}$ & $\Lambda$ & \\
\hline 告江 & $\begin{array}{l}\text { OPEN SV }-* 63 \\
\text { CLOSE SV }-{ }^{*} 64\end{array}$ & $N / A$ & $N / A$ & A & \\
\hline 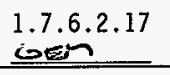 & $\begin{array}{l}\text { RECORD A IN } \\
\text { PDIT }-* 60\end{array}$ & -26.15 & $\begin{array}{l}-15 \text { to }-50 \\
" \mathrm{H}_{2} \mathrm{O}\end{array}$ & $A$ & \\
\hline $\begin{array}{c}1.7 .6 .2 .18 \\
\end{array}$ & $\begin{array}{l}\text { INSTALL } \\
\text { JUMPER }\end{array}$ & $N / A$ & N/A & $\Delta$ & \\
\hline $\begin{array}{c}1.7 .6 .2 .19 \\
\text { Geism } \\
\end{array}$ & CLOSE FU-15 & $N / A$ & N/A & $A$ & \\
\hline $\begin{array}{l}1.7 .6 .2 .20 \\
6.4\end{array}$ & $\begin{array}{l}\text { ADJ SV }-* 61 \% \\
\text { RECORD } \\
\text { PDIT-*60\% }\end{array}$ & -23.3 & $\begin{array}{l} \pm 5^{\prime \prime} \mathrm{H}_{2} \mathrm{O} \text { OF } \\
1.7 .6 .2 .17\end{array}$ & A & \\
\hline
\end{tabular}


APPENDIX $N$

TEST DATA SHEET

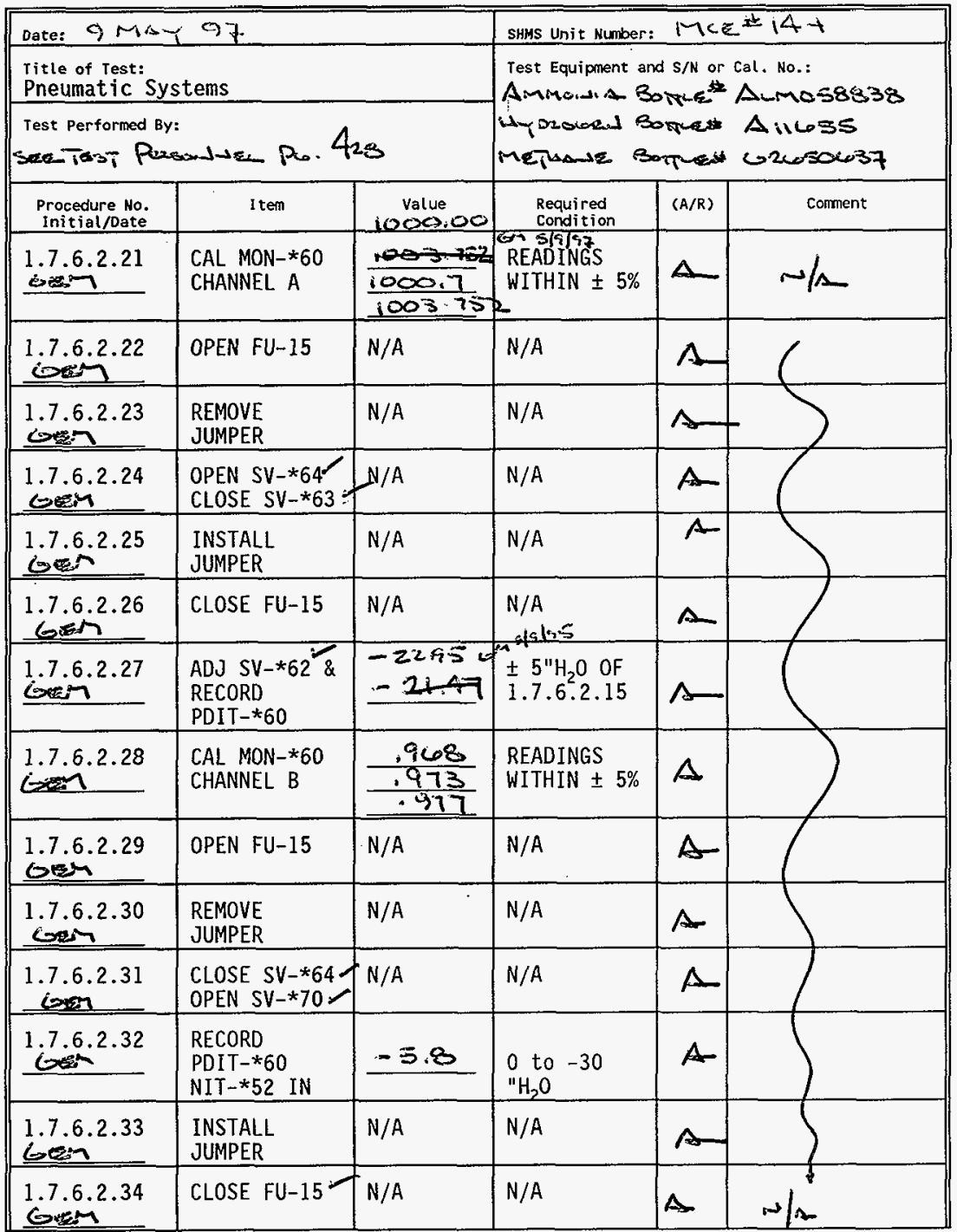


APPENDIX N

$1 \ldots$

HNF-SD-WM-ATR- 191

PAGE N-27

TEST DATA SHEET

\begin{tabular}{|c|c|c|c|c|c|}
\hline \multicolumn{3}{|c|}{ Date: 9 MAY 97} & \multicolumn{3}{|c|}{ SHMS Unit Number: MaE $14+$} \\
\hline \multicolumn{3}{|c|}{$\begin{array}{l}\text { Title of Test: } \\
\text { Pneumatic Systems }\end{array}$} & \multirow{2}{*}{\multicolumn{3}{|c|}{ 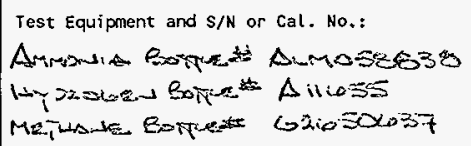 }} \\
\hline \multicolumn{3}{|c|}{ 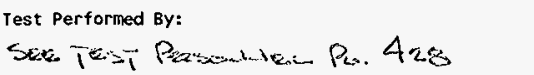 } & & & \\
\hline $\begin{array}{l}\text { Procedure No. } \\
\text { Initial/Date }\end{array}$ & Item & value & $\begin{array}{l}\text { Required } \\
\text { Condition }\end{array}$ & $(A / R)$ & Comment \\
\hline 1.7 .6 .2 .35 & $\begin{array}{l}\text { ESTABLISH } \\
\text { BYPASS FLOW } \\
\end{array}$ & $i .5$ & $\begin{array}{l}\text { FIV }-* 70 \\
1.5 \pm .2 \mathrm{cfh} \\
\end{array}$ & $A$ & $\begin{array}{l}a \\
\sin p\end{array}$ \\
\hline 1.7 .6 .2 .36 & $\begin{array}{l}\text { ADJ SV-*71 } \\
\text { NIT } * 52 \\
\text { SAMPLE IN } \\
\end{array}$ & -7.4 & $\begin{array}{l} \pm 5 \quad \mathrm{H}_{2} \mathrm{O} \text { OF } \\
1.7 .6 .2 .32\end{array}$ & $A$ & \\
\hline 1.7 .6 .2 .37 & $\begin{array}{l}\text { RECORD } \\
\text { NIT } * 52 \\
\text { SAMPLES }\end{array}$ & $\frac{32.9}{32.6} \frac{33.0}{32}$ & $\begin{array}{l}\text { READINGS } \\
\text { WITHIN } \pm 5 \%\end{array}$ & $A$ & \\
\hline $\begin{array}{l}1.7 .6 .2 .38 \\
\sin \end{array}$ & OPEN FU-15 & $N / A$ & N/A & $A$ & \\
\hline 1.7 .6 .2 .39 & $\begin{array}{l}\text { REMOVE } \\
\text { JUMPER }\end{array}$ & $N / A$ & $\mathrm{~N} / \mathrm{A}$ & $\Delta$ & \\
\hline \multicolumn{6}{|c|}{ Section 1.7.7 provides for test system shutdown. } \\
\hline 1.7.7.1 & $\begin{array}{l}\text { DISCONNECT } \\
\mathrm{H}_{2} \text { CAL GAS }\end{array}$ & $N / A$ & N/A & $A$ & \\
\hline Gein & $\begin{array}{l}\text { DISCONNECT } \\
\mathrm{CH}_{6} \text { CAL GAS }\end{array}$ & N/A & N/A & 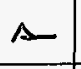 & \\
\hline $\begin{array}{l}1.7 .7 .3 \\
601\end{array}$ & $\begin{array}{l}\text { DISCONNECT } \\
\mathrm{NH}_{3} \text { CAL GAS }\end{array}$ & N/A & N/A & $A$ & \\
\hline 6.7.7 & $\begin{array}{l}\text { SECURE \& } \\
\text { TURN OFF } \\
\text { ANA. INST.S }\end{array}$ & N/A & N/A & $\Delta$ & \\
\hline $\begin{array}{l}1.7 .7 .5 \\
62.7 \\
\end{array}$ & $\begin{array}{l}\text { DISCONNECT } \\
\mathrm{N}_{2} \text { CARRIER } \\
\end{array}$ & N/A & N/A & $\Delta$ & \\
\hline $\begin{array}{l}1.7 .7 .6 \\
\text { gin }\end{array}$ & $\begin{array}{l}\text { DISCONNECT } \\
\text { He CARRIER }\end{array}$ & N/A & N/A & $\approx$ & \\
\hline 1.7.7 & $\begin{array}{l}\text { OPEN ALL } \\
\text { BREAKERS \& } \\
\text { FUSES } \\
\end{array}$ & N/A & N/A & $A$ & \\
\hline G.7.7. & $\begin{array}{l}\text { CLOSE LISTED } \\
\text { SYS. VALVES }\end{array}$ & $N / A$ & $\mathrm{~N} / \mathrm{A}$ & $\Delta$ & $M / A$ \\
\hline
\end{tabular}

bern awe $5 / 9 / 97$ 
APPENDIX N

PAGE N-28 homo

$\mathrm{CSPY}$
HNF-SD-WM-ATR- 191

Rev. 0

Page 454

TEST DATA SHEET

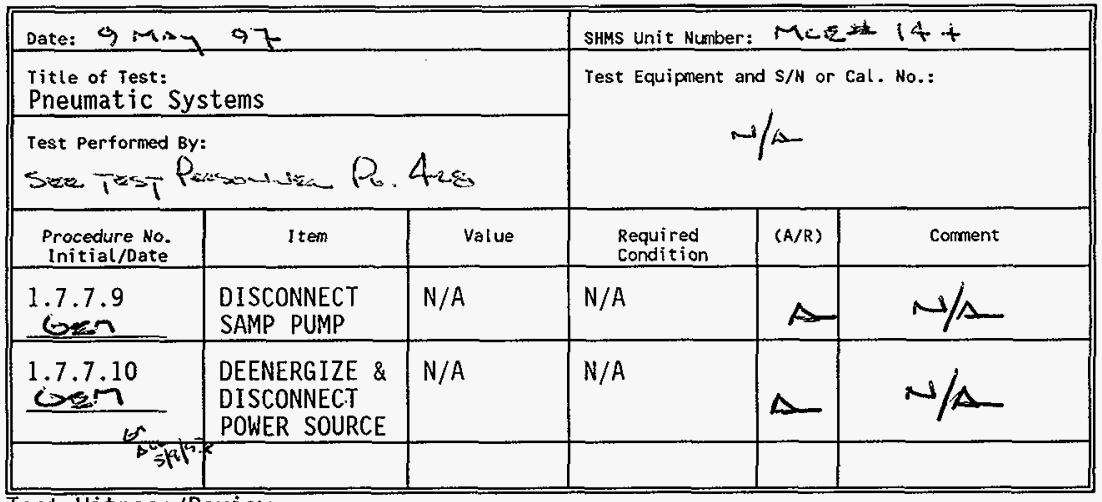

Test Witness/Review:
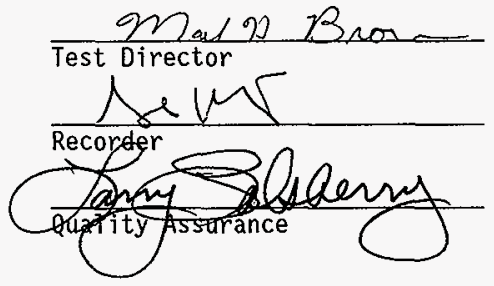

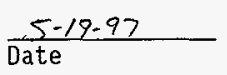

$\frac{19 \mathrm{Msy} 97}{\text { Date }}$

$\frac{5 / 28 / 97}{\text { Date }}$ 
APPENDIX $N$

PAGE N-29

HWF-SD-WM-ATR- 191
ReV. 0
Page 455

TEST EXCEPTION SHEET

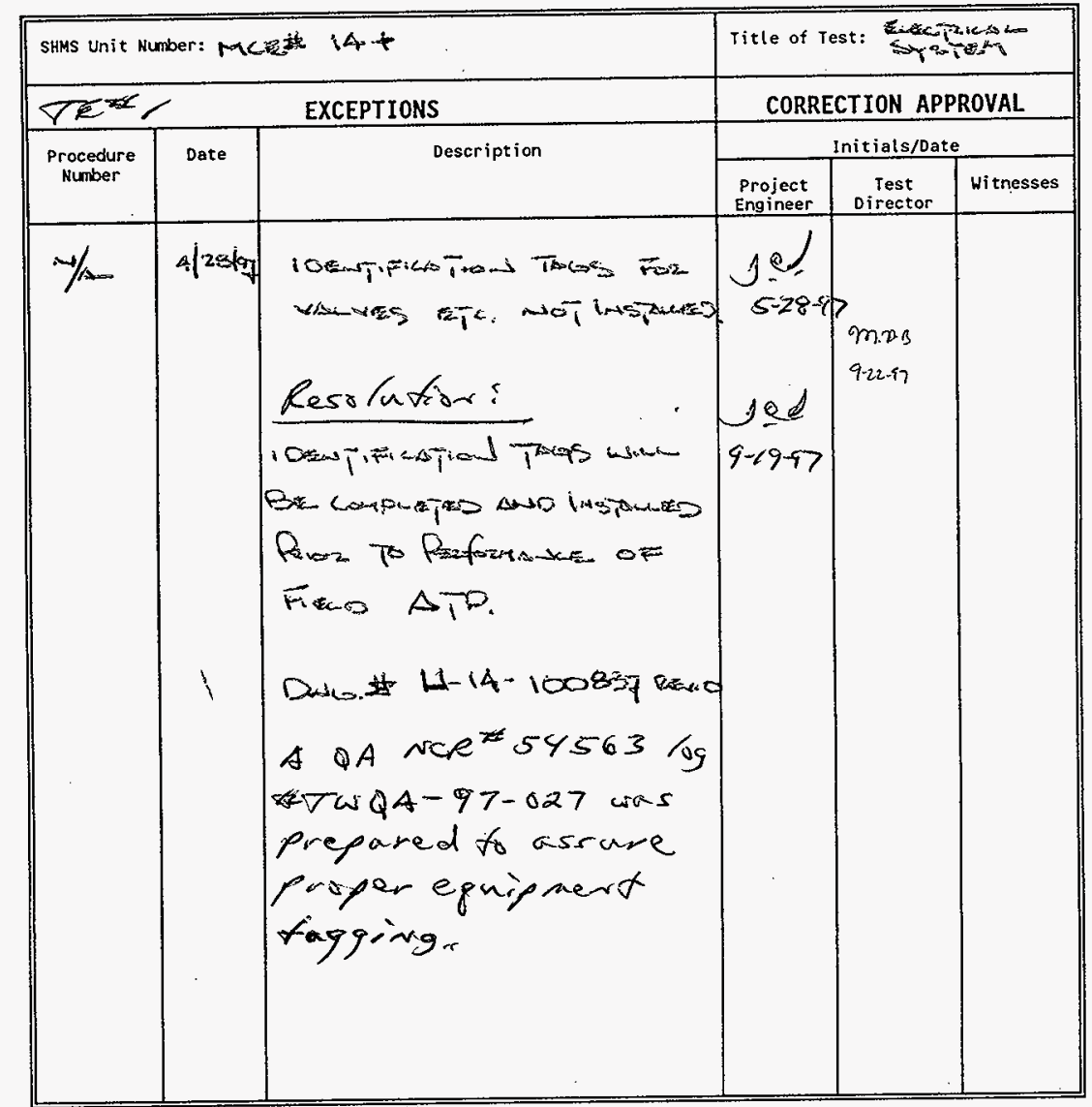

TEST APPROVED WITH EXCEPTIONS

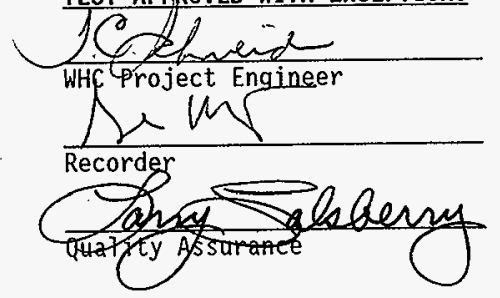

$$
\begin{aligned}
& \frac{5-28-97}{\text { Date }} \\
& \frac{28 \text { Man } 97}{\text { Date }} \\
& \frac{5 / 28 / 977}{\text { Date }}
\end{aligned}
$$


APPENDIX N

PAGE N-30
HNF-SD-WM-ATR- 191

Rev.
Page 456

TEST LOG

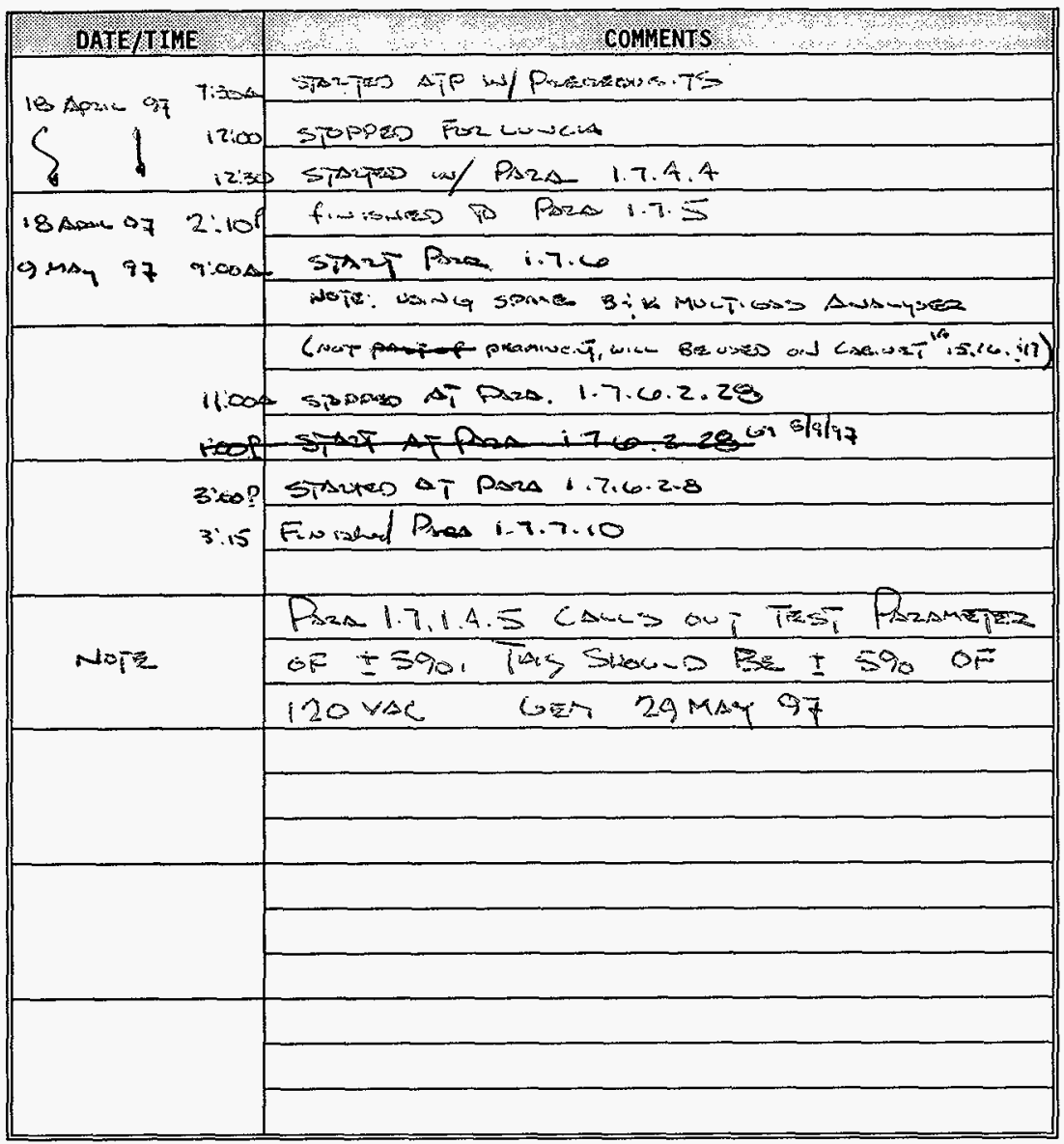


APPENDIX 0

PAGE 0-1 nenos

ho
HNF - SD - WM-ATR - 191

Rev. 0

Page 457

APPENDIX 0 
TEST EXECUTION SHEET

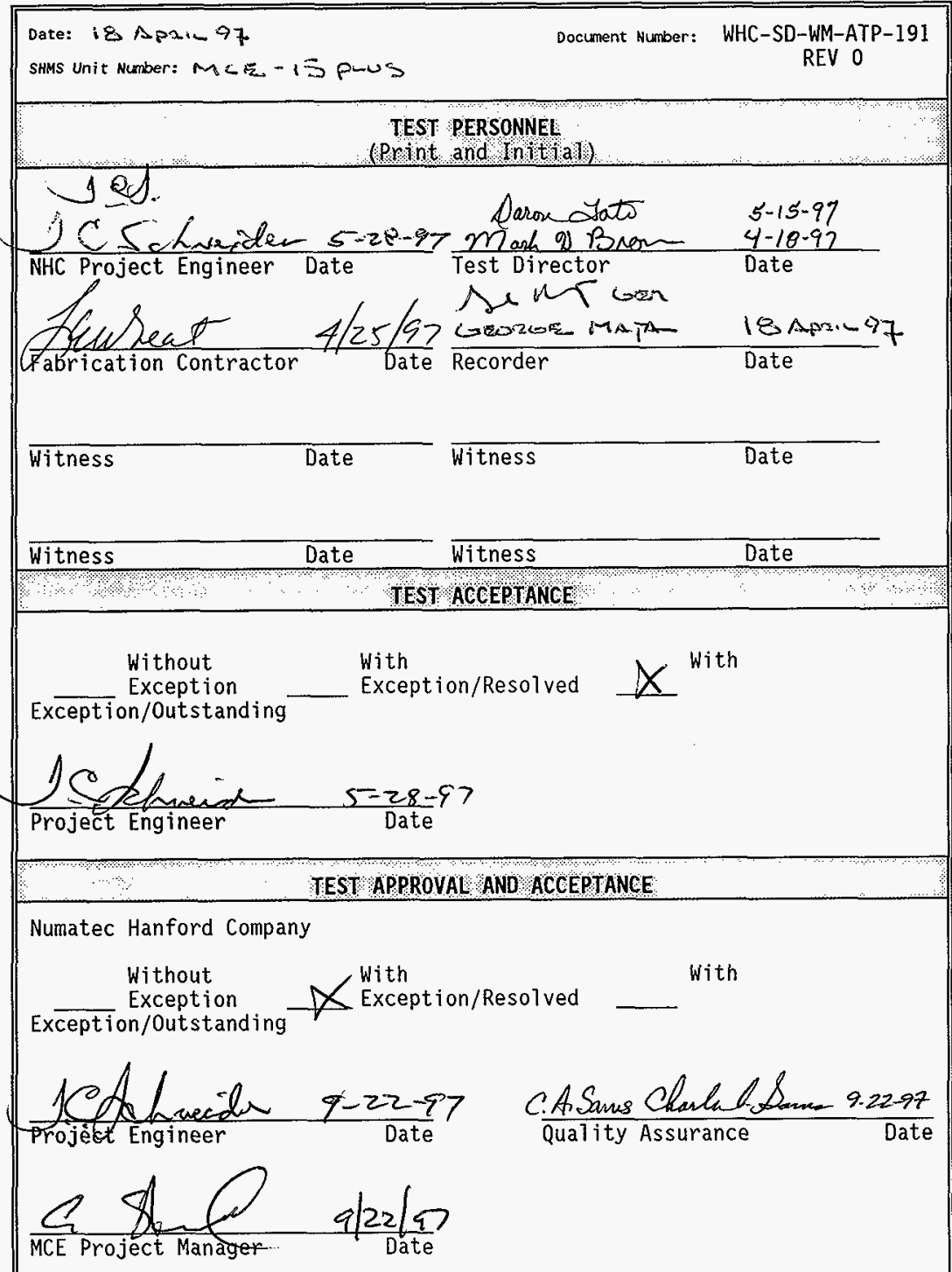


APPENDIX 0

PAGE $0-3$

SHMS Unit Number:

\section{PREREQUISITES AND INITIAL TEST CONDITIONS}

The following conditions shail exist at the start of the acceptance testing. Initial and date to verify that each of the following items have been accomplished.

$\leftrightarrow 4 / \mathrm{l}_{3}$ Systems being tested have been inspected for workmanship and for compliance with design.

sines continuity and megger tests have been performed on portions of the electrical and instrument systems being tested, as required.

$6 x A / 4$ leak tests on the pneumatic systems have been performed.

en 8 The following circuit breakers and fuses are installed per the specified size and are open

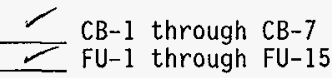

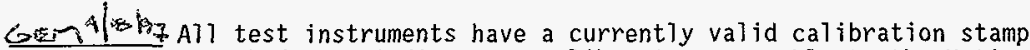
attached that indicates a calibration traceable to the National Institute of Standards and Technology.

Sum $4 \mid$ is ig following process and control instrument systems have been initially configured and aligned for proper operation.

FIT-*57, FSL $-* 57$, NIT-*54, NIT-*55, (NR-*54), PDIT-*60, TIC-*50, TIC $-* 56$, TIS $* 62, Y Y C-* 01$ NOTE: NR $-* 54$ is not used in the ANALYTICAL configuration.

Ges 4 is Personnel responsible for directing and witnessing the performance of the tests described in this ATP have read and understand appropriate certified vendor information (CVI) pertaining to the operation of the equipment to be tested.

$G+2 / 8 i_{4}$ CLOSE all system manual valves with the exception of the following:

$$
\begin{array}{ll}
\text { VTP-FIV-*52 } \\
\text { VTP-FIV-*56 } \\
\text { VTP-FIV-*70 } \\
\text { VTP-SV-*24 } \\
\text { VTP-SV-*61 } \\
\text { VTP-SV-*62 } \\
\text { VTP-SV-*67 } \\
\text { VTP-SV-*71 }
\end{array}
$$

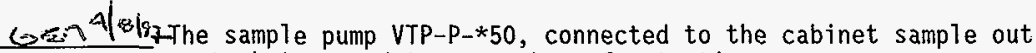
port, but vented to atmosphere for testing. 
S $1 / b_{1}$. 208 Vac 1 phase 15-20 ampere temporary power source has been connected, but not energized, to the appropriate TB1 terminals per H-14-100838.

Verify by signature and date that all prerequisites have been met.

$\frac{\text { Manh 2) Bnor }}{\text { Test Director Signature }} \frac{4-18-97}{\text { Date }}$


TEST DATA SHEET

\begin{tabular}{|c|c|c|c|c|c|}
\hline \multicolumn{3}{|c|}{ Date: 18 Aprz. -97} & \multicolumn{3}{|c|}{ SHMS Unit Number: $M C^{2}$ is pus } \\
\hline \multicolumn{3}{|c|}{$\begin{array}{l}\text { Title of rest: } \\
\text { Electrical Systems }\end{array}$} & \multirow{2}{*}{\multicolumn{3}{|c|}{ 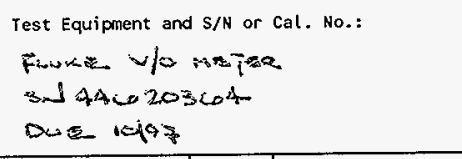 }} \\
\hline \multicolumn{3}{|c|}{ 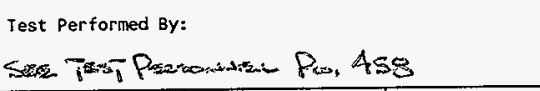 } & & & \\
\hline $\begin{array}{l}\text { Procedure No. } \\
\text { Initial/Date }\end{array}$ & Item & value & $\begin{array}{l}\text { Required } \\
\text { condition } \\
\end{array}$ & $(A / R)$ & Comment \\
\hline \multicolumn{6}{|c|}{ Section 1.7.1.1 verifies the system Mains Power. } \\
\hline 1.7.1.1.1 & Resistance & 0.1 & $<1$ ohm & $A$ & $N$ \\
\hline $\begin{array}{l}1.7 .1 .1 .2 \\
\text { GE.7 }\end{array}$ & $\begin{array}{l}\text { Temp power } \\
208 \mathrm{Vac}\end{array}$ & $N / A$ & Energized & $A$ & \\
\hline $\begin{array}{l}1.7 .1 .1 .3 \\
\text { Gen }\end{array}$ & $\begin{array}{l}\text { Line Vac: } \\
\text { L1-L2 } \\
\text { L1-Gnd } \\
\text { L2-Gnd } \\
\mathrm{N}-\text { Gnd }\end{array}$ & $\frac{\frac{209.5}{119.8}}{\frac{119.60}{0}}$ & $\begin{array}{l}L 1-L 2: 208 \\
\text { Vac }+10 /-1 \% \\
L 1, L 2: 120 \\
\text { Vac } \pm 5 \% \\
N: 0 \text { Vac }\end{array}$ & $A$ & \\
\hline \multicolumn{6}{|c|}{ Section 1.7.1.2 verifies the enclosure HVAC system. } \\
\hline $\begin{array}{c}1.7 .1 .2 .1 \\
0.257 \\
\end{array}$ & AC Adjusted & N/A & N/A & $\Delta$ & \\
\hline $\begin{array}{c}1.7 .1 .2 .2 \\
\end{array}$ & $\begin{array}{l}\text { CLOSE } \\
\mathrm{CB}-1 / 2 \\
\end{array}$ & $N / A$ & N/A & $\Delta$ & \\
\hline 1.7 .1 .2 .3 & Heater ON & $\checkmark$ & Heater ON & $A$ & \\
\hline $\begin{array}{l}1.7 .1 .2 .4 \\
\text { ien }\end{array}$ & AC Adjusted & $N / A$ & $N / A$ & $A$ & \\
\hline 1.7 .1 .2 .5 & $\begin{array}{l}\text { Heater OfF } \\
\text { Cooling ON } \\
\end{array}$ & $\frac{r}{\alpha}$ & $\begin{array}{l}\text { Heater OFF } \\
\text { Cooling ON } \\
\end{array}$ & $\Delta$ & \\
\hline $\begin{array}{l}1.7 .1 .2 .6 \\
\end{array}$ & AC Adjusted & $N / A$ & $\mathrm{~N} / \mathrm{A}$ & $A$ & \\
\hline $\begin{array}{l}1.7 .1 .2 .7 \\
\end{array}$ & $\begin{array}{l}\text { OPEN } \\
\text { CB-1/2 }\end{array}$ & $N / A$ & N/A & $A$ & \\
\hline \multicolumn{6}{|c|}{ Section 1.7.1.3 verifies the sample pump is properiy connected. } \\
\hline $\begin{array}{l}1.7 .1 .3 .1 \\
\text { iofen }\end{array}$ & \begin{tabular}{|l} 
CLOSE \\
CB-3/4 \\
\end{tabular} & N/A & $N / A$ & A- & \\
\hline 1.7 .1 .3 .2 & $\begin{array}{l}\text { VTP-P } * 50 \\
\text { ON }\end{array}$ & 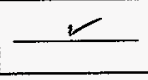 & $\begin{array}{l}\text { VTP-P-*50 } \\
\text { ON }\end{array}$ & A & $N^{\Downarrow}$ \\
\hline
\end{tabular}


APPENDIX 0

TEST DATA SHEET

\begin{tabular}{|c|c|c|c|c|c|}
\hline \multicolumn{3}{|c|}{ Date: is $\Delta_{\text {mom }}{ }_{7} 7$} & \multicolumn{3}{|c|}{ SHMS Unit Number: Mas $* 15+$} \\
\hline \multicolumn{3}{|c|}{$\begin{array}{l}\text { Iitte of rest: } \\
\text { Electrical Systems }\end{array}$} & \multirow{2}{*}{\multicolumn{3}{|c|}{ 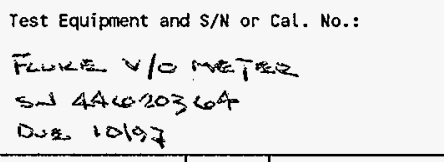 }} \\
\hline \multicolumn{3}{|c|}{ 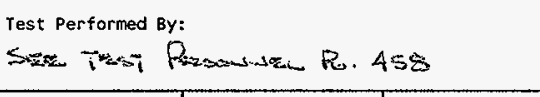 } & & & \\
\hline $\begin{array}{l}\text { Procedure No. } \\
\text { Initial/Date } \\
\end{array}$ & Item & Value & $\begin{array}{l}\text { Required } \\
\text { Condition } \\
\end{array}$ & $(A / R)$ & nt \\
\hline $\begin{array}{l}1.7 .1 .3 .3 \\
\text { Lxin }\end{array}$ & $\begin{array}{l}\text { OPEN } \\
\text { CB-3/4 }\end{array}$ & N/A & N/A & 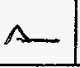 & \\
\hline \multicolumn{6}{|c|}{ Section 1.7.1.4 verifies the enclosure lighting and GFCI receptacles. } \\
\hline $\begin{array}{l}1.7 .1 .4 .1 \\
\text { Gen }\end{array}$ & $\begin{array}{l}\text { CLOSE } \\
\text { CB-6 }\end{array}$ & $\mathrm{N} / \mathrm{A}$ & N/A & $\AA$ & \\
\hline $\begin{array}{c}1: 7.1 .4 .2 \\
4\end{array}$ & $\begin{array}{l}\text { ENCLOSURE } \\
\text { LIGHT }\end{array}$ & N/A & $\mathrm{N} / \mathrm{A}$ & $\therefore$ & \\
\hline $\mathrm{sin}^{1.7 .1 .4 .3}$ & LIGHT ON & 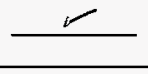 & LIGHT ON & A & \\
\hline $\begin{array}{c}1.7 .1 .4 .4 \\
6 \text { isin }\end{array}$ & $\begin{array}{l}\text { GFCI RECP. } \\
\text { 1A. L-N } \\
\text { 1B. L-GND } \\
2 A . \text { L-N } \\
2 B . \text { L-GND }\end{array}$ & $\frac{\frac{119.9}{119.9}}{\frac{119.9}{119.9}}$ & $\begin{array}{l}\text { 1A. } 120 \mathrm{Vac} \\
1 \mathrm{~B} .120 \mathrm{Vac} \\
2 \mathrm{~A} .120 \mathrm{Vac} \\
2 \mathrm{~B} .120 \mathrm{Vac} \\
\text { ALL } \pm 5 \%\end{array}$ & $\Delta$ & \\
\hline 1.7 .1 .4 .5 & $\begin{array}{l}\text { GFCI TEST } \\
\text { IA. L-N } \\
1 B . \text { L-GND } \\
\text { 2A. L-N } \\
\text { 2B. L-GND } \\
\end{array}$ & $\frac{\frac{.115}{.276}}{\frac{.07}{1.18}}$ & $\begin{array}{l}\text { IA. } 0 \text { Vac } \\
1 B .0 \text { Vac } \\
2 A .0 \text { Vac } \\
2 B .0 \text { Vac } \\
\text { ALL } \pm 5 \%\end{array}$ & $\Delta$ & \\
\hline $\begin{array}{c}1.7 .1 .4 .6 \\
\text { grign }\end{array}$ & $\begin{array}{l}\text { ENCLOSURE } \\
\text { LIGHT OFF }\end{array}$ & N/A & N/A & $\wedge$ & \\
\hline $\begin{array}{c}1.7 .1 .4 .7 \\
\text { coeis }\end{array}$ & $\begin{array}{l}\text { OPEN } \\
\text { CB-6 }\end{array}$ & $N / A$ & $N / A$ & $A$ & $1 / A$ \\
\hline \multicolumn{6}{|c|}{ Section 1.7.1.5 verifies the instrument power source isolation transforme } \\
\hline $\begin{array}{l}1.7 .1 .5 .1 \\
6{ }^{2} 17\end{array}$ & $\begin{array}{l}\text { CLOSE } \\
\text { CB-7 }\end{array}$ & $N / A$ & N/A & A & $N / A$ \\
\hline $\begin{array}{l}1.7 .1 .5 .2 \\
\text { cision }\end{array}$ & $\begin{array}{l}\text { XFRM Vac } \\
\text { PRI. L-N } \\
\text { SEC. L-N } \\
\text { SEC. N-GND } \\
\end{array}$ & $\frac{\frac{119.5}{123,3}}{0}$ & $\begin{array}{l}\text { PRI, \& SEC. } \\
\text { L-N } 120 \pm 5 \% \\
\text { SEC. } \\
\text { N-GND } 0 \text { VaC }\end{array}$ & $\Delta$ & \\
\hline $\begin{array}{c}1.7 .1 .5 .3 \\
\text { exin }\end{array}$ & CLOSE FU-1 & $N / A$ & $N / A$ & A & $\pi / s$ \\
\hline
\end{tabular}


TEST DATA SHEET

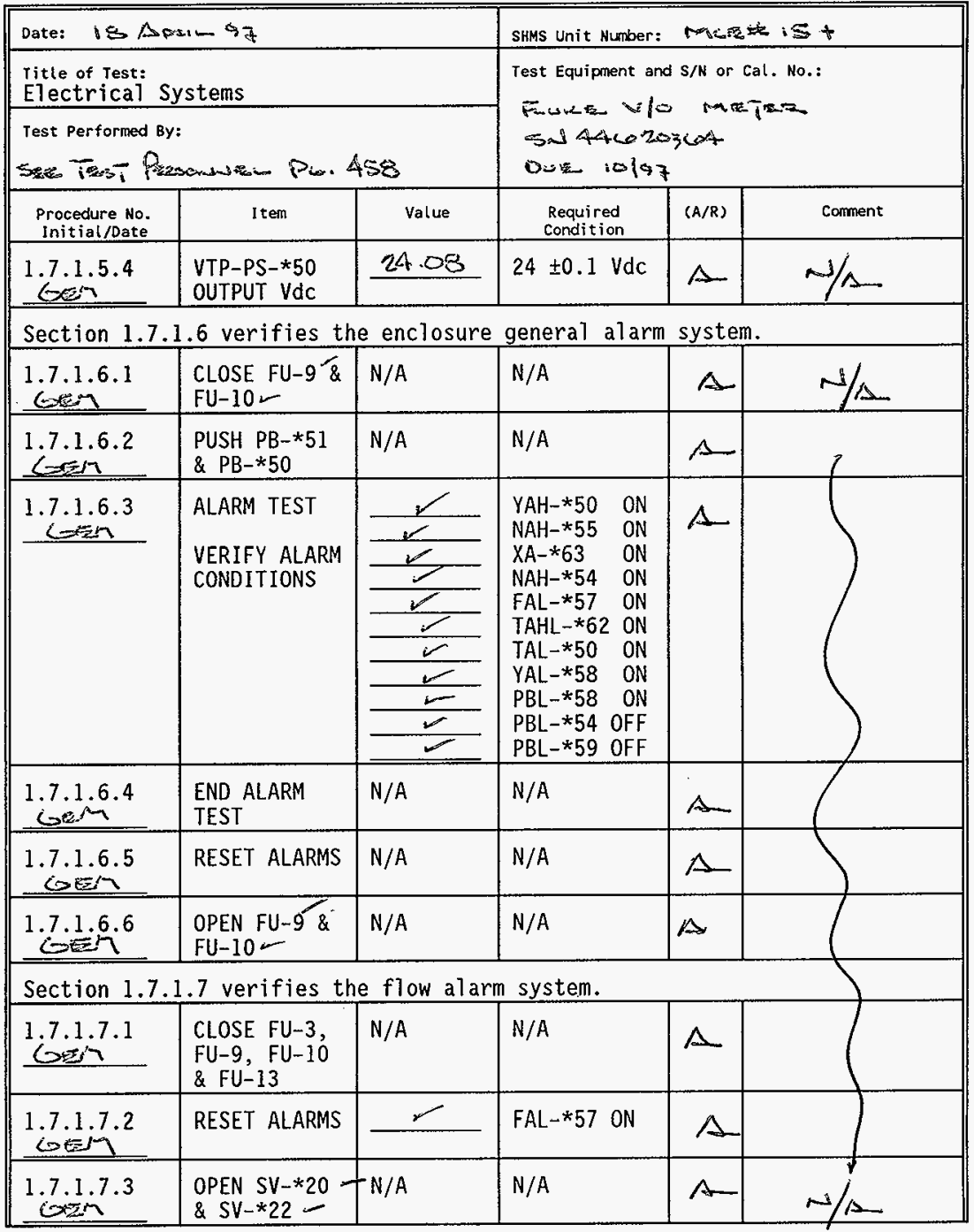


TEST DATA SHEET

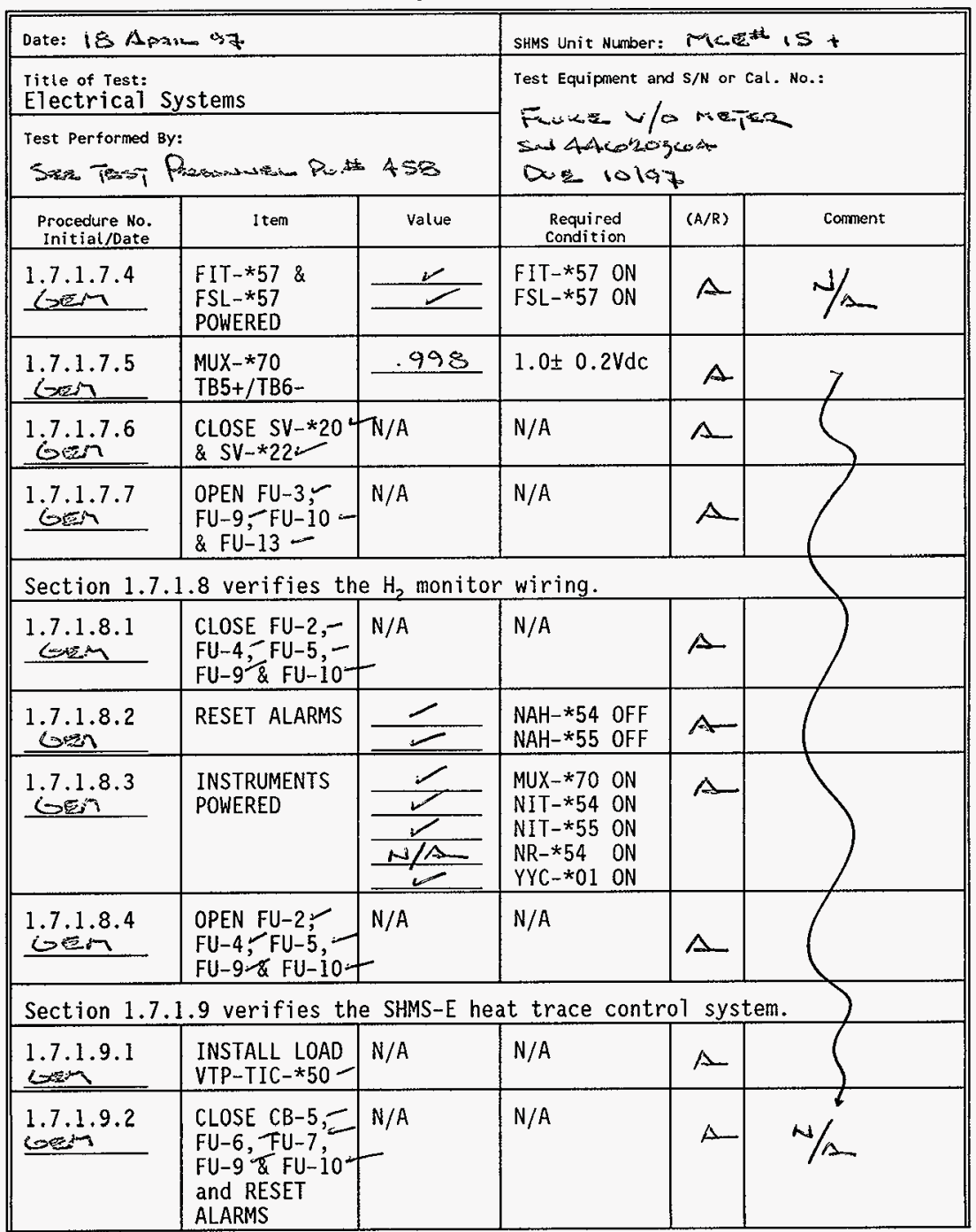


TEST DATA SHEET

\begin{tabular}{|c|c|c|c|c|c|}
\hline \multicolumn{3}{|c|}{ Date: $18 \Delta$ pris 97} & \multicolumn{3}{|c|}{ SHMS Unit Number: race. $15+$} \\
\hline \multicolumn{3}{|c|}{$\begin{array}{l}\text { Title of rest: } \\
\text { Electrical Systems }\end{array}$} & \multirow{2}{*}{\multicolumn{3}{|c|}{ Test Equipment and $\mathrm{S} / \mathrm{N}$ or Cal. No.: }} \\
\hline \multicolumn{3}{|c|}{ 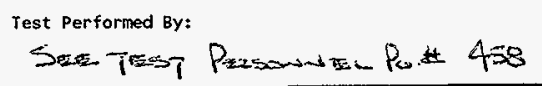 } & & & \\
\hline $\begin{array}{l}\text { Procedure No. } \\
\text { Initial/Date }\end{array}$ & item & & 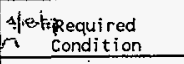 & $(A / R)$ & ent \\
\hline 1.7.1.9.3 & $\begin{array}{l}\text { TIC-*50 \& } \\
\text { TIC-*56 } \\
\text { NOMINAL TEMP }\end{array}$ & $\frac{\frac{\cos }{\cos }}{\cos }$ & $\begin{array}{l}\text { TIC }-* 50 \\
\text { TIC }-* 56 \\
\text { TEMPERATURE } \\
\end{array}$ & $\Delta$ & \\
\hline $\mathrm{Bg}^{1.7 .1 .9 .4}$ & $\begin{array}{l}\text { ADJ TIC }-* 50 \\
\& \text { TIC } * 56 \\
\text { SP2 \& RESET } \\
\text { ALARMS }\end{array}$ & $N / A$ & $N / A$ & $\Delta$ & \\
\hline $\begin{array}{c}1.7 .1 .9 .5 \\
6 E .7 \\
\end{array}$ & $\begin{array}{l}\text { ADJ TIC-*50 } \\
\text { SP } 1\end{array}$ & $N / A$ & $N / A$ & $\Lambda$ & \\
\hline $\begin{array}{l}1.7 .1 .9 .6 \\
\text { boin- }\end{array}$ & $\begin{array}{l}\text { ADJ } T I C-* 50 \\
\text { SP } 2\end{array}$ & $N / A$ & N/A & $\triangle$ & \\
\hline 1.7 .1 .9 .7 & $\begin{array}{l}\text { TAL-*50 \& } \\
\text { HORN ACTIVE }\end{array}$ & $\frac{r}{2}$ & $\begin{array}{l}\text { TAL-*50 ON } \\
\text { HORN ON } \\
\text { ACKNOWLEDGE } \\
\end{array}$ & $\Delta$ & \\
\hline $\begin{array}{l}1.7 .1 .9 .8 \\
\text { csen }\end{array}$ & $\begin{array}{l}\text { TIC }-* 50 \\
\text { OPERATES }\end{array}$ & $r$ & \begin{tabular}{|l|l} 
TIC $-* 50$ \\
OPERATES \\
\end{tabular} & $\Delta$ & \\
\hline $\begin{array}{l}1.7 .1 .9 .9 \\
\mathrm{sin}\end{array}$ & $\begin{array}{l}\text { ADJ TIC } * 50 \\
\text { SP } 2\end{array}$ & $N / A$ & $N / A$ & $\Delta$ & \\
\hline $\operatorname{con}^{1.7 .1 .9 .10}$ & RESET ALARM & 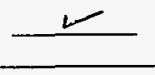 & $\mathrm{TAL}-\star 50$ OFF & $A$ & \\
\hline $\operatorname{cosin}^{7.1 .9 .11}$ & $\begin{array}{l}A D J \text { TIC }-* 50 \\
S P 1 \& S P 2\end{array}$ & $N / A$ & $N / A$ & A & \\
\hline 1.7.1.9.12 & $\begin{array}{l}\text { OPEN CB-5 \& } \\
\text { REMOVE LOAD }\end{array}$ & N/A & $N / A$ & A & \\
\hline$\underline{6.7 .9 .13}$ & $\begin{array}{l}\text { INSTALL LOAD } \\
\text { VTP-TIC-*56 }\end{array}$ & N/A & N/A & $\Delta$ & \\
\hline 1.7 .1 .9 .14 & CLOSE CB-5 & $N / A$ & N/A & $\Delta$ & \\
\hline $\begin{array}{l}1.7 .1 .9 .15 \\
\text { gen }\end{array}$ & $\begin{array}{l}\text { ADJ TIC }-* 56 \\
\text { SP } 1\end{array}$ & N/A & $N / A$ & $\Delta$ & \\
\hline $\sin ^{1.7 .1 .9 .16}$ & $\begin{array}{l}\text { ADJ TIC-*56 } \\
\text { SP } 2\end{array}$ & $\mathrm{~N} / \mathrm{A}$ & N/A & $\Delta$ & \\
\hline
\end{tabular}


TEST DATA SHEET

\begin{tabular}{|c|c|c|c|c|c|}
\hline \multicolumn{3}{|c|}{ Date: $18 \Delta_{p a n} \Delta_{4}$} & SHMS Unit Number: & \multicolumn{2}{|c|}{ mest $.5 t$} \\
\hline \multicolumn{3}{|c|}{$\begin{array}{l}\text { Title of Test: } \\
\text { Electrical Systems }\end{array}$} & \multirow{2}{*}{\multicolumn{3}{|c|}{ Test Equipnent and $\mathrm{s} / \mathrm{N}$ or Cal. No.: }} \\
\hline \multicolumn{3}{|c|}{$\begin{array}{l}\text { Test Performed By: } \\
\text { Stan Tersi Pesonde-Po. } 458\end{array}$} & & & \\
\hline $\begin{array}{l}\text { Procedure No. } \\
\text { Initial/Date }\end{array}$ & Item & value & $\begin{array}{l}\text { Required } \\
\text { Condition }\end{array}$ & $(A / R)$ & corment \\
\hline $\operatorname{lic}_{i \rightarrow 0}^{1.7 .1 .9 .17}$ & $\begin{array}{l}\text { TAL. }-* 50 \& \\
\text { HORN ACTIVE }\end{array}$ & $\frac{r}{L}$ & $\begin{array}{l}\text { TAL-*50 ON } \\
\text { HORN ON } \\
\text { ACKNOWLEDGE- }\end{array}$ & $A$ & \\
\hline $\mathrm{cxp}^{1.7 .1 .9 .18}$ & $\begin{array}{l}\text { TIC-*56 } \\
\text { OPERATES }\end{array}$ & & $\begin{array}{l}\text { TIC }-* 56 \\
\text { OPERATES }\end{array}$ & 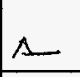 & \\
\hline $\begin{array}{l}1.7 .1 .9 .19 \\
6.20\end{array}$ & $\begin{array}{l}\text { ADJ TIC-*56 } \\
\text { SP } 2\end{array}$ & N/A & $N / A$ & $\Delta$ & \\
\hline$\underbrace{1.7 .1 .9 .20}_{\cos }$ & RESET ALARM & $\varnothing$ & $\mathrm{TAL}-* 50$ OFF & $A$ & \\
\hline $\begin{array}{l}1.7 .1 .9 .21 \\
\operatorname{con}\end{array}$ & $\begin{array}{l}\text { ADJ TIC } * 56 \\
S P 1 \& S P 2\end{array}$ & $N / A$ & $N / A$ & $A$ & \\
\hline $\begin{array}{c}1.7 .1 .9 .22 \\
\text { Eizan }\end{array}$ & $\begin{array}{l}\text { OPEN CB-5, } \\
\text { FU-6-6 FU-7 }\end{array}$ & $N / A$ & $N / A$ & $\Delta$ & \\
\hline 1.7 .1 .9 .23 & $\begin{array}{l}\text { REMOVE LOAD } \\
\text { TIC-*56 }\end{array}$ & $N / A$ & $\mathrm{~N} / \mathrm{A}$ & A & \\
\hline 1.7 .1 .9 .24 & $\begin{array}{l}\text { CLOSE FU-8 } \\
\text { TIS-*62 } \\
\text { NOMINAL TEMP }\end{array}$ & $63 F$ & $\begin{array}{l}\text { TIS-*62 } \\
\text { TEMP }\end{array}$ & $\Delta$ & \\
\hline 6.7.1.9.25 & $\begin{array}{l}\text { ADJ TIS-*62 } \\
\text { SP } 1\end{array}$ & $N / A$ & $N / A$ & $A$ & \\
\hline $\begin{array}{l}1.7 .1 .9 .26 \\
\cos \end{array}$ & $\begin{array}{l}\text { ADJ TIS-*62 } \\
\text { SP } 2\end{array}$ & $\mathrm{~N} / \mathrm{A}$ & $N / A$ & $\Delta$ & \\
\hline $\begin{array}{l}1.7 .1 .9 .27 \\
<-2.2 \\
\end{array}$ & RESET ALARMS & 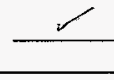 & $\begin{array}{l}\text { TAHL-*62 } \\
\text { OFF }\end{array}$ & $A$ & \\
\hline $\sin ^{1.7 .1 .9 .28}$ & $\begin{array}{l}\text { ADJ TIS-*62 } \\
\text { SP } 2\end{array}$ & N/A & $N / A$ & $\Delta$ & \\
\hline $\begin{array}{l}1.7 .1 .9 .29 \\
\end{array}$ & $\begin{array}{l}\text { TAHL }-* 62 \& \\
\text { HORN ACTIVE }\end{array}$ & $\frac{2}{2}$ & $\begin{array}{l}\text { TAHL-*62 ON } \\
\text { HORN ON } \\
\text { ACKNOWLEDGE }\end{array}$ & $A$ & \\
\hline $\operatorname{cis}^{1.7 .1 .9 .30}$ & $\begin{array}{l}\text { ADJ TIS }-\star 62 \\
\text { SP } 2\end{array}$ & N/A & N/A & $A$ & $1 / 2$ \\
\hline
\end{tabular}


APPENDIX 0

HNF - SD - WM - ATR - 191

PAGE 0- /l

TEST DATA SHEET

\begin{tabular}{|c|c|c|c|c|c|}
\hline \multicolumn{3}{|c|}{ Date: is ApriL 07} & \multicolumn{3}{|c|}{ SHMS Unit Number: MCE $15+$} \\
\hline \multicolumn{3}{|c|}{$\begin{array}{l}\text { Iitle of Test: } \\
\text { Electrical Systems }\end{array}$} & \multirow{2}{*}{\multicolumn{3}{|c|}{ 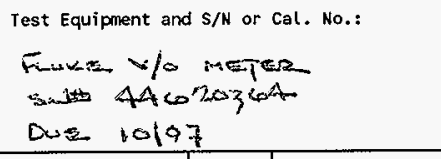 }} \\
\hline \multicolumn{3}{|c|}{ 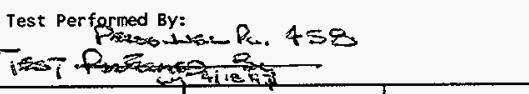 } & & & \\
\hline $\begin{array}{l}\text { Procedure No. } \\
\text { Initial/Date }\end{array}$ & Item & Value & $\begin{array}{c}\text { Required } \\
\text { Condition } \\
\end{array}$ & $(A / R)$ & Comment \\
\hline $\begin{array}{c}1.7 .1 .9 .31 \\
6.1 \\
\end{array}$ & RESET ALARMS & $\ldots$ & $\begin{array}{l}\text { TAHL-*62 } \\
\text { OFF } \\
\end{array}$ & $A$ & a \\
\hline 1.7 .1 .9 .32 & $\begin{array}{l}\text { ADJ TIS-*62 } \\
\text { SP } 1\end{array}$ & $N / A$ & N/A & A & \\
\hline $\begin{array}{c}1.7 .1 .9 .33 \\
\cos \\
\end{array}$ & $\begin{array}{l}\text { TAHL-*62 \& } \\
\text { HORN ACTIVE }\end{array}$ & $\frac{r}{r}$ & $\begin{array}{l}\text { TAHL-*62 ON } \\
\text { HORN ON } \\
\text { ACKNOWLEDGE } \\
\end{array}$ & $\triangle$ & \\
\hline $\begin{array}{l}1.7 .1 .9 .34 \\
0.27 \\
\end{array}$ & $\begin{array}{l}\text { ADJ TIS-*62 } \\
\text { SP } 1 \& S P 2\end{array}$ & $N / A$ & N/A & Ar & \\
\hline $\begin{array}{c}1.7 .1 .9 .35 \\
6.57^{6} \\
\end{array}$ & $\begin{array}{l}\text { OPEN FU-8, } \\
\text { FU-9 \& FU-10- }\end{array}$ & $N / A$ & $N / A$ & $\Delta$ & \\
\hline \multicolumn{6}{|c|}{ Section 1.7.1.10 verifies the differential pressure transmitter system } \\
\hline $\mathrm{ose}^{1.7 .1 .10 .1}$ & $\begin{array}{l}\text { REMOVE PLUGS } \\
\text { BAL PDIT }-* 60 \\
\end{array}$ & N/A & $N / A$ & $\Delta$ & \\
\hline$\frac{1.7 .1 .10 .2}{6 \operatorname{lan}^{2}}$ & $\begin{array}{l}\text { CLOSE FU-14 } \\
\text { REC PDIT-*60 } \\
\text { PRESSURE } \\
\end{array}$ & .030 & $\begin{array}{l}\text { PDIT-*60 } \\
\text { PRESSURE } \\
0 \pm 1 " \mathrm{H}_{2} 0 \\
\end{array}$ & A & \\
\hline 1.7 .1 .10 .3 & $\begin{array}{l}\text { MUX-*70 VdC } \\
\text { TB7+ \& TB8- }\end{array}$ & 2.15 & $\begin{array}{l}\text { MUX }-* 70 \mathrm{Vdc} \\
2.2 \pm 0.1\end{array}$ & A & \\
\hline $\begin{array}{l}1.7 .1 .10 .4 \\
6 e^{-2}\end{array}$ & $\begin{array}{l}\text { REPLACE } \\
\text { PLUGS ON } \\
\text { PDIT-*60 } \\
\end{array}$ & N/A & $N / A$ & $A$ & \\
\hline $\begin{array}{l}1.7 .1 .10 .5 \\
2.0 .0 \\
\end{array}$ & OPEN FU-14 & N/A & $N / A$ & $\Delta$ & \\
\hline \multicolumn{6}{|c|}{ Section 1.7.1.11 verifies the gas chromatograph power. } \\
\hline $\begin{array}{l}1.7 .1 .11 .1 \\
\text { gen }\end{array}$ & CLOSE FU-11 & N/A & $N / A$ & $\Lambda$ & \\
\hline $\begin{array}{l}1.7 .1 .11 .2 \\
\end{array}$ & $\begin{array}{l}\text { POWER } \\
\text { MON-*60 }\end{array}$ & $\checkmark$ & $\begin{array}{l}\text { MON-*60 } \\
\text { POWERED } \\
\end{array}$ & $\Lambda$ & \\
\hline $\operatorname{coz}^{1.7 .1}$ & OPEN FU-11 & $N / A$ & $N / A$ & $\Delta$ & \\
\hline
\end{tabular}


APPENDIX 0

TEST DATA SHEET

\begin{tabular}{|c|c|c|c|c|c|}
\hline \multicolumn{3}{|c|}{ Date: is Apri: 97} & \multicolumn{3}{|c|}{ SHMS Unit Number: MCR is ist } \\
\hline \multicolumn{3}{|c|}{$\begin{array}{l}\text { Iitle of Test: } \\
\text { Electrical Systems }\end{array}$} & \multirow{2}{*}{\multicolumn{3}{|c|}{ 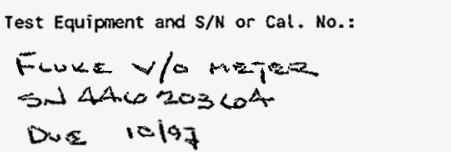 }} \\
\hline \multicolumn{3}{|c|}{ 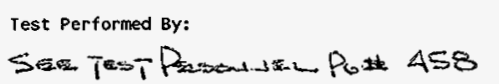 } & & & \\
\hline $\begin{array}{l}\text { Procedure No. } \\
\text { Initial/Date }\end{array}$ & Item & value & $\begin{array}{l}\text { Required } \\
\text { Condition }\end{array}$ & $(A / R)$ & Corment \\
\hline \multicolumn{6}{|c|}{ Section 1.7.1.12 verifies the multi gas analyzer power. } \\
\hline $\begin{array}{l}1.7 .1 .12 .1 \\
\text { Sofent? } \\
\end{array}$ & CLOSE FU-12 & $N / A$ & N/A & $\wedge$ & \\
\hline $\begin{array}{l}1.7 .1 .12 .2 \\
\end{array}$ & $\begin{array}{l}\text { POWER } \\
\text { NIT-*52 }\end{array}$ & & $\begin{array}{l}\text { NIT }-* 52 \\
\text { POWERED } \\
\end{array}$ & $A$ & $123:=$ \\
\hline $\begin{array}{l}1.7 .1 .12 .3 \\
\text { Cot.12 } \\
\end{array}$ & OPEN FU-12 & $N / A$ & $N / A$ & $\Delta$ & \\
\hline \multicolumn{6}{|c|}{ Section 1.7.1.13 verifies the personnel computer power. } \\
\hline $\begin{array}{l}1.7 .1 .13 .1 \\
6 \times 1 \\
\end{array}$ & CLOSE CB-6 & $N / A$ & $N / A$ & $\Delta$ & $\begin{array}{l}\text { ReE Dass } \\
1.7 .1 .4\end{array}$ \\
\hline 1.7 .1 .13 .2 & $\begin{array}{l}\text { POWER PC }-* 60 \\
\& P C-* 70\end{array}$ & 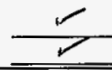 & $\begin{array}{ll}P C-* 60 & 0 N \\
P C-* 70 & O N\end{array}$ & $\Delta$ & \\
\hline 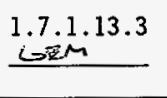 & $\begin{array}{l}\text { TURN INST. } \\
\text { OFF \& } \\
\text { OPEN CB-6 }\end{array}$ & $\stackrel{r}{2}$ & $\begin{array}{ll}P C-* 60 & \text { OFF } \\
P C-\star 70 & \text { OFF }\end{array}$ & A & 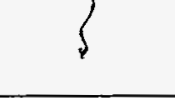 \\
\hline
\end{tabular}

cor ALC $4\{i s k j$ 
APPENDIX 0

PAGE 0-/3

$$
\begin{array}{r}
H N F-S D-W M-A T R-191 \\
\text { ReV. } 0 \\
\text { Page } 469
\end{array}
$$

TEST DATA SHEET

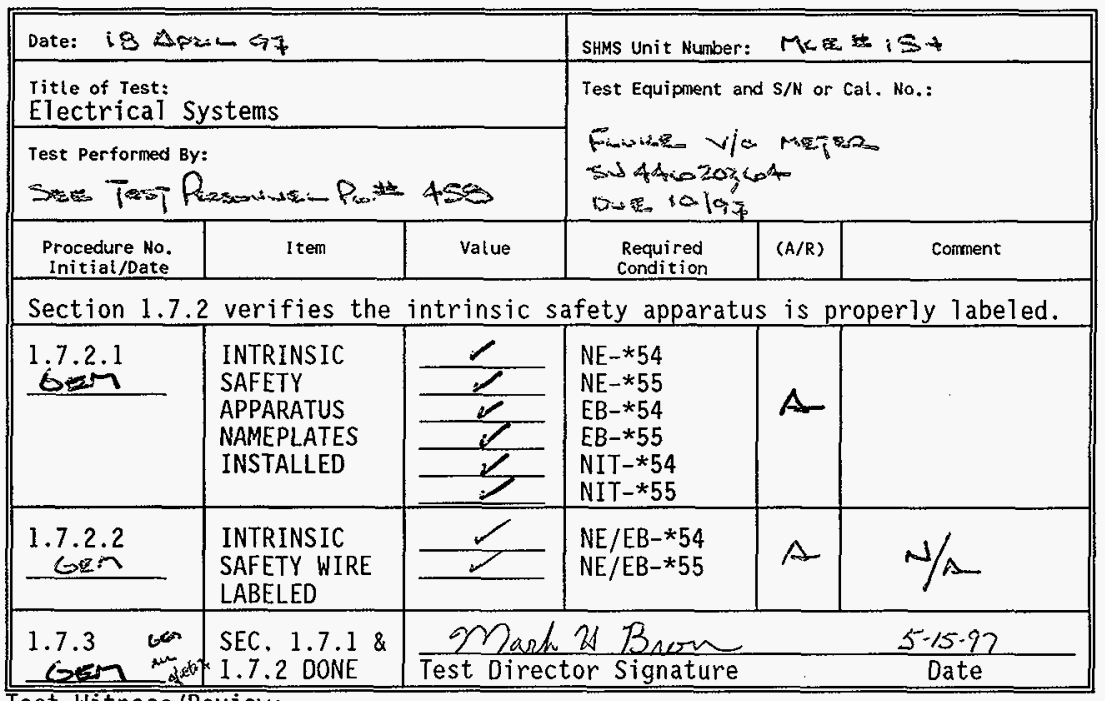

Test Witness/Review:

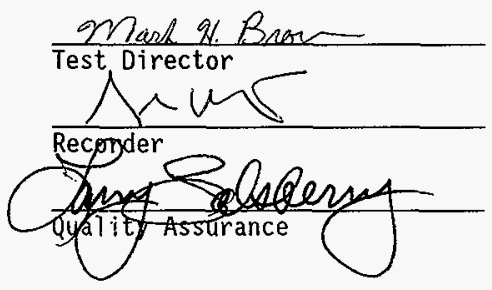

$$
\frac{5-15-97}{\text { Date }}
$$$$
\frac{\text { iq ms }}{\text { Date } 97}
$$$$
\frac{5 / 28 / 97}{\text { Date }}
$$ 
TEST DATA SHEET

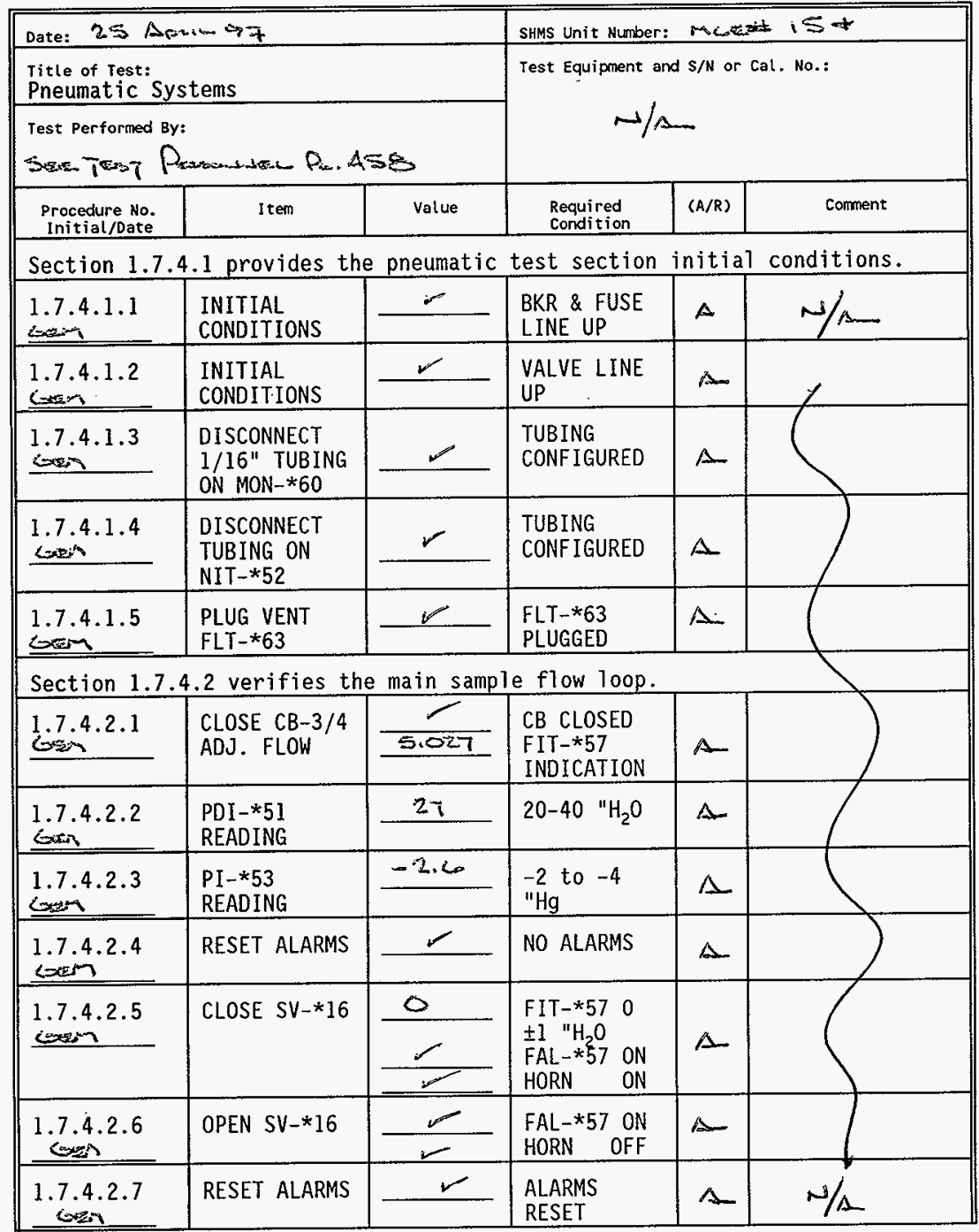


TEST DATA SHEET

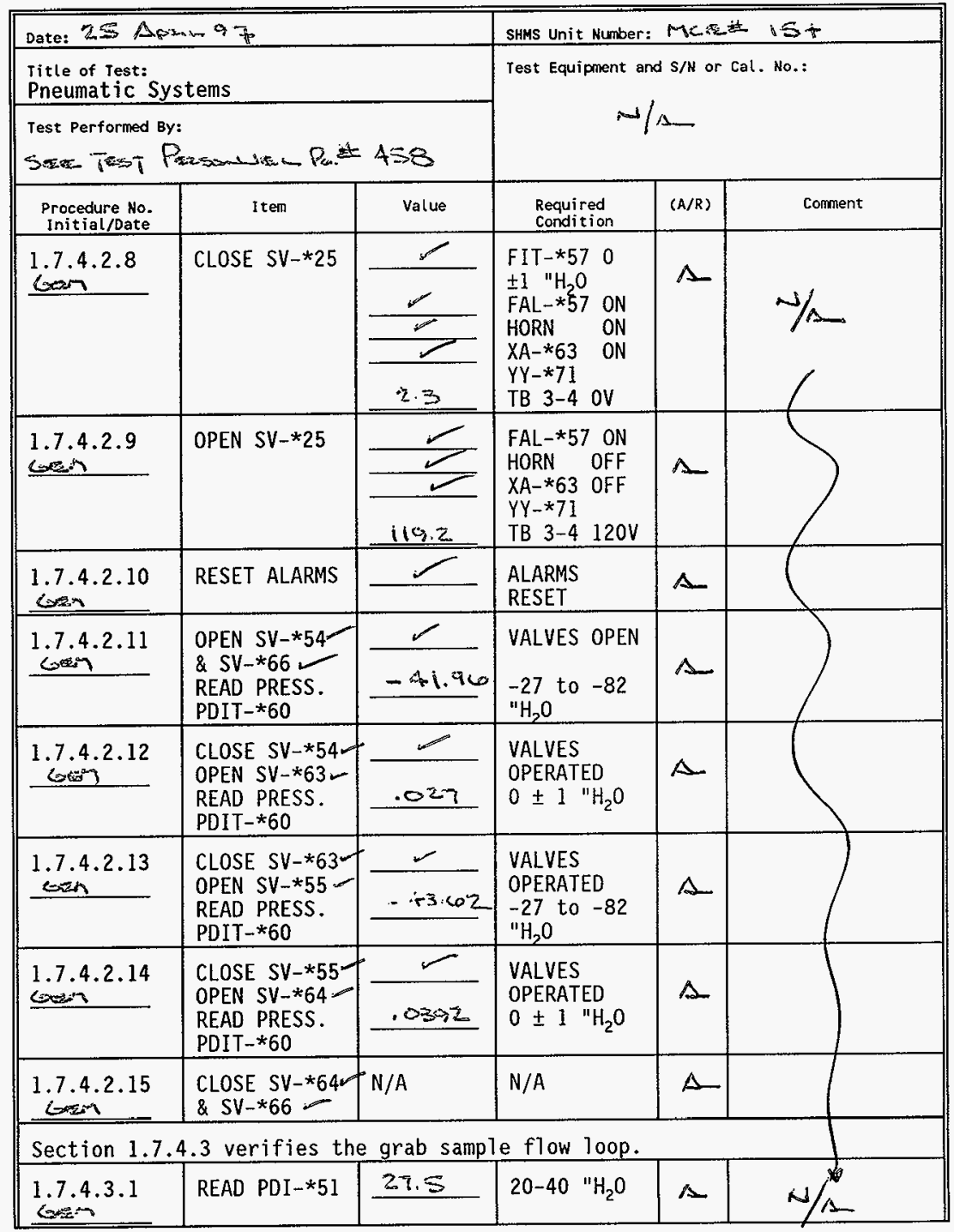


TEST DATA SHEET

\begin{tabular}{|c|c|c|c|c|c|}
\hline \multicolumn{3}{|c|}{ Date: 25 Apar 97} & SHMS Unit Number: & \multicolumn{2}{|c|}{ Mctis ISt } \\
\hline \multicolumn{3}{|c|}{$\begin{array}{l}\text { Title of rest: } \\
\text { Pneumatic Systems }\end{array}$} & \multirow{2}{*}{\multicolumn{3}{|c|}{$\begin{array}{l}\text { Test Equipnent and } S / \mathrm{N} \text { or Cal. No.: } \\
\text { N/A }\end{array}$}} \\
\hline \multicolumn{3}{|c|}{ 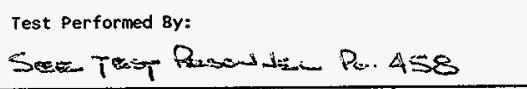 } & & & \\
\hline $\begin{array}{l}\text { Procedure No. } \\
\text { Initial/Date }\end{array}$ & Item & Value & $\begin{array}{l}\text { Required } \\
\text { condition }\end{array}$ & $(A / R)$ & Comment \\
\hline $\begin{array}{l}1.7 .4 .3 .2 \\
\cos \end{array}$ & $\begin{array}{l}\text { PUSH PB-*59 } \\
\text { GRAB SAMPLE }\end{array}$ & $\leftarrow$ & $\begin{array}{l}\text { START } \\
\text { STOPWATCH }\end{array}$ & $\Delta$ & $\mathrm{N} / \mathrm{s}$ \\
\hline 1.7.4.3.3 & $\begin{array}{l}\text { GRAB SAMPLE } \\
\text { LAMPS ON } \\
\text { READ FIV }-* 52\end{array}$ & $\frac{r}{\infty}$ & $\begin{array}{l}\text { PBL-*58 ON } \\
\text { PBL-*59 ON } \\
\text { YAL-*58 ON } \\
\text { FIV-*52 } \\
\end{array}$ & A. & \\
\hline $\begin{array}{l}1.7 .4 .3 .4 \\
\end{array}$ & $\begin{array}{l}\text { OPEN SV-*15 } \\
\& \text { ADJ. FIV- } \\
* 52\end{array}$ & $\mathscr{L}$ & $\begin{array}{l}\text { SV }-* 15 \text { OPEN } \\
\text { FIV }-* 52 \\
\text { FLOW } 10 \text { CFH } \\
\end{array}$ & $\Delta$ & \\
\hline $\begin{array}{c}1.7 .4 .3 .5 \\
\end{array}$ & READ PDI $-* 51$ & 32 & $\begin{array}{l}\text { PDI }-* 51 \\
\text { HIGHER THAN } \\
1.7 .4 .3 .1 \\
\end{array}$ & $\Delta$ & \\
\hline $\begin{array}{l}1.7 .4 .3 .6 \\
\sin ^{2}\end{array}$ & CLOSE SV-* $15^{\circ}$ & 0 & FIV- $* 520$ & A & \\
\hline $\begin{array}{l}1.7 .4 .3 .7 \\
\text { 요 }\end{array}$ & OPEN SV-*15 & 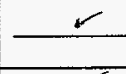 & $\begin{array}{l}\text { FIV-*52 } \\
\text { FLOW }\end{array}$ & $\Delta$ & \\
\hline $\begin{array}{c}1.7 .4 .3 .8 \\
\end{array}$ & $\begin{array}{l}\text { PBL- } * 59 \\
\text { GRAB SAMPLE } \\
\text { TIME }\end{array}$ & $4 ! 59$ & $\begin{array}{l}\text { PBL- } * 59 \text { OFF } \\
\text { STOP WATCH } \\
5 \pm .5 \mathrm{MIN} \\
\end{array}$ & $A$ & \\
\hline $\begin{array}{l}1.7 .4 .3 .9 \\
\end{array}$ & $\begin{array}{l}\text { PB-*58 RESET } \\
\text { SAMPLER } \\
\end{array}$ & $\frac{\mathscr{L}}{\not}$ & $\begin{array}{l}P B L-* 58 \text { OFF } \\
\text { YAL-*58 OFF }\end{array}$ & $\Delta$ & \\
\hline \multicolumn{6}{|c|}{ Section 1.7 .4 .4 verifies the $\mathrm{H}_{2}$ cell calibration loop. } \\
\hline $\begin{array}{l}1.7 .4 .4 .1 \\
\text { (6) }\end{array}$ & $\begin{array}{l}\text { CONNECT } \mathrm{H}_{2} \\
\text { CAL GAS }\end{array}$ & N/A & N/A & A. & \\
\hline $\begin{array}{c}1.7 .4 .4 .2 \\
\end{array}$ & $\begin{array}{l}\text { OPEN ISO } \\
\text { VALVE }\end{array}$ & 5 & $<10$ PSIG & $A$ & \\
\hline 1.7 .4 .4 .3 & $\begin{array}{l}\text { OPEN SV }-* 18 \\
\text { ADJ. FIV }-* 56\end{array}$ & 2 & $2 \pm .1 \mathrm{CFH}$ & $\Delta$ & $\mathrm{N} / \mathrm{A}^{2}$ \\
\hline
\end{tabular}


TEST DATA SHEET

\begin{tabular}{|c|c|c|c|c|c|}
\hline \multicolumn{3}{|c|}{ Date: $2 S \Delta A_{p 2}-97$} & \multicolumn{3}{|c|}{ SHMS Unit Number: Mcez ist } \\
\hline \multicolumn{3}{|c|}{$\begin{array}{l}\text { Title of rest: } \\
\text { Pneumat ic Systems }\end{array}$} & \multirow{2}{*}{\multicolumn{3}{|c|}{ 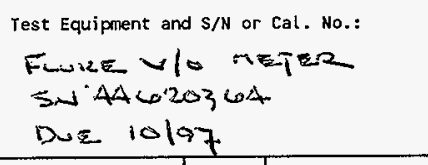 }} \\
\hline \multirow{2}{*}{$\begin{array}{l}\text { Test Perforned By } \\
\text { Sezer TESsT } \\
\begin{array}{c}\text { Procedure No. } \\
\text { Jnitial/Date }\end{array} \\
\end{array}$} & Pensondanch & Po.4t 438 & & & \\
\hline & item & Value & $\begin{array}{l}\text { Required } \\
\text { Condition } \\
\end{array}$ & $(A / R)$ & Comment \\
\hline $\cos ^{1.7 .4 .4 .4}$ & $\begin{array}{l}\text { VERIFY } \\
\text { CONDITIONS }\end{array}$ & $\frac{5}{\frac{5}{L}}$ & $\begin{array}{l}\text { NIT-*54 } \\
>.625 \% \mathrm{H}_{2} \\
\text { NAH-*55 ON } \\
\text { NAH-*54 ON } \\
\mathrm{PBL}-* 59 \text { ON } \\
\mathrm{PBL}-* 58 \text { ON } \\
\text { YAL-*58 ON } \\
\text { FIV }-* 52 \quad \mathrm{FLO} \\
\text { HORN ON } \\
\text { TB2-21/22 } \\
\text { OPEN } \\
\end{array}$ & $A$ & \\
\hline $\begin{array}{l}1.7 .4 .4 .5 \\
\text { sen }\end{array}$ & $\begin{array}{l}\text { ACKNOWLEDGE } \\
\text { HORN }\end{array}$ & $N / A$ & N/A & $\Delta$ & \\
\hline $\begin{array}{l}1.7 .4 .4 .6 \\
\text { gem }\end{array}$ & $\begin{array}{l}\text { CLOSE SV }-* 18 \gamma \\
\text { VERIFY } \\
\text { CONDITIONS }\end{array}$ & $\frac{\sigma}{-\frac{1}{\sigma}}$ & $\begin{array}{l}\text { FIV-*56 } 0 \\
\text { NIT-*54 } \\
<.625 \% \mathrm{H}_{2} \\
\text { NAH-*55 OFF } \\
\text { NAH-*54 ON } \\
\text { PBL-*58 ON } \\
\text { YAL-*58 ON } \\
\text { TB2-21/22 } \\
\text { CLOSED } \\
\end{array}$ & $A$ & \\
\hline $\begin{array}{l}1.7 .4 .4 .7 \\
6\end{array}$ & $\begin{array}{l}\text { PUSH RESET } \\
\text { SAMPLER PB- } \\
* 58\end{array}$ & $\leftarrow$ & $\begin{array}{l}\text { PBL } * 558 \text { OFF } \\
\text { YAL }-* 58 \text { OFF }\end{array}$ & A- & \\
\hline $\cos ^{1.7 .4 .8}$ & RESET ALARM & 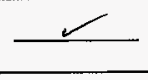 & $\mathrm{NAH}-\star 54$ OFF & $\Delta$ & \\
\hline 1.7 .4 .4 .9 & $\begin{array}{l}\text { OPEN SV-*19 } \\
\text { ADJ. FIV-*56 }\end{array}$ & 2 & $2 \pm .1 \mathrm{CFH}$ & $\Delta \rightarrow$ & $N / \alpha$ \\
\hline
\end{tabular}

Sor Aw tiz:liq 
TEST DATA SHEET

\begin{tabular}{|c|c|c|c|c|c|}
\hline \multicolumn{3}{|c|}{ Date: $25 \Delta p_{2}: n_{-}=$} & \multicolumn{3}{|c|}{ Mcte } \\
\hline \multicolumn{3}{|c|}{$\begin{array}{l}\text { Title of Test: } \\
\text { Pneumatic Systems }\end{array}$} & \multirow{2}{*}{\multicolumn{3}{|c|}{ 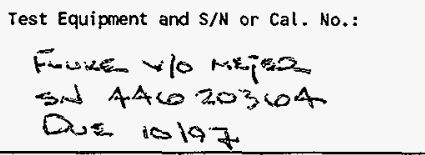 }} \\
\hline \multicolumn{3}{|c|}{ 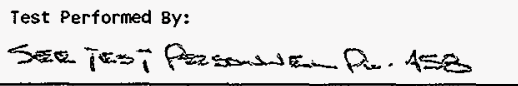 } & & & \\
\hline $\begin{array}{l}\text { Procedure No. } \\
\text { Initial/Date }\end{array}$ & I tem & Value & $\begin{array}{l}\text { Required } \\
\text { Condition }\end{array}$ & $(A / R)$ & Comment \\
\hline $\begin{array}{l}1.7 .4 .4 .10 \\
<.4\end{array}$ & $\begin{array}{l}\text { VERIFY } \\
\text { CONDITIONS }\end{array}$ & 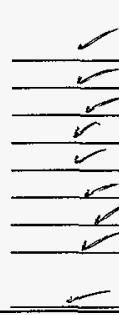 & $\begin{array}{l}\text { NIT-*55 } \\
>.625 \% \mathrm{H}_{2} \\
\text { NAH-*55 ON } \\
\text { NAH-*54 ON } \\
\mathrm{PBL}-* 59 \text { ON } \\
\mathrm{PBL}-* 58 \text { ON } \\
\text { YAL-*58 ON } \\
\text { FIV-*52 FL0 } \\
\text { HORN ON } \\
\text { TB2-21/22 } \\
\text { OPEN }\end{array}$ & $\Delta$ & \\
\hline $\begin{array}{c}1.7 .4 .4 .11 \\
\end{array}$ & $\begin{array}{l}\text { ACKNOWLEDGE } \\
\text { HORN }\end{array}$ & $N / A$ & $N / A$ & $\Delta$ & \\
\hline $\begin{array}{l}1.7 .4 .4 .12 \\
0 \times 1 \\
\end{array}$ & $\begin{array}{l}\text { CLOSE SV-*19: } \\
\text { VERIFY } \\
\text { CONDITIONS }\end{array}$ & $=$ & $\begin{array}{l}\text { FIV-*56 0 } \\
\text { NIT-*55 } \\
<.625 \% \mathrm{H}_{2} \\
\mathrm{NAH}-* 55 \text { OFF } \\
\text { NAH-*54 ON } \\
\text { PBL-*58 ON } \\
\text { YAL-*58 ON } \\
\text { TB2-21/22 } \\
\text { CLOSED }\end{array}$ & A- & \\
\hline $\begin{array}{l}1.7 .4 .4 .13 \\
\operatorname{Lstan} \\
\end{array}$ & $\begin{array}{l}\text { PUSH RESET } \\
\text { SAMPLER PB- } \\
* 58\end{array}$ & $\infty$ & $\begin{array}{l}\mathrm{PBL}-* 58 \text { OFF } \\
\text { YAL } * 58 \text { OFF }\end{array}$ & $\Delta$ & \\
\hline $\begin{array}{l}1.7 .4 .4 .14 \\
\end{array}$ & RESET ALARM & & $\mathrm{NAH}-* 54$ OFF & $A$ & \\
\hline $\begin{array}{l}1.7 .4 .4 .15 \\
6 \\
\end{array}$ & $\begin{array}{l}\text { DISCONNECT } \\
\text { CAL. GAS }\end{array}$ & $N / A$ & $N / A$ & $\Delta$ & \\
\hline$\underline{\sin }^{7.4 .16}$ & $\begin{array}{l}\text { PUSH PB } * 54 \\
\text { VERIFY } \\
\text { CONDITIONS }\end{array}$ & 2 & $\begin{array}{l}X A-* 63 \text { ON } \\
\text { PBL-*54 ON } \\
\text { TB2-23/24 } \\
\text { OPEN }\end{array}$ & $\Delta$ & \\
\hline $\begin{array}{l}1.7 .4 .4 .17 \\
\end{array}$ & $\begin{array}{l}\text { PUSH PB-*54 } \\
\text { VERIFY } \\
\text { CONDITIONS }\end{array}$ & 2 & $\begin{array}{l}\text { XA-*63 OFF } \\
\text { PBL-*54 OFF } \\
\text { TB2-23/24 } \\
\text { CLOSED }\end{array}$ & A & \\
\hline
\end{tabular}


TEST DATA SHEET

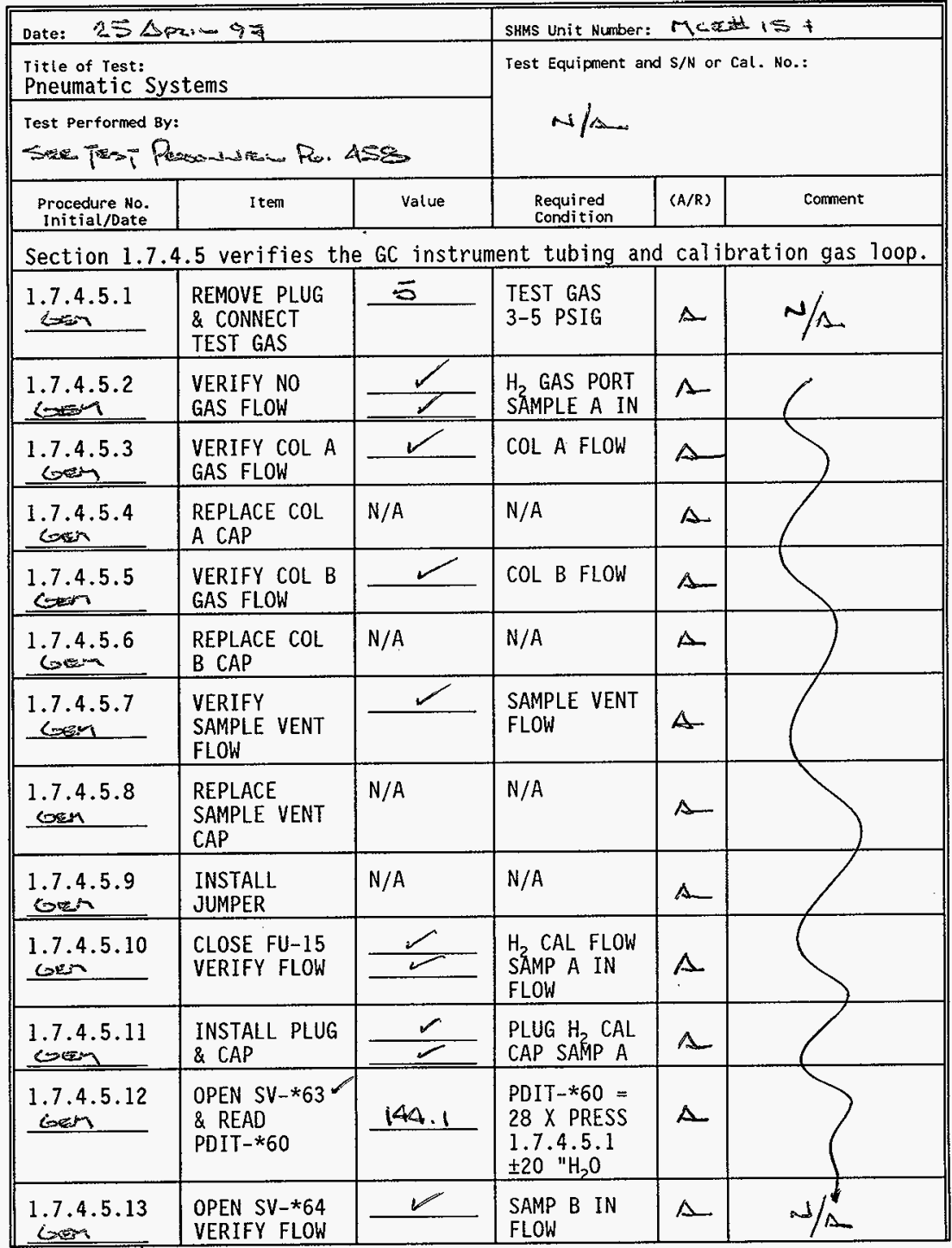


TEST DATA SHEET

\begin{tabular}{|c|c|c|c|c|c|}
\hline \multicolumn{3}{|c|}{ Date: 25 Aprim 9t } & \multicolumn{3}{|c|}{ ShMS Unit Number: most is $5+$} \\
\hline \multicolumn{3}{|c|}{$\begin{array}{l}\text { Title of Test: } \\
\text { Pneumatic Systems }\end{array}$} & \multirow{2}{*}{\multicolumn{3}{|c|}{ Test Equipnent and $\mathrm{S} / \mathrm{N}$ or Cal. No.: }} \\
\hline \multicolumn{3}{|c|}{ 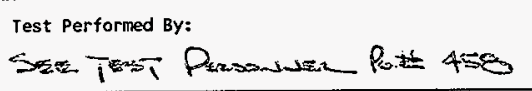 } & & & \\
\hline $\begin{array}{c}\text { Procedure No. } \\
\text { Initial/Date }\end{array}$ & Item & Value & $\begin{array}{l}\text { Required } \\
\text { Condition }\end{array}$ & $(A / R)$ & Comment \\
\hline $\begin{array}{l}1.7 .4 .5 .14 \\
6 \text { बen } \\
\end{array}$ & $\begin{array}{l}\text { REMOVE TEST } \\
\text { GAS }\end{array}$ & $\begin{array}{l}\text { N/A } \\
\therefore\end{array}$ & $N / A$ & A & \\
\hline $\begin{array}{l}1.7 .4 .5 .15 \\
\text { G5.7 }\end{array}$ & $\begin{array}{l}\text { CLOSE SV-*63- } \\
\& \text { SV }-* 64-\end{array}$ & N/A & $N / A$ & $\Delta$ & \\
\hline $\begin{array}{l}1.7 .4 .5 .16 \\
\text { cosen }\end{array}$ & $\begin{array}{l}\text { OPEN FU-15 } \\
\text { REMOVE } \\
\text { JUMPER }\end{array}$ & $N / A$ & $N / A$ & $\Delta$ & \\
\hline $\begin{array}{l}1.7 .4 .5 .17 \\
\end{array}$ & $\begin{array}{l}\text { INSTALL } \\
\text { SAMP A UNION }\end{array}$ & $N / A$ & $N / A$ & A. & \\
\hline $\begin{array}{l}1.7 .4 .5 .18 \\
\operatorname{logh} \\
\end{array}$ & $\begin{array}{l}\text { OPEN SV-*60 } \\
\text { VERIFY FLOW }\end{array}$ & & $\begin{array}{l}F I-* 60 \\
\text { NO FLOW }\end{array}$ & $\Delta$ & \\
\hline$\frac{1.7 .4 .5 .19}{\sin }$ & $\begin{array}{l}\text { POWER SOV- } \\
\text { *60, VERIFY } \\
\text { NO FLOW } \\
\end{array}$ & 1 & $\begin{array}{l}\text { FI }-* 60 \\
\text { NO FLOW }\end{array}$ & $\Delta$ & \\
\hline 1.7 .4 .5 .20 & $\begin{array}{l}\text { OPEN SV-*68 } \\
\text { ADJ SV }-* 67 \\
\end{array}$ & 50 & $\begin{array}{l}\mathrm{FI}-* 60 \\
50 \mathrm{CCM}\end{array}$ & $\Delta$ & \\
\hline $\begin{array}{l}1.7 .4 .5 .21 \\
\end{array}$ & $\begin{array}{l}\text { DEENERGIZE } \\
\text { SOV }-* 60\end{array}$ & $\checkmark$ & $\begin{array}{l}\text { FI-*60 FLOW } \\
\text { DECREASES }\end{array}$ & A & \\
\hline 1.7 .4 .5 .22 & $\begin{array}{l}\text { CLOSE SV-*60 } \\
\& S V-* 68\end{array}$ & N/A & N/A & $\Delta$ & \\
\hline 1.7 .4 .5 .23 & $\begin{array}{l}\text { REMOVE } \\
\text { SAMP A UNION }\end{array}$ & N/A & N/A & $\Delta$ & \\
\hline $\operatorname{seg}^{1.7 .4 .5 .24}$ & $\begin{array}{l}\text { CONNECT TEST } \\
\text { GAS } \mathrm{N}_{2} 0 \text { PORT }\end{array}$ & $\Phi$ & $\begin{array}{l}\text { TEST GAS } \\
3-5 \text { PSIG }\end{array}$ & $\Delta$ & \\
\hline$\frac{1.7 .4 .5 .25}{6 \times 2.7}$ & $\begin{array}{l}\text { VERIFY NO } \\
\text { FLOW }\end{array}$ & $\frac{\alpha}{2}$ & $\begin{array}{l}\text { SAMP B IN \& } \\
\text { FLT-*63 } \\
\text { NO FLOW- }\end{array}$ & $\Delta$ & \\
\hline 1.7 .4 .5 .26 & $\begin{array}{l}\text { INSTALL } \\
\text { JUMPER } \\
\end{array}$ & $N / A$ & N/A & $A$ & \\
\hline $\operatorname{sen}^{1.7 .4 .5 .27}$ & $\begin{array}{l}\text { CLOSE FU-15 } \\
\text { VERIFY FLOW }\end{array}$ & $\frac{r}{r}$ & $\begin{array}{l}\text { SAMP B IN } \\
\text { FLT-*63 } \\
\text { FLOW }\end{array}$ & $\Delta$ & \\
\hline
\end{tabular}


APPENDIX 0

PAGE 0-2r
HNF-SD-WM-ATR-191

Rev. 0
Page 477

TEST DATA SHEET

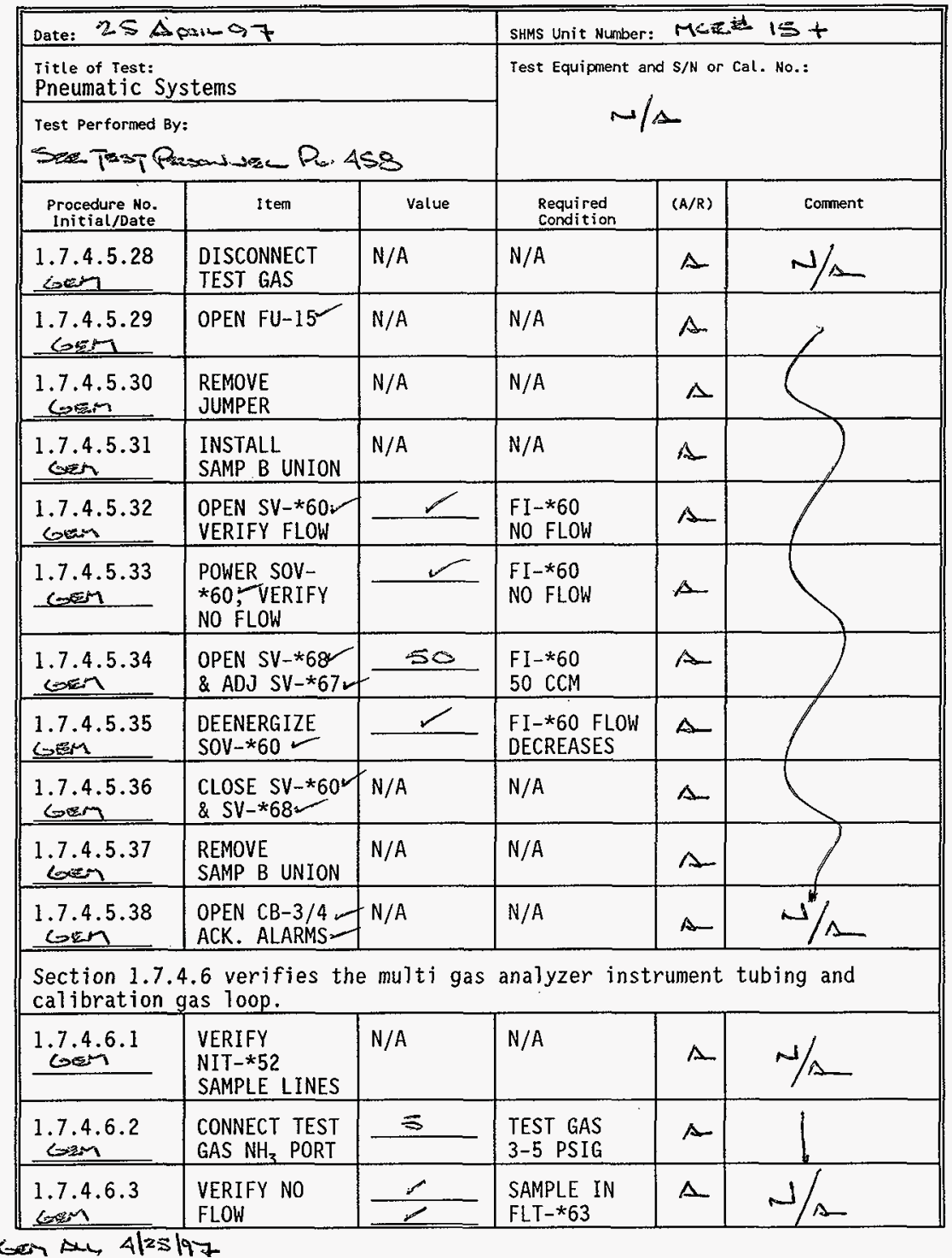


TEST DATA SHEET

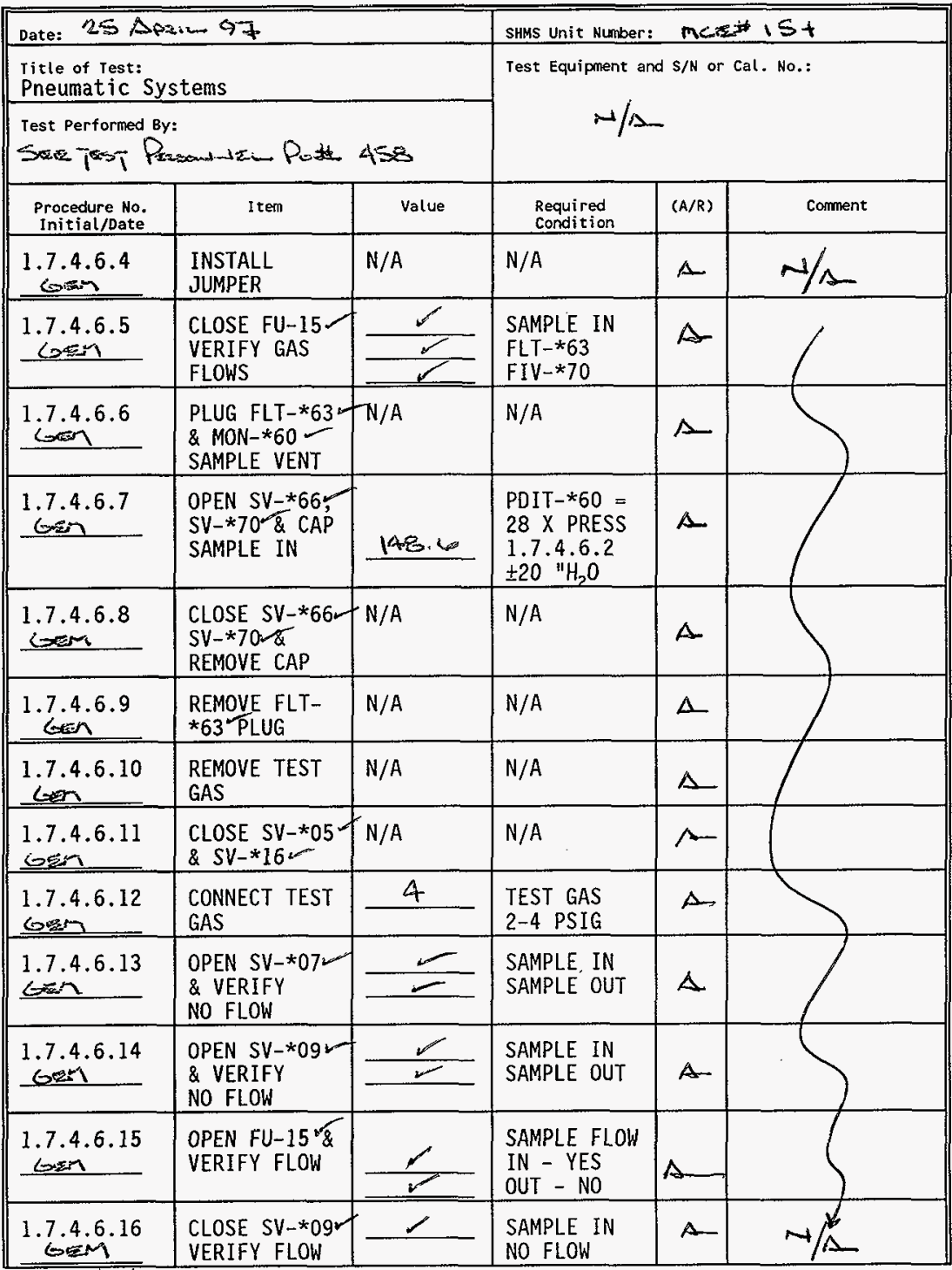


TEST DATA SHEET

\begin{tabular}{|c|c|c|c|c|c|}
\hline \multicolumn{3}{|c|}{ Date: 25 Aprim 97} & \multicolumn{3}{|c|}{ SHMS Unit Number: Mus $\$$ ist } \\
\hline \multicolumn{3}{|c|}{$\begin{array}{l}\text { Title of Test: } \\
\text { Pneumatic Systems }\end{array}$} & \multirow{2}{*}{\multicolumn{3}{|c|}{ Test Equipment and $S / \mathrm{N}$ or Cal. No.: }} \\
\hline \multicolumn{3}{|c|}{ 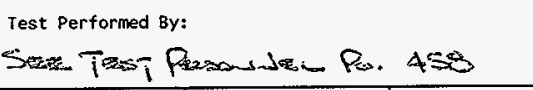 } & & & \\
\hline $\begin{array}{l}\text { Procedure No. } \\
\text { Initial/oate }\end{array}$ & Item & Value & $\begin{array}{l}\text { Required } \\
\text { Condition }\end{array}$ & $(A / R)$ & Comment \\
\hline $\begin{array}{l}1.7 .4 .6 .17 \\
\text { cosing }\end{array}$ & $\begin{array}{l}\text { OPEN SV-*10 } \\
\text { VERIFY FLOW }\end{array}$ & $\frac{\swarrow}{\swarrow}$ & $\begin{array}{l}\text { SAMPLE FLOW } \\
\text { IN - NO } \\
\text { OUT - YES } \\
\end{array}$ & $\Delta$ & \\
\hline $\sin ^{1.7 .4 .6 .18}$ & $\begin{array}{l}\text { REMOVE TEST } \\
\text { GAS \& PLUG } \\
\text { SV }-* 07\end{array}$ & $N / A$ & N/A & A & \\
\hline $\begin{array}{l}1.7 .4 .6 .19 \\
\text { gen }\end{array}$ & $\begin{array}{l}\text { CLOSE SV-*07w } \\
\& \text { SV-*10- }\end{array}$ & $N / A$ & $N / A$ & $A$ & \\
\hline $\begin{array}{l}1.7 .4 .6 .20 \\
\text { G.8R }\end{array}$ & $\begin{array}{l}\text { OPEN SV-*05- } \\
\& \text { SV-*16- }\end{array}$ & $N / A$ & N/A & 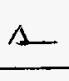 & \\
\hline 1.7 .4 .6 .21 & $\begin{array}{l}\text { REMOVE } \\
\text { JUMPER }\end{array}$ & N/A & N/A & $\Delta$ & \\
\hline \multicolumn{6}{|c|}{ Section 1.7.4.7 verifies the carrier gas supply lines. } \\
\hline 1.7.4.7.1 & $\begin{array}{l}\text { CONNECT TEST } \\
\text { GAS }\end{array}$ & 4 & 3-5 PSIG & $\Delta$ & \\
\hline $\begin{array}{l}1.7 .4 .7 .2 \\
\cos 1\end{array}$ & $\begin{array}{l}\text { REMOVE PLUG } \\
\& \text { VERIFY } \\
\text { FLOW }\end{array}$ & $\sim$ & $\begin{array}{l}\mathrm{N}_{2} \text { CARRIER } \\
\text { GAS FLOWS }\end{array}$ & $\Delta$ & \\
\hline 1.7 .4 .7 .3 & $\begin{array}{l}\text { DISCONNECT } \\
\text { TEST GAS }\end{array}$ & $N / A$ & $N / A$ & $s$ & 7 \\
\hline$\underline{6.2 .7}^{1.7 .4}$ & $\begin{array}{l}\text { CONNECT TEST } \\
\text { GAS }\end{array}$ & 4 & 3-5 PSIG & $\wedge$ & $1 / 1$ \\
\hline $\begin{array}{l}1.7 .4 .7 .5 \\
\text { ion }\end{array}$ & $\begin{array}{l}\text { REMOVE PLUG } \\
\& \text { VERIFY } \\
\text { FLOW }\end{array}$ & $\checkmark$ & $\begin{array}{l}\text { He CARRIER } \\
\text { GAS FLOWS }\end{array}$ & $\Delta$ & 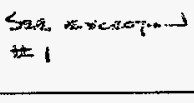 \\
\hline$\underbrace{1.7 .4 .7 .6}$ & $\begin{array}{l}\text { DISCONNECT } \\
\text { TEST GAS }\end{array}$ & $N / A$ & $N / A$ & $\Delta$ & $\mathrm{N} / \mathrm{a}$ \\
\hline $\begin{array}{l}1.7 .5 \\
\cos n \\
\end{array}$ & $\begin{array}{l}\text { TEST SEC. } \\
1.7 .4 \text { DONE }\end{array}$ & \multicolumn{4}{|c|}{$\begin{array}{cc}\text { Morh D Bnos } & 4.25 .97 \\
\text { Test Director Signature } & \text { Date }\end{array}$} \\
\hline
\end{tabular}

ex AM $4.12=177$ 
APPENDIX 0

TEST DATA SHEET

\begin{tabular}{|c|c|c|c|c|c|}
\hline \multicolumn{3}{|c|}{ Date: 9 MAY 97} & \multicolumn{3}{|c|}{ SHMS Unit Number: MCBS ist } \\
\hline \multicolumn{3}{|c|}{$\begin{array}{l}\text { Title of Test: } \\
\text { Pneumatic Systems }\end{array}$} & \multirow{2}{*}{\multicolumn{3}{|c|}{ Test Equipment and $\mathrm{S} / \mathrm{N}$ or Cal. No.: }} \\
\hline \multicolumn{3}{|c|}{ 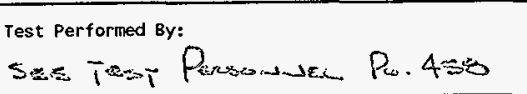 } & & & \\
\hline $\begin{array}{l}\text { Procedure No. } \\
\text { Initial/Date }\end{array}$ & Item & Value & $\begin{array}{l}\text { Required } \\
\text { Condition }\end{array}$ & $(A / R)$ & conment \\
\hline $\begin{array}{l}1.7 .6 \\
\text { G.E-1 }\end{array}$ & \multicolumn{5}{|c|}{$\begin{array}{l}\text { THIS SECTION IS ONLY FOR THE SHMS-E IN THE ANALYTICAL MODE. } \\
\text { IF NOT APPLICABLE, N/A STEPS AND TEST DIRECTOR WILL SIGN. }\end{array}$} \\
\hline & \multicolumn{3}{|c|}{ Test Director Signature } & Date & \\
\hline $\begin{array}{c}1.7 .6 .1 .1 \\
\end{array}$ & $\begin{array}{l}\text { VERIFY BKR } \\
\text { LINE UP }\end{array}$ & $N / A$ & $N / A$ & $\Delta$ & \\
\hline $\begin{array}{c}1.7 .6 .1 .2 \\
\text { coxin }\end{array}$ & $\begin{array}{l}\text { VERIFY VALVE } \\
\text { LINE UP }\end{array}$ & $N / A$ & $N / A$ & $A$ & \\
\hline $\begin{array}{l}1.7 .6 .1 .3 \\
0.1\end{array}$ & $\begin{array}{l}\text { VERIFY MON- } \\
\star 60 \text { INSTALL }\end{array}$ & $N / A$ & $N / A$ & $\boldsymbol{A}$ & \\
\hline 1.6.1.4 & $\begin{array}{l}\text { VERIFY NIT- } \\
\star 52 \text { INSTALL }\end{array}$ & $N / A$ & $N / A$ & $\Delta$ & \\
\hline 1.7 .6 .1 .5 & $\begin{array}{l}\text { CONNECT } \mathrm{H}_{2} \\
\text { CAL GAS }\end{array}$ & $N / A$ & $N / A$ & $\Delta$ & \\
\hline $\begin{array}{l}1.7 .6 .1 .6 \\
6+2 \times 1\end{array}$ & $\begin{array}{l}\text { CONNECT } \mathrm{CH}_{4} \\
\text { CAL GAS }\end{array}$ & $N / A$ & $N / A$ & 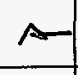 & \\
\hline 1.7 .6 .1 .7 & $\begin{array}{l}\text { CONNECT } \mathrm{NH}_{3} \\
\text { CAL GAS }\end{array}$ & $N / A$ & $N / A$ & 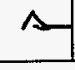 & \\
\hline \multicolumn{6}{|c|}{ Section 1.7.6.2 establishes the main and auxiliary loop flows. } \\
\hline$i^{1.7 .6 .2 .1}$ & CLOSE FU-1I & 7 & $\begin{array}{l}\text { MON-*60 } \\
\text { POWERED }\end{array}$ & $\hat{A}$ & \\
\hline $\begin{array}{l}1.7 .6 .2 .2 \\
6.2\end{array}$ & POWER PC $-* 60$ & $\sim$ & $\begin{array}{l}\text { ESTABLISH } \\
\text { SAMPLING } \\
\text { ROUTINE }\end{array}$ & $\Delta$ & \\
\hline $\begin{array}{l}1.7 .6 .2 .3 \\
\end{array}$ & CLOSE FU-12 & 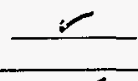 & $\begin{array}{l}\text { NIT }-* 52 \\
\text { POWERED }\end{array}$ & 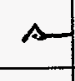 & \\
\hline $\begin{array}{l}1.7 .6 .2 .4 \\
6 \sin \end{array}$ & POWER PC-*70 & 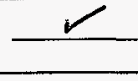 & $\begin{array}{l}\text { PC-*70 } \\
\text { BOOTED UP }\end{array}$ & $\mathcal{A}$ & \\
\hline $\begin{array}{l}1.7 .6 .2 .5 \\
\text { GEm }\end{array}$ & $\begin{array}{l}\text { CLOSE CB- } 3 / 4 \\
\text { ADJUST FLOW }\end{array}$ & $i .1$ & $\begin{array}{l}\text { FIT- } * 57 \\
1 \pm .1 \quad \mathrm{H}_{2} 0\end{array}$ & & \\
\hline
\end{tabular}


APPENDIX 0

TEST DATA SHEET

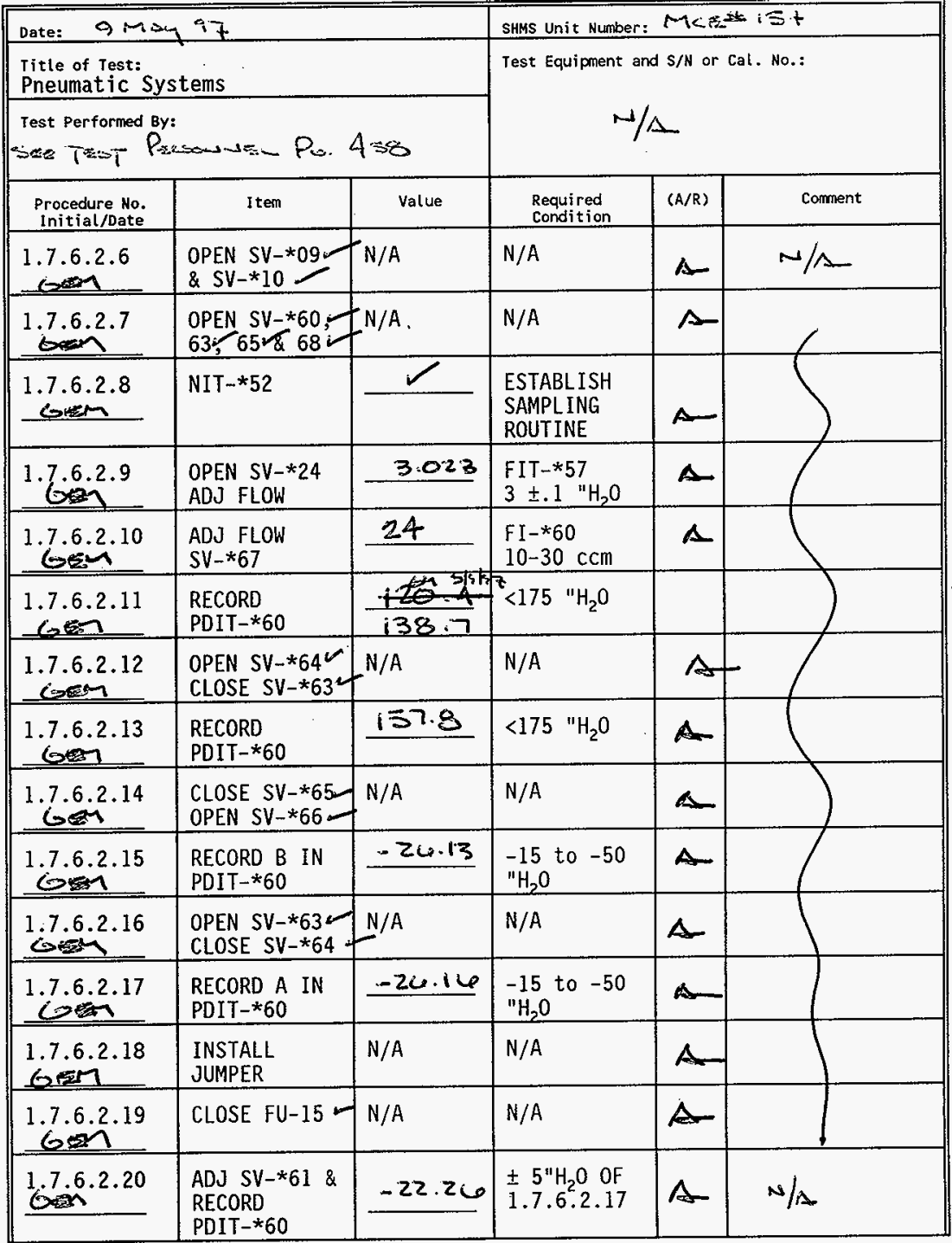


APPENDIX 0

TEST DATA SHEET

\begin{tabular}{|c|c|c|c|c|c|}
\hline \multicolumn{3}{|c|}{ Date: 9 May 97} & \multicolumn{3}{|c|}{ SHMS Unit Number: $M<\pi^{ \pm 1}$ ist } \\
\hline \multicolumn{3}{|c|}{$\begin{array}{l}\text { ritle of rest: } \\
\text { Pneumatic Systems }\end{array}$} & \multirow{2}{*}{\multicolumn{3}{|c|}{ 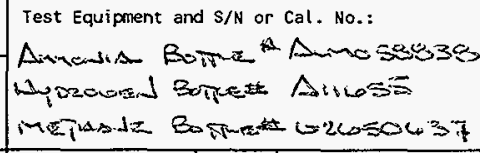 }} \\
\hline \multicolumn{3}{|c|}{ 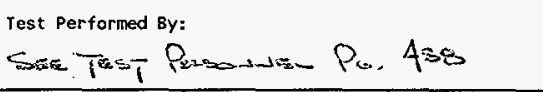 } & & & \\
\hline $\begin{array}{l}\text { Procedure No. } \\
\text { initial/Date }\end{array}$ & Item & value & $\begin{array}{l}\text { Required } \\
\text { Condition }\end{array}$ & $(A / R)$ & Comment \\
\hline $\begin{array}{l}1.7 .6 .2 .21 \\
\text { Gen }\end{array}$ & $\begin{array}{l}\text { CAL MON-*60 } \\
\text { CHANNEL A }\end{array}$ & $\begin{array}{l}1030.082 \\
1031.313 \\
1033.175\end{array}$ & $\begin{array}{l}\text { READINGS } \\
\text { WITHIN } \pm 5 \%\end{array}$ & 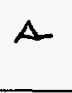 & $-1 / A$ \\
\hline $\begin{array}{l}1.7 .6 .2 .22 \\
6 x^{2}\end{array}$ & OPEN FU-15 & $N / A$ & $N / A$ & 1 & \\
\hline $\begin{array}{l}1.7 .6 .2 .23 \\
\text { ges.7 } \\
\end{array}$ & $\begin{array}{l}\text { REMOVE } \\
\text { JUMPER }\end{array}$ & N/A & N/A & 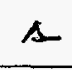 & \\
\hline $\begin{array}{l}1.7 .6 .2 .24 \\
\text { sim } \\
\end{array}$ & $\begin{array}{l}\text { OPEN SV-*64 } \\
\text { CLOSE SV-*63\% }\end{array}$ & $N / A$ & N/A & $\mathcal{A}$ & \\
\hline $\begin{array}{l}1.7 .6 .2 .25 \\
6 \mathrm{gm} \\
\end{array}$ & $\begin{array}{l}\text { INSTALL } \\
\text { JUMPER }\end{array}$ & $N / A$ & N/A & $A$ & \\
\hline $\operatorname{con}^{1.7 .6 .2 .26}$ & CLOSE FU-15 & $N / A$ & $N / A$ & A & \\
\hline $\begin{array}{l}1.7 .6 .2 .27 \\
6.92\end{array}$ & $\begin{array}{l}\text { ADJ SV }-* 62 \& \\
\text { RECORD } \\
\text { PDIT-*60 }\end{array}$ & -23.13 & $\begin{array}{l} \pm 5 " \mathrm{H}_{2} \mathrm{O} \text { OF } \\
1.7 .6 .2 .15\end{array}$ & A & \\
\hline$\frac{1.7 .6 .2 .28}{6 n^{-1}}$ & $\begin{array}{l}\text { CAL MON-*60 } \\
\text { CHANNEL B }\end{array}$ & $\frac{1.000}{1.999}$ & $\begin{array}{l}\text { READINGS } \\
\text { WITHIN } \pm 5 \%\end{array}$ & 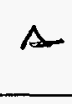 & \\
\hline $\begin{array}{l}1.7 .6 .2 .29 \\
\underline{6 m}\end{array}$ & OPEN FU-15 & N/A & N/A & $\triangle$ & \\
\hline $\begin{array}{l}1.7 .6 .2 .30 \\
\text { Ger }\end{array}$ & $\begin{array}{l}\text { REMOVE } \\
\text { JUMPER }\end{array}$ & N/A & $N / A$ & $\Delta$ & \\
\hline 1.7 .6 .2 .31 & $\begin{array}{l}\text { CLOSE SV-*64 } \\
\text { OPEN SV-*70 }\end{array}$ & $N / A$ & $N / A$ & $A$ & \\
\hline $\begin{array}{l}1.7 .6 .2 .32 \\
\end{array}$ & $\begin{array}{l}\text { RECORD } \\
\text { PDIT }-* 60 \\
\text { NIT } * 52 \text { IN } \\
\end{array}$ & -14.19 & $\begin{array}{l}0 \text { to }-30 \\
\text { " } \mathrm{H}_{2} \mathrm{O}\end{array}$ & $\Delta$ & \\
\hline $\begin{array}{l}1.7 .6 .2 .33 \\
\end{array}$ & $\begin{array}{l}\text { INSTALL } \\
\text { JUMPER }\end{array}$ & $N / A$ & $N / A$ & $A$ & \\
\hline $\begin{array}{c}1.6 .2 .34 \\
6 \mathrm{en}\end{array}$ & CLOSE FU-15 & N/A & $N / A$ & $A$ & \\
\hline
\end{tabular}


TEST DATA SHEET

\begin{tabular}{|c|c|c|c|c|c|}
\hline \multicolumn{3}{|c|}{ Date: $15 \mathrm{MAY} 97$} & \multicolumn{3}{|c|}{ SHMS Unit Number: McE些 ist } \\
\hline \multicolumn{3}{|c|}{$\begin{array}{l}\text { ritle of rest: } \\
\text { Pneumatic Systems }\end{array}$} & \multirow{2}{*}{\multicolumn{3}{|c|}{ 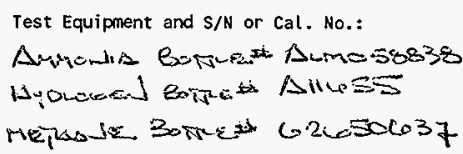 }} \\
\hline \multicolumn{3}{|c|}{ 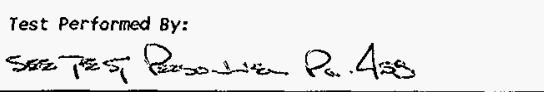 } & & & \\
\hline $\begin{array}{l}\text { Procedure No. } \\
\text { Initial/Date }\end{array}$ & Item & Value & $\begin{array}{l}\text { Required } \\
\text { Condition } \\
\end{array}$ & $(A / R)$ & Comnent \\
\hline $\begin{array}{l}1.7 .6 .2 .35 \\
\operatorname{cosin}^{2} \\
\end{array}$ & $\begin{array}{l}\text { ESTABLISH } \\
\text { BYPASS FLOW }\end{array}$ & 1.7 & $\begin{array}{l}\text { FIV }-* 70 \\
1.5 \pm .2 \mathrm{cfh}\end{array}$ & $A$ & $1 / 1$ \\
\hline$\frac{1.7 .6 .2 .36}{\mathrm{ges}^{-1}}$ & $\begin{array}{l}\text { ADJ SV }-* 71 \\
\text { NIT-*52 } \\
\text { SAMPLE IN }\end{array}$ & -10.8 & $\begin{array}{l} \pm 5 \quad \mathrm{H}_{2} \mathrm{O} \text { OF } \\
1.7 .6 .2 .32\end{array}$ & $A$ & \\
\hline$\frac{1.7 .6 .2 .37}{1}$ & $\begin{array}{l}\text { RECORD } \\
\text { NIT-*52 } \\
\text { SAMPLES }\end{array}$ & $\frac{36.7}{36.8}$ & $\begin{array}{l}\text { READINGS } \\
\text { WITHIN } \pm 5 \%\end{array}$ & $\Delta$ & \\
\hline $\begin{array}{r}1.7 .6 .2 .38 \\
\end{array}$ & OPEN FU-15 & N/A & $N / A$ & $A$ & \\
\hline 1.7 .6 .2 .39 & $\begin{array}{l}\text { REMOVE } \\
\text { JUMPER }\end{array}$ & N/A & $N / A$ & $\Lambda$ & \\
\hline \multicolumn{6}{|c|}{ Section 1.7 .7 provides for test system shutdown. } \\
\hline $\begin{array}{l}1.7 .7 .1 \\
\end{array}$ & $\begin{array}{l}\text { DISCONNECT } \\
\mathrm{H}_{2} \text { CAL GAS }\end{array}$ & N/A & N/A & A & \\
\hline $\begin{array}{r}1.7 .7 .2 \\
-60.7 \\
\end{array}$ & $\begin{array}{l}\text { DISCONNECT } \\
\mathrm{CH}_{6} \text { CAL GAS } \\
\end{array}$ & N/A & N/A & A & \\
\hline $\begin{array}{r}1.7 .7 .3 \\
\mathrm{Gxm}\end{array}$ & $\begin{array}{l}\text { DISCONNECT } \\
\mathrm{NH}_{3} \text { CAL GAS }\end{array}$ & N/A & N/A & $\Delta$ & \\
\hline $\begin{array}{l}1.7 .7 .4 \\
\end{array}$ & $\begin{array}{l}\text { SECURE \& } \\
\text { TURN OFF } \\
\text { ANA. INST.S }\end{array}$ & N/A & $N / A$ & $A$ & \\
\hline $\begin{array}{l}1.7 .7 .5 \\
8 x^{5}-1 \\
\end{array}$ & $\begin{array}{l}\text { DISCONNECT } \\
\mathrm{N}_{2} \text { CARRIER } \\
\end{array}$ & $N / A$ & $N / A$ & A & \\
\hline $\begin{array}{l}1.7 .7 .6 \\
\text { sem }\end{array}$ & $\begin{array}{l}\text { DISCONNECT } \\
\text { He CARRIER }\end{array}$ & N/A & N/A & $\triangle$ & \\
\hline $\begin{array}{l}1.7 .7 .7 \\
\text { sen }\end{array}$ & $\begin{array}{l}\text { OPEN ALL } \\
\text { BREAKERS \& } \\
\text { FUSES } \\
\end{array}$ & N/A & $N / A$ & $A$ & \\
\hline $\begin{array}{c}1.7 .7 .8 \\
\text { G.in } \\
\end{array}$ & $\begin{array}{l}\text { CLOSE LISTED } \\
\text { SYS. VALVES }\end{array}$ & N/A & N/A & $\Delta$ & 7 \\
\hline
\end{tabular}


APPENDIX 0

PAGE $0-28$
HNF-SD-WM-ATR-191
REV.

ReV. 0
Page 484

TEST DATA SHEET

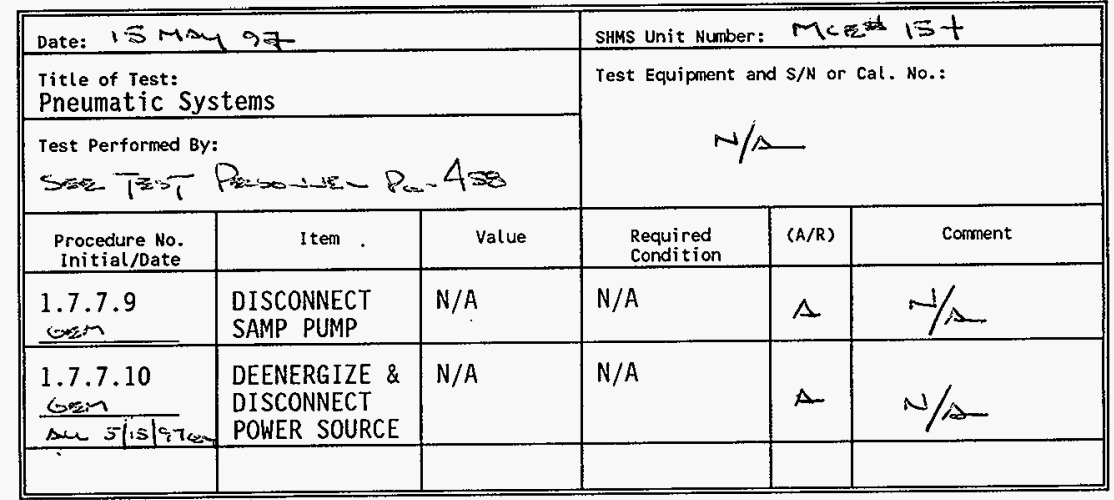

Test Witness/Review:

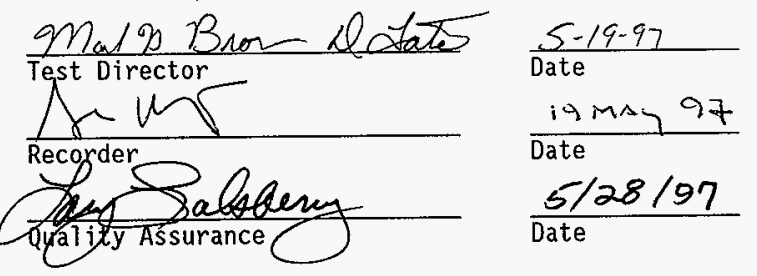


APPENDIX 0

PAGE 0-29
HNF-SD- HM-ATR- 191

Rev. 0

TEST EXCEPTION SHEET

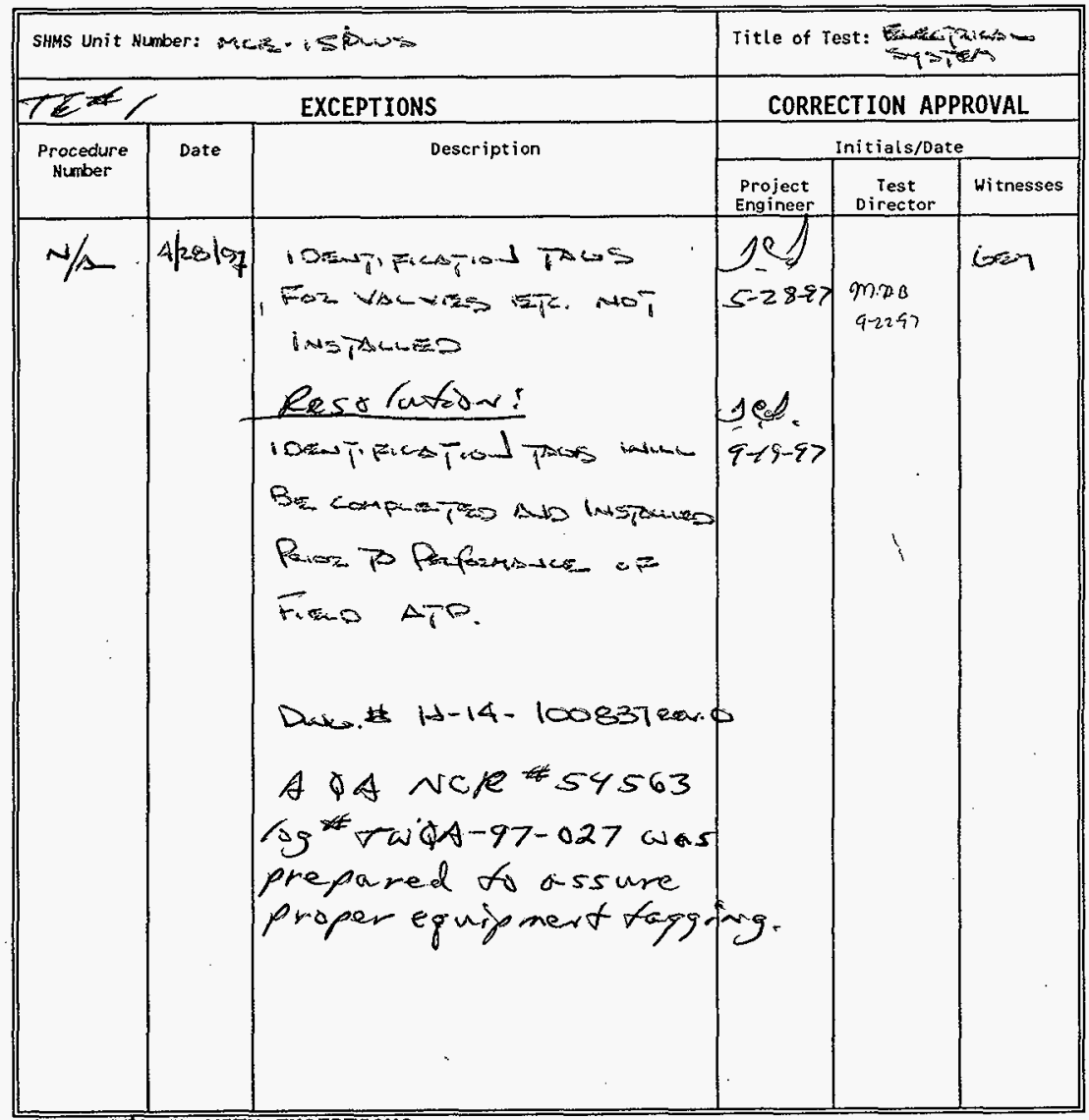

TEST APPROVED WITH EXCEPTIONS

ithenids

WHG Project Engineer

$\frac{5-29-97}{\text { Date }}$

Recorder

$\frac{28 \text { May } 97}{\text { Date }}$

$5 / 28 / 97$ 
APPENDIX 0

PAGE 0-30
HNF-SD-WW-ATR- 191
Rev. 0
Page 486

TEST LOG

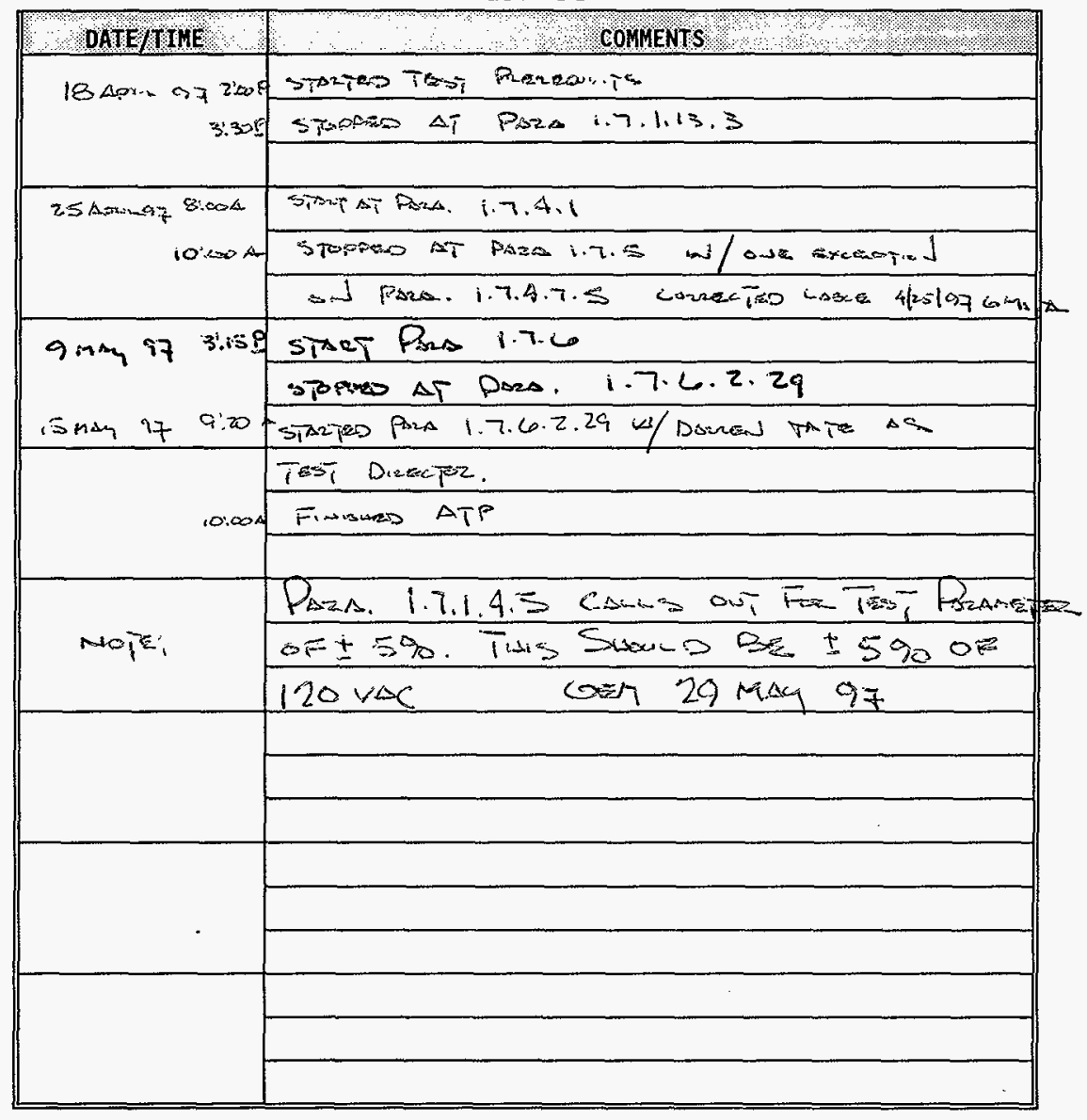


Rev. 0

Page 487

APPENDIX P 
TEST EXECUTION SHEET

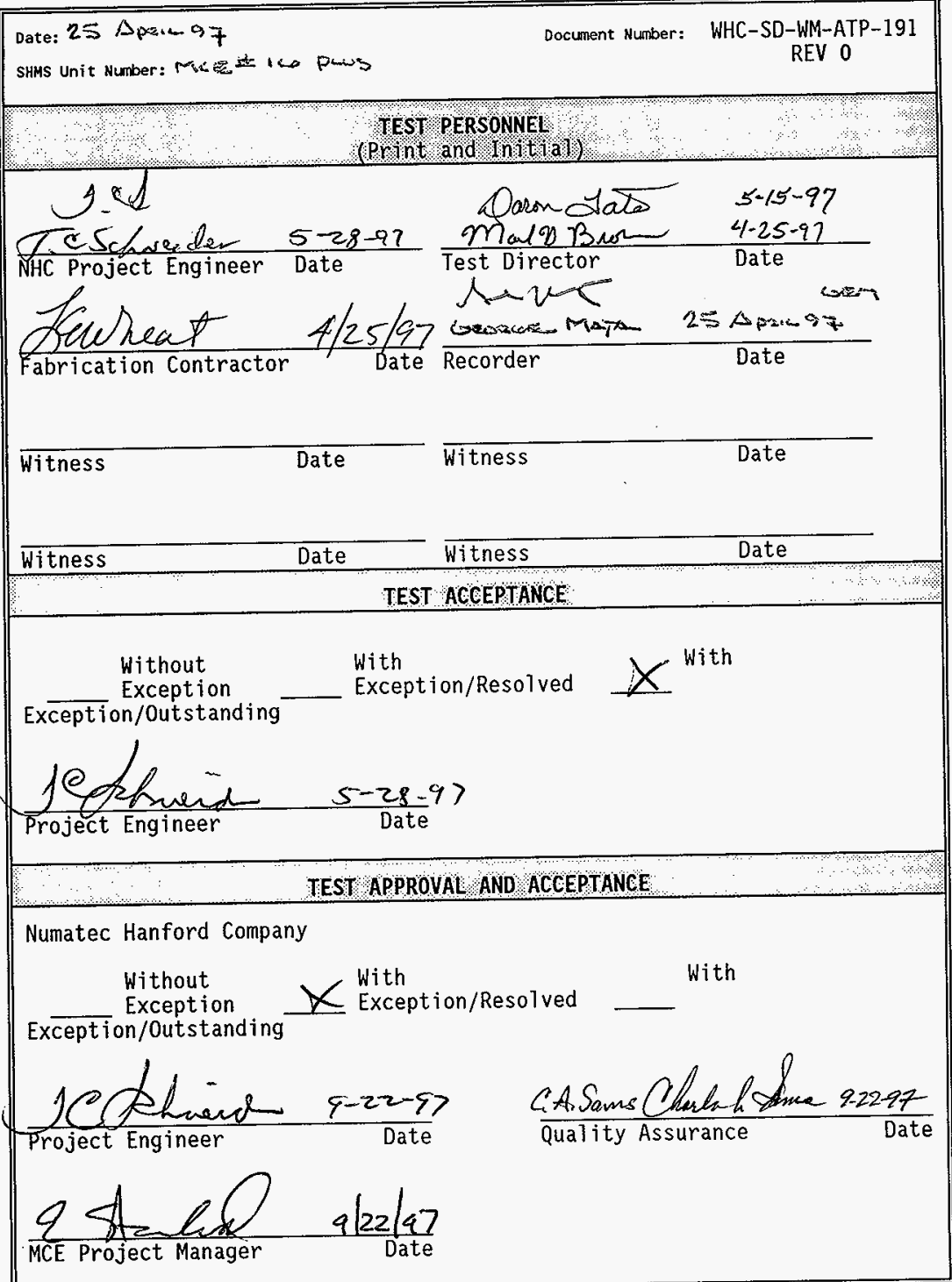


SHMS Unit Number: Mces 120 Pus

\section{PREREQUISITES AND INITIAL TEST CONDITIONS}

The following conditions shall exist at the start of the acceptance testing. Initial and date to verify that each of the following items have been accomplished.

s compliance with design.

$\cos 4: 3+13 z$ Continuity and megger tests have been performed on portions of the electrical and instrument systems being tested, as required.

Len andistis Leak tests on the pneumatic systems have been performed.

The following circuit breakers and fuses are installed per the specified size and are open

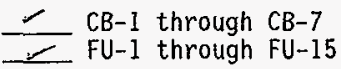

allos test instruments have a currently valid calibration stamp attached that indicates a calibration traceable to the National Institute of Standards and Technology.

calosiz The following process and control instrument systems have been initially configured and aligned for proper operation.

FIT-*57, FSL-*57, NIT-*54, NIT-*55, (NR-*54), PDIT-*60, TIC-*50, TIC-*56, TIS-*62, YYC $* 01$ NOTE: NR-*54 is not used in the ANALYTICAL configuration.

Ge, $4 \mid \omega ; a_{y}$ Personnel responsible for directing and witnessing the performance of the tests described in this ATP have read and understand appropriate certified vendor information (CVI) pertaining to the operation of the equipment to be tested.

isen of 2 fay CLOSE a1\} system manual valves with the exception of the following:

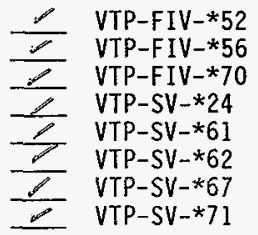

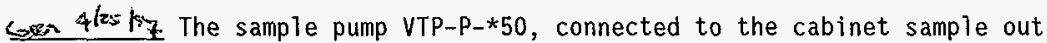
port, but vented to atmosphere for testing. 
$S_{2}-425 h_{2} 208$ Vac 1 phase 15-20 ampere temporary power source has been connected, but not energized, to the appropriate TB1 terminals per H-14-100838.

Verify by signature and date that all prerequisites have been met.

$\frac{\text { Mad op } / 3 \text { nen }}{\text { Test Director Signature }} \frac{4-25.97}{\text { Date }}$


TEST DATA SHEET

\begin{tabular}{|c|c|c|c|c|c|}
\hline \multicolumn{3}{|c|}{ Date: $25 \Delta$ sow- 97} & \multicolumn{3}{|c|}{ SHMS Unit Number: MCEF $16+$} \\
\hline \multicolumn{3}{|c|}{$\begin{array}{l}\text { Title of Test: } \\
\text { Electrical Systems }\end{array}$} & \multirow{2}{*}{\multicolumn{3}{|c|}{ 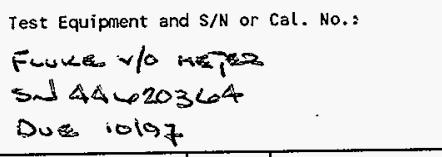 }} \\
\hline \multicolumn{3}{|c|}{ 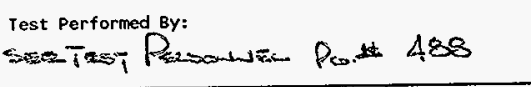 } & & & \\
\hline $\begin{array}{l}\text { Procedure No. } \\
\text { Initial/Date }\end{array}$ & - Item & value & $\begin{array}{l}\text { Required } \\
\text { Condition } \\
\end{array}$ & $(A / R)$ & Comment \\
\hline \multicolumn{6}{|c|}{ Section 1.7.1.1 verifies the system Mains Power. } \\
\hline $\operatorname{sen}^{1.7 .1 .1}$ & Resistance & .01 & $<1 \mathrm{ohm}$ & A & \\
\hline $\begin{array}{l}1.7 .1 .1 .2 \\
\end{array}$ & $\begin{array}{l}\text { Temp power } \\
208 \mathrm{Vac}\end{array}$ & $N / A$ & Energized & $\Delta$ & \\
\hline $\operatorname{con}^{1.7 .1 .1 .3}$ & $\begin{array}{l}\text { Line Vac: } \\
\text { LI-L2 } \\
\text { L1-Gnd } \\
\text { L2-Gnd } \\
N-\text { Gnd }\end{array}$ & $\frac{\frac{207.8}{118.7}}{\frac{118.8}{.46}}$ & $\begin{array}{l}L 1-L 2: 208 \\
\operatorname{Vac}+10 /-1 \% \\
\mathrm{~L} 1, \mathrm{~L} 2: 120 \\
\mathrm{Vac} \pm 5 \% \\
\mathrm{~N}: 0 \mathrm{Vac}\end{array}$ & $A$ & \\
\hline \multicolumn{6}{|c|}{ Section 1.7.1.2 verifies the enclosure HVAC system. } \\
\hline $\begin{array}{l}1.7 .1 .2 .1 \\
6 x=15\end{array}$ & AC Adjusted & $N / A$ & $N / A$ & $\Delta$ & \\
\hline $\begin{array}{l}1.7 .1 .2 .2 \\
\end{array}$ & $\begin{array}{l}\text { CLOSE } \\
\text { CB- } 1 / 2 \\
\end{array}$ & $N / A$ & $\mathrm{~N} / \mathrm{A}$ & $\Delta$ & \\
\hline $\begin{array}{l}1.7 .1 .2 .3 \\
6+2 \\
\end{array}$ & Heater ON & $\checkmark$ & Heater ON & A & \\
\hline 1.7 .1 .2 .4 & AC Adjusted & $\mathrm{N} / \mathrm{A}$ & $N / A$ & $\Delta$ & \\
\hline 1.7 .1 .2 .5 & $\begin{array}{l}\text { Heater OFF } \\
\text { Cooling ON }\end{array}$ & $\frac{1}{2}$ & $\begin{array}{l}\text { Heater OFF } \\
\text { Cooling ON } \\
\end{array}$ & $A$ & \\
\hline $\operatorname{son}^{1.7 .1 .2 .6}$ & AC Adjusted & $N / A$ & $N / A$ & $\Delta$ & \\
\hline $\operatorname{con}^{1.7 .1 .2 .7}$ & $\begin{array}{l}\text { OPEN } \\
\mathrm{CB}-1 / 2 \\
\end{array}$ & N/A & N/A & A & - \\
\hline \multicolumn{6}{|c|}{ Section 1.7.1.3 verifies the sample pump is properly connected. } \\
\hline $\begin{array}{c}1.7 .1 .3 .1 \\
\cos \end{array}$ & $\begin{array}{l}\text { CLOSE } \\
\text { CB-3/4 }\end{array}$ & $N / A$ & $N / A$ & A- & $\omega / \Lambda$ \\
\hline 1.7 .1 .3 .2 & $\begin{array}{l}\text { VTP-P } * \text { *50 } \\
\text { ON }\end{array}$ & $\leftarrow$ & $\begin{array}{l}\text { VTP-P-*50 } \\
\text { ON }\end{array}$ & s. & $s / \Delta$ \\
\hline
\end{tabular}


APPENDIX $P$

HNF-SD-WM-ATR- 191.
Rev 0
Page 492

PAGE PD

TEST DATA SHEET

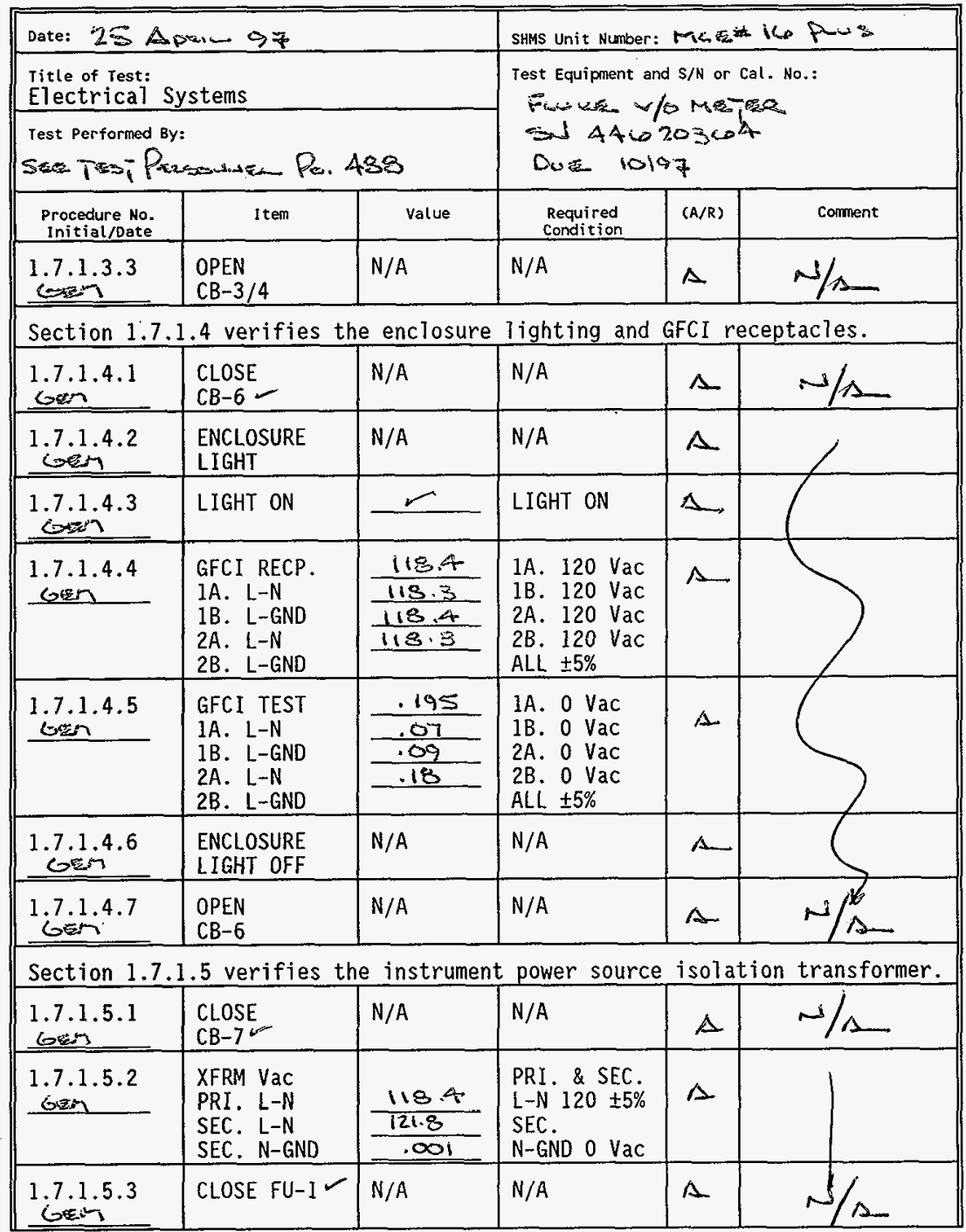

A. Alzalor 
TEST DATA SHEET

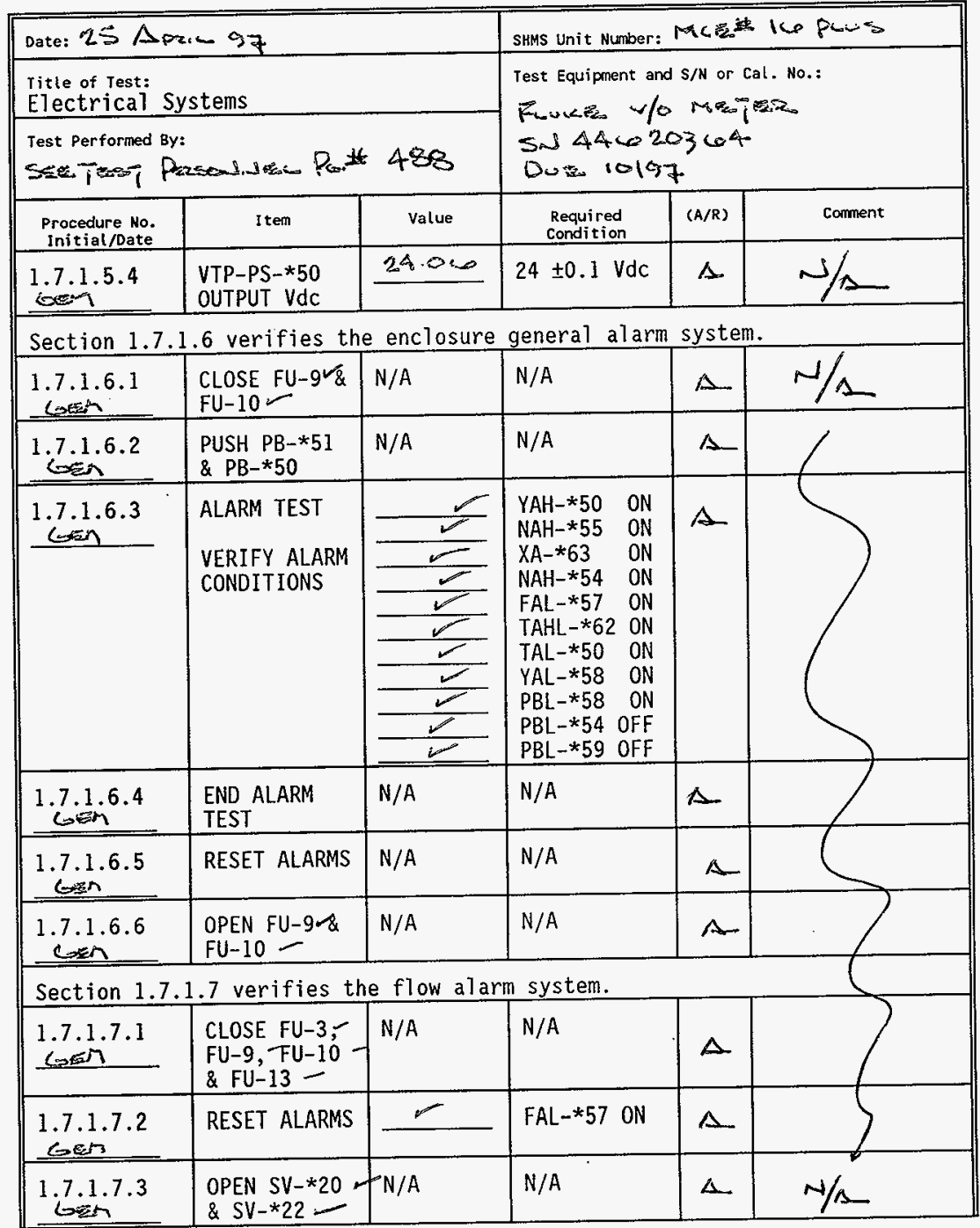


TEST DATA SHEET

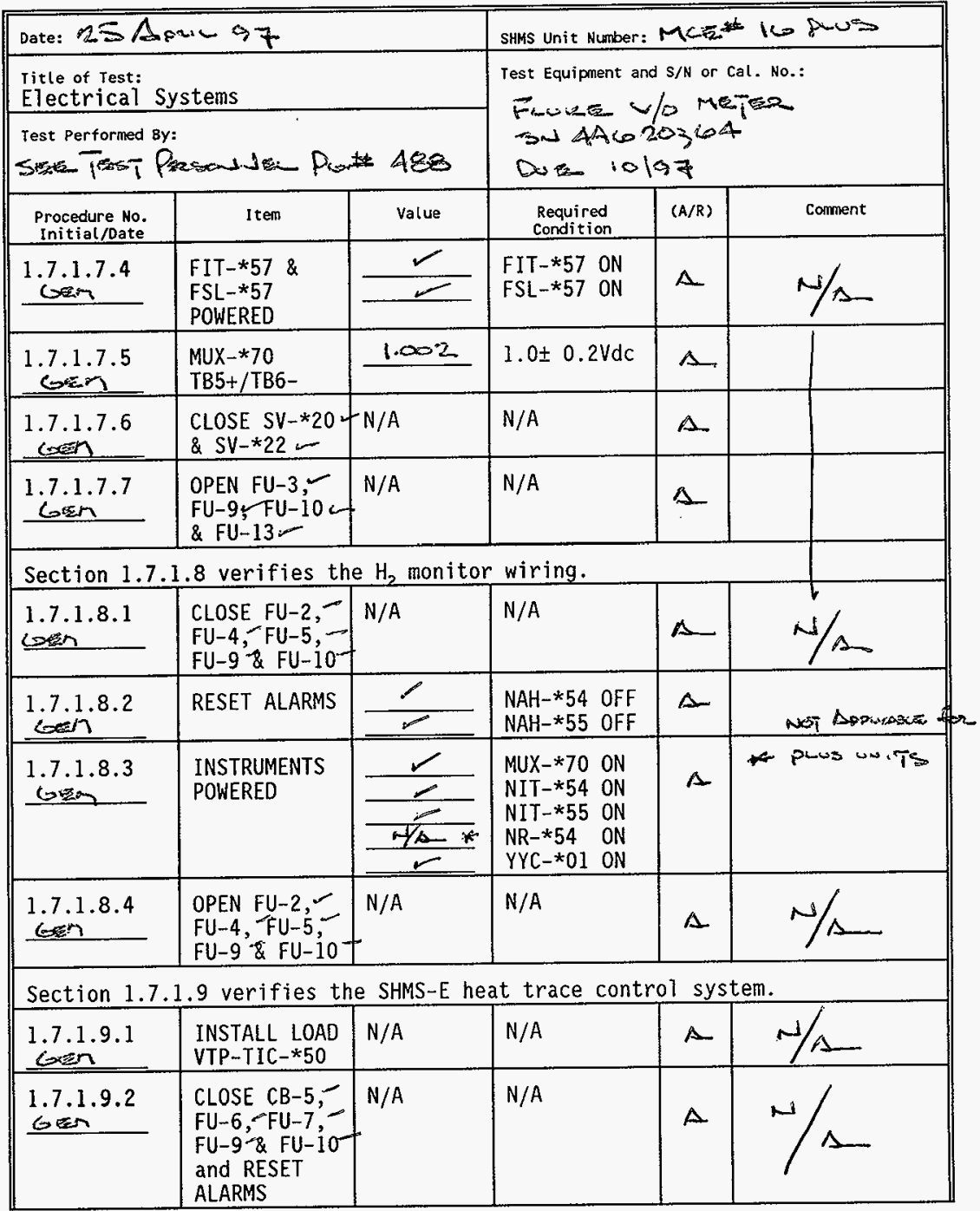

Gem Ain 4/25/iz 
TEST DATA SHEET

\begin{tabular}{|c|c|c|c|c|c|}
\hline \multicolumn{3}{|c|}{ Date: $2 \leq A$ prat -97} & \multicolumn{3}{|c|}{ 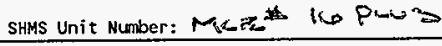 } \\
\hline \multicolumn{3}{|c|}{$\begin{array}{l}\text { Title of Test: } \\
\text { Electrical Systems }\end{array}$} & \multirow{2}{*}{\multicolumn{3}{|c|}{ Test Equipment and $\mathrm{S} / \mathrm{N}$ or Cal. No.: }} \\
\hline \multicolumn{3}{|c|}{$\begin{array}{l}\text { Test Performed By: } \\
\text { Sez Torm, }\end{array}$} & & & \\
\hline $\begin{array}{l}\text { Procedure No. } \\
\text { Initial/Date } \\
\end{array}$ & Item & Value & $\begin{array}{c}\text { Required } \\
\text { Condition }\end{array}$ & $(A / R)$ & Comment \\
\hline 1.7 .1 .9 .3 & $\begin{array}{l}\text { TIC-*50/\& } \\
\text { TIC-*56 } \\
\text { NOMINAL TEMP }\end{array}$ & $\frac{r}{r}$ & $\begin{array}{l}\text { TIC-*50 col } \\
\text { TIC-*56 SO } \\
\text { TEMPERATURE }\end{array}$ & $\Delta$ & \\
\hline $\begin{array}{l}1.7 .1 .9 .4 \\
\text { exen }\end{array}$ & $\begin{array}{l}\text { ADJ TIC-*50- } \\
\& \text { TIC }-* 56 \\
\text { SP2 \& RESET } \\
\text { ALARMS }\end{array}$ & $N / A$ & $N / A$ & A & \\
\hline $\begin{array}{l}1.7 .1 .9 .5 \\
\mathrm{exp}^{2}\end{array}$ & $\begin{array}{l}\text { ADJ TIC }-* 50 \\
\text { SP } 1\end{array}$ & $N / A$ & $N / A$ & $\Delta$ & \\
\hline $\begin{array}{l}1.7 .1 .9 .6 \\
\sin \end{array}$ & $\begin{array}{l}\text { ADJ TIC-*50 } \\
\text { SP } 2\end{array}$ & $N / A$ & $N / A$ & $\Delta$ & \\
\hline $\begin{array}{l}1.7 .1 .9 .7 \\
\text { con }\end{array}$ & $\begin{array}{l}\text { TAL } * 50 \& \\
\text { HORN ACTIVE }\end{array}$ & $\frac{r}{2}$ & $\begin{array}{l}\text { TAL }-\star 50 \text { ON } \\
\text { HORN ON } \\
\text { ACKNOWLEDGE }\end{array}$ & A & \\
\hline $\begin{array}{l}1.7 .1 .9 .8 \\
\operatorname{con}\end{array}$ & $\begin{array}{l}\text { TIC }-* 50 \\
\text { OPERATES }\end{array}$ & $r$ & $\begin{array}{l}\text { TIC }-* 50 \\
\text { OPERATES }\end{array}$ & $\Delta$ & \\
\hline $\begin{array}{l}1.7 .1 .9 .9 \\
\end{array}$ & $\begin{array}{l}\text { ADJ TIC }-* 50 \\
S P{ }_{2}\end{array}$ & $N / A$ & $N / A$ & $A$ & \\
\hline 1.7 .1 .9 .10 & RESET ALARM & 工 & $\mathrm{TAL}-\star 50$ OFF & $\Delta$ & \\
\hline 1.7 .1 .9 .11 & $\begin{array}{l}\text { ADJ TIC } * 50 \\
S P 1 \& S P 2\end{array}$ & $N / A$ & $N / A$ & A & \\
\hline $\begin{array}{l}1.7 .1 .9 .12 \\
\end{array}$ & $\begin{array}{l}\text { OPEN CB-5 \& } \\
\text { REMOVE LOAD }\end{array}$ & $N / A$ & N/A & $A$ & \\
\hline $\begin{array}{l}1.7 .1 .9 .13 \\
\text { sen }\end{array}$ & $\begin{array}{l}\text { INSTALL LOAD } \\
\text { VTP-TIC-*56- }\end{array}$ & N/A & N/A & A & \\
\hline 1.7 .1 .9 .14 & CLOSE CB-5 & N/A & $\mathrm{N} / \mathrm{A}$ & A & \\
\hline $\begin{array}{l}1.7 .1 .9 .15 \\
\text { Gern }\end{array}$ & $\begin{array}{l}\text { ADJ TIC-*56 } \\
\text { SP } 1\end{array}$ & $N / A$ & N/A & $\Delta$ & \\
\hline $\begin{array}{l}1.7 .1 .9 .16 \\
\text { gem }\end{array}$ & $\begin{array}{l}\text { ADJ TIC }-* 56 \\
\text { SP } 2\end{array}$ & N/A & $\mathrm{N} / \mathrm{A}$ & $\Delta$ & \\
\hline
\end{tabular}


TEST DATA SHEET

\begin{tabular}{|c|c|c|c|c|c|}
\hline \multicolumn{3}{|c|}{ Date: $23 \Delta 0 x-97$} & \multicolumn{3}{|c|}{ ShMS Unit Number: MC的 } \\
\hline \multicolumn{3}{|c|}{$\begin{array}{l}\text { Title of rest: } \\
\text { Electrical Systems }\end{array}$} & \multirow{2}{*}{\multicolumn{3}{|c|}{ Test Equipment and $\mathrm{S} / \mathrm{N}$ or Cal. No.: }} \\
\hline \multicolumn{3}{|c|}{ 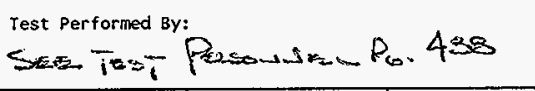 } & & & \\
\hline $\begin{array}{l}\text { Procedure No. } \\
\text { Initial/Date }\end{array}$ & Item & Value & $\begin{array}{l}\text { Required } \\
\text { Condition }\end{array}$ & $(A / R)$ & Comment \\
\hline$\frac{1.7 .1 .9 .17}{\operatorname{sen}}$ & $\begin{array}{l}\text { TAL } * 50 \& \& \\
\text { HORN ACTIVE }\end{array}$ & $\frac{2}{\square}$ & $\begin{array}{l}\text { TAL }-* 50 \text { ON } \\
\text { HORN ON } \\
\text { ACKNOWLEDGE }\end{array}$ & $\Delta$ & \\
\hline $\begin{array}{l}1.7 .1 .9 .18 \\
\end{array}$ & $\begin{array}{l}\text { TIC }-* 56 \\
\text { OPERATES } \\
\end{array}$ & 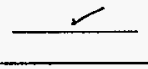 & $\begin{array}{l}\text { TIC }-* 56 \\
\text { OPERATES }\end{array}$ & $\Delta$ & \\
\hline $\begin{array}{l}1.7 .1 .9 .19 \\
\end{array}$ & $\begin{array}{l}\text { ADJ TIC-*56 } \\
\text { SP 2 }\end{array}$ & $N / A$ & $N / A$ & $A$ & \\
\hline 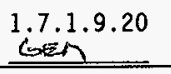 & RESET ALARM & $\sim$ & TAL- $\star 50$ OFF & s. & \\
\hline $\begin{array}{l}1.7 .1 .9 .21 \\
\text { Gen }\end{array}$ & $\begin{array}{l}\text { ADJ TIC-*56 } \\
\text { SP } 1 \& \text { SP } 2 \\
\end{array}$ & $N / A$ & N/A & $s$ & \\
\hline $\begin{array}{l}1.7 .1 .9 .22 \\
\end{array}$ & $\begin{array}{l}\text { OPEN CB-5-5 } \\
\text { FU-6-\& FU-7 }\end{array}$ & $N / A$ & N/A & $\Delta$ & \\
\hline $\begin{array}{l}1.7 .1 .9 .23 \\
\end{array}$ & $\begin{array}{l}\text { REMOVE LOAD } \\
\text { TIC }-* 56\end{array}$ & $N / A$ & $N / A$ & $\wedge$ & \\
\hline $\cosh ^{1.7 .1 .9 .24}$ & $\begin{array}{l}\text { CLOSE FU-8 } \\
\text { TIS }-* 62 \\
\text { NOMINAL TEMP } \\
\end{array}$ & 600 & $\begin{array}{l}\text { TIS }-\star 62 \\
\text { TEMP }\end{array}$ & $\triangle$ & \\
\hline $\begin{array}{l}1.7 .1 .9 .25 \\
\end{array}$ & $\begin{array}{l}\text { ADJ TIS }-* 62 \\
\text { SP } 1\end{array}$ & N/A & N/A & A. & \\
\hline $\begin{array}{l}1.7 .1 .9 .26 \\
\text { geam }\end{array}$ & $\begin{array}{l}\text { ADJ TIS-*62 } \\
\text { SP 2 }\end{array}$ & $N / A$ & $N / A$ & 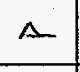 & \\
\hline$\underbrace{1.7 .1 .9 .27}_{\text {ixin }}$ & RESET ALARMS & 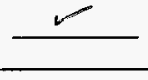 & $\begin{array}{l}\text { TAHL-*62 } \\
\text { OFF }\end{array}$ & A & \\
\hline $\begin{array}{c}1.7 .1 .9 .28 \\
\end{array}$ & $\begin{array}{l}\text { ADJ TIS-*62 } \\
\text { SP 2 }\end{array}$ & $N / A$ & $N / A$ & $\Delta$ & \\
\hline $\cos ^{1.7 .1 .9 .29}$ & $\begin{array}{l}\text { TAHL-*62 \& } \\
\text { HORN ACTIVE }\end{array}$ & $\stackrel{r}{\check{L}}$ & $\begin{array}{l}\text { TAHL-*62 ON } \\
\text { HORN ON } \\
\text { ACKNOWLEDGE } \\
\end{array}$ & $A$ & \\
\hline 1.7 .1 .9 .30 & $\begin{array}{l}\text { ADJ TIS }-* 62 \\
\text { SP } 2\end{array}$ & $N / A$ & $N / A$ & A & \\
\hline
\end{tabular}


APPENDIX $P$

HNF-SD-LM-ATR-191
Rev. 0
Page 497

TEST DATA SHEET

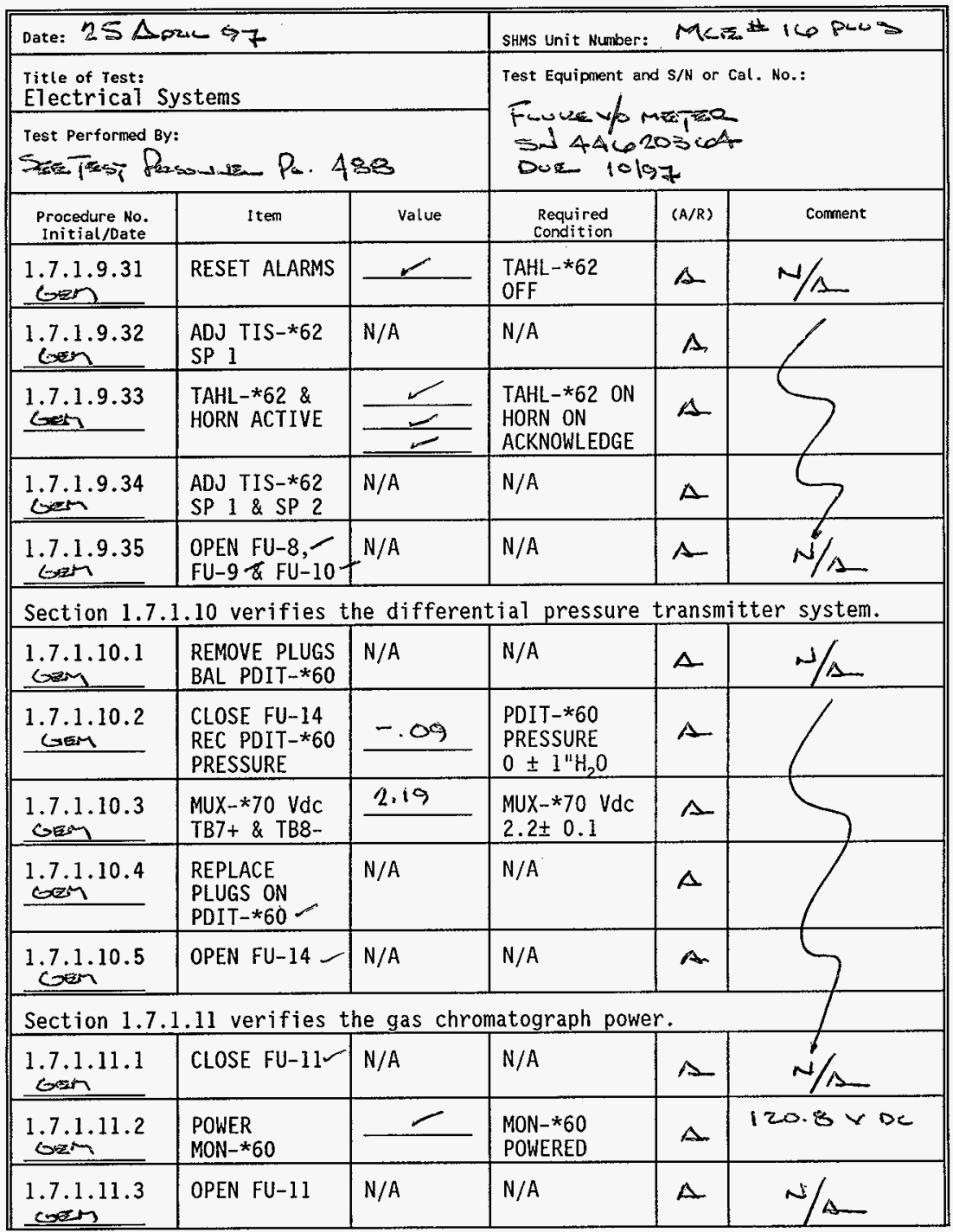


APPENDIX $P$

PAGE $P-C$
HNF-SD-WH-ATR- 191
ReV. 0
Page 498

TEST DATA SHEET

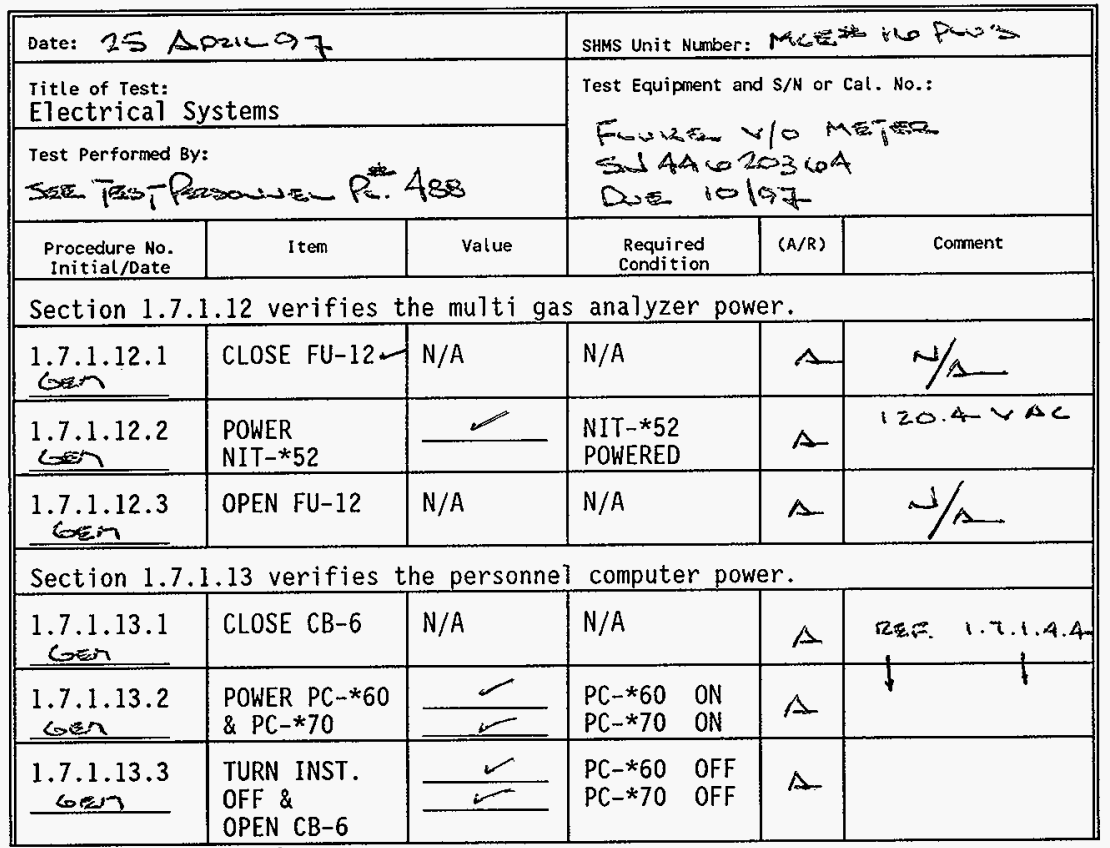

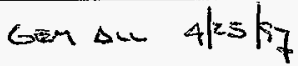


APPENDIX $P$

PAGE $P-13$

$$
\begin{array}{r}
\text { HNF-SD-WM-ATR- } 191 \\
\text { Rev. } 0 \\
\text { Page } 499
\end{array}
$$

TEST DATA SHEET

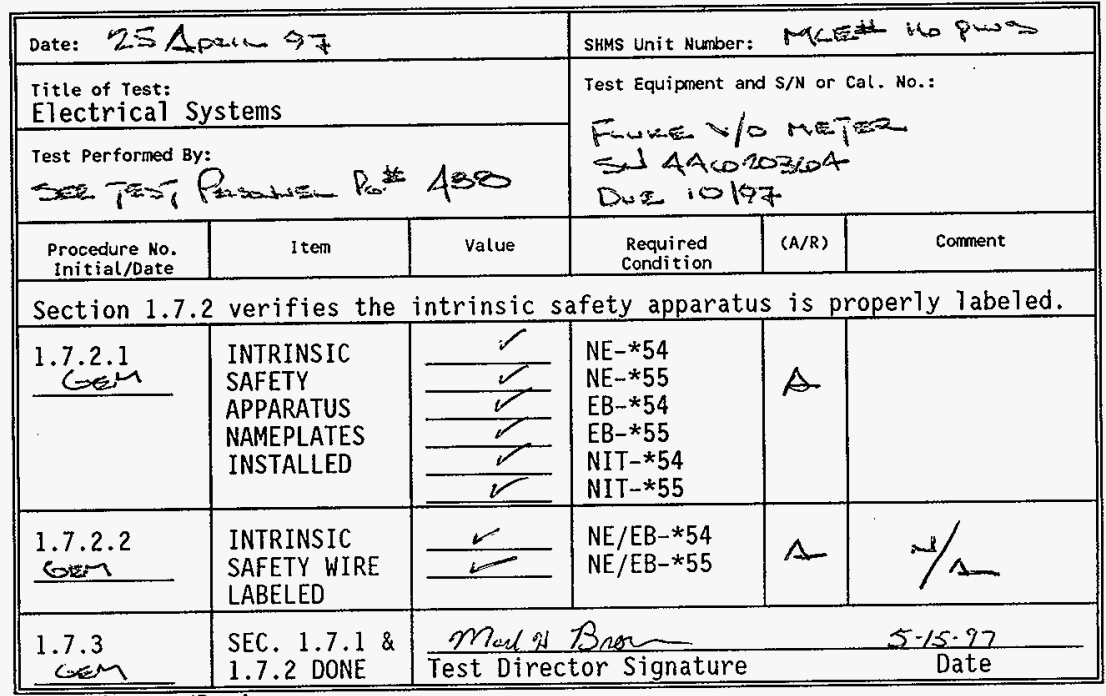

Test Witness/Review:

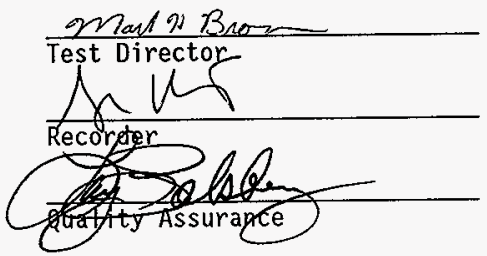

$$
\begin{aligned}
& \frac{5-15.97}{\text { Date }} \\
& 5 / 19197 \\
& \text { Date } \\
& 5 / 28 / 97 \\
& \text { Date }
\end{aligned}
$$


TEST DATA SHEET

\begin{tabular}{|c|c|c|c|c|c|}
\hline \multicolumn{3}{|c|}{ Date: $25 \Delta$ Arat-97 } & \multicolumn{3}{|c|}{ 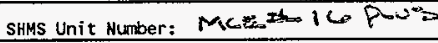 } \\
\hline \multicolumn{3}{|c|}{$\begin{array}{l}\text { Title of rest: } \\
\text { Pneumatic Systems }\end{array}$} & \multirow{2}{*}{\multicolumn{3}{|c|}{ 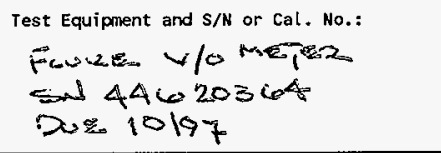 }} \\
\hline \multicolumn{3}{|c|}{ 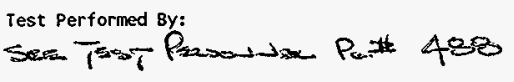 } & & & \\
\hline $\begin{array}{l}\text { Procedure No. } \\
\text { Initial/Date }\end{array}$ & Item & value & $\begin{array}{l}\text { Required } \\
\text { Condition }\end{array}$ & $(A / R)$ & ent \\
\hline \multicolumn{6}{|c|}{ Section 1.7.4.1 provides the pneumatic test section initial conditions. } \\
\hline $\begin{array}{l}1.7 .4 .1 .1 \\
\operatorname{cosin}\end{array}$ & $\begin{array}{l}\text { INITIAL } \\
\text { CONDITIONS }\end{array}$ & 2 & $\begin{array}{l}\text { BKR \& FUSE } \\
\text { LINE UP }\end{array}$ & $\Delta$ & \\
\hline $\begin{array}{l}1.7 .4 .1 .2 \\
\cos \end{array}$ & $\begin{array}{l}\text { INITIAL } \\
\text { CONDITIONS }\end{array}$ & 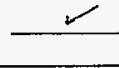 & $\begin{array}{l}\text { VALVE LINE } \\
\text { UP }\end{array}$ & A & \\
\hline G.7.4.1.3 & $\begin{array}{l}\text { DISCONNECT } \\
1 / 16^{\prime \prime} \text { TUBING } \\
\text { ON MON-*60 } \\
\end{array}$ & 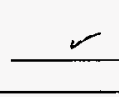 & $\begin{array}{l}\text { TUBING } \\
\text { CONFIGURED }\end{array}$ & $\Delta$ & \\
\hline $\begin{array}{l}1.7 .4 .1 .4 \\
\text { cosen }\end{array}$ & $\begin{array}{l}\text { DISCONNECT } \\
\text { TUBING ON } \\
\text { NIT }-\star 52\end{array}$ & $\zeta$ & $\begin{array}{l}\text { TUBING } \\
\text { CONFIGURED }\end{array}$ & 今 & \\
\hline $\begin{array}{l}1.7 .4 .1 .5 \\
\cos 20\end{array}$ & $\begin{array}{l}\text { PLUG VENT } \\
\text { FLT-*63 }\end{array}$ & \llcorner & $\begin{array}{l}\text { FLT } * 63 \\
\text { PLUGGED }\end{array}$ & $\AA$ & \\
\hline \multicolumn{6}{|c|}{ Section 1.7 .4 .2 verifies the main sample flow loop. } \\
\hline $\begin{array}{l}1.7 .4 .2 .1 \\
60\end{array}$ & $\begin{array}{l}\text { CLOSE CB-3/4 } \\
\text { ADJ. FLOW }\end{array}$ & $\frac{r}{r}$ & $\begin{array}{l}\text { CB CLOSED } \\
\text { FIT } * 57 \\
\text { INDICATION }\end{array}$ & $\Delta$ & $\begin{array}{l}\text { Sox } \\
\text { 210 }\end{array}$ \\
\hline 1.7 .4 .2 .2 & $\begin{array}{l}\text { PDI } * \text { *51 } \\
\text { READING }\end{array}$ & 27.5 & $20-40 \quad \mathrm{H}_{2} \mathrm{O}$ & A & \\
\hline 1.7 .4 .2 .3 & $\begin{array}{l}\text { PI } * 53 \\
\text { READING } \\
\end{array}$ & -2.8 & $\begin{array}{l}-2 \text { to }-4 \\
\text { "Hg }\end{array}$ & s & \\
\hline $\begin{array}{l}1.7 .4 .2 .4 \\
\text { Gen }\end{array}$ & RESET ALARMS & r & NO ALARMS & 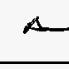 & \\
\hline $\begin{array}{l}1.7 .4 .2 .5 \\
\text { Lexn }\end{array}$ & CLOSE SV-*16- & $\begin{array}{l}0 \\
r \\
r\end{array}$ & 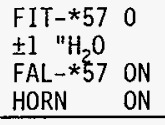 & $\Delta$ & \\
\hline t.7.4.2.6 & OPEN SV-*16- & 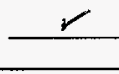 & $\begin{array}{l}\text { FAL-*57 ON } \\
\text { HORN OFF }\end{array}$ & $\Delta$ & \\
\hline $\begin{array}{l}1.7 .4 .2 .7 \\
6 x\end{array}$ & RESET ALARMS & 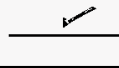 & $\begin{array}{l}\text { ALARMS } \\
\text { RESET }\end{array}$ & $A$ & \\
\hline
\end{tabular}


TEST DATA SHEET

\begin{tabular}{|c|c|c|c|c|c|}
\hline \multicolumn{3}{|c|}{ Date: $z=\Delta$ Foris -97} & \multicolumn{3}{|c|}{ SHMS Unit Number: Meth in Puns } \\
\hline \multicolumn{3}{|c|}{$\begin{array}{l}\text { Title of Test: } \\
\text { Pneumatic Systems }\end{array}$} & \multirow{2}{*}{\multicolumn{3}{|c|}{ 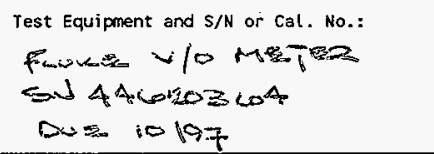 }} \\
\hline \multicolumn{3}{|c|}{$\begin{array}{l}\text { Test Performed By: } \\
\text { Sestant } 480\end{array}$} & & & \\
\hline $\begin{array}{l}\text { Procedure No. } \\
\text { Initial/Date }\end{array}$ & Item & Value & $\begin{array}{l}\text { Required } \\
\text { Condition }\end{array}$ & (A/R) & Comment \\
\hline $\begin{array}{l}1.7 .4 .2 .8 \\
\qquad .5 \\
\end{array}$ & CLOSE SV-*25 & 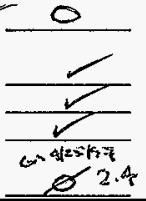 & 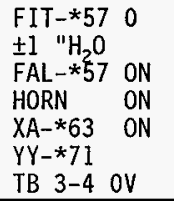 & $\Delta$ & \\
\hline $\begin{array}{l}1.7 .4 .2 .9 \\
\text { Ges }\end{array}$ & OPEN SV-*25 & $\frac{7}{118}$ & $\begin{array}{l}\text { FAL }-* 57 \text { ON } \\
\text { HORN OFF } \\
\text { XA-*63 OFF } \\
\text { YY } * 71 \\
\text { TB } 3-4 \\
\end{array}$ & $\Delta$ & \\
\hline $\begin{array}{l}1.7 .4 .2 .10 \\
\cos \\
\end{array}$ & RESET ALARMS & 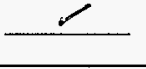 & $\begin{array}{l}\text { ALARMS } \\
\text { RESET }\end{array}$ & $A$ & \\
\hline 1.7 .4 .2 .11 & $\begin{array}{l}\text { OPEN SV-*54- } \\
\text { \& SV-*66- } \\
\text { READ PRESS, } \\
\text { PDIT-*60 }\end{array}$ & -41.6 & $\begin{array}{l}\text { VALVES OPEN } \\
-27 \text { to }-82 \\
" \mathrm{H}_{2} \mathrm{O}\end{array}$ & $\Delta$ & \\
\hline 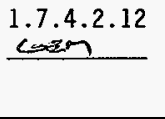 & $\begin{array}{l}\text { CLOSE SV-*54 } \\
\text { OPEN SV-*63- } \\
\text { READ PRESS. } \\
\text { PDIT-*60 }\end{array}$ & -0.13 & $\begin{array}{l}\text { VALVES } \\
\text { OPERATED } \\
0 \pm 1 \quad " \mathrm{H}_{2} \mathrm{O}\end{array}$ & $1 /$ & \\
\hline $\begin{array}{l}1.7 .4 .2 .13 \\
\text { sin }\end{array}$ & $\begin{array}{l}\text { CLOSE SV }-* 63 \\
\text { OPEN SV } * 55 \\
\text { READ PRESS. } \\
\text { PDIT-*60 } \\
\end{array}$ & $\frac{-43.17}{-17}$ & $\begin{array}{l}\text { VALVES } \\
\text { OPERATED } \\
-27 \text { to }-82 \\
" \mathrm{H}_{2} \mathrm{O}\end{array}$ & $A$ & \\
\hline $\begin{array}{l}1.7 .4 .2 .14 \\
\operatorname{sen} \\
\end{array}$ & $\begin{array}{l}\text { CLOSE SV }-* 55 \\
\text { OPEN SV-*64 } \\
\text { READ PRESS. } \\
\text { PDIT }-* 60\end{array}$ & $\Delta$ & $\begin{array}{l}\text { VALVES } \\
\text { OPERATED } \\
0 \pm 1 " \mathrm{H}_{2} \mathrm{O}\end{array}$ & $A$ & \\
\hline $\begin{array}{l}1.7 .4 .2 .15 \\
\text { Sish }\end{array}$ & $\begin{array}{l}\text { CLOSE SV-*64 } \\
\& S V-* 66\end{array}$ & $N / A$ & $N / A$ & $\Delta$ & \\
\hline \multicolumn{6}{|c|}{ Section 1.7 .4 .3 verifies the grab sample flow loop. } \\
\hline $\begin{array}{l}1.7 .4 .3 .1 \\
\text { cotis }\end{array}$ & READ PDI $-\star 51$ & 27.0 & $20-40 \quad " \mathrm{H}_{2} \mathrm{O}$ & $\Delta$ & \\
\hline
\end{tabular}


TEST DATA SHEET

\begin{tabular}{|c|c|c|c|c|c|}
\hline \multicolumn{3}{|c|}{ Date: $2 S \Delta$ pari-gF } & SHMS Unit Number: & \multicolumn{2}{|c|}{ 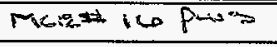 } \\
\hline \multicolumn{3}{|c|}{$\begin{array}{l}\text { Title of Test: } \\
\text { Pneumatic Systems }\end{array}$} & \multirow{2}{*}{\multicolumn{3}{|c|}{ Test Equipment and $\mathrm{S} / \mathrm{N}$ or Cal. No.: }} \\
\hline \multicolumn{3}{|c|}{ 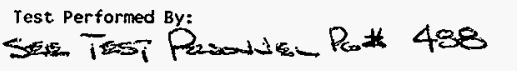 } & & & \\
\hline $\begin{array}{l}\text { Procedure No. } \\
\text { Initial/Date } \\
\end{array}$ & Item & Value & $\begin{array}{l}\text { Required } \\
\text { Condition }\end{array}$ & $(A / R)$ & Corment \\
\hline $\begin{array}{l}1.7 .4 .3 .2 \\
\end{array}$ & $\begin{array}{l}\text { PUSH PB }-* 59 \\
\text { GRAB SAMPLE } \\
\end{array}$ & 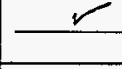 & $\begin{array}{l}\text { START } \\
\text { STOPWATCH }\end{array}$ & A & \\
\hline $\begin{array}{l}1.7 .4 .3 .3 \\
6.25\end{array}$ & $\begin{array}{l}\text { GRAB SAMPLE } \\
\text { LAMPS ON } \\
\text { READ FIV }-\star 52\end{array}$ & $\frac{r}{\frac{r}{2}}$ & $\begin{array}{l}\text { PBL-*58 ON } \\
\text { PBL-*59 ON } \\
\text { YAL-*58 ON } \\
\text { FIV-*52 } 0 \\
\end{array}$ & $\alpha$ & \\
\hline 1.7 .4 .3 .4 & $\begin{array}{l}\text { OPEN SV }-* 15 n \\
\& \text { ADJ. FIV- } \\
* 52 \\
\end{array}$ & 10 & $\begin{array}{l}\text { SV-* } 15 \text { OPEN } \\
\text { FIV } * 52 \\
\text { FLOW } 10 \quad \mathrm{CFH}\end{array}$ & A & $\operatorname{sim}_{\text {Find }} \Delta<i \rightarrow \infty$ \\
\hline 1.7 .4 .3 .5 & READ PDI $-* 51$ & 30 & $\begin{array}{l}\text { PDI }-* 51 \\
\text { HIGHER THAN } \\
1.7 .4 .3 .1\end{array}$ & A & \\
\hline $\begin{array}{l}1.7 .4 .3 .6 \\
\end{array}$ & CLOSE SV-*15 & 0 & FIV $-* 52 \quad 0$ & $\Delta$ & \\
\hline $\begin{array}{l}1.7 .4 .3 .7 \\
6 \operatorname{lon}\end{array}$ & OPEN SV-*15 & $r$ & $\begin{array}{l}\text { FIV-*52 } \\
\text { FLOW }\end{array}$ & A & \\
\hline $\begin{array}{l}1.7 .4 .3 .8 \\
\operatorname{sis}^{2}\end{array}$ & $\begin{array}{l}\text { PBL }-* 59 \\
\text { GRAB SAMPLE } \\
\text { TIME }\end{array}$ & $\begin{array}{l}1 \\
\equiv .0 \\
\end{array}$ & $\begin{array}{l}\text { PBL-*59 OFF } \\
\text { STOP WATCH } \\
5 \pm .5 \mathrm{MIN} \\
\end{array}$ & A. & \\
\hline $\begin{array}{l}1.7 .4 .3 .9 \\
\text { exin }^{2}\end{array}$ & $\begin{array}{l}\text { PB-*58 RESET } \\
\text { SAMPLER } \\
\end{array}$ & -2 & $\begin{array}{l}\mathrm{PBL}-* 58 \quad \text { OFF } \\
\text { YAL-*58 OFF }\end{array}$ & A & \\
\hline \multicolumn{6}{|c|}{ Section 1.7.4.4 verifies the $\mathrm{H}_{2}$ cell calibration loop. } \\
\hline 1.7 .4 .4 .1 & $\begin{array}{l}\text { CONNECT } \mathrm{H}_{2} \\
\text { CAL GAS }\end{array}$ & N/A & N/A & 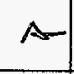 & \\
\hline $\begin{array}{l}1.7 .4 .4 .2 \\
\end{array}$ & $\begin{array}{l}\text { OPEN ISO } \\
\text { VALVE } \\
\end{array}$ & 5 & $<10$ PSIG & 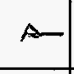 & \\
\hline $\mathrm{exin}^{1.7 .4 .4 .3}$ & $\begin{array}{l}\text { OPEN SV-*18- } \\
\text { ADJ. FIV-*56- }\end{array}$ & 2.0 & $2 \pm .1 \mathrm{CFH}$ & $\Delta$ & $1 / 1$ \\
\hline
\end{tabular}

corm bu 4listif 
TEST DATA SHEET

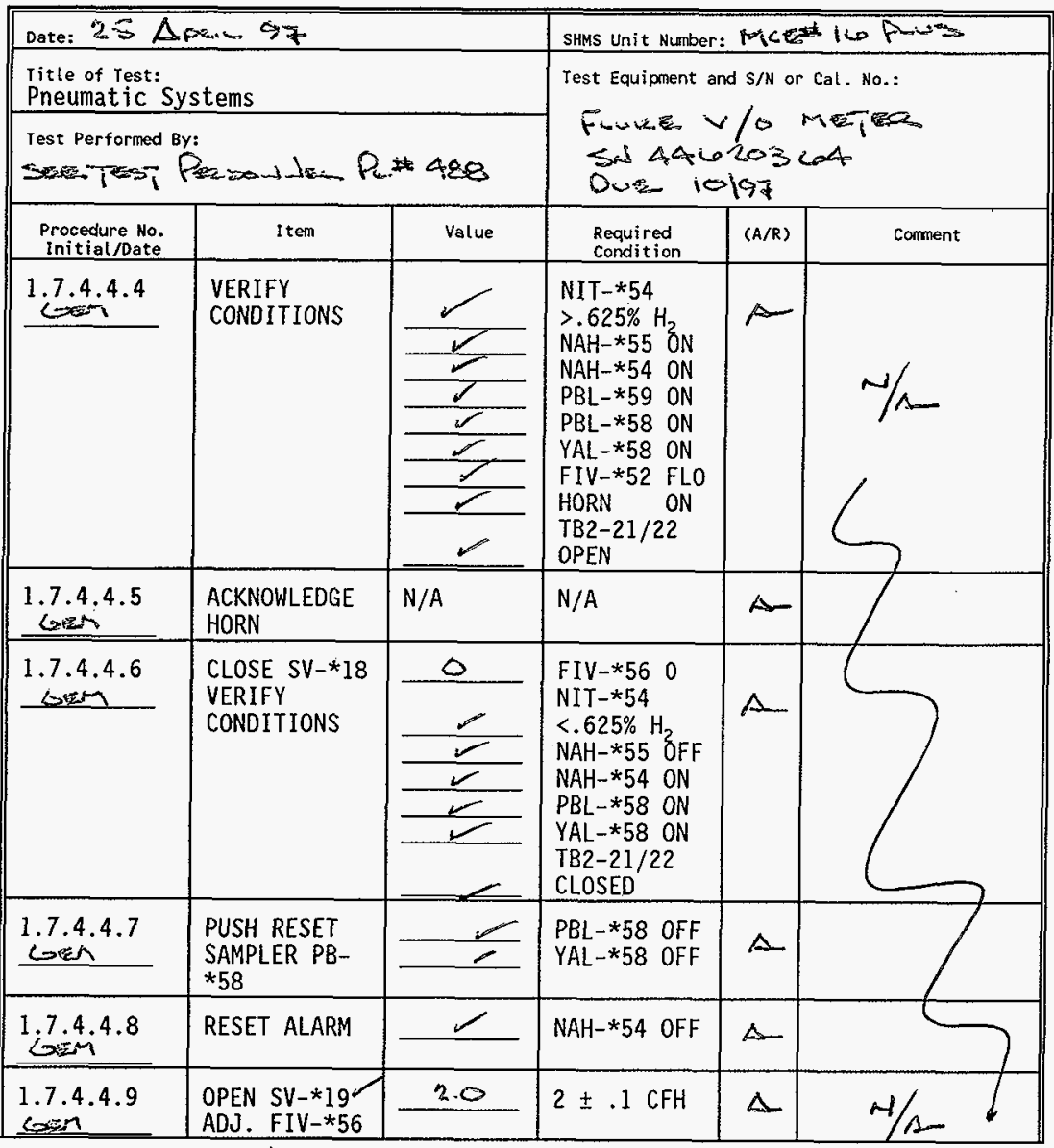

eon Aur lopslio 
TEST DATA SHEET

\begin{tabular}{|c|c|c|c|c|c|}
\hline \multicolumn{3}{|c|}{ Date: 25 Apri- 97} & \multicolumn{3}{|c|}{ SHMS Unit Number: MCK ICO PLW" } \\
\hline \multicolumn{3}{|c|}{$\begin{array}{l}\text { Title of Test: } \\
\text { Pneumat ic Systems }\end{array}$} & \multirow{2}{*}{\multicolumn{3}{|c|}{ 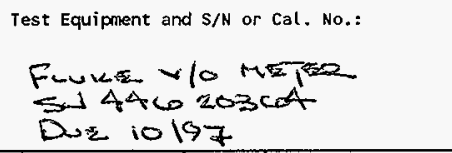 }} \\
\hline \multicolumn{3}{|c|}{ 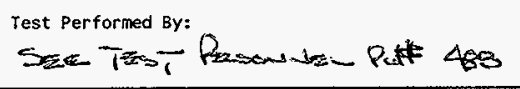 } & & & \\
\hline $\begin{array}{l}\text { Procedure No. } \\
\text { Initial/Date }\end{array}$ & I tem & Value & $\begin{array}{l}\text { Required } \\
\text { Condition }\end{array}$ & $(A / R)$ & Comment \\
\hline $\begin{array}{l}1.7 .4 .4 .10 \\
\text { Gean } \\
\end{array}$ & $\begin{array}{l}\text { VERIFY } \\
\text { CONDITIONS }\end{array}$ & $\frac{2}{2}$ & $\begin{array}{l}\text { NIT-*55 } \\
>.625 \% \mathrm{H}_{2} \\
\text { NAH-*55 ON } \\
\text { NAH-*54 ON } \\
\text { PBL-*59 ON } \\
\text { PBL-*58 ON } \\
\text { YAL-*58 ON } \\
\text { FIV-*52 FL0 } \\
\text { HORN ON } \\
\text { TB2-21/22 } \\
\text { OPEN }\end{array}$ & $\Delta$ & \\
\hline $\begin{array}{l}1.7 .4 .4 .11 \\
\text { exth }\end{array}$ & $\begin{array}{l}\text { ACKNOWLEDGE } \\
\text { HORN }\end{array}$ & $N / A$ & $N / A$ & $\Delta$ & \\
\hline $\begin{array}{l}1.7 .4 .4 .12 \\
\Leftrightarrow 4.7 \\
\end{array}$ & $\begin{array}{l}\text { CLOSE SV-*19*} \\
\text { VERIFY } \\
\text { CONDITIONS }\end{array}$ & & $\begin{array}{l}\text { FIV-*56 O } \\
\text { NIT-*55 } \\
<.625 \% \mathrm{H}_{2} \\
\mathrm{NAH}-* 55 \text { OFF } \\
\text { NAH-*54 ON } \\
\text { PBL-*58 ON } \\
\text { YAL-*58 ON } \\
\text { TB2-21/22 } \\
\text { CLOSED }\end{array}$ & $A$ & \\
\hline $\begin{array}{l}1.7 .4 .4 .13 \\
6=7 \\
\end{array}$ & $\begin{array}{l}\text { PUSH RESET } \\
\text { SAMPLER PB- } \\
* 58\end{array}$ & & $\begin{array}{l}\mathrm{PBL}-* 58 \text { OFF } \\
\text { YAL-*58 OFF }\end{array}$ & $\alpha$ & \\
\hline $\begin{array}{l}1.7 .4 .4 .14 \\
\text { rosn } \\
\end{array}$ & RESET ALARM & & $\mathrm{NAH}-* 54$ OFF & $A$ & \\
\hline $\begin{array}{l}1.7 .4 .4 .15 \\
\text { Genim } \\
\end{array}$ & $\begin{array}{l}\text { DISCONNECT } \\
\text { CAL. GAS }\end{array}$ & $\mathrm{N} / \mathrm{A}$ & $N / A$ & $\Delta$ & \\
\hline $\begin{array}{l}1.7 .4 .4 .16 \\
\end{array}$ & $\begin{array}{l}\text { PUSH PB- } * 54 \\
\text { VERIFY } \\
\text { CONDITIONS }\end{array}$ & $\mathscr{L}$ & $\begin{array}{l}X A-* 63 \text { ON } \\
\text { PBL }-* 54 \text { ON } \\
\text { TB2-23/24 } \\
\text { OPEN }\end{array}$ & $A$ & \\
\hline $\begin{array}{l}1.7 .4 .4 .17 \\
6 \mathrm{E}^{-} \\
\end{array}$ & $\begin{array}{l}\text { PUSH PB } * 54 \\
\text { VERIFY } \\
\text { CONDITIONS }\end{array}$ & $r$ & $\begin{array}{l}X A-* 63 \text { OFF } \\
\text { PBL }-* 54 \text { OFF } \\
\text { TB2-23/24 } \\
\text { CLOSED }\end{array}$ & $A$ & \\
\hline
\end{tabular}


TEST DATA SHEET

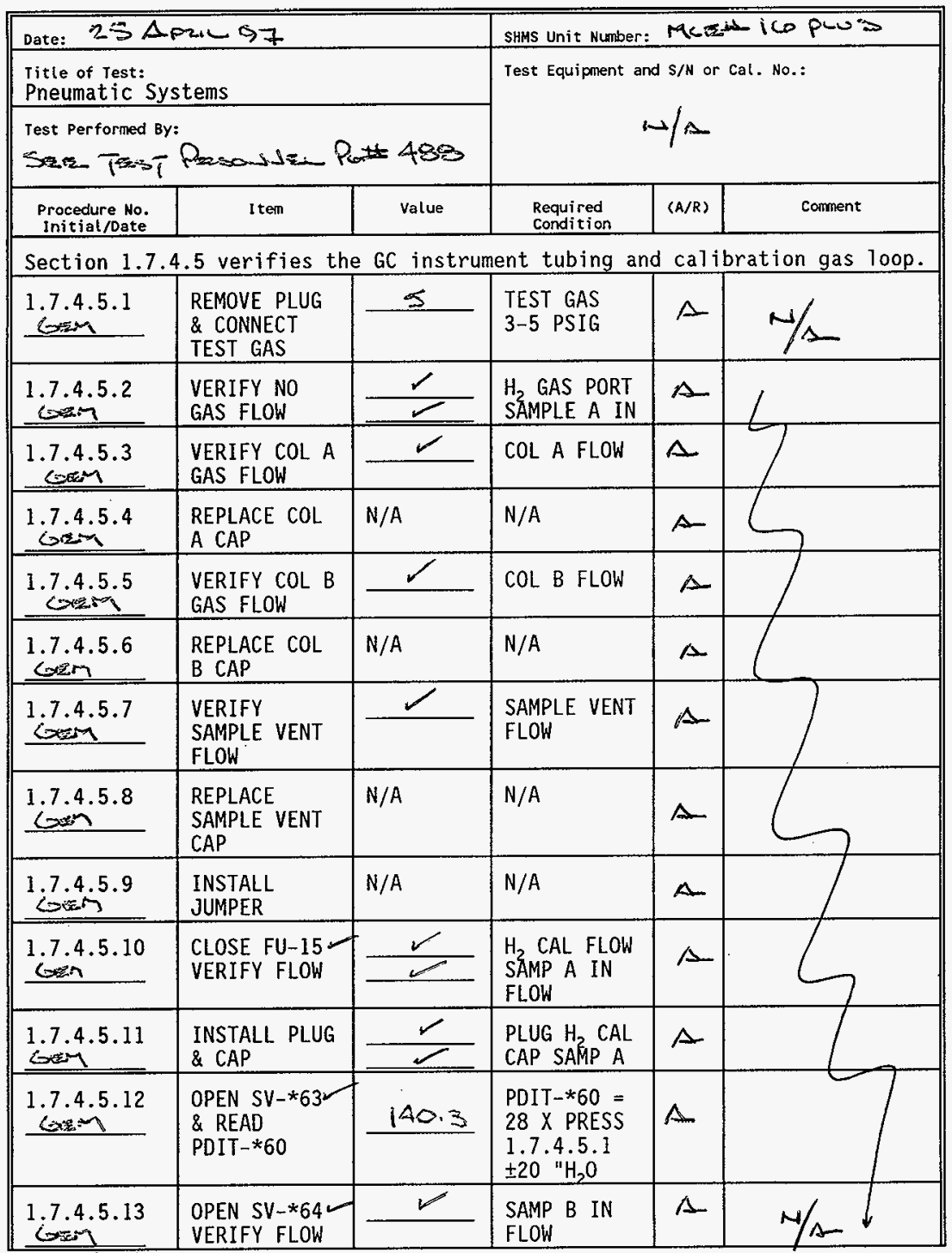


TEST DATA SHEET

\begin{tabular}{|c|c|c|c|c|c|}
\hline Date: $2 S A$ & $x-97$ & & SHMS Unit Number & $M<8$ & 16e P-Nas \\
\hline $\begin{array}{l}\text { Iitle of Test: } \\
\text { Pneumatic } S\end{array}$ & tems & & Test Equipment ar & $S / N$ or & Cal. No.: \\
\hline 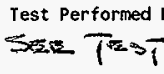 & Bosomand $P_{\infty}=$ & 1488 & & & \\
\hline $\begin{array}{l}\text { Procedure No. } \\
\text { Initial/Date }\end{array}$ & Item & value & $\begin{array}{l}\text { Required } \\
\text { Condition }\end{array}$ & $(A / R)$ & Corment \\
\hline $\begin{array}{c}1.7 .4 .5 .14 \\
0.00 \\
\end{array}$ & $\begin{array}{l}\text { REMOVE TEST } \\
\text { GAS }\end{array}$ & $N / A$ & $N / A$ & $\Delta$ & $N / \Delta$ \\
\hline 1.7 .4 .5 .15 & $\begin{array}{l}\text { CLOSE SV-*63 } \\
\& \text { SV-*64- }\end{array}$ & N/A & $N / A$ & $\Delta$ & 1 \\
\hline$\sum_{0.1 .7}^{1.7 .4 .5}$ & $\begin{array}{l}\text { OPEN FU-15- } \\
\text { REMOVE } \\
\text { JUMPER }\end{array}$ & $N / A$ & $N / A$ & $\Delta$ & \\
\hline 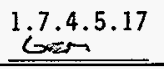 & $\begin{array}{l}\text { INSTALL } \\
\text { SAMP A UNION }\end{array}$ & $N / A$ & $N / A$ & $\wedge$ & \\
\hline $\begin{array}{l}1.7 .4 .5 .18 \\
\text { owan. }\end{array}$ & $\begin{array}{l}\text { OPEN SV-*60 } \\
\text { VERIFY FL.OW }\end{array}$ & & $\begin{array}{l}\text { FI-*60 } \\
\text { NO FLOW }\end{array}$ & $\Delta$ & \\
\hline is.7. & $\begin{array}{l}\text { POWER SOV- } \\
* 60 \text {, VERIFY } \\
\text { NO FLOW }\end{array}$ & $\sigma$ & $\begin{array}{l}\text { FI-*60 } \\
\text { NO FLOW }\end{array}$ & $\Delta$ & \\
\hline 1.7 .4 .5 .20 & $\begin{array}{l}\text { OPEN SV-*68 } \\
\text { ADJ SV-*67 }\end{array}$ & 50 & $\begin{array}{l}\mathrm{FI}-* 60 \\
50 \mathrm{CCM} \\
\end{array}$ & $\Delta$ & \\
\hline $\begin{array}{l}1.7 .4 .5 .21 \\
\end{array}$ & $\begin{array}{l}\text { DEENERGIZE } \\
\text { SOV-*60 } \\
\end{array}$ & 2 & $\begin{array}{l}\text { FI-*60 FLOW } \\
\text { DECREASES }\end{array}$ & $\Delta$ & \\
\hline $\begin{array}{l}1.7 .4 .5 .22 \\
\operatorname{gen} \\
\end{array}$ & $\begin{array}{l}\text { CLOSE SV-*60- } \\
\& \text { SV }-* 68-\end{array}$ & $N / A$ & $N / A$ & $\Delta$ & \\
\hline $\begin{array}{l}1.7 .4 .5 .23 \\
\text { Cosin }\end{array}$ & $\begin{array}{l}\text { REMOVE } \\
\text { SAMP A UNION }\end{array}$ & $N / A$ & $N / A$ & $\Delta$ & \\
\hline 1.7 .4 .5 .24 & $\begin{array}{l}\text { CONNECT TEST } \\
\text { GAS } N_{2} 0 \text { PORT } \\
\end{array}$ & 5 & $\begin{array}{l}\text { TEST GAS } \\
3-5 \text { PSIG }\end{array}$ & A & \\
\hline$\frac{1.7 .4 .5 .25}{\cos 2 \pi}$ & $\begin{array}{l}\text { VERIFY NO } \\
\text { FLOW }\end{array}$ & $\frac{r}{r}$ & $\begin{array}{l}\text { SAMP B IN \& } \\
\text { FLT-*63 } \\
\text { NO FLOW }\end{array}$ & $\Delta$ & \\
\hline $\operatorname{con}^{1.7 .4 .5 .26}$ & $\begin{array}{l}\text { INSTALL } \\
\text { JUMPER }\end{array}$ & $N / A$ & $N / A$ & $A$ & \\
\hline $\begin{array}{l}1.7 .4 .5 .27 \\
629\end{array}$ & $\begin{array}{l}\text { CLOSE FU-15 } \\
\text { VERIFY FLOW }\end{array}$ & $\infty$ & $\begin{array}{l}\text { SAMP B IN } \\
\text { FLT }-* 63 \\
\text { FLOW }\end{array}$ & A & $N / \Omega$ \\
\hline
\end{tabular}


APPENDIX $P$

PAGE P- 2 \%
HNF-SD-WM-ATR-191

Rev. 0

TEST DATA SHEET

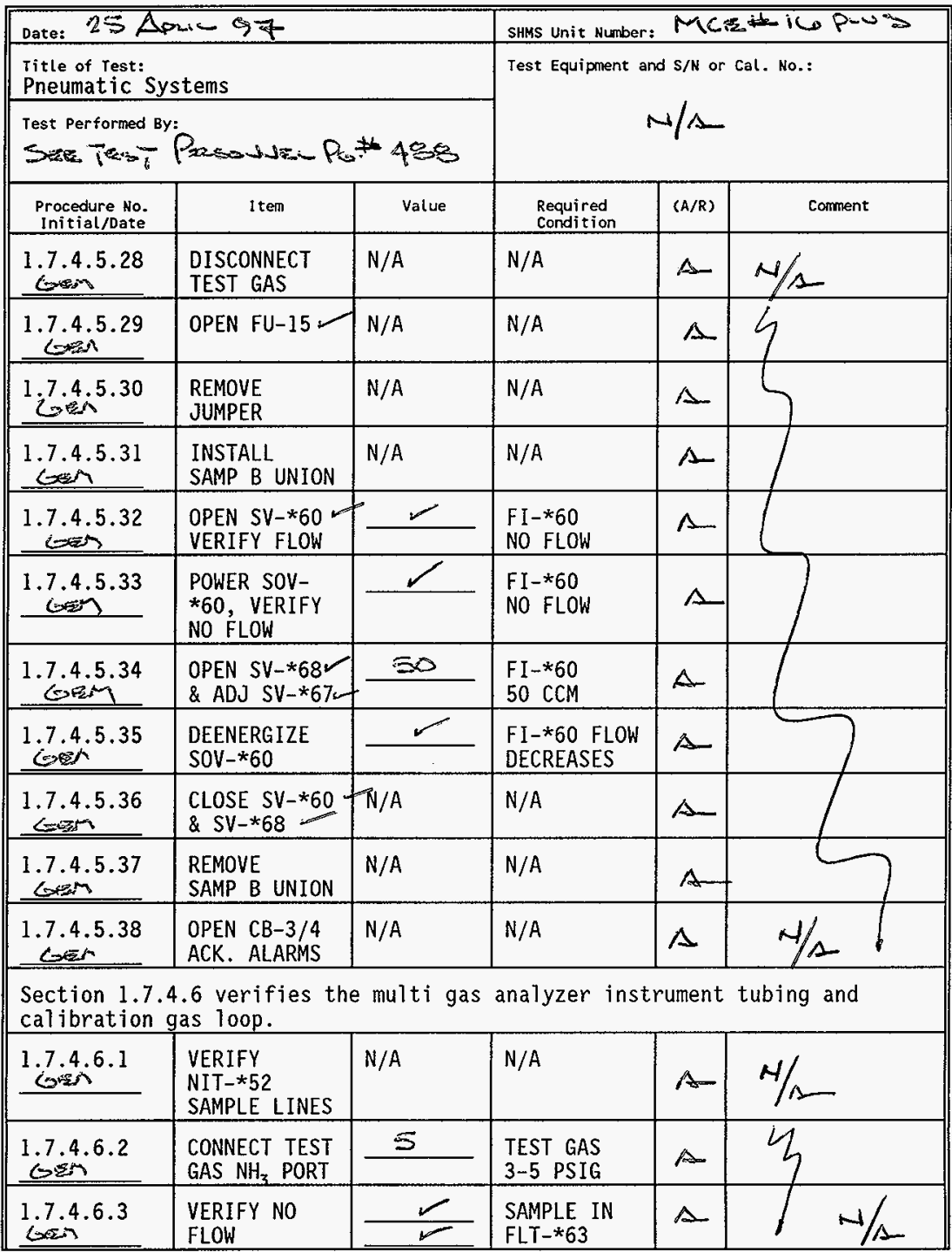


TEST DATA SHEET

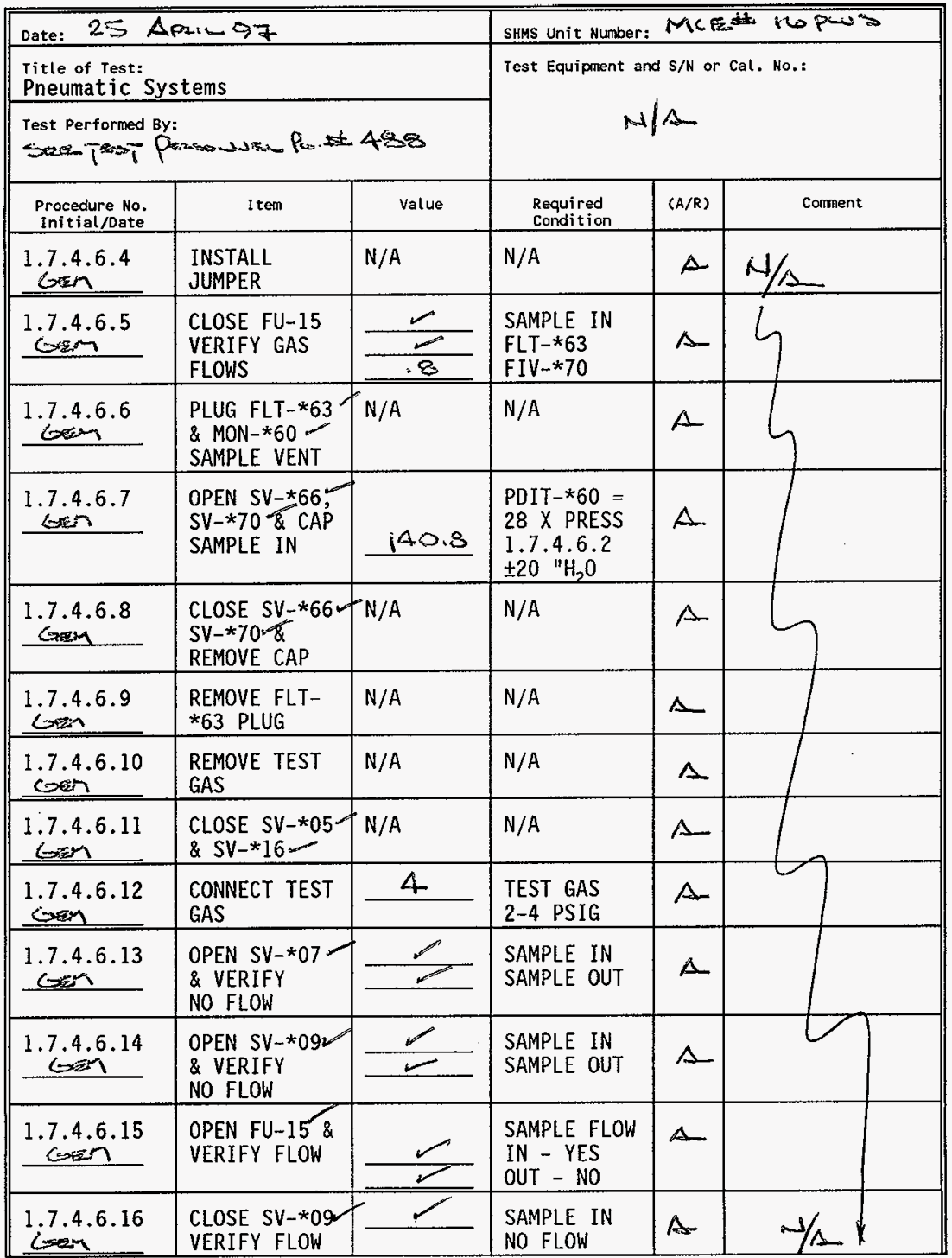


APPENDIX P

HNF-SD-WM-ATR- 199
ReV. 0
Page 509

TEST DATA SHEET

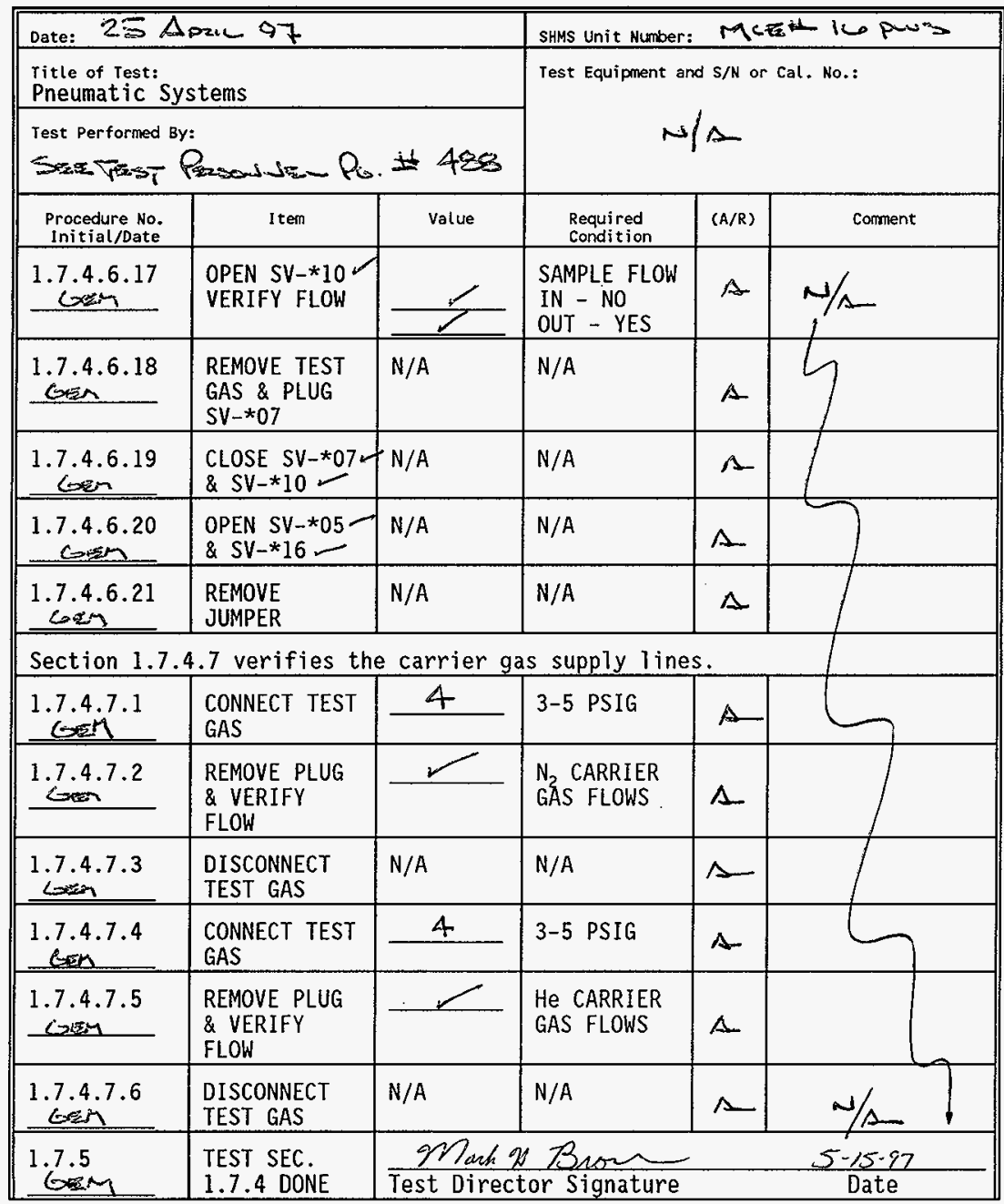

con An A/2s/87 
TEST DATA SHEET

\begin{tabular}{|c|c|c|c|c|c|}
\hline \multicolumn{3}{|c|}{ Date: $15 \mathrm{MAY} 97$} & \multicolumn{3}{|c|}{ SHMS Unit Nunber: Mceziticot } \\
\hline \multicolumn{3}{|c|}{$\begin{array}{l}\text { Title of Test: } \\
\text { Pneumat ic Systems }\end{array}$} & \multirow{2}{*}{\multicolumn{3}{|c|}{ Test Equipment and $\mathrm{S} / \mathrm{N}$ or Cal. No.: }} \\
\hline \multicolumn{3}{|c|}{ 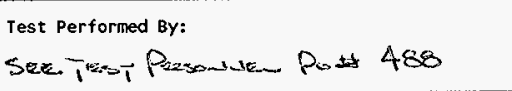 } & & & \\
\hline $\begin{array}{l}\text { Procedure No. } \\
\text { Initial/Date }\end{array}$ & Item & Value & $\begin{array}{l}\text { Required } \\
\text { Condition }\end{array}$ & $(A / R)$ & Comment \\
\hline \multirow[t]{2}{*}{$\begin{array}{l}1.7 .6 \\
\text { \&e.m }\end{array}$} & \multirow{2}{*}{\multicolumn{5}{|c|}{$\begin{array}{l}\text { THIS SECTION IS ONLY FOR THE SHMS-E IN THE ANALYTICAL MODE } \\
\text { IF NOT APPLICABLE, N/A STEPS AND TEST DIRECTOR WILL SIGN. }\end{array}$}} \\
\hline & & & & & \\
\hline 1.7 .6 .1 .1 & $\begin{array}{l}\text { VERIFY BKR } \\
\text { LINE UP }\end{array}$ & N/A & N/A & A & \\
\hline $\begin{array}{l}1.7 .6 .1 .2 \\
\text { sem }\end{array}$ & $\begin{array}{l}\text { VERIFY VALVE } \\
\text { LINE UP }\end{array}$ & N/A & N/A & $A$ & \\
\hline$\frac{1.7 .6 .1 .3}{6 \operatorname{sen}^{2}}$ & $\begin{array}{l}\text { VERIFY MON- } \\
\text { *60 INSTALL }\end{array}$ & N/A & N/A & A & \\
\hline 1.7 .6 .1 .4 & $\begin{array}{l}\text { VERIFY NIT- } \\
* 52 \text { INSTALL } \\
\end{array}$ & N/A & $\mathrm{N} / \mathrm{A}$ & A & \\
\hline $\begin{array}{l}1.7 .6 .1 .5 \\
\end{array}$ & $\begin{array}{l}\text { CONNECT } \mathrm{H}_{2} \\
\text { CAL GAS }\end{array}$ & $N / A$ & N/A & $\Delta$ & \\
\hline $\begin{array}{l}1.7 .6 .1 .6 \\
\end{array}$ & $\begin{array}{l}\text { CONNECT } \mathrm{CH}_{4} \\
\text { CAL GAS }\end{array}$ & N/A & N/A & $\Delta$ & \\
\hline $\begin{array}{l}1.7 .6 .1 .7 \\
\text { Gen }\end{array}$ & $\begin{array}{l}\text { CONNECT } \mathrm{NH}_{3} \\
\text { CAL GAS }\end{array}$ & N/A & N/A & A & \\
\hline \multicolumn{6}{|c|}{ Section 1.7.6.2 establishes the main and auxiliary loop flows. } \\
\hline $\begin{array}{l}1.7 .6 .2 .1 \\
\text { Gen }\end{array}$ & CLOSE FU-11 & 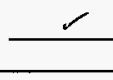 & $\begin{array}{l}\text { MON-*60 } \\
\text { POWERED }\end{array}$ & $A$ & \\
\hline $\begin{array}{l}1.7 .6 .2 .2 \\
\operatorname{sen}\end{array}$ & POWER PC $-* 60$ & 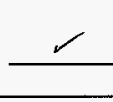 & $\begin{array}{l}\text { ESTABLISH } \\
\text { SAMPLING } \\
\text { ROUTINE }\end{array}$ & $A$ & \\
\hline $\mathrm{Gen}^{1.7 .6 .2 .3}$ & CLOSE FU-12 & $r$ & $\begin{array}{l}\text { NIT } * 52 \\
\text { POWERED } \\
\end{array}$ & $A$ & \\
\hline $\begin{array}{c}1.7 .6 .2 .4 \\
\cos \end{array}$ & POWER PC-*70 & $r$ & $\begin{array}{l}\text { PC-*70 } \\
\text { BOOTED UP }\end{array}$ & $\wedge$ & \\
\hline $\begin{array}{l}1.7 .6 .2 .5 \\
\text { Gem } \\
\end{array}$ & $\begin{array}{l}\text { CLOSE CB-3/4 } \\
\text { ADJUST FLOW }\end{array}$ & 1.0 & $\begin{array}{l}\text { FIT-*577 } \\
1 \pm .1 \quad \mathrm{H}_{2} \mathrm{O}\end{array}$ & $A$ & \\
\hline
\end{tabular}


TEST DATA SHEET

\begin{tabular}{|c|c|c|c|c|c|}
\hline \multirow{2}{*}{\multicolumn{3}{|c|}{$\begin{array}{l}\text { Date: is } \\
\text { Title of Te } \\
\text { Pneumati }\end{array}$}} & \multicolumn{3}{|c|}{ Mciz $160+$} \\
\hline & & & \multirow{2}{*}{\multicolumn{3}{|c|}{ Test Equipment and S/N or Cal. No.: }} \\
\hline \multicolumn{3}{|c|}{ 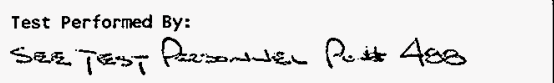 } & & & \\
\hline $\begin{array}{l}\text { Procedure No. } \\
\text { Initial/Date }\end{array}$ & item & value & $\begin{array}{l}\text { Required } \\
\text { Condition }\end{array}$ & $(A / R)$ & Comment \\
\hline $\begin{array}{l}1.7 .6 .2 .6 \\
\text { Sesen }\end{array}$ & $\begin{array}{l}\text { OPEN SV-*09- } \\
\& S V-* 10\end{array}$ & $N / A$ & $N / A$ & $A$ & \\
\hline $\begin{array}{l}1.7 .6 .2 .7 \\
\text { gest }\end{array}$ & $\begin{array}{l}\text { OPEN SV }-* 60 \\
63,65 \& 68\end{array}$ & $N / A$ & N/A & $A$ & \\
\hline $\begin{array}{l}1.7 .6 .2 .8 \\
\cosh ^{2}\end{array}$ & NIT $-* 52$ & 2 & $\begin{array}{l}\text { ESTABL ISH } \\
\text { SAMPLING } \\
\text { ROUTINE }\end{array}$ & $A$ & \\
\hline $\begin{array}{c}1.7 .6 .2 .9 \\
x^{-4}\end{array}$ & $\begin{array}{l}\text { OPEN SV-*24 } \\
\text { ADJ FLOW }\end{array}$ & 2.99 & $\begin{array}{l}\text { FIT-*57 } \\
3 \pm .1 \quad \mathrm{H}_{2} \mathrm{O}\end{array}$ & $A$ & \\
\hline $\begin{array}{l}1.7 .6 .2 .10 \\
\text { geven } \\
\end{array}$ & $\begin{array}{l}\text { ADJ FLOW } \\
\text { SV-*67 }\end{array}$ & $\frac{28}{25}$ & $\begin{array}{l}F I-* 60 \\
10-30 \mathrm{ccm}\end{array}$ & $A$ & \\
\hline 1.7 .6 .2 .11 & $\begin{array}{l}\text { RECORD } \\
\text { PDIT-*60 }\end{array}$ & $\frac{74+14}{110}$ & $<175 \quad$ " $\mathrm{H}_{2} \mathrm{O}$ & $A$ & \\
\hline $\begin{array}{l}1.7 .6 .2 .12 \\
\tan \end{array}$ & $\begin{array}{l}\text { OPEN SV } * 64 \\
\text { CLOSE SV-*63- }\end{array}$ & $N / A$ & N/A & $A$ & \\
\hline $\begin{array}{c}1.7 .6 .2 .13 \\
\operatorname{sen}\end{array}$ & $\begin{array}{l}\text { RECORD } \\
\text { PDIT }-* 60\end{array}$ & 111.7 & $<175 \quad " \mathrm{H}_{2} \mathrm{O}$ & $A$ & \\
\hline$\underset{62 n^{1.74}}{1.7 .2 .14}$ & $\begin{array}{l}\text { CLOSE SV }-* 65- \\
\text { OPEN SV }-* 66\end{array}$ & $N / A$ & N/A & $A$ & \\
\hline $\begin{array}{c}1.7 .6 .2 .15 \\
\end{array}$ & $\begin{array}{l}\text { RECORD B IN } \\
\text { PDIT }-* 60\end{array}$ & -23.77 & $\begin{array}{l}-15 \text { to }-50 \\
\mathrm{IH}_{2} \mathrm{O}\end{array}$ & $A$ & \\
\hline 1.7 .6 .2 .16 & $\begin{array}{l}\text { OPEN SV }-* 63 \\
\text { CLOSE SV }-* 64-\end{array}$ & $N / A$ & N/A & A & \\
\hline 1.7 .6 .2 .17 & $\begin{array}{l}\text { RECORD A IN } \\
\text { PDIT }-* 60\end{array}$ & -23.78 & $\begin{array}{l}-15 \text { to }-50 \\
\mathrm{NH}_{2} \mathrm{O}\end{array}$ & $A$ & \\
\hline $\begin{array}{c}1.7 .6 .2 .18 \\
\end{array}$ & $\begin{array}{l}\text { INSTALL } \\
\text { JUMPER }\end{array}$ & N/A & N/A & $A$ & \\
\hline$\underset{\mathrm{Gin}}{1.7 .6 .2 .19}$ & CLOSE FU-15 & $N / A$ & $N / A$ & $A$ & \\
\hline$\frac{1.7 .6 .2 .20}{\cos }$ & $\begin{array}{l}\text { ADJ SV-*61 \& } \\
\text { RECORD } \\
\text { PDIT }-\star 60\end{array}$ & -23.12 & $\begin{array}{l} \pm 5^{\prime \prime} \mathrm{H}_{2} \mathrm{O} \text { OF } \\
1.7 .6 .2 .17\end{array}$ & $\Lambda$ & \\
\hline
\end{tabular}


TEST DATA SHEET

\begin{tabular}{|c|c|c|c|c|c|}
\hline \multirow{2}{*}{\multicolumn{3}{|c|}{$\begin{array}{l}\text { Date: IS MAY } 97 \\
\text { Title of Test: } \\
\text { Pneumatic Systems }\end{array}$}} & \multicolumn{3}{|c|}{ SHMs Unit Nunber: Mce $15+$} \\
\hline & & & \multirow{2}{*}{\multicolumn{3}{|c|}{ 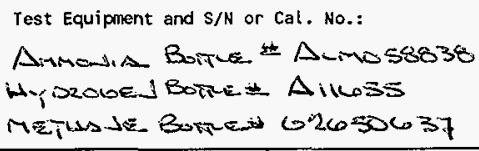 }} \\
\hline \multicolumn{3}{|c|}{ 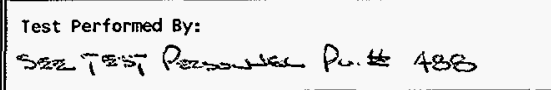 } & & & \\
\hline $\begin{array}{l}\text { Procedure No. } \\
\text { Initial/Date }\end{array}$ & Item & Value & $\begin{array}{l}\begin{array}{l}\text { Required } \\
\text { condition }\end{array} \\
\end{array}$ & $(\mathrm{A} / \mathrm{R})$ & Comment \\
\hline $\begin{array}{l}1.7 .6 .2 .21 \\
\end{array}$ & $\begin{array}{l}\text { CAL MON-*60 } \\
\text { CHANNEL A }\end{array}$ & $\frac{1.05}{1.073}$ & $\begin{array}{l}\text { READINGS } \\
\text { WITHIN } \pm 5 \%\end{array}$ & A & $\sim / \Omega$ \\
\hline$\frac{1.7 .6 .2 .22}{6 \mathrm{gin}^{-}}$ & OPEN FU-15 & N/A & N/A & $A$ & 1 \\
\hline $\begin{array}{l}1.7 .6 .2 .23 \\
\end{array}$ & $\begin{array}{l}\text { REMOVE } \\
\text { JUMPER }\end{array}$ & $N / A$ & N/A & $\Delta$ & \\
\hline 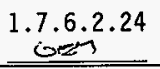 & $\begin{array}{l}\text { OPEN SV-*64 } \\
\text { CLOSE SV-*63- }\end{array}$ & $N / A$ & N/A & $\Delta$ & \\
\hline $\begin{array}{l}1.7 .6 .2 .25 \\
\text { Goin }\end{array}$ & $\begin{array}{l}\text { INSTALL } \\
\text { JUMPER }\end{array}$ & $\mathrm{N} / \mathrm{A}$ & N/A & $A$ & \\
\hline $\begin{array}{l}1.7 .6 .2 .26 \\
\operatorname{sen}\end{array}$ & CLOSE FU-15 & N/A & N/A & $A$ & \\
\hline$\frac{1.7 .6 .2 .27}{\log ^{2}}$ & $\begin{array}{l}\text { ADJ SV }-* 62 \& \\
\text { RECORD } \\
\text { PDIT }-* 60\end{array}$ & -20.42 & $\begin{array}{l} \pm 5^{\prime \prime} \mathrm{H}_{2} \mathrm{O} \text { OF } \\
1.7 .6 .2 .15\end{array}$ & $\Delta$ & \\
\hline$\frac{1.7 .6 .2 .28}{\operatorname{stg}}$ & $\begin{array}{l}\text { CAL MON-*60 } \\
\text { CHANNEL B }\end{array}$ & $\frac{\frac{1.004}{1.005}}{1.007}$ & $\begin{array}{l}\text { READINGS } \\
\text { WITHIN } \pm 5 \%\end{array}$ & $A$ & \\
\hline $\begin{array}{l}1.7 .6 .2 .29 \\
\end{array}$ & OPEN FU-15 & $N / A$ & N/A & $A$ & \\
\hline $\begin{array}{c}1.7 .6 .2 .30 \\
\end{array}$ & $\begin{array}{l}\text { REMOVE } \\
\text { JUMPER }\end{array}$ & N/A & N/A & $A$ & \\
\hline $\begin{array}{l}1.7 .6 .2 .31 \\
\text { ixti }\end{array}$ & $\begin{array}{l}\text { CLOSE SV-*64 } \\
\text { OPEN SV-*70 }\end{array}$ & EN/A & $\mathrm{N} / \mathrm{A}$ & $A$ & \\
\hline $\begin{array}{l}1.7 .6 .2 .32 \\
\text { Gin }\end{array}$ & $\begin{array}{l}\text { RECORD } \\
\text { PDIT-*60 } \\
\text { NIT-*52 IN }\end{array}$ & -11.16 & $\begin{array}{l}0 \text { to }-30 \\
" \mathrm{H}_{2} \mathrm{O}\end{array}$ & $A$ & \\
\hline $\operatorname{tgm}^{1.7 .6 .2 .33}$ & $\begin{array}{l}\text { INSTALL } \\
\text { JUMPER }\end{array}$ & N/A & N/A & $A$ & \\
\hline $\begin{array}{l}1.7 .6 .2 .34 \\
\text { som }\end{array}$ & CLOSE FU-15 - & N/A & N/A & $A$ & $N / \Delta$ \\
\hline
\end{tabular}

$\Leftrightarrow A_{-}=1=187$ 
TEST DATA SHEET

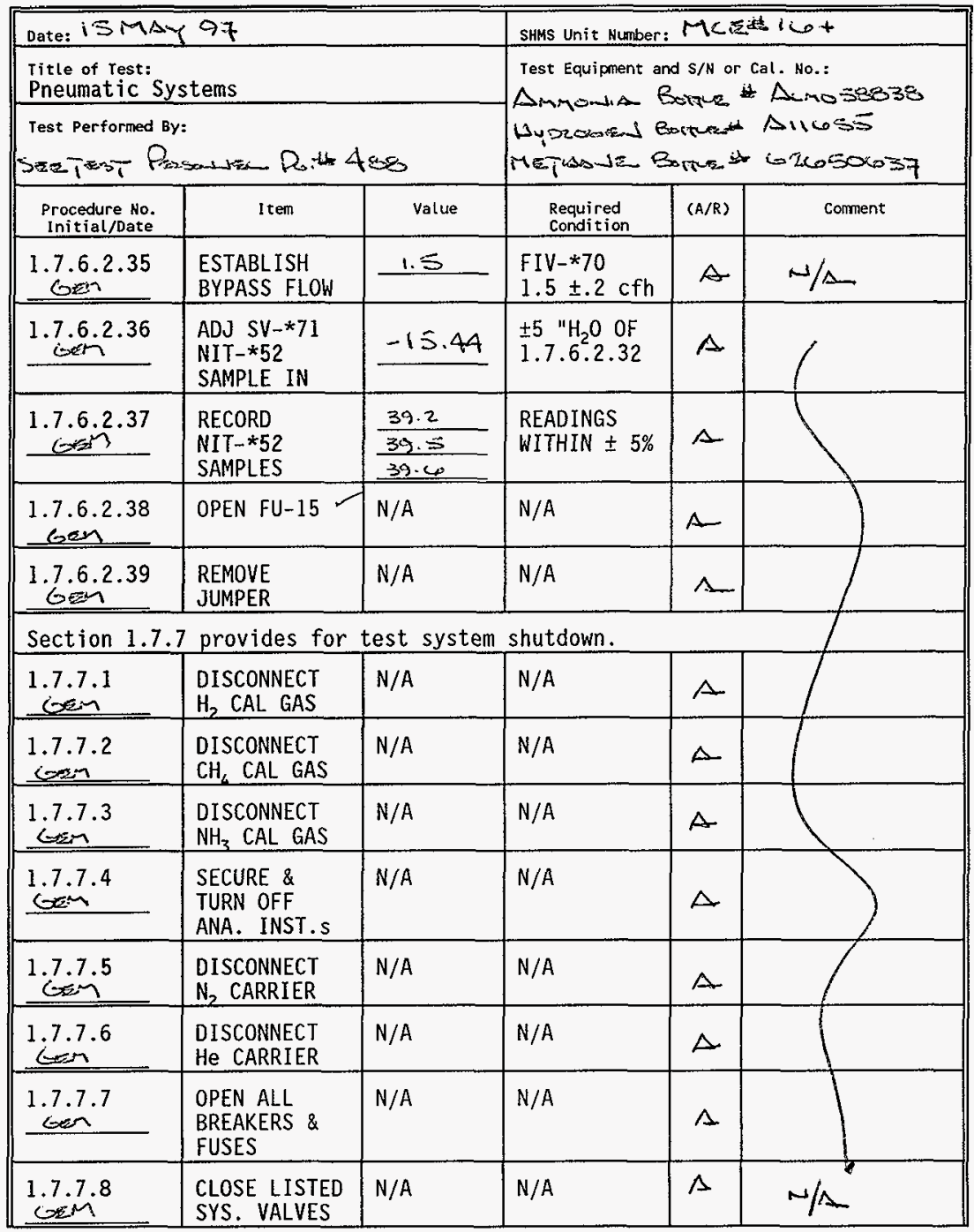

Gen an s/sh 
APPENDIX P

PAGE P- 28
HNF-SD-WH-ATR-191
ReV. 0

Page 514

TEST DATA SHEET

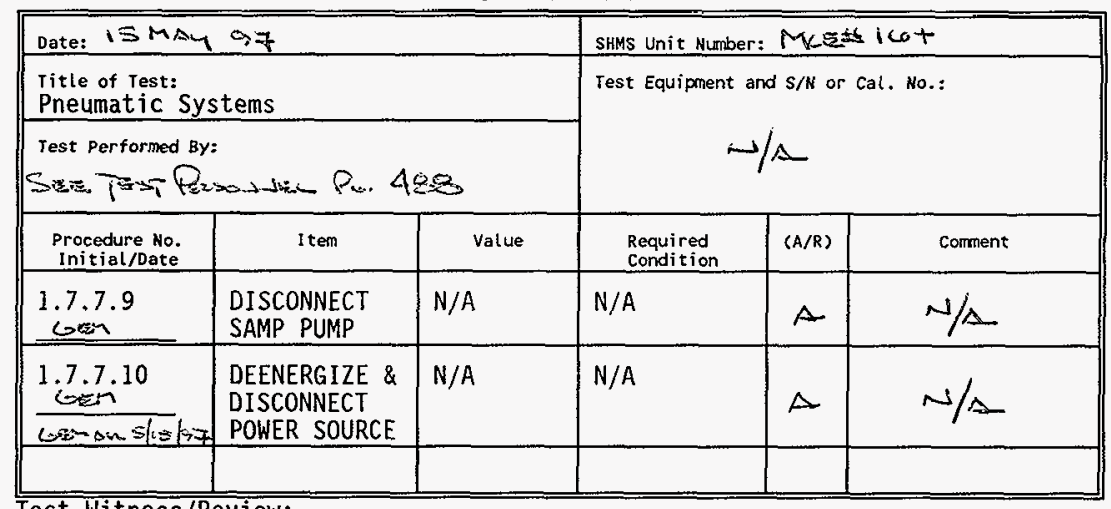

Test Witness/Review:

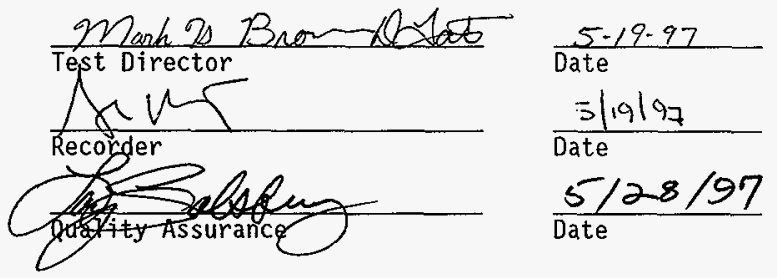


APPENDIX $P$

HNF-SD-WM-ATR- 191

PAGE P-Z.?

Rev. 0
Page 515

TEST EXCEPTION SHEET

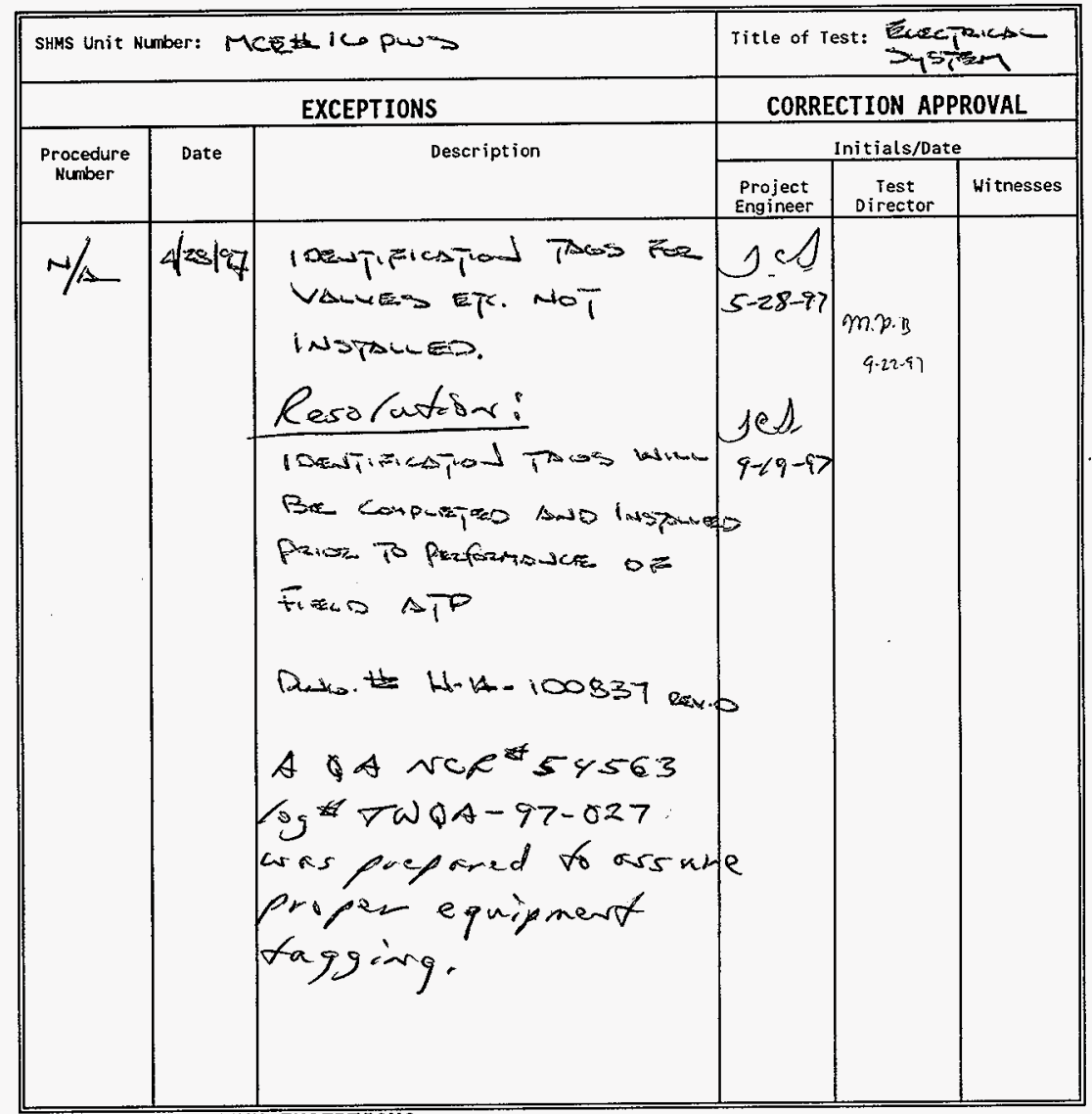

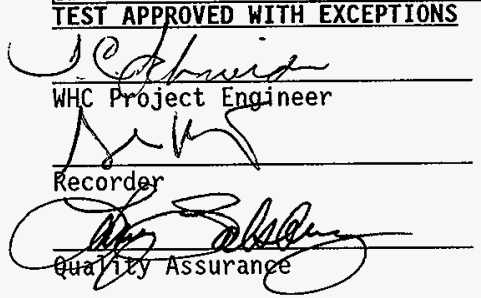

$$
\begin{aligned}
& \frac{5-2 p-97}{\text { Date }} \\
& \frac{28 \text { may } 97}{\text { Date }} \\
& \frac{5 / 28 / 97}{\text { Date }}
\end{aligned}
$$


APPENDIX $P$

PAGE P-30
HNF-SD-WM-ATR- 191

Rev. 0
Page 516

TEST LOG

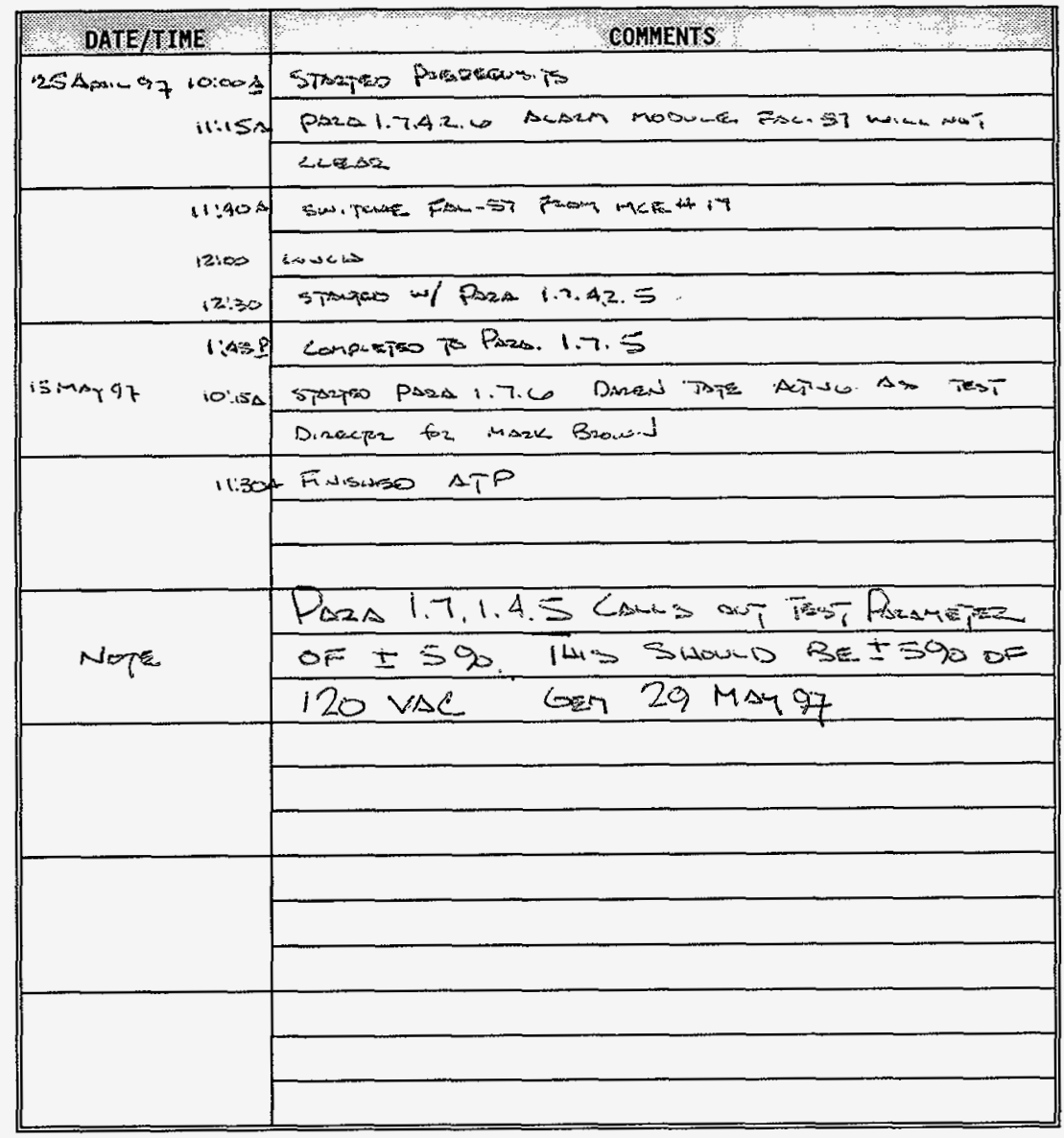


APPENDIX Q

PAGE Q-I

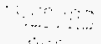

$\therefore \because$

$\ldots$

APPENDIX 0

HNF-SD-WM-ATR-191

Rev. 0 
TEST EXECUTION SHEET

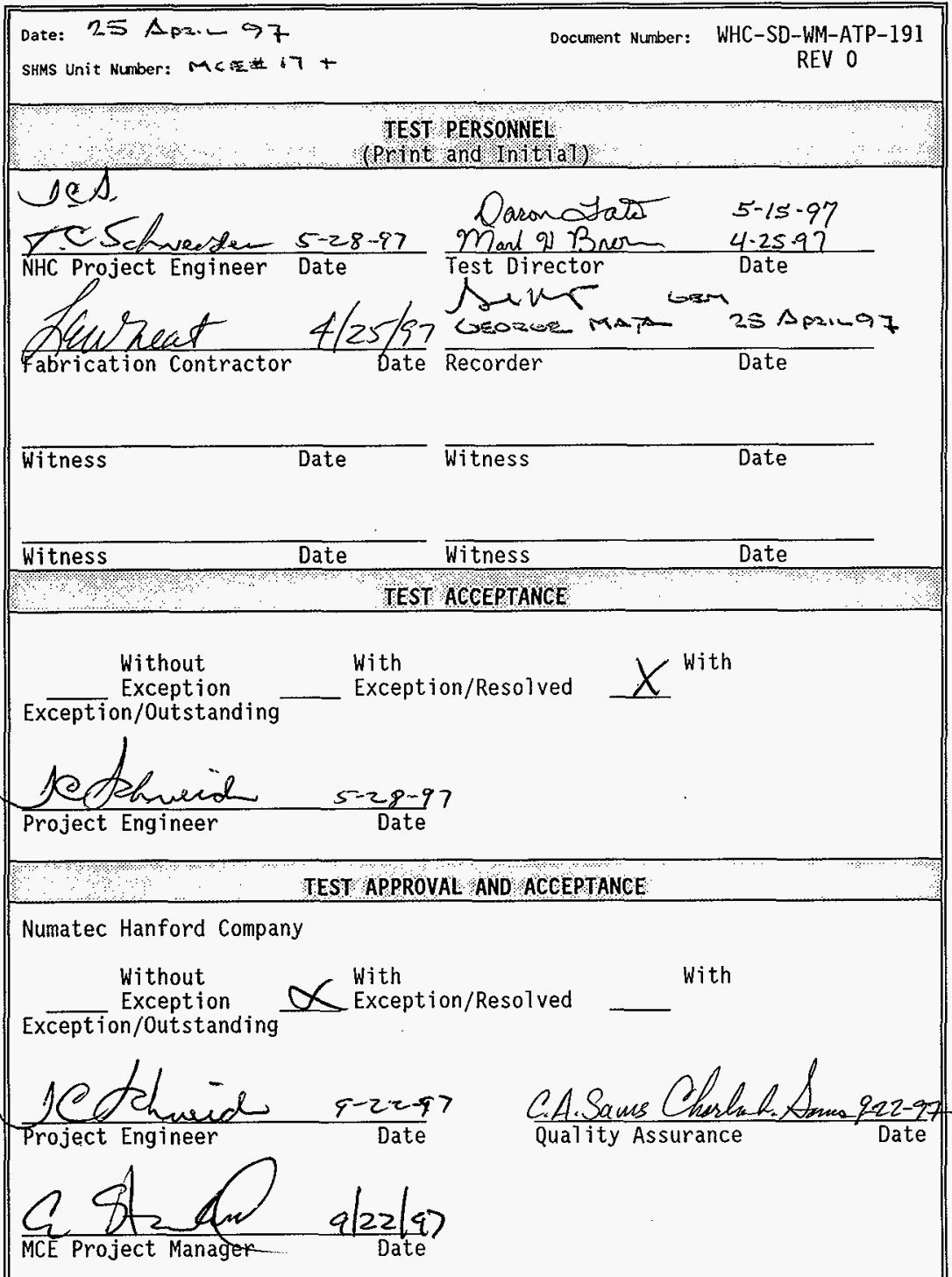


SHMS Unit Number: Mentat $17+$

\section{PREREQUISITES AND INITIAL TEST CONDITIONS}

The following conditions shall exist at the start of the acceptance testing. Initial and date to verify that each of the following items have been accomplished.

Gran 42517 systems being tested have been inspected for workmanship and for compliance with design.

Sorstinuity and megger tests have been performed on portions of the electrical and instrument systems being tested, as required.

$4|25| 9.7$ Leak tests on the pneumatic systems have been performed.

$\Leftrightarrow 4 / 25197$ The following circuit breakers and fuses are installed per the specified size and are open

CB-1 through CB-7
FU-1 through FU-15

Grem Alasly A11 test instruments have a currently valid calibration stamp attached that indicates a calibration traceable to the National Institute of Standards and Technology.

$\cos 4 \mid 25107$

The following process and control instrument systems have been initially configured and aligned for proper operation.

FIT-*57, FSL $-* 57$, NIT-*54, NIT-*55, (NR-*54), PDIT-*60, TIC-*50, TIC $-* 56$, TIS $-* 62$, YYC $-* 01$ NOTE: NR-*54 is not used in the ANALYTICAL configuration.

Ger $412=197$ Personnel responsible for directing and witnessing the performance of the tests described in this ATP have read and understand appropriate certified vendor information (CVI) pertaining to the operation of the equipment to be tested.

$\cos 4 / 2=177$

CLOSE all system manual valves with the exception of the following:

$$
\begin{array}{ll}
\checkmark & \text { VTP-FIV-*52 } \\
\text { VTP-FIV-*56 } \\
\text { VTP-FIV-*70 } \\
\text { VTP-SV-*24 } \\
\text { VTP-SV-*61 } \\
\text { VTP-SV-*62 } \\
\text { VTP-SV-*67 } \\
\text { VTP-SV-*71 }
\end{array}
$$

SAs 187 The sample pump VTP-P-*50, connected to the cabinet sample out port, but vented to atmosphere for testing. 
$\Leftrightarrow$ atezhit208 Vac 1 phase 15-20 ampere temporary power source has been connected, but not energized, to the appropriate TBl terminals per H-14-100838.

Verify by signature and date that all prerequisites have been met.

$\frac{M \operatorname{mox} q \mathrm{Bnon}}{\text { Test Director Signature }} \frac{4-25.97}{\text { Date }}$


TEST DATA SHEET

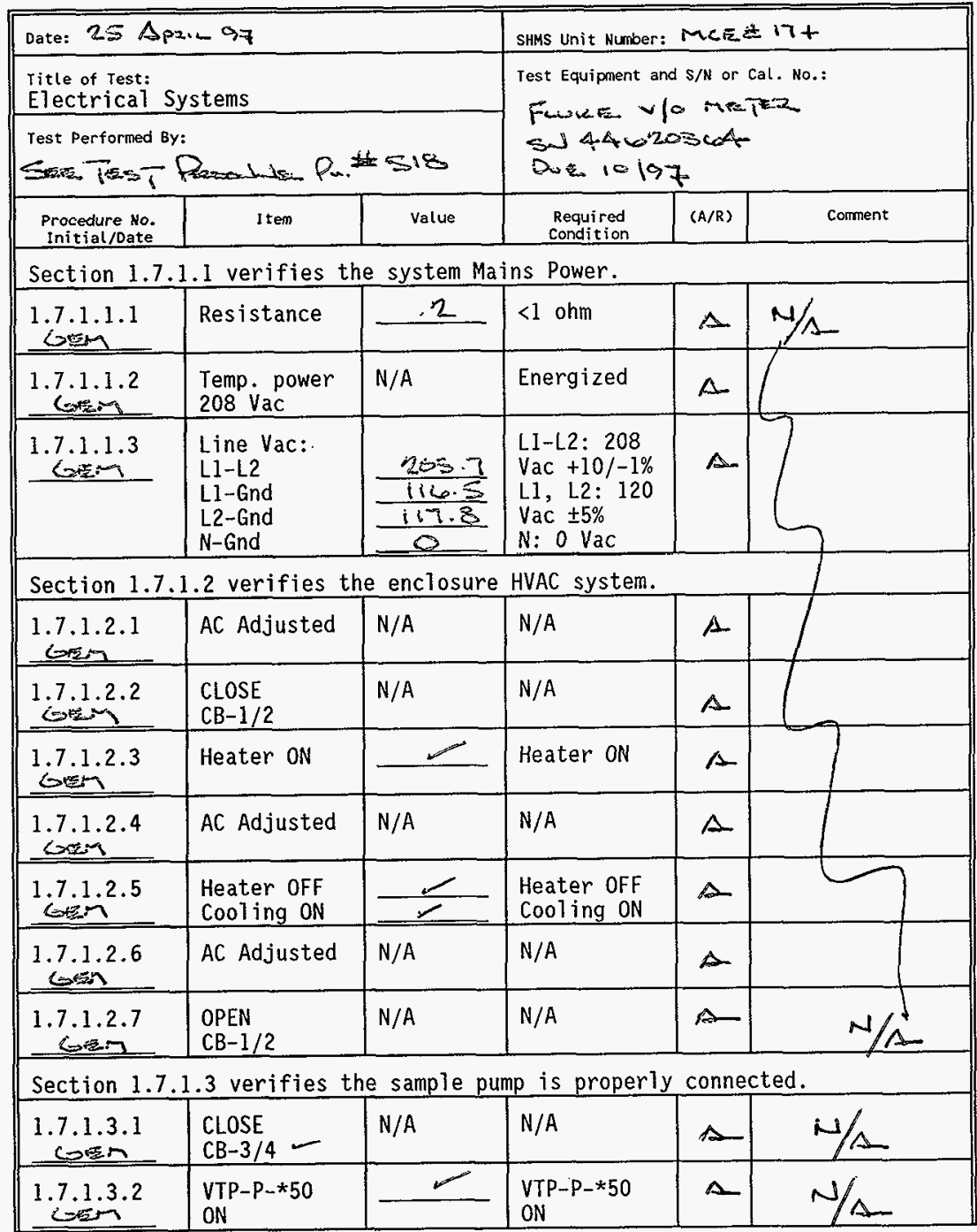

Gory sec $4 / 25 / 87$ 
TEST DATA SHEET

\begin{tabular}{|c|c|c|c|c|c|}
\hline \multicolumn{3}{|c|}{ Date: $25 A_{\text {man }} 97$} & \multicolumn{3}{|c|}{ 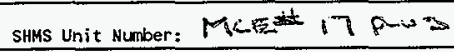 } \\
\hline \multicolumn{3}{|c|}{$\begin{array}{l}\text { Iitle of rest: } \\
\text { Electrical Systems }\end{array}$} & \multirow{2}{*}{\multicolumn{3}{|c|}{ 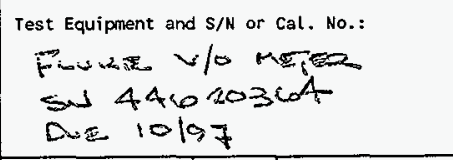 }} \\
\hline \multicolumn{3}{|c|}{ 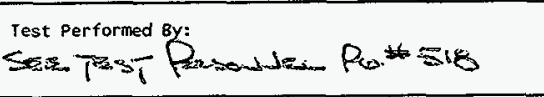 } & & & \\
\hline $\begin{array}{l}\text { Procedure No. } \\
\text { Initial/Date }\end{array}$ & Item & value & $\begin{array}{l}\text { Required } \\
\text { Condition } \\
\end{array}$ & $(A / R)$ & Comment \\
\hline $\begin{array}{l}1.7 .1 .3 .3 \\
\end{array}$ & $\begin{array}{l}\text { OPEN } \\
\text { CB-3/4 }\end{array}$ & N/A & N/A & $A$ & \\
\hline \multicolumn{6}{|c|}{ Section 1.7.1.4 verifies the enclosure lighting and GFCI receptacles. } \\
\hline $\begin{array}{l}1.7 .1 .4 .1 \\
\text { oen }\end{array}$ & $\begin{array}{l}\text { CLOSE } \\
\text { CB-6 }\end{array}$ & N/A & N/A & $\Delta$ & \\
\hline $\begin{array}{l}1.7 .1 .4 .2 \\
\text { Gen } \\
\end{array}$ & $\begin{array}{l}\text { ENCLOSURE } \\
\text { LIGHT }\end{array}$ & N/A & N/A & $\Delta$ & \\
\hline $\begin{array}{l}1.7 .1 .4 .3 \\
\end{array}$ & LIGHT ON & 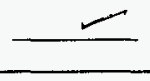 & LIGHT ON & $\Delta$ & \\
\hline$\underbrace{1.7 .1 .4 .4}$ & $\begin{array}{l}\text { GFCI RECP. } \\
\text { IA. L-N } \\
1 B . L-G N D \\
2 A . L-N \\
2 B . L-G N D \\
\end{array}$ & $\frac{\frac{116 \cdot 2}{116 \cdot 2}}{\frac{116 \cdot 1}{116 \cdot 1}}$ & $\begin{array}{l}1 \mathrm{~A} .120 \mathrm{Vac} \\
1 \mathrm{~B} .120 \mathrm{Vac} \\
2 \mathrm{~A} .120 \mathrm{VaC} \\
2 B .120 \mathrm{Vac} \\
\mathrm{ALL} \pm 5 \% \\
\end{array}$ & A. & \\
\hline 1.7 .1 .4 .5 & $\begin{array}{l}\text { GFCI TEST } \\
1 A . L-N \\
1 B . L-G N D \\
2 A . L-N \\
2 B . L-G N D\end{array}$ & $\frac{\frac{0}{1.6}}{\frac{.49}{1.1}}$ & $\begin{array}{l}\text { 1A. } 0 \text { Vac } \\
1 B .00 \mathrm{Vac} \\
2 \mathrm{~A} .0 \mathrm{Vac} \\
2 \mathrm{~B} .00 \mathrm{Vac} \\
\text { ALL } \pm 5 \% \\
\end{array}$ & $\Delta$ & \\
\hline $\begin{array}{l}1.7 .1 .4 .6 \\
\end{array}$ & $\begin{array}{l}\text { ENCLOSURE } \\
\text { LIGHT OFF }\end{array}$ & N/A & N/A & s & \\
\hline s.1. $^{1.7}$ & $\begin{array}{l}\text { OPEN } \\
\text { CB-6 - }\end{array}$ & N/A & N/A & $\Delta$ & $N / \alpha$ \\
\hline \multicolumn{6}{|c|}{ Section 1.7.1.5 verifies the instrument power source isolation transforme } \\
\hline $\begin{array}{l}1.7 .1 .5 .1 \\
6 \times 25 n \\
\end{array}$ & $\begin{array}{l}\text { CLOSE } \\
\text { CB-7 }\end{array}$ & N/A & N/A & $A$ & $N /$. \\
\hline $\begin{array}{l}1.7 .1 .5 .2 \\
6 x^{2}\end{array}$ & $\begin{array}{l}\text { XFRM Vac } \\
\text { PRI. L-N } \\
\text { SEC. L-N } \\
\text { SEC. N-GND } \\
\end{array}$ & $\frac{\frac{118.0}{120.4}}{.001}$ & $\begin{array}{l}\text { PRI. \& SEC. } \\
\text { L-N } 120 \pm 5 \% \\
\text { SEC. } \\
\text { N-GND O Vac }\end{array}$ & $A$ & \\
\hline 1.7 .1 .5 .3 & CLOSE FU-1 & $N / A$ & $N / A$ & $\Delta$ & $N / \mathrm{s}$ \\
\hline
\end{tabular}


TEST DATA SHEET

\begin{tabular}{|c|c|c|c|c|c|}
\hline \multicolumn{3}{|c|}{ Date: $25 A_{p a i n} 97$} & \multicolumn{3}{|c|}{ shms unit Number: MkE 71 pus } \\
\hline \multicolumn{3}{|c|}{$\begin{array}{l}\text { Title of rest: } \\
\text { Electrical Systems }\end{array}$} & \multirow{2}{*}{\multicolumn{3}{|c|}{ 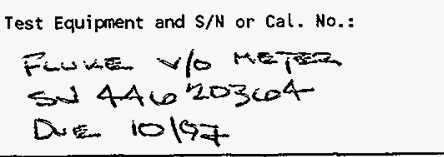 }} \\
\hline \multicolumn{3}{|c|}{ 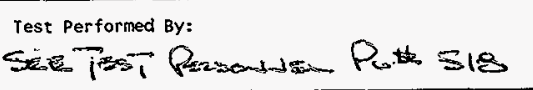 } & & & \\
\hline $\begin{array}{l}\text { Procedure No. } \\
\text { Initial/Date }\end{array}$ & Item & Value & $\begin{array}{l}\text { Required } \\
\text { Condition } \\
\end{array}$ & $(A / R)$ & Comment \\
\hline $\begin{array}{l}1.7 .1 .5 .4 \\
6 x .4\end{array}$ & $\begin{array}{l}\text { VTP-PS-*50 } \\
\text { OUTPUT Vdc }\end{array}$ & 24.02 & $24 \pm 0.1 \mathrm{Vdc}$ & $\Delta$ & $N / s$ \\
\hline \multicolumn{6}{|c|}{ Section 1.7.1.6 verifies the enclosure general alarm system. } \\
\hline $\begin{array}{c}1.7 .1 .6 .1 \\
0 \times+1 \\
\end{array}$ & $\begin{array}{l}\text { CLOSE FU-9 \& } \\
\text { FU-10 - }\end{array}$ & N/A & N/A & A & . \\
\hline $\begin{array}{c}1.7 .1 .6 .2 \\
\text { Sogn } \\
\end{array}$ & $\begin{array}{l}\text { PUSH PB-*51 } \\
\& P B-* 50\end{array}$ & $N / A$ & $N / A$ & $\Lambda$ & \\
\hline 1.7 .1 .6 .3 & $\begin{array}{l}\text { ALARM TEST } \\
\text { VERIFY ALARM } \\
\text { CONDITIONS }\end{array}$ & 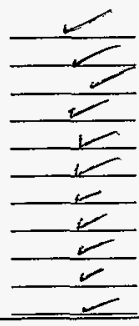 & $\begin{array}{ll}\text { YAH-*50 } & \text { ON } \\
\text { NAH-*55 } & \text { ON } \\
\text { XA-*63 } & \text { ON } \\
\text { NAH-*54 } & \text { ON } \\
\text { FAL-*57 } & \text { ON } \\
\text { TAHL-*62 ON } & \text { ON } \\
\text { TAL-*50 } & \text { ON } \\
\text { YAL-*58 } & \text { ON } \\
\text { PBL-*58 } & \text { ON } \\
\text { PBL-*54 } & \text { OFF } \\
\text { PBL-*59 } & \text { OFF } \\
\end{array}$ & $A$ & \\
\hline $\begin{array}{l}1.7 .1 .6 .4 \\
\end{array}$ & $\begin{array}{l}\text { END ALARM } \\
\text { TEST } \\
\end{array}$ & $\mathrm{N} / \mathrm{A}$ & N/A & 1 & \\
\hline $\begin{array}{l}1.7 .1 .6 .5 \\
6.2\end{array}$ & RESET ALARMSS & N/A & $N / A$ & $\alpha$ & \\
\hline 1.7 .1 .6 .6 & $\begin{array}{l}\text { OPEN FU-9 \& } \\
\text { FU-10 }\end{array}$ & $N / A$ & $N / A$ & A & \\
\hline \multicolumn{6}{|c|}{ Section 1.7.1.7 verifies the flow alarm system. } \\
\hline $\begin{array}{l}1.7 .1 .7 .1 \\
\dot{g}\end{array}$ & $\begin{array}{l}\text { CLOSE FU-3,- } \\
\text { FU-9, FU-10- } \\
\& \text { FU-13- }\end{array}$ & $N / A$ & N/A & s & \\
\hline $\begin{array}{l}1.7 .1 .7 .2 \\
\end{array}$ & RESET ALARMS & $\infty$ & $\mathrm{FAL}-\star 57$ ON & $\Delta$ & \\
\hline $\begin{array}{l}1.7 .1 .7 .3 \\
\text { sin }\end{array}$ & $\begin{array}{l}\text { OPEN SV-*20 } \\
\& \text { SV-*22 }\end{array}$ & $N / A$ & N/A & $\Delta$ & $N / \Delta$ \\
\hline
\end{tabular}

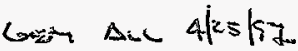


TEST DATA SHEET

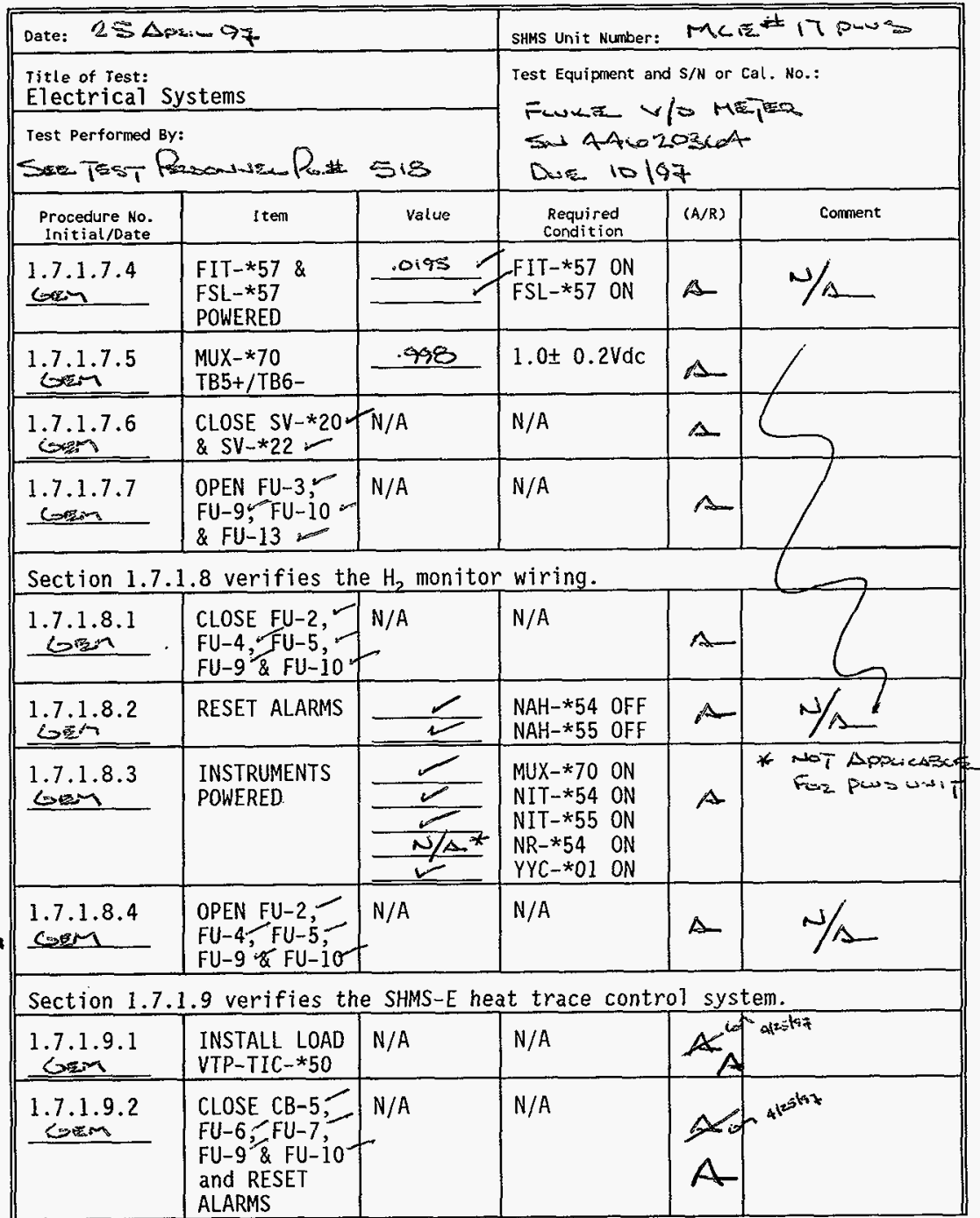


TEST DATA SHEET

\begin{tabular}{|c|c|c|c|c|c|}
\hline \multicolumn{3}{|c|}{ Date: $25 \Delta p \pm 1.97$} & SHMS Unit Number: & \multicolumn{2}{|c|}{ Mcet 17 puss } \\
\hline \multicolumn{3}{|c|}{$\begin{array}{l}\text { Title of Test: } \\
\text { Electrical Systems }\end{array}$} & \multirow{2}{*}{\multicolumn{3}{|c|}{ Test Equipment and $\mathrm{S} / \mathrm{N}$ or Cal. No.: }} \\
\hline \multicolumn{3}{|c|}{ 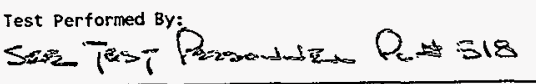 } & & & \\
\hline $\begin{array}{l}\text { Procedure No. } \\
\text { Initial/Oate }\end{array}$ & item & Value & $\begin{array}{l}\text { Required } \\
\text { Condition } \\
\end{array}$ & $(A / R)$ & Comment \\
\hline 1.7.1.9.3 & $\begin{array}{l}\text { TIC-*50 \& } \\
\text { TIC-*56 } \\
\text { NOMINAL TEMP } \\
\end{array}$ & $\frac{\cos F}{\cos F}$ & $\begin{array}{l}\text { TIC }-* 50 \\
\text { TIC-*56 } \\
\text { TEMPERATURE }\end{array}$ & $\begin{array}{c}N^{n+2} \\
A\end{array}$ & \\
\hline $\begin{array}{l}1.7 .1 .9 .4 \\
0.1\end{array}$ & $\begin{array}{l}\text { ADJ TIC }-* 50 \\
\& \text { TIC }-* 56 \\
\text { SP2 \& RESET } \\
\text { ALARMS } \\
\end{array}$ & $N / A$ & $N / A$ & $\Lambda$ & \\
\hline $\begin{array}{l}1.7 .1 .9 .5 \\
0200\end{array}$ & $\begin{array}{l}\text { ADJ TIC }-\star 50 \\
\text { SP } 1\end{array}$ & $N / A$ & $N / A$ & A & \\
\hline G.1.9.6 & $\begin{array}{l}\text { ADJ } T I C-* 50 \\
\text { SP } 2\end{array}$ & $N / A$ & $N / A$ & & \\
\hline 1.7 .1 .9 .7 & $\begin{array}{l}\text { TAL-*50\& } \\
\text { HORN ACTIVE }\end{array}$ & $\frac{1}{2}$ & $\begin{array}{l}\text { TAL } * 50 \text { ON } \\
\text { HORN ON } \\
\text { ACKNOWLEDGE }\end{array}$ & $A$ & \\
\hline 1.7 .1 .9 .8 & $\begin{array}{l}\text { TIC }-* 50 \\
\text { OPERATES }\end{array}$ & 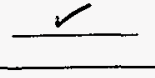 & $\begin{array}{l}\text { TIC }-* 50 \\
\text { OPERATES } \\
\end{array}$ & $A$ & \\
\hline $\mathrm{Gien}^{1.7 .1 .9 .9}$ & $\begin{array}{l}\text { ADJ TIC }-* 50 \\
\text { SP } 2\end{array}$ & $N / A$ & $N / A$ & $\curvearrowright$ & \\
\hline 6.7 .1 .9 .10 & RESET ALARM & $\nearrow$ & TAL $-\star 50$ OFF & ser & \\
\hline $\operatorname{sen}^{1.7 .1 .9 .11}$ & $\begin{array}{l}A D J \text { TIC-*50 } \\
S P 1 \& S P 2\end{array}$ & $N / A$ & $N / A$ & 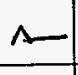 & \\
\hline $\begin{array}{l}1.7 .1 .9 .12 \\
\end{array}$ & $\begin{array}{l}\text { OPEN CB-5 \& } \\
\text { REMOVE LOAD }\end{array}$ & $N / A$ & $N / A$ & $A$ & \\
\hline $\begin{array}{r}1.7 .1 .9 .13 \\
6 \cos _{4} .425 b_{7} \\
\end{array}$ & $\begin{array}{l}\text { INSTALL LOAD } \\
\text { VTP-TIC-*56 } \\
\end{array}$ & $N / A$ & N/A & A & $N / A$ \\
\hline $\begin{array}{c}1.7 .1 .9 .14 \\
\end{array}$ & CLOSE CB-5 & $\mathrm{N} / \mathrm{A}$ & N/A & $\Delta$ & \\
\hline $\begin{array}{l}1.7 .1 .9,15 \\
\operatorname{sen} 4.20157 \\
\end{array}$ & $\begin{array}{l}\text { ADJ TIC-*56 } \\
\text { SP } 1\end{array}$ & $N / A$ & $N / A$ & $A$ & TiF \\
\hline 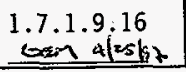 & $\begin{array}{l}\text { ADJ TIC }-* 56 \\
\text { SP } 2\end{array}$ & $N / A$ & $N / A$ & $\Delta$ & \\
\hline
\end{tabular}


TEST DATA SHEET

\begin{tabular}{|c|c|c|c|c|c|}
\hline \multicolumn{3}{|c|}{ Date: $25 \Delta$ bs:- 97} & SHMS Unit Number: & \multicolumn{2}{|c|}{ 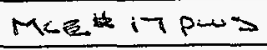 } \\
\hline \multicolumn{3}{|c|}{$\begin{array}{l}\text { Titie of Test: } \\
\text { Electrical Systems }\end{array}$} & \multirow{2}{*}{\multicolumn{3}{|c|}{ Test Equipment and $\mathrm{S} / \mathrm{N}$ or Cal. No.: }} \\
\hline \multicolumn{3}{|c|}{ 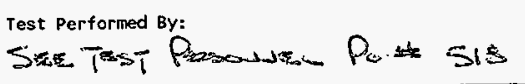 } & & & \\
\hline $\begin{array}{l}\text { Procedure No. } \\
\text { Initial/Date }\end{array}$ & It ten & value & $\begin{array}{l}\text { Required } \\
\text { Condition }\end{array}$ & $(A / R)$ & Comment \\
\hline $\begin{array}{l}1.7 .1 .9 .17 \\
\text { sem }\end{array}$ & $\begin{array}{l}\text { TAL-*50 \& } \\
\text { HORN ACTIVE }\end{array}$ & $\frac{\swarrow}{2}$ & $\begin{array}{l}\text { TAL } * 50 \text { ON } \\
\text { HORN ON } \\
\text { ACKNOWLEDGE }\end{array}$ & A & \\
\hline 1.7 .1 .9 .18 & $\begin{array}{l}\text { TIC }-* 56 \\
\text { OPERATES } \\
\end{array}$ & $r$ & $\begin{array}{l}\text { TIC }-\star 56 \\
\text { OPERATES }\end{array}$ & $\Delta$ & \\
\hline $\begin{array}{l}1.7 .1 .9 .19 \\
\end{array}$ & $\begin{array}{l}\text { ADJ TIC-*56 } \\
\text { SP } 2\end{array}$ & $N / A$ & N/A & $\Delta$ & \\
\hline$\underbrace{1.7 .1 .9 .20}$ & RESET ALARM & 2 & TAL- $* 50$ OFF & $A$ & \\
\hline $\begin{array}{l}1.7 .1 .9 .21 \\
6.9 .0 \\
\end{array}$ & $\begin{array}{l}A D J \text { TIC }-* 56 \\
S P \text { I \& SP 2 }\end{array}$ & N/A & N/A & $n$ & \\
\hline $\begin{array}{c}1.7 .1 .9 .22 \\
\end{array}$ & $\begin{array}{l}\text { OPEN CB-5, } \\
\text { FU-6 \& FU-7 }\end{array}$ & $N / A$ & N/A & 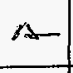 & \\
\hline $\begin{array}{l}1.7 .1 .9 .23 \\
\end{array}$ & $\begin{array}{l}\text { REMOVE LOAD } \\
\text { TIC-*56}\end{array}$ & $N / A$ & $N / A$ & $\Delta$ & \\
\hline 1.7.1.9.24 & $\begin{array}{l}\text { CLOSE FU-8 } \\
\text { TIS-*62 } \\
\text { NOMINAL TEMP }\end{array}$ & $71^{\circ}$ & $\begin{array}{l}\text { TIS-*62 } \\
\text { TEMP }\end{array}$ & $A$ & \\
\hline $\begin{array}{l}1.7 .1 .9 .25 \\
6.5 \\
\end{array}$ & $\begin{array}{l}\text { ADJ TIS-*62 } \\
\text { SP } 1\end{array}$ & $N / A$ & $N / A$ & $\wedge$ & \\
\hline $\begin{array}{l}1.7 .1 .9 .26 \\
\end{array}$ & $\begin{array}{l}\text { ADJ TIS-*62 } \\
\text { SP } 2 \\
\end{array}$ & $N / A$ & $N / A$ & $\Delta$ & \\
\hline $\begin{array}{l}1.7 .1 .9 .27 \\
6 \cosh \\
\end{array}$ & RESET ALARMS & $\infty$ & $\begin{array}{l}\text { TAHL }-* 62 \\
\text { OFF }\end{array}$ & 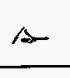 & \\
\hline 1.7 .1 .9 .28 & $\begin{array}{l}\text { ADJ TIS-*62 } \\
\text { SP } 2 \\
\end{array}$ & $N / A$ & $N / A$ & $\Delta$ & \\
\hline $\begin{array}{l}1.7 .1 .9 .29 \\
0.7\end{array}$ & $\begin{array}{l}\text { TAHL }-* 62 \& \\
\text { HORN ACTIVE }\end{array}$ & $\frac{r}{2}$ & $\begin{array}{l}\text { TAHL-*62 ON } \\
\text { HORN ON } \\
\text { ACKNOWLEDGE }\end{array}$ & A & \\
\hline $\begin{array}{l}1.7 .1 .9 .30 \\
\operatorname{sen}\end{array}$ & $\begin{array}{l}\text { ADJ TIS }-* 62 \\
\text { SP } 2\end{array}$ & $N / A$ & $N / A$ & $\Delta$ & $\omega / \Delta$ \\
\hline
\end{tabular}


APPENDIX $Q$

HNF-SD-WM-ATR - 191

PAGE Q- /

Rev. 0

TEST DATA SHEET

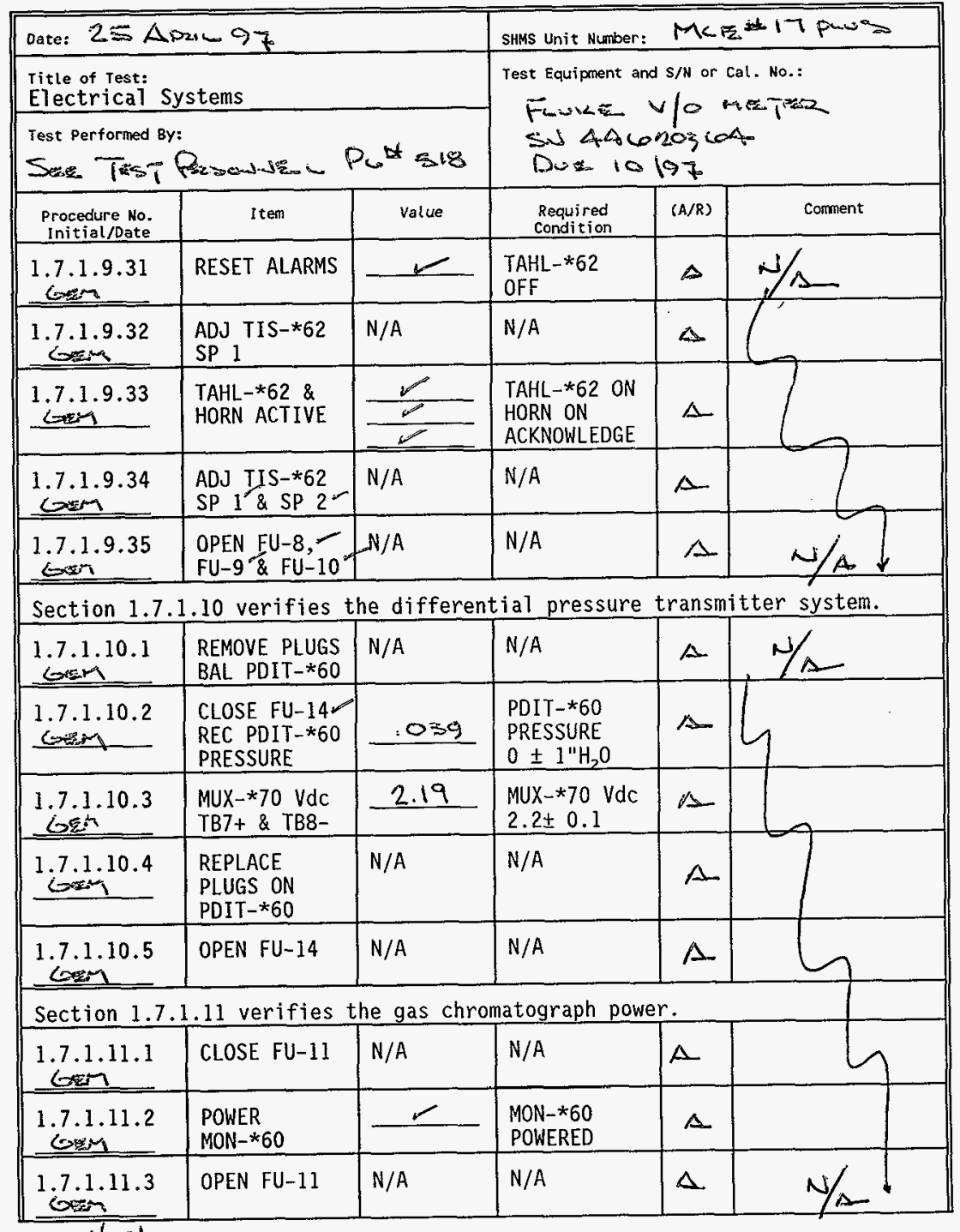


APPENDIX Q

PAGE $Q-12$

$$
\begin{array}{r}
\text { HNF-SO-WM-ATR-191 } \\
\text { ReV. } 0 \\
\text { Page } 528
\end{array}
$$

TEST DATA SHEET

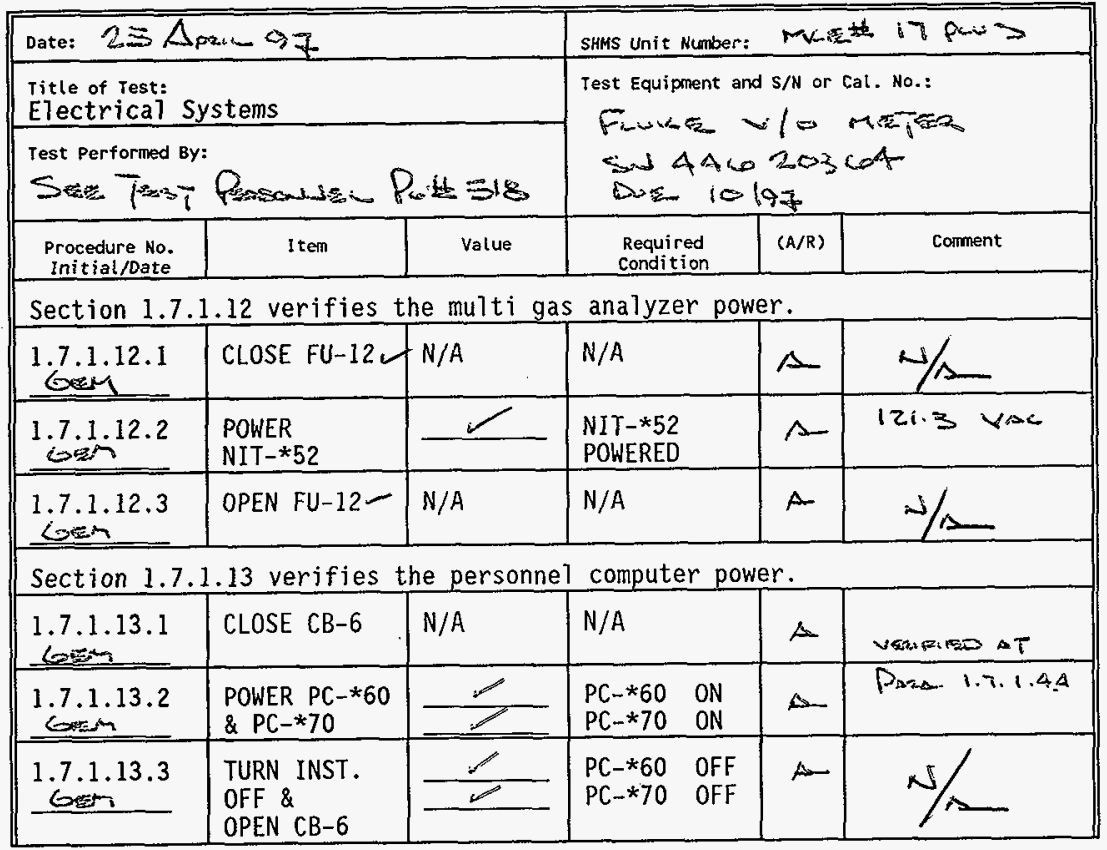

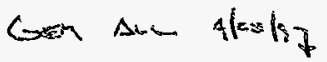


TEST DATA SHEET

\begin{tabular}{|c|c|c|c|c|c|}
\hline \multicolumn{3}{|c|}{ Date: $2 \equiv \Delta p$ pri- 97} & SHMS Unit Numb & \multicolumn{2}{|c|}{ MLE iT PUS } \\
\hline \multicolumn{3}{|c|}{$\begin{array}{l}\text { Titie of Test: } \\
\text { Electrical Systems }\end{array}$} & \multirow{2}{*}{\multicolumn{3}{|c|}{ 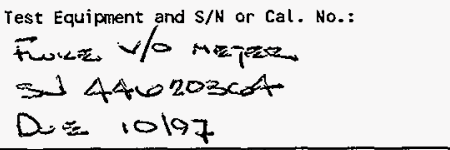 }} \\
\hline \multicolumn{3}{|c|}{ 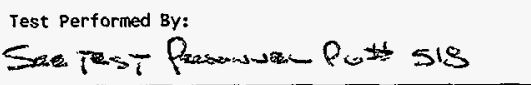 } & & & \\
\hline $\begin{array}{l}\text { Procedure No. } \\
\text { Initial/Date }\end{array}$ & Item & Value & $\begin{array}{l}\text { Required } \\
\text { Condition } \\
\end{array}$ & $(A / R)$ & Comment \\
\hline \multicolumn{6}{|c|}{ Section 1.7 .2 verifies the intrinsic safety apparatus is properly labelec } \\
\hline $\operatorname{sen}$ & $\begin{array}{l}\text { INTRINSIC } \\
\text { SAFETY } \\
\text { APPARATUS } \\
\text { NAMEPLATES } \\
\text { INSTALLED }\end{array}$ & $\frac{-\frac{1}{1}}{\frac{1}{1}}$ & $\begin{array}{l}\text { NE }-* 54 \\
\text { NE }-* 55 \\
E B-* 54 \\
E B-* 55 \\
\text { NIT } * 54 \\
\text { NIT }-* 55 \\
\end{array}$ & A & \\
\hline $\begin{array}{l}1.7 .2 .2 \\
\sin \end{array}$ & $\begin{array}{l}\text { INTRINSIC } \\
\text { SAFETY WIRE } \\
\text { LABELED }\end{array}$ & 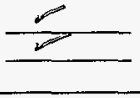 & $\begin{array}{l}N E / E B-* 54 \\
N E / E B-* 55\end{array}$ & 今 & \\
\hline $\begin{array}{l}1.7 .3 \\
6 \sin \sin 2\end{array}$ & $\begin{array}{l}\text { SEC. } 1.7 .1 \text { \& } \\
1.7 .2 \text { DONE }\end{array}$ & groud 11 & Brom & & $\frac{-s 7}{\text { Date }}$ \\
\hline
\end{tabular}

Test Witness/Review:

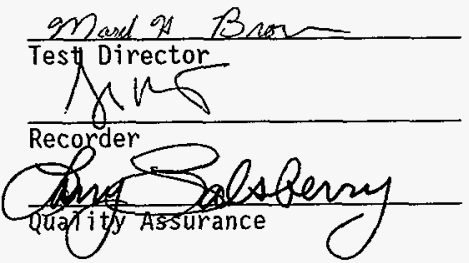

$\frac{5 \cdot 15-97}{\text { Date }}$
s/.9197
Date
$\frac{5 / 28 / 97}{\text { Date }}$


TEST DATA SHEET

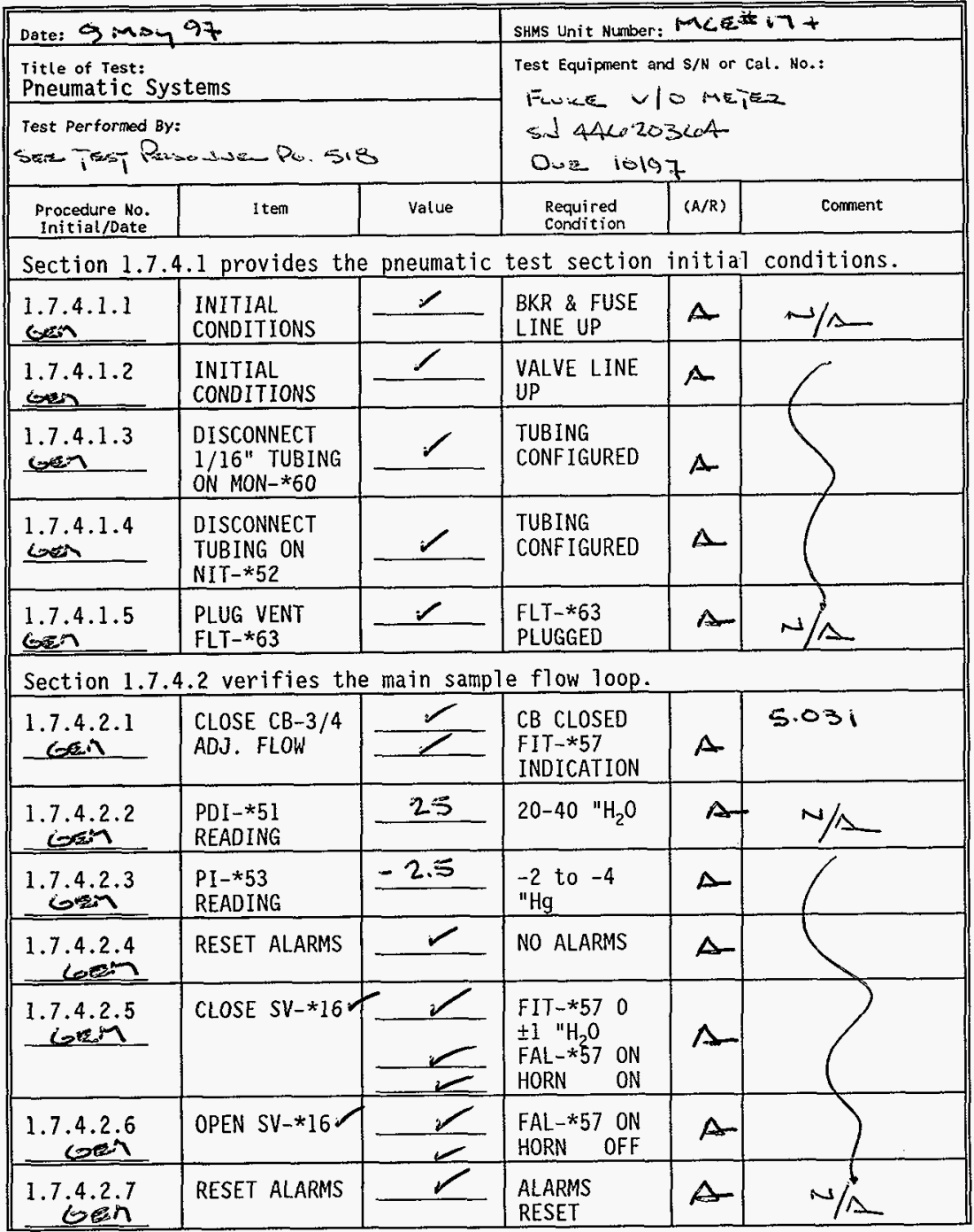


TEST DATA SHEET

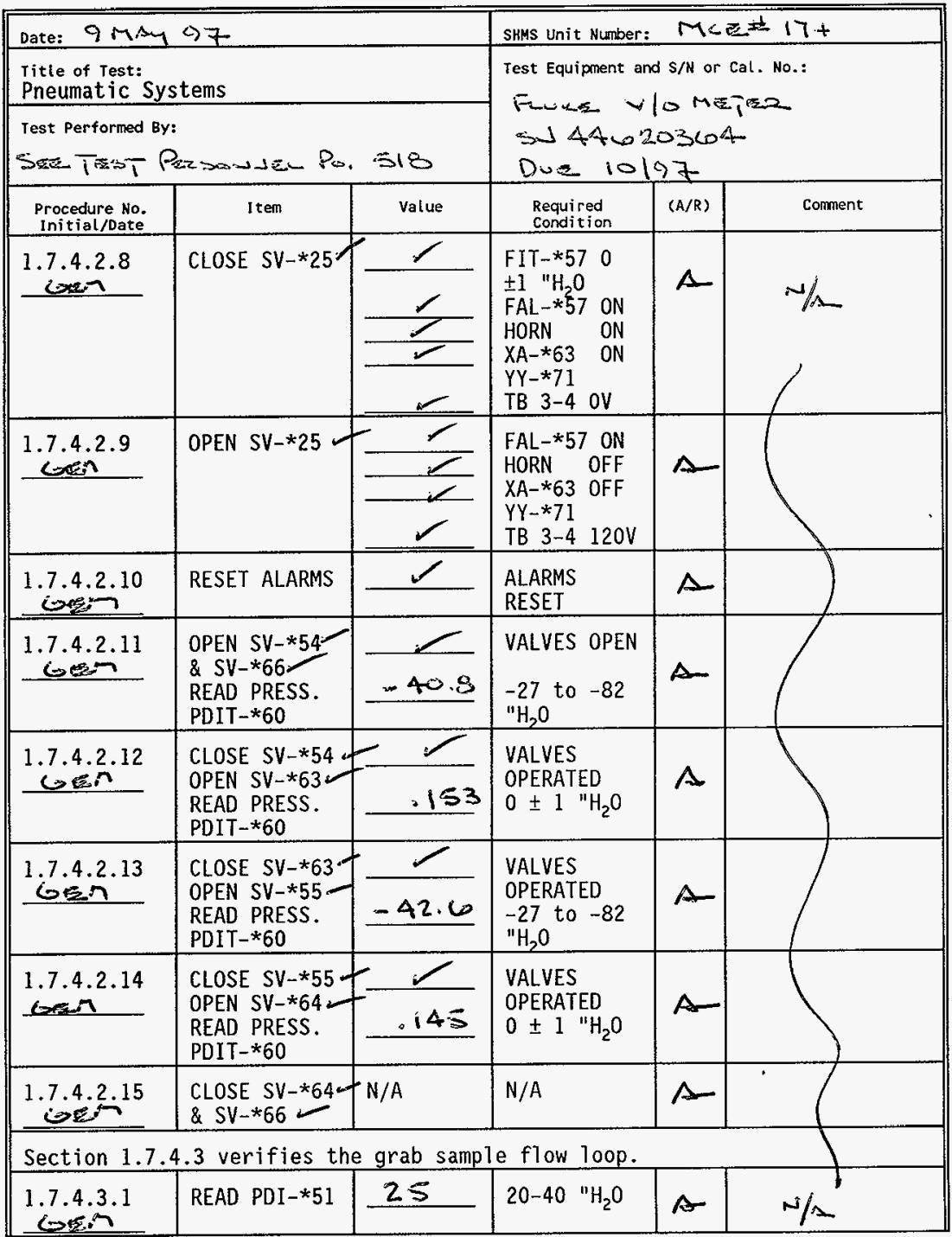


TEST DATA SHEET

\begin{tabular}{|c|c|c|c|c|c|}
\hline Date: $9 M A$ & 97 & & SHMS Unit Number: & Mr & $17+$ \\
\hline $\begin{array}{l}\text { Title of Test: } \\
\text { Pneumatic } S\end{array}$ & tems & & Test Equipment an & $S / N$ or & No.: \\
\hline $\begin{array}{l}\text { Test Performed } \\
\text { Sez TES }\end{array}$ & Passond. Pen & SIS & $\omega$ & & \\
\hline $\begin{array}{l}\text { Procedure No. } \\
\text { Initial/Date }\end{array}$ & Iten & Value & $\begin{array}{l}\text { Required } \\
\text { Condition }\end{array}$ & $(A / R)$ & Comment \\
\hline $\begin{array}{c}1.7 .4 .3 .2 \\
4 .-7 \\
\end{array}$ & $\begin{array}{l}\text { PUSH PB- } * 59 \\
\text { GRAB SAMPLE }\end{array}$ & $r$ & $\begin{array}{l}\text { START } \\
\text { STOPWATCH }\end{array}$ & $\Omega$ & \\
\hline $\begin{array}{l}1.7 .4 .3 .3 \\
6 \cos \end{array}$ & $\begin{array}{l}\text { GRAB SAMPLE } \\
\text { LAMPS ON } \\
\text { READ FIV-*52 }\end{array}$ & $\frac{2}{2}$ & $\begin{array}{l}\text { PBL-*58 ON } \\
\text { PBL-*59 ON } \\
\text { YAL }-* 58 \text { ON } \\
\text { FIV }-* 52 \quad 0 \\
\end{array}$ & 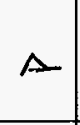 & \\
\hline 1.7 .4 .3 .4 & $\begin{array}{l}\text { OPEN SV }-* 15 \\
\& \text { ADJ. FIV- } \\
* 52\end{array}$ & $\begin{array}{l}1 \\
10 \\
\end{array}$ & $\begin{array}{l}\text { SV-*15 OPEN } \\
\text { FIV-*52 } \\
\text { FLOW } 10 \text { CFH }\end{array}$ & 1 & \\
\hline G.7.3.5 & READ PDI $-\star 51$ & 20 & $\begin{array}{l}\text { PDI-*51 } \\
\text { HIGHER THAN } \\
1.7 .4 .3 .1\end{array}$ & $A$ & \\
\hline $\cos ^{1.7 .4 .3 .6}$ & CLOSE SV $-* 154$ & 0 & $\mathrm{FIV}-* 520$ & $\Delta$ & \\
\hline $\begin{array}{l}1.7 .4 .3 .7 \\
\end{array}$ & OPEN SV-*15 & 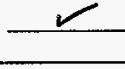 & $\begin{array}{l}\text { FIV-*52 } \\
\text { FLOW }\end{array}$ & $A$ & \\
\hline 1.7 .4 .3 .8 & $\begin{array}{l}\text { PBL }-* 59 \\
\text { GRAB SAMPLE } \\
\text { TIME }\end{array}$ & $S$ & $\begin{array}{l}\text { PBL-*59 OFF } \\
\text { STOP WATCH } \\
5 \pm .5 \text { MIN }\end{array}$ & $\Delta$ & \\
\hline${ }^{1.7 .4 .3 .9}$ & $\begin{array}{l}\text { PB-*58 RESET } \\
\text { SAMPLER }\end{array}$ & 2 & $\begin{array}{l}\mathrm{PBL}-* 58 \text { OFF } \\
\text { YAL-*58 OFF } \\
\end{array}$ & $\Delta$ & \\
\hline Section 1.7 & 4 verifies th & $\mathrm{H}_{2}$ cell & Jibration 100 & & \\
\hline 1.7 .4 .4 .1 & $\begin{array}{l}\text { CONNECT } \mathrm{H}_{2} \\
\text { CAL GAS }\end{array}$ & $N / A$ & $N / A$ & 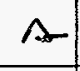 & \\
\hline $\begin{array}{l}1.7 .4 .4 .2 \\
x^{2}\end{array}$ & $\begin{array}{l}\text { OPEN ISO } \\
\text { VALVE }\end{array}$ & 5 & $<10$ PSIG & $A$ & \\
\hline$\underline{L}^{1.7 .4 .4 .3}$ & $\begin{array}{l}\text { OPEN SV-*18 } \\
\text { ADJ. FIV }-* 56 *\end{array}$ & 2 & $2 \pm .1 \mathrm{CFH}$ & $\triangle$ & $\perp$ \\
\hline
\end{tabular}


TEST DATA SHEET

\begin{tabular}{|c|c|c|c|c|c|}
\hline Date: $9 \mathrm{Ms}$ & 97 & & SHMS Unit Number & MLE & 177 \\
\hline $\begin{array}{l}\text { Title of Test: } \\
\text { Pneumatic S }\end{array}$ & tems & & rest Equipment a & $S / N$ or & \\
\hline $\begin{array}{l}\text { Test Performed } \\
\text { Saz Trist }\end{array}$ & sossta- $P_{\omega}$ & 518 & $\begin{array}{l}\text { Sud } 446 \\
\text { Due } 10\end{array}$ & 203 & \\
\hline $\begin{array}{l}\text { Procedure No. } \\
\text { Initial/Date }\end{array}$ & Item & value & $\begin{array}{l}\text { Required } \\
\text { Condition } \\
\end{array}$ & $(A / R)$ & Comment \\
\hline $\begin{array}{l}1.7 .4 .4 .4 \\
1\end{array}$ & $\begin{array}{l}\text { VERIFY } \\
\text { CONDITIONS }\end{array}$ & $\frac{2}{2}$ & $\begin{array}{l}\text { NIT-*554 } \\
>>625 \% H_{3} \\
\text { NAH-*55 ON } \\
\text { NAH-*54 ON } \\
\text { PBL-*59 ON } \\
\text { PBL-*58 ON } \\
\text { YAL-*58 ON } \\
\text { FIV-*52 FLO } \\
\text { HORN ON } \\
\text { TB2-21/22 } \\
\text { OPEN }\end{array}$ & $\Lambda$ & \\
\hline 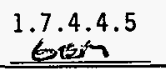 & $\begin{array}{l}\text { ACKNOWLEDGE } \\
\text { HORN }\end{array}$ & $N / A$ & $N / A$ & $\Delta$ & \\
\hline $\begin{array}{l}1.7 .4 .4 .6 \\
\end{array}$ & $\begin{array}{l}\text { CLOSE SV-*18. } \\
\text { VERIFY } \\
\text { CONDITIONS }\end{array}$ & 2 & $\begin{array}{l}\text { FIV-*56 O } \\
\text { NIT-*54 } \\
<.625 \% \mathrm{H}_{2} \\
\text { NAH-*55 OFF } \\
\text { NAH- } * 54 \text { ON } \\
\text { PBL-*58 ON } \\
\text { YAL-*58 ON } \\
\text { TB2-21/22 } \\
\text { CLOSED }\end{array}$ & A & \\
\hline $\begin{array}{c}1.7 .4 .4 .7 \\
20.7\end{array}$ & $\begin{array}{l}\text { PUSH RESET } \\
\text { SAMPLER PB- } \\
* 58 \\
\end{array}$ & $\swarrow$ & $\begin{array}{l}\text { PBL-*58 OFF } \\
\text { YAL-*58 OFF }\end{array}$ & 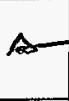 & \\
\hline $\begin{array}{c}1.7 .4 .4 .8 \\
60^{-7} \\
\end{array}$ & RESET ALARM & 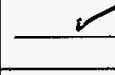 & $\mathrm{NAH}-* 54$ OFF & $A$ & \\
\hline $\begin{array}{l}1.7 .4 .4 .9 \\
\text { Gein }\end{array}$ & $\begin{array}{l}\text { OPEN SV }-* 19 \\
\text { ADJ. FIV }-* 56\end{array}$ & 2 & $2 \pm .1 \mathrm{CFH}$ & $\Lambda$ & $\omega / \Delta$ \\
\hline
\end{tabular}


TEST DATA SHEET

\begin{tabular}{|c|c|c|c|c|c|}
\hline \multicolumn{3}{|c|}{ Date: 9 mon 97} & \multicolumn{3}{|c|}{ SHMS Unit Number: MCE仿 $17+$} \\
\hline \multicolumn{3}{|c|}{$\begin{array}{l}\text { Title of Test: } \\
\text { Pneumatic Systems }\end{array}$} & \multirow{2}{*}{\multicolumn{3}{|c|}{ 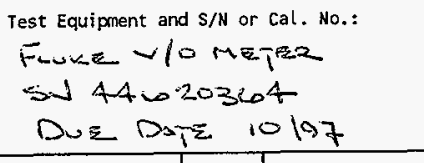 }} \\
\hline \multicolumn{3}{|c|}{ 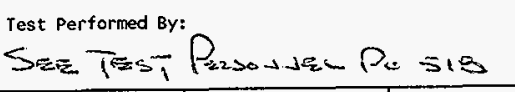 } & & & \\
\hline $\begin{array}{l}\text { Procedure No. } \\
\text { Initial/Date }\end{array}$ & Item & value & $\begin{array}{l}\text { Required } \\
\text { Condition } \\
\end{array}$ & $(A / R)$ & Comment \\
\hline 6. & $\begin{array}{l}\text { VERIFY } \\
\text { CONDITIONS }\end{array}$ & $\square$ & $\begin{array}{l}\mathrm{NIT}-* 55 \\
>.625 \% \mathrm{H}_{2} \\
\mathrm{NAH}-\star 55 \text { ON } \\
\text { NAH-*54 ON } \\
\text { PBL-*59 ON } \\
\mathrm{PBL}-* 58 \text { ON } \\
\text { YAL-*58 ON } \\
\text { FIV-*52 FLO } \\
\text { HORN ON } \\
\text { TB2-21/22 } \\
\text { OPEN } \\
\end{array}$ & $A$ & \\
\hline $\begin{array}{l}1.7 .4 .4 .11 \\
6.4 \\
\end{array}$ & $\begin{array}{l}\text { ACKNOWLEDGE } \\
\text { HORN }\end{array}$ & N/A & N/A & $\Delta$ & \\
\hline 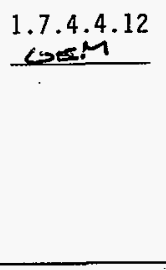 & $\begin{array}{l}\text { CLOSE SV }-* 19 \\
\text { VERIFY } \\
\text { CONDITIONS }\end{array}$ & & $\begin{array}{l}\text { FIV- } * 560 \\
\text { NIT-*55 } \\
<.625 \% \mathrm{H}_{2} \\
\text { NAH- } * 55 \mathrm{OFF} \\
\text { NAH- } * 54 \text { ON } \\
\text { PBL-*58 ON } \\
\text { YAL-*58 ON } \\
\text { TB2-21/22 } \\
\text { CLOSED } \\
\end{array}$ & $\Delta$ & \\
\hline$\frac{1.7 .4 .4 .13}{8}$ & $\begin{array}{l}\text { PUSH RESET } \\
\text { SAMPLER PB- } \\
\star 58=\end{array}$ & & $\begin{array}{l}\mathrm{PBL}-* 58 \text { OFF } \\
\mathrm{YAL}-* 58 \text { OFF }\end{array}$ & A & \\
\hline $\begin{array}{l}1.7 .4 .4 .14 \\
\end{array}$ & RESET ALARM & & $\mathrm{NAH}-\star 54$ OFF & $\Delta$ & \\
\hline 1.7 .4 .4 .15 & $\begin{array}{l}\text { DISCONNECT } \\
\text { CAL. GAS }\end{array}$ & $N / A$ & $N / A$ & $A$ & \\
\hline 1.7 .4 .4 .16 & $\begin{array}{l}\text { PUSH PB-*54- } \\
\text { VERIFY } \\
\text { CONDITIONS }\end{array}$ & & $\begin{array}{l}\text { XA-*63 ON } \\
\text { PBL-*54 ON } \\
\text { TB2-23/24 } \\
\text { OPEN } \\
\end{array}$ & $\Delta$ & \\
\hline $\begin{array}{l}1.7 .4 .4 .17 \\
6 \times 2.17\end{array}$ & $\begin{array}{l}\text { PUSH PB-*54- } \\
\text { VERIFY } \\
\text { CONDITIONS }\end{array}$ & & $\begin{array}{l}X A-* 63 \quad 0 F F \\
P B L-* 54 \text { OFF } \\
\text { TB2-23/24 } \\
\text { CLOSED }\end{array}$ & $A$ & $\mathrm{~N} / \mathrm{s}^{\mathrm{h}}$ \\
\hline
\end{tabular}


TEST DATA SHEET

\begin{tabular}{|c|c|c|c|c|c|}
\hline Date: $9 \mathrm{MA}$ & 07 & & SHMS Unit Number & MCD & $17+$ \\
\hline $\begin{array}{l}\text { Title of rest: } \\
\text { Pneumatic } S\end{array}$ & tems & & Test Equipment a & $\mathrm{S} / \mathrm{N}$ or & No.: \\
\hline $\begin{array}{l}\text { Test Performed } \\
\text { Sis. TES }\end{array}$ & Persoustal $P$ & 518 & & $1 / \Delta$ & \\
\hline $\begin{array}{l}\text { Procedure No. } \\
\text { Initial/Date }\end{array}$ & Item & value & $\begin{array}{l}\text { Required } \\
\text { Condition } \\
\end{array}$ & $(A / R)$ & Comment \\
\hline Section 1.7 & 5 verifies th & GC i & nt tubing ar & calit & 701 \\
\hline$\underbrace{1.7 .4 .5 .1}$ & $\begin{array}{l}\text { REMOVE PLUG } \\
\text { \& CONNECT } \\
\text { TEST GAS } \\
\end{array}$ & 5 & $\begin{array}{l}\text { TEST GAS } \\
3-5 \text { PSIG }\end{array}$ & $\Lambda$ & \\
\hline $\begin{array}{c}1.7 .4 .5 .2 \\
6\end{array}$ & $\begin{array}{l}\text { VERIFY NO } \\
\text { GAS FLOW }\end{array}$ & 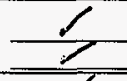 & $\begin{array}{l}\mathrm{H}_{2} \text { GAS PORT } \\
\text { SAMPLE A IN } \\
\end{array}$ & $\Delta$ & \\
\hline $\begin{array}{c}1.4 .5 .3 \\
\end{array}$ & $\begin{array}{l}\text { VERIFY COL A } \\
\text { GAS FLOW }\end{array}$ & 1 & COL A FLOW & $\Delta$ & \\
\hline G.7.4. & $\begin{array}{l}\text { REPLACE COL } \\
\text { A CAP }\end{array}$ & $N / A$ & $N / A$ & $\mathbf{A}$ & \\
\hline $\mathrm{Cx}^{1.7 .4}$ & $\begin{array}{l}\text { VERIFY COL B } \\
\text { GAS FLOW }\end{array}$ & $\angle$ & COL B FLOW & 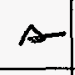 & \\
\hline $\begin{array}{l}1.7 .4 .5 .6 \\
\end{array}$ & $\begin{array}{l}\text { REPLACE COL } \\
\text { B CAP }\end{array}$ & $N / A$ & $N / A$ & & \\
\hline $\mathrm{ing}^{1.7 .4 .5 .7}$ & $\begin{array}{l}\text { VERIFY } \\
\text { SAMPLE VENT } \\
\text { FLOW }\end{array}$ & $\swarrow$ & $\begin{array}{l}\text { SAMPLE VENT } \\
\text { FLOW }\end{array}$ & $\Delta$ & \\
\hline $\begin{array}{l}1.7 .4 .5 .8 \\
\text { ges. }\end{array}$ & $\begin{array}{l}\text { REPLACE } \\
\text { SAMPLE VENT } \\
\text { CAP }\end{array}$ & $N / A$ & $N / A$ & $A$ & \\
\hline 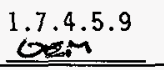 & $\begin{array}{l}\text { INSTALL } \\
\text { JUMPER } \\
\end{array}$ & $N / A$ & $N / A$ & $A$ & \\
\hline $\begin{array}{l}1.7 .4 .5 .10 \\
\cos ^{2}\end{array}$ & $\begin{array}{l}\text { CLOSE FU-15 } \\
\text { VERIFY FLOW }\end{array}$ & 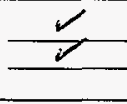 & $\begin{array}{l}\mathrm{H}_{2} \text { CAL FLOW } \\
\text { SAMP A IN } \\
\text { FLOW }\end{array}$ & $A$ & \\
\hline$\underbrace{1.7 .4 .5 .11}$ & $\begin{array}{l}\text { INSTALL PLUG } \\
\& \text { CAP }\end{array}$ & $\frac{1}{L}$ & $\begin{array}{l}\text { PLUG } \mathrm{H}_{2} \text { CAL } \\
\text { CAP SAMP A }\end{array}$ & $A$ & \\
\hline 1.7 .4 .5 .12 & $\begin{array}{l}\text { OPEN SV-*63 } \\
\& \text { READ } \\
\text { PDIT }-^{\star} 60\end{array}$ & 135.1 & $\begin{array}{l}\text { PDIT-*60 = } \\
28 \times \text { PRESS } \\
1.7 .4 .5 .1 \\
\pm 20 " \mathrm{H}_{2} \mathrm{O}\end{array}$ & $\Lambda$ & \\
\hline $\begin{array}{l}1.7 .4 .5 .13 \\
6 \text {. }\end{array}$ & $\begin{array}{l}\text { OPEN SV-*64- } \\
\text { VERIFY FLOW }\end{array}$ & $\checkmark$ & $\begin{array}{l}\text { SAMP B IN } \\
\text { FLOW }\end{array}$ & $\Leftrightarrow$ & $N / \Lambda^{1}$ \\
\hline
\end{tabular}


TEST DATA SHEET

\begin{tabular}{|c|c|c|c|c|c|}
\hline Date: $\triangle M A$ & 97 & & SHMS Unit Number: & $M G 8$ & $17+$ \\
\hline $\begin{array}{l}\text { Title of Test: } \\
\text { Pneumatic }\end{array}$ & tems & & Test Equipment ar & $\mathrm{S} / \mathrm{N}$ or & Ho, \\
\hline $\begin{array}{l}\text { Test Performed } \\
\text { Seze Tas }\end{array}$ & terso-isect $P_{0}$ & 518 & & $1 / \Delta$ & \\
\hline $\begin{array}{l}\text { Procedure No. } \\
\text { Initial/Date }\end{array}$ & Item & value & $\begin{array}{l}\text { Required } \\
\text { Condition }\end{array}$ & $(A / R)$ & ment \\
\hline$\underbrace{1.74}_{1.7 .5}$ & $\begin{array}{l}\text { REMOVE TEST } \\
\text { GAS }\end{array}$ & $N / A$ & $N / A$ & $\Lambda$ & \\
\hline $\operatorname{lnm}^{1.7 .4 .5 .15}$ & $\begin{array}{l}\text { CLOSE SV }-* 63- \\
\& \text { SV }-* 64\end{array}$ & $N / A$ & $\mathrm{~N} / \mathrm{A}$ & $\Delta$ & \\
\hline $\begin{array}{l}1.7 .4 .5 .16 \\
\end{array}$ & $\begin{array}{l}\text { OPEN FU-15 } \\
\text { REMOVE } \\
\text { JUMPER }\end{array}$ & $N / A$ & $N / A$ & $\Lambda$ & \\
\hline $\mathrm{cos}^{1.7 .4 .5}$ & $\begin{array}{l}\text { INSTALL } \\
\text { SAMP A UNION }\end{array}$ & $N / A$ & $N / A$ & $A$ & \\
\hline $\begin{array}{l}1.7 .4 .5 .18 \\
\end{array}$ & $\begin{array}{l}\text { OPEN SV-*60 } \\
\text { VERIFY FLOW }\end{array}$ & & $\begin{array}{l}\text { FI-*60 } \\
\text { NO FLOW }\end{array}$ & $A$ & \\
\hline 1.7 .4 .5 .19 & $\begin{array}{l}\text { POWER SOV- } \\
* 60 \text {, VERIFY } \\
\text { NO FLOW }\end{array}$ & 1 & $\begin{array}{l}\text { FI-*60 } \\
\text { NO FLOW }\end{array}$ & $\triangle 1$ & \\
\hline $\begin{array}{l}1.7 .4 .5 .20 \\
\text { s.m. }\end{array}$ & $\begin{array}{l}\text { OPEN SV-*68- } \\
\text { ADJ SV-*67- }\end{array}$ & 50 & $\begin{array}{l}\mathrm{FI}-* 60 \\
50 \mathrm{CCM}\end{array}$ & $\Delta$ & \\
\hline$\underbrace{1.7 .4 .5 .21}$ & $\begin{array}{l}\text { DEENERGIZE } \\
\text { SOV }-* 60\end{array}$ & 2 & $\begin{array}{l}\text { FI-*60 FLOW } \\
\text { DECREASES }\end{array}$ & $\Delta$ & \\
\hline $\begin{array}{l}1.7 .4 .5 .22 \\
\text { S.2in }\end{array}$ & $\begin{array}{l}\text { CLOSE SV-*60 } \\
\& \text { SV-*68 }\end{array}$ & $N / A$ & N/A & $\Delta$ & \\
\hline gen & $\begin{array}{l}\text { REMOVE } \\
\text { SAMP A UNION }\end{array}$ & $N / A$ & $N / A$ & A & \\
\hline oinin $^{1.7 .4 .5 .24}$ & $\begin{array}{l}\text { CONNECT TEST } \\
\text { GAS } N_{2} O \text { PORT }\end{array}$ & 5 & $\begin{array}{l}\text { TEST GAS } \\
3-5 \text { PSIG }\end{array}$ & $\Delta$ & \\
\hline 1.7 .4 .5 .25 & $\begin{array}{l}\text { VERIFY NO } \\
\text { FLOW }\end{array}$ & $\frac{1}{2}$ & $\begin{array}{l}\text { SAMP B IN \& } \\
\text { FLT-*63 } \\
\text { NO FLOW }\end{array}$ & $\Delta$ & \\
\hline $\begin{array}{l}1.7 .4 .5 .26 \\
\end{array}$ & $\begin{array}{l}\text { INSTALL } \\
\text { JUMPER }\end{array}$ & $N / A$ & N/A & $A$ & \\
\hline $\begin{array}{l}1.7 .4 .5 .27 \\
\cos \end{array}$ & $\begin{array}{l}\text { CLOSE FU-15 } \\
\text { VERIFY FLOW }\end{array}$ & $\frac{1}{2}$ & $\begin{array}{l}\text { SAMP B IN } \\
\text { FLT-*63 } \\
\text { FLOW }\end{array}$ & $\Delta$ & \\
\hline
\end{tabular}


TEST DATA SHEET

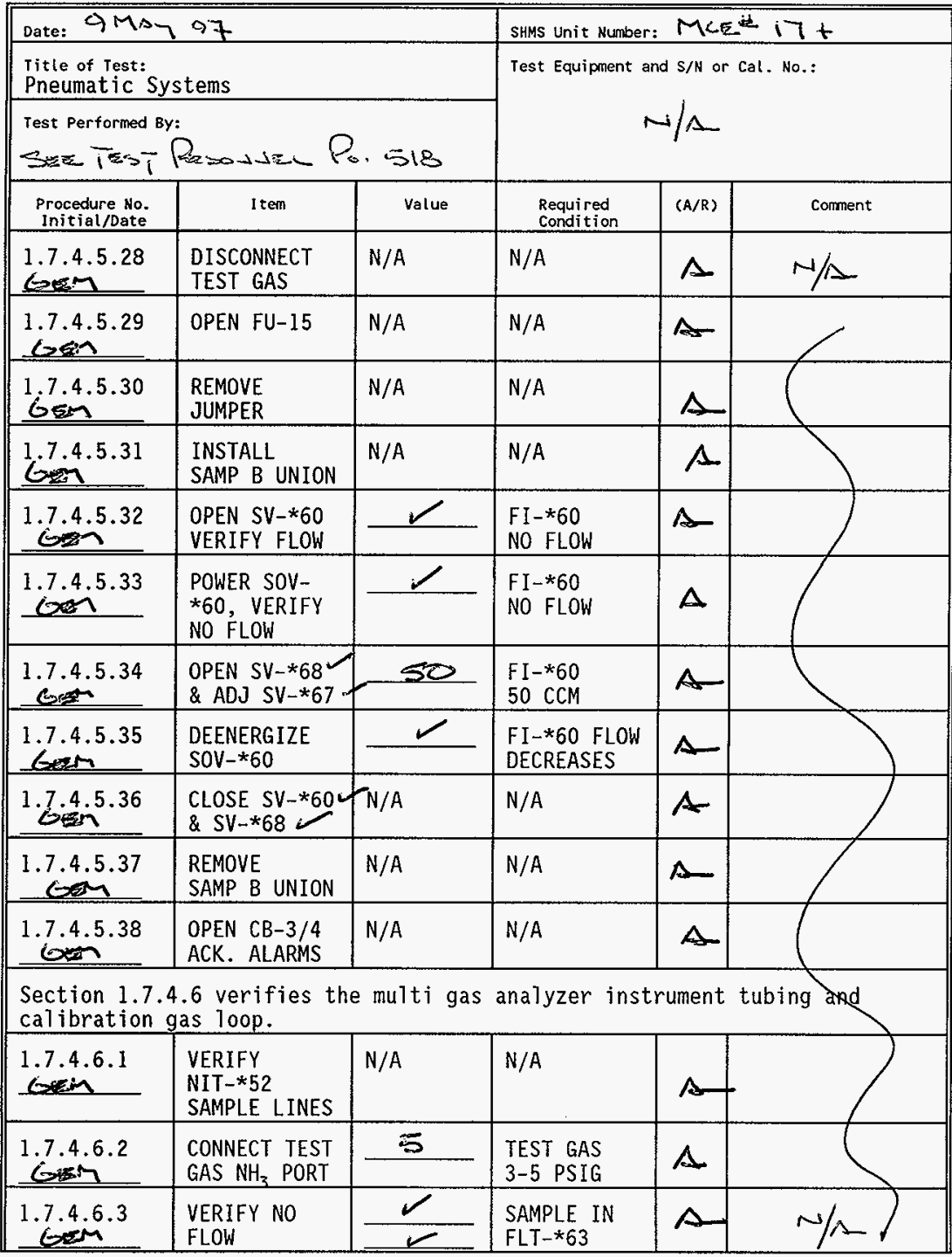


APPENDIX Q

PAGE Q-ZZ

TEST DATA SHEET

\begin{tabular}{|c|c|c|c|c|c|}
\hline Date: $\triangle M$ & 497 & & SHMS Unit Number & $M C$ & $17+$ \\
\hline $\begin{array}{l}\text { ritle of rest: } \\
\text { Pneumatic } S\end{array}$ & tems & & Test Equipment a & $\mathrm{S} / \mathrm{N}$ or & No.: \\
\hline $\begin{array}{l}\text { Test Performed } \\
\text { See } T T^{2}-T\end{array}$ & zoseste- Po. & \pm 19 & & $/ \Delta$ & \\
\hline $\begin{array}{l}\text { Procedure No. } \\
\text { Initial/Date }\end{array}$ & I tem & value & $\begin{array}{l}\text { Required } \\
\text { Condition }\end{array}$ & $(A / R)$ & corment \\
\hline $0^{1.7 .4 .6 .4}$ & $\begin{array}{l}\text { INSTALL } \\
\text { JUMPER } \\
\end{array}$ & $N / A$ & N/A & A. & \\
\hline$i^{7.4 .6 .5}$ & $\begin{array}{l}\text { CLOSE FU-15 } \\
\text { VERIFY GAS } \\
\text { FLOWS }\end{array}$ & 2 & $\begin{array}{l}\text { SAMPLE IN } \\
\text { FLT-*63 } \\
\text { FIV-*70 }\end{array}$ & $\Lambda$ & \\
\hline 1.7 .4 .6 .6 & $\begin{array}{l}\text { PLUG FLT-*63 } \\
\& \text { MON-*60 } \\
\text { SAMPLE VENT }\end{array}$ & N/A & $\mathrm{N} / \mathrm{A}$ & $A$ & \\
\hline 1.7.4.6.7 & $\begin{array}{l}\text { OPEN SV-*66, } \\
\text { SV-*70 \& CAP } \\
\text { SAMPLE IN }\end{array}$ & 141.3 & $\begin{array}{l}\text { PDIT }-* 60= \\
28 \times \text { PRESS } \\
1.7 .4 .6 .2 \\
\pm 20 \quad \mathrm{H}_{3} \mathrm{O}\end{array}$ & $A$ & \\
\hline 1.7 .4 .6 .8 & $\begin{array}{l}\text { CLOSE SV-*66- } \\
\text { SV -*70\&. } \\
\text { REMOVE CAP }\end{array}$ & $N / A$ & $N / A$ & $\Delta$ & \\
\hline $\begin{array}{l}1.7 .4 .6 .9 \\
\text { cosin }\end{array}$ & $\begin{array}{l}\text { REMOVE FLT- } \\
* 63 \text { PLUG }\end{array}$ & $N / A$ & $N / A$ & $\Lambda$ & \\
\hline 1.7 .4 .6 .10 & $\begin{array}{l}\text { REMOVE TEST } \\
\text { GAS }\end{array}$ & N/A & $\mathrm{N} / \mathrm{A}$ & $A$ & \\
\hline 1.7.4.6.11 & $\begin{array}{l}\text { CLOSE SV-*05 } \\
\& \text { SV-*16 }\end{array}$ & N/A & N/A & 1 & \\
\hline $\begin{array}{l}1.7 .4 .6 .12 \\
6 \mathrm{i}^{2}\end{array}$ & $\begin{array}{l}\text { CONNECT TEST } \\
\text { GAS }\end{array}$ & 4 & $\begin{array}{l}\text { TEST GAS } \\
2-4 \text { PSIG }\end{array}$ & $A$ & \\
\hline$\frac{1.7 .4 .6 .13}{6 \operatorname{con}^{-1}}$ & $\begin{array}{l}\text { OPEN SV }-* 07- \\
\text { \& VERIFY } \\
\text { NO FLOW } \\
\end{array}$ & 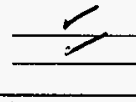 & $\begin{array}{l}\text { SAMPLE IN } \\
\text { SAMPLE OUT }\end{array}$ & $\Delta$ & \\
\hline $\sin ^{1.7 .6 .14}$ & $\begin{array}{l}\text { OPEN SV-*09 } \\
\text { \& VERIFY } \\
\text { NO FLOW }\end{array}$ & 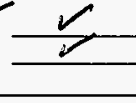 & $\begin{array}{l}\text { SAMPLE IN } \\
\text { SAMPLE OUT }\end{array}$ & $\Delta$ & \\
\hline$\frac{1.7 .4 .6 .15}{2.7}$ & $\begin{array}{l}\text { OPEN FU-15 \& } \\
\text { VERIFY FLOW }\end{array}$ & 2 & $\begin{array}{l}\text { SAMPLE FLOW } \\
\text { IN - YES } \\
\text { OUT - NO } \\
\end{array}$ & $\Lambda$ & \\
\hline $\begin{array}{l}1.7 .4 .6 .16 \\
\end{array}$ & $\begin{array}{l}\text { CLOSE SV-*09- } \\
\text { VERIFY FLOW }\end{array}$ & ' & $\begin{array}{l}\text { SAMPLE IN } \\
\text { NO FLOW }\end{array}$ & 1 & $b$ \\
\hline
\end{tabular}


TEST DATA SHEET

\begin{tabular}{|c|c|c|c|c|c|}
\hline \multicolumn{3}{|c|}{ Date: 9 Msy 97} & \multicolumn{3}{|c|}{ SHMS Unit Number: MCE $17+$} \\
\hline \multicolumn{3}{|c|}{$\begin{array}{l}\text { Title of rest: } \\
\text { Pneumatic Systems }\end{array}$} & \multirow{2}{*}{\multicolumn{3}{|c|}{ Test Equipment and $\mathrm{S} / \mathrm{N}$ or Cal. No.: }} \\
\hline \multicolumn{3}{|c|}{ 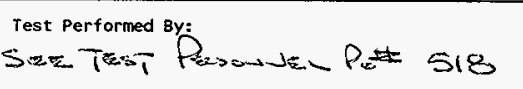 } & & & \\
\hline $\begin{array}{l}\text { Procedure No. } \\
\text { Initial/Date }\end{array}$ & Item & value & $\begin{array}{l}\text { Required } \\
\text { Condition }\end{array}$ & $(A / R)$ & Comment \\
\hline$\frac{1.7 .4 .6 .17}{\cos }$ & $\begin{array}{l}\text { OPEN SV }-* 10 \\
\text { VERIFY FLOW }\end{array}$ & & $\begin{array}{l}\text { SAMPLE FLOW } \\
\text { IN - NO } \\
\text { OUT - YES } \\
\end{array}$ & 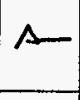 & \\
\hline $\begin{array}{l}1.7 .4 .6 .18 \\
8 \mathrm{E}^{-18}\end{array}$ & $\begin{array}{l}\text { REMOVE TEST } \\
\text { GAS \& PLUG } \\
\text { SV-*07 }\end{array}$ & $N / A$ & N/A & $\triangle$ & \\
\hline $\begin{array}{c}1.7 .4 .6 .19 \\
\cos \\
\end{array}$ & $\begin{array}{l}\text { CLOSE SV-*07 } \\
\& \text { SV-*10 }\end{array}$ & $\pi / A$ & $\mathrm{~N} / \mathrm{A}$ & $\Lambda$ & \\
\hline $\begin{array}{r}1.7 .4 .6 .20 \\
620 \\
\end{array}$ & $\begin{array}{l}\text { OPEN SV-*05 } \\
\& \text { SV-*16. }\end{array}$ & N/A & N/A & $A$ & \\
\hline $\begin{array}{l}1.7 .4 .6 .21 \\
6 \log \end{array}$ & $\begin{array}{l}\text { REMOVE } \\
\text { JUMPER }\end{array}$ & $N / A$ & N/A & $\Lambda$ & \\
\hline \multicolumn{6}{|c|}{ Section 1.7.4.7 verifies the carrier gas supply lines. } \\
\hline $\begin{array}{l}1.7 .4 .7 .1 \\
\cos \end{array}$ & $\begin{array}{l}\text { CONNECT TEST } \\
\text { GAS }\end{array}$ & 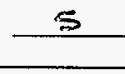 & 3-5 PSIG & $A$ & \\
\hline 1.7 .4 .7 .2 & $\begin{array}{l}\text { REMOVE PLUG } \\
\text { \& VERIFY } \\
\text { FLOW } \\
\end{array}$ & 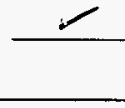 & $\begin{array}{l}\mathrm{N}_{2} \text { CARRIER } \\
\text { GAS FLOWS }\end{array}$ & A & \\
\hline $\begin{array}{l}1.7 .4 .7 .3 \\
6.7 .7\end{array}$ & $\begin{array}{l}\text { DISCONNECT } \\
\text { TEST GAS } \\
\end{array}$ & N/A & N/A & A & \\
\hline$\underline{0.7 .4 .4}$ & $\begin{array}{l}\text { CONNECT TEST } \\
\text { GAS }\end{array}$ & 5 & 3-5 PSIG & $A$ & \\
\hline $\begin{array}{l}1.7 .4 .7 .5 \\
\operatorname{sen}^{-1}\end{array}$ & $\begin{array}{l}\text { REMOVE PLUG } \\
\& \text { VERIFY } \\
\text { FLOW }\end{array}$ & 2 & $\begin{array}{l}\text { He CARRIER } \\
\text { GAS FLOWS }\end{array}$ & 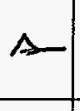 & \\
\hline $\begin{array}{l}1.7 .4 .7 .6 \\
\text { isis. }\end{array}$ & $\begin{array}{l}\text { DISCONNECT } \\
\text { TEST GAS } \\
\end{array}$ & $N / A$ & $N / A$ & $A$ & 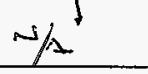 \\
\hline 1.7 .5 & $\begin{array}{l}\text { TEST SEC. } \\
1.7 .4 \text { DONE }\end{array}$ & \multicolumn{4}{|c|}{$\begin{array}{cc}\text { 9r7and of Bner } & 5-19-97 \\
\text { Test Director Signature } & \text { Date }\end{array}$} \\
\hline
\end{tabular}


TEST DATA SHEET

\begin{tabular}{|c|c|c|c|c|c|}
\hline \multicolumn{3}{|c|}{ Date: is MAY 97} & \multicolumn{3}{|c|}{ SHMS Unit Number: MCE $17 t$} \\
\hline \multicolumn{3}{|c|}{$\begin{array}{l}\text { Title of Test: } \\
\text { Pneumatic Systems }\end{array}$} & \multirow{2}{*}{\multicolumn{3}{|c|}{ Test Equipment and $S / \mathrm{N}$ or Cal. No.: }} \\
\hline \multicolumn{3}{|c|}{ 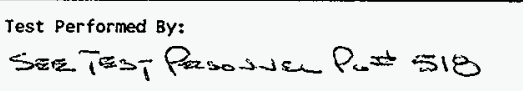 } & & & \\
\hline $\begin{array}{c}\text { Procedure No. } \\
\text { Initial/Date } \\
\end{array}$ & Item & Value & $\begin{array}{l}\text { Required } \\
\text { Condition }\end{array}$ & $(A / R)$ & Comment \\
\hline $\begin{array}{ll}1.7 .6 \\
62+7\end{array}$ & \multicolumn{5}{|c|}{$\begin{array}{l}\text { THIS SECTION IS ONLY FOR THE SHMS-E IN THE ANALYTICAL MODE. } \\
\text { IF NOT APPLICABLE, N/A STEPS AND TEST DIRECTOR WILL SIGN. }\end{array}$} \\
\hline & \multicolumn{3}{|c|}{ Test Director Signature } & Date & \\
\hline 1.7 .6 .1 .1 & $\begin{array}{l}\text { VERIFY BKR } \\
\text { LINE UP }\end{array}$ & $N / A$ & N/A & $\therefore$ & $\mathrm{N} / \mathrm{\Delta}$ \\
\hline $\cos ^{1.7 .6 .1 .2}$ & $\begin{array}{l}\text { VERIFY VALVE } \\
\text { LINE UP }\end{array}$ & $N / A$ & $N / A$ & $\Delta$ & \\
\hline$\frac{1.7 .6 .1 .3}{6 x^{2}}$ & $\begin{array}{l}\text { VERIFY MON- } \\
* 60 \text { INSTALL }\end{array}$ & $N / A$ & $N / A$ & A & \\
\hline $\begin{array}{l}1.7 .6 .1 .4 \\
\end{array}$ & $\begin{array}{l}\text { VERIFY NIT- } \\
\star 52 \text { INSTALL } \\
\end{array}$ & $N / A$ & $N / A$ & $\Delta$ & \\
\hline 1.7 .6 .1 .5 & $\begin{array}{l}\text { CONNECT } \mathrm{H}_{2} \\
\text { CAL GAS }\end{array}$ & $N / A$ & $N / A$ & $A$ & \\
\hline $\operatorname{sen}^{1.7 .6 .1 .6}$ & $\begin{array}{l}\text { CONNECT } \mathrm{CH}_{4} \\
\text { CAL GAS }\end{array}$ & $N / A$ & N/A & $A$ & \\
\hline $\begin{array}{ll}1.7 .6 .1 .7 \\
\end{array}$ & $\begin{array}{l}\text { CONNECT NH } \\
\text { CAL GAS }\end{array}$ & $N / A$ & N/A & A & \\
\hline \multicolumn{6}{|c|}{ Section 1.7.6.2 establishes the main and auxiliary loop flows. } \\
\hline $\begin{array}{l}1.7 .6 .2 .1 \\
6 \cos \\
\end{array}$ & CLOSE FU-11 & $<$ & $\begin{array}{l}\text { MON-*60 } \\
\text { POWERED }\end{array}$ & $\Delta$ & \\
\hline $\operatorname{com}^{1.7 .6 .2 .2}$ & POWER PC-*60 & l & $\begin{array}{l}\text { ESTABLISH } \\
\text { SAMPLING } \\
\text { ROUTINE }\end{array}$ & A & \\
\hline $\begin{array}{l}1.7 .6 .2 .3 \\
\end{array}$ & CLOSE FU-12 & 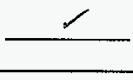 & $\begin{array}{l}\text { NIT-*52 } \\
\text { POWERED } \\
\end{array}$ & $A$ & \\
\hline $\begin{array}{l}1.7 .6 .2 .4 \\
\end{array}$ & POWER PC-*70 & $\checkmark$ & $\begin{array}{l}\text { PC-*70 } \\
\text { BOOTED UP }\end{array}$ & $A$ & \\
\hline $\begin{array}{l}1.7 .6 .2 .5 \\
\text { Gen }\end{array}$ & $\begin{array}{l}\text { CLOSE CB-3/4 } \\
\text { ADJUST FLOW }\end{array}$ & 1.08 & $\begin{array}{l}\mathrm{FIT}-* 57 \\
1 \pm .1 \mathrm{H} \mathrm{H}_{2} \mathrm{O}\end{array}$ & $A$ & \\
\hline
\end{tabular}

wer su 515197 
APPENDIX Q

TEST DATA SHEET

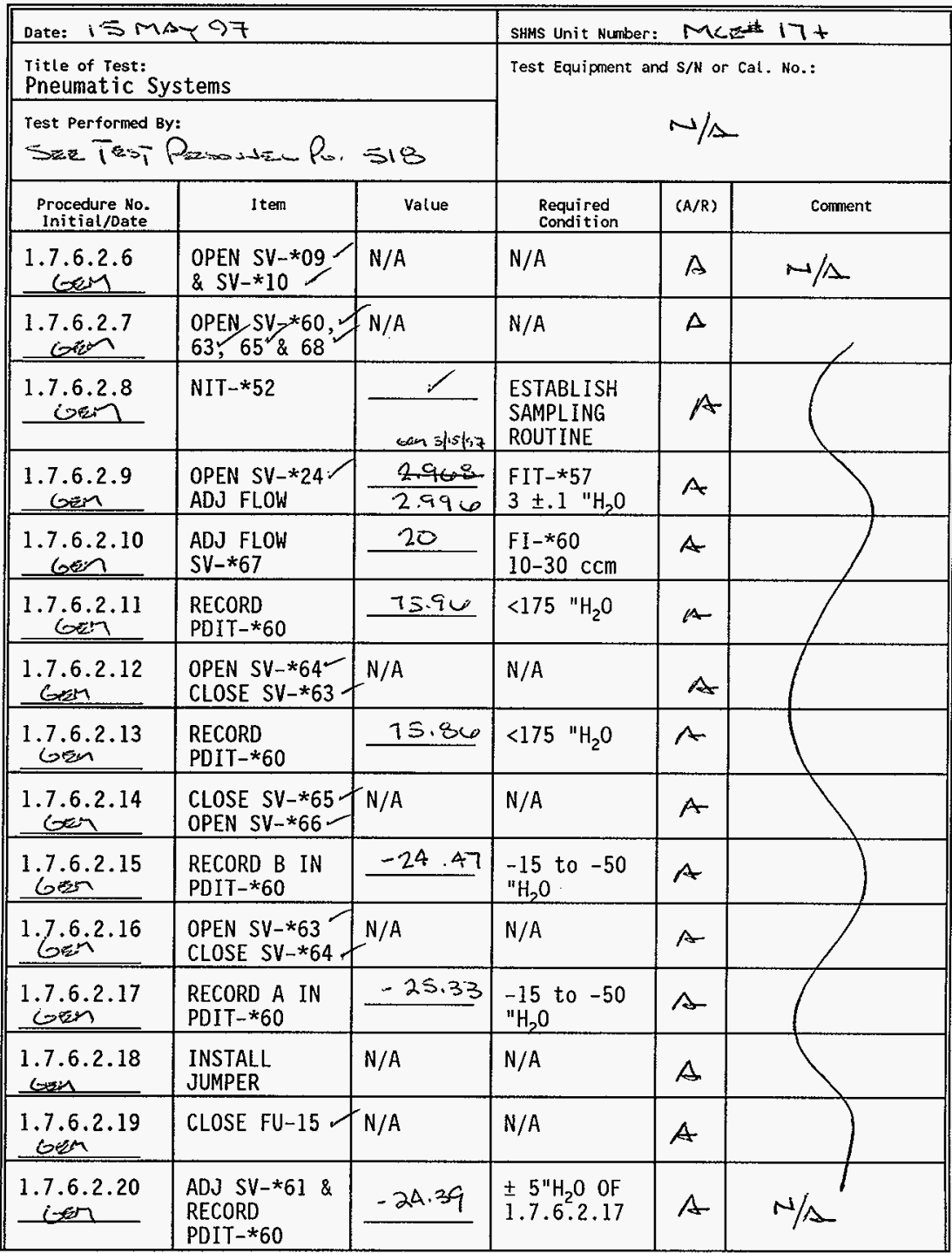


TEST DATA SHEET

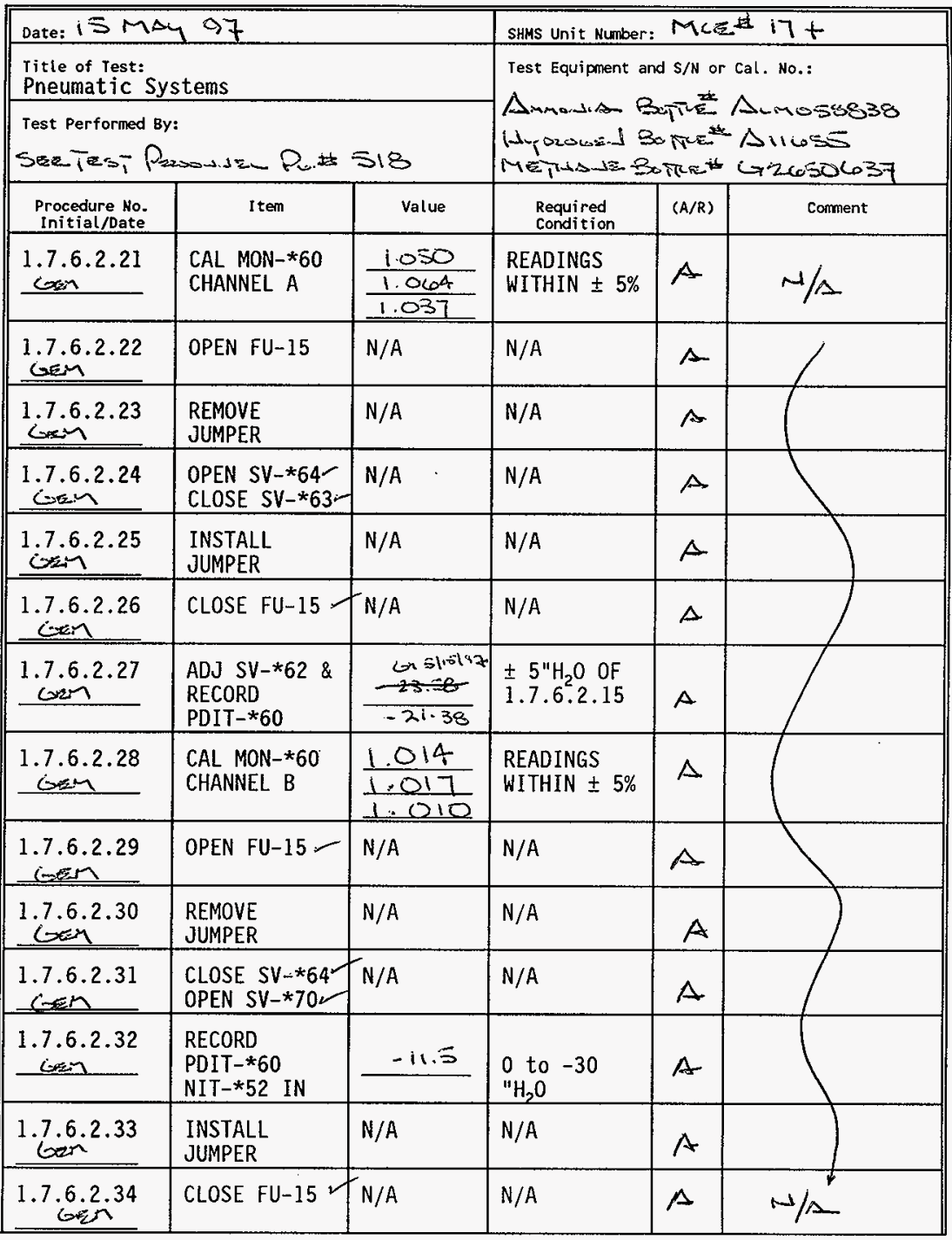


APPENDIX Q

TEST DATA SHEET

\begin{tabular}{|c|c|c|c|c|c|}
\hline \multicolumn{3}{|c|}{ Date: 15 MAY 97} & \multicolumn{3}{|c|}{ SHMS Unit Number: MCE $17+$} \\
\hline \multicolumn{3}{|c|}{$\begin{array}{l}\text { Title of Test: } \\
\text { Pneumatic Systems }\end{array}$} & \multirow{2}{*}{\multicolumn{3}{|c|}{ 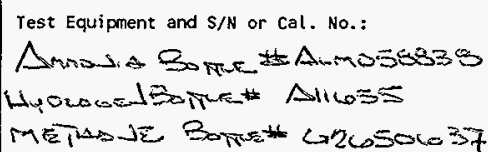 }} \\
\hline \multicolumn{2}{|c|}{ 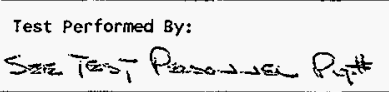 } & \multirow{2}{*}{$\frac{518}{\text { value }}$} & & & \\
\hline $\begin{array}{l}\text { Procedure No. } \\
\text { Initial/Date }\end{array}$ & Iten & & $\begin{array}{l}\text { Required } \\
\text { Condition } \\
\end{array}$ & $(\mathrm{A} / \mathrm{R})$ & Comment \\
\hline $\begin{array}{l}1.7 .6 .2 .35 \\
6 \text { Sin }^{2} \\
\end{array}$ & $\begin{array}{l}\text { ESTABLISH } \\
\text { BYPASS FLOW }\end{array}$ & 1.3 & $\begin{array}{l}\text { FIV }-* 70 \\
1.5 \pm .2 \mathrm{cfh}\end{array}$ & $A$ & \\
\hline$\frac{1.7 .6 .2 .36}{6 e^{24}}$ & $\begin{array}{l}\text { ADJ SV-*71 } \\
\text { NIT-*52 } \\
\text { SAMPLE IN }\end{array}$ & $\frac{-14.300}{60131.5620}$ & $\begin{array}{l} \pm 5 \quad \mathrm{H}_{2} \mathrm{O} \quad \mathrm{OF} \\
1.7 .6 .2 .32\end{array}$ & $A$ & \\
\hline $\begin{array}{c}1.7 .6 .2 .37 \\
0.74 \\
\end{array}$ & $\begin{array}{l}\text { RECORD } \\
\text { NIT }-* 52 \\
\text { SAMPLES }\end{array}$ & $\frac{40.39 .0}{\frac{39.7}{40.4}}$ & $\begin{array}{l}\text { READINGS } \\
\text { WITHIN } \pm 5 \%\end{array}$ & $A$ & \\
\hline $\begin{array}{l}1.7 .6 .2 .38 \\
\cos \end{array}$ & OPEN FU-15 & N/A & $N / A$ & A & \\
\hline 1.7 .6 .2 .39 & $\begin{array}{l}\text { REMOVE } \\
\text { JUMPER } \\
\end{array}$ & N/A & $N / A$ & A & \\
\hline \multicolumn{6}{|c|}{ Section 1.7.7 provides for test system shutdown. } \\
\hline $\begin{array}{c}1.7 .7 .1 \\
0.5 \\
\end{array}$ & $\begin{array}{l}\text { DISCONNECT } \\
\mathrm{H}_{2} \text { CAL GAS }\end{array}$ & $N / A$ & $N / A$ & $\Delta$ & \\
\hline $\begin{array}{c}1.7 .7 .2 \\
\end{array}$ & $\begin{array}{l}\text { DISCONNECT } \\
\mathrm{CH}_{G} \text { CAL GAS }\end{array}$ & $N / A$ & N/A & $\Delta$ & \\
\hline $\begin{array}{l}1.7 .7 .3 \\
\mathrm{sin}\end{array}$ & $\begin{array}{l}\text { DISCONNECT } \\
\mathrm{NH}_{3} \text { CAL GAS }\end{array}$ & $N / A$ & $N / A$ & $A$ & \\
\hline $\begin{array}{l}1.7 .7 .4 \\
\text { sin }\end{array}$ & $\begin{array}{l}\text { SECURE \& } \\
\text { TURN OFF } \\
\text { ANA. INST.S }\end{array}$ & N/A & $N / A$ & $\Lambda$ & \\
\hline $\begin{array}{c}1.7 .7 .5 \\
\end{array}$ & $\begin{array}{l}\text { DISCONNECT } \\
\mathrm{N}_{2} \text { CARRIER }\end{array}$ & N/A & $N / A$ & $\Delta$ & \\
\hline $\begin{array}{l}1.7 .7 .6 \\
\text { - }\end{array}$ & $\begin{array}{l}\text { DISCONNECT } \\
\text { He CARRIER }\end{array}$ & N/A & $N / A$ & $\Delta$ & \\
\hline $\begin{array}{l}1.7 .7 .7 \\
\cos \end{array}$ & $\begin{array}{l}\text { OPEN ALL } \\
\text { BREAKERS \& } \\
\text { FUSES }\end{array}$ & $N / A$ & N/A & 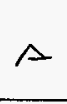 & \\
\hline $\begin{array}{l}1.7 .7 .8 \\
6.7 \\
\end{array}$ & $\begin{array}{l}\text { CLOSE LISTED } \\
\text { SYS. VALVES }\end{array}$ & $N / A$ & N/A & $\Delta$ & \\
\hline
\end{tabular}


APPENDIX Q

PAGE Q-28

$$
\begin{array}{r}
\text { HNF-SD-WM-ATR-191 } \\
\text { Rev. } 0 \\
\text { Page } 544
\end{array}
$$

TEST DATA SHEET

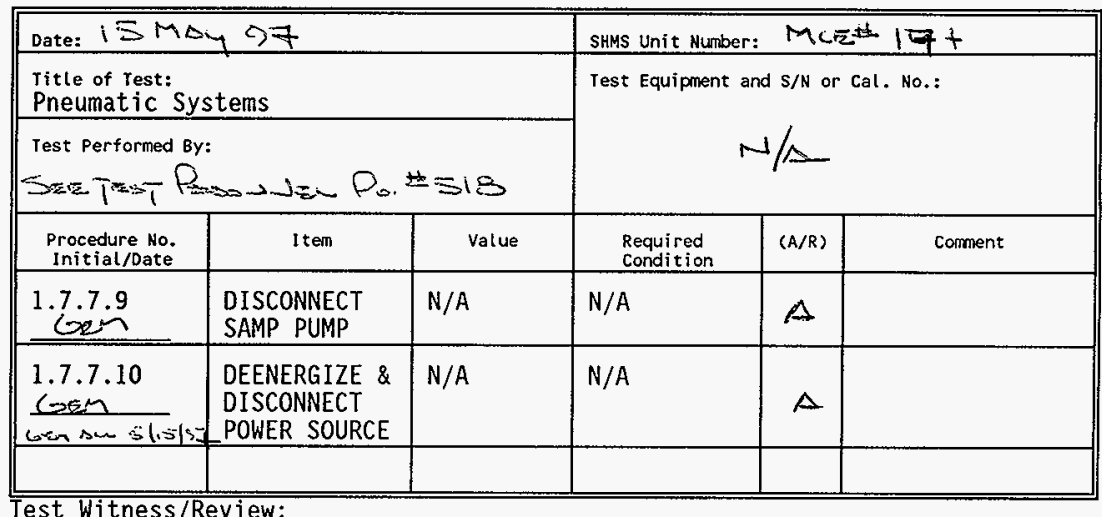

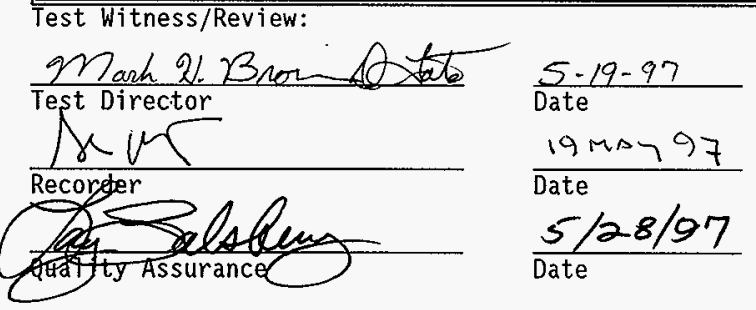


APPENDIX $Q$

HNF-SD-WM-ATR- 191

PAGE Q-29

Rev.
Page 545

TEST EXCEPTION SHEET

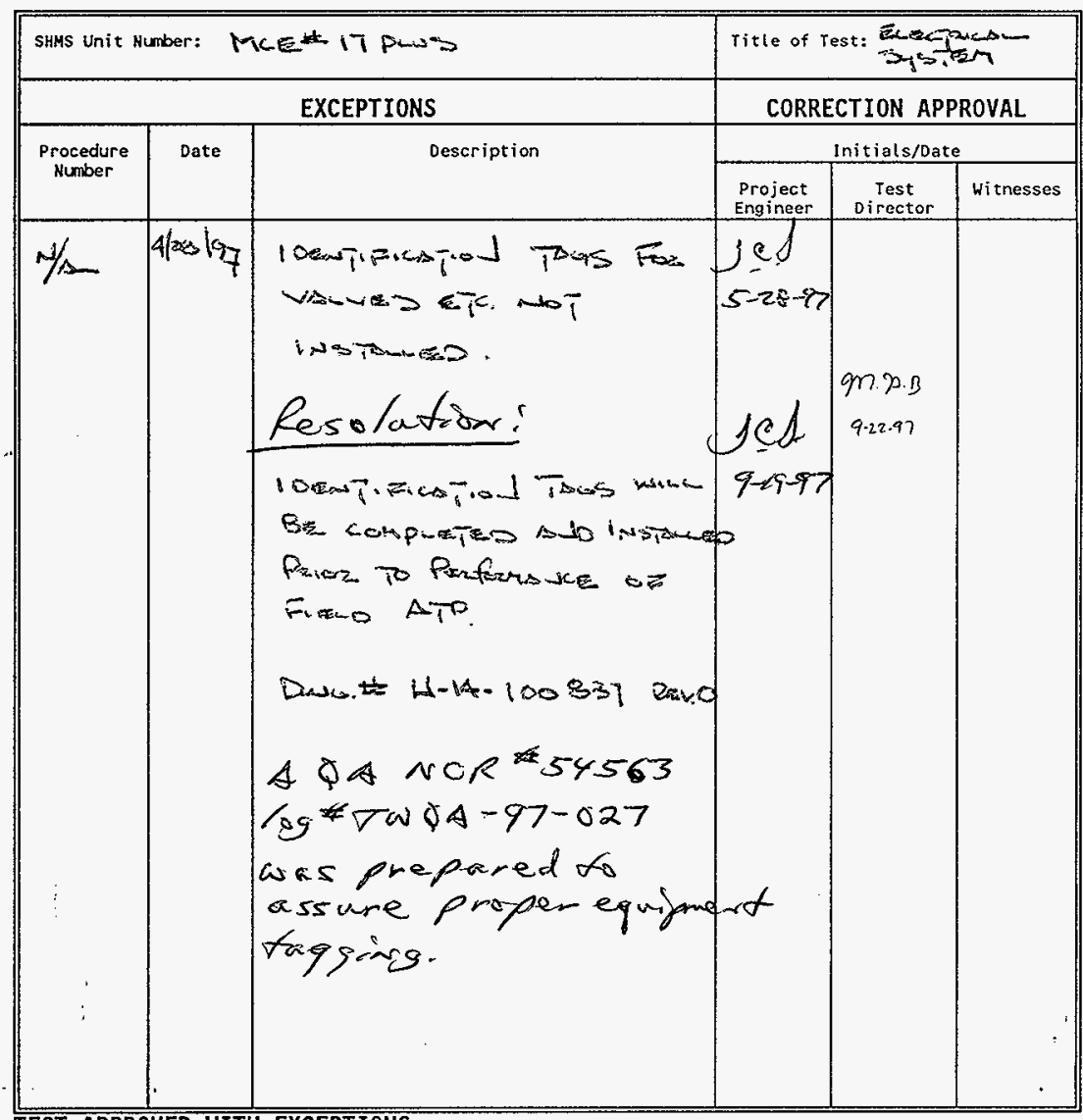

TEST APPROVED WITH EXCEPTIONS

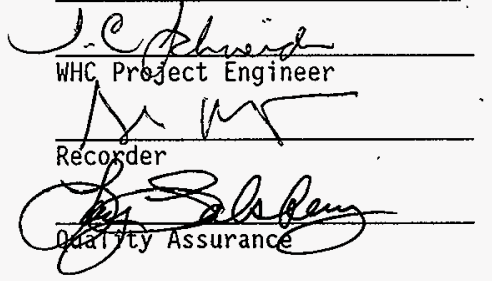

$$
\begin{aligned}
& \frac{5-28-77}{\text { Date }} \\
& \frac{23 \mathrm{Date}}{5 / 28 / 97} \\
& \text { Date }
\end{aligned}
$$


APPENDIX Q

PAGE Q-30
HNF-SD-WIK-ATR-191

Rev. 0
Page 546

TEST LOG

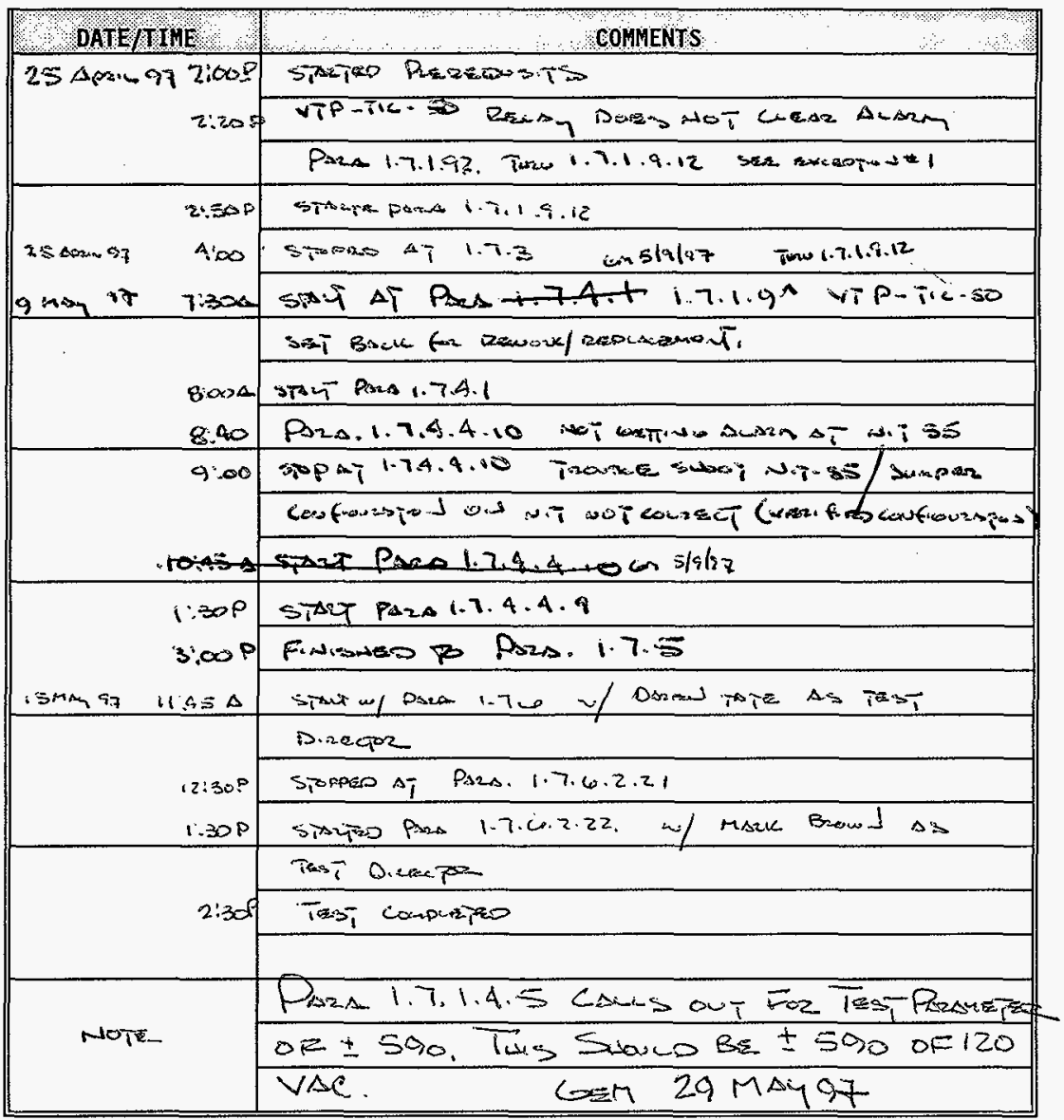




\begin{tabular}{|c|c|c|c|c|c|}
\hline \multicolumn{6}{|c|}{ DISTRIBUTION SHEET } \\
\hline To & \multirow{2}{*}{\multicolumn{3}{|c|}{$\begin{array}{l}\text { From } \\
\text { Mechanical Systems Engineering }\end{array}$}} & \multicolumn{2}{|l|}{ Page 1 of 1} \\
\hline DISTRIBUTION & & & & \multicolumn{2}{|c|}{ Date Sept. 26,1997} \\
\hline \multirow{2}{*}{\multicolumn{4}{|c|}{$\begin{array}{l}\text { Project Title/Work Order } \\
94 \mathrm{C}-\mathrm{EWW}-369 \text {, Hydrogen Monitoring / N2022 }\end{array}$}} & \multirow{2}{*}{\multicolumn{2}{|c|}{$\begin{array}{ll}\text { EDT No. } & 600197 \\
\text { ECN No. } & N / A \\
\end{array}$}} \\
\hline & & & & & \\
\hline Name & MSIN & $\begin{array}{l}\text { Text } \\
\text { With All } \\
\text { Attach. }\end{array}$ & Text Only & $\begin{array}{l}\text { Attach./ } \\
\text { Appendix } \\
\text { Only }\end{array}$ & $\begin{array}{l}\text { EDT/ECN } \\
\text { Only }\end{array}$ \\
\hline $\begin{array}{l}\text { MH Brown } \\
\text { KE Carpenter } \\
\text { DW Crass } \\
\text { M Cremonini } \\
\text { MF Erhart } \\
\text { GD Johnson } \\
\text { RL Schlosser } \\
\text { TC Schneider } \\
\text { DD Tate } \\
\text { MC Tipps } \\
\text { JJ Verderber }\end{array}$ & $\begin{array}{l}S 2-56 \\
R 3-47 \\
R 3-25 \\
H 5-61 \\
R 1-51 \\
S 7-15 \\
R 1-56 \\
L 6-37 \\
L 6-37 \\
T 4-07 \\
S 5-421\end{array}$ & $x$ & & $\begin{array}{l}X \\
X \\
X \\
X \\
X\end{array}$ & \\
\hline Central Files & A3-88 & $x$ & & & \\
\hline
\end{tabular}

